PARABOLIC LAYER POTENTIALS AND

INITIAL BOUNDARY VALUE PROBLEMS

IN LIPSCHITZ CYLINDERS

WITH DATA IN BESOV SPACES

\begin{abstract}
A Dissertation
presented to

the Faculty of the Graduate School

University of Missouri-Columbia

In Partial Fulfillment

of the Requirements for the Degree

Doctor of Philosophy

by

TUNDE JAKAB

Dr. Marius Mitrea, Dissertation Supervisor
\end{abstract}

MAY 2006 
The undersigned, appointed by the Dean $\mathrm{f}$ the Graduate School, have examined the dissertation entitled

\author{
PARABOLIC LAYER POTENTIALS AND \\ INITIAL BOUNDARY VALUE PROBLEMS
}

IN LIPSCHITZ CYLINDERS

WITH DATA IN BESOV SPACES

Presented by Tunde Jakab

A candidate for the degree of Doctor of Philosophy

And hereby certify that in their opinion it is worthy of acceptance.

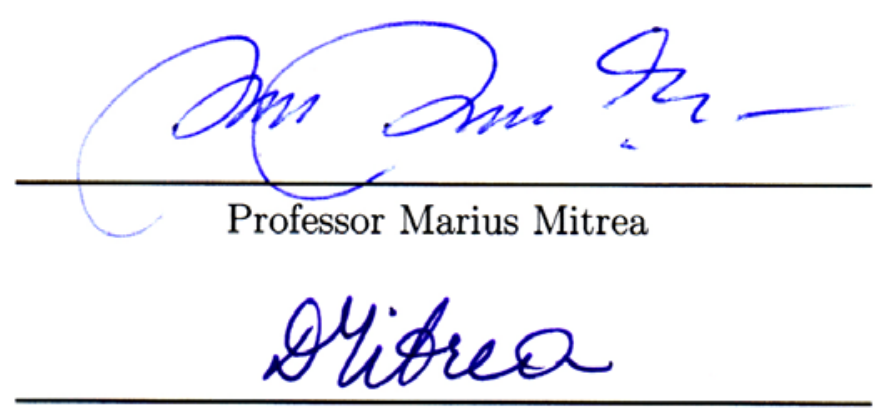

Professor Dorina Mitrea

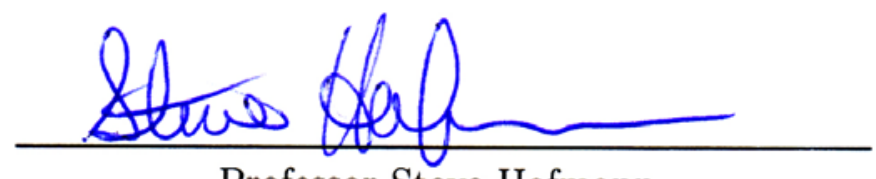

Professor Steve Hofmann

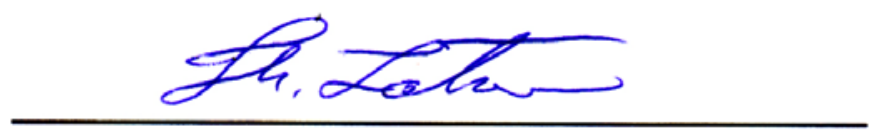

Professor Yuri Latushkin

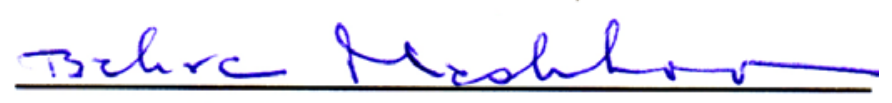

Professor Bahram Mashhoon 


\section{ACKNOWLEDGEMENTS}

Over the past few years I had the privilege to work and study under the guidance of Professor Marius Mitrea. It is a distinct pleasure to express my sincere thanks and deepest appreciation to him for not only sharing his knowledge, but his invaluable insights, motivation, encouragement, and constant support, as well. Benefiting from his expertise, professional advice, and learning from the way he handled mathematical issues has been a decisive experience for me.

I would also like to express my gratitude to the members of my doctoral committee, Professor Steve Hofmann, Professor Dorina Mitrea, Professor Yuri Latushkin from the Mathematics Department, as well as Professor Bahram Mashhoon and Professor Brian DeFacio from the Department of Physics and Astronomy. Special thanks go to Professor Dorina Mitrea, who sparked my interest in partial differential equations with her expertise and enthusiasm while teaching graduate PDE classes.

During my graduate studies I had constant support and encouragement from my husband and parents. Their patience, understanding and belief in me have greatly contributed to the completion of this dissertation. 


\section{Contents}

$\begin{array}{ll}\text { Acknowledgements } & \text { ii }\end{array}$

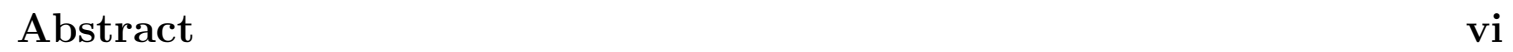

1 Introduction 1

1.1 History and description of main results . . . . . . . . . . . 1

1.2 Basic definitions, notation and preliminary results . . . . . . . 12

1.2.1 Notation and basic conventions . . . . . . . . . . . . 12

1.2.2 Lipschitz domain . . . . . . . . . . . . . . . 14

1.2.3 Estimates for the caloric fundamental solution . . . . . . . . 18

1.2.4 Function spaces . . . . . . . . . . . . . . . . 21

1.2.5 Review of Fourier transform . . . . . . . . . . . . . 26

1.2.6 Review of multipliers . . . . . . . . . . . . . . . . . . 29

1.2.7 Real and complex interpolation methods . . . . . . . . . . . 37

1.2.8 Parabolic sequence spaces _. . . . . . . . . . . . . . . 61

1.2.9 Envelopes of Hardy spaces . . . . . . . . . . . . . . . 66

2 Parabolic function spaces $\quad 76$ 
2.1 Euclidean setting . . . . . . . . . . . . . . . 76

2.1.1 Besov and Triebel-Lizorkin spaces . . . . . . . . . . . 76

2.1.2 Sobolev spaces . . . . . . . . . . . . . . 100

2.1.3 Hardy spaces . . . . . . . . . . . . . . . . . 113

2.1.4 Interpolation . . . . . . . . . . . . . . . 131

2.2 Spaces on Lipschitz cylinders . . . . . . . . . . . . . . . . . . . . . 140

2.3 Spaces defined on the lateral boundary . . . . . . . . . . . . . . 155

2.3.1 Atomic decompositions of Besov spaces . . . . . . . . . 186

2.3.2 Atomic decompositions of Hardy spaces . . . . . . . . . . . . . 189

2.4 Duality results . . . . . . . . . . . . . . . . . 193

2.5 Envelopes of parabolic Hardy spaces . . . . . . . . . . . . . . . 200

3 Spaces with built-in initial conditions 211

3.1 The interior of the cylinder . . . . . . . . . . . . . 211

3.2 The lateral side of the cylinder . . . . . . . . . . . . . . . . 238

3.3 Banach envelopes of Hardy spaces with built-in initial conditions . . . 249

3.4 Interpolation scales of spaces with built-in initial conditions . . . . . 260

4 Mapping properties of the double layer potential 286

4.1 Two basic estimates . . . . . . . . . . . . . . . 286

4.2 General case . . . . . . . . . . . . . . . . . 306

5 Mapping properties of the single layer potential 321

5.1 Basic estimates ......................... 321 
5.2 General case . . . . . . . . . . . . . . . . . 399

6 Invertibility of boundary potential operators 428

6.1 Case of an unbounded cylinder . . . . . . . . . . . . . . . . 430

6.2 Case of a bounded cylinder . . . . . . . . . . . . . . . . . 438

7 The boundary trace operator, the normal derivative and the Newtonian potential $\quad 462$

7.1 Mapping properties of the trace operator . . . . . . . . . . . . . 462

7.2 The normal derivative . . . . . . . . . . . . . . . . . 471

7.3 The caloric Newtonian potential . . . . . . . . . . . . . . . . . 484

8 Boundary value problems $\quad 504$

8.1 The Poisson problem with Dirichlet boundary conditions . . . . . . . 505

8.2 The Poisson problem with Neumann boundary conditions . . . . . . . 509

$\begin{array}{ll}\text { Bibliography } & 513\end{array}$

$\begin{array}{ll}\text { Index } & 527\end{array}$

$\begin{array}{ll}\text { Vita } & 527\end{array}$ 


\title{
PARABOLIC LAYER POTENTIALS AND INITIAL BOUNDARY VALUE PROBLEMS IN LIPSCHITZ CYLINDERS WITH DATA IN BESOV SPACES
}

\author{
Tunde Jakab
}

Dr. Marius Mitrea, Dissertation Supervisor

\begin{abstract}
We adapt the method of boundary layer potentials to the Poisson problem for the heat operator $\partial_{t}-\Delta$ in a bounded Lipschitz cylinder, with Dirichlet and Neumann boundary conditions. When the lateral datum has a fractional amount of smoothness measured at the Besov scale with parabolic anisotropy, the well-posedness of these problems is obtained in a constructive fashion. More specifically, the solution can be represented as a double layer potential in the Dirichlet case, and as a single layer potential in the Neumann case.

The main theorems we prove extend, generalize and bring together many earlier results, such as the work of E. Fabes and N. Rivière (1978) for $C^{1}$ domains; R. Brown (1990) for integer amount of smoothness; D. Jerison and C. Kenig (1995), and E. Fabes, O. Mendez and M. Mitrea (1998) who dealt with the case of the Laplacian.
\end{abstract}




\section{Chapter 1}

\section{Introduction}

\subsection{History and description of main results}

Due to the numerous applications of the theory of initial boundary value problems, they have been extensively studied over the past decades.

In 1968 E. Fabes and M. Jodeit considered parabolic boundary value problems in smooth cylinders, as well as Sobolev-Besov spaces exhibiting parabolic anisotropy. Using parabolic singular integral operators, they obtained the existence, uniqueness, and a priori estimates of the solutions of the homogeneous parabolic boundary value problem, with Dirichlet and Neumann type boundary conditions.

Considering the same problem with boundary datum from $L^{p}\left(\mathbb{R}^{n}\right)$, in 1972 E. Fabes utilized the a priori estimates to uniquely solve the boundary value problem.

In 1978 E. Fabes and N. Rivière ([FaRi]) considered the initial Dirichlet and Neumann problems for the heat operator in the cylindrical domain $\Omega \times(0, T)$ for a bounded $C^{1}$ domain $\Omega$ in $\mathbb{R}^{n}$. They constructed solutions to these problems satisfying the zero initial condition in the form of a caloric double layer potential in the Dirichlet case and a caloric single layer potential in the Neumann case. While the 
Neumann datum is taken from the Lebesgue space $L^{p}(\partial \Omega \times(0, T)), 1<p<\infty$, the Dirichlet datum $g$ is assumed to be either from $L^{p}(\partial \Omega \times(0, T)), 1<p<\infty$, or from the anisotropic Sobolev space $L_{1}^{p}(\partial \Omega \times(0, T))$, where the anisotropy is dictated by the parabolic nature of the heat operator. More precisely, $L_{1}^{p}(\partial \Omega \times(0, T))$ contains functions which belong to $L^{p}(\partial \Omega \times(0, T))$ together with their first-order spacial and "half-order" time derivatives. The authors also prove that, in the Dirichlet case, the solution $u$ satisfies the following estimates:

$$
\|\mathcal{N}(u)\|_{L^{p}(\partial \Omega \times(0, T))} \leq c\|g\|_{L^{p}(\partial \Omega \times(0, T))}
$$

and

$$
\|\mathcal{N}(\nabla u)\|_{L^{p}(\partial \Omega \times(0, T))}+\left\|\mathcal{N}\left(D_{\text {time }}^{1 / 2} u\right)\right\|_{L^{p}(\partial \Omega \times(0, T))} \leq c\|g\|_{L_{1}^{p}(\partial \Omega \times(0, T))}
$$

where $\mathcal{N}(u)(x, t):=\sup _{(y, s) \in \Gamma(x, t)}|u(y, s)|$ is the parabolic nontangential maximal function of $u$ at the boundary point $(x, t) \in \partial \Omega \times(0, T)$, and $\Gamma(x, t) \subset \Omega$ is a parabolic nontangential cone at $(x, t)$. Also, the family of fractional time-derivative operators are introduced as

$$
D_{\text {time }}^{\theta}:=\frac{\partial}{\partial t} I_{\text {time }}^{1-\theta}, \quad 0 \leq \theta \leq 1
$$

where, for $f \in L^{1}(-\infty, T)$ which decays fast enough at $-\infty$, and for $0<\theta \leq 1$, the fractional integral operator of order $\theta$ is defined by

$$
I_{\text {time }}^{\theta} f(t):=\frac{1}{\Gamma(\theta)} \int_{-\infty}^{t} \frac{f(s)}{(t-s)^{1-\theta}} d s
$$

In this context $\Gamma$ is the usual Euler's gamma function. For $\theta=0, I_{\text {time }}^{0}$ is taken to be the identity operator. In particular, $D_{\text {time }}^{1}=\partial / \partial t$, is the ordinary time-derivative. 
The technique used by E. Fabes and N. Rivière relies on the method of layer potentials. Tipically, solving a Dirichlet or Neumann problem amounts to inverting an operator of the form "identity $+K$ ", where $K$ is a singular integral operator, acting on appropriate boundary function spaces. When the domain is of class $C^{1}$, it has been observed that the operator $K$ is compact on the Lebesgue scale $L^{p}$, first in [FJR] for the case of the Laplace operator, then sortly thereafter in [FaRi] for the case of the heat operator. For domains whose boundary is only Lipschitz, $K$ may fail to be compact and this creates difficulties of a fundamental nature. In the case of the Laplacian these have been overcome by G. Verchota in [Ver1] and B. Dahlberg and C. Kenig in [DaKe].

In 1990 R. Brown ([Bro2]) was able to adapt this analysis to the case of the initial Dirichlet and Neumann boundary problems for the heat equation in Lipschitz cylinders, with zero initial data. The key ingredient in Brown's approach was establishing the invertibility of the appropriate caloric boundary layer potentials on Sobolev spaces with an integer amount of smoothness. In the case of the Neumann problem, the boundary datum is taken from $L^{p}(\partial \Omega \times(0, T))$ for $1<p \leq 2$. When considering the initial Dirichlet problem, the lateral datum belongs to the space $L_{1}^{p}(\partial \Omega \times(0, T))$ of functions having one spatial gradient and half of a time derivative in $L^{p}(\partial \Omega \times(0, T))$ for $1<p \leq 2$.

The inhomogeneous Dirichlet problem for the elliptic case, in bounded Lipschitz domains was studied in 1995 by D. Jerison and C. Kenig ([JeKe]), via harmonic measure techniques, emphasizing the difference between smooth domains and domains 
having only a Lipschitz boundary. The optimal range of solvability and smoothness of the solution of

$$
\begin{cases}\Delta u=f & \text { in } \Omega, \\ \operatorname{Tr} u=0 & \text { on } \partial \Omega,\end{cases}
$$

and

$$
\begin{cases}\Delta u=0 & \text { in } \Omega, \\ \operatorname{Tr} u=g & \text { on } \partial \Omega,\end{cases}
$$

is presented, where $f$ and $g$ is allowed to have fractional order of smoothness.

A natural continuation of the work in [JeKe] is presented by E. Fabes, O. Mendez and M. Mitrea in [FMM], where Poisson's equation is considered for the Laplacian, with data in Sobolev-Besov spaces. The main results are establishing the invertibility of boundary layer potential operators on the Sobolev-Besov scale for optimal ranges of indices. It is also shown that the (inhomogeneous) Laplace equation with Dirichlet and Neumann boundary conditions can be treated via single and double layer potentials.

Other, more recent, related developments can be found in Communications of Partial Differential Equations ([Mit2]), where M. Mitrea studies variable coefficient parabolic systems in nonsmooth cylinders, and in the Illinois Juornal of Mathematics ([HLM]), where S. Hofmann, J. Lewis and M. Mitrea consider transmission initial boundary value problems in nonsmooth domains.

As we have seen, a lot of work has been done in particular for data in Lebesgue and Sobolev spaces (the latter with an integer amount of smoothness). This work aims to generalize and bring together all the previously mentioned major results in such a way that the earlier results appear as special limiting cases of our theory. 
The main results of this present work are establishing well-posedness of the Poisson problem for the heat operator with Dirichlet and Neumann boundary conditions, when the boundary datum has a fractional amount of smoothness (Theorems 8.1.1 and 8.2.1). The solutions can be represented as a double layer potential, in the Dirichlet case, and as a single layer potential, in the Neumann case. Also, there is a precise correlation between the smoothness of the solution and that of the boundary data.

Chapter 1 contains basic definitions, notation needed throughout the dissertation. We also discuss isotropic function spaces, Fourier transforms and multipliers, the real and complex interpolation methods, as well as parabolic sequence spaces and envelopes of (isotropic) Hardy spaces.

In Chapter 2 we introduce and study the anisotropic function spaces, where the anisotropy is dictated by the heat operator. More precisely, in the Littlewood-Paley characterization of anisotropic Besov and Triebel-Lizorkin spaces we replace the usual norm by the parabolic norm $\|(x, t)\|_{\text {par }}:=\left(|x|^{2}+|t|\right)^{1 / 2}$ for $x \in \mathbb{R}^{n}$ and $t \in \mathbb{R}$. Then the parabolic Besov scale is well-suited for measuring smoothness. One of the most impotrant properties of the diagonal Besov scale $B_{\alpha, p a r}^{p, p} \equiv B_{\alpha, p a r}^{p}$ is Fubini's property (Proposition 2.1.19), which allows us to decompose the amount of smoothness separately in time and space. This is used in obtaining an intrinsic characterization of $B_{\alpha, \text { par }}^{p}\left(\mathbb{R}^{n} \times \mathbb{R}\right)$, whose proper isotropic analogue can be found on p. 189 in [Tri4]. Several real and complex interpolation results are presented when the underlying space is the interior of a Lipschitz cylinder or its lateral boundary. The key ingredient in 
the first case is finding a well-suited extension operator from the interior of the cylin$\operatorname{der} \Omega \times I$ to $\mathbb{R}^{n} \times \mathbb{R}$, where $\Omega$ is a Lipschitz domain in $\mathbb{R}^{n}$ and $I \subseteq \mathbb{R}$ is an interval. We also describe the atomic decompositions of (homogeneous and inhomogeneous) parabolic Besov and Hardy spaces defined on the lateral side of a Lipschitz cylinder $\partial \Omega \times I$. Finally, dulaity results for the anisotropic Besov and Triebel-Lizorkin scale are presented, as well as envelopes of parabolic Hardy spaces.

The novelty of Chapter 3 is the construction of parabolic function spaces with built-in initial conditions. More precisely, for $1<p, q<\infty, \alpha \in \mathbb{R} \backslash\{0\}$, and a Lipschitz domain $\Omega$ in $\mathbb{R}^{n}, 0<T<\infty$, we define (cf. Definition 3.1.5 and Remark 3.1.6)

$$
\begin{gathered}
{ }_{0} B_{\alpha, p a r}^{p, q}(\Omega \times(0, T)):=\left\{\left.F\right|_{\Omega \times(-\infty, T)}: F \in B_{\alpha, p a r}^{p, q}\left(\mathbb{R}^{n} \times \mathbb{R}\right), \operatorname{supp} F \subseteq \mathbb{R}^{n} \times[0, \infty)\right\}, \\
{ }^{0} B_{\alpha, p a r}^{p, q}(\Omega \times(0, T)):=\left\{\left.F\right|_{\Omega \times(0, \infty)}: F \in B_{\alpha, p a r}^{p, q}\left(\mathbb{R}^{n} \times \mathbb{R}\right), \operatorname{supp} F \subseteq \mathbb{R}^{n} \times(-\infty, T]\right\},
\end{gathered}
$$

and

$$
\begin{aligned}
{ }_{z} B_{\alpha, p a r}^{p, q}(\Omega \times(0, T)):=\left\{\left.F\right|_{\mathbb{R}^{n} \times(-\infty, T)}:\right. & F \in B_{\alpha, p a r}^{p, q}\left(\mathbb{R}^{n} \times \mathbb{R}\right), \\
& \left.\quad \operatorname{supp} F \subseteq(\bar{\Omega} \times[0, \infty)) \cup\left(\mathbb{R}^{n} \times[T, \infty)\right)\right\} .
\end{aligned}
$$

One of the main duality results of this chapter is Theorem 3.1.11, which states that, under the same assumptions as above,

$$
\left.\left({ }^{0} B_{\alpha, p a r}^{p, q}(\Omega \times(0, T))\right)^{*}={ }_{z} B_{-\alpha, p a r}^{p^{\prime}, q^{\prime}} \Omega \times(0, T)\right) .
$$

With regard to the lateral side of a Lipschitz cylinder, $\partial \Omega \times(0, T)$, for $1<p, q<\infty$ and $\alpha \in(-1,1) \backslash\{0\}$, we define

$$
{ }_{0} B_{\alpha, p a r}^{p, q}(\partial \Omega \times(0, T)):=\left\{\left.F\right|_{\partial \Omega \times(-\infty, T)}: F \in B_{\alpha, p a r}^{p, q}(\partial \Omega \times \mathbb{R}), \operatorname{supp} F \subseteq \partial \Omega \times[0, \infty)\right\}
$$


${ }^{0} B_{\alpha, p a r}^{p, q}(\partial \Omega \times(0, T)):=\left\{\left.F\right|_{\partial \Omega \times(0, \infty)}: F \in B_{\alpha, p a r}^{p, q}(\partial \Omega \times \mathbb{R}), \operatorname{supp} F \subseteq \partial \Omega \times(-\infty, T]\right\}$

A remarkable duality property of these spaces is the following:

$$
\left({ }_{0} B_{\alpha, p a r}^{p, q}(\partial \Omega \times(0, T))\right)^{*}={ }^{0} B_{-\alpha, p a r}^{p^{\prime}, q^{\prime}}(\partial \Omega \times(0, T)),
$$

where $1<p, q<\infty, \alpha \in(-1,1) \backslash\{0\}$, and $p, p^{\prime}$, as well as $q, q^{\prime}$ are conjugate indices, i.e. $1 / p+1 / p^{\prime}=1$ and $1 / q+1 / q^{\prime}=1$.

Chapter 4 deals with the mapping properties of the double layer heat potential, which is defined by

$$
\mathcal{D} f(x, t):=\int_{\partial \Omega \times \mathbb{R}} \partial_{\nu_{y}}[E(x-y, t-s)] f(y, s) d \sigma_{y} d s,
$$

where $(x, t) \in\left(\mathbb{R}^{n} \backslash \partial \Omega\right) \times \mathbb{R}$ and $d \sigma$ is the canonical surface measure on $\partial \Omega$. First we treat the two endpoint cases separately, namely $p=1$ in Theorem 4.1 .2 and $p=\infty$ in Theorem 4.1.4. Then the idea is to use the real interpolation method to obtain the most general result:

Consider a bounded Lipschitz domain $\Omega$ in $\mathbb{R}^{n}, 0<T<\infty$ and indices $0<\alpha<1$ and $1<p \leq \infty$ such that $\left(\alpha, \frac{1}{p}\right)$ belongs to the interior of $O A B C D E$ in the following diagram. 


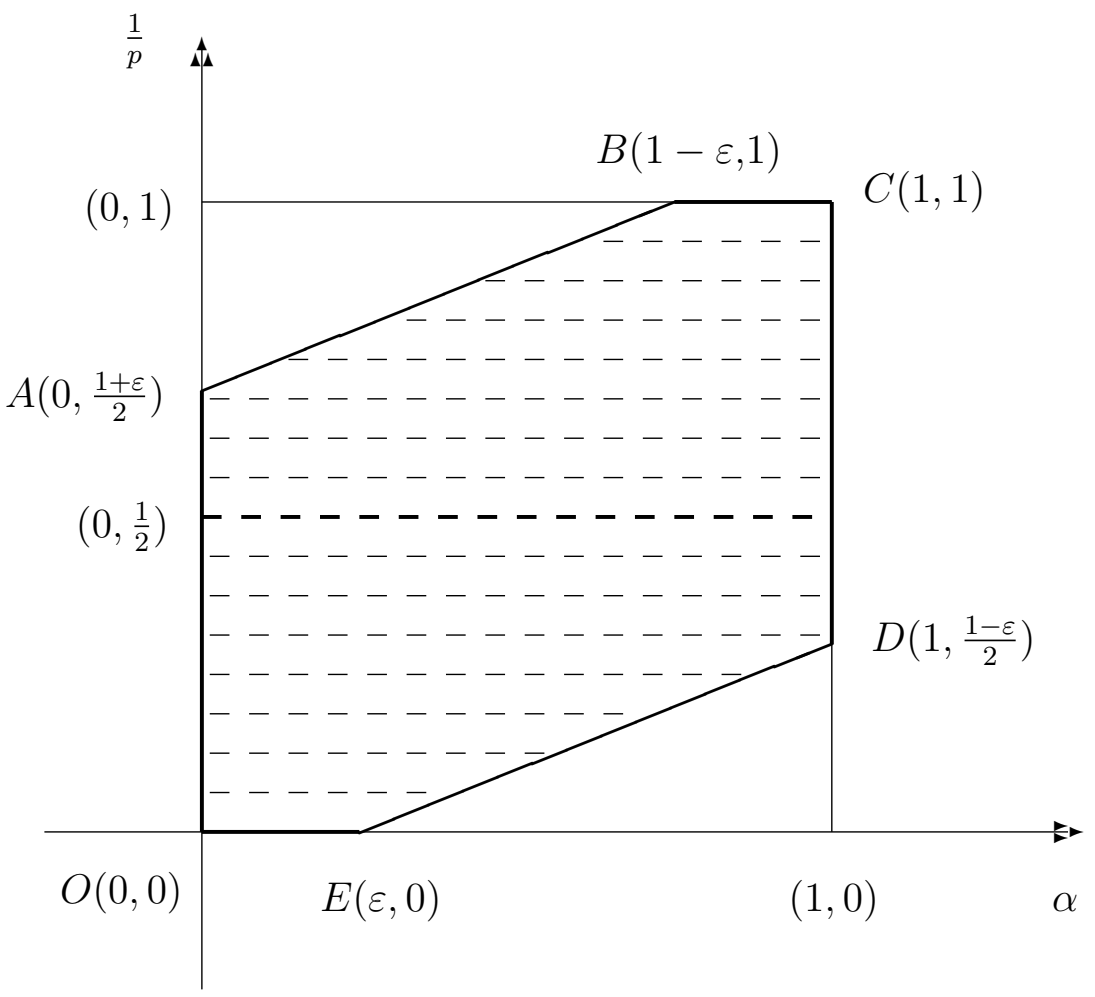

Then

$$
\mathcal{D}:{ }_{0} B_{\alpha, p a r}^{p}(\partial \Omega \times(0, T)) \longrightarrow{ }_{0} B_{\alpha+\frac{1}{p}, p a r}^{p}(\Omega \times(0, T))
$$

is bounded.

Similarly, in Chapter 5 we present the mapping properties of the single layer heat potential, which is defined, for $(x, t) \in\left(\mathbb{R}^{n} \backslash \partial \Omega\right) \times \mathbb{R}$, by

$$
\mathcal{S} f(x, t):=\int_{\partial \Omega \times \mathbb{R}} E(x-y, t-s) f(y, s) d \sigma_{y} d s .
$$

Interpolating, this time by the complex method, between the endpoint cases $p=1$ (Theorem 5.1.9) and $p=\infty$ (Theorem 5.1.10), the final conclusion of this chapter is the following:

Let $\Omega$ be a bounded Lipschitz domain in $\mathbb{R}^{n}, 0<T<\infty$, and $1<p<\infty$, 
$0<\alpha<1$, the caloric single layer potential operator

$$
\mathcal{S}:{ }_{0} B_{-\alpha, p a r}^{p}(\partial \Omega \times(0, T)) \longrightarrow{ }_{0} B_{1-\alpha+1 / p, p a r}^{p}(\Omega \times(0, T))
$$

is bounded.

In Chapter 6 we study the invertibility of boundary layer operators on Besov spaces with fractional amount of smoothness. Similar results were obtained in [Bro2] for integer order of smoothness, i.e. for $L^{p}(\partial \Omega \times(0, T))$ and $L_{1}^{p}(\partial \Omega \times(0, T))$. The main result of this chapter is that for a bounded Lipschitz domain $\Omega \subset \mathbb{R}^{n}, 0<T<\infty$, and indices $\alpha$ (representing smoothness), $p$ (representing integrability) such that $\left(\alpha, \frac{1}{p}\right)$ belongs to the interior of the hexagon $O A B C D E$, the operators

$$
\pm \frac{1}{2} I+K:{ }_{0} B_{\alpha, p a r}^{p}(\partial \Omega \times(0, T)) \stackrel{\sim}{\longrightarrow}{ }_{0} B_{\alpha, p a r}^{p}(\partial \Omega \times(0, T))
$$

are invertible. Here $I$ represents the identity operator and for a boundary point $(x, t) \in \partial \Omega \times \mathbb{R}$

$$
K f(x, t):=\text { p.v. } \int_{\partial \Omega \times \mathbb{R}} \partial_{\nu_{y}}[E(x-y, t-s)] f(y, s) d \sigma_{y} d s,
$$

where p.v. indicates that the above integral is taken in the principal value sense.

Also, if $K^{\prime}$ is introduced as in [Bro2], i.e. $K^{\prime}=R \circ K^{*} \circ R$, where $K^{*}$ is the formal adjoint of $K$, and $R$ is the reflection operator in time, then

$$
\pm \frac{1}{2} I+K^{\prime}:{ }_{0} B_{-\alpha, p a r}^{p}(\partial \Omega \times(0, T)) \stackrel{\sim}{\longrightarrow}{ }_{0} B_{-\alpha, p a r}^{p}(\partial \Omega \times(0, T))
$$

are invertible for $\left(\alpha, 1-\frac{1}{p}\right)$ in the interior of $O A B C D E$, which is the proper range of solvability of the Neumann problem. 
Chapter 7 deals with the mapping properties of the boundary trace operator and the definition of the normal derivative, as well as with the caloric Newtonian potential. The trace operator is originaly given in the nontangential limit sense, i.e. for almost every $(x, t) \in \partial \Omega \times \mathbb{R}$,

$$
\operatorname{Tr} u(x, t)=\left.u\right|_{\partial \Omega \times \mathbf{R}}(x, t):=\lim _{\substack{(y, s) \rightarrow(x, t) \\(y, s) \in \Gamma(x, t)}} u(y, s)
$$

where $\Gamma(x, t)$ is a nontangential cone at the boundary point $(x, t)$ as in (1.2.8). According to Theorem 7.1.2,

$$
\operatorname{Tr}:{ }_{0} B_{\alpha, p a r}^{p}(\Omega \times(0, T)) \longrightarrow{ }_{0} B_{\alpha-\frac{1}{p}, p a r}^{p}(\partial \Omega \times(0, T)) \quad \text { is bounded, }
$$

where $\Omega$ is a bounded Lipschitz domain in $\mathbb{R}^{n}, 0<T<\infty$, and the pair of indices $\left(\alpha, \frac{1}{p}\right)$ belongs to a region that contains the hexagon $O A B C D E$.

For $u \in{ }_{0} B_{1-\alpha+\frac{1}{p}, p a r}^{p}(\Omega \times(0, T))$ we define the normal derivative of $u$ as a linear functional, i.e.

$$
\partial_{\nu} u \in{ }_{0} B_{-\alpha, p a r}^{p}(\partial \Omega \times(0, T))=\left({ }^{0} B_{\alpha, p a r}^{p^{\prime}}(\partial \Omega \times(0, T))\right)^{*}, \quad \frac{1}{p}+\frac{1}{p^{\prime}}=1 .
$$

The actual definition depends on the interval $\alpha+\frac{1}{p}$ belongs to (see Definition 7.2.3).

The parabolic Newtonian potential is defined by formal convolution with the fundamental solution of the heat operator (Definition 7.3.1), i.e. for $(x, t) \in \mathbb{R}^{n} \times \mathbb{R}$,

$$
\left(\Pi_{\text {par }} f\right)(x, t) ;=\int_{\mathbb{R}^{n} \times \mathbb{R}} E(x-y, t-s) f(y, s) d y d s
$$

When smoothness is measured on $L^{p}$-based spaces, the (parabolic) Newtonian potential has a smoothing effect of order 2 (Theorem 7.3.13). 
Finally, the inhomogeneous Poisson problem for the heat operator (and for the adjoint heat operator) with Dirichlet and Neumann boundary conditions are formulated and solved in Chapter 8. More specifically, one of the main results of this work is the following.

Theorem 8.1.1. For each bounded Lipschitz domain $\Omega$ in $\mathbb{R}^{n}$ there exists some small constant $\varepsilon>0$ with the following significance. Let $0<T<\infty$ and assume that the indices $1<p<\infty$ and $0<\alpha<1$ are such that the point with coordinates $\left(\alpha, \frac{1}{p}\right)$ belongs to the interior of the hexagon $O A B C D E$. Then there exists a unique solution $u \in{ }_{0} B_{\alpha+\frac{1}{p}, p a r}^{p}(\Omega \times(0, T))$ to the problem

$$
(\mathrm{PD})\left\{\begin{array}{l}
\left(\partial_{t}-\Delta\right) u=f \in{ }_{0} B_{\alpha+\frac{1}{p}-2, p a r}^{p}(\Omega \times(0, T)), \\
\left.u\right|_{\partial \Omega \times(0, T)}=g \in{ }_{0} B_{\alpha, p a r}^{p}(\partial \Omega \times(0, T)) .
\end{array}\right.
$$

Furthermore, there is an integral representation of the solution and

$$
\|u\|_{0 B_{\alpha+\frac{1}{p}, p a r}^{p}(\Omega \times(0, T))} \leq c\left(\|f\|_{0 B_{\alpha+\frac{1}{p}-2, p a r}^{p}(\Omega \times(0, T))}+\|g\|_{0} B_{\alpha, p a r}^{p}(\partial \Omega \times(0, T))\right) .
$$

In particular, we shall prove that the solution $u$ can be represented in the following form:

$$
u=\Pi_{p a r} f+\mathcal{D}\left[\left(\frac{1}{2} I+K\right)^{-1}\left(g-\operatorname{Tr}\left(\Pi_{p a r} f\right)\right)\right] .
$$

The other main result of this chapter (and dissertation) is as follows.

Theorem 8.2.1. Given a bounded Lipschitz domain $\Omega$ in $\mathbb{R}^{n}$, there exists $\varepsilon>0$ such that if $0<T<\infty$ and $1<p<\infty, 0<\alpha<1$ with the property that the point with coordinates $\left(\alpha, 1-\frac{1}{p}\right)$ belongs to the interior of the hexagon $O A B C D E$, then there exists a unique solution $u \in{ }_{0} B_{1-\alpha+\frac{1}{p}, \text { par }}^{p}(\Omega \times(0, T))$ to the problem

$$
(\mathrm{PN})\left\{\begin{array}{l}
\left(\partial_{t}-\Delta\right) u=\left.f\right|_{\Omega \times(0, T)}, f \in{ }_{z} B_{-1-\alpha+\frac{1}{p}, p a r}^{p}(\Omega \times(0, T)), \\
\partial_{\nu} u=g \in{ }_{0} B_{-\alpha, p a r}^{p}(\partial \Omega \times(0, T)) .
\end{array}\right.
$$


In addition, $u$ can be represented in terms of caloric layer potentials and

$$
\|u\|_{0 B_{1-\alpha+\frac{1}{p}, p a r}^{p}(\Omega \times(0, T))} \leq c\left(\|f\|_{z B_{-1-\alpha+\frac{1}{p}, p a r}^{p}}(\Omega \times(0, T))+\|g\|_{\left.0 B_{-\alpha, p a r}^{p}(\partial \Omega \times(0, T))\right)} .\right.
$$

Generally, $\partial_{\nu} u$ is understood as the normal derivative of $u$, relative to the extension $\operatorname{Ext}\left(\left(\partial_{t}-\Delta\right) u\right)$ of $\left(\partial_{t}-\Delta\right) u$. In Theorem 8.3.1, $\partial_{\nu} u$ means the normal derivative of $u$, relative to the functional $\left(\partial_{t}-\Delta\right) u=f$.

We shall prove that the solution of (PN) can be represented in the form

$$
u=\Pi_{p a r} f+\mathcal{S}\left[\left(-\frac{1}{2} I+K^{\prime}\right)^{-1}\left(g-\partial_{\nu}\left(\Pi_{p a r} f, f\right)\right)\right] .
$$

The proper analogue of these theorems for the adjoint heat operator are formulated in Theorems 8.1.2 and 8.2.2.

\subsection{Basic definitions, notation and preliminary re- sults}

\subsubsection{Notation and basic conventions}

$$
\begin{aligned}
& \mathbb{N}=\{1,2, \ldots\}, \mathbb{N}_{0}=\mathbb{N} \cup\{0\}, \mathbb{Z}=\{\ldots,-2,-1,0,1,2, \ldots\}, \\
& \mathbb{C}=\text { the complex plane, } \\
& \mathbb{R}^{n}=\text { the } n \text {-dimensional Euclidean space, } \\
& \bar{z}=\text { the complex conjugate of } z, \\
& |x|=\sqrt{x_{1}^{2}+\ldots+x_{n}^{2}} \text { for } x \in \mathbb{R}^{n}, \\
& {[a]=\text { the integer part of } a \in \mathbb{R},} \\
& (a)_{+}:=\max \{a, 0\}, \quad \text { for } a<0, \\
& \operatorname{sign}(a):=\left\{\begin{aligned}
-1 & \text { for } a=0, \\
1 & \text { for } a>0,
\end{aligned}\right.
\end{aligned}
$$


$\oplus=$ direct sum,

$\partial \Omega=$ the boundary of $\Omega$,

$\bar{A}=$ the closure of a set $A$,

$A^{c}=$ the complement of a set $A$,

$|A|=$ the volume of the set $A$,

$\chi_{A}=$ the characteristic function of the set $A$,

$\operatorname{supp} f=\operatorname{support}$ of $f$,

$(f * g)(x):=\int_{\mathbb{R}^{n}} f(y) g(x-y) d y=$ convolution of $f$ with $g,\left(x \in \mathbb{R}^{n}\right)$,

$C^{\infty}\left(\mathbb{R}^{n}\right)=$ class of infinitely differentiable functions on $\mathbb{R}^{n}$,

$C_{c}^{\infty}\left(\mathbb{R}^{n}\right)=$ class of infinitely differentiable, compactly supported functions on $\mathbb{R}^{n}$,

$I$ represents the identity operator unless otherwise noted,

$f_{A} f(x) d x:=\frac{1}{|A|} \int_{A} f(x) d x=$ average of $f$ over the set $A$,

$T^{*}$ denotes the adjoint of the operator $T$,

$\hookrightarrow$ stands for continuous embedding, i.e. $A_{1} \hookrightarrow A_{2}$ if and only if there exists a positive finite constant such that $\|a\|_{A_{1}} \leq c\|a\|_{A_{2}}$ for all $a \in A_{1}$.

Given two Banach spaces $X$ and $Y$, the space of bounded linear operators from $X$ to $Y$ is denoted by $\mathcal{L}(X, Y)$. By $X^{*}$ we denote the dual of the Banach space $X$. The duality pairing between $f \in X^{*}$ and $g \in X$ is denoted by $\langle f, g\rangle_{X^{*}, X}$ or $\langle f, g\rangle$. Given an operator $M \in \mathcal{L}(X, Y)$, its adjoint is denoted by $M^{*}$.

Let $U$ and $V$ be open subsets of $\mathbb{R}^{n}$. If $U \subset \bar{U} \subset V$ and $\bar{U}$ is compact, then we write $U \subset \subset V$ and say that $U$ is compactly contained in $V$. 
For any muiltiindex $\alpha=\left(\alpha_{1}, \ldots, \alpha_{n}\right) \in \mathbb{N}_{0}^{n}$ and $x \in \mathbb{R}^{n},|\alpha|:=\alpha_{1}+\ldots+\alpha_{n}$

$$
x^{\alpha}:=x_{1}^{\alpha_{1}} \ldots x_{n}^{\alpha_{n}}, \quad \partial_{x}^{\alpha}:=\partial_{x_{1}}^{\alpha_{1}} \ldots \partial_{x_{n}}^{\alpha_{n}} .
$$

By $\ell^{p}, 1 \leq p \leq \infty$, we denote the colletion of all sequences $s=\left\{s_{j}\right\}_{j=1}^{\infty}$ of real (or complex) numbers such that

$$
\|s\|_{\ell^{p}}:= \begin{cases}\left(\sum_{j=1}^{\infty}\left|s_{j}\right|^{p}\right)^{\frac{1}{p}}<\infty, & \text { if } 1 \leq p<\infty, \\ \sup _{j \in \mathbb{N}_{0}}\left|s_{j}\right|<\infty, & \text { if } p=\infty .\end{cases}
$$

For $(x, t) \in \mathbb{R}^{n} \times \mathbb{R}$, the parabolic norm of $(x, t)$ is defined by

$$
\|(x, t)\|_{p a r}:=\left(|x|^{2}+|t|\right)^{1 / 2} .
$$

Throughout this dissertation S stands for the Schwartz class of rapidly decreasing functions and $\mathrm{S}^{\prime}$, its dual, represents the space of tempered distributions. $\mathcal{D}^{\prime}$ and $\mathcal{E}^{\prime}$ will denote, respectively, the class of distributions and the class of distributions with compact support. We let $c$ and $C$ denote finite positive constants which may vary from one occurence to another. By $A \approx B$ we mean that $c \leq A / B \leq C$.

\subsubsection{Lipschitz domain}

An open set $\Omega \subset \mathbb{R}^{n}$ is called a Lipschitz domain if for each $x^{*} \in \partial \Omega$ there exists a rectangular coordinate system, $\left(x^{\prime}, x_{n}\right), x^{\prime} \in \mathbb{R}^{n-1}, x_{n} \in \mathbb{R}$, assumed to be a rotation and translation of the standard rectangular coordinates for $\mathbb{R}^{n}$, a neighborhood of $x^{*}, U \equiv U\left(x^{*}\right) \subset \mathbb{R}^{n}$, and a function $\varphi \equiv \varphi_{x^{*}}: \mathbb{R}^{n-1} \rightarrow \mathbb{R}$ such that

(i) $\left|\varphi\left(x^{\prime}\right)-\varphi\left(y^{\prime}\right)\right| \leq C_{x^{*}}\left|x^{\prime}-y^{\prime}\right|$ for all $x^{\prime}, y^{\prime} \in \mathbb{R}^{n-1}, C_{x^{*}}<\infty$,

(ii) $U \cap \Omega=\left\{\left(x^{\prime}, x_{n}\right): x_{n}>\varphi\left(x^{\prime}\right)\right\} \cap U$. 
Let $\Omega \subset \mathbb{R}^{n}, n \geq 2$, be a bounded Lipschitz domain with boundary $\partial \Omega$. By a cylinder, $Z(x, r)$, we mean an open, right circular, doubly truncated cylinded centered at $x \in \mathbb{R}^{n}$ with radius $r$. A coordinate cylinder for $\Omega, Z=Z\left(x^{*}, r\right), x^{*} \in \partial \Omega$, will be defined by the following properties. First, the bases of $Z$ are some positive distance from $\partial \Omega$. Second, there exists a rectangular coordinate system for $\mathbb{R}^{n}$, $\left(x^{\prime}, x_{n}\right), x \in \mathbb{R}^{n-1}, x_{n} \in \mathbb{R}$, with the $x_{n}$-axis containing the axis of $Z$, and there exists an associated function $\varphi=\varphi_{Z}: \mathbb{R}^{n-1} \rightarrow \mathbb{R}$ that is Lipschitz, i.e. $\left|\varphi\left(x^{\prime}\right)-\varphi\left(y^{\prime}\right)\right| \leq$ $c\left|x^{\prime}-y^{\prime}\right|, c=c_{Z}<\infty$, for all $x^{\prime}, y^{\prime} \in \mathbb{R}^{n-1}$ and satisfies

(i) $Z \cap \Omega=Z \cap\left\{\left(x^{\prime}, x_{n}\right): x_{n}>\varphi\left(x^{\prime}\right)\right\}$,

(ii) $x^{*}=(0, \varphi(0))$.

The pair $(Z, \varphi)$ will be called a coordinate pair. In addition we will occasionally write $Z_{e}=Z \backslash \bar{\Omega}$ and $Z_{i}=Z \cap \Omega$ and call these the exterior and interior cylinders, respectively. For any positive number $t, t Z(x, r)$ will denote the cylinder $\left\{z \in \mathbb{R}^{n}\right.$ : $x+(z-x) / t \in Z\}$, i.e. the dilation of $Z$ about $x$ by a factor of $t$.

By compactness, it is possible to cover $\partial \Omega$ with a finite number of coordinate cylinders $Z_{1}, \ldots, Z_{N}$. Furthermore, it is possible and often convenient to do this in such a way that for each $Z_{j}$ there is a coordinate pair $\left(Z_{j}^{*}, \varphi_{j}\right)$ with $Z_{j}^{*}=t_{j} Z_{j}$, where $t_{j}$ is some sufficiently large positive number. For example, it can be useful to take $t_{j}>10\left(1+\left\|\nabla \varphi_{j}\right\|_{L^{\infty}\left(\mathbb{R}^{n-1}\right)}^{2}\right)^{1 / 2}$, for $1 \leq j \leq N$. Whenever we cover $\partial \Omega$ with coordinate cylinders we will also assume that the coordinate cylinders $Z^{*}$ exist. Note that $\varphi_{j}$ may be taken to have compact support in $\mathbb{R}^{n-1}$.

For a Lipschitz domain $\Omega$, there exists a number $M<\infty$, so that for any covering 
of $\partial \Omega$ with coordinate pairs $\left(Z_{j}, \varphi_{j}\right)$, the $\varphi_{j}$ 's have Lipschitz constants less then or equal to $M$. The smallest such number is called the Lipschitz constant for $\Omega$.

By a cone we mean an open, circular, doubly truncated cone with two non-empty, convex components. If $x \in \partial \Omega, \Gamma(x)$ will denote a cone with vertex at $x$ and one component in $\Omega$ and the other in $\mathbb{R}^{n} \backslash \bar{\Omega}$. The component interior to $\Omega$ will be denoted by $\Gamma_{i}(x)$ and the component exterior to $\bar{\Omega}$ will be denoted by $\Gamma_{e}(x)$. Assigning one cone, $\Gamma(x)$, to each $x \in \partial \Omega$, we call the resulting family, $\{\Gamma(x): x \in \partial \Omega\}$, regular if there is a finite covering of $\partial \Omega$ by coordinate pairs $(Z, \varphi)$, as described above, such that for each $(Z, \varphi)$ there are three cones, $\Gamma_{1}, \Gamma_{2}$, and $\Gamma_{3}$, each with vertex at the origin and axis along the axis of $Z$ enjoying the following properties:

$$
\Gamma_{1} \subset \overline{\Gamma_{2}} \backslash\{0\} \subset \Gamma_{3},
$$

and for all $\left(x^{\prime}, \varphi\left(x^{\prime}\right)\right)=x \in \frac{4}{5} Z^{*} \cap \partial \Omega$, where $x^{\prime} \in \mathbb{R}^{n-1}$,

$$
\begin{gathered}
\Gamma_{1}+x \subset \Gamma(x) \subset \overline{\Gamma(x)} \backslash\{x\} \subset \Gamma_{2}+x, \\
\left(\Gamma_{3}+x\right)_{i} \subset \Omega \cap Z^{*}, \quad \text { and } \quad\left(\Gamma_{3}+x\right)_{e} \subset Z^{*} \backslash \bar{\Omega} .
\end{gathered}
$$

A basic observation is that for any bounded Lipschitz domain $\Omega \subset \mathbb{R}^{n}$ there exists a regular family of cones $\{\Gamma\}$ as described above. See [Ver1].

Remark 1.2.1. For the applications we have in mind, we are going to use the following approach. For any bounded Lipschitz domain $\Omega \subset \mathbb{R}^{n}$ there exist $V_{i}$ ( $i=$ $1, \ldots, N, N$ finite) open, bounded subsets of $\mathbb{R}^{n}$ such that $\partial \Omega \subseteq \cup_{i=1}^{N} V_{i}$, and there are, in some coordinate system, Lipschitz graphs $\partial \Omega_{i}$ with $V_{i} \cap \partial \Omega \subset \subset \partial \Omega_{i} \cap \partial \Omega$. 
Also, there exists a finite partition of unity $\psi_{i} \in C_{c}^{\infty}\left(V_{i}\right)$ (i.e. $\sum_{i=1}^{N} \psi_{i}=1$ ) on $\partial \Omega$ such that supp $\psi_{i} \subseteq V_{i}$. The above mentioned coordinate system is obtained from the standard one via translation and rotation.

We next introduce the parabolic approach (cone-like) regions to the lateral boundary of $\Omega_{ \pm} \times I$, where $\Omega_{+}:=\Omega$ is a Lipschitz domain in $\mathbb{R}^{n}, \Omega_{-}:=\mathbb{R}^{n} \backslash \bar{\Omega}$, and $I$ is an interval in $\mathbb{R}$. Fix some $\kappa=\kappa(\partial \Omega, I)>1$, sufficiently large. Then for a boundary point $(x, t) \in \partial \Omega \times I$ these regions, lying in $\Omega_{+} \times I$ and $\Omega_{-} \times I$, respectively, are:

$$
\Gamma_{ \pm}(x, t):=\left\{(y, s) \in \Omega_{ \pm} \times I: \operatorname{dist}((x, t),(y, s))<\kappa \operatorname{dist}((y, s), \partial \Omega \times I)\right\} .
$$

For an arbitrary function $u: \Omega_{ \pm} \times I \rightarrow \mathbb{R}$ we define the parabolic non-tangential maximal operator $\mathcal{N}$ by

$$
\mathcal{N}(u)(x, t):=\sup _{(y, s) \in \Gamma_{ \pm}(x, t)}|u(y, s)|, \quad(x, t) \in \partial \Omega \times I .
$$

The non-tangential restrictions to the boundary are defined as

$$
\left.u\right|_{\partial \Omega \times I}(x, t):=\lim _{\substack{(y, s) \rightarrow(x, t) \\(y, s) \in \Gamma_{ \pm}(x, t)}} u(y, s), \quad \text { for a.e. }(x, t) \in \partial \Omega \times I,
$$

the choice of the sign depending on whether the function $u$ is defined in $\Omega_{+} \times I$ or $\Omega_{-} \times I$. Similarly, for the normal derivative of $u, \partial_{\nu} u$ we set

$$
\partial_{\nu} u(x, t):=\nu(x) \cdot\left(\lim _{\substack{(y, s) \rightarrow(x, t) \\(y, s) \in \Gamma_{ \pm}(x, t)}}(\nabla u)(y, s)\right), \quad \text { for a.e. }(x, t) \in \partial \Omega \times I,
$$

where $\nu$ is the outward unit normal to $\Omega$. Also, $\nabla_{\text {tan }}:=\nabla-\nu \partial_{\nu}$ and $d \sigma$ will denote the tangential gradient and the canonical surface measure, respectively, on $\partial \Omega$. 


\subsubsection{Estimates for the caloric fundamental solution}

Denote by $E(x, t)$ the fundamental solution of the heat operator

$$
\partial_{t}-\Delta:=\partial_{t}-\sum_{i=1}^{n} \frac{\partial^{2}}{\partial x_{i}^{2}}
$$

in $\mathbb{R}^{n} \times \mathbb{R}$, i.e.

$$
E(x, t):=\frac{1}{(4 \pi t)^{n / 2}} \exp \left\{\frac{-|x|^{2}}{4 t}\right\} \chi_{(0, \infty)}(t), \quad x \in \mathbb{R}^{n}, t \in \mathbb{R} .
$$

Proposition 1.2.2. For every multiindex $\alpha=\left(\alpha_{1}, \ldots, \alpha_{n}\right)$ and every $k \in \mathbb{N}_{0}$ there exists a constant $c_{n, k, \alpha}$ such that

$$
\left|\partial_{t}^{k} \partial_{x}^{\alpha} E(x, t)\right| \leq \frac{c_{n, k, \alpha}}{\left(|x|^{2}+|t|\right)^{(n+|\alpha|+2 k) / 2}}
$$

uniformly for $(x, t) \in \mathbb{R}^{n} \times \mathbb{R}$.

Proof. We first claim that for every $k \in \mathbb{N}_{0}$ there exist constants $\left\{c_{n, k, j}\right\}_{j}$ such that

$$
\partial_{t}^{k} E(x, t)=E(x, t) \sum_{j=k}^{2 k} c_{n, k, j} \frac{|x|^{2(j-k)}}{t^{j}}, \quad(x, t) \in \mathbb{R}^{n} \times \mathbb{R} .
$$

In order to show (1.2.14) we proceed by induction and note that for $k=0$ there is nothing to prove. Next, assume that (1.2.14) holds for some $k \in \mathbb{N}_{0}$. Then differentiating the identity (1.2.14) with respect to time gives

$$
\begin{aligned}
\partial_{t}\left(\partial_{t}^{k} E(x, t)\right) & =E(x, t)\left(\sum_{j=k}^{2 k} c_{n, k, j} \frac{|x|^{2(j-k)}}{t^{j+1}}+\sum_{j=k}^{2 k} c_{n, k, j} \frac{|x|^{2(j-k+1)}}{t^{j+2}}\right) \\
& =E(x, t)\left(\sum_{l=k+1}^{2 k+1} c_{n, k, l} \frac{|x|^{2[l-(k+1)]}}{t^{l}}+\sum_{m=k+2}^{2 k+2} c_{n, k, m} \frac{|x|^{2[m-(k+1)]}}{t^{m}}\right) \\
& =E(x, t) \sum_{l=k+1}^{2 k+2} c_{n, k, l} \frac{|x|^{2[l-(k+1)]}}{t^{l}} .
\end{aligned}
$$


Thus, by induction, (1.2.14) holds for any $k \in \mathbb{N}_{0}$. Our second claim is that for any $\alpha \in \mathbb{N}_{0}^{n}$ there are constants $\left\{c_{n, \alpha, \beta, j}\right\}_{j, \beta}$ such that

$$
\partial_{x}^{\alpha} E(x, t)=E(x, t) \sum_{j=\left[\frac{|\alpha|+1}{2}\right]}^{|\alpha|} \sum_{|\beta|=2 j-|\alpha|} c_{n, \alpha, \beta, j} \frac{x^{\beta}}{t^{j}} .
$$

To prove (1.2.15) first observe that if $|\alpha|=0$, then (1.2.15) naturally holds. Second, assume (1.2.15) holds for some $\alpha \in \mathbb{N}_{0}^{n}$. Then, differentiating (1.2.15) with respect to $x_{i}, 1 \leq i \leq n$, we get

$$
\begin{aligned}
\partial_{x_{i}}\left(\partial_{x}^{\alpha} E(x, t)\right) & \\
=E(x, t) & \left(\sum_{j=\left[\frac{|\alpha|+2}{2}\right]}^{|\alpha|} \sum_{|\beta|=2 j-(|\alpha|+1)} c_{n, \alpha, \beta, j} \frac{x^{\beta}}{t^{j}}+\sum_{j=\left[\frac{|\alpha|+1}{2}\right]} \sum_{|\beta|=2 j-|\alpha|} c_{n, \alpha, \beta, j} \frac{x^{\beta+1}}{t^{j+1}}\right) \\
& =E(x, t)\left(\sum_{j=\left[\frac{|\alpha|+2}{2}\right]}^{|\alpha|} \sum_{|\beta|=2 j-(|\alpha|+1)} c_{n, \alpha, \beta, j} \frac{x^{\beta}}{t^{j}}+\sum_{s=\left[\frac{|\alpha|+1}{2}\right]+1} \sum_{|\gamma|=2 s-(|\alpha|+1)} c_{n, \alpha, \gamma, s} \frac{x^{\gamma}}{t^{s}}\right) \\
& =E(x, t) \sum_{j=\left[\frac{|\alpha|+2}{2}\right]|\beta|=2 j-(|\alpha|+1)}^{|\alpha|+1} c_{n, \alpha, \beta, j} \frac{x^{\beta}}{t^{j}} .
\end{aligned}
$$

Hence, by induction, (1.2.15) holds for any multiindex $\alpha$.

Consequently, for $k \in \mathbb{N}_{0}$ and $\alpha \in \mathbb{N}_{0}^{n}$, applying $\partial_{t}^{k}$ to (1.2.15) and writing out the result using Leibnitz's formula, we obtain that

$$
\begin{aligned}
\partial_{t}^{k} \partial_{x}^{\alpha} E(x, t) & =\sum_{l=0}^{k}\left[\partial_{t}^{l} E(x, t) \partial_{t}^{k-l}\left(\sum_{j=\left[\frac{|\alpha|+1}{2}\right]|\beta|=2 j-|\alpha|}^{|\alpha|} c_{n, \alpha, \beta, j} \frac{x^{\beta}}{t^{j}}\right)\right] \\
& =E(x, t) \sum_{l=0}^{k}\left(\sum_{r=l}^{2 l} c_{n, r, l} \frac{|x|^{2(r-l)}}{t^{r}}\right)\left(\sum_{j=\left[\frac{|\alpha|+1}{2}\right]} \sum_{|\beta|=2 j-|\alpha|} c_{n, \alpha, \beta, j} \frac{x^{\beta}}{t^{j+k-l}}\right) \\
& =E(x, t) \sum_{l=0}^{k} \sum_{r=l}^{2 l} \sum_{j=\left[\frac{|\alpha|+1}{2}\right]}^{|\alpha|} \sum_{|\beta|=2 j-|\alpha|} \sum_{|\delta|=2 r-2 l+|\beta|} c_{n, \alpha, \delta, \beta, j, r, l} \frac{x^{\delta}}{t^{r+j+k-l}} .
\end{aligned}
$$


Note that $0 \leq r-l \leq l$. Moreover, if $q:=r+j+k-l$, then the above identity can be rewritten in the form

$$
\partial_{t}^{k} \partial_{x}^{\alpha} E(x, t)=E(x, t) \sum_{q=\left[\frac{|\alpha|+1}{2}\right]+k}^{|\alpha|+2 k} \sum_{|\delta|=2 q-2 k-|\alpha|} c_{n, k, \alpha, \delta, q} \frac{x^{\delta}}{t^{q}}
$$

Having justified (1.2.16), we now turn out attention to the task of proving (1.2.13). Granted (1.2.16), in order to show the estimate (1.2.13), it is enough to prove that

$$
|E(x, t)| \sum_{q=\left[\frac{|\alpha|+1}{2}\right]+k}^{|\alpha|+2 k} c_{n, k, \alpha, q} \frac{\left(|x|^{2}\right)^{q-k-\frac{|\alpha|}{2}}}{t^{q}} \leq \frac{C_{n, k, \alpha}}{\left(|x|^{2}+t\right)^{\frac{n}{2}+\frac{|\alpha|}{2}+k}} \quad \text { for } \quad x \in \mathbb{R}^{n}, t>0 .
$$

This follows, in turn, since obviously $\left(|x|^{2}\right)^{q-k-\frac{|\alpha|}{2}}\left(|x|^{2}+t\right)^{\frac{n}{2}+\frac{|\alpha|}{2}+k} \leq\left(|x|^{2}+t\right)^{q+\frac{n}{2}}$, as soon as we prove that

$$
\exp \left\{-\frac{|x|^{2}}{4 t}\right\} \sum_{q=\left[\frac{|\alpha|+1}{2}\right]+k}^{|\alpha|+2 k} c_{n, k, \alpha, q}\left(\frac{|x|^{2}+t}{t}\right)^{q+\frac{n}{2}} \leq C_{n, k, \alpha}
$$

uniformly for $x \in \mathbb{R}^{n}$ and $t>0$. Each term in the left-hand side of (1.2.17) can be handled individually, using the fact that $\lim _{s \rightarrow \infty}\left(P(s) \mathrm{e}^{-s}\right)=0$ for any polynomial $P$ on $\mathbb{R}$. Hence the proof of $(1.2 .13)$ is complete.

We shall also make use of estimates for partial derivatives of $E(x, t)$ with fractional order. To state these, recall the fractional time-derivative $D_{\text {time }}^{\theta}$ and the fractional integral operator $I_{\text {time }}^{\theta}$.

$$
D_{\text {time }}^{\theta}:=\frac{\partial}{\partial t} I_{\text {time }}^{1-\theta}, \quad 0 \leq \theta \leq 1
$$

where, for $f \in L^{1}(-\infty, T)$ which decays fast enough at $-\infty$, and for $0<\theta \leq 1$,

$$
I_{\text {time }}^{\theta} f(t):=\frac{1}{\Gamma(\theta)} \int_{-\infty}^{t} \frac{f(s)}{(t-s)^{1-\theta}} d s
$$


In this context $\Gamma$ is the usual Euler's gamma function. For $\theta=0, I_{\text {time }}^{0}$ is taken to be the identity operator. In particular, $D_{\text {time }}^{1}=\partial / \partial t$, is the ordinary time-derivative.

Proposition 1.2.3. According to Proposition 5.2 of [Mit2], for $0<\theta<1, \alpha \in \mathbb{N}_{0}^{n}$, $\beta \in \mathbb{N}_{0}$, we have the following estimates:

$$
\begin{gathered}
\left|D_{\text {time }}^{\theta} \partial_{x}^{\alpha} \partial_{\text {time }}^{\beta} E(x, t)\right| \leq c_{\alpha, \beta, \theta} \frac{|t|^{1-\theta}}{|x|^{n+2+|\alpha|+2 \beta}}\left(\min \left\{1, \frac{|x|^{2}}{|t|}\right\}\right)^{\beta+2}, \\
\left|I_{\text {time }}^{\theta} \partial_{x}^{\alpha} \partial_{\text {time }}^{\beta} E(x, t)\right| \leq c_{\alpha, \beta, \theta} \frac{|t|^{\theta}}{|x|^{n+|\alpha|+2 \beta}}\left(\min \left\{1, \frac{|x|^{2}}{|t|}\right\}\right)^{\beta+1} .
\end{gathered}
$$

\subsubsection{Function spaces}

Definition 1.2.4. Given $U \subseteq \mathbb{R}^{n}$ and $V \subseteq \mathbb{R}$, we define the space $\operatorname{Lip}(U \times V)$ as the collection of all Lipschitz functions $f$ with respect to the parabolic norm, i.e. functions with the following property: there exists a finite positive constant $\mathrm{c}$ such that

$$
|f(x, t)-f(y, s)| \leq c\left(|x-y|+|t-s|^{1 / 2}\right)
$$

for every $x, y \in U$ and $t, s \in V$.

Definition 1.2.5. For a measure space $(\Omega, d \mu)$, a Banach space $X$ and $0<p \leq \infty$ we define

$$
L^{p}(\Omega ; X):=\left\{f: \Omega \rightarrow X:\|f\|_{L^{p}(\Omega ; X)}<\infty\right\}
$$

where, for $0<p<\infty$,

$$
\|f\|_{L^{p}(\Omega ; X)}:=\left(\int_{\Omega}\|f(x)\|_{X}^{p} d \mu_{x}\right)^{1 / p},
$$

and, for $p=\infty$,

$$
\|f\|_{L^{\infty}(\Omega ; X)}:=\sup _{x \in \mathbb{R}^{n}}\|f(x)\|_{X} .
$$


Note that in particular, when $\Omega=\mathbb{R}^{n}$ and $d \mu$ is the Lebesgue measure $d x$, then Definition 1.2.5 gives the usual Lebegue space defined as

$$
L^{p}\left(\mathbb{R}^{n}\right):=\left\{f: \mathbb{R}^{n} \rightarrow \mathbb{R}:\|f\|_{L^{p}\left(\mathbb{R}^{n}\right)}<\infty\right\}
$$

where, for $0<p<\infty$,

$$
\|f\|_{L^{p}\left(\mathbb{R}^{n}\right)}:=\left(\int_{\mathbb{R}^{n}}|f(x)|^{p} d x\right)^{1 / p}
$$

and, for $p=\infty$,

$$
\|f\|_{L^{\infty}\left(\mathbb{R}^{n}\right)}:=\operatorname{ess} \sup _{x \in \mathbb{R}^{n}}|f(x)|
$$

Definition 1.2.6. For a measure space $(\Omega, d \mu)$, a Banach space $X$ and $0<p \leq \infty$, $0<\alpha<1$ we define

$$
B_{\alpha}^{p}(\Omega ; X):=\left\{f: \Omega \rightarrow X:\|f\|_{B_{\alpha}^{p}(\Omega ; X)}<\infty\right\}
$$

where, for $0<p<\infty$,

$$
\|f\|_{B_{\alpha}^{p}(\Omega ; X)}:=\|f\|_{L^{p}(\Omega ; X)}+\left(\int_{\Omega} \int_{\Omega} \frac{\|f(x)-f(y)\|_{X}^{p}}{|x-y|^{n+\alpha p}} d \mu_{x} d \mu_{y}\right)^{1 / p},
$$

and, for $p=\infty$,

$$
\|f\|_{B_{\alpha}^{\infty}(\Omega ; X)}:=\|f\|_{L^{\infty}(\Omega ; X)}+\sup _{\substack{x, y \in \mathbb{R}^{n} \\ x \neq y}} \frac{\|f(x)-f(y)\|_{X}}{|x-y|^{\alpha}} .
$$

Remark 1.2.7. For $1 \leq p<\infty$ and $0<\alpha<1$ we have

$$
B_{\alpha}^{p}\left(\mathbb{R}^{n} ; L^{p}(\mathbb{R})\right)=L^{p}\left(\mathbb{R} ; B_{\alpha}^{p}\left(\mathbb{R}^{n}\right)\right)
$$

Proof. We first observe that by definition, $f$ belongs to $B_{\alpha}^{p}\left(\mathbb{R}^{n} ; L^{p}(\mathbb{R})\right)$ if and only if

$$
\|f\|_{L^{p}\left(\mathbb{R}^{n} \times \mathbb{R}\right)}<\infty \quad \text { and } \quad \int_{\mathbb{R}^{n}} \int_{\mathbb{R}^{n}} \int_{\mathbb{R}} \frac{|f(x, t)-f(y, t)|^{p} d t}{|x-y|^{n+\alpha p}} d x d y<\infty
$$


Onthe other hand, by definition,

$$
f \in L^{p}\left(\mathbb{R} ; B_{\alpha}^{p}\left(\mathbb{R}^{n}\right)\right) \Longleftrightarrow \int_{\mathbb{R}}\|f(\cdot, t)\|_{B_{\alpha}^{p}\left(\mathbb{R}^{n}\right)}^{p} d t<\infty
$$

Since (1.2.20) and (1.2.21) are equivalent with each other, the proof of the remark is complete.

Next we give a Littlewood-Paley type definition of the (isotropic) Besov and Triebel-Lizorkin spaces (cf. p. 45 of [Tri4]). Fix $\varphi_{0} \in C^{\infty}\left(\mathbb{R}^{n}\right)$ such that $\varphi_{0}(x)=1$ if $|x| \leq 1$ and $\operatorname{supp} \varphi_{0} \subset\left\{x \in \mathbb{R}^{n}:|x| \leq 2\right\}$. Then introduce

$$
\varphi_{j}(x):=\varphi_{0}\left(2^{-j} x\right)-\varphi_{0}\left(2^{-j+1} x\right), \quad x \in \mathbb{R}^{n}, j \in \mathbb{N} .
$$

It follows that $\left\{\varphi_{j}\right\}_{j=0}^{\infty}$ is a partition of unity, i.e.

$$
\sum_{j=0}^{\infty} \varphi_{j}(x)=1 \quad \text { for } \quad x \in \mathbb{R}^{n}
$$

Definition 1.2.8. For $0<p, q \leq \infty, \alpha \in \mathbb{R}$, define

$$
B_{\alpha}^{p, q}\left(\mathbb{R}^{n}\right):=\left\{f \in S^{\prime}\left(\mathbb{R}^{n}\right):\|f\|_{B_{\alpha}^{p, q}\left(\mathbb{R}^{n}\right)}:=\left(\sum_{j=0}^{\infty} 2^{j \alpha q}\left\|\mathcal{F}^{-1}\left(\varphi_{j} \mathcal{F} f\right)\right\|_{L^{p}\left(\mathbb{R}^{n}\right)}^{q}\right)^{1 / q}<\infty\right\}
$$

and, for $p \neq \infty$,

$$
F_{\alpha}^{p, q}\left(\mathbb{R}^{n}\right):=\left\{f \in \mathrm{S}^{\prime}\left(\mathbb{R}^{n}\right):\|f\|_{F_{\alpha}^{p, q}\left(\mathbb{R}^{n}\right)}:=\left\|\left(\sum_{j=0}^{\infty} 2^{j \alpha q}\left|\mathcal{F}^{-1}\left(\varphi_{j} \mathcal{F} f\right)\right|^{q}\right)^{1 / q}\right\|_{L^{p}\left(\mathbb{R}^{n}\right)}<\infty\right\}
$$

with the usual modification if $q=\infty$, i.e.

$$
B_{\alpha}^{p, \infty}\left(\mathbb{R}^{n}\right):=\left\{f \in \mathrm{S}^{\prime}\left(\mathbb{R}^{n}\right):\|f\|_{B_{\alpha}^{p, \infty}\left(\mathbb{R}^{n}\right)}:=\sup _{j \in \mathbb{N}_{0}}\left[2^{j \alpha}\left\|\mathcal{F}^{-1}\left(\varphi_{j} \mathcal{F} f\right)\right\|_{L^{p}\left(\mathbb{R}^{n}\right)}\right]<\infty\right\}
$$

and, for $p \neq \infty$,

$$
F_{\alpha}^{p, \infty}\left(\mathbb{R}^{n}\right):=\left\{f \in \mathrm{S}^{\prime}\left(\mathbb{R}^{n}\right):\|f\|_{F_{\alpha}^{p, \infty}\left(\mathbb{R}^{n}\right)}:=\left\|\sup _{j \in \mathbb{N}_{0}}\left(2^{j \alpha}\left|\mathcal{F}^{-1}\left(\varphi_{j} \mathcal{F} f\right)\right|\right)\right\|_{L^{p}\left(\mathbb{R}^{n}\right)}<\infty\right\}
$$


Note that different choices of $\varphi_{0}$ yield the same function spaces with equivalent norms. For this, we refer the reader to Proposition 1 in 2.3.2 of [Tri4].

As is well known (see, e.g. Theorem on p. 48 and Proposition 2 on p. 47 in [Tri4]), for $0<p, q \leq \infty, \alpha \in \mathbb{R}$ the spaces $B_{\alpha}^{p, q}\left(\mathbb{R}^{n}\right)$ and $F_{\alpha}^{p, q}\left(\mathbb{R}^{n}\right)(p \neq \infty$ for the $F$-scale $)$ are complete, for $p, q \geq 1$ they are Banach spaces, and in general

$$
\begin{aligned}
& \mathrm{S}\left(\mathbb{R}^{n}\right) \hookrightarrow B_{\alpha}^{p, q}\left(\mathbb{R}^{n}\right) \hookrightarrow \mathrm{S}^{\prime}\left(\mathbb{R}^{n}\right), \\
& \mathrm{S}\left(\mathbb{R}^{n}\right) \hookrightarrow F_{\alpha}^{p, q}\left(\mathbb{R}^{n}\right) \hookrightarrow \mathrm{S}^{\prime}\left(\mathbb{R}^{n}\right) \text { if } \quad p \neq \infty
\end{aligned}
$$

are continuous, while the first inclusions in (1.2.22) and (1.2.23) are dense provided $0<p, q<\infty$. Moreover, the following simple embedding results hold for $0<p \leq \infty$ $\left(p \neq \infty\right.$ for the Triebel-Lizorkin scale) and $\alpha, \alpha_{0}, \alpha_{1} \in \mathbb{R}$.

$$
\begin{gathered}
B_{\alpha}^{p, q_{0}}\left(\mathbb{R}^{n}\right) \hookrightarrow B_{\alpha}^{p, q_{1}}\left(\mathbb{R}^{n}\right), \quad F_{\alpha}^{p, q_{0}}\left(\mathbb{R}^{n}\right) \hookrightarrow F_{\alpha}^{p, q_{1}}\left(\mathbb{R}^{n}\right) \quad \text { for } 0<q_{0} \leq q_{1} \leq \infty, \\
B_{\alpha_{0}}^{p, q}\left(\mathbb{R}^{n}\right) \hookrightarrow B_{\alpha_{1}}^{p, r}\left(\mathbb{R}^{n}\right), F_{\alpha_{0}}^{p, q}\left(\mathbb{R}^{n}\right) \hookrightarrow F_{\alpha_{1}}^{p, r}\left(\mathbb{R}^{n}\right) \quad \text { for } \alpha_{0}>\alpha_{1}, 0<q, r \leq \infty, \\
B_{\alpha}^{p, \min \{p, q\}}\left(\mathbb{R}^{n}\right) \hookrightarrow F_{\alpha}^{p, q}\left(\mathbb{R}^{n}\right) \hookrightarrow B_{\alpha}^{p, \max \{p, q\}}\left(\mathbb{R}^{n}\right), \quad 0<q \leq \infty
\end{gathered}
$$

In order to simplify the notation, we will write $B_{\alpha}^{p}$ in place of $B_{\alpha}^{p, p}$ for $0<p \leq \infty$ and $\alpha \in \mathbb{R}$.

Proposition 1.2.9. (Part (i) of Definition in 2.5.13 of [Tri4])

Let $0<p \leq \infty$ and $\alpha>n\left(\frac{1}{\min (p, 1)}-1\right)$. Then $B_{\alpha}^{p}\left(\mathbb{R}^{n}\right)$ satisfies the Fubini property, i.e.

$$
\sum_{j=1}^{n}\|\| f\left(x_{1}, \ldots, x_{j-1}, \cdot, x_{j+1}, \ldots, x_{n}\right)\left\|_{B_{\alpha}^{p}(\mathbb{R})}\right\|_{L^{p}\left(\mathbb{R}^{n-1}\right)}
$$

is an equivalent quasi-norm in $B_{\alpha}^{p}\left(\mathbb{R}^{n}\right)$. 
Definition 1.2.10. For $1<p<\infty$ and $\alpha \in \mathbb{R}$ we define the (isotropic) Sobolev spaces as

$$
L_{\alpha}^{p}\left(\mathbb{R}^{n}\right):=F_{\alpha}^{p, 2}\left(\mathbb{R}^{n}\right)
$$

As on p. 333 in [Gri2], for $1 \leq p \leq \infty, k \in \mathbb{N}_{0}$ and a Banach space $\mathrm{X}$, the space $L_{k}^{p}(\mathbb{R} ; X)$ is defined as

$$
L_{k}^{p}(\mathbb{R} ; X):=\left\{f \in L^{p}(\mathbb{R} ; X): \frac{d^{j}}{d t^{j}} f \in L^{p}(\mathbb{R} ; X), 1 \leq i \leq k\right\}
$$

Next, for $1 \leq p \leq \infty, \alpha \notin \mathbb{N}, \alpha>0$ and a Banach space $\mathrm{X}$, we define

$$
L_{\alpha}^{p}(\mathbb{R} ; X):=\left[L_{k}^{p}(\mathbb{R} ; X), L_{k+1}^{p}(\mathbb{R} ; X)\right]_{\theta},
$$

where $[\cdot, \cdot]_{\theta}$ denotes the complex interpolation method, $k:=[\alpha], 0<\theta<1$ and $\alpha=k+\theta$

Proposition 1.2.11. For $1<p<\infty$ and $\alpha>0$, there holds

$$
L_{\alpha}^{p}\left(\mathbb{R} ; L^{p}\left(\mathbb{R}^{n}\right)\right)=L^{p}\left(\mathbb{R}^{n} ; L_{\alpha}^{p}(\mathbb{R})\right) .
$$

Proof. For $\alpha \in \mathbb{N}_{0}$ there is nothing to prove. When $\alpha>0$ is not an integer, by (1.2.28) and the result of this proposition for integer $\alpha$, we obtain that

$$
\begin{aligned}
L_{\alpha}^{p}\left(\mathbb{R} ; L^{p}\left(\mathbb{R}^{n}\right)\right) & =\left[L^{p}\left(\mathbb{R}^{n} ; L_{k}^{p}(\mathbb{R})\right), L^{p}\left(\mathbb{R}^{n} ; L_{k+1}^{p}(\mathbb{R})\right)\right]_{\theta} \\
& =L^{p}\left(\mathbb{R}^{n} ;\left[L_{k}^{p}(\mathbb{R}), L_{k+1}^{p}(\mathbb{R})\right]_{\theta}\right) \\
& =L^{p}\left(\mathbb{R}^{n} ; L_{\alpha}^{p}(\mathbb{R})\right),
\end{aligned}
$$

where $\alpha=k+\theta$ and $0<\theta<1$. Here we have also used Theorems 5.1.2 and 6.4.5 from $[\mathrm{BeL} \ddot{\mathrm{O}}]$. 


\subsubsection{Review of Fourier transform}

Given $f \in \mathrm{S}\left(\mathbb{R}^{n}\right)$, we define its Fourier transform by

$$
\mathcal{F} f(\xi)=\hat{f}(\xi):=\frac{1}{(2 \pi)^{n / 2}} \int_{\mathbb{R}^{n}} e^{-i x \cdot \xi} f(x) d x, \quad \xi \in \mathbb{R}^{n}
$$

and its invers Fourier transform by

$$
\mathcal{F}^{-1} f(\xi)=\check{f}(\xi):=\frac{1}{(2 \pi)^{n / 2}} \int_{\mathbb{R}^{n}} e^{i x \cdot \xi} f(x) d x, \quad \xi \in \mathbb{R}^{n} .
$$

Some of the basic properties of these operators are as follows. For $f, g \in \mathbf{S}\left(\mathbb{R}^{n}\right)$,

1. $\mathcal{F}\left(\mathcal{F}^{-1} f\right)=f=\mathcal{F}^{-1}(\mathcal{F} f)$,

2. (Plancherel) $\|f\|_{L^{2}\left(\mathbb{R}^{n}\right)}=\|\mathcal{F} f\|_{L^{2}\left(\mathbb{R}^{n}\right)}=\left\|\mathcal{F}^{-1} f\right\|_{L^{2}\left(\mathbb{R}^{n}\right)}$,

3. $\mathcal{F}\left(\partial^{\alpha} f\right)(\xi)=(i \xi)^{\alpha} \mathcal{F} f(\xi)$, where $\xi \in \mathbb{R}^{n}, \alpha \in \mathbb{N}_{0}^{n}$,

4. $\mathcal{F}(f * g)=(2 \pi)^{n / 2} \mathcal{F} f \mathcal{F} g$,

5. $\mathcal{F}(f g)=(2 \pi)^{-n / 2}(\mathcal{F} f * \mathcal{F} g)$,

6. $\int_{\mathbb{R}^{n}} f(x) \overline{g(x)} d x=\int_{\mathbb{R}^{n}} \mathcal{F} f(\xi) \overline{\mathcal{F} g(\xi)} d \xi$.

Definition 1.2.12. Given $f \in \mathrm{S}\left(\mathbb{R}^{n}\right)$ we define its Fourier transform in the $j$-th component $(1 \leq j \leq n), \mathcal{F}_{j} f$, by $\left(\mathcal{F}_{j} f\right)\left(x_{1}, \ldots, x_{j-1}, \xi, x_{j+1}, \ldots, x_{n}\right):=\frac{1}{\sqrt{2 \pi}} \int_{\mathbb{R}} e^{-i x_{j} \xi} f\left(x_{1}, \ldots, x_{j-1}, x_{j}, x_{j+1}, \ldots, x_{n}\right) d x_{j}$, where $\xi \in \mathbb{R}$.

Proposition 1.2.13. With the above notation we have $\mathcal{F}=\mathcal{F}_{1} \circ \mathcal{F}_{2} \circ \ldots \circ \mathcal{F}_{n}$, where $\mathcal{F}$ represents the (global) Fourier transform in $\mathbb{R}^{n}$, introduced in (1.2.29) . 
Proof. The proof is straightforward. Consider $f \in \mathrm{S}\left(\mathbb{R}^{n}\right)$ and $\xi \in \mathbb{R}^{n}$. Then

$$
\begin{aligned}
& (\mathcal{F} f)(\xi)=\frac{1}{(2 \pi)^{n / 2}} \int_{\mathbb{R}^{n}} \prod_{j=1}^{n} e^{-i \xi_{j} x_{j}} f\left(x_{1}, \ldots, x_{n}\right) d x_{1} \ldots d x_{n} \\
& =\frac{1}{\sqrt{2 \pi}} \int_{\mathbb{R}} e^{-i \xi_{1} x_{1}}\left(\frac{1}{\sqrt{2 \pi}} \int_{\mathbb{R}} e^{-i \xi_{2} x_{2}}\left(\ldots\left(\frac{1}{\sqrt{2 \pi}} \int_{\mathbb{R}} e^{-i \xi_{n} x_{n}} f\left(x_{1}, \ldots, x_{n}\right) d x_{n}\right) \ldots\right) d x_{2}\right) d x_{1} \\
& =\mathcal{F}_{1}\left(\mathcal{F}_{2}\left(\ldots\left(\mathcal{F}_{n} f\right) \ldots\right)\right)\left(\xi_{1}, \xi_{2}, \ldots, \xi_{n}\right)=\left(\mathcal{F}_{1} \circ \mathcal{F}_{2} \circ \ldots \circ \mathcal{F}_{n} f\right)(\xi),
\end{aligned}
$$

which is what we wanted to show.

Definition 1.2.14. For $f \in \mathrm{S}\left(\mathbb{R}^{n} \times \mathbb{R}\right)$ define the Fourier transform in the time variable, $\mathcal{F}_{\text {time }}$, by

$$
\left(\mathcal{F}_{\text {time }} f\right)(x, \tau):=\frac{1}{\sqrt{2 \pi}} \int_{\mathbb{R}} e^{-i t \tau} f(x, t) d t, \quad x \in \mathbb{R}^{n}, \tau \in \mathbb{R}
$$

and recall from (1.2.29) the definition of the (global) Fourier transform in $\mathbb{R}^{n} \times \mathbb{R}$

$$
(\mathcal{F} f)(\xi, \tau):=\frac{1}{(2 \pi)^{(n+1) / 2}} \int_{\mathbb{R}^{n} \times \mathbb{R}} e^{-i(x, t) \cdot(\xi, \tau)} f(x, t) d x d t, \quad \xi \in \mathbb{R}^{n}, \tau \in \mathbb{R} .
$$

The corresponding invers Fourier transforms are defined as

$$
\left(\mathcal{F}_{\text {time }}^{-1} f\right)(x, \tau):=\frac{1}{\sqrt{2 \pi}} \int_{\mathbb{R}} e^{i t \tau} f(x, t) d t, \quad x \in \mathbb{R}^{n}, \tau \in \mathbb{R}
$$

and

$$
\left(\mathcal{F}^{-1} f\right)(\xi, \tau):=\frac{1}{(2 \pi)^{(n+1) / 2}} \int_{\mathbb{R}^{n} \times \mathbb{R}} e^{i(x, t) \cdot(\xi, \tau)} f(x, t) d x d t, \quad \xi \in \mathbb{R}^{n} \tau \in \mathbb{R}
$$

A word of warning is in order here. The Fourier transform (1.2.29) on $\mathbb{R}^{n}$ will be used in the context of distributions in $\mathbb{R}^{n} \times \mathbb{R}$. We shall nonetheless continue to employ the same symbol $\mathcal{F}$. Also, typically, the Fourier variables corresponding to $(x, t) \in \mathbb{R}^{n} \times \mathbb{R}$ will be denoted by $(\xi, \tau)$. 
Definition 1.2.15. Consider $f \in \mathrm{S}\left(\mathbb{R}^{n} \times \mathbb{R}\right)$ and $\theta \in \mathbb{R}$. For $x, \xi \in \mathbb{R}^{n}$ and $t, \tau \in \mathbb{R}$ define the following operators:

$$
\begin{aligned}
\left(\partial_{\text {time }}^{\theta} f\right)(x, t) & :=\mathcal{F}_{\text {time }}^{-1}\left[\left(1+\tau^{2}\right)^{\theta / 2}\left(\mathcal{F}_{\text {time }} f(x, \cdot)\right)(\tau)\right](t), \\
\left(\partial_{t}^{\theta} f\right)(x, t) & :=\mathcal{F}^{-1}\left[\left(1+\tau^{2}\right)^{\theta / 2}(\mathcal{F} f)(\xi, \tau)\right](x, t) .
\end{aligned}
$$

Proposition 1.2.16. The operators introduced in Definition 1.2.15 are equal in the sense that for any complex-valued test function $\varphi \in \mathrm{S}\left(\mathbb{R}^{n} \times \mathbb{R}\right)$, the following holds:

$$
\int_{\mathbb{R}^{n} \times \mathbb{R}}\left(\partial_{t}^{\theta} f\right)(x, t) \overline{\varphi(x, t)} d x d t=\int_{\mathbb{R}^{n} \times \mathbb{R}}\left(\partial_{t i m e}^{\theta} f\right)(x, t) \overline{\varphi(x, t)} d x d t
$$

Proof. The proof consists of a direct calculation. According to Definition 1.2.15, using Parseval's theorem and Proposition 1.2.13, we have

$$
\begin{aligned}
& \int_{\mathbb{R}^{n} \times \mathbb{R}}\left(\partial_{t}^{\theta} f\right)(x, t) \overline{\varphi(x, t)} d x d t \\
& =\int_{\mathbb{R}^{n} \times \mathbb{R}}\left(1+\tau^{2}\right)^{\theta / 2}(\mathcal{F} f)(\xi, \tau) \overline{(\mathcal{F} \varphi)(\xi, \tau)} d \xi d \tau \\
& =\int_{\mathbb{R}}\left(1+\tau^{2}\right)^{\theta / 2}\left[\int_{\mathbb{R}^{n}} \mathcal{F}_{1}\left(\ldots\left(\mathcal{F}_{n}\left(\mathcal{F}_{\text {time }} f\right)\right) \ldots\right)\left(\xi_{1}, \ldots, \xi_{n}, \tau\right)\right. \\
& \left.\quad \times \overline{\mathcal{F}_{1}\left(\ldots\left(\mathcal{F}_{n}\left(\mathcal{F}_{\text {time }} \varphi\right)\right) \ldots\right)\left(\xi_{1}, \ldots, \xi_{n}, \tau\right)} d \xi_{1} \ldots d \xi_{n}\right] d \tau \\
& =\int_{\mathbb{R}}\left(1+\tau^{2}\right)^{\theta / 2}\left[\int_{\mathbb{R}^{n}}\left(\mathcal{F}_{\text {time }} f\right)(x, \tau) \overline{\left(\mathcal{F}_{\text {time }} \varphi\right)(x, \tau)} d x\right] d \tau \\
& =\int_{\mathbb{R}^{n}} \int_{\mathbb{R}} \mathcal{F}_{\text {time }}\left\{\mathcal{F}_{\text {time }}^{-1}\left[\left(1+\tau^{2}\right)^{\theta / 2}\left(\mathcal{F}_{\text {time }} f(x, \cdot)\right)(\tau)\right](t)\right\}(\tau) \overline{\left(\mathcal{F}_{\text {time }} \varphi\right)(x, \tau)} d \tau d x \\
& =\int_{\mathbb{R}^{n} \times \mathbb{R}}\left(\partial_{\text {time }}^{\theta} f\right)(x, t) \overline{\varphi(x, t)} d x d t
\end{aligned}
$$

This completes the proof of the proposition.

Proposition 1.2.17. The operator $\partial_{\text {time }}^{\theta}$ described in (1.2.31) is self-adjoint, i.e.

$$
\int_{\mathbb{R}^{n} \times \mathbb{R}}\left(\partial_{\text {time }}^{\theta} f\right)(x, t) \overline{g(x, t)} d x d t=\int_{\mathbb{R}^{n} \times \mathbb{R}} f(x, t)\left(\partial_{\text {time }}^{\theta} \bar{g}\right)(x, t) d x d t
$$


for any complex-valued functions $f, g \in \mathrm{S}\left(\mathbb{R}^{n} \times \mathbb{R}\right)$.

Proof. If we denote the left-hand side of (1.2.33) by LHS then, by the identity already established in Proposition 1.2.16 and Parseval's theorem, we obtain that

$$
L H S=\int_{\mathbb{R}^{n} \times \mathbb{R}}\left(1+\tau^{2}\right)^{\theta / 2} \mathcal{F} f(\xi, \tau) \overline{\mathcal{F} g(\xi, \tau)} d \xi d \tau .
$$

Using the same ingredients as before, and denoting the right-hand side of (1.2.33) by $R H S$, we get the following.

$$
\begin{aligned}
R H S & =\int_{\mathbb{R}^{n} \times \mathbb{R}} f(x, t) \mathcal{F}\left[\left(1+\tau^{2}\right)^{\theta / 2} \overline{\mathcal{F} g(-\xi,-\tau)}\right](x, t) d x d t \\
& =\int_{\mathbb{R}^{n} \times \mathbb{R}} \mathcal{F}\left(\mathcal{F}^{-1} f\right)(x, t) \overline{\mathcal{F}\left[\left(1+\tau^{2}\right)^{\theta / 2}(\mathcal{F} g)(-\xi,-\tau)\right]}(x, t) d x d t \\
& =\int_{\mathbb{R}^{n} \times \mathbb{R}} \mathcal{F}^{-1} f(\xi, \tau)\left(1+\tau^{2}\right)^{\theta / 2} \overline{\mathcal{F} g(-\xi,-\tau)} d \xi d \tau .
\end{aligned}
$$

Making the change of variables $-\xi=\eta \in \mathbb{R}^{n}$ and $-\tau=r \in \mathbb{R}$, we further obtain that

$$
\begin{aligned}
R H S & =\int_{\mathbb{R}^{n} \times \mathbb{R}} \mathcal{F}^{-1} f(-\eta,-r)\left(1+r^{2}\right)^{\theta / 2} \overline{\mathcal{F} g(\eta, r)} d \eta d r \\
& =\int_{\mathbb{R}^{n} \times \mathbb{R}}\left(1+r^{2}\right)^{\theta / 2} \mathcal{F} f(\eta, r) \overline{\mathcal{F} g(\eta, r)} d \eta d r .
\end{aligned}
$$

Finally, (1.2.33) follows from (1.2.34) and 1.2.35).

\subsubsection{Review of multipliers}

Let $m$ be a bounded measurable function on $\mathbb{R}^{n}$. For $f \in L^{2}\left(\mathbb{R}^{n}\right) \cap L^{p}\left(\mathbb{R}^{n}\right)$, $1<p<\infty$, one can define the linear transformation $T_{m}$ by

$$
\mathcal{F}\left(T_{m} f\right)(\xi)=m(\xi) \mathcal{F} f(\xi)
$$

We say that $m$ is a multiplier for $L^{p}\left(\mathbb{R}^{n}\right), 1 \leq p \leq \infty$, if $f \in L^{2}\left(\mathbb{R}^{n}\right) \cap L^{p}\left(\mathbb{R}^{n}\right)$ implies $T_{m} f \in L^{p}\left(\mathbb{R}^{n}\right)$, and $\left\|T_{m} f\right\|_{L^{p}\left(\mathbb{R}^{n}\right)} \leq c\|f\|_{L^{p}\left(\mathbb{R}^{n}\right)}$, with $c$ independent of $f$. 
Remark 1.2.18. If $m_{1}$ and $m_{2}$ are multipliers for $L^{p}\left(\mathbb{R}^{n}\right)$, then their product $m_{1} m_{2}$ is a multiplier for $L^{p}\left(\mathbb{R}^{n}\right)$ as well.

Theorem 1.2.19. As a consequence of the Marcinkiewicz multiplier theorem, $m$ is a multiplier for $L^{p}\left(\mathbb{R}^{n}\right)(1<p<\infty)$ if one of the following criteria holds:

$$
\begin{aligned}
& \sup _{|\alpha| \leq[n / 2]+1, \xi \in \mathbb{R}^{n}}|\xi|^{|\alpha|}\left|D_{\xi}^{\alpha} m(\xi)\right|<\infty \quad \text { (Mihlin, Theorem 2 of [Mih]), } \\
& \sup _{\alpha_{i} \in\{0,1\}, \xi \in \mathbb{R}^{n}}\left|\xi^{\alpha} D_{\xi}^{\alpha} m(\xi)\right|<\infty \quad \text { (Lizorkin, Theorem } 8 \text { in [LizQ]). }
\end{aligned}
$$

In what follows we shall give several examples of multipliers for $L^{p}\left(\mathbb{R}^{n} \times \mathbb{R}\right)$, where $1<p<\infty$. These multipliers are going to be especially useful for us in Chapter 2 .

\section{Examples of multipliers for $L^{p}\left(\mathbb{R}^{n} \times \mathbb{R}\right), 1<p<\infty$.}

\section{Example 1.}

$$
m_{1}(\xi, \tau):=\frac{\sqrt{|\xi|^{2}+i \tau}}{\sum_{j=1}^{n}\left|\xi_{j}\right|+|\tau|^{1 / 2}}, \quad \xi \in \mathbb{R}^{n}, \tau \in \mathbb{R} .
$$

Proof. Based on Lizorkin's citerion (1.2.37) we need to prove that

$$
\sup _{\substack{\alpha, \beta \in\{0,1\} \\(\xi, \tau) \in \mathbb{R}^{n} \times \mathbb{R}}}\left|\xi_{1}^{\alpha_{1}} \ldots \xi_{n}^{\alpha_{n}} \tau^{\beta} \partial_{\xi_{1}}^{\alpha_{1}} \ldots \partial_{\xi_{n}}^{\alpha_{n}} \partial_{\tau}^{\beta} m_{1}(\xi, \tau)\right|<\infty .
$$

We consider several separate cases starting with:

CASE 1. $\alpha_{j}=\beta=0$ for all $j \in\{1, \ldots, n\}$. Then, using the fact that $|\sqrt{z}|=\sqrt{|z|}$ for any complex number $z$,

$$
\left|m_{1}(\xi, \tau)\right| \leq c \frac{\left(|\xi|^{4}+|\tau|^{2}\right)^{1 / 4}}{|\xi|+|\tau|^{1 / 2}}<\infty
$$

CASE 2. $\alpha_{j}=0$ for all $j \in\{1, \ldots, n\}, \beta=1$. Then

$$
\left|\tau \partial_{\tau} m_{1}(\xi, \tau)\right| \leq c \frac{|\tau|}{\left(|\xi|+|\tau|^{1 / 2}\right)\left(|\xi|^{4}+|\tau|^{2}\right)^{1 / 4}}+c \frac{|\tau|^{1 / 2}\left(|\xi|^{4}+|\tau|^{2}\right)^{1 / 4}}{\left(|\xi|+|\tau|^{1 / 2}\right)^{2}}<\infty
$$


CASE 3. $\alpha_{1}=1, \alpha_{j}=0$ for $j \in\{2, \ldots, n\}, \beta=1$. Along the same lines as before,

$$
\begin{aligned}
\left|\xi_{1} \tau \partial_{\xi_{1}} \partial_{\tau} m_{1}(\xi, \tau)\right| \leq & c \frac{|\tau||\xi|^{2}}{\left(|\xi|+|\tau|^{1 / 2}\right)^{4}}+c \frac{|\tau||\xi|}{\left(|\xi|+|\tau|^{1 / 2}\right)^{3}} \\
& +c \frac{|\tau|^{1 / 2}|\xi|^{2}}{\left(|\xi|+|\tau|^{1 / 2}\right)^{3}}+c \frac{|\tau|^{1 / 2}|\xi|}{\left(|\xi|+|\tau|^{1 / 2}\right)^{2}}<\infty .
\end{aligned}
$$

All the other cases are handled in a similar fashion.

\section{Example 2.}

$$
m_{2}(\xi, \tau):=\frac{\sum_{j=1}^{n}\left|\xi_{j}\right|+|\tau|^{1 / 2}}{\sqrt{|\xi|^{2}+i \tau}}, \quad \xi \in \mathbb{R}^{n}, \tau \in \mathbb{R} .
$$

Proof. We will show that $m_{2}$ satisfies

$$
\sup _{\substack{\alpha_{j}, \beta \in\{0,1\} \\(\xi, \tau) \in \mathbb{R}^{n} \times \mathbb{R}}}\left|\xi_{1}^{\alpha_{1}} \ldots \xi_{n}^{\alpha_{n}} \tau^{\beta} \partial_{\xi_{1}}^{\alpha_{1}} \ldots \partial_{\xi_{n}}^{\alpha_{n}} \partial_{\tau}^{\beta} m_{2}(\xi, \tau)\right|<\infty
$$

CAsE $1 . \alpha_{j}=\beta=0$ for all $j \in\{1, \ldots, n\}$. Then, using the fact that $|\sqrt{z}|=\sqrt{|z|}$ for any complex number $z$,

$$
\left|m_{2}(\xi, \tau)\right| \leq c \frac{|\xi|+|\tau|^{1 / 2}}{\left(|\xi|^{4}+|\tau|^{2}\right)^{1 / 4}}<\infty
$$

CASE 2. $\alpha_{j}=0$ for all $j \in\{1, \ldots, n\}, \beta=1$. Then

$$
\left|\tau \partial_{\tau} m_{2}(\xi, \tau)\right| \leq c \frac{|\tau|^{1 / 2}}{\left(|\xi|^{4}+|\tau|^{2}\right)^{1 / 4}}+c \frac{|\tau|\left(|\xi|+|\tau|^{1 / 2}\right)}{\left(|\xi|^{4}+|\tau|^{2}\right)^{3 / 4}}<\infty
$$

CASE 3. $\alpha_{1}=1, \alpha_{j}=0$ for $j \in\{2, \ldots, n\}, \beta=1$. Along the same lines as before,

$$
\left|\xi_{1} \tau \partial_{\xi_{1}} \partial_{\tau} m_{2}(\xi, \tau)\right| \leq c \frac{|\tau|^{1 / 2}|\xi|^{2}}{\left(|\xi|^{4}+|\tau|^{2}\right)^{3 / 4}}+c \frac{|\tau||\xi|}{\left(|\xi|^{4}+|\tau|^{2}\right)^{3 / 4}}+c \frac{|\tau||\xi|^{2}}{\left(|\xi|+|\tau|^{1 / 2}\right)^{4}}<\infty
$$

The other cases are handled in a similar way.

\section{Example 3.}

$$
m_{3}(\xi, \tau):=\frac{\left(1+|\xi|^{4}+\tau^{2}\right)^{\alpha / 4}}{\sum_{j=1}^{n}\left(1+\xi_{j}^{2}\right)^{\alpha / 2}+\left(1+\tau^{2}\right)^{\alpha / 4}}, \quad \xi \in \mathbb{R}^{n}, \tau \in \mathbb{R}
$$


Proof. Based on Lizorkin's criterion (1.2.37) we need to show that

$$
\sup _{\substack{\beta_{i}, \gamma \in\{0,1\} \\(\xi, \tau) \in \mathbb{R}^{n} \times \mathbb{R}}}\left|\xi_{1}^{\beta_{1}} \ldots \xi_{n}^{\beta_{n}} \tau^{\gamma} \partial_{\xi_{1}}^{\beta_{1}} \ldots \partial_{\xi_{n}}^{\beta_{n}} \partial_{\tau}^{\gamma} m_{3}(\xi, \tau)\right|<\infty
$$

CASE $1 . \beta_{j}=\gamma=0$ for all $j \in\{1, \ldots, n\}$. The inequality $\left|m_{3}(\xi, \tau)\right|<\infty$ is then equivalent with

$$
1+|\xi|^{4}+\tau^{2} \leq c\left(\sum_{k=1}^{n}\left(1+\xi_{k}^{2}\right)^{2}+1+\tau^{2}\right)
$$

or, with

$$
|\xi|^{4} \leq c\left(n+|\xi|^{2}\right)^{2}
$$

which is certainly true.

CAsE 2. $\beta_{j}=0$ for all $j \in\{1, \ldots, n\}, \gamma=1$. Then $\left|\tau \partial_{\tau} m_{3}(\xi, \tau)\right|<\infty$ if

$$
\frac{\left(1+|\xi|^{4}+\tau^{2}\right)^{\alpha / 4-1} \tau^{2}}{\sum_{j=1}^{n}\left(1+\xi_{j}^{2}\right)^{\alpha / 2}+\left(1+\tau^{2}\right)^{\alpha / 4}}<\infty
$$

and

$$
\frac{\tau^{2}\left(1+\tau^{2}\right)^{\alpha / 4-1}\left(1+|\xi|^{4}+\tau^{2}\right)^{\alpha / 4}}{\left(\sum_{j=1}^{n}\left(1+\xi_{j}^{2}\right)^{\alpha / 2}+\left(1+\tau^{2}\right)^{\alpha / 4}\right)^{2}}<\infty .
$$

The inequality (1.2.42) can be rewritten in the following form:

$$
m_{3}(\xi, \tau) \frac{\tau^{2}}{1+|\xi|^{4}+\tau^{2}}<\infty
$$

which, based on Case 1, holds. The left-hand side of (1.2.43) is bounded by

$$
\frac{\left(1+\tau^{2}\right)^{\alpha / 4}\left(1+|\xi|^{4}+\tau^{2}\right)^{\alpha / 4}}{\left(\sum_{j=1}^{n}\left(1+\xi_{j}^{2}\right)^{\alpha / 2}+\left(1+\tau^{2}\right)^{\alpha / 4}\right)^{2}}
$$

which, in turn, is controlled by $m_{3}(\xi, \tau)$. Therefore, with the help of Case 1 , the inequality (1.2.43) holds, as well. 
CASE 3. $\beta_{1}=1, \beta_{j}=0$ for $j \in\{2, \ldots, n\}, \gamma=1$. Then $\left|\xi_{1} \tau \partial_{\xi_{1}} \partial_{\tau} m_{3}(\xi, \tau)\right|<\infty$ if

$$
\begin{gathered}
\frac{\tau^{2}\left(1+|\xi|^{4}+\tau^{2}\right)^{\alpha / 4-2}|\xi|^{2}\left|\xi_{1}\right|^{2}}{\sum_{j=1}^{n}\left(1+\xi_{j}^{2}\right)^{\alpha / 2}+\left(1+\tau^{2}\right)^{\alpha / 4}}<\infty, \\
\frac{\tau^{2}\left(1+|\xi|^{4}+\tau^{2}\right)^{\alpha / 4-1}\left(1+\xi_{1}^{2}\right)^{\alpha / 2-1}\left|\xi_{1}\right|^{2}}{\left(\sum_{j=1}^{n}\left(1+\xi_{j}^{2}\right)^{\alpha / 2}+\left(1+\tau^{2}\right)^{\alpha / 4}\right)^{2}}<\infty, \\
\frac{|\tau|\left(1+\tau^{2}\right)^{\alpha / 4-1}\left(1+|\xi|^{4}+\tau^{2}\right)^{\alpha / 4-1}|\xi|^{2}\left|\xi_{1}\right|^{2}}{\left(\sum_{j=1}^{n}\left(1+\xi_{j}^{2}\right)^{\alpha / 2}+\left(1+\tau^{2}\right)^{\alpha / 4}\right)^{2}}<\infty,
\end{gathered}
$$

and

$$
\frac{|\tau|\left(1+\tau^{2}\right)^{\alpha / 4-1}\left(1+|\xi|^{4}+\tau^{2}\right)^{\alpha / 4}\left(1+\xi_{1}^{2}\right)^{\alpha / 2-1}\left|\xi_{1}\right|^{2}}{\left(\sum_{j=1}^{n}\left(1+\xi_{j}^{2}\right)^{\alpha / 2}+\left(1+\tau^{2}\right)^{\alpha / 4}\right)^{3}}<\infty .
$$

The left-hand side of (1.2.44) does not exceed

$$
\frac{\left(1+|\xi|^{4}+\tau^{2}\right)^{\alpha / 4-1}|\xi|^{4}}{\sum_{j=1}^{n}\left(1+\xi_{j}^{2}\right)^{\alpha / 2}+\left(1+\tau^{2}\right)^{\alpha / 4}} \leq\left|m_{3}(\xi, \tau)\right|<\infty
$$

Similarly, the left-hand side of (1.2.45) is controlled by

$$
\frac{\left(1+|\xi|^{4}+\tau^{2}\right)^{\alpha / 4}\left(1+\xi_{1}^{2}\right)^{\alpha / 2}}{\left(\sum_{j=1}^{n}\left(1+\xi_{j}^{2}\right)^{\alpha / 2}+\left(1+\tau^{2}\right)^{\alpha / 4}\right)^{2}} \leq\left|m_{3}(\xi, \tau)\right|<\infty
$$

Going further, using (1.2.48) and the fact that $2|\tau| \leq 1+\tau^{2}$, we can conclude that the left-hand side of (1.2.46) is bounded by

$$
\frac{\left(1+\tau^{2}\right)^{\alpha / 4}\left(1+|\xi|^{4}+\tau^{2}\right)^{\alpha / 4-1}|\xi|^{4}}{\left(\sum_{j=1}^{n}\left(1+\xi_{j}^{2}\right)^{\alpha / 2}+\left(1+\tau^{2}\right)^{\alpha / 4}\right)^{2}} \leq \frac{\left(1+|\xi|^{4}+\tau^{2}\right)^{\alpha / 4-1}|\xi|^{4}}{\sum_{j=1}^{n}\left(1+\xi_{j}^{2}\right)^{\alpha / 2}+\left(1+\tau^{2}\right)^{\alpha / 4}}<\infty .
$$

Finally, using some elementary estimates and Case 1, the left-hand side of (1.2.47) is controlled by

$$
\frac{\left(1+\tau^{2}\right)^{\alpha / 4}\left(1+|\xi|^{4}+\tau^{2}\right)^{\alpha / 4}\left(1+\xi_{1}^{2}\right)^{\alpha / 2}}{\left(\sum_{j=1}^{n}\left(1+\xi_{j}^{2}\right)^{\alpha / 2}+\left(1+\tau^{2}\right)^{\alpha / 4}\right)^{3}} \leq\left|m_{3}(\xi, \tau)\right|<\infty,
$$


which completes the proof in Case 3. All the other possible cases can be reduced to the first one (as we could observe in Cases 2 and 3).

\section{Example 4.}

$$
m_{4}(\xi, \tau):=\frac{\sum_{j=1}^{n}\left(1+\xi_{j}^{2}\right)^{\alpha / 2}+\left(1+\tau^{2}\right)^{\alpha / 4}}{\left(1+|\xi|^{4}+\tau^{2}\right)^{\alpha / 4}}, \quad \xi \in \mathbb{R}^{n}, \tau \in \mathbb{R}
$$

Proof. We will show that $m_{4}$ satisfies Lizorkin's criterion (1.2.37), i.e.

$$
\sup _{\substack{\beta_{i}, \gamma \in\{0,1\} \\(\xi, \tau) \in \mathbb{R}^{n} \times \mathbb{R}}}\left|\xi_{1}^{\beta_{1}} \ldots \xi_{n}^{\beta_{n}} \tau^{\gamma} \partial_{\xi_{1}}^{\beta_{1}} \ldots \partial_{\xi_{n}}^{\beta_{n}} \partial_{\tau}^{\gamma} m_{4}(\xi, \tau)\right|<\infty
$$

CASE 1. $\beta_{j}=\gamma=0$ for all $j \in\{1, \ldots, n\}$. Then

$$
\begin{aligned}
\left|m_{4}(\xi, \tau)\right| & \leq c\left(\frac{\left(\sum_{j=1}^{n}\left(1+\xi_{j}^{2}\right)\right)^{2}+1+\tau^{2}}{1+|\xi|^{4}+\tau^{2}}\right)^{\alpha / 4} \leq c\left(\frac{1+|\xi|^{2}+|\xi|^{4}+\tau^{2}}{1+|\xi|^{4}+\tau^{2}}\right)^{\alpha / 4} \\
& <\infty
\end{aligned}
$$

CASE 2. $\beta_{j}=0$ for all $j \in\{1, \ldots, n\}, \gamma=1$. In a similar way, using also Case 1 , we have

$$
\begin{aligned}
\left|\tau \partial_{\tau} m_{4}(\xi, \tau)\right| & \leq c\left(\frac{\left(1+\tau^{2}\right)^{\alpha / 4}}{\left(1+|\xi|^{4}+\tau^{2}\right)^{\alpha / 4}}+\frac{\left[\sum_{j=1}^{n}\left(1+\xi_{j}^{2}\right)^{\alpha / 2}+\left(1+\tau^{2}\right)^{\alpha / 4}\right] \tau^{2}}{\left(1+|\xi|^{4}+\tau^{2}\right)^{\alpha / 4}\left(1+|\xi|^{4}+\tau^{2}\right)}\right) \\
& <\infty
\end{aligned}
$$

CASE 3. $\beta_{1}=1, \beta_{j}=0$ for $j \in\{2, \ldots, n\}, \gamma=1$. Then one can easily show that the inequality $\left|\xi_{1} \tau \partial_{\xi_{1}} \partial_{\tau} m_{4}(\xi, \tau)\right|<\infty$ holds if

$$
\frac{\tau^{2}\left(1+\tau^{2}\right)^{\alpha / 4-1}|\xi|^{2} \xi_{1}^{2}}{\left(1+|\xi|^{4}+\tau^{2}\right)^{\alpha / 4+1}}<\infty, \quad \frac{\tau^{2}\left(1+\xi_{1}^{2}\right)^{\alpha / 2-1} \xi_{1}^{2}}{\left(1+|\xi|^{4}+\tau^{2}\right)^{\alpha / 4+1}}<\infty
$$

and

$$
\frac{\tau^{2}\left(\sum_{j=1}^{n}\left(1+\xi_{j}^{2}\right)^{\alpha / 2}+\left(1+\tau^{2}\right)^{\alpha / 4}\right)|\xi|^{2} \xi_{1}^{2}}{\left(1+|\xi|^{4}+\tau^{2}\right)^{\alpha / 4+2}}<\infty
$$


Both ineqalities in (1.2.49) can be handled using elemantary estimates. The lefthand side in (1.2.50) is controlled by $\left|m_{4}(\xi, \tau)\right|$, which, in turn, based on Case 1, is bounded by a finite constant. All the other cases are treated in a similar fashion to

Cases 1, 2 and 3.

\section{Example 5.}

$$
m_{5}(\xi, \tau):=\frac{\left(1+\tau^{2}\right)^{\theta / 4}}{\left(1+|\xi|^{4}+\tau^{2}\right)^{\theta / 4}}, \quad \xi \in \mathbb{R}^{n}, \tau \in \mathbb{R} .
$$

Proof. We will show that $m_{5}$ verifies Lizorkin's criterion (1.2.37), i.e. that

$$
\sup _{\substack{\beta_{i}, \gamma \in\{0,1\} \\(\xi, \tau) \in \mathbb{R}^{n} \times \mathbb{R}}}\left|\xi_{1}^{\beta_{1}} \ldots \xi_{n}^{\beta_{n}} \tau^{\gamma} \partial_{\xi_{1}}^{\beta_{1}} \ldots \partial_{\xi_{n}}^{\beta_{n}} \partial_{\tau}^{\gamma} m_{5}(\xi, \tau)\right|<\infty .
$$

CASE 1. $\beta_{j}=\gamma=0$ for all $j \in\{1, \ldots, n\}$. The inequality $\left|m_{5}(\xi, \tau)\right| \leq 1$ is immediate.

CASE 2. $\beta_{j}=0$ for all $j \in\{1, \ldots, n\}, \gamma=1$. Then we can write

$$
\begin{aligned}
\left|\tau \partial_{\tau} m_{5}(\xi, \tau)\right| & \leq c\left(\frac{\left(1+\tau^{2}\right)^{\theta / 4}}{\left(1+|\xi|^{4}+\tau^{2}\right)^{\theta / 4}}+\frac{\left(1+\tau^{2}\right)^{\theta / 4+1}}{\left(1+|\xi|^{4}+\tau^{2}\right)^{\theta / 4+1}}\right) \\
& <\infty
\end{aligned}
$$

CASE 3. $\beta_{1}=1, \beta_{j}=0$ for $j \in\{2, \ldots, n\}, \gamma=1$. Then

$$
\begin{aligned}
\left|\xi_{1} \tau \partial_{\xi_{1}} \partial_{\tau} m_{5}(\xi, \tau)\right| & \leq c\left(\frac{\tau^{2}\left(1+\tau^{2}\right)^{\theta / 4-1}|\xi|^{2} \xi_{1}^{2}}{\left(1+|\xi|^{4}+\tau^{2}\right)^{\theta / 4+1}}+\frac{\tau^{2}\left(1+\tau^{2}\right)^{\theta / 4}|\xi|^{2} \xi_{1}^{2}}{\left(1+|\xi|^{4}+\tau^{2}\right)^{\theta / 4+2}}\right) \\
& <\infty
\end{aligned}
$$

The other cases are handled in a similar way.

\section{Example 6.}

$$
m_{6}(\xi, \tau):=\frac{\sqrt{|\xi|^{2}+i \tau}}{\left(1+|\xi|^{2}\right)^{1 / 2}+|\tau|^{1 / 2}}, \quad(\xi, \tau) \in \mathbb{R}^{n} \times \mathbb{R} .
$$

Proof. We will show that $m_{6}$ satisfies

$$
\sup _{\substack{\beta_{i}, \gamma \in\{0,1\} \\(\xi, \tau) \in \mathbb{R}^{n} \times \mathbb{R}}}\left|\xi_{1}^{\beta_{1}} \ldots \xi_{n}^{\beta_{n}} \tau^{\gamma} \partial_{\xi_{1}}^{\beta_{1}} \ldots \partial_{\xi_{n}}^{\beta_{n}} \partial_{\tau}^{\gamma} m_{6}(\xi, \tau)\right|<\infty
$$


CASE 1. $\beta_{j}=\gamma=0$ for all $j \in\{1, \ldots, n\}$. It is easy to see that $\left|m_{6}(\xi, \tau)\right|<\infty$ holds.

CASE 2. $\beta_{j}=0$ for all $j \in\{1, \ldots, n\}, \gamma=1$. Then $\left|\tau \partial_{\tau} m_{6}(\xi, \tau)\right| \leq c \frac{|\tau|}{\left(|\xi|^{4}+\tau^{2}\right)^{1 / 4}\left[\left(1+|\xi|^{2}\right)^{1 / 2}+|\tau|^{1 / 2}\right]}+c \frac{|\tau|^{1 / 2}\left(|\xi|^{4}+\tau^{2}\right)^{1 / 4}}{\left[\left(1+|\xi|^{2}\right)^{1 / 2}+|\tau|^{1 / 2}\right]^{2}}<\infty$

CASE 3. $\beta_{1}=1, \beta_{j}=0$ for $j \in\{2, \ldots, n\}, \gamma=0$. In this case we obtain that

$$
\begin{aligned}
\left|\xi_{1} \partial_{\xi_{1}} m_{6}(\xi, \tau)\right| \leq & c \frac{|\xi|^{2}}{\left(|\xi|^{4}+\tau^{2}\right)^{1 / 4}\left[\left(1+|\xi|^{2}\right)^{1 / 2}+|\tau|^{1 / 2}\right]} \\
& +c \frac{|\xi|^{2}\left(|\xi|^{4}+\tau^{2}\right)^{1 / 4}}{\left(1+|\xi|^{2}\right)^{1 / 2}\left[\left(1+|\xi|^{2}\right)^{1 / 2}+|\tau|^{1 / 2}\right]^{2}} \\
& <\infty .
\end{aligned}
$$

Similar considerations apply to all the other cases.

The remaining examples are handled in a similar fashion as the above ones.

\section{Example 7.}

$$
m_{7}(\xi, \tau):=\frac{\left(1+|\xi|^{4}+\tau^{2}\right)^{1 / 4}}{1+\left(|\xi|^{2}+|\tau|\right)^{1 / 2}}, \quad(\xi, \tau) \in \mathbb{R}^{n} \times \mathbb{R}
$$

\section{Example 8.}

$$
m_{8}(\xi, \tau):=\frac{\left(|\xi|^{2}+|\tau|\right)^{1 / 2}}{\left(1+|\xi|^{4}+\tau^{2}\right)^{1 / 4}}, \quad(\xi, \tau) \in \mathbb{R}^{n} \times \mathbb{R}
$$

\section{Example 9.}

$$
m_{9}(\xi, \tau):=\frac{\sum_{j=1}^{n}\left(1+\xi_{j}^{2}\right)^{1 / 2}+\left(1+\tau^{2}\right)^{1 / 4}}{1+\sum_{j=1}^{n}\left|\xi_{j}\right|+|\tau|^{1 / 2}}, \quad(\xi, \tau) \in \mathbb{R}^{n} \times \mathbb{R}
$$

\section{Example 10.}

$$
m_{10}(\xi, \tau):=\frac{\sum_{j=1}^{n}\left|\xi_{j}\right|+|\tau|^{1 / 2}}{\sum_{j=1}^{n}\left(1+\xi_{j}^{2}\right)^{1 / 2}+\left(1+\tau^{2}\right)^{1 / 4}}, \quad(\xi, \tau) \in \mathbb{R}^{n} \times \mathbb{R}
$$




\subsubsection{Real and complex interpolation methods}

In this subsection we present only the definitions and results regarding the real and complex interpolation methods, which are needed in our further study. A more comprehensive treatment of these methods is given in [Cal2], [Peet], [BeLö] and [Tri2]. Initially, both interpolation methods were given for Banach spaces, but it is wellknown that the real method can be extended to quasi-Banach spaces.

Definition 1.2.20. Let $A_{0}$ and $A_{1}$ be two topological vector spaces. $A_{0}$ and $A_{1}$ are called compatible if there is a Hausdorff topological vector space $\mathcal{H}$ such that $A_{0}$ and $A_{1}$ are subspaces of $\mathcal{H}$. Then we can form their sum $A_{0}+A_{1}$ consisting of all elements $a \in \mathcal{H}$ which can be represented as $a=a_{0}+a_{1}$ for some $a_{0} \in A_{0}$ and $a_{1} \in A_{1}$. A compatible pair $\left(A_{0}, A_{1}\right)$ is called an interpolation couple. For $0<t<\infty$ and $a \in A_{0}+A_{1}$, J. Peetre's $K$-functional is given by

$$
K\left(t, a ; A_{0}, A_{1}\right):=\inf \left(\left\|a_{0}\right\|_{A_{0}}+t\left\|a_{1}\right\|_{A_{1}}\right),
$$

where the infimum is taken over all representations of $a=a_{0}+a_{1}$ with $a_{0} \in A_{0}$ and $a_{1} \in A_{1}$. Let $0<\theta<1$ and $0<q \leq \infty$. Then

$$
\left(A_{0}, A_{1}\right)_{\theta, q}:=\left\{a \in A_{0}+A_{1}:\|a\|_{\left(A_{0}, A_{1}\right)_{\theta, q}}<\infty\right\}
$$

where

$$
\|a\|_{\left(A_{0}, A_{1}\right)_{\theta, q}}:= \begin{cases}\left(\int_{0}^{\infty}\left[t^{-\theta} K\left(t, a ; A_{0}, A_{1}\right)\right]^{q} \frac{d t}{t}\right)^{1 / q} & \text { if } 1<q<\infty \\ \sup _{0<t<\infty}\left[t^{-\theta} K\left(t, a ; A_{0}, A_{1}\right)\right] & \text { if } q=\infty .\end{cases}
$$

We next prove some abstract results needed in the following chapters. 
Lemma 1.2.21. For any $N \in \mathbb{N}$ and interpolation couples $\left(A_{0}^{i}, A_{1}^{i}\right), i=1, \ldots, N$, we have

$$
\left(\bigoplus_{i=1}^{N} A_{0}^{i}, \bigoplus_{i=1}^{N} A_{1}^{i}\right)_{\theta, p}=\bigoplus_{i=1}^{N}\left(A_{0}^{i}, A_{1}^{i}\right)_{\theta, p},
$$

where $0<\theta<1$ and $0<p \leq \infty$.

Proof. It is enough to show that, for interpolation pairs $\left(A_{0}, A_{1}\right)$ and $\left(B_{0}, B_{1}\right)$, $0<\theta<1$ and $0<p \leq \infty$

$$
\left(A_{0} \oplus B_{0}, A_{1} \oplus B_{1}\right)_{\theta, p}=\left(A_{0}, A_{1}\right)_{\theta, p} \oplus\left(B_{0}, B_{1}\right)_{\theta, p},
$$

since (1.2.51) will follow from (1.2.52) by induction.

Let us consider the projection $\pi^{A}: A_{j} \oplus B_{j} \rightarrow A_{j}, j=0,1$ defined by

$$
\pi^{A}(a, b):=a \quad \text { for } \quad(a, b) \in A_{j} \oplus B_{j} .
$$

Then, by real interpolation, we have

$$
\pi^{A}:\left(A_{0} \oplus B_{0}, A_{1} \oplus B_{1}\right)_{\theta, p} \longrightarrow\left(A_{0}, A_{1}\right)_{\theta, p} .
$$

Similarly, the projection $\pi^{B}: A_{j} \oplus B_{j} \rightarrow B_{j}, j=0,1$, defined by

$$
\pi^{B}(a, b):=b \quad \text { for } \quad(a, b) \in A_{j} \oplus B_{j}
$$

has the property that

$$
\pi^{B}:\left(A_{0} \oplus B_{0}, A_{1} \oplus B_{1}\right)_{\theta, p} \longrightarrow\left(B_{0}, B_{1}\right)_{\theta, p}
$$

is bounded. Going further,

$$
\begin{aligned}
& \Psi:\left(A_{0} \oplus B_{0}, A_{1} \oplus B_{1}\right)_{\theta, p} \longrightarrow\left(A_{0}, A_{1}\right)_{\theta, p} \oplus\left(B_{0}, B_{1}\right)_{\theta, p} \\
& \Psi(x):=\left(\pi^{A} x, \pi^{B} x\right) \quad \text { for } \quad x \in\left(A_{0} \oplus B_{0}, A_{1} \oplus B_{1}\right)_{\theta, p}
\end{aligned}
$$


is well-defined, linear and bounded.

On the other hand, for $\iota_{A}: A_{j} \rightarrow A_{j} \oplus B_{j}, j=0,1$, given by

$$
\iota_{A}(a):=(a, 0) \text { for } a \in A_{j}
$$

by real interpolation, we have that

$$
\iota_{A}:\left(A_{0}, A_{1}\right)_{\theta, p} \longrightarrow\left(A_{0} \oplus B_{0}, A_{1} \oplus B_{1}\right)_{\theta, p}
$$

is bounded. Similarly, $\iota_{B}: B_{j} \rightarrow A_{j} \oplus B_{j}, j=0,1$, defined by

$$
\iota_{B}(b):=(0, b) \quad \text { for } \quad b \in B_{j}
$$

has the property that

$$
\iota_{B}:\left(B_{0}, B_{1}\right)_{\theta, p} \longrightarrow\left(A_{0} \oplus B_{0}, A_{1} \oplus B_{1}\right)_{\theta, p}
$$

is bounded. Finally,

$$
\begin{aligned}
& \Phi:\left(A_{0}, A_{1}\right)_{\theta, p} \oplus\left(B_{0}, B_{1}\right)_{\theta, p} \longrightarrow\left(A_{0} \oplus B_{0}, A_{1} \oplus B_{1}\right)_{\theta, p} \\
& \Phi(a, b):=\iota_{A}(a)+\iota_{B}(b) \quad \text { for } \quad(a, b) \in\left(A_{0}, A_{1}\right)_{\theta, p} \oplus\left(B_{0}, B_{1}\right)_{\theta, p}
\end{aligned}
$$

is well-defined, linear and bounded. Moreover, it is easy to check that

$$
(\Psi \circ \Phi)(a, b)=(a, b) \quad \text { for any }(a, b) \in\left(A_{0}, A_{1}\right)_{\theta, p} \oplus\left(B_{0}, B_{1}\right)_{\theta, p}
$$

and

$$
(\Phi \circ \Psi)(x)=x \quad \text { for any } x \in\left(A_{0} \oplus B_{0}, A_{1} \oplus B_{1}\right)_{\theta, p},
$$

consequently, $\Phi$ is an isomorphism on $\left(A_{0}, A_{1}\right)_{\theta, p} \oplus\left(B_{0}, B_{1}\right)_{\theta, p}=\left(A_{0} \oplus B_{0}, A_{1} \oplus B_{1}\right)_{\theta, p}$. This finishes the proof of the lemma. 
Lemma 1.2.22. Let $X_{i}, Y_{i}, Z_{i}, i=0,1$, be Banach spaces. Assume that $X_{0}$ and $X_{1}$ are contained in a larger Banach space such that $X_{0} \cap X_{1}$ is dense in both $X_{0}$ and $X_{1}$, similarly for $Z_{0}$ and $Z_{1}$. Suppose that $Y_{i} \hookrightarrow Z_{i}, i=0,1$, and that there is a continuous linear mapping $D: X_{i} \rightarrow Z_{i}, i=0,1$. Define the spaces

$$
X_{i}(D):=\left\{u \in X_{i}: D u \in Y_{i}\right\}, \quad i=0,1,
$$

equipped with the graph norm, i.e. $\|u\|_{X_{i}(D)}:=\|u\|_{X_{i}}+\|D u\|_{Y_{i}}, i=0,1$. Suppose that there exist continuous linear mappings $G: Z_{i} \rightarrow X_{i}$ and $K: Z_{i} \rightarrow Y_{i}$ such that $D \circ G f=f+K f$ for all $f \in Z_{i}$. Then we can define $D$ by real interpolation, for $0<\theta<1$ and $1 \leq p \leq \infty$, as a mapping from $\left(X_{0}, X_{1}\right)_{\theta, p}$ to $\left(Z_{0}, Z_{1}\right)_{\theta, p}$ and the natural inclusion $\left(Y_{0}, Y_{1}\right)_{\theta, p} \hookrightarrow\left(Z_{0}, Z_{1}\right)_{\theta, p}$. It follows that, for $0<\theta<1$ and $1 \leq p \leq \infty$

$$
\left(X_{0}(D), X_{1}(D)\right)_{\theta, p}=\left\{u \in\left(X_{0}, X_{1}\right)_{\theta, p}: D u \in\left(Y_{0}, Y_{1}\right)_{\theta, p}\right\}
$$

Proof. In order to show the left-to-right inclusion in (1.2.53), note that $X_{0}(D) \subset X_{0}$, $X_{1}(D) \subset X_{1}$, hence

$$
\left(X_{0}(D), X_{1}(D)\right)_{\theta, p} \hookrightarrow\left(X_{0}, X_{1}\right)_{\theta, p} \quad \text { for } 0<\theta<1 \text { and } 1 \leq p \leq \infty
$$

Also, since $D: X_{i} \rightarrow Z_{i}$ and $X_{i}(D) \subset X_{i}$, we have that $D: X_{i}(D) \rightarrow Z_{i}$. Moreover, if $u \in X_{i}(D)$, then $D u \in Y_{i}$, therefore $D: X_{i}(D) \rightarrow Y_{i}$. Consequently, by real interpolation,

$$
D:\left(X_{0}(D), X_{1}(D)\right)_{\theta, p} \longrightarrow\left(Y_{0}, Y_{1}\right)_{\theta, p}
$$

Now the left-to-right inclusion in (1.2.53) follows from (1.2.54) and (1.2.55). 
Conversely, let $u \in\left(X_{0}, X_{1}\right)_{\theta, p}$ so that $D u \in\left(Y_{0}, Y_{1}\right)_{\theta, p}$. By Definition 1.2 from [Zaf] this amounts to

$$
u=\int_{0}^{\infty} t^{\theta} f(t) \frac{d t}{t}, \quad \text { where } f \in W\left(p, X_{0}, X_{1}\right)
$$

and

$$
D u=\int_{0}^{\infty} t^{\theta} g(t) \frac{d t}{t}, \quad \text { where } g \in W\left(p, X_{0}, X_{1}\right) .
$$

To clarify the terms we are using we invoke the following: according to Definition 1.1 from [Zaf], for an interpolation pair $\left(B_{0}, B_{1}\right)$ and for $1 \leq p \leq \infty$, the set $W\left(p, B_{0}, B_{1}\right)$ is the collection of all strongly measurable functions $F:(0, \infty) \rightarrow B_{0} \cap B_{1}$ for which

$$
\|F\|_{W}:=\max \left\{\|F(t)\|_{L_{*}^{p}\left(B_{0}\right)},\|t F(t)\|_{L_{*}^{p}\left(B_{1}\right)}\right\}<\infty
$$

Here $L_{*}^{p}(B)$ stands for the space of functions $F:(0, \infty) \rightarrow B$, where $B$ is a quasiBanach space, such that $F$ is strongly measurable with respect to the measure $\frac{d t}{t}$ and

$$
\|F\|_{L_{*}^{p}(B)}:= \begin{cases}\left(\int_{0}^{\infty}\|F\|_{B}^{p} \frac{d t}{t}\right)^{1 / p}<\infty, & \text { if } 1 \leq p<\infty \\ \sup _{t>0}\|F(t)\|_{B}<\infty, & \text { if } p=\infty\end{cases}
$$

Returning to the proof, for $t>0$ let us define

$$
h(t):=f(t)-G D f(t)+G g(t) .
$$

Then, by (1.2.56) and (1.2.57),

$$
\int_{0}^{\infty} t^{\theta} h(t) \frac{d t}{t}=u
$$

which amounts to $u \in\left(X_{0}(D), X_{1}(D)\right)_{\theta, p}$ provided $h \in W\left(p, X_{0}(D), X_{1}(D)\right)$. This, in turn, holds if

$$
h:(0, \infty) \longrightarrow X_{0}(D) \cap X_{1}(D) \text { is strongly measurable, }
$$




$$
\begin{gathered}
\int_{0}^{\infty}\|h(t)\|_{X_{0}(D)}^{p} \frac{d t}{t}<\infty \quad \text { if } 1 \leq p<\infty, \\
\sup _{t>0}\|h(t)\|_{X_{0}(D)}<\infty \quad \text { if } p=\infty, \\
\int_{0}^{\infty}\|t h(t)\|_{X_{1}(D)}^{p} \frac{d t}{t}<\infty \quad \text { if } 1 \leq p<\infty
\end{gathered}
$$

and

$$
\sup _{t>0}\|t h(t)\|_{X_{1}(D)}<\infty \quad \text { if } p=\infty .
$$

To show (1.2.59) and (1.2.60), first observe that $\|h(t)\|_{X_{0}(D)}=\|h(t)\|_{X_{0}}+\|D h(t)\|_{Y_{0}}$. Second, one can easily see that

$$
\begin{aligned}
\|h(t)\|_{X_{0}} & \leq\left(1+\|G D\|_{\mathcal{L}\left(X_{0}, X_{0}\right)}\right)\|f(t)\|_{X_{0}}+\|G\|_{\mathcal{L}\left(Y_{0}, X_{0}\right)}\|g(t)\|_{Y_{0}} \\
& \leq c\|f(t)\|_{X_{0}}+c\|g(t)\|_{Y_{0}} .
\end{aligned}
$$

Rewriting $D h$ we get that $D h=g+K g-K D f$. Then

$$
\begin{aligned}
\|D h(t)\|_{Y_{0}} & \leq\left(1+\|K\|_{\mathcal{L}\left(Y_{0}, Y_{0}\right)}\right)\|g(t)\|_{Y_{0}}+\|K D\|_{\mathcal{L}\left(X_{0}, Y_{0}\right)}\|f(t)\|_{X_{0}} \\
& \leq c\|f(t)\|_{X_{0}}+c\|g(t)\|_{Y_{0}} .
\end{aligned}
$$

As a consequence of (1.2.63) and (1.2.64) we obtain that

$$
\|h(t)\|_{X_{0}(D)} \leq c\|f(t)\|_{X_{0}}+c\|g(t)\|_{Y_{0}} .
$$

Hence, using the facts that $f \in W\left(p, X_{0}, X_{1}\right)$ and $g \in W\left(p, Y_{0}, Y_{1}\right)$,

$$
\int_{0}^{\infty}\|h(t)\|_{X_{0}(D)}^{p} \frac{d t}{t} \leq c \int_{0}^{\infty}\|f(t)\|_{X_{0}}^{p} \frac{d t}{t}+c \int_{0}^{\infty}\|g(t)\|_{Y_{0}}^{p} \frac{d t}{t}<\infty \text { if } 1 \leq p<\infty,
$$

and

$$
\sup _{t>0}\|h(t)\|_{X_{0}(D)} \leq c \sup _{t>0}\|f(t)\|_{X_{0}}+c \sup _{t>0}\|g(t)\|_{Y_{0}}<\infty \quad \text { if } p=\infty,
$$


finishing the proof of (1.2.59) and (1.2.60).

Next, to handle (1.2.61) and (1.2.62), we proceed in a similar way, first observing that $\|t h(t)\|_{X_{1}(D)}=\|t h(t)\|_{X_{1}}+\|t D h(t)\|_{Y_{1}}$. Furthermore,

$$
\begin{aligned}
\|t h(t)\|_{X_{1}} & \leq\left(1+\|G D\|_{\mathcal{L}\left(X_{1}, X_{1}\right)}\right)\|t f(t)\|_{X_{1}}+\|G\|_{\mathcal{L}\left(Y_{1}, X_{1}\right)}\|t g(t)\|_{Y_{1}} \\
& \leq c\|t f(t)\|_{X_{1}}+c\|t g(t)\|_{Y_{1}},
\end{aligned}
$$

and, as before, $t D h(t)=t g(t)+t K g(t)-t K D f(t)$. Then

$$
\begin{aligned}
\|t D h(t)\|_{Y_{1}} & \leq\left(1+\|K\|_{\mathcal{L}\left(Y_{1}, Y_{1}\right)}\right)\|t g(t)\|_{Y_{1}}+\|K D\|_{\mathcal{L}\left(X_{1}, Y_{1}\right)}\|t f(t)\|_{X_{1}} \\
& \leq c\|t f(t)\|_{X_{1}}+c\|t g(t)\|_{Y_{1}} .
\end{aligned}
$$

As a consequence of (1.2.65) and (1.2.66) we obtain that

$$
\|t h(t)\|_{X_{1}(D)} \leq c\|t f(t)\|_{X_{1}}+c\|t g(t)\|_{Y_{1}} .
$$

Hence, using that $f \in W\left(p, X_{0}, X_{1}\right)$ and $g \in W\left(p, Y_{0}, Y_{1}\right)$, for $1 \leq p<\infty$,

$$
\int_{0}^{\infty}\|t h(t)\|_{X_{1}(D)}^{p} \frac{d t}{t} \leq c \int_{0}^{\infty}\|t f(t)\|_{X_{1}}^{p} \frac{d t}{t}+c \int_{0}^{\infty}\|t g(t)\|_{Y_{1}}^{p} \frac{d t}{t}<\infty
$$

and, for $p=\infty$,

$$
\sup _{t>0}\|t h(t)\|_{X_{1}(D)} \leq c \sup _{t>0}\|t f(t)\|_{X_{1}}+c \sup _{t>0}\|t g(t)\|_{Y_{1}}<\infty .
$$

This completes the proof of (1.2.61), (1.2.62) and of the lemma.

Remark 1.2.23. Lemma 1.2 .22 holds true if $X_{i}, Y_{i}, Z_{i}, i=0,1$ are quasi-Banach spaces. Since this aspect is not going to be important for us, we only include the idea in the proof, and leave the details to the interested reader. The goal is to establish the following equivalence:

$$
K\left(t, a ; X_{0}(D), X_{1}(D)\right) \approx K\left(t, a ; X_{0}, X_{1}\right)+K\left(t, D a ; Y_{0}, Y_{1}\right) .
$$


In order to do so we consider $a \in X_{0}+X_{1}$ with

$$
a=x_{0}+x_{1}, \quad x_{i} \in X_{i}, \quad \text { and } \quad D a=y_{0}+y_{1}, \quad y_{i} \in Y_{i}, \quad i=0,1
$$

such that

$$
\left\|x_{0}\right\|_{X_{0}}+t\left\|x_{1}\right\|_{X_{1}} \approx K\left(t, a ; X_{0}, X_{1}\right) \quad \text { and } \quad\left\|y_{0}\right\|_{Y_{0}}+t\left\|y_{1}\right\|_{Y_{1}} \approx K\left(t, D a ; Y_{0}, Y_{1}\right)
$$

It is possible to split $a$ in a different way than in (1.2.68), namely

$$
a=x_{0}^{\prime}+x_{1}^{\prime}, \quad \text { where } \quad x_{i}:=x_{i}-G D x_{i}+G y_{i}, \quad i=0,1 .
$$

Then one could easily check that for $i=0,1$

$$
\left\|x_{i}^{\prime}\right\|_{X_{i}} \leq c\left(\left\|x_{i}\right\|_{X_{i}}+\left\|y_{i}\right\|_{Y_{i}}\right) \quad \text { and } \quad\left\|D x_{i}^{\prime}\right\|_{Y_{i}} \leq c\left(\left\|x_{i}\right\|_{X_{i}}+\left\|y_{i}\right\|_{Y_{i}}\right)
$$

which, in turn, yield (1.2.67).

Lemma 1.2.24. Let us consider $\mathcal{X}$ and $\mathcal{Y}$ quasi-Banach spaces, $\left\{X_{\alpha}^{p}\right\}_{p, \alpha \in \mathbb{R}} \subset \mathcal{X}$ and $\left\{Y_{\alpha}^{p, q}\right\}_{p, q, \alpha \in \mathbb{R}} \subset \mathcal{Y}$ function spaces on a domain $\Omega \subset \mathbb{R}^{n}$ such that, for $p_{i}, \alpha_{i} \in \mathbb{R}$, $i=0,1$,

$$
\left(X_{\alpha_{0}}^{p_{0}}, X_{\alpha_{1}}^{p_{1}}\right)_{\theta, q}=Y_{\alpha}^{p, q}
$$

where $0<\theta<1,1 \leq q \leq \infty, \frac{1}{p}=\frac{1-\theta}{p_{0}}+\frac{\theta}{p_{1}}$ and $\alpha=(1-\theta) \alpha_{0}+\theta \alpha_{1}$. Let $D_{1} \subseteq \Omega$ open and $D_{2} \subset \Omega$ closed subsets, and define

$$
X_{\alpha, D_{1}}^{p}:=\left\{\left.f\right|_{D 1}: f \in X_{\alpha}^{p}\right\} \quad \text { and } \quad X_{\alpha, D_{2}, *}^{p}:=\left\{f \in X_{\alpha}^{p}: \text { supp } f \subseteq D_{2}\right\}
$$

Similarly,

$$
Y_{\alpha, D_{1}}^{p, q}:=\left\{\left.f\right|_{D_{1}}: f \in Y_{\alpha}^{p, q}\right\} \quad \text { and } \quad Y_{\alpha, D_{2}, *}^{p, q}:=\left\{f \in Y_{\alpha}^{p, q}: \text { supp } f \subseteq D_{2}\right\} \text {. }
$$


(i) If there exists a universal extension operator $E_{D_{1}}: X_{\alpha, D_{1}}^{p} \rightarrow X_{\alpha}^{p}$ for all $p, \alpha$ in $\mathbb{R}$, then

$$
\left(X_{\alpha_{0}, D_{1}}^{p_{0}}, X_{\alpha_{1}, D_{1}}^{p_{1}}\right)_{\theta, q}=Y_{\alpha, D_{1}}^{p, q}
$$

where $p_{i}, \alpha_{i} \in \mathbb{R}, i=0,1,0<\theta<1,1 \leq q \leq \infty, \frac{1}{p}=\frac{1-\theta}{p_{0}}+\frac{\theta}{p_{1}}$ and $\alpha=$ $(1-\theta) \alpha_{0}+\theta \alpha_{1}$

(ii) If for any $p_{i}, \alpha_{i} \in \mathbb{R}, i=0,1, X_{\alpha_{0}}^{p_{0}} \cap X_{\alpha_{1}}^{p_{1}}$ is dense in both $X_{\alpha_{0}}^{p_{0}}$ and $X_{\alpha_{1}}^{p_{1}}$, and if there exists a universal extension operator $E_{D_{2}^{c}}: X_{\alpha, D_{2}^{c}}^{p} \rightarrow X_{\alpha}^{p}$ for all $p, \alpha \in \mathbb{R}$, where $D_{2}^{c}:=\Omega \backslash D_{2}$, then

$$
\left(X_{\alpha_{0}, D_{2}, *}^{p_{0}}, X_{\alpha_{1}, D_{2}, *}^{p_{1}}\right)_{\theta, q}=Y_{\alpha, D_{2}, *}^{p, q}
$$

where $0<\theta<1,1 \leq q \leq \infty, \frac{1}{p}=\frac{1-\theta}{p_{0}}+\frac{\theta}{p_{1}}$ and $\alpha=(1-\theta) \alpha_{0}+\theta \alpha_{1}$.

(iii) Assume (1.2.72) holds and, for $p, q, \alpha \in \mathbb{R}$, define

$$
\tilde{X}_{\alpha, D_{1}}^{p}:=\left\{\left.f\right|_{D_{1}}: f \in X_{\alpha, D_{2}, *}^{p}\right\} \quad \text { and } \quad \tilde{Y}_{\alpha, D_{1}}^{p, q}:=\left\{\left.f\right|_{D_{1}}: f \in Y_{\alpha, D_{2}, *}^{p, q}\right\}
$$

If there exists a universal extension operator $E: \tilde{X}_{\alpha, D_{1}}^{p} \rightarrow X_{\alpha, D_{2}, *}^{p}$ for all $p, \alpha \in \mathbb{R}$, then

$$
\left(\tilde{X}_{\alpha_{0}, D_{1}}^{p_{0}}, \tilde{X}_{\alpha_{1}, D_{1}}^{p_{1}}\right)_{\theta, q}=\tilde{Y}_{\alpha, D_{1}}^{p, q}
$$

where $0<\theta<1,1 \leq q \leq \infty, \frac{1}{p}=\frac{1-\theta}{p_{0}}+\frac{\theta}{p_{1}}$ and $\alpha=(1-\theta) \alpha_{0}+\theta \alpha_{1}$.

Proof. (i) Define the restriction operator $R_{D_{1}} f=\left.f\right|_{D_{1}}$. Then $R_{D_{1}}: X_{\alpha}^{p} \rightarrow X_{\alpha, D_{1}}^{p}$, hence $R_{D_{1}} \circ E_{D_{1}}=I$ on $X_{\alpha, D_{1}}^{p}$. By real interpolation we also have

$$
R_{D_{1}}:\left(X_{\alpha_{0}}^{p_{0}}, X_{\alpha_{1}}^{p_{1}}\right)_{\theta, q} \longrightarrow\left(X_{\alpha_{0}, D_{1}}^{p_{0}}, X_{\alpha_{1}, D_{1}}^{p_{1}}\right)_{\theta, q}
$$

i.e. $R_{D_{1}}: Y_{\alpha}^{p, q} \rightarrow\left(X_{\alpha_{0}, D_{1}}^{p_{0}}, X_{\alpha_{1}, D_{1}}^{p_{1}}\right)_{\theta, q}$. Since $R_{D_{1}}$ maps $Y_{\alpha}^{p, q}$ onto $Y_{\alpha, D_{1}}^{p, q}$, we get that

$$
Y_{\alpha, D_{1}}^{p, q} \hookrightarrow\left(X_{\alpha_{0}, D_{1}}^{p_{0}}, X_{\alpha_{1}, D_{1}}^{p_{1}}\right)_{\theta, q}
$$


On the other hand, $E_{D_{1}}:\left(X_{\alpha_{0}, D_{1}}^{p_{0}}, X_{\alpha_{1}, D_{1}}^{p_{1}}\right)_{\theta, q} \rightarrow Y_{\alpha}^{p, q}$ and $R_{D_{1}}: Y_{\alpha}^{p, q} \rightarrow Y_{\alpha, D_{1}}^{p, q}$. Their composition $R_{D_{1}} \circ E_{D_{1}}=I$, therefore

$$
\left(X_{\alpha_{0}, D_{1}}^{p_{0}}, X_{\alpha_{1}, D_{1}}^{p_{1}}\right)_{\theta, q} \hookrightarrow Y_{\alpha, D_{1}}^{p, q}
$$

and the proof of (i) is complete.

(ii) In the case when $\mathcal{X}, \mathcal{Y}$ are Banach spaces, we can apply Lemma 1.2.22 with $X_{0}=X_{\alpha_{0}}^{p_{0}}, X_{1}=X_{\alpha_{1}}^{p_{1}}, Z_{0}=X_{\alpha_{0}, D_{2}^{c}}^{p_{0}}, Z_{1}=X_{\alpha_{1}, D_{2}^{c}}^{p_{1}}, Y_{0}=Y_{1}=0, K=0, D=R_{D_{2}^{c}}$, and $G=E_{D_{2}^{c}}$. Then $R_{D_{2}^{c}} \circ E_{D_{2}^{c}}=I$ on $X_{\alpha, D_{2}^{c}}^{p}$,

$$
X_{0}(D)=X_{\alpha_{0}, D_{2}, *}^{p_{0}} \quad \text { and } \quad X_{1}(D)=X_{\alpha_{1}, D_{2}, *}^{p_{1}} \cdot
$$

Consequently,

$$
\begin{aligned}
\left(X_{\alpha_{0}, D_{2}, *}^{p_{0}}, X_{\alpha_{1}, D_{2}, *}^{p_{1}}\right)_{\theta, q} & =\left\{f \in\left(X_{\alpha_{0}}^{p_{0}}, X_{\alpha_{1}}^{p_{1}}\right)_{\theta, q}:\left.f\right|_{D_{2}^{c}}=0\right\} \\
& =\left\{f \in Y_{\alpha}^{p, q}: \operatorname{supp} f \subseteq D_{2}\right\} \\
& =Y_{\alpha, D_{2}, *}^{p, q}
\end{aligned}
$$

where $p_{i}, \alpha_{i} \in \mathbb{R}(i=0,1), 0<\theta<1,1 \leq q \leq \infty, \frac{1}{p}=\frac{1-\theta}{p_{0}}+\frac{\theta}{p_{1}}$ and $\alpha=$ $(1-\theta) \alpha_{0}+\theta \alpha_{1}$

If $\mathcal{X}, \mathcal{Y}$ are quasi-Banach spaces, the proof follows from the following.

Claim. Consider $A_{j}, B_{j}, j=0,1$, quasi-Banach spaces such that $B_{j} \hookrightarrow A_{j}$, and let the operator $T: A_{j} \rightarrow B_{j}$ be such that $T^{2}=T$ and $\left.T\right|_{B_{j}}=I$. Then, for $0<\theta<1$ and $1 \leq q \leq \infty$,

$$
T\left(A_{0}, A_{1}\right)_{\theta, q}=\left(B_{0}, B_{1}\right)_{\theta, q}
$$


Proof of Claim. Since $T:\left(A_{0}, A_{1}\right)_{\theta, q} \rightarrow\left(B_{0}, B_{1}\right)_{\theta, q}$, the left-to-right inclusion in (1.2.73) is immediate. To show the opposite inclusion in (1.2.73) first note that, for $j=0,1$, the operator $T-I: B_{j} \rightarrow 0$, hence $T-I:\left(B_{0}, B_{1}\right)_{\theta, q} \rightarrow 0$ for $0<\theta<1$. Therefore,

$$
\forall x \in\left(B_{0}, B_{1}\right)_{\theta, q} \hookrightarrow\left(A_{0}, A_{1}\right)_{\theta, q} \text { we have } x=T x \in T\left(A_{0}, A_{1}\right)_{\theta, q}
$$

i.e. $\left(B_{0}, B_{1}\right)_{\theta, q} \hookrightarrow T\left(A_{0}, A_{1}\right)_{\theta, q}$, which completes the proof of the claim.

In order to show that the proof of (ii) follows from the above Claim, let us consider the operator $P$ defined as

$$
P:=I-E_{D_{2}^{c}} \circ R_{D_{2}^{c}}
$$

Take $f \in X_{\alpha}^{p}$. Then $R_{D_{2}^{c}} f \in X_{\alpha, D_{2}^{c}}^{p}$, and $\left(E_{D_{2}^{c}} \circ R_{D_{2}^{c}}\right) f \in X_{\alpha}^{p}$, hence $\operatorname{Pf} \in X_{\alpha}^{p}$. Moreover, since $R_{D_{2}^{c}} \circ E_{D_{2}^{c}}=I$,

$$
R_{D_{2}^{c}}(P f)=R_{D_{2}^{c}} f-\left(R_{D_{2}^{c}} \circ E_{D_{2}^{c}} \circ R_{D_{2}^{c}}\right) f=0
$$

which implies that supp $P f \subseteq D_{2}$. Consequently,

$$
P: X_{\alpha}^{p} \longrightarrow X_{\alpha, D_{2}, *}^{p}
$$

Going further, for any $f \in X_{\alpha, D_{2}, *}^{p}$ we have $f \in X_{\alpha}^{p}$ with supp $f \subseteq D_{2}$, implying $P f=f$. This amounts to $P: X_{\alpha}^{p} \rightarrow X_{\alpha, D_{2}, *}^{p}$ being onto.

To show that $P^{2}=P$ on $X_{\alpha}^{p}$, let $f \in X_{\alpha}^{p}$. Then $P f \in X_{\alpha}^{p}$ with $\operatorname{supp}(P f) \subseteq D_{2}$, and

$$
P^{2} f=P(P f)=P f-\left(E_{D_{2}^{c}} \circ R_{D_{2}^{c}}\right)(P f)=P f .
$$


Taking also into account the fact that $\left.P\right|_{X_{\alpha, D_{2}, *}^{p}}=I$, the operator $P$ and the spaces $X_{\alpha}^{p}, X_{\alpha, D_{2}, *}^{p}$ satisfy the conditions of the Claim. Therefore,

$$
P\left(X_{\alpha_{0}}^{p_{0}}, X_{\alpha_{1}}^{p_{1}}\right)_{\theta, q}=\left(X_{\alpha_{0}, D_{2}, *}^{p_{0}}, X_{\alpha_{1}, D_{2}, *}^{p_{1}}\right)_{\theta, q} .
$$

The left-hand side of (1.2.74) is $P\left(Y_{\alpha}^{p, q}\right)$, and since $P$ is onto, $P\left(Y_{\alpha}^{p, q}\right)=Y_{\alpha, D_{2}, *}^{p, q}$, consequently,

$$
\left(X_{\alpha_{0}, D_{2}, *}^{p_{0}}, X_{\alpha_{1}, D_{2}, *}^{p_{1}}\right)_{\theta, q}=Y_{\alpha, D_{2}, *}^{p, q}
$$

where $0<\theta<1,1 \leq q \leq \infty, \frac{1}{p}=\frac{1-\theta}{p_{0}}+\frac{\theta}{p_{1}}$ and $\alpha=(1-\theta) \alpha_{0}+\theta \alpha_{1}$.

Note. In view of Remark 1.2.23, we can justify (ii) for quasi-Banach spaces in the same spirit as we did for Banach spaces (as above).

(iii) Consider the restriction operator $R_{D_{1}}$ defined in the proof of (i). Then clearly $R_{D_{1}}: X_{\alpha, D_{2}, *}^{p} \rightarrow \tilde{X}_{\alpha, D_{1}}^{p}$, hence $R_{D_{1}} \circ E=I$ on $\tilde{X}_{\alpha, D_{1}}^{p}$. Also,

$$
R_{D_{1}}: Y_{\alpha, D_{2}, *}^{p, q} \longrightarrow\left(\tilde{X}_{\alpha_{0}, D_{1}}^{p_{0}}, \tilde{X}_{\alpha_{1}, D_{1}}^{p_{1}}\right)_{\theta, q}
$$

Since $R_{D_{1}}$ maps $Y_{\alpha, D_{2}, *}^{p, q}$ onto $\tilde{Y}_{\alpha, D_{1}}^{p, q}$, we obtain that, for the appropriate indices,

$$
\tilde{Y}_{\alpha, D_{1}}^{p, q} \hookrightarrow\left(\tilde{X}_{\alpha_{0}, D_{1}}^{p_{0}}, \tilde{X}_{\alpha_{1}, D_{1}}^{p_{1}}\right)_{\theta, q}
$$

On the other hand,

$$
\left(\tilde{X}_{\alpha_{0}, D_{1}}^{p_{0}}, \tilde{X}_{\alpha_{1}, D_{1}}^{p_{1}}\right)_{\theta, q} \stackrel{E}{\longrightarrow} Y_{\alpha, D_{2}, *}^{p, q} \stackrel{R_{D_{1}}}{\longrightarrow} \tilde{Y}_{\alpha, D_{1}}^{p, q},
$$

and $R_{D_{1}} \circ E=I$, therefore, $\left(\tilde{X}_{\alpha_{0}, D_{1}}^{p_{0}}, \tilde{X}_{\alpha_{1}, D_{1}}^{p_{1}}\right)_{\theta, q} \hookrightarrow \tilde{Y}_{\alpha, D_{1}}^{p, q}$, which completes the proof of (iii) and the lemma.

Next, we invoke a result describing the way Banach space valued $L^{p}$-spaces interpolate via the real method. 
Theorem 1.2.25. (Theorem 1.1 on p. 47 in [LiPe])

Let $\left(A_{0}, A_{1}\right)$ be an interpolation couple of Banach spaces, and consider $p_{0}$ and $p_{1}$ such that $1 \leq p_{0}, p_{1} \leq \infty$. Then

$$
\left(L^{p_{0}}\left(A_{0}\right), L^{p_{1}}\left(A_{1}\right)\right)_{\theta, p}=L^{p}\left(\left(A_{0}, A_{1}\right)_{\theta, p}\right),
$$

where $0<\theta<1$ and $\frac{1}{p}=\frac{1-\theta}{p_{0}}+\frac{\theta}{p_{1}}$.

We now turn our attention to the complex interpolation method, which, in contrast to the real method, cannot be extended immediately from Banach spaces to quasi-Banach spaces. However, Calderón's original definition for Banach spaces has several (at least three) modifications. We first present the most general one, which is also suited for the applications we have in mind, and it is due to N. Kalton and M. Mitrea.

Following [KaMi] and [MeMi], let us invoke that if $X$ is a quasi-Banach space and $\Omega \subseteq \mathbb{C}$ is an open subset of the complex plane, then $f: \Omega \rightarrow X$ is called analytic if for each $z_{0} \in \Omega$ there exists $r>0$ such that there is a power series expantion $f(z)=\sum_{n=0}^{\infty} x_{n} z^{n}, x_{n} \in X$, converging uniformly for $\left|z-z_{0}\right|<r$.

We let $\left(X_{0}, X_{1}\right)$ be an interpolation couple of quasi-Banach spaces, i.e. assume that $X_{j}$ with $j=0,1$ are continuously embedded into a larger topological vector space, and $X_{0} \cap X_{1}$ is dense in $X_{j}, j=0,1$. We then define $\mathrm{F}$, the class of admissible functions, as the collection of all bounded, analytic functions

$$
f:\{x \in \mathbb{C}: 0<\operatorname{Re} z<1\} \longrightarrow X_{0}+X_{1},
$$

which extend continuously to the closure of the strip such that the traces $t \mapsto f(j+i t)$ 
are bounded continuous functions into $X_{j}, j=0,1$.

As in Claderón's original definition for Banach spaces, we equip F with the quasinorm

$$
\|f\|_{\mathrm{F}}:=\max \left\{\sup _{t \in \mathbb{R}}\|f(i t)\|_{X_{0}}, \sup _{t \in \mathbb{R}}\|f(1+i t)\|_{X_{1}}\right\} .
$$

Then, for $0<\theta<1$ we define

$$
\left[X_{0}, X_{1}\right]_{\theta}:=\left\{x \in X_{0}+X_{1}: x=f(\theta) \text { for some } f \in \mathrm{F}\right\}
$$

and endow it with the quasi-norm

$$
\|x\|_{\left[X_{0}, X_{1}\right]_{\theta}}:=\inf \left\{\|f\|_{\mathrm{F}}: f \in \mathrm{F}, f(\theta)=x\right\}, \quad \text { for } x \in\left[X_{0}, X_{1}\right]_{\theta}
$$

The difficulties with the above definition is that $F$ and the intermediate spaces $\left[X_{0}, X_{1}\right]_{\theta}$ are not necessarily complete. This is due to the fact that, in general, there is no analogue of the Maximum Modulus Principle in quasi-Banach spaces. However, there exists an important subclass of quasi-Banach spaces, called $A$-convex (analytically convex) in [Kal] (see pp. 299 - 300 and Theorem 4.1) in which the Maximum Modulus Principle does hold.

A quasi-Banach space $\left(X,\|\cdot\|_{X}\right)$ is called $A$-convex if there exists a constant $C$ such that for every polynomial $P: \mathbb{C} \rightarrow X$ we have $\|P(0)\|_{X} \leq C \max _{|z|=1}\|P(z)\|_{X}$. For further applications we also note that a quasi-Banach space $\left(X,\|\cdot\|_{X}\right)$ is $A$-convex if and only if there exists a positive finite constant $C$ such that

$$
\max _{0<\operatorname{Re} z<1}\|f(z)\|_{X} \leq C \max _{\operatorname{Re} z=0,1}\|f(z)\|_{X}
$$

for any analytic function $f:\{z \in \mathbb{C}: 0<\operatorname{Re} z<1\} \rightarrow X$ which is continuous and bounded on the closed strip $\{z \in \mathbb{C}: 0 \leq \operatorname{Re} z \leq 1\}$. 
With the additional assumption that $X_{0}+X_{1}$ is $A$-convex, we have

$$
\sup \left\{\|f(z)\|_{X_{0}+X_{1}}: 0<\operatorname{Re} z<1\right\} \leq C\|f\|_{\mathrm{F}}
$$

uniformly for $f \in \mathrm{F}$. This rectifies all the shortcomings of the above definition of the complex interpolation method. Moreover, the abstract stability theory developed in $[\mathrm{KaMi}]$ is valid. In particular, the following result is going to be of basic importance for us.

Proposition 1.2.26. (Corollary 2 in [MeMi])

Assume that the family of A-convex quasi-Banach spaces $\left\{X_{\alpha}^{p, q}\right\}$, indexed by triplets $\{p, q, \alpha\}$ in some open connected subset $\mathcal{U}$ of $(0, \infty) \times(0, \infty) \times \mathbb{R}$, is a complex interpolation scale, i.e. for any $\left(p_{j}, q_{j}, \alpha_{j}\right) \in \mathcal{U}, j=0,1$, there holds

$$
\left[X_{\alpha_{0}}^{p_{0}, q_{0}}, X_{\alpha_{1}}^{p_{1}, q_{1}}\right]_{\theta}=X_{\alpha}^{p, q}, \quad \forall \theta \in(0,1)
$$

where $1 / p:=(1-\theta) / p_{0}+\theta / p_{1}, 1 / q:=(1-\theta) / q_{0}+\theta / q_{1}$, and $\alpha:=(1-\theta) \alpha_{0}+\theta \alpha_{1}$. Also, suppose that $T$ is a linear operator mapping each $X_{\alpha}^{p, q}$ boundedly into itself and so that there exists $\left(p^{*}, q^{*}, \alpha^{*}\right) \in \mathcal{U}$ for which $T$ is an isomorphism when considered from $X_{\alpha^{*}}^{p^{*}, q^{*}}$ onto itself.

Then there exists an open neighborhood $\mathcal{V}$ of $\left(p^{*}, q^{*}, \alpha^{*}\right)$ in $\mathcal{U}$ such that $T$ continues to be an isomorphism of $X_{\alpha}^{p, q}$ for each $(p, q, \alpha) \in \mathcal{V}$.

We also invoke two other results from [MeMi], which are essential for the applications we have in mind. 
Lemma 1.2.27. (Lemma 1 in $[\mathrm{MeMi}])$

Assume that $(\Omega, d \mu)$ is a measure space, $0<p \leq \infty$ and that $\left(X,\|\cdot\|_{X}\right)$ is an A-convex quasi-Banach space. Then $L^{p}(\Omega ; X)$ is an A-convex quasi-Banach space.

Proposition 1.2.28. (Proposition 7 in $[\mathrm{MeMi}]$ )

For any $0<p, q \leq \infty, \alpha \in \mathbb{R}$, the spaces $B_{\alpha}^{p, q}\left(\mathbb{R}^{n}\right)$ and $F_{\alpha}^{p, q}\left(\mathbb{R}^{n}\right)(p \neq \infty$ for the Triebel-Lizorkin scale) are A-convex. The same is true for their homogeneous versions and for the associated (homogeneous and inhomogeneous) discrete spaces.

For the definition of the discrete Besov and Triebel-Lizorkin spaces we refer the reader to p. 46 in Section 5 of [FJW] and pp. 47, 71, 130 in [FrJa2], respectively (which correspond to the isotropic analogue of Definition 1.2.38). The parabolic counterpart of Proposition 1.2.28 can be proved in a very similar fashion, obtaining:

Proposition 1.2.29. For any $0<p, q \leq \infty$ and any $\alpha \in \mathbb{R}$, the anisotropic spaces $B_{\alpha, p a r}^{p, q}\left(\mathbb{R}^{n} \times \mathbb{R}\right)$ and $F_{\alpha, p a r}^{p, q}\left(\mathbb{R}^{n} \times \mathbb{R}\right)(p \neq \infty$ for the Triebel-Lizorkin scale) exhibiting parabolic anisotropy are A-convex. The same is true for their homogeneous versions and for the associated (homogeneous and inhomogeneous) discrete spaces.

The parabolic spaces are introduced in Definitions 2.1.2, 2.1.4, 2.1.6 and 2.1.8. Their discrete versions can be found in Definition 1.2.38.

In order to state the next result, we invoke some definitions from pp. $516-517$ of [MeMi]. Consider a $\sigma$-finite measure space $(\Omega, \Sigma, \mu)$ and let $L$ be the collection of all complex-valued, $\mu$-measurable functions on $\Omega$. Then a quasi-Banach function space $X$ on $(\Omega, \Sigma, \mu)$ is an order-ideal in the space $\mathrm{L}$ containing a strictly positive 
function, equipped with a quasi-norm $\|\cdot\|_{X}$ so that $\left(X,\|\cdot\|_{X}\right)$ is complete, and $f \in X$ and $g \in L$ with $|g| \leq|f|$ a.e. implies $g \in X$ with $\|g\|_{X} \leq\|f\|_{X}$.

Given two quasi-Banach lattices of functions $\left(X_{j},\|\cdot\|_{X_{j}}\right), j=0,1$, the Calderón product $X_{0}^{1-\theta} X_{1}^{\theta}$, where $0<\theta<1$, is the function space defined by

$$
X_{0}^{1-\theta} X_{1}^{\theta}:=\left\{g \in L: \exists f_{0} \in X_{0}, f_{1} \in X_{1} \text { such that }|g| \leq\left|f_{0}\right|^{1-\theta}\left|f_{1}\right|^{\theta}\right\}
$$

with the quasi-norm

$$
\|g\|_{X_{0}^{1-\theta} X_{1}^{\theta}}:=\inf \left\{\left\|f_{0}\right\|_{X_{0}}^{1-\theta}\left\|f_{1}\right\|_{X_{1}}^{\theta}:|g| \leq\left|f_{0}\right|^{1-\theta}\left|f_{1}\right|^{\theta}, f_{j} \in X_{j}, j=0,1\right\}
$$

The erarlier advertised result is as follows.

Theorem 1.2.30. (Theorem 3.4 in $[\mathrm{KaMi}]$ )

Let $\Omega$ be a Polish space (i.e. complete, separable, metric space), and let $\mu$ be a $\sigma$-finite Borel measure on $\Omega$. Assume that $X_{0}$ and $X_{1}$ are quasi-Banach function spaces on $(\Omega, \mu)$, they are $A$-convex and separable. Then $X_{0}+X_{1}$ is $A$-convex and $\left[X_{0}, X_{1}\right]_{\theta}=X_{0}^{1-\theta} X_{1}^{\theta}$ for each $0<\theta<1$, in the sense of equivalence of quasi-norms.

Remark 1.2.31. The complex method described above was applied, by O. Mendez and M. Mitrea, to pairs of Besov and Triebel-Lizorkin spaces (see p. 520 in [MeMi]). Formally, there is a resemblance between their results and the ones proved in Section 2.4.7 of [Tri4]. However, the reader should be aware that in [Tri4] there is a different definition of the complex interpolation method, which is not general enough (for our purposes), and which does not have the so-called interpolation property (i.e. preservation of the boundedness of linear operators (see the comment at the beginning of Section 2.4.8 of [Tri4]). This promted us to adapt the above approach. 
For the convenience of the reader, we also give the definition of the modified complex method due to A. P. Calderón and A. Torchinsky (cf. [CaTo1], [CaTo2], see also Section 2.4.4 in [Tri4]), which then can be applied to some quasi-Banach spaces, in particular, to Besov and Triebel-Lizorkin spaces. For brevity we present this method for the Besov scale $B_{\alpha}^{p, q}\left(\mathbb{R}^{n}\right), 0<p, q \leq \infty, \alpha \in \mathbb{R}$, but similar considerations apply to the Triebel-Lizorkin scale $F_{\alpha}^{p, q}\left(\mathbb{R}^{n}\right)$ with $0<p<\infty, 0<q \leq \infty$ and $\alpha \in \mathbb{R}$.

Definition 1.2.32. Let $A:=\{z \in \mathbb{C}: 0<$ Re $z<1\}$ be a strip in the complex plane, and consider $f$ such that:

(i) $f(z) \in \mathrm{S}^{\prime}\left(\mathbb{R}^{n}\right)$ for every $z \in \bar{A}$,

(ii) for every $\varphi \in \mathrm{S}\left(\mathbb{R}^{n}\right)$ with compact support in $\mathbb{R}^{n},\left(\mathcal{F}^{-1} \varphi \mathcal{F} f\right)(x, z)$ is a uniformly continuous bounded function in $\mathbb{R}^{n} \times \bar{A}$,

(iii) for every $\varphi \in \mathrm{S}\left(\mathbb{R}^{n}\right)$ with compact support in $\mathbb{R}^{n}$ and every fixed $x \in \mathbb{R}^{n}$, $\left(\mathcal{F}^{-1} \varphi \mathcal{F} f\right)(x, z)$ is an analytic function in $A$.

Assume that $0<p_{0}, p_{1}, q_{0}, q_{1} \leq \infty, \alpha_{0}, \alpha_{1} \in \mathbb{R}, 0<\theta<1$, and define

$$
\begin{aligned}
& {\left[B_{\alpha_{0}}^{p_{0}, q_{0}}\left(\mathbb{R}^{n}\right), B_{\alpha_{1}}^{p_{1}, q_{1}}\left(\mathbb{R}^{n}\right)\right]_{\theta}:=\left\{g \in \mathrm{S}^{\prime}\left(\mathbb{R}^{n}\right): \exists f(z)\right. \text { satisfying (i), (ii) and (iii) with }} \\
& f(i t) \in B_{\alpha_{0}}^{p_{0}, q_{0}}\left(\mathbb{R}^{n}\right), f(1+i t) \in B_{\alpha_{1}}^{p_{1}, q_{1}}\left(\mathbb{R}^{n}\right) \\
& \text { for every } t \in \mathbb{R} \text {, such that } g=f(\theta)\} \text {, }
\end{aligned}
$$

and

$$
\|g\|_{\left[B_{\alpha_{0}}^{p_{0}, q_{0}}\left(\mathbb{R}^{n}\right), B_{\alpha_{1}}^{p_{1}, q_{1}}\left(\mathbb{R}^{n}\right)\right]_{\theta}}:=\inf _{f(z)}\left\{\max \left[\sup _{t \in \mathbb{R}}\|f(i t)\|_{B_{\alpha_{0}}^{p_{0}, q_{0}}\left(\mathbb{R}^{n}\right)}, \sup _{t \in \mathbb{R}}\|f(1+i t)\|_{B_{\alpha_{1}}^{p_{1}, q_{1}}\left(\mathbb{R}^{n}\right)}\right]\right\} .
$$

The following abstract results will be needed in our further discussion. 
Lemma 1.2.33. (Theorem 14.3 of [LiMa], Vol. I, see also Lemma 2.12 of [JeKe]) Let $X_{i}, Y_{i}, Z_{i}, i=0,1$, be Banach spaces. Assume that $X_{0}$ and $X_{1}$ are contained in a larger Banach space such that $X_{0} \cap X_{1}$ is dense in both $X_{0}$ and $X_{1}$, similarly for $Z_{0}$ and $Z_{1}$. Suppose that $Y_{i} \hookrightarrow Z_{i}, i=0,1$, and that there is a continuous linear mapping $D: X_{i} \rightarrow Z_{i}$. Define the spaces

$$
X_{i}(D):=\left\{u \in X_{i}: D u \in Y_{i}\right\}, \quad i=0,1
$$

equipped with the graph norm, i.e. $\|u\|_{X_{i}(D)}:=\|u\|_{X_{i}}+\|D u\|_{Y_{i}}, i=0,1$. Suppose that there exist continuous linear mappings $G: Z_{i} \rightarrow X_{i}$ and $K: Z_{i} \rightarrow Y_{i}$ such that $D \circ G f=f+K f$ for all $f \in Z_{i}$. Then we can define $D$ by complex interpolation, for $0<\theta<1$, as a mapping from $\left[X_{0}, X_{1}\right]_{\theta}$ to $\left[Z_{0}, Z_{1}\right]_{\theta}$ and the natural inclusion $\left[Y_{0}, Y_{1}\right]_{\theta} \hookrightarrow\left[Z_{0}, Z_{1}\right]_{\theta}$. It follows that, for $0<\theta<1$,

$$
\left[X_{0}(D), X_{1}(D)\right]_{\theta}=\left\{u \in\left[X_{0}, X_{1}\right]_{\theta}: D u \in\left[Y_{0}, Y_{1}\right]_{\theta}\right\}
$$

Remark 1.2.34. With the additional assumption that the spaces $X_{0}+X_{1}$ and $Y_{0}+Y_{1}$ are $A$-convex, Lemma 1.2.33 holds true for $X_{i}, Y_{i}, Z_{i}, i=0,1$, quasi-Banach spaces.

Inded, it is not hard to see that the proof of Theorem 14.3 in [LiMa] can be carried out, for quasi-Banach spaces, with minor adjustments. The only aspect we need to verify is that the space $X_{0}(D)+X_{1}(D)$ is $A$-convex. In order to see this we first note that based on the decompositions (1.2.68) and (1.2.70), one can easily check that $X_{0}(D)+X_{1}(D)=\left(X_{0}+X_{1}\right)(D)$, where

$$
\left(X_{0}+X_{1}\right)(D):=\left\{u \in X_{0}+X_{1}: D u \in Y_{0}+Y_{1}\right\}
$$


equipped with the natural graph norm. We shall prove that the space $\left(X_{0}+X_{1}\right)(D)$ is $A$-convex. To do so, let us consider the open strip $U:=\{x \in \mathbb{C}: 0<\operatorname{Re} z<1\}$ and an analytic function $f: U \rightarrow\left(X_{0}+X_{1}\right)(D)$ which is continuous and bounded on the closed strip $\bar{U}$. Since the operator $D:\left(X_{0}+X_{1}\right)(D) \rightarrow Y_{0}+Y_{1}$ is linear and bounded, $D f$ is a $Y_{0}+Y_{1}$-valued analytic function in $U$, which extends by continuity to $\bar{U}$. Thus, employing our hypotheses that the spaces $X_{0}+X_{1}$ and $Y_{0}+Y_{1}$ are $A$-convex, we obtain the following:

$$
\begin{aligned}
\max _{0<\operatorname{Re} z<1}\|f(z)\|_{\left(X_{0}+X_{1}\right)(D)} & \approx \max _{0<\operatorname{Re} z<1}\|f(z)\|_{X_{0}+X_{1}}+\max _{0<\operatorname{Re} z<1}\|D f(z)\|_{Y_{0}+Y_{1}} \\
& \leq C \max _{\operatorname{Re} z=0,1}\|f(z)\|_{X_{0}+X_{1}}+C \max _{\operatorname{Re} z=0,1}\|D f(z)\|_{Y_{0}+Y_{1}} \\
& \approx \max _{\operatorname{Re} z=0,1}\|f(z)\|_{\left(X_{0}+X_{1}\right)(D)} .
\end{aligned}
$$

In view of $(1.2 .78)$ this amounts to $\left(X_{0}+X_{1}\right)(D)$ being $A$-convex, which is what we wanted to show.

Lemma 1.2.35. Let us consider $\mathcal{X}, \mathcal{Y}$ quasi-Banach spaces, $\left\{X_{\alpha}^{p, q}\right\}_{p, q, \alpha \in \mathbb{R}} \subset \mathcal{X}$ and $\left\{Y_{\alpha}^{p, q}\right\}_{p, q, \alpha \in \mathbb{R}} \subset \mathcal{Y}$ function spaces on a domain $\Omega \subset \mathbb{R}^{n}$ such that, for $p_{i}, q_{i}, \alpha_{i} \in \mathbb{R}$, $i=0,1$, the sum $X_{\alpha_{0}}^{p_{0}, q_{0}}+X_{\alpha_{1}}^{p_{1}, q_{1}}$ is A-convex, and

$$
\left[X_{\alpha_{0}}^{p_{0}, q_{0}}, X_{\alpha_{1}}^{p_{1}, q_{1}}\right]_{\theta}=Y_{\alpha}^{p, q}
$$

where $0<\theta<1, \frac{1}{p}=\frac{1-\theta}{p_{0}}+\frac{\theta}{p_{1}}, \frac{1}{q}=\frac{1-\theta}{q_{0}}+\frac{\theta}{q_{1}}$, and $\alpha=(1-\theta) \alpha_{0}+\theta \alpha_{1}$. Let $D_{1} \subseteq \Omega$ open and $D_{2} \subset \Omega$ closed subspaces, and define

$$
X_{\alpha, D_{1}}^{p, q}:=\left\{\left.f\right|_{D_{1}}: f \in X_{\alpha}^{p, q}\right\} \quad \text { and } \quad X_{\alpha, D_{2}, *}^{p, q}:=\left\{f \in X_{\alpha}^{p, q}: \operatorname{supp} f \subseteq D_{2}\right\}
$$

Similarly,

$$
Y_{\alpha, D_{1}}^{p, q}:=\left\{\left.f\right|_{D_{1}}: f \in Y_{\alpha}^{p, q}\right\} \quad \text { and } \quad Y_{\alpha, D_{2}, *}^{p, q}:=\left\{f \in Y_{\alpha}^{p, q}: \text { supp } f \subseteq D_{2}\right\} \text {. }
$$


(i) If there exists a universal extension operator $E_{D_{1}}: X_{\alpha, D_{1}}^{p, q} \rightarrow X_{\alpha}^{p, q}$ for all $p, q, \alpha \in \mathbb{R}$, then

$$
\left[X_{\alpha_{0}, D_{1}}^{p_{0}, q_{0}}, X_{\alpha_{1}, D_{1}}^{p_{1}, q_{1}}\right]_{\theta}=Y_{\alpha, D_{1}}^{p, q}
$$

where $0<\theta<1, \frac{1}{p}=\frac{1-\theta}{p_{0}}+\frac{\theta}{p_{1}}, \frac{1}{q}=\frac{1-\theta}{q_{0}}+\frac{\theta}{q_{1}}$ and $\alpha=(1-\theta) \alpha_{0}+\theta \alpha_{1}$.

(ii) If $X_{\alpha_{0}}^{p_{0}, q_{0}} \cap X_{\alpha_{1}}^{p_{1}, q_{1}}$ is dense in both $X_{\alpha_{0}}^{p_{0}, q_{0}}$ and $X_{\alpha_{1}}^{p_{1}, q_{1}}$, and if there exists $a$ universal extension operator $E_{D_{2}^{c}}: X_{\alpha, D_{2}^{c}}^{p, q} \rightarrow X_{\alpha}^{p, q}$ for all indices $p, q, \alpha \in \mathbb{R}$, where $D_{2}^{c}:=\Omega \backslash D_{2}$, then

$$
\left[X_{\alpha_{0}, D_{2}, *}^{p_{0}, q_{0}}, X_{\alpha_{1}, D_{2}, *}^{p_{1}, q_{1}}\right]_{\theta}=Y_{\alpha, D_{2}, *}^{p, q}
$$

where $0<\theta<1, \frac{1}{p}=\frac{1-\theta}{p_{0}}+\frac{\theta}{p_{1}}, \frac{1}{q}=\frac{1-\theta}{q_{0}}+\frac{\theta}{q_{1}}$ and $\alpha=(1-\theta) \alpha_{0}+\theta \alpha_{1}$.

(iii) Assume (1.2.83) holds and, for $p, q, \alpha \in \mathbb{R}$, define

$$
\tilde{X}_{\alpha, D_{1}}^{p, q}:=\left\{\left.f\right|_{D_{1}}: f \in X_{\alpha, D_{2}, *}^{p, q}\right\} \quad \text { and } \quad \tilde{Y}_{\alpha, D_{1}}^{p, q}:=\left\{\left.f\right|_{D_{1}}: f \in Y_{\alpha, D_{2}, *}^{p, q}\right\}
$$

If there exists a universal extension operator $E: \tilde{X}_{\alpha, D_{1}}^{p, q} \rightarrow X_{\alpha, D_{2}, *}^{p, q}$ for all $p, q, \alpha \in \mathbb{R}$, then

$$
\left[\tilde{X}_{\alpha_{0}, D_{1}}^{p_{0}, q_{0}}, \tilde{X}_{\alpha_{1}, D_{1}}^{p_{1}, q_{1}}\right]_{\theta}=\tilde{Y}_{\alpha, D_{1}}^{p, q}
$$

where $0<\theta<1, \frac{1}{p}=\frac{1-\theta}{p_{0}}+\frac{\theta}{p_{1}}, \frac{1}{q}=\frac{1-\theta}{q_{0}}+\frac{\theta}{q_{1}}$ and $\alpha=(1-\theta) \alpha_{0}+\theta \alpha_{1}$.

Remark 1.2.36. Before we proceed to prove the above lemma, a comment is in order here. First note that in part (ii) of Lemma 1.2.35 the $A$-convexity of the space $X_{\alpha_{0}, D_{2}, *}^{p_{0}, q_{0}}+X_{\alpha_{1}, D_{2}, *}^{p_{1}, q_{1}}$ is automatically satisfied (see the proof of (ii) below and Remark 1.2.34 above). Second, we observe that given two quasi-Banach function spaces $X_{0}, X_{1}$ on a domain $\Omega \subset \mathbb{R}^{n}$ such that $X_{0}+X_{1}$ is $A$-convex, the space $X_{0, D_{1}}+X_{1, D_{1}}$ is $A$-convex, as well, where $X_{i, D_{1}}:=\left\{\left.f\right|_{D_{1}}: f \in X_{i}\right\}, i=0,1$, and $D_{1}$ 
is a closed subset of $\Omega$. In order to justify this we can show that $X_{0, D_{1}}+X_{1, D_{1}}=$ $\left(X_{0}+X_{1}\right)_{D_{1}}$ and, using $(1.2 .78)$, that $\left(X_{0}+X_{1}\right)_{D_{1}}$ is $A$-convex. This property will guarantee that the complex interpolation method described in this subsection can be applied, without additional assumption, to the pairs $\left(X_{\alpha_{0}, D_{1}}^{p_{0}, q_{0}}, X_{\alpha_{1}, D_{1}}^{p_{1}, q_{1}}\right)$ and $\left(\tilde{X}_{\alpha_{0}, D_{1}}^{p_{0}, q_{0}}, \tilde{X}_{\alpha_{1}, D_{1}}^{p_{1}, q_{1}}\right)$ in parts (i) and (iii), respectively, of Lemma 1.2.35.

Proof of Lemma 1.2.35. (i) Define the restriction operator $R_{D_{1}} f=\left.f\right|_{D_{1}}$. Then $R_{D_{1}}: X_{\alpha}^{p, q} \rightarrow X_{\alpha, D_{1}}^{p, q}$, hence $R_{D_{1}} \circ E_{D_{1}}=I$ on $X_{\alpha, D_{1}}^{p, q}$. By complex interpolation we also have

$$
R_{D_{1}}:\left[X_{\alpha_{0}}^{p_{0}, q_{0}}, X_{\alpha_{1}}^{p_{1}, q_{1}}\right]_{\theta} \longrightarrow\left[X_{\alpha_{0}, D_{1}}^{p_{0}, q_{0}}, X_{\alpha_{1}, D_{1}}^{p_{1}, q_{1}}\right]_{\theta}
$$

i.e. $R_{D_{1}}: Y_{\alpha}^{p, q} \rightarrow\left[X_{\alpha_{0}, D_{1}}^{p_{0}, q_{0}}, X_{\alpha_{1}, D_{1}}^{p_{1}, q_{1}}\right]_{\theta}$. Since $R_{D_{1}}$ maps $Y_{\alpha}^{p, q}$ onto $Y_{\alpha, D_{1}}^{p, q}$, we get that

$$
Y_{\alpha, D_{1}}^{p, q} \hookrightarrow\left[X_{\alpha_{0}, D_{1}}^{p_{0}, q_{0}}, X_{\alpha_{1}, D_{1}}^{p_{1}, q_{1}}\right]_{\theta}
$$

On the other hand, $E_{D_{1}}:\left[X_{\alpha_{0}, D_{1}}^{p_{0}, q_{0}}, X_{\alpha_{1}, D_{1}}^{p_{1}, q_{1}}\right]_{\theta} \rightarrow Y_{\alpha}^{p, q}$ and $R_{D_{1}}: Y_{\alpha}^{p, q} \rightarrow Y_{\alpha, D_{1}}^{p, q}$. Their composition $R_{D_{1}} \circ E_{D_{1}}=I$, therefore

$$
\left[X_{\alpha_{0}, D_{1}}^{p_{0}, q_{0}}, X_{\alpha_{1}, D_{1}}^{p_{1}, q_{1}}\right]_{\theta} \hookrightarrow Y_{\alpha, D_{1}}^{p, q}
$$

and the proof of (i) is complete.

(ii) In the case when $\mathcal{X}, \mathcal{Y}$ are Banach spaces, we can apply Lemma 1.2.33 with $X_{0}=X_{\alpha_{0}}^{p_{0}, q_{0}}, X_{1}=X_{\alpha_{1}}^{p_{1}, q_{1}}, Z_{0}=X_{\alpha_{0}, D_{2}^{c}}^{p_{0}, q_{0}}, Z_{1}=X_{\alpha_{1}, D_{2}^{c}}^{p_{1}, q_{1}}, Y_{0}=Y_{1}=0, K=0, D=R_{D_{2}^{c}}$, and $G=E_{D_{2}^{c}}$. Then $R_{D_{2}^{c}} \circ E_{D_{2}^{c}}=I$ on $X_{\alpha, D_{2}^{c}}^{p, q}$,

$$
X_{0}(D)=X_{\alpha_{0}, D_{2}, *}^{p_{0}, q_{0}} \quad \text { and } \quad X_{1}(D)=X_{\alpha_{1}, D_{2}, *}^{p_{1}, q_{1}} .
$$


Consequently,

$$
\begin{aligned}
{\left[X_{\alpha_{0}, D_{2}, *}^{p_{0}, q_{0}}, X_{\alpha_{1}, D_{2}, *}^{p_{1}, q_{1}}\right]_{\theta} } & =\left\{f \in\left[X_{\alpha_{0}}^{p_{0}, q_{0}}, X_{\alpha_{1}}^{p_{1}, q_{1}}\right]_{\theta}:\left.f\right|_{D_{2}^{c}}=0\right\} \\
& =\left\{f \in Y_{\alpha}^{p, q}: \operatorname{supp} f \subseteq D_{2}\right\} \\
& =Y_{\alpha, D_{2}, *}^{p, q},
\end{aligned}
$$

where $0<\theta<1, \frac{1}{p}=\frac{1-\theta}{p_{0}}+\frac{\theta}{p_{1}}, \frac{1}{q}=\frac{1-\theta}{q_{0}}+\frac{\theta}{q_{1}}$ and $\alpha=(1-\theta) \alpha_{0}+\theta \alpha_{1}$.

If $\mathcal{X}, \mathcal{Y}$ are quasi-Banach spaces, we will show that the proof follows from the following.

Claim. Let $A_{j}, B_{j}, j=0,1$, be quasi-Banach spaces such that $B_{j} \hookrightarrow A_{j}$, and consider the operator $T: A_{j} \rightarrow B_{j}$ with the property that $T^{2}=T$ and $\left.T\right|_{B_{j}}=I$. Then, for $0<\theta<1$,

$$
T\left[A_{0}, A_{1}\right]_{\theta}=\left[B_{0}, B_{1}\right]_{\theta} .
$$

Proof of Claim. Since $T:\left[A_{0}, A_{1}\right]_{\theta} \rightarrow\left[B_{0}, B_{1}\right]_{\theta}$, the left-to-right inclusion in (1.2.84) is immediate. To show the opposite inclusion in (1.2.84) note that for $j=0,1$ the operator $T-I: B_{j} \rightarrow 0$, hence $T-I:\left[B_{0}, B_{1}\right]_{\theta} \rightarrow 0$ for $0<\theta<1$. Therefore,

$$
\forall x \in\left[B_{0}, B_{1}\right]_{\theta} \hookrightarrow\left[A_{0}, A_{1}\right]_{\theta} \text { we have } x=T x \in T\left[A_{0}, A_{1}\right]_{\theta},
$$

i.e. $\left[B_{0}, B_{1}\right]_{\theta} \hookrightarrow T\left[A_{0}, A_{1}\right]_{\theta}$, which completes the proof of the claim.

In order to show that the proof of (ii) follows from the above Claim, let us consider the operator $P$ defined as

$$
P:=I-E_{D_{2}^{c}} \circ R_{D_{2}^{c}}
$$

Take $f \in X_{\alpha}^{p, q}$. Then $R_{D_{2}^{c}} f \in X_{\alpha, D_{2}^{c}}^{p, q}$, and $\left(E_{D_{2}^{c}} \circ R_{D_{2}^{c}}\right) f \in X_{\alpha}^{p, q}$, hence $\operatorname{Pf} \in X_{\alpha}^{p, q}$. 
Moreover, since $R_{D_{2}^{c}} \circ E_{D_{2}^{c}}=I$,

$$
R_{D_{2}^{c}}(P f)=R_{D_{2}^{c}} f-\left(R_{D_{2}^{c}} \circ E_{D_{2}^{c}} \circ R_{D_{2}^{c}}\right) f=0
$$

which implies that supp $P f \subseteq D_{2}$. Consequently,

$$
P: X_{\alpha}^{p, q} \longrightarrow X_{\alpha, D_{2}, *}^{p, q}
$$

Going further, for any $f \in X_{\alpha, D_{2}, *}^{p, q}$ we have $f \in X_{\alpha}^{p, q}$ with supp $f \subseteq D_{2}$, implying $P f=f$. This amounts to $P: X_{\alpha}^{p, q} \rightarrow X_{\alpha, D_{2}, *}^{p, q}$ being onto.

To show that $P^{2}=P$ on $X_{\alpha}^{p, q}$, let $f \in X_{\alpha}^{p, q}$. Then $P f \in X_{\alpha}^{p, q}$ with $\operatorname{supp}(P f) \subseteq$ $D_{2}$, and

$$
P^{2} f=P(P f)=P f-\left(E_{D_{2}^{c}} \circ R_{D_{2}^{c}}\right)(P f)=P f .
$$

Taking also into account the fact that $\left.P\right|_{X_{\alpha, D_{2}, *}^{p, q}}=I$, the operator $P$ and the spaces $X_{\alpha}^{p, q}, X_{\alpha, D_{2}, *}^{p, q}$ satisfy the conditions of the Claim. Therefore,

$$
P\left[X_{\alpha_{0}}^{p_{0}, q_{0}}, X_{\alpha_{1}}^{p_{1}, q_{1}}\right]_{\theta}=\left[X_{\alpha_{0}, D_{2}, *}^{p_{0}, q_{0}}, X_{\alpha_{1}, D_{2}, *}^{p_{1}, q_{1}}\right]_{\theta}
$$

The left-hand side of (1.2.85) is $P\left(Y_{\alpha}^{p, q}\right)$, and since $P$ is onto, $P\left(Y_{\alpha}^{p, q}\right)=Y_{\alpha, D_{2}, *}^{p, q}$, consequently,

$$
\left[X_{\alpha_{0}, D_{2}, *}^{p_{0}, q_{0}}, X_{\alpha_{1}, D_{2}, *}^{p_{1}, q_{1}}\right]_{\theta}=Y_{\alpha, D_{2}, *}^{p, q}
$$

where $0<\theta<1, \frac{1}{p}=\frac{1-\theta}{p_{0}}+\frac{\theta}{p_{1}}, \frac{1}{q}=\frac{1-\theta}{q_{0}}+\frac{\theta}{q_{1}}$ and $\alpha=(1-\theta) \alpha_{0}+\theta \alpha_{1}$.

Note. In view of Remark 1.2.34 the proof of (ii) for quasi-Banach spaces can be carried out in the same spirit as the proof of (ii) for Banach spaces (as above).

(iii) Consider the restriction operator $R_{D_{1}}$ defined in the proof of (i). Then, naturaly, $R_{D_{1}}: X_{\alpha, D_{2}, *}^{p, q} \rightarrow \tilde{X}_{\alpha, D_{1}}^{p, q}$, hence $R_{D_{1}} \circ E=I$ on $\tilde{X}_{\alpha, D_{1}}^{p, q}$. Moreover, by 
(1.2.83), we have

$$
R_{D_{1}}: Y_{\alpha, D_{2}, *}^{p, q} \longrightarrow\left[\tilde{X}_{\alpha_{0}, D_{1}}^{p_{0}, q_{0}}, \tilde{X}_{\alpha_{1}, D_{1}}^{p_{1}, q_{1}}\right]_{\theta}
$$

Since $R_{D_{1}}$ maps $Y_{\alpha, D_{2}, *}^{p, q}$ onto $\tilde{Y}_{\alpha, D_{1}}^{p, q}$, we obtain that

$$
\tilde{Y}_{\alpha, D_{1}}^{p, q} \hookrightarrow\left[\tilde{X}_{\alpha_{0}, D_{1}}^{p_{0}, q_{0}}, \tilde{X}_{\alpha_{1}, D_{1}}^{p_{1}, q_{1}}\right]_{\theta} .
$$

On the other hand,

$$
\left[\tilde{X}_{\alpha_{0}, D_{1}}^{p_{0}, q_{0}}, \tilde{X}_{\alpha_{1}, D_{1}}^{p_{1}, q_{1}}\right]_{\theta} \stackrel{E}{\longrightarrow} Y_{\alpha, D_{2}, *}^{p, q} \stackrel{R_{D_{1}}}{\longrightarrow} \tilde{Y}_{\alpha, D_{1}}^{p, q}
$$

and $R_{D_{1}} \circ E=I$, therefore, $\left[\tilde{X}_{\alpha_{0}, D_{1}}^{p_{0}, q_{0}}, \tilde{X}_{\alpha_{1}, D_{1}}^{p_{1}, q_{1}}\right]_{\theta} \hookrightarrow \tilde{Y}_{\alpha, D_{1}}^{p, q}$, which completes the proof of (iii) and the lemma.

The argument in the proof of Lemma 1.2.21 with complex interpolation instead of the real one yields the following.

Lemma 1.2.37. For any $N \in \mathbb{N}$ and interpolation couples $\left(A_{0}^{i}, A_{1}^{i}\right), i=1, \ldots, N$, such that $A_{0}^{i}+A_{1}^{i}$ are $A$-convex, there holds

$$
\left[\bigoplus_{i=1}^{N} A_{0}^{i}, \bigoplus_{i=1}^{N} A_{1}^{i}\right]_{\theta}=\bigoplus_{i=1}^{N}\left[A_{0}^{i}, A_{1}^{i}\right]_{\theta},
$$

where $0<\theta<1$.

\subsubsection{Parabolic sequence spaces}

In this subsection we concentrate on the connection between parabolic function spaces (defined in Chapter 2) and their discrete counterparts. This correlation plays a crucial role when we try to ensure that the above described complex interpolation method can be applied to parabolic quasi-Banach spaces. We start with the definition of the discrete version of the parabolic Besov and Triebel-Lizorkin spaces (cf. 
Definitions 3.1 and 3.3 in [Bow2], Definitions 3.1 and 3.3 of [BoHo] for the anisotropic case, and pp. 47, 71, 130 of [FrJa2] for the $f$-scale, p. 46 of [FJW] for the $b$-scale in the isotropic setting).

Definition 1.2.38. For $\nu \in \mathbb{Z}$ and $(k, l) \in \mathbb{Z}^{n} \times \mathbb{Z}$ consider the parabolic dyadic cube

$$
\begin{gathered}
Q=Q_{\nu, k, l}:=\left\{(x, t) \in \mathbb{R}^{n} \times \mathbb{R}: 2^{-\nu} k_{i} \leq x_{i} \leq 2^{-\nu}\left(k_{i}+1\right), 1 \leq i \leq n,\right. \\
\left.2^{-2 \nu} l \leq t \leq 2^{-2 \nu}(l+1)\right\}
\end{gathered}
$$

Consider $0<p, q \leq \infty$ and $\alpha \in \mathbb{R}$. The sequence space $\dot{b}_{\alpha, p a r}^{p, q}$ is the collection of all complex-valued sequences $s=\left\{s_{Q}\right\}_{Q}$ such that

$$
\|s\|_{\dot{b}_{\alpha, p a r}^{p, q}}:=\left[\sum_{\nu \in \mathbb{Z}}\left(\left\|\sum_{l(Q)=2^{-\nu}}|Q|^{\frac{-\alpha}{n+2}-\frac{1}{2}}\left|s_{Q}\right| \chi_{Q}\right\|_{L^{p}\left(\mathbb{R}^{n} \times \mathbb{R}\right)}\right)^{q}\right]^{1 / q}<\infty .
$$

For the same indices the sequence space $\dot{f}_{\alpha, p a r}^{p, q}$ is the collection of all complex-valued sequences $s=\left\{s_{Q}\right\}_{Q}$ such that

$$
\|s\|_{\dot{f}_{\alpha, p a r}^{p, q}}:=\left\|\left[\sum_{Q}\left(|Q|^{\frac{-\alpha}{n+2}-\frac{1}{2}}\left|s_{Q}\right| \chi_{Q}\right)^{q}\right]^{1 / q}\right\|_{L^{p}\left(\mathbb{R}^{n} \times \mathbb{R}\right)}<\infty, \quad \text { if } \quad p<\infty,
$$

and

$$
\|s\|_{f_{\alpha, p a r}^{\infty, q}}:=\sup _{P \text { dyadic }}\left[\frac{1}{|P|} \int_{P} \sum_{Q \subset P}\left(|Q|^{\frac{-\alpha}{n+2}-\frac{1}{2}}\left|s_{Q}\right| \chi_{Q}(x, t)\right)^{q} d x d t\right]^{1 / q}<\infty .
$$

The spaces $b_{\alpha, p a r}^{p, q}$ and $f_{\alpha, p a r}^{p, q}$ of sequences associated with the inhomogeneous case are defined in a similar manner, except that the sum only involves cubes with sidelength $l(Q) \leq 1$.

Remark 1.2.39. Writing the sum in (1.2.89) as a double sum (as in (1.2.88)), and observing that with $l(Q)=2^{-\nu}$ the $\chi_{Q}$ 's have disjoint support, one can see that the 
$\dot{b}$ and $\dot{f}$ norms are obtained from each other by interchanging the $L^{p}\left(\mathbb{R}^{n} \times \mathbb{R}\right)$ and $\ell^{q}$ norms (as in the case of Besov and Triebel-Lizorkin spaces).

Also, note that $\dot{b}_{\alpha, p a r}^{p, q}$ and $\dot{f}_{\alpha, p a r}^{p, q}$ coincide for $p=q$. In this case, for simplicity, we shall write $\dot{b}_{\alpha, p a r}^{p}$ instead of $\dot{b}_{\alpha, p a r}^{p, q}$. Similar considerations apply to the inhomogeneous case, as well.

Definition 1.2.40. Consider the parabolic dyadic cube $Q$ as above (in (1.2.87)) and $\varphi \in \mathrm{S}\left(\mathbb{R}^{n} \times \mathbb{R}\right)$ with

$$
\begin{gathered}
\operatorname{supp} \hat{\varphi} \subset\left([-\pi, \pi]^{n} \backslash\{0\}\right) \times\left(\left[-\pi^{2}, \pi^{2}\right] \backslash\{0\}\right), \\
\sup _{m \in \mathbb{Z}}\left|\hat{\varphi}\left(A^{m}(\xi, \tau)\right)\right|>0 \text { for all }(\xi, \tau) \in \mathbb{R}^{n} \times \mathbb{R} \backslash\{(0,0)\}
\end{gathered}
$$

and

$$
\sum_{m \in \mathbb{Z}}\left|\hat{\varphi}\left(A^{m}(\xi, \tau)\right)\right|^{2}=1 \quad \forall(\xi, \tau) \in \mathbb{R}^{n} \times \mathbb{R} \backslash\{(0,0)\}
$$

where $A=\left(a_{i j}\right)_{1 \leq i, j \leq n+1}$ is a matrix defined by $a_{i i}=2$ if $i \leq n, a_{i i}=4$ if $i=n+1$, and $a_{i j}=0$ for $i \neq j$. Also, let $\nu \in \mathbb{Z},(k, l) \in \mathbb{Z}^{n} \times \mathbb{Z}$, and set

$$
\varphi_{Q}(x, t):=2^{\frac{\nu(n+2)}{2}} \varphi\left(2^{\nu} x-k, 2^{2 \nu} t-l\right), \quad(x, t) \in \mathbb{R}^{n} \times \mathbb{R} .
$$

We next invoke the definition of the operators $T_{\varphi}$ and $S_{\varphi}$ (see Definition 3.2 in [Bow2] or in [BoHo])

$$
T_{\varphi} \lambda:=\sum_{Q} \lambda_{Q} \varphi_{Q} \quad \text { for } \lambda=\left\{\lambda_{Q}\right\}_{Q}
$$

and

$$
S_{\varphi} f:=\left\{\left\langle f, \varphi_{Q}\right\rangle\right\}_{Q} \quad \text { for } f \in S^{\prime}\left(\mathbb{R}^{n} \times \mathbb{R}\right) / P
$$

where $P=P\left(\mathbb{R}^{n} \times \mathbb{R}\right)$ is the collection of all polynomials in $\mathbb{R}^{n} \times \mathbb{R}$. 
In what follows we record some important results (see Theorem 3.5 of [Bow2] and $[\mathrm{BoHo}])$.

Theorem 1.2.41. Consider the operators $T_{\varphi}$ and $S_{\varphi}$ as in Definition 1.2.40. Then, for $0<p, q \leq \infty(p \neq \infty$ for the $F$ and $f$ scales $)$ and $\alpha \in \mathbb{R}$,

$$
\begin{aligned}
& T_{\varphi}: \dot{b}_{\alpha, p a r}^{p, q} \longrightarrow \dot{B}_{\alpha, p a r}^{p, q}\left(\mathbb{R}^{n} \times \mathbb{R}\right), \\
& T_{\varphi}: \dot{f}_{\alpha, p a r}^{p, q} \longrightarrow \dot{F}_{\alpha, p a r}^{p, q}\left(\mathbb{R}^{n} \times \mathbb{R}\right)
\end{aligned}
$$

and

$$
\begin{gathered}
S_{\varphi}: \dot{B}_{\alpha, p a r}^{p, q}\left(\mathbb{R}^{n} \times \mathbb{R}\right) \longrightarrow \dot{b}_{\alpha, p a r}^{p, q}, \\
S_{\varphi}: \dot{F}_{\alpha, p a r}^{p, q}\left(\mathbb{R}^{n} \times \mathbb{R}\right) \longrightarrow \dot{f}_{\alpha, p a r}^{p, q}
\end{gathered}
$$

are bounded. Also,

$$
T_{\varphi} \circ S_{\varphi}=I \quad \text { on } \dot{B}_{\alpha, p a r}^{p, q}\left(\mathbb{R}^{n} \times \mathbb{R}\right) \quad \text { and } \quad T_{\varphi} \circ S_{\varphi}=I \quad \text { on } \dot{F}_{\alpha, p a r}^{p, q}\left(\mathbb{R}^{n} \times \mathbb{R}\right) \text {. }
$$

Remark 1.2.42. According to [Bow2] (p. 558) and [BoHo] (p. 20), the inhomogeneous counterpart of Theorem 1.2.41 is also valid. In this case the correspoding operators are defined by

$$
T_{\varphi, \Phi} \lambda:=\sum_{|Q|=1} \lambda_{Q} \Phi_{Q}+\sum_{|Q|<1} \lambda_{Q} \varphi_{Q} \quad \text { for } \lambda=\left\{\lambda_{Q}\right\}_{|Q| \leq 1}
$$

and

$$
S_{\varphi, \Phi} f:=\left\{\left(S_{\varphi, \Phi} f\right)_{Q}\right\}_{|Q| \leq 1} \quad \text { for } f \in \mathrm{S}^{\prime}\left(\mathbb{R}^{n} \times \mathbb{R}\right),
$$

where

$$
\left(S_{\varphi, \Phi} f\right)_{Q}:= \begin{cases}\left\langle f, \Phi_{Q}\right\rangle, & \text { if }|Q|=1 \\ \left\langle f, \varphi_{Q}\right\rangle, & \text { if }|Q|<1\end{cases}
$$


In this scenario the assumptions on $\varphi, \Phi \in \mathrm{S}\left(\mathbb{R}^{n} \times \mathbb{R}\right)$ are as follows:

$$
\begin{gathered}
\operatorname{supp} \hat{\varphi} \subset\left([-\pi, \pi]^{n} \backslash\{0\}\right) \times\left(\left[-\pi^{2}, \pi^{2}\right] \backslash\{0\}\right), \\
\operatorname{supp} \hat{\Phi} \subset[-\pi, \pi]^{n} \times\left[-\pi^{2}, \pi^{2}\right], \\
\sup _{m \in \mathbb{Z}}\left|\hat{\varphi}\left(A^{m}(\xi, \tau)\right)\right|>0 \quad \text { for all }(\xi, \tau) \in \mathbb{R}^{n} \times \mathbb{R} \backslash\{(0,0)\}, \\
\sup _{m \geq 1}\left\{\left|\hat{\varphi}\left(A^{-m}(\xi, \tau)\right)\right|,|\hat{\Phi}(\xi, \tau)|\right\}>0 \quad \text { for all }(\xi, \tau) \in \mathbb{R}^{n} \times \mathbb{R} \backslash\{(0,0)\},
\end{gathered}
$$

where the matrix $A$ is the same as in Definition 1.2.40.

In the remaining part of the current subsection, following the proof of Theorem 11 in $[\mathrm{MeMi}]$, we show that the above described complex interpolation method can be applied to the parabolic Besov and Triebel-Lizorkin scales defined in Section 2.1.1.

Theorem 1.2.43. Let $0<p_{0}, p_{1}, q_{0}, q_{1} \leq \infty\left(0<p_{0}, p_{1}<\infty\right.$ for the Triebel-Lizorkin scale), and $\alpha_{0}, \alpha_{1} \in \mathbb{R}$. Then

$$
\begin{aligned}
& B_{\alpha_{0}, \text { par }}^{p_{0}, q_{0}}\left(\mathbb{R}^{n} \times \mathbb{R}\right)+B_{\alpha_{1}, \text { par }}^{p_{1}, q_{1}}\left(\mathbb{R}^{n} \times \mathbb{R}\right) \quad \text { is A-convex }, \\
& F_{\alpha_{0}, \text { par }}^{p_{0}, q_{0}}\left(\mathbb{R}^{n} \times \mathbb{R}\right)+F_{\alpha_{1}, \text { par }}^{p_{1}, q_{1}}\left(\mathbb{R}^{n} \times \mathbb{R}\right) \quad \text { is A-convex. }
\end{aligned}
$$

The same is true for their homogeneous versions.

Proof. We sketch a proof of (1.2.97) only, since the same argument works for (1.2.98) and for the homogeneous scales.

We first observe that, by Remark $1.2 .42, B_{\alpha_{i}, p a r}^{p_{i}, q_{i}}\left(\mathbb{R}^{n} \times \mathbb{R}\right)$ with $0<p_{i}, q_{i} \leq \infty$, $\alpha_{i} \in \mathbb{R}, i=0,1$, can be identified with the discrete scale $b_{\alpha_{i}, \text { par }}^{p_{i}, q_{i}}$ with the same indices. Hence, in order to prove (1.2.97), it suffices to show that, for the indices specified 
above, $b_{\alpha_{0}, p a r}^{p_{0}, q_{0}}+b_{\alpha_{1}, p a r}^{p_{1}, q_{1}}$ is $A$-convex. This, in turn, follows from the fact that each $b_{\alpha_{i}, p a r}^{p_{i}, q_{i}}, i=0,1$, is A-convex (cf. Proposition 1.2.29) and from Theorem 1.2.30.

\subsubsection{Envelopes of Hardy spaces}

Following [Mit3], this subsection is an introduction to the envelopes of quasinormed spaces. In particular, the envelope of the (homogeneous) Hardy space $\dot{H}^{p}(\mathbb{R})$ is computed. We present only the information needed for our purposes, for more details we refer the reader to [Mit3] and [MeMi].

Let $0<p \leq 1$. A set $S$ (in a vector space $X$ ) is called $p$-convex if $S$ coincides with its $p$-convex hull, i.e.

$$
\left\{\sum_{\text {finite }} \lambda_{j} a_{j}: a_{j} \in S, \lambda \geq 0, \sum \lambda^{p} \leq 1\right\} .
$$

Also, call $S$ absolutely $p$-convex if $S$ coincides with its absolutely $p$-convex hull, i.e.

$$
\left\{\sum_{\text {finite }} \lambda_{j} a_{j}: a_{j} \in S, \sum|\lambda|^{p} \leq 1\right\} .
$$

Let $X$ be a linear, topological space and, for each $0<p \leq 1$, let $W_{X, p}$ be the absolutely $p$-convex hull of the unit ball in $X$. Set

$$
\||x|\|_{p}:=\inf \left\{\lambda>0: x / \lambda \in W_{X, p}\right\}
$$

Recall that $X^{*}$, the dual of $X$, is defined as the collection of all linear, continuous functionals on $X$. The latter condition means that $f: X \rightarrow \mathbb{R}$ satisfies

$$
\forall \varepsilon>0 \quad \exists \delta>0 \text { such that }\|x\|_{X}<\delta \Rightarrow|f(x)|<\varepsilon .
$$


If $X$ is locally bounded, with a topology given by a quasi-norm $\|\cdot\|_{X}$, the above condition is equivalent to

$$
\|f\|:=\sup \left\{|f(x)|:\|x\|_{X} \leq 1\right\}<+\infty .
$$

We say that $X^{*}$ separates the points in $X$ if $x=0 \Leftrightarrow f(x)=0, \forall f \in X^{*}$.

Proposition 1.2.44. If $X$ is a quasi-normed space whose dual separates its points, then (1.2.101) is a p-norm. That is, $\||x|\|_{p}=0$ if and only if $x=0,\||\lambda x|\|_{p}=$ $|\lambda|\||x|\|_{p}$, and

$$
\||x+y|\|_{p}^{p} \leq\||x|\|_{p}^{p}+\||y|\|_{p}^{p}, \quad \forall x, y \in X .
$$

For each $X$ as above, we denote by $\mathcal{E}_{p}(X)$ the $p$-envelope of $X$, i.e. the completion of $X$ in the quasi-norm $\||\cdot|\|_{p}$.

Comments. (i) The $\||\cdot|\|_{p}$ "norm" generates a locally $p$-convex topology, weaker that the original topology on $X$.

(ii) $\mathcal{E}_{p}(X)$ is a $p$-Banach space, i.e. a complete metric space whose metric is naturally induced by a $p$-norm.

(iii) $\mathcal{E}_{p}(X)$ should be thought of as the "smallest" locally $p$-convex topological space containing $X$. In fact, if $X$ is locally bounded, then $\mathcal{E}_{p}(X)$ is the "smallest" $p$-Banach space containing $X$. In particular, if $X$ is a $p$-Banach space to begin with, then $\mathcal{E}_{p}(X)=X$.

(vi) When $p=1, \mathcal{E}_{p}(X)$ corresponds to the so-called Banach envelope of $X$, i.e. the "smallest" Banach space containing $X$. See [MeMi]. 
Throughout, all vector spaces considered are assumed to be quasi-normed, whose duals separate points.

Proposition 1.2.45. Any bounded, linear operator $T: X \rightarrow Y$ extends to a bounded operator $\hat{T}: \mathcal{E}_{p}(X) \rightarrow \mathcal{E}_{p}(Y)$.

Proof. This is carried out much as in the case $p=1$ treated in [MeMi].

Proposition 1.2.46. For each $0<p \leq 1$,

$$
\mathcal{E}_{p}(X)^{*}=X^{*}
$$

Proof. Since $X \hookrightarrow \mathcal{E}_{p}(X)$, the mapping $\Phi:\left(\mathcal{E}_{p}(X)\right)^{*} \rightarrow X^{*}$, defined by $\Phi(f):=\left.f\right|_{X}$ is well defined, linear and bounded. Also, by Proposition 1.2.44, any $f \in X^{*}$ extends to a unique element $\hat{f} \in\left(\mathcal{E}_{p}(X)\right)^{*}$, and we define $\Psi: X^{*} \rightarrow\left(\mathcal{E}_{p}(X)\right)^{*}$ by $\Psi(f):=\hat{f}$. Then, clearly, $\Phi(\Psi(f))=\left.\hat{f}\right|_{X}=f$.

Also, for any $f \in\left(\mathcal{E}_{p}(X)\right)^{*}$, the fact that $\left.\widehat{\left(\left.f\right|_{X}\right)}\right|_{X}=\left.f\right|_{X}$, in concert with the fact that $X \hookrightarrow \mathcal{E}_{p}(X)$ densely, implies that $\widehat{\left(\left.f\right|_{X}\right)}=f$. Thus, $\Psi(\Phi(f))=f$.

This proves that $\Psi$ and $\Phi$ are inverses to each other, justifying (1.2.105).

Remark 1.2.47. Fix $0<p \leq p^{*} \leq 1$. Since $W_{X, p} \subseteq W_{X, p^{*}}$, it follows that $\||x|\|_{p^{*}} \leq$ $\||x|\|_{p}$ for any $x \in X$. In particular, any Cauchy sequence in $\||\cdot|\|_{p}$ is also Cauchy in $\||\cdot|\|_{p^{*}}$. In turn, (in concert with the abstract definition of compeletion as the vector space of equivalence classes of Cauchy sequences) implies that the identity map $I: X \rightarrow X$ extends to a linear, bounded operator $I: \mathcal{E}_{p}(X) \rightarrow \mathcal{E}_{p^{*}}(X)$. However, even though $\mathcal{E}_{p}(X)^{*}=\mathcal{E}_{p^{*}}(X)^{*}\left(=X^{*}\right)$, there are no guarantees that this map is one-to-one! Also, generally speaking, the dual of $\mathcal{E}_{p}(X)$ may not separate its points. 
Theorem 1.2.48. Let E, F be two quasi-Banach spaces whose duals separate points, and fix $0<p \leq 1$. Suppose that $F$ is a $p$-Banach space, $E \subseteq F$ and the inclusion $\iota: E \hookrightarrow F$ is continuous with dense range. Also, assume that $E$ has a good approximation of identity, in the sense that there exists a sequence of operators $\left\{A_{m}\right\}_{m}$, $A_{m}: E \rightarrow E$, such that

$$
\left\|A_{m} x\right\|_{\mathcal{E}_{p}(E)} \leq C\|x\|_{F}, \text { uniformly in } m \text {, for each } x \in E \text {, }
$$

and

$$
\left\|A_{m} x-x\right\|_{E} \rightarrow 0 \text { as } m \rightarrow \infty, \text { for each fixed } x \in E \text {. }
$$

Then $F$ is the p-envelope of $E$, i.e. $F=\mathcal{E}_{p}(E)$, if and only if the closure in $F$ of the absolutely p-convex hull of $B_{E}(0,1)$, the unit ball of $E$, is a neighborhood of the origin in $F$.

Proof. Assume that $F=\mathcal{E}_{p}(E)$. Then, if $W$ denotes the absolutely $p$-convex hull of $B_{E}(0,1)$,

$$
\|x\|_{F}<1 \Rightarrow \exists \lambda \in(0,1) \text { such that } x / \lambda \in W \Rightarrow x \in \lambda W \subseteq W \subseteq \bar{W}^{\|\cdot\|_{F}} .
$$

This proves the left-to-right implication.

Conversely, assume that the closure in $F$ of the absolutely $p$-convex hull of $B_{E}(0,1)$ is a neighborhood of the origin in $F$. First, by Proposition 1.2.44, the inclusion $\iota: E \hookrightarrow F$ extends to a bounded, linear operator

$$
\hat{\iota}: \mathcal{E}_{p}(E) \longrightarrow F \text {. }
$$

Our goal is to show that this extension is, in fact, an isomorphism. 
As a preliminary step, we note that

$E \cap B_{\mathcal{E}_{p}(E)}(0,1)$ coincides with $W_{E, p}$, the absolutely $p$-convex hull of $B_{E}(0,1)$.

Indeed, if $x \in W_{E, p}$ then $y \in E$ and $\|x\|_{\mathcal{E}_{p}(E)}<1$, proving the left-to-right inclusion in (1.2.109). As for the opposite one, we note that if $x \in E$ and $\|x\|_{\mathcal{E}_{p}(E)}<1$ then there exits $\lambda \in(0,1)$ such that $x / \lambda \in W_{E, p}$. In particular, $x \in \lambda W_{E, p} \subseteq W_{E, p}$, as desired.

Having justified (1.2.109) we then observe that

$$
W_{E, p}=\hat{\imath}\left(E \cap B_{\mathcal{E}_{p}(E)}(0,1)\right) \subseteq \hat{\imath}\left(B_{\mathcal{E}_{p}(E)}(0,1)\right) .
$$

Thus,

$$
{\overline{W_{E, p}}}^{\|\cdot\|_{F}} \subseteq \overline{\hat{\imath}\left(B_{\mathcal{E}_{p}(E)}(0,1)\right)}\|\cdot\|_{F} .
$$

Since the set in the left side of (1.2.111) is, by the current hypotheses, a neighborhood of 0 in $F$, it follows from the Open Mapping Theorem on p. 9 in $[\mathrm{KPR}]$ that $\hat{\iota}$ in (1.2.108) is onto.

There remains to show that this operator is also one-to-one. A moment's reflection shows that this is equivalent to proving that

$$
x_{j} \in E,\left\{x_{j}\right\}_{j} \text { Cauchy in } \mathcal{E}_{p}(E), x_{j} \rightarrow 0 \text { in } F \Rightarrow x_{j} \rightarrow 0 \text { in } \mathcal{E}_{p}(E) \text {. }
$$

Granted this, and recalling the approximate identity operators $A_{m}$, we write

$$
\left\|x_{j}\right\|_{\mathcal{E}_{p}(E)} \leq C\left\|x_{j}-A_{m} x_{j}\right\|_{\mathcal{E}_{p}(E)}+C\left\|A_{m} x_{j}\right\|_{\mathcal{E}_{p}(E)} .
$$

Under the current hypotheses on $\left\{x_{j}\right\}_{j}$ and $\left\{A_{m}\right\}_{m}$, given any $\varepsilon>0$ there exists $j_{\varepsilon}$ such that $C\left\|x_{j_{\varepsilon}}\right\|_{F} \leq \varepsilon$ and $C\left\|x_{j_{\varepsilon}}-A_{m} x_{j_{\varepsilon}}\right\|_{E} \leq \varepsilon / 2$ provided $m$ is large enough. Thus, 
$\left\|A_{m} x_{j_{\varepsilon}}\right\|_{\mathcal{E}_{p}(E)} \leq C\left\|x_{j_{\varepsilon}}\right\|_{F} \leq \varepsilon / 2$ and $C\left\|A_{m} x_{j_{\varepsilon}}\right\|_{\mathcal{E}_{p}(E)} \leq C\left\|A_{m} x_{j_{\varepsilon}}\right\|_{E} \leq \varepsilon / 2$. Hence, by (1.2.113), $\left\|x_{j_{\varepsilon}}\right\|_{\mathcal{E}_{p}(E)} \leq \varepsilon$. This proves that $\left\{x_{j}\right\}_{j}$ contains a subsequence which is convergent to zero in $\mathcal{E}_{p}(E)$. Being Cauchy, then the entire sequence converges to zero in $\mathcal{E}_{p}(E)$, as desired.

As is well-known (cf. Theorem I from [Coi], pp. $591-593$ of [CoWe2], Theorem in [Lat]), for $0<p \leq 1$, the homogeneous atomic Hardy space $\dot{H}^{p}\left(\mathbb{R}^{n}\right)$ is defined as the $\ell^{p}$-span of atoms, i.e.

$$
\begin{aligned}
& \text { supp } a \subset Q, \quad\|a\|_{L^{\infty}\left(\mathbb{R}^{n}\right)} \leq|Q|^{-1 / p} \text {, for some cube } Q \subseteq \mathbb{R}^{n}, \\
& \text { and } \int_{\mathbb{R}^{n}} x^{\beta} a(x) d x=0, \quad \forall \beta \in \mathbb{N}_{0}^{n} \text { with }|\beta| \leq[n(1 / p-1)] .
\end{aligned}
$$

On the other hand, if $0<p^{*} \leq 1$ and $\alpha \in \mathbb{R}$, then the homogeneous Besov space $\dot{B}_{\alpha}^{p^{*}}\left(\mathbb{R}^{n}\right)$ is defined (cf. Theorems 2.6 and 3.1 in [FrJa1], or Theorem 4.1 in [FrJa2]) as the $\ell^{p^{*}}$-span of atoms, i.e. functions satisfying

$$
\operatorname{supp} a \subset Q,
$$

$$
\begin{aligned}
& \left\|\partial^{\gamma} a\right\|_{L^{\infty}\left(\mathbb{R}^{n}\right)} \leq|Q|^{\alpha / n-1 / p^{*}-|\gamma| / n}, \quad \text { if }|\gamma| \leq([\alpha]+1)_{+}, \gamma \in \mathbb{N}_{0}^{n}, \\
& \int_{\mathbb{R}^{n}} x^{\beta} a(x) d x=0, \quad \forall \beta \in \mathbb{N}_{0}^{n},|\beta| \leq \max \left\{\left[n\left(1 / p^{*}-1\right)_{+}-\alpha\right],-1\right\} .
\end{aligned}
$$

Remark 1.2.49. If $0<p<p^{*} \leq 1$ and $\alpha=n\left(1 / p^{*}-1 / p\right)$ then the atoms defined as in (1.2.114) coincide with the atoms defined in (1.2.115).

Remark 1.2.50. If $0<p<p^{*} \leq 1$ and $\alpha=n\left(1 / p^{*}-1 / p\right)$ then $\dot{H}^{p}\left(\mathbb{R}^{n}\right) \hookrightarrow \dot{B}_{\alpha}^{p^{*}}\left(\mathbb{R}^{n}\right)$.

Our goal is to eventually prove that

Theorem 1.2.51. For $0<p<p^{*} \leq 1$ and $\alpha:=n\left(1 / p^{*}-1 / p\right)$, 


$$
\mathcal{E}_{p^{*}}\left(\dot{H}^{p}\left(\mathbb{R}^{n}\right)\right)=\dot{B}_{\alpha}^{p^{*}}\left(\mathbb{R}^{n}\right)
$$

Proof. The strategy is to use Theorem 1.2.48. We proceed in steps, starting with

Step I. The closure in $\dot{B}_{\alpha}^{p^{*}}\left(\mathbb{R}^{n}\right)$ of the $p^{*}$-convex hull of the standard unit ball of $\dot{H}^{p}\left(\mathbb{R}^{n}\right)$ contains a ball centered at the origin in $\dot{B}_{\alpha}^{p^{*}}\left(\mathbb{R}^{n}\right)$.

To see this, fix $u \in \dot{B}_{\alpha}^{p^{*}}\left(\mathbb{R}^{n}\right)$ with $\|u\|_{\dot{B}_{\alpha}^{p^{*}}\left(\mathbb{R}^{n}\right)}<1$. Then there exist $\lambda_{j} \in \mathbb{R}$ and $a_{j}$ atoms so that

$$
u=\sum_{j} \lambda_{j} a_{j}, \quad \sum_{j}\left|\lambda_{j}\right|^{p^{*}}<1 .
$$

Now, by Remark 1.2.49 above, each partial sum $\sum_{j=1}^{N} \lambda_{j} a_{j}$ belongs to the $p^{*}$-convex hull of the standard unit ball in $\dot{H}^{p}\left(\mathbb{R}^{n}\right)$, so that $u$ can be approximated (in $\dot{B}_{\alpha}^{p^{*}}\left(\mathbb{R}^{n}\right)$ ) by elements from this set. This clearly justifies the claim made in Step I.

Step II. The space $\dot{H}^{p}\left(\mathbb{R}^{n}\right)$ has the approximate identity property, relative to $\dot{B}_{s}^{p^{*}, p^{*}}\left(\mathbb{R}^{n}\right)$.

Given a real-valued function $\varphi$ and, for $\nu \in \mathbb{Z}, k \in \mathbb{Z}^{n}$, a dyadic cube $Q=Q_{\nu, k}$,

$$
Q_{\nu, k}:=\left\{x \in \mathbb{R}^{n}: 2^{-\nu} k_{i} \leq x_{i} \leq 2^{-\nu}\left(k_{i}+1\right), 1 \leq i \leq n\right\},
$$

we set, as usual, $\varphi_{Q}(x):=2^{\nu n / 2} \varphi\left(2^{\nu} x-k\right)$.

Let $\left\{\varphi^{\ell}\right\}_{\ell}$ be a family of "mother wavelets" and, for each $m \in \mathbb{N}$, set

$$
A_{m} f:=\sum_{\ell=1}^{2^{n}-1} \sum_{|\nu| \leq m} \sum_{\operatorname{dist}(Q, 0) \leq m}\left\langle f, \varphi_{Q}^{\ell}\right\rangle \varphi_{Q}^{\ell},
$$

i.e. a truncated wavelet expansion of $f$. According to Theorems 2.6 and 3.1 in [FrJa1], Theorem 4.1 of [FrJa2],

$$
\|f\|_{\dot{B}_{\alpha}^{p^{*}}\left(\mathbb{R}^{n}\right)} \approx \sum_{\ell=1}^{2^{n}-1}\left\|\left\{\tilde{f}_{Q}^{\ell}\right\}_{Q}\right\|_{\ell p^{*}},
$$


where

$$
\tilde{f}_{Q}^{\ell}:=|Q|^{1 / p-1 / 2}\left\langle f, \varphi_{Q}^{\ell}\right\rangle
$$

Also,

$$
\tilde{\varphi}_{Q}^{\ell}:=|Q|^{1 / 2-1 / p} \varphi_{Q}^{\ell}
$$

is a $\dot{H}^{p}$-atom, and

$$
A_{m} f=\sum \sum \sum \tilde{f}_{Q}^{\ell} \tilde{\varphi}_{Q}^{\ell}
$$

Consequently, if $\lambda:=\sum_{\ell=1}^{2^{n}-1}\left\|\left\{\tilde{f}_{Q}^{\ell}\right\}_{Q}\right\|_{\ell^{p^{*}}}$, then $A_{m} f / \lambda$ belongs to the absolute $p^{*}$-convex hull of the unit ball in $\dot{H}^{p}\left(\mathbb{R}^{n}\right)$, so that

$$
\left\|A_{m} f\right\|_{\mathcal{E}_{p^{*}}\left(\dot{H}^{p}\left(\mathbb{R}^{n}\right)\right)} \leq \lambda \approx\|f\|_{\dot{B}_{\alpha}^{p^{*}}\left(\mathbb{R}^{n}\right)}
$$

uniformly in $m$. This justifies (1.2.106) in the present context.

Since it is well known that

$$
A_{m} f \longrightarrow f \text { as } m \rightarrow \infty, \text { in } \dot{H}^{p}\left(\mathbb{R}^{n}\right)
$$

(cf., e.g., Theorem 7.20 in [FJW]), it follows that the analogue of (1.2.107) also holds.

The proof of the Theorem 1.2.51 is therefore finished.

The following abstract lemma will be useful in order to prove Theorems 3.3.1, 3.3.3 and Corollaries 3.3.2, 3.3.4.

Lemma 1.2.52. Consider $X_{i}, i=1,2$, quasi-Banach spaces, $Y_{i}, i=1,2$, Banach spaces, so that the incusions $X_{i} \hookrightarrow Y_{i}$ are well-defined, continuous and dense. Assume that $P \in \mathcal{L}\left(Y_{1}, Y_{2}\right)$ is well-defiened, continuous and has a continuous inverse to the right, as well as $\left.P\right|_{X_{1}} \in \mathcal{L}\left(X_{1}, X_{2}\right)$. Then, if $\mathcal{E}_{p}\left(X_{1}\right)=Y_{1}, 0<p \leq 1$, then we also have $\mathcal{E}_{p}\left(X_{2}\right)=Y_{2}$. 


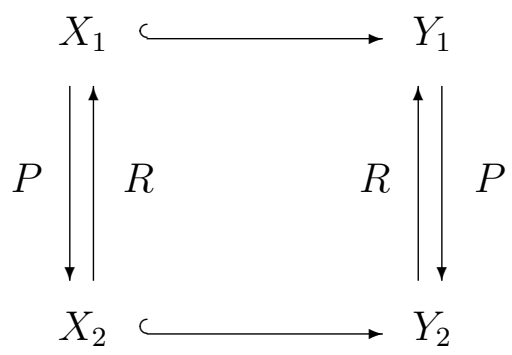

Proof. Denote the right-inverse of $P$ by $R$. Then $P \circ R=I$ on $Y_{2}$ and $\left.P \circ R\right|_{X_{2}}=I$ on $X_{2}$. Moreover, $P: X_{1} \rightarrow X_{2}$ and $R: X_{2} \rightarrow X_{1}$ extend linearly and boundedly as

$$
\hat{P}: \mathcal{E}_{p}\left(X_{1}\right) \longrightarrow \mathcal{E}_{p}\left(X_{2}\right) \text { and } \hat{R}: \mathcal{E}_{p}\left(X_{2}\right) \longrightarrow \mathcal{E}_{p}\left(X_{1}\right) \text {, }
$$

where $0<p \leq 1$. Since $\mathcal{E}_{p}\left(X_{1}\right)=Y_{1}$,

$$
\hat{P} \circ R: Y_{2} \longrightarrow \mathcal{E}_{p}\left(X_{2}\right), \quad P \circ \hat{R}: \mathcal{E}_{p}\left(X_{2}\right) \longrightarrow Y_{2}
$$

are bounded. In order to prove that $\mathcal{E}_{p}\left(X_{2}\right)=Y_{2}$, we will show that

$$
(\hat{P} \circ R) \circ(P \circ \hat{R})=I \quad \text { on } \quad \mathcal{E}_{p}\left(X_{2}\right)
$$

and

$$
(P \circ \hat{R}) \circ(\hat{P} \circ R)=I \quad \text { on } \quad Y_{2} .
$$

For (1.2.124) it is enough to consider a dense subclass of $\mathcal{E}_{p}\left(X_{2}\right)$, in particular, $X_{2}$. It is not hard to see that $(\hat{P} \circ R) \circ(P \circ \hat{R})$ is the identity operatr on $X_{2}$. Similarly, for (1.2.125), we consider $X_{2}$, a dense subclass of $Y_{2}$, and we show that $(P \circ \hat{R}) \circ(\hat{P} \circ R)$ is the identity on $X_{2}$. This completes the proof of the lemma.

Remark 1.2.53. In Theorem 1.2.52 it is enough to assume the density of the inclusion $X_{1} \hookrightarrow Y_{1}$ only, since, in this case, the density of $X_{2} \hookrightarrow Y_{2}$ follows. 
Proof. Let $y \in Y_{2}$. Then $R y \in Y_{1}$ and, since $X_{1}$ is dense in $Y_{1}$, there exists a sequence $\left\{x_{k}\right\}_{k \in \mathbb{N}}$ in $X_{1}$ such that $\left\|x_{k}-R y\right\|_{Y_{1}} \rightarrow 0$ as $k \rightarrow 0$. Moreover, $P x_{k} \in X_{2}$ for each $k \in \mathbb{N}$ and

$$
\left\|P x_{k}-y\right\|_{Y_{2}}=\left\|P x_{k}-(P \circ R) y\right\|_{Y_{2}}=\left\|P\left(x_{k}-R y\right)\right\|_{Y_{2}} \leq c\left\|x_{k}-R y\right\|_{Y_{1}} .
$$

Therefore, $X_{2}$ is dense in $Y_{2}$. 


\section{Chapter 2}

\section{Parabolic function spaces}

In this chapter we discuss Sobolev, Besov, Hardy and Triebel-Lizorkin spaces with parabolic anisotropy. Section 2.1 deals with the Euclidean setting $\mathbb{R}^{n} \times \mathbb{R}$. In Sections 2.2 and 2.3 we adapt these definitions to the case of a Lipschitz cylinder and its lateral boundary, respectively. Section 2.4 contains duality results on different geometrical settings. In Section 2.5 we extend the theory of envelopes of isotropic Hardy spaces to the parabolic setting.

\subsection{Euclidean setting}

\subsubsection{Besov and Triebel-Lizorkin spaces}

We first give the definition of the parabolic Besov and Triebel-Lizorkin spaces, then we shall present several important features of these spaces.

Recall that $\|(x, t)\|_{\text {par }}:=\left(|x|^{2}+|t|\right)^{1 / 2}$ for $x \in \mathbb{R}^{n}, t \in \mathbb{R}$.

Definition 2.1.1. Define the system $\Phi$ of complex-valued functions $\left\{\varphi_{j}\right\}_{j=0}^{\infty}$ with the following properties:

(i) $\varphi_{j} \in C_{c}^{\infty}\left(\mathbb{R}^{n} \times \mathbb{R}\right)$; 
(ii) For $\kappa>1$ an appropriate number,

$$
\begin{aligned}
& \operatorname{supp} \varphi_{0} \subset\left\{(x, t) \in \mathbb{R}^{n} \times \mathbb{R}:\|(x, t)\|_{\text {par }} \leq \kappa 2\right\}, \\
& \operatorname{supp} \varphi_{j} \subset\left\{(x, t) \in \mathbb{R}^{n} \times \mathbb{R}: \frac{1}{\kappa} 2^{j} \leq\|(x, t)\|_{p a r} \leq \kappa 2^{j+1}\right\}, \quad j \in \mathbb{N} ;
\end{aligned}
$$

(iii) There exist positive constants $c_{0}$ and $c_{1}$ such that

$$
c_{0} \leq\left|\sum_{j=0}^{\infty} \varphi_{j}(x, t)\right| \leq c_{1}, \quad(x, t) \in \mathbb{R}^{n} \times \mathbb{R} ;
$$

(iv) For every multiindex $\gamma \in \mathbb{N}_{0}^{n}$ and every $k \in \mathbb{N}_{0}$ there exists a positive constant $C_{\gamma, k}$ such that

$$
\left|\partial_{x}^{\gamma} \partial_{t}^{k} \varphi_{j}(x, t)\right| \leq \frac{C_{\gamma, k}}{2^{j(|\gamma|+2 k)}}
$$

An example of the family $\left\{\varphi_{j}\right\}_{j=0}^{\infty}$ is as follows. Fix $\varphi_{0} \in C^{\infty}\left(\mathbb{R}^{n} \times \mathbb{R}\right)$ such that $\varphi_{0}(x, t)=1$ if $\|(x, t)\|_{\text {par }} \leq 1$ and supp $\varphi_{0} \subset\left\{(x, t) \in \mathbb{R}^{n} \times \mathbb{R}:\|(x, t)\|_{\text {par }} \leq 2\right\}$. Then introduce

$$
\varphi_{j}(x, t):=\varphi_{0}\left(2^{-j} x, 2^{-2 j} t\right)-\varphi_{0}\left(2^{-j+1} x, 2^{2(-j+1)} t\right), \quad(x, t) \in \mathbb{R}^{n} \times \mathbb{R}, j \in \mathbb{N} .
$$

It follows that $\left\{\varphi_{j}\right\}_{j=0}^{\infty}$ is a partition of unity, i.e.

$$
\sum_{j=0}^{\infty} \varphi_{j}(x, t)=1 \quad \text { for } \quad(x, t) \in \mathbb{R}^{n} \times \mathbb{R}
$$

and, clearly, $\left\{\varphi_{j}\right\}_{j=0}^{\infty}$ satisfies all of the conditions (i)-(iv) of Definition 2.1.1, hence $\left\{\varphi_{j}\right\}_{j=0}^{\infty} \in \Phi$

Based on this system $\Phi$, we can then give a Littlewood-Paley type definition of parabolic Besov and Triebel-Lizorkin spaces much as in [Stö1] (pp. 790 - 793) and in the isotropic setting (cf. pp. 171 - 172 of [Tri2]). 
Definition 2.1.2. For $0<p, q \leq \infty, \alpha \in \mathbb{R}$ and $\left\{\varphi_{j}\right\}_{j=0}^{\infty} \in \Phi$ define

$$
\begin{aligned}
B_{\alpha, p a r}^{p, q}\left(\mathbb{R}^{n} \times \mathbb{R}\right):= & \left\{f \in \mathrm{S}^{\prime}\left(\mathbb{R}^{n} \times \mathbb{R}\right):\right. \\
& \left.\|f\|_{B_{\alpha, p a r}^{p, q}\left(\mathbb{R}^{n} \times \mathbb{R}\right)}:=\left(\sum_{j=0}^{\infty} 2^{j \alpha q}\left\|\mathcal{F}^{-1}\left(\varphi_{j} \mathcal{F} f\right)\right\|_{L^{p}\left(\mathbb{R}^{n} \times \mathbb{R}\right)}^{q}\right)^{1 / q}<\infty\right\}
\end{aligned}
$$

and, for $p \neq \infty$,

$$
\begin{aligned}
F_{\alpha, p a r}^{p, q}\left(\mathbb{R}^{n} \times \mathbb{R}\right):=\left\{f \in \mathrm{S}^{\prime}\left(\mathbb{R}^{n} \times \mathbb{R}\right)\right. & : \\
& \left.\|f\|_{F_{\alpha, p a r}^{p, q}\left(\mathbb{R}^{n} \times \mathbb{R}\right)}:=\left\|\left(\sum_{j=0}^{\infty} 2^{j \alpha q}\left|\mathcal{F}^{-1}\left(\varphi_{j} \mathcal{F} f\right)\right|^{q}\right)^{1 / q}\right\|_{L^{p}\left(\mathbb{R}^{n} \times \mathbb{R}\right)}<\infty\right\}
\end{aligned}
$$

with the usual modification if $q=\infty$, i.e.

$$
\begin{aligned}
B_{\alpha, p a r}^{p, \infty}\left(\mathbb{R}^{n} \times \mathbb{R}\right):=\left\{f \in \mathrm{S}^{\prime}\left(\mathbb{R}^{n} \times \mathbb{R}\right):\right. & \\
& \left.\|f\|_{B_{\alpha, p a r}^{p, \infty}\left(\mathbb{R}^{n} \times \mathbb{R}\right)}:=\sup _{j \in \mathbb{N}_{0}}\left[2^{j \alpha}\left\|\mathcal{F}^{-1}\left(\varphi_{j} \mathcal{F} f\right)\right\|_{L^{p}\left(\mathbb{R}^{n} \times \mathbb{R}\right)}\right]<\infty\right\}
\end{aligned}
$$

and, for $p \neq \infty$,

$$
\begin{aligned}
F_{\alpha, p a r}^{p, \infty}\left(\mathbb{R}^{n} \times \mathbb{R}\right):=\left\{f \in \mathrm{S}^{\prime}\left(\mathbb{R}^{n} \times \mathbb{R}\right):\right. & \\
& \left.\|f\|_{F_{\alpha, p a r}^{p, \infty}\left(\mathbb{R}^{n} \times \mathbb{R}\right)}:=\left\|\sup _{j \in \mathbb{N}_{0}}\left(2^{j \alpha}\left|\mathcal{F}^{-1}\left(\varphi_{j} \mathcal{F} f\right)\right|\right)\right\|_{L^{p}\left(\mathbb{R}^{n} \times \mathbb{R}\right)}<\infty\right\} .
\end{aligned}
$$

When emphasizing the system $\Phi$, we shall use the notation $B_{\alpha, p a r}^{p, q, \Phi}$ and $F_{\alpha, \text { par }}^{p, q}$ instead of $B_{\alpha, p a r}^{p, q}$ and $F_{\alpha, p a r}^{p, q}$, respectively.

Note that a different choice of $\left\{\varphi_{j}\right\}_{j=0}^{\infty} \in \Phi$ yields a vector space equipped with an equivalent quasi-norm. For this, we refer the reader to pp. 790 - 793 in [Stö1], Theorem 3.6 of [Yam], Corollary 3.7 of [BoHo] and Theorem 3 of [DaTr].

Remark 2.1.3. There exist other natural possibilities of introducing the systems $\Phi$ 's. For example, we can define $\Phi^{\prime}$ much as in Definition 2.1.1, except that 
(iii) $\sum_{j=0}^{\infty} \varphi_{j}$ is real-valued and there exist positive constants $c_{0}$ and $c_{1}$ such that

$$
c_{0} \leq \sum_{j=0}^{\infty} \varphi_{j}(x, t) \leq c_{1}, \quad(x, t) \in \mathbb{R}^{n} \times \mathbb{R} .
$$

Similarly, we can introduce $\Phi^{\prime \prime}$ as in Definition 2.1.1, except that (iii) $\sum_{j=0}^{\infty} \varphi_{j}$ is real-valued and, for $(x, t) \in \mathbb{R}^{n} \times \mathbb{R}$, we have $\sum_{j=0}^{\infty} \varphi_{j}(x, t)=1$.

Nonetheless, one can show that these definitions lead to the same Besov and Triebel-Lizorkin spaces when $\Phi^{\prime}$ or $\Phi^{\prime \prime}$ is used in place of $\Phi$ (although their norms change up to an equivalence).

A sketch of proof is based on the following ideas. Here we only consider the Besov scale with $0<p<\infty$, however a similar argument can be easily carried out for $p=\infty$ and for the Triebel-Lizorkin space, as well. Clearly, $\Phi^{\prime \prime} \subset \Phi^{\prime} \subset \Phi$. Hence

$$
B_{\alpha, p a r}^{p, q, \Phi^{\prime \prime}}\left(\mathbb{R}^{n} \times \mathbb{R}\right) \hookrightarrow B_{\alpha, p a r}^{p, q, \Phi}\left(\mathbb{R}^{n} \times \mathbb{R}\right) .
$$

In order to show the opposite inclusion in (2.1.1), let $f \in B_{\alpha, p a r}^{p, q}\left(\mathbb{R}^{n} \times \mathbb{R}\right),\left\{\varphi_{j}\right\}_{j=0}^{\infty} \in \Phi$ and set

$$
\varphi:=\sum_{j=0}^{\infty} \varphi_{j}, \quad \widetilde{\varphi}_{j}:=\frac{\varphi_{j}}{\varphi} .
$$

Then $\left\{\widetilde{\varphi}_{j}\right\}_{j=0}^{\infty} \in \Phi^{\prime \prime}$ and, by definition,

$$
\begin{aligned}
\|f\|_{B_{\alpha, p a r}^{p, q, \Phi^{\prime \prime}}\left(\mathbb{R}^{n} \times \mathbb{R}\right)} & =\left(\sum_{j=0}^{\infty} 2^{j \alpha q}\left\|\mathcal{F}^{-1}\left(\tilde{\varphi}_{j} \mathcal{F} f\right)\right\|_{L^{p}\left(\mathbb{R}^{n} \times \mathbb{R}\right)}^{q}\right)^{1 / q} \\
& =\left(\sum_{j=0}^{\infty} 2^{j \alpha q}\left\|\mathcal{F}^{-1}\left[\varphi_{j} \mathcal{F}\left(\mathcal{F}^{-1}\left(\frac{1}{\varphi} \mathcal{F} f\right)\right)\right]\right\|_{L^{p}\left(\mathbb{R}^{n} \times \mathbb{R}\right)}^{q}\right)^{1 / q} \\
& =\left\|\mathcal{F}^{-1}\left(\frac{1}{\varphi} \mathcal{F} f\right)\right\|_{B_{\alpha, p a r}^{p, q, \Phi}\left(\mathbb{R}^{n} \times \mathbb{R}\right)} \\
& \leq c\|f\|_{B_{\alpha, p a r}^{p, q, \Phi}\left(\mathbb{R}^{n} \times \mathbb{R}\right)} .
\end{aligned}
$$


The inequality in (2.1.3) holds provided that $1 / \varphi$ is a multiplier in $B_{\alpha, p a r}^{p, q, \Phi}\left(\mathbb{R}^{n} \times \mathbb{R}\right)$ for any $0<p, q \leq \infty$ and any $\alpha \in \mathbb{R}$. This, in turn, can be verified by the following (cf. Theorem 2.1 and Remark 2.1 of [Stö1]). For every multiindex $\gamma \in \mathbb{N}_{0}^{n}$ and every $k \in \mathbb{N}_{0}$ there exists a positive finite constant $C_{\gamma, k}$ such that

$$
\left|\partial_{\xi}^{\gamma} \partial_{\tau}^{k}\left[\left(\sum_{j=0}^{\infty} \varphi_{j}(\xi, \tau)\right)^{-1}\right]\right| \leq \frac{C_{\gamma, k}}{\left(1+\|(\xi, \tau)\|_{p a r}\right)^{\gamma+2 k}}, \quad(\xi, \tau) \in \mathbb{R}^{n} \times \mathbb{R} .
$$

Taking into account the support conditions on $\varphi_{j}, j \in \mathbb{N}_{0}$ (see part (ii) of Definition 2.1.1) and using part (iv) of Definition 2.1.1, as well, one can check that (2.1.4) holds. Therefore (2.1.3) is true, which further implies the opposite inclusion in (2.1.1), finishing the proof of Remark 2.1.3.

For further applications we find it useful to give yet another definition.

Definition 2.1.4. Let $\varphi \in \mathrm{S}\left(\mathbb{R}^{n} \times \mathbb{R}\right)$ be such that

$$
\begin{gathered}
\varphi(x, t)>0 \quad \text { for } 2^{-1}<\|(x, t)\|_{\text {par }}<2, \\
\operatorname{supp} \varphi=\left\{(x, t) \in \mathbb{R}^{n} \times \mathbb{R}: 2^{-1} \leq\|(x, t)\|_{\text {par }} \leq 2\right\}
\end{gathered}
$$

and

$$
\sum_{j=-\infty}^{\infty} \varphi\left(2^{-j} x, 2^{-2 j} t\right)=1 \quad \text { for } \quad(x, t) \neq 0 .
$$

Define the functions $\varphi_{j}$ and $\psi_{j}$ by

$$
\begin{gathered}
\left(\mathcal{F} \varphi_{j}\right)(x, t)=\varphi\left(2^{-j} x, 2^{-2 j} t\right) \quad \text { for } \quad j \in \mathbb{Z}, \\
\left(\mathcal{F} \psi_{0}\right)(x, t)=1-\sum_{j=1}^{\infty} \varphi\left(2^{-j} x, 2^{-2 j} t\right), \quad \psi_{j}=\varphi_{j} \quad \text { for } j \geq 1 .
\end{gathered}
$$


Then, for $0<p, q \leq \infty, \alpha \in \mathbb{R}$, the parabolic Besov and Triebel-Lizorkin spaces are defined by

$$
\begin{aligned}
B_{\alpha, p a r}^{p, q}\left(\mathbb{R}^{n} \times \mathbb{R}\right):=\left\{f \in \mathrm{S}^{\prime}\left(\mathbb{R}^{n} \times \mathbb{R}\right):\right. & \\
& \left.\|f\|_{B_{\alpha, p a r}^{p, q}\left(\mathbb{R}^{n} \times \mathbb{R}\right)}:=\left(\sum_{j=0}^{\infty} 2^{j \alpha q}\left\|\psi_{j} * f\right\|_{L^{p}\left(\mathbb{R}^{n} \times \mathbb{R}\right)}^{q}\right)^{1 / q}<\infty\right\}
\end{aligned}
$$

and, for $p \neq \infty$,

$$
\begin{aligned}
F_{\alpha, \text { par }}^{p, q}\left(\mathbb{R}^{n} \times \mathbb{R}\right):= & \left\{f \in \mathrm{S}^{\prime}\left(\mathbb{R}^{n} \times \mathbb{R}\right):\right. \\
& \left.\|f\|_{F_{\alpha, \text { par }}^{p, q}\left(\mathbb{R}^{n} \times \mathbb{R}\right)}:=\left\|\left(\sum_{j=0}^{\infty} 2^{j \alpha q}\left|\left(\psi_{j} * f\right)(\cdot)\right|^{q}\right)^{1 / q}\right\|_{L^{p}\left(\mathbb{R}^{n} \times \mathbb{R}\right)}<\infty\right\},
\end{aligned}
$$

with the usual modification if $q=\infty$, i.e.

$$
\begin{aligned}
B_{\alpha, \text { par }}^{p, \infty}\left(\mathbb{R}^{n} \times \mathbb{R}\right):=\left\{f \in \mathrm{S}^{\prime}\left(\mathbb{R}^{n} \times \mathbb{R}\right):\right. & \\
& \left.\|f\|_{B_{\alpha, \text { par }}^{p, \infty}\left(\mathbb{R}^{n} \times \mathbb{R}\right)}:=\sup _{j \in \mathbb{N}_{0}}\left(2^{j \alpha} \|\left.\left(\psi_{j} * f\right)\right|_{L^{p}\left(\mathbb{R}^{n} \times \mathbb{R}\right)}\right)<\infty\right\}
\end{aligned}
$$

and, for $p \neq \infty$,

$$
\begin{aligned}
F_{\alpha, p a r}^{p, \infty}\left(\mathbb{R}^{n} \times \mathbb{R}\right):=\left\{f \in S^{\prime}\left(\mathbb{R}^{n} \times \mathbb{R}\right):\right. & \\
& \left.\|f\|_{F_{\alpha, p a r}^{p, \infty}\left(\mathbb{R}^{n} \times \mathbb{R}\right)}:=\left\|\sup _{j \in \mathbb{N}_{0}}\left(2^{j \alpha}\left|\left(\psi_{j} * f\right)(\cdot)\right|\right)\right\|_{L^{p}\left(\mathbb{R}^{n} \times \mathbb{R}\right)}<\infty\right\} .
\end{aligned}
$$

This definition is equivalent with Definition 2.1.4, since $\left\{\mathcal{F} \psi_{j}\right\}_{j \in \mathbb{N}_{0}}$ satisfies similar properties to $\left\{\varphi_{j}\right\}_{j \in \mathbb{N}_{0}}$ from Definition 2.1.2.

As is well known (see, e.g. Remark 3.1, Proposition 3.5 of [Yam], and p. 11 of $[\mathrm{Joh}])$, for $0<p, q \leq \infty, \alpha \in \mathbb{R}$ the spaces $B_{\alpha, p a r}^{p, q}\left(\mathbb{R}^{n} \times \mathbb{R}\right)$ and $F_{\alpha, p a r}^{p, q}\left(\mathbb{R}^{n} \times \mathbb{R}\right)$ ( $p \neq \infty$ for the $F$-scale) are complete, for $p, q \geq 1$ they are Banach spaces, and in general

$$
\begin{aligned}
& \mathrm{S}\left(\mathbb{R}^{n} \times \mathbb{R}\right) \hookrightarrow B_{\alpha, \text { par }}^{p, q}\left(\mathbb{R}^{n} \times \mathbb{R}\right) \hookrightarrow \mathrm{S}^{\prime}\left(\mathbb{R}^{n} \times \mathbb{R}\right), \\
& \mathrm{S}\left(\mathbb{R}^{n} \times \mathbb{R}\right) \hookrightarrow F_{\alpha, \text { par }}^{p, q}\left(\mathbb{R}^{n} \times \mathbb{R}\right) \hookrightarrow \mathrm{S}^{\prime}\left(\mathbb{R}^{n} \times \mathbb{R}\right) \text { if } p \neq \infty
\end{aligned}
$$


are continuous, while the first inclusions in (2.1.5) and (2.1.6) are dense provided $0<p, q<\infty$. Moreover, the following simple embedding results hold for $0<p \leq \infty$ $(p \neq \infty$ for the Triebel-Lizorkin scale $)$ and $\alpha, \alpha_{0}, \alpha_{1} \in \mathbb{R}$.

$$
\begin{aligned}
& B_{\alpha, p a r}^{p, q_{0}}\left(\mathbb{R}^{n} \times \mathbb{R}\right) \hookrightarrow B_{\alpha, p a r}^{p, q_{1}}\left(\mathbb{R}^{n} \times \mathbb{R}\right), \quad F_{\alpha, \text { par }}^{p, q_{0}}\left(\mathbb{R}^{n} \times \mathbb{R}\right) \hookrightarrow F_{\alpha, \text { par }}^{p, q_{1}}\left(\mathbb{R}^{n} \times \mathbb{R}\right), \\
& \text { when } 0<q_{0} \leq q_{1} \leq \infty, \\
& B_{\alpha_{0}, p a r}^{p, q}\left(\mathbb{R}^{n} \times \mathbb{R}\right) \hookrightarrow B_{\alpha_{1}, p a r}^{p, r}\left(\mathbb{R}^{n} \times \mathbb{R}\right), \quad F_{\alpha_{0}, p a r}^{p, q}\left(\mathbb{R}^{n} \times \mathbb{R}\right) \hookrightarrow F_{\alpha \alpha_{1}, p a r}^{p, r}\left(\mathbb{R}^{n} \times \mathbb{R}\right) \\
& \qquad \text { for } \alpha_{0}>\alpha_{1} \text { and every } 0<q, r \leq \infty, \\
& B_{\alpha, p a r}^{p, \min \{p, q\}}\left(\mathbb{R}^{n} \times \mathbb{R}\right) \hookrightarrow F_{\alpha, p a r}^{p, q}\left(\mathbb{R}^{n} \times \mathbb{R}\right) \hookrightarrow B_{\alpha, \text { par }}^{p, \max \{p, q\}}\left(\mathbb{R}^{n} \times \mathbb{R}\right), \quad 0<q \leq \infty .
\end{aligned}
$$

Next we turn our attention to the homogeneous Besov and Triebel-Lizorkin spaces, starting with the counterpart of Definitions 2.1.1 and 2.1.2 for the homogeneous scale.

Definition 2.1.5. Define the system $\dot{\Phi}$ of complex-valued functions $\left\{\varphi_{j}\right\}_{j=-\infty}^{\infty}$ with the following properties:

(i) $\varphi_{j} \in C_{c}^{\infty}\left(\mathbb{R}^{n} \times \mathbb{R} \backslash\{(0,0)\}\right)$;

(ii) For $\kappa>1$ an appropriate number,

$$
\operatorname{supp} \varphi_{j} \subset\left\{(x, t) \in \mathbb{R}^{n} \times \mathbb{R}: \frac{1}{\kappa} 2^{j} \leq\|(x, t)\|_{\text {par }} \leq \kappa 2^{j+1}\right\}
$$

(iii) There exist positive constants $c_{0}$ and $c_{1}$ such that

$$
c_{0} \leq\left|\sum_{j=-\infty}^{\infty} \varphi_{j}(x, t)\right| \leq c_{1}, \quad(x, t) \in \mathbb{R}^{n} \times \mathbb{R} \backslash\{(0,0)\}
$$


(iv) For every multiindex $\gamma \in \mathbb{N}_{0}^{n}$ and every $k \in \mathbb{N}_{0}$ there exists a positive constant $C_{\gamma, k}$ such that

$$
\left|\partial_{x}^{\gamma} \partial_{t}^{k} \varphi_{j}(x, t)\right| \leq \frac{C_{\gamma, k}}{2^{j(|\gamma|+2 k)}} .
$$

Definition 2.1.6. For $0<p, q \leq \infty, \alpha \in \mathbb{R}$ and $\left\{\varphi_{j}\right\}_{j=-\infty}^{\infty} \in \dot{\Phi}$ define

$$
\begin{aligned}
& \dot{B}_{\alpha, p a r}^{p, q}\left(\mathbb{R}^{n} \times \mathbb{R}\right):=\left\{f \in \mathrm{S}^{\prime}\left(\mathbb{R}^{n} \times \mathbb{R}\right):\right. \\
&\left.\|f\|_{\dot{B}_{\alpha, p a r}^{p, q}\left(\mathbb{R}^{n} \times \mathbb{R}\right)}:=\left(\sum_{j=-\infty}^{\infty} 2^{j \alpha q}\left\|\mathcal{F}^{-1}\left(\varphi_{j} \mathcal{F} f\right)\right\|_{L^{p}\left(\mathbb{R}^{n} \times \mathbb{R}\right)}^{q}\right)^{1 / q}<\infty\right\}
\end{aligned}
$$

and, for $p \neq \infty$,

$$
\begin{aligned}
& \dot{F}_{\alpha, p a r}^{p, q}\left(\mathbb{R}^{n} \times \mathbb{R}\right):=\left\{f \in \mathrm{S}^{\prime}\left(\mathbb{R}^{n} \times \mathbb{R}\right):\right. \\
&\left.\|f\|_{\dot{F}_{\alpha, p a r}^{p, q}\left(\mathbb{R}^{n} \times \mathbb{R}\right)}:=\left\|\left(\sum_{j=-\infty}^{\infty} 2^{j \alpha q}\left|\mathcal{F}^{-1}\left(\varphi_{j} \mathcal{F} f\right)\right|^{q}\right)^{1 / q}\right\|_{L^{p}\left(\mathbb{R}^{n} \times \mathbb{R}\right)}<\infty\right\}
\end{aligned}
$$

with the usual modification if $q=\infty$, i.e.

$$
\begin{aligned}
\dot{B}_{\alpha, p a r}^{p, \infty}\left(\mathbb{R}^{n} \times \mathbb{R}\right):= & \left\{f \in \mathrm{S}^{\prime}\left(\mathbb{R}^{n} \times \mathbb{R}\right):\right. \\
& \left.\|f\|_{\dot{B}_{\alpha, p a r}^{p, \infty}\left(\mathbb{R}^{n} \times \mathbb{R}\right)}:=\sup _{j \in \mathbb{Z}}\left[2^{j \alpha}\left\|\mathcal{F}^{-1}\left(\varphi_{j} \mathcal{F} f\right)\right\|_{L^{p}\left(\mathbb{R}^{n} \times \mathbb{R}\right)}\right]<\infty\right\}
\end{aligned}
$$

and, for $p \neq \infty$,

$$
\begin{aligned}
& \dot{F}_{\alpha, p a r}^{p, \infty}\left(\mathbb{R}^{n} \times \mathbb{R}\right):=\left\{f \in \mathrm{S}^{\prime}\left(\mathbb{R}^{n} \times \mathbb{R}\right):\right. \\
&\left.\|f\|_{\dot{F}_{\alpha, p a r}^{p, \infty}\left(\mathbb{R}^{n} \times \mathbb{R}\right)}:=\left\|\sup _{j \in \mathbb{Z}}\left(2^{j \alpha}\left|\mathcal{F}^{-1}\left(\varphi_{j} \mathcal{F} f\right)\right|\right)\right\|_{L^{p}\left(\mathbb{R}^{n} \times \mathbb{R}\right)}<\infty\right\} .
\end{aligned}
$$

Different sequences $\left\{\varphi_{j}\right\}_{j=-\infty}^{\infty} \in \dot{\Phi}$ yield equivalent quasi-norms (cf. Theorem 1.1 of [Stö1]).

Remark 2.1.7. A remark similar is spirit to Remark 2.1 .3 can be made in connection with Definition 2.1.5.

The proper analogue of Defnition 2.1.4 for homogeneous spaces is as follows. 
Definition 2.1.8. In the context of Definition 2.1.4, for $0<p, q \leq \infty$ and $\alpha \in \mathbb{R}$, the homogeneous parabolic Besov space is given by

$$
\begin{aligned}
& \dot{B}_{\alpha, p a r}^{p, q}\left(\mathbb{R}^{n} \times \mathbb{R}\right):=\left\{f \in \mathrm{S}^{\prime}\left(\mathbb{R}^{n} \times \mathbb{R}\right):\right. \\
&\left.\|f\|_{\dot{B}_{\alpha, p a r}^{p, q}\left(\mathbb{R}^{n} \times \mathbb{R}\right)}:=\left(\sum_{j=-\infty}^{\infty} 2^{j \alpha q}\left\|\varphi_{j} * f\right\|_{L^{p}\left(\mathbb{R}^{n} \times \mathbb{R}\right)}^{q}\right)^{1 / q}<\infty\right\} .
\end{aligned}
$$

For $p \neq \infty$ the homogeneous parabolic Triebel-Lizorkin space is given by

$$
\begin{aligned}
& \dot{F}_{\alpha, p a r}^{p, q}\left(\mathbb{R}^{n} \times \mathbb{R}\right):=\left\{f \in \mathrm{S}^{\prime}\left(\mathbb{R}^{n} \times \mathbb{R}\right):\right. \\
&\left.\|f\|_{\dot{F}_{\alpha, p a r}^{p, q}\left(\mathbb{R}^{n} \times \mathbb{R}\right)}:=\left\|\left(\sum_{j=-\infty}^{\infty} 2^{j \alpha q}\left|\left(\varphi_{j} * f\right)(\cdot)\right|^{q}\right)^{1 / q}\right\|_{L^{p}\left(\mathbb{R}^{n} \times \mathbb{R}\right)}<\infty\right\} .
\end{aligned}
$$

The usual modifications for $q=\infty$ are as follows:

$$
\begin{aligned}
\dot{B}_{\alpha, p a r}^{p, \infty}\left(\mathbb{R}^{n} \times \mathbb{R}\right):=\left\{f \in \mathrm{S}^{\prime}\left(\mathbb{R}^{n} \times \mathbb{R}\right):\right. & \\
& \|f\|_{\dot{B}_{\alpha, \text { par }}^{p, \infty}\left(\mathbb{R}^{n} \times \mathbb{R}\right)}:=\sup _{j \in \mathbb{N}_{0}}\left(2^{j \alpha} \|\left(\varphi_{j} * f \|_{L^{p}\left(\mathbb{R}^{n} \times \mathbb{R}\right)}\right)<\infty\right\}
\end{aligned}
$$

and, for $p \neq \infty$,

$$
\begin{aligned}
& \dot{F}_{\alpha, p a r}^{p, \infty}\left(\mathbb{R}^{n} \times \mathbb{R}\right):=\left\{f \in \mathrm{S}^{\prime}\left(\mathbb{R}^{n} \times \mathbb{R}\right):\right. \\
&\left.\|f\|_{\dot{F}_{\alpha, p a r}^{p, \infty}\left(\mathbb{R}^{n} \times \mathbb{R}\right)}:=\left\|\sup _{j \in \mathbb{N}_{0}}\left(2^{j \alpha}\left|\left(\varphi_{j} * f\right)(\cdot)\right|\right)\right\|_{L^{p}\left(\mathbb{R}^{n} \times \mathbb{R}\right)}<\infty\right\} .
\end{aligned}
$$

Proposition 2.1.9. According to [See] (p.391), for $1<p<\infty, 1<q \leq \infty$ and $\alpha>0$

$$
F_{\alpha, p a r}^{p, q}\left(\mathbb{R}^{n} \times \mathbb{R}\right)=\dot{F}_{\alpha, p a r}^{p, q}\left(\mathbb{R}^{n} \times \mathbb{R}\right) \cap L^{p}\left(\mathbb{R}^{n} \times \mathbb{R}\right)
$$

with equivalent norms, meaning

$$
\|f\|_{F_{\alpha, p a r}^{p, q}\left(\mathbb{R}^{n} \times \mathbb{R}\right)} \approx\|f\|_{\dot{F}_{\alpha, p a r}^{p, q}\left(\mathbb{R}^{n} \times \mathbb{R}\right)}+\|f\|_{L^{p}\left(\mathbb{R}^{n} \times \mathbb{R}\right)}
$$

In particular,

$$
\|f\|_{B_{\alpha, p a r}^{p}\left(\mathbb{R}^{n} \times \mathbb{R}\right)} \approx\|f\|_{\dot{B}_{\alpha, p a r}^{p}\left(\mathbb{R}^{n} \times \mathbb{R}\right)}+\|f\|_{L^{p}\left(\mathbb{R}^{n} \times \mathbb{R}\right)} \quad \text { for } \quad 1<p<\infty, \alpha>0 .
$$


We next present a few lifting operators on inhomogeneous and homogeneous Besov and Triebel-Lizorkin scales, starting with the operators already in the literature.

Theorem 2.1.10. Let $\sigma$ be a real number and define the lifting operator $\mathcal{I}_{\sigma}$ by

$$
\mathcal{I}_{\sigma} f:=\mathcal{F}^{-1}\left\{\left[\left(1+|\xi|^{2}\right)^{\frac{1}{2}}+|\tau|^{\frac{1}{2}}\right]^{-\sigma} \mathcal{F} f(\xi, \tau)\right\}, \quad(\xi, \tau) \in \mathbb{R}^{n} \times \mathbb{R}, \quad \sigma \in \mathbb{R},
$$

for $f \in \mathrm{S}^{\prime}\left(\mathbb{R}^{n} \times \mathbb{R}\right)$. Then, for $0<p, q \leq \infty(p \neq \infty$ in the case of the TriebelLizorkin space) and $\alpha \in \mathbb{R}$, the operators

$$
\begin{aligned}
& \mathcal{I}_{\sigma}: B_{\alpha, p a r}^{p, q}\left(\mathbb{R}^{n} \times \mathbb{R}\right) \stackrel{\sim}{\longrightarrow} B_{\alpha+\sigma, p a r}^{p, q}\left(\mathbb{R}^{n} \times \mathbb{R}\right), \\
& \mathcal{I}_{\sigma}: F_{\alpha, p a r}^{p, q}\left(\mathbb{R}^{n} \times \mathbb{R}\right) \stackrel{\sim}{\longrightarrow} F_{\alpha+\sigma, p a r}^{p, q}\left(\mathbb{R}^{n} \times \mathbb{R}\right)
\end{aligned}
$$

are isomorphisms.

Proof. According to Proposition 3.3 of [Yam], the lifting operator $\mathcal{I}_{\sigma}$ maps the space $B_{\alpha, \text { par }}^{p, q}\left(\mathbb{R}^{n} \times \mathbb{R}\right)$ boundedly into $B_{\alpha-\sigma, p a r}^{p, q}\left(\mathbb{R}^{n} \times \mathbb{R}\right)$. Since $\mathcal{I}_{\sigma} \circ \mathcal{I}_{-\sigma}=\mathcal{I}_{-\sigma} \circ \mathcal{I}_{\sigma}=I$ on $B_{\alpha, \text { par }}^{p, q}\left(\mathbb{R}^{n} \times \mathbb{R}\right)$, the map $\mathcal{I}_{\sigma}$ is an isomorphism. The same argument can be carried out for the Triebel-Lizorkin scale.

Theorem 2.1.11. (Theorem 6 in [StTr], see also Remark 3.1 in [Stö1]) Let $0<p, q \leq \infty(0<p<\infty$ for the $F$-scale $)$ and $\sigma \in \mathbb{R}$. Define the operator $\mathrm{I}_{\sigma}$ by

$$
\mathrm{I}_{\sigma} f:=\mathcal{F}^{-1}\left[\left(\sum_{j=1}^{n}\left(1+\xi_{j}^{2}\right)^{\sigma / 2}+\left(1+\tau^{2}\right)^{\sigma / 4}\right)(\mathcal{F} f)(\xi, \tau)\right], \quad(\xi, \tau) \in \mathbb{R}^{n} \times \mathbb{R}
$$

for $f \in \mathrm{S}^{\prime}\left(\mathbb{R}^{n} \times \mathbb{R}\right)$. Then the operator $\mathrm{I}_{\sigma}$ is an isomorphism in the following instances:

$$
\begin{aligned}
& \mathrm{I}_{\sigma}: B_{\sigma, p a r}^{p, q}\left(\mathbb{R}^{n} \times \mathbb{R}\right) \stackrel{\sim}{\longrightarrow} B_{0, p a r}^{p, q}\left(\mathbb{R}^{n} \times \mathbb{R}\right), \\
& \mathrm{I}_{\sigma}: F_{\sigma, p a r}^{p, q}\left(\mathbb{R}^{n} \times \mathbb{R}\right) \stackrel{\sim}{\longrightarrow} F_{0, p a r}^{p, q}\left(\mathbb{R}^{n} \times \mathbb{R}\right) .
\end{aligned}
$$


Theorem 2.1.12. ((4.3) of [StTr], see also Theorem 3.1 in [Stö1])

Let $0<p, q \leq \infty(0<p<\infty$ for the F-scale $)$ and $\sigma \in \mathbb{R}$. Define the operator $\dot{\mathrm{I}}_{\sigma}$ by

$$
\dot{\mathrm{I}}_{\sigma} f:=\mathcal{F}^{-1}\left[\left(\sum_{j=1}^{n}\left|\xi_{j}\right|^{\sigma}+|\tau|^{\sigma / 2}\right)(\mathcal{F} f)(\xi, \tau)\right], \quad(\xi, \tau) \in \mathbb{R}^{n} \times \mathbb{R}
$$

for $f \in \mathrm{S}^{\prime}\left(\mathbb{R}^{n} \times \mathbb{R}\right)$. Then the operators

$$
\begin{aligned}
& \dot{\mathrm{I}}_{\sigma}: \dot{B}_{\sigma, p a r}^{p, q}\left(\mathbb{R}^{n} \times \mathbb{R}\right) \stackrel{\sim}{\longrightarrow} \dot{B}_{0, p a r}^{p, q}\left(\mathbb{R}^{n} \times \mathbb{R}\right), \\
& \dot{\mathrm{I}}_{\sigma}: \dot{F}_{\sigma, p a r}^{p, q}\left(\mathbb{R}^{n} \times \mathbb{R}\right) \stackrel{\sim}{\longrightarrow} \dot{F}_{0, p a r}^{p, q}\left(\mathbb{R}^{n} \times \mathbb{R}\right)
\end{aligned}
$$

are isomorphisms.

We find it useful to introduce other lifting operators, since they will play important roles in some of our further results.

Theorem 2.1.13. For $f \in \mathrm{S}^{\prime}\left(\mathbb{R}^{n} \times \mathbb{R}\right)$ define the operator

$$
\dot{L} f:=\mathcal{F}^{-1}\left[\sqrt{|\xi|^{2}+i \tau} \mathcal{F} f(\xi, \tau)\right], \quad(\xi, \tau) \in \mathbb{R}^{n} \times \mathbb{R}
$$

For $0<p<\infty$,

$$
\dot{L}: \dot{F}_{1, \text { par }}^{p, 2}\left(\mathbb{R}^{n} \times \mathbb{R}\right) \stackrel{\sim}{\longrightarrow} \dot{F}_{0, \text { par }}^{p, 2}\left(\mathbb{R}^{n} \times \mathbb{R}\right)
$$

is an isomorphism.

Proof. We take a sequence $\left\{\varphi_{j}\right\}_{j=-\infty}^{\infty} \in \dot{\Phi}$ such that $\sum_{j=-\infty}^{\infty} \varphi_{j}=1$ and $0 \leq \varphi_{j} \leq 1$. See the example on p.93 in [RuSi] for a construction of such a sequence in the isotropic setting. For the parabolic case this is carried out in a similar fashion. Next, for $j \in \mathbb{Z}$ we define

$$
\psi_{j}(\xi, \tau):=\varphi_{j}(\xi, \tau) \frac{2^{j}}{\sqrt{|\xi|^{2}+i \tau}}, \quad(\xi, \tau) \in \mathbb{R}^{n} \times \mathbb{R}
$$


As a preliminary step we shall prove that the sequence $\left\{\psi_{j}\right\}_{j=-\infty}^{\infty} \in \dot{\Phi}$.

Parts (i) and (ii) in Definition 2.1.5 are obvious. Based on the finite overlap of the support of $\varphi_{j}$ 's, on the fact that

$$
\left|\frac{2^{j}}{\sqrt{|\xi|^{2}+i \tau}}\right| \approx 1 \quad \text { on the support of } \varphi_{j}
$$

and on the way the sequence $\left\{\varphi_{j}\right\}_{j=-\infty}^{\infty}$ was introduced, it is not too difficult to check that (iii) in Definition 2.1.5 holds.

In order to show part (iv) of the same definition, note that it suffices to prove that for every $\gamma \in \mathbb{N}_{0}^{n}$ and every $k \in \mathbb{N}_{0}$ there exists a positive constant $C_{\gamma, k}$ such that

$$
\left|\partial_{\xi}^{\gamma} \partial_{\tau}^{k}\left(\frac{1}{\sqrt{|\xi|^{2}+i \tau}}\right)\right| \leq \frac{C_{\gamma, k}}{2^{j(|\gamma|+2 k+1)}}
$$

for $(\xi, \tau) \in \mathbb{R}^{n} \times \mathbb{R}$ with $\|(\xi, \tau)\|_{\text {par }} \approx 2^{j}$. One can check that, in general, for every $\gamma \in \mathbb{N}_{0}^{n}$ and every $k \in \mathbb{N}_{0}$ there exists a positive constant $C_{\gamma, k}$ such that

$$
\left|\partial_{\xi}^{\gamma} \partial_{\tau}^{k}\left(\frac{1}{\sqrt{|\xi|^{2}+i \tau}}\right)\right| \leq C_{\gamma, k}\left|\frac{1}{\sqrt{|\xi|^{2}+i \tau}}\right|^{|\gamma|+2 k+1}, \quad(\xi, \tau) \in \mathbb{R}^{n} \times \mathbb{R} .
$$

Then (2.1.16) follows from (2.1.17) and (2.1.15), hence part (iv) of Definition 2.1.5 holds . Therefore, $\left\{\psi_{j}\right\}_{j=-\infty}^{\infty} \in \dot{\Phi}$. Using this, as well as the fact that, under the current assumptions,

$$
\mathcal{F}^{-1}\left[\psi_{j}(\xi, \tau) \mathcal{F}(\dot{L} f)(\xi, \tau)\right]=2^{j} \mathcal{F}^{-1}\left[\varphi_{j}(\xi, \tau) \mathcal{F} f(\xi, \tau)\right]
$$


we obtain the following: for $f \in \dot{F}_{1, p a r}^{p, 2}\left(\mathbb{R}^{n} \times \mathbb{R}\right)$,

$$
\begin{aligned}
\|f\|_{\dot{F}_{1, p a r}^{p, 2}\left(\mathbb{R}^{n} \times \mathbb{R}\right)} & \stackrel{\text { def }}{=}\left\|\left(\sum_{j=-\infty}^{\infty} 2^{2 j}\left|\mathcal{F}^{-1}\left(\varphi_{j} \mathcal{F} f\right)\right|^{2}\right)^{\frac{1}{2}}\right\|_{L^{p\left(\mathbb{R}^{n} \times \mathbb{R}\right)}} \\
& =\left\|\left(\sum_{j=-\infty}^{\infty}\left|\mathcal{F}^{-1}\left(\psi_{j} \mathcal{F}(\dot{L} f)\right)\right|^{2}\right)^{\frac{1}{2}}\right\|_{L^{p}\left(\mathbb{R}^{n} \times \mathbb{R}\right)} \\
& \stackrel{\text { def }}{=}\|\dot{L} f\|_{\dot{F}_{0, p a r}^{p, 2}\left(\mathbb{R}^{n} \times \mathbb{R}\right)} .
\end{aligned}
$$

This completes the proof of the theorem.

Theorem 2.1.14. For $\sigma \in \mathbb{R}$ and $f \in \mathrm{S}^{\prime}\left(\mathbb{R}^{n} \times \mathbb{R}\right)$ define the operator $\dot{I}_{\sigma}$ by

$$
\dot{I}_{\sigma} f:=\mathcal{F}^{-1}\left[\left(|\xi|^{2}+|\tau|\right)^{-\frac{\sigma}{2}} \mathcal{F} f(\xi, \tau)\right], \quad(\xi, \tau) \in \mathbb{R}^{n} \times \mathbb{R}
$$

Then, if $0<p, q \leq \infty(p \neq \infty$ for the F-scale $)$ and $\alpha \in \mathbb{R}$,

$$
\begin{gathered}
\dot{I}_{\sigma}: \dot{B}_{\alpha, p a r}^{p, q}\left(\mathbb{R}^{n} \times \mathbb{R}\right) \stackrel{\sim}{\longrightarrow} \dot{B}_{\alpha+\sigma, p a r}^{p, q}\left(\mathbb{R}^{n} \times \mathbb{R}\right), \\
\dot{I}_{\sigma}: \dot{F}_{\alpha, p a r}^{p, q}\left(\mathbb{R}^{n} \times \mathbb{R}\right) \stackrel{\sim}{\longrightarrow} \dot{F}_{\alpha+\sigma, p a r}^{p, q}\left(\mathbb{R}^{n} \times \mathbb{R}\right)
\end{gathered}
$$

are isomorhisms.

Proof. Let $\left\{\varphi_{j}\right\}_{j=-\infty}^{\infty} \in \dot{\Phi}$ such that $\sum_{j=-\infty}^{\infty} \varphi_{j}=1$ and $0 \leq \varphi_{j} \leq 1$. For a construction of such sequence in the isotropic case, see the example on p. 93 in [RuSi]. In the parabolic setting this is carried out in a similar fashion. Next, for $j \in \mathbb{Z}$ and $\sigma \in \mathbb{R}$ we define

$$
\psi_{j}(\xi, \tau):=\varphi_{j}(\xi, \tau) \frac{\left(|\xi|^{2}+|\tau|\right)^{\frac{\sigma}{2}}}{2^{j \sigma}}, \quad(\xi, \tau) \in \mathbb{R}^{n} \times \mathbb{R}
$$

First we will show that $\left\{\psi_{j}\right\}_{j=-\infty}^{\infty}$ belongs to the system $\dot{\Phi}$. 
Parts (i) and (ii) of Definition 2.1.5 are immediate. On the support of $\varphi_{j}$ we have that $\|(\xi, \tau)\|_{p a r} \approx 2^{j}$ and

$$
\frac{\left(|\xi|^{2}+|\tau|\right)^{\frac{\sigma}{2}}}{2^{j \sigma}} \approx 1
$$

Based on the finite overlap of the support of $\varphi_{j}$ 's, on (2.1.15) and on the way $\left\{\varphi_{j}\right\}_{j=-\infty}^{\infty}$ was defined, it is not difficult to check that part (iii) of Definition 2.1.5 holds.

To prove part (iv) of the same definition it is enough to show that for every $\gamma \in \mathbb{N}_{0}^{n}$ and every $k \in \mathbb{N}_{0}$ there exists a positive constant $C_{\sigma, \gamma, k}$ such that

$$
\left|\partial_{\xi}^{\gamma} \partial_{\tau}^{k}\left[\left(|\xi|^{2}+|\tau|\right)^{\frac{\sigma}{2}}\right]\right| \leq \frac{C_{\sigma, \gamma, k}}{2^{j(|\gamma|+2 k-\sigma)}}
$$

where $(\xi, \tau) \in \mathbb{R}^{n} \times \mathbb{R}$ with $\|(\xi, \tau)\|_{\text {par }} \approx 2^{j}$. One can check that, in general, for every $\gamma \in \mathbb{N}_{0}^{n}$ and every $k \in \mathbb{N}_{0}$ there exists a positive constant $C_{\sigma, \gamma, k}$ such that

$$
\left|\partial_{\xi}^{\gamma} \partial_{\tau}^{k}\left[\left(|\xi|^{2}+|\tau|\right)^{\frac{\sigma}{2}}\right]\right| \leq C_{\sigma, \gamma, k}\left(|\xi|^{2}+|\tau|\right)^{\frac{\sigma-|\gamma|-2 k}{2}}, \quad(\xi, \tau) \in \mathbb{R}^{n} \times \mathbb{R}
$$

Then (2.1.19) follows from (2.1.20) and (2.1.18), proving part (iv) of Definition 2.1.5, hence, $\left\{\psi_{j}\right\}_{j=-\infty}^{\infty} \in \dot{\Phi}$ holds. With this in hand,

$$
\mathcal{F}^{-1}\left[\psi_{j}(\xi, \tau) \mathcal{F}\left(\dot{I}_{\sigma} f\right)(\xi, \tau)\right]=2^{-j \sigma} \mathcal{F}^{-1}\left[\varphi_{j}(\xi, \tau) \mathcal{F} f(\xi, \tau)\right]
$$

implies the following: for $f \in \dot{B}_{\alpha, p a r}^{p, q}\left(\mathbb{R}^{n} \times \mathbb{R}\right)$,

$$
\begin{aligned}
\|f\|_{\dot{B}_{\alpha, p a r}^{p, q}\left(\mathbb{R}^{n} \times \mathbb{R}\right)} & \stackrel{\text { def }}{=}\left(\sum_{j=-\infty}^{\infty} 2^{j \alpha q}\left\|\mathcal{F}^{-1}\left(\varphi_{j} \mathcal{F} f\right)\right\|_{L^{p}\left(\mathbb{R}^{n} \times \mathbb{R}\right)}^{q}\right)^{1 / q} \\
& =\left(\sum_{j=-\infty}^{\infty} 2^{j(\alpha+\sigma) q}\left\|\mathcal{F}^{-1}\left(\psi_{j} \mathcal{F}\left(\dot{I}_{\sigma} f\right)\right)\right\|_{L^{p}\left(\mathbb{R}^{n} \times \mathbb{R}\right)}^{q}\right)^{1 / q} \\
& \stackrel{\text { def }}{=}\left\|\dot{I}_{\sigma} f\right\|_{\dot{B}_{\alpha+\sigma, p a r}^{p, q}\left(\mathbb{R}^{n} \times \mathbb{R}\right)} .
\end{aligned}
$$


Similarly, for $f \in \dot{F}_{\alpha, p a r}^{p, q}\left(\mathbb{R}^{n} \times \mathbb{R}\right)$,

$$
\begin{aligned}
\|f\|_{\dot{F}_{\alpha, p a r}^{p, q}\left(\mathbb{R}^{n} \times \mathbb{R}\right)} & \stackrel{\text { def }}{=}\left\|\left(\sum_{j=-\infty}^{\infty} 2^{j \alpha q}\left|\mathcal{F}^{-1}\left(\varphi_{j} \mathcal{F} f\right)\right|^{q}\right)^{\frac{1}{q}}\right\|_{L^{p}\left(\mathbb{R}^{n} \times \mathbb{R}\right)} \\
& =\left\|\left(\sum_{j=-\infty}^{\infty} 2^{j(\alpha+\sigma) q}\left|\mathcal{F}^{-1}\left(\psi_{j} \mathcal{F}\left(\dot{I}_{\sigma} f\right)\right)\right|^{q}\right)^{\frac{1}{q}}\right\|_{L^{p}\left(\mathbb{R}^{n} \times \mathbb{R}\right)} \\
& \stackrel{\text { def }}{=}\left\|\dot{I}_{\sigma} f\right\|_{\dot{F}_{\alpha+\sigma, p a r}^{p, q}\left(\mathbb{R}^{n} \times \mathbb{R}\right)} .
\end{aligned}
$$

This completes the proof of the theorem.

Theorem 2.1.15. For $\sigma \in \mathbb{R}$ and $f \in \mathrm{S}^{\prime}\left(\mathbb{R}^{n} \times \mathbb{R}\right)$ define the operator $\Theta_{\sigma}$ by

$$
\Theta_{\sigma} f:=\mathcal{F}^{-1}\left[\left(1+|\xi|^{4}+\tau^{2}\right)^{\frac{\sigma}{4}} \mathcal{F} f(\xi, \tau)\right], \quad(\xi, \tau) \in \mathbb{R}^{n} \times \mathbb{R}
$$

Then, if $0<p, q \leq \infty(p \neq \infty$ for the $F$-scale $)$ and $\alpha \in \mathbb{R}$,

$$
\begin{aligned}
& \Theta_{\sigma}: B_{\alpha, \text { par }}^{p, q}\left(\mathbb{R}^{n} \times \mathbb{R}\right) \stackrel{\sim}{\longrightarrow} B_{\alpha-\sigma, p a r}^{p, q}\left(\mathbb{R}^{n} \times \mathbb{R}\right), \\
& \Theta_{\sigma}: F_{\alpha, p a r}^{p, q}\left(\mathbb{R}^{n} \times \mathbb{R}\right) \stackrel{\sim}{\longrightarrow} F_{\alpha-\sigma, p a r}^{p, q}\left(\mathbb{R}^{n} \times \mathbb{R}\right)
\end{aligned}
$$

are isomorhisms.

Proof. We omit the proof, since it can be carried out along the same lines as the proof of Theorem 2.1.14.

We next invoke some results due to M. Yamazaki, which will be needed in our further discussion.

Theorem 2.1.16. (Proposition 3.3 of [Yam], see also (A.12) from [Gru1])

Let $\beta \in \mathbb{N}_{0}^{n}, k \in \mathbb{N}_{0}$ and $f \in B_{\alpha, \text { par }}^{p, q}\left(\mathbb{R}^{n} \times \mathbb{R}\right)$, where $0<p, q \leq \infty, \alpha \in \mathbb{R}$ (respectively $\left.f \in F_{\alpha, p a r}^{p, q}\left(\mathbb{R}^{n-1} \times \mathbb{R}\right), p \neq \infty\right)$. Then

$$
\partial_{x}^{\beta} \partial_{t}^{k} f \in B_{\alpha-|\beta|-2 k, p a r}^{p, q}\left(\mathbb{R}^{n} \times \mathbb{R}\right)
$$


(respectively $\left.\partial_{x}^{\beta} \partial_{t}^{k} f \in F_{\alpha-|\beta|-2 k, p a r}^{p, q}\left(\mathbb{R}^{n} \times \mathbb{R}\right), p \neq \infty\right)$ and a natural estimate holds.

Theorem 2.1.17. (Theorem 3.4 from [Yam])

1. Suppose $0<p_{0}<p_{1} \leq \infty, 0<q \leq \infty$ and $\alpha \in \mathbb{R}$. Then the inclusion

$$
B_{\alpha, p a r}^{p_{0}, q}\left(\mathbb{R}^{n} \times \mathbb{R}\right) \hookrightarrow B_{\alpha-(n+2)\left(1 / p_{0}-1 / p_{1}\right), p a r}^{p_{1}, q}\left(\mathbb{R}^{n} \times \mathbb{R}\right)
$$

holds in the sense of quasi-normed spaces.

2. Suppose $0<p_{0}<p_{1}<\infty, 0<q_{0}, q_{1} \leq \infty$ and $\alpha \in \mathbb{R}$. Then the inclusion

$$
F_{\alpha, p a r}^{p_{0}, q_{0}}\left(\mathbb{R}^{n} \times \mathbb{R}\right) \hookrightarrow F_{\alpha-(n+2)\left(1 / p_{0}-1 / p_{1}\right), p a r}^{p_{1}, q_{1}}\left(\mathbb{R}^{n} \times \mathbb{R}\right)
$$

holds in the sense of quasi-normed spaces.

Remark 2.1.18. For $0<p<\infty$ and $\alpha \in \mathbb{R}$ one has

$$
B_{\alpha, p a r}^{p, p}\left(\mathbb{R}^{n} \times \mathbb{R}\right) \equiv F_{\alpha, p a r}^{p, p}\left(\mathbb{R}^{n} \times \mathbb{R}\right)
$$

In order to simplify the notation, we will write $B_{\alpha, p a r}^{p}$ and $\dot{B}_{\alpha, p a r}^{p}$ in place of $B_{\alpha, p a r}^{p, p}$ and $\dot{B}_{\alpha, p a r}^{p, p}$, respectively, for $0<p \leq \infty$ and $\alpha \in \mathbb{R}$.

Prior to presenting our next result let us recall the definition of the class $L^{p}(\Omega ; X)$ and of the isotropic Besov space from Defnitions 1.2.5 and 1.2.8, respectively.

Proposition 2.1.19. For $1 \leq p \leq \infty, \alpha>0$ the diagonal Besov scale has the Fubini property:

$$
B_{\alpha, p a r}^{p}\left(\mathbb{R}^{n} \times \mathbb{R}\right)=L^{p}\left(\mathbb{R}^{n} ; B_{\alpha / 2}^{p}(\mathbb{R})\right) \cap L^{p}\left(\mathbb{R} ; B_{\alpha}^{p}\left(\mathbb{R}^{n}\right)\right)
$$

Proof. For notational convenience we present an argument for $p<\infty$. The case $p=\infty$ is treated analogously. 
By Definition 5.1 and Theorem 5.1 from [Dac1], $f$ belongs to $B_{\alpha, p a r}^{p}\left(\mathbb{R}^{n} \times \mathbb{R}\right)$ if and only if

$$
\sum_{j=1}^{n}\|\| f\left(x_{1}, \ldots, x_{j-1}, \cdot, x_{j+1}, \ldots, x_{n}, t\right)\left\|_{B_{\alpha}^{p}(\mathbb{R})}\right\|_{L^{p}\left(\mathbb{R}^{n}\right)}<\infty
$$

and

$$
\|\| f(x, \cdot)\left\|_{B_{\alpha / 2}^{p}(\mathbb{R})}\right\|_{L^{p}\left(\mathbb{R}^{n}\right)}<\infty .
$$

On the other hand, by definition,

$$
f \in L^{p}\left(\mathbb{R}^{n} ; B_{\alpha / 2}^{p}(\mathbb{R})\right) \Longleftrightarrow\left[\int_{\mathbb{R}^{n}}\|f(x, \cdot)\|_{B_{\alpha / 2}^{p}(\mathbb{R})}^{p} d x\right]^{\frac{1}{p}}<\infty \Longleftrightarrow(2.1 .24),
$$

and

$$
f \in L^{p}\left(\mathbb{R} ; B_{\alpha}^{p}\left(\mathbb{R}^{n}\right)\right) \Longleftrightarrow \int_{\mathbb{R}}\|f(\cdot, t)\|_{B_{\alpha}^{p}\left(\mathbb{R}^{n}\right)}^{p} d t<\infty
$$

Using the Fubini property of the isotropic space $B_{\alpha}^{p}\left(\mathbb{R}^{n}\right)$ (see Proposition 1.2.9), we obtain that

$$
\begin{aligned}
\int_{\mathbb{R}} & \|f(\cdot, t)\|_{B_{\alpha}^{p}\left(\mathbb{R}^{n}\right)}^{p} d t \approx \int_{\mathbb{R}}\left[\sum_{j=1}^{n}\|\| f\left(x_{1}, \ldots, x_{j-1}, \cdot, x_{j+1}, \ldots, x_{n}, t\right)\left\|_{B_{\alpha}^{p}(\mathbb{R})}\right\|_{L^{p}\left(\mathbb{R}^{n-1}\right)}\right]^{p} d t \\
& \approx \int_{\mathbb{R}} \sum_{j=1}^{n}\|\| f\left(x_{1}, \ldots, x_{j-1}, \cdot, x_{j+1}, \ldots, x_{n}, t\right)\left\|_{B_{\alpha}^{p}(\mathbb{R})}\right\|_{L^{p}\left(\mathbb{R}^{n-1}\right)}^{p} d t \\
& =\sum_{j=1}^{n} \int_{\mathbb{R}} \int_{\mathbb{R}^{n-1}}\left\|f\left(x_{1}, \ldots, x_{j-1}, \cdot, x_{j+1}, \ldots, x_{n}, t\right)\right\|_{B_{\alpha}^{p}(\mathbb{R})}^{p} d x_{1} \cdots d x_{j-1} d x_{j+1} \cdots d x_{n} d t \\
& =\sum_{j=1}^{n}\|\| f\left(x_{1}, \ldots, x_{j-1}, \cdot, x_{j+1}, \ldots, x_{n}, t\right)\left\|_{B_{\alpha}^{p}(\mathbb{R})}\right\|_{L^{p}\left(\mathbb{R}^{n}\right)}^{p} \\
& \approx\left[\sum_{j=1}^{n}\|\| f\left(x_{1}, \ldots, x_{j-1}, \cdot, x_{j+1}, \ldots, x_{n}, t\right)\left\|_{B_{\alpha}^{p}(\mathbb{R})}\right\|_{L^{p}\left(\mathbb{R}^{n}\right)}\right]^{p} .
\end{aligned}
$$

This shows that $f \in L^{p}\left(\mathbb{R} ; B_{\alpha}^{p}\left(\mathbb{R}^{n}\right)\right)$ if and only if (2.1.23) holds, completing the proof of the proposition. 
We next discuss the identification of the parabolic Besov space $B_{\alpha, p a r}^{\infty}\left(\mathbb{R}^{n} \times \mathbb{R}\right)$ with the Hölder space $C_{\text {par }}^{\alpha}\left(\mathbb{R}^{n} \times \mathbb{R}\right)$ with parabolic anisotropy. We begin by defining the latter.

Definition 2.1.20. For $0<\alpha<1$ we define the (parabolic) space of Hölder continuous functions of order $\alpha$ in the following way.

$$
C_{\text {par }}^{\alpha}\left(\mathbb{R}^{n} \times \mathbb{R}\right):=\left\{f \in \mathrm{S}^{\prime}\left(\mathbb{R}^{n} \times \mathbb{R}\right):\|f\|_{C_{p a r}^{\alpha}\left(\mathbb{R}^{n} \times \mathbb{R}\right)}<\infty\right\}
$$

where

$$
\|f\|_{C_{p a r}^{\alpha}\left(\mathbb{R}^{n} \times \mathbb{R}\right)}:=\|f\|_{L^{\infty}\left(\mathbb{R}^{n} \times \mathbb{R}\right)}+\sup _{\substack{(x, t),(y, s) \in \mathbb{R}^{n} \times \mathbb{R} \\(x, t) \neq(y, s)}} \frac{|f(x, t)-f(y, s)|}{\left(|x-y|+|t-s|^{1 / 2}\right)^{\alpha}} .
$$

Lemma 2.1.21. Let $0<\alpha<1$. Then

$$
B_{\alpha, p a r}^{\infty}\left(\mathbb{R}^{n} \times \mathbb{R}\right)=C_{\text {par }}^{\alpha}\left(\mathbb{R}^{n} \times \mathbb{R}\right)
$$

Proof. Proposition 2.1.19 and p. 189 of [Tri4] give the following equivalences:

$$
\begin{aligned}
f \in B_{\alpha, p a r}^{\infty} & \left(\mathbb{R}^{n} \times \mathbb{R}\right) \Longleftrightarrow f \in L^{\infty}\left(\mathbb{R}^{n} ; B_{\alpha / 2}^{\infty}(\mathbb{R})\right) \cap L^{\infty}\left(\mathbb{R} ; B_{\alpha}^{\infty}\left(\mathbb{R}^{n}\right)\right) \\
& \Longleftrightarrow\left\{\begin{array}{l}
\sup _{x \in \mathbb{R}^{n}} \sup _{t \in \mathbb{R}}|f(x, t)|<\infty, \quad \sup _{t \in \mathbb{R}} \sup _{x \in \mathbb{R}^{n}}|f(x, t)|<\infty, \\
\sup _{x \in \mathbb{R}^{n}} \sup _{\substack{t, s \in \mathbb{R} \\
t \neq s}} \frac{|f(x, t)-f(x, s)|}{|t-s|^{\alpha / 2}}<\infty, \\
\sup _{t \in \mathbb{R}} \sup _{\substack{x, y \in \mathbb{R}^{n} \\
x \neq y}} \frac{|f(x, t)-f(y, t)|}{|x-y|^{\alpha}}<\infty .
\end{array}\right.
\end{aligned}
$$

First assume that $f \in B_{\alpha, \text { par }}^{\infty}\left(\mathbb{R}^{n} \times \mathbb{R}\right)$. Then $f \in L^{\infty}\left(\mathbb{R}^{n} \times \mathbb{R}\right)$ and, for any $(x, t),(y, s) \in \mathbb{R}^{n} \times \mathbb{R}$ with $(x, t) \neq(y, s)$, we may write

$$
\frac{|f(x, t)-f(y, s)|}{\left[|x-y|+|t-s|^{1 / 2}\right]^{\alpha}} \leq \frac{|f(x, t)-f(x, s)|}{\left(|x-y|+|t-s|^{1 / 2}\right)^{\alpha}}+\frac{|f(x, s)-f(y, s)|}{\left(|x-y|+|t-s|^{1 / 2}\right)^{\alpha}}
$$




$$
\leq \frac{|f(x, t)-f(x, s)|}{|t-s|^{\alpha / 2}}+\frac{|f(x, s)-f(y, s)|}{|x-y|^{\alpha}} .
$$

Hence, by the last two inequalities in (2.1.27), we have

$$
\begin{aligned}
& \sup _{\substack{(x, t),(y, s) \in \mathbb{R}^{n} \times \mathbb{R} \\
(x, t) \neq(y, s)}} \frac{|f(x, t)-f(y, s)|}{\left[|x-y|+|t-s|^{1 / 2}\right]^{\alpha}} \\
& \quad \leq \sup _{\substack{x \in \mathbb{R}^{n}, t, s \in \mathbb{R} \\
t \neq s}} \frac{|f(x, t)-f(x, s)|}{|t-s|^{\alpha / 2}}+\sup _{\substack{x, y \in \mathbb{R}^{n}, s \in \mathbb{R} \\
x \neq y}} \frac{|f(x, s)-f(y, s)|}{|x-y|^{\alpha}}<\infty .
\end{aligned}
$$

This proves the left-to-right inclusion in (2.1.25).

Turning to the oposite one, assume that $f \in L^{\infty}\left(\mathbb{R}^{n} \times \mathbb{R}\right)$ and

$$
\sup _{\substack{(x, t),(y, s) \in \mathbb{R}^{n} \times \mathbb{R} \\(x, t) \neq(y, s)}} \frac{|f(x, t)-f(y, s)|}{\left(|x-y|+|t-s|^{1 / 2}\right)^{\alpha}}<\infty .
$$

Suitably specializing (2.1.28) yields

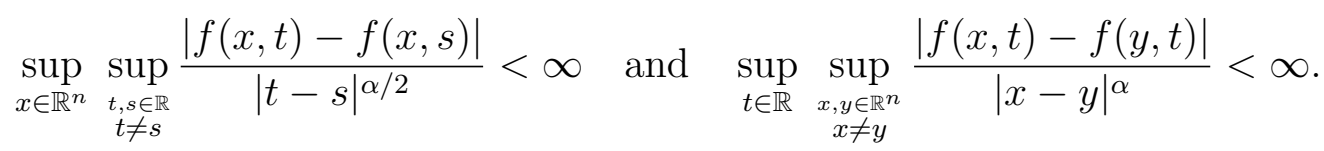

Now

$$
f \in L^{\infty}\left(\mathbb{R}^{n} ; L^{\infty}(\mathbb{R})\right)=L^{\infty}\left(\mathbb{R} ; L^{\infty}\left(\mathbb{R}^{n}\right)\right),
$$

in concert with (2.1.29) ultimately amounts to

$$
f \in L^{\infty}\left(\mathbb{R}^{n} ; B_{\alpha / 2}^{\infty}(\mathbb{R})\right) \cap L^{\infty}\left(\mathbb{R} ; B_{\alpha}^{\infty}\left(\mathbb{R}^{n}\right)\right)=B_{\alpha, p a r}^{p}\left(\mathbb{R}^{n} \times \mathbb{R}\right),
$$

where the last equality has been noted in (2.1.26).

Next, we present an intrinsic characterization of $B_{\alpha, p a r}^{p}\left(\mathbb{R}^{n} \times \mathbb{R}\right)$ with $p<\infty$.

Lemma 2.1.22. Let $1 \leq p<\infty$ and $0<\alpha<1$. Then $f \in B_{\alpha, p a r}^{p}\left(\mathbb{R}^{n} \times \mathbb{R}\right) \Longleftrightarrow$

$$
f \in L^{p}\left(\mathbb{R}^{n} \times \mathbb{R}\right) \text { and } \int_{\mathbb{R}^{n} \times \mathbb{R}} \int_{\mathbb{R}^{n} \times \mathbb{R}} \frac{|f(x, t)-f(y, s)|^{p}}{\left(|x-y|+|t-s|^{1 / 2}\right)^{n+2+\alpha p}} d y d s d x d t<\infty .
$$


Proof. Using Proposition 2.1.19 and p. 189 of [Tri4], membership of $f$ to the space $B_{\alpha, p a r}^{p}\left(\mathbb{R}^{n} \times \mathbb{R}\right)$ is equivalent to

$$
\left\{\begin{array}{l}
f \in L^{p}\left(\mathbb{R}^{n} \times \mathbb{R}\right), \\
\int_{\mathbb{R}^{n}} \int_{\mathbb{R}} \int_{\mathbb{R}} \frac{|f(x, t)-f(x, s)|^{p}}{|t-s|^{1+\frac{\alpha p}{2}}} d s d t d x<\infty, \\
\int_{\mathbb{R}} \int_{\mathbb{R}^{n}} \int_{\mathbb{R}^{n}} \frac{|f(x, t)-f(y, t)|^{p}}{|x-y|^{n+\alpha p}} d y d x d t<\infty .
\end{array}\right.
$$

To prove the direct implication in the statement of the lemma, let us assume that $f \in B_{\alpha, p a r}^{p}\left(\mathbb{R}^{n} \times \mathbb{R}\right)$. Then, by the triangle inequality,

$$
\begin{aligned}
\int_{\mathbb{R}^{n} \times \mathbb{R}} \int_{\mathbb{R}^{n} \times \mathbb{R}} & \frac{|f(x, t)-f(y, s)|^{p}}{\left(|x-y|+|t-s|^{1 / 2}\right)^{n+2+\alpha p}} d y d s d x d t \\
\leq & C \int_{\mathbb{R}^{n} \times \mathbb{R}} \int_{\mathbb{R}^{n} \times \mathbb{R}} \frac{|f(x, t)-f(x, s)|^{p}}{\left(|x-y|+|t-s|^{1 / 2}\right)^{n+2+\alpha p}} d y d s d x d t \\
& +C \int_{\mathbb{R}^{n} \times \mathbb{R}} \int_{\mathbb{R}^{n} \times \mathbb{R}} \frac{|f(x, s)-f(y, s)|^{p}}{\left(|x-y|+|t-s|^{1 / 2}\right)^{n+2+\alpha p}} d y d s d x d t \\
= & : I+I I .
\end{aligned}
$$

Making the change of variables $x-y=z \in \mathbb{R}^{n}, z=z^{\prime}|t-s|^{1 / 2} \in \mathbb{R}^{n}$ in $I$, and $t-s=r \in \mathbb{R}, r=r^{\prime}|x-y|^{2} \in \mathbb{R}$ in $I I$ yields

$$
\begin{aligned}
I+I I \leq & C\left(\int_{\mathbb{R}^{n}} \frac{d z^{\prime}}{\left(\left|z^{\prime}\right|+1\right)^{n+2+\alpha p}}\right) \int_{\mathbb{R}^{n}} \int_{\mathbb{R}} \int_{\mathbb{R}} \frac{|f(x, t)-f(x, s)|^{p}}{|t-s|^{1+\frac{\alpha p}{2}}} d s d t d x \\
& +C\left(\int_{\mathbb{R}} \frac{d r^{\prime}}{\left(\left|r^{\prime}\right|+1\right)^{n+2+\alpha p}}\right) \int_{\mathbb{R}} \int_{\mathbb{R}^{n}} \int_{\mathbb{R}^{n}} \frac{|f(x, s)-f(y, s)|^{p}}{|x-y|^{n+\alpha p}} d x d y d s \\
\leq & C<\infty .
\end{aligned}
$$

Since $B_{\alpha, p a r}^{p}\left(\mathbb{R}^{n} \times \mathbb{R}\right) \hookrightarrow L^{p}\left(\mathbb{R}^{n} \times \mathbb{R}\right)$ is immediate by definition, we have proved the direct implication. 
To prove the oposite one, assume $f \in L^{p}\left(\mathbb{R}^{n} \times \mathbb{R}\right)$ and

$$
\int_{\mathbb{R}^{n} \times \mathbb{R}} \int_{\mathbb{R}^{n} \times \mathbb{R}} \frac{|f(x, t)-f(y, s)|^{p}}{\left(|x-y|+|t-s|^{1 / 2}\right)^{n+2+\alpha p}} d y d s d x d t<\infty .
$$

The strategy is to rely on $(2.1 .12)$ so we need to be able to control $\|f\|_{\dot{B}_{\alpha, p a r}^{p}\left(\mathbb{R}^{n} \times \mathbb{R}\right)}$ uniformly in $f$. To this end, we shall invoke Theorem 1 from [See] with $m=1$, $r=1$ and $p=q$ (for $1 \leq p<\infty, 0<\alpha<1$ ), to the effect that

$$
\|f\|_{\dot{B}_{\alpha, p a r}^{p}\left(\mathbb{R}^{n} \times \mathbb{R}\right)} \approx\left\|\mathcal{D}_{p, 1,1}^{\alpha} f\right\|_{L^{p}\left(\mathbb{R}^{n} \times \mathbb{R}\right)}
$$

where, for $X:=(x, t) \in \mathbb{R}^{n} \times \mathbb{R}$ and $Y:=(y, s) \in \mathbb{R}^{n} \times \mathbb{R}$,

$$
\begin{aligned}
& \mathcal{D}_{p, 1,1}^{\alpha} f(X):=\left(\int_{0}^{\infty}\left[\operatorname{osc}_{1}^{0}(f, X, \theta)\right]^{p} \frac{d \theta}{\theta^{1+\alpha p}}\right)^{\frac{1}{p}}, \\
& \operatorname{osc}_{1}^{0}(f, X, \theta):=\inf _{c \in \mathbb{R}} f_{\|X-Y\|_{p a r} \leq \theta}|f(Y)-c| d Y .
\end{aligned}
$$

There remains to estimate $\left\|\mathcal{D}_{p, 1,1}^{\alpha} f\right\|_{L^{p}\left(\mathbb{R}^{n} \times \mathbb{R}\right)}$ in terms of (2.1.32) and (2.1.33). As a preliminary step in this direction we observe that, if $p^{\prime}$ denotes the conjugate of $p$ (i.e. $1 / p+1 / p^{\prime}=1$ ), by Hölder's inequality we have

$$
\begin{aligned}
{\left[\operatorname{osc}_{1}^{0}(f, X, \theta)\right]^{p} } & \leq\left[f_{\|X-Y\|_{p a r} \leq \theta}|f(Y)-f(X)| d Y\right]^{p} \\
& \leq\left(\frac{1}{\theta^{n+2}}\right)^{p}\left(\theta^{n+2}\right)^{\frac{p}{p^{\prime}}} \int_{\|X-Y\|_{p a r} \leq \theta}|f(Y)-f(X)|^{p} d Y \\
& =\left(\theta^{n+2}\right)^{\frac{p}{p^{\prime}}-p+1} \frac{1}{\theta^{n+1}} \int_{\|X-Y\|_{p a r} \leq \theta}|f(Y)-f(X)|^{p} d Y \\
& =f_{\|X-Y\|_{p a r} \leq \theta}|f(Y)-f(X)|^{p} d Y .
\end{aligned}
$$


Then, using (2.1.34) we obtain that

$$
\begin{aligned}
& \left\|\mathcal{D}_{p, 1,1}^{\alpha} f\right\|_{L^{p}\left(\mathbb{R}^{n} \times \mathbb{R}\right)}^{p} \\
& \quad=C \int_{X \in \mathbb{R}^{n} \times \mathbb{R}}\left(\int_{0}^{\infty}\left[\operatorname{osc}_{1}^{0}(f, X, \theta)\right]^{p} \frac{d \theta}{\theta^{1+\alpha p}}\right) d X \\
& \quad \leq C \int_{X \in \mathbb{R}^{n} \times \mathbb{R}}\left[\int_{0}^{\infty} \frac{1}{\theta^{n+2}}\left(\int_{\|X-Y\|_{p a r} \leq \theta}|f(X)-f(Y)|^{p} d Y\right) \frac{d \theta}{\theta^{1+\alpha p}}\right] d X \\
& \quad \leq C \int_{X \in \mathbb{R}^{n} \times \mathbb{R}} \int_{Y \in \mathbb{R}^{n} \times \mathbb{R}}|f(X)-f(Y)|^{p}\left(\int_{\|X-Y\|_{p a r}}^{\infty} \frac{d \theta}{\theta^{n+3+\alpha p}}\right) d Y d X \\
& \quad \leq C \int_{X \in \mathbb{R}^{n} \times \mathbb{R}} \int_{Y \in \mathbb{R}^{n} \times \mathbb{R}} \frac{|f(X)-f(Y)|^{p}}{\|X-Y\|_{p a r}^{n+2+\alpha p}} d Y d X \\
& \quad=C \int_{\mathbb{R}^{n} \times \mathbb{R}} \int_{\mathbb{R}^{n} \times \mathbb{R}} \frac{|f(x, t)-f(y, s)|^{p}}{\left(|x-y|+|t-s|^{1 / 2}\right)^{n+2+\alpha p}} d y d s d x d t<\infty,
\end{aligned}
$$

Hence

$$
\begin{aligned}
& \|f\|_{B_{\alpha, p a r}^{p}\left(\mathbb{R}^{n} \times \mathbb{R}\right)} \\
& \approx\|f\|_{L^{p}\left(\mathbb{R}^{n} \times \mathbb{R}\right)}+\|f\|_{\dot{B}_{\alpha, p a r}^{p}\left(\mathbb{R}^{n} \times \mathbb{R}\right)} \\
& \approx\|f\|_{L^{p}\left(\mathbb{R}^{n} \times \mathbb{R}\right)}+\left\|\mathcal{D}_{p, 1,1}^{\alpha} f\right\|_{L^{p}\left(\mathbb{R}^{n} \times \mathbb{R}\right)} \\
& \approx\|f\|_{L^{p}\left(\mathbb{R}^{n} \times \mathbb{R}\right)}+\left(\int_{\mathbb{R}^{n} \times \mathbb{R}} \int_{\mathbb{R}^{n} \times \mathbb{R}} \frac{|f(x, t)-f(y, s)|^{p}}{\left(|x-y|+|t-s|^{1 / 2}\right)^{n+2+\alpha p}} d y d s d x d t\right)^{1 / p}<\infty,
\end{aligned}
$$

as desired.

In the last part of this subsection we present the atomic decompositions of homogeneous and inhomogeneous anisotropic Besov spaces with parabolic anisotropy.

Remark 2.1.23. Recall that a parabolic cube $Q$ in $\mathbb{R}^{n} \times \mathbb{R}$ with sidelength $r$ has the form $Q=Q^{\prime} \times I$, where $Q^{\prime} \subseteq \mathbb{R}^{n}$ is a (regular) cube of sidelength $r$ and $I \subseteq \mathbb{R}$ is an 
open interval of length $r^{2}$. We note that $|Q|=r^{n+2}$. Instead of parabolic (regular) cubes one may consider corresponging balls as well. In this case the resulting vector space is equipped with a quasi-norm which is equivalent with the quasi-norm of the space obtained using cubes.

At the beginning of our discussion we focus on spaces with positive amount of smoothness.

Definition 2.1.24. Let $Q$ be a parabolic cube in $\mathbb{R}^{n} \times \mathbb{R}$ of sidelength $r$ and suppose that $\frac{n+2}{n+3}<p \leq 1,(n+2)\left(\frac{1}{p}-1\right)<\alpha<1$. Then $a_{Q}$ is called a $(p, \alpha)$-atom if

$$
\begin{aligned}
& \operatorname{supp}_{Q} \subset Q, \\
& \left\|a_{Q}\right\|_{L^{\infty}\left(\mathbb{R}^{n} \times \mathbb{R}\right)} \leq r^{\alpha-\frac{n+2}{p}}, \\
& \left\|\partial_{x_{i}} a_{Q}\right\|_{L^{\infty}\left(\mathbb{R}^{n} \times \mathbb{R}\right)} \leq r^{\alpha-1-\frac{n+2}{p}}, \\
& \left\|\partial_{t} a_{Q}\right\|_{L^{\infty}\left(\mathbb{R}^{n} \times \mathbb{R}\right)} \leq r^{\alpha-2-\frac{n+2}{p}} .
\end{aligned}
$$

Recall that the spaces $\dot{B}_{\alpha, p a r}^{p}\left(\mathbb{R}^{n} \times \mathbb{R}\right)$ and $B_{\alpha, p a r}^{p}\left(\mathbb{R}^{n} \times \mathbb{R}\right)$ were introduced (both for positive and negative order of smoothness) in Definitions 2.1.6 and 2.1.2, respectively.

Theorem 2.1.25. (Theorem 5.8 of [BoHo])

In the context of Definition 2.1.24, $f$ belongs to the homogeneous parabolic Besov space $\dot{B}_{\alpha, p a r}^{p}\left(\mathbb{R}^{n} \times \mathbb{R}\right)$ if and only if $f$ admits the representation $f=\sum_{Q} s_{Q} a_{Q}$, where $\left\{s_{Q}\right\}_{Q} \in \ell^{p}$ and $a_{Q}$ are $(p, \alpha)$-atoms. Moreover, if $f \in \dot{B}_{\alpha, p a r}^{p}\left(\mathbb{R}^{n} \times \mathbb{R}\right)$, then

$$
\|f\|_{\dot{B}_{\alpha, p a r}^{p}\left(\mathbb{R}^{n} \times \mathbb{R}\right)} \approx \inf \left(\sum_{Q}\left|s_{Q}\right|^{p}\right)^{1 / p}
$$

where the infimum is taken over all atomic decompositions of $f$. 
Theorem 2.1.26. (Theorem 5.11 of $[\mathrm{BoHo}]$ )

Using the notation of Definition 2.1.24, $f \in B_{\alpha, p a r}^{p}\left(\mathbb{R}^{n} \times \mathbb{R}\right)$ if and only if $f$ admits the representation $f=\sum_{Q} s_{Q} a_{Q}$, where $\left\{s_{Q}\right\}_{Q} \in \ell^{p}$ and $a_{Q}$ are $(p, \alpha)$-atoms with $|Q| \leq 1$. Moreover, if $f \in B_{\alpha, p a r}^{p}\left(\mathbb{R}^{n} \times \mathbb{R}\right)$, then

$$
\|f\|_{B_{\alpha, p a r}^{p}\left(\mathbb{R}^{n} \times \mathbb{R}\right)} \approx \inf \left(\sum_{|Q| \leq 1}\left|s_{Q}\right|^{p}\right)^{1 / p},
$$

where the infimum is taken over all representations of $f$.

When the order of smoothness is negative, the following characterizations are going to be useful.

Definition 2.1.27. Consider a parabolic cube $Q$ in $\mathbb{R}^{n} \times \mathbb{R}$ with sidelength $r$. Suppose

$$
\begin{aligned}
& \frac{n+2}{n+3}<p \leq 1 \text { and }(n+2)\left(\frac{1}{p}-1\right)<1-\alpha<1 \text {. Then we call } a_{Q} a(p,-\alpha) \text {-atom if } \\
& \qquad \begin{array}{c}
\operatorname{supp} a_{Q} \subset Q, \\
\left\|a_{Q}\right\|_{L^{\infty}\left(\mathbb{R}^{n} \times \mathbb{R}\right)} \leq r^{-\alpha-\frac{n+2}{p}}, \\
\int_{\mathbb{R}^{n} \times \mathbb{R}} a_{Q}(x, t) d x d t=0 .
\end{array}
\end{aligned}
$$

Theorem 2.1.28. (Theorem 5.8 of $[\mathrm{BoHo}]$ )

In the conext of Definition 2.1.27, $f$ belongs to the homogeneous parabolic Besov space $\dot{B}_{-\alpha, p a r}^{p}\left(\mathbb{R}^{n} \times \mathbb{R}\right)$ if and only if $f$ admits the representation $f=\sum_{Q} s_{Q} a_{Q}$, where $\left\{s_{Q}\right\}_{Q} \in \ell^{p}$ and $a_{Q}$ are $(p,-\alpha)$-atoms. Moreover, if $f \in \dot{B}_{-\alpha, p a r}^{p}\left(\mathbb{R}^{n} \times \mathbb{R}\right)$, then

$$
\|f\|_{\dot{B}_{-\alpha, p a r}^{p}\left(\mathbb{R}^{n} \times \mathbb{R}\right)} \approx \inf \left(\sum_{Q}\left|s_{Q}\right|^{p}\right)^{1 / p},
$$

where the infimum is taken over all representations of $f$. 
Theorem 2.1.29. (Theorem 5.11 of $[\mathrm{BoHo}]$ )

Using the notation of Definition 2.1.27, $f \in B_{-\alpha, p a r}^{p}\left(\mathbb{R}^{n} \times \mathbb{R}\right)$ if and only if $f$ admits the representation $f=\sum_{Q} s_{Q} a_{Q}$, where $\left\{s_{Q}\right\}_{Q} \in \ell^{p}$ and $a_{Q}$ are $(p,-\alpha)$ atoms with $|Q| \leq 1$. Moreover, if $f \in B_{-\alpha, p a r}^{p}\left(\mathbb{R}^{n} \times \mathbb{R}\right)$, then

$$
\|f\|_{B_{-\alpha, p a r}^{p}\left(\mathbb{R}^{n} \times \mathbb{R}\right)} \approx \inf \left(\sum_{|Q| \leq 1}\left|s_{Q}\right|^{p}\right)^{1 / p}
$$

where the infimum is taken over all atomic decompositions of $f$.

\subsubsection{Sobolev spaces}

In this subsection we focus our attention on a distinguished subclass of the TriebelLizorkin scale, namely the Sobolev/Bessel potential spaces.

Definition 2.1.30. We define the parabolic Sobolev space for $1<p<\infty$ and $\alpha \in \mathbb{R}$ by

$$
L_{\alpha, p a r}^{p}\left(\mathbb{R}^{n} \times \mathbb{R}\right):=F_{\alpha, p a r}^{p, 2}\left(\mathbb{R}^{n} \times \mathbb{R}\right)
$$

By virtue of Proposition 3.11 in [Yam], if $1<p<\infty$ then

$$
F_{0, p a r}^{p, 2}\left(\mathbb{R}^{n} \times \mathbb{R}\right)=L^{p}\left(\mathbb{R}^{n} \times \mathbb{R}\right)
$$

so that

$$
L_{0, p a r}^{p}\left(\mathbb{R}^{n} \times \mathbb{R}\right)=L^{p}\left(\mathbb{R}^{n} \times \mathbb{R}\right), \quad 1<p<\infty .
$$

A characterization, via a Fourier multiplier, of these parabolic Sobolev spaces (which is a special case of Theorem 2.1.15), is as follows.

Corollary 2.1.31. Let $1<p<\infty$ and $\alpha \in \mathbb{R}$. Then, for $f \in \mathrm{S}^{\prime}\left(\mathbb{R}^{n} \times \mathbb{R}\right)$, the following are equivalent: 
(1) $f \in L_{\alpha, p a r}^{p}\left(\mathbb{R}^{n} \times \mathbb{R}\right)$;

(2) $\Theta_{\alpha} f \in L^{p}\left(\mathbb{R}^{n} \times \mathbb{R}\right)$,

where recall that

$$
\Theta_{\alpha} f:=\mathcal{F}^{-1}\left[\left(1+|\xi|^{4}+\tau^{2}\right)^{\alpha / 4} \mathcal{F} f(\xi, \tau)\right], \quad(\xi, \tau) \in \mathbb{R}^{n} \times \mathbb{R}
$$

Remark. In order to prove Corollary 2.1 .31 for a restricted range of $\alpha$, i.e. for $\alpha \geq 0$, we could have also used Proposition 2.1.38 (which is proved independently of Corollary 2.1.31) in the following way. First, we assume that $f \in L_{\alpha, p a r}^{p}\left(\mathbb{R}^{n} \times \mathbb{R}\right)$. By Proposition 2.1.38,

$$
g:=\mathcal{F}^{-1}\left[\left(\sum_{k=1}^{n}\left(1+\xi_{k}^{2}\right)^{\alpha / 2}+\left(1+\tau^{2}\right)^{\alpha / 4}\right) \mathcal{F} f(\xi, \tau)\right] \in L^{p}\left(\mathbb{R}^{n} \times \mathbb{R}\right) .
$$

Notice that we can rewrite $\Theta_{\alpha} f$ as $\Theta_{\alpha} f=\mathcal{F}^{-1}[q(\xi, \tau) \mathcal{F} g(\xi, \tau)]$, where

$$
q(\xi, \tau):=\frac{\left(1+|\xi|^{4}+\tau^{2}\right)^{\alpha / 4}}{\sum_{k=1}^{n}\left(1+\xi_{k}^{2}\right)^{\alpha / 2}+\left(1+\tau^{2}\right)^{\alpha / 4}}, \quad \xi \in \mathbb{R}^{n}, \tau \in \mathbb{R}
$$

and $g$ is given in (2.1.38). Since $g \in L^{p}\left(\mathbb{R}^{n} \times \mathbb{R}\right)$ and $q$ is a Fourier multiplier for $L^{p}\left(\mathbb{R}^{n} \times \mathbb{R}\right)$ (see $m_{3}$ in Example 3 of 1.2.6), we can conclude that $\Theta_{\alpha} f \in L^{p}\left(\mathbb{R}^{n} \times \mathbb{R}\right)$, which proves (2).

Conversely, assume (2), i.e. that $h:=\Theta_{\alpha} f \in L^{p}\left(\mathbb{R}^{n} \times \mathbb{R}\right)$. In order to show that $\Theta_{-\alpha} h=f$ belongs to the Sobolev space $L_{\alpha, p a r}^{p}\left(\mathbb{R}^{n} \times \mathbb{R}\right)$, according to Proposition 2.1.38, it is enough to prove that

$$
G:=\mathcal{F}^{-1}\left[\left(\sum_{k=1}^{n}\left(1+\xi_{k}^{2}\right)^{\alpha / 2}+\left(1+\tau^{2}\right)^{\alpha / 4}\right) \mathcal{F}\left(\Theta_{-\alpha} h\right)(\xi, \tau)\right] \in L^{p}\left(\mathbb{R}^{n} \times \mathbb{R}\right),
$$

or, equivalently, using the description of the operator $\Theta_{\alpha}$ in (2.1.37), that

$$
r(\xi, \tau):=\frac{\sum_{k=1}^{n}\left(1+\xi_{k}^{2}\right)^{\alpha / 2}+\left(1+\tau^{2}\right)^{\alpha / 4}}{\left(1+|\xi|^{4}+\tau^{2}\right)^{\alpha / 4}}, \quad \xi \in \mathbb{R}^{n}, \tau \in \mathbb{R},
$$


is a Fourier multiplier for $L^{p}\left(\mathbb{R}^{n} \times \mathbb{R}\right)$. This, in turn, was proved in Example 4 of 1.2.6.

Remark 2.1.32. In [Gru1] (pp. 84 - 85) the parabolic Sobolev space $L_{\alpha, p a r}^{p}\left(\mathbb{R}^{n} \times \mathbb{R}\right)$ is defined as the collection of all $f \in \mathrm{S}^{\prime}\left(\mathbb{R}^{n} \times \mathbb{R}\right)$ so that

$$
\mathcal{F}^{-1}\left[\left(1+|\xi|^{4}+\tau^{2}\right)^{\alpha / 4} \mathcal{F} f(\xi, \tau)\right] \in L^{p}\left(\mathbb{R}^{n} \times \mathbb{R}\right)
$$

for $1<p<\infty$ and $\alpha \in \mathbb{R}$.

Proposition 2.1.33. For $1<p<\infty$ and $\alpha \geq 0$ the following Fubini property for the parabolic Sobolev scale holds:

$$
L_{\alpha, p a r}^{p}\left(\mathbb{R}^{n} \times \mathbb{R}\right)=L^{p}\left(\mathbb{R} ; L_{\alpha}^{p}\left(\mathbb{R}^{n}\right)\right) \cap L^{p}\left(\mathbb{R}^{n} ; L_{\alpha / 2}^{p}(\mathbb{R})\right)
$$

Proof. For $\alpha=0$ there is nothing to prove. When $\alpha>0$, according to (i) in (A.10) of [Gru1],

$$
L_{\alpha, p a r}^{p}\left(\mathbb{R}^{n} \times \mathbb{R}\right)=L^{p}\left(\mathbb{R} ; L_{\alpha}^{p}\left(\mathbb{R}^{n}\right)\right) \cap L_{\alpha / 2}^{p}\left(\mathbb{R} ; L^{p}\left(\mathbb{R}^{n}\right)\right)
$$

Recall from Proposition 1.2.11 that

$$
L_{\alpha / 2}^{p}\left(\mathbb{R} ; L^{p}\left(\mathbb{R}^{n}\right)\right)=L^{p}\left(\mathbb{R}^{n} ; L_{\alpha / 2}^{p}(\mathbb{R})\right)
$$

Now (2.1.40) and (2.1.41) readily imply what we wanted to show.

Definition 2.1.34. The homogeneous parabolic Sobolev (Bessel potential) space, for $1<p<\infty$ and $\alpha \in \mathbb{R}$, is defined as

$$
\dot{L}_{\alpha, p a r}^{p}\left(\mathbb{R}^{n} \times \mathbb{R}\right):=\dot{F}_{\alpha, p a r}^{p, 2}\left(\mathbb{R}^{n} \times \mathbb{R}\right) .
$$


Corollary 2.1.35. As a consequence of (2.1.10) and (2.1.11), for $1<p<\infty$ and $\alpha>0$

$$
L_{\alpha, p a r}^{p}\left(\mathbb{R}^{n} \times \mathbb{R}\right)=\dot{L}_{\alpha, p a r}^{p}\left(\mathbb{R}^{n} \times \mathbb{R}\right) \cap L^{p}\left(\mathbb{R}^{n} \times \mathbb{R}\right)
$$

with equivalent norms.

Since the case of (2.1.42) with $\alpha=1$ is going to be particularly useful for us, we also sketch a direct proof of it. In order to do so, we shall use an alternative characterization of $\dot{L}_{1, p a r}^{p}\left(\mathbb{R}^{n} \times \mathbb{R}\right)$ inspired by [Bro2], pp. $5-6$. First we make a definition.

Definition 2.1.36. For $1<p<\infty$

$$
f \in \mathcal{L}_{1}^{p}\left(\mathbb{R}^{n-1} \times \mathbb{R}\right) \Longleftrightarrow \mathcal{F}^{-1}\left[\sqrt{|\xi|^{2}+i \tau}(\mathcal{F} f)(\xi, \tau)\right] \in L^{p}\left(\mathbb{R}^{n-1} \times \mathbb{R}\right) .
$$

As a preliminary step we prove the following.

Lemma 2.1.37. The spaces $\mathcal{L}_{1}^{p}\left(\mathbb{R}^{n} \times \mathbb{R}\right)$ and $\dot{L}_{1, p a r}^{p}\left(\mathbb{R}^{n} \times \mathbb{R}\right)$ coincide for each value of $p$ with $1<p<\infty$.

Proof. According to Theorem 2.1.12, in order to prove the inclusion $\mathcal{L}_{1}^{p}\left(\mathbb{R}^{n} \times \mathbb{R}\right) \supseteq$ $\dot{L}_{1, p a r}^{p}\left(\mathbb{R}^{n} \times \mathbb{R}\right)$, it is enough to show that

$$
m(\xi, \tau):=\frac{\sqrt{|\xi|^{2}+i \tau}}{\sum_{j=1}^{n}\left|\xi_{j}\right|+|\tau|^{1 / 2}}, \quad \xi \in \mathbb{R}^{n}, \tau \in \mathbb{R},
$$

is a multiplier for $L^{p}\left(\mathbb{R}^{n} \times \mathbb{R}\right)$. This, in turn, was proved in Example 1 of 1.2.6, i.e. $m=m_{1}$ for $m_{1}$ as in (1.2.38).

Conversely, we need to show that

$$
q(\xi, \tau):=\frac{\sum_{j=1}^{n}\left|\xi_{j}\right|+|\tau|^{1 / 2}}{\sqrt{|\xi|^{2}+i \tau}}, \quad \xi \in \mathbb{R}^{n}, \tau \in \mathbb{R}
$$


is a multiplier for $L^{p}\left(\mathbb{R}^{n} \times \mathbb{R}\right)$. Since $q=m_{2}$ for $m_{2}$ as in (1.2.39), and we showed in Example 2 of 1.2.6 that $m_{2}$ is a multiplier for $L^{p}\left(\mathbb{R}^{n} \times \mathbb{R}\right)$, the proof of the lemma is complete.

Proof of (2.1.42) when $\alpha=1$.

We shall employ lifting operators, and several natural choices present themselves. Our reasoning is based on Theorems 2.1.14 and 2.1.15, although we could have also used Theorems 2.1.12 and 2.1.11 (and Examples 9 and 10 in 1.2.6).

First assume that $f \in \dot{L}_{1, p a r}^{p}\left(\mathbb{R}^{n} \times \mathbb{R}\right)$ and $f \in L^{p}\left(\mathbb{R}^{n} \times \mathbb{R}\right)$. Our goal is to show that $f \in L_{1, p a r}^{p}\left(\mathbb{R}^{n} \times \mathbb{R}\right)$. According to Theorem 2.1.14, the first hypothesis implies that, for $(\xi, \tau) \in \mathbb{R}^{n} \times \mathbb{R}$, we have the following:

$$
h:=\mathcal{F}^{-1}\left[\left(|\xi|^{2}+|\tau|\right)^{1 / 2} \mathcal{F} f(\xi, \tau)\right] \in L^{p}\left(\mathbb{R}^{n} \times \mathbb{R}\right) .
$$

Since $f \in L^{p}\left(\mathbb{R}^{n} \times \mathbb{R}\right)$ by hypothesis, (2.1.43) yields

$$
f+h=\mathcal{F}^{-1}\left[\left(1+\left(|\xi|^{2}+|\tau|\right)^{1 / 2}\right) \mathcal{F} f(\xi, \tau)\right] \in L^{p}\left(\mathbb{R}^{n} \times \mathbb{R}\right)
$$

In order to show that $f \in L_{1, p a r}^{p}\left(\mathbb{R}^{n} \times \mathbb{R}\right)$, by Theorem 2.1.15, it suffices to prove that, for $(\xi, \tau) \in \mathbb{R}^{n} \times \mathbb{R}$,

$$
g:=\mathcal{F}\left[\left(1+|\xi|^{4}+\tau^{2}\right)^{1 / 4} \mathcal{F} f(\xi, \tau)\right] \in L^{p}\left(\mathbb{R}^{n} \times \mathbb{R}\right)
$$

Recall from Example 7 of 1.2.6 that $m$ defined as

$$
m(\xi, \tau):=\frac{\left(1+|\xi|^{4}+\tau^{2}\right)^{1 / 4}}{1+\left(|\xi|^{2}+|\tau|\right)^{1 / 2}}, \quad(\xi, \tau) \in \mathbb{R}^{n} \times \mathbb{R}
$$

is a multiplier for $L^{p}\left(\mathbb{R}^{n} \times \mathbb{R}\right), 1<p<\infty$. With this and (2.1.44) in hand, (2.1.45) immediately follows, proving the right-to-left inclusion in (2.1.42) for $\alpha=1$. 
Conversely, suppose that $f$ is contained in $L_{1, p a r}^{p}\left(\mathbb{R}^{n} \times \mathbb{R}\right)$. We shall prove that $f \in \dot{L}_{1, p a r}^{p}\left(\mathbb{R}^{n} \times \mathbb{R}\right)$ and $f \in L^{p}\left(\mathbb{R}^{n} \times \mathbb{R}\right)$. In view of Theorem 2.1.15, our assumption amounts to

$$
\mathcal{F}\left[\left(1+|\xi|^{4}+\tau^{2}\right)^{1 / 4} \mathcal{F} f(\xi, \tau)\right] \in L^{p}\left(\mathbb{R}^{n} \times \mathbb{R}\right),
$$

where $(\xi, \tau) \in \mathbb{R}^{n} \times \mathbb{R}$. To prove that $f \in \dot{L}_{1, p a r}^{p}\left(\mathbb{R}^{n} \times \mathbb{R}\right)$, by Theorem 2.1.14, it is enough to show the following: for $(\xi, \tau) \in \mathbb{R}^{n} \times \mathbb{R}$,

$$
\mathcal{F}^{-1}\left[\left(|\xi|^{2}+|\tau|\right)^{1 / 2} \mathcal{F} f(\xi, \tau)\right] \in L^{p}\left(\mathbb{R}^{n} \times \mathbb{R}\right) .
$$

From Example 8 in 1.2.6 we have that

$$
q(\xi, \tau):=\frac{\left(|\xi|^{2}+|\tau|\right)^{1 / 2}}{\left(1+|\xi|^{4}+\tau^{2}\right)^{1 / 4}}, \quad(\xi, \tau) \in \mathbb{R}^{n} \times \mathbb{R}
$$

is a multiplier for $L^{p}\left(\mathbb{R}^{n} \times \mathbb{R}\right), 1<p<\infty$. Therefore, (2.1.47) follows from (2.1.46), proving that $f$ belongs to $\dot{L}_{1, p a r}^{p}\left(\mathbb{R}^{n} \times \mathbb{R}\right)$.

To see that $f \in L^{p}\left(\mathbb{R}^{n} \times \mathbb{R}\right)$, we recall the Fubini property of the Sobolev scale from Proposition 2.1.33. Then our current assumption implies that

$$
f \in L^{p}\left(\mathbb{R} ; L_{1}^{p}\left(\mathbb{R}^{n}\right)\right)
$$

hence, by a particular case of $(1.2 .25), f \in L^{p}\left(\mathbb{R} ; L^{p}\left(\mathbb{R}^{n}\right)\right)=L^{p}\left(\mathbb{R}^{n} \times \mathbb{R}\right)$. This proves the left-to-right inclusion in (2.1.42) for $\alpha=1$.

We next give a Fourier multiplier characterization of the parabolic Sobolev space $L_{\alpha, p a r}^{p}\left(\mathbb{R}^{n} \times \mathbb{R}\right)$. 
Proposition 2.1.38. For any $1<p<\infty$ and $\alpha \in \mathbb{R}$ the following holds:

$$
\begin{aligned}
& F \in L_{\alpha, p a r}^{p}\left(\mathbb{R}^{n} \times \mathbb{R}\right) \Longleftrightarrow F \in \mathrm{S}^{\prime}\left(\mathbb{R}^{n} \times \mathbb{R}\right) \quad \text { and } \\
& \mathcal{F}^{-1}\left[\left(\sum_{k=1}^{n}\left(1+\xi_{k}^{2}\right)^{\alpha / 2}+\left(1+\tau^{2}\right)^{\alpha / 4}\right) \mathcal{F} F(\xi, \tau)\right] \in L^{p}\left(\mathbb{R}^{n} \times \mathbb{R}\right) .
\end{aligned}
$$

Moreover,

$$
\|F\|_{L_{\alpha, p a r}^{p}\left(\mathbb{R}^{n} \times \mathbb{R}\right)} \approx\left\|\mathcal{F}^{-1}\left[\left(\sum_{k=1}^{n}\left(1+\xi_{k}^{2}\right)^{\alpha / 2}+\left(1+\tau^{2}\right)^{\alpha / 4}\right) \mathcal{F} F(\xi, \tau)\right]\right\|_{L^{p}\left(\mathbb{R}^{n} \times \mathbb{R}\right)}
$$

Proof. In order to show that we can reduce matters to prove a simpler statement, we recall the definition and a property of certain anisotropic (in our case parabolic) Sobolev spaces from [Far] (cf. (2.7) on p. 89 in $[\mathrm{Far}]$ ). For each $k \in\{1,2, \ldots, n\}$, define $L_{\alpha, p a r}^{p, x_{k}}\left(\mathbb{R}^{n} \times \mathbb{R}\right)$ as the collection of $f \in \mathrm{S}^{\prime}\left(\mathbb{R}^{n} \times \mathbb{R}\right)$ such that

$$
g_{k}:=\mathcal{F}_{k}^{-1}\left\{\left(1+\xi_{k}^{2}\right)^{\alpha / 2}\left[\mathcal{F}_{k} f\left(\xi_{1}, \ldots, \xi_{k-1}, \cdot, \xi_{k+1}, \ldots, \xi_{n}, \tau\right)\right]\left(\xi_{k}\right)\right\} \in L^{p}\left(\mathbb{R}^{n} \times \mathbb{R}\right),
$$

and equip it with the norm

$$
\|f\|_{L_{\alpha, p a r}^{p, x_{k}\left(\mathbb{R}^{n} \times \mathbb{R}\right)}}:=\left\|g_{k}\right\|_{L^{p}\left(\mathbb{R}^{n} \times \mathbb{R}\right)}
$$

Similarly, $L_{\alpha, \text { par }}^{p, t}\left(\mathbb{R}^{n} \times \mathbb{R}\right)$ is defined as the set of all $f \in \mathrm{S}^{\prime}\left(\mathbb{R}^{n} \times \mathbb{R}\right)$ for which

$$
g_{t}:=\mathcal{F}_{\text {time }}^{-1}\left\{\left(1+\tau^{2}\right)^{\alpha / 4}\left[\mathcal{F}_{\text {time }} f(\xi, \cdot)\right](\tau)\right\} \in L^{p}\left(\mathbb{R}^{n} \times \mathbb{R}\right),
$$

and it is equipped with the norm

$$
\|f\|_{L_{\alpha, p a r}^{p, t}\left(\mathbb{R}^{n} \times \mathbb{R}\right)}:=\left\|g_{t}\right\|_{L^{p}\left(\mathbb{R}^{n} \times \mathbb{R}\right)} .
$$

Then, according to (9.1) of [Nik] (see also (2.8) on p. 89 of [Far]),

$$
L_{\alpha, p a r}^{p}\left(\mathbb{R}^{n} \times \mathbb{R}\right)=\left(\bigcap_{k=1}^{n} L_{\alpha, p a r}^{p, x_{k}}\left(\mathbb{R}^{n} \times \mathbb{R}\right)\right) \bigcap L_{\alpha, p a r}^{p, t}\left(\mathbb{R}^{n} \times \mathbb{R}\right),
$$


and

$$
\|f\|_{L_{\alpha, p a r}^{p}\left(\mathbb{R}^{n} \times \mathbb{R}\right)} \approx \sum_{k=1}^{n}\|f\|_{L_{\alpha, p a r}^{p, x_{k}\left(\mathbb{R}^{n} \times \mathbb{R}\right)}}+\|f\|_{L_{\alpha, p a r}^{p, t}\left(\mathbb{R}^{n} \times \mathbb{R}\right)} .
$$

The relevance of the above discussion is the following. In order to prove the proposition, it is enough to show that given $f \in L_{\alpha}^{p}\left(\mathbb{R}^{2}\right)$ and

$$
\begin{aligned}
& g:=\mathcal{F}^{-1}\left\{\left[\left(1+\xi_{1}^{2}\right)^{\alpha / 2}+\left(1+\xi_{2}^{2}\right)^{\alpha / 2}\right] \mathcal{F} f\left(\xi_{1}, \xi_{2}\right)\right\} \\
& g_{1}:=\mathcal{F}_{1}^{-1}\left\{\left(1+\xi_{1}^{2}\right)^{\alpha / 2}\left[\mathcal{F}_{1} f\left(\cdot, \xi_{2}\right)\right]\left(\xi_{1}\right)\right\} \\
& g_{2}:=\mathcal{F}_{2}^{-1}\left\{\left(1+\xi_{2}^{2}\right)^{\alpha / 2}\left[\mathcal{F}_{2} f\left(\xi_{1}, \cdot\right)\right]\left(\xi_{2}\right)\right\},
\end{aligned}
$$

we have

$$
g \in L^{p}\left(\mathbb{R}^{2}\right) \Longleftrightarrow g_{1}, g_{2} \in L^{p}\left(\mathbb{R}^{2}\right)
$$

and

$$
\|g\|_{L^{p}\left(\mathbb{R}^{2}\right)} \approx\left\|g_{1}\right\|_{L^{p}\left(\mathbb{R}^{2}\right)}+\left\|g_{2}\right\|_{L^{p}\left(\mathbb{R}^{2}\right)}
$$

To see this, we use Proposition 1.2.13 to rewrite $g$ in the following way

$$
\begin{aligned}
g & =\left(\mathcal{F}_{1} \circ \mathcal{F}_{2}\right)^{-1}\left\{\left[\left(1+\xi_{1}^{2}\right)^{\alpha / 2}+\left(1+\xi_{2}^{2}\right)^{\alpha / 2}\right] \mathcal{F}_{1} \circ \mathcal{F}_{2} f\left(\xi_{1}, \xi_{2}\right)\right\} \\
& =\mathcal{F}_{2}^{-1} \circ \mathcal{F}_{1}^{-1}\left[\left(1+\xi_{1}^{2}\right)^{\alpha / 2} \mathcal{F}_{1} \circ \mathcal{F}_{2} f\left(\xi_{1}, \xi_{2}\right)\right]+\mathcal{F}_{2}^{-1} \circ \mathcal{F}_{1}^{-1}\left[\left(1+\xi_{2}^{2}\right)^{\alpha / 2} \mathcal{F}_{1} \circ \mathcal{F}_{2} f\left(\xi_{1}, \xi_{2}\right)\right] \\
& =\mathcal{F}_{1}^{-1}\left[\left(1+\xi_{1}^{2}\right)^{\alpha / 2} \mathcal{F}_{1} f\left(\xi_{1}, \xi_{2}\right)\right]+\mathcal{F}_{2}^{-1}\left[\left(1+\xi_{2}^{2}\right)^{\alpha / 2} \mathcal{F}_{2} f\left(\xi_{1}, \xi_{2}\right)\right] \\
& =g_{1}+g_{2} .
\end{aligned}
$$

With this in hand, we immediately obtain (2.1.48) and (2.1.49).

Before we present another characteriation of the Sobolev space $L_{1, p a r}^{p}\left(\mathbb{R}^{n} \times \mathbb{R}\right)$, we need a few preliminary results.

Lemma 2.1.39. ( [Ste1], Lemma 2, p. 133) Let $\alpha>0$. 
(i) There exists a finite measure $\mu_{\alpha}$ on $\mathbb{R}^{n}$ such that its Fourier transform $\widehat{\mu_{\alpha}}$ is given by

$$
\widehat{\mu_{\alpha}}(x)=\frac{(2 \pi|x|)^{\alpha}}{\left(1+4 \pi^{2}|x|^{2}\right)^{\alpha / 2}}
$$

(ii) There exist a pair of finite measures $\nu_{\alpha}$ and $\lambda_{\alpha}$ on $\mathbb{R}^{n}$ such that

$$
\left(1+4 \pi^{2}|x|^{2}\right)^{\alpha / 2}=\widehat{\nu_{\alpha}}(x)+(2 \pi|x|)^{\alpha} \widehat{\lambda_{\alpha}}(x) .
$$

Proposition 2.1.40. Let $f \in L^{p}\left(\mathbb{R}^{n}\right), 1<p<\infty, \alpha>0$ and $1 \leq i \leq n$. Then the following are equivalent:

(1) $g:=\mathcal{F}^{-1}\left[\left(1+\xi_{i}^{2}\right)^{\alpha / 2} \mathcal{F} f(\xi)\right] \in L^{p}\left(\mathbb{R}^{n}\right)$;

(2) $h:=\mathcal{F}^{-1}\left[\left|\xi_{i}\right|^{\alpha} \mathcal{F} f(\xi)\right] \in L^{p}\left(\mathbb{R}^{n}\right)$.

Moreover, there are natural accompanying estimates.

Proof. In order to show that (1) implies (2), we first rewrite $h$ in the following way.

$$
h=\mathcal{F}^{-1}\left[\frac{\left|\xi_{i}\right|^{\alpha}}{\left(1+\xi_{i}^{2}\right)^{\alpha / 2}} \mathcal{F} \mathcal{F}^{-1}\left(1+\xi_{i}^{2}\right)^{\alpha / 2} \mathcal{F} f(\xi)\right] .
$$

Then, by part (i) of Lemma 2.1.39,

$$
h=\mathcal{F}^{-1}[\mathcal{F} \mu \mathcal{F} g]
$$

where $\mu$ is a finite measure on $\mathbb{R}$ and $g=\mathcal{F}^{-1}\left[\left(1+\xi_{i}^{2}\right)^{\alpha / 2} \mathcal{F} f(\xi)\right] \in L^{p}\left(\mathbb{R}^{n}\right)$. Moreover, $h=\mathcal{F}^{-1}\left[\mathcal{F}\left(g *_{i} \mu\right)\right]=g *_{i} \mu$, the convolution acting in the $i$-th variable.

Next, with $x_{1}, \ldots, x_{i-1}, x_{i+1}, \ldots, x_{n} \in \mathbb{R}$ fixed, define the operator $T$ as

$$
T g^{i}:=g^{i} *_{i} \mu \quad \text { for } \quad g^{i}:=g\left(x_{1}, \ldots, x_{i-1}, \cdot, x_{i+1}, \ldots, x_{n}\right) \text {. }
$$


We will show that $T$ is a bounded operator on $L^{p}(\mathbb{R})$ for $1<p<\infty$. In ordere to do so, we first consider the case when $g^{i} \in L^{1}(\mathbb{R})$. Then

$$
\begin{aligned}
\left\|T g^{i}\right\|_{L^{1}(\mathbb{R})} & =\int_{\mathbb{R}}\left|\int_{\mathbb{R}} g^{i}(z-y) d \mu(y)\right| d z \\
& \leq \int_{\mathbb{R}} \int_{\mathbb{R}}\left|g^{i}(z-y)\right| d z d \mu(y) \\
& \leq\left\|g^{i}\right\|_{L^{1}(\mathbb{R})} \mu(\mathbb{R})<\infty
\end{aligned}
$$

hence $T$ is bounded on $L^{1}(\mathbb{R})$. Second, we let $g^{i} \in L^{\infty}(\mathbb{R})$. Then for almost every $z \in \mathbb{R}$

$$
\left|T g^{i}(z)\right| \leq \int_{\mathbb{R}}\left|g^{i}(z-y)\right| d \mu(y) \leq\left\|g^{i}\right\|_{L^{\infty}(\mathbb{R})} \mu(\mathbb{R})<\infty
$$

therefore $T$ is bounded on $L^{\infty}(\mathbb{R})$. Consequently, by real (or complex) interpolation, $T$ is bounded on $L^{p}(\mathbb{R})$ for $1<p<\infty$. Using this fact, after we integrate with respect to the variable $x_{i}$, we get that

$$
\begin{aligned}
\|h\|_{L^{p}\left(\mathbb{R}^{n}\right)}^{p} & =\int_{\mathbb{R}^{n}}\left|\int_{\mathbb{R}} g\left(x_{1}, \ldots, x_{i-1}, x_{i}-t, x_{i+1}, \ldots, x_{n}\right) d \mu(t)\right|^{p} d x \\
& =\int_{\mathbb{R}^{n-1}}\left\|T g^{i}\right\|_{L^{p}(\mathbb{R})}^{p} d x_{1} \ldots d x_{i-1} d x_{i+1} \ldots d x_{n} \\
& \leq c \int_{\mathbb{R}^{n-1}}\left\|g^{i}\right\|_{L^{p}(\mathbb{R})}^{p} d x_{1} \ldots d x_{i-1} d x_{i+1} \ldots d x_{n} \\
& \leq c\|g\|_{L^{p}\left(\mathbb{R}^{n}\right)}^{p}<\infty .
\end{aligned}
$$

Hence, $h \in L^{p}\left(\mathbb{R}^{n}\right)$ and the implication $(1) \Rightarrow(2)$ is proved.

Conversely, we assume (2) and we will show that (1) holds. By part (ii) of Lemma 2.1.39 we know that there exist $\mu_{1}, \mu_{2}$ finite measures on $\mathbb{R}$ such that

$$
\left(1+\xi_{i}^{2}\right)^{\alpha / 2}=\widehat{\mu_{1}}\left(\xi_{i}\right)+\left|\xi_{i}\right|^{\alpha} \widehat{\mu_{2}}\left(\xi_{i}\right)
$$


Then

$$
\begin{aligned}
g & =\mathcal{F}^{-1}\left(\mathcal{F} \mu_{1} \mathcal{F} f\right)+\mathcal{F}^{-1}\left(\mathcal{F} \mu_{2}\left|\xi_{i}\right|^{\alpha} \mathcal{F} f\right) \\
& =f *_{i} \mu_{1}+\mathcal{F}^{-1}\left(\mathcal{F} \mu_{2} \mathcal{F} h\right) \\
& =f *_{i} \mu_{1}+h *_{i} \mu_{2},
\end{aligned}
$$

where the convolution $*_{i}$ acts in the $i$-th variable. By hypothesis, $f, h \in L^{p}\left(\mathbb{R}^{n}\right)$, and we have seen that the operator of convolution with a finite measure is bounded on $L^{p}$ for $1<p<\infty$. Now reasoning as we did in the first part of the proof, we may conclude that $g \in L^{p}\left(\mathbb{R}^{n}\right)$. This finishes the proof of the proposition.

Now we are ready to give the characterization - via derivatives - of the Sobolev space $L_{1, p a r}^{p}\left(\mathbb{R}^{n} \times \mathbb{R}\right)$.

Theorem 2.1.41. For any $1<p<\infty$ we have

$$
L_{1, p a r}^{p}\left(\mathbb{R}^{n} \times \mathbb{R}\right)=\left\{f \in L^{p}\left(\mathbb{R}^{n} \times \mathbb{R}\right): \partial_{x_{i}} f, \partial_{\text {time }}^{1 / 2} f \in L^{p}\left(\mathbb{R}^{n} \times \mathbb{R}\right), 1 \leq i \leq n\right\}
$$

Proof. For the left-to-right inclusion in (2.1.50) we observe that, by Proposition 2.1.33,

$$
f \in L^{p}\left(\mathbb{R} ; L_{1}^{p}\left(\mathbb{R}^{n}\right)\right)
$$

and

$$
f \in L^{p}\left(\mathbb{R}^{n} ; L_{1 / 2}^{p}(\mathbb{R})\right)
$$

Now (2.1.51) implies that $f, \nabla f \in L^{p}\left(\mathbb{R}^{n} \times \mathbb{R}\right)$, while from (2.1.52) we obtain that

$$
\int_{\mathbb{R}^{n}}\|f(x, \cdot)\|_{L_{1 / 2}^{p}(\mathbb{R})}^{p} d x<\infty
$$

Rewriting the left-hand side of the above inequality, using the characterization of the space $L_{1 / 2}^{p}(\mathbb{R})$ given in $(1.2)$ on p. 168 of $[\mathrm{GrKo}]$, we get that

$$
\int_{\mathbb{R}^{n}} \int_{\mathbb{R}}\left|\left\{\mathcal{F}_{\text {time }}^{-1}\left[\left(1+\tau^{2}\right)^{1 / 4}\left(\mathcal{F}_{\text {time }} f(\xi, \cdot)\right)(\tau)\right](x, \cdot)\right\}(t)\right|^{p} d t d x<\infty
$$


or, equivalently, that

$$
\mathcal{F}_{\text {time }}^{-1}\left[\left(1+\tau^{2}\right)^{1 / 4}\left(\mathcal{F}_{\text {time }} f(\xi, \cdot)\right)(\tau)\right] \in L^{p}\left(\mathbb{R}^{n} \times \mathbb{R}\right)
$$

This, in turn, by Definition 1.2.15, amounts to $\partial_{\text {time }}^{1 / 2} f \in L^{p}\left(\mathbb{R}^{n} \times \mathbb{R}\right)$, finishing the proof of the left-to-right inclusion in (2.1.50).

Conversely, assume that $f, \nabla f, \partial_{\text {time }}^{1 / 2} f \in L^{p}\left(\mathbb{R}^{n} \times \mathbb{R}\right)$. Our goal is to prove that $f \in L_{1, \text { par }}^{p}\left(\mathbb{R}^{n} \times \mathbb{R}\right)$. According to Proposition 2.1.38, it is enough to show that

$$
\mathcal{F}^{-1}\left[\left(1+\tau^{2}\right)^{1 / 4} \mathcal{F} f(\xi, \tau)\right] \in L^{p}\left(\mathbb{R}^{n} \times \mathbb{R}\right)
$$

and

$$
\mathcal{F}^{-1}\left[\left(1+\xi_{i}^{2}\right)^{1 / 2} \mathcal{F} f(\xi, \tau)\right] \in L^{p}\left(\mathbb{R}^{n} \times \mathbb{R}\right), \quad \text { for all } \quad i=1, \ldots, n
$$

By Proposition 1.2.16 we have $\partial_{\text {time }}^{1 / 2}=\partial_{t}^{1 / 2}$. Definition 1.2 .15 and the assumption that $\partial_{\text {time }}^{1 / 2} f \in L^{p}\left(\mathbb{R}^{n} \times \mathbb{R}\right)$ readily yield (2.1.53). To prove (2.1.54), based on Proposition 2.1.40, it is enough to show that

$$
\mathcal{F}^{-1}\left(\left|\xi_{i}\right| \mathcal{F} f(\xi, \tau)\right) \in L^{p}\left(\mathbb{R}^{n} \times \mathbb{R}\right), \quad \text { for all } \quad i=1, \ldots, n
$$

Observe that $\mathcal{F}^{-1}\left(\left|\xi_{i}\right| \mathcal{F} f(\xi, \tau)\right)$ can be expressed as

$$
\begin{aligned}
\mathcal{F}_{\text {space }}^{-1} \circ \mathcal{F}_{\text {time }}^{-1}\left(\left|\xi_{i}\right|\left(\mathcal{F}_{\text {time }} \circ \mathcal{F}_{\text {space }} f\right)(\xi, \tau)\right) & =\mathcal{F}_{i}^{-1}\left(\left|\xi_{i}\right|\left(\mathcal{F}_{i} f\right)(\xi, \tau)\right) \\
& =\mathcal{F}_{i}^{-1}\left[\left(\operatorname{sign}\left(\xi_{i}\right)\right) \xi_{i}\left(\mathcal{F}_{i} f\right)(\xi, \tau)\right] \\
& =c \mathcal{F}_{i}^{-1}\left[\left(\operatorname{sign}\left(\xi_{i}\right)\right) \mathcal{F}_{i}\left(\partial_{x_{i}} f\right)(\xi, \tau)\right] \\
& =c H\left(\partial_{x_{i}} f\right),
\end{aligned}
$$


where $\mathcal{F}_{\text {space }}$ and $\mathcal{F}_{\text {space }}^{-1}$ are the Fourier transform and the invers Fourier transform, respectively, in the spacial variable in $\mathbb{R}^{n}, c \in \mathbb{R}$ is a suitable constant, and $H$ stands for the Hilbert transform with respect to the $i$-th spacial variable. Since $\nabla f \in L^{p}\left(\mathbb{R}^{n} \times \mathbb{R}\right)$ and $H$ is bounded on $L^{p}(\mathbb{R})$ for $1<p<\infty$, we may conclude that (2.1.55) holds, and the proof of the theorem is complete.

In the last part of this subsection we record some mapping properties of the operator $\partial_{\text {time }}^{\theta / 2}$ defined in (1.2.31).

Theorem 2.1.42. For any $\alpha, \theta \in \mathbb{R}$ and $1<p<\infty$ the operators

$$
\partial_{\text {time }}^{\theta / 2}: L_{\alpha, p a r}^{p}\left(\mathbb{R}^{n} \times \mathbb{R}\right) \longrightarrow L_{\alpha-\theta, p a r}^{p}\left(\mathbb{R}^{n} \times \mathbb{R}\right)
$$

and

$$
\partial_{\text {time }}^{\theta / 2}: B_{\alpha, p a r}^{p}\left(\mathbb{R}^{n} \times \mathbb{R}\right) \longrightarrow B_{\alpha-\theta, p a r}^{p}\left(\mathbb{R}^{n} \times \mathbb{R}\right)
$$

are bounded.

Proof. First we will show that

$$
\Theta_{\alpha-\theta} \partial_{\text {time }}^{\theta / 2} \Theta_{-\alpha}: L^{p}\left(\mathbb{R}^{n} \times \mathbb{R}\right) \longrightarrow L^{p}\left(\mathbb{R}^{n} \times \mathbb{R}\right)
$$

is bounded, where $\Theta_{\alpha}$ is given in (2.1.37) and $\alpha, \theta, p$ are as in the theorem. Consider $f$ from $L^{p}\left(\mathbb{R}^{n} \times \mathbb{R}\right)$. Then, combining the definitions of $\Theta_{\alpha}$ and $\partial_{\text {time }}^{\theta}, \partial_{t}^{\theta}$ (see Definition 1.2.15), using also the equivalence between the operators $\partial_{\text {time }}^{\theta}$ and $\partial_{t}^{\theta}(\mathrm{cf}$. Proposition 1.2.16), we obtain that

$$
\Theta_{\alpha-\theta} \partial_{\text {time }}^{\theta / 2} \Theta_{-\alpha} f=\mathcal{F}^{-1}[m(\xi, \tau) \mathcal{F} f(\xi, \tau)]
$$


with

$$
m(\xi, \tau):=\frac{\left(1+\tau^{2}\right)^{\theta / 4}}{\left(1+|\xi|^{4}+\tau^{2}\right)^{\theta / 4}} .
$$

In order to show that $\Theta_{\alpha-\theta} \partial_{\text {time }}^{\theta / 2} \Theta_{-\alpha} f \in L^{p}\left(\mathbb{R}^{n} \times \mathbb{R}\right)$, it is enough to prove that $m$ is a multiplier for $L^{p}\left(\mathbb{R}^{n} \times \mathbb{R}\right)$. This, in turn, was proved in Example 5 in 1.2.6., therefore we can conclude that (2.1.58) holds for the appropriate range of parameters.

Going further we note that, based on Lemma 2.1.31, the mapping property (2.1.58) is equivalent with the following:

$$
\partial_{\text {time }}^{\theta / 2}: L_{\alpha, p a r}^{p}\left(\mathbb{R}^{n} \times \mathbb{R}\right) \longrightarrow L_{\alpha-\theta, p a r}^{p}\left(\mathbb{R}^{n} \times \mathbb{R}\right)
$$

is bounded for $\alpha, \theta \in \mathbb{R}$ and $1<p<\infty$. This proves (2.1.56).

In order to show (2.1.57) we pick $\alpha_{0}, \alpha_{1} \in \mathbb{R}$ such that $\alpha_{0} \neq \alpha_{1}$. Then real interpolation of parabolic Sobolev spaces (cf. part (i) of Theorem 2.1.60) yields

$$
\left(L_{\alpha_{0}, p a r}^{p}\left(\mathbb{R}^{n} \times \mathbb{R}\right), L_{\alpha_{1}, p a r}^{p}\left(\mathbb{R}^{n} \times \mathbb{R}\right)\right)_{\eta, p}=B_{\alpha, p a r}^{p}\left(\mathbb{R}^{n} \times \mathbb{R}\right)
$$

where $0<\eta<1$ and $\alpha=(1-\eta) \alpha_{0}+\eta \alpha_{1}$. With this in hand we can conclude that

$$
\partial_{\text {time }}^{\theta / 2}: B_{\alpha, p a r}^{p}\left(\mathbb{R}^{n} \times \mathbb{R}\right) \longrightarrow B_{\alpha-\theta, p a r}^{p}\left(\mathbb{R}^{n} \times \mathbb{R}\right)
$$

is bounded for any $\alpha, \theta \in \mathbb{R}$ and $1<p<\infty$, finishing the proof of the theorem.

\subsubsection{Hardy spaces}

We shall now specialize the Triebel-Lizorkin scale to yet another distinguished subclass, that of Hardy spaces. 
Definition 2.1.43. The inhomogeneous and homogeneous parabolic Hardy scales are defined as

$$
H_{\text {par }}^{p}\left(\mathbb{R}^{n} \times \mathbb{R}\right):=F_{0, \text { par }}^{p, 2}\left(\mathbb{R}^{n} \times \mathbb{R}\right) \quad \text { and } \quad \dot{H}_{\text {par }}^{p}\left(\mathbb{R}^{n} \times \mathbb{R}\right):=\dot{F}_{0, \text { par }}^{p, 2}\left(\mathbb{R}^{n} \times \mathbb{R}\right)
$$

respectively, for $0<p \leq 1$.

We first present the atomic characterizations of these spaces, and we continue to employ the parabolic cubes discussed in Remark 2.1.23. We begin with the description of two types of atoms.

Definition 2.1.44. Let $Q$ be a parabolic cube in $\mathbb{R}^{n} \times \mathbb{R}$ with sidelength $r$, and let $\frac{n+2}{n+3}<p \leq 1$. Then $a_{Q}$ is a p-atom if

$$
\begin{aligned}
& \text { supp } a_{Q} \subset Q, \\
& \left\|a_{Q}\right\|_{L^{\infty}\left(\mathbb{R}^{n} \times \mathbb{R}\right)} \leq r^{-\frac{n+2}{p}}, \\
& \int_{\mathbb{R}^{n} \times \mathbb{R}} a_{Q}(x, t) d x d t=0 .
\end{aligned}
$$

Definition 2.1.45. Let $Q$ be a parabolic cube in $\mathbb{R}^{n} \times \mathbb{R}$ with sidelength $r$, and let $\frac{n+2}{n+3}<p \leq 1,1 \leq q \leq \infty$ such that $p<q$. Then $a_{Q}$ is a $(p, q)$-atom if

$$
\begin{aligned}
& \operatorname{supp}_{Q} \subset Q, \\
& \left\|a_{Q}\right\|_{L^{q}\left(\mathbb{R}^{n} \times \mathbb{R}\right)} \leq r^{(n+2)\left(\frac{1}{q}-\frac{1}{p}\right)}, \\
& \int_{\mathbb{R}^{n} \times \mathbb{R}} a_{Q}(x, t) d x d t=0 .
\end{aligned}
$$

The atomic decomposition of the homogeneous Hardy space $\dot{H}_{p a r}^{p}\left(\mathbb{R}^{n} \times \mathbb{R}\right)$, introduced in Definition 2.1.43, is given in the following theorem. 
Theorem 2.1.46. (Theorem 1 of [LaUc], or Theorem 6.5 and Definition 4.3 from Chapter 1 of $[$ Bow1])

Let $\frac{n+2}{n+3}<p \leq 1$ and $1 \leq q \leq \infty$ such that $p<q$. Then $f \in \dot{H}_{\text {par }}^{p}\left(\mathbb{R}^{n} \times \mathbb{R}\right)$ if and only if $f$ admits the representation $f=\sum_{Q} s_{Q} a_{Q}$, where $\left\{s_{Q}\right\}_{Q} \in \ell^{p}$ and $a_{Q}$ are p-atoms (or $(p, q)$-atoms). Moreover, if $f \in \dot{H}_{\text {par }}^{p}\left(\mathbb{R}^{n} \times \mathbb{R}\right)$, then

$$
\|f\|_{\dot{H}_{p a r}^{p}\left(\mathbb{R}^{n} \times \mathbb{R}\right)} \approx \inf \left(\sum_{Q}\left|s_{Q}\right|^{p}\right)^{1 / p},
$$

where the infimum is taken over all atomic decompositions of $f$.

In what follows we focus on the inhomogeneous atoms and local Hardy spaces.

Definition 2.1.47. Consider a parabolic cube $Q$ in $\mathbb{R}^{n} \times \mathbb{R}$ with sidelength $r$, and let $\frac{n+2}{n+3}<p \leq 1$. Then a bounded measurable function $a_{Q}$ is called an inhomogeneous (local) p-atom if

$$
\operatorname{supp}_{Q} \subset Q, \quad\left\|a_{Q}\right\|_{L^{\infty}\left(\mathbb{R}^{n} \times \mathbb{R}\right)} \leq r^{-\frac{n+2}{p}},
$$

and either

$$
|Q|<1 \quad \text { and } \quad \int_{\mathbb{R}^{n} \times \mathbb{R}} a_{Q}(x, t) d x d t=0
$$

or

$$
|Q|=1
$$

In analogy with the elliptic case described in Lemma 5 of [Gol] (see also Theorem 2.3 in $[\mathrm{CKS}])$, we obtain the atomic decomposition of the parabolic inhomogeneous Hardy space $H_{p a r}^{p}\left(\mathbb{R}^{n} \times \mathbb{R}\right)$, defined in (2.1.60). 
Theorem 2.1.48. Suppose $\frac{n+2}{n+3}<p \leq 1$. Then $f \in H_{\text {par }}^{p}\left(\mathbb{R}^{n} \times \mathbb{R}\right)$ if and only if $f$ admits the representation $f=\sum_{Q} s_{Q} a_{Q}$, where $\left\{s_{Q}\right\}_{Q} \in \ell^{p}$ and $a_{Q}$ are local (inhomogeneous) p-atoms. Moreover, if $f \in H_{\text {par }}^{p}\left(\mathbb{R}^{n} \times \mathbb{R}\right)$, then

$$
\|f\|_{H_{p a r}^{p}\left(\mathbb{R}^{n} \times \mathbb{R}\right)} \approx \inf \left(\sum_{|Q| \leq 1}\left|s_{Q}\right|^{p}\right)^{1 / p},
$$

where the infimum is taken over all representations of $f$.

Remark 2.1.49. Adapting Goldberg's original description of an inhomogeneous (local) p-atom (for the elliptic case, see pp.36-37 of [Gol]) to the parabolic setting, we obtain that a bounded measurable function $a_{Q}$ is called an inhomogeneous (local) $p$-atom if

$$
\operatorname{supp} a_{Q} \subset Q, \quad\left\|a_{Q}\right\|_{L^{\infty}\left(\mathbb{R}^{n} \times \mathbb{R}\right)} \leq r^{-\frac{n+2}{p}}, \quad|Q|=r^{n+2},
$$

and either

$$
|Q|<1 \quad \text { and } \quad \int_{\mathbb{R}^{n} \times \mathbb{R}} a_{Q}(x, t) d x d t=0
$$

or

$$
|Q| \geq 1
$$

In order to show that the $\ell^{p}$-span of these atoms yields the same parabolic Hardy space $H_{p a r}^{p}\left(\mathbb{R}^{n} \times \mathbb{R}\right)$ as the $\ell^{p}$-span of the atoms described in Definition 2.1.47, the only case we have to analyze is when $|Q|>1$.

Since $|Q|=r^{n+2}$, we know that $2^{N-1}<r \leq 2^{N}$ for some $N \in \mathbb{N}$. Take a cube with sidelength $2^{N}$ which contains $Q$, and divide it into $2^{N(n+2)}$ cubes $Q_{i}$. Then each $Q_{i}$ will have sidelength $r_{i}=1$ and $\left|Q_{i}\right|=1$. Also,

$$
a_{Q}=\sum_{i=1}^{2^{N(n+2)}} a_{Q} \chi_{Q_{i}}=\sum_{i=1}^{2^{N(n+2)}} a_{Q} \chi_{Q_{i}} r^{\frac{n+2}{p}} r^{-\frac{n+2}{p}}=\sum_{i=1}^{2^{N(n+2)}} b_{Q_{i}} \beta_{Q_{i}},
$$


where $b_{Q_{i}}:=a_{Q} \chi_{Q_{i}} r^{\frac{n+2}{p}}$ and $\beta_{Q_{i}}:=r^{-\frac{n+2}{p}}$. Then

$$
\operatorname{supp} b_{Q_{i}} \subset Q_{i}, \quad\left|Q_{i}\right|=1, \quad\left\|b_{Q_{i}}\right\|_{L^{\infty}\left(\mathbb{R}^{n} \times \mathbb{R}\right)} \leq 1=r_{i}^{-\frac{n+2}{p}}
$$

hence $b_{Q_{i}}$ are inhomogeneous (local) p-atoms in the sense of Definition 2.1.47. As far as the coefficients are concerned, we observe that for $f \in H_{p a r}^{p}\left(\mathbb{R}^{n} \times \mathbb{R}\right)$,

$$
f=\sum_{Q} a_{Q} s_{Q}=\sum_{|Q| \leq 1} a_{Q} s_{Q}+\sum_{|Q|>1} a_{Q} s_{Q}=\sum_{|Q| \leq 1} a_{Q} s_{Q}+\sum_{|Q|>1}\left(\sum_{i=1}^{2^{N(n+2)}} b_{Q_{i}} \beta_{Q_{i}}\right) s_{Q}
$$

and that

$$
\sum_{|Q|>1}\left(\sum_{i=1}^{2^{N(n+2)}}\left|\beta_{Q_{i}}\right|^{p}\right)\left|s_{Q}\right|^{p}<\sum_{|Q|>1} 2^{n+2}\left|s_{Q}\right|^{p}<\infty
$$

as desired.

Remark 2.1.50. Similar to the elliptic case considered by D.-C. Chang, S. Krantz and E. Stein (see Definition 2.2. in $[\mathrm{CKS}]$ ), a bounded measurable function $a_{Q}$ is called an inhomogeneous (local) $p$-atom if

$$
\operatorname{supp} a_{Q} \subset Q, \quad\left\|a_{Q}\right\|_{L^{\infty}\left(\mathbb{R}^{n} \times \mathbb{R}\right)} \leq r^{-\frac{n+2}{p}}, \quad|Q|=r^{n+2}
$$

and either

$$
|Q| \leq 1 \quad \text { and } \quad \int_{\mathbb{R}^{n} \times \mathbb{R}} a_{Q}(x, t) d x d t=0
$$

or

$$
|Q|>1
$$

In order to show that the $\ell^{p}$-span of these atoms yields the same parabolic Hardy space $H_{p a r}^{p}\left(\mathbb{R}^{n} \times \mathbb{R}\right)$ as the $\ell^{p}$-span of the atoms described in Remark 2.1.49 (hence the $\ell^{p}$-span of the atoms described in Definition 2.1.47), we need to show that Goldberg's 
description implies the characterization given by D.-C. Chang, S. Krantz and E. Stein. Assume $|Q|=1$ (the proof in the other cases is immediate). We will show that in this case $a_{Q}$ is a fixed constant multiple of an inhomogeneous (local) p-atom in the sense of Goldberg's description (see Remark 2.1.49). Let us consider a cube $Q^{\prime}$ with sidelength $l\left(Q^{\prime}\right)=r^{\prime}=2$ which contains $Q$. Then

$$
\left\|a_{Q}\right\|_{L^{\infty}\left(\mathbb{R}^{n} \times \mathbb{R}\right)} \leq 1=c_{n, p} 2^{-\frac{n+2}{p}}
$$

or, equivalently,

$$
\left\|\tilde{a}_{Q}\right\|_{L^{\infty}\left(\mathbb{R}^{n} \times \mathbb{R}\right)} \leq\left(r^{\prime}\right)^{-\frac{n+2}{p}}
$$

where $\tilde{a}_{Q}:=\frac{a_{Q}}{c_{n, p}}$. Also, the support of $\tilde{a}_{Q}$ is included in $Q^{\prime}$ and $\left|Q^{\prime}\right| \geq 1$, therefore $a_{Q}$ is a fixed constant multiple of an atom described in Remark 2.1.49.

The second part of our discussion deals with the homogeneous Hardy scale $\dot{H}_{\text {par }}^{1, p}\left(\mathbb{R}^{n} \times\right.$ $\mathbb{R})$.

Definition 2.1.51. In the spirit of p. 7 in [Bro2], we say that, for $\frac{n+2}{n+3}<p \leq 1, f$ belongs to the space $\dot{H}_{\text {par }}^{1, p}\left(\mathbb{R}^{n} \times \mathbb{R}\right)$ if and only if

$$
\mathcal{F}^{-1}\left[\sqrt{|\xi|^{2}+i \tau} \mathcal{F} f(\xi, \tau)\right] \in \dot{H}_{\text {par }}^{p}\left(\mathbb{R}^{n} \times \mathbb{R}\right)
$$

where $\xi \in \mathbb{R}^{n}$ and $\tau \in \mathbb{R}$.

It is important to note the following equivalence between $\dot{H}_{\text {par }}^{1, p}\left(\mathbb{R}^{n} \times \mathbb{R}\right)$ and the Triebel-Lizorkin space $\dot{F}_{1, p a r}^{p, 2}\left(\mathbb{R}^{n} \times \mathbb{R}\right)$.

Proposition 2.1.52. For $\frac{n+2}{n+3}<p \leq 1$,

$$
\dot{H}_{\text {par }}^{1, p}\left(\mathbb{R}^{n} \times \mathbb{R}\right) \equiv \dot{F}_{1, \text { par }}^{p, 2}\left(\mathbb{R}^{n} \times \mathbb{R}\right)
$$


with equivalent norms.

Proof. By Definitions 2.1.51 and 2.1.43

$$
f \in \dot{H}_{\text {par }}^{1, p}\left(\mathbb{R}^{n} \times \mathbb{R}\right) \Longleftrightarrow \dot{L} f \in \dot{H}_{\text {par }}^{p}\left(\mathbb{R}^{n} \times \mathbb{R}\right) \equiv \dot{F}_{0, p a r}^{p, 2}\left(\mathbb{R}^{n} \times \mathbb{R}\right),
$$

where the operator $\dot{L}$ was defined in Theorem 2.1.13, i.e.

$$
\dot{L} f=\mathcal{F}^{-1}\left[\sqrt{|\xi|^{2}+i \tau} \mathcal{F} f(\xi, \tau)\right] \quad \text { for } \quad(\xi, \tau) \in \mathbb{R}^{n} \times \mathbb{R} .
$$

From the same theorem we obtain that

$$
\dot{L} f \in \dot{F}_{0, \text { par }}^{p, 2}\left(\mathbb{R}^{n} \times \mathbb{R}\right) \Longleftrightarrow f \in \dot{F}_{1, \text { par }}^{p, 2}\left(\mathbb{R}^{n} \times \mathbb{R}\right)
$$

Consequently, (2.1.62) and (2.1.63) yield the desired result.

Since Proposition 2.1.52 is particulary important to us, for the reader's convenience we include yet another proof of this result.

Alternative proof of Proposition 2.1.52. According to Theorem 2.1.14, for $0<p \leq 1$,

$$
f \in \dot{F}_{1, p a r}^{p, 2}\left(\mathbb{R}^{n} \times \mathbb{R}\right) \Longleftrightarrow \mathcal{F}^{-1}\left[\left(|\xi|^{2}+|\tau|\right)^{1 / 2} \mathcal{F} f(\xi, \tau)\right] \in \dot{H}_{\text {par }}^{p}\left(\mathbb{R}^{n} \times \mathbb{R}\right)
$$

In view of (2.1.64), Definitions 2.1.51 and 2.1.43, in order to show the left-to-right inclusion in (2.1.61) it is enough to prove that

$$
m(\xi, \tau):=\frac{\left(|\xi|^{2}+|\tau|\right)^{1 / 2}}{\sqrt{|\xi|^{2}+i|\tau|}}, \quad \xi \in \mathbb{R}^{n}, \tau \in \mathbb{R}
$$

is a multiplier for $\dot{H}_{p a r}^{p}\left(\mathbb{R}^{n} \times \mathbb{R}\right)$. According to $[\mathrm{StTr}](4.2)$ (see also [Stö1], Theorem 2.1), we need to show that for every multiindex $\gamma=\left(\gamma_{1}, \ldots, \gamma_{n}\right), \gamma_{j} \in \mathbb{N}_{0}$ and every $k \in \mathbb{N}_{0}$, there exists a constant $c_{\gamma, k}$ such that

$$
\left|\partial_{\xi}^{\gamma} \partial_{\tau}^{k} m(\xi, \tau)\right| \leq \frac{c_{\gamma, k}}{\left(|\xi|+|\tau|^{1 / 2}\right)^{|\gamma|+2 k}} .
$$


Case 1. $|\gamma|=0, k=0$. Using the fact that $|\sqrt{z}|=\sqrt{|z|}$ for every $z \in \mathbb{C}$, we obtain that

$$
|m(\xi, \tau)| \leq \frac{\left(|\xi|^{2}+|\tau|\right)^{1 / 2}}{\left(|\xi|^{4}+|\tau|^{2}\right)^{1 / 4}} \leq c .
$$

Case 2. $|\gamma|=0, k=1$. Then

$$
\left|\partial_{\tau} m(\xi, \tau)\right| \leq c|m(\xi, \tau)|^{-1} \frac{\left(|\xi|^{4}+|\tau|^{2}\right)^{1 / 2}}{|\xi|^{4}+|\tau|^{2}} \leq \frac{c}{\left(|\xi|+|\tau|^{1 / 2}\right)^{2}}
$$

Case 3. $\gamma_{j}=1, \gamma_{1}=\ldots=\gamma_{j-1}=\gamma_{j+1}=\ldots=\gamma_{n}=0, k=0$. First note that

$$
\partial_{\xi_{j}} m(\xi, \tau)=\left(\frac{|\xi|^{2}+|\tau|}{|\xi|^{2}+i|\tau|}\right)^{-1 / 2} \frac{\xi_{j}|\tau|(i-1)}{\left(|\xi|^{2}+i|\tau|\right)^{2}}
$$

Therefore,

$$
\left|\partial_{\xi_{j}} m(\xi, \tau)\right| \leq c \frac{|\xi||\tau|}{\left(|\xi|^{2}+|\tau|\right)^{2}} \leq \frac{c}{|\xi|+|\tau|^{1 / 2}}
$$

Case 4. $\gamma_{j}=2, \gamma_{1}=\ldots=\gamma_{j-1}=\gamma_{j+1}=\ldots=\gamma_{n}=0, k=0$. Using the previous case as a starting point, we get that

$$
\begin{aligned}
\left|\partial_{\xi_{j}}^{2} m(\xi, \tau)\right| & \leq c|m(\xi, \tau)|^{-3} \frac{|\xi|^{2}|\tau|^{2}}{\left(|\xi|^{2}+|\tau|\right)^{4}}+c|m(\xi, \tau)|^{-1} \frac{|\tau|\left(|\xi|^{2}+|\tau|\right)+|\xi|^{2}|\tau|}{\left(|\xi|^{2}+|\tau|\right)^{3}} \\
& \leq \frac{c}{\left(|\xi|+|\tau|^{1 / 2}\right)^{2}}
\end{aligned}
$$

Case 5. $\gamma_{j}=1, \gamma_{1}=\ldots=\gamma_{j-1}=\gamma_{j+1}=\ldots=\gamma_{n}=0, k=1$. Along the same lines as before,

$$
\begin{aligned}
\left|\partial_{\tau} \partial_{\xi_{j}} m(\xi, \tau)\right| & \leq c|m(\xi, \tau)|^{-3} \frac{\left|\xi_{j}\right||\tau|}{\left(|\xi|^{2}+|\tau|\right)^{3}}+c|m(\xi, \tau)|^{-1} \frac{\left|\xi_{j}\right|}{\left(|\xi|^{2}+|\tau|\right)^{2}} \\
& \leq \frac{c}{\left(|\xi|+|\tau|^{1 / 2}\right)^{3}} .
\end{aligned}
$$

All the other cases are treated in a similar fashion. This proves the left-to-right inclusion in (2.1.61). 
For the opposite one (based on a similar argument as before) it is enough to show that

$$
q(\xi, \tau):=\frac{\sqrt{|\xi|^{2}+i|\tau|}}{\left(|\xi|^{2}+|\tau|\right)^{1 / 2}}, \quad \xi \in \mathbb{R}^{n}, \tau \in \mathbb{R},
$$

is a multiplier for $\dot{H}_{p a r}^{p}\left(\mathbb{R}^{n} \times \mathbb{R}\right)$. According to [StTr] (4.2) (see also [Stö1], Theorem 2.1), we need to show that for every multiindex $\gamma=\left(\gamma_{1}, \ldots, \gamma_{n}\right), \gamma_{j} \in \mathbb{N}_{0}$ and every $k \in \mathbb{N}_{0}$, there exists a constant $c_{\gamma, k}$ such that

$$
\left|\partial_{\xi}^{\gamma} \partial_{\tau}^{k} q(\xi, \tau)\right| \leq \frac{c_{\gamma, k}}{\left(|\xi|+|\tau|^{1 / 2}\right)^{|\gamma|+2 k}} .
$$

Case 1. $|\gamma|=0, k=0$. Using the fact that $|\sqrt{z}|=\sqrt{|z|}$ for every $z \in \mathbb{C}$, we obtain that

$$
|q(\xi, \tau)| \leq \frac{\left(|\xi|^{4}+|\tau|^{2}\right)^{1 / 4}}{\left(|\xi|^{2}+|\tau|\right)^{1 / 2}} \leq c .
$$

Case 2. $|\gamma|=0, k=1$. Then

$$
\left|\partial_{\tau} q(\xi, \tau)\right| \leq c|q(\xi, \tau)|^{-1} \frac{1}{|\xi|^{2}+|\tau|} \leq \frac{c}{\left(|\xi|+|\tau|^{1 / 2}\right)^{2}} .
$$

Case 3. $\gamma_{j}=1, \gamma_{1}=\ldots=\gamma_{j-1}=\gamma_{j+1}=\ldots=\gamma_{n}=0, k=0$. First observe that

$$
\partial_{\xi_{j}} q(\xi, \tau)=\left(\frac{|\xi|^{2}+i|\tau|}{|\xi|^{2}+|\tau|}\right)^{-1 / 2} \frac{\xi_{j}|\tau|(1-i)}{\left(|\xi|^{2}+|\tau|\right)^{2}} .
$$

Then,

$$
\left|\partial_{\xi_{j}} q(\xi, \tau)\right| \leq c \frac{|\xi||\tau|}{\left(|\xi|^{2}+|\tau|\right)^{2}} \leq \frac{c}{|\xi|+|\tau|^{1 / 2}} .
$$

Case 4. $\gamma_{j}=2, \gamma_{1}=\ldots=\gamma_{j-1}=\gamma_{j+1}=\ldots=\gamma_{n}=0, k=0$. Using the previous case we get that

$$
\begin{aligned}
\left|\partial_{\xi_{j}}^{2} q(\xi, \tau)\right| & \leq c|q(\xi, \tau)|^{-3} \frac{|\xi|^{2}|\tau|^{2}}{\left(|\xi|^{2}+|\tau|\right)^{4}}+c|q(\xi, \tau)|^{-1} \frac{|\tau|\left(|\xi|^{2}+|\tau|\right)+|\xi|^{2}|\tau|}{\left(|\xi|^{2}+|\tau|\right)^{3}} \\
& \leq \frac{c}{\left(|\xi|+|\tau|^{1 / 2}\right)^{2}} .
\end{aligned}
$$


Case 5. $\gamma_{j}=1, \gamma_{1}=\ldots=\gamma_{j-1}=\gamma_{j+1}=\ldots=\gamma_{n}=0, k=1$. Similar to the previous cases,

$$
\begin{aligned}
\left|\partial_{\tau} \partial_{\xi_{j}} q(\xi, \tau)\right| & \leq c|q(\xi, \tau)|^{-3} \frac{|\xi||\tau|}{\left(|\xi|^{2}+|\tau|\right)^{3}}+c|q(\xi, \tau)|^{-1} \frac{|\xi|}{\left(|\xi|^{2}+|\tau|\right)^{2}} \\
& \leq \frac{c}{\left(|\xi|+|\tau|^{1 / 2}\right)^{3}} .
\end{aligned}
$$

All the other cases are treated in a similar way. This proves the right-to-left inclusion in (2.1.61), completing the alternative proof of Proposition 2.1.52.

In the last part of this subsection we show the equivalence between the TriebelLizorkin scale $F_{1, \text { par }}^{p, 2}\left(\mathbb{R}^{n} \times \mathbb{R}\right)$ and the space $H_{\text {par }}^{1, p}\left(\mathbb{R}^{n} \times \mathbb{R}\right)$. We start with the definition of the latter, in which the parabolic cubes $Q$ retain the same signficance as in Remark 2.1.23.

Definition 2.1.53. For $\frac{n+2}{n+3}<p \leq 1$ we define the space $H_{\text {par }}^{1, p}\left(\mathbb{R}^{n} \times \mathbb{R}\right)$ as the $\ell^{p}$-span of local (inhomogeneous) $(1, p)$-atoms, where $a_{Q}$ is called a local $(1, p)$-atom if

$$
\begin{aligned}
& \text { supp } a_{Q} \subset Q, \quad|Q|=r^{n+2} \leq 1, \\
& \left\|a_{Q}\right\|_{\dot{L}_{1, p a r}^{2}\left(\mathbb{R}^{n} \times \mathbb{R}\right)} \leq r^{(n+2)(1 / 2-1 / p)} .
\end{aligned}
$$

The associated norm is given by

$$
\begin{aligned}
&\|f\|_{H_{p a r}^{1, p}\left(\mathbb{R}^{n} \times \mathbb{R}\right)}:=\inf \left\{\left(\sum_{Q}\left|s_{Q}\right|^{p}\right)^{1 / p}:\right. \\
&\left.\quad f=\sum_{Q} s_{Q} a_{Q},\left\{s_{Q}\right\}_{Q} \in \ell^{p}, a_{Q} \text { local }(1, p) \text {-atoms }\right\} .
\end{aligned}
$$

Remark 2.1.54. Recall the spaces $\mathcal{L}_{1}^{q}\left(\mathbb{R}^{n} \times \mathbb{R}\right), 1<q<\infty$, from Definition 2.1.36 and the result from Lemma 2.1.37 to the effect that the spaces $\mathcal{L}_{1}^{q}\left(\mathbb{R}^{n} \times \mathbb{R}\right)$ and $\dot{L}_{1, p a r}^{q}\left(\mathbb{R}^{n} \times \mathbb{R}\right)$ coincide for each $1<q<\infty$. Using this with $q=2$, we observe that the space $H_{\text {par }}^{1,1}\left(\mathbb{R}^{n} \times \mathbb{R}\right)$ is denoted by $\mathcal{L}_{1}^{1}\left(\mathbb{R}^{n} \times \mathbb{R}\right)$ on p. 7 in $[$ Bro2]. 
Remark 2.1.55. A closer inspection of a local $(1, p)$-atom $a_{Q}$ reveals that

$$
\left\|a_{Q}\right\|_{L_{1, p a r}^{2}\left(\mathbb{R}^{n} \times \mathbb{R}\right)} \leq r^{(n+2)(1 / 2-1 / p)}
$$

Indeed, since $\left\|\nabla a_{Q}\right\|_{L^{2}\left(\mathbb{R}^{n} \times \mathbb{R}\right)} \leq r^{(n+2)(1 / 2-1 / p)}$ and $r \leq 1$, we obtain that

$$
\left\|a_{Q}\right\|_{L^{2}\left(\mathbb{R}^{n} \times \mathbb{R}\right)} \leq r^{(n+2)(1 / 2-1 / p)} .
$$

Due to the fact that $L_{1, p a r}^{2}\left(\mathbb{R}^{n} \times \mathbb{R}\right)=\dot{L}_{1, \text { par }}^{2}\left(\mathbb{R}^{n} \times \mathbb{R}\right) \cap L^{2}\left(\mathbb{R}^{n} \times \mathbb{R}\right)($ cf. Theorem 2.1.35), the proof of (2.1.66) is complete.

The following result will be useful in order to give an atomic decomposition of the Triebel-Lizorkin space $F_{1, p a r}^{p, 2}\left(\mathbb{R}^{n} \times \mathbb{R}\right)$, when $\frac{n+2}{n+3}<p \leq 1$.

Lemma 2.1.56. Consider $\frac{n+2}{n+3}<p \leq 1$ and $Q \subset \mathbb{R}^{n} \times \mathbb{R}$ a parabolic cube with sidelength $l(Q) \leq 1$. Assume that $A \in L_{1, \text { par }}^{2}\left(\mathbb{R}^{n} \times \mathbb{R}\right)$ satisfies

$$
\operatorname{supp} A \subset Q, \quad\|A\|_{L_{1, p a r}^{2}\left(\mathbb{R}^{n} \times \mathbb{R}\right)} \leq|Q|^{1 / 2-1 / p} .
$$

Then there exists a positive constant $c$ such that

$$
\|A\|_{F_{1, p a r}^{p, 2}\left(\mathbb{R}^{n} \times \mathbb{R}\right)} \leq c .
$$

Proof. Without loss of generality we can assume that the cube $Q$ is centered at 0 . Recall from Defnition 2.1.4 the ingredients needed in order to give a Littlewood-Paley type definition of the space $F_{1, \text { par }}^{p, 2}\left(\mathbb{R}^{n} \times \mathbb{R}\right)$. Fix $\varphi \in \mathrm{S}\left(\mathbb{R}^{n} \times \mathbb{R}\right)$ such that

$$
\begin{gathered}
\varphi(x, t)>0 \quad \text { for } \quad 2^{-1}<\|(x, t)\|_{\text {par }}<2, \\
\operatorname{supp} \varphi=\left\{(x, t) \in \mathbb{R}^{n} \times \mathbb{R}: 2^{-1} \leq\|(x, t)\|_{\text {par }} \leq 2\right\}
\end{gathered}
$$


and

$$
\sum_{j=-\infty}^{\infty} \varphi\left(2^{-j} x, 2^{-2 j} t\right)=1 \quad \text { for } \quad(x, t) \neq 0 .
$$

Define the functions $\varphi_{j}$ and $\psi_{j}$ by

$$
\begin{aligned}
& \left(\mathcal{F} \varphi_{j}\right)(x, t)=\varphi\left(2^{-j} x, 2^{-2 j} t\right) \quad \text { for } \quad j \in \mathbb{Z}, \\
& \left(\mathcal{F} \psi_{0}\right)(x, t)=1-\sum_{j=1}^{\infty} \varphi\left(2^{-j} x, 2^{-2 j} t\right), \quad \psi_{j}=\varphi_{j} \quad \text { for } j \geq 1 .
\end{aligned}
$$

Based on the comments on p. 236 in [HPW] and Definition 3.3 in [BoHo], the definition of $F_{1, p a r}^{p, 2}\left(\mathbb{R}^{n} \times \mathbb{R}\right)$ can also involve a compactly supported $\mathcal{F}^{-1} \varphi$ and $\psi_{0}$, meaning that $\mathcal{F}^{-1} \varphi \in \mathrm{S}\left(\mathbb{R}^{n} \times \mathbb{R}\right)$ such that

$$
\begin{gathered}
\mathcal{F}^{-1} \varphi(x, t)>0 \text { for } 2^{-1}<\|(x, t)\|_{\text {par }}<2, \\
\operatorname{supp} \mathcal{F}^{-1} \varphi=\left\{(x, t) \in \mathbb{R}^{n} \times \mathbb{R}: 2^{-1} \leq\|(x, t)\|_{\text {par }} \leq 2\right\}, \\
\sum_{j=-\infty}^{\infty}\left(\mathcal{F}^{-1} \varphi\left(2^{-j} \cdot, 2^{-2 j} \cdot\right)\right)(x, t)=1 \quad \text { for } \quad(x, t) \neq 0,
\end{gathered}
$$

and with $\varphi_{j}(j \in \mathbb{Z}), \psi_{j}\left(j \in \mathbb{N}_{0}\right)$ defined as in (2.1.69), $\psi_{0}$ having compact support.

We next decompose

$$
\begin{aligned}
\|A\|_{F_{1, p a r}^{p, 2}\left(\mathbb{R}^{n} \times \mathbb{R}\right)}^{p}= & \left\|\left(\sum_{j=0}^{\infty} 2^{2 j}\left|\psi_{j} * A\right|^{2}\right)^{1 / 2}\right\|_{L^{p}\left(\mathbb{R}^{n} \times \mathbb{R}\right)}^{p} \\
= & \int_{c_{0} Q}\left(\sum_{j=0}^{\infty} 2^{2 j}\left|\left(\psi_{j} * A\right)(x, t)\right|^{2}\right)^{p / 2} d x d t \\
& +\int_{\mathbb{R}^{n} \times \mathbb{R} \backslash c_{0} Q}\left(\sum_{j=0}^{\infty} 2^{2 j}\left|\left(\psi_{j} * A\right)(x, t)\right|^{2}\right)^{p / 2} d x d t \\
= & I+I I
\end{aligned}
$$

for a fixed $c_{0}>0$ to be specified later. 
Expressing $\varphi_{j}$ in terms of $\varphi$ gives that for $j \in \mathbb{Z}$ and $(x, t) \in \mathbb{R}^{n} \times \mathbb{R}$,

$$
\begin{aligned}
\varphi_{j}(x, t) & =\left(\mathcal{F}^{-1} \varphi\left(2^{-j} \cdot, 2^{-2 j} \cdot\right)\right)(x, t) \\
& =2^{j(n+2)}\left(\mathcal{F}^{-1} \varphi\right)\left(2^{j} x, 2^{2 j} t\right)
\end{aligned}
$$

with $\|(x, t)\|_{p a r} \leq 2^{-j+1}$. Hence, for $j \in \mathbb{N}$,

$$
\operatorname{supp} \psi_{j} \subset B_{\text {par }}\left(0, c 2^{-j}\right)
$$

where $B_{\text {par }}\left(\left(x_{0}, t_{0}\right), r\right)$ denotes a parabolic ball centered at $\left(x_{0}, t_{0}\right) \in \mathbb{R}^{n} \times \mathbb{R}$ with radius $r$. Since $\mathcal{F}^{-1} \varphi \in \mathrm{S}\left(\mathbb{R}^{n} \times \mathbb{R}\right) \hookrightarrow L^{\infty}\left(\mathbb{R}^{n} \times \mathbb{R}\right),(2.1 .71)$ also implies that

$$
\left\|\psi_{j}\right\|_{L^{\infty}\left(\mathbb{R}^{n} \times \mathbb{R}\right)} \leq c 2^{j(n+2)} \quad \text { for } \quad j \in \mathbb{N}
$$

Taking into account that $\mathcal{F}^{-1} \varphi>0$ on its support, for $(x, t) \neq 0$ we have

$$
\begin{aligned}
\psi_{0}(x, t) & =\left[\mathcal{F}^{-1}\left(\sum_{j=-\infty}^{0} \varphi\left(2^{-j} \cdot, 2^{-2 j} \cdot\right)\right)\right](x, t)=\sum_{j=-\infty}^{0}\left(\mathcal{F}^{-1} \varphi\left(2^{-j} \cdot, 2^{-2 j} \cdot\right)\right)(x, t) \\
& \leq \sum_{j=-\infty}^{\infty}\left(\mathcal{F}^{-1} \varphi\left(2^{-j} \cdot, 2^{-2 j} \cdot\right)\right)(x, t)=1 .
\end{aligned}
$$

Therefore,

$$
\left\|\psi_{0}\right\|_{L^{\infty}\left(\mathbb{R}^{n} \times \mathbb{R}\right)} \leq 1
$$

Going further, fix $r:=\frac{2}{p} \in(1, \infty)$ and let $r^{\prime}$ be the conjugate index of $r$, i.e. $\frac{1}{r}+\frac{1}{r^{\prime}}=1$. Then by Hölder's inequality,

$$
\begin{aligned}
|I| & \leq\left\{\int_{c_{0} Q}\left(\sum_{j=0}^{\infty} 2^{2 j}\left|\left(\psi_{j} * A\right)(x, t)\right|^{2}\right)^{\frac{p}{2} r} d x d t\right\}^{\frac{1}{r}}\left\{\int_{c_{0} Q} d x d t\right\}^{\frac{1}{r^{\prime}}} \\
& \leq c\|A\|_{F_{1, p a r}^{2,2}\left(\mathbb{R}^{n} \times \mathbb{R}\right)}|Q|^{\frac{2-p}{p}} .
\end{aligned}
$$


Consequently, (2.1.75) and (2.1.67) imply that $|I| \leq c$. In order to show that $|I I| \leq c$, our goal is to prove the following estimate.

$$
\left\|\psi_{j} * A\right\|_{L^{\infty}\left(\mathbb{R}^{n} \times \mathbb{R}\right)} \leq c 2^{j(n+2)}|Q|^{1+\frac{1}{n+2}-\frac{1}{p}}, \quad j \in \mathbb{N}_{0}
$$

Pick $\kappa \in C_{c}^{\infty}(2 Q)$ such that $\kappa \equiv 1$ on $Q$ and $0 \leq \kappa \leq 1$. Then, by the duality result given in Theorem 2.4.2, for $(x, t) \in \mathbb{R}^{n} \times \mathbb{R}$ and $j \in \mathbb{N}_{0}$, we obtain that

$$
\begin{aligned}
\left|\left(\psi_{j} * A\right)(x, t)\right| & =\left|\left\langle\psi_{j}(x-\cdot, t-\cdot), A\right\rangle\right|=\left|\left\langle\psi_{j}(x-\cdot, t-\cdot) \kappa(\cdot, \cdot), A\right\rangle\right| \\
& \leq\left\|\psi_{j}(x-\cdot, t-\cdot) \kappa(\cdot, \cdot)\right\|_{L_{-1, p a r}^{2}\left(\mathbb{R}^{n} \times \mathbb{R}\right)}\|A\|_{L_{1, p a r}^{2}\left(\mathbb{R}^{n} \times \mathbb{R}\right)} .
\end{aligned}
$$

Since $\|A\|_{L_{1, p a r}^{2}\left(\mathbb{R}^{n} \times \mathbb{R}\right)} \leq|Q|^{1 / 2-1 / p}$ (by hypothesis (2.1.67)), with (2.1.77) in hand, in order to prove (2.1.76), it is enough to show that for all $j \in \mathbb{N}_{0}$,

$$
\left\|\psi_{j}(x-\cdot, t-\cdot) \kappa(\cdot, \cdot)\right\|_{L_{-1, p a r}^{2}\left(\mathbb{R}^{n} \times \mathbb{R}\right)} \leq c 2^{j(n+2)}|Q|^{\frac{1}{2}+\frac{1}{n+2}}
$$

uniformly in $(x, t) \in \mathbb{R}^{n} \times \mathbb{R}$.

To simplify the notation, for $(x, t) \in \mathbb{R}^{n} \times \mathbb{R}$ and a fixed $j \in \mathbb{N}_{0}$ we introduce $\Phi:=\psi_{j}(x-\cdot, t-\cdot) \kappa(\cdot, \cdot)$. Using the properties of the lifting operator defined in (2.1.13) (cf. Theorem 2.1.10), we obtain that

$$
\begin{aligned}
\|\Phi\|_{L_{-1, p a r}^{2}\left(\mathbb{R}^{n} \times \mathbb{R}\right)} & \approx\left\|\mathcal{F}^{-1}\left[\frac{1}{\left(1+|\xi|^{2}\right)^{1 / 2}+|\tau|^{1 / 2}} \mathcal{F} \Phi(\xi, \tau)\right]\right\|_{L^{2}\left(\mathbb{R}^{n} \times \mathbb{R}\right)} \\
& =\left\|\mathcal{F}^{-1}\left[m(\xi, \tau) \frac{1}{\sqrt{|\xi|^{2}+i \tau}} \mathcal{F} \Phi(\xi, \tau)\right]\right\|_{L^{2}\left(\mathbb{R}^{n} \times \mathbb{R}\right)},
\end{aligned}
$$

where

$$
m(\xi, \tau):=\frac{\sqrt{|\xi|^{2}+i \tau}}{\left(1+|\xi|^{2}\right)^{1 / 2}+|\tau|^{1 / 2}}, \quad(\xi, \tau) \in \mathbb{R}^{n} \times \mathbb{R} .
$$

Observe that $m \in L^{\infty}\left(\mathbb{R}^{n} \times \mathbb{R}\right)$ (in fact, $m$ is a multiplier for $L^{p}\left(\mathbb{R}^{n} \times \mathbb{R}\right), 1<p<\infty$, 
see Example 6 in 1.2.6), hence the last expression in (2.1.79) is equivalent with

$$
\begin{aligned}
\| \mathcal{F}^{-1} & {\left[\frac{1}{\sqrt{|\xi|^{2}+i \tau}} \mathcal{F} \Phi(\xi, \tau)\right] \|_{L^{2}\left(\mathbb{R}^{n} \times \mathbb{R}\right)} } \\
& \approx\left\|\mathcal{F}^{-1}[\mathcal{F} \lambda(\xi, \tau) \mathcal{F} \Phi(\xi, \tau)]\right\|_{L^{2}\left(\mathbb{R}^{n} \times \mathbb{R}\right)} \approx\|\lambda * \Phi\|_{L^{2}\left(\mathbb{R}^{n} \times \mathbb{R}\right)} \\
& =\|\Lambda \Phi\|_{L^{2}\left(\mathbb{R}^{n} \times \mathbb{R}\right)},
\end{aligned}
$$

where, for $(x, t) \in \mathbb{R}^{n} \times \mathbb{R}$,

$$
\lambda(x, t):= \begin{cases}\frac{2}{(4 \pi t)^{\frac{n+1}{2}}} e^{-\frac{|x|^{2}}{4 t}}, & \text { if } t>0 \\ 0, & \text { if } t \leq 0\end{cases}
$$

and

$$
\Lambda \Phi(x, t):=(\lambda * \Phi)(x, t)=\int_{-\infty}^{t} \int_{\mathbb{R}^{n}} \lambda(x-y, t-s) \Phi(y, s) d y d s
$$

We have also used the fact that, for $(\xi, \tau) \in \mathbb{R}^{n} \times \mathbb{R}$,

$$
\mathcal{F} \lambda(\xi, \tau)=c \frac{1}{\sqrt{|\xi|^{2}+i \tau}} .
$$

According to $\left[\right.$ Bro2] (p. 6), the operator $\Lambda: L^{p_{0}}\left(\mathbb{R}^{n} \times \mathbb{R}\right) \longrightarrow L^{q_{0}}\left(\mathbb{R}^{n} \times \mathbb{R}\right)$ is bounded for $q_{0}=\frac{(n+2) p_{0}}{n+2-p_{0}}$ and $1<p_{0}<n+2$. In particular, if $q_{0}=2$, then $p_{0}=\frac{2(n+2)}{n+4}$, hence

$$
\Lambda: L^{p_{0}}\left(\mathbb{R}^{n} \times \mathbb{R}\right) \longrightarrow L^{2}\left(\mathbb{R}^{n} \times \mathbb{R}\right) \quad \text { for } \quad p_{0}=\frac{2(n+2)}{n+4}
$$

As a consequence of (2.1.79), (2.1.80) and (2.1.81), we have

$$
\|\Phi\|_{L_{-1, p a r}^{2}\left(\mathbb{R}^{n} \times \mathbb{R}\right)} \leq c\|\Phi\|_{L^{p_{0}\left(\mathbb{R}^{n} \times \mathbb{R}\right)}}
$$

Moreover, by $(2.1 .73),(2.1 .74)$ and the properties of $\kappa$ we obtain that for all $j \in \mathbb{N}_{0}$,

$$
\begin{aligned}
\|\Phi\|_{L^{p_{0}\left(\mathbb{R}^{n} \times \mathbb{R}\right)}} \stackrel{\text { def }}{=}\left\|\psi_{j}(c-\cdot, t-\cdot) \kappa(\cdot, \cdot)\right\|_{L^{p_{0}\left(\mathbb{R}^{n} \times \mathbb{R}\right)}} \leq c\left\|\psi_{j}\right\|_{L^{\infty}\left(\mathbb{R}^{n} \times \mathbb{R}\right)}\|\kappa\|_{L^{p_{0}\left(\mathbb{R}^{n} \times \mathbb{R}\right)}} \\
\leq c 2^{j(n+2)}|Q|^{\frac{1}{p_{0}}}=c 2^{j(n+2)}|Q|^{\frac{1}{2}+\frac{1}{n+2}} .
\end{aligned}
$$


This, together with (2.1.82), proves (2.1.78), therefore (2.1.76) holds.

Going further, to estimate $I I$, first note that

$$
I I=\int_{\mathbb{R}^{n} \times \mathbb{R} \backslash c_{0} Q}\left(\sum_{\substack{j=0 \\(x, t) \in \operatorname{supp}\left(\psi_{j} * A\right)}}^{\infty} 2^{2 j}\left|\left(\psi_{j} * A\right)(x, t)\right|^{2}\right)^{p / 2} d x d t
$$

and that $\left(\psi_{j} * A\right)(x, t)=\left\langle\psi_{j}((x, t)-(y, s)), A(y, s)\right\rangle$ with $(y, s) \in \operatorname{supp} A \subset Q$. Taking also into account (2.1.72) and using the triangle inequality, we obtain that, for all $j \in \mathbb{N}_{0}$,

$$
\|(x, t)\|_{p a r} \leq\|(x, t)-(y, s)\|_{p a r}+\|(y, s)\|_{p a r} \leq c 2^{-j}+\sqrt{n+2} l(Q) .
$$

Also, $(x, t) \in \mathbb{R}^{n} \times \mathbb{R} \backslash c_{0} Q$ implies that

$$
c_{0} l(Q) \leq\|(x, t)\|_{p a r}
$$

Consequently, (2.1.85) and (2.1.86) gives

$$
c_{0} l(Q) \leq c 2^{-j}+\sqrt{n+2} l(Q)
$$

or, equivalently,

$$
l(Q) \leq \frac{c}{c_{0}-\sqrt{n+2}} 2^{-j} \quad \text { if } \quad c_{0}>\sqrt{n+2}
$$

Having specified $c_{0}$, from $(2.1 .85)$ and $(2.1 .87)$ we also obtain that there exists a positive constant $c$ such that

$$
\|(x, t)\|_{p a r} \leq c 2^{-j}, \quad j \in \mathbb{N}_{0}
$$

Solving (2.1.88) for $j$ yields

$$
j \leq \log _{2}\left(\frac{c}{\|(x, t)\|_{\text {par }}}\right) \quad \text { for some } \quad c>0
$$


With (2.1.89) and (2.1.76) in hand, we estimate $I I$ in the following way.

$$
\begin{aligned}
I I & \leq c \int_{\mathbb{R}^{n} \times \mathbb{R} \backslash c_{0} Q}\left\{\sum_{j \leq \log _{2}\left(\frac{c}{\|(x, t)\|_{p a r}}\right)} 2^{2 j}\left(2^{j(n+2)}|Q|^{1+\frac{1}{n+2}-\frac{1}{p}}\right)^{2}\right\}^{\frac{p}{2}} d x d t \\
& \leq c|Q|^{p+\frac{p}{n+2}-1} \int_{\mathbb{R}^{n} \times \mathbb{R} \backslash c_{0} Q}\left(\frac{c}{\|(x, t)\|_{p a r}}\right)^{p(n+3)} d x d t \\
& \leq c\left(l(Q)^{n+2}\right)^{p+\frac{p}{n+2}-1} \frac{1}{l(Q)^{p(n+3)-(n+2)}}=c .
\end{aligned}
$$

This shows that $I I \leq c$, and the proof of the lemma is complete.

We now define the concept of a rough atom, which will play an important role shortly.

Definition 2.1.57. Let $Q$ be a parabolic cube in $\mathbb{R}^{n} \times \mathbb{R}$ with $|Q| \leq 1$ and sidelength $l(Q)$, and fix $\frac{n+2}{n+3}<p \leq 1$. Then $A$ is called a local (inhomogeneous) rough p-atom if

$$
\operatorname{supp} A \subset 3 Q \quad \text { and } \quad\|A\|_{L_{1, p a r}^{2}\left(\mathbb{R}^{n} \times \mathbb{R}\right)} \leq|Q|^{1 / 2-1 / p},
$$

where $3 Q$ is the parabolic cube in $\mathbb{R}^{n} \times \mathbb{R}$ concentric with $Q$ and with sidelength $3 l(Q)$.

Armed with Definition 2.1.57, we proceed to establish a decomposition of the space $F_{1, \text { par }}^{p, 2}\left(\mathbb{R}^{n} \times \mathbb{R}\right)$ into rough atoms.

Theorem 2.1.58. Let $\frac{n+2}{n+3}<p \leq 1$. Then $f \in F_{1, p a r}^{p, 2}\left(\mathbb{R}^{n} \times \mathbb{R}\right)$ if and only if $f$ can be represented as

$$
f=\sum_{j=0}^{\infty} \lambda_{j} A_{j}, \quad \text { where } \quad\left\{\lambda_{j}\right\}_{j \in \mathbb{N}_{0}} \in \ell^{p} \text { and } A_{j} \text { are local rough p-atoms }
$$

Proof. First assume that $f=\sum_{j=0}^{\infty} \lambda_{j} A_{j}$, where $\left\{\lambda_{j}\right\}_{j \in \mathbb{N}_{0}} \in \ell^{p}$ and $A_{j}$ are local rough $p$-atoms. Since $\|\cdot\|_{F_{1, p a r}^{p, 2}\left(\mathbb{R}^{n} \times \mathbb{R}\right)}$ is a $p$-norm (see Proposition 1.2.44), using also 
Lemma 2.1.56 for the atoms $A_{j}$, we obtain that

$$
\begin{aligned}
\|f\|_{F_{1, p a r}^{p, 2}\left(\mathbb{R}^{n} \times \mathbb{R}\right)}^{p} & =\left\|\sum_{j=0}^{\infty} \lambda_{j} A_{j}\right\|_{F_{1, p a r}^{p, 2}\left(\mathbb{R}^{n} \times \mathbb{R}\right)}^{p} \leq \sum_{j=0}^{\infty}\left|\lambda_{j}\right|^{p}\left\|A_{j}\right\|_{F_{1, p a r}^{p, 2}\left(\mathbb{R}^{n} \times \mathbb{R}\right)}^{p} \\
& \leq c \sum_{j=0}^{\infty}\left|\lambda_{j}\right|^{p}<\infty .
\end{aligned}
$$

This proves the left-to-right implication in the theorem.

The opposite one follows in the same fashion as its elliptic counterpart (cf. [FrJa2], p. 133).

One of the main result of this subsection is as follows.

Theorem 2.1.59. Let $\frac{n+2}{n+3}<p \leq 1$. Then

$$
H_{\text {par }}^{1, p}\left(\mathbb{R}^{n} \times \mathbb{R}\right) \equiv F_{1, \text { par }}^{p, 2}\left(\mathbb{R}^{n} \times \mathbb{R}\right)
$$

with equivalent quasi-norms.

Proof. Since every local (inhomogeneous) $(1, p)$-atom for $H_{\text {par }}^{1, p}\left(\mathbb{R}^{n} \times \mathbb{R}\right)$ (see Definition 2.1.53 and Remark 2.1.55) is also a local rough $p$-atom for $F_{1, p a r}^{p, 2}\left(\mathbb{R}^{n} \times \mathbb{R}\right)$ (see Definition 2.1.57 and Theorem 2.1.58), the left-to-right inclusion in (2.1.91) obviously holds.

For the opposite one, consider $f \in F_{1, p a r}^{p, 2}\left(\mathbb{R}^{n} \times \mathbb{R}\right)$ with the representation

$$
f=\sum_{|Q| \leq 1} \lambda_{Q} A_{Q}
$$

where $\left\{\lambda_{Q}\right\}_{Q} \in \ell^{p}, \operatorname{supp} A_{Q} \subset 3 Q$ and $\left\|A_{Q}\right\|_{L_{1, p a r}^{2}\left(\mathbb{R}^{n} \times \mathbb{R}\right)} \leq|Q|^{1 / 2-1 / p}$. This is possible due to Theorem 2.1.58 and Definition 2.1.57. We can decompose $3 Q=\sum_{i=1}^{3^{n+2}} Q_{i}$ with $\left|Q_{i}\right|=|Q| \leq 1$ and write

$$
A_{Q}=\sum_{i=1}^{3^{n+2}} A_{Q} \chi_{Q_{i}}=\sum_{i=1}^{3^{n+2}} A_{Q_{i}}, \quad A_{Q_{i}}:=A_{Q} \chi_{Q_{i}} .
$$


Then, naturally, supp $A_{Q_{i}} \subset Q_{i}$ with $\left|Q_{i}\right| \leq 1$. Moreover,

$$
\left\|A_{Q_{i}}\right\|_{\dot{L}_{1, p a r}^{2}\left(\mathbb{R}^{n} \times \mathbb{R}\right)} \leq\left\|A_{Q_{i}}\right\|_{L_{1, p a r}^{2}\left(\mathbb{R}^{n} \times \mathbb{R}\right)} \leq\left\|A_{Q}\right\|_{L_{1, p a r}^{2}\left(\mathbb{R}^{n} \times \mathbb{R}\right)} \leq|Q|^{1 / 2-1 / p},
$$

hence, each $A_{Q_{i}}$ is a local $(1, p)$-atom for $H_{\text {par }}^{1, p}\left(\mathbb{R}^{n} \times \mathbb{R}\right)$. Therefore we have

$$
f=\sum_{|Q| \leq 1} \lambda_{Q}\left(\sum_{i=1}^{3^{n+2}} A_{Q_{i}}\right)=\sum_{i=1}^{3^{n+2}} \sum_{|Q| \leq 1} \lambda_{Q} A_{Q_{i}} \in H_{\text {par }}^{1, p}\left(\mathbb{R}^{n} \times \mathbb{R}\right),
$$

which proves the right-to-left inclusion in (2.1.91).

\subsubsection{Interpolation}

In this subsection we collect all the real and complex interpolation results regarding the parabolic Besov and Triebel-Lizorkin spaces defined on the Euclidean setting.

Theorem 2.1.60. The following interpolation formulas hold.

(a) (Theorem 1(i) in [Stö2], Lemma 2 on p. 30 in [FJSi]) Suppose $0<p<\infty$, $0<q_{0}, q_{1}, q \leq \infty$ and $\alpha_{0}, \alpha_{1} \in \mathbb{R}$ such that $\alpha_{0} \neq \alpha_{1}$. Then

$$
\begin{aligned}
\left(F_{\alpha_{0}, p a r}^{p, q_{0}}\left(\mathbb{R}^{n} \times \mathbb{R}\right), F_{\alpha_{1}, p a r}^{p, q_{1}}\left(\mathbb{R}^{n} \times \mathbb{R}\right)\right)_{\theta, q} & =\left(F_{\alpha_{0}, p a r}^{p, q_{0}}\left(\mathbb{R}^{n} \times \mathbb{R}\right), B_{\alpha_{1}, p a r}^{p, q_{1}}\left(\mathbb{R}^{n} \times \mathbb{R}\right)\right)_{\theta, q} \\
& =B_{\alpha, p a r}^{p, q}\left(\mathbb{R}^{n} \times \mathbb{R}\right)
\end{aligned}
$$

for $0<\theta<1$ and $\alpha=(1-\theta) \alpha_{0}+\theta \alpha_{1}$.

(b) If $0<\theta<1, \frac{1}{p}=\frac{1-\theta}{p_{0}}+\frac{\theta}{p_{1}}=\frac{1-\theta}{q_{0}}+\frac{\theta}{q_{1}}$ and $\alpha=(1-\theta) \alpha_{0}+\theta \alpha_{1}$, then

$$
\left(B_{\alpha_{0}, p a r}^{p_{0}, q_{0}}\left(\mathbb{R}^{n} \times \mathbb{R}\right), B_{\alpha_{1}, p a r}^{p_{1}, q_{1}}\left(\mathbb{R}^{n} \times \mathbb{R}\right)\right)_{\theta, p}=B_{\alpha, p a r}^{p}\left(\mathbb{R}^{n} \times \mathbb{R}\right)
$$

either for $0<p_{0}<p_{1}<\infty, 0<q_{0}<q_{1}<\infty$ and $\alpha_{0}, \alpha_{1} \in \mathbb{R}$ (on account of Theorem 1(ii) in [Stö2]), or for $0<p_{0}, p_{1}, q_{0}, q_{1}<\infty$ and $\alpha_{0}, \alpha_{1}>0, \alpha_{0} \neq \alpha_{1}$ (according to Corollary 4.5 in [GHT1]). 
(c) (Theorem 1(i) in [Stö2], Lemma 2 on p. 30 in [FJSi]) If $0<p, q_{0}, q_{1} \leq \infty$ and $\alpha_{0}, \alpha_{1} \in \mathbb{R}$ such that $\alpha_{0} \neq \alpha_{1}$, then

$$
\left(B_{\alpha_{0}, p a r}^{p, q_{0}}\left(\mathbb{R}^{n} \times \mathbb{R}\right), B_{\alpha_{1}, p a r}^{p, q_{1}}\left(\mathbb{R}^{n} \times \mathbb{R}\right)\right)_{\theta, q}=B_{\alpha, p a r}^{p, q}\left(\mathbb{R}^{n} \times \mathbb{R}\right)
$$

where $0<\theta<1,0<q \leq \infty$ and $\alpha=(1-\theta) \alpha_{0}+\theta \alpha_{1}$.

(d) (Proposition 5 of [FJSi]) If $0<p_{0}<p_{1}<\infty, 0<q \leq \infty$ and $\alpha \in \mathbb{R}$, then

$$
\left(F_{\alpha, p a r}^{p_{0}, q}\left(\mathbb{R}^{n} \times \mathbb{R}\right), F_{\alpha, \text { par }}^{p_{1}, q}\left(\mathbb{R}^{n} \times \mathbb{R}\right)\right)_{\theta, p}=F_{\alpha, p a r}^{p, q}\left(\mathbb{R}^{n} \times \mathbb{R}\right)
$$

for $0<\theta<1$ and $\frac{1}{p}=\frac{1-\theta}{p_{0}}+\frac{\theta}{p_{1}}$.

(e) (Corollary 2.6.4 from [Joh]) If $0<p_{0}, p_{1}<\infty, 0<q_{0}, q_{1} \leq \infty$ and $\alpha_{0}, \alpha_{1} \in \mathbb{R}$, then

$$
\left[F_{\alpha_{0}, p a r}^{p_{0}, q_{0}}\left(\mathbb{R}^{n} \times \mathbb{R}\right), F_{\alpha_{1}, p a r}^{p_{1}, q_{1}}\left(\mathbb{R}^{n} \times \mathbb{R}\right)\right]_{\theta}=F_{\alpha, p a r}^{p, q}\left(\mathbb{R}^{n} \times \mathbb{R}\right)
$$

for $0<\theta<1, \frac{1}{p}=\frac{1-\theta}{p_{0}}+\frac{\theta}{p_{1}}, \frac{1}{q}=\frac{1-\theta}{q_{0}}+\frac{\theta}{q_{1}}$ and $\alpha=(1-\theta) \alpha_{0}+\theta \alpha_{1}$.

(f) (Corollary 2.6.4 from [Joh]) If $0<p_{0}, p_{1}, q_{0}, q_{1} \leq \infty$ and $\alpha_{0}, \alpha_{1} \in \mathbb{R}$, then

$$
\left[B_{\alpha_{0}, p a r}^{p_{0}, q_{0}}\left(\mathbb{R}^{n} \times \mathbb{R}\right), B_{\alpha_{1}, p a r}^{p_{1}, q_{1}}\left(\mathbb{R}^{n} \times \mathbb{R}\right)\right]_{\theta}=B_{\alpha, p a r}^{p, q}\left(\mathbb{R}^{n} \times \mathbb{R}\right)
$$

for $0<\theta<1, \frac{1}{p}=\frac{1-\theta}{p_{0}}+\frac{\theta}{p_{1}}, \frac{1}{q}=\frac{1-\theta}{q_{0}}+\frac{\theta}{q_{1}}$ and $\alpha=(1-\theta) \alpha_{0}+\theta \alpha_{1}$. For the same range of indices except that $1 \leq p_{0}, p_{1}, q_{0}, q_{1} \leq \infty$, the result can also be invoked from Theorem 6.1 in [Gri1].

(g) (Theorem 6.2 in [Gri1]) If $1 \leq p_{0}, p_{1} \leq \infty$ and $\alpha_{0}, \alpha_{1} \in \mathbb{R}$, then

$$
\left(B_{\alpha_{0}, p a r}^{p_{0}}\left(\mathbb{R}^{n} \times \mathbb{R}\right), B_{\alpha_{1}, p a r}^{p_{1}}\left(\mathbb{R}^{n} \times \mathbb{R}\right)\right)_{\theta, p}=B_{\alpha, p a r}^{p}\left(\mathbb{R}^{n} \times \mathbb{R}\right)
$$

for $0<\theta<1, \frac{1}{p}=\frac{1-\theta}{p_{0}}+\frac{\theta}{p_{1}}$ and $\alpha=(1-\theta) \alpha_{0}+\theta \alpha_{1}$. 
(h) ((A.4) from [Gru1]) If $1<p<\infty$ and $\alpha_{0}, \alpha_{1} \in \mathbb{R}$, then

$$
\left[L_{\alpha_{0}, p a r}^{p}\left(\mathbb{R}^{n} \times \mathbb{R}\right), L_{\alpha_{1}, p a r}^{p}\left(\mathbb{R}^{n} \times \mathbb{R}\right)\right]_{\theta}=L_{\alpha, p a r}^{p}\left(\mathbb{R}^{n} \times \mathbb{R}\right)
$$

for $0<\theta<1$ and $\alpha=(1-\theta) \alpha_{0}+\theta \alpha_{1}$.

(i) ((A.7) in [Gru1]) If $1<p<\infty$ and $\alpha_{0}, \alpha_{1} \in \mathbb{R}$ with $\alpha_{0} \neq \alpha_{1}$, then

$$
\left(L_{\alpha_{0}, p a r}^{p}\left(\mathbb{R}^{n} \times \mathbb{R}\right), L_{\alpha_{1}, p a r}^{p}\left(\mathbb{R}^{n} \times \mathbb{R}\right)\right)_{\theta, p}=B_{\alpha, p a r}^{p}\left(\mathbb{R}^{n} \times \mathbb{R}\right)
$$

for $0<\theta<1$ and $\alpha=(1-\theta) \alpha_{0}+\theta \alpha_{1}$.

(j) (Theorem 3.1 of [CaTo2], particular case of part (e) above)

For indices $\frac{n+2}{n+3}<p_{0}, p_{1} \leq 1$,

$$
\left[H_{\text {par }}^{p_{0}}\left(\mathbb{R}^{n} \times \mathbb{R}\right), H_{\text {par }}^{p_{1}}\left(\mathbb{R}^{n} \times \mathbb{R}\right)\right]_{\theta}=H_{\text {par }}^{p}\left(\mathbb{R}^{n} \times \mathbb{R}\right),
$$

where $0<\theta<1$ and $\frac{1}{p}=\frac{1-\theta}{p_{0}}+\frac{\theta}{p_{1}}$.

Remark 2.1.61. An inspection of the arguments in [Stö2] and [BeLö] also shows that parts (a), (e), (f) and (i) (not only, but these are the results needed later on) of Theorem 2.1.60 hold for the homogeneous scales, as well. More specifically, one has

(a) For $0<p<\infty, 0<q_{0}, q_{1}, q \leq \infty, \alpha_{0}, \alpha_{1} \in \mathbb{R}, \alpha_{0} \neq \alpha_{1}, 0<\theta<1$ and $\alpha=(1-\theta) \alpha_{0}+\theta \alpha_{1}$,

$$
\left(\dot{F}_{\alpha_{0}, p a r}^{p, q_{0}}\left(\mathbb{R}^{n} \times \mathbb{R}\right), \dot{F}_{\alpha_{1}, p a r}^{p, q_{1}}\left(\mathbb{R}^{n} \times \mathbb{R}\right)\right)_{\theta, q}=\dot{B}_{\alpha, p a r}^{p, q}\left(\mathbb{R}^{n} \times \mathbb{R}\right) .
$$

(b) For $0<p_{0}, p_{1}<\infty, 0<q_{0}, q_{1} \leq \infty, \alpha_{0}, \alpha_{1} \in \mathbb{R}, 0<\theta<1, \frac{1}{p}=\frac{1-\theta}{p_{0}}+\frac{\theta}{p_{1}}$, $\frac{1}{q}=\frac{1-\theta}{q_{0}}+\frac{\theta}{q_{1}}$ and $\alpha=(1-\theta) \alpha_{0}+\theta \alpha_{1}$,

$$
\left[\dot{F}_{\alpha_{0}, p a r}^{p_{0}, q_{0}}\left(\mathbb{R}^{n} \times \mathbb{R}\right), \dot{F}_{\alpha_{1}, p a r}^{p_{1}, q_{1}}\left(\mathbb{R}^{n} \times \mathbb{R}\right)\right]_{\theta}=\dot{F}_{\alpha, p a r}^{p, q}\left(\mathbb{R}^{n} \times \mathbb{R}\right) .
$$


(c) For $0<p_{0}, p_{1}, q_{0}, q_{1} \leq \infty, \alpha_{0}, \alpha_{1} \in \mathbb{R}, 0<\theta<1, \frac{1}{p}=\frac{1-\theta}{p_{0}}+\frac{\theta}{p_{1}}, \frac{1}{q}=\frac{1-\theta}{q_{0}}+\frac{\theta}{q_{1}}$ and $\alpha=(1-\theta) \alpha_{0}+\theta \alpha_{1}$,

$$
\left[\dot{B}_{\alpha_{0}, p a r}^{p_{0}, q_{0}}\left(\mathbb{R}^{n} \times \mathbb{R}\right), \dot{B}_{\alpha_{1}, p a r}^{p_{1}, q_{1}}\left(\mathbb{R}^{n} \times \mathbb{R}\right)\right]_{\theta}=\dot{B}_{\alpha, p a r}^{p, q}\left(\mathbb{R}^{n} \times \mathbb{R}\right)
$$

(d) For $1<p<\infty, \alpha_{0}, \alpha_{1} \in \mathbb{R}, \alpha_{0} \neq \alpha_{1}, 0<\theta<1$ and $\alpha=(1-\theta) \alpha_{0}+\theta \alpha_{1}$,

$$
\left(\dot{L}_{\alpha_{0}, p a r}^{p}\left(\mathbb{R}^{n} \times \mathbb{R}\right), \dot{L}_{\alpha_{1}, p a r}^{p}\left(\mathbb{R}^{n} \times \mathbb{R}\right)\right)_{\theta, p}=\dot{B}_{\alpha, p a r}^{p}\left(\mathbb{R}^{n} \times \mathbb{R}\right)
$$

In the last part of this subsection we present a proof of one of the interpolation results invoked above, following the proof of the corresponding result in the isotropic setting (cf. Theorem 6.4.5 in [BeLö]). This approach can be used to show other interpolations formulas, as well, but since it yields some of the above results for a smaller range of indices, we only give the proof of part (f) in Theorem 2.1.60 with $1 \leq p_{0}, p_{1}, q_{0}, q_{1} \leq \infty$. In order to do this, we shall invoke some definitions and known results.

Definition 2.1.62. For $0<q \leq \infty, \alpha \in \mathbb{R}$, and any Banach space $A$, let us introduce the space $\ell_{\alpha}^{q}(A)$ of all sequences $\left\{a_{j}\right\}_{j=0}^{\infty}, a_{j} \in A$, such that, for $0<q<\infty$,

$$
\left\|\left\{a_{j}\right\}\right\|_{\ell_{\alpha}^{q}(A)}=\left(\sum_{j=0}^{\infty} 2^{j \alpha q}\left\|a_{j}\right\|_{A}^{q}\right)^{\frac{1}{q}}<\infty
$$

and, for $q=\infty$,

$$
\left\|\left\{a_{j}\right\}\right\|_{\ell_{\alpha}^{\infty}(A)}=\sup _{j \in \mathbb{N}_{0}}\left(2^{j \alpha}\left\|a_{j}\right\|_{A}\right)<\infty .
$$

We invoke from Theorem 5.6.3 in [BeLö] a complex interpolation result for these spaces:

$$
\left[\ell_{\alpha_{0}}^{q_{0}}\left(A_{0}\right), \ell_{\alpha_{1}}^{q_{1}}\left(A_{1}\right)\right]_{\theta}=\ell_{\alpha}^{q}\left(\left[A_{0}, A_{1}\right]_{\theta}\right)
$$


for $1 \leq q_{0}, q_{1} \leq \infty, \alpha_{0}, \alpha_{1} \in \mathbb{R}$, where $A_{0}, A_{1}$ are Banach spaces, $0<\theta<1$, $\frac{1}{q}=\frac{1-\theta}{q_{0}}+\frac{\theta}{q_{1}}$ and $\alpha=(1-\theta) \alpha_{0}+\theta \alpha_{1}$

Definition 2.1.63. (Definition 6.4.1 from [BeLö])

We say that the space $B$ is a retract of the space $A$ if there are morphisms

$$
\mathcal{I}: B \rightarrow A \quad \text { and } \quad \mathcal{P}: A \rightarrow B
$$

such that $\mathcal{P} \circ \mathcal{I}=I$ on $B$.

Theorem 2.1.64. (Theorem 6.4 .2 of [BeLö])

If the couple $\left(B_{0}, B_{1}\right)$ is a retract of $\left(A_{0}, A_{1}\right)$ with mappings $\mathcal{I}$ and $\mathcal{P}$, then $\left[B_{0}, B_{1}\right]_{\theta}$ and $\left(B_{0}, B_{1}\right)_{\theta, q}$ are retracts of $\left[A_{0}, A_{1}\right]_{\theta}$ and $\left(A_{0}, A_{1}\right)_{\theta, q}$, respectively, with the same mappings.

Sketch of Proof. Since $\mathcal{I}: B_{i} \rightarrow A_{i}, i=0,1$, we have that $\mathcal{I}:\left[B_{0}, B_{1}\right]_{\theta} \rightarrow\left[A_{0}, A_{1}\right]_{\theta}$ and $\mathcal{I}:\left(B_{0}, B_{1}\right)_{\theta, q} \rightarrow\left(A_{0}, A_{1}\right)_{\theta, q}$. Similarly, $\mathcal{P}:\left[A_{0}, A_{1}\right]_{\theta} \rightarrow\left[B_{0}, B_{1}\right]_{\theta}$ and $\mathcal{P}$ : $\left(A_{0}, A_{1}\right)_{\theta, q} \rightarrow\left(B_{0}, B_{1}\right)_{\theta, q}$. Moreover, $\mathcal{P} \circ \mathcal{I}=I$ on $\left[B_{0}, B_{1}\right]_{\theta}$ and on $\left(B_{0}, B_{1}\right)_{\theta, q}$. Now in view of Definition 2.1.63 the conclusion holds.

We next introduce two mappings, $\mathcal{I}$ and $\mathcal{P}$, as follows: $\mathcal{I}$ maps $S^{\prime}\left(\mathbb{R}^{n} \times \mathbb{R}\right)$ to the space of all sequences of tempered disrtibutions, and it is given by

$$
(\mathcal{I} f)_{j}:=\mathcal{F}^{-1}\left(\varphi_{j} \mathcal{F} f\right), \quad j=0,1,2, \ldots
$$

We define $\mathcal{P}$ by

$$
\mathcal{P} a:=\sum_{j=1}^{\infty} \mathcal{F}^{-1}\left(\tilde{\varphi}_{j} \mathcal{F} a_{j}\right), \quad \text { where } a=\left\{a_{j}\right\}_{j=0}^{\infty}, a_{j} \in \mathrm{S}^{\prime}\left(\mathbb{R}^{n} \times \mathbb{R}\right)
$$


for those sequences $\left\{a_{j}\right\}_{j=0}^{\infty}$ of tempered distributions for which the series defining $\mathcal{P} a$ converges in $\mathrm{S}^{\prime}\left(\mathbb{R}^{n} \times \mathbb{R}\right)$. Here $\tilde{\varphi}_{0}:=\varphi_{0}+\varphi_{1}$ and $\tilde{\varphi}_{j}:=\varphi_{j-1}+\varphi_{j}+\varphi_{j+1}$ for $j \geq 1$, where $\varphi_{j}(j=0,1,2, \ldots)$ are introduced in the following way: $\varphi_{0} \in C^{\infty}\left(\mathbb{R}^{n} \times \mathbb{R}\right)$, $\varphi_{0}(x, t)=1$ if $\|(x, t)\|_{p a r} \leq 1$ and $\operatorname{supp} \varphi_{0} \subset\left\{(x, t) \in \mathbb{R}^{n} \times \mathbb{R}:\|(x, t)\| \leq 2\right\}$, and $\varphi_{j}(x, t):=\varphi_{0}\left(2^{-j} x, 2^{-2 j} t\right)-\varphi_{0}\left(2^{-j+1} x, 2^{2(-j+1)} t\right)$ for $j \in \mathbb{N}$.

Lemma 2.1.65. Let $\mathcal{I}$ and $\mathcal{P}$ as above. Then $\mathcal{P} \circ \mathcal{I}=I$ on $\mathrm{S}^{\prime}\left(\mathbb{R}^{n} \times \mathbb{R}\right)$.

Proof. For $f \in \mathrm{S}^{\prime}\left(\mathbb{R}^{N} \times \mathbb{R}\right)$ we have

$$
\begin{aligned}
(\mathcal{P} \circ \mathcal{I})(f) & =\sum_{j=0}^{\infty} \mathcal{F}^{-1}\left(\tilde{\varphi}_{j} \varphi_{j} \mathcal{F} f\right)=\mathcal{F}^{-1}\left(\sum_{j=0}^{\infty} \tilde{\varphi}_{j} \varphi_{j} \mathcal{F} f\right) \\
& =\mathcal{F}^{-1}\left\{\mathcal{F} f\left[\left(\varphi_{0}+\varphi_{1}\right) \varphi_{0}+\sum_{j=1}^{\infty}\left(\varphi_{j-1}+\varphi_{j}+\varphi_{j+1}\right) \varphi_{j}\right]\right\} .
\end{aligned}
$$

Since $\left(\varphi_{0}+\varphi_{1}\right)(x, t)=\varphi\left(2^{-1} x, 2^{-2} t\right)$, it follows that $\varphi_{0}+\varphi_{1}=1$ if $\|(x, t)\| \leq 2$ and $\operatorname{supp}\left(\varphi_{0}+\varphi_{1}\right) \subset\left\{(x, t) \in \mathbb{R}^{n} \times \mathbb{R}:\|(x, t)\| \leq 2^{2}\right\}$. Now one can conclude that $\left(\varphi_{0}+\varphi_{1}\right) \varphi_{0}=\varphi_{0}$. For the second term, $\sum_{j=1}^{\infty}\left(\varphi_{j-1}+\varphi_{j}+\varphi_{j-1}\right) \varphi_{j}$, let us rewrite $\tilde{\varphi}_{j}$ as follows

$$
\tilde{\varphi}_{j}(x, t)=\varphi_{0}\left(2^{-j-1} x, 2^{2(-j-1)} t\right)-\varphi_{0}\left(2^{-j+2} x, 2^{2(-j+2)} t\right)
$$

Then, by the size and support conditions of $\varphi_{0}$, we can see that

$$
\tilde{\varphi}_{j}=1 \text { if } 2^{j-1} \leq\|(x, t)\|_{p a r} \leq 2^{j+1}
$$

and

$$
\operatorname{supp} \tilde{\varphi}_{j} \subset\left\{(x, t) \in \mathbb{R}^{n} \times \mathbb{R}: 2^{j-2} \leq\|(x, t)\|_{p a r} \leq 2^{j+2}\right\} .
$$

By the properties of $\varphi_{j}$ this amounts to $\tilde{\varphi}_{j} \varphi_{j}=\varphi_{j}$ for each $j \geq 1$. Consequently,

$$
\left(\varphi_{0}+\varphi_{1}\right) \varphi_{0}+\sum_{j=1}^{\infty}\left(\varphi_{j-1}+\varphi_{j}+\varphi_{j+1}\right) \varphi_{j}=\varphi_{0}+\sum_{j=1}^{\infty} \varphi_{j}=\sum_{j=0}^{\infty} \varphi_{j}=1
$$


and $\mathcal{P} \circ \mathcal{I}=I$ on $\mathrm{S}^{\prime}\left(\mathbb{R}^{n} \times \mathbb{R}\right)$.

Theorem 2.1.66. Consider $1 \leq p, q \leq \infty$ and $\alpha \in \mathbb{R}$. Then the parabolic Besov space $B_{\alpha, p a r}^{p, q}\left(\mathbb{R}^{n} \times \mathbb{R}\right)$ is a retract of $\ell_{\alpha}^{q}\left(L^{p}\left(\mathbb{R}^{n} \times \mathbb{R}\right)\right)$.

Proof. Case 1. $1 \leq q<\infty$. Let $f \in B_{\alpha, \text { par }}^{p, q}\left(\mathbb{R}^{n} \times \mathbb{R}\right)$. By the definitions of the spaces inviolved, taking also into account that $\left\{\varphi_{j}\right\}_{j=0}^{\infty} \in \Phi$ (see the example after Defnition 2.1.1), we have

$$
\begin{aligned}
\|\mathcal{I} f\|_{\ell_{\alpha}^{q}\left(L^{p}\left(\mathbb{R}^{n} \times \mathbb{R}\right)\right)} & =\left(\sum_{j=0}^{\infty} 2^{j \alpha q}\left\|(\mathcal{I} f)_{j}\right\|_{L^{p}\left(\mathbb{R}^{n} \times \mathbb{R}\right)}^{q}\right)^{\frac{1}{q}} \\
& =\left(\sum_{j=0}^{\infty} 2^{j \alpha q}\left\|\mathcal{F}^{-1}\left(\varphi_{j} \mathcal{F} f\right)\right\|_{L^{p}\left(\mathbb{R}^{n} \times \mathbb{R}\right)}^{q}\right)^{\frac{1}{q}} \\
& =\|f\|_{B_{\alpha, p a r}^{p, q}\left(\mathbb{R}^{n} \times \mathbb{R}\right)},
\end{aligned}
$$

therefore $\mathcal{I}: B_{\alpha, p a r}^{p, q}\left(\mathbb{R}^{n} \times \mathbb{R}\right) \rightarrow \ell_{\alpha}^{q}\left(L^{p}\left(\mathbb{R}^{n} \times \mathbb{R}\right)\right)$ is bounded.

We next let $a=\left\{a_{k}\right\}_{k=0}^{\infty} \subset \ell_{\alpha}^{q}\left(L^{p}\left(\mathbb{R}^{n} \times \mathbb{R}\right)\right)$. On account of definitions we can write

$$
\mathcal{P} a=\sum_{k=0}^{\infty} \mathcal{F}^{-1}\left(\tilde{\tilde{\varphi}_{k}} \mathcal{F} a_{k}\right)=\mathcal{F}^{-1}\left(\sum_{k=0}^{\infty} \tilde{\varphi_{k}} \mathcal{F} a_{k}\right)
$$

and

$$
\begin{aligned}
\|a\|_{B_{\alpha, p a r}^{p, q}\left(\mathbb{R}^{n} \times \mathbb{R}\right)} & =\left(\sum_{j=0}^{\infty} 2^{j \alpha q}\left\|\mathcal{F}^{-1}\left(\varphi_{j} \mathcal{F}(\mathcal{P} a)\right)\right\|_{L^{p}\left(\mathbb{R}^{n} \times \mathbb{R}\right)}^{q}\right)^{\frac{1}{q}} \\
& =\left(\sum_{j=0}^{\infty} 2^{j \alpha q}\left\|\mathcal{F}^{-1}\left(\varphi_{j} \sum_{k=0}^{\infty} \tilde{\varphi_{k}} \mathcal{F} a_{k}\right)\right\|_{L^{p}\left(\mathbb{R}^{n} \times \mathbb{R}\right)}^{q}\right)
\end{aligned}
$$

At this stage we observe that, for $j \geq 2$,

$$
\sum_{k=0}^{\infty} \tilde{\varphi}_{k} \varphi_{j} \mathcal{F} a_{k}=\sum_{k=j-2}^{j+2} \tilde{\varphi}_{k} \varphi_{j} \mathcal{F} a_{k} \leq \sum_{k=j-2}^{j+2} \mathcal{F} a_{k}
$$


For $j=0,1$, taking into account the support conditions on $\tilde{\varphi}_{k}$ and $\varphi_{j}$, the sum in the leftmost of (2.1.96) contains even less terms then in the case $j \geq 2$, each of them is less then or equal to $\mathcal{F} a_{k}$, with $k$ at most 1 . With the contribution of one of the terms from the sum $\sum_{k=j-2}^{j+2} \mathcal{F} a_{k}$, for example for $k=j-1$, the expression in (2.1.95) is bounded by

$$
\begin{aligned}
\left(\sum_{j=2}^{\infty} 2^{j \alpha q}\left\|a_{j-1}\right\|_{L^{p}\left(\mathbb{R}^{n} \times \mathbb{R}\right)}^{q}\right)^{\frac{1}{q}} & =\left(\sum_{i=1}^{\infty} 2^{i \alpha q} 2^{\alpha q}\left\|a_{i}\right\|_{L^{p}\left(\mathbb{R}^{n} \times \mathbb{R}\right)}^{q}\right)^{\frac{1}{q}} \\
& \leq c\left(\sum_{i=0}^{\infty} 2^{i \alpha q}\left\|a_{i}\right\|_{L^{p}\left(\mathbb{R}^{n} \times \mathbb{R}\right)}^{q}\right)^{\frac{1}{q}} \\
& \leq c\left\|\left\{a_{j}\right\}\right\|_{\ell_{\alpha}^{q}\left(L^{p}\left(\mathbb{R}^{n} \times \mathbb{R}\right)\right)} .
\end{aligned}
$$

Similar considerations apply to the other terms of the sum $\sum_{k=j-2}^{j+2} \mathcal{F} a_{k}$, as well as to the case when $j=0,1$. Consequently, (2.1.94)-(2.1.97) imply that

$$
\|a\|_{B_{\alpha, p a r}^{p, q}\left(\mathbb{R}^{n} \times \mathbb{R}\right)} \leq c\left\|\left\{a_{j}\right\}\right\|_{\ell_{\alpha}^{q}\left(L^{p}\left(\mathbb{R}^{n} \times \mathbb{R}\right)\right)}
$$

hence $\mathcal{P}: \ell_{\alpha}^{q}\left(L^{p}\left(\mathbb{R}^{n} \times \mathbb{R}\right)\right) \rightarrow B_{\alpha, p a r}^{p, q}\left(\mathbb{R}^{n} \times \mathbb{R}\right)$ is bounded.

Finally, since $\mathcal{P} \circ \mathcal{I}$ is the identity operator on $B_{\alpha, p a r}^{p, q}\left(\mathbb{R}^{n} \times \mathbb{R}\right)$, in view of Definition 2.1.63, the space $B_{\alpha, p a r}^{p, q}\left(\mathbb{R}^{n} \times \mathbb{R}\right)$ is a retract of $\ell_{\alpha}^{q}\left(L^{p}\left(\mathbb{R}^{n} \times \mathbb{R}\right)\right)$ for $1 \leq p \leq \infty$, $1 \leq q<\infty$ and $\alpha \in \mathbb{R}$

Case 2. $q=\infty$. Following the steps of the previous case, we have

$$
\begin{aligned}
\|\mathcal{I} f\|_{\ell_{\alpha}^{\infty}\left(L^{p}\left(\mathbb{R}^{n} \times \mathbb{R}\right)\right)} & =\sup _{j \in \mathbb{N}_{0}}\left(2^{j \alpha}\left\|(\mathcal{I} f)_{j}\right\|_{L^{p}\left(\mathbb{R}^{n} \times \mathbb{R}\right)}\right) \\
& =\sup _{j \in \mathbb{N}_{0}}\left(2^{j \alpha}\left\|\mathcal{F}^{-1}\left(\varphi_{j} \mathcal{F} f\right)\right\|_{L^{p}\left(\mathbb{R}^{n} \times \mathbb{R}\right)}\right) \\
& =\|f\|_{B_{\alpha, p a r}^{p, \infty}\left(\mathbb{R}^{n} \times \mathbb{R}\right)},
\end{aligned}
$$


which implies that $\mathcal{I}: B_{\alpha, p a r}^{p, \infty}\left(\mathbb{R}^{n} \times \mathbb{R}\right) \rightarrow \ell_{\alpha}^{\infty}\left(L^{p}\left(\mathbb{R}^{n} \times \mathbb{R}\right)\right)$ is bounded.

For $a=\left\{a_{k}\right\}_{k=0}^{\infty} \subset \ell_{\alpha}^{\infty}\left(L^{p}\left(\mathbb{R}^{n} \times \mathbb{R}\right)\right)$ we obtain that

$$
\begin{aligned}
\|\mathcal{P} a\|_{B_{\alpha, p a r}^{\left.p, \mathbb{R}^{n} \times \mathbb{R}\right)}} & =\sup _{j \in \mathbb{N}_{0}}\left(2^{j \alpha}\left\|\mathcal{F}^{-1}\left(\varphi_{j} \sum_{k=0}^{\infty} \tilde{\varphi}_{k} \mathcal{F} a_{k}\right)\right\|_{L^{p\left(\mathbb{R}^{n} \times \mathbb{R}\right)}}\right) \\
& \leq c \sup _{j \in \mathbb{N}_{0}}\left(2^{j \alpha}\left\|a_{j}\right\|_{L^{p}\left(\mathbb{R}^{n} \times \mathbb{R}\right)}\right) \\
& =c\|a\|_{\ell_{\alpha}^{\infty}\left(L^{p}\left(\mathbb{R}^{n} \times \mathbb{R}\right)\right)},
\end{aligned}
$$

hence $\mathcal{P}: \ell_{\alpha}^{q}\left(L^{p}\left(\mathbb{R}^{n} \times \mathbb{R}\right)\right) \rightarrow B_{\alpha, p a r}^{p, q}\left(\mathbb{R}^{n} \times \mathbb{R}\right)$ is bounded.

Finally, since $\mathcal{P} \circ \mathcal{I}=I$ on $B_{\alpha, p a r}^{p, \infty}\left(\mathbb{R}^{n} \times \mathbb{R}\right)$, by Definition 2.1.63, the space $B_{\alpha, p a r}^{p, \infty}\left(\mathbb{R}^{n} \times \mathbb{R}\right)$ is a retract of $\ell_{\alpha}^{\infty}\left(L^{p}\left(\mathbb{R}^{n} \times \mathbb{R}\right)\right)$ for $1 \leq p \leq \infty$ and $\alpha \in \mathbb{R}$. This finishes the proof of the theorem.

The result of the next theorem can be found in Theorem 6.1 of [Gri1] for the same range of indices, and in Corollary 2.6.4 of [Joh] for the same indices except that $0<p_{0}, p_{1}, q_{0}, q_{1} \leq \infty$. Here we present a (different) proof based on the above results in this section.

Theorem 2.1.67. For $1 \leq p_{0}, p_{1}, q_{0}, q_{1} \leq \infty, \alpha_{0}, \alpha_{1} \in \mathbb{R}$ and $0<\theta<1$ we have

$$
\left[B_{\alpha_{0}, p a r}^{p_{0}, q_{0}}\left(\mathbb{R}^{n} \times \mathbb{R}\right), B_{\alpha_{1}, p a r}^{p_{1}, q_{1}}\left(\mathbb{R}^{n} \times \mathbb{R}\right)\right]_{\theta}=B_{\alpha, p a r}^{p, q}\left(\mathbb{R}^{n} \times \mathbb{R}\right),
$$

where $\frac{1}{p}=\frac{1-\theta}{p_{0}}+\frac{\theta}{p_{1}}, \frac{1}{q}=\frac{1-\theta}{q_{0}}+\frac{\theta}{q_{1}}$, and $\alpha=(1-\theta) \alpha_{0}+\theta \alpha_{1}$.

Proof. On account of Theorems 2.1.64 and 2.1.66, the intermediate space

$$
\left[B_{\alpha_{0}, p a r}^{p_{0}, q_{0}}\left(\mathbb{R}^{n} \times \mathbb{R}\right), B_{\alpha_{1}, p a r}^{p_{1}, q_{1}}\left(\mathbb{R}^{n} \times \mathbb{R}\right)\right]_{\theta}
$$

is a retract of

$$
\left[\ell_{\alpha_{0}}^{q_{0}}\left(L^{p_{0}}\left(\mathbb{R}^{n} \times \mathbb{R}\right)\right), \ell_{\alpha_{1}}^{q_{1}}\left(L^{p_{1}}\left(\mathbb{R}^{n} \times \mathbb{R}\right)\right)\right]_{\theta},
$$


which in turn, by Theorem 5.6.3 from [BeLö], is

$$
\ell_{\alpha}^{q}\left(\left[L^{p_{0}}\left(\mathbb{R}^{n} \times \mathbb{R}\right), L^{p_{1}}\left(\mathbb{R}^{n} \times \mathbb{R}\right)\right]_{\theta}\right)=\ell_{\alpha}^{q}\left(L^{p}\left(\mathbb{R}^{n} \times \mathbb{R}\right)\right)
$$

for $\frac{1}{p}=\frac{1-\theta}{p_{0}}+\frac{\theta}{p_{1}}, \frac{1}{q}=\frac{1-\theta}{q_{0}}+\frac{\theta}{q_{1}}$, and $\alpha=(1-\theta) \alpha_{0}+\theta \alpha_{1}$. On the other hand, we know from Theorem 2.1.66 that $B_{\alpha, p a r}^{p, q}\left(\mathbb{R}^{n} \times \mathbb{R}\right)$ is a retract of $\ell_{\alpha}^{q}\left(L^{p}\left(\mathbb{R}^{n} \times \mathbb{R}\right)\right)$ for any $1 \leq p, q \leq \infty$ and $\alpha \in \mathbb{R}$.

Summarizing the properties of the operators $\mathcal{I}$ and $\mathcal{P}$ introduced in (2.1.92) and 2.1.93), respectively, we get that

$$
\begin{gathered}
\mathcal{I}: B_{\alpha, \text { par }}^{p, q}\left(\mathbb{R}^{n} \times \mathbb{R}\right) \longrightarrow \ell_{\alpha}^{q}\left(L^{p}\left(\mathbb{R}^{n} \times \mathbb{R}\right)\right), \\
\mathcal{I}:\left[B_{\alpha_{0}, \text { par }}^{p_{0}, q_{0}}\left(\mathbb{R}^{n} \times \mathbb{R}\right), B_{\alpha_{1}, \text { par }}^{p_{1}, q_{1}}\left(\mathbb{R}^{n} \times \mathbb{R}\right)\right]_{\theta} \longrightarrow \ell_{\alpha}^{q}\left(L^{p}\left(\mathbb{R}^{n} \times \mathbb{R}\right)\right), \\
\mathcal{P}: \ell_{\alpha}^{q}\left(L^{p}\left(\mathbb{R}^{n} \times \mathbb{R}\right)\right) \longrightarrow\left[B_{\alpha_{0}, \text { par }}^{p_{0}, q_{0}}\left(\mathbb{R}^{n} \times \mathbb{R}\right), B_{\alpha_{1}, p_{\text {par }}}^{p_{1}, q_{1}}\left(\mathbb{R}^{n} \times \mathbb{R}\right)\right]_{\theta}
\end{gathered}
$$

for $0<\theta<1, \frac{1}{p}=\frac{1-\theta}{p_{0}}+\frac{\theta}{p_{1}}, \frac{1}{q}=\frac{1-\theta}{q_{0}}+\frac{\theta}{q_{1}}$ and $\alpha=(1-\theta) \alpha_{0}+\theta \alpha_{1}$ with $\alpha_{0}, \alpha_{1} \in \mathbb{R}$, and

$$
\mathcal{P} \text { maps } \ell_{\alpha}^{q}\left(L^{p}\left(\mathbb{R}^{n} \times \mathbb{R}\right)\right) \text { onto } B_{\alpha, p a r}^{p, q}\left(\mathbb{R}^{n} \times \mathbb{R}\right)
$$

for $1 \leq p, q \leq \infty$ and $\alpha \in \mathbb{R}$. Consequently, (2.1.99)-(2.1.102) readily imply

$$
\left[B_{\alpha_{0}, p a r}^{p_{0}, q_{0}}\left(\mathbb{R}^{n} \times \mathbb{R}\right), B_{\alpha_{1}, \text { par }}^{p_{1}, q_{1}}\left(\mathbb{R}^{n} \times \mathbb{R}\right)\right]_{\theta}=B_{\alpha, p a r}^{p, q}\left(\mathbb{R}^{n} \times \mathbb{R}\right)
$$

for $1 \leq p_{0}, p_{1}, q_{0}, q_{1} \leq \infty, \alpha_{0}, \alpha_{1} \in \mathbb{R}, 0<\theta<1, \frac{1}{p}=\frac{1-\theta}{p_{0}}+\frac{\theta}{p_{1}}, \frac{1}{q}=\frac{1-\theta}{q_{0}}+\frac{\theta}{q_{1}}$ and $\alpha=(1-\theta) \alpha_{0}+\theta \alpha_{1}$, completing the proof of the theorem.

\subsection{Spaces on Lipschitz cylinders}

In this section we adapt earlier definitions to the case when the underlying space is the interior of a Lipschitz cylinder, then we discuss several properties of these 
spaces.

Definition 2.2.1. Consider an open set $\mathcal{O} \subseteq \mathbb{R}^{n} \times \mathbb{R}$. Then for $0<p, q \leq \infty$, $\alpha \in \mathbb{R}$, we define

$$
B_{\alpha, p a r}^{p, q}(\mathcal{O}):=\left.B_{\alpha, p a r}^{p, q}\left(\mathbb{R}^{n} \times \mathbb{R}\right)\right|_{\mathcal{O}}
$$

with

$$
\|f\|_{B_{\alpha, p a r}^{p, q}(\mathcal{O})}:=\inf \left\{\|F\|_{B_{\alpha, p a r}^{p, q}\left(\mathbb{R}^{n} \times \mathbb{R}\right)}: F \in B_{\alpha, p a r}^{p, q}\left(\mathbb{R}^{n} \times \mathbb{R}\right),\left.F\right|_{\mathcal{O}}=f\right\}
$$

and, for $p \neq \infty$,

$$
F_{\alpha, p a r}^{p, q}(\mathcal{O}):=\left.F_{\alpha, p a r}^{p, q}\left(\mathbb{R}^{n} \times \mathbb{R}\right)\right|_{\mathcal{O}}
$$

with

$$
\|f\|_{F_{\alpha, p a r}^{p, q}(\mathcal{O})}:=\inf \left\{\|F\|_{F_{\alpha, p a r}^{p, q}\left(\mathbb{R}^{n} \times \mathbb{R}\right)}: F \in F_{\alpha, p a r}^{p, q}\left(\mathbb{R}^{n} \times \mathbb{R}\right),\left.F\right|_{\mathcal{O}}=f\right\}
$$

The above conventions apply to the Sobolev scale $L_{\alpha, p a r}^{p}(\mathcal{O})$ for $1<p<\infty$, to the Hardy scales $H_{\alpha, p a r}^{p}(\mathcal{O})$ for $0<p \leq 1$ and $H_{\alpha, p a r}^{1, p}(\mathcal{O})$ for $\frac{n+2}{n+3}<p \leq 1$, and to the Hölder class $C_{\text {par }}^{\alpha}(\mathcal{O})$ for $0<\alpha<1$, as well as to the homogeneous Besov and Triebel-Lizorkin (hence Sobolev, Hardy and Hölder) scales. In particular, all the inclusions in $(2.1 .7),(2.1 .8)$ and $(2.1 .9)$ continue to hold with $\mathbb{R}^{n} \times \mathbb{R}$ replaced by an open subset $\mathcal{O}$ of $\mathbb{R}^{n} \times \mathbb{R}$.

Using the same argument as in the proof of Proposition 2.1.33 but for some interval $I \subseteq \mathbb{R}$ instead of $\mathbb{R}$, we obtain the following.

Proposition 2.2.2. For $1<p<\infty$ and $\alpha \geq 0$ the following Fubini property for the parabolic Sobolev scale holds:

$$
L_{\alpha, p a r}^{p}\left(\mathbb{R}^{n} \times I\right)=L^{p}\left(I ; L_{\alpha}^{p}\left(\mathbb{R}^{n}\right)\right) \cap L^{p}\left(\mathbb{R}^{n} ; L_{\alpha / 2}^{p}(I)\right)
$$


where $I$ is an arbitrary interval in $\mathbb{R}$.

We shall find this result particularly useful when $I=(-\infty, 0)$ and $I=(-\infty, T)$ for some $0<T<\infty$.

Proposition 2.2.3. Let $\Omega$ be a Lipschitz domain in $\mathbb{R}^{n}, I \subseteq \mathbb{R}$ an interval, and $1 \leq p \leq \infty, \alpha>0$. Then the following identification holds:

$$
B_{\alpha, p a r}^{p}(\Omega \times I)=L^{p}\left(\Omega ; B_{\alpha / 2}^{p}(I)\right) \cap L^{p}\left(I ; B_{\alpha}^{p}(\Omega)\right) .
$$

Proof. First let $f \in B_{\alpha, p a r}^{p}(\Omega \times I)$ be arbitrary. Then by Definition 2.2.1 there exists a function $F$ in $B_{\alpha, p a r}^{p}\left(\mathbb{R}^{n} \times \mathbb{R}\right)$ such that $f=\left.F\right|_{\Omega \times I}$. Based on Proposition 2.1.19 we have $F \in L^{p}\left(\mathbb{R}^{n} ; B_{\alpha / 2}^{p}(\mathbb{R})\right) \cap L^{p}\left(\mathbb{R} ; B_{\alpha}^{p}\left(\mathbb{R}^{n}\right)\right)$ and, hence, $f=\left.F\right|_{\Omega \times I}$ belongs to $L^{p}\left(\Omega ; B_{\alpha / 2}^{p}(I)\right) \cap L^{p}\left(I ; B_{\alpha}^{p}(\Omega)\right)$. This proves the left-to-right inclusion in(2.2.103).

For the opposite one, if $f \in L^{p}\left(\Omega ; B_{\alpha / 2}^{p}(I)\right) \cap L^{p}\left(I ; B_{\alpha}^{p}(\Omega)\right)$, then

$$
\int_{\Omega}\|f(x, \cdot)\|_{B_{\alpha / 2}^{p}(I)}^{p} d x<\infty
$$

and

$$
\int_{I}\|f(\cdot, t)\|_{B_{\alpha}^{p}(\Omega)}^{p} d t<\infty
$$

In order to prove that $f \in B_{\alpha, p a r}^{p}(\Omega \times I)$, we make use of the following difference operators defined for $X:=(x, t) \in \mathbb{R}^{n} \times \mathbb{R}, h \in \mathbb{R}$ and $m \in \mathbb{N}, m>1$, by

$$
\begin{gathered}
\Delta_{i}(h) f(X):=f\left(X+h e_{i}\right)-f(X), \quad i=1,2, \ldots, n, n+1, \\
\Delta_{i}^{m}(h) f(X):=\Delta_{i}(h)\left[\Delta_{i}^{m-1}(h) f(X)\right]=\sum_{j=0}^{m}(-1)^{m-j}\left(\begin{array}{c}
j \\
m
\end{array}\right) f\left(X+j h e_{i}\right) .
\end{gathered}
$$


Here $\left(\begin{array}{c}j \\ m\end{array}\right)$ is the usual binomial coefficient. The concept of finite differences on a set $G \subset \mathbb{R}^{n} \times \mathbb{R}$ is defined as follows. If $f: G \rightarrow \mathbb{R}$ and $X=(x, t) \in G$, then for $1 \leq i \leq n+1, h \in \mathbb{R}$ and $m \in \mathbb{N}, m>1$ we set

$$
\Delta_{i}^{m}(h ; G) f(X):= \begin{cases}\Delta_{i}^{m}(h) f(X) & \text { if }\left[X, X+m h e_{i}\right] \subset G \\ 0 & \text { if }\left[X, X+m h e_{i}\right] \not \subset G .\end{cases}
$$

We employ the notation $\Delta_{\text {time }}$ when these differences are taken in the time component, that is $\Delta_{\text {time }}=\Delta_{n+1}$. Our goal is to use Definition 18.1 and Theorem 18.2 from $[\mathrm{BIN}]\left(\right.$ pp. $58-60$ in Vol. II) with $p^{0}=p^{1}=\ldots=p^{n}=\theta=p, h_{0}=1, m_{i} \in \mathbb{N}$,

$$
k_{i}=0, \quad m_{i}>1>l_{i}=\alpha>0 \quad \text { for } \quad i=1, \ldots, n,
$$

and

$$
k_{n+1}=0, \quad m_{n+1}=m>1>l_{n+1}=\frac{\alpha}{2}>0 .
$$

One can show that (2.2.104) implies

$$
\int_{\Omega} \int_{0}^{1} \frac{\left\|\Delta_{\text {time }}^{m}(h ; I) f(x, \cdot)\right\|_{L^{p}(I)}^{p}}{h^{\frac{\alpha}{2} p}} \frac{d h}{h} d x<\infty .
$$

For further use let us point out that (2.2.104) also entails

$$
\int_{\Omega} \int_{I}|f(x, t)|^{p} d t d x=\int_{\Omega}\|f(x, \cdot)\|_{L^{p}(I)}^{p} d x<\infty .
$$

Next, (2.2.105) implies that

$$
\begin{aligned}
\int_{I}\left\{\sum_{i=1}^{n}\left[\int_{0}^{1} \frac{\left\|\Delta_{i}^{m_{i}}(h ; \Omega) f(\cdot, t)\right\|_{L^{p}(\Omega)}^{p}}{h^{\alpha p}} \frac{d h}{h}\right]^{\frac{1}{p}}\right\}^{p} d t \\
\approx \sum_{i=1}^{n} \int_{I} \int_{0}^{1} \frac{\left\|\Delta_{i}^{m_{i}}(h ; \Omega) f(\cdot, t)\right\|_{L^{p}(\Omega)}^{p}}{h^{\alpha p}} \frac{d h}{h} d t<\infty .
\end{aligned}
$$


Going further, use (2.2.106) to write

$$
\begin{aligned}
\int_{0}^{1} \frac{\int_{\Omega}\left\|\Delta_{\text {time }}^{m}(h ; I) f(x, \cdot)\right\|_{L^{p}(I)}^{p} d x}{h^{\frac{\alpha}{2} p}} \frac{d h}{h} \\
\approx \int_{0}^{1} \frac{\left\|\Delta_{\text {time }}^{m}(h ; \Omega \times I) f\right\|_{L^{p}(\Omega \times I)}^{p}}{h^{\frac{\alpha}{2} p}} \frac{d h}{h}<\infty,
\end{aligned}
$$

and use (2.2.107) to write

$$
\begin{aligned}
& \sum_{i=1}^{n} \int_{0}^{1} \frac{\int_{I}\left\|\Delta_{i}^{m_{i}}(h ; \Omega) f(\cdot, t)\right\|_{L^{p}(\Omega)}^{p} d t}{h^{\alpha p}} \frac{d h}{h} \\
& \approx \sum_{i=1}^{n} \int_{0}^{1} \frac{\left\|\Delta_{i}^{m_{i}}(h ; \Omega \times I) f\right\|_{L^{p}(\Omega \times I)}^{p}}{h^{\alpha p}} \frac{d h}{h} \\
& \approx \sum_{i=1}^{n}\left[\int_{0}^{1} \frac{\left\|\Delta_{i}^{m_{i}}(h ; \Omega \times I) f\right\|_{L^{p}(\Omega \times I)}^{p}}{h^{\alpha p}} \frac{d h}{h}\right]^{\frac{1}{p}}<\infty .
\end{aligned}
$$

Now (2.2.108) and (2.2.109) together with $f \in L^{p}(\Omega \times I)$ imply $f \in B_{p}^{\alpha, p a r}(\Omega \times I)$, where membership to the space $B_{p}^{\alpha, p a r}(\Omega \times I)$ is defined in [BIN] (Definition 18.1) by requiring

$$
\begin{gathered}
\|f\|_{B_{p}^{\alpha, p a r}(\Omega \times I)}:=\|f\|_{L^{p}(\Omega \times I)}+\sum_{i=1}^{n}\left[\int_{0}^{1} \frac{\left\|\Delta_{i}^{m_{i}}(h ; \Omega \times I) f\right\|_{L^{p}(\Omega \times I)}^{p}}{h^{\alpha p}} \frac{d h}{h}\right]^{\frac{1}{p}} \\
+\left[\int_{0}^{1} \frac{\left\|\Delta_{\text {time }}^{m}(h ; \Omega \times I) f\right\|_{L^{p}(\Omega \times I)}^{p}}{h} \frac{d h}{h}\right]^{\frac{1}{p}}<\infty .
\end{gathered}
$$

Consequently, in order to prove the proposition, we need to show that

$$
B_{p}^{\alpha, p a r}(\Omega \times I)=B_{\alpha, p a r}^{p}(\Omega \times I) \quad \text { for } \quad 1 \leq p \leq \infty \text { and } \alpha>0 .
$$

With this in mind fix some $f \in B_{p}^{\alpha, p a r}(\Omega \times I)$. Then, by Theorem 18.5 from [BIN], there exists an extension operator $E$ such that for $1 \leq p \leq \infty$ and $\alpha>0$

$$
E: B_{p}^{\alpha, p a r}(\Omega \times I) \longrightarrow B_{p}^{\alpha, p a r}\left(\mathbb{R}^{n} \times \mathbb{R}\right)
$$


is linear, bounded and $\left.(E f)\right|_{\Omega \times I}=f$. Here we were also using the fact that a Lipschitz cylinder fits into the more general class of domains considered by O. Besov, V. Il'in and S. M. Nikol'skiü (who require that $\Omega \times I$ satisfies the strong $l$-horn condition, cf. pp. 153 - 157 in Vol. I of [BIN] for more details). The next step is to observe that, according to Theorem 4.3 of [Dac1] we have

$$
B_{p}^{\alpha, p a r}\left(\mathbb{R}^{n} \times \mathbb{R}\right)=B_{\alpha, p a r}^{p}\left(\mathbb{R}^{n} \times \mathbb{R}\right) \quad \text { for } \quad 1 \leq p \leq \infty \text { and } \alpha>0
$$

Thus, $F:=E f \in B_{\alpha, p a r}^{p}\left(\mathbb{R}^{n} \times \mathbb{R}\right)$ and $\left.F\right|_{\Omega \times I}=f$, which, by Definition 2.2.1, further implies that $f \in B_{\alpha, p a r}^{p}(\Omega \times I)$. This proves the left-to-right inclusion in (2.2.110).

For the opposite inclusion in (2.2.110) consider $f$ in $B_{\alpha, p a r}^{p}(\Omega \times I)$. By Definition 2.2.1, there exists a function $F$ in $B_{\alpha, p a r}^{p}\left(\mathbb{R}^{n} \times \mathbb{R}\right)$ such that $\left.F\right|_{\Omega \times I}=f$. Now using Theorem 4.3 from [Dac1] and Definition 18.1 from [BIN], we can conclude that

$$
F \in B_{p}^{\alpha, p a r}\left(\mathbb{R}^{n} \times \mathbb{R}\right)
$$

which, in view of $\left.F\right|_{\Omega \times I}=f$, implies that $f \in B_{p}^{\alpha, p a r}(\Omega \times I)$. Hence the right-to-left inclusion in (2.2.110) holds. This finishes the proof of Proposition 2.2.3.

We next note the identification of $B_{\alpha, p a r}^{\infty}(\Omega \times I)$ with the Hölder space $C_{p a r}^{\alpha}(\Omega \times I)$.

Lemma 2.2.4. Consider a Lipschitz domain $\Omega$ in $\mathbb{R}^{n}$, let $I \subseteq \mathbb{R}$ be an interval, and $0<\alpha<1$. Then

$$
B_{\alpha, p a r}^{\infty}(\Omega \times I)=C_{p a r}^{\alpha}(\Omega \times I)
$$

Proof. We follow the same argument as in the proof of Lemma 2.1.21, and use Proposition 2.2.3 (Fubini property for $B_{\alpha, p a r}^{p}(\Omega \times I)$ ), as well as the fact (cf. p. 189 
of [Tri4]) that, for $0<\alpha<1$,

$$
\|f\|_{B_{\alpha}^{\infty}(\Omega)} \approx\|f\|_{L^{\infty}(\Omega)}+\sup _{\substack{x, y \in \Omega \\ x \neq y}} \frac{|f(x)-f(y)|}{|x-y|^{\alpha}}
$$

This finishes the proof of the lemma.

In what follows we discuss an intrinsic characterization of $B_{\alpha, p a r}^{p}(\Omega \times I)$ for $p<\infty$.

Lemma 2.2.5. Let $\Omega$ be a Lipschitz domain in $\mathbb{R}^{n}, I \subseteq \mathbb{R}$ an interval, $1 \leq p<\infty$ and $0<\alpha<1$. Then $f \in B_{\alpha, p a r}^{p}(\Omega \times I)$ if and only if

$$
f \in L^{p}(\Omega \times I) \text { and } \int_{\Omega \times I} \int_{\Omega \times I} \frac{|f(x, t)-f(y, s)|^{p}}{\left(|x-y|+|t-s|^{1 / 2}\right)^{n+2+\alpha p}} d y d s d x d t<\infty .
$$

Furthermore, there is a natural norm estimate associated with this statement.

Proof. First assume that $f \in B_{\alpha, p a r}^{p}(\Omega \times I)$. Then, by Definition 2.2.1, there exists a function $F$ in $B_{\alpha, p a r}^{p}\left(\mathbb{R}^{n} \times \mathbb{R}\right)$ such that $\left.F\right|_{\Omega \times I}=f$, and by Lemma 2.1.22 (the intrinsic characterization of parabolic Besov spaces on $\left.\mathbb{R}^{n} \times \mathbb{R}\right), F \in L^{p}\left(\mathbb{R}^{n} \times \mathbb{R}\right)$ and

$$
\int_{\mathbb{R}^{n} \times \mathbb{R}} \int_{\mathbb{R}^{n} \times \mathbb{R}} \frac{|F(x, t)-F(y, s)|^{p}}{\left(|x-y|+|t-s|^{1 / 2}\right)^{n+2+\alpha p}} d y d s d x d t<\infty .
$$

Consequently,

$$
\begin{aligned}
& \int_{\Omega \times I} \int_{\Omega \times I} \frac{|f(x, t)-f(y, s)|^{p}}{\left(|x-y|+|t-s|^{1 / 2}\right)^{n+2+\alpha p}} d y d s d x d t \\
& =\int_{\Omega \times I} \int_{\Omega \times I} \frac{|F(x, t)-F(y, s)|^{p}}{\left(|x-y|+|t-s|^{1 / 2}\right)^{n+2+\alpha p}} d y d s d x d t \\
& \leq \int_{\mathbb{R}^{n} \times \mathbb{R}} \int_{\mathbb{R}^{n} \times \mathbb{R}} \frac{|F(x, t)-F(y, s)|^{p}}{\left(|x-y|+|t-s|^{1 / 2}\right)^{n+2+\alpha p}} d y d s d x d t<\infty,
\end{aligned}
$$

as desired. Since $B_{\alpha, p a r}^{p}(\Omega \times I) \hookrightarrow L^{p}(\Omega \times I)$ is immediate by definition, we have proved the direct implication. 
To show the opposite one, assume that $f \in L^{p}(\Omega \times I)$ and

$$
\int_{\Omega \times I} \int_{\Omega \times I} \frac{|f(x, t)-f(y, s)|^{p}}{\left(|x-y|+|t-s|^{1 / 2}\right)^{n+2+\alpha p}} d y d s d x d t<\infty .
$$

Relying on (2.1.12), it remains to control $\|f\|_{\dot{B}_{\alpha, p a r}^{p}(\Omega \times I)}$ uniformly in $f$. In order to do so, we first invoke Corollary 1 from [See] with $m=1$ and $r=1$, to the effect that

$$
\|f\|_{\dot{B}_{\alpha, p a r}^{p}(\Omega \times I)}^{p} \leq c \int_{X \in \Omega \times I}\left(\int_{0}^{\delta_{X}}\left[\operatorname{osc}_{1}^{0}(f, X, \theta)\right]^{p} \frac{d \theta}{\theta^{1+\alpha p}}\right) d X,
$$

where, for $X:=(x, t) \in \Omega \times I$, we define $\delta_{X}:=\operatorname{dist}\left(X,\left(\mathbb{R}^{n} \times \mathbb{R}\right) \backslash(\Omega \times I)\right)$, and $o s c_{1}^{0}$ was introduced in (2.1.33). Then expressing the right-hand side of (2.2.111) in a more explicit fashion, and integrating with respect to $\theta$, we obtain that

$$
\begin{aligned}
\|f\|_{\dot{B}_{\alpha, p a r}^{p}(\Omega \times I)}^{p} & \leq c \int_{X \in \Omega \times I}\left(\int_{0}^{\infty} \frac{1}{\theta^{n+2}} \int_{\substack{\|X-Y\|_{\text {par }} \leq \theta \\
Y \in \Omega \times I}}|f(X)-f(Y)|^{p} d Y \frac{d \theta}{\theta^{1+\alpha p}}\right) d X \\
& \leq c \int_{X \in \Omega \times I} \int_{Y \in \Omega \times I}|f(X)-f(Y)|^{p}\left(\int_{\|X-Y\|_{p a r}}^{\infty} \frac{d \theta}{\theta^{n+3+\alpha p}}\right) d Y d X \\
& \leq c \int_{\Omega \times I} \int_{\Omega \times I} \frac{|f(X)-f(Y)|^{p}}{\|X-Y\|_{p a r}^{n+2+\alpha p}} d Y d X \\
& =c \int_{\Omega \times I} \int_{\Omega \times I} \frac{|f(x, t)-f(y, s)|^{p}}{\left[|x-y|+|t-s|^{1 / 2}\right]^{n+2+\alpha p}} d y d s d x d t<\infty,
\end{aligned}
$$

which completes the proof of the lemma.

As a consequence of Theorem 2.1.42, we record the following useful result.

Corollary 2.2.6. Let $\Omega$ be a Lipschitz domain in $\mathbb{R}^{n}, 1<p<\infty$ and $0<\theta<\alpha$.

Then the operator

$$
\partial_{\text {time }}^{\theta / 2}: B_{\alpha, p a r}^{p}(\Omega \times \mathbb{R}) \longrightarrow B_{\alpha-\theta, p a r}^{p}(\Omega \times \mathbb{R})
$$

is bounded. 
Proof. Let a function $f$ be from $B_{\alpha, p a r}^{p}(\Omega \times \mathbb{R})$. Then, by Definition 2.2.1, there exists $F \in B_{\alpha, p a r}^{p}\left(\mathbb{R}^{n} \times \mathbb{R}\right)$ such that $\left.F\right|_{\Omega \times \mathbb{R}}=f$. Consider $\varphi_{0} \in C_{c}^{\infty}\left(\mathbb{R}^{n}\right)$ with $\operatorname{supp} \varphi_{0} \subset K$ for some compact set $K \subset \Omega, \varphi_{1} \in C_{c}^{\infty}(\mathbb{R})$, and $\varphi: \mathbb{R}^{n} \times \mathbb{R} \rightarrow \mathbb{R}$ defined by $\varphi(x, t):=\varphi_{0}(x) \varphi_{1}(t)$.

Due to the fact that for any $\theta \in \mathbb{R}$, the operator $\partial_{\text {time }}^{\theta}$ is self-adjoint (cf. Proposition 1.2.17), using also the properties of $\varphi$ and $F$, we obtain the following.

$$
\begin{aligned}
\left\langle\partial_{\text {time }}^{\theta / 2} F, \bar{\varphi}\right\rangle & =\int_{\mathbb{R}^{n} \times \mathbb{R}}\left(\partial_{\text {time }}^{\theta / 2} F\right)(x, t) \bar{\varphi}(x, t) d x d t \\
& =\int_{\mathbb{R}^{n} \times \mathbb{R}} F(x, t)\left(\partial_{\text {time }}^{\theta / 2} \bar{\varphi}\right)(x, t) d x d t \\
& =\int_{\Omega \times \mathbb{R}} f(x, t) \overline{\varphi_{0}}(x)\left(\partial_{\text {time }}^{\theta / 2} \varphi_{1}\right)(t) d x d t \\
& =\int_{\mathbb{R}^{n} \times \mathbb{R}} f(x, t)\left(\partial_{\text {time }}^{\theta / 2} \bar{\varphi}\right)(x, t) d x d t \\
& =\int_{\mathbb{R}^{n} \times \mathbb{R}}\left(\partial_{\text {time }}^{\theta / 2} f\right)(x, t) \bar{\varphi}(x, t) d x d t \\
& =\left\langle\partial_{\text {time }}^{\theta / 2} f, \bar{\varphi}\right\rangle .
\end{aligned}
$$

Consequently, $\left.\partial_{\text {time }}^{\theta / 2} F\right|_{\Omega \times \mathbb{R}}=\partial_{\text {time }}^{\theta / 2} f$. With this in hand, using also the fact that $\partial_{\text {time }}^{\theta / 2} F \in B_{\alpha-\theta, p a r}^{p}\left(\mathbb{R}^{n} \times \mathbb{R}\right)$ (by Theorem 2.1.42), we can conclude that

$$
\partial_{\text {time }}^{\theta / 2} f \in B_{\alpha-\theta, p a r}^{p}(\Omega \times \mathbb{R}) .
$$

This finishes the proof of the corollary.

In order to present the last part of this section we make a comment regarding an extension operator.

Remark 2.2.7. To prove interpolation results for Triebel-Lizorkin spaces defined on a Lipschitz cylinder, it is convenient to use an extension operator $E$ introduced by A. Seeger (Theorem 2 of [See]). We also note that Lipschitz cylinders $\Omega \times I$, 
where $\Omega$ is a Lipschitz domain in $\mathbb{R}^{n}$ and $I \subseteq \mathbb{R}$ is an interval, do fit into the more general domains considered by A. Seeger. Therefore, in the context of the domains we consider, Theorem 2 of [See] reads as follows:

(i) Let $\Omega$ be a Lipschitz domain in $\mathbb{R}^{n}, I \subseteq \mathbb{R}$ an interval, and $0<p, q<\infty$,

$$
\alpha>\sigma_{p, q}:=\max \left\{0,(n+2)\left(\frac{1}{p}-1\right),(n+2)\left(\frac{1}{q}-1\right)\right\} .
$$

Then there exists a linear extension operator E such that

$$
E: F_{\alpha, p a r}^{p, q}(\Omega \times I) \longrightarrow F_{\alpha, p a r}^{p, q}\left(\mathbb{R}^{n} \times \mathbb{R}\right)
$$

In particular, this extension operator can be applied to the parabolic Sobolev space $L_{\alpha, p a r}^{p}(\Omega \times I)$ for $1<p<\infty, \alpha>0$, and to the Hölder class $H_{\text {par }}^{1, p}(\Omega \times I)$ for $\frac{n+2}{n+3}<p \leq 1$. After we show part (a) of Theorem 2.2.8, we shall prove that $E$ can be applied to the parabolic Besov scale, as well. More specifically, we will show the following:

(ii) Let $\Omega$ be a Lipschitz domain in $\mathbb{R}^{n}, I \subseteq \mathbb{R}$ an interval, $0<p<\infty, 0<q \leq \infty$ and

$$
\alpha>\sigma_{p}:=\max \left\{0,(n+2)\left(\frac{1}{p}-1\right)\right\}
$$

Then there exists a linear extension operator $E$ such that

$$
E: B_{\alpha, p a r}^{p, q}(\Omega \times I) \longrightarrow B_{\alpha, p a r}^{p, q}\left(\mathbb{R}^{n} \times \mathbb{R}\right)
$$

We now are ready to record several useful results pertaining to the way anisotropic Besov and Triebel-Lizorkin spaces interpolate. Recall from 1.2.7 that given a pair of compatible Banach spaces $(X, Y)$, we let $(X, Y)_{\theta, q}$ and $[X, Y]_{\theta}$ denote the intermediate spaces obtained by the real and complex interpolation methods, respectively. 
Theorem 2.2.8. Recall the defnitions of $\sigma_{p, q}$ and $\sigma_{p}$ from (2.2.112) and (2.2.114), respectively. Let $\Omega$ be a Lipschitz domain in $\mathbb{R}^{n}$ and $I \subseteq \mathbb{R}$ an interval. Then the following interpolation formulas hold.

(a) If $0<p, q_{0}, q_{1}<\infty$ and $\alpha_{i}>\sigma_{p, q_{i}}, i=0,1$, such that $\alpha_{0} \neq \alpha_{1}$, then

$$
\left(F_{\alpha_{0}, p a r}^{p, q_{0}}(\Omega \times I), F_{\alpha_{1}, p a r}^{p, q_{1}}(\Omega \times I)\right)_{\theta, q}=B_{\alpha, p a r}^{p, q}(\Omega \times I)
$$

for $0<\theta<1,0<q \leq \infty$ and $\alpha=(1-\theta) \alpha_{0}+\theta \alpha_{1}$.

(b) If $0<p, q_{0}<\infty, 0<q_{1} \leq \infty$ and $\alpha_{0}>\sigma_{p, q_{0}}, \alpha_{1}>\sigma_{p}$ with $\alpha_{0} \neq \alpha_{1}$, then

$$
\left(F_{\alpha_{0}, p a r}^{p, q_{0}}(\Omega \times I), B_{\alpha_{1}, p a r}^{p, q_{1}}(\Omega \times I)\right)_{\theta, q}=B_{\alpha, p a r}^{p, q}(\Omega \times I),
$$

where $0<\theta<1,0<q \leq \infty$ and $\alpha=(1-\theta) \alpha_{0}+\theta \alpha_{1}$.

(c) If $\alpha_{i}>\sigma_{p_{i}}, i=0,1$, and $0<\theta<1, \frac{1}{p}=\frac{1-\theta}{p_{0}}+\frac{\theta}{p_{1}}=\frac{1-\theta}{q_{0}}+\frac{\theta}{q_{1}}, \alpha=$ $(1-\theta) \alpha_{0}+\theta \alpha_{1}$, then

$$
\left(B_{\alpha_{0}, p a r}^{p_{0}, q_{0}}(\Omega \times I), B_{\alpha_{1}, p a r}^{p_{1}, q_{1}}(\Omega \times I)\right)_{\theta, p}=B_{\alpha, p a r}^{p}(\Omega \times I)
$$

either for $0<p_{0}<p_{1}<\infty, 0<q_{0}<q_{1}<\infty$, or for $0<p_{0}, p_{1}, q_{0}, q_{1}<\infty, \alpha_{0} \neq \alpha_{1}$.

(d) If $0<p<\infty, 0<q_{0}, q_{1} \leq \infty, \alpha_{i}>\sigma_{p_{i}}, i=0,1$, with $\alpha_{0} \neq \alpha_{1}$, then

$$
\left(B_{\alpha_{0}, p a r}^{p, q_{0}}(\Omega \times I), B_{\alpha_{1}, p a r}^{p, q_{1}}(\Omega \times I)\right)_{\theta, q}=B_{\alpha, p a r}^{p, q}(\Omega \times I),
$$

where $0<\theta<1,0<q \leq \infty$ and $\alpha=(1-\theta) \alpha_{0}+\theta \alpha_{1}$.

(e) If $0<p_{0}<p_{1}<\infty, 0<q<\infty$ and $\alpha>\sigma_{p_{0}, q}$, then

$$
\left(F_{\alpha, \text { par }}^{p_{0}, q}(\Omega \times I), F_{\alpha, p a r}^{p_{1}, q}(\Omega \times I)\right)_{\theta, p}=F_{\alpha, \text { par }}^{p, q}(\Omega \times I)
$$

for $0<\theta<1$ and $\frac{1}{p}=\frac{1-\theta}{p_{0}}+\frac{\theta}{p_{1}}$. 
(f) If $0<p_{i}, q_{i}<\infty$ and $\alpha_{i}>\sigma_{p_{i}, q_{i}}, i=0,1$, then

$$
\left[F_{\alpha_{0}, p a r}^{p_{0}, q_{0}}(\Omega \times I), F_{\alpha_{1}, p a r}^{p_{1}, q_{1}}(\Omega \times I)\right]_{\theta}=F_{\alpha, p a r}^{p, q}(\Omega \times I)
$$

for $0<\theta<1, \frac{1}{p}=\frac{1-\theta}{p_{0}}+\frac{\theta}{p_{1}}, \frac{1}{q}=\frac{1-\theta}{q_{0}}+\frac{\theta}{q_{1}}$ and $\alpha=(1-\theta) \alpha_{0}+\theta \alpha_{1}$.

(g) If $0<p_{i}<\infty, 0<q_{i} \leq \infty$ and $\alpha_{i}>\sigma_{p_{i}}, i=0,1$, then

$$
\left[B_{\alpha_{0}, p a r}^{p_{0}, q_{0}}(\Omega \times I), B_{\alpha_{1}, p a r}^{p_{1}, q_{1}}(\Omega \times I)\right]_{\theta}=B_{\alpha, p a r}^{p, q}(\Omega \times I)
$$

for $0<\theta<1, \frac{1}{p}=\frac{1-\theta}{p_{0}}+\frac{\theta}{p_{1}}, \frac{1}{q}=\frac{1-\theta}{q_{0}}+\frac{\theta}{q_{1}}$ and $\alpha=(1-\theta) \alpha_{0}+\theta \alpha_{1}$.

(h) If $0<p_{i}<\infty$ and $\alpha_{i}>\sigma_{p_{i}}, i=0,1$, then

$$
\left(B_{\alpha_{0}, p a r}^{p_{0}}(\Omega \times I), B_{\alpha_{1}, p a r}^{p_{1}}(\Omega \times I)\right)_{\theta, p}=B_{\alpha, p a r}^{p}(\Omega \times I)
$$

for $0<\theta<1, \frac{1}{p}=\frac{1-\theta}{p_{0}}+\frac{\theta}{p_{1}}$ and $\alpha=(1-\theta) \alpha_{0}+\theta \alpha_{1}$.

(i) If $1<p<\infty$ and $\alpha_{0}, \alpha_{1}>0$, then

$$
\left[L_{\alpha_{0}, p a r}^{p}(\Omega \times I), L_{\alpha_{1}, p a r}^{p}(\Omega \times I)\right]_{\theta}=L_{\alpha, p a r}^{p}(\Omega \times I)
$$

for $0<\theta<1$ and $\alpha=(1-\theta) \alpha_{0}+\theta \alpha_{1}$.

(j) If $1<p<\infty$ and $\alpha_{0}, \alpha_{1}>0$ with $\alpha_{0} \neq \alpha_{1}$, then

$$
\left(L_{\alpha_{0}, p a r}^{p}(\Omega \times I), L_{\alpha_{1}, p a r}^{p}(\Omega \times I)\right)_{\theta, p}=B_{\alpha, p a r}^{p}(\Omega \times I)
$$

for $0<\theta<1$ and $\alpha=(1-\theta) \alpha_{0}+\theta \alpha_{1}$.

Proof. We shall prove parts (a) and (b) only, since the rest of the theorem follows by the same argument using Remark 2.2.7 and the corresponding interpolation results on $\mathbb{R}^{n} \times \mathbb{R}$ (Theorem 2.1.60). 
Proof of (a). According to part (i) of Remark 2.2.7 there exists a linear, bounded operator $E$ such that

$$
E: F_{\alpha_{i}, p a r}^{p, q_{i}}(\Omega \times I) \longrightarrow F_{\alpha_{i}, p a r}^{p, q_{i}}\left(\mathbb{R}^{n} \times \mathbb{R}\right)
$$

and $\left.\left(E f_{i}\right)\right|_{\Omega \times I}=f_{i}$ for $f_{i} \in F_{\alpha_{i}, p a r}^{p, q_{i}}(\Omega \times I), i=0,1$, where $0<p, q_{0}, q_{1}<\infty$ and

$$
\alpha_{i}>\sigma_{p, q_{i}}:=\max \left\{0,(n+2)\left(\frac{1}{p}-1\right),(n+2)\left(\frac{1}{q_{i}}-1\right)\right\}, \quad i=0,1
$$

Then using a real interpolation result, more specifically the first part in (a) of Theorem 2.1.60, we get that

$$
E:\left(F_{\alpha_{0}, p a r}^{p, q_{0}}(\Omega \times I), F_{\alpha_{1}, p a r}^{p, q_{1}}(\Omega \times I)\right)_{\theta, q} \longrightarrow B_{\alpha, p a r}^{p, q}\left(\mathbb{R}^{n} \times \mathbb{R}\right)
$$

for

$$
\begin{aligned}
& 0<p, q_{0}, q_{1}<\infty \\
& \alpha_{i}>\sigma_{p, q_{i}}:=\max \left\{0,(n+2)\left(\frac{1}{p}-1\right),(n+2)\left(\frac{1}{q_{i}}-1\right)\right\}, \quad i=0,1, \\
& 0<\theta<1, \quad 0<q \leq \infty \quad \text { and } \quad \alpha=(1-\theta) \alpha_{0}+\theta \alpha_{1} .
\end{aligned}
$$

A moment's reflection allows one to deduce from (2.2.126) that the left-to-right inclusion in (2.2.116) holds for the range of indices as in (2.2.127).

As for the opposite one, by reasoning in a similar fashion, the restriction operator maps

$$
\left.\cdot\right|_{\Omega \times I}: F_{\alpha_{i}, p a r}^{p, q_{i}}\left(\mathbb{R}^{n} \times \mathbb{R}\right) \longrightarrow F_{\alpha_{i}, p a r}^{p, q_{i}}(\Omega \times I), \quad i=0,1,
$$

hence, by the first part in (a) of Theorem 2.1.60,

$$
\left.\cdot\right|_{\Omega \times I}: B_{\alpha, p a r}^{p, q}\left(\mathbb{R}^{n} \times \mathbb{R}\right) \longrightarrow\left(F_{\alpha_{0}, p a r}^{p, q_{0}}(\Omega \times I), F_{\alpha_{1}, p a r}^{p, q_{1}}(\Omega \times I)\right)_{\theta, q}
$$


for

$$
\begin{aligned}
& 0<p<\infty, \quad 0<q_{0}, q_{1}, q \leq \infty, \quad \alpha_{0}, \alpha_{1} \in \mathbb{R}, \quad \alpha_{0} \neq \alpha_{1}, \\
& 0<\theta<1 \quad \text { and } \quad \alpha=(1-\theta) \alpha_{0}+\theta \alpha_{1} .
\end{aligned}
$$

On the other hand

$$
\left.\cdot\right|_{\Omega \times I}: B_{\alpha, p a r}^{p, q}\left(\mathbb{R}^{n} \times \mathbb{R}\right) \longrightarrow B_{\alpha, p a r}^{p, q}(\Omega \times I)
$$

is, by definition, onto. This, in concert with (2.2.128), proves the right-to-left inclusion in (2.2.116) for the range of indices specified in (2.2.129). Consequently, (2.2.116) holds with indices as in (2.2.127), proving part (a) of the theorem.

Proof of (ii) in Remark 2.2.7. Given $\alpha>\sigma_{p}:=\max \left\{0,(n+2)\left(\frac{1}{p}-1\right)\right\}$ and $0<\theta<1$, there exist

$$
\alpha_{0}, \alpha_{1}>\sigma_{p}=\max \left\{0,(n+2)\left(\frac{1}{p}-1\right)\right\}
$$

such that $\alpha=(1-\theta) \alpha_{0}+\theta \alpha_{1}$. Part (i) of Remark 2.2.7 with $0<p<\infty$ and $1 \leq q_{0}, q_{1}<\infty$ (hence with $\alpha_{0}, \alpha_{1}$ as in (2.2.130)) implies that

$$
E: F_{\alpha_{i}, p a r}^{p, q_{i}}(\Omega \times I) \longrightarrow F_{\alpha_{i}, p a r}^{p, q_{i}}\left(\mathbb{R}^{n} \times \mathbb{R}\right), \quad i=0,1, \quad \text { is bounded }
$$

Taking into account part (a) of Theorem 2.2.8 and the first part of (a) in Theorem 2.1.60, as well as the fact that $E$ is a linear operator, real interpolation yields

$$
E: B_{\alpha, \text { par }}^{p, q}(\Omega \times I) \longrightarrow B_{\alpha, \text { par }}^{p, q}\left(\mathbb{R}^{n} \times \mathbb{R}\right)
$$

is bounded for $0<p<\infty, 0<q \leq \infty$ and $\alpha>\max \left\{0,(n+2)\left(\frac{1}{p}-1\right)\right\}$. This proves (ii) in Remark 2.2.7. 
Proof of (b). With parts (i) and (ii) of Remark 2.2.7 in hand, real interpolation gives that

$$
E:\left(F_{\alpha_{0}, p a r}^{p, q_{0}}(\Omega \times I), B_{\alpha_{1}, p a r}^{p, q_{1}}(\Omega \times I)\right)_{\theta, q} \longrightarrow\left(F_{\alpha_{0}, p a r}^{p, q_{0}}\left(\mathbb{R}^{n} \times \mathbb{R}\right), B_{\alpha_{1}, p a r}^{p, q_{1}}\left(\mathbb{R}^{n} \times \mathbb{R}\right)\right)_{\theta, q}
$$

is bounded for the range of indices as in (b). By the second part of (a) in Theorem 2.1.60,

$$
\left(F_{\alpha_{0}, p a r}^{p, q_{0}}\left(\mathbb{R}^{n} \times \mathbb{R}\right), B_{\alpha_{1}, p a r}^{p, q_{1}}\left(\mathbb{R}^{n} \times \mathbb{R}\right)\right)_{\theta, q}=B_{\alpha, p a r}^{p, q}\left(\mathbb{R}^{n} \times \mathbb{R}\right)
$$

for $0<p<\infty, 0<q_{0}, q_{1}, q \leq \infty, \alpha_{0}, \alpha_{1} \in \mathbb{R}$ with $\alpha_{0} \neq \alpha_{1}$, and $0<\theta<1$, $\alpha=(1-\theta) \alpha_{0}+\theta \alpha_{1}$. This shows the left-to-right inclusion in (2.2.117) for indices as in (b).

For the opposite one, note that the restriction operators map

$$
\begin{aligned}
& \left.\cdot\right|_{\Omega \times I}: F_{\alpha_{0}, p a r}^{p, q_{0}}\left(\mathbb{R}^{n} \times \mathbb{R}\right) \longrightarrow F_{\alpha_{0}, p a r}^{p, q_{0}}(\Omega \times I), \\
& \left.\cdot\right|_{\Omega \times I}: B_{\alpha_{1}, p a r}^{p, q_{1}}\left(\mathbb{R}^{n} \times \mathbb{R}\right) \longrightarrow B_{\alpha_{1}, p a r}^{p, q_{1}}(\Omega \times I),
\end{aligned}
$$

for $0<p<\infty, 0<q_{0}, q_{1} \leq \infty$ and $\alpha_{0}, \alpha_{1} \in \mathbb{R}$. Hence, by the second part in (a) of Theorem 2.1.60,

$$
\left.\cdot\right|_{\Omega \times I}: B_{\alpha, p a r}^{p, q}\left(\mathbb{R}^{n} \times \mathbb{R}\right) \longrightarrow\left(F_{\alpha_{0}, p a r}^{p, q_{0}}(\Omega \times I), B_{\alpha_{1}, p a r}^{p, q_{1}}(\Omega \times I)\right)_{\theta, q}
$$

for

$$
\begin{aligned}
& 0<p<\infty, \quad 0<q_{0}, q_{1}, q \leq \infty, \quad \alpha_{0}, \alpha_{1} \in \mathbb{R}, \quad \alpha_{0} \neq \alpha_{1}, \\
& 0<\theta<1 \quad \text { and } \quad \alpha=(1-\theta) \alpha_{0}+\theta \alpha_{1} .
\end{aligned}
$$

On the other hand

$$
\left.\cdot\right|_{\Omega \times I}: B_{\alpha, p a r}^{p, q}\left(\mathbb{R}^{n} \times \mathbb{R}\right) \longrightarrow B_{\alpha, p a r}^{p, q}(\Omega \times I)
$$


is, by definition, onto. This, in concert with (2.2.132), proves the right-to-left inclusion in (2.2.117) for the range of indices specified in (2.2.133). Consequently, (2.2.117) holds with indices as in (b), completing the proof of part (b) of Theorem 2.2.8.

Remark 2.2.9. In order to prove interpolation results for parabolic Besov spaces defined on a Lipschitz cylinder we could also employ the linear extension operator given by O. Besov, V.Il'in and S. M. Nikol'skiu in Theorem 18.5 (p.63 in Vol. II) of $[\mathrm{BIN}]$, for $1 \leq p \leq \infty$ and $\alpha>0$. We also note that Lipschitz cylinders fit into the more general class of domains (satisfying the strong l-horn condition), considered on pp. 153 - 155 in Vol. I of [BIN]. Using this extension operator we obtain similar results to parts (c), (d), (g) and (h) of Theorem 2.2.8. More specifically:

(c') The range of indices in (c) is larger then the one obtained using the extension operator from $[\mathrm{BIN}]$.

(d') If $1 \leq p, q_{0}, q_{1} \leq \infty, \alpha_{0}, \alpha_{1}>0$ with $\alpha_{0} \neq \alpha_{1}$, then (2.2.119) holds for $0<\theta<1,1 \leq q \leq \infty$ and $\alpha=(1-\theta) \alpha_{0}+\theta \alpha_{1}$.

(g') If $1 \leq p_{i}, q_{i} \leq \infty$ and $\alpha_{i}>0, i=0,1$, then (2.2.122) holds for $0<\theta<1$, $\frac{1}{p}=\frac{1-\theta}{p_{0}}+\frac{\theta}{p_{1}}, \frac{1}{q}=\frac{1-\theta}{q_{0}}+\frac{\theta}{q_{1}}$ and $\alpha=(1-\theta) \alpha_{0}+\theta \alpha_{1}$.

(h') If $1 \leq p_{0}, p_{1} \leq \infty$ and $\alpha_{0}, \alpha_{1}>0$, then (2.2.123) holds for $0<\theta<1$, $\frac{1}{p}=\frac{1-\theta}{p_{0}}+\frac{\theta}{p_{1}}$ and $\alpha=(1-\theta) \alpha_{0}+\theta \alpha_{1}$.

\subsection{Spaces defined on the lateral boundary}

This section contains the definitions and various properties of the parabolic Besov and Triebel-Lizorkin spaces on the lateral boundary of a Lipschitz cylinder. 
Here we give the precise definitions only for the inhomogeneous scale, their homogeneous counterparts are definied in an analogous way. We also note that for simplicity we shall write $B_{\alpha, p a r}^{p}(\partial \Omega \times \mathbb{R})$ in place of $B_{\alpha, p a r}^{p, p}(\partial \Omega \times \mathbb{R})$. Similar considerations apply to the homogeneous Besov scale and for spaces defined on $\partial \Omega \times I$, where $I$ is any open interval in $\mathbb{R}$.

Recall that $(a)_{+}:=\max \{a, 0\}$.

Definition 2.3.1. Let $\frac{n+1}{n+2}<p, q \leq \infty$ and $(n+1)\left(\frac{1}{p}-1\right)_{+}<\alpha<1$.

(i) For an unbounded Lipschitz domain $\Omega \subset \mathbb{R}^{n}$ above the graph of (a Lipschitz function) $\varphi: \mathbb{R}^{n-1} \rightarrow \mathbb{R}$, define the space

$$
B_{\alpha, p a r}^{p, q}(\partial \Omega \times \mathbb{R}):=\left\{f \in L^{p}(\partial \Omega \times \mathbb{R}): \quad f \circ \Phi \in B_{\alpha, p a r}^{p, q}\left(\mathbb{R}^{n-1} \times \mathbb{R}\right)\right\}
$$

where

$$
\Phi: \mathbb{R}^{n-1} \times \mathbb{R} \longrightarrow \partial \Omega \times \mathbb{R}, \quad \Phi\left(x^{\prime}, t\right):=\left(x^{\prime}, \varphi\left(x^{\prime}\right), t\right)
$$

and

$$
\|f\|_{B_{\alpha, p a r}^{p, q}(\partial \Omega \times \mathbb{R})}:=\|f \circ \Phi\|_{B_{\alpha, p a r}^{p, q}\left(\mathbb{R}^{n-1} \times \mathbb{R}\right)} .
$$

(ii) Let $\Omega$ be a bounded Lipschitz domain in $\mathbb{R}^{n}$ as in Remark 1.2.1. By definition,

$$
f \in B_{\alpha, p a r}^{p, q}(\partial \Omega \times \mathbb{R}) \Longleftrightarrow g_{i}:={\widetilde{\psi_{i} f}}^{\partial \Omega_{i}} \in B_{\alpha, p a r}^{p, q}\left(\partial \Omega_{i} \times \mathbb{R}\right) \quad \forall i=1, \ldots, N
$$

where $\sim \partial \Omega_{i}$ stands for the extension by 0 to $\partial \Omega_{i}$, and

$$
\|f\|_{B_{\alpha, p a r}^{p, q}(\partial \Omega \times \mathbb{R})}:=\sum_{i=1}^{N}\left\|g_{i}\right\|_{B_{\alpha, p a r}^{p, q}\left(\partial \Omega_{i} \times \mathbb{R}\right)}
$$


Remark 2.3.2. 1. Observe that

$$
\|f\|_{B_{\alpha, p a r}^{p, q}(\partial \Omega \times \mathbb{R})} \approx \inf \left[\sum_{i=1}^{N}\left\|g_{i}\right\|_{B_{\alpha, p a r}^{p, q}\left(\partial \Omega_{i} \times \mathbb{R}\right)}\right],
$$

where the infimum is taken over all smooth coverings $\left\{V_{i}\right\}_{i=1}^{N}$ as in Remark 1.2.1 and all partitions of unity $\left\{\psi_{i}\right\}_{i=1}^{N}$ subordinated to this cover.

2. The definition of $B_{\alpha, p a r}^{p, q}(\partial \Omega \times \mathbb{R})$ is independent of $\left\{V_{i}\right\}_{1 \leq i \leq N}$ and $\left\{\psi_{i}\right\}_{1 \leq i \leq N}$, meaning that different choices of $\left\{V_{i}\right\}_{i}$ and $\left\{\psi_{i}\right\}_{i}$ yield isomorphic spaces.

3. If $f \in B_{\alpha, p a r}^{p, q}(\partial \Omega \times \mathbb{R})$ and $\xi \in C^{\infty}\left(\mathbb{R}^{n}\right)$, then $\xi f$ belongs to $B_{\alpha, p a r}^{p, q}(\partial \Omega \times \mathbb{R})$.

4. If supp $f \subset \subset \partial \Omega_{i} \cap \partial \Omega$, then the following are equivalent:

$$
\begin{gathered}
\tilde{f}^{\partial \Omega} \in B_{\alpha, p a r}^{p, q}(\partial \Omega \times \mathbb{R}), \\
\widetilde{f}^{\partial \Omega_{i}} \in B_{\alpha, p a r}^{p, q}\left(\partial \Omega_{i} \times \mathbb{R}\right) .
\end{gathered}
$$

Definition 2.3.3. (Besov spaces with negative amount of smoothness)

(i) If $\Omega$ is an unbounded Lipschitz domain in $\mathbb{R}^{n}$ with $\partial \Omega$ being the graph of the function $\varphi: \mathbb{R}^{n-1} \rightarrow \mathbb{R}$, and $\frac{n+1}{n+2}<p, q<\infty,(n+1)\left(\frac{1}{p}-1\right)_{+}<\alpha<1$, then

$$
f \in B_{\alpha-1, p a r}^{p, q}(\partial \Omega \times \mathbb{R}) \Longleftrightarrow g \in B_{\alpha-1, p a r}^{p, q}\left(\mathbb{R}^{n-1} \times \mathbb{R}\right)
$$

where

$$
g\left(x^{\prime}, t\right):=f\left(x^{\prime}, \varphi\left(x^{\prime}\right), t\right) \sqrt{1+\left|\nabla \varphi\left(x^{\prime}\right)\right|^{2}}, \quad x^{\prime} \in \mathbb{R}^{n-1}, t \in \mathbb{R}
$$

and

$$
\|f\|_{B_{\alpha-1, p a r}^{p, q}(\partial \Omega \times \mathbb{R})}:=\|g\|_{B_{\alpha-1, p a r}^{p, q}\left(\mathbb{R}^{n-1} \times \mathbb{R}\right)} .
$$

(ii) For a bounded domain $\Omega$ in $\mathbb{R}^{n}$ as in Remark 1.2.1, by definition,

$$
f \in B_{\alpha-1, p a r}^{p, q}(\partial \Omega \times \mathbb{R}) \Longleftrightarrow g_{i}:=\widetilde{\psi_{i} f}{ }^{\partial \Omega_{i}} \in B_{\alpha-1, p a r}^{p, q}\left(\partial \Omega_{i} \times \mathbb{R}\right) \quad \forall i=1, \ldots, N
$$


where $\sim \partial \Omega_{i}$ represents the extension by 0 to $\partial \Omega_{i}$, and

$$
\|f\|_{B_{\alpha-1, p a r}^{p, q}(\partial \Omega \times \mathbb{R})}:=\inf \left[\sum_{i=1}^{N}\left\|g_{i}\right\|_{B_{\alpha-1, p a r}^{p, q}\left(\partial \Omega_{i} \times \mathbb{R}\right)}\right]
$$

where the infimum is taken over all smooth coverings $\left\{V_{i}\right\}_{i=1}^{N}$ and all partitions of unity $\left\{\psi_{i}\right\}_{i=1}^{N}$ subordinated to this cover.

Definition 2.3.4. Let $\Omega$ be a Lipschitz domain in $\mathbb{R}^{n}, I \subseteq \mathbb{R}$ an interval, and

$$
\begin{aligned}
& \text { either } \frac{n+1}{n+2}<p, q \leq \infty, \quad(n+1)\left(\frac{1}{p}-1\right)_{+}<\alpha<1, \\
& \text { or } \quad \frac{n+1}{n+2}<p, q<\infty, \quad(n+1)\left(\frac{1}{p}-1\right)_{+}-1<\alpha<0 .
\end{aligned}
$$

Then

$$
B_{\alpha, p a r}^{p, q}(\partial \Omega \times I):=\left.B_{\alpha, p a r}^{p, q}(\partial \Omega \times \mathbb{R})\right|_{\partial \Omega \times I}
$$

and

$$
\|f\|_{B_{\alpha, p a r}^{p, q a}(\partial \Omega \times I)}:=\inf \left\{\|F\|_{B_{\alpha, p a r}^{p, q}(\partial \Omega \times \mathbb{R})}: \quad F \in B_{\alpha, p a r}^{p, q}(\partial \Omega \times \mathbb{R}),\left.F\right|_{\partial \Omega \times I}=f\right\} .
$$

Definition 2.3.5. Suppose that either

$$
\frac{n+1}{n+2}<p<\infty, \quad \frac{n+1}{n+2}<q \leq \infty, \quad(n+1)\left(\frac{1}{\min \{p, q\}}-1\right)_{+}<\alpha<1,
$$

or

$$
\frac{n+1}{n+2}<p<\infty, \quad q=2, \quad \alpha=1 .
$$

(i) Consider an unbounded Lipschitz domain $\Omega \subseteq \mathbb{R}^{n}$ and, using the notation of Definition 2.3.1, define

$$
F_{\alpha, p a r}^{p, q}(\partial \Omega \times \mathbb{R}):=\left\{f \in L^{p}(\partial \Omega \times \mathbb{R}): \quad f \circ \Phi \in F_{\alpha, p a r}^{p, q}\left(\mathbb{R}^{n-1} \times \mathbb{R}\right)\right\},
$$




$$
\|f\|_{F_{\alpha, p a r}^{p, q}(\partial \Omega \times \mathbb{R})}:=\|f \circ \Phi\|_{F_{\alpha, p a r}^{p, q}\left(\mathbb{R}^{n-1} \times \mathbb{R}\right)}
$$

(ii) Let $\Omega$ be a bounded Lipschitz domain in $\mathbb{R}^{n}$. Then, in the context of Definition 2.3.1 and Remark 1.2.1,

$$
f \in F_{\alpha, p a r}^{p, q}(\partial \Omega \times \mathbb{R}) \Longleftrightarrow g_{i} \in F_{\alpha, p a r}^{p, q}\left(\partial \Omega_{i} \times \mathbb{R}\right) \quad \forall i=1, \ldots, N
$$

and

$$
\|f\|_{F_{\alpha, p a r}^{p, q}(\partial \Omega \times \mathbb{R})}:=\sum_{i=1}^{N}\left\|g_{i}\right\|_{F_{\alpha, p a r}^{p, q}\left(\partial \Omega_{i} \times \mathbb{R}\right)} .
$$

Remark 2.3.6. Note that

$$
\|f\|_{F_{\alpha, p a r}^{p, q}(\partial \Omega \times \mathbb{R})} \approx \inf \left[\sum_{i=1}^{N}\left\|g_{i}\right\|_{F_{\alpha, p a r}^{p, q}\left(\partial \Omega_{i} \times \mathbb{R}\right)}\right]
$$

where the infimum is taken over all smooth coverings $\left\{V_{i}\right\}_{i=1}^{N}$ as in Remark 1.2.1 and all partitions of unity $\left\{\psi_{i}\right\}_{i=1}^{N}$ subordinated to this cover.

Definition 2.3.7. (Triebel-Lizorkin spaces with negative amount of smoothness) Consider either

$$
\frac{n+1}{n+2}<p, q<\infty, \quad(n+1)\left(\frac{1}{\min \{p, q\}}-1\right)_{+}<\alpha<1
$$

or

$$
\frac{n+1}{n+2}<p<\infty, \quad q=2, \quad \alpha=1
$$

(i) If $\Omega$ is an unbounded Lipschitz domain in $\mathbb{R}^{n}$ with $\partial \Omega$ being the graph of $\varphi: \mathbb{R}^{n-1} \rightarrow \mathbb{R}$, then

$$
f \in F_{\alpha-1, p a r}^{p, q}(\partial \Omega \times \mathbb{R}) \Longleftrightarrow g \in F_{\alpha-1, p a r}^{p, q}\left(\mathbb{R}^{n-1} \times \mathbb{R}\right)
$$


where

$$
g\left(x^{\prime}, t\right):=f\left(x^{\prime}, \varphi\left(x^{\prime}\right), t\right) \sqrt{1+\left|\nabla \varphi\left(x^{\prime}\right)\right|^{2}}, \quad x^{\prime} \in \mathbb{R}^{n-1}, t \in \mathbb{R}
$$

and

$$
\|f\|_{F_{\alpha-1, p a r}^{p, q}(\partial \Omega \times \mathbb{R})}:=\|g\|_{F_{\alpha-1, p a r}^{p, q}\left(\mathbb{R}^{n-1} \times \mathbb{R}\right)} .
$$

(ii) For a bounded domain $\Omega$, with the notation of Definition 2.3 .3 and Remark 1.2.1,

$$
f \in F_{\alpha-1, p a r}^{p, q}(\partial \Omega \times \mathbb{R}) \Longleftrightarrow g_{i}={\widetilde{\psi_{i} f}}^{\partial \Omega_{i}} \in F_{\alpha-1, p a r}^{p, q}\left(\partial \Omega_{i} \times \mathbb{R}\right) \quad \forall i=1, \ldots, N
$$

and

$$
\|f\|_{F_{\alpha-1, p a r}^{p, q}(\partial \Omega \times \mathbb{R})}:=\inf \left[\sum_{i=1}^{N}\left\|g_{i}\right\|_{F_{\alpha-1, p a r}^{p, q}\left(\partial \Omega_{i} \times \mathbb{R}\right)}\right],
$$

where the infimum is taken over all smooth coverings $\left\{V_{i}\right\}_{i=1}^{N}$ and all partitions of unity $\left\{\psi_{i}\right\}_{i=1}^{N}$ subordinated to this cover.

We next introduce the notation we are going to employ for some of the distingiushed subclasses of the Triebel-Lizorkin scale.

Definition 2.3.8. Let $\Omega$ be a Lipschitz domain in $\mathbb{R}^{n}$ and $0 \leq \alpha \leq 1$. Then

$$
L_{ \pm \alpha, p a r}^{p}(\partial \Omega \times \mathbb{R}):=F_{ \pm \alpha, p a r}^{p, 2}(\partial \Omega \times \mathbb{R}) \quad \text { and } \quad \dot{L}_{ \pm \alpha, p a r}^{p}(\partial \Omega \times \mathbb{R}):=\dot{F}_{ \pm \alpha, p a r}^{p, 2}(\partial \Omega \times \mathbb{R})
$$

for $1<p<\infty$.

Definition 2.3.9. Consider a Lipschitz domain $\Omega$ in $\mathbb{R}^{n}$ and $\frac{n+1}{n+2}<p \leq 1$. Then

$$
H_{\text {par }}^{1, p}(\partial \Omega \times \mathbb{R}):=F_{1, \text { par }}^{p, 2}(\partial \Omega \times \mathbb{R}) \quad \text { and } \quad \dot{H}_{\text {par }}^{1, p}(\partial \Omega \times \mathbb{R}):=\dot{F}_{1, \text { par }}^{p, 2}(\partial \Omega \times \mathbb{R}) .
$$


Definition 2.3.10. Let $\Omega$ be a Lipschitz domain in $\mathbb{R}^{n}, I \subseteq \mathbb{R}$ an interval, and the indices $p, q, \alpha$ satisfying one of the following conditions:

(a) $\frac{n+1}{n+2}<p<\infty, \quad \frac{n+1}{n+2}<q \leq \infty, \quad(n+1)\left(\frac{1}{\min \{p, q\}}-1\right)_{+}<\alpha<1$;

(b) $\frac{n+1}{n+2}<p, q<\infty, \quad(n+1)\left(\frac{1}{\min \{p, q\}}-1\right)_{+}-1<\alpha<0$;

(c) $\frac{n+1}{n+2}<p<\infty, \quad q=2, \quad \alpha \in\{0,1\}$.

Then

$$
F_{\alpha, p a r}^{p, q}(\partial \Omega \times I):=\left.F_{\alpha, p a r}^{p, q}(\partial \Omega \times \mathbb{R})\right|_{\partial \Omega \times I}
$$

and

$$
\|f\|_{F_{\alpha, p a r}^{p, q}(\partial \Omega \times I)}:=\inf \left\{\|F\|_{F_{\alpha, p a r}^{p, q}(\partial \Omega \times \mathbb{R})}: \quad F \in F_{\alpha, p a r}^{p, q}(\partial \Omega \times \mathbb{R}),\left.F\right|_{\partial \Omega \times I}=f\right\} .
$$

Definition 2.3.11. For $1<p<\infty$ and $0 \leq \alpha \leq 1$ we define

$$
L_{\alpha, p a r}^{p}(\partial \Omega \times I):=F_{\alpha, p a r}^{p, 2}(\partial \Omega \times I)
$$

Based on (2.1.7), (2.1.8), (2.1.9) and Definitions 2.3.1, 2.3.3, 2.3.5 and 2.3.7 the following simple embedding results hold for any Lipschitz domain $\Omega$ in $\mathbb{R}^{n}$, when the corresponding indices satisfy (2.3.135) in the case of the Besov scale, and one of the conditions in (2.3.140) in the case of the Triebel-Lizorkin scale:

$$
B_{\alpha, p a r}^{p, q_{0}}(\partial \Omega \times \mathbb{R}) \hookrightarrow B_{\alpha, p a r}^{p, q_{1}}(\partial \Omega \times \mathbb{R}), \quad F_{\alpha, p a r}^{p, q_{0}}(\partial \Omega \times \mathbb{R}) \hookrightarrow F_{\alpha, p a r}^{p, q_{1}}(\partial \Omega \times \mathbb{R}), \quad \text { when } q_{0} \leq q_{1},
$$

$$
\begin{gathered}
B_{\alpha_{0}, p a r}^{p, q}(\partial \Omega \times \mathbb{R}) \hookrightarrow B_{\alpha_{1}, p a r}^{p, r}(\partial \Omega \times \mathbb{R}), \quad F_{\alpha_{0}, p a r}^{p, q}(\partial \Omega \times \mathbb{R}) \hookrightarrow F_{\alpha_{1}, p a r}^{p, r}(\partial \Omega \times \mathbb{R}) \\
\text { for } \alpha_{0}>\alpha_{1},
\end{gathered}
$$




$$
B_{\alpha, p a r}^{p, \min \{p, q\}}(\partial \Omega \times \mathbb{R}) \hookrightarrow F_{\alpha, p a r}^{p, q}(\partial \Omega \times \mathbb{R}) \hookrightarrow B_{\alpha, p a r}^{p, \max \{p, q\}}(\partial \Omega \times \mathbb{R})
$$

In view of Definitions 2.3.4 and 2.3.10, in (2.3.141), (2.3.142) and (2.3.143) we can replace $\mathbb{R}$ with an arbitrary interval $I \subseteq \mathbb{R}$.

As a consequence of Theorem 2.1.10 we obtain the following.

Proposition 2.3.12. Let $\Omega$ be an unbounded domain (in $\mathbb{R}^{n}$ ) above the graph of the Lipschitz function $\varphi: \mathbb{R}^{n-1} \rightarrow \mathbb{R}$. For any real number $\sigma$ and any $f \in \mathrm{S}^{\prime}(\partial \Omega \times \mathbb{R})$ define the operator

$$
\mathcal{I}_{\sigma}:=\Phi \circ I_{\sigma} \circ \Phi^{-1}
$$

where $I_{\sigma}$ is the operator introduced in (2.1.13) and

$$
\Phi: \mathbb{R}^{n-1} \times \mathbb{R} \longrightarrow \partial \Omega \times \mathbb{R}, \quad \Phi\left(x^{\prime}, t\right):=\left(x^{\prime}, \varphi\left(x^{\prime}\right), t\right)
$$

Then

$$
\begin{aligned}
& \mathcal{I}_{\sigma}: B_{\alpha, p a r}^{p, q}(\partial \Omega \times \mathbb{R}) \stackrel{\sim}{\longrightarrow} B_{\alpha+\sigma, p a r}^{p, q}(\partial \Omega \times \mathbb{R}), \\
& \mathcal{I}_{\sigma}: F_{\alpha, p a r}^{p, q}(\partial \Omega \times \mathbb{R}) \stackrel{\sim}{\longrightarrow} F_{\alpha+\sigma, p a r}^{p, q}(\partial \Omega \times \mathbb{R})
\end{aligned}
$$

are isomorphisms, provided that the corresponding indices satisfy (2.3.135) in the case of the Besov scale, and one of the conditions in (2.3.140) in the case of the Triebel-Lizorkin scale.

The next result is an immediate consequence of Theorem 2.1.17 and part (i) of Definitions 2.3.1, 2.3.3, 2.3.5 and 2.3.7.

Proposition 2.3.13. Let $\Omega$ be an unbounded Lipschitz domain in $\mathbb{R}^{n}, p_{0}<p_{1}$ and $\alpha_{1}:=\alpha_{0}-(n+1)\left(1 / p_{0}-1 / p_{1}\right)$. 
1. Then

$$
B_{\alpha_{0}, p a r}^{p_{0}, q}(\partial \Omega \times \mathbb{R}) \hookrightarrow B_{\alpha_{1}, p a r}^{p_{1}, q}(\partial \Omega \times \mathbb{R})
$$

as long as (2.3.135) holds for the indices $\left(p_{0}, q, \alpha_{0}\right)$ and $\left(p_{1}, q, \alpha_{1}\right)$.

2. In a similar spirit,

$$
F_{\alpha_{0}, p a r}^{p_{0}, q_{0}}(\partial \Omega \times \mathbb{R}) \hookrightarrow F_{\alpha_{1}, p a r}^{p_{1}, q_{1}}(\partial \Omega \times \mathbb{R})
$$

provided that the indices $\left(p_{0}, q, \alpha_{0}\right)$ and $\left(p_{1}, q, \alpha_{1}\right)$ satisfy one of the conditions in (2.3.140).

In the following lemmas we give characterizations of the parabolic Besov space $B_{\alpha, p a r}^{p, q}(\partial \Omega \times I)$ and of the parabolic Triebel-Lizorkin scale $F_{\alpha, p a r}^{p, q}(\partial \Omega \times I)$.

Lemma 2.3.14. Let $I \subseteq \mathbb{R}$ be an interval and consider either $\frac{n+1}{n+2}<p, q \leq \infty$, $(n+1)\left(\frac{1}{p}-1\right)_{+}<\alpha<1$ or $\frac{n+1}{n+2}<p, q<\infty,(n+1)\left(\frac{1}{p}-1\right)_{+}-1<\alpha<0$.

(i) If $\Omega$ is an unbounded domain (in $\mathbb{R}^{n}$ ) above the graph of the Lipschitz function $\varphi: \mathbb{R}^{n-1} \rightarrow \mathbb{R}$, then

$$
B_{\alpha, p a r}^{p, q}(\partial \Omega \times I)=\left\{f \in L^{p}(\partial \Omega \times I): \quad g \in B_{\alpha, p a r}^{p, q}\left(\mathbb{R}^{n-1} \times I\right)\right\}
$$

where, for $\left(x^{\prime}, t\right) \in \mathbb{R}^{n-1} \times I$,

$$
g\left(x^{\prime}, t\right):= \begin{cases}f\left(x^{\prime}, \varphi\left(x^{\prime}\right), t\right), & \text { if } \alpha>0, \\ f\left(x^{\prime}, \varphi\left(x^{\prime}\right), t\right) \sqrt{1+\left|\nabla \varphi\left(x^{\prime}\right)\right|^{2}}, & \text { if } \alpha<0 .\end{cases}
$$

(ii) If $\Omega$ is a bounded Lipschitz domain, in the context of Definition 2.3.1 and Remark 1.2.1, we have

$$
B_{\alpha, p a r}^{p, q}(\partial \Omega \times I)=\left\{f \in L^{p}(\partial \Omega \times I):\left.\quad g_{i}\right|_{I} \in B_{\alpha, p a r}^{p, q}\left(\partial \Omega_{i} \times I\right) \text { for each } i=1, \ldots, N\right\}
$$


Proof. We shall carry out the proof only for spaces with positive amount of smoothness, since the case with negative smoothness is treated in a similar fashion.

(i) Let $f \in B_{\alpha, \text { par }}^{p, q}(\partial \Omega \times I)$. Then, by Definition 2.3 .4 , there exists a function $F \in B_{\alpha, p a r}^{p, q}(\partial \Omega \times \mathbb{R})$ with $\left.F\right|_{\partial \Omega \times I}=f$, and, from Definition 2.3.1 we have that

$$
F \in L^{p}(\partial \Omega \times \mathbb{R}) \text { and } F \circ \Phi \in B_{\alpha, p a r}^{p, q}\left(\mathbb{R}^{n-1} \times \mathbb{R}\right)
$$

where

$$
\Phi: \mathbb{R}^{n-1} \times \mathbb{R} \longrightarrow \partial \Omega \times \mathbb{R}, \quad \Phi\left(x^{\prime}, t\right):=\left(x^{\prime}, \varphi\left(x^{\prime}\right), t\right)
$$

For $\left(x^{\prime}, t\right) \in \mathbb{R}^{n} \times I$ we can write

$$
\left(\left.f \circ \Phi\right|_{\mathbb{R}^{n-1} \times I}\right)\left(x^{\prime}, t\right)=f\left(x^{\prime}, \varphi\left(x^{\prime}\right), t\right)=\left(\left.F\right|_{\partial \Omega \times I}\right)\left(x^{\prime}, \varphi\left(x^{\prime}\right), t\right)=\left.(F \circ \Phi)\right|_{\mathbb{R}^{n-1} \times I}\left(x^{\prime}, t\right),
$$

and, since $F \circ \Phi \in B_{\alpha, p a r}^{p, q}\left(\mathbb{R}^{n} \times \mathbb{R}\right)$, we obtain that

$$
g=\left.f \circ \Phi\right|_{\mathbb{R}^{n-1} \times I} \in B_{\alpha, p a r}^{p, q}\left(\mathbb{R}^{n} \times I\right) .
$$

Moreover, $F \in L^{p}(\partial \Omega \times \mathbb{R})$ and $\left.F\right|_{\partial \Omega \times I}=f$ readily yield $f \in L^{p}(\partial \Omega \times I)$. This, in concert with (2.3.148) implies that $f$ belongs to the right-hand side of (2.3.145).

Conversely, suppose $f \in L^{p}(\partial \Omega \times I)$ and $g \in B_{\alpha, \text { par }}^{p, q}\left(\mathbb{R}^{n-1} \times I\right)$. Then, by Definition 2.2.1, there exists a function $F \in B_{\alpha, p a r}^{p, q}\left(\mathbb{R}^{n-1} \times \mathbb{R}\right)$ with $\left.F\right|_{\mathbb{R}^{n-1} \times I}=g$. For $\left(x^{\prime}, t\right) \in \mathbb{R}^{n-1} \times I$ we have

$$
f\left(x^{\prime}, \varphi\left(x^{\prime}\right), t\right)=g\left(x^{\prime}, t\right)=F\left(x^{\prime}, t\right)=\left(F \circ \pi_{0}\right)\left(x^{\prime}, \varphi\left(x^{\prime}\right), t\right),
$$

where

$$
\pi_{0}: \partial \Omega \times I \longrightarrow \mathbb{R}^{n-1} \times I, \quad \pi_{0}\left(x^{\prime}, \varphi\left(x^{\prime}\right), t\right):=\left(x^{\prime}, t\right) .
$$


Since $\pi: \partial \Omega \times \mathbb{R} \rightarrow \mathbb{R}^{n-1} \times \mathbb{R}$ is defined by $\pi\left(x^{\prime}, \varphi\left(x^{\prime}\right), t\right):=\left(x^{\prime}, t\right)$, we obtain that $\left.(F \circ \pi)\right|_{\partial \Omega \times I}=F \circ \pi_{0}=f$ and $F \circ \pi \in B_{\alpha, p a r}^{p, q}(\partial \Omega \times \mathbb{R})$. This amounts to $f \in B_{\alpha, p a r}^{p, q}(\partial \Omega \times I)$, completing the proof of (i).

(ii) First, consider $f$ from $B_{\alpha, \text { par }}^{p, q}(\partial \Omega \times I)$. Then, by Definition 2.3.4, there exists a function $F \in B_{\alpha, p a r}^{p, q}(\partial \Omega \times \mathbb{R})$ such that $\left.F\right|_{\partial \Omega \times I}=f$. Moreover, by Definition 2.3.1, with the notation of Remark 1.2.1, $F \in L^{p}(\partial \Omega \times \mathbb{R})$ and $G_{i}={\widetilde{\psi_{i} F}}^{\partial \Omega_{i}}$ belongs to $B_{\alpha, p a r}^{p, q}\left(\partial \Omega_{i} \times \mathbb{R}\right)$, where recall that $\left\{\psi_{i}\right\}_{i=1}^{N}$ is a partition of unity on $\partial \Omega$ and $\sim \partial \Omega_{i}$ stands for the extension by 0 to $\partial \Omega_{i}$. For $(y, t) \in\left(\partial \Omega \cap V_{i}\right) \times I, i=1, \ldots, N$, we have

$$
G_{i}(y, t)={\widetilde{\psi_{i} F}}^{\partial \Omega_{i}}(y, t)={\widetilde{\psi_{i} f}}^{\partial \Omega_{i}}(y, t)=g_{i}(y, t)
$$

therefore, $\left.g_{i}\right|_{I} \in B_{\alpha, \text { par }}^{p, q}\left(\partial \Omega_{i} \times I\right)$, for each $i=1, \ldots, N$. This, together with the fact that $f \in L^{p}(\partial \Omega \times I)$ (which is immediate), implies that $f$ belongs to the right-hand side of (2.3.147).

Conversely, let $f \in L^{p}(\partial \Omega \times I)$ and $\left.g_{i}\right|_{I} \in B_{\alpha, p a r}^{p, q}\left(\partial \Omega_{i} \times I\right)$ for each $i=1, \ldots, N$. By definition, $g_{i} \in B_{\alpha, p a r}^{p, q}\left(\partial \Omega_{i} \times \mathbb{R}\right)$, and $g_{i}={\widetilde{\psi_{i} F}}^{\partial \Omega_{i}}$ with $F \in B_{\alpha, p a r}^{p, q}(\partial \Omega \times \mathbb{R})$. Since

$$
{\widetilde{\psi_{i} f}}^{\partial \Omega_{i}}=\left.g_{i}\right|_{I}=\psi_{i}{\widetilde{\left(\left.F\right|_{\partial \Omega \times I}\right)}}^{\partial \Omega_{i}}
$$

we also have that $\left.F\right|_{\partial \Omega \times I}=f$. With this in hand, taking also into account that $F$ belongs to the space $B_{\alpha, p a r}^{p, q}(\partial \Omega \times \mathbb{R})$, based on Definition 2.3.4 we can conclude that $f \in B_{\alpha, p a r}^{p, q}(\partial \Omega \times I)$, completing the proof of (ii) and of the lemma.

In a very similar spirit to the proof of Lemma 2.3.14, using Definitions 2.3.5 and 2.3.10, one can show the following. 
Lemma 2.3.15. Let $I \subseteq \mathbb{R}$ be an interval and assume that the indices $p, q, \alpha$ are such that one of the conditions in (2.3.140) is satisfied.

(i) Consider an unbounden Lipschitz domain $\Omega \subseteq \mathbb{R}^{n}$, whose boundary $\partial \Omega$ is the graph of (the Lipschitz function) $\varphi: \mathbb{R}^{n-1} \rightarrow \mathbb{R}$. Then

$$
F_{\alpha, p a r}^{p, q}(\partial \Omega \times I)=\left\{f \in L^{p}(\partial \Omega \times I): \quad g \in F_{\alpha, p a r}^{p, q}\left(\mathbb{R}^{n-1} \times I\right)\right\}
$$

where $g$ was given in (2.3.146).

(ii) Let $\Omega$ be a bounded Lipschitz domain in $\mathbb{R}^{n}$. Then, using the notation of Definition 2.3.1 and Remark 1.2.1,

$F_{\alpha, \text { par }}^{p, q}(\partial \Omega \times I)=\left\{f \in L^{p}(\partial \Omega \times I):\left.\quad g_{i}\right|_{I} \in F_{\alpha, p a r}^{p, q}\left(\partial \Omega_{i} \times I\right)\right.$ for each $\left.i=1, \ldots, N\right\}$.

We next present the Fubini property of the Besov scale $B_{\alpha, p a r}^{p}(\partial \Omega \times I)$.

Proposition 2.3.16. Let $\Omega$ be a Lipschitz domain in $\mathbb{R}^{n}, I \subseteq \mathbb{R}$ an interval, and assume that $1 \leq p \leq \infty$ and $0<\alpha<1$. There holds

$$
B_{\alpha, p a r}^{p}(\partial \Omega \times I)=L^{p}\left(\partial \Omega ; B_{\alpha / 2}^{p}(I)\right) \cap L^{p}\left(I ; B_{\alpha}^{p}(\partial \Omega)\right) .
$$

Proof. (i) First consider the unbounded case (i.e. $\partial \Omega$ is the graph of a Lipschitz function $\varphi: \mathbb{R}^{n-1} \rightarrow \mathbb{R}$ ). Using the first part of Lemma 2.3.14 and Proposition 2.2.3 with $\Omega=\mathbb{R}^{n-1}$, we obtain the following equivalences 


$$
\begin{aligned}
& f \in B_{\alpha, p a r}^{p}(\partial \Omega \times I) \Longleftrightarrow f \in L^{p}(\partial \Omega \times I) \quad \text { and } \quad f \circ \Phi_{0} \in B_{\alpha, p a r}^{p}\left(\mathbb{R}^{n-1} \times I\right) \\
& \Longleftrightarrow f \circ \Phi_{0} \in L^{p}\left(\mathbb{R}^{n-1} ; B_{\alpha / 2}^{p}(I)\right) \quad \text { and } \quad f \circ \Phi_{0} \in L^{p}\left(I ; B_{\alpha}^{p}\left(\mathbb{R}^{n-1}\right)\right) \\
& \Longleftrightarrow \int_{\mathbb{R}^{n-1}}\left\|f\left(\Phi_{0}\left(x^{\prime}, \cdot\right)\right)\right\|_{B_{\alpha / 2}^{p}(I)}^{p} d x^{\prime}<\infty \quad \text { and } \quad \int_{I}\left\|f\left(\Phi_{0}(\cdot, t)\right)\right\|_{B_{\alpha}^{p}\left(\mathbb{R}^{n-1}\right)}^{p} d t<\infty \\
& \Longleftrightarrow \int_{\mathbb{R}^{n-1}}\left\|f\left(x^{\prime}, \varphi\left(x^{\prime}\right), \cdot\right)\right\|_{B_{\alpha / 2}^{p}(I)}^{p} d x^{\prime}<\infty \text { and } \int_{I}\|f(\cdot, \varphi(\cdot), t)\|_{B_{\alpha}^{p}\left(\mathbb{R}^{n-1}\right)}^{p} d t<\infty \\
& \stackrel{\substack{y=\left(x^{\prime}, \varphi\left(x^{\prime}\right)\right) \in \partial \Omega \\
d \sigma y \approx d x^{\prime}}}{\Longleftrightarrow} \int_{\partial \Omega}\|f(y, \cdot)\|_{B_{\alpha / 2}^{p}(I)}^{p} d x \sigma_{y}<\infty \quad \text { and } \quad \int_{I}\|f(\cdot, t)\|_{B_{\alpha}^{p}(\partial \Omega)}^{p} d t<\infty \\
& \Longleftrightarrow f \in L^{p}\left(\partial \Omega ; B_{\alpha / 2}^{p}(I)\right) \text { and } f \in L^{p}\left(I ; B_{\alpha}^{p}(\partial \Omega)\right) \text {. }
\end{aligned}
$$

This completes the proof of the proposition in case (i).

(ii) Second, let $\Omega$ be a bounded Lipschitz domain in $\mathbb{R}^{n}$, with the notation of Remark 1.2.1. Then Lemma 2.3.14 and the previous (unbounded) case of this proposition for each $\Omega_{i}, i=1, \ldots, N$, yield

$$
\begin{aligned}
f \in B_{\alpha, p a r}^{p}(\partial \Omega \times I) & \Longleftrightarrow f \in L^{p}(\partial \Omega \times I) \quad \text { and }\left.\quad g_{i}\right|_{I} \in B_{\alpha, p a r}^{p}\left(\partial \Omega_{i} \times I\right), \quad 1 \leq i \leq N \\
& \left.\Longleftrightarrow g_{i}\right|_{I} \in L^{p}\left(\partial \Omega_{i} ; B_{\alpha / 2}^{p}(I)\right) \cap L^{p}\left(I ; B_{\alpha}^{p}\left(\partial \Omega_{i}\right)\right), \quad 1 \leq i \leq N,
\end{aligned}
$$

which is further equivalent with

$$
\int_{\partial \Omega_{i}}\left\|g_{i}(y, \cdot)\right\|_{B_{\alpha / 2}^{p}(I)}^{p} d \sigma_{y}<\infty, \quad i=1, \ldots, N
$$

and

$$
\int_{I}\left\|g_{i}(\cdot, t)\right\|_{B_{\alpha}^{p}\left(\partial \Omega_{i}\right)}^{p} d t<\infty, \quad i=1, \ldots, N
$$

Since (2.3.149) holds for each $i=1, \ldots, N$,

$$
\int_{\partial \Omega}\|f(y, \cdot)\|_{B_{\alpha / 2}^{p}(I)}^{p} d \sigma_{y} \approx \sum_{i=1}^{N} \int_{\partial \Omega_{i}}\left\|g_{i}(y, \cdot)\right\|_{B_{\alpha / 2}^{p}(I)}^{p} d \sigma_{y}<\infty
$$


and this amounts to

$$
f \in L^{p}\left(\partial \Omega ; B_{\alpha / 2}^{p}(I)\right)
$$

Moreover,

$$
\sum_{i=1}^{N} \int_{I}\left\|g_{i}(\cdot, t)\right\|_{B_{\alpha}^{p}\left(\partial \Omega_{i}\right)}^{p} d t \approx \int_{I}\left[\sum_{i=1}^{N}\left\|g_{i}(\cdot, t)\right\|_{B_{\alpha}^{p}\left(\partial \Omega_{i}\right)}\right]^{p} d t \approx \int_{I}\|f(\cdot, t)\|_{B_{\alpha}^{p}(\partial \Omega)}^{p} d t
$$

and by (2.3.150),

$$
f \in L^{p}\left(I ; B_{\alpha}^{p}(\partial \Omega)\right)
$$

In view of (2.3.151) and (2.3.152) the proof of the proposition is finished.

Our next discussion is about the identification of $B_{\alpha, p a r}^{\infty}(\partial \Omega \times I)$ with the parabolic Hölder space $C_{\text {par }}^{\alpha}(\partial \Omega \times I)$. We start with the definition of the latter.

Definition 2.3.17. Let $\Omega$ be a Lipschitz domain in $\mathbb{R}^{n}, I \subseteq \mathbb{R}$ an interval. For $0<\alpha<1$ we define

$$
C_{p a r}^{\alpha}(\partial \Omega \times I):=\left\{f:\|f\|_{C_{p a r}^{\alpha}(\partial \Omega \times I)}<\infty\right\}
$$

where

$$
\|f\|_{C_{p a r}^{\alpha}(\partial \Omega \times I)}:=\|f\|_{\left.L^{\infty}(\partial \Omega \times I)\right)}+\sup _{\substack{(x, t),(y, s) \in \partial \Omega \times I \\(x, t) \neq(y, s)}} \frac{|f(x, t)-f(y, s)|}{\left(|x-y|+|t-s|^{1 / 2}\right)^{\alpha}} .
$$

Lemma 2.3.18. Let $\Omega$ be a Lipschitz domain in $\mathbb{R}^{n}, I \subseteq \mathbb{R}$ an interval, and consider $0<\alpha<1$. Then

$$
B_{\alpha, p a r}^{\infty}(\partial \Omega \times I)=C_{p a r}^{\alpha}(\partial \Omega \times I)
$$

Proof. First let us invoke the definition of the space of Hölder continuous functions on $\partial \Omega$ in the isotropic setting: $B_{\alpha}^{\infty}(\partial \Omega), 0<\alpha<1$, is defined as the Banach space 
of measurable functions $f$ on $\partial \Omega$ such that

$$
\|f\|_{B_{\alpha}^{\infty}(\partial \Omega)}:=\|f\|_{L^{\infty}(\partial \Omega)}+\sup _{\substack{x, y \in \partial \Omega \\ x \neq y}} \frac{|f(x)-f(y)|}{|x-y|^{\alpha}}<\infty .
$$

Assume that $f$ belongs to $B_{\alpha, \text { par }}^{\infty}(\partial \Omega \times I)$. On account of Proposition 2.3.16 with $p=\infty$ and of the definition in (2.3.154), it follows that $\sup _{x \in \partial \Omega, t \in I}|f(x, t)|<\infty$,

$$
\sup _{x \in \partial \Omega} \sup _{\substack{t, s \in I \\ t \neq s}} \frac{|f(x, t)-f(x, s)|}{|t-s|^{\alpha / 2}}<\infty
$$

and

$$
\sup _{t \in I} \sup _{\substack{x, y \in \partial \Omega \\ x \neq y}} \frac{|f(x, t)-f(y, t)|}{|x-y|^{\alpha}}<\infty .
$$

From (2.3.155) and (2.3.156), by adding and subtracting $f(y, t)$, and using elementary estimates, we obtain that

$$
\sup _{\substack{(x, t),(y, s) \in \partial \Omega \times I \\(x, t) \neq(y, s)}} \frac{|f(x, t)-f(y, s)|}{\left(|x-y|+|t-s|^{1 / 2}\right)^{\alpha}}<\infty,
$$

which proves the left-to-right inclusion in (2.3.153).

Conversely, assume that $f$ belongs to the right-hand side of (2.3.153). Then, by definition,

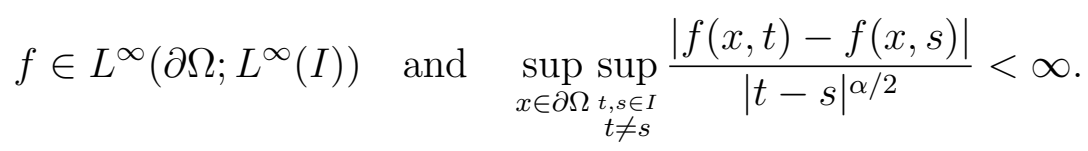

With (2.3.154) in hand, (2.3.157) further implies that

$$
f \in L^{\infty}\left(\partial \Omega ; B_{\alpha / 2}^{\infty}(I)\right)
$$

Also, by definition, $f \in L^{\infty}\left(I ; L^{\infty}(\partial \Omega)\right)$ and

$$
\sup _{t \in I} \sup _{\substack{x, y \in \partial \Omega \\ x \neq y}} \frac{|f(x, t)-f(y, t)|}{|x-y|^{\alpha}}<\infty
$$


from which we can deduce that

$$
f \in L^{\infty}\left(I ; B_{\alpha}^{\infty}(\partial \Omega)\right)
$$

Consequently, (2.3.158), (2.3.159) and Proposition 2.3.16 (with $p=\infty$ ) yield the right-to-left inclusion in (2.3.153), completing the proof of the lemma.

Next, we note an intrinsic characterization of $B_{\alpha, p a r}^{p}(\partial \Omega \times I)$ for $p<\infty$.

Lemma 2.3.19. Let $\Omega$ be a Lipschitz domain in $\mathbb{R}^{n}, I \subseteq \mathbb{R}$ an interval, $1 \leq p<\infty$ and $0<\alpha<1$. Then $f \in B_{\alpha, p a r}^{p}(\partial \Omega \times I)$ if and only if

$$
f \in L^{p}(\partial \Omega \times I) \quad \text { and } \quad \int_{\partial \Omega \times I} \int_{\partial \Omega \times I} \frac{|f(x, t)-f(y, s)|^{p}}{\left(|x-y|+|t-s|^{1 / 2}\right)^{n+1+\alpha p}} d \sigma_{y} d s d \sigma_{x} d t<\infty .
$$

Proof. Case (i) $\Omega$ is unbounded. Consider $f$ from the Besov space $B_{\alpha, p a r}^{p}(\partial \Omega \times I)$. Then, by definition, there exists a function $F$ from $B_{\alpha, p a r}^{p}(\partial \Omega \times \mathbb{R})$ such that its restriction to $\partial \Omega \times I$ is $f$. Moreover, $F \in L^{p}(\partial \Omega \times \mathbb{R})$ and $F \circ \Phi \in B_{\alpha, p a r}^{p}\left(\mathbb{R}^{n-1} \times \mathbb{R}\right)$ with $\left.F\right|_{\partial \Omega \times I}=f$. By Lemma 2.2 .5 with $\Omega=\mathbb{R}^{n-1}$ and $I=\mathbb{R}$ we have

$$
\int_{\mathbb{R}^{n-1} \times \mathbb{R}} \int_{\mathbb{R}^{n-1} \times \mathbb{R}} \frac{\left|(F \circ \Phi)\left(x^{\prime}, t\right)-(F \circ \Phi)\left(y^{\prime}, s\right)\right|^{p}}{\left(\left|x^{\prime}-y^{\prime}\right|+|t-s|^{1 / 2}\right)^{n+1+\alpha p}} d y^{\prime} d s d x^{\prime} d t<\infty,
$$

or, equivalently,

$$
\int_{\mathbb{R}^{n-1} \times \mathbb{R}} \int_{\mathbb{R}^{n-1} \times \mathbb{R}} \frac{\left|F\left(x^{\prime}, \varphi\left(x^{\prime}\right), t\right)-F\left(y^{\prime}, \varphi\left(y^{\prime}\right), s\right)\right|^{p}}{\left(\left|x^{\prime}-y^{\prime}\right|+|t-s|^{1 / 2}\right)^{n+1+\alpha p}} d y^{\prime} d s d x^{\prime} d t<\infty .
$$

We make the change of variables $x=\left(x^{\prime}, \varphi\left(x^{\prime}\right)\right) \in \partial \Omega$ and $y=\left(y^{\prime}, \varphi\left(y^{\prime}\right)\right) \in \partial \Omega$. Then $\left|x^{\prime}-y^{\prime}\right| \approx|x-y|, d x^{\prime} \approx d \sigma_{x}, d y^{\prime} \approx d \sigma_{y}$ and the above integral inequality becomes

$$
\int_{\partial \Omega \times \mathbb{R}} \int_{\partial \Omega \times \mathbb{R}} \frac{|F(x, t)-F(y, s)|^{p}}{\left(|x-y|+|t-s|^{1 / 2}\right)^{n+1+\alpha p}} d \sigma_{y} d s d \sigma_{x} d t<\infty
$$


Taking into account (2.3.161) and the fact that $f=\left.F\right|_{\partial \Omega \times I}$, we obtain $f \in L^{p}(\partial \Omega \times I)$ and

$$
\begin{aligned}
& \int_{\partial \Omega \times I} \int_{\partial \Omega \times I} \frac{|f(x, t)-f(y, s)|^{p}}{\left(|x-y|+|t-s|^{1 / 2}\right)^{n+1+\alpha p}} d \sigma_{y} d s d \sigma_{x} d t \\
& \leq \int_{\partial \Omega \times \mathbb{R}} \int_{\partial \Omega \times \mathbb{R}} \frac{|F(x, t)-F(y, s)|^{p}}{\left(|x-y|+|t-s|^{1 / 2}\right)^{n+1+\alpha p}} d \sigma_{y} d s d \sigma_{x} d t<\infty,
\end{aligned}
$$

as desired.

Conversely, assume $f$ satisfies (2.3.160) and define the function $G$ on $\mathbb{R}^{n-1} \times I$ by $G\left(x^{\prime}, t\right):=f\left(x^{\prime}, \varphi\left(x^{\prime}\right), t\right)$. Then $G \in L^{p}\left(\mathbb{R}^{n-1} \times I\right)$ and

$$
\int_{\mathbb{R}^{n-1} \times I} \int_{\mathbb{R}^{n-1} \times I} \frac{\left|G\left(x^{\prime}, t\right)-G\left(y^{\prime}, s\right)\right|^{p}}{\left(\left|x^{\prime}-y^{\prime}\right|+|t-s|^{1 / 2}\right)^{n+1+\alpha p}} d y^{\prime} d s d x^{\prime} d t<\infty,
$$

which, according to Lemma 2.2 .5 (with $\Omega=\mathbb{R}^{n-1}$ ), amounts to $G \in B_{\alpha, p a r}^{p}\left(\mathbb{R}^{n-1} \times I\right)$. By Definition 2.2.1 (with $n-1$ in place of $n$ and $\mathcal{O}=\mathbb{R}^{n-1} \times I$ ), there exists $\tilde{G}$ belonging to the Besov space $B_{\alpha, p a r}^{p}\left(\mathbb{R}^{n-1} \times \mathbb{R}\right)$ whose restriction to $\mathbb{R}^{n-1} \times I$ is $G$. Finally, define $\tilde{f}\left(x^{\prime}, \varphi\left(x^{\prime}\right), t\right):=\tilde{G}\left(x^{\prime}, t\right)$. Then $\tilde{f} \in B_{\alpha, p a r}^{p}(\partial \Omega \times \mathbb{R})$ and $\left.\tilde{f}\right|_{\partial \Omega \times I}=f$, which implies that $f \in B_{\alpha, p a r}^{p}(\partial \Omega \times I)$.

Case (ii) $\Omega$ is bounded. According to Definitions 2.3.4 and 2.3.1, a function $f$ belongs to the space $B_{\alpha, p a r}^{p}(\partial \Omega \times I)$ if and only if there exists a function $F$ from $B_{\alpha, p a r}^{p}(\partial \Omega \times \mathbb{R})$ such that $\left.F\right|_{\partial \Omega \times I}=f$, and $G_{i}:=\widetilde{\psi_{i} F}{ }^{\partial \Omega_{i}} \in B_{\alpha, p a r}^{p}\left(\partial \Omega_{i} \times \mathbb{R}\right)$ for each $i=1, \ldots, N$. Now using Case (i) for each unbounded domain $\Omega_{i}, i=1, \ldots, N$,

$$
\int_{\partial \Omega_{i} \times \mathbb{R}} \int_{\partial \Omega_{i} \times \mathbb{R}} \frac{\left|G_{i}(x, t)-G_{i}(y, s)\right|^{p}}{\left(|x-y|+|t-s|^{1 / 2}\right)^{n+1+\alpha p}} d \sigma_{y} d s d \sigma_{x} d t<\infty
$$

for each $i=1, \ldots, N$, which is equivalent with

$$
\int_{\partial \Omega \times \mathbb{R}} \int_{\partial \Omega \times \mathbb{R}} \frac{|F(x, t)-F(y, s)|^{p}}{\left(|x-y|+|t-s|^{1 / 2}\right)^{n+1+\alpha p}} d \sigma_{y} d s d \sigma_{x} d t<\infty .
$$


Since $\left.F\right|_{\partial \Omega \times I}=f$, and $F \in L^{p}(\partial \Omega \times \mathbb{R})$, we readily obtain (2.3.160). The other direction can be carried out in the same way as in the unbounded case, each step is justified by definitions and properties given earlier.

In the next part of this section we record several interpolation properties of anisotropic Besov and Triebel-Lizorkin spaces defined on the lateral boundary of a Lipschitz cylinder. Recall that, this time,

$$
\sigma_{p, q}:=\max \left\{0,(n+1)\left(\frac{1}{p}-1\right),(n+1)\left(\frac{1}{q}-1\right)\right\}
$$

and

$$
\sigma_{p}:=\max \left\{0,(n+1)\left(\frac{1}{p}-1\right)\right\} .
$$

We also note that the equivalent forms of $\sigma_{p, q}$ and $\sigma_{p}$ used in the definition of TriebelLizorkin and Besov spaces on the lateral side of a Lipschitz cylinder were

$$
\sigma_{p, q}=(n+1)\left(\frac{1}{\min \{p, q\}}-1\right)_{+} \quad \text { and } \quad \sigma_{p}=(n+1)\left(\frac{1}{p}-1\right)_{+},
$$

where $(a)_{+}:=\max \{a, 0\}$.

Theorem 2.3.20. Let $\Omega$ be a Lipschitz domain in $\mathbb{R}^{n}$ and consider $I \subseteq \mathbb{R}$ an interval. Then the following interpolation formulas hold.

(a) Assume that

$$
\begin{array}{ll}
\text { either } & \frac{n+1}{n+2}<p, q_{0}<\infty, \quad \sigma_{p, q_{0}}<\alpha_{0}<1, \\
\text { or } \quad & \frac{n+1}{n+2}<p<\infty, \quad q_{0}=2, \quad \alpha_{0}=1,
\end{array}
$$

and

$$
\begin{array}{ll}
\text { either } & \frac{n+1}{n+2}<p, q_{1}<\infty, \quad \sigma_{p, q_{1}}<\alpha_{1}<1, \\
\text { or } \quad & \frac{n+1}{n+2}<p<\infty, \quad q_{1}=2, \quad \alpha_{1}=1,
\end{array}
$$


with the additional property that $\alpha_{0} \neq \alpha_{1}$. Then

$$
\left(F_{\alpha_{0}, p a r}^{p, q_{0}}(\partial \Omega \times I), F_{\alpha_{1}, p a r}^{p, q_{1}}(\partial \Omega \times I)\right)_{\theta, q}=B_{\alpha, p a r}^{p, q}(\partial \Omega \times I)
$$

for $0<\theta<1, \frac{n+1}{n+2}<q \leq \infty$ and $\alpha=(1-\theta) \alpha_{0}+\theta \alpha_{1}$.

(b) Suppose that

$$
\begin{array}{ll}
\text { either } & \frac{n+1}{n+2}<p, q_{0}<\infty, \quad \sigma_{p, q_{0}}<\alpha_{0}<1, \\
\text { or } \quad & \frac{n+1}{n+2}<p<\infty, \quad q_{0}=2, \quad \alpha_{0}=1,
\end{array}
$$

and $\frac{n+1}{n+2}<p<\infty, \frac{n+1}{n+2}<q_{1} \leq \infty, \sigma_{p}<\alpha_{1}<1$ such that $\alpha_{0} \neq \alpha_{1}$. Then

$$
\left(F_{\alpha_{0}, p a r}^{p, q_{0}}(\partial \Omega \times I), B_{\alpha_{1}, p a r}^{p, q_{1}}(\partial \Omega \times I)\right)_{\theta, q}=B_{\alpha, p a r}^{p, q}(\partial \Omega \times I),
$$

where $0<\theta<1, \frac{n+1}{n+2}<q \leq \infty$ and $\alpha=(1-\theta) \alpha_{0}+\theta \alpha_{1}$.

(c) If $\sigma_{p_{i}}<\alpha_{i}<1, i=0,1$, and $0<\theta<1$, $\frac{1}{p}=\frac{1-\theta}{p_{0}}+\frac{\theta}{p_{1}}=\frac{1-\theta}{q_{0}}+\frac{\theta}{q_{1}}$, $\alpha=(1-\theta) \alpha_{0}+\theta \alpha_{1}$, then

$$
\left(B_{\alpha_{0}, p a r}^{p_{0}, q_{0}}(\partial \Omega \times I), B_{\alpha_{1}, p a r}^{p_{1}, q_{1}}(\partial \Omega \times I)\right)_{\theta, p}=B_{\alpha, p a r}^{p}(\partial \Omega \times I)
$$

either for $\frac{n+1}{n+2}<p_{0}<p_{1}<\infty, \frac{n+1}{n+2}<q_{0}<q_{1}<\infty$, or for $\frac{n+1}{n+2}<p_{0}, p_{1}, q_{0}, q_{1}<\infty$, $\alpha_{0} \neq \alpha_{1}$.

(d) If $\frac{n+1}{n+2}<p<\infty, \frac{n+1}{n+2}<q_{0}, q_{1} \leq \infty$, and $\sigma_{p_{i}}<\alpha_{i}<1, i=0,1$, with $\alpha_{0} \neq \alpha_{1}$, then

$$
\left(B_{\alpha_{0}, p a r}^{p, q_{0}}(\partial \Omega \times I), B_{\alpha_{1}, p a r}^{p, q_{1}}(\partial \Omega \times I)\right)_{\theta, q}=B_{\alpha, p a r}^{p, q}(\partial \Omega \times I),
$$

where $0<\theta<1, \frac{n+1}{n+2}<q \leq \infty$ and $\alpha=(1-\theta) \alpha_{0}+\theta \alpha_{1}$.

(e) Assume that

$$
\begin{aligned}
& \text { either } \frac{n+1}{n+2}<p_{0}, p_{1}, q<\infty, \quad \sigma_{p_{0}, q}<\alpha<1, \quad \sigma_{p_{1}, q}<\alpha, \\
& \text { or } \quad \frac{n+1}{n+2}<p_{0}, p_{1}<\infty, \quad q=2, \quad \alpha=1,
\end{aligned}
$$


with the additional property that $p_{0}<p_{1}$. Then

$$
\left(F_{\alpha, p a r}^{p_{0}, q}(\partial \Omega \times I), F_{\alpha, p a r}^{p_{1}, q}(\partial \Omega \times I)\right)_{\theta, p}=F_{\alpha, p a r}^{p, q}(\partial \Omega \times I)
$$

for $0<\theta<1$ and $\frac{1}{p}=\frac{1-\theta}{p_{0}}+\frac{\theta}{p_{1}}$.

(f) Suppose that

$$
\begin{aligned}
& \text { either } \frac{n+1}{n+2}<p_{0}, q_{0}<\infty, \quad \sigma_{p_{0}, q_{0}}<\alpha_{0}<1, \\
& \text { or } \quad \frac{n+1}{n+2}<p_{0}<\infty, \quad q_{0}=2, \quad \alpha_{0}=1
\end{aligned}
$$

and

$$
\begin{array}{ll}
\text { either } & \frac{n+1}{n+2}<p_{1}, q_{1}<\infty, \quad \sigma_{p_{1}, q_{1}}<\alpha_{1}<1, \\
\text { or } \quad & \frac{n+1}{n+2}<p_{1}<\infty, \quad q_{1}=2, \quad \alpha_{1}=1 .
\end{array}
$$

Then

$$
\left[F_{\alpha_{0}, p a r}^{p_{0}, q_{0}}(\partial \Omega \times I), F_{\alpha_{1}, p a r}^{p_{1}, q_{1}}(\partial \Omega \times I)\right]_{\theta}=F_{\alpha, p a r}^{p, q}(\partial \Omega \times I)
$$

for $0<\theta<1, \frac{1}{p}=\frac{1-\theta}{p_{0}}+\frac{\theta}{p_{1}}, \frac{1}{q}=\frac{1-\theta}{q_{0}}+\frac{\theta}{q_{1}}$ and $\alpha=(1-\theta) \alpha_{0}+\theta \alpha_{1}$.

(g) If $\frac{n+1}{n+2}<p_{i}<\infty, \frac{n+1}{n+2}<q_{i} \leq \infty$ and $\sigma_{p_{i}}<\alpha_{i}<1$, $i=0$, 1, then

$$
\left[B_{\alpha_{0}, p a r}^{p_{0}, q_{0}}(\partial \Omega \times I), B_{\alpha_{1}, p a r}^{p_{1}, q_{1}}(\partial \Omega \times I)\right]_{\theta}=B_{\alpha, p a r}^{p, q}(\partial \Omega \times I)
$$

for $0<\theta<1, \frac{1}{p}=\frac{1-\theta}{p_{0}}+\frac{\theta}{p_{1}}, \frac{1}{q}=\frac{1-\theta}{q_{0}}+\frac{\theta}{q_{1}}$ and $\alpha=(1-\theta) \alpha_{0}+\theta \alpha_{1}$.

(h) If $\frac{n+1}{n+2}<p_{i}<\infty$ and $\sigma_{p_{i}}<\alpha_{i}<1, i=0,1$, then

$$
\left(B_{\alpha_{0}, p a r}^{p_{0}}(\partial \Omega \times I), B_{\alpha_{1}, p a r}^{p_{1}}(\partial \Omega \times I)\right)_{\theta, p}=B_{\alpha, p a r}^{p}(\partial \Omega \times I)
$$

for $0<\theta<1, \frac{1}{p}=\frac{1-\theta}{p_{0}}+\frac{\theta}{p_{1}}$ and $\alpha=(1-\theta) \alpha_{0}+\theta \alpha_{1}$.

(i) If $1<p<\infty$ and $0 \leq \alpha_{0}, \alpha_{1} \leq 1$, then

$$
\left[L_{\alpha_{0}, p a r}^{p}(\partial \Omega \times I), L_{\alpha_{1}, p a r}^{p}(\partial \Omega \times I)\right]_{\theta}=L_{\alpha, p a r}^{p}(\partial \Omega \times I)
$$


for $0<\theta<1$ and $\alpha=(1-\theta) \alpha_{0}+\theta \alpha_{1}$.

(j) If $1<p<\infty$ and $0 \leq \alpha_{0}, \alpha_{1} \leq 1$ with $\alpha_{0} \neq \alpha_{1}$, then

$$
\left(L_{\alpha_{0}, p a r}^{p}(\partial \Omega \times I), L_{\alpha_{1}, p a r}^{p}(\partial \Omega \times I)\right)_{\theta, p}=B_{\alpha, p a r}^{p}(\partial \Omega \times I)
$$

for $0<\theta<1$ and $\alpha=(1-\theta) \alpha_{0}+\theta \alpha_{1}$

Before we proceed with the proof, a few observations are in order here. In each part of the above theorem we have to make sure that the indices of the resulting space (after interpolation, i.e. right-hand sides) belong to the correct range, where the space in question is well-defined. In particular, in part (a) we have to ensure that $\frac{n+1}{n+2}<p, q \leq \infty$ and $\sigma_{p}<\alpha<1$. Similar conditions need to be verified in each case. Since the argument proving these conditions is elementary, we omit the details.

Proof. The proofs of parts (a)-(h) are very similar, here we give the reasoning for part (d). Also, (i) and (j) are handled in the same way, we shall prove part (i) only. The reason we need to treat $(\mathrm{i})$ and $(\mathrm{j})$ separately is that we cannot use the corresonding parts of Theorem 2.2.8 (as we do in cases (a)-(h)), since they do not hold for $\alpha_{0}=\alpha_{1}=0$. Instead, we shall employ results when the underlying space is $\mathbb{R}^{n-1} \times \mathbb{R}\left(\right.$ not $\mathbb{R}^{n-1} \times I$, as in parts $\left.(\mathrm{a})-(\mathrm{h})\right)$.

Proof of $(d)$. When $\Omega$ is an unbounded domain above the graph of (a Lipschitz function) $\varphi: \mathbb{R}^{n-1} \rightarrow \mathbb{R}$, we define the operators

$$
T_{1}: B_{\alpha_{k}, p a r}^{p, q_{k}}(\partial \Omega \times I) \longrightarrow B_{\alpha_{k}, p a r}^{p, q_{k}}\left(\mathbb{R}^{n-1} \times I\right), \quad k=0,1,
$$


by $T_{1} f:=f \circ \Phi_{0}$, where $\Phi_{0}: \mathbb{R}^{n-1} \times I \rightarrow \partial \Omega \times I, \Phi_{0}\left(x^{\prime}, t\right):=\left(x^{\prime}, \varphi\left(x^{\prime}\right), t\right)$ and

$$
R_{1}: B_{\alpha_{k}, p a r}^{p, q_{k}}\left(\mathbb{R}^{n-1} \times I\right) \longrightarrow B_{\alpha_{k}, p a r}^{p, q_{k}}(\partial \Omega \times I), \quad k=0,1,
$$

by $R_{1} g:=g \circ \Phi_{0}^{-1}$. Then, for $0<\theta<1, \frac{n+1}{n+2}<q \leq \infty$,

$$
\begin{aligned}
T_{1}:\left(B_{\alpha_{0}, p a r}^{p, q_{0}}(\partial \Omega \times I), B_{\alpha_{1}, p a r}^{p, q_{1}}(\partial \Omega \times I)\right)_{\theta, q} & \\
& \longrightarrow\left(B_{\alpha_{0}, p a r}^{p, q_{0}}\left(\mathbb{R}^{n-1} \times I\right), B_{\alpha_{1}, p a r}^{p, q_{1}}\left(\mathbb{R}^{n-1} \times I\right)\right)_{\theta, q}
\end{aligned}
$$

and using part (d) of Theorem 2.2.8 with $\Omega=\mathbb{R}^{n-1}$,

$$
T_{1}:\left(B_{\alpha_{0}, p a r}^{p, q_{0}}(\partial \Omega \times I), B_{\alpha_{1}, p a r}^{p, q_{1}}(\partial \Omega \times I)\right)_{\theta, q} \longrightarrow B_{\alpha, p a r}^{p, q}\left(\mathbb{R}^{n-1} \times I\right),
$$

where $\alpha=(1-\theta) \alpha_{0}+\theta \alpha_{1}$. This shows the left-to-right inclusion in (2.3.168).

For the opposite one, using part (d) of Theorem 2.2 .8 with $\Omega=\mathbb{R}^{n-1}$, a similar argument yields

$$
R_{1}: B_{\alpha, p a r}^{p, q}\left(\mathbb{R}^{n-1} \times I\right) \longrightarrow\left(B_{\alpha_{0}, p a r}^{p, q_{0}}(\partial \Omega \times I), B_{\alpha_{1}, p a r}^{p, q_{1}}(\partial \Omega \times I)\right)_{\theta, q}
$$

is bounded, where $0<\theta<1, \frac{n+1}{n+2}<q \leq \infty$ and $\alpha=(1-\theta) \alpha_{0}+\theta \alpha_{1}$.

On the other hand, by definition, $R_{1}$ maps $B_{\alpha, p a r}^{p, q}\left(\mathbb{R}^{n-1} \times I\right)$ onto $B_{\alpha, p a r}^{p, q}(\partial \Omega \times I)$. This, together with (2.3.178), further implies the right-to-left inclusion in (2.3.168) for $0<\theta<1, \frac{n+1}{n+2}<q \leq \infty$ and $\alpha=(1-\theta) \alpha_{0}+\theta \alpha_{1}$, completing the proof of $(\mathrm{d})$ in the case when $\Omega$ is a graph domain.

If $\Omega$ is bounded, we retain the notation from part (ii) of Definition 2.3.1 and Remark 1.2.1. Let us introduce the operator

$$
\left.T_{2}: B_{\alpha_{k}, p a r}^{p, q_{k}}(\partial \Omega \times I) \longrightarrow \bigoplus_{i=1}^{N} B_{\alpha_{k}, p a r}^{p, q_{k}}\left(\partial \Omega_{i} \times I\right)\right), \quad k=0,1
$$


$T_{2} f:=\left\{{\widetilde{\xi_{i} f}}^{\partial \Omega_{i}}\right\}_{i=1}^{N}$, where $\xi_{i} \in C_{c}^{\infty}\left(\mathbb{R}^{n-1}\right)$, supp $\psi_{i} \subset \operatorname{supp} \xi_{i} \subseteq V_{i}, \xi_{i} \equiv 1$ on the $\operatorname{supp} \psi_{i}$, and $\sim \partial \Omega_{i}$ means extension by 0 to $\partial \Omega_{i}$. We also define

$$
R_{2}: \bigoplus_{i=1}^{N} B_{\alpha_{k}, p a r}^{p, q_{k}}\left(\partial \Omega_{i} \times I\right) \longrightarrow B_{\alpha_{k}, p a r}^{p, q_{k}}(\partial \Omega \times I), \quad k=0,1
$$

by $R_{2}\left(\left\{f_{i}\right\}_{i=1}^{N}\right):=\sum_{i=1}^{N} \widetilde{\psi_{i} f_{i}} \partial \Omega$, where $\sim \partial \Omega$ means extension by 0 to $\partial \Omega$. Then it is easy to check that

$$
\left(R_{2} \circ T_{2}\right)(f)=R_{2}\left(\left\{{\widetilde{\xi_{i} f}}^{\partial \Omega_{i}}\right\}_{i=1}^{N}\right)=\sum_{i=1}^{N}\left(\widetilde{\psi_{i}{\widetilde{\xi_{i} f}}^{\partial \Omega_{i}}}\right)^{\partial \Omega}=f \sum_{i=1}^{N} \psi_{i}=f .
$$

Since, for $0<\theta<1$ and $\frac{n+1}{n+2}<q \leq \infty$,

$$
\begin{aligned}
T_{2}:\left(B_{\alpha_{0}, p a r}^{p, q_{0}}(\partial \Omega \times I), B_{\alpha_{1}, p a r}^{p, q_{1}}(\partial \Omega \times I)\right)_{\theta, q} & \\
& \longrightarrow\left(\bigoplus_{i=1}^{N} B_{\alpha_{0}, p a r}^{p, q_{0}}\left(\partial \Omega_{i} \times I\right), \bigoplus_{i=1}^{N} B_{\alpha_{1}, p a r}^{p, q_{1}}\left(\partial \Omega_{i} \times I\right)\right)_{\theta, q},
\end{aligned}
$$

by Lemma 1.2.21 we have

$$
\begin{aligned}
T_{2}:\left(B_{\alpha_{0}, p a r}^{p, q_{0}}(\partial \Omega \times I), B_{\alpha_{1}, p a r}^{p, q_{1}}(\partial \Omega \times I)\right)_{\theta, q} \\
\\
\longrightarrow \bigoplus_{i=1}^{N}\left(B_{\alpha_{0}, p a r}^{p, q_{0}}\left(\partial \Omega_{i} \times I\right), B_{\alpha_{1}, p a r}^{p, q_{1}}\left(\partial \Omega_{i} \times I\right)\right)_{\theta, q} .
\end{aligned}
$$

On account of the previous (graph domain) case, this further implies that

$$
T_{2}:\left(B_{\alpha_{0}, p a r}^{p, q_{0}}(\partial \Omega \times I), B_{\alpha_{1}, p a r}^{p, q_{1}}(\partial \Omega \times I)\right)_{\theta, q} \longrightarrow \bigoplus_{i=1}^{N} B_{\alpha_{0}, p a r}^{p, q}\left(\partial \Omega_{i} \times I\right),
$$

where $\alpha=(1-\theta) \alpha_{0}+\theta \alpha_{1}$, showing the left-to-right inclusion in (2.3.168).

After a similar reasoning as before we arrive at

$$
R_{2}: \bigoplus_{i=1}^{N} B_{\alpha, p a r}^{p, q}\left(\partial \Omega_{i} \times I\right) \longrightarrow\left(B_{\alpha_{0}, p a r}^{p, q_{0}}(\partial \Omega \times I), B_{\alpha_{1}, p a r}^{p, q_{1}}(\partial \Omega \times I)\right)_{\theta, q} \quad \text { is bounded }
$$


for $0<\theta<1, \frac{n+1}{n+2}<q \leq \infty$ and $\alpha=(1-\theta) \alpha_{0}+\theta \alpha_{1}$. Since $R_{2}$ maps $\bigoplus_{i=1}^{N} B_{\alpha, p a r}^{p, q}\left(\partial \Omega_{i} \times I\right)$ onto $B_{\alpha, p a r}^{p, q}(\partial \Omega \times I)$, the right-to-left inclusion in (2.3.168) holds, completing the proof of $(d)$.

Proof of (i). When $\Omega$ is an unbounded domain above the graph of (a Lipschitz function) $\varphi: \mathbb{R}^{n-1} \rightarrow \mathbb{R}$, we define the operators

$$
T_{3}: L_{\alpha_{k}, p a r}^{p}(\partial \Omega \times I) \longrightarrow L_{\alpha_{k}, p a r}^{p}\left(\mathbb{R}^{n-1} \times \mathbb{R}\right), \quad k=0,1
$$

by $T_{3} f:=E\left(f \circ \Phi_{0}\right)$, where $\Phi_{0}: \mathbb{R}^{n-1} \times I \longrightarrow \partial \Omega \times I$ is given by $\Phi_{0}\left(x^{\prime}, t\right):=$ $\left(x^{\prime}, \varphi\left(x^{\prime}\right), t\right)$, and $E$ is the extension operator introduced in Proposition 3.4.2. We also consider

$$
R_{3}: L_{\alpha_{k}, p a r}^{p}\left(\mathbb{R}^{n-1} \times \mathbb{R}\right) \longrightarrow L_{\alpha_{k}, p a r}^{p}(\partial \Omega \times I), \quad k=0,1
$$

by $R_{3} g:=\left.g\right|_{\mathbb{R}^{n-1} \times I} \circ \Phi_{0}^{-1}$. Then for $0<\theta<1$,

$T_{3}:\left[L_{\alpha_{0}, p a r}^{p}(\partial \Omega \times I), L_{\alpha_{1}, p a r}^{p}(\partial \Omega \times I)\right]_{\theta} \longrightarrow\left[L_{\alpha_{0}, p a r}^{p}\left(\mathbb{R}^{n-1} \times \mathbb{R}\right), L_{\alpha_{1}, p a r}^{p}\left(\mathbb{R}^{n-1} \times \mathbb{R}\right)\right]_{\theta}$.

By part (h) of Theorem 2.1.60 this further implies that

$$
T_{3}:\left[L_{\alpha_{0}, p a r}^{p}(\partial \Omega \times I), L_{\alpha_{1}, p a r}^{p}(\partial \Omega \times I)\right]_{\theta} \longrightarrow L_{\alpha, p a r}^{p}\left(\mathbb{R}^{n-1} \times \mathbb{R}\right),
$$

where $\alpha=(1-\theta) \alpha_{0}+\theta \alpha_{1}$. Hence the left-to-right inclusion in (2.3.176) holds.

For the opposite inclusion in (2.3.176), by a similar reasoning as above, one can easily see that for $0<\theta<1$,

$$
R_{3}: L_{\alpha, p a r}^{p}\left(\mathbb{R}^{n-1} \times \mathbb{R}\right) \longrightarrow\left[L_{\alpha_{0}, p a r}^{p}(\partial \Omega \times I), L_{\alpha_{1}, p a r}^{p}(\partial \Omega \times I)\right]_{\theta}
$$


where $\alpha=(1-\theta) \alpha_{0}+\theta \alpha_{1}$. We also note that $R_{3} \circ T_{3}$ is the identity operator on $L_{\alpha, p a r}^{p}(\partial \Omega \times I)$, and $R_{3}$ maps $L_{\alpha, p a r}^{p}\left(\mathbb{R}^{n-1} \times \mathbb{R}\right)$ onto $L_{\alpha, p a r}^{p}(\partial \Omega \times I)$. This further implies the right-to-left inclsion in (2.3.176), completing the proof of (i) when $\Omega$ is a graph domain.

If $\Omega$ is bounded, one follows the same steps as in the proof of part (d) in Theorem 2.3.20 and obtains the desired result.

Remark 2.3.21. Based on Remark 2.2.9, similar to the proof of Theorem 2.3.20, we obtain the results of parts (c), (d), (g) and (h) of Theorem 2.3.20 for a different range of indices. More specifically:

(c') Let $0<\alpha_{i}<1, i=0,1$, and $0<\theta<1, \frac{1}{p}=\frac{1-\theta}{p_{0}}+\frac{\theta}{p_{1}}=\frac{1-\theta}{q_{0}}+\frac{\theta}{q_{1}}$, $\alpha=(1-\theta) \alpha_{0}+\theta \alpha_{1}$. Then (2.3.167) holds either for $1 \leq p_{0}<p_{1}<\infty$ and $1 \leq q_{0}<q_{1}<\infty$, or for $1 \leq p_{0}, p_{1}, q_{0}, q_{1}<\infty$ and $\alpha_{0} \neq \alpha_{1}$.

(d') If $1 \leq p, q_{0}, q_{1} \leq \infty, 0<\alpha_{0}, \alpha_{1}<1$ with $\alpha_{0} \neq \alpha_{1}$, then (2.3.168) holds for $0<\theta<1,1 \leq q \leq \infty$ and $\alpha=(1-\theta) \alpha_{0}+\theta \alpha_{1}$.

(g') If $1 \leq p_{i}, q_{i} \leq \infty$ and $0<\alpha_{i}<1, i=0,1$, then (2.3.174) holds for $0<\theta<1$, $\frac{1}{p}=\frac{1-\theta}{p_{0}}+\frac{\theta}{p_{1}}, \frac{1}{q}=\frac{1-\theta}{q_{0}}+\frac{\theta}{q_{1}}$ and $\alpha=(1-\theta) \alpha_{0}+\theta \alpha_{1}$.

(h') If $1 \leq p_{0}, p_{1} \leq \infty$ and $0<\alpha_{0}, \alpha_{1}<1$, then (2.3.175) holds for $0<\theta<1$, $\frac{1}{p}=\frac{1-\theta}{p_{0}}+\frac{\theta}{p_{1}}$ and $\alpha=(1-\theta) \alpha_{0}+\theta \alpha_{1}$.

In the last part of this section we give the counterpart of Theorem 2.1.16 for spaces defined on $\partial \Omega \times \mathbb{R}$. Recall that by definition $\partial_{\tau_{j k}}:=\nu_{j} \partial_{k}-\nu_{k} \partial_{j}$.

Proposition 2.3.22. If $\Omega$ is a Lipschitz domain in $\mathbb{R}^{n}$ and $1<p<\infty$, then for all 
$j, k \in\{1,2, \ldots, n\}$, the operators

$$
\partial_{\tau_{j k}}: B_{\alpha, p a r}^{p}(\partial \Omega \times \mathbb{R}) \longrightarrow B_{\alpha-1, p a r}^{p}(\partial \Omega \times \mathbb{R}), \quad 0<\alpha<1
$$

and

$$
\partial_{\tau_{j k}}: L_{\alpha, p a r}^{p}(\partial \Omega \times \mathbb{R}) \longrightarrow L_{\alpha-1, p a r}^{p}(\partial \Omega \times \mathbb{R}), \quad 0 \leq \alpha \leq 1
$$

are bounded.

Proof. Step 1. Show that matters can be reduced to the graph domain case.

Let $0<\alpha<1$ and assume that $\Omega$ is a bounded Lipschitz domain in $\mathbb{R}^{n}$. With the notation of Remark 1.2.1, by definition,

$$
\begin{aligned}
& f \in B_{\alpha, p a r}^{p}(\partial \Omega \times \mathbb{R}) \quad \text { if and only if } \\
& {\widetilde{\psi_{i} f}}^{\partial \Omega_{i}} \in B_{\alpha, p a r}^{p}\left(\partial \Omega_{i} \times \mathbb{R}\right) \text { for each } i=1, \ldots, N .
\end{aligned}
$$

Similarly,

$$
\begin{aligned}
& \partial_{\tau_{j k}} f \in B_{\alpha-1, p a r}^{p}(\partial \Omega \times \mathbb{R}) \text { if and only if } \\
& {\widetilde{\psi_{i}\left(\partial_{\tau_{j k}} f\right)}}^{\partial \Omega_{i}} \in B_{\alpha-1, p a r}^{p}\left(\partial \Omega_{i} \times \mathbb{R}\right) \text { for each } i=1, \ldots, N .
\end{aligned}
$$

The observation we make is that

$$
\partial_{\tau_{j k}}\left(\psi_{i} f\right)=\left(\partial_{\tau_{j k}} \psi_{i}\right) f+\psi_{i}\left(\partial_{\tau_{j k}} f\right)
$$

hence

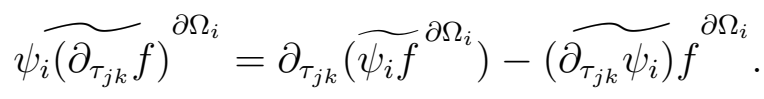

Given $f \in B_{\alpha, \text { par }}^{p}(\partial \Omega \times \mathbb{R})$, the second term in the right-hand side of (2.3.184) belongs to $B_{\alpha, p a r}^{p}\left(\partial \Omega_{i} \times \mathbb{R}\right) \hookrightarrow B_{\alpha-1, p a r}^{p}\left(\partial \Omega_{i} \times \mathbb{R}\right)$ for each $i=1, \ldots, N$. With this in mind, from $(2.3 .181),(2.3 .182)$ and (2.3.184) we can concude that

$$
\partial_{\tau_{j k}}: B_{\alpha, p a r}^{p}(\partial \Omega \times \mathbb{R}) \longrightarrow B_{\alpha-1, p a r}^{p}(\partial \Omega \times \mathbb{R}) \quad \text { is bounded }
$$


if and only if

$$
\partial_{\tau_{j k}}: B_{\alpha, p a r}^{p}\left(\partial \Omega_{i} \times \mathbb{R}\right) \longrightarrow B_{\alpha-1, p a r}^{p}\left(\partial \Omega_{i} \times \mathbb{R}\right) \quad \text { is bounded for each } i=1, \ldots, N \text {. }
$$

A similar argument for the Sobolev space $L_{\alpha, p a r}^{p}(\partial \Omega \times \mathbb{R})$ with $0 \leq \alpha \leq 1$ completes the proof of Step 1.

Step 2. Consider a graph Lipschitz domain $\Omega$ in $\mathbb{R}^{n}$ and $1<p<\infty$. Then for any $j, k \in\{1,2, \ldots, n\}$ show that

$$
\partial_{\tau_{j k}}: L_{1, p a r}^{p}(\partial \Omega \times \mathbb{R}) \longrightarrow L^{p}(\partial \Omega \times \mathbb{R}) \quad \text { is bounded }
$$

Let $\varphi: \mathbb{R}^{n-1} \rightarrow \mathbb{R}$ be the Lipschitz function such that the graph of $\varphi$ represents the boundary of $\Omega$. Recall that the outward normal unit vector at the boundary point $\left(x^{\prime}, \varphi\left(x^{\prime}\right)\right) \in \partial \Omega$ is

$$
\nu\left(x^{\prime}, \varphi\left(x^{\prime}\right)\right)=\frac{\left(\nabla \varphi\left(x^{\prime}\right),-1\right)}{\sqrt{1+\mid \nabla \varphi\left(x^{\prime}\right)^{2}}}, \quad x^{\prime} \in \mathbb{R}^{n-1} .
$$

We let $f \in L_{1, p a r}^{p}(\partial \Omega \times \mathbb{R})$. Then by definition, $g$ belongs to $L_{1, p a r}^{p}\left(\mathbb{R}^{n-1} \times \mathbb{R}\right)$, where $g\left(x^{\prime}, t\right):=f\left(x^{\prime}, \varphi\left(x^{\prime}\right), t\right)$ for $\left(x^{\prime}, t\right) \in \mathbb{R}^{n-1} \times \mathbb{R}$. On account of Theorem 2.1.16 (or Theorem 2.1.41) this further implies that

$$
\partial_{x_{j}} g \in L^{p}\left(\mathbb{R}^{n-1} \times \mathbb{R}\right) \quad \text { for each } j \text { such that } 1 \leq j \leq n-1 \text {. }
$$

Note also that

$$
\partial_{x_{j}} g\left(x^{\prime}, t\right)=\partial_{j} f\left(x^{\prime}, \varphi\left(x^{\prime}\right), t\right)+\partial_{j} \varphi\left(x^{\prime}\right) \partial_{n} f\left(x^{\prime}, \varphi\left(x^{\prime}\right), t\right), \quad 1 \leq j \leq n-1 .
$$

Our goal in this step is to show that $\partial_{\tau_{j k}} \in L^{p}(\partial \Omega \times \mathbb{R})$ for all $j, k \in\{1,2, \ldots, n\}$. If $j=k$, there is nothing to prove, hence we may assume, without loss of generality, that $j \neq k$. We shall consider two cases: 
- $1 \leq j \leq n-1$ and $k=n$, and

- $1 \leq j, k \leq n-1$.

When $1 \leq k \leq n-1$ and $j=n$, using symmetry, one proceeds as in the case when $1 \leq j \leq n-1$ and $k=n$, therefore it suffices to analyze the above two cases.

If $1 \leq j \leq n-1$ and $k=n$, then using the definition of the operator $\partial_{\tau_{j k}}$ we obtain that

$$
\partial_{\tau_{j k}} f\left(x^{\prime}, \varphi\left(x^{\prime}\right), t\right)=\frac{\partial_{j} \varphi\left(x^{\prime}\right) \partial_{n} f\left(x^{\prime}, \varphi\left(x^{\prime}\right), t\right)+\partial_{j} f\left(x^{\prime}, \varphi\left(x^{\prime}\right), t\right)}{\sqrt{1+\left|\nabla \varphi\left(x^{\prime}\right)\right|^{2}}} .
$$

In concert with (2.3.186) this further implies that

$$
\partial_{\tau_{j k}} f\left(x^{\prime}, \varphi\left(x^{\prime}\right), t\right)=\frac{\partial_{x_{j}} g\left(x^{\prime}, t\right)}{\sqrt{1+\left|\nabla \varphi\left(x^{\prime}\right)\right|^{2}}} .
$$

Consequently, (2.3.185) and (2.3.188) imply that $\partial_{\tau_{j k}} f \in L^{p}\left(\mathbb{R}^{n-1} \times \mathbb{R}\right)$ in the case when $1 \leq j \leq n-1$ and $k=n$.

When $1 \leq j, k \leq n-1$, similar to the previous case, we have

$$
\partial_{\tau_{j k}} f\left(x^{\prime}, \varphi\left(x^{\prime}\right), t\right)=\frac{\partial_{j} \varphi\left(x^{\prime}\right) \partial_{k} f\left(x^{\prime}, \varphi\left(x^{\prime}\right), t\right)-\partial_{k} \varphi\left(x^{\prime}\right) \partial_{j} f\left(x^{\prime}, \varphi\left(x^{\prime}\right), t\right)}{\sqrt{1+\left|\nabla \varphi\left(x^{\prime}\right)\right|^{2}}} .
$$

Adding and subtracting the mixt term $\partial_{j} \varphi\left(x^{\prime}\right) \partial_{k} \varphi\left(x^{\prime}\right) \partial_{n} f\left(x^{\prime}, \varphi\left(x^{\prime}\right), t\right)$ in the numerator of the right-hand side in (2.3.189) yields

$$
\begin{aligned}
\partial_{\tau_{j k}} f\left(x^{\prime}, \varphi\left(x^{\prime}\right), t\right)= & \frac{\partial_{j} \varphi\left(x^{\prime}\right)}{\sqrt{1+\left|\nabla \varphi\left(x^{\prime}\right)\right|^{2}}}\left[\partial_{k} f\left(x^{\prime}, \varphi\left(x^{\prime}\right), t\right)+\partial_{n} f\left(x^{\prime}, \varphi\left(x^{\prime}\right), t\right) \partial_{k} \varphi\left(x^{\prime}\right)\right] \\
& -\frac{\partial_{k} \varphi\left(x^{\prime}\right)}{\sqrt{1+\left|\nabla \varphi\left(x^{\prime}\right)\right|^{2}}}\left[\partial_{j} f\left(x^{\prime}, \varphi\left(x^{\prime}\right), t\right)+\partial_{n} f\left(x^{\prime}, \varphi\left(x^{\prime}\right), t\right) \partial_{j} \varphi\left(x^{\prime}\right)\right] .
\end{aligned}
$$


Taking into account (2.3.186), we can rewrite (2.3.190) as follows:

$$
\begin{aligned}
\partial_{\tau_{j k}} f\left(x^{\prime}, \varphi\left(x^{\prime}\right), t\right)= & \frac{\partial_{j} \varphi\left(x^{\prime}\right)}{\sqrt{1+\left|\nabla \varphi\left(x^{\prime}\right)\right|^{2}}} \partial_{x_{k}} g\left(x^{\prime}, t\right) \\
& -\frac{\partial_{k} \varphi\left(x^{\prime}\right)}{\sqrt{1+\left|\nabla \varphi\left(x^{\prime}\right)\right|^{2}}} \partial_{x_{j}} g\left(x^{\prime}, t\right) .
\end{aligned}
$$

From (2.3.185), (2.3.191) and the fact that $\varphi$ is a Lipschitz function one concludes that $\partial_{\tau_{j k}} f \in L^{p}\left(\mathbb{R}^{n-1} \times \mathbb{R}\right)$ for $1 \leq j, k \leq n-1$, finishing the proof of Step 2 .

Step 3. Consider a graph Lipschitz domain $\Omega$ in $\mathbb{R}^{n}$ and $1<p<\infty$. Then for any $j, k \in\{1,2, \ldots, n\}$ show that

$$
\partial_{\tau_{j k}}: L^{p}(\partial \Omega \times \mathbb{R}) \longrightarrow L_{-1, p a r}^{p}(\partial \Omega \times \mathbb{R}) \quad \text { is bounded }
$$

Our goal is to find the dual of the operator $\partial_{\tau_{j k}}$, then to dualize the resut found in Step 2. In order to compute $\left(\partial_{\tau_{j k}}\right)^{*}$ we let $f, g \in C^{1}(\bar{\Omega} \times \mathbb{R})$. Then by the Gauss-Green theorem applied to $\left(\partial_{k} f\right) g$ and $\left(\partial_{j} f\right) g$, we deduce that for any $j, k \in\{1,2, \ldots, n\}$,

$$
\begin{aligned}
\int_{\partial \Omega \times \mathbb{R}}\left(\partial_{\tau_{j k}} f\right) g d \sigma d t & =\int_{\partial \Omega \times \mathbb{R}} \nu_{j}\left(\partial_{k} f\right) g d \sigma d t-\int_{\partial \Omega \times \mathbb{R}} \nu_{k}\left(\partial_{j} f\right) g d \sigma d t \\
& =\int_{\Omega \times \mathbb{R}}\left\{\partial_{j}\left[\left(\partial_{k} f\right) g\right]-\partial_{k}\left[\left(\partial_{j} f\right) g\right]\right\} d x d t
\end{aligned}
$$

This simplifies to

$$
\int_{\partial \Omega \times \mathbb{R}}\left(\partial_{\tau_{j k}} f\right) g d \sigma d t=\int_{\Omega \times \mathbb{R}}\left[\left(\partial_{k} f\right)\left(\partial_{j} g\right)-\left(\partial_{j} f\right)\left(\partial_{k} g\right)\right] d x d t
$$

Interchanging $f$ and $g$, as well as $j$ and $k$ in (2.3.193) yields

$$
\int_{\partial \Omega \times \mathbb{R}} f\left(\partial_{\tau_{k j}} g\right) d \sigma d t=\int_{\partial \Omega \times \mathbb{R}}\left(\partial_{\tau_{j k}} f\right) g d \sigma d t
$$

therefore $\left(\partial_{\tau_{j k}}\right)^{*}=\partial_{\tau_{k j}}$ for all $j, k \in\{1,2, \ldots, n\}$. With this in hand, the result of Step 2 implies that for any $j, k \in\{1,2, \ldots, n\}$, the operator 


$$
\partial_{\tau_{k j}}:\left(L^{p}(\partial \Omega \times \mathbb{R})\right)^{*} \longrightarrow\left(L_{1, p a r}^{p}(\partial \Omega \times \mathbb{R})\right)^{*} \quad \text { is bounded }
$$

where $\Omega$ is a graph Lipschitz domain in $\mathbb{R}^{n}$ and $1<p<\infty$. Since $\left(L^{p}(\partial \Omega \times \mathbb{R})\right)^{*}=$ $L^{p^{\prime}}(\partial \Omega \times \mathbb{R})$ and , by Remark 2.4.7,

$$
\left(L_{1, p a r}^{p}(\partial \Omega \times \mathbb{R})\right)^{*}=L_{-1, p a r}^{p^{\prime}}(\partial \Omega \times \mathbb{R})
$$

for $1 / p+1 / p^{\prime}=1$, the boundedness of the operator in (2.3.195) readily implies what we wanted to show in Step 3.

Step 4. For a graph Lipschitz domain $\Omega$ in $\mathbb{R}^{n}$ show (2.3.179) and (2.3.180) in Proposition 2.3.22.

Since $\partial_{\tau_{k j}}$ is a linear operator, real interpolation between the results of Steps 2 and 3 yields the following: for a graph Lipschitz domain $\Omega$ in $\mathbb{R}^{n}, 1<p<\infty$, and any $j, k \in\{1,2, \ldots, n\}$,

$$
\partial_{\tau_{j k}}:\left(L_{1, p a r}^{p}(\partial \Omega \times \mathbb{R}), L^{p}(\partial \Omega \times \mathbb{R})\right)_{\theta, p} \longrightarrow\left(L^{p}(\partial \Omega \times \mathbb{R}), L_{-1, p a r}^{p}(\partial \Omega \times \mathbb{R})\right)_{\theta, p}
$$

is bounded, where $0<\theta<1$. On account of part $(\mathrm{j})$ of Theorem 2.3.20,

$$
\left(L_{1, p a r}^{p}(\partial \Omega \times \mathbb{R}), L^{p}(\partial \Omega \times \mathbb{R})\right)_{\theta, p}=B_{1-\theta, p a r}^{p}(\partial \Omega \times \mathbb{R}) .
$$

With regard to the target space, using Remark 2.4 .7 with $1 / p+1 / p^{\prime}=1$ and the duality theorem for the real method (Theorem 3.7.1 in [BeLö]), then part (j) of Theorem 2.3.20 and Proposition 2.4.5, we deduce that 


$$
\begin{aligned}
\left(L^{p}(\partial \Omega \times \mathbb{R}),\right. & \left.L_{-1, p a r}^{p}(\partial \Omega \times \mathbb{R})\right)_{\theta, p} \\
& =\left(\left(L^{p^{\prime}}(\partial \Omega \times \mathbb{R})\right)^{*},\left(L_{1, p a r}^{p^{\prime}}(\partial \Omega \times \mathbb{R})\right)^{*}\right)_{\theta, p} \\
& =\left[\left(L^{p^{\prime}}(\partial \Omega \times \mathbb{R}), L_{1, p a r}^{p^{\prime}}(\partial \Omega \times \mathbb{R})\right)_{\theta, p^{\prime}}\right]^{*} \\
& =\left(B_{\theta, p a r}^{p^{\prime}}(\partial \Omega \times \mathbb{R})\right)^{*} \\
& =B_{-\theta, p a r}^{p}(\partial \Omega \times \mathbb{R}) .
\end{aligned}
$$

From (2.3.196)-(2.3.198), denoting $1-\theta$ by $\alpha$, we conclude that for all $j, k \in \mathbb{N}$ such that $1 \leq j, k \leq n$,

$$
\partial_{\tau_{j k}}: B_{\alpha, p a r}^{p}(\partial \Omega \times \mathbb{R}) \longrightarrow B_{\alpha-1, p a r}^{p}(\partial \Omega \times \mathbb{R})
$$

is bounded, where $\Omega$ is a graph Lipschitz domain in $\mathbb{R}^{n}$ and $1<p<\infty, 0<\alpha<1$.

Similarly, complex interpolation between the results of Steps 2 and 3 yields that for a graph Lipschitz domain $\Omega$ in $\mathbb{R}^{n}, 1<p<\infty$, and any $j, k \in\{1,2, \ldots, n\}$,

$$
\partial_{\tau_{j k}}:\left[L_{1, p a r}^{p}(\partial \Omega \times \mathbb{R}), L^{p}(\partial \Omega \times \mathbb{R})\right]_{\theta} \longrightarrow\left[L^{p}(\partial \Omega \times \mathbb{R}), L_{-1, p a r}^{p}(\partial \Omega \times \mathbb{R})\right]_{\theta}
$$

is bounded, where $0<\theta<1$. On account of part (i) of Theorem 2.3.20,

$$
\left[L_{1, p a r}^{p}(\partial \Omega \times \mathbb{R}), L^{p}(\partial \Omega \times \mathbb{R})\right]_{\theta}=L_{1-\theta, p a r}^{p}(\partial \Omega \times \mathbb{R})
$$

Regarding the target space, we are using Remark 2.4 .7 with $1 / p+1 / p^{\prime}=1$, the duality theorem for the complex method (Corollary 4.5.2 in [BeLö]) along with the fact that $L^{p^{\prime}}(\partial \Omega \times \mathbb{R})$ is reflexive for $1<p^{\prime}<\infty$, then part (i) of Theorem 2.3.20 
and Remark 2.4.7, we deduce that

$$
\begin{aligned}
{\left[L^{p}(\partial \Omega \times \mathbb{R}),\right.} & \left.L_{-1, p a r}^{p}(\partial \Omega \times \mathbb{R})\right]_{\theta} \\
& =\left[\left(L^{p^{\prime}}(\partial \Omega \times \mathbb{R})\right)^{*},\left(L_{1, p a r}^{p^{\prime}}(\partial \Omega \times \mathbb{R})\right)^{*}\right]_{\theta} \\
& =\left(\left[L^{p^{\prime}}(\partial \Omega \times \mathbb{R}), L_{1, p a r}^{p^{\prime}}(\partial \Omega \times \mathbb{R})\right]_{\theta}\right)^{*} \\
& =\left(L_{\theta, p a r}^{p^{\prime}}(\partial \Omega \times \mathbb{R})\right)^{*} \\
& =L_{-\theta, p a r}^{p}(\partial \Omega \times \mathbb{R}) .
\end{aligned}
$$

From (2.3.199)-(2.3.201), with $1-\theta=\alpha$, we ultimately obtain that for all $j, k \in \mathbb{N}$ such that $1 \leq j, k \leq n$,

$$
\partial_{\tau_{j k}}: L_{\alpha, p a r}^{p}(\partial \Omega \times \mathbb{R}) \longrightarrow L_{\alpha-1, p a r}^{p}(\partial \Omega \times \mathbb{R})
$$

is bounded, where $\Omega$ is a graph Lipschitz domain in $\mathbb{R}^{n}$ and $1<p<\infty, 0<\alpha<1$. The endpoint cases $\alpha=0$ and $\alpha=1$ of (2.3.202) are covered by Steps 3 and 2, respectively. This completes the proof of Step 4.

In view of Steps 1 and 4 the proof of Proposition 2.3.22 is finished.

\subsubsection{Atomic decompositions of Besov spaces}

Relying on the atomic characterizations of Besov spaces in the Euclidean setting described in Section 2.1.1, we next give the decompositions of parabolic Besov spaces when the underlying space is $\partial \Omega \times \mathbb{R}, \Omega$ being a Lipshitz graph domain in $\mathbb{R}^{n}$.

Remark 2.3.23. Given that $\Omega$ is a (Lipschitz) domain in $\mathbb{R}^{n}$ and $I \subseteq \mathbb{R}$ an interval, we call $\mathrm{Q}$ a parabolic surface cube on $\partial \Omega \times I$ with sidelength $r$ if $\mathrm{Q}$ has the form $\mathrm{Q}=\mathrm{Q}^{\prime} \times J$, where $\mathrm{Q}^{\prime} \subseteq \partial \Omega$ is a (regular) surface cube of sidelength $r$ and $I \subseteq J$ is 
an open interval of length $r^{2}$. Note that $|\mathrm{Q}|=r^{n+1}$. Instead of parabolic (regular, surface) cubes one may consider corresponging balls as well. In this case the resulting vector space is equipped with a quasi-norm which is equivalent with the quasi-norm of the space obtained using cubes.

We start with the counterparts of Definition 2.1.24 and Theorems 2.1.25 and 2.1.26.

Definition 2.3.24. Let us consider an unbounded Lipschitz domain $\Omega \subseteq \mathbb{R}^{n}$, and a parabolic surface cube $\mathrm{Q}$ on $\partial \Omega \times \mathbb{R}$. Further assume that $\frac{n+1}{n+2}<p \leq 1$ and $(n+1)\left(\frac{1}{p}-1\right)<\alpha<1$. Then we call $a_{\mathrm{Q}} a(p, \alpha)$-atom for $\dot{B}_{\alpha, p a r}^{p}(\partial \Omega \times \mathbb{R})$ if $\tilde{a}_{Q}$ is a $(p, \alpha)$-atom for $\dot{B}_{\alpha, p a r}^{p}\left(\mathbb{R}^{n-1} \times \mathbb{R}\right)$, where

$$
a_{\mathrm{Q}}\left(x^{\prime}, \varphi\left(x^{\prime}\right), t\right)=\tilde{a}_{Q}\left(x^{\prime}, t\right), \quad\left(x^{\prime}, t\right) \in \mathbb{R}^{n-1} \times \mathbb{R}
$$

and the graph of $\varphi: \mathbb{R}^{n-1} \rightarrow \mathbb{R}$ is the boundary of $\Omega$.

With the extra assumption that $|\mathrm{Q}| \leq 1$, a $a_{\mathrm{Q}}$ is a $(p, \alpha)$-atom for $B_{\alpha, p a r}^{p}(\partial \Omega \times \mathbb{R})$, if $\tilde{a}_{Q}$ is a $(p, \alpha)$-atom for $B_{\alpha, p a r}^{p}\left(\mathbb{R}^{n-1} \times \mathbb{R}\right)$.

Definition 2.3.25. Let $\Omega$ be an unbounded Lipschitz domain $\mathbb{R}^{n}, \frac{n+1}{n+2}<p \leq 1$ and $(n+1)\left(\frac{1}{p}-1\right)<\alpha<1$. Then

$\dot{B}_{\alpha, p a r}^{p}(\partial \Omega \times \mathbb{R}):=\left\{\sum_{\mathrm{Q}} s_{\mathrm{Q}} a_{\mathrm{Q}}:\left\{s_{\mathrm{Q}}\right\}_{\mathrm{Q}} \in \ell^{p}, a_{\mathrm{Q}}\right.$ are $(p, \alpha)$-atoms for $\left.\dot{B}_{\alpha, p a r}^{p}(\partial \Omega \times \mathbb{R})\right\}$

The associated norm is given by

$$
\|f\|_{\dot{B}_{\alpha, p a r}^{p}(\partial \Omega \times \mathbb{R})}:=\inf \left\{\left(\sum_{\mathrm{Q}}\left|s_{\mathrm{Q}}\right|^{p}\right)^{1 / p}: f=\sum_{\mathrm{Q}} s_{\mathrm{Q}} a_{\mathrm{Q}}\right\} .
$$


Recall that the space $B_{\alpha, p a r}^{p}(\partial \Omega \times \mathbb{R})$ with positive $\alpha$ was introduced in Definition 2.3.1.

Proposition 2.3.26. Let $\Omega$ be an unbounded Lipschitz domain $\mathbb{R}^{n}, \frac{n+1}{n+2}<p \leq 1$ and $(n+1)\left(\frac{1}{p}-1\right)<\alpha<1$. Then $f \in B_{\alpha, p a r}^{p}(\partial \Omega \times \mathbb{R})$ if and only if $f=\sum_{\mathrm{Q}} s_{\mathrm{Q}} a_{\mathrm{Q}}$, where $\left\{s_{\mathrm{Q}}\right\}_{\mathrm{Q}} \in \ell^{p}$ and $a_{\mathrm{Q}}$ are $(p, \alpha)$-atoms introduced in Definition 2.3.24 with $|\mathrm{Q}| \leq 1$. Also, if $f \in B_{\alpha, p a r}^{p}(\partial \Omega \times \mathbb{R})$, then

$$
\|f\|_{B_{\alpha, p a r}^{p}(\partial \Omega \times \mathbb{R})} \approx \inf \left(\sum_{|Q| \leq 1}\left|s_{Q}\right|^{p}\right)^{1 / p}
$$

where the infimum is taken over all representations of $f$.

When the smoothness is of negative order, the corresponding definitions and result to Definition 2.1.27 and Theorems 2.1.28 and 2.1.29 (on the geometrical setting $\partial \Omega \times \mathbb{R})$ are the following.

Definition 2.3.27. Consider an unbounded Lipschitz domain $\Omega \subseteq \mathbb{R}^{n}$, and a parabolic surface cube $\mathrm{Q}$ on $\partial \Omega \times \mathbb{R}$. Further assume that the indices $p, \alpha$ are such that $\frac{n+1}{n+2}<p \leq 1$ and $(n+1)\left(\frac{1}{p}-1\right)<\alpha<1$. We then call $a_{\mathrm{Q}} a(p,-\alpha)$-atom for $\dot{B}_{-\alpha, p a r}^{p}(\partial \Omega \times \mathbb{R})$ if $\tilde{a}_{Q}$ is a $(p,-\alpha)$-atom for $\dot{B}_{-\alpha, p a r}^{p}\left(\mathbb{R}^{n-1} \times \mathbb{R}\right)$, where

$$
a_{\mathrm{Q}}\left(x^{\prime}, \varphi\left(x^{\prime}\right), t\right) \sqrt{1+\left|\nabla \varphi\left(x^{\prime}\right)\right|^{2}}=\tilde{a}_{Q}\left(x^{\prime}, t\right), \quad\left(x^{\prime}, t\right) \in \mathbb{R}^{n-1} \times \mathbb{R},
$$

and the graph of $\varphi: \mathbb{R}^{n-1} \rightarrow \mathbb{R}$ is the boundary of $\Omega$.

With the extra assumption that $|\mathrm{Q}| \leq 1$, a $a_{\mathrm{Q}}$ is a $(p,-\alpha)$-atom for $B_{-\alpha, p a r}^{p}(\partial \Omega \times \mathbb{R})$, if $\tilde{a}_{Q}$ is a $(p,-\alpha)$-atom for $B_{-\alpha, p a r}^{p}\left(\mathbb{R}^{n-1} \times \mathbb{R}\right)$. 
Definition 2.3.28. Let $\Omega$ be an unbounded Lipschitz domain $\mathbb{R}^{n}, \frac{n+1}{n+2}<p \leq 1$ and $(n+1)\left(\frac{1}{p}-1\right)<\alpha<1$. Then

$\dot{B}_{-\alpha, p a r}^{p}(\partial \Omega \times \mathbb{R}):=\left\{\sum_{\mathrm{Q}} s_{\mathrm{Q}} a_{\mathrm{Q}}:\left\{s_{\mathrm{Q}}\right\}_{\mathrm{Q}} \in \ell^{p}, a_{\mathrm{Q}}\right.$ are $(p,-\alpha)$-atoms for $\left.\dot{B}_{-\alpha, p a r}^{p}(\partial \Omega \times \mathbb{R})\right\}$.

The associated norm is given by

$$
\|f\|_{\dot{B}_{-\alpha, p a r}^{p}(\partial \Omega \times \mathbb{R})}:=\inf \left\{\left(\sum_{\mathbf{Q}}\left|s_{\mathbf{Q}}\right|^{p}\right)^{1 / p}: f=\sum_{\mathbf{Q}} s_{\mathrm{Q}} a_{\mathrm{Q}}\right\} .
$$

Recall that for negative order of smoothness the parabolic Besov spaces on $\partial \Omega \times \mathbb{R}$ were introduced in Definition 2.3.3.

Proposition 2.3.29. Let $\Omega$ be an unbounded Lipschitz domain $\mathbb{R}^{n}, \frac{n+1}{n+2}<p \leq 1$ and $(n+1)\left(\frac{1}{p}-1\right)<\alpha<1$. Then $f \in B_{-\alpha, p a r}^{p}(\partial \Omega \times \mathbb{R})$ if and only if $f=\sum_{\mathrm{Q}} s_{\mathrm{Q}} a_{\mathrm{Q}}$, where $\left\{s_{\mathrm{Q}}\right\}_{\mathrm{Q}} \in \ell^{p}$ and $a_{\mathrm{Q}}$ are $(p,-\alpha)$-atoms introduced in Definition 2.3.27 with $|\mathrm{Q}| \leq 1$. Moreover, if $f \in B_{-\alpha, p a r}^{p}(\partial \Omega \times \mathbb{R})$, then

$$
\|f\|_{B_{-\alpha, p a r}^{p}(\partial \Omega \times \mathbb{R})} \approx \inf \left(\sum_{|Q| \leq 1}\left|s_{Q}\right|^{p}\right)^{1 / p}
$$

where the infimum is taken over all representations of $f$.

\subsubsection{Atomic decompositions of Hardy spaces}

This subsection contains the atomic decompositions of homogeneous and inhomogeneous Hardy spaces defined on $\partial \Omega \times \mathbb{R}$, where $\Omega$ is a Lipschitz domain in $\mathbb{R}^{n}$. Recall the parabolic surface cubes from Remark 2.3.23 and the definition and properties of Hardy spaces in the Euclidean setting from Section 2.1.3. 
Definition 2.3.30. Consider an unbounded Lipschitz domain $\Omega \subseteq \mathbb{R}^{n}$, and a parabolic surface cube $\mathrm{Q}$ on $\partial \Omega \times \mathbb{R}$. Further assume that $\frac{n+1}{n+2}<p \leq 1$ and $1 \leq q \leq \infty$ with $p<q$. We then call $a_{\mathrm{Q}}$ a p-atom (respectively, a $(p, q)$-atom) for $\dot{H}_{\text {par }}^{p}(\partial \Omega \times \mathbb{R})$, if $\tilde{a}_{Q}$ is a p-atom (respectively, a $(p, q)$-atom) for $\dot{H}_{\text {par }}^{p}\left(\mathbb{R}^{n-1} \times \mathbb{R}\right)$, where

$$
a_{\mathrm{Q}}\left(x^{\prime}, \varphi\left(x^{\prime}\right), t\right) \sqrt{1+\left|\nabla \varphi\left(x^{\prime}\right)\right|^{2}}=\tilde{a}_{Q}\left(x^{\prime}, t\right), \quad\left(x^{\prime}, t\right) \in \mathbb{R}^{n-1} \times \mathbb{R},
$$

and the graph of $\varphi: \mathbb{R}^{n-1} \rightarrow \mathbb{R}$ is the boundary of $\Omega$.

A bounded, measurable function $a_{\mathrm{Q}}$ is an inhomogeneous (local) p-atom for the space $H_{\text {par }}^{p}(\partial \Omega \times \mathbb{R})$, if $\tilde{a}_{Q}$ is an inhomogeneous (local) p-atom for $H_{\text {par }}^{p}\left(\mathbb{R}^{n-1} \times \mathbb{R}\right)$.

Definition 2.3.31. Let $\Omega$ be an unbounded Lipschitz domain $\mathbb{R}^{n}, \frac{n+1}{n+2}<p \leq 1$ and $1 \leq q \leq \infty$ with $p<q$. Then the space $\dot{H}_{p a r}^{p}(\partial \Omega \times \mathbb{R})$ is defined as

$$
\left\{\sum_{\mathrm{Q}} s_{\mathrm{Q}} a_{\mathrm{Q}}:\left\{s_{\mathrm{Q}}\right\}_{\mathrm{Q}} \in \ell^{p}, a_{\mathrm{Q}} \text { are p-atoms (or }(p, q) \text {-atoms) for } \dot{H}_{p a r}^{p}(\partial \Omega \times \mathbb{R})\right\} \text {. }
$$

The associated norm is given by

$$
\|f\|_{\dot{H}_{p a r}^{p}(\partial \Omega \times \mathbb{R})}:=\inf \left\{\left(\sum_{\mathrm{Q}}\left|s_{\mathrm{Q}}\right|^{p}\right)^{1 / p}: f=\sum_{\mathrm{Q}} s_{\mathrm{Q}} a_{\mathrm{Q}}\right\} .
$$

Under the same assumptions on $\Omega$ and $p$ as above, $H_{\text {par }}^{p}(\partial \Omega \times \mathbb{R})$ is defined by

$\left\{\sum_{\mathrm{Q}} s_{\mathrm{Q}} a_{\mathrm{Q}}:\left\{s_{\mathrm{Q}}\right\}_{\mathrm{Q}} \in \ell^{p}, a_{\mathrm{Q}}\right.$ are inhomogeneous (local) p-atoms for $\left.H_{\text {par }}^{p}(\partial \Omega \times \mathbb{R})\right\}$,

and the associated norm is given by

$$
\|f\|_{H_{p a r}^{p}(\partial \Omega \times \mathbb{R})}:=\inf \left\{\left(\sum_{\mathrm{Q}}\left|s_{\mathrm{Q}}\right|^{p}\right)^{1 / p}: f=\sum_{\mathrm{Q}} s_{\mathrm{Q}} a_{\mathrm{Q}}\right\}
$$


In the case when $\Omega$ is a bounded Lipschitz domain and $I$ is an interval in $\mathbb{R}$, we define the parabolic homogeneous and inhomogeneous (local) Hardy spaces, $\dot{H}_{\text {par }}^{p}(\partial \Omega \times$ $I)$ and $H_{p a r}^{p}(\partial \Omega \times I)$, respectively, as follows.

Definition 2.3.32. Consider a bounded Lipschitz domain $\Omega$ in $\mathbb{R}^{n}$ and $I \subseteq \mathbb{R}$ an open interval. Let $\mathrm{Q}$ be a parabolic surface cube on $\partial \Omega \times I$ with sidelength $r$, and $\frac{n+1}{n+2}<p \leq 1,1 \leq q \leq \infty$ such that $p<q$. A measurable function $a_{\mathrm{Q}}$ is called $a$ homogeneous $(p, q)$-atom for $\dot{H}_{p a r}^{p}(\partial \Omega \times I)$ if it satisfies the following conditions:

$$
\operatorname{supp} a_{\mathrm{Q}} \subset \mathrm{Q}, \quad\left\|a_{\mathrm{Q}}\right\|_{L^{q}(\partial \Omega \times I)} \leq r^{(n+1)\left(\frac{1}{q}-\frac{1}{p}\right)} \quad \text { and } \quad \int_{\partial \Omega \times I} a_{\mathrm{Q}}(x, t) d \sigma_{x} d t=0 .
$$

Definition 2.3.33. Let $\Omega$ be a bounded Lipschitz domain in $\mathbb{R}^{n}, I \subseteq \mathbb{R}$ an interval, and $\frac{n+1}{n+2}<p \leq 1$. Then

$$
\dot{H}_{p a r}^{p}(\partial \Omega \times I):=\left\{\sum_{\mathrm{Q}} s_{\mathrm{Q}} a_{\mathrm{Q}}:\left\{s_{\mathrm{Q}}\right\}_{\mathrm{Q}} \in \ell^{p}, a_{\mathrm{Q}} \text { 's are p-atoms satisfying (2.3.207) }\right\}
$$

and the associated norm is given by

$$
\|f\|_{\dot{H}_{p a r}^{p}(\partial \Omega \times I)}:=\inf \left\{\left(\sum_{\mathrm{Q}}\left|s_{\mathrm{Q}}\right|^{p}\right)^{1 / p}: f=\sum_{\mathrm{Q}} s_{\mathrm{Q}} a_{\mathrm{Q}}\right\} .
$$

Definition 2.3.34. Consider a bounded Lipschitz domain $\Omega$ in $\mathbb{R}^{n}$ and $I \subseteq \mathbb{R}$ an open interval. Let $\mathrm{Q}$ be a parabolic surface cube on $\partial \Omega \times I$ with sidelength $r$, and $\frac{n+1}{n+2}<p \leq 1,1 \leq q \leq \infty$ such that $p<q$. In this setting a measurable function $a_{\mathrm{Q}}$ is called a local (inhmogeneous) $(p, q)$-atom for $H_{p a r}^{p}(\partial \Omega \times I)$ if it satisfies the following conditions:

$$
\operatorname{supp} a_{\mathrm{Q}} \subset \mathrm{Q}, \quad\left\|a_{\mathrm{Q}}\right\|_{L^{q}(\partial \Omega \times I)} \leq r^{(n+1)\left(\frac{1}{q}-\frac{1}{p}\right)}
$$


and

$$
\begin{aligned}
& \text { either }|\mathrm{Q}|<\eta \quad \text { and } \int_{\partial \Omega \times I} a_{\mathrm{Q}}(x, t) d \sigma_{x} d t=0, \\
& \text { or } \quad|\mathrm{Q}| \geq \eta \text {. }
\end{aligned}
$$

Above $\eta=\eta(\partial \Omega, I)>0$ is a fixed, sufficiently small constant.

Definition 2.3.35. Consider a bounded Lipschitz domain $\Omega$ in $\mathbb{R}^{n}, I \subseteq \mathbb{R}$ an interval, and $\frac{n+1}{n+2}<p \leq 1$. Then

$$
H_{p a r}^{p}(\partial \Omega \times I):=\left\{\sum_{\mathrm{Q}} s_{\mathrm{Q}} a_{\mathrm{Q}}:\left\{s_{\mathrm{Q}}\right\}_{\mathrm{Q}} \in \ell^{p}, a_{\mathrm{Q}} \text { 's satisfy (2.3.208) and (2.3.209) }\right\}
$$

equipped with the usual infimum norm.

Remark 2.3.36. i. The choice of $q$ in (2.3.208) does not affect the definition of $H_{p a r}^{p}(\partial \Omega \times I)($ cf. Theorem A on p. 592 in [CoWe1] $)$.

ii. We note that, as in the ellipic setting (cf. p. 147 of [EsMi]), for a bounded Lipschitz domain $\Omega$ in $\mathbb{R}^{n}, I \subset \mathbb{R}$ a bounded interval, and $\frac{n+1}{n+2}<p \leq 1$,

$$
H_{\text {par }}^{p}(\partial \Omega \times I)=\dot{H}_{\text {par }}^{p}(\partial \Omega \times I)+L^{q}(\partial \Omega \times I) \quad \forall q>1
$$

In order to see the left-to-right inclusion in (2.3.210), let $f \in H_{p a r}^{p}(\partial \Omega \times I)$. Then, by definition, $f$ can be decomposed as

$$
f=\sum_{\mathrm{Q}} s_{\mathrm{Q}} a_{\mathrm{Q}}=\sum_{|\mathrm{Q}|<\eta} s_{\mathrm{Q}} a_{\mathrm{Q}}+\sum_{|\mathrm{Q}| \geq \eta} s_{\mathrm{Q}} a_{\mathrm{Q}}=: f_{1}+f_{2},
$$

where $\left\{s_{\mathrm{Q}}\right\}_{\mathrm{Q}} \in \ell^{p}$ and $a_{\mathrm{Q}}$ are local (inhomogeneous) $(p, q)$-atoms for $H_{p a r}^{p}(\partial \Omega \times I)$. Since $a_{\mathrm{Q}}$ with $|\mathrm{Q}|<\eta$ are homogeneous $(p, q)$-atoms for $\dot{H}_{p a r}^{p}(\partial \Omega \times I)$, we readily have $f_{1} \in \dot{H}_{\text {par }}^{p}(\partial \Omega \times I)$. It is straightforward to check that $f_{2} \in L^{q}(\partial \Omega \times I)$, which completes the proof of the left-to-right inclusion in (2.3.210). 
For the opposite one we first note that $\dot{H}_{p a r}^{p}(\partial \Omega \times I) \hookrightarrow H_{p a r}^{p}(\partial \Omega \times I)$, based on their atomic decomposition. Therefore it suffices to show that for any $q>1$, $L^{q}(\partial \Omega \times I) \hookrightarrow H_{p a r}^{p}(\partial \Omega \times I)$. In order to do so, depending whether $|\partial \Omega| \geq|I|^{(n-1) / 2}$ or $|\partial \Omega|<|I|^{(n-1) / 2}$, we divide either $\partial \Omega=\cup_{j=1}^{N_{1}} \Delta_{j}$ such that $\Delta_{j} \times I$ are parabolic surface cubes of sidelength $r_{j}$, or $I=\cup_{j=1}^{N_{2}} I_{j}$ such that $\partial \Omega \times I_{j}$ are parabolic surface cubes of sidelength $R_{j}$. Note that since $\partial \Omega \times I$ is bounded, $N_{1}$ and $N_{2}$ are finite. Also,

$$
f=\sum_{j=1}^{N_{1}} \chi_{\Delta_{j} \times I} f=\sum_{j=1}^{N_{1}} s_{j} a_{j}
$$

where

$$
s_{j}:=\frac{\|f\|_{L^{q}\left(\Delta_{j} \times I\right)}}{r_{j}^{(n+1)\left(\frac{1}{q}-\frac{1}{p}\right)}}, \quad r_{j}^{n+1}=\left|\Delta_{j} \times I\right|,
$$

and

$$
a_{j}(x, t):=s_{j}^{-1} \chi_{\Delta_{j} \times I}(x, t) f(x, t), \quad(x, t) \in \partial \Omega \times I .
$$

A similar argument applies in the case when the interval $I$ is divided. Then it is not hard to check that $a_{j}$ are local $(p, q)$-atoms for $H_{p a r}^{p}(\partial \Omega \times I)$ and that $\left\{s_{j}\right\}_{j} \in \ell^{p}$. Consequently, $f \in H_{p a r}^{p}(\partial \Omega \times I)$, finishing the proof of the right-to-left inclusion in (2.3.210).

\subsection{Duality results}

In this section we identify the duals of parabolic Besov and Triebel-Lizorkin spaces on different geometrical settings. 
Lemma 2.4.1. For any real number $\sigma$ and $f \in \mathrm{S}^{\prime}\left(\mathbb{R}^{n} \times \mathbb{R}\right)$ recall from (2.1.13) the definition of the lifting operator $\mathcal{I}_{\sigma}$, i.e.

$$
\mathcal{I}_{\sigma} f:=\mathcal{F}^{-1}\left\{\left[\left(1+|\xi|^{2}\right)^{\frac{1}{2}}+|\tau|^{\frac{1}{2}}\right]^{-\sigma} \mathcal{F} f(\xi, \tau)\right\}, \quad(\xi, \tau) \in \mathbb{R}^{n} \times \mathbb{R}, \quad \sigma \in \mathbb{R},
$$

Then, with $\mathcal{I}_{\sigma}^{*}$ denoting the adjoint of the operator $\mathcal{I}_{\sigma}$, one has

$$
\mathcal{I}_{\sigma}^{*}=\mathcal{I}_{\sigma}
$$

Proof. For complex-valued $f, g \in \mathrm{S}^{\prime}\left(\mathbb{R}^{n} \times \mathbb{R}\right)$ we may write

$$
\begin{aligned}
\left\langle\mathcal{I}_{\sigma}^{*} f, \bar{g}\right\rangle & =\int_{\mathbb{R}^{n} \times \mathbb{R}} f(\xi, \tau) \mathcal{I}_{\sigma} \bar{g}(\xi, \tau) d \xi d \tau \\
& =\int_{\mathbb{R}^{n} \times \mathbb{R}} f(\xi, \tau) \mathcal{F}^{-1}\left\{\left[\left(1+|x|^{2}\right)^{1 / 2}+|t|^{1 / 2}\right]^{-\sigma} \overline{\mathcal{F} g(-x,-t)}\right\}(\xi, \tau) d \xi d \tau \\
& =\int_{\mathbb{R}^{n} \times \mathbb{R}} \mathcal{F}\left(\mathcal{F}^{-1} f\right)(\xi, \tau) \overline{\mathcal{F}\left\{\left[\left(1+|x|^{2}\right)^{1 / 2}+|t|^{1 / 2}\right]^{-\sigma} \mathcal{F} g(-x,-t)\right\}(\xi, \tau)} d \xi d \tau .
\end{aligned}
$$

Now using Parseval's Theorem, then the change of variables $-x=y \in \mathbb{R}^{n}$ and $-t=s \in \mathbb{R}$, we obtain that

$$
\begin{aligned}
\left\langle\mathcal{I}_{\sigma}^{*} f, \bar{g}\right\rangle & =\int_{\mathbb{R}^{n} \times \mathbb{R}} \mathcal{F}^{-1} f(x, t)\left[\left(1+|x|^{2}\right)^{1 / 2}+|t|^{1 / 2}\right]^{-\sigma} \overline{\mathcal{F} g(-x,-t)} d x d t \\
& =\int_{\mathbb{R}^{n} \times \mathbb{R}} \mathcal{F} f(y, s)\left[\left(1+|y|^{2}\right)^{1 / 2}+|s|^{1 / 2}\right]^{-\sigma} \overline{\mathcal{F} g(y, s)} d y d s \\
& =\int_{\mathbb{R}^{n} \times \mathbb{R}} \mathcal{F} \mathcal{F}^{-1}\left\{\left[\left(1+|y|^{2}\right)^{1 / 2}+|s|^{1 / 2}\right]^{-\sigma} \mathcal{F} f(y, s)\right\} \overline{\mathcal{F} \mathcal{F}^{-1}(\mathcal{F} g(y, s))} d y d s .
\end{aligned}
$$

Using again Parseval's Theorem, we can conclude that

$$
\left\langle\mathcal{I}_{\sigma}^{*} f, \bar{g}\right\rangle=\left\langle\mathcal{I}_{\sigma} f, \bar{g}\right\rangle,
$$

as desired. 
Theorem 2.4.2. Consider $1<p<\infty, \sigma \in \mathbb{R}$, and $p^{\prime}$ the dual index of $p$, i.e. $1 / p+1 / p^{\prime}=1$. Then

$$
\left(F_{\sigma, p a r}^{p, 2}\left(\mathbb{R}^{n} \times \mathbb{R}\right)\right)^{*}=F_{-\sigma, p a r}^{p^{\prime}, 2}\left(\mathbb{R}^{n} \times \mathbb{R}\right) .
$$

Proof. An argument relying on isotropic spaces can be found in [ScTr] 4.2.4. The result itself (without a proof) can also be found in the first part of (A.9) from [Gru1].

Here we present a direct proof using the lifting operator recalled in (2.4.214). First note that, by Theorem 2.1.10,

$$
\mathcal{I}_{\sigma}: F_{\alpha, p a r}^{p, q}\left(\mathbb{R}^{n} \times \mathbb{R}\right) \stackrel{\sim}{\longrightarrow} F_{\alpha+\sigma, p a r}^{p, q}\left(\mathbb{R}^{n} \times \mathbb{R}\right)
$$

for any $0<p<\infty, 0<q \leq \infty$ and $\alpha, \sigma \in \mathbb{R}$. If $1<p<\infty, q=2$ and $\alpha=0$, then for any $\sigma \in \mathbb{R}$,

$$
\mathcal{I}_{\sigma}: L^{p}\left(\mathbb{R}^{n} \times \mathbb{R}\right) \stackrel{\sim}{\longrightarrow} F_{\sigma, \text { par }}^{p, 2}\left(\mathbb{R}^{n} \times \mathbb{R}\right)
$$

and dualizing the above result, we have

$$
\mathcal{I}_{\sigma}^{*}:\left(F_{\sigma, \text { par }}^{p, 2}\left(\mathbb{R}^{n} \times \mathbb{R}\right)\right)^{*} \stackrel{\sim}{\longrightarrow} L^{p^{\prime}}\left(\mathbb{R}^{n} \times \mathbb{R}\right) .
$$

Therefore, with Lemma 2.4.1 in hand, we obtain that

$$
\begin{aligned}
\left(F_{\sigma, p a r}^{p, 2}\left(\mathbb{R}^{n} \times \mathbb{R}\right)\right)^{*} & =\left(\mathcal{I}_{\sigma}^{*}\right)^{-1}\left(L^{p^{\prime}}\left(\mathbb{R}^{n} \times \mathbb{R}\right)\right)=\left(\mathcal{I}_{\sigma}\right)^{-1}\left(L^{p^{\prime}}\left(\mathbb{R}^{n} \times \mathbb{R}\right)\right) \\
& =\mathcal{I}_{-\sigma}\left(L^{p^{\prime}}\left(\mathbb{R}^{n} \times \mathbb{R}\right)\right) \\
& =F_{-\sigma, p a r}^{p^{\prime}, 2}\left(\mathbb{R}^{n} \times \mathbb{R}\right),
\end{aligned}
$$

which is what we wanted to show.

A particular case (i.e when $p=q$ ) of the following result (without a proof), can be found in the second part of (A.9) from [Gru1]. 
Theorem 2.4.3. Let $1<p<\infty, 1 \leq q<\infty$ and $\sigma \in \mathbb{R}$. Then

$$
\left(B_{\sigma, p a r}^{p, q}\left(\mathbb{R}^{n} \times \mathbb{R}\right)\right)^{*}=B_{-\sigma, p a r}^{p^{\prime}, q^{\prime}}\left(\mathbb{R}^{n} \times \mathbb{R}\right)
$$

where $1 / p+1 / p^{\prime}=1$ and $1 / q+1 / q^{\prime}=1$.

Proof. For two different real numbers $\sigma_{0}$ and $\sigma_{1}$ the real interpolation of TriebelLizorkin spaces (cf. first part of (a) in Theorem 2.1.60) gives a Besov space, more precisely,

$$
\left(F_{\sigma_{0}, p a r}^{p, 2}\left(\mathbb{R}^{n} \times \mathbb{R}\right), F_{\sigma_{1}, p a r}^{p, 2}\left(\mathbb{R}^{n} \times \mathbb{R}\right)\right)_{\theta, q}=B_{\sigma, p a r}^{p, q}\left(\mathbb{R}^{n} \times \mathbb{R}\right)
$$

where $0<\theta<1,1 \leq q<\infty$ and $\sigma=(1-\theta) \sigma_{0}+\theta \sigma_{1}$. Now, by the Duality Theorem for the real method (Theorem 3.7.1 from [BeLö]), and by Theorem 2.4.2, we obtain that

$$
\begin{aligned}
\left(B_{\sigma, p a r}^{p, q}\left(\mathbb{R}^{n} \times \mathbb{R}\right)\right)^{*} & =\left(\left(F_{\sigma_{0}, p a r}^{p, 2}\left(\mathbb{R}^{n} \times \mathbb{R}\right)\right)^{*},\left(F_{\sigma_{1}, p a r}^{p, 2}\left(\mathbb{R}^{n} \times \mathbb{R}\right)\right)^{*}\right)_{\theta, q^{\prime}} \\
& =\left(F_{-\sigma_{0}, p a r}^{p^{\prime}, 2}\left(\mathbb{R}^{n} \times \mathbb{R}\right), F_{-\sigma_{1}, p a r}^{p^{\prime}, 2}\left(\mathbb{R}^{n} \times \mathbb{R}\right)\right)_{\theta, q^{\prime}} \\
& =B_{-\sigma, p a r}^{p^{\prime}, q^{\prime}}\left(\mathbb{R}^{n} \times \mathbb{R}\right) .
\end{aligned}
$$

This finishes the proof of the theorem.

Next we give a more general result regarding the dual of a parabolic TriebelLizorkin space.

Theorem 2.4.4. Consider $\sigma \in \mathbb{R}$ and $1<p, q<\infty$ so that the point with coordinates $\left(\frac{1}{p}, \frac{1}{q}\right)$ belongs to the interior of $O M N P$ in the following diagram: 


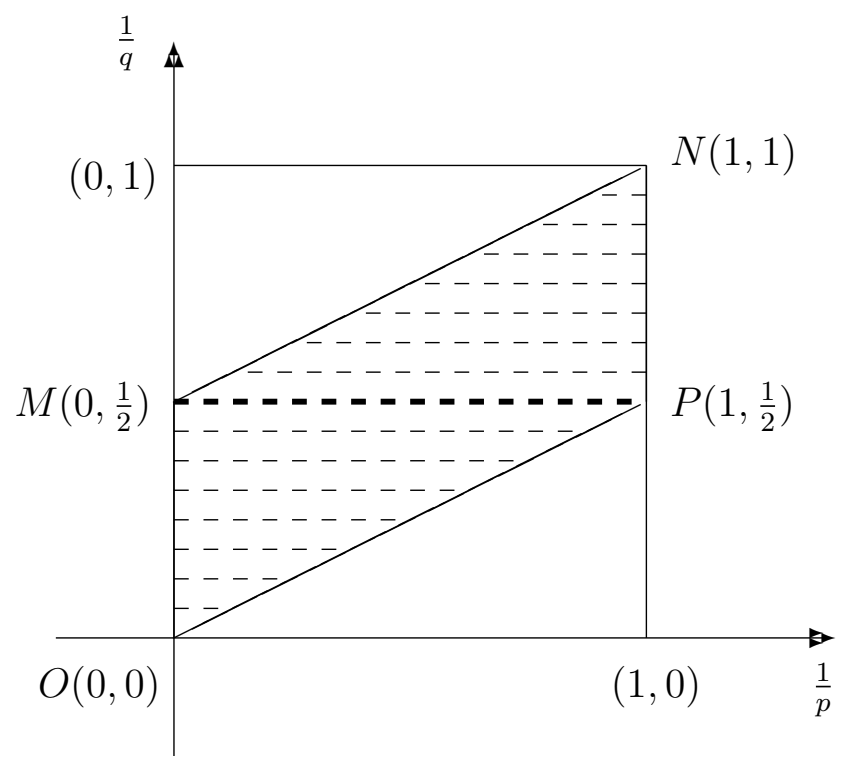

Then

$$
\left(F_{\sigma, p a r}^{p, q}\left(\mathbb{R}^{n} \times \mathbb{R}\right)\right)^{*}=F_{-\sigma, p a r}^{p^{\prime}, q^{\prime}}\left(\mathbb{R}^{n} \times \mathbb{R}\right),
$$

where $1 / p+1 / p^{\prime}=1$ and $1 / q+1 / q^{\prime}=1$.

Proof. Given $p$ and $q$ as in the theorem, geometrical considerations yield to the existence of $p_{0}$ and $p_{1}$ such that $1<p_{0}, p_{1}<\infty, \frac{1}{p_{0}} \in(M P), \frac{1}{p_{1}} \in(O N)$, and

$$
\frac{1}{p}=\frac{1-\theta}{p_{0}}+\frac{\theta}{p_{1}} \text { and } \quad \frac{1}{q}=\frac{1-\theta}{2}+\frac{\theta}{p_{1}}
$$

for $0<\theta<1$. For $p, q, \theta$ as above, and $\sigma_{0}, \sigma_{1} \in \mathbb{R}$, recall from part (e) of Theorem 2.1.60 the following complex interpolation result:

$$
\left[F_{\sigma_{0}, p a r}^{p_{0}, 2}\left(\mathbb{R}^{n} \times \mathbb{R}\right), F_{\sigma_{1}, p a r}^{p_{1}, p_{1}}\left(\mathbb{R}^{n} \times \mathbb{R}\right)\right]_{\theta}=F_{\sigma, p a r}^{p, q}\left(\mathbb{R}^{n} \times \mathbb{R}\right),
$$

where $\sigma=(1-\theta) \sigma_{0}+\theta \sigma_{1}$. Due to the reflexivity of the space $F_{\sigma_{0}, p a r}^{p_{0}, 2}\left(\mathbb{R}^{n} \times \mathbb{R}\right)$ (which follows from Theorem 2.4.2), we can use the Duality Theorem of the complex 
interpolation method (Corollary 4.5.2 from [BeLö]), and then Theorems 2.4.2 and 2.4.3, to obtain that

$$
\begin{aligned}
\left(F_{\sigma, p a r}^{p, q}\left(\mathbb{R}^{n} \times \mathbb{R}\right)\right)^{*} & =\left[\left(F_{\sigma_{0}, \text { par }}^{p_{0}, 2}\left(\mathbb{R}^{n} \times \mathbb{R}\right)\right)^{*},\left(F_{\sigma_{1}, \text { par }}^{p_{1}, p_{1}}\left(\mathbb{R}^{n} \times \mathbb{R}\right)\right)^{*}\right]_{\theta} \\
& =\left[F_{-\sigma_{0}, \text { par }}^{p_{0}^{\prime}, 2}\left(\mathbb{R}^{n} \times \mathbb{R}\right), F_{-\sigma_{1}, p a r}^{p_{1}^{\prime}, p_{1}^{\prime}}\left(\mathbb{R}^{n} \times \mathbb{R}\right)\right]_{\theta} \\
& =F_{-\sigma, p a r}^{p^{\prime}, q^{\prime}}\left(\mathbb{R}^{n} \times \mathbb{R}\right),
\end{aligned}
$$

where $\frac{1}{p_{0}}+\frac{1}{p_{0}^{\prime}}=1$ and $\frac{1}{p_{1}}+\frac{1}{p_{1}^{\prime}}=1$. We can easily check that

$$
\frac{1}{p^{\prime}}=\frac{1-\theta}{p_{0}^{\prime}}+\frac{\theta}{p_{1}^{\prime}} \quad \text { and } \quad \frac{1}{q^{\prime}}=\frac{1-\theta}{2}+\frac{\theta}{p_{1}^{\prime}},
$$

which is necessary in the last equality of (2.4.215).

In what follows, we specialize Theorems 2.4.3 and 2.4.4 to the case when the underlying space is $\partial \Omega \times \mathbb{R}$ for any Lipschitz domain $\Omega \subseteq \mathbb{R}^{n}$.

Proposition 2.4.5. Let $\Omega$ be a Lipschitz domain in $\mathbb{R}^{n}$ and consider the indices $1<p<\infty, 1 \leq q<\infty$ and $0<|\alpha|<1$. Then one has

$$
B_{-\alpha, p a r}^{p, q}(\partial \Omega \times \mathbb{R})=\left(B_{\alpha, p a r}^{p^{\prime}, q^{\prime}}(\partial \Omega \times \mathbb{R})\right)^{*}
$$

where $1 / p+1 / p^{\prime}=1$ and $1 / q+1 / q^{\prime}=1$.

Proof. We present only the proof of the case when $0<\alpha<1$, since if $-1<\alpha<0$, one proceeds analogously, employing Definition 2.3.1 in place of Definition 2.3.3.

Case (i) $\Omega$ is unbounded. Theorem 2.4.3 and Definition 2.3.3 yield the desired result.

Case (ii) $\Omega$ is bounded. Let us recall the notation from Remark 1.2.1 and introduce the following mappings.

$$
\Phi: B_{-\alpha, p a r}^{p, q}(\partial \Omega \times \mathbb{R}) \longrightarrow\left(B_{\alpha, p a r}^{p^{\prime}, q^{\prime}}(\partial \Omega \times \mathbb{R})\right)^{*}
$$




$$
[\Phi(f)](g):=\sum_{i=1}^{N}\left\langle\widetilde{\eta_{i} f}{ }^{\partial \Omega_{i}}, \widetilde{\psi_{i} g}{ }^{\partial \Omega_{i}}\right\rangle
$$

for $f \in B_{-\alpha, p a r}^{p, q}(\partial \Omega \times \mathbb{R})$ and $g \in B_{\alpha, p a r}^{p^{\prime}, q^{\prime}}(\partial \Omega \times \mathbb{R})$, where $\eta_{i} \in C_{c}^{\infty}\left(V_{i}\right)$ and $\eta_{i} \equiv 1$ on the support of $\psi_{i}, i=1, \ldots, N$. Also, let

$$
\Psi:\left(B_{\alpha, p a r}^{p^{\prime}, q^{\prime}}(\partial \Omega \times \mathbb{R})\right)^{*} \longrightarrow B_{-\alpha, p a r}^{p, q}(\partial \Omega \times \mathbb{R})
$$

defined by

$$
\Psi(\Lambda):=\sum_{i=1}^{N} \widetilde{\psi_{i} \cdot \Lambda} \partial \Omega, \quad \Lambda \in\left(B_{\alpha, p a r}^{p^{\prime}, q^{\prime}}(\partial \Omega \times \mathbb{R})\right)^{*}
$$

where

$$
\left(\psi_{i} \cdot \Lambda\right)(f):=\Lambda\left({\widetilde{\psi_{i} f}}^{\partial \Omega}\right) \text { for } f \in B_{\alpha, p a r}^{p^{\prime}, q^{\prime}}\left(\partial \Omega_{i} \times \mathbb{R}\right)
$$

Note that matters have been arranged so that, when $\Phi$ is acting on $L_{c}^{\infty}$-functions on $\partial \Omega \times \mathbb{R}$, and $\Psi$ is considered on functionals which are pairings with $L_{c}^{\infty}$-functions on $\partial \Omega \times \mathbb{R}, \Phi$ and $\Psi$ are the identity operator.

According to Definition 2.3.3 (partition of unity and pull-back), $f$ belongs to $B_{-\alpha, p a r}^{p, q}(\partial \Omega \times \mathbb{R})$ if and only if for each $i=1, \ldots, N$,

$$
F_{i} \in B_{-\alpha, p a r}^{p, q}\left(\mathbb{R}^{n-1} \times \mathbb{R}\right),
$$

where $F_{i}\left(x^{\prime}, t\right):={\widetilde{\psi_{i}}}^{\partial \Omega_{i}}\left(x^{\prime}, \varphi_{i}\left(x^{\prime}\right), t\right) \sqrt{1+\left|\nabla \varphi_{i}\left(x^{\prime}\right)\right|^{2}}$ for $\left(x^{\prime}, t\right) \in \mathbb{R}^{n-1} \times \mathbb{R}$, and the graph of the Lipschitz function $\varphi_{i}: \mathbb{R}^{n-1} \rightarrow \mathbb{R}$ represents the boundary of $\Omega_{i}$.

Since $L_{c}^{\infty}\left(\mathbb{R}^{n-1} \times \mathbb{R}\right)$ is dense in $B_{-\alpha, p a r}^{p, q}\left(\mathbb{R}^{n-1} \times \mathbb{R}\right)$, the class of functionals, which are pairings with $L_{c}^{\infty}$-functions on $\mathbb{R}^{n-1} \times \mathbb{R}$, is dense in $\left(B_{\alpha, p a r}^{p^{\prime}, q^{\prime}}\left(\mathbb{R}^{n-1} \times \mathbb{R}\right)\right)^{*}=$ $B_{-\alpha, p a r}^{p, q}\left(\mathbb{R}^{n-1} \times \mathbb{R}\right)$. 
In order to show that $\Phi \circ \Psi=I$ on $\left(B_{\alpha, p a r}^{p^{\prime}, q^{\prime}}(\partial \Omega \times \mathbb{R})\right)^{*}$, and that $\Psi \circ \Phi=I$ on $B_{-\alpha, p a r}^{p, q}(\partial \Omega \times \mathbb{R})$, it is enough to work with dense subclasses, in which case, given the above observation, the verification is straightforward.

In a very similar fashion, relying on Theorem 2.4.4 and Definitions 2.3.5 and 2.3.7, we obtain the following.

Proposition 2.4.6. For a Lipschitz domain $\Omega \subseteq \mathbb{R}^{n}$, indices $1<p, q<\infty$ such that the pair $\left(\frac{1}{p}, \frac{1}{q}\right)$ belongs to the interior of $O M N P$, and $0<|\alpha|<1$, we have

$$
F_{-\alpha, p a r}^{p, q}(\partial \Omega \times \mathbb{R})=\left(F_{\alpha, p a r}^{p^{\prime}, q^{\prime}}(\partial \Omega \times \mathbb{R})\right)^{*}
$$

where $1 / p+1 / p^{\prime}=1$ and $1 / q+1 / q^{\prime}=1$.

Remark 2.4.7. In particular, if we take $q=2$ in Proposition 2.4.6, then $1<p<\infty$ and one may consider $0 \leq|\alpha| \leq 1$. In this case, for a Lipschitz domain $\Omega \subseteq \mathbb{R}^{n}$, the previous result reads as

$$
L_{-\alpha, p a r}^{p}(\partial \Omega \times \mathbb{R})=\left(L_{\alpha, p a r}^{p^{\prime}}(\partial \Omega \times \mathbb{R})\right)^{*}
$$

where $1 / p+1 / p^{\prime}=1$.

\subsection{Envelopes of parabolic Hardy spaces}

We now present the parabolic analogue of Theorem 1.2.51 and its inhomogeneous counterpart, then a related result involving the same type of spaces, but with different amount of smoothness. Their consequence, Corollary 2.5.3 will play a key role in the proof of our main result in Section 6.1, Theorem 6.1.4. 
Theorem 2.5.1. For $\frac{n+2}{n+3}<p<p^{*} \leq 1$ and $\alpha=(n+2)\left(\frac{1}{p}-\frac{1}{p^{*}}\right)$ one has

$$
\begin{aligned}
& \mathcal{E}_{p^{*}}\left(\dot{H}_{\text {par }}^{p}\left(\mathbb{R}^{n} \times \mathbb{R}\right)\right)=\dot{B}_{-\alpha, p a r}^{p^{*}}\left(\mathbb{R}^{n} \times \mathbb{R}\right), \\
& \mathcal{E}_{p^{*}}\left(H_{p a r}^{p}\left(\mathbb{R}^{n} \times \mathbb{R}\right)\right)=B_{-\alpha, p a r}^{p^{*}}\left(\mathbb{R}^{n} \times \mathbb{R}\right) .
\end{aligned}
$$

Proof. Step I. Show that the closure in $\dot{B}_{-\alpha, p a r}^{p^{*}}\left(\mathbb{R}^{n} \times \mathbb{R}\right)$ of the $p^{*}$-convex hull of the parabolic unit ball of $\dot{H}_{\text {par }}^{p}\left(\mathbb{R}^{n} \times \mathbb{R}\right)$ contains a ball centered at the origin in $\dot{B}_{-\alpha, p a r}^{p^{*}}\left(\mathbb{R}^{n} \times \mathbb{R}\right)$

Let $u \in \dot{B}_{-\alpha, p a r}^{p^{*}}\left(\mathbb{R}^{n} \times \mathbb{R}\right)$ such that $\|u\|_{\dot{B}_{-\alpha, p a r}^{p^{*}}\left(\mathbb{R}^{n} \times \mathbb{R}\right)}<1$. Then there exist $\left\{\lambda_{Q}\right\}_{Q} \in$ $\ell^{p^{*}}$ and $a_{Q}$ atoms for $\dot{B}_{-\alpha, p a r}^{p^{*}}\left(\mathbb{R}^{n} \times \mathbb{R}\right)$ such that

$$
u=\sum_{Q} \lambda_{Q} a_{Q} \quad \text { and } \quad \inf \left(\sum_{Q}\left|\lambda_{Q}\right|^{p^{*}}\right)^{1 / p^{*}}<1 .
$$

For the given range of indices one can easily check that the atoms for the space $\dot{B}_{-\alpha, p a r}^{p^{*}}\left(\mathbb{R}^{n} \times \mathbb{R}\right)$ coincide with the atoms for $\dot{H}_{p a r}^{p}\left(\mathbb{R}^{n} \times \mathbb{R}\right)$, hence $a_{Q}$ are also atoms for $\dot{H}_{p a r}^{p}\left(\mathbb{R}^{n} \times \mathbb{R}\right)$. Therefore, each partial sum $\sum_{Q} \lambda_{Q} a_{Q}$ belongs to the $p^{*}$-convex hull of the parabolic unit ball in $\dot{H}_{\text {par }}^{p}\left(\mathbb{R}^{n} \times \mathbb{R}\right)$, thus $u$ can be approximated (in $\left.\dot{B}_{-\alpha, p a r}^{p^{*}}\left(\mathbb{R}^{n} \times \mathbb{R}\right)\right)$ by elements from $\mathcal{E}_{p^{*}}\left(\dot{H}_{p a r}^{p}\left(\mathbb{R}^{n} \times \mathbb{R}\right)\right)$.

Step II. Show that the space $\dot{H}_{\text {par }}^{p}\left(\mathbb{R}^{n} \times \mathbb{R}\right)$ has the approximate identity property, relative to $\dot{B}_{-\alpha, p a r}^{p^{*}}\left(\mathbb{R}^{n} \times \mathbb{R}\right)$.

In order to apply a result of $[\mathrm{BoHo}]$, let us consider $\varphi \in \mathrm{S}\left(\mathbb{R}^{n} \times \mathbb{R}\right)$ with the following characteristics:

$$
\begin{gathered}
\operatorname{supp} \hat{\varphi} \subset\left([-\pi, \pi]^{n} \backslash\{0\}\right) \times\left(\left[-\pi^{2}, \pi^{2}\right] \backslash\{0\}\right), \\
\sup _{m \in \mathbb{Z}}\left|\hat{\varphi}\left(A^{m}(\xi, \tau)\right)\right|>0 \text { for all }(\xi, \tau) \in \mathbb{R}^{n} \times \mathbb{R} \backslash\{(0,0)\}
\end{gathered}
$$


and

$$
\sum_{m \in \mathbb{Z}}\left|\hat{\varphi}\left(A^{m}(\xi, \tau)\right)\right|^{2}=1 \quad \forall(\xi, \tau) \in \mathbb{R}^{n} \times \mathbb{R} \backslash\{(0,0)\}
$$

where $A=\left(a_{i j}\right)_{1 \leq i, j \leq n+1}$ is a matrix defined by $a_{i i}=2$ if $i \leq n, a_{i i}=4$ if $i=n+1$, and $a_{i j}=0$ for $i \neq j$. Also, let $\nu \in \mathbb{Z},(k, l) \in \mathbb{Z}^{n} \times \mathbb{Z}$, and recall the definition of the parabolic dyadic cube from (1.2.87):

$$
\begin{gathered}
Q=Q_{\nu, k, l}:=\left\{(x, t) \in \mathbb{R}^{n} \times \mathbb{R}: 2^{-\nu} k_{i} \leq x_{i} \leq 2^{-\nu}\left(k_{i}+1\right), 1 \leq i \leq n,\right. \\
\left.2^{-2 \nu} l \leq t \leq 2^{-2 \nu}(l+1)\right\} .
\end{gathered}
$$

We also recall from Definition 1.2.40 the way the operators $T_{\varphi}$ and $S_{\varphi}$ are defined, and, as in (1.2.91), we set

$$
\varphi_{Q}(x, t):=2^{\frac{\nu(n+2)}{2}} \varphi\left(2^{\nu} x-k, 2^{2 \nu} t-l\right), \quad(x, t) \in \mathbb{R}^{n} \times \mathbb{R} .
$$

According to Theorem 1.2.41, the operators

$$
T_{\varphi}: \dot{b}_{-\alpha, p a r}^{p^{*}}\left(\mathbb{R}^{n} \times \mathbb{R}\right) \longrightarrow \dot{B}_{-\alpha, p a r}^{p^{*}}\left(\mathbb{R}^{n} \times \mathbb{R}\right)
$$

and

$$
S_{\varphi}: \dot{B}_{-\alpha, p a r}^{p^{*}}\left(\mathbb{R}^{n} \times \mathbb{R}\right) \longrightarrow \dot{b}_{-\alpha, p a r}^{p^{*}}\left(\mathbb{R}^{n} \times \mathbb{R}\right)
$$

are bounded, and

$$
T_{\varphi} \circ S_{\varphi}=I \quad \text { on } \dot{B}_{-\alpha, p a r}^{p^{*}}\left(\mathbb{R}^{n} \times \mathbb{R}\right) .
$$

Therefore, given $f \in \dot{B}_{-\alpha, p a r}^{p^{*}}\left(\mathbb{R}^{n} \times \mathbb{R}\right)$,

$$
\|f\|_{\dot{B}_{-\alpha, p a r}^{p^{*}}\left(\mathbb{R}^{n} \times \mathbb{R}\right)} \leq\left\|\left(T_{\varphi} \circ S_{\varphi}\right) f\right\|_{\dot{B}_{-\alpha, p a r}^{p^{*}}\left(\mathbb{R}^{n} \times \mathbb{R}\right)} \leq c\left\|S_{\varphi} f\right\|_{\dot{b}_{-\alpha, p a r}^{p^{*}}\left(\mathbb{R}^{n} \times \mathbb{R}\right)} .
$$

On the other hand

$$
\left\|S_{\varphi} f\right\|_{\dot{b}_{-\alpha, p a r}^{p^{*}}\left(\mathbb{R}^{n} \times \mathbb{R}\right)} \leq c\|f\|_{\dot{B}_{-\alpha, p a r}^{p^{*}}\left(\mathbb{R}^{n} \times \mathbb{R}\right)} .
$$


Now from (2.5.218) and (2.5.219) we obtain that

$$
\begin{aligned}
& \|f\|_{\dot{B}_{-\alpha, p a r}^{p^{*}}\left(\mathbb{R}^{n} \times \mathbb{R}\right)} \approx\left\|S_{\varphi} f\right\|_{\dot{b}_{-\alpha, p a r}^{p^{*}}\left(\mathbb{R}^{n} \times \mathbb{R}\right)} \\
& =\left\|\left(\sum_{Q}|Q|^{\frac{\alpha p^{*}}{n+2}-\frac{p^{*}}{2}}\left|\left\langle f, \varphi_{Q}\right\rangle\right|^{p^{*}} \chi_{Q}\right)^{1 / p^{*}}\right\|_{L^{p^{*}\left(\mathbb{R}^{n} \times \mathbb{R}\right)}} \\
& =\left(\sum_{Q}|Q|^{\frac{\alpha p^{*}}{n+2}-\frac{p^{*}}{2}+1}\left|\left\langle f, \varphi_{Q}\right\rangle\right|^{p^{*}}\right)^{1 / p^{*}} \\
& =\left\|\left\{|Q|^{\frac{\alpha}{n+2}-\frac{1}{2}+\frac{1}{p^{*}}}\left\langle f, \varphi_{Q}\right\rangle\right\}\right\|_{\ell^{p^{*}}} \\
& =\left\|\left\{\left\langle|Q|^{\frac{1}{p}-\frac{1}{2}} f, \varphi_{Q}\right\rangle\right\}_{Q}\right\|_{\ell^{p^{*}}}=\left\|\left\{\left\langle\tilde{f}_{Q}, \varphi_{Q}\right\rangle\right\}_{Q}\right\|_{\ell^{p^{*}}}=\left\|\left\{\tilde{f}_{Q}^{l}\right\}_{Q}\right\|_{\ell^{p^{*}}},
\end{aligned}
$$

where, by definition, $\tilde{f}_{Q}:=|Q|^{\frac{1}{p}-\frac{1}{2}} f$ and $\tilde{f}_{Q}^{l}:=\left\langle\tilde{f}_{Q}, \varphi_{Q}\right\rangle$. We can also write

$$
f=\left(T_{\varphi} \circ S_{\varphi}\right) f=\sum_{Q}\left\langle f, \varphi_{Q}\right\rangle \varphi_{Q}=\sum_{Q} \tilde{f}_{Q}^{l} \tilde{\varphi}_{Q}^{l}
$$

where $\tilde{\varphi}_{Q}^{l}:=|Q|^{\frac{1}{2}-\frac{1}{p}} \varphi_{Q}$. We are left with showing that $\tilde{\varphi}_{Q}^{l}$ belongs to $\dot{H}_{p a r}^{p}\left(\mathbb{R}^{n} \times \mathbb{R}\right)$. In particular, we claim that $\tilde{\varphi}_{Q}^{l}$ is a fixed multiple of a molecule for $\dot{H}_{p a r}^{p}\left(\mathbb{R}^{n} \times \mathbb{R}\right)$.

Based on Lemma 9.3 and the Remark on p. 63 of [Bow1], a molecule $b$ for the space $\dot{H}_{\text {par }}^{p}\left(\mathbb{R}^{n} \times \mathbb{R}\right)$ localized around the parabolic ball $\left(x_{0}, t_{0}\right)+B_{k}$ (where $B_{k}:=A^{k} \Delta$, $k \in \mathbb{Z}$ and $\left.\Delta:=\left\{(x, t) \in \mathbb{R}^{n} \times \mathbb{R}:|x|+|t|^{1 / 2}<1\right\}\right)$ should satisfy the following conditions:

$$
\begin{gathered}
\int_{\mathbb{R}^{n} \times \mathbb{R}} b(x, t) d x d t=0, \\
\left\{\begin{array}{l}
\left(\frac{1}{\left|B_{k}\right|} \int_{\left(x_{0}, t_{0}\right)+B_{k}}|b(x, t)|^{q} d x d t\right)^{1 / q} \leq c\left|B_{k}\right|^{-1 / p} \quad \text { if } 1 \leq q<\infty \\
\|b\|_{L^{\infty}\left(\mathbb{R}^{n} \times \mathbb{R}\right)} \leq c\left|B_{k}\right|^{-1 / p} \quad \text { if } q=\infty
\end{array}\right.
\end{gathered}
$$




$$
|b(x, t)| \leq\left|B_{k}\right|^{-1 / p} \rho\left(A^{-k}\left[(x, t)-\left(x_{0}, t_{0}\right)\right]\right)^{-\delta} \quad \text { for } \quad(x, t) \in\left(x_{0}, t_{0}\right)+B_{k}^{c},
$$

where $1 \leq q \leq \infty, \frac{n+2}{n+3}<p \leq 1, p<q, \rho(x, t)=\left(|x|^{2}+|t|\right)^{\frac{n+2}{2}}$ and $\delta>\frac{1}{p}$.

In order to see that $\tilde{\varphi}_{Q}^{l}$ satisfies (2.5.220) we note that under the current assumptions, cf. Definition 3.2 of [BoHo],

$$
\int_{\mathbb{R}^{n} \times \mathbb{R}} x^{\beta} t^{s} \varphi_{Q}(x, t) d x d t=0 \quad \text { for any multiindex } \beta \text { and any } s \in \mathbb{N}_{0} \text {. }
$$

To verify $(2.5 .221)$ for $\tilde{\varphi}_{Q}^{l}$ we proceed as follows. Using Section 2.2 from [BoHo] and the fact that $|\operatorname{det} A|^{-\nu}=|Q|$, for some $\left(x_{0}, t_{0}\right) \in \mathbb{R}^{n} \times \mathbb{R}$ we have

$$
\begin{aligned}
\tilde{\varphi}_{Q}^{l}(x, t) & =|Q|^{1-\frac{1}{p}}|\operatorname{det} A|^{\nu} \varphi\left(A^{\nu}\left[(x, t)-\left(x_{0}, t_{0}\right)\right]\right) \\
& =|Q|^{-\frac{1}{p}} \varphi\left(A^{\nu}\left[(x, t)-\left(x_{0}, t_{0}\right)\right]\right) .
\end{aligned}
$$

With this in hand one can easily see that

$$
\begin{aligned}
\left\|\tilde{\varphi}_{Q}^{l}\right\|_{L^{\infty}\left(\mathbb{R}^{n} \times \mathbb{R}\right)} \leq c|Q|^{-1 / p} & \Longleftrightarrow \sup _{(x, t) \in \mathbb{R}^{n} \times \mathbb{R}}\left|\varphi\left(A^{\nu}\left[(x, t)-\left(x_{0}, t_{0}\right)\right]\right)\right| \leq c \\
& \Longleftrightarrow\|\varphi\|_{L^{\infty}\left(\mathbb{R}^{n} \times \mathbb{R}\right)} \leq c .
\end{aligned}
$$

Since $\varphi \in \mathrm{S}\left(\mathbb{R}^{n} \times \mathbb{R}\right)$ by hypothesis, (2.5.224) holds, proving the second part of (2.5.221), i.e. when $q=\infty$. In the case when $1 \leq q<\infty$, using the fact that $\left\|\tilde{\varphi}_{Q}^{l}\right\|_{L^{\infty}\left(\mathbb{R}^{n} \times \mathbb{R}\right)} \leq c|Q|^{-1 / p}$, we obtain that

$$
\left(\frac{1}{|Q|} \int_{\left(x_{0}, t_{0}\right)+A^{-\nu} \Delta}\left|\tilde{\varphi}_{Q}^{l}(x, t)\right|^{q} d x d t\right)^{1 / q} \leq c|Q|^{-1 / p} \Longleftrightarrow\left(\frac{1}{|Q|}|Q|^{-q / p}|Q|\right)^{1 / q} \leq c|Q|^{-1 / p} .
$$

This clearly shows that $\tilde{\varphi}_{Q}^{l}$ satisfies the first part of (2.5.221) as well. 
Finally, we are going to show that $\tilde{\varphi}_{Q}^{l}$ satisfies (2.5.222) with a fixed mutiplicative constant. In order to do so we first observe that for $\rho(x, t)=\left(|x|^{2}+|t|\right)^{\frac{n+2}{2}}$ and $(x, t) \notin\left(x_{0}, t_{0}\right)+Q$, the following equivalences hold

$$
\begin{aligned}
\left|\tilde{\varphi}_{Q}^{l}(x, t)\right| & \leq c|Q|^{-1 / p} \rho\left(A^{\nu}\left[(x, t)-\left(x_{0}, t_{0}\right)\right]\right)^{-\delta} \\
& \Longleftrightarrow\left|\varphi\left(A^{\nu}\left[(x, t)-\left(x_{0}, t_{0}\right)\right]\right)\right| \leq c \rho\left(A^{\nu}\left[(x, t)-\left(x_{0}, t_{0}\right)\right]\right)^{-\delta} \\
& \Longleftrightarrow\left(|y|^{2}+|s|\right)^{\frac{n+2}{2} \delta}|\varphi(y, s)| \leq c .
\end{aligned}
$$

Since $\varphi \in \mathrm{S}\left(\mathbb{R}^{n} \times \mathbb{R}\right), \varphi$ decays faster then any polynomial at infinity, in particular $|\varphi(y, s)| \leq \frac{c}{\left(|y|^{2}+|t|\right)^{\frac{n+2}{2} \delta}}$, therefore (2.5.226) holds, and $\tilde{\varphi}_{Q}^{l}$ satisfies (2.5.222) with a multiplicative constant.

We now can conclude that $\tilde{\varphi}_{Q}^{l}$ is a fixed multiple of a molecule for $\dot{H}_{p a r}^{p}\left(\mathbb{R}^{n} \times \mathbb{R}\right)$. Therefore, $\tilde{\varphi}_{Q}^{l}$ belongs to $\dot{H}_{p a r}^{p}\left(\mathbb{R}^{n} \times \mathbb{R}\right)$, and the proof of (2.5.216) is complete.

In order to show (2.5.217), we follow the above argument with some modifications. More precisely, in Step I we use the fact that, for the range of indices we consider, atoms for $B_{-\alpha, p a r}^{p^{*}}\left(\mathbb{R}^{n} \times \mathbb{R}\right)$ are also atoms for $H_{\text {par }}^{p}\left(\mathbb{R}^{n} \times \mathbb{R}\right)$. In Step II we assume that $\varphi, \Phi \in \mathrm{S}\left(\mathbb{R}^{n} \times \mathbb{R}\right)$ have the following properties:

$$
\begin{gathered}
\operatorname{supp} \hat{\varphi} \subset\left([-\pi, \pi]^{n} \backslash\{0\}\right) \times\left(\left[-\pi^{2}, \pi^{2}\right] \backslash\{0\}\right), \\
\operatorname{supp} \hat{\Phi} \subset[-\pi, \pi]^{n} \times\left[-\pi^{2}, \pi^{2}\right], \\
\sup _{m \in \mathbb{Z}}\left|\hat{\varphi}\left(A^{m}(\xi, \tau)\right)\right|>0 \quad \text { for all }(\xi, \tau) \in \mathbb{R}^{n} \times \mathbb{R} \backslash\{(0,0)\}, \\
\sup _{m \geq 1}\left\{\left|\hat{\varphi}\left(A^{-m}(\xi, \tau)\right)\right|,|\hat{\Phi}(\xi, \tau)|\right\}>0 \quad \text { for all }(\xi, \tau) \in \mathbb{R}^{n} \times \mathbb{R} \backslash\{(0,0)\} .
\end{gathered}
$$


We next recall form Remark 1.2.42 the definition of the operators $T_{\varphi, \Phi}$ and $S_{\varphi, \Phi}$, as well as their important property that $T_{\varphi, \Phi} \circ S_{\varphi, \Phi}$ is the identity operator on $B_{-\alpha, p a r}^{p^{*}}\left(\mathbb{R}^{n} \times \mathbb{R}\right)$. According to Section 3.2 of $[\mathrm{BoHo}]$, for any $f$ that belongs to $\mathrm{S}^{\prime}\left(\mathbb{R}^{n} \times \mathbb{R}\right)$

$$
f=\sum_{|Q|=1}\left\langle f, \Phi_{Q}\right\rangle \Phi_{Q}+\sum_{|Q|<1}\left\langle f, \varphi_{Q}\right\rangle \varphi_{Q}
$$

where

$$
\Phi_{Q}(x, t):=2^{\frac{\nu(n+2)}{2}} \Phi\left(2^{\nu} x-k, 2^{2 \nu} t-l\right), \quad(x, t) \in \mathbb{R}^{n} \times \mathbb{R},
$$

and, as in the first part of this proof, $\nu \in \mathbb{Z},(k, l) \in \mathbb{Z}^{n} \times \mathbb{Z}$. Similar to the argument in the proof of (2.5.216), the second sum in the right-hand side of (2.5.227) can be expressed as

$$
\sum_{|Q|<1}\left\langle f, \varphi_{Q}\right\rangle \varphi_{Q}=\sum_{|Q|<1} \tilde{f}_{Q}^{l} \tilde{\varphi}_{Q}^{l}
$$

where $\tilde{f}_{Q}^{l}:=\left\langle\tilde{f}_{Q}, \varphi_{Q}\right\rangle, \tilde{f}_{Q}:=|Q|^{1 / p-1 / 2} f$ and $\tilde{\varphi}_{Q}^{l}:=|Q|^{1 / 2-1 / p} \varphi_{Q}$. In the spirit of the proof of (2.5.216) we can show that $\tilde{\varphi}_{Q}^{l} \in \dot{H}_{\text {par }}^{p}\left(\mathbb{R}^{n} \times \mathbb{R}\right)$, hence $\tilde{\varphi}_{Q}^{l} \in H_{\text {par }}^{p}\left(\mathbb{R}^{n} \times \mathbb{R}\right)$.

As far as the first sum in the right-hand side of (2.5.227) is concerned, we are left with showing that $\Phi_{Q}$ belongs to $H_{p a r}^{p}\left(\mathbb{R}^{n} \times \mathbb{R}\right)$, in particular, we claim that $\Phi_{Q}$ is a fixed multiple of a molecule for $H_{p a r}^{p}\left(\mathbb{R}^{n} \times \mathbb{R}\right)$. Much as in the elliptic case, molecules for $H_{\text {par }}^{p}\left(\mathbb{R}^{n} \times \mathbb{R}\right)$ are as those for $\dot{H}_{\text {par }}^{p}\left(\mathbb{R}^{n} \times \mathbb{R}\right)$ if $|Q|<1$, and with no vanishing moment condition if $|Q|=1$.

Note that in order to check the size conditions (2.5.221) and (2.5.222) for $\Phi_{Q}$ with 
$|Q|=1$, one needs to show that

$$
\left\{\begin{array}{l}
\left(\int_{\left(x_{0}, t_{0}\right)+A^{-\nu} \Delta}\left|\Phi_{Q}(x, t)\right|^{q} d x d t\right)^{1 / q} \leq c \quad \text { if } 1 \leq q<\infty \\
\left\|\Phi_{Q}\right\|_{L^{\infty}\left(\mathbb{R}^{n} \times \mathbb{R}\right)} \leq c \quad \text { if } \quad q=\infty
\end{array}\right.
$$

and

$$
\left|\Phi_{Q}(x, t)\right| \leq \frac{c}{\left(\left|x-x_{0}\right|^{2}+\left|t-t_{0}\right|\right)^{\frac{n+2}{2} \delta}} \quad \text { for some } \delta>\frac{1}{p} .
$$

We can easily verify the inequalities in (2.5.229) and (2.5.230) using only the definition of $\Phi_{Q}$ (see (2.5.228) ) and the hypothesis that $\Phi \in \mathrm{S}\left(\mathbb{R}^{n} \times \mathbb{R}\right)$. Hence $\Phi_{Q}$ is a fixed multiple of a molecule for $H_{p a r}^{p}\left(\mathbb{R}^{n} \times \mathbb{R}\right)$. This completes the proof of $(2.5 .217)$ and of Theorem 2.5.1.

Our next goal is to establish an analogue of Theorem 2.5.1, involving spaces which have an additional unit of smoothness.

Theorem 2.5.2. For $\frac{n+2}{n+3}<p<p^{*} \leq 1$ and $\alpha=(n+2)\left(\frac{1}{p}-\frac{1}{p^{*}}\right)$ the following holds:

$$
\begin{aligned}
& \mathcal{E}_{p^{*}}\left(\dot{H}_{\text {par }}^{1, p}\left(\mathbb{R}^{n} \times \mathbb{R}\right)\right)=\dot{B}_{1-\alpha, \text { par }}^{p^{*}}\left(\mathbb{R}^{n} \times \mathbb{R}\right), \\
& \mathcal{E}_{p^{*}}\left(H_{\text {par }}^{1, p}\left(\mathbb{R}^{n} \times \mathbb{R}\right)\right)=B_{1-\alpha, \text { par }}^{p^{*}}\left(\mathbb{R}^{n} \times \mathbb{R}\right) .
\end{aligned}
$$

Proof. In order to prove (2.5.231), first let us recall from Theorem 2.1.14 the definition of the operator $\dot{I}_{\sigma}$ : for $\sigma \in \mathbb{R}$ and $f \in \mathrm{S}^{\prime}\left(\mathbb{R}^{n} \times \mathbb{R}\right)$,

$$
\dot{I}_{\sigma} f:=\mathcal{F}^{-1}\left[\left(|\xi|^{2}+|\tau|\right)^{-\frac{\sigma}{2}} \mathcal{F} f(\xi, \tau)\right], \quad(\xi, \tau) \in \mathbb{R}^{n} \times \mathbb{R}
$$

From the same theorem we know that, if $0<p, q \leq \infty(p \neq \infty$ for the $F$-scale $)$ and $\alpha \in \mathbb{R}$, then

$$
\dot{I}_{\sigma}: \dot{B}_{\alpha, p a r}^{p, q}\left(\mathbb{R}^{n} \times \mathbb{R}\right) \stackrel{\sim}{\longrightarrow} \dot{B}_{\alpha+\sigma, p a r}^{p, q}\left(\mathbb{R}^{n} \times \mathbb{R}\right),
$$




$$
\dot{I}_{\sigma}: \dot{F}_{\alpha, p a r}^{p, q}\left(\mathbb{R}^{n} \times \mathbb{R}\right) \stackrel{\sim}{\longrightarrow} \dot{F}_{\alpha+\sigma, p a r}^{p, q}\left(\mathbb{R}^{n} \times \mathbb{R}\right)
$$

are isomorhisms. In particular, for $q=2, \alpha=0$ and $\sigma=1,(2.5 .234)$ reads as

$$
\dot{I}_{1}: \dot{F}_{0, p a r}^{p, 2}\left(\mathbb{R}^{n} \times \mathbb{R}\right) \stackrel{\sim}{\longrightarrow} \dot{F}_{1, p a r}^{p, 2}\left(\mathbb{R}^{n} \times \mathbb{R}\right)
$$

for any $0<p<\infty$. According to Definition 2.1.43 and Proposition 2.1.52, respectively, for $\frac{n+2}{n+3}<p \leq 1$, one has the following equivalences:

$$
\dot{F}_{0, p a r}^{p, 2}\left(\mathbb{R}^{n} \times \mathbb{R}\right) \equiv \dot{H}_{\text {par }}^{p}\left(\mathbb{R}^{n} \times \mathbb{R}\right) \quad \text { and } \quad \dot{F}_{1, p a r}^{p, 2}\left(\mathbb{R}^{n} \times \mathbb{R}\right) \equiv \dot{H}_{\text {par }}^{1, p}\left(\mathbb{R}^{n} \times \mathbb{R}\right)
$$

Now (2.5.235) in concert with (2.5.236) yields

$$
\dot{I}_{1}: \dot{H}_{\text {par }}^{p}\left(\mathbb{R}^{n} \times \mathbb{R}\right) \stackrel{\sim}{\longrightarrow} \dot{H}_{\text {par }}^{1, p}\left(\mathbb{R}^{n} \times \mathbb{R}\right), \quad \text { where } \quad \frac{n+2}{n+3}<p \leq 1
$$

With this in hand, thanks to Proposition 1.2.45, we can conclude that, for any $0<p^{*} \leq 1$, the operator $\dot{I}_{1}$ extends to

$$
\dot{I}_{1}: \mathcal{E}_{p^{*}}\left(\dot{H}_{\text {par }}^{p}\left(\mathbb{R}^{n} \times \mathbb{R}\right)\right) \stackrel{\sim}{\longrightarrow} \mathcal{E}_{p^{*}}\left(\dot{H}_{\text {par }}^{1, p}\left(\mathbb{R}^{n} \times \mathbb{R}\right)\right)
$$

Identifying the envelope of $\dot{H}_{\text {par }}^{p}\left(\mathbb{R}^{n} \times \mathbb{R}\right)$, in view of Theorem 2.5.1, the isomorphism in $(2.5 .238)$ becomes

$$
\dot{I}_{1}: \dot{B}_{-\alpha, p a r}^{p^{*}}\left(\mathbb{R}^{n} \times \mathbb{R}\right) \stackrel{\sim}{\longrightarrow} \mathcal{E}_{p^{*}}\left(\dot{H}_{\text {par }}^{1, p}\left(\mathbb{R}^{n} \times \mathbb{R}\right)\right)
$$

where $\frac{n+2}{n+3}<p<p^{*} \leq 1$ and $\alpha=(n+2)\left(\frac{1}{p}-\frac{1}{p^{*}}\right)$.

Keepig this in mind, we now employ a particular case of (2.5.233). More concretely, for $\frac{n+2}{n+3}<p<p^{*} \leq 1$ and $\alpha=(n+2)\left(\frac{1}{p}-\frac{1}{p^{*}}\right)$, the operator

$$
\dot{I}_{1}: \dot{B}_{-\alpha, p a r}^{p^{*}}\left(\mathbb{R}^{n} \times \mathbb{R}\right) \stackrel{\sim}{\longrightarrow} \dot{B}_{1-\alpha, p a r}^{p^{*}}\left(\mathbb{R}^{n} \times \mathbb{R}\right)
$$


is an isomorphism. One can now see that (2.5.239) and (2.5.240) yield the first part of the theorem, i.e. (2.5.231).

In order to show the second part of the statement, i.e. (2.5.232), we start with recalling from Theorem 2.1.10 that the lifting operator $\mathcal{I}_{\sigma}$ has the following property: for $0<p, q \leq \infty(p \neq \infty$ for the $F$-scale $)$ and $\alpha, \sigma \in \mathbb{R}$,

$$
\begin{aligned}
& \mathcal{I}_{\sigma}: B_{\alpha, p a r}^{p, q}\left(\mathbb{R}^{n} \times \mathbb{R}\right) \stackrel{\sim}{\longrightarrow} B_{\alpha+\sigma, p a r}^{p, q}\left(\mathbb{R}^{n} \times \mathbb{R}\right), \\
& \mathcal{I}_{\sigma}: F_{\alpha, \text { par }}^{p, q}\left(\mathbb{R}^{n} \times \mathbb{R}\right) \stackrel{\sim}{\longrightarrow} F_{\alpha+\sigma, p a r}^{p, q}\left(\mathbb{R}^{n} \times \mathbb{R}\right)
\end{aligned}
$$

are isomorphisms. By Definition 2.1.43 and Theorem 2.1.59, respectively, for the range $\frac{n+2}{n+3}<p \leq 1$, one has the equivalences

$$
F_{0, \text { par }}^{p, 2}\left(\mathbb{R}^{n} \times \mathbb{R}\right) \equiv H_{\text {par }}^{p}\left(\mathbb{R}^{n} \times \mathbb{R}\right) \quad \text { and } \quad F_{1, \text { par }}^{p, 2}\left(\mathbb{R}^{n} \times \mathbb{R}\right) \equiv H_{\text {par }}^{1, p}\left(\mathbb{R}^{n} \times \mathbb{R}\right)
$$

With (2.5.242) and (2.5.243) in hand, according to Proposition 1.2.45, the operator $\mathcal{I}_{1}$ extends to an isomorphism from $\mathcal{E}_{p^{*}}\left(H_{\text {par }}^{p}\left(\mathbb{R}^{n} \times \mathbb{R}\right)\right)$ to $\mathcal{E}_{p^{*}}\left(H_{\text {par }}^{1, p}\left(\mathbb{R}^{n} \times \mathbb{R}\right)\right)$ for any $0<p^{*} \leq 1$. Applying the second part of Theorem 2.5.1 in order to identify the envelope of $H_{\text {par }}^{p}\left(\mathbb{R}^{n} \times \mathbb{R}\right)$, this further implies that

$$
\mathcal{I}_{1}: B_{-\alpha, p a r}^{p^{*}}\left(\mathbb{R}^{n} \times \mathbb{R}\right) \stackrel{\sim}{\longrightarrow} \mathcal{E}_{p^{*}}\left(H_{\text {par }}^{1, p}\left(\mathbb{R}^{n} \times \mathbb{R}\right)\right)
$$

is invertible for the range of indices $\frac{n+2}{n+3}<p<p^{*} \leq 1$ and $\alpha=(n+2)\left(\frac{1}{p}-\frac{1}{p^{*}}\right)$.

For the same indices as above, a special case of (2.5.241) is the following:

$$
\mathcal{I}_{1}: B_{-\alpha, p a r}^{p^{*}}\left(\mathbb{R}^{n} \times \mathbb{R}\right) \stackrel{\sim}{\longrightarrow} B_{1-\alpha, p a r}^{p^{*}}\left(\mathbb{R}^{n} \times \mathbb{R}\right)
$$

is invertible. Consequently, (2.5.244) and (2.5.245) imply the second part of the statement, hence the proof of Theorem 2.5.2 is complete. 
As a direct consequence of a bi-Lipschitz change of variables in Theorems 2.5.1 and 2.5.2 one obtains similar results involving spaces defined on $\partial \Omega \times \mathbb{R}$ where $\Omega$ is a graph Lipschitz domain in $\mathbb{R}^{n}$. More specifically, we have the following.

Corollary 2.5.3. Let $\Omega$ be an unbounded (graph) Lipschitz domain in $\mathbb{R}^{n}$. Suppose also that $\frac{n+1}{n+2}<p<p^{*} \leq 1$ and $\alpha=(n+1)\left(\frac{1}{p}-\frac{1}{p^{*}}\right)$. Then

$$
\begin{aligned}
& \mathcal{E}_{p^{*}}\left(\dot{H}_{p a r}^{p}(\partial \Omega \times \mathbb{R})\right)=\dot{B}_{-\alpha, p a r}^{p^{*}}(\partial \Omega \times \mathbb{R}), \\
& \mathcal{E}_{p^{*}}\left(H_{p a r}^{p}(\partial \Omega \times \mathbb{R})\right)=B_{-\alpha, p a r}^{p^{*}}(\partial \Omega \times \mathbb{R}),
\end{aligned}
$$

and

$$
\begin{aligned}
& \mathcal{E}_{p^{*}}\left(\dot{H}_{\text {par }}^{1, p}(\partial \Omega \times \mathbb{R})\right)=\dot{B}_{1-\alpha, \text { par }}^{p^{*}}(\partial \Omega \times \mathbb{R}), \\
& \mathcal{E}_{p^{*}}\left(H_{\text {par }}^{1, p}(\partial \Omega \times \mathbb{R})\right)=B_{1-\alpha, \text { par }}^{p^{*}}(\partial \Omega \times \mathbb{R}) .
\end{aligned}
$$




\section{Chapter 3}

\section{Spaces with built-in initial conditions}

In this chapter we introduce and explore various properties of parabolic Besov and Triebel-Lizorkin spaces with several different built-in initial conditions. Sections 3.1 and 3.2 deal with these spaces defined on the interior of a Lipschitz cylinder and on its lateral boundary, respectively. Relying on the foundation developed in Section 2.5, Envelopes of parabolic Hardy spaces, as well as on the abstract result formulated in Lemma 1.2.52, in Section 3.3 we compute Banach enelopes of Hardy spaces with a certain type of built-in initial condition. Finally, Section 3.4 contains several real and complex interpolation results involving Besov and Triebel-Lizorkin spaces with built-in initial conditions.

\subsection{The interior of the cylinder}

We first present the case when the underlying space is the interior of a Lipschitz cylinder. In preparation we need some additional introductory definitions and preliminary results. 
Definition 3.1.1. For $0<p, q \leq \infty$ and $\alpha \in \mathbb{R}$ define

$$
B_{\alpha, p a r, 0}^{p, q}\left(\mathbb{R}^{n} \times(0, \infty)\right):=\left\{f \in B_{\alpha, p a r}^{p, q}\left(\mathbb{R}^{n} \times \mathbb{R}\right): \operatorname{supp} f \subseteq \mathbb{R}^{n} \times[0, \infty)\right\}
$$

and, for $0<T<\infty$,

$$
B_{\alpha, p a r, T}^{p, q}\left(\mathbb{R}^{n} \times(-\infty, T)\right):=\left\{f \in B_{\alpha, p a r}^{p, q}\left(\mathbb{R}^{n} \times \mathbb{R}\right): \operatorname{supp} f \subseteq \mathbb{R}^{n} \times(-\infty, T]\right\}
$$

The norms are given by

$$
\|f\|_{B_{\alpha, p a r, 0}^{p, q}\left(\mathbb{R}^{n} \times(0, \infty)\right)}:=\inf \left\{\|f\|_{B_{\alpha, p a r}^{p, q}\left(\mathbb{R}^{n} \times \mathbb{R}\right)}: \operatorname{supp} f \subseteq \mathbb{R}^{n} \times[0, \infty)\right\}
$$

and

$$
\|f\|_{B_{\alpha, p a r, T}^{p, q}\left(\mathbb{R}^{n} \times(-\infty, T)\right)}:=\inf \left\{\|f\|_{B_{\alpha, p a r}^{p, q}\left(\mathbb{R}^{n} \times \mathbb{R}\right)}: \operatorname{supp} f \subseteq \mathbb{R}^{n} \times(-\infty, T]\right\}
$$

respectively.

The spaces $F_{\alpha, \text { par, } 0}^{p, q}\left(\mathbb{R}^{n} \times(0, \infty)\right)$ and $F_{\alpha, p a r, T}^{p, q}\left(\mathbb{R}^{n} \times(-\infty, T)\right)$, for the same indices except $p \neq \infty$, are defined in a similar fashion.

Remark 3.1.2. Resembling the notation introduced in Remark 2.1.18, we shall write $B_{\alpha, p a r, 0}^{p}$ and $B_{\alpha, p a r, T}^{p}$ in place of $B_{\alpha, p a r, 0}^{p, p}$ and $B_{\alpha, p a r, T}^{p, p}$, respectively.

Lemma 3.1.3. Let $1<p, q<\infty$ and $\alpha \in \mathbb{R} \backslash\{0\}$. Then

$$
\left(B_{\alpha, p a r, 0}^{p, q}\left(\mathbb{R}^{n} \times(0, \infty)\right)\right)^{*}=\left.B_{-\alpha, p a r}^{p^{\prime}, q^{\prime}}\left(\mathbb{R}^{n} \times \mathbb{R}\right)\right|_{\mathbb{R}^{n} \times(0, \infty)},
$$

where $1 / p+1 / p^{\prime}=1$ and $1 / q+1 / q^{\prime}=1$. Under the extra assumption that the pair $(1 / p, 1 / q)$ belongs to the interior of $O M N P$ in the figure below, the analogue of (3.1.1) holds true for the Triebel-Lizorkin scale, as well. 


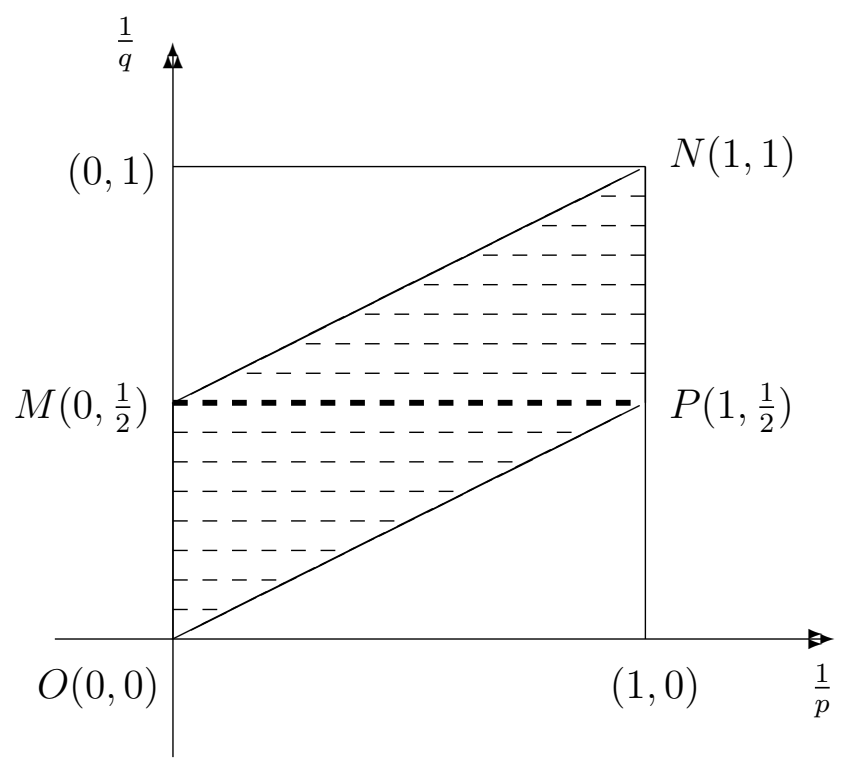

Proof. In order to show the right-to-left inclusion in the indentification (3.1.1) let us consider $f$ from $\left.B_{-\alpha, p a r}^{p^{\prime}, q^{\prime}}\left(\mathbb{R}^{n} \times \mathbb{R}\right)\right|_{\mathbb{R}^{n} \times(0, \infty)}$. Then there exists $F \in B_{-\alpha, p a r}^{p^{\prime}, q^{\prime}}\left(\mathbb{R}^{n} \times \mathbb{R}\right)$ such that $\left.F\right|_{\mathbb{R}^{n} \times(0, \infty)}=f$. Since $B_{-\alpha, p a r}^{p^{\prime}, q^{\prime}}\left(\mathbb{R}^{n} \times \mathbb{R}\right)=\left(B_{\alpha, p a r}^{p, q}\left(\mathbb{R}^{n} \times \mathbb{R}\right)\right)^{*}($ cf. Theorem 2.4.3), we can define the functional $\Lambda$ by

$$
\Lambda(g):=\langle F, g\rangle
$$

for $g \in B_{\alpha, p a r}^{p, q}\left(\mathbb{R}^{n} \times \mathbb{R}\right)$ with supp $g \subseteq \mathbb{R}^{n} \times[0, \infty)$. To show that $\Lambda(g)$ is independent of the choice of $F$, we pick $F$ and $F^{\prime}$ from $B_{-\alpha, p a r}^{p^{\prime}, q^{\prime}}\left(\mathbb{R}^{n} \times \mathbb{R}\right)$ such that $\left.F\right|_{\mathbb{R}^{n} \times(0, \infty)}=$ $\left.F^{\prime}\right|_{\mathbb{R}^{n} \times(0, \infty)}=f$. For $g_{j}(x, t):=g\left(x, t-\frac{1}{j}\right)$ with $j \in \mathbb{R}_{+}$we have supp $g_{j} \subseteq \mathbb{R}^{n} \times\left[\frac{1}{j}, \infty\right)$ and $\lim _{j \rightarrow \infty}\left\|g_{j}-g\right\|_{B_{\alpha, p a r}^{p, q}\left(\mathbb{R}^{n} \times \mathbb{R}\right)}=0$. Moreover,

$$
\left\langle F-F^{\prime}, g\right\rangle=\lim _{j \rightarrow \infty}\left\langle F-F^{\prime}, g_{j}\right\rangle=0,
$$

since the support of $F-F^{\prime}$ and the support of $g_{j}$ have no common points. Therefore, the mapping

$$
\left.B_{-\alpha, p a r}^{p^{\prime}, q^{\prime}}\left(\mathbb{R}^{n} \times \mathbb{R}\right)\right|_{\mathbb{R}^{n} \times(0, \infty)} \ni f \longmapsto \Lambda \in\left(B_{\alpha, p a r, 0}^{p, q}\left(\mathbb{R}^{n} \times(0, \infty)\right)\right)^{*}
$$


is independent of the choice of $F$, in particular, for given $f_{1}, f_{2}$ with extensions $F_{1}$ and $F_{2}$, respectively, we can choose $F_{1}+F_{2}$ to be the extension of $f_{1}+f_{2}$, hence the mapping $f \longmapsto \Lambda$ is linear.

Conversely, if $\Lambda$ is a linear functional defined on $B_{\alpha, p a r, 0}^{p, q}\left(\mathbb{R}^{n} \times(0, \infty)\right)$, which is a closed subspace of $B_{\alpha, p a r}^{p, q}\left(\mathbb{R}^{n} \times \mathbb{R}\right)$, then, by the Hahn-Banach theorem, there exists an extension of $\Lambda, \tilde{\Lambda}$, that is a linear functional defined on $B_{\alpha, p a r}^{p, q}\left(\mathbb{R}^{n} \times \mathbb{R}\right)$, i.e., by the duality Theorem 2.4.3, $\tilde{\Lambda} \in B_{-\alpha, p a r}^{p^{\prime}, q^{\prime}}\left(\mathbb{R}^{n} \times \mathbb{R}\right)$. We next define

$$
f:=\left.\tilde{\Lambda}\right|_{\mathbb{R}^{n} \times(0, \infty)}
$$

Then, clearly, $f$ belongs to the right-hand side of (3.1.1). To show that the definition of $f$ is independent of the particular choice of the extension $\tilde{\Lambda}$, let us pick two different extensions of $\Lambda, \tilde{\Lambda}$ and $\tilde{\tilde{\Lambda}}$ from $B_{-\alpha, p a r}^{p^{\prime}, q^{\prime}}\left(\mathbb{R}^{n} \times \mathbb{R}\right)$, and let $\varphi$ be from $C_{c}^{\infty}\left(\mathbb{R}^{n} \times(0, \infty)\right)$. Also, for $(x, t) \in \mathbb{R}^{n} \times \mathbb{R}$, consider

$$
\tilde{\varphi}(x, t):= \begin{cases}\varphi(x, t), & \text { for } \left.(x, t) \in \mathbb{R}^{n} \times(0, \infty)\right), \\ 0, & \text { for } \left.(x, t) \in \mathbb{R}^{n} \times(-\infty, 0]\right) .\end{cases}
$$

Then $\tilde{\varphi} \in B_{\alpha, p a r}^{p, q}\left(\mathbb{R}^{n} \times \mathbb{R}\right)$ with $\operatorname{supp} \tilde{\varphi} \subseteq \mathbb{R}^{n} \times[0, \infty)$, hence $\tilde{\varphi}$ belongs to the space $B_{\alpha, p a r, 0}^{p, q}\left(\mathbb{R}^{n} \times(0, \infty)\right)$. Moreover

$$
\left\langle\left.\tilde{\Lambda}\right|_{\mathbb{R}^{n} \times(0, \infty)}, \varphi\right\rangle=\langle\Lambda, \tilde{\varphi}\rangle=\left\langle\left.\tilde{\tilde{\Lambda}}\right|_{\mathbb{R}^{n} \times(0, \infty)}, \varphi\right\rangle
$$

which completes the proof of the left-to-right inclusion in (3.1.1).

In order to prove that the two maps constructed above are inverses to each other, on the one hand, let $\left.f \in B_{-\alpha, p a r}^{p^{\prime}, q^{\prime}}\left(\mathbb{R}^{n} \times \mathbb{R}\right)\right|_{\mathbb{R}^{n} \times(0, \infty)}$ and consider the mappings

$$
\left.f \longmapsto \Lambda \longmapsto \tilde{\Lambda}\right|_{\mathbb{R}^{n} \times(0, \infty)}
$$


defined earlier. For $\varphi \in C_{c}^{\infty}\left(\mathbb{R}^{n} \times(0, \infty)\right)$, we obtain that

$$
\left\langle\left.\tilde{\Lambda}\right|_{\mathbb{R}^{n} \times(0, \infty)}, \varphi\right\rangle=\langle\tilde{\Lambda}, \tilde{\varphi}\rangle=\langle\Lambda, \tilde{\varphi}\rangle=\langle F, \tilde{\varphi}\rangle=\left\langle\left. F\right|_{\mathbb{R}^{n} \times(0, \infty)}, \varphi\right\rangle=\langle f, \varphi\rangle
$$

as desired. On the other hand, let $\Lambda \in\left(B_{\alpha, p a r, 0}^{p, q}\left(\mathbb{R}^{n} \times(0, \infty)\right)\right)^{*}$ and, retaining the above notation,

$$
\left.\Lambda \longmapsto \tilde{\Lambda}\right|_{\mathbb{R}^{b} \times(0, \infty)}=f \longmapsto\langle F, \cdot\rangle
$$

As we have established earlier in the present proof, the pairing $\langle F, \cdot\rangle$ is independent of the particular choice of the extension $F$ of $f$, hence we can take $F=\tilde{\Lambda}$. Then $\langle\tilde{\Lambda}, \cdot\rangle=\Lambda$ on $B_{\alpha, p a r, 0}^{p, q}\left(\mathbb{R}^{n} \times(0, \infty)\right)$, which suits our goal, finishing the proof of (3.1.1).

In a similar manner, using Theorem 2.4.4 in place of Theorem 2.4.3, we arrive at (3.1.1) with $B$ replaced by $F$. The additional assumption that $(1 / p, 1 / q) \in O M N P$ is necessary, since Theorem 2.4.4 is valid for this range of indices. Therefore, the proof of Lemma 3.1.3 is complete.

The proper analogue of Lemma 3.1 .3 for the spaces $B_{\alpha, p a r, T}^{p, q}\left(\mathbb{R}^{n} \times(-\infty, T)\right)$ and $\left.F_{\alpha, p a r, T}^{p, q}\left(\mathbb{R}^{n} \times(-\infty, T)\right)\right)$ introduced in Definition 3.1.1 is as follows.

Lemma 3.1.4. Let $1<p, q<\infty, \alpha \in \mathbb{R} \backslash\{0\}$, and $0<T<\infty$. Then

$$
\left(B_{\alpha, p a r, T}^{p, q}\left(\mathbb{R}^{n} \times(-\infty, T)\right)\right)^{*}=\left.B_{-\alpha, p a r}^{p^{\prime}, q^{\prime}}\left(\mathbb{R}^{n} \times \mathbb{R}\right)\right|_{\mathbb{R}^{n} \times(-\infty, T)},
$$

where $1 / p+1 / p^{\prime}=1$ and $1 / q+1 / q^{\prime}=1$. With the additional hypothesis that the pair $(1 / p, 1 / q)$ belongs to the interior of $O M N P$ in the figure in Lemma 3.1.3, the analogue of (3.1.2) is valid for the Triebel-Lizorkin scale, as well.

Proof. With minor modifications, one could follow the same steps as in the proof of Lemma 3.1.3 to obtain the desired results. However, a simpler proof can be given by 
using the reflection operator in time, defined by $R f(x, t)=f(x, T-t)$ for $x \in \mathbb{R}^{n}$ and $t \in \mathbb{R}$. Since this proof relies on Lemma 3.1.3, we only present the case of the Besov scale. The same argument yields (3.1.2) with $B$ replaced by $F$.

We first let $\left.f \in B_{-\alpha, p a r}^{p^{\prime}, q^{\prime}}\left(\mathbb{R}^{n} \times \mathbb{R}\right)\right|_{\mathbb{R}^{n} \times(-\infty, T)}$. Then $\left.R \circ f \in B_{-\alpha, p a r}^{p^{\prime}, q^{\prime}}\left(\mathbb{R}^{n} \times \mathbb{R}\right)\right|_{\mathbb{R}^{n} \times(0, \infty)}$, and, according to Lemma 3.1.3,

$$
R \circ f \in\left(B_{\alpha, p a r, 0}^{p, q}\left(\mathbb{R}^{n} \times(0, \infty)\right)\right)^{*},
$$

hence, for $\varphi \in B_{\alpha, p a r, T}^{p, q}\left(\mathbb{R}^{n} \times(-\infty, T)\right)$, we can define

$$
\langle f, \varphi\rangle:=\langle R \circ f, R \circ \varphi\rangle
$$

Therefore, $f$ belongs to $\left(B_{\alpha, \text { par,T }}^{p, q}\left(\mathbb{R}^{n} \times(-\infty, T)\right)\right)^{*}$, proving the right-to-left inclusion in $(3.1 .2)$.

Conversely, let $\Lambda \in\left(B_{\alpha, p a r, T}^{p, q}\left(\mathbb{R}^{n} \times(-\infty, T)\right)\right)^{*}$. Then, for any $\varphi$ from the space $B_{\alpha, p a r, T}^{p, q}\left(\mathbb{R}^{n} \times(-\infty, T)\right)$

$$
\langle\Lambda, \varphi\rangle=\langle\Lambda, R \circ R \circ \varphi\rangle=\langle R \circ \Lambda, R \circ \varphi\rangle
$$

where we have used the fact that $R$ is self-adjoint, i.e. $R^{*}=R$. Since $R \circ \varphi$ belongs to the space $B_{\alpha, p a r, 0}^{p, q}\left(\mathbb{R}^{n} \times(0, \infty)\right)$, in concert with Lemma 3.1.3, we obtain that

$$
R \circ \Lambda \in\left(B_{\alpha, p a r, 0}^{p, q}\left(\mathbb{R}^{n} \times(0, \infty)\right)\right)^{*}=\left.B_{-\alpha, p a r}^{p^{\prime}, q^{\prime}}\left(\mathbb{R}^{n} \times \mathbb{R}\right)\right|_{\mathbb{R}^{n} \times(0, \infty)}
$$

for the appropriate indices. This yields

$$
\left.\Lambda \in B_{-\alpha, p a r}^{p^{\prime}, q^{\prime}}\left(\mathbb{R}^{n} \times \mathbb{R}\right)\right|_{\mathbb{R}^{n} \times(-\infty, T)},
$$

proving the left-to-right inclusion in (3.1.2). 
Finally, it is easy to see that the two maps constructed above, i.e.

$$
f \mapsto\langle f, \cdot\rangle \quad \text { for }\left.f \in B_{-\alpha, p a r}^{p^{\prime}, q^{\prime}}\left(\mathbb{R}^{n} \times \mathbb{R}\right)\right|_{\mathbb{R}^{n} \times(-\infty, T)}
$$

and

$$
\langle\Lambda, \cdot\rangle \mapsto \Lambda \quad \text { for } \Lambda \in\left(B_{\alpha, p a r, T}^{p, q}\left(\mathbb{R}^{n} \times(-\infty, T)\right)\right)^{*}
$$

are inverses to each other. This finishes the proof of the lemma.

In the following Definitions (3.1.5, 3.1.7 and 3.1.9) we construct parabolic Besov and Triebel-Lizorkin spaces with specific types of built-in initial conditions.

Definition 3.1.5. For indices $1<p, q<\infty, \alpha>0$, and $\Omega$ Lipschitz domain in $\mathbb{R}^{n}$, $0<T<\infty$, define the following spaces.

(a) ${ }_{0} B_{\alpha, p a r}^{p, q}(\Omega \times(0, T)):=\left\{\left.F\right|_{\Omega \times(0, T)}: F \in B_{\alpha, p a r}^{p, q}\left(\mathbb{R}^{n} \times \mathbb{R}\right)\right.$, supp $\left.F \subseteq \mathbb{R}^{n} \times[0, \infty)\right\}$,

(b) ${ }^{0} B_{\alpha, p a r}^{p, q}(\Omega \times(0, T)):=\left\{\left.F\right|_{\Omega \times(0, T)}: F \in B_{\alpha, \text { par }}^{p, q}\left(\mathbb{R}^{n} \times \mathbb{R}\right)\right.$, supp $\left.F \subseteq \mathbb{R}^{n} \times(-\infty, T]\right\}$,

(c) $\quad{ }_{0} B_{-\alpha, p a r}^{p, q}(\Omega \times(0, T)):=\left\{\left.F\right|_{\Omega \times(-\infty, T)}: F \in B_{-\alpha, p a r}^{p, q}\left(\mathbb{R}^{n} \times \mathbb{R}\right)\right.$,

$$
\text { supp } \left.F \subseteq \mathbb{R}^{n} \times[0, \infty)\right\},
$$

$$
\begin{aligned}
{ }^{0} B_{-\alpha, p a r}^{p, q}(\Omega \times(0, T)):=\left\{\left.F\right|_{\Omega \times(0, \infty)}: F \in B_{-\alpha, p a r}^{p, q}\left(\mathbb{R}^{n} \times \mathbb{R}\right),\right. \\
\left.\operatorname{supp} F \subseteq \mathbb{R}^{n} \times(-\infty, T]\right\} .
\end{aligned}
$$

The norms are given by

$$
\|f\|_{0 B_{\alpha, p a r}^{p, q}(\Omega \times(0, T))}:=\inf \left\{\|F\|_{B_{\alpha, p a r}^{p, q}\left(\mathbb{R}^{n} \times \mathbb{R}\right)}:\left.F\right|_{\Omega \times(0, T)}=f, \operatorname{supp} F \subseteq \mathbb{R}^{n} \times[0, \infty)\right\}
$$

and similarly for the other spaces.

For $p \neq \infty$ the classes ${ }_{0} F_{ \pm \alpha, p a r}^{p, q}(\Omega \times(0, T))$ and ${ }^{0} F_{ \pm \alpha, p a r}^{p, q}(\Omega \times(0, T))$ are defined as above, using $F$ in place of $B$. 
In order to simplify the notation, we shall use ${ }_{0} B_{ \pm \alpha, p a r}^{p}$ and ${ }^{0} B_{ \pm \alpha, p a r}^{p}$ instead of ${ }_{0} B_{ \pm \alpha, p a r}^{p, p}$ and ${ }^{0} B_{ \pm \alpha, p a r}^{p, p}$, respectively.

Remark 3.1.6. For indices $1<p, q<\infty, \alpha>0$, and $\Omega$ Lipschitz domain in $\mathbb{R}^{n}$, $0<T<\infty$, another way to look at the spaces with positive smoothness, introduced in Definition 3.1.5 (a) and (b), is the following.

$$
\begin{aligned}
& { }_{0} B_{\alpha, p a r}^{p, q}(\Omega \times(0, T))=\left\{\left.F\right|_{\Omega \times(-\infty, T)}: F \in B_{\alpha, p a r}^{p, q}\left(\mathbb{R}^{n} \times \mathbb{R}\right), \operatorname{supp} F \subseteq \mathbb{R}^{n} \times[0, \infty)\right\}, \\
& { }^{0} B_{\alpha, \text { par }}^{p, q}(\Omega \times(0, T))=\left\{\left.F\right|_{\Omega \times(0, \infty)}: F \in B_{\alpha, \text { par }}^{p, q}\left(\mathbb{R}^{n} \times \mathbb{R}\right), \operatorname{supp} F \subseteq \mathbb{R}^{n} \times(-\infty, T]\right\} .
\end{aligned}
$$

When $p \neq \infty$, a similar statement holds true for the Triebel-Lizorkin scale.

Definition 3.1.7. Consider a Lipschitz domain $\Omega$ in $\mathbb{R}^{n}, 0<T<\infty$ and indices $1<p, q<\infty, \alpha \in \mathbb{R} \backslash\{0\}$. By definition,

$$
\begin{aligned}
{ }_{z} B_{\alpha, p a r}^{p, q}(\Omega \times(0, T)) & :=\left\{\left.F\right|_{\mathbb{R}^{n} \times(-\infty, T)}: F \in B_{\alpha, p a r}^{p, q}\left(\mathbb{R}^{n} \times \mathbb{R}\right),\right. \\
& \left.\operatorname{supp} F \subseteq(\bar{\Omega} \times[0, \infty)) \cup\left(\mathbb{R}^{n} \times[T, \infty)\right)\right\}
\end{aligned}
$$

with

$\|f\|_{z B_{\alpha, p a r}^{p, q}(\Omega \times(0, T))}:=\inf \left\{\|F\|_{B_{\alpha, p a r}^{p, q}\left(\mathbb{R}^{n} \times \mathbb{R}\right)}:\left.F\right|_{\mathbb{R}^{n} \times(-\infty, T)}=f\right.$,

$$
\text { supp } \left.F \subseteq(\bar{\Omega} \times[0, \infty)) \cup\left(\mathbb{R}^{n} \times[T, \infty)\right)\right\} .
$$

The space ${ }_{z} F_{\alpha, p a r}^{p, q}(\Omega \times(0, T))$, for $p \neq \infty$, is defined in a similar manner.

Remark 3.1.8. The region $(\bar{\Omega} \times[0, \infty)) \cup\left(\mathbb{R}^{n} \times[T, \infty)\right)$ is given in the diagram below: 


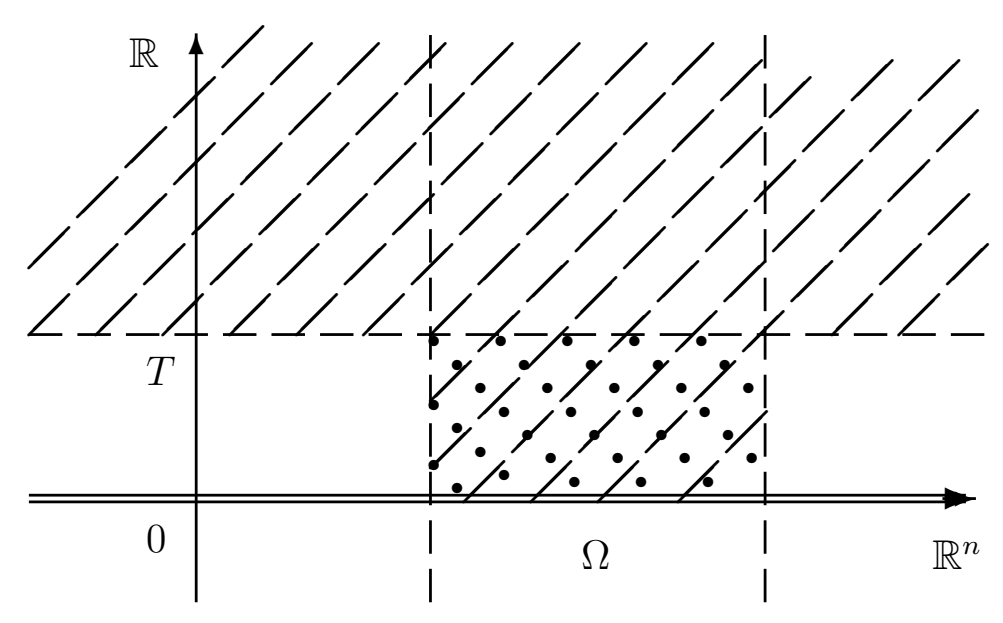

Definition 3.1.9. Consider a Lipschitz domain $\Omega$ in $\mathbb{R}^{n}, 0<T<\infty$ and assume that $1<p, q<\infty, \alpha \in \mathbb{R} \backslash\{0\}$. By definition,

$$
\begin{aligned}
{ }^{z} B_{\alpha, p a r}^{p, q}(\Omega \times(0, T)):=\left\{\left.F\right|_{\mathbb{R}^{n} \times(0, \infty)}: F\right. & \in B_{\alpha, p a r}^{p, q}\left(\mathbb{R}^{n} \times \mathbb{R}\right), \\
& \left.\operatorname{supp} F \subseteq(\bar{\Omega} \times(-\infty, T]) \cup\left(\mathbb{R}^{n} \times(-\infty, 0]\right)\right\}
\end{aligned}
$$

with

$$
\begin{aligned}
\|f\|_{{ }_{B_{\alpha, p a r}(\Omega \times(0, T))}^{p, q}}:=\inf \left\{\|F\|_{B_{\alpha, p a r}^{p, q}\left(\mathbb{R}^{n} \times \mathbb{R}\right)}:\left.F\right|_{\mathbb{R}^{n} \times(0, \infty)}=f,\right. \\
\left.\operatorname{supp} F \subseteq(\bar{\Omega} \times(-\infty, T]) \cup\left(\mathbb{R}^{n} \times(-\infty, 0]\right)\right\} .
\end{aligned}
$$

The space ${ }^{z} F_{\alpha, p a r}^{p, q}(\Omega \times(0, T))$, for $p \neq \infty$, is defined in a similar fashion.

Remark 3.1.10. The region $(\bar{\Omega} \times(-\infty, T]) \cup\left(\mathbb{R}^{n} \times(-\infty, 0]\right)$ is given in the following diagram: 


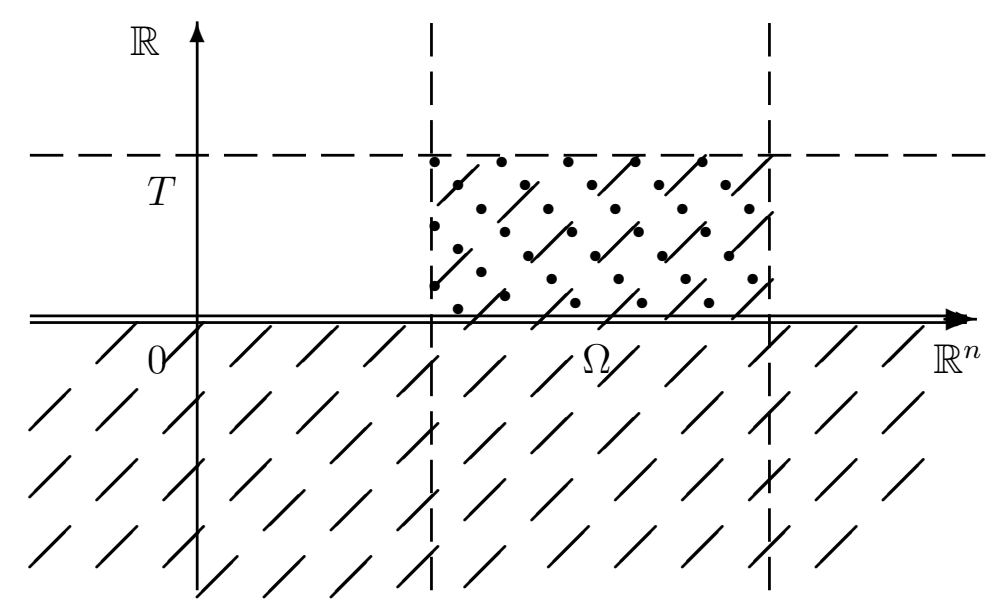

For notational convenience, we are going to use ${ }_{z} B_{\alpha, p a r}^{p}$ and ${ }^{z} B_{\alpha, p a r}^{p}$ in place of ${ }_{z} B_{\alpha, p a r}^{p, p}$ and ${ }^{z} B_{\alpha, p a r}^{p, p}$, respectively.

We now are in a position to present one of our duality results which will play an important role later on.

Theorem 3.1.11. Consider a Lipschitz domain $\Omega$ in $\mathbb{R}^{n}, 0<T<\infty$, and the indices $1<p, q<\infty, \alpha \in \mathbb{R} \backslash\{0\}$. Then

$$
\left({ }^{0} B_{\alpha, p a r}^{p, q}(\Omega \times(0, T))\right)^{*}={ }_{z} B_{-\alpha, p a r}^{p^{\prime}, q^{\prime}}(\Omega \times(0, T))
$$

where $1 / p+1 / p^{\prime}=1$ and $1 / q+1 / q^{\prime}=1$. With the additional assumption that the pair $(1 / p, 1 / q)$ belongs to the interior of $O M N P$ in the figure in Lemma 3.1.3, the analogue of (3.1.3) is valid for the Triebel-Lizorkin scale, as well.

Proof. We present the proof of (3.1.3) only, since the same argument, using Theorem 2.4.4 instead of Theorem 2.4.3, yields the desired result. 
Let us first assume that $f \in\left({ }^{0} B_{\alpha, p a r}^{p, q}(\Omega \times(0, T))\right)^{*}$, and consider the restriction operator $R_{\Omega \times(0, \infty)}$. According to Definitions 3.1.1 and 3.1.5, $R_{\Omega \times(0, \infty)}$ maps the space $B_{\alpha, p a r, T}^{p, q}\left(\mathbb{R}^{n} \times(-\infty, T)\right)$ into ${ }^{0} B_{\alpha, p a r}^{p, q}(\Omega \times(0, T))$, therefore

$$
f \circ R_{\Omega \times(0, \infty)} \in\left(B_{\alpha, p a r, T}^{p, q}\left(\mathbb{R}^{n} \times(-\infty, T)\right)\right)^{*}=\left.B_{-\alpha, p a r}^{p^{\prime}, q^{\prime}}\left(\mathbb{R}^{n} \times \mathbb{R}\right)\right|_{\mathbb{R}^{n} \times(-\infty, T)},
$$

where we have also used Lemma 3.1.4. Hence there exists $F$ from the $B_{-\alpha, p a r}^{p^{\prime}, q^{\prime}}\left(\mathbb{R}^{n} \times \mathbb{R}\right)$ such that $\left.F\right|_{\mathbb{R}^{n} \times(-\infty, T)}=f \circ R_{\Omega \times(0, \infty)}$. Then $\operatorname{supp} F \subseteq(\bar{\Omega} \times[0, \infty)) \cup\left(\mathbb{R}^{n} \times[T, \infty)\right)$, which further implies

$$
f \circ R_{\Omega \times(0, \infty)} \in{ }_{z} B_{-\alpha, p a r}^{p^{\prime}, q^{\prime}}(\Omega \times(0, T)) .
$$

Conversely, let $f \in{ }_{z} B_{-\alpha, p a r}^{p^{\prime}, q^{\prime}}(\Omega \times(0, T))$. By Definition 3.1.7, there exists $F$ in $B_{-\alpha, p a r}^{p^{\prime}, q^{\prime}}\left(\mathbb{R}^{n} \times \mathbb{R}\right)$ so that $f=\left.F\right|_{\mathbb{R}^{n} \times(-\infty, T)}$ and the support of $F$ belongs to $(\bar{\Omega} \times[0, \infty)) \cup\left(\mathbb{R}^{n} \times[T, \infty)\right)$. We take $u \in{ }^{0} B_{\alpha, p a r}^{p, q}(\Omega \times(0, T))$, which, by Definition 3.1.5 and Remark 3.1.6, implies the existence of $U \in B_{\alpha, p a r}^{p, q}\left(\mathbb{R}^{n} \times \mathbb{R}\right)$ with $\left.U\right|_{\Omega \times(0, \infty)}$ and $\operatorname{supp} U \subseteq \mathbb{R}^{n} \times(-\infty, T]$. For $f$ and $u$ as above we define the duality pairing

$$
\langle f, u\rangle:=\langle F, U\rangle_{\left(B_{\alpha, p a r}^{p, q}\left(\mathbb{R}^{n} \times \mathbb{R}\right)\right)^{*}, B_{\alpha, p a r}^{p, q}\left(\mathbb{R}^{n} \times \mathbb{R}\right)^{*}}
$$

In order to show that the above definition is independent of the particular choice of the extension $U$ of $u$, let $U, U^{\prime} \in B_{\alpha, p a r}^{p, q}\left(\mathbb{R}^{n} \times \mathbb{R}\right)$ such that $\left.U\right|_{\Omega \times(0, \infty)}=u=\left.U^{\prime}\right|_{\Omega \times(0, \infty)}$ and both $U$ and $U^{\prime}$ are supported in $\mathbb{R}^{n} \times(-\infty, T]$. Then

$$
\operatorname{supp}\left(U-U^{\prime}\right) \subseteq\left(\mathbb{R}^{n} \times(-\infty, T]\right) \backslash(\Omega \times(0, T)) .
$$

To this end, we introduce $V_{j} \in B_{\alpha, p a r}^{p, q}\left(\mathbb{R}^{n} \times \mathbb{R}\right), j \in \mathbb{N}$, so that

$$
\lim _{j \rightarrow \infty}\left\|\left(U-U^{\prime}\right)-V_{j}\right\|_{B_{\alpha, p a r}^{p, q}\left(\mathbb{R}^{n} \times \mathbb{R}\right)}=0
$$


and

$$
\operatorname{supp} V_{j} \subseteq \text { interior }\left[\left(\mathbb{R}^{n} \times(-\infty, T]\right) \backslash(\Omega \times(0, T))\right]
$$

Then we obtain that

$$
\left\langle F, U-U^{\prime}\right\rangle=\lim _{j \rightarrow \infty}\left\langle F, V_{j}\right\rangle=0
$$

since the supports of $F$ and $V_{j}$ are disjoint.

To show that the pairing defined in (3.1.4) is independent of the choice of the extension $F$ of $f$, we consider $F, F^{\prime} \in B_{-\alpha, p a r}^{p^{\prime}, q^{\prime}}\left(\mathbb{R}^{n} \times \mathbb{R}\right)=\left(B_{\alpha, p a r}^{p, q}\left(\mathbb{R}^{n} \times \mathbb{R}\right)\right)^{*}(\mathrm{cf}$. Theorem 2.4.3 for this last identification) such that $\left.F\right|_{\mathbb{R}^{n} \times(-\infty, T)}=f=\left.F^{\prime}\right|_{\mathbb{R}^{n} \times(-\infty, T)}$, and both $F$ and $F^{\prime}$ are supported in $(\bar{\Omega} \times[0, \infty)) \cup\left(\mathbb{R}^{n} \times[T, \infty)\right)$. Let us also introduce $U_{j} \in B_{\alpha, p a r}^{p, q}\left(\mathbb{R}^{n} \times \mathbb{R}\right), j \in \mathbb{N}$, such that

$$
\lim _{j \rightarrow \infty}\left\|U-U_{j}\right\|_{B_{\alpha, p a r}^{p, q}\left(\mathbb{R}^{n} \times \mathbb{R}\right)}=0
$$

and $\operatorname{supp} U_{j} \subseteq \mathbb{R}^{n} \times(-\infty, T)$. Therefore the supports of $F-F^{\prime}$ and $U_{j}$ are disdoint, and

$$
\left\langle F-F^{\prime}, U\right\rangle=\lim _{j \rightarrow \infty}\left\langle F-F^{\prime}, U_{j}\right\rangle=0
$$

One could follow a similar approximation argument for $F-F^{\prime}$ instead of $U$ to obtain that (3.1.4) is independent of the particular choice of the extension $F$ of $f$.

Finally, we claim that the two maps constructed above, i.e.

$$
f \longmapsto f \circ R_{\Omega \times(0, \infty)} \quad \text { for } f \in\left({ }^{0} B_{\alpha, p a r}^{p, q}(\Omega \times(0, T))\right)^{*}
$$

and

$$
f \longmapsto\langle f, \cdot\rangle \quad \text { for } f \in{ }_{z} B_{-\alpha, p a r}^{p^{\prime}, q^{\prime}}(\Omega \times(0, T))
$$


are inverses to each other.

First, let $f \in\left({ }^{0} B_{\alpha, p a r}^{p, q}(\Omega \times(0, T))\right)^{*}$. Then there exists $F \in B_{-\alpha, p a r}^{p^{\prime}, q^{\prime}}\left(\mathbb{R}^{n} \times \mathbb{R}\right)$ such that $\left.F\right|_{\mathbb{R}^{n} \times(-\infty, T)}=f \circ R_{\Omega \times(0, \infty)}$, and we have seen earlier that $f \circ R_{\Omega \times(0, \infty)}$ belongs to ${ }_{z} B_{-\alpha, p a r}^{p^{\prime}, q^{\prime}}(\Omega \times(0, T))$. Consider $u \in{ }^{0} B_{\alpha, p a r}^{p, q}(\Omega \times(0, T))$ and $U \in B_{\alpha, p a r}^{p, q}\left(\mathbb{R}^{n} \times \mathbb{R}\right)$ so that $\left.U\right|_{\Omega \times(0, \infty)}=u$ and $\operatorname{supp} U \subseteq \mathbb{R}^{n} \times(-\infty, T]$. For $U_{j} \in C_{c}^{\infty}\left(\mathbb{R}^{n} \times \mathbb{R}\right), j \in \mathbb{N}$, such that $\lim _{j \rightarrow \infty}\left\|U-U_{j}\right\|_{B_{\alpha, p a r}^{p, q}\left(\mathbb{R}^{n} \times \mathbb{R}\right)}=0$ and $\operatorname{supp} U_{j} \subseteq \mathbb{R}^{n} \times(-\infty, T)$, we obtain that

$$
\begin{aligned}
\left\langle f \circ R_{\Omega \times(0, \infty)}, u\right\rangle & =\langle F, U\rangle=\lim _{j \rightarrow \infty}\left\langle F, U_{j}\right\rangle=\lim _{j \rightarrow \infty}\left\langle\left. F\right|_{\mathbb{R}^{n} \times(-\infty, T)}, U_{j}\right\rangle \\
& =\lim _{j \rightarrow \infty}\left\langle f \circ R_{\Omega \times(0, \infty)}, U_{j}\right\rangle=\lim _{j \rightarrow \infty}\left\langle\left\langle f,\left.U_{j}\right|_{\Omega \times(0, \infty)}\right\rangle\right\rangle \\
& =\langle\langle f, u\rangle\rangle,
\end{aligned}
$$

where $\langle\langle\cdot, \cdot\rangle\rangle$ stands for a $\left({ }^{0} B_{\alpha, p a r}^{p, q}(\Omega \times(0, T))\right)^{*},{ }^{0} B_{\alpha, p a r}^{p, q}(\Omega \times(0, T))$ duality pairing, and we were using that $\lim _{j \rightarrow \infty}\left\|\left.U_{j}\right|_{\Omega \times(0, \infty)}-u\right\|_{B_{\alpha, p a r}^{p, q}(\Omega \times(0, T))}=0$.

Second, let $f \in{ }_{z} B_{-\alpha, p a r}^{p^{\prime}, q^{\prime}}(\Omega \times(0, T))$. We have seen that $\langle f, \cdot\rangle$ defined in (3.1.4) belongs to $\left({ }^{0} B_{\alpha, p a r}^{p, q}(\Omega \times(0, T))\right)^{*}$, and that $\langle f, \cdot\rangle \circ R_{\Omega \times(0, \infty)} \in{ }_{z} B_{-\alpha, p a r}^{p^{\prime}, q^{\prime}}(\Omega \times(0, T))$. For $\varphi \in C_{c}^{\infty}\left(\mathbb{R}^{n} \times(-\infty, T)\right)$ and $F \in B_{-\alpha, p a r}^{p^{\prime}, q^{\prime}}\left(\mathbb{R}^{n} \times \mathbb{R}\right)$ with $\left.F\right|_{\mathbb{R}^{n} \times(-\infty, T)}=f$ and support included in $(\bar{\Omega} \times[0, \infty)) \cup\left(\mathbb{R}^{n} \times[T, \infty)\right)$, we have the folowing:

$$
\langle f, \varphi\rangle \circ R_{\Omega \times(0, \infty)}=\left\langle f,\left.\varphi\right|_{\Omega \times(0, \infty)}\right\rangle=\langle F, \varphi\rangle=\left\langle\left. F\right|_{\mathbb{R}^{n} \times(-\infty, T)}, \varphi\right\rangle=f(\varphi),
$$

which completes the proof of (3.1.3).

A relatively simple, yet important consequence of Theorem 3.1.11 is as follows.

Corollary 3.1.12. Consider a Lipschitz domain $\Omega \subseteq \mathbb{R}^{n}, 0<T<\infty$, and indices $1<p, q<\infty, \alpha \in \mathbb{R} \backslash\{0\}$. Then

$$
\left({ }_{z} B_{\alpha, p a r}^{p, q}(\Omega \times(0, T))\right)^{*}={ }^{0} B_{-\alpha, p a r}^{p^{\prime}, q^{\prime}}(\Omega \times(0, T)),
$$


where $1 / p+1 / p^{\prime}=1$ and $1 / q+1 / q^{\prime}=1$. Under the additional assumption that the pair $(1 / p, 1 / q)$ belongs to the interior of $O M N P$ in the figure in Lemma 3.1.3, the analogue of (3.1.5) for the Triebel-Lizorkin scale is also valid.

Proof. Our first observation is that thanks to Theorem 2.4.3, the space $B_{\alpha, p a r}^{p, q}\left(\mathbb{R}^{n} \times \mathbb{R}\right)$ is reflexive. Taking also into account that ${ }^{0} B_{\alpha, p a r}^{p, q}(\Omega \times(0, T))$ is a closed subspace of $B_{\alpha, \text { par }}^{p, q}\left(\mathbb{R}^{n} \times \mathbb{R}\right)$, by the Eberlein-Shmulyan Theorem (see p.141 of [Yos]), the space ${ }^{0} B_{\alpha, p a r}^{p, q}(\Omega \times(0, T))$ is also reflexive. With this at hand, dualizing the result of Theorem 3.1.11, we altogether obtain (3.1.5). For the $F$-scale one follows the same argument.

The analogue of Theorem 3.1.11 and Corollary 3.1.12 (in the sense of having opposite initial conditions) are the next two results.

Theorem 3.1.13. Let $\Omega$ be a Lipschitz domain in $\mathbb{R}^{n}, 0<T<\infty$, and consider $1<p, q<\infty, \alpha \in \mathbb{R} \backslash\{0\}$. Then

$$
\left({ }_{0} B_{\alpha, p a r}^{p, q}(\Omega \times(0, T))\right)^{*}={ }^{z} B_{-\alpha, p a r}^{p^{\prime}, q^{\prime}}(\Omega \times(0, T)),
$$

where $1 / p+1 / p^{\prime}=1$ and $1 / q+1 / q^{\prime}=1$. Under the additional hypothesis that the pair $(1 / p, 1 / q)$ belongs to the interior of $O M N P$ in the figure in Lemma 3.1.3, a similar equivalence to (3.1.6) holds true for the Triebel-Lizorkin scale.

Proof. Note that once (3.1.6) is established, the same follows for the $F$-scale, similar to the cases we have encountered earlier in the present section.

In order to show (3.1.6), one possibility is to follow, with minor modifications, each step in the proof of Theorem 3.1.11. However, a simpler proof can be obtained 
by using the reflection operator in time, defined by $R f(x, t)=f(x, T-t)$ for $x \in \mathbb{R}^{n}$ and $t \in \mathbb{R}$.

On the one hand, we let $f \in{ }^{z} B_{-\alpha, p a r}^{p^{\prime}, q^{\prime}}(\Omega \times(0, T))$. Then $R \circ f$ belongs to ${ }_{z} B_{-\alpha, p a r}^{p^{\prime}, q^{\prime}}(\Omega \times(0, T))$ and, according to Theorem 3.1.11,

$$
R \circ f \in\left({ }^{0} B_{\alpha, p a r}^{p, q}(\Omega \times(0, T))\right)^{*},
$$

hence, for $\varphi \in{ }_{0} B_{\alpha, p a r}^{p, q}(\Omega \times(0, T))$, we can define

$$
\langle f, \varphi\rangle:=\langle R \circ f, R \circ \varphi\rangle .
$$

Therefore, $f$ belongs to $\left({ }_{0} B_{\alpha, p a r}^{p, q}(\Omega \times(0, T))\right)^{*}$, as desired.

On the other hand, let $\Lambda \in\left({ }_{0} B_{\alpha, p a r}^{p, q}(\Omega \times(0, T))\right)^{*}$. Then, for any $\varphi$ from the space ${ }_{0} B_{\alpha, p a r}^{p, q}(\Omega \times(0, T))$,

$$
\langle\Lambda, \varphi\rangle=\langle\Lambda, R \circ R \circ \varphi\rangle=\langle R \circ \Lambda, R \circ \varphi\rangle
$$

where we have used that $R$ is self-adjoint, i.e. $R^{*}=R$. Since $R \circ \varphi$ belongs to the space ${ }^{0} B_{\alpha, p a r}^{p, q}(\Omega \times(0, T))$, on account of Theorem 3.1 .11 we arrive at

$$
R \circ \Lambda \in\left({ }^{0} B_{\alpha, p a r}^{p, q}(\Omega \times(0, T))\right)^{*}={ }_{z} B_{-\alpha, p a r}^{p^{\prime}, q^{\prime}}(\Omega \times(0, T))
$$

for the appropriate indices. This yields

$$
\Lambda \in{ }^{z} B_{-\alpha, p a r}^{p^{\prime}, q^{\prime}}(\Omega \times(0, T)) .
$$

Finally, it is easy to see that the two maps constructed above, i.e.

$$
f \mapsto\langle f, \cdot\rangle \quad \text { for } f \in{ }^{z} B_{-\alpha, p a r}^{p^{\prime}, q^{\prime}}(\Omega \times(0, T))
$$


and

$$
\langle\Lambda, \cdot\rangle \mapsto \Lambda \quad \text { for } \Lambda \in\left({ }_{0} B_{\alpha, p a r}^{p, q}(\Omega \times(0, T))\right)^{*}
$$

are inverses to each other. This finishes the proof of the theorem.

Corollary 3.1.14. Consider a Lipschitz domain $\Omega \subseteq \mathbb{R}^{n}, 0<T<\infty$, and indices $1<p, q<\infty, \alpha \in \mathbb{R} \backslash\{0\}$. Then

$$
\left({ }^{z} B_{\alpha, p a r}^{p, q}(\Omega \times(0, T))\right)^{*}={ }_{0} B_{-\alpha, p a r}^{p^{\prime}, q^{\prime}}(\Omega \times(0, T)),
$$

where $1 / p+1 / p^{\prime}=1$ and $1 / q+1 / q^{\prime}=1$. With the additional assumption that the pair $(1 / p, 1 / q)$ belongs to the interior of $O M N P$ in the figure in Lemma 3.1.3, the proper counterpart of (3.1.7) for the Triebel-Lizorkin scale holds, as well.

Proof. We first note that, by Theorem 2.4 .3 , the space $B_{\alpha, p a r}^{p, q}\left(\mathbb{R}^{n} \times \mathbb{R}\right)$ is reflexive. Considering also the fact that ${ }_{0} B_{\alpha, p a r}^{p, q}(\Omega \times(0, T))$ is a closed subspace of $B_{\alpha, \text { par }}^{p, q}\left(\mathbb{R}^{n} \times \mathbb{R}\right)$, on account of the Eberlein-Shmulyan Theorem (see p. 141 of [Yos]), the space ${ }_{0} B_{\alpha, p a r}^{p, q}(\Omega \times(0, T))$ is also reflexive. With this at hand, dualizing the result of Theorem 3.1.13, yields the desired conclusion. As before, the case of the $F$-scale is treated in a similar fashion.

Our next goal is to identify the range of indices for which the Besov spaces ${ }_{0} B_{\alpha, p a r}^{p, q}(\Omega \times(0, T))$ and ${ }_{\square} B_{\alpha, p a r}^{p, q}(\Omega \times(0, T))$ (and their counterpart in the sense of opposite initial conditions) are equivalent. We begin by defining the latter.

Definition 3.1.15. For a Lipschitz domain $\Omega \subseteq \mathbb{R}^{n}, 0<T<\infty$, and indices 
$1<p, q<\infty, \alpha>0$ let us introduce the following function spaces.

(a) $\square^{B_{\alpha, p a r}}(\Omega \times(0, T)):=\left\{\left.F\right|_{\Omega \times(0, T)}: F \in B_{\alpha, p a r}^{p, q}(\Omega \times \mathbb{R})\right.$, supp $\left.F \subseteq \Omega \times[0, \infty)\right\}$

and

(b) $\square_{B_{\alpha, p a r}^{p, q}}(\Omega \times(0, T)):=\left\{\left.F\right|_{\Omega \times(0, T)}: F \in B_{\alpha, p a r}^{p, q}(\Omega \times \mathbb{R})\right.$, supp $\left.F \subseteq \Omega \times(-\infty, T]\right\}$.

The norms are given by

$$
\|f\|_{\square B_{\alpha, p a r}^{p, q}(\Omega \times(0, T))}:=\inf \left\{\|F\|_{B_{\alpha, p a r}^{p, q}(\Omega \times \mathbb{R})}:\left.F\right|_{\Omega \times(0, T)}=f, \operatorname{supp} F \subseteq \Omega \times[0, \infty)\right\}
$$

and

$$
\|f\|_{\square_{\alpha, p a r}^{p, q}(\Omega \times(0, T))}:=\inf \left\{\|F\|_{B_{\alpha, p a r}^{p, q}(\Omega \times \mathbb{R})}:\left.F\right|_{\Omega \times(0, T)}=f, \operatorname{supp} F \subseteq \Omega \times(-\infty, T]\right\}
$$

respectively.

For the same indices as above except $p \neq \infty$, the spaces $\square F_{\alpha, p a r}^{p, q}(\Omega \times(0, T))$ and $\square_{F_{\alpha, p a r}^{p, q}}(\Omega \times(0, T))$ are defined in a similar manner.

Choosing to use $\square B_{\alpha, p a r}^{p}$ and $\square^{\square} B_{\alpha, p a r}^{p}$ in place of $\square B_{\alpha, p a r}^{p, p}$ and $\square_{B_{\alpha, p a r}^{p, p}}$, respectively, will simplify the notation later on.

Remark 3.1.16. Under the same assumptions as in Definition 3.1.15, we may characterize the spaces just introduced as

(a) $\square B_{\alpha, p a r}^{p, q}(\Omega \times(0, T))=\left\{\left.F\right|_{\Omega \times(-\infty, T)}: F \in B_{\alpha, p a r}^{p, q}(\Omega \times \mathbb{R}), \operatorname{supp} F \subseteq \Omega \times[0, \infty)\right\}$

and

(b) $\square_{B_{\alpha, p a r}^{p, q}}(\Omega \times(0, T))=\left\{\left.F\right|_{\Omega \times(0, \infty)}: F \in B_{\alpha, p a r}^{p, q}(\Omega \times \mathbb{R})\right.$, supp $\left.F \subseteq \Omega \times(-\infty, T]\right\}$. 
Naturally, similar considerations apply to $\square F_{\alpha, p a r}^{p, q}(\Omega \times(0, T))$ and $\square_{\alpha, p a r}^{p, q}(\Omega \times(0, T))$, as well.

Our next result is isotropic in nature, and its parabolic analogue is established in Lemma 3.1.18. As a preamble to the statement of this isotropic result, let us invoke that the trace operator, denoted by $\operatorname{Tr}$ and initially defined on $C^{\infty}(\bar{\Omega})$ as the restriction to $\partial \Omega$, extends to a bounded linear operator from $B_{\alpha}^{p}(\Omega)$ to $B_{\alpha-1 / p}^{p}(\partial \Omega)$ for any Lipschitz domain $\Omega$ in $\mathbb{R}^{n}$ and indices $1 \leq p \leq \infty, \frac{1}{p}<\alpha<1+\frac{1}{p}$ (cf. Theorem 3.1 from [JeKe], which is a special case of Theorem 2 on p. 209 in [JoWa]).

Lemma 3.1.17. (Isotropic result)

Let $1<p<\infty, \frac{1}{p}<\alpha<1+\frac{1}{p}$, and $u^{ \pm} \in B_{\alpha}^{p}\left(\Omega_{ \pm}\right)$, where $\Omega_{+}:=\Omega$ is a bounded Lipschitz domain in $\mathbb{R}^{n}$ and $\Omega_{-}:=\mathbb{R}^{n} \backslash \bar{\Omega}$. Further assume that $\operatorname{Tr}\left(u^{+}\right)=\operatorname{Tr}\left(u^{-}\right)$ and, for $x \in \mathbb{R}^{n}$, define

$$
u(x):= \begin{cases}u^{+}(x) & \text { for } x \in \Omega_{+}, \\ u^{-}(x) & \text { for } x \in \Omega_{-} .\end{cases}
$$

Then $u \in B_{\alpha}^{p}\left(\mathbb{R}^{n}\right)$ and

$$
\|u\|_{B_{\alpha}^{p}\left(\mathbb{R}^{n}\right)} \approx\left\|u^{+}\right\|_{B_{\alpha}^{p}\left(\Omega_{+}\right)}+\left\|u^{-}\right\|_{B_{\alpha}^{p}\left(\Omega_{-}\right)} .
$$

Proof. Since $u^{+} \in B_{\alpha}^{p}\left(\Omega_{+}\right)$, there exists $U^{+} \in B_{\alpha}^{p}\left(\mathbb{R}^{n}\right)$ so that $\left.U^{+}\right|_{\Omega_{+}}=u^{+}$. Define

$$
v:=\left.U^{+}\right|_{\Omega_{-}}-u^{-} .
$$

Then $v \in B_{\alpha}^{p}\left(\Omega_{-}\right)$and

$$
\operatorname{Tr} v=\operatorname{Tr}\left(\left.U^{+}\right|_{\Omega_{+}}\right)-\operatorname{Tr}\left(u^{-}\right)=\operatorname{Tr}\left(u^{+}\right)-\operatorname{Tr}\left(u^{-}\right)=0 .
$$


According to Corollary 1.4.4.5 of [Gri3], $v \in\left\{v \in B_{\alpha}^{p}\left(\Omega_{-}\right): \tilde{v} \in B_{\alpha}^{p}\left(\mathbb{R}^{n}\right)\right\}$, where $\tilde{v}$ is the extension of $v$ by 0 outside $\Omega_{-}$, i.e. for $x \in \mathbb{R}^{n}$,

$$
\tilde{v}(x):=\left\{\begin{array}{l}
v(x), \quad \text { if } x \in \Omega_{-}, \\
0, \quad \text { if } x \in \mathbb{R}^{n} \backslash \bar{\Omega}_{-} .
\end{array}\right.
$$

Then

$$
U^{+}(x)-\tilde{v}(x)=\left\{\begin{array}{l}
u^{+}(x) \quad \text { for } x \in \Omega_{+}, \\
\left.U^{+}\right|_{\Omega_{-}}(x)-v(x) \text { for } x \in \Omega_{-},
\end{array}\right.
$$

or, equivalently, $U^{+}-\tilde{v}=u$. Since both $U^{+}$and $\tilde{v}$ belong to $B_{\alpha}^{p}\left(\mathbb{R}^{n}\right)$, we also have that $u \in B_{\alpha}^{p}\left(\mathbb{R}^{n}\right)$. Moreover,

$$
\begin{aligned}
\|u\|_{B_{\alpha}^{p}\left(\mathbb{R}^{n}\right)} & =\left\|U^{+}-\tilde{v}\right\|_{B_{\alpha}^{p}\left(\mathbb{R}^{n}\right)} \\
& \leq\left\|\left.\left(U^{+}-\tilde{v}\right)\right|_{\Omega_{+}}\right\|_{B_{\alpha}^{p}\left(\Omega_{+}\right)}+\left\|\left.\left(U^{+}-\tilde{v}\right)\right|_{\Omega_{-}}\right\|_{B_{\alpha}^{p}\left(\Omega_{-}\right)} \\
& =\left\|u^{+}\right\|_{B_{\alpha}^{p}\left(\Omega_{+}\right)}+\left\|u^{-}\right\|_{B_{\alpha}^{p}\left(\Omega_{-}\right)} .
\end{aligned}
$$

This establishes one direction in the equivalence (3.1.10).

Conversely, by the continuity of the restriction operator $\left.\cdot\right|_{\Omega_{ \pm}}$, we have that

$$
\left\|u^{+}\right\|_{B_{\alpha}^{p}\left(\Omega_{+}\right)}+\left\|u^{-}\right\|_{B_{\alpha}^{p}\left(\Omega_{-}\right)} \leq c\|u\|_{B_{\alpha}^{p}\left(\mathbb{R}^{n}\right)} .
$$

With this in hand, in concert with (3.1.11), we ultimately obtain (3.1.10), completing the proof of the lemma.

The parabolic counterpart of the above result is as follows.

Lemma 3.1.18. Let $1<p<\infty, \frac{1}{p}<\alpha<1+\frac{1}{p}$, and $u^{ \pm} \in B_{\alpha, p a r}^{p}\left(\Omega_{ \pm} \times I\right)$, where $\Omega_{+}:=\Omega$ is a bounded open subset of $\mathbb{R}^{n}$ with a Lipschitz boundary, $\Omega_{-}:=\mathbb{R}^{n} \backslash \bar{\Omega}$, and $I \subseteq \mathbb{R}$ is an interval. Further assume that $\operatorname{Tr}\left(u^{+}\right)=\operatorname{Tr}\left(u^{-}\right)$and, for $(x, t) \in \mathbb{R}^{n} \times I$, define

$$
u(x, t):= \begin{cases}u^{+}(x, t) & \text { for }(x, t) \in \Omega_{+} \times I, \\ u^{-}(x, t) & \text { for }(x, t) \in \Omega_{-} \times I .\end{cases}
$$


Then $u \in B_{\alpha, p a r}^{p}\left(\mathbb{R}^{n} \times I\right)$ and

$$
\|u\|_{B_{\alpha, p a r}^{p}\left(\mathbb{R}^{n} \times I\right)} \approx\left\|u^{+}\right\|_{B_{\alpha, p a r}^{p}\left(\Omega_{+} \times I\right)}+\left\|u^{-}\right\|_{B_{\alpha, p a r}^{p}\left(\Omega_{-} \times I\right)} .
$$

Proof. Our first observation is that by Proposition 2.2.3,

$$
\|u\|_{B_{\alpha, p a r}^{p}\left(\mathbb{R}^{n} \times I\right)} \approx\|u\|_{L^{p}\left(\mathbb{R}^{n} ; B_{\alpha / 2}^{p}(I)\right)}+\|u\|_{L^{p}\left(I ; B_{\alpha}^{p}\left(\mathbb{R}^{n}\right)\right)}
$$

On the one hand,

$$
\begin{aligned}
\|u\|_{L^{p}\left(\mathbb{R}^{n} ; B_{\alpha / 2}^{p}(I)\right)}^{p} & =\int_{\Omega_{+}}\|u(x, \cdot)\|_{B_{\alpha / 2}^{p}(I)}^{p} d x+\int_{\Omega_{-}}\|u(x, \cdot)\|_{B_{\alpha / 2}^{p}(I)}^{p} d x \\
& =\left\|u^{+}\right\|_{L^{p}\left(\Omega_{+} ; B_{\alpha / 2}^{p}(I)\right)}^{p}+\left\|u^{-}\right\|_{L^{p}\left(\Omega_{-} ; B_{\alpha / 2}^{p}(I)\right)}^{p} \\
& \leq\left\|u^{+}\right\|_{B_{\alpha, p a r}^{p}\left(\Omega_{+} \times I\right)}^{p}+\left\|u^{-}\right\|_{B_{\alpha, p a r}^{p}\left(\Omega_{-} \times I\right)}^{p} .
\end{aligned}
$$

On the other hand,

$$
\|u\|_{L^{p}\left(I ; B_{\alpha}^{p}\left(\mathbb{R}^{n}\right)\right)}^{p}=\int_{I}\|u(\cdot, t)\|_{B_{\alpha}^{p}\left(\mathbb{R}^{n}\right)}^{p} d t
$$

where

$$
u(\cdot, t):= \begin{cases}u^{+}(\cdot, t) & \text { in } \Omega_{+}, \\ u^{-}(\cdot, t) & \text { in } \Omega_{-} .\end{cases}
$$

Also, since $u^{ \pm}(\cdot, t) \in B_{\alpha}^{p}\left(\Omega_{ \pm}\right)$for almost every $t \in I$, and $\operatorname{Tr} u^{+}(\cdot, t)=\operatorname{Tr} u^{-}(\cdot, t)$, we can apply the isotropic case, Lemma 3.1.17, for $u^{ \pm}(\cdot, t)$ with fixed $t \in I$. This yields $u(\cdot, t) \in B_{\alpha}^{p}\left(\mathbb{R}^{n}\right)$ for almost every $t \in I$, and

$$
\|u(\cdot, t)\|_{B_{\alpha}^{p}\left(\mathbb{R}^{n}\right)} \approx\left\|u^{+}(\cdot, t)\right\|_{B_{\alpha}^{p}\left(\Omega_{+}\right)}+\left\|u^{-}(\cdot, t)\right\|_{B_{\alpha}^{p}\left(\Omega_{-}\right)} .
$$

Going further, using Fubini's property (Proposition 2.2.3) for $u^{ \pm}$, we obtain that

$$
\begin{aligned}
\|u\|_{L^{p}\left(I ; B_{\alpha}^{p}\left(\mathbb{R}^{n}\right)\right)}^{p} & \leq c\left(\int_{I}\left\|u^{+}(\cdot, t)\right\|_{B_{\alpha}^{p}\left(\Omega_{+}\right)}^{p} d t+\int_{I}\left\|u^{-}(\cdot, t)\right\|_{B_{\alpha}^{p}\left(\Omega_{-}\right)}^{p} d t\right) \\
& \leq c\left(\left\|u^{+}\right\|_{L^{p}\left(I ; B_{\alpha}^{p}\left(\Omega_{+}\right)\right)}^{p}+\left\|u^{-}\right\|_{L^{p}\left(I ; B_{\alpha}^{p}\left(\Omega_{-}\right)\right)}^{p}\right) \\
& \leq c\left(\left\|u^{+}\right\|_{B_{\alpha, p a r}^{p}\left(\Omega_{+} \times I\right)}^{p}+\left\|u^{-}\right\|_{B_{\alpha, p a r}^{p}\left(\Omega_{-} \times I\right)}^{p}\right) .
\end{aligned}
$$


From (3.1.13), (3.1.14) and (3.1.15) we obtain one direction in the equivalence (3.1.12). The opposite direction readily follows from the continuity of the restriction operator $\left.\cdot\right|_{\Omega_{ \pm} \times I}$. This finishes the proof of the lemma.

We now are able to start the discussion on the equivalence between the spaces ${ }_{0} B_{\alpha, \text { par }}^{p, q}(\Omega \times(0, T))$ and ${ }_{\square} B_{\alpha, p a r}^{p, q}(\Omega \times(0, T))$ (and their proper analogues in the sense of opposite initial conditions). In the first this type of result we consider an arbitrary Lipschitz domain.

Theorem 3.1.19. Let $\Omega$ be a Lipschitz domain in $\mathbb{R}^{n}, 0<T<\infty$ and $1<p<\infty$.

(i) For $1<q<\infty$ and $\alpha>0$ we have the natural inclusions

$$
{ }_{0} B_{\alpha, p a r}^{p, q}(\Omega \times(0, T)) \hookrightarrow \square B_{\alpha, p a r}^{p, q}(\Omega \times(0, T))
$$

and

$$
{ }^{0} B_{\alpha, p a r}^{p, q}(\Omega \times(0, T)) \hookrightarrow{ }^{\square} B_{\alpha, p a r}^{p, q}(\Omega \times(0, T)) .
$$

Similar results are valid for the Triebel-Lizorkin scale, as well.

(ii) If $0<\alpha<2 / p$, then

$$
{ }_{0} B_{\alpha, p a r}^{p}(\Omega \times(0, T))=\square B_{\alpha, p a r}^{p}(\Omega \times(0, T))
$$

and

$$
{ }^{0} B_{\alpha, p a r}^{p}(\Omega \times(0, T))=\square^{\square} B_{\alpha, p a r}^{p}(\Omega \times(0, T)) .
$$

Proof. (i) In order to show (3.1.16), we let $f \in{ }_{0} B_{\alpha, p a r}^{p, q}(\Omega \times(0, T))$. By part (a) of Definition 3.1.5, there exists a function $F \in B_{\alpha, p a r}^{p, q}\left(\mathbb{R}^{n} \times \mathbb{R}\right)$ such that $\left.F\right|_{\Omega \times(0, T)}=f$ and $\operatorname{supp} F \subseteq \mathbb{R}^{n} \times[0, \infty)$. If we now define $F^{\prime}:=\left.F\right|_{\Omega \times \mathbb{R}}$, then

$$
\left.F^{\prime}\right|_{\Omega \times(0, T)}=\left.F\right|_{\Omega \times(0, T)}=f,
$$


and, naturally, $F^{\prime} \in B_{\alpha, p a r}^{p, q}(\Omega \times \mathbb{R})$ with $\operatorname{supp} F^{\prime} \subseteq \Omega \times[0, \infty)$. Therefore, based on Definition 3.1.15 (a), $f$ belongs to $\square B_{\alpha, p a r}^{p, q}(\Omega \times(0, T))$. This completes the proof of (3.1.16). A similar argument yields (3.1.17) and the appropriate results for the Triebel-Lizorkin spaces.

(ii) The left-to-right inclusion in (3.1.18) narurally follows from (3.1.16). In order to show the opposite inclusion, we let $f \in \square B_{\alpha, p a r}^{p}(\Omega \times(0, T))$. Then, on account of Definition 3.1.15 (a), we have $f=\left.F\right|_{\Omega \times(0, T)}$ for some $F \in B_{\alpha, p a r}^{p}(\Omega \times \mathbb{R})$ with $\operatorname{supp} F \subseteq \Omega \times[0, \infty)$. Going further, by definition, there exists a function $G$ that belongs to $B_{\alpha, p a r}^{p}\left(\mathbb{R}^{n} \times \mathbb{R}\right)$ such that $\left.G\right|_{\Omega \times \mathbb{R}}=F$. With $G$ in hand, we now define

$$
\tilde{F}:=G \chi_{\mathbb{R}^{n} \times(0, \infty)} .
$$

Since $G$ belongs to $L^{p}\left(\mathbb{R}^{n} ; B_{\alpha / 2}^{p}(\mathbb{R})\right.$ ) (by the Fubini type property of the parabolic Besov scale, Proposition 2.1.19), and $\chi_{(0, \infty)}$ is a pointwise multiplier for $B_{\alpha / 2}^{p}(\mathbb{R})$ if $0<\alpha<2 / p$ (cf. Proposition 5.1 of [Tri5]), we obtain that

$$
\tilde{F} \in L^{p}\left(\mathbb{R}^{n} ; B_{\alpha / 2}^{p}(\mathbb{R})\right)
$$

Using again Proposition 2.1.19 we obtain that $G \in L^{p}\left(\mathbb{R} ; B_{\alpha}^{p}\left(\mathbb{R}^{n}\right)\right)$, which further implies that

$$
\tilde{F} \in L^{p}\left(\mathbb{R} ; B_{\alpha}^{p}\left(\mathbb{R}^{n}\right)\right) .
$$

Finally, (3.1.20) and (3.1.21), in concert with Proposition 2.1.19, this time used for $\tilde{F}$, yield

$$
\tilde{F} \in B_{\alpha, p a r}^{p}\left(\mathbb{R}^{n} \times \mathbb{R}\right) .
$$


Taking also into account the other properties of $\tilde{F}$, i.e. that

$$
\operatorname{supp} \tilde{F} \subseteq \mathbb{R}^{n} \times[0, \infty) \quad \text { and }\left.\quad \tilde{F}\right|_{\Omega \times(0, T)}=\left.G\right|_{\Omega \times(0, T)}=\left.F\right|_{\Omega \times(0, T)}=f
$$

we obtain that $f \in{ }_{0} B_{\alpha, p a r}^{p}(\Omega \times(0, T))$, as desired. Similar considerations and reasoning imply (3.1.19), completing the proof of the theorem.

In the next result we shall consider a bounded Lipschitz domain.

Theorem 3.1.20. Let $\Omega$ be a bounded open subset of $\mathbb{R}^{n}$ with Lipschitz boundary, and $0<T<\infty$. Also, consider $1<p<\infty$ and $\alpha>0$ such that the pair $\left(\alpha-\frac{1}{p}, \frac{1}{p}\right)$ belongs to the interior of the hexagon $O A B C D E$ in the figure below.

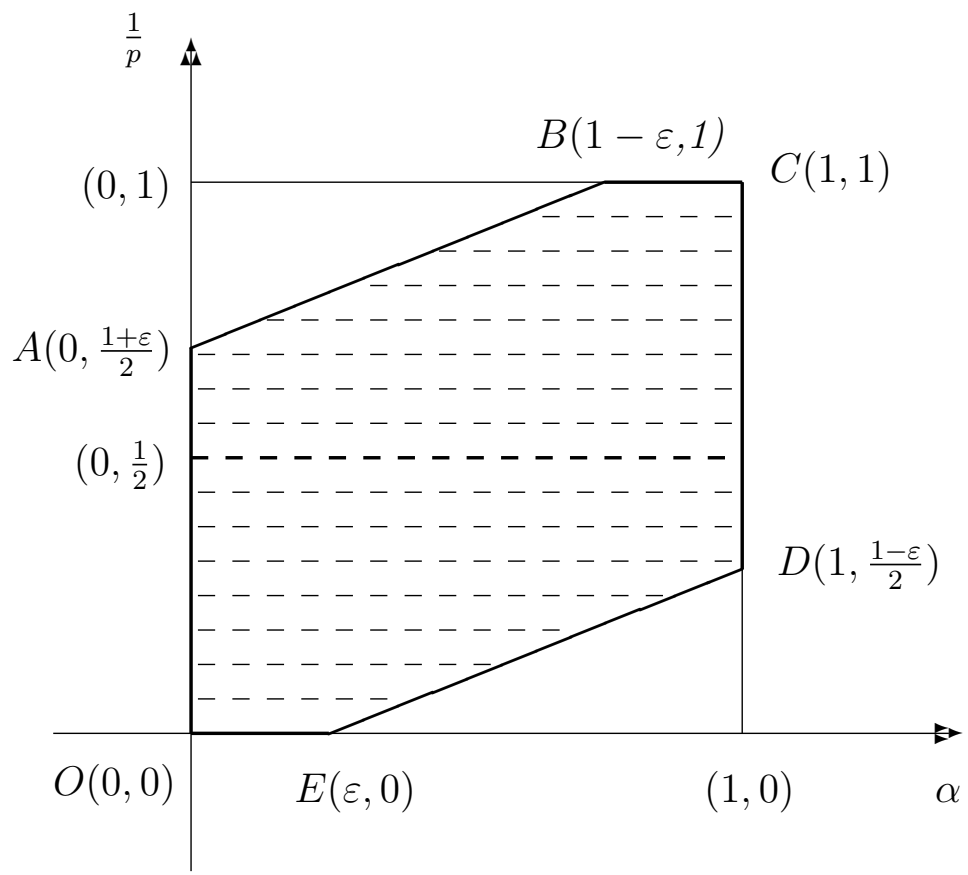

Then

$$
{ }_{0} B_{\alpha, p a r}^{p}(\Omega \times(0, T))=\square B_{\alpha, p a r}^{p}(\Omega \times(0, T))
$$


and

$$
{ }^{0} B_{\alpha, p a r}^{p}(\Omega \times(0, T))={ }^{\square} B_{\alpha, p a r}^{p}(\Omega \times(0, T)) .
$$

Proof. We first observe the folowing: the hypothesis that $\left(\alpha-\frac{1}{p}, \frac{1}{p}\right)$ belongs to the interior of $O A B C D E$ is equivalent with saying that $\left(\alpha, \frac{1}{p}\right) \in$ interior of $O M N P Q R$ in the following diagram.

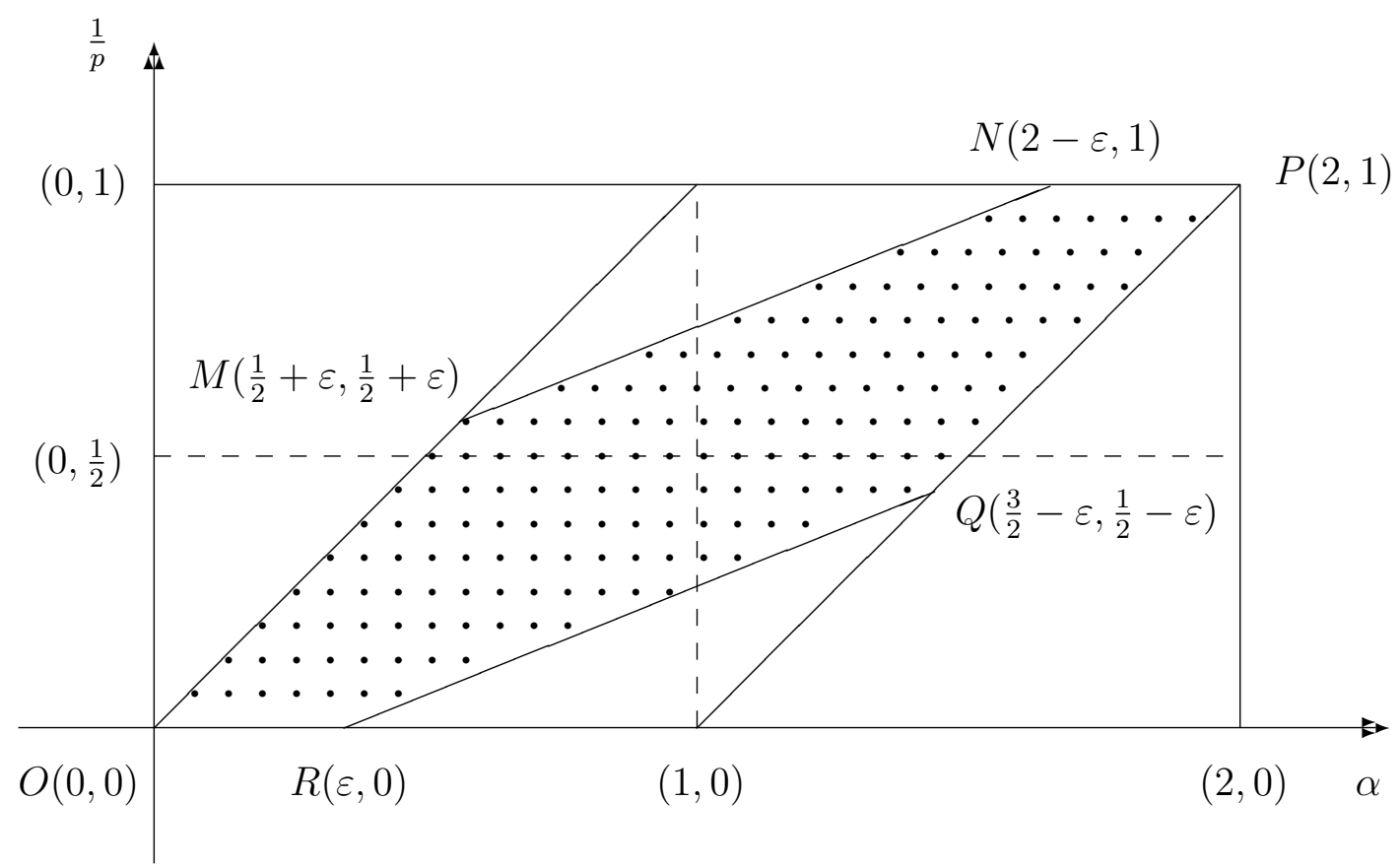

Therefore, the current assumptions on the indices imply that $\frac{1}{p}<\alpha<1+\frac{1}{p}$. Since the proofs of (3.1.22) and (3.1.23) are very similar to each other, we restrict out attention to (3.1.22). Note that the left-to-right inclusion in (3.1.22) holds for a larger range of indices, as in (3.1.16). In order to show the opposite inclusion in (3.1.22), suppose that $f \in \square B_{\alpha, p a r}^{p}(\Omega \times(0, T))$. Then, by definition, $f=\left.F\right|_{\Omega \times(0, T)}$ for some $F \in B_{\alpha, p a r}^{p}(\Omega \times \mathbb{R})$ with support included in $\Omega \times[0, \infty)$. Let us introduce 
$\psi \in C_{c}^{\infty}\left(\mathbb{R}^{n} \times \mathbb{R}\right)$ with $\operatorname{supp} \psi \subseteq \mathbb{R}^{n} \times[-T, 2 T]$ and such that $\psi \equiv 1$ in a neighborhood of $\Omega \times(0, T)$. Then

$\psi F \in B_{\alpha, p a r}^{p}(\Omega \times \mathbb{R}), \quad \operatorname{supp}(\psi F) \subseteq \Omega \times[0, \infty) \quad$ and $\left.\quad(\psi F)\right|_{\Omega \times(0, T)}=\left.F\right|_{\Omega \times(0, T)}=f$

According to Theorem 7.1.1 (which is independent of Theorem 3.1.20) and the fact $\frac{1}{p}<\alpha<1+\frac{1}{p}$ established earlier, we obtain that the boundary trace of $F, \operatorname{Tr} F$, belongs to $B_{\alpha-\frac{1}{p}, p a r}^{p}(\partial \Omega \times \mathbb{R})$. Also, the support of $\operatorname{Tr}(\psi F)$ is included in $\partial \Omega \times[0, \infty)$ and $\operatorname{Tr}(\psi F)=\left.[\operatorname{Tr}(\psi F)]\right|_{\partial \Omega \times(0,2 T)}$, therefore,

$$
\operatorname{Tr}(\psi F) \in{ }_{0} B_{\alpha-\frac{1}{p}, p a r}^{p}(\partial \Omega \times(0,2 T)) .
$$

To this end, let us define

$$
h:=\left(-\frac{1}{2} I+K\right)^{-1} \operatorname{Tr}(\psi F),
$$

where $I$ is the identity operator and $K$ is the boundary potential operator defined in (1.1.6). On account of the invertibility of the operator $-\frac{1}{2} I+K$ on the space ${ }_{0} B_{\alpha-\frac{1}{p}, p a r}^{p}(\partial \Omega \times(0,2 T))$ for the range of indices satisfying $\left(\alpha-\frac{1}{p}, \frac{1}{p}\right) \in$ interior of the hexagon $O A B C D E$ (cf. 6.2.77 established independently of all the consequences of the presenet result), we may conclude that $h \in{ }_{0} B_{\alpha-\frac{1}{p}, p a r}^{p}(\partial \Omega \times(0,2 T))$. Hence, by definition, there exists $H$ from $B_{\alpha-\frac{1}{p}, p a r}^{p}(\partial \Omega \times \mathbb{R})$ such that supp $H \subseteq \partial \Omega \times[0, \infty)$ and $\left.H\right|_{\partial \Omega \times(0,2 T)}=h$.

Going further, denote $\Omega_{+}:=\Omega$ and $\Omega_{-}:=\mathbb{R}^{n} \backslash \bar{\Omega}$, and consider

$$
G:=\varphi \mathcal{D} H \quad \text { in } \Omega_{-} \times \mathbb{R}
$$


where $\varphi \in C^{\infty}\left(\mathbb{R}^{n} \times \mathbb{R}\right)$ is such that there exists a constant $k>0$ with $\operatorname{supp} \varphi \subseteq$ $\left\{x \in \mathbb{R}^{n}: \operatorname{dist}(x, \partial \Omega)<k\right\} \times \mathbb{R}$, and $\varphi \equiv 1$ in a neighborhood of $\partial \Omega \times \mathbb{R}$. Here $\mathcal{D}$ stands for the caloric double layer potential operator introduced in (1.1.5). Now Theorem 4.2.8 implies that $G \in B_{\alpha, p a r}^{p}\left(\Omega_{-} \times(0,2 T)\right)$. Moreover,

$$
\operatorname{supp} G \subseteq \Omega_{-} \times[0, \infty)
$$

and, on $\partial \Omega \times(-\infty, 2 T)$ we have the following:

$$
\operatorname{Tr} G=\operatorname{Tr}(\mathcal{D} H)=\left(-\frac{1}{2} I+K\right) H=\left(-\frac{1}{2} I+K\right) h=\operatorname{Tr}(\psi F),
$$

(see part (2) of Theorem 4.17 in [Bro2] for the trace of $\mathcal{D}$ ).

We next define

$$
W:= \begin{cases}\psi F & \text { in } \Omega_{+} \times(-\infty, 2 T), \\ G & \text { in } \Omega_{-} \times(-\infty, 2 T) .\end{cases}
$$

Then, by Lemma 3.1.18, $W$ belongs to the space $B_{\alpha, p a r}^{p}\left(\mathbb{R}^{n} \times(-\infty, 2 T)\right)$. Also, note that $W$ is supported in $\mathbb{R}^{n} \times[0, \infty)$ and $\left.W\right|_{\Omega \times(0, T)}=\left.(\psi F)\right|_{\Omega \times(0, T)}=f$.

Finally, consider

$$
U(x, t):= \begin{cases}\xi(x, t) W(x, t), & \text { if } \quad(x, t) \in \mathbb{R}^{n} \times(-\infty, 2 T), \\ 0, & \text { if } \quad(x, t) \in \mathbb{R}^{n} \times(2 T, \infty),\end{cases}
$$

where $\xi \in C_{c}^{\infty}\left(\mathbb{R}^{n} \times \mathbb{R}\right)$ with $\operatorname{supp} \xi \subseteq \mathbb{R}^{n} \times(-\infty, 3 T / 2]$ and such that $\xi \equiv 1$ in a neighborhood of $\bar{\Omega} \times[0, T]$. Then

$$
U \in B_{\alpha, p a r}^{p}\left(\mathbb{R}^{n} \times \mathbb{R}\right)
$$

with $\operatorname{supp} U \subseteq \mathbb{R}^{n} \times[0, \infty)$ and $\left.U\right|_{\Omega \times(0, T)}=\left.W\right|_{\Omega \times(0, T)}=f$. Hence, we can conclude that $f$ belongs to ${ }_{0} B_{\alpha, p a r}^{p}(\Omega \times(0, T))$, as desired, completing the proof of Theorem 3.1.20. 
As a consequence of Theorems 3.1.19 and 3.1.20, for a bounded Lipschitz domain $\Omega$ we obtain the following.

Corollary 3.1.21. Let $\Omega$ be a bounded open subset of $\mathbb{R}^{n}$ with Lipschitz boundary, and $0<T<\infty$. Also, consider $1<p<\infty$ and $\alpha>0$ such that the pair $\left(\alpha, \frac{1}{p}\right)$ belongs to the interior of OSPQR in the following diagram.

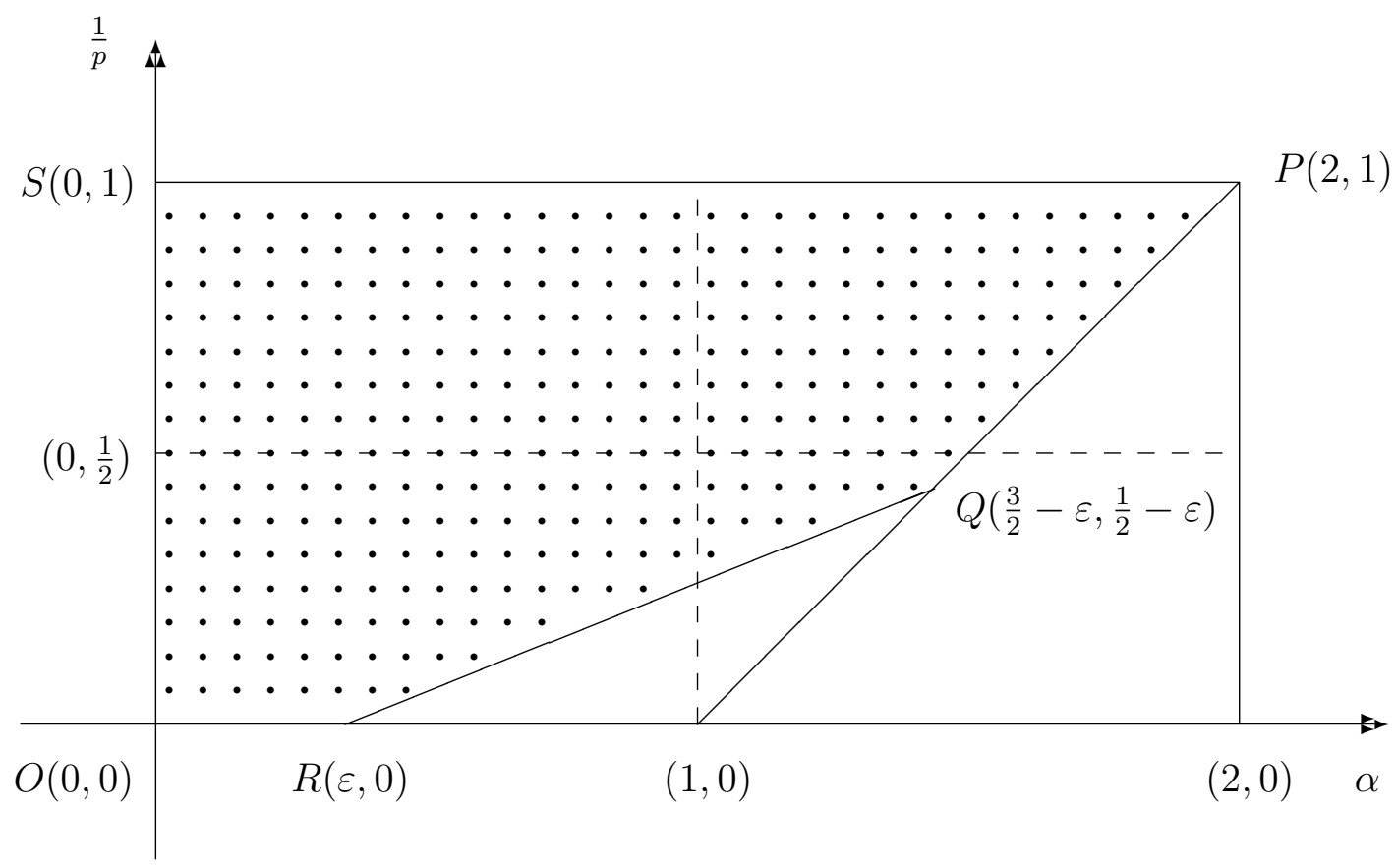

Then

$$
{ }_{0} B_{\alpha, p a r}^{p}(\Omega \times(0, T))=\square B_{\alpha, p a r}^{p}(\Omega \times(0, T))
$$

and

$$
{ }^{0} B_{\alpha, p a r}^{p}(\Omega \times(0, T))={ }^{\square} B_{\alpha, p a r}^{p}(\Omega \times(0, T)) .
$$




\subsection{The lateral side of the cylinder}

In the present section we discuss parabolic Besov and Triebel-Lizorkin spaces with built-in initial conditions, defined on the lateral boundary of a Lipschitz cylinder.

Definition 3.2.1. Similar to Definition 3.1.1, for an unbounded Lipschitz domain $\Omega$ in $\mathbb{R}^{n}$, and indices $p, q, \alpha$ such that

$$
\begin{aligned}
& \text { either } \frac{n+1}{n+2}<p, q \leq \infty, \quad(n+1)\left(\frac{1}{p}-1\right)_{+}<\alpha<1, \\
& \text { or } \quad \frac{n+1}{n+2}<p, q<\infty, \quad(n+1)\left(\frac{1}{p}-1\right)_{+}-1<\alpha<0,
\end{aligned}
$$

we define

$$
B_{\alpha, p a r, 0}^{p, q}(\partial \Omega \times(0, \infty)):=\left\{f \in B_{\alpha, p a r}^{p, q}(\partial \Omega \times \mathbb{R}): \operatorname{supp} f \subseteq \partial \Omega \times[0, \infty)\right\}
$$

and

$$
B_{\alpha, p a r, T}^{p, q}(\partial \Omega \times(-\infty, T)):=\left\{f \in B_{\alpha, p a r}^{p, q}(\partial \Omega \times \mathbb{R}): \operatorname{supp} f \subseteq \partial \Omega \times(-\infty, T]\right\}
$$

The norms are given by

$$
\|f\|_{B_{\alpha, p a r, 0}^{p, q}(\partial \Omega \times(0, \infty))}:=\inf \left\{\|f\|_{B_{\alpha, p a r}^{p, q}(\partial \Omega \times \mathbb{R})}: \operatorname{supp} f \subseteq \partial \Omega \times[0, \infty)\right\}
$$

and

$$
\|f\|_{B_{\alpha, p a r, T}^{p, q}(\partial \Omega \times(-\infty, T))}:=\inf \left\{\|f\|_{B_{\alpha, p a r}^{p, q}(\partial \Omega \times \mathbb{R})}: \operatorname{supp} f \subseteq \partial \Omega \times(-\infty, T]\right\}
$$

respectively.

The spaces $F_{\alpha, p a r, 0}^{p, q}(\partial \Omega \times(0, \infty))$ and $F_{\alpha, p a r, T}^{p, q}(\partial \Omega \times(-\infty, T))$ are defined in a 
similar fashion as long as $p, q$ and $\alpha$ satisfy one of the following conditions:
(a) $\frac{n+1}{n+2}<p<\infty, \quad \frac{n+1}{n+2}<q \leq \infty, \quad(n+1)\left(\frac{1}{\min \{p, q\}}-1\right)_{+}<\alpha<1$;
(b) $\frac{n+1}{n+2}<p, q<\infty, \quad(n+1)\left(\frac{1}{\min \{p, q\}}-1\right)_{+}-1<\alpha<0$;
(c) $\frac{n+1}{n+2}<p<\infty, \quad q=2, \quad \alpha \in\{0,1\}$.

Definition 3.2.2. Let $\Omega$ be a Lipschitz domain in $\mathbb{R}^{n}, 0<T<\infty$, and indices $p, q, \alpha$ with the property that

$$
\begin{aligned}
& \text { either } \frac{n+1}{n+2}<p, q \leq \infty, \quad(n+1)\left(\frac{1}{p}-1\right)_{+}<\alpha<1, \\
& \text { or } \quad \frac{n+1}{n+2}<p, q<\infty, \quad 0<\alpha<1-(n+1)\left(\frac{1}{p}-1\right)_{+} .
\end{aligned}
$$

We then define

(a) ${ }_{0} B_{\alpha, p a r}^{p, q}(\partial \Omega \times(0, T)):=\left\{\left.F\right|_{\partial \Omega \times(0, T)}: F \in B_{\alpha, p a r}^{p, q}(\partial \Omega \times \mathbb{R})\right.$, supp $\left.F \subseteq \partial \Omega \times[0, \infty)\right\}$,

$$
\begin{array}{r}
{ }^{0} B_{\alpha, p a r}^{p, q}(\partial \Omega \times(0, T)):=\left\{\left.F\right|_{\partial \Omega \times(0, T)}: F \in B_{\alpha, p a r}^{p, q}(\partial \Omega \times \mathbb{R}),\right. \\
\quad \operatorname{supp} F \subseteq \partial \Omega \times(-\infty, T]\},
\end{array}
$$

(c) $\quad{ }_{0} B_{-\alpha, p a r}^{p, q}(\partial \Omega \times(0, T)):=\left\{\left.F\right|_{\partial \Omega \times(-\infty, T)}: F \in B_{-\alpha, p a r}^{p, q}(\partial \Omega \times \mathbb{R})\right.$,

$$
\text { supp } F \subseteq \partial \Omega \times[0, \infty)\},
$$

$(d)$

$$
\begin{array}{r}
{ }^{0} B_{-\alpha, p a r}^{p, q}(\partial \Omega \times(0, T)):=\left\{\left.F\right|_{\partial \Omega \times(0, \infty)}: F \in B_{-\alpha, p a r}^{p, q}(\partial \Omega \times \mathbb{R}),\right. \\
\operatorname{supp} F \subseteq \partial \Omega \times(-\infty, T]\},
\end{array}
$$


where the spaces $B_{ \pm \alpha, p a r}^{p, q}(\partial \Omega \times \mathbb{R})$ were introduced in Definitions 2.3.1 and 2.3.3. The norms are given by

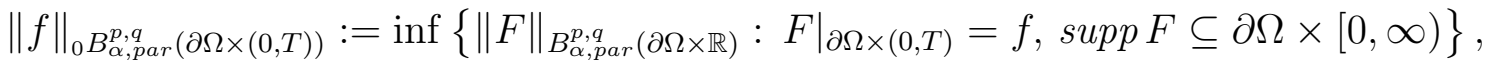

and similarly for the other spaces.

The classes ${ }_{0} F_{\alpha, p a r}^{p, q}(\partial \Omega \times(0, T))$ and ${ }^{0} F_{\alpha, p a r}^{p, q}(\partial \Omega \times(0, T))$ are introduced in a similar manner for the range of $p, q$ and $\alpha$ specified in (3.2.28). Note that this covers the spaces ${ }_{0} H_{\text {par }}^{1, p}(\partial \Omega \times(0, T))$ and ${ }^{0} H_{\text {par }}^{1, p}(\partial \Omega \times(0, T)), \frac{n+1}{n+2}<p \leq 1$, due to the identification proved in Theorem 2.1.59 and the notation introduced in Definition 2.3.9. As far as the Hardy scale $H_{p a r}^{p}$ is concerned, relying on the definitions of $H^{p}(\partial \Omega \times \mathbb{R})$ and $\dot{H}^{p}(\partial \Omega \times \mathbb{R})$ form Section 2.3.2, we define the spaces ${ }_{0} H_{\text {par }}^{p}(\partial \Omega \times(0, T))$ and ${ }^{0} H_{p a r}^{p}(\partial \Omega \times(0, T)), \frac{n+1}{n+2}<p \leq 1$, (and their homogeneous counterparts) as we did for the Besov scale above.

Remark 3.2.3. We shall use the notation ${ }_{0} B_{ \pm \alpha}^{p}$ and ${ }^{0} B_{ \pm \alpha}^{p}$ instead of ${ }_{0} B_{ \pm \alpha}^{p, p}$ and ${ }^{0} B_{ \pm \alpha}^{p, p}$, respectively.

Note that for any Lipschitz domain $\Omega$ in $\mathbb{R}^{n}, 0<T<\infty$, and $1<p, q<\infty$, $0<\alpha<1$, the following identifications hold.

$$
\begin{array}{r}
{ }_{0} B_{\alpha, p a r}^{p, q}(\partial \Omega \times(0, T))=\left\{\left.F\right|_{\partial \Omega \times(-\infty, T)}: F \in B_{\alpha, p a r}^{p, q}(\partial \Omega \times \mathbb{R}),\right. \\
\text { supp } F \subseteq \partial \Omega \times[0, \infty)\}
\end{array}
$$

and

$$
\begin{aligned}
&{ }^{0} B_{\alpha, p a r}^{p, q}(\partial \Omega \times(0, T))=\left\{\left.F\right|_{\partial \Omega \times(0, \infty)}: F \in B_{\alpha, p a r}^{p, q}(\partial \Omega \times \mathbb{R})\right. \\
&\text { supp } F \subseteq \partial \Omega \times(-\infty, T]\} .
\end{aligned}
$$

A similar statement is valid for the F-scale. 
Remark 3.2.4. Consider a bounded Lipschitz domain $\Omega \subset \mathbb{R}^{n}, 0<T<\infty$ and $\frac{n+1}{n+2}<p \leq 1$

i. Based on the definition of the spaces we consider, using the extension operator by 0 (similar to the proof of $(6.2 .43))$, there holds

$$
\begin{aligned}
& { }_{0} H_{p a r}^{p}(\partial \Omega \times(0, T)) \equiv H_{p a r}^{p}(\partial \Omega \times(0, T)), \\
& { }_{0} \dot{H}_{p a r}^{p}(\partial \Omega \times(0, T)) \equiv \dot{H}_{p a r}^{p}(\partial \Omega \times(0, T)) .
\end{aligned}
$$

ii. One also has

$$
{ }_{0} H_{\text {par }}^{p}(\partial \Omega \times(0, T))={ }_{0} \dot{H}_{\text {par }}^{p}(\partial \Omega \times(0, T))+L^{q}(\partial \Omega \times(0, T)) \quad \forall q>1
$$

The right-to-left inclusion in (3.2.32) is a consequence of Remark 2.3.36 and (3.2.30), (3.2.31). The opposite one follows along the same lines as Remark 2.3.36 if one keeps in mind a special atomic decomposition (i.e. atoms supported in $\partial \Omega \times[0, \infty)$, as in Step 6 of Theorem 6.2.6) of the space $H_{p a r}^{p}(\partial \Omega \times \mathbb{R})$.

In order to use (later on) some of the results form [Bro2], first we have to identify the spaces in [Bro2] with the corresponding ones in our notation.

Lemma 3.2.5. For a bounded Lipschitz domain $\Omega \subset \mathbb{R}^{n}$ and $0<T<\infty$,

$$
{ }_{0} H_{\text {par }}^{1,1}(\partial \Omega \times(0, T)) \equiv \mathcal{L}_{1}^{1}(\partial \Omega \times(0, T))
$$

where the space in the right-hand side of (3.2.33) is defined on p. 10 in [Bro2].

Proof. First let us consider $f \in \mathcal{L}_{1}^{1}(\partial \Omega \times(0, T))$. Then, by definition, $f=g \chi_{(0, T)}$, where $g \in \mathcal{L}_{1}^{1}(\partial \Omega \times(0, \infty))$ and

$$
\left(\psi_{i} \tilde{g}\right) \circ \pi_{i}^{-1} \in \mathcal{L}_{1}^{1}\left(\mathbb{R}^{n-1} \times \mathbb{R}\right)
$$


Here $\left\{\psi_{i}\right\}_{i=1}^{N}$ is a partition of unity on $\partial \Omega$, for more details the reader is referred to Remark 1.2.1. Also, for each $i=1, \ldots, N$, the function $\pi_{i}^{-1}$ is given by

$$
\begin{aligned}
& \pi_{i}^{-1}: \mathbb{R}^{n-1} \times \mathbb{R} \longrightarrow \partial \Omega_{i} \times \mathbb{R}, \\
& \pi_{i}^{-1}\left(x^{\prime}, t\right):=\left(x^{\prime}, \psi_{i}\left(x^{\prime}\right), t\right),
\end{aligned}
$$

and $\tilde{g}$ is the extension by 0 of $g$ to $\partial \Omega \times(-\infty, 0)$. Recall from Remark 2.1.54 that $\mathcal{L}_{1}^{1}\left(\mathbb{R}^{n-1} \times \mathbb{R}\right)$ coincides with $H_{\text {par }}^{1,1}\left(\mathbb{R}^{n-1} \times \mathbb{R}\right)$. Then (3.2.34) yields

$$
\left(\psi_{i} \tilde{g}\right) \circ \pi_{i}^{-1} \in H_{\text {par }}^{1,1}\left(\mathbb{R}^{n-1} \times \mathbb{R}\right)
$$

for $i=1, \ldots, N$. This furher implies that $\psi_{i} \tilde{g} \in H_{\text {par }}^{1,1}\left(\partial \Omega_{i} \times \mathbb{R}\right)$ for each $i=1, \ldots, N$, hence we can conclude that $\tilde{g} \in H_{\text {par }}^{1,1}(\partial \Omega \times \mathbb{R})$ and $\left.\tilde{g}\right|_{\partial \Omega \times(0, T)}=f$ with supp $\tilde{g} \subseteq[0, \infty)$, which amounts to $f \in{ }_{0} H_{\text {par }}^{1,1}(\partial \Omega \times(0, T))$, as desired. This shows the right-to-left inclusion in (3.2.33).

Conversely, let $f \in{ }_{0} H_{\text {par }}^{1,1}(\partial \Omega \times(0, T))$. Then, by definition, there exists a function $F \in H_{\text {par }}^{1,1}(\partial \Omega \times \mathbb{R})$ such that $\left.F\right|_{\partial \Omega \times(0, T)}=f$ and $\operatorname{supp} F \subseteq[0, \infty)$. With the above notation,

$$
\left(\psi_{i} F\right) \circ \pi_{i}^{-1} \in H_{\text {par }}^{1,1}\left(\mathbb{R}^{n-1} \times \mathbb{R}\right),
$$

and by the equivalence between the spaces $H_{\text {par }}^{1,1}$ and $\mathcal{L}_{1}^{1}$ on $\mathbb{R}^{n-1} \times \mathbb{R}$ (see Remark 2.1.54), we obtain that

$$
\left(\psi_{i} F\right) \circ \pi_{i}^{-1} \in \mathcal{L}_{1}^{1}\left(\mathbb{R}^{n-1} \times \mathbb{R}\right) .
$$

Therefore, $\psi_{i} F \in \mathcal{L}_{1}^{1}\left(\partial \Omega_{i} \times \mathbb{R}\right)$ for each $i=1, \ldots, N$, which further implies that $F \in \mathcal{L}_{1}^{1}(\partial \Omega \times(0, \infty))$ and $f=F \chi_{(0, T)}$. Hence, $f \in \mathcal{L}_{1}^{1}(\partial \Omega \times(0, T))$, which proves the left-to-right inclusion in (3.2.33). 
Lemma 3.2.6. Consider a bounded Lipschitz domain $\Omega$ in $\mathbb{R}^{n}, 0<T<\infty$ and $1<p<n+1$. Then

$$
{ }_{0} L_{1, p a r}^{p}(\partial \Omega \times(0, T)) \equiv \mathcal{L}_{1}^{p}(\partial \Omega \times(0, T)),
$$

where the space on the right-hand side of (3.2.36) is defined on p.10 in [Bro2].

Proof. First, let us consider $f \in \mathcal{L}_{1}^{p}(\partial \Omega \times(0, T))$. Then, by definition, $f=g \chi_{(0, T)}$, where $g \in \mathcal{L}_{1}^{p}(\partial \Omega \times(0, \infty))$ and

$$
\left(\psi_{i} \tilde{g}\right) \circ \pi_{i}^{-1} \in \mathcal{L}_{1}^{p}\left(\mathbb{R}^{n-1} \times \mathbb{R}\right)
$$

Here $\left\{\psi_{i}\right\}_{i=1}^{N}$ is a partition of unity on $\partial \Omega$, for more details the reader is referred to Remark 1.2.1. Also, for each $i=1, \ldots, N$, the function $\pi_{i}^{-1}$ is given by

$$
\begin{aligned}
& \pi_{i}^{-1}: \mathbb{R}^{n-1} \times \mathbb{R} \longrightarrow \partial \Omega_{i} \times \mathbb{R}, \\
& \pi_{i}^{-1}\left(x^{\prime}, t\right):=\left(x^{\prime}, \psi_{i}\left(x^{\prime}\right), t\right)
\end{aligned}
$$

and $\tilde{g}$ is the extension by 0 of $g$ to $\partial \Omega \times(-\infty, 0)$. Without loss of generality we can assume that $g=G \psi$, where $G \in \mathcal{L}_{1}^{p}(\partial \Omega \times(0, \infty))$, and $\psi \in C_{c}^{\infty}(0, \infty)$ such that $\psi \equiv 1$ on $(0, T), \operatorname{supp} \psi \subseteq(0,2 T)$. Therefore, according to [Bro2] (p.10), $\left(\psi_{i} \tilde{g}\right) \circ \pi_{i}^{-1} \in L^{\frac{(n+1) p}{n+1-p}}\left(\mathbb{R}^{n-1} \times \mathbb{R}\right)$ for $1<p<n+1$ and $i=1, \ldots, N$, which further implies that $\psi_{i} \tilde{g} \in L^{\frac{(n+1) p}{n+1-p}}\left(\partial \Omega_{i} \times \mathbb{R}\right)$ for $1<p<n+1$ and $i=1, \ldots, N$. Summing up from $i=1$ to $N$, we get that $\tilde{g} \in L^{\frac{(n+1) p}{n+1-p}}(\partial \Omega \times \mathbb{R})$ for $1<p<n+1$. Hence $f=g \chi_{(0, T)} \in L^{\frac{(n+1) p}{n+1-p}}(\partial \Omega \times(0, T)) \hookrightarrow L^{p}(\partial \Omega \times(0, T))$, and we may then write $g \in L^{p}(\partial \Omega \times(0, \infty))$. With this in hand, we also have

$$
\left(\psi_{i} \tilde{g}\right) \circ \pi_{i}^{-1} \in L^{p}\left(\mathbb{R}^{n-1} \times \mathbb{R}\right)
$$


for $1<p<n+1$ and $i=1, \ldots, N$. Now, form (3.2.37) and (3.2.38), relying on the equivalence between the spaces $\mathcal{L}_{1}^{p} \cap L^{p}$ and $L_{1, p a r}^{p}$ on $\mathbb{R}^{n-1} \times \mathbb{R}$ established in Chapter 2 (see Theorem 2.1.35), we obtain that

$$
\left(\psi_{i} \tilde{g}\right) \circ \pi_{i}^{-1} \in L_{1, p a r}^{p}\left(\mathbb{R}^{n-1} \times \mathbb{R}\right)
$$

for $1<p<n+1$ and $i=1, \ldots, N$. This furher implies that $\psi_{i} \tilde{g} \in L_{1, p a r}^{p}\left(\partial \Omega_{i} \times \mathbb{R}\right)$ for each $i=1, \ldots, N$ and $1<p<n+1$, hence we can conclude that $\tilde{g} \in L_{1, p a r}^{p}(\partial \Omega \times \mathbb{R})$ and $\left.\tilde{g}\right|_{\partial \Omega \times(0, T)}=f$ with supp $\tilde{g} \subseteq[0, \infty)$, which amounts to $f \in{ }_{0} L_{1, p a r}^{p}(\partial \Omega \times(0, T))$, as desired. This proves the right-to-left inclusion in (3.2.36).

Conversely, let $f \in{ }_{0} L_{1, p a r}^{p}(\partial \Omega \times(0, T))$. Then, by definition, there exists a function $F \in L_{1, \text { par }}^{p}(\partial \Omega \times \mathbb{R})$ such that $\left.F\right|_{\partial \Omega \times(0, T)}=f$ and $\operatorname{supp} F \subseteq[0, \infty)$. With the above notation,

$$
\left(\psi_{i} F\right) \circ \pi_{i}^{-1} \in L_{1, p a r}^{p}\left(\mathbb{R}^{n-1} \times \mathbb{R}\right)
$$

and by the equivalence between the spaces $\mathcal{L}_{1}^{p} \cap L^{p}$ and $L_{1, p a r}^{p}$ on $\mathbb{R}^{n-1} \times \mathbb{R}$, established in Chapter 2 (see Theorem 2.1.35), we obtain that

$$
\left(\psi_{i} F\right) \circ \pi_{i}^{-1} \in \mathcal{L}_{1}^{p}\left(\mathbb{R}^{n-1} \times \mathbb{R}\right)
$$

Therefore, $\psi_{i} F \in \mathcal{L}_{1}^{p}\left(\partial \Omega_{i} \times \mathbb{R}\right)$ for each $i=1, \ldots, N$, which further implies that $F \in \mathcal{L}_{1}^{p}(\partial \Omega \times(0, \infty))$ and $f=F \chi_{(0, T)}$. Hence, $f \in \mathcal{L}_{1}^{p}(\partial \Omega \times(0, T))$, showing the left-to-right inclusion in (3.2.36).

We now are ready to explore the duality properties of the classes introduced in Definition 3.2.2. These results will play an important role in Chapters 6-8. 
Theorem 3.2.7. Consider a Lipschitz domain $\Omega \subseteq \mathbb{R}^{n}, 0<T<\infty$, and indices $1<p, q<\infty, 0<\alpha<1$. Then

$$
\left({ }^{0} B_{\alpha, p a r}^{p, q}(\partial \Omega \times(0, T))\right)^{*}={ }_{0} B_{-\alpha, p a r}^{p^{\prime}, q^{\prime}}(\partial \Omega \times(0, T))
$$

and

$$
\left({ }_{0} B_{\alpha, p a r}^{p, q}(\partial \Omega \times(0, T))\right)^{*}={ }^{0} B_{-\alpha, p a r}^{p^{\prime}, q^{\prime}}(\partial \Omega \times(0, T)),
$$

where $1 / p+1 / p^{\prime}=1$ and $1 / q+1 / q^{\prime}=1$. With the additional assumption that the pair $(1 / p, 1 / q)$ belongs to the interior of $O M N P$ in the figure in Lemma 3.1.3, similar results hold for the Triebel-Lizorkin scale, as well.

Proof. Our first goal is to show (3.2.40).

Case (i) $\Omega$ is unbounded. Applying Theorem 3.1.11 with $\Omega=\mathbb{R}^{n-1}$ and using the definitions of the appropriate function spaces, we obtain that

$$
\begin{aligned}
& \left({ }^{0} B_{\alpha, p a r}^{p, q}\left(\mathbb{R}^{n-1} \times(0, T)\right)\right)^{*} \\
& \quad={ }_{z} B_{-\alpha, p a r}^{p^{\prime}, q^{\prime}}\left(\mathbb{R}^{n-1} \times(0, T)\right) \\
& \quad=\left\{\left.F\right|_{\mathbb{R}^{n-1} \times(-\infty, T)}: F \in B_{-\alpha, p a r}^{p^{\prime}, q^{\prime}}\left(\mathbb{R}^{n-1} \times \mathbb{R}\right), \text { supp } F \subseteq \mathbb{R}^{n-1} \times[0, \infty)\right\} \\
& \quad={ }_{0} B_{-\alpha, p a r}^{p^{\prime}, q^{\prime}}\left(\mathbb{R}^{n-1} \times(0, T)\right) .
\end{aligned}
$$

This, in turn, due to a bi-Lipschitz change of variables, is equivalent with (3.2.40) in the setting of a graph domain.

Case (ii) $\Omega$ is bounded. We consider a partition of unity $\left\{\psi_{i}\right\}_{1 \leq i \leq N}$ subordinated to a smooth covering of $\partial \Omega$ as in Remark 1.2.1. Based on Definitions 3.2.2 and 2.3.3 we then arrive at the following equivalence:

$$
f \in{ }_{0} B_{-\alpha, p a r}^{p^{\prime}, q^{\prime}}(\partial \Omega \times(0, T)) \Longleftrightarrow g_{i} \in{ }_{0} B_{-\alpha, p a r}^{p^{\prime}, q^{\prime}}\left(\mathbb{R}^{n-1} \times(0, T)\right) \quad \forall i=1, \ldots, N,
$$


where, for $\left(x^{\prime}, t\right) \in \mathbb{R}^{n-1} \times(0, T)$,

$$
g_{i}\left(x^{\prime}, t\right):={\widetilde{\psi_{i} f}}^{\partial \Omega_{i}}\left(x^{\prime}, \varphi\left(x^{\prime}\right), t\right) \sqrt{1+\left|\nabla \varphi_{i}\left(x^{\prime}\right)\right|^{2}} .
$$

With this in hand, we proceed much as in the proof of Proposition 2.4.5 and use the previous case with $\Omega=\mathbb{R}^{n-1}$. This completes the proof of the first part of the theorem.

In order to show (3.2.41) when $\Omega$ is unbounded, we apply Theorem 3.1.13 with $\Omega=\mathbb{R}^{n-1}$. In view of the definitions of the spaces involved, we arrive at

$$
\begin{aligned}
& \left({ }_{0} B_{\alpha, p a r}^{p, q}\left(\mathbb{R}^{n-1} \times(0, T)\right)\right)^{*} \\
& \quad={ }^{z} B_{-\alpha, p a r}^{p^{\prime}, q^{\prime}}\left(\mathbb{R}^{n-1} \times(0, T)\right) \\
& \quad=\left\{\left.F\right|_{\mathbb{R}^{n-1} \times(0, \infty)}: F \in B_{-\alpha, p a r}^{p^{\prime}, q^{\prime}}\left(\mathbb{R}^{n-1} \times \mathbb{R}\right), \text { supp } F \subseteq \mathbb{R}^{n-1} \times(-\infty, T]\right\} \\
& \quad={ }^{0} B_{-\alpha, p a r}^{p^{\prime}, q^{\prime}}\left(\mathbb{R}^{n-1} \times(0, T)\right),
\end{aligned}
$$

which, thanks to a bi-Lipschitz change of variables, amounts to (3.2.41) with a graph domain. When $\Omega$ is bounded, we use the same approach as we did in the case of $(3.2 .40)$.

Using the appropriate parts of Theorems 3.1.11 and 3.1.13, a similar reasoning yields the desired result for the Triebel-Lzorkin scale. This finishes the proof of the theorem.

Theorem 3.2.8. Consider a Lipschitz domain $\Omega$ in $\mathbb{R}^{n}, 0<T<\infty$, and indices $1<p, q<\infty, 0<\alpha<1$. Then

$$
\left({ }^{0} B_{-\alpha, p a r}^{p, q}(\partial \Omega \times(0, T))\right)^{*}={ }_{0} B_{\alpha, p a r}^{p^{\prime}, q^{\prime}}(\partial \Omega \times(0, T))
$$


and

$$
\left({ }_{0} B_{-\alpha, p a r}^{p, q}(\partial \Omega \times(0, T))\right)^{*}={ }^{0} B_{\alpha, p a r}^{p^{\prime}, q^{\prime}}(\partial \Omega \times(0, T)),
$$

where $1 / p+1 / p^{\prime}=1$ and $1 / q+1 / q^{\prime}=1$. Under the additional hypothesis that the pair $(1 / p, 1 / q)$ belongs to the interior of $O M N P$ in the figure in Lemma 3.1.3, similar results hold for the Triebel-Lizorkin scale, as well.

Proof. Since the ingredients necessary to prove (3.2.42) and (3.2.43) are the same, we will present only the proof of the first duality result, (3.2.42).

Case (i) $\Omega$ is unbounded. The main idea is to show that the Besov space ${ }_{0} B_{\alpha, p a r}^{p, q}(\partial \Omega \times(0, T))$ is reflexive, then to use the duality result (3.2.41). First, we note that

$$
{ }_{0} B_{\alpha, p a r}^{p, q}(\partial \Omega \times(0, T))=\left\{\left.F\right|_{\partial \Omega \times(0, T)}: F \in B_{\alpha, p a r, 0}^{p, q}(\partial \Omega \times(0, \infty))\right\}
$$

In order to construct an extension operator from the Besov space ${ }_{0} B_{\alpha, p a r}^{p, q}(\partial \Omega \times(0, T))$ to $B_{\alpha, p a r, 0}^{p, q}(\partial \Omega \times(0, \infty))$, we let $f$ be a function from ${ }_{0} B_{\alpha, p a r}^{p, q}\left(\mathbb{R}^{n-1} \times(0, T)\right)$. Then, by definition, there exists a function $F \in B_{\alpha, \text { par }}^{p, q}\left(\mathbb{R}^{n-1} \times \mathbb{R}\right)$ such that $\operatorname{supp} F$ is included in $\mathbb{R}^{n-1} \times[0, \infty)$ and $f=\left.F\right|_{\mathbb{R}^{n-1} \times(0, T)}$. Let us introduce $\psi_{0}, \psi_{1} \in C^{\infty}(\mathbb{R})$ with $\psi_{0}+\psi_{1}=1$, so that

$$
\begin{aligned}
& \psi_{0} \equiv 1 \text { on }[T, \infty), \quad \operatorname{supp} \psi_{0} \subseteq(0, \infty), \\
& \psi_{1} \equiv 1 \text { on }(-\infty, 0], \quad \operatorname{supp} \psi_{1} \subseteq(-\infty, T] .
\end{aligned}
$$

We also define $\tilde{E} f:=\psi_{1} F+\psi_{0} E f$, where $E$ is the extension operator introduced in Remark 2.2.7. 
Then $\tilde{E} f \in B_{\alpha, p a r}^{p, q}\left(\mathbb{R}^{n-1} \times \mathbb{R}\right)$ and supp $\tilde{E} f \subseteq \mathbb{R}^{n-1} \times[0, \infty)$, hence

$$
\tilde{E} f \in B_{\alpha, p a r, 0}^{p, q}\left(\mathbb{R}^{n-1} \times(0, \infty)\right) .
$$

Moreover, $\left.\tilde{E} f\right|_{\mathbb{R}^{n} \times(0, T)}=\left(\psi_{0}+\psi_{1}\right) f=f \in{ }_{0} B_{\alpha, p a r}^{p, q}\left(\mathbb{R}^{n-1} \times(0, T)\right)$. To show that the definition of $\tilde{E}$ is independent of the choice of $F$, we pick $F, F^{\prime}$ from $B_{\alpha, \text { par }}^{p, q}\left(\mathbb{R}^{n-1} \times \mathbb{R}\right)$ so that $\operatorname{supp} F \subseteq \mathbb{R}^{n-1} \times[0, \infty), \operatorname{supp} F^{\prime} \subseteq \mathbb{R}^{n-1} \times[0, \infty)$, and $f=\left.F\right|_{\mathbb{R}^{n-1} \times(0, T)}=$ $\left.F^{\prime}\right|_{\mathbb{R}^{n-1} \times(0, T)}$. Then we can see that $\psi_{1}\left(F-F^{\prime}\right) \equiv 0$ for a.e. $t \in \mathbb{R}$, which means that the definition of $\tilde{E}$ is independent of the particular choice of $F$. Hence, for given $f, g \in{ }_{0} B_{\alpha, p a r}^{p, q}\left(\mathbb{R}^{n-1} \times(0, T)\right)$ with extensions $F$ and $G$, respectively, from $B_{\text {par }}^{p, q}\left(\mathbb{R}^{n-1} \times \mathbb{R}\right)$, we can choose $F+G$ to be the extension of $f+g$. Consequently, $\tilde{E}$ is linear.

Using a bi-Lipschitz change of variables we obtain that there exists a linear, bounded extension operator

$$
\tilde{\tilde{E}}:{ }_{0} B_{\alpha, p a r}^{p, q}(\partial \Omega \times(0, T)) \longrightarrow B_{\alpha, p a r, 0}^{p, q}(\partial \Omega \times(0, \infty)) .
$$

If we denote $\tilde{\tilde{E}} \circ R_{\partial \Omega \times(0, T)}$ by $P$, where $R_{\partial \Omega \times(0, T)}$ is the restriction operator, then $P^{2}=P$, i.e. $P$ is a projection, and

$$
{ }_{0} B_{\alpha, p a r}^{p, q}(\partial \Omega \times(0, T)) \simeq P\left(B_{\alpha, p a r, 0}^{p, q}(\partial \Omega \times(0, \infty))\right) \hookrightarrow B_{\alpha, p a r, 0}^{p, q}(\partial \Omega \times(0, \infty))
$$

Since $B_{\alpha, p a r, 0}^{p, q}(\partial \Omega \times(0, \infty))$ is a closed subspace of $B_{\alpha, p a r}^{p, q}(\partial \Omega \times \mathbb{R})$, which is reflexive (according to Proposition 2.4.5), then by the Eberlein-Shmulyan Theorem (p. 141 in $[$ Yos $])$, the space $B_{\alpha, p a r, 0}^{p, q}(\partial \Omega \times(0, \infty))$ is also reflexive. Moreover, (3.2.44) and a similar argument as above yields the reflexivity of ${ }_{0} B_{\alpha, p a r}^{p, q}(\partial \Omega \times(0, T))$, which, in concert with (3.2.41), implies the desired result. 
Case (ii) $\Omega$ is bounded. We can use the same approach as in the proof of Theorem 3.2.7 and Proposition 2.4.5.

As far as the Triebel-Lizorkin scale is concerned, one proceeds as above, using Proposition 2.4.6 and an analogue of (3.2.41) in place of Proposition 2.4.5 and (3.2.41), respectively. This concludes the proof of the theorem.

\subsection{Banach envelopes of Hardy spaces with built- in initial conditions}

Based on Theorems 2.5.1 and 2.5.2, in this section we present a few direct applications of Lemma 1.2.52 and their consequences, which are going to play a significant role in our discussion on the invertibility of boundary potential operators on bounded Lipschitz cylinders.

Theorem 3.3.1. Consider the indices $\frac{n+2}{n+3}<p<1$ and $\alpha=(n+2)\left(\frac{1}{p}-1\right)$ and let $0<T<\infty$. Then the following holds:

$$
\mathcal{E}_{1}\left({ }_{0} H_{p a r}^{p}\left(\mathbb{R}^{n} \times(0, T)\right)\right)={ }_{0} B_{-\alpha, p a r}^{1}\left(\mathbb{R}^{n} \times(0, T)\right) .
$$

Proof. Our goal is to apply Lemma 1.2.52 with

$$
\begin{gathered}
X_{1}=H_{p a r}^{p}\left(\mathbb{R}^{n} \times \mathbb{R}\right), \quad Y_{1}=B_{-\alpha, p a r}^{1}\left(\mathbb{R}^{n} \times \mathbb{R}\right), \\
X_{2}={ }_{0} H_{p a r}^{p}\left(\mathbb{R}^{n} \times(0, T)\right), \quad Y_{2}={ }_{0} B_{-\alpha, p a r}^{1}\left(\mathbb{R}^{n} \times(0, T)\right) .
\end{gathered}
$$

For the range of indices we consider, the inclusion

$$
H_{\text {par }}^{p}\left(\mathbb{R}^{n} \times \mathbb{R}\right) \hookrightarrow B_{-\alpha, p a r}^{1}\left(\mathbb{R}^{n} \times \mathbb{R}\right)
$$


is well-defined, continuous and dense. Indeed, according to Theorem 2.1.17 with $p_{0}=p, q_{0}=2, \alpha=0$ and $p_{1}=q_{1}=1$, the inclusion in (3.3.45) is continuous. Next, recall from (2.1.5) and (2.1.6) that $\mathrm{S}\left(\mathbb{R}^{n} \times \mathbb{R}\right) \hookrightarrow B_{-\alpha, p a r}^{1}\left(\mathbb{R}^{n} \times \mathbb{R}\right)$ densly, and that $\mathrm{S}\left(\mathbb{R}^{n} \times \mathbb{R}\right) \hookrightarrow H_{\text {par }}^{p}\left(\mathbb{R}^{n} \times \mathbb{R}\right)$. Consequently, the inclusion in (3.3.45) is dense, as well.

Going further, we shall define the operators $P$ and $R$ from Lemma 1.2.52 in a few of steps, starting with

Step 1. Definition of an extension operator

$$
\text { ext : } B_{-\alpha, p a r}^{1}\left(\mathbb{R}^{n} \times(-\infty, 0)\right) \longrightarrow B_{-\alpha, p a r}^{1}\left(\mathbb{R}^{n} \times \mathbb{R}\right) \text {. }
$$

For $(x, t) \in \mathbb{R}^{n} \times \mathbb{R}$ let us introduce

$$
(\operatorname{ext} f)(x, t):=\left\{\begin{array}{cl}
f(x, t), & \text { if } t<0 \\
-f(x,-t), & \text { if } t>0
\end{array}\right.
$$

and choose $f \in B_{-\alpha, p a r}^{1}\left(\mathbb{R}^{n} \times(-\infty, 0)\right)$. Then, by definition, there exists $g$ from $B_{-\alpha, p a r}^{1}\left(\mathbb{R}^{n} \times \mathbb{R}\right)$ so that $\left.g\right|_{\mathbb{R}^{n} \times(-\infty, 0)}=f$. According to the atomic decomposition of the space $B_{-\alpha, p a r}^{1}\left(\mathbb{R}^{n} \times \mathbb{R}\right)$ (see Theorem 2.1.29),

$$
g=\sum_{j=0}^{\infty} \lambda_{j} a_{j}
$$

where the sequence $\left\{\lambda_{j}\right\}_{j \in \mathbb{N}_{0}} \in \ell^{1}$ and $a_{j}$ are $(1,-\alpha)$-atoms satisfying the following conditions:

- $\operatorname{supp} a_{j} \subset Q,|Q|=r^{n+2} \leq 1$,

- $\left\|a_{j}\right\|_{L^{\infty}\left(\mathbb{R}^{n} \times \mathbb{R}\right)} \leq r^{-\alpha-(n+2)}$,

- $\int_{\mathbb{R}^{n} \times \mathbb{R}} a_{j}(x, t) d x d t=0$. 
Also, note that

$$
\operatorname{ext} f=\sum_{j=0}^{\infty} \lambda_{j} \operatorname{ext}\left(\left.a_{j}\right|_{\mathbb{R}^{n} \times(-\infty, 0)}\right) .
$$

The claim we make is that $\operatorname{ext}\left(\left.a_{j}\right|_{\mathbb{R}^{n} \times(-\infty, 0)}\right)$ is a fixed constant multiple of a $(1,-\alpha)$ atom. In order to see this, we analyze three different situations. When $a_{j}$ is supported in $\mathbb{R}^{n} \times[0, \infty)$, then, obviously, $\operatorname{ext}\left(\left.a_{j}\right|_{\mathbb{R}^{n} \times(-\infty, 0)}\right)=0$ is a $(1,-\alpha)$-atom. If $\operatorname{supp} a_{j}$ is in $\mathbb{R}^{n} \times(-\infty, 0]$, then $\left.a_{j}\right|_{\mathbb{R}^{n} \times(-\infty, 0)}=a_{j}$, and, for $(x, t) \in \mathbb{R}^{n} \times \mathbb{R}$,

$$
\left(\operatorname{ext} a_{j}\right)(x, t)=\left\{\begin{array}{cc}
a_{j}(x, t), & \text { if } t<0 \\
-a_{j}(x,-t), & \text { if } t>0
\end{array}\right.
$$

Therefore, $\operatorname{supp}\left(\operatorname{ext} a_{j}\right) \subset 2 Q$,

$$
\left\|\operatorname{ext} a_{j}\right\|_{L^{\infty}\left(\mathbb{R}^{n} \times \mathbb{R}\right)} \leq 2\left\|a_{j}\right\|_{L^{\infty}\left(\mathbb{R}^{n} \times \mathbb{R}\right)} \leq 2 r^{-\alpha-(n+2)}
$$

and

$$
\int_{\mathbb{R}^{n} \times \mathbb{R}} \operatorname{ext} a_{j}(x, t) d x d t=\int_{\mathbb{R}^{n} \times(-\infty, 0)} a_{j}(x, t) d x d t-\int_{\mathbb{R}^{n} \times(0, \infty)} a_{j}(x,-t) d x d t=0 .
$$

Hence, in this case, $\operatorname{ext}\left(\left.a_{j}\right|_{\mathbb{R}^{n} \times(-\infty, 0)}\right)$ is a fixed constant multiple of a $(1,-\alpha)$-atom. Finally, when the support of $a_{j}$ intersects the plane $\mathbb{R}^{n} \times\{t=0\}$, we denote $\left.a_{j}\right|_{\mathbb{R}^{n} \times(-\infty, 0)}$ by $b_{j}$. Then $\operatorname{supp}\left(\operatorname{ext} b_{j}\right) \subset 2 Q$,

$$
\left\|\operatorname{ext} b_{j}\right\|_{L^{\infty}\left(\mathbb{R}^{n} \times \mathbb{R}\right)} \leq 2\left\|b_{j}\right\|_{L^{\infty}\left(\mathbb{R}^{n} \times \mathbb{R}\right)} \leq 2\left\|a_{j}\right\|_{L^{\infty}\left(\mathbb{R}^{n} \times \mathbb{R}\right)} \leq 2 r^{-\alpha-(n+2)}
$$

and

$$
\int_{\mathbb{R}^{n} \times \mathbb{R}} \operatorname{ext} b_{j}(x, t) d x d t=\int_{\mathbb{R}^{n} \times(-\infty, 0)} b_{j}(x, t) d x d t-\int_{\mathbb{R}^{n} \times(0, \infty)} b_{j}(x,-t) d x d t=0 .
$$

Therefore, in this scenario (and, as we have seen above, in the other two cases, as well) $\operatorname{ext}\left(\left.a_{j}\right|_{\mathbb{R}^{n} \times(-\infty, 0)}\right)$ is a fixed constant multiple of a $(1,-\alpha)$-atom. 
Consequently, for $f \in B_{-\alpha, p a r}^{1}\left(\mathbb{R}^{n} \times(-\infty, 0)\right)$, we have ext $f \in B_{-\alpha, p a r}^{1}\left(\mathbb{R}^{n} \times \mathbb{R}\right)$, which is what we wanted to show in Step 1.

Step 2. Definition of $P$.

Let us consider the operator $P$ defined by

$$
P:=\left.\left(I-\operatorname{ext} \circ R_{\mathbb{R}^{n} \times(-\infty, 0)}\right)\right|_{\mathbb{R}^{n} \times(-\infty, T)} .
$$

For $g \in B_{-\alpha, p a r}^{1}\left(\mathbb{R}^{n} \times \mathbb{R}\right)$ we have

$$
\begin{gathered}
g-\left.\operatorname{ext} \circ R\right|_{\mathbb{R}^{n} \times(-\infty, 0)} g \in B_{-\alpha, p a r}^{1}\left(\mathbb{R}^{n} \times \mathbb{R}\right), \\
\operatorname{supp}\left(g-\left.\operatorname{ext} \circ R\right|_{\mathbb{R}^{n} \times(-\infty, 0)} g\right) \subseteq \mathbb{R}^{n} \times[0, \infty),
\end{gathered}
$$

therefore, by definition,

$$
P g=\left.\left(g-\left.\operatorname{ext} \circ R\right|_{\mathbb{R}^{n} \times(-\infty, 0)} g\right)\right|_{\mathbb{R}^{n} \times(-\infty, T)} \in{ }_{0} B_{-\alpha, p a r}^{1}\left(\mathbb{R}^{n} \times(0, T)\right) \text {. }
$$

Consequently, $P: B_{-\alpha, p a r}^{1}\left(\mathbb{R}^{n} \times \mathbb{R}\right) \rightarrow{ }_{0} B_{-\alpha, p a r}^{1}\left(\mathbb{R}^{n} \times(0, T)\right)$ is well-defined and continuous.

Step 3. Definition of the right inverse of $P$.

We introduce the operator Ext given by

$$
\text { Ext } f:=\psi_{1} F+\psi_{0} E f
$$

where $\psi_{0}, \psi_{1} \in C^{\infty}(\mathbb{R})$ with $\psi_{0}+\psi_{1}=1$ and such that

$$
\begin{gathered}
\psi_{0} \equiv 1 \quad \text { on } \quad[T, \infty), \quad \operatorname{supp} \psi_{0} \subseteq[0, \infty), \\
\psi_{1} \equiv 1 \quad \text { on } \quad(-\infty, 0], \quad \operatorname{supp} \psi_{1} \subseteq(-\infty, T] .
\end{gathered}
$$


For $(x, t) \in \mathbb{R}^{n} \times \mathbb{R}$, the operator $E$ is defined by

$$
E f(x, t):=\left\{\begin{array}{cl}
f(x, t), & \text { if } t<T, \\
-f(x,-t), & \text { if } t>T,
\end{array}\right.
$$

and $F$ is given in the following way: for $f \in{ }_{0} B_{-\alpha, p a r}^{1}\left(\mathbb{R}^{n} \times(0, T)\right)$, by definition, there exists $F$ from $B_{-\alpha, p a r}^{1}\left(\mathbb{R}^{n} \times \mathbb{R}\right)$ with $\operatorname{supp} F \subseteq \mathbb{R}^{n} \times[0, \infty)$ and such that $\left.F\right|_{\mathbb{R}^{n} \times(-\infty, T)}=f$

Following the idea in Step 1, we can easily show that the operator $E$ maps $B_{-\alpha, p a r}^{1}\left(\mathbb{R}^{n} \times(-\infty, T)\right)$ linearly and boundedly into $B_{-\alpha, p a r}^{1}\left(\mathbb{R}^{n} \times \mathbb{R}\right)$. Using also the fact that ${ }_{0} B_{-\alpha, p a r}^{1}\left(\mathbb{R}^{n} \times(0, T)\right) \hookrightarrow B_{-\alpha, p a r}^{1}\left(\mathbb{R}^{n} \times(-\infty, T)\right)$, we obtain that

$$
\text { Ext : }{ }_{0} B_{-\alpha, p a r}^{1}\left(\mathbb{R}^{n} \times(0, T)\right) \longrightarrow B_{-\alpha, p a r}^{1}\left(\mathbb{R}^{n} \times \mathbb{R}\right)
$$

is bounded. By the definition of the operator Ext we also have supp(Ext $f) \subseteq$ $\mathbb{R}^{n} \times[0, \infty)$ and $\left.($ Ext $f)\right|_{\mathbb{R}^{n} \times(-\infty, T)}=f$

To show that the definition of Ext is independent of the choice of $F$, we pick $F$ and $F^{\prime}$ from $B_{-\alpha, p a r}^{1}\left(\mathbb{R}^{n} \times \mathbb{R}\right)$ so that $\operatorname{supp} F \subseteq \mathbb{R}^{n} \times[0, \infty), \operatorname{supp} F^{\prime} \subseteq \mathbb{R}^{n} \times[0, \infty)$, and $\left.F\right|_{\mathbb{R}^{n} \times(-\infty, T)}=f=\left.F^{\prime}\right|_{\mathbb{R}^{n} \times(-\infty, T)}$. Then $\psi_{1}\left(F-F^{\prime}\right) \equiv 0$ for almost every $t \in \mathbb{R}$, hence, the definition of the operator Ext is independent of the particular choice if $F$. Therefore, for given $f, g \in{ }_{0} B_{-\alpha, p a r}^{1}\left(\mathbb{R}^{n} \times(0, T)\right)$ with extensions $F$ and $G$, respectively, from $B_{-\alpha, p a r}^{1}\left(\mathbb{R}^{n} \times \mathbb{R}\right)$, we can choose $F+G$ to be the extension of $f+g$. Consequently, Ext is linear.

In order to prove that Ext is the right inverse of $P$, we observe that for $f$ in ${ }_{0} B_{-\alpha, p a r}^{1}\left(\mathbb{R}^{n} \times(0, T)\right)$, there holds

$$
(P \circ \operatorname{Ext}) f=\left.(\text { Ext } f)\right|_{\mathbb{R}^{n} \times(-\infty, T)}=f,
$$


i.e. $P \circ$ Ext is the identity operator on ${ }_{0} B_{-\alpha, p a r}^{1}\left(\mathbb{R}^{n} \times(0, T)\right)$. This concludes Step 3 .

Steps 1, 2 and 3 for the parabolic Hardy scale (instead of the Besov scale) can be carried out in a very similar fashion. Also, according to Theorem 2.5.1, for indices $\frac{n+2}{n+3}<p<1$ and $\alpha=(n+2)\left(\frac{1}{p}-1\right)$, and for $0<T<\infty$, we have

$$
\mathcal{E}_{1}\left(H_{\text {par }}^{p}\left(\mathbb{R}^{n} \times \mathbb{R}\right)\right)=B_{-\alpha, p a r}^{1}\left(\mathbb{R}^{n} \times \mathbb{R}\right) .
$$

Now we can apply Lemma 1.2.52 to conclude that for indices $\frac{n+2}{n+3}<p<1$, $\alpha=(n+2)\left(\frac{1}{p}-1\right)$, and for $0<T<\infty$, we have

$$
\mathcal{E}_{1}\left({ }_{0} H_{p a r}^{p}\left(\mathbb{R}^{n} \times(0, T)\right)\right)={ }_{0} B_{-\alpha, p a r}^{1}\left(\mathbb{R}^{n} \times(0, T)\right) .
$$

This finishes the proof of the theorem.

As a consequence of Theorem 3.3.1 we obtain the following.

Corollary 3.3.2. For any Lipschitz domain $\Omega$ in $\mathbb{R}^{n}, 0<T<\infty$ and fixed $p$ and $\alpha$ such that $\frac{n+1}{n+2}<p<1$ and $\alpha=(n+1)\left(\frac{1}{p}-1\right)$ there holds

$$
\mathcal{E}_{1}\left({ }_{0} H_{p a r}^{p}(\partial \Omega \times(0, T))\right)={ }_{0} B_{-\alpha, p a r}^{1}(\partial \Omega \times(0, T)) .
$$

Proof. The case when $\Omega$ is a graph domain immediately follows from Theorem 3.3.1, therefore we assume that $\Omega$ is a bounded domain. The idea is to apply Lemma 1.2.52.

Recall from Remark 1.2.1 that, for a bounded Lipschitz domain $\Omega \subset \mathbb{R}^{n}$, there exist $V_{i}(i=1, \ldots, N, N$ finite $)$ open, bounded subsets of $\mathbb{R}^{n}$ such that $\partial \Omega \subseteq \cup_{i=1}^{N} V_{i}$, and there are, in some coordinate system, Lipschitz graphs $\partial \Omega_{i}$ with $V_{i} \cap \partial \Omega \subset \subset$ $\partial \Omega_{i} \cap \partial \Omega$. Also, there exists a finite partition of unity $\psi_{i} \in C_{c}^{\infty}\left(V_{i}\right)\left(\sum_{i=1}^{N} \psi_{i}=1\right)$ on $\partial \Omega$ such that $\operatorname{supp} \psi_{i} \subseteq V_{i}$. 
We define the operators $\mathcal{P}$ and $\mathcal{R}$ by

$$
\mathcal{P}\left(\left\{f_{i}\right\}_{i=1}^{N}\right):=\sum_{i=1}^{N} \widetilde{\psi_{i} f_{i}} \partial \Omega, \quad f_{i} \in \mathrm{S}^{\prime}\left(\mathbb{R}^{n-1} \times \mathbb{R}\right), i=1, \ldots, N
$$

and

$$
\mathcal{R} f:=\left\{{\widetilde{\xi_{i} f}}^{\partial \Omega_{i}}\right\}_{i=1}^{N}, \quad f \in \mathrm{S}^{\prime}\left(\mathbb{R}^{n-1} \times \mathbb{R}\right)
$$

where $\xi_{i} \in C_{c}^{\infty}\left(\mathbb{R}^{n-1}\right)$, supp $\psi_{i} \subset \operatorname{supp} \xi_{i} \subseteq V_{i}, \xi_{i} \equiv 1$ on the support of $\psi_{i}$, and the symbols $\sim \partial \Omega, \sim \partial \Omega_{i}$ stand for extensions by 0 on $\partial \Omega$ and $\partial \Omega_{i}$, respectively. In particular,

$$
\begin{aligned}
& \mathcal{P}: \bigoplus_{i=1}^{N}{ }_{0} H_{\text {par }}^{p}\left(\partial \Omega_{i} \times(0, T)\right) \longrightarrow{ }_{0} H_{\text {par }}^{p}(\partial \Omega \times(0, T)) \\
& \mathcal{P}: \bigoplus_{i=1}^{N}{ }_{0} B_{-\alpha, p a r}^{1}\left(\partial \Omega_{i} \times(0, T)\right) \longrightarrow{ }_{0} B_{-\alpha, p a r}^{1}(\partial \Omega \times(0, T))
\end{aligned}
$$

and

$$
\begin{aligned}
& \mathcal{R}:{ }_{0} H_{\text {par }}^{p}(\partial \Omega \times(0, T)) \longrightarrow \bigoplus_{i=1}^{N}{ }_{0} H_{\text {par }}^{p}\left(\partial \Omega_{i} \times(0, T)\right), \\
& \mathcal{R}:{ }_{0} B_{-\alpha, p a r}^{1}(\partial \Omega \times(0, T)) \longrightarrow \bigoplus_{i=1}^{N}{ }_{0} B_{-\alpha, p a r}^{1}\left(\partial \Omega_{i} \times(0, T)\right)
\end{aligned}
$$

are bounded. Moreover, for $f \in \mathrm{S}^{\prime}\left(\mathbb{R}^{n-1} \times \mathbb{R}\right)$,

$$
(\mathcal{P} \circ \mathcal{R})(f)=\mathcal{P}\left(\left\{{\widetilde{\xi_{i} f}}^{\partial \Omega_{i}}\right\}_{i=1}^{N}\right)=\sum_{i=1}^{N}{\widetilde{\psi_{i} \tilde{\xi}_{i} \partial \Omega_{i}}}^{\partial \Omega}=f \sum_{i=1}^{N} \psi_{i}=f
$$

hence $\mathcal{R}$ is the right-inverse of $\mathcal{P}$. In order to show that

$$
\bigoplus_{i=1}^{N}{ }_{0} H_{p a r}^{p}\left(\partial \Omega_{i} \times(0, T)\right) \hookrightarrow \bigoplus_{i=1}^{N}{ }_{0} B_{-\alpha, p a r}^{1}\left(\partial \Omega_{i} \times(0, T)\right)
$$

is well-defined, continuous and dense, it suffices to show that

$$
{ }_{0} H_{\text {par }}^{p}\left(\mathbb{R}^{n-1} \times(0, T)\right) \hookrightarrow{ }_{0} B_{-\alpha, p a r}^{1}\left(\mathbb{R}^{n-1} \times(0, T)\right)
$$


is well-defined, continuous and dense. First, let $f \in{ }_{0} H_{p a r}^{p}\left(\mathbb{R}^{n-1} \times(0, T)\right)$. Then, by definition, there exists an $F \in H_{\text {par }}^{p}\left(\mathbb{R}^{n-1} \times \mathbb{R}\right)$ such that $f=\left.F\right|_{\mathbb{R}^{n-1} \times(0, T)}$ and $\operatorname{supp} F \subseteq \mathbb{R}^{n-1} \times[0, \infty)$. Due to the continuity of the inclusion in (3.3.45), we have that $F \in B_{-\alpha, p a r}^{1}\left(\mathbb{R}^{n-1} \times \mathbb{R}\right)$, hence, by definition, $f \in{ }_{0} B_{-\alpha, p a r}^{1}\left(\mathbb{R}^{n-1} \times(0, T)\right)$. The density of the inclusion in (3.3.47) follows from Remark 1.2.53 and the proof of Theorem 3.3.1. We now apply Lemma 1.2.52 with

$$
\begin{gathered}
X_{1}=\bigoplus_{i=1}^{N}{ }_{0} H_{p a r}^{p}\left(\partial \Omega_{i} \times(0, T)\right), \quad Y_{1}=\bigoplus_{i=1}^{N}{ }_{0} B_{-\alpha, p a r}^{1}\left(\partial \Omega_{i} \times(0, T)\right), \\
X_{2}={ }_{0} H_{p a r}^{p}(\partial \Omega \times(0, T)), \quad Y_{2}={ }_{0} B_{-\alpha, p a r}^{1}(\partial \Omega \times(0, T)) .
\end{gathered}
$$

Using the fact that the present corollary holds true for the graph domains $\Omega_{i}, i=$ $1, \ldots, N$, the conclusion of Lemma 1.2 .52 follows. This proves the corollary for bounded domains $\Omega$.

Our next result is the proper analogue of Theorem 3.3.1, this time involving spaces that have an additional unit of smoothness.

Theorem 3.3.3. Consider the indices $\frac{n+2}{n+3}<p<1$ and $\alpha=(n+2)\left(\frac{1}{p}-1\right)$, and let $0<T<\infty$. Then the following holds:

$$
\mathcal{E}_{1}\left({ }_{0} H_{\text {par }}^{1, p}\left(\mathbb{R}^{n} \times(0, T)\right)\right)={ }_{0} B_{1-\alpha, p a r}^{1}\left(\mathbb{R}^{n} \times(0, T)\right) .
$$

Proof. The general idea is to apply Lemma 1.2 .52 with

$$
\begin{gathered}
X_{1}=H_{\text {par }}^{1, p}\left(\mathbb{R}^{n} \times \mathbb{R}\right), \quad Y_{1}=B_{1-\alpha, \text { par }}^{1}\left(\mathbb{R}^{n} \times \mathbb{R}\right), \\
X_{2}={ }_{0} H_{\text {par }}^{1, p}\left(\mathbb{R}^{n} \times(0, T)\right), \quad Y_{2}={ }_{0} B_{1-\alpha, \text { par }}^{1}\left(\mathbb{R}^{n} \times(0, T)\right) .
\end{gathered}
$$


For the range of indices we consider, the inclusion

$$
H_{\text {par }}^{1, p}\left(\mathbb{R}^{n} \times \mathbb{R}\right) \hookrightarrow B_{1-\alpha, p a r}^{1}\left(\mathbb{R}^{n} \times \mathbb{R}\right)
$$

is well-defined, continuous and dense. Indeed, according to Theorem 2.1.17 with $p_{0}=p, q_{0}=2, \alpha=1$ and $p_{1}=q_{1}=1$, the inclusion in (3.3.48) is continuous. Next, recall from $(2.1 .5)$ and $(2.1 .6)$ that $\mathrm{S}\left(\mathbb{R}^{n} \times \mathbb{R}\right) \hookrightarrow B_{1-\alpha, p a r}^{1}\left(\mathbb{R}^{n} \times \mathbb{R}\right)$ densly, and that $\mathrm{S}\left(\mathbb{R}^{n} \times \mathbb{R}\right) \hookrightarrow H_{\text {par }}^{1, p}\left(\mathbb{R}^{n} \times \mathbb{R}\right)$. Consequently, the inclusion in (3.3.48) is dense, as well.

We shall define the operators $P$ and $R$ from Lemma 1.2.52 in a few of steps, starting with

Step 1. Definition of an extension operator

$$
\text { ext }: H_{\text {par }}^{1, p}\left(\mathbb{R}^{n} \times(-\infty, 0)\right) \longrightarrow H_{\text {par }}^{1, p}\left(\mathbb{R}^{n} \times \mathbb{R}\right) \text {. }
$$

For $(x, t) \in \mathbb{R}^{n} \times \mathbb{R}$ let us introduce

$$
(\operatorname{ext} f)(x, t):=\left\{\begin{array}{cl}
f(x, t), & \text { if } t<0 \\
-f(x,-t), & \text { if } t>0
\end{array}\right.
$$

and choose $f \in H_{\text {par }}^{1, p}\left(\mathbb{R}^{n} \times(-\infty, 0)\right)$. Then, by definition, there exists $g$ from $H_{\text {par }}^{1, p}\left(\mathbb{R}^{n} \times \mathbb{R}\right)$ such that $\left.g\right|_{\mathbb{R}^{n} \times(-\infty, 0)}=f$. According to the atomic decomposition of the space $H_{\text {par }}^{1, p}\left(\mathbb{R}^{n} \times \mathbb{R}\right)$ (cf. Definition 2.1.53 and Remark 2.1.55),

$$
g=\sum_{|Q| \leq 1} s_{Q} a_{Q}
$$

where $\left\{s_{Q}\right\}_{Q} \in \ell^{p}$ and $a_{Q}$ are local (inhomogeneous) (1,p)-atoms satisfying

$$
\operatorname{supp} a_{Q} \subset Q, \quad|Q| \leq 1, \quad \text { and } \quad\left\|a_{Q}\right\|_{L_{1, p a r}^{2}\left(\mathbb{R}^{n} \times \mathbb{R}\right)} \leq|Q|^{1 / 2-1 / p}
$$


Also, note that

$$
\operatorname{ext} f=\sum_{|Q| \leq 1} s_{Q} \operatorname{ext}\left(\left.a_{Q}\right|_{\mathbb{R}^{n} \times(-\infty, 0)}\right) .
$$

In order to show that ext $f \in H_{\text {par }}^{1, p}\left(\mathbb{R}^{n} \times \mathbb{R}\right)$, we have to differentiate three cases. If $\operatorname{supp} a_{Q} \subset \mathbb{R}^{n} \times[0, \infty)$, then, naturally, $\operatorname{ext}\left(\left.a_{Q}\right|_{\mathbb{R}^{n} \times(-\infty, 0)}\right)=0$ is a local $(1, p)$-atom. When $\operatorname{supp} a_{Q} \subset \mathbb{R}^{n} \times(-\infty, 0]$, then $\left.a_{Q}\right|_{\mathbb{R}^{n} \times(-\infty, 0)}=a_{Q}$, and, for $(x, t) \in \mathbb{R}^{n} \times \mathbb{R}$,

$$
\left(\operatorname{ext} a_{Q}\right)(x, t)=\left\{\begin{array}{cc}
a_{Q}(x, t), & \text { if } t<0 \\
-a_{Q}(x,-t), & \text { if } t>0
\end{array}\right.
$$

Therefore, $\operatorname{supp}\left(\operatorname{ext} a_{Q}\right) \subset 2 Q$. Next recall from Theorem 2.1.41 a characterization of the Sobolev space $L_{1, \text { par }}^{2}\left(\mathbb{R}^{n} \times \mathbb{R}\right)$, i.e.

$$
L_{1, \text { par }}^{2}\left(\mathbb{R}^{n} \times \mathbb{R}\right)=\left\{f \in L^{2}\left(\mathbb{R}^{n} \times \mathbb{R}\right): \forall i=1, \ldots, n, \partial_{i} f, \partial_{\text {time }}^{1 / 2} f \in L^{2}\left(\mathbb{R}^{n} \times \mathbb{R}\right)\right\}
$$

Based on (3.3.49) - (3.3.51) we obtain the following:

$$
\begin{aligned}
& \left\|\operatorname{ext} a_{Q}\right\|_{L^{2}\left(\mathbb{R}^{n} \times \mathbb{R}\right)} \leq 2\left\|a_{Q}\right\|_{L^{2}\left(\mathbb{R}^{n} \times \mathbb{R}\right)} \leq 2|Q|^{1 / 2-1 / p} \\
& \left\|\nabla\left(\operatorname{ext} a_{Q}\right)\right\|_{L^{2}\left(\mathbb{R}^{n} \times \mathbb{R}\right)} \leq 2\left\|\nabla a_{Q}\right\|_{L^{2}\left(\mathbb{R}^{n} \times \mathbb{R}\right)} \leq 2|Q|^{1 / 2-1 / p}, \\
& \left\|\partial_{\text {time }}^{1 / 2}\left(\operatorname{ext} a_{Q}\right)\right\|_{L^{2}\left(\mathbb{R}^{n} \times \mathbb{R}\right)} \leq 2\left\|\partial_{\text {time }}^{1 / 2} a_{Q}\right\|_{L^{2}\left(\mathbb{R}^{n} \times \mathbb{R}\right)} \leq 2|Q|^{1 / 2-1 / p} .
\end{aligned}
$$

In view of (3.3.51) this further implies that

$$
\left\|\operatorname{ext} a_{Q}\right\|_{\dot{L}_{1, p a r}^{2}\left(\mathbb{R}^{n} \times \mathbb{R}\right)} \leq\left\|\operatorname{ext} a_{Q}\right\|_{L_{1, p a r}^{2}\left(\mathbb{R}^{n} \times \mathbb{R}\right)} \leq c|Q|^{1 / 2-1 / p}
$$

therefore, in this case, ext $a_{Q}$ is a fixed constant multiple of a local $(1, p)$-atom for $H_{\text {par }}^{1, p}\left(\mathbb{R}^{n} \times \mathbb{R}\right)$. When the support of $a_{Q}$ intersects the plane $\mathbb{R}^{n} \times\{t=0\}$, we denote 
$\left.a_{Q}\right|_{\mathbb{R}^{n} \times(-\infty, 0)}$ by $b_{Q}$. Then $\operatorname{supp}\left(\operatorname{ext} b_{Q}\right) \subset 2 Q$ and

$$
\begin{aligned}
& \left\|\operatorname{ext} b_{Q}\right\|_{L^{2}\left(\mathbb{R}^{n} \times \mathbb{R}\right)} \leq 2\left\|b_{Q}\right\|_{L^{2}\left(\mathbb{R}^{n} \times \mathbb{R}\right)} \leq 2\left\|a_{Q}\right\|_{L^{2}\left(\mathbb{R}^{n} \times \mathbb{R}\right)} \leq 2|Q|^{1 / 2-1 / p} \\
& \left\|\nabla\left(\operatorname{ext} b_{Q}\right)\right\|_{L^{2}\left(\mathbb{R}^{n} \times \mathbb{R}\right)} \leq 2\left\|\nabla b_{Q}\right\|_{L^{2}\left(\mathbb{R}^{n} \times \mathbb{R}\right)} \leq 2\left\|\nabla a_{Q}\right\|_{L^{2}\left(\mathbb{R}^{n} \times \mathbb{R}\right)} \leq 2|Q|^{1 / 2-1 / p} \\
& \left\|\partial_{\text {time }}^{1 / 2}\left(\operatorname{ext} b_{Q}\right)\right\|_{L^{2}\left(\mathbb{R}^{n} \times \mathbb{R}\right)} \leq 2\left\|\partial_{\text {time }}^{1 / 2} b_{Q}\right\|_{L^{2}\left(\mathbb{R}^{n} \times \mathbb{R}\right)} \leq 2\left\|\partial_{\text {time }}^{1 / 2} a_{Q}\right\|_{L^{2}\left(\mathbb{R}^{n} \times \mathbb{R}\right)} \leq 2|Q|^{1 / 2-1 / p} .
\end{aligned}
$$

Now (3.3.53) and (3.3.51) imply that

$$
\left\|\operatorname{ext} b_{Q}\right\|_{\dot{L}_{1, p a r}^{2}\left(\mathbb{R}^{n} \times \mathbb{R}\right)} \leq\left\|\operatorname{ext} b_{Q}\right\|_{L_{1, p a r}^{2}\left(\mathbb{R}^{n} \times \mathbb{R}\right)} \leq c|Q|^{1 / 2-1 / p},
$$

hence, in this scenario, ext $b_{Q}$ is a fixed constant multiple of a local $(1, p)$-atom for $H_{\text {par }}^{1, p}\left(\mathbb{R}^{n} \times \mathbb{R}\right)$.

Consequently, ext $f \in H_{\text {par }}^{1, p}\left(\mathbb{R}^{n} \times \mathbb{R}\right)$ for $f \in H_{\text {par }}^{1, p}\left(\mathbb{R}^{n} \times(-\infty, 0)\right)$, which completes the proof of Step 1.

In a very similar fashion we can show that ext $f$ belongs to $B_{1-\alpha, p a r}^{1}\left(\mathbb{R}^{n} \times \mathbb{R}\right)$ for $f \in B_{1-\alpha, p a r}^{1}\left(\mathbb{R}^{n} \times(-\infty, 0)\right)$, using the atomic decomposition of the parabolic Besov space $B_{1-\alpha, p a r}^{1}\left(\mathbb{R}^{n} \times \mathbb{R}\right)$.

Steps 2, 3 can be carried out exactly as in the proof of Theorem 3.3.1, for both the Hardy and Besov scales.

Now using Lemma 1.2.52 and the fact that, for the current range of indices,

$$
\mathcal{E}_{1}\left(H_{\text {par }}^{1, p}\left(\mathbb{R}^{n} \times \mathbb{R}\right)\right)=B_{1-\alpha, p a r}^{1}\left(\mathbb{R}^{n} \times \mathbb{R}\right),
$$

(cf. (2.5.232) with $p^{*}=1$ ) we may conclude that

$$
\mathcal{E}_{1}\left({ }_{0} H_{\text {par }}^{1, p}\left(\mathbb{R}^{n} \times(0, T)\right)\right)={ }_{0} B_{1-\alpha, p a r}^{1}\left(\mathbb{R}^{n} \times(0, T)\right) .
$$


This finishes the proof of Theorem 3.3.3.

As a consequence of Theorem 3.3.3, in a similar fashion as Corollary 3.3.2, we obtain the following.

Corollary 3.3.4. For any Lipschitz domain $\Omega$ in $\mathbb{R}^{n}, 0<T<\infty$ and fixed $p$ and $\alpha$ such that $\frac{n+1}{n+2}<p<1$ and $\alpha=(n+1)\left(\frac{1}{p}-1\right)$ the following holds:

$$
\mathcal{E}_{1}\left({ }_{0} H_{\text {par }}^{1, p}(\partial \Omega \times(0, T))\right)={ }_{0} B_{1-\alpha, p a r}^{1}(\partial \Omega \times(0, T))
$$

\subsection{Interpolation scales of spaces with built-in ini- tial conditions}

In this section we collect all the real and complex interpolation results involving spaces with built-in initial conditions, that are needed in our further discussion in Chapters $6-8$. Recall that $(\cdot, \cdot)_{\theta, p}$ and $[\cdot, \cdot]_{\theta}$ denote the real and complex interpolation methods, respectively. These methods were described in 1.2.7.

Lemma 3.4.1. Let $\Omega$ be a Lipschitz domain in $\mathbb{R}^{n}, 0<T<\infty$ and $\frac{n+1}{n+2}<p \leq 1$. Then ${ }_{0} H_{p a r}^{p}(\partial \Omega \times(0, T))$ is a complex interpolation scale, i.e. for $\frac{n+1}{n+2}<p_{0}, p_{1} \leq 1$,

$$
\left[{ }_{0} H_{\text {par }}^{p_{0}}(\partial \Omega \times(0, T)),{ }_{0} H_{\text {par }}^{p_{1}}(\partial \Omega \times(0, T))\right]_{\theta}={ }_{0} H_{\text {par }}^{p}(\partial \Omega \times(0, T)),
$$

with equivalent quasi-norms, where $0<\theta<1$ and $\frac{1}{p}=\frac{1-\theta}{p_{0}}+\frac{\theta}{p_{1}}$.

The same is true for the homogeneous scale ${ }_{0} \dot{H}_{\text {par }}^{p}(\partial \Omega \times(0, T))$.

Proof. Here we present the proof of (3.4.54) only, since the homogeneous scale is treated in a similar fashion. 
Case (i) $\Omega$ is unbounded. Due to a bi-Lipschitz change of variables, it is enough to show that, for $p_{0}$ and $p_{1}$ such that $\frac{n+2}{n+3}<p_{0}, p_{1} \leq 1$,

$$
\left[{ }_{0} H_{\text {par }}^{p_{0}}\left(\mathbb{R}^{n} \times(0, T)\right),{ }_{0} H_{\text {par }}^{p_{1}}\left(\mathbb{R}^{n} \times(0, T)\right)\right]_{\theta}={ }_{0} H_{\text {par }}^{p}\left(\mathbb{R}^{n} \times(0, T)\right),
$$

where $0<\theta<1$ and $\frac{1}{p}=\frac{1-\theta}{p_{0}}+\frac{\theta}{p_{1}}$. In order to prove (3.4.55), we proceed in two stages, starting with

Step I. Show that for the appropriate parameters

$$
\left[H_{\text {par }, 0}^{p_{0}}\left(\mathbb{R}^{n} \times(0, \infty)\right), H_{\text {par }, 0}^{p_{1}}\left(\mathbb{R}^{n} \times(0, \infty)\right)\right]_{\theta}=H_{\text {par }, 0}^{p}\left(\mathbb{R}^{n} \times(0, \infty)\right),
$$

where, by definition,

$$
H_{\text {par }, 0}^{p}\left(\mathbb{R}^{n} \times(0, \infty)\right):=\left\{f \in H_{\text {par }}^{p}\left(\mathbb{R}^{n} \times \mathbb{R}\right): \operatorname{supp} f \subseteq \mathbb{R}^{n} \times[0, \infty)\right\}
$$

We apply part (ii) of Lemma 1.2 .35 with $X=Y, X^{p_{i}}=H_{p a r}^{p_{i}}\left(\mathbb{R}^{n} \times \mathbb{R}\right), i=0,1$, $D_{2}=\mathbb{R}^{n} \times[0, \infty)$, and with

$$
E_{D_{2}^{c}}=E: H_{\text {par }}^{p_{i}}\left(\mathbb{R}^{n} \times(-\infty, 0)\right) \longrightarrow H_{\text {par }}^{p_{i}}\left(\mathbb{R}^{n} \times \mathbb{R}\right)
$$

an extension operator defined by

$$
(E f)(x, t):=\left\{\begin{aligned}
f(x, t), & \text { if } t<0 \\
-f(x,-t), & \text { if } t>0
\end{aligned}\right.
$$

for $(x, t) \in \mathbb{R}^{n} \times \mathbb{R}$. To show that $E f \in H_{\text {par }}^{p}\left(\mathbb{R}^{n} \times \mathbb{R}\right)$ for $f \in H_{\text {par }}^{p}\left(\mathbb{R}^{n} \times(-\infty, 0)\right)$ and $p \in\left(\frac{n+2}{n+3}, 1\right]$, first note that, by definition, there exists $F$ from $H_{p a r}^{p}\left(\mathbb{R}^{n} \times \mathbb{R}\right)$ such that $\left.F\right|_{\mathbb{R}^{n} \times(-\infty, 0)}=f$. According to the atomic decomposition of the parabolic Hardy space $H_{\text {par }}^{p}\left(\mathbb{R}^{n} \times \mathbb{R}\right)$

$$
F=\sum_{j=0}^{\infty} \lambda_{j} a_{j}
$$

where $\left\{\lambda_{j}\right\}_{j} \in \ell^{p}$ and $a_{j}$ 's are inhomogeneous (local) p-atoms satisfying 
- $\operatorname{supp} a_{j} \subset Q, \quad|Q|=r^{n+2}$,

- $\left\|a_{j}\right\|_{L^{\infty}\left(\mathbb{R}^{n} \times \mathbb{R}\right)} \leq r^{-\frac{n+2}{p}}$,

- either $|Q|<1$ and $\int_{\mathbb{R}^{n} \times \mathbb{R}} a_{j}(x, t) d x d t=0$, or $|Q|=1$.

Here $Q$ is a parabolic cube in $\mathbb{R}^{n} \times \mathbb{R}$ (see Definition 2.1.44 and Theorem 2.1.46).

We can easily see that

$$
E f=\sum_{j=0}^{\infty} \lambda_{j} E\left(\left.a_{j}\right|_{\mathbb{R}^{n} \times(-\infty, 0)}\right) .
$$

Our next goal is to show that $E\left(\left.a_{j}\right|_{\mathbb{R}^{n} \times(-\infty, 0)}\right)$ is a fixed constant multiple of an inhomogeneous (local) p-atom for $H_{\text {par }}^{p}\left(\mathbb{R}^{n} \times \mathbb{R}\right)$. We have three different situations. If $\operatorname{supp} a_{j} \subset \mathbb{R}^{n} \times[0, \infty)$, then, evidently, $E\left(\left.a_{j}\right|_{\mathbb{R}^{n} \times(-\infty, 0)}\right)=0$ is an inhomogeneous (local) p-atom. When $\operatorname{supp} a_{j} \subset \mathbb{R}^{n} \times(-\infty, 0]$ (but supp $a_{j}$ does not intersect the plane $\left.\mathbb{R}^{n} \times\{t=0\}\right)$, then $\left.a_{j}\right|_{\mathbb{R}^{n} \times(-\infty, 0)}=a_{j}$, and, for $(x, t) \in \mathbb{R}^{n} \times \mathbb{R}$,

$$
\left(E a_{j}\right)(x, t)=\left\{\begin{aligned}
a_{j}(x, t), & \text { if } t<0 \\
-a_{j}(x,-t), & \text { if } t>0
\end{aligned}\right.
$$

Then $\operatorname{supp}\left(E a_{j}\right) \subset 2 Q$, and

$$
\left\|E a_{j}\right\|_{L^{\infty}\left(\mathbb{R}^{n} \times \mathbb{R}\right)} \leq 2\left\|a_{j}\right\|_{L^{\infty}\left(\mathbb{R}^{n} \times \mathbb{R}\right)} \leq 2 r^{-\frac{n+2}{p}}
$$

If $|2 Q|=1$, then, at this stage, we may conclude that $E\left(\left.a_{j}\right|_{\mathbb{R}^{n} \times(-\infty, 0)}\right)$ is a fixed constant multiple of an inhomogeneous (local) p-atom for $H_{p a r}^{p}\left(\mathbb{R}^{n} \times \mathbb{R}\right)$. If $|2 Q|<1$, we have to make sure that the vanishing moment condition holds. To this end, note that

$$
\int_{\mathbb{R}^{n} \times \mathbb{R}} E a_{j}(x, t) d x d t=\int_{\mathbb{R}^{n} \times(-\infty, 0)} a_{j}(x, t) d x d t-\int_{\mathbb{R}^{n} \times(0, \infty)} a_{j}(x,-t) d x d t=0,
$$


where we have used the change of variables $t=-s$ for $t \in(0, \infty)$. Therefore, in this scenario, $E\left(\left.a_{j}\right|_{\mathbb{R}^{n} \times(-\infty, 0)}\right)$ is a fixed constant multiple of an inhomogeneous (local) $p$-atom for $H_{p a r}^{p}\left(\mathbb{R}^{n} \times \mathbb{R}\right)$. Finally, when the support of $a_{j}$ inersects the plane $\mathbb{R}^{n} \times\{t=0\}$, we let $\left.a_{j}\right|_{\mathbb{R}^{n} \times(-\infty, 0)}=: b_{j}$. Then, $\operatorname{supp}\left(E b_{j}\right) \subset 2 Q$, and

$$
\left\|E b_{j}\right\|_{L^{\infty}\left(\mathbb{R}^{n} \times \mathbb{R}\right)} \leq 2\left\|b_{j}\right\|_{L^{\infty}\left(\mathbb{R}^{n} \times \mathbb{R}\right)} \leq 2\left\|a_{j}\right\|_{L^{\infty}\left(\mathbb{R}^{n} \times \mathbb{R}\right)} \leq 2 r^{-\frac{n+2}{p}}
$$

If $|2 Q|=1$, then we may conclude that $E\left(a_{j}{\mid \mathbb{R}^{n} \times(-\infty, 0)}\right)$ is a fixed constant multiple of an inhomogeneous (local) $p$-atom for $H_{p a r}^{p}\left(\mathbb{R}^{n} \times \mathbb{R}\right)$. If $|2 Q|<1$, we have to ensure that the vanishing moment condition holds. One can easily see that

$$
\int_{\mathbb{R}^{n} \times \mathbb{R}} E b_{j}(x, t) d x d t=\int_{\mathbb{R}^{n} \times(-\infty, 0)} b_{j}(x, t) d x d t-\int_{\mathbb{R}^{n} \times(0, \infty)} b_{j}(x,-t) d x d t=0,
$$

where we have used the change of variables $t=-s$ for $t \in(0, \infty)$. Therefore, in this case (and, as we have seen above, in the other cases, as well) $E\left(\left.a_{j}\right|_{\mathbb{R}^{n} \times(-\infty, 0)}\right)$ is a fixed constant multiple of an inhomogeneous (local) p-atom for $H_{p a r}^{p}\left(\mathbb{R}^{n} \times \mathbb{R}\right)$.

Consequently, $E f \in H_{\text {par }}^{p}\left(\mathbb{R}^{n} \times \mathbb{R}\right)$ for $f \in H_{\text {par }}^{p}\left(\mathbb{R}^{n} \times(-\infty, 0)\right)$, which is what we wanted to show.

Going further, granted that $\left[H_{\text {par }}^{p_{0}}\left(\mathbb{R}^{n} \times \mathbb{R}\right), H_{\text {par }}^{p_{1}}\left(\mathbb{R}^{n} \times \mathbb{R}\right)\right]_{\theta}=H_{\text {par }}^{p}\left(\mathbb{R}^{n} \times \mathbb{R}\right)$ for indices $\frac{n+2}{n+3}<p_{0}, p_{1} \leq 1,0<\theta<1$ and $\frac{1}{p}=\frac{1-\theta}{p_{0}}+\frac{\theta}{p_{1}}$ (Theorem 2.1.60, $(\mathrm{j})$ ), the conclusion of part (ii) of Lemma 1.2.35 holds, i.e. we have proved the claim of Step I. Step II. Show (3.4.55).

We apply Lemma 1.2.35, this time part (iii), with $X=Y, D_{1}=\mathbb{R}^{n} \times(0, T)$, and $X_{D_{2}, *}^{p_{i}}=H_{\text {par }, 0}^{p_{i}}\left(\mathbb{R}^{n} \times(0, \infty)\right), i=0,1$. In view of Step I and the fact that, based on 
the definitions of the spaces involved,

$$
{ }_{0} H_{p a r}^{p}\left(\mathbb{R}^{n} \times(0, T)\right)=\left\{\left.f\right|_{\mathbb{R}^{n} \times(0, T)}: f \in H_{p a r, 0}^{p}\left(\mathbb{R}^{n} \times(0, \infty)\right)\right\},
$$

in order to prove (3.4.55), it suffices to show that there exists a universal extension operator

$$
\tilde{E}:{ }_{0} H_{p a r}^{p}\left(\mathbb{R}^{n} \times(0, T)\right) \longrightarrow H_{p a r, 0}^{p}\left(\mathbb{R}^{n} \times(0, \infty)\right)
$$

To construct $\tilde{E}$, we first consider $f$ from ${ }_{0} H_{p a r}^{p}\left(\mathbb{R}^{n} \times(0, T)\right)$. Then there exists a function $F \in H_{\text {par }}^{p}\left(\mathbb{R}^{n} \times \mathbb{R}\right)$ such that $\operatorname{supp} F \subseteq \mathbb{R}^{n} \times[0, \infty)$ and $f=\left.F\right|_{\mathbb{R}^{n} \times(0, T)}$. Let us introduce $\psi_{0}, \psi_{1} \in C^{\infty}(\mathbb{R})$ with $\psi_{0}+\psi_{1}=1$, so that

$$
\begin{aligned}
& \psi_{0} \equiv 1 \text { on }[T, \infty), \quad \text { supp } \psi_{0} \subseteq[0, \infty), \\
& \psi_{1} \equiv 1 \text { on }(-\infty, 0], \quad \operatorname{supp} \psi_{1} \subseteq(-\infty, T] .
\end{aligned}
$$

Now we define $\tilde{E} f:=\psi_{1} F+\psi_{0} \mathrm{E} f$, where $\mathrm{E}$ is the extension operator introduced as follows. For $(x, t) \in \mathbb{R}^{n} \times \mathbb{R}$,

$$
(\mathrm{E} f)(x, t):=\left\{\begin{aligned}
& f(x, t), \quad \text { if } t<T, \\
&-f(x, 2 T-t), \text { if } t>T .
\end{aligned}\right.
$$

Strictly speaking we need

$$
\mathrm{E}:{ }_{0} H_{p a r}^{p}\left(\mathbb{R}^{n} \times(0, T)\right) \longrightarrow H_{p a r}^{p}\left(\mathbb{R}^{n} \times \mathbb{R}\right)
$$

to be bounded, but since ${ }_{0} H_{\text {par }}^{p}\left(\mathbb{R}^{n} \times(0, T)\right) \hookrightarrow H_{\text {par }}^{p}\left(\mathbb{R}^{n} \times(-\infty, T)\right)$, it is enough to prove that

$$
\mathrm{E}: H_{p a r}^{p}\left(\mathbb{R}^{n} \times(-\infty, T)\right) \longrightarrow H_{p a r}^{p}\left(\mathbb{R}^{n} \times \mathbb{R}\right)
$$


is bounded. To show that $\mathrm{E} f \in H_{\text {par }}^{p}\left(\mathbb{R}^{n} \times \mathbb{R}\right)$ for $f \in H_{\text {par }}^{p}\left(\mathbb{R}^{n} \times(-\infty, T)\right)$ and $p \in\left(\frac{n+2}{n+3}, 1\right]$, first note that, by definition, there exists $F$ from $H_{p a r}^{p}\left(\mathbb{R}^{n} \times \mathbb{R}\right)$ such that $\left.F\right|_{\mathbb{R}^{n} \times(-\infty, T)}=f$. According to the atomic decomposition of the parabolic Hardy space $H_{\text {par }}^{p}\left(\mathbb{R}^{n} \times \mathbb{R}\right)$,

$$
F=\sum_{j=0}^{\infty} \lambda_{j} a_{j}
$$

where $\left\{\lambda_{j}\right\}_{j} \in \ell^{p}$ and $a_{j}$ 's are inhomogeneous (local) p-atoms satisfying

- $\operatorname{supp} a_{j} \subset Q, \quad|Q|=r^{n+2}$,

- $\left\|a_{j}\right\|_{L^{\infty}\left(\mathbb{R}^{n} \times \mathbb{R}\right)} \leq r^{-\frac{n+2}{p}}$,

- either $|Q|<1$ and $\int_{\mathbb{R}^{n} \times \mathbb{R}} a_{j}(x, t) d x d t=0$, or $|Q|=1$.

Here $Q$ is a parabolic cube in $\mathbb{R}^{n} \times \mathbb{R}$ (see Definition 2.1.44 and Theorem 2.1.46). Then

$$
\mathrm{E} f=\sum_{j=0}^{\infty} \lambda_{j} \mathrm{E}\left(\left.a_{j}\right|_{\mathbb{R}^{n} \times(-\infty, T)}\right) .
$$

The claim that we make is that $\mathrm{E}\left(\left.a_{j}\right|_{\mathbb{R}^{n} \times(-\infty, T)}\right)$ is a fixed constant multiple of an inhomogeneous (local) p-atom for $H_{p a r}^{p}\left(\mathbb{R}^{n} \times \mathbb{R}\right)$. There are three different scenarios. If $\operatorname{supp} a_{j} \subset \mathbb{R}^{n} \times[T, \infty)$, then, evidently, $\mathrm{E}\left(\left.a_{j}\right|_{\mathbb{R}^{n} \times(-\infty, T)}\right)=0$ is an inhomogeneous (local) p-atom. When $\operatorname{supp} a_{j} \subset \mathbb{R}^{n} \times(-\infty, T]$ (but the support of $a_{j}$ does not intersect the plane $\left.\mathbb{R}^{n} \times\{t=T\}\right)$, then $\left.a_{j}\right|_{\mathbb{R}^{n} \times(-\infty, T)}=a_{j}$, and, for $(x, t) \in \mathbb{R}^{n} \times \mathbb{R}$,

$$
\left(\mathrm{E} a_{j}\right)(x, t)=\left\{\begin{array}{c}
a_{j}(x, t), \quad \text { if } t<T \\
-a_{j}(x, 2 T-t), \text { if } t>T
\end{array}\right.
$$

Since the transformation $t \mapsto 2 T-t$ amounts to a reflection about the line $t=T$, 
$\operatorname{supp} \mathrm{E} a_{j} \subset 2 Q$, and

$$
\left\|\mathrm{E} a_{j}\right\|_{L^{\infty}\left(\mathbb{R}^{n} \times \mathbb{R}\right)} \leq 2\left\|a_{j}\right\|_{L^{\infty}\left(\mathbb{R}^{n} \times \mathbb{R}\right)} \leq 2 r^{-\frac{n+2}{p}}
$$

If $|2 Q|=1$, then, at this stage, we may conclude that $\mathrm{E}\left(\left.a_{j}\right|_{\mathbb{R}^{n} \times(-\infty, T)}\right)$ is a fixed constant multiple of an inhomogeneous (local) $p$-atom for $H_{p a r}^{p}\left(\mathbb{R}^{n} \times \mathbb{R}\right)$. If $|2 Q|<1$, we have to ensure that the vanishing moment condition holds. To this end, note that

$$
\int_{\mathbb{R}^{n} \times \mathbb{R}} \mathrm{E} a_{j}(x, t) d x d t=\int_{\mathbb{R}^{n} \times(-\infty, T)} a_{j}(x, t) d x d t-\int_{\mathbb{R}^{n} \times(T, \infty)} a_{j}(x, 2 T-t) d x d t=0,
$$

where we have used the change of variables $2 T-t=s$ for $t \in(T, \infty)$. Therefore, in this case, $\mathrm{E}\left(\left.a_{j}\right|_{\mathbb{R}^{n} \times(-\infty, T)}\right)$ is a fixed constant multiple of an inhomogeneous (local) $p$ atom for $H_{\text {par }}^{p}\left(\mathbb{R}^{n} \times \mathbb{R}\right)$. Finally, if the support of $a_{j}$ inersects the plane $\mathbb{R}^{n} \times\{t=T\}$, we let $\left.a_{j}\right|_{\mathbb{R}^{n} \times(-\infty, T)}=: b_{j}$. Then, $\operatorname{supp}\left(\mathrm{E} b_{j}\right) \subset 2 Q$, and

$$
\left\|\mathrm{E} b_{j}\right\|_{L^{\infty}\left(\mathbb{R}^{n} \times \mathbb{R}\right)} \leq 2\left\|b_{j}\right\|_{L^{\infty}\left(\mathbb{R}^{n} \times \mathbb{R}\right)} \leq 2\left\|a_{j}\right\|_{L^{\infty}\left(\mathbb{R}^{n} \times \mathbb{R}\right)} \leq 2 r^{-\frac{n+2}{p}}
$$

If $|2 Q|=1$, then we may conclude that $\mathrm{E}\left(a_{j}{\mid \mathbb{R}^{n} \times(-\infty, T)}\right)$ is a fixed constant multiple of an inhomogeneous (local) $p$-atom for $H_{p a r}^{p}\left(\mathbb{R}^{n} \times \mathbb{R}\right)$. If $|2 Q|<1$, we have to make sure that the vanishing moment condition holds. It is easy to see that

$$
\int_{\mathbb{R}^{n} \times \mathbb{R}} \mathrm{E} b_{j}(x, t) d x d t=\int_{\mathbb{R}^{n} \times(-\infty, T)} b_{j}(x, t) d x d t-\int_{\mathbb{R}^{n} \times(T, \infty)} b_{j}(x, 2 T-t) d x d t=0,
$$

where we have used the change of variables $2 T-t=s$ for $t \in(T, \infty)$. Therefore, in this scenario (as well as in the other ones above) $\mathrm{E}\left(\left.a_{j}\right|_{\mathbb{R}^{n} \times(-\infty, T)}\right)$ is a fixed constant multiple of an inhomogeneous (local) $p$-atom for $H_{\text {par }}^{p}\left(\mathbb{R}^{n} \times \mathbb{R}\right)$. This proves the claim we made earlier in Step II. 
Consequently, $\mathrm{E} f \in H_{\text {par }}^{p}\left(\mathbb{R}^{n} \times \mathbb{R}\right)$, which is what we wanted to show. Also, note that $\left.\mathrm{E} f\right|_{\mathbb{R}^{n} \times(0, T)}=f \in{ }_{0} H_{p a r}^{p}\left(\mathbb{R}^{n} \times(0, T)\right)$.

Going further, we turn out attention to the operator $\tilde{E}$. From the definition of $\tilde{E}$ one can easily see that $\tilde{E} f \in H_{p a r}^{p}\left(\mathbb{R}^{n} \times \mathbb{R}\right)$ and supp $\tilde{E} f \subseteq \mathbb{R}^{n} \times[0, \infty)$. Hence

$$
\tilde{E} f \in H_{p a r, 0}^{p}\left(\mathbb{R}^{n} \times(0, \infty)\right) .
$$

Moreover, $\left.\tilde{E} f\right|_{\mathbb{R}^{n} \times(0, T)}=\left(\psi_{0}+\psi_{1}\right) f=f \in{ }_{0} H_{p a r}^{p}\left(\mathbb{R}^{n} \times(0, T)\right)$, as desired.

In order to show that the definition of $\tilde{E}$ is independent of the choice of $F$, we pick $F$ and $F^{\prime}$ from $H_{\text {par }}^{p}\left(\mathbb{R}^{n} \times \mathbb{R}\right)$ such that $\operatorname{supp} F \subseteq \mathbb{R}^{n} \times[0, \infty)$, supp $F^{\prime} \subseteq \mathbb{R}^{n} \times[0, \infty)$, and $\left.F\right|_{\mathbb{R}^{n} \times(0, T)}=f=\left.F^{\prime}\right|_{\mathbb{R}^{n} \times(0, T)}$. Then we can see that $\psi_{1}\left(F-F^{\prime}\right) \equiv 0$ for a.e. $t \in \mathbb{R}$. This shows that the definition of $\tilde{E}$ is independent of the particular choice of $F$. Hence, for given $f, g \in{ }_{0} H_{p a r}^{p}\left(\mathbb{R}^{n} \times(0, T)\right)$ with extensions $F$ and $G$, respectively, from $H_{p a r}^{p}\left(\mathbb{R}^{n} \times \mathbb{R}\right)$, we can choose $F+G$ to be the extension of $f+g$. Consequently, $\tilde{E}$ is linear. This concludes Step II and the proof of (3.4.55).

Case (ii) $\Omega$ is bounded. This can be carried out in a similar fashion to the proof of part (d) of Theorem 2.3.20 for a bounded domain.

Prior to our next interpolation theorem, we present an independent result, needed in Theorems 3.4.3, 3.4.4 and 2.3.20.

Proposition 3.4.2. Let $1<p<\infty, 0 \leq \alpha \leq 1$ and I an interval in $\mathbb{R}$. Then there exists a linear extension operator

$$
E: L_{\alpha, p a r}^{p}\left(\mathbb{R}^{n} \times I\right) \longrightarrow L_{\alpha, p a r}^{p}\left(\mathbb{R}^{n} \times \mathbb{R}\right) .
$$


Proof. Let us consider $f \in L_{\alpha, p a r}^{p}\left(\mathbb{R}^{n} \times I\right)$ and, for $x \in \mathbb{R}^{n}, t \in \mathbb{R}$, we define

$$
(E f)(x, t):=\mathcal{E}[f(x, \cdot)](t),
$$

where $\mathcal{E}$ is Stein's extension operator. More specifically, $\mathcal{E}$ is introduced as follows. Based on pp. $181-182$ of [Ste1], there exists an infinitely differentiable function $\delta^{*}(t)$ defined on $\mathbb{R} \backslash \bar{I}$ such that $\delta^{*}(t) \approx \operatorname{dist}(t, \bar{I})$, and

$$
\left|\frac{d^{\beta}}{d t^{\beta}} \delta^{*}(t)\right| \leq c_{\beta}(\operatorname{dist}(t, \bar{I}))^{1-\beta} \quad \text { for every } \quad \beta \in \mathbb{N}_{0} .
$$

Also, there exists a continuous function $\psi$ defined on $[1, \infty)$ such that $\psi(\lambda)=O\left(\lambda^{-N}\right)$ as $\lambda \rightarrow \infty$ for every $N$ ( $\psi$ is rapidly decreasing at $\infty$ ), and

$$
\int_{1}^{\infty} \psi(\lambda) d \lambda=1, \quad \int_{1}^{\infty} \lambda^{k} \psi(\lambda) d \lambda=0 \quad \text { for } \quad k=1,2, \ldots
$$

Then the extension operator $\mathcal{E}$ is given by

$$
\mathcal{E} u(t):= \begin{cases}\int_{1}^{\infty} u\left(t+\lambda \delta^{*}(t)\right) \psi(\lambda) d \lambda, & \text { if } t \in \mathbb{R} \backslash \bar{I}, \\ u(t), & \text { if } t \in I .\end{cases}
$$

Let us invoke Theorem 5 on p. 181 in [Ste1] to the effect that for all $k \in \mathbb{N}_{0}$ and all $p$ with $1 \leq p \leq \infty$, the operator

$$
\mathcal{E}: L_{k}^{p}(I) \rightarrow L_{k}^{p}(\mathbb{R}) \quad \text { is linear and bounded. }
$$

As is well-known in the theory of complex interpolation of isotropic Sobolev spaces (cf., e.g., p. 317 in [Tri2] or Proposition 2.4 on p. 170 in [JeKe]), for any $k \in \mathbb{N}_{0}$ and $1<p<\infty$,

$$
\left[L_{k}^{p}(I), L_{k+1}^{p}(I)\right]_{\theta}=L_{k+\theta}^{p}(I), \quad 0<\theta<1,
$$


and (cf. part (7) of Theorem 6.4.5 in [BeLö]), for the same range of indices as above,

$$
\left[L_{k}^{p}(\mathbb{R}), L_{k+1}^{p}(\mathbb{R})\right]_{\theta}=L_{k+\theta}^{p}(\mathbb{R}), \quad 0<\theta<1
$$

Now (3.4.59)-(3.4.61) further imply that, for $1<p<\infty$ and $\alpha \geq 0$,

$$
\mathcal{E}: L_{\alpha}^{p}(I) \longrightarrow L_{\alpha}^{p}(\mathbb{R}) \text { is bounded. }
$$

In our particular case we take $u:=f(x, \cdot)$, where $x \in \mathbb{R}^{n}$. Then for $(x, t) \in \mathbb{R}^{n} \times \mathbb{R}$,

$$
\mathcal{E}[f(x, \cdot)](t)= \begin{cases}\int_{1}^{\infty} f\left(x, t+\lambda \delta^{*}(t)\right) \psi(\lambda) d \lambda, & \text { if } t \in \mathbb{R} \backslash \bar{I}, \\ f(x, t), & \text { if } t \in I .\end{cases}
$$

and, for any $1 \leq j \leq n$, when $t \in \mathbb{R} \backslash \bar{I}$,

$$
\begin{aligned}
\partial_{x_{j}} \mathcal{E}[f(x, \cdot)](t) & =\int_{1}^{\infty} \partial_{x_{j}} f\left(x, t+\lambda \delta^{*}(t)\right) \psi(\lambda) d \lambda \\
& =\mathcal{E}\left[\partial_{x_{j}} f(x, \cdot)\right](t) .
\end{aligned}
$$

Hence $\partial_{x_{j}}(\mathcal{E} f)=\mathcal{E}\left(\partial_{x_{j}} f\right)$ for any $1 \leq j \leq n$.

We next claim that, for $1<p<\infty$,

$$
E: L^{p}\left(\mathbb{R}^{n} \times I\right) \longrightarrow L^{p}\left(\mathbb{R}^{n} \times \mathbb{R}\right)
$$

is bounded. Indeed, if we consider $f \in L^{p}\left(\mathbb{R}^{n} \times I\right)$, then

$$
\begin{aligned}
\|E f\|_{L^{p}\left(\mathbb{R}^{n} \times \mathbb{R}\right)}^{p} & =\int_{\mathbb{R}^{n}} \int_{\mathbb{R}}|\mathcal{E}[f(x, \cdot)](t)|^{p} d t d x \\
& \leq c \int_{\mathbb{R}^{n}} \int_{I}|f(x, t)|^{p} d t d x \\
& =c\|f\|_{L^{p}\left(\mathbb{R}^{n} \times I\right)}^{p} .
\end{aligned}
$$

As a next step, we shall prove that, for $1<p<\infty$, E maps $L_{1, p a r}^{p}\left(\mathbb{R}^{n} \times I\right)$ boundedly into $L_{1, p a r}^{p}\left(\mathbb{R}^{n} \times \mathbb{R}\right)$. To do so, let $f \in L_{1, p a r}^{p}\left(\mathbb{R}^{n} \times I\right)$. Then, according to 
the Fubini property of $L_{1, \text { par }}^{p}\left(\mathbb{R}^{n} \times \mathbb{R}\right)$ (see Proposition 2.1.33),

$$
\begin{aligned}
\|E f\|_{L_{1, p a r}^{p}\left(\mathbb{R}^{n} \times \mathbb{R}\right)}^{p} & \approx \int_{\mathbb{R}^{n}}\|(E f)(x, \cdot)\|_{L_{1 / 2}^{p}(\mathbb{R})}^{p} d x+\int_{\mathbb{R}}\|(E f)(\cdot, t)\|_{L_{1}^{p}\left(\mathbb{R}^{n}\right)}^{p} d t \\
& =: I+I I .
\end{aligned}
$$

The Fubini property of $L_{1, p a r}^{p}\left(\mathbb{R}^{n} \times I\right)$ (cf. Proposition 2.2.2), in concert with (3.4.62) with $\alpha=1 / 2$, imply that

$$
\begin{aligned}
I & =\int_{\mathbb{R}^{n}}\|(\mathcal{E} f)(x, \cdot)\|_{L_{1 / 2}^{p}(\mathbb{R})}^{p} d x \leq c \int_{\mathbb{R}^{n}}\|f(x, \cdot)\|_{L_{1 / 2}^{p}(I)}^{p} d x \\
& \leq c\|f\|_{L_{1, p a r}^{p}\left(\mathbb{R}^{n} \times I\right)}^{p} .
\end{aligned}
$$

Using (1.2.27) with $X=\mathbb{R},(3.4 .63)$ with $\alpha=0$ for $f$ and $\nabla f$, and the Fubini property of $L_{1, p a r}^{p}\left(\mathbb{R}^{n} \times I\right)$, we obtain that

$$
\begin{aligned}
I I & =\int_{\mathbb{R}}\|(\mathcal{E} f)(\cdot, t)\|_{L_{1}^{p}\left(\mathbb{R}^{n}\right)}^{p} d t \\
& \leq c \int_{\mathbb{R}}\|(\mathcal{E} f)(\cdot, t)\|_{L^{p}\left(\mathbb{R}^{n}\right)}^{p} d t+c \int_{\mathbb{R}^{\prime}}\|\nabla(\mathcal{E} f)(\cdot, t)\|_{L^{p}\left(\mathbb{R}^{n}\right)}^{p} d t \\
& \leq c \int_{I} \int_{\mathbb{R}^{n}}|f(x, t)|^{p} d x d t+c \int_{I} \int_{\mathbb{R}^{n}}|\nabla f(x, t)|^{p} d x d t \\
& \leq c \int_{I}\|f(\cdot, t)\|_{L_{1}^{p}\left(\mathbb{R}^{n}\right)}^{p} d t \\
& \leq c\|f\|_{L_{1, p a r}^{p}}^{p}\left(\mathbb{R}^{n} \times I\right)
\end{aligned}
$$

On account of $(3.4 .64)-(3.4 .66)$, the operator

$$
E: L_{1, p a r}^{p}\left(\mathbb{R}^{n} \times I\right) \longrightarrow L_{1, p a r}^{p}\left(\mathbb{R}^{n} \times \mathbb{R}\right)
$$

is bounded for all $1<p<\infty$. In view of (3.4.63), (3.4.67) and the fact that $E$ is a linear operator, complex interpolation of Sobolev spaces (see p. 88 of [Gru1] for the spaces defined on $\mathbb{R}^{n} \times I$, and part (h) of Theorem 2.1.60 for the target spaces) we 
obtain that, for all $1<p<\infty$ and $0 \leq \alpha \leq 1$, the operator $E$ introduced in (3.4.58) has the following property:

$$
E: L_{\alpha, p a r}^{p}\left(\mathbb{R}^{n} \times I\right) \longrightarrow L_{\alpha, p a r}^{p}\left(\mathbb{R}^{n} \times \mathbb{R}\right)
$$

is bounded.

We also note that $E$ defined in (3.4.58) is an extension operator, since for any function $f$ defined on $\mathbb{R}^{n} \times I$,

$$
\left.(E f)\right|_{\mathbb{R}^{n} \times I}=\left.(\mathcal{E} f)\right|_{\mathbb{R}^{n} \times I}=f .
$$

In view of (3.4.68) and (3.4.69) the proof of the proposition is complete.

We now are ready to present our next interpolation result, this time involving parabolic Sobolev spaces.

Theorem 3.4.3. Let $\Omega$ be a Lipschitz domain in $\mathbb{R}^{n}, 0<T<\infty$, and indices $1<p_{0}, p_{1}<\infty, 0 \leq \alpha_{0}, \alpha_{1} \leq 1$. Then

$$
\left[{ }_{0} L_{\alpha_{0}, p a r}^{p_{0}}(\partial \Omega \times(0, T)),{ }_{0} L_{\alpha_{1}, p a r}^{p_{1}}(\partial \Omega \times(0, T))\right]_{\theta}={ }_{0} L_{\alpha, p a r}^{p}(\partial \Omega \times(0, T)),
$$

with equivalent norms, where $0<\theta<1, \frac{1}{p}=\frac{1-\theta}{p_{0}}+\frac{\theta}{p_{1}}$ and $\alpha=(1-\theta) \alpha_{0}+\theta \alpha_{1}$.

Proof. Case (i) $\Omega$ is unbounded. Thanks to a bi-Lipschitz change of variables, it is enough to show that, for the appropriate indices,

$$
\left[{ }_{0} L_{\alpha_{0}, p a r}^{p_{0}}\left(\mathbb{R}^{n} \times(0, T)\right),{ }_{0} L_{\alpha_{1}, p a r}^{p_{1}}\left(\mathbb{R}^{n} \times(0, T)\right)\right]_{\theta}={ }_{0} L_{\alpha, p a r}^{p}\left(\mathbb{R}^{n} \times(0, T)\right) .
$$

Step I. Show that for the parameters as in the theorem,

$$
\left[L_{\alpha_{0}, p a r, 0}^{p_{0}}\left(\mathbb{R}^{n} \times(0, \infty)\right), L_{\alpha_{1}, p a r, 0}^{p_{1}}\left(\mathbb{R}^{n} \times(0, \infty)\right)\right]_{\theta}=L_{\alpha, p a r, 0}^{p}\left(\mathbb{R}^{n} \times(0, \infty)\right),
$$


where recall that

$$
L_{\alpha, p a r, 0}^{p}\left(\mathbb{R}^{n} \times(0, \infty)\right):=\left\{f \in L_{\alpha, p a r}^{p}\left(\mathbb{R}^{n} \times \mathbb{R}\right): \text { supp } f \subseteq \mathbb{R}^{n} \times[0, \infty)\right\}
$$

We apply part (ii) of Lemma 1.2.35 with $X=Y, X_{\alpha_{i}}^{p_{i}}=L_{\alpha_{i}, p a r}^{p_{i}}\left(\mathbb{R}^{n} \times \mathbb{R}\right), i=0,1$, $D_{2}=\mathbb{R}^{n} \times[0, \infty)$, and

$$
E_{D_{2}^{c}}=E: L_{\alpha_{i}, p a r}^{p_{i}}\left(\mathbb{R}^{n} \times(-\infty, 0)\right) \longrightarrow L_{\alpha_{i}, p a r}^{p_{i}}\left(\mathbb{R}^{n} \times \mathbb{R}\right),
$$

where $E$ is the extension operator introduced in Proposition 3.4.2 with $I=(-\infty, 0)$.

Granted that $\left[L_{\alpha_{0}, p a r}^{p_{0}}\left(\mathbb{R}^{n} \times \mathbb{R}\right), L_{\alpha_{1}, p a r}^{p_{1}}\left(\mathbb{R}^{n} \times \mathbb{R}\right)\right]_{\theta}=L_{\alpha, p a r}^{p}\left(\mathbb{R}^{n} \times \mathbb{R}\right)$ for indices $1<p_{0}, p_{1}<\infty, \alpha_{0}, \alpha_{1} \in \mathbb{R}, 0<\theta<1, \frac{1}{p}=\frac{1-\theta}{p_{0}}+\frac{\theta}{p_{1}}$ and $\alpha=(1-\theta) \alpha_{0}+\theta \alpha_{1}$ (cf. part (e) of Theorem 2.1.60), the conclusion of Lemma 1.2.35, part (ii), holds. This finishes the proof of Step I.

Step II. Show (3.4.70).

We apply Lemma 1.2.35, this time part (iii), with $X=Y, D_{1}=\mathbb{R}^{n} \times(0, T)$, $X_{\alpha_{i}, D_{2}, *}^{p_{i}}=L_{\alpha_{i}, p a r, 0}^{p_{i}}\left(\mathbb{R}^{n} \times(0, \infty)\right), i=0,1$. In view of Step I and the fact that, based on the definition of the spaces involved,

$$
{ }_{0} L_{\alpha, p a r}^{p}\left(\mathbb{R}^{n} \times(0, T)\right)=\left\{\left.f\right|_{\mathbb{R}^{n} \times(0, T)}: f \in L_{\alpha, p a r, 0}^{p}\left(\mathbb{R}^{n} \times(0, \infty)\right)\right\},
$$

in order to prove (3.4.70), it suffices to show that there exists a universal extension operator

$$
\tilde{E}:{ }_{0} L_{\alpha, p a r}^{p}\left(\mathbb{R}^{n} \times(0, T)\right) \longrightarrow L_{\alpha, p a r, 0}^{p}\left(\mathbb{R}^{n} \times(0, \infty)\right) .
$$

To construct $\tilde{E}$, we first let $f \in{ }_{0} L_{\alpha, p a r}^{p}\left(\mathbb{R}^{n} \times(0, T)\right)$. Then, by definition, there exists a function $F \in L_{\alpha, p a r}^{p}\left(\mathbb{R}^{n} \times \mathbb{R}\right)$ such that $\operatorname{supp} F \subseteq \mathbb{R}^{n} \times[0, \infty)$ and $f=\left.F\right|_{\mathbb{R}^{n} \times(0, T)}$. 
Let us introduce $\psi_{0}, \psi_{1} \in C^{\infty}(\mathbb{R})$ with $\psi_{0}+\psi_{1}=1$, so that

$$
\begin{aligned}
& \psi_{0} \equiv 1 \text { on }[T, \infty), \quad \operatorname{supp} \psi_{0} \subseteq[0, \infty), \\
& \psi_{1} \equiv 1 \text { on }(-\infty, 0], \quad \operatorname{supp} \psi_{1} \subseteq(-\infty, T] .
\end{aligned}
$$

We now define $\tilde{E} f:=\psi_{1} F+\psi_{0} E f$, where $E$ is the extension operator introduced in Proposition 3.4.2 with $I=(-\infty, T)$. Note that

$$
{ }_{0} L_{\alpha, p a r}^{p}\left(\mathbb{R}^{n} \times(0, T)\right) \hookrightarrow L_{\alpha, p a r}^{p}\left(\mathbb{R}^{n} \times(-\infty, T)\right)
$$

and $\left.E f\right|_{\mathbb{R}^{n} \times(0, T)}=f \in{ }_{0} L_{\alpha, p a r}^{p}\left(\mathbb{R}^{n} \times(0, T)\right)$. We can easily show that $\tilde{E} f$ belongs to $L_{\alpha, p a r}^{p}\left(\mathbb{R}^{n} \times \mathbb{R}\right)$ and supp $\tilde{E} f \subseteq \mathbb{R}^{n} \times[0, \infty)$, hence

$$
\tilde{E} f \in L_{\alpha, p a r, 0}^{p}\left(\mathbb{R}^{n} \times(0, \infty)\right) .
$$

Moreover, $\left.\tilde{E} f\right|_{\mathbb{R}^{n} \times(0, T)}=\left(\psi_{0}+\psi_{1}\right) f=f \in{ }_{0} L_{\alpha, p a r}^{p}\left(\mathbb{R}^{n} \times(0, T)\right)$, as desired.

To show that the definition of $\tilde{E}$ is independent of the choice of $F$, we choose $F$ and $F^{\prime}$ from $L_{\alpha, p a r}^{p}\left(\mathbb{R}^{n} \times \mathbb{R}\right)$ so that $\operatorname{supp} F \subseteq \mathbb{R}^{n} \times[0, \infty)$, supp $F^{\prime} \subseteq \mathbb{R}^{n} \times[0, \infty)$, and $\left.F\right|_{\mathbb{R}^{n} \times(0, T)}=f=\left.F^{\prime}\right|_{\mathbb{R}^{n} \times(0, T)}$. Then one can see that $\psi_{1}\left(F-F^{\prime}\right) \equiv 0$ for a.e. $t \in \mathbb{R}$, i.e. the definition of $\tilde{E}$ is independent of the particular choice of $F$. Hence, for given $f, g \in{ }_{0} L_{\alpha, p a r}^{p}\left(\mathbb{R}^{n} \times(0, T)\right)$ with extensions $F$ and $G$, respectively, from $L_{\alpha, p a r}^{p}\left(\mathbb{R}^{n} \times \mathbb{R}\right)$, we can choose $F+G$ to be the extension of $f+g$. Consequently, $\tilde{E}$ is linear. This concludes Step II and the proof of (3.4.70).

Case (ii) $\Omega$ is bounded. This can be carried out in a similar fashion to the proof of part (d) of Theorem 2.3.20 for a bounded domain.

The analogue of Theorem 3.4.3, with the complex interpolation method replaced by the real one, is as follows. 
Theorem 3.4.4. Let $\Omega$ be a Lipschitz domain in $\mathbb{R}^{n}, 0<T<\infty$, and indices $1<p<\infty, 0 \leq \alpha_{0}, \alpha_{1} \leq 1$ so that $\alpha_{0} \neq \alpha_{1}$. Then

$$
\left({ }_{0} L_{\alpha_{0}, p a r}^{p}(\partial \Omega \times(0, T)),{ }_{0} L_{\alpha_{1}, p a r}^{p}(\partial \Omega \times(0, T))\right)_{\theta, p}={ }_{0} B_{\alpha, p a r}^{p}(\partial \Omega \times(0, T)),
$$

with equivalent norms, where $0<\theta<1$ and $\alpha=(1-\theta) \alpha_{0}+\theta \alpha_{1}$.

Proof. Case (i) $\Omega$ is unbounded. In this scenario, in order to prove the theorem, it is enough to show (due to a bi-Lipschitz change of variables), that

$$
\left({ }_{0} L_{\alpha_{0}, p a r}^{p}\left(\mathbb{R}^{n} \times(0, T)\right),{ }_{0} L_{\alpha_{1}, p a r}^{p}\left(\mathbb{R}^{n} \times(0, T)\right)\right)_{\theta, p}={ }_{0} B_{\alpha, p a r}^{p}\left(\mathbb{R}^{n} \times(0, T)\right)
$$

for the appropriate range of indices.

Step I. Show that under the current assumptions on the indices,

$$
\left(L_{\alpha_{0}, p a r, 0}^{p}\left(\mathbb{R}^{n} \times(0, \infty)\right), L_{\alpha_{1}, p a r, 0}^{p}\left(\mathbb{R}^{n} \times(0, \infty)\right)\right)_{\theta, p}=B_{\alpha, p a r, 0}^{p}\left(\mathbb{R}^{n} \times(0, \infty)\right),
$$

where recall that

$$
L_{\alpha, p a r, 0}^{p}\left(\mathbb{R}^{n} \times(0, \infty)\right):=\left\{f \in L_{\alpha, p a r}^{p}\left(\mathbb{R}^{n} \times \mathbb{R}\right): \text { supp } f \subseteq \mathbb{R}^{n} \times[0, \infty)\right\}
$$

and

$$
B_{\alpha, p a r, 0}^{p}\left(\mathbb{R}^{n} \times(0, \infty)\right):=\left\{f \in B_{\alpha, p a r}^{p}\left(\mathbb{R}^{n} \times \mathbb{R}\right): \text { supp } f \subseteq \mathbb{R}^{n} \times[0, \infty)\right\}
$$

We apply part (ii) of Lemma 1.2.24 with $X_{\alpha_{i}}^{p}=L_{\alpha_{i}, p a r}^{p}\left(\mathbb{R}^{n} \times \mathbb{R}\right), i=0,1, Y_{\alpha}^{p}=$ $B_{\alpha, p a r}^{p}\left(\mathbb{R}^{n} \times \mathbb{R}\right), D_{2}=\mathbb{R}^{n} \times[0, \infty)$, and

$$
E_{D_{2}^{c}}=E: L_{\alpha_{i}, p a r}^{p}\left(\mathbb{R}^{n} \times(-\infty, 0)\right) \longrightarrow L_{\alpha_{i}, p a r}^{p}\left(\mathbb{R}^{n} \times \mathbb{R}\right)
$$

where $E$ is the extension operator introduced in Proposition 3.4 .2 with $I=(-\infty, 0)$. 
Granted that $\left(L_{\alpha_{0}, p a r}^{p}\left(\mathbb{R}^{n} \times \mathbb{R}\right), L_{\alpha_{1}, p a r}^{p}\left(\mathbb{R}^{n} \times \mathbb{R}\right)\right)_{\theta, p}=B_{\alpha, p a r}^{p}\left(\mathbb{R}^{n} \times \mathbb{R}\right)$ for indices $1<p<\infty, \alpha_{0}, \alpha_{1} \in \mathbb{R}, \alpha_{0} \neq \alpha_{1}, 0<\theta<1$, and $\alpha=(1-\theta) \alpha_{0}+\theta \alpha_{1}$ (cf. part (i) of Theorem 2.1.60), the conclusion of Lemma 1.2.24, part (ii), holds. This finishes the proof of the claim in Step I.

Step II. Show (3.4.71).

To this end, we invoke part (iii) of Lemma 1.2.24 with $D_{1}=\mathbb{R}^{n} \times(0, T), X_{\alpha_{i}, D_{2}, *}^{p}=$ $L_{\alpha_{i}, p a r, 0}^{p}\left(\mathbb{R}^{n} \times(0, \infty)\right), i=0,1$, and $Y_{\alpha, D_{2}, *}^{p}=B_{\alpha, p a r, 0}^{p}\left(\mathbb{R}^{n} \times(0, \infty)\right)$. Since

$$
{ }_{0} L_{\alpha, p a r}^{p}\left(\mathbb{R}^{n} \times(0, T)\right)=\left\{\left.f\right|_{\mathbb{R}^{n} \times(0, T)}: f \in L_{\alpha, p a r, 0}^{p}\left(\mathbb{R}^{n} \times(0, \infty)\right)\right\}
$$

and

$$
{ }_{0} B_{\alpha, p a r}^{p}\left(\mathbb{R}^{n} \times(0, T)\right)=\left\{\left.f\right|_{\mathbb{R}^{n} \times(0, T)}: f \in B_{\alpha, p a r, 0}^{p}\left(\mathbb{R}^{n} \times(0, \infty)\right)\right\},
$$

with Step I in hand, in order to prove (3.4.71), it suffices to show that there exists a universal extension operator

$$
\tilde{E}:{ }_{0} L_{\alpha, p a r}^{p}\left(\mathbb{R}^{n} \times(0, T)\right) \longrightarrow L_{\alpha, p a r, 0}^{p}\left(\mathbb{R}^{n} \times(0, \infty)\right) .
$$

We take $\tilde{E}$ to be the same operator as in the proof of Theorem 3.4.3. This concludes the proof of (3.4.71).

Case (ii) $\Omega$ is bounded. An argument similar to the one in the proof of Theorem 2.3.20, part (d) for a bounded domain, yields the desired conclusion.

We now turn our attention to the complex interpolation of parabolic Besov and Triebel-Lizorkin spaces. Since an extension operator applied to these spaces will be often used, we recall from Remark 2.2.7 the following. 
Remark 3.4.5. Let $\Omega$ be a Lipschitz domain in $\mathbb{R}^{n}, I \subseteq \mathbb{R}$ an interval. Then there exists a linear extension operator $E$ such that

$$
\|E f\|_{F_{\alpha, p a r}^{p, q}\left(\mathbb{R}^{n} \times \mathbb{R}\right)} \leq c\|f\|_{F_{\alpha, p a r}^{p, q}(\Omega \times I)},
$$

where $0<p, q<\infty$ and

$$
\alpha>\sigma_{p, q}:=\max \left\{0,(n+2)\left(\frac{1}{p}-1\right),(n+2)\left(\frac{1}{q}-1\right)\right\} .
$$

The same extension operator $E$ has the property that

$$
E: B_{\alpha, p a r}^{p, q}(\Omega \times I) \longrightarrow B_{\alpha, p a r}^{p, q}\left(\mathbb{R}^{n} \times \mathbb{R}\right)
$$

is bounded for $1 \leq p<\infty, 0<q \leq \infty$ and $\alpha>0$. For more details on $E$ the reader is referred to Remark 2.2.7.

Theorem 3.4.6. Consider a Lipschitz domain $\Omega \subset \mathbb{R}^{n}, 0<T<\infty$, and indices $1 \leq p_{0}, p_{1}<\infty, 1 \leq q_{0}, q_{1} \leq \infty, 0<\alpha_{0}, \alpha_{1}<1$. Then

$$
\left[{ }_{0} B_{\alpha_{0}, p a r}^{p_{0}, q_{0}}(\partial \Omega \times(0, T)),{ }_{0} B_{\alpha_{1}, p a r}^{p_{1}, q_{1}}(\partial \Omega \times(0, T))\right]_{\theta}={ }_{0} B_{\alpha, p a r}^{p, q}(\partial \Omega \times(0, T)),
$$

with equivalent norms, where $0<\theta<1, \frac{1}{p}=\frac{1-\theta}{p_{0}}+\frac{\theta}{p_{1}}, \frac{1}{q}=\frac{1-\theta}{q_{0}}+\frac{\theta}{q_{1}}$ and $\alpha=$ $(1-\theta) \alpha_{0}+\theta \alpha_{1}$

Proof. Case (i) $\Omega$ is unbounded. In order to prove the theorem in this scenario, it suffices to show that, for the appropriate indices

$$
\left[{ }_{0} B_{\alpha_{0}, p a r}^{p_{0}, q_{0}}\left(\mathbb{R}^{n} \times(0, T)\right),{ }_{0} B_{\alpha_{1}, p a r}^{p_{1}, q_{1}}\left(\mathbb{R}^{n} \times(0, T)\right)\right]_{\theta}={ }_{0} B_{\alpha, p a r}^{p, q}\left(\mathbb{R}^{n} \times(0, T)\right) .
$$

Step I. Show that for the parameters as in the theorem,

$$
\left[B_{\alpha_{0}, \text { par }, 0}^{p_{0}, q_{0}}\left(\mathbb{R}^{n} \times(0, \infty)\right), B_{\alpha_{1}, p a r, 0}^{p_{1}, q_{1}}\left(\mathbb{R}^{n} \times(0, \infty)\right)\right]_{\theta}=B_{\alpha, p a r, 0}^{p, q}\left(\mathbb{R}^{n} \times(0, \infty)\right),
$$


where recall that by definition

$$
B_{\alpha, p a r, 0}^{p, q}\left(\mathbb{R}^{n} \times(0, \infty)\right):=\left\{f \in B_{\alpha, p a r}^{p, q}\left(\mathbb{R}^{n} \times \mathbb{R}\right): \text { supp } f \subseteq \mathbb{R}^{n} \times[0, \infty)\right\}
$$

We first recall part (ii) of Lemma 1.2 .35 with $X=Y, X_{\alpha_{i}}^{p_{i}, q_{i}}=B_{\alpha_{i}, p a r}^{p_{i}, q_{i}}\left(\mathbb{R}^{n} \times \mathbb{R}\right)$, $i=0,1, D_{2}=\mathbb{R}^{n} \times[0, \infty)$, and

$$
E_{D_{2}^{c}}=E: B_{\alpha_{i}, p a r}^{p_{i}, q_{i}}\left(\mathbb{R}^{n} \times(-\infty, 0)\right) \longrightarrow B_{\alpha_{i}, p a r}^{p_{i}, q_{i}}\left(\mathbb{R}^{n} \times \mathbb{R}\right)
$$

where $E$ is the extension operator as in either Remark 3.4.5 or Remark 2.2.9. Second, granted that

$$
\left[B_{\alpha_{0}, p a r}^{p_{0}, q_{0}}\left(\mathbb{R}^{n} \times \mathbb{R}\right), B_{\alpha_{1}, p a r}^{p_{1}, q_{1}}\left(\mathbb{R}^{n} \times \mathbb{R}\right)\right]_{\theta}=B_{\alpha, \text { par }}^{p, q}\left(\mathbb{R}^{n} \times \mathbb{R}\right)
$$

for indices $0<p_{0}, p_{1}, q_{0}, q_{1} \leq \infty, \alpha_{0}, \alpha_{1} \in \mathbb{R}, 0<\theta<1, \frac{1}{p}=\frac{1-\theta}{p_{0}}+\frac{\theta}{p_{1}}, \frac{1}{q}=\frac{1-\theta}{q_{0}}+\frac{\theta}{q_{1}}$ and $\alpha=(1-\theta) \alpha_{0}+\theta \alpha_{1}$ (cf. Theorem 2.1.60, (f)), the conclusion of part (ii) of Lemma 1.2.35 holds. Therefore, the proof of the claim made in Step I is complete.

Step II. Show (3.4.72).

We apply part (iii) of Lemma 1.2 .35 with $X=Y, D_{1}=\mathbb{R}^{n} \times(0, T), X_{\alpha_{i}, D_{2}, *}^{p_{i}, q_{i}}=$ $B_{\alpha_{i}, p_{i}, 0}^{p_{i}, q_{i}}\left(\mathbb{R}^{n} \times(0, \infty)\right), i=0,1$. Considering also the result proved in Step I and the fact that, on account of definitions,

$$
{ }_{0} B_{\alpha, p a r}^{p, q}\left(\mathbb{R}^{n} \times(0, T)\right)=\left\{\left.f\right|_{\mathbb{R}^{n} \times(0, T)}: f \in B_{\alpha, p a r, 0}^{p, q}\left(\mathbb{R}^{n} \times(0, \infty)\right)\right\},
$$

in order to prove (3.4.72), it is enough to show that there exists a universal extension operator

$$
\tilde{E}:{ }_{0} B_{\alpha, p a r}^{p, q}\left(\mathbb{R}^{n} \times(0, T)\right) \longrightarrow B_{\alpha, p a r, 0}^{p, q}\left(\mathbb{R}^{n} \times(0, \infty)\right) .
$$


To construct $\tilde{E}$, we first let $f \in{ }_{0} B_{\alpha, p a r}^{p, q}\left(\mathbb{R}^{n} \times(0, T)\right)$. Then there exists a function $F \in B_{\alpha, p a r}^{p, q}\left(\mathbb{R}^{n} \times \mathbb{R}\right)$ such that $\operatorname{supp} F \subseteq \mathbb{R}^{n} \times[0, \infty)$ and $f=\left.F\right|_{\mathbb{R}^{n} \times(0, T)}$. Let us introduce $\psi_{0}, \psi_{1} \in C^{\infty}(\mathbb{R})$ with $\psi_{0}+\psi_{1}=1$, so that

$$
\begin{aligned}
& \psi_{0} \equiv 1 \text { on }[T, \infty), \quad \operatorname{supp} \psi_{0} \subseteq[0, \infty) \\
& \psi_{1} \equiv 1 \text { on }(-\infty, 0], \quad \operatorname{supp} \psi_{1} \subseteq(-\infty, T] .
\end{aligned}
$$

We then define $\tilde{E} f:=\psi_{1} F+\psi_{0} E f$, where $E$ is the extension operator from Remark 3.4.5. Note that $\tilde{E} f \in B_{\alpha, p a r}^{p, q}\left(\mathbb{R}^{n} \times \mathbb{R}\right)$ and $\operatorname{supp} \tilde{E} f \subseteq \mathbb{R}^{n} \times[0, \infty)$, hence

$$
\tilde{E} f \in B_{\alpha, p a r, 0}^{p, q}\left(\mathbb{R}^{n} \times(0, \infty)\right)
$$

One also has the following: ${ }_{0} B_{\alpha, p a r}^{p, q}\left(\mathbb{R}^{n} \times(0, T)\right) \hookrightarrow B_{\alpha, p a r}^{p, q}\left(\mathbb{R}^{n} \times(-\infty, T)\right)$, and $\left.E f\right|_{\mathbb{R}^{n} \times(0, T)}=f \in{ }_{0} B_{\alpha, p a r}^{p, q}\left(\mathbb{R}^{n} \times(0, T)\right)$. This further implies that $\left.\tilde{E} f\right|_{\mathbb{R}^{n} \times(0, T)}=$ $\left(\psi_{0}+\psi_{1}\right) f=f \in{ }_{0} B_{\alpha, p a r}^{p, q}\left(\mathbb{R}^{n} \times(0, T)\right)$.

To show that the definition of $\tilde{E}$ is independent of the choice of $F$, we pick $F$ and $F^{\prime}$ from $B_{\alpha, p a r}^{p, q}\left(\mathbb{R}^{n} \times \mathbb{R}\right)$ such that $\operatorname{supp} F \subseteq \mathbb{R}^{n} \times[0, \infty)$, supp $F^{\prime} \subseteq \mathbb{R}^{n} \times[0, \infty)$, and $\left.F\right|_{\mathbb{R}^{n} \times(0, T)}=f=\left.F^{\prime}\right|_{\mathbb{R}^{n} \times(0, T)}$. Then one can see that $\psi_{1}\left(F-F^{\prime}\right) \equiv 0$ for a.e. $t \in \mathbb{R}$, i.e. the definition of $\tilde{E}$ is independent of the particular choice of $F$. Hence, for given $f, g \in{ }_{0} B_{\alpha, p a r}^{p, q}\left(\mathbb{R}^{n} \times(0, T)\right)$ with extensions $F$ and $G$, respectively, from $B_{\alpha, p a r}^{p, q}\left(\mathbb{R}^{n} \times \mathbb{R}\right)$, one can choose $F+G$ to be the extension of $f+g$. Consequently, $\tilde{E}$ is linear. This concludes Step II and the proof of (3.4.72).

Case (ii) $\Omega$ is bounded. The same reasoning as in the proof of Theorem 2.3.20, part (d) for a bounded domain, yields the desired result, concluding the proof of Theorem 3.4.6. 
In a very similar fashion, using the complex interpolation of Triebel-Lizorkin spaces on the Euclidean setting (part (e) of Theorem 2.1.60) and the extension operator from Remark 3.4.5, we obtain the following.

Theorem 3.4.7. Consider a Lipschitz domain $\Omega \subset \mathbb{R}^{n}, 0<T<\infty$, and indices $0<p_{0}, p_{1}, q_{0}, q_{1}<\infty$ and

$$
\alpha_{i}>\sigma_{p_{i}, q_{i}}:=\max \left\{0,(n+1)\left(\frac{1}{p_{i}}-1\right),(n+1)\left(\frac{1}{q_{i}}-1\right)\right\}, \quad i=0,1 .
$$

Then

$$
\left[{ }_{0} F_{\alpha_{0}, p a r}^{p_{0}, q_{0}}(\partial \Omega \times(0, T)),{ }_{0} F_{\alpha_{1}, p a r}^{p_{1}, q_{1}}(\partial \Omega \times(0, T))\right]_{\theta}={ }_{0} F_{\alpha, p a r}^{p, q}(\partial \Omega \times(0, T)),
$$

with equivalent quasi-norms, where $0<\theta<1, \frac{1}{p}=\frac{1-\theta}{p_{0}}+\frac{\theta}{p_{1}}, \frac{1}{q}=\frac{1-\theta}{q_{0}}+\frac{\theta}{q_{1}}$ and $\alpha=(1-\theta) \alpha_{0}+\theta \alpha_{1}$.

Remark 3.4.8. We first note that the condition (3.4.73) ensures the existence of the extension operator as in Remark 3.4.5, that are needed in the proof of Theorem 3.4.7.

Second, we observe that in particular, if $1 \leq p_{i}, q_{i}<\infty, i=0,1$, in Theorem 3.4.7, then $\sigma_{p_{i}, q_{i}}=0, i=0,1$. Therefore, in this scenario, the condition (3.4.73) reduces to $\alpha_{i}>0, i=0,1$.

Third, if $\frac{n+1}{n+2}<p_{0}, p_{1} \leq 1$ and $q_{0}=q_{1}=2$ in Theorem 3.4.7, then

$$
1>\sigma_{p_{i}}=\max \left\{0,(n+1)\left(\frac{1}{p_{i}}-1\right)\right\} .
$$

Therefore, we may consider the special case when $\alpha_{0}=\alpha_{1}=1$, and, on account of Theorem 2.1.59 to the effect that $H_{\text {par }}^{1, p}\left(\mathbb{R}^{n} \times \mathbb{R}\right) \equiv F_{1, p a r}^{p, 2}\left(\mathbb{R}^{n} \times \mathbb{R}\right)$ for $\frac{n+2}{n+3}<p \leq 1$, we arrive at the following theorem, which will be useful later on. 
Theorem 3.4.9. Consider a Lipschitz domain $\Omega \subset \mathbb{R}^{n}, 0<T<\infty$, and indices $\frac{n+1}{n+2}<p_{0}, p_{1} \leq 1$. Then

$$
\left[{ }_{0} H_{\text {par }}^{1, p_{0}}(\partial \Omega \times(0, T)),{ }_{0} H_{\text {par }}^{1, p_{1}}(\partial \Omega \times(0, T))\right]_{\theta}={ }_{0} H_{\text {par }}^{1, p}(\partial \Omega \times(0, T)),
$$

where $0<\theta<1$ and $\frac{1}{p}=\frac{1-\theta}{p_{0}}+\frac{\theta}{p_{1}}$.

In the last part of this section we prove three complex interpolation results which involve parabolic Besov spaces with particular amounts of smoothness, and with two different types of built-in initial conditions. These properties will play important roles in Chapter 7. Recall the hexagon OABCDE from our earlier discussion.

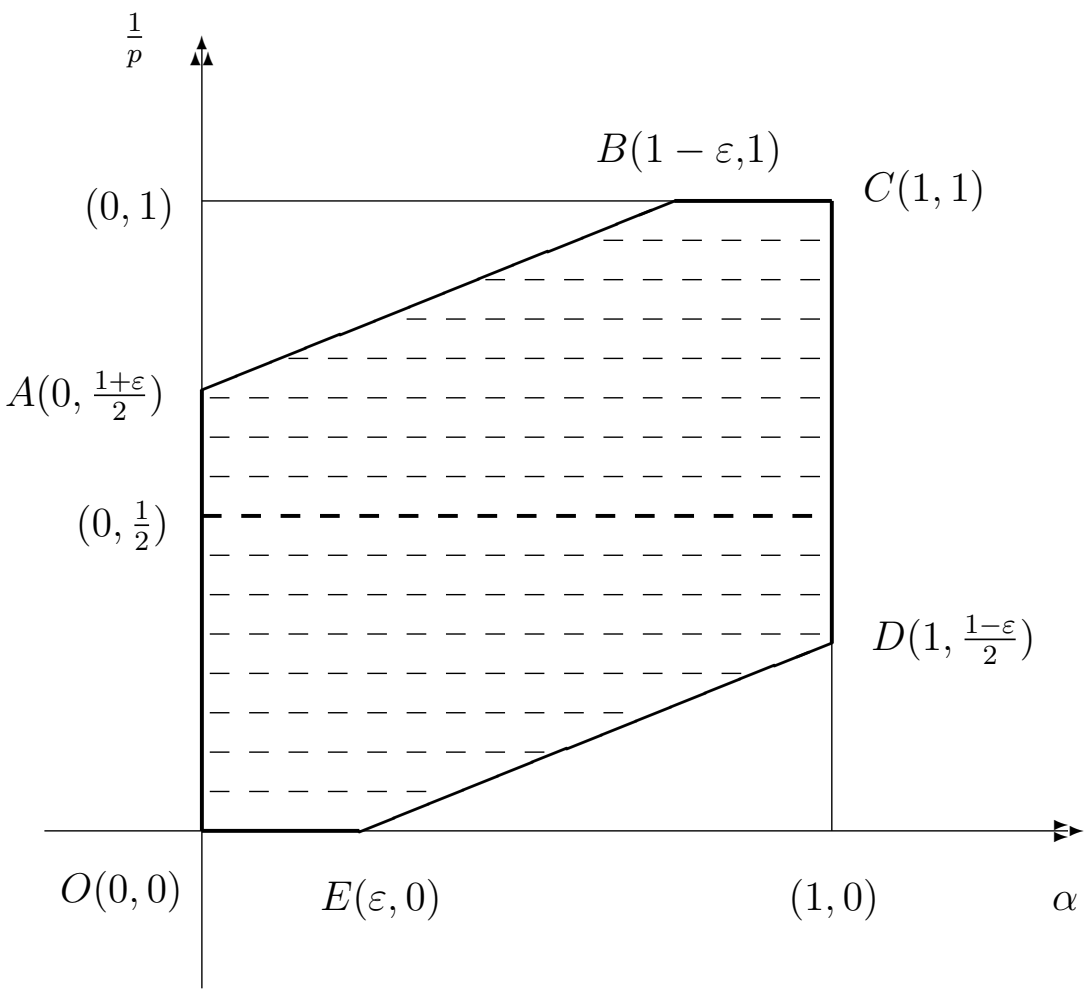


Theorem 3.4.10. Consider a bounded Lipschitz domain $\Omega \subset \mathbb{R}^{n}, 0<T<\infty$, and indices $1<p_{0}, p_{1}<\infty, 0<\alpha_{0}, \alpha_{1}<1$ such that the pairs $\left(1-\alpha_{i}, \frac{1}{p_{i}}\right), i=0,1$, belong to the interior of the hexagon $O A B C D E$. Then

$$
\left[{ }_{0} B_{1-\alpha_{0}+\frac{1}{p_{0}}, p a r}^{p_{0}}(\Omega \times(0, T)),{ }_{0} B_{1-\alpha_{1}+\frac{1}{p_{1}}, p a r}^{p_{1}}(\Omega \times(0, T))\right]_{\theta}={ }_{0} B_{1-\alpha+\frac{1}{p}, p a r}^{p}(\Omega \times(0, T)),
$$

with equivalent norms, where $0<\theta<1, \frac{1}{p}=\frac{1-\theta}{p_{0}}+\frac{\theta}{p_{1}}$, and $\alpha=(1-\theta) \alpha_{0}+\theta \alpha_{1}$.

Proof. Step I. Show that for the appropriate parameters,

$$
\left[B_{1-\alpha_{0}+\frac{1}{p_{0}}, p a r, 0}^{p_{0}}(\Omega \times(0, \infty)), B_{1-\alpha_{1}+\frac{1}{p_{1}}, p a r, 0}^{p_{1}}(\Omega \times(0, \infty))\right]_{\theta}=B_{1-\alpha+\frac{1}{p}, p a r, 0}^{p}(\Omega \times(0, \infty)),
$$

where, by definition,

$$
B_{1-\alpha+\frac{1}{p}, p a r, 0}^{p}(\Omega \times(0, \infty)):=\left\{f \in B_{1-\alpha+\frac{1}{p}, p a r}^{p}(\Omega \times \mathbb{R}): \operatorname{supp} f \subseteq \Omega \times[0, \infty)\right\} .
$$

We first consider part (ii) of Lemma 1.2.35 in the special case when $X=Y, X_{\alpha_{i}}^{p_{i}, p_{i}}=$ $B_{1-\alpha_{i}+\frac{1}{p}, p a r}^{p_{i}}(\Omega \times \mathbb{R}), i=0,1, D_{2}=\Omega \times[0, \infty)$, and

$$
E_{D_{2}^{c}}=\left.R\right|_{\Omega \times \mathbb{R}} \circ E: B_{1-\alpha_{i}+\frac{1}{p}, p a r}^{p_{i}}(\Omega \times(-\infty, 0)) \longrightarrow B_{1-\alpha_{i}+\frac{1}{p}, p a r}^{p_{i}}(\Omega \times \mathbb{R}),
$$

where $E$ is the extension operator from Remark 3.4.5.

Granted that $\left[B_{1-\alpha_{0}+\frac{1}{p}, p a r}^{p_{0}}(\Omega \times \mathbb{R}), B_{1-\alpha_{1}+\frac{1}{p}, p a r}^{p_{1}}(\Omega \times \mathbb{R})\right]_{\theta}=B_{1-\alpha+\frac{1}{p}, p a r}^{p}(\Omega \times \mathbb{R})$ for indices $1<p_{0}, p_{1}<\infty, 0<\alpha_{0}, \alpha_{1}<1,0<\theta<1, \frac{1}{p}=\frac{1-\theta}{p_{0}}+\frac{\theta}{p_{1}}$, and $\alpha=(1-\theta) \alpha_{0}+\theta \alpha_{1}$ (cf. part (g) of Theorem 2.2.8), the conclusion of Lemma 1.2.35 holds. This finishes the proof of he claim made in Step I.

Step II. Show (3.4.74). 
Our goal is to apply part (iii) of Lemma 1.2 .35 with $X=Y, D_{1}=\Omega \times(0, T)$, $X_{\alpha_{i}, D_{2}, *}^{p_{i}, p_{i}}=B_{1-\alpha_{i}+\frac{1}{p}, p a r, 0}^{p_{i}}(\Omega \times(0, \infty)), i=0,1$. We recall Theorem 3.1 .20 regarding the equivalence between the classes ${ }_{0} B_{\beta, p a r}^{q}(\Omega \times(0, T))$ and $\square B_{\beta, p a r}^{q}(\Omega \times(0, T))$ for a bounded Lipschitz domain, where $\left(\beta-\frac{1}{q}, \frac{1}{q}\right)$ belongs to the interior of the hexagon $O A B C D E$. In our situation, i.e. with $1-\alpha_{i}+\frac{1}{p_{i}}, i=0,1$, and $1-\alpha+\frac{1}{p}$ in place of $\beta$, by hypotheses and convex combination, the conditions $\left(1-\alpha_{i}, \frac{1}{p_{i}}\right),\left(1-\alpha, \frac{1}{p}\right) \in$ interior of the hexagon $O A B C D E$ are naturally satisfied. Then, by Theorem 3.1.20 and by the definitions of the spaces involved,

$$
{ }_{0} B_{1-\alpha_{i}+\frac{1}{p_{i}}, p a r}^{p_{i}}(\Omega \times(0, T))=\left\{\left.f\right|_{\Omega \times(0, T)}: f \in B_{1-\alpha_{i}+\frac{1}{p_{i}}, p a r, 0}^{p_{i}}(\Omega \times(0, \infty))\right\}, \quad i=0,1,
$$

for the range of indices we consider. There is a similar characterization of the space ${ }_{0} B_{1-\alpha+\frac{1}{p}, p a r}^{p}(\Omega \times(0, T))$, as well. In view of (3.4.75), Step I and the particular case of Lema 1.2.35 mentioned above, in order to show (3.4.74), it suffices to prove that there exists a universal extension operator

$$
\tilde{E}:{ }_{0} B_{1-\alpha+\frac{1}{p}, p a r}^{p}(\Omega \times(0, T)) \longrightarrow B_{1-\alpha+\frac{1}{p}, p a r, 0}^{p}(\Omega \times(0, \infty)) .
$$

To construct $\tilde{E}$, we first let $f \in{ }_{0} B_{1-\alpha+\frac{1}{p}, p a r}^{p}(\Omega \times(0, T))$. Then, by definition, there exists a function $F \in B_{1-\alpha+\frac{1}{p}, p a r}^{p}(\Omega \times \mathbb{R})$ such that supp $F \subseteq \Omega \times[0, \infty)$ and $f=\left.F\right|_{\Omega \times(0, T)}$. Let us introduce $\psi_{0}, \psi_{1} \in C^{\infty}(\mathbb{R})$ with $\psi_{0}+\psi_{1}=1$, such that

$$
\begin{aligned}
& \psi_{0} \equiv 1 \text { on }[T, \infty), \quad \operatorname{supp} \psi_{0} \subseteq[0, \infty), \\
& \psi_{1} \equiv 1 \text { on }(-\infty, 0], \quad \operatorname{supp} \psi_{1} \subseteq(-\infty, T] .
\end{aligned}
$$


We now define $\tilde{E} f:=\psi_{1} F+\psi_{0}\left(\left.R\right|_{\Omega \times \mathbb{R}} \circ E\right) f$, where $E$ is the extension operator as in Remark 3.4.5.

Note that $\tilde{E} f \in B_{1-\alpha+\frac{1}{p}, p a r}^{p}(\Omega \times \mathbb{R})$ and supp $\tilde{E} f \subseteq \Omega \times[0, \infty)$, hence

$$
\tilde{E} f \in B_{1-\alpha+\frac{1}{p}, p a r, 0}^{p}(\Omega \times(0, \infty)) .
$$

Also, it is easy to see that ${ }_{0} B_{1-\alpha+\frac{1}{p}, p a r}^{p}(\Omega \times(0, T)) \hookrightarrow B_{1-\alpha+\frac{1}{p}, p a r}^{p}(\Omega \times(-\infty, T))$, and $\left.E f\right|_{\Omega \times(0, T)}=f \in{ }_{0} B_{1-\alpha+\frac{1}{p}, p a r}^{p}(\Omega \times(0, T))$. In addition to that, $\left.\tilde{E} f\right|_{\Omega \times(0, T)}=$ $\left(\psi_{0}+\psi_{1}\right) f=f \in{ }_{0} B_{1-\alpha+\frac{1}{p}, p a r}^{p}(\Omega \times(0, T))$.

To show that the definition of $\tilde{E}$ is independent of the choice of $F$, we pick $F$ and $F^{\prime}$ from $B_{1-\alpha+\frac{1}{p}, p a r}^{p}(\Omega \times \mathbb{R})$ so that $\operatorname{supp} F \subseteq \Omega \times[0, \infty), \operatorname{supp} F^{\prime} \subseteq \Omega \times[0, \infty)$, and $\left.F\right|_{\Omega \times(0, T)}=f=\left.F^{\prime}\right|_{\Omega \times(0, T)}$. Then one can see that $\psi_{1}\left(F-F^{\prime}\right) \equiv 0$ for a.e. $t \in \mathbb{R}$, i.e. the definition of $\tilde{E}$ is independent of the particular choice of $F$. Hence, for given $f, g \in{ }_{0} B_{1-\alpha+\frac{1}{p}, p a r}^{p}(\Omega \times(0, T))$ with extensions $F$ and $G$, respectively, from $B_{1-\alpha+\frac{1}{p}, \text { par }}^{p}(\Omega \times \mathbb{R})$, we can choose $F+G$ to be the extension of $f+g$. Consequently, $\tilde{E}$ is linear. This concludes Step II and the proof of Theorem 3.4.10.

Contrary to the results we have presented above in this section, we now consider parabolic Besov spaces with a different type of built-in initial condition. The hexagon OABCDE retains the same significance as above.

Theorem 3.4.11. Let $\Omega$ be a bounded Lipschitz domain in $\mathbb{R}^{n}, 0<T<\infty$, and indices $1<p_{0}, p_{1}<\infty, 0<\alpha_{0}, \alpha_{1}<1$ such that the pairs $\left(\alpha_{i}, 1-\frac{1}{p_{i}}\right), i=0,1$, belong to the interior of the hexagon $O A B C D E$. Then

$$
\left[{ }_{z} B_{-1-\alpha_{0}+\frac{1}{p_{0}}, p a r}^{p_{0}}(\Omega \times(0, T)),{ }_{z} B_{-1-\alpha_{1}+\frac{1}{p_{1}}, p a r}^{p_{1}}(\Omega \times(0, T))\right]_{\theta}={ }_{z} B_{-1-\alpha+\frac{1}{p}, p a r}^{p}(\Omega \times(0, T)),
$$


with equivalent norms, where $0<\theta<1, \frac{1}{p}=\frac{1-\theta}{p_{0}}+\frac{\theta}{p_{1}}$ and $\alpha=(1-\theta) \alpha_{0}+\theta \alpha_{1}$.

Proof. As a first step, similar to the proof of Theorem 3.4.10, we show that the space ${ }^{0} B_{1-\alpha+\frac{1}{p}, p a r}^{p}(\Omega \times(0, T))$ is a complex interpolation scale for $\left(1-\alpha, \frac{1}{p}\right) \in$ interior of the hexagon $O A B C D E$, or, equivalently,

$$
\begin{gathered}
{ }^{0} B_{\beta+\frac{1}{p}, p a r}^{p}(\Omega \times(0, T)) \text { is a complex interpolation scale for } \\
\left(\beta, \frac{1}{p}\right) \in \text { interior of the hexagon } O A B C D E
\end{gathered}
$$

We next recall from Theorem 3.1.11 and Corollary 3.1.12 that for any $p, \beta \in \mathbb{R}$ such that $1<p<\infty$ and $\beta+\frac{1}{p} \neq 0$,

$$
\left({ }^{0} B_{\beta+\frac{1}{p}, p a r}^{p}(\Omega \times(0, T))\right)^{*}={ }_{z} B_{-\beta-\frac{1}{p}, p a r}^{p^{\prime}}(\Omega \times(0, T))
$$

and

$$
\left({ }_{z} B_{-\beta-\frac{1}{p}, p a r}^{p^{\prime}}(\Omega \times(0, T))\right)^{*}={ }^{0} B_{\beta+\frac{1}{p}, p a r}^{p}(\Omega \times(0, T))
$$

respectively, where $1 / p+1 / p^{\prime}=1$. Based on (3.4.77) and (3.4.78), it is easy to see that ${ }^{0} B_{\beta+\frac{1}{p}, p a r}^{p}(\Omega \times(0, T))$ is reflexive. With this and (3.4.76) in hand, we can use the Duality Theorem for the complex interpolation method (cf., e.g., Theorem 4.5.1 and Corollary 4.5.2 in [BeLö]), and we ultimately obtain that ${ }_{z} B_{-\beta-\frac{1}{p}, p a r}^{p^{\prime}}(\Omega \times(0, T))$ is a complex interpolation scale for the range $\left(\beta, \frac{1}{p}\right) \in$ interior of the hexagon $O A B C D E$. This, in turn, is equivalent with the fact that ${ }_{z} B_{-1-\beta+\frac{1}{p^{\prime}}, p a r}^{p^{\prime}}(\Omega \times(0, T))$ is a complex interpolation scale for $\left(\beta, 1-\frac{1}{p^{\prime}}\right) \in$ interior of the hexagon $O A B C D E$, as desired.

In a very similar fashion, this time relying on Theorem 3.1.13 and Corollary 3.1.14 (instead of Theorem 3.1.11 and Corollary 3.1.12), we obtain the following. 
Theorem 3.4.12. Let $\Omega$ be a bounded Lipschitz domain in $\mathbb{R}^{n}, 0<T<\infty$, and indices $1<p_{0}, p_{1}<\infty, 0<\alpha_{0}, \alpha_{1}<1$ such that the pairs $\left(\alpha_{i}, 1-\frac{1}{p_{i}}\right), i=0,1$, belong to the interior of the hexagon $O A B C D E$. Then

$$
\left[{ }^{z} B_{-1-\alpha_{0}+\frac{1}{p_{0}}, p a r}^{p_{0}}(\Omega \times(0, T)),{ }^{z} B_{-1-\alpha_{1}+\frac{1}{p_{1}}, p a r}^{p_{1}}(\Omega \times(0, T))\right]_{\theta}={ }^{z} B_{-1-\alpha+\frac{1}{p}, p a r}^{p}(\Omega \times(0, T)),
$$

with equivalent norms, where $0<\theta<1, \frac{1}{p}=\frac{1-\theta}{p_{0}}+\frac{\theta}{p_{1}}$ and $\alpha=(1-\theta) \alpha_{0}+\theta \alpha_{1}$. 


\section{Chapter 4}

\section{Mapping properties of the double layer potential}

Our goal in this chapter is to prove that for a bounded Lipschitz domain $\Omega \subset \mathbb{R}^{n}$, $0<T<\infty$ and appropriate indices, the caloric double layer potential $\mathcal{D}$ is a bounded linear map from ${ }_{0} B_{\alpha, p a r}^{p}(\partial \Omega \times(0, T))$ into ${ }_{0} B_{\alpha+\frac{1}{p}, p a r}^{p}(\Omega \times(0, T))$ (see Theorem 4.2.11). This can be achieved via interpolation between the endpoint cases $p=1$ (Theorem 4.1.2) and $p=\infty$ (Theorem 4.1.4).

\subsection{Two basic estimates}

This section contains the discussion of the above mentioned endpoint cases $p=1$ and $p=\infty$.

Recall from (1.1.5) that the caloric double layer potential operator is defined by

$$
\mathcal{D} f(x, t):=\int_{\partial \Omega \times \mathbb{R}} \partial_{\nu_{y}}[E(x-y, t-s)] f(y, s) d \sigma_{y} d s
$$

for $(x, t) \in \Omega \times \mathbb{R}$, where $E(x, t)$ is the fundamental solution of the heat operator and $\Omega$ is a domain in $\mathbb{R}^{n}$ with outward unit notmal $\nu$ and surface measure $d \sigma$. Before stating our first result, we need to fix some notation. By $\delta(x)$, for $x \in \Omega$, we mean 
the distance from $x$ to the boundary of the domain $\Omega$ in $\mathbb{R}^{n}$, i.e. $\delta(x):=\operatorname{dist}(x, \partial \Omega)$. The symbol $\nabla^{k}$ denotes any combination of mixed spacial partial derivatives of order $k$. Also, recall the definition of the fractional integral operator from (1.1.4).

The most significant result regarding one of the endpoint cases (when $p=1$ ) is as follows.

Theorem 4.1.1. Let $\Omega$ be a Lipschitz domain in $\mathbb{R}^{n}$, and consider $N:=k+2 \gamma-$ $2 \theta-\alpha-1>-1$, where $k, \gamma \in \mathbb{N}_{0}, 0 \leq \theta<1,0<\alpha<1$ such that $2 \gamma-2 \theta-\alpha>0$ for $\theta \neq 0$. Then

$$
\delta^{N} \nabla^{k} \partial_{\text {time }}^{\gamma} I_{\text {time }}^{\theta} \mathcal{D}: B_{\alpha, p a r}^{1}(\partial \Omega \times \mathbb{R}) \longrightarrow L^{1}(\Omega \times \mathbb{R})
$$

is bounded.

Proof. First let us consider the case $0<\theta<1$.

Let $f \in B_{\alpha, p a r}^{1}(\partial \Omega \times \mathbb{R})$ and fix $(x, t) \in \Omega \times \mathbb{R}$. Also let $\hat{x} \in \partial \Omega$ such that $\delta(x)=\operatorname{dist}(x, \partial \Omega)=|x-\hat{x}|$ and denote this distance by $\tau$. Then we have

$$
\begin{aligned}
& \left(\delta^{N} \nabla^{k} \partial_{\text {time }}^{\gamma} I_{\text {time }}^{\theta} \mathcal{D} f\right)(x, t) \\
& \quad=\int_{\mathbb{R}} \int_{\partial \Omega} \delta(x)^{N}\left(\partial_{\nu(y)} \nabla^{k} \partial_{\text {time }}^{\gamma} I_{\text {time }}^{\theta}\right)[E(x-y, t-s)] f(y, s) d \sigma_{y} d s \\
& \quad=\delta(x)^{N} \int_{\mathbb{R}} \int_{\partial \Omega}\left(\nabla^{k+1} \partial_{\text {time }}^{\gamma} I_{\text {time }}^{\theta}\right)[E(x-y, t-s)]\{f(y, s)-f(\hat{x}, t)\} d \sigma_{y} d s \\
& \quad+\delta(x)^{N} f(\hat{x}, t)\left(\nabla^{k} \partial_{\text {time }}^{\gamma} I_{\text {time }}^{\theta}\right)\left\{\int_{\mathbb{R}} \int_{\partial \Omega} \partial_{\nu(y)}[E(x-y, t-s)] d \sigma_{y} d s\right\},
\end{aligned}
$$

where the last term equals zero, since the expression inside the braces is $\mathcal{D} 1=1$, and we cannot have $k=0$ and $\gamma=0$ at the same time. In view of the Fubini property 
of the space $B_{\alpha, p a r}^{1}(\partial \Omega \times \mathbb{R})$ (see Proposition 2.3.16), we need to prove that

$$
\left\|\delta^{N} \nabla^{k} \partial_{\text {time }}^{\gamma} I_{\text {time }}^{\theta} \mathcal{D} f\right\|_{L^{1}(\Omega \times \mathbb{R})} \leq \int_{\partial \Omega}\|f(y, \cdot)\|_{B_{\alpha / 2}^{1}(\mathbb{R})} d \sigma_{y}+\int_{\mathbb{R}}\|f(\cdot, t)\|_{B_{\alpha}^{1}(\partial \Omega)} d t
$$

We will show that

$$
\begin{gathered}
\int_{\Omega \times \mathbb{R}} \int_{\partial \Omega \times \mathbb{R}} \delta(x)^{N}\left|\left(\nabla^{k+1} \partial_{\text {time }}^{\gamma} I_{\text {time }}^{\theta}\right)[E(x-y, t-s)]\right||f(y, s)-f(y, t)| d \sigma_{y} d s d x d t \\
\leq c \int_{\partial \Omega} \int_{\mathbb{R}} \int_{\mathbb{R}} \frac{|f(y, s)-f(y, t)|}{|t-s|^{1+\frac{\alpha}{2}}} d t d s d \sigma_{y},
\end{gathered}
$$

and that

$$
\begin{gathered}
\int_{\Omega \times \mathbb{R}} \int_{\partial \Omega \times \mathbb{R}} \delta(x)^{N}\left|\left(\nabla^{k+1} \partial_{\text {time }}^{\gamma} I_{\text {time }}^{\theta}\right)[E(x-y, t-s)]\right||f(y, t)-f(\hat{x}, t)| d \sigma_{y} d s d x d t \\
\leq c \int_{\mathbb{R}} \int_{\partial \Omega} \int_{\partial \Omega} \frac{|f(y, t)-f(z, t)|}{|y-z|^{n-1+\alpha}} d \sigma_{y} d \sigma_{z} d t,
\end{gathered}
$$

which yield to a slightly stronger inequality than (4.1.2).

To show (4.1.3) and (4.1.4), we split the domain of integration into two parts, first we consider the case when $|x-y|^{2} \geq|t-s|$, second, when $|x-y|^{2}<|t-s|$.

Using (1.2.19), the left-hand side of (4.1.3), when $|x-y|^{2} \geq|t-s|$, is bounded by

$$
c \int_{D_{1}} \tau^{N} \frac{|t-s|^{\theta}}{|x-y|^{n+k+1+2 \gamma}}\left[\min \left\{1, \frac{|x-y|^{2}}{|t-s|}\right\}\right]^{\gamma+1}|f(y, s)-f(y, t)| d \sigma_{y} d s d x d t
$$

where

$$
D_{1}:=\left\{(x, t, y, s) \in \Omega \times \mathbb{R} \times \partial \Omega \times \mathbb{R}:|x-y|^{2} \geq|t-s|\right\} .
$$

By the change of variables $x=(\hat{x}, \tau) \in \partial \Omega \times \mathbb{R}_{+},(4.1 .5)$ is bounded by

$$
c \int_{D_{2}} \tau^{N} \frac{|t-s|^{\theta}}{(|\hat{x}-y|+\tau)^{n+k+1+2 \gamma}}|f(y, s)-f(y, t)| d \sigma_{y} d s d \sigma_{\hat{x}} d \tau d t
$$


where

$$
D_{2}:=\left\{(\hat{x}, \tau, t, y, s) \in \partial \Omega \times \mathbb{R}_{+} \times \mathbb{R} \times \partial \Omega \times \mathbb{R}:|\hat{x}-y|+\tau \geq|t-s|^{1 / 2}\right\}
$$

Note that $|x-y| \approx|\hat{x}-y|+\tau$ (we were also using that $d x \approx d \sigma_{\hat{x}} d \tau$ ). Making the translation $\hat{x}-y=x^{\prime} \in \mathbb{R}^{n-1}$, the expresion in (4.1.6) is controlled by

$$
c \int_{D_{3}} \tau^{N} \frac{|t-s|^{\theta}|f(y, s)-f(y, t)|}{\left(\left|x^{\prime}\right|+\tau\right)^{n+k+1+2 \gamma}} d \sigma_{y} d s d t d x^{\prime} d \tau
$$

Here

$$
D_{3}:=\left\{\left(x^{\prime}, \tau, t, y, s\right) \in \mathbb{R}^{n-1} \times \mathbb{R}_{+} \times \mathbb{R} \times \partial \Omega \times \mathbb{R}:\left|x^{\prime}\right|+\tau \geq|t-s|^{1 / 2}\right\} .
$$

Now if we rescale (4.1.7) in $x^{\prime}$ and $\tau$, by setting $x^{\prime}=|t-s|^{1 / 2} x^{\prime \prime}$ and $\tau=|t-s|^{1 / 2} \tau^{\prime}$, we'll obtain that (4.1.7) is bounded by

$$
\left(\int_{\partial \Omega} \int_{\mathbb{R}} \int_{\mathbb{R}} \frac{|f(y, s)-f(y, t)|}{|t-s|^{1+\alpha / 2}} d s d t d \sigma_{y}\right)\left[\int_{D_{4}} \frac{\left(\tau^{\prime}\right)^{N}}{\left(\left|x^{\prime \prime}\right|+\tau^{\prime}\right)^{n+k+1+2 \gamma}} d x^{\prime \prime} d \tau^{\prime}\right]
$$

where $D_{4}:=\left\{\left(x^{\prime \prime}, \tau^{\prime}\right) \in \mathbb{R}^{n-1} \times \mathbb{R}_{+}:\left|x^{\prime \prime}\right|+\tau^{\prime} \geq c\right\}$. Denote the integral in the brackets in (4.1.8) by $I_{1}$. Comparing (4.1.3) and (4.1.8), we are left with showing that $I_{1}$ is finite.

When $\tau^{\prime} \geq\left|x^{\prime \prime}\right|$, we make the change of variables $x^{\prime \prime}=\tau^{\prime} \tilde{x}, \tilde{x} \in \mathbb{R}^{n-1}$. Then from the conditions we have for $\tau^{\prime}$ and $x^{\prime \prime}$ it follows that $\tau^{\prime}>c$ and $|\tilde{x}| \leq 1$. The integral

$$
I_{1} \leq c\left(\int_{\substack{\tau^{\prime} \in \mathbb{R}_{+} \\ \tau^{\prime}>c}} \frac{d \tau^{\prime}}{\left(\tau^{\prime}\right)^{2 \theta+\alpha+3}}\right) \int_{\substack{\tilde{x} \in \mathbb{R}^{n-1} \\|\tilde{x}| \leq 1}} \frac{d \tilde{x}}{(|\tilde{x}|+1)^{n+k+1+2 \gamma}}<\infty
$$

since $2 \theta+\alpha+3>1$. 
If $\left|x^{\prime \prime}\right|>\tau^{\prime}$, we let $\tau^{\prime}=\left|x^{\prime \prime}\right| r, r \in \mathbb{R}_{+}$. Hence

$$
I_{1} \leq c\left(\int_{\substack{r \in \mathbb{R}_{+} \\ r \leq 1}} \frac{r^{N}}{(1+r)^{n+k+1+2 \gamma}} d r \int_{\substack{x^{\prime \prime} \in \mathbb{R}^{n-1} \\\left|x^{\prime \prime}\right|>c}} \frac{d x^{\prime \prime}}{\left|x^{\prime \prime}\right|^{n+2 \theta+\alpha+1}}<\infty .\right.
$$

Here we were using that the power of $\left|x^{\prime \prime}\right|$ is greater than $n-1$, and that for $r \leq 1$ the expression $1+r$ behaves like 1 , and $\int_{0<r \leq 1} r^{N} d r$ is finite for $N>-1$.

As of now we proved that (4.1.3) holds true when $|x-y|^{2} \geq|t-s|$. For the other domain of integration, following the same steps as before $\left(x=(\hat{x}, \tau), \hat{x}-y=x^{\prime}\right)$, we obtain that, when $|x-y|^{2}<|t-s|$, the left-hand side of (4.1.3) is bounded by

$$
\begin{aligned}
& c \int_{D_{1}^{\prime}} \tau^{N} \frac{|t-s|^{\theta}}{|x-y|^{n+k+1+2 \gamma}}\left[\min \left\{1, \frac{|x-y|^{2}}{|t-s|}\right\}\right]^{\gamma+1}|f(y, s)-f(y, t)| d \sigma_{y} d s d x d t \\
& \leq c \int_{D_{2}^{\prime}} \tau^{N} \frac{|f(y, s)-f(y, t)|}{(|\hat{x}-y|+\tau)^{n+k-1}|t-s|^{\gamma-\theta+1}} d \sigma_{y} d s d \sigma_{\hat{x}} d \tau d t \\
& \leq c \int_{D_{3}^{\prime}} \frac{\tau^{N}|f(y, s)-f(y, t)|}{\left(\left|x^{\prime}\right|+\tau\right)^{n+k-1}|t-s|^{\gamma-\theta+1}} d \sigma_{y} d s d x^{\prime} d \tau d t
\end{aligned}
$$

where

$$
\begin{gathered}
D_{1}^{\prime}:=\left\{(x, t, y, s) \in \Omega \times \mathbb{R} \times \partial \Omega \times \mathbb{R}:|x-y|^{2}<|t-s|\right\}, \\
D_{2}^{\prime}:=\left\{(\hat{x}, \tau, t, y, s) \in \partial \Omega \times \mathbb{R}_{+} \times \mathbb{R} \times \partial \Omega \times \mathbb{R}:|\hat{x}-y|+\tau<|t-s|^{1 / 2}\right\},
\end{gathered}
$$

and

$$
D_{3}^{\prime}:=\left\{\left(x^{\prime}, \tau, t, y, s\right) \in \mathbb{R}^{n-1} \times \mathbb{R}_{+} \times \mathbb{R} \times \partial \Omega \times \mathbb{R}:\left|x^{\prime}\right|+\tau<|t-s|^{1 / 2}\right\} .
$$

As in the previous case, let $x^{\prime}=|t-s|^{1 / 2} x^{\prime \prime}$ and $\tau=|t-s|^{1 / 2} \tau^{\prime}$. Then the integral 
in (4.1.9) is controlled by

$$
c\left(\int_{\partial \Omega} \int_{\mathbb{R}} \int_{\mathbb{R}} \frac{|f(y, s)-f(y, t)|}{|t-s|^{1+\alpha / 2}} d t d s d \sigma_{y}\right)\left[\int_{D_{4}^{\prime}} \frac{\left(\tau^{\prime}\right)^{N}}{\left(\left|x^{\prime \prime}\right|+\tau^{\prime}\right)^{n+k-1}} d x^{\prime \prime} d \tau^{\prime}\right],
$$

where $D_{4}^{\prime}:=\left\{\left(x^{\prime \prime}, \tau^{\prime}\right) \in \mathbb{R}^{n-1} \times \mathbb{R}_{+}:\left|x^{\prime \prime}\right|+\tau^{\prime}<c\right\}$. We denote the integral in the brackets in (4.1.10) by $I_{1}^{\prime}$, and we will show that $I_{1}^{\prime}$ is finite.

First, suppose that $\left|x^{\prime \prime}\right| \leq \tau^{\prime}$ and set $x^{\prime \prime}=\tilde{x} \tau^{\prime}, \tilde{x} \in \mathbb{R}^{n-1}$. Then $|\tilde{x}| \leq 1$, $0<\tau^{\prime}<c$, and

$$
I_{1}^{\prime} \leq c\left(\int_{0<\tau^{\prime}<c}\left(\tau^{\prime}\right)^{2 \gamma-2 \theta-\alpha-1} d \tau^{\prime}\right) \int_{\substack{\tilde{x} \in \mathbb{R}^{n-1} \\|\tilde{x}| \leq 1}} \frac{d \tilde{x}}{(1+|\tilde{x}|)^{n+k-1}}<\infty
$$

since $2 \gamma-2 \theta-\alpha-1>-1$.

Second, assume $\left|x^{\prime \prime}\right|>\tau^{\prime}$, and set $\tau^{\prime}=\left|x^{\prime \prime}\right| r, r \in \mathbb{R}_{+}$. Then

$$
I_{1}^{\prime} \leq c\left(\int_{0<r<1} \frac{r^{N}}{(1+r)^{n+k-1}} d r\right) \int_{\substack{x^{\prime \prime} \in \mathbb{R}^{n-1} \\\left|x^{\prime \prime}\right|<c}} \frac{d x^{\prime \prime}}{\left|x^{\prime \prime}\right|^{n-2 \gamma+2 \theta+\alpha-1}}<\infty,
$$

where we were using that $1+r \approx 1, \int_{0<r<1} r^{N} d r<\infty$ for $N>-1$, and that $n-2 \gamma+2 \theta+\alpha-1<n-1, x^{\prime \prime} \in \mathbb{R}^{n-1}$. Therefore, $I_{1}^{\prime}$ is finite, and by comparing (4.1.10) with (4.1.3), we can see that (4.1.3) is proved.

Now we turn our attention to (4.1.4). Using again (1.2.19), the left-hand side of (4.1.4), when $|x-y|^{2} \geq|t-s|$, is bounded by

$$
c \int_{D_{1}} \tau^{N} \frac{|t-s|^{\theta}}{|x-y|^{n+k+1+2 \gamma}}\left[\min \left\{1, \frac{|x-y|^{2}}{|t-s|}\right\}\right]^{\gamma+1}|f(y, t)-f(\hat{x}, t)| d \sigma_{y} d s d x d t
$$

where $D_{1}$ is as before, i.e.

$$
D_{1}=\left\{(x, t, y, s) \in \Omega \times \mathbb{R} \times \partial \Omega \times \mathbb{R}:|x-y|^{2} \geq|t-s|\right\}
$$


By the change of variables $x=(\hat{x}, \tau) \in \partial \Omega \times \mathbb{R}_{+},(4.1 .11)$ is bounded by

$$
c \int_{D_{2}} \tau^{N} \frac{|t-s|^{\theta}}{(|\hat{x}-y|+\tau)^{n+k+1+2 \gamma}}|f(y, t)-f(\hat{x}, t)| d \sigma_{y} d s d \sigma_{\hat{x}} d \tau d t .
$$

Here

$$
D_{2}=\left\{(\hat{x}, \tau, t, y, s) \in \partial \Omega \times \mathbb{R}_{+} \times \mathbb{R} \times \partial \Omega \times \mathbb{R}:|\hat{x}-y|+\tau \geq|t-s|^{1 / 2}\right\}
$$

as before (we were also using that $|x-y| \approx|\hat{x}-y|+\tau$ and $d x \approx d \sigma_{\hat{x}} d \tau$ ). Let $t-s=s^{\prime}$. Then the above integral is equivalent with

$$
c \int_{D_{5}} \tau^{N} \frac{\left|s^{\prime}\right|^{\theta}}{(|\hat{x}-y|+\tau)^{n+k+1+2 \gamma}}|f(y, t)-f(\hat{x}, t)| d \sigma_{y} d s^{\prime} d \sigma_{\hat{x}} d \tau d t
$$

where

$$
D_{5}:=\left\{\left(\hat{x}, \tau, t, y, s^{\prime}\right) \in \partial \Omega \times \mathbb{R}_{+} \times \mathbb{R} \times \partial \Omega \times \mathbb{R}:|\hat{x}-y|+\tau \geq\left|s^{\prime}\right|^{1 / 2}\right\} .
$$

Now we rescale in $\tau$ and $s^{\prime}$, by setting $\tau=\tau^{\prime}|\hat{x}-y|$ and $s^{\prime}=s^{\prime \prime}|\hat{x}-y|$, to obtain that (4.1.12) is controlled by

$$
\begin{gathered}
c\left(\int_{\partial \Omega} \int_{\partial \Omega} \int_{\mathbb{R}} \frac{|f(y, t)-f(\hat{x}, t)|}{|\hat{x}-y|^{n-1+\alpha}} d t d \sigma_{y} d \sigma_{\hat{x}}\right) \times \\
\times \int_{\tau^{\prime} \in \mathbb{R}_{+}} \frac{\left(\tau^{\prime}\right)^{N}}{\left(1+\tau^{\prime}\right)^{n+k+1+2 \gamma}}\left(\int_{\substack{s^{\prime \prime} \in \mathbb{R} \\
\left|s^{\prime \prime}\right|^{1 / 2} \leq 1+\tau^{\prime}}}\left|s^{\prime \prime}\right|^{\theta} d s^{\prime \prime}\right) d \tau^{\prime} \\
\leq c\left(\int_{\partial \Omega} \int_{\partial \Omega} \frac{|f(y, t)-f(\hat{x}, t)|}{|\hat{x}-y|^{n-1+\alpha}} d t d \sigma_{y} d \sigma_{\hat{\mathbb{x}}}\right) \int_{\tau^{\prime} \in \mathbb{R}_{+}} \frac{\left(\tau^{\prime}\right)^{N}}{\left(1+\tau^{\prime}\right)^{n+k-1+2 \gamma-2 \theta}} d \tau^{\prime} . \quad(4.1 .13
\end{gathered}
$$

Let $I_{2}$ be the integral in the brackets in (4.1.13). If $0<\tau^{\prime} \leq 1$, then $1+\tau^{\prime} \approx 1$ and $\int_{0<\tau^{\prime} \leq 1}\left(\tau^{\prime}\right)^{N} d \tau^{\prime}$ is bounded for $N>-1$. On the other hand, when $\tau^{\prime}>1$, 
$1+\tau^{\prime} \approx \tau^{\prime}$, and $I_{2} \leq c \int_{\tau^{\prime}>1} \frac{d \tau^{\prime}}{\left(\tau^{\prime}\right)^{n+\alpha}}<\infty$, since $n+\alpha>1$. This proves (4.1.4) when $|x-y|^{2} \geq|t-s|$.

In the other case, when $|x-y|^{2}<|t-s|$, the left-hand side of (4.1.4) is bounded by

$$
c \int_{D_{1}^{\prime}} \frac{\tau^{N}}{|x-y|^{n+k-1}|t-s|^{\gamma-\theta+1}}|f(y, t)-f(\hat{x}, t)| d \sigma_{y} d s d x d t
$$

with

$$
D_{1}^{\prime}=\left\{(x, t, y, s) \in \Omega \times \mathbb{R} \times \partial \Omega \times \mathbb{R}:|x-y|^{2}<|t-s|\right\} .
$$

Following the steps of the previous case, i.e. $x=(\hat{x}, t), t-s=s^{\prime}$, and the rescaling $\tau=\tau^{\prime}|\hat{x}-y|, s^{\prime}=s^{\prime \prime}|\hat{x}-y|^{2}$, we can control (4.1.14) by

$$
\begin{gathered}
c \int_{D_{2}^{\prime}} \frac{\tau^{N}}{(|\hat{x}-y|+\tau)^{n+k-1}|t-s|^{\gamma-\theta+1}}|f(y, t)-f(\hat{x}, t)| d \sigma_{y} d s d t d \sigma_{\hat{x}} d \tau \\
\leq c \int_{D_{5}^{\prime}} \frac{\tau^{N}}{(|\hat{x}-y|+\tau)^{n+k-1}\left|s^{\prime}\right|^{\gamma-\theta+1}}|f(y, t)-f(\hat{x}, t)| d \sigma_{y} d s^{\prime} d t d \sigma_{\hat{x}} d \tau \\
\leq c\left(\int_{\partial \Omega} \int_{\partial \Omega} \int_{\mathbb{R}} \frac{|f(y, t)-f(\hat{x}, t)|}{|\hat{x}-y|^{n-1+\alpha}} d \sigma_{y} d \sigma_{\hat{x}} d t\right) \times \\
\times \int_{\tau^{\prime} \in \mathbb{R}_{+}} \frac{\left(\tau^{\prime}\right)^{N}}{\left(1+\tau^{\prime}\right)^{n+k-1}}\left(\int_{\substack{s^{\prime \prime} \in \mathbb{R} \\
\left|s^{\prime \prime}\right|^{1 / 2}>1+\tau^{\prime}}} \frac{d s^{\prime \prime}}{\left|s^{\prime \prime}\right|^{\gamma-\theta+1}}\right) d \tau^{\prime},
\end{gathered}
$$

where

$$
D_{2}^{\prime}=\left\{(\hat{x}, \tau, t, y, s) \in \partial \Omega \times \mathbb{R}_{+} \times \mathbb{R} \times \partial \Omega \times \mathbb{R}:|\hat{x}-y|+\tau<|t-s|^{1 / 2}\right\},
$$

and

$$
D_{5}^{\prime}=\left\{\left(\hat{x}, \tau, t, y, s^{\prime}\right) \in \partial \Omega \times \mathbb{R}_{+} \times \mathbb{R} \times \partial \Omega \times \mathbb{R}:|\hat{x}-y|+\tau<\left|s^{\prime}\right|^{1 / 2}\right\} .
$$


Denoting the integral in the brackets in (4.1.15) by $I_{2}^{\prime}$, and observing that $\gamma-$ $\theta+1>1$ we can see that

$$
I_{2}^{\prime} \leq c \int_{\tau^{\prime} \in \mathbb{R}_{+}} \frac{\left(\tau^{\prime}\right)^{N}}{\left(1+\tau^{\prime}\right)^{n+k_{1}}} \frac{1}{\left(1+\tau^{\prime}\right)^{2(\gamma-\theta)}} d \tau^{\prime}=\int_{\tau^{\prime} \in \mathbb{R}_{+}} \frac{\left(\tau^{\prime}\right)^{N}}{\left(1+\tau^{\prime}\right)^{n+k+2 \gamma-2 \theta-1}} d \tau^{\prime} .
$$

If $0<\tau^{\prime} \leq 1$, then $1+\tau^{\prime} \approx 1$ and $\int_{0<\tau^{\prime} \leq 1}\left(\tau^{\prime}\right)^{N} d \tau^{\prime}<\infty$ for $N>-1$. In the other case, when $\tau^{\prime}>1$, we have $1+\tau^{\prime} \approx \tau^{\prime}$ and $I_{2}^{\prime} \leq c \int_{\tau^{\prime}>1} \frac{d \tau^{\prime}}{\left(\tau^{\prime}\right)^{n+\alpha}}<\infty$, since $n+\alpha>1$, $\tau^{\prime} \in \mathbb{R}_{+}$. This proves the inequality (4.1.4) for $|x-y|^{2}<|t-s|$. We showed that (4.1.4) holds when $|x-y|^{2} \geq|t-s|$, hence (4.1.4) holds in general. This completes the proof of the theorem for $0<\theta<1$.

Finally, let us consider the case $\theta=0$. Similar to the other case, when $0<\theta<1$, let $f \in B_{\alpha, p a r}^{1}(\partial \Omega \times \mathbb{R})$ and fix $(x, t) \in \Omega \times \mathbb{R}$. Also let $\hat{x} \in \partial \Omega$ such that $\delta(x)=$ $\operatorname{dist}(x, \partial \Omega)=|x-\hat{x}|$ and denote this distance by $\tau$. Then we have the following

$$
\begin{aligned}
\left(\delta^{N} \nabla^{k} \partial_{\text {time }}^{\gamma} \mathcal{D} f\right) & (x, t)=\int_{\mathbb{R}} \int_{\partial \Omega} \delta(x)^{N}\left(\partial_{\nu(y)} \nabla^{k} \partial_{\text {time }}^{\gamma}\right)[E(x-y, t-s)] f(y, s) d \sigma_{y} d s \\
= & \delta(x)^{N} \int_{\mathbb{R}} \int_{\partial \Omega}\left(\nabla^{k+1} \partial_{\text {time }}^{\gamma}\right)[E(x-y, t-s)]\{f(y, s)-f(\hat{x}, t)\} d \sigma_{y} d s \\
& +\delta(x)^{N} f(\hat{x}, t)\left(\nabla^{k} \partial_{\text {time }}^{\gamma}\right)\left\{\int_{\mathbb{R}} \int_{\partial \Omega} \partial_{\nu(y)}[E(x-y, t-s)] d \sigma_{y} d s\right\},
\end{aligned}
$$

where the last term equals zero, since the expression inside the braces is $\mathcal{D} 1=1$, and we cannot have $k=0$ and $\gamma=0$ at the same time. Therefore,

$$
\begin{aligned}
& \left\|\delta^{N} \nabla^{k} \partial_{\text {time }}^{\gamma} \mathcal{D} f\right\|_{L^{1}(\Omega \times \mathbb{R})} \\
& =\int_{\Omega \times \mathbb{R}} \int_{\partial \Omega \times \mathbb{R}} \delta(x)^{N}\left|\left(\nabla^{k+1} \partial_{\text {time }}^{\gamma}\right)[E(x-y, t-s)]\right||f(y, s)-f(\hat{x}, t)| d \sigma_{y} d s d x d t \\
& \leq c \int_{\Omega \times \mathbb{R}} \int_{\partial \Omega \times \mathbb{R}} \frac{\tau^{N}|f(y, s)-f(\hat{x}, t)|}{\left(|x-y|+|t-s|^{1 / 2}\right)^{n+k+1+2 \gamma}} d \sigma_{y} d s d x d t=: I_{3},
\end{aligned}
$$


where we have used (1.2.13) to bound the derivatives of the fundamental solution $E$ of the heat operator. Making once again the change of variables $x=(\hat{x}, \tau) \in \partial \Omega \times \mathbb{R}_{+}$, then $\tau=\tau^{\prime}\left(|\hat{x}-y|+|t-s|^{1 / 2}\right)$, we obtain that

$$
\begin{aligned}
I_{3} & \leq c \int_{\partial \Omega \times \mathbb{R}_{+}} \int_{\mathbb{R}} \int_{\partial \Omega \times \mathbb{R}} \frac{\tau^{N}|f(y, s)-f(\hat{x}, t)|}{\left(|\hat{x}-y|+\tau+|t-s|^{1 / 2}\right)^{n+k+1+2 \gamma}} d \sigma_{y} d s d t d \sigma_{\hat{x}} d \tau \\
& \leq c\left(\int_{\partial \Omega \times \mathbb{R}} \int_{\partial \Omega \times \mathbb{R}} \frac{|f(y, s)-f(\hat{x}, t)|}{\left(|\hat{x}-y|+|t-s|^{1 / 2}\right)^{n+1+\alpha}} d \sigma_{y} d s d \sigma_{\hat{x}} d t\right) \int_{\tau^{\prime} \in \mathbb{R}_{+}} \frac{\left(\tau^{\prime}\right)^{N}}{\left(1+\tau^{\prime}\right)^{n+k+1+2 \gamma}} d \tau^{\prime} \\
& \leq c\|f\|_{B_{\alpha, p a r}^{1}(\partial \Omega \times \mathbb{R})} I_{3}^{\prime},
\end{aligned}
$$

where $I_{3}^{\prime}:=\int_{\tau^{\prime} \in \mathbb{R}_{+}} \frac{\left(\tau^{\prime}\right)^{N}}{\left(1+\tau^{\prime}\right)^{n+k+1+2 \gamma}} d \tau^{\prime}$, and we were using the fact that the expression in parentheses is a part of the norm $\|f\|_{B_{\alpha, p a r}^{1}(\partial \Omega \times \mathbb{R})}(\mathrm{cf}$. Lemma 2.3.19, the intrinsic characterization of Besov spaces).

If $0<\tau^{\prime} \leq 1$, then $I_{3}^{\prime} \leq \int_{0<\tau^{\prime} \leq 1}\left(\tau^{\prime}\right)^{N} d \tau^{\prime}<\infty$, since $N>-1$. In the other case, when $\tau^{\prime}>1$, we obtain that $I_{3}^{\prime} \leq \int_{\tau^{\prime}>1} \frac{d \tau^{\prime}}{\left(\tau^{\prime}\right)^{n+2+\alpha}}<\infty$, since $n+2+\alpha>1$.

Consequently, (4.1.16) and the fact we have just proved to the effect that $I_{3}^{\prime}<\infty$, imply that $\left\|\delta^{N} \nabla^{k} \partial_{\text {time }}^{\gamma} \mathcal{D} f\right\|_{L^{1}(\Omega \times \mathbb{R})} \leq c\|f\|_{B_{\alpha, p a r}^{1}(\partial \Omega \times \mathbb{R})}$, which completes the proof of Theorem 4.1.1.

In order to state the next result let us recall from (1.1.3) the definition of the fractional time-derivative operator . If we replace $I_{\text {time }}^{\theta}$ with $D_{\text {time }}^{\theta}$ in Theorem 4.1.1, then we obtain the following.

Theorem 4.1.2. Consider a Lipschitz domain $\Omega$ in $\mathbb{R}^{n}$, and set $N^{\prime}:=k+2 \gamma+2 \theta-$ $\alpha-1>-1$, where $k, \gamma \in \mathbb{N}_{0}, 0 \leq \theta<1,0<\alpha<1$ such that $2 \gamma+2 \theta-\alpha>0$ for 
$\theta \neq 0$. Then

$$
\delta^{N^{\prime}} \nabla^{k} \partial_{\text {time }}^{\gamma} D_{\text {time }}^{\theta} \mathcal{D}: B_{\alpha, p a r}^{1}(\partial \Omega \times \mathbb{R}) \longrightarrow L^{1}(\Omega \times \mathbb{R})
$$

is bounded.

Proof. In view of the definition of the fractional time-derivative (and integral) operator $D_{\text {time }}^{\theta}\left(\right.$ and $\left.I_{\text {time }}^{\theta}\right)$ (see (1.1.3) and (1.1.4), respectively), we have that

$$
\delta^{N^{\prime}} \nabla^{k} \partial_{\text {time }}^{\gamma} D_{\text {time }}^{\theta}=\delta^{N^{\prime}} \nabla^{k} \partial_{\text {time }}^{\gamma+1} I_{\text {time }}^{1-\theta}
$$

For $0<\theta<1$, we use Theorem 4.1.1 and (4.1.18) to obtain (4.1.17). If $\theta=0$, notice that $D_{\text {time }}^{0}=\partial_{\text {time }} I_{\text {time }}^{1}=I=I_{\text {time }}^{0}$, and we can use the case $\theta=0$ of Theorem 4.1.1 to conclude the desired result.

In the second part of this section we turn our attention to the other endpoint case, i.e. when $p=\infty$.

Theorem 4.1.3. Let $\Omega$ be a Lipschitz domain in $\mathbb{R}^{n}$, and consider $N_{0}:=k+2 \gamma-$ $2 \theta-\alpha>0$, where $k, \gamma \in \mathbb{N}_{0}, 0 \leq \theta<1,0<\alpha<1$. Then

$$
\delta^{N_{0}} \nabla^{k} \partial_{\text {time }}^{\gamma} I_{\text {time }}^{\theta} \mathcal{D}: B_{\alpha, p a r}^{\infty}(\partial \Omega \times \mathbb{R}) \longrightarrow L^{\infty}(\Omega \times \mathbb{R})
$$

is bounded.

Proof. First let us consider the case $0<\theta<1$.

Let $f \in B_{\alpha, p a r}^{\infty}(\partial \Omega \times \mathbb{R})$ and fix $(x, t) \in \Omega \times \mathbb{R}$. Also let $\hat{x} \in \partial \Omega$ such that $\delta(x)=\operatorname{dist}(x, \partial \Omega)=|x-\hat{x}|$ and denote this distance by $\tau$. Then we have 


$$
\begin{aligned}
\left(\delta^{N_{0}} \nabla^{k} \partial_{\text {time }}^{\gamma} I_{\text {time }}^{\theta} \mathcal{D} f\right)(x, t) & \\
= & \int_{\mathbb{R}} \int_{\partial \Omega} \delta(x)^{N_{0}}\left(\partial_{\nu(y)} \nabla^{k} \partial_{\text {time }}^{\gamma} I_{\text {time }}^{\theta}\right)[E(x-y, t-s)] f(y, s) d \sigma_{y} d s \\
= & \delta(x)^{N_{0}} \int_{\mathbb{R}} \int_{\partial \Omega}\left(\nabla^{k+1} \partial_{\text {time }}^{\gamma} I_{\text {time }}^{\theta}\right)[E(x-y, t-s)]\{f(y, s)-f(\hat{x}, t)\} d \sigma_{y} d s \\
& +\delta(x)^{N_{0}} f(\hat{x}, t)\left(\nabla^{k} \partial_{\text {time }}^{\gamma} I_{\text {time }}^{\theta}\right)\left\{\int_{\mathbb{R}} \int_{\partial \Omega} \partial_{\nu(y)}[E(x-y, t-s)] d \sigma_{y} d s\right\},
\end{aligned}
$$

where the last term equals zero, since the expression inside the braces is $\mathcal{D} 1=1$, and we cannot have $k=0$ and $\gamma=0$ at the same time. Then, by an equivalent characterization of $B_{\alpha, p a r}^{\infty}(\partial \Omega \times \mathbb{R})$ (see Lemma 2.3.18), we obtain that

$$
\begin{aligned}
& \left\|\delta^{N_{0}} \nabla^{k} \partial_{\text {time }}^{\gamma} I_{\text {time }}^{\theta} \mathcal{D} f\right\|_{L^{\infty}(\Omega \times \mathbb{R})} \\
& =\sup _{(x, t) \in \Omega \times \mathbb{R}}\left\{\delta(x)^{N_{0}}\left|\int_{\mathbb{R}} \int_{\partial \Omega}\left(\nabla^{k+1} \partial_{\text {time }}^{\gamma} I_{\text {time }}^{\theta}\right)[E(x-y, t-s)][f(y, s)-f(\hat{x}, t)] d \sigma_{y} d s\right|\right\} \\
& \leq \sup _{(x, t) \in \Omega \times \mathbb{R}}\left\{\delta(x)^{N_{0}} \int_{\mathbb{R}} \int_{\partial \Omega}\left|\left(\nabla^{k+1} \partial_{\text {time }}^{\gamma} I_{\text {time }}^{\theta}\right)[E(x-y, t-s)]\right| \times\right. \\
& \left.\times\|f\|_{B_{\alpha, p a r}^{\infty}(\partial \Omega \times \mathbb{R})}\left(|\hat{x}-y|+|t-s|^{1 / 2}\right)^{\alpha} d \sigma_{y} d s\right\} .
\end{aligned}
$$

Since we need to prove that $\left\|\delta^{N_{0}} \nabla^{k} \partial_{\text {time }}^{\gamma} I_{\text {time }}^{\theta} \mathcal{D} f\right\|_{L^{\infty}(\Omega \times \mathbb{R})} \leq\|f\|_{B_{\alpha, p a r}^{\infty}(\partial \Omega \times \mathbb{R})}$, it is enough to show that there exists a positive constant $c<\infty$ such that for any pair

$$
\begin{aligned}
& (x, t) \in \Omega \times \mathbb{R}, \\
& \left.\int_{\mathbb{R}} \int_{\partial \Omega} \mid \nabla^{k+1} \partial_{\text {time }}^{\gamma} I_{\text {time }}^{\theta}\right)[E(x-y, t-s)] \mid\left(|\hat{x}-y|+|t-s|^{1 / 2}\right)^{\alpha} d \sigma_{y} d s \leq c \delta(x)^{-N_{0}} .
\end{aligned}
$$

We devide the domain of integration in four parts. First, let

$$
D_{1}:=\left\{(y, s) \in \partial \Omega \times \mathbb{R}:|\hat{x}-y|>c \tau \text { and }|t-s|^{1 / 2}>c \tau\right\}
$$


and set

$$
\left.I_{1}:=\iint_{D_{1}} \mid \nabla^{k+1} \partial_{\text {time }}^{\gamma} I_{\text {time }}^{\theta}\right)[E(x-y, t-s)] \mid\left(|\hat{x}-y|+|t-s|^{1 / 2}\right)^{\alpha} d \sigma_{y} d s .
$$

According to (1.2.19), we obtain the following.

$$
\begin{aligned}
I_{1} & \leq c \iint_{D_{1}} \frac{|t-s|^{\theta}\left(|\hat{x}-y|+|t-s|^{1 / 2}\right)^{\alpha}}{|x-y|^{n+k+1+2 \gamma}}\left[\min \left\{1, \frac{|x-y|^{2}}{|t-s|}\right\}\right]^{\gamma+1} d \sigma_{y} d s \\
& =c \iint_{D_{1}} \frac{|t-s|^{\theta}\left(|\hat{x}-y|+|t-s|^{1 / 2}\right)^{\alpha}}{|x-y|^{n+k-1}}\left[\min \left\{\frac{1}{|x-y|^{2}}, \frac{1}{|t-s|}\right\}\right]^{\gamma+1} d \sigma_{y} d s .
\end{aligned}
$$

Since $|x-\hat{x}| \leq|x-y|$ for any $y \in \partial \Omega$, we have that $|\hat{x}-y| \leq 2|x-y|$, and

$$
I_{1} \leq c \iint_{D_{1}} \frac{|t-s|^{\theta}\left(|\hat{x}-y|+|t-s|^{1 / 2}\right)^{\alpha}}{|\hat{x}-y|^{n+k-1}}\left[\min \left\{\frac{1}{|\hat{x}-y|^{2}}, \frac{1}{|t-s|}\right\}\right]^{\gamma+1} d \sigma_{y} d s .
$$

Let $y=\left(y^{\prime}, \varphi\left(y^{\prime}\right)\right)$ and $\hat{x}=\left(x^{\prime}, \varphi\left(x^{\prime}\right)\right)$, where the graph of the Lipschitz function $\varphi: \mathbb{R}^{n-1} \rightarrow \mathbb{R}$ is the boundary of $\Omega$ (if $\Omega$ is a bounded Lipschitz domain, then we take a finite partition of unity on $\partial \Omega$ and consider a piece of the boundary which, after extending by 0 , is the boundary of an unbounded Lipschitz domain). Then $|\hat{x}-y| \approx\left|x^{\prime}-y^{\prime}\right|, d \sigma_{y} \approx d y^{\prime}$, and $I_{1}$ will be bounded by

$$
c \iint_{D_{1}^{\prime}} \frac{|t-s|^{\theta}\left(\left|x^{\prime}-y^{\prime}\right|+|t-s|^{1 / 2}\right)^{\alpha}}{\left|x^{\prime}-y^{\prime}\right|^{n+k-1}}\left[\min \left\{\frac{1}{\left|x^{\prime}-y^{\prime}\right|^{2}}, \frac{1}{|t-s|}\right\}\right]^{\gamma+1} d y^{\prime} d s,
$$

where $D_{1}^{\prime}:=\left\{\left(y^{\prime}, s\right) \in \mathbb{R}^{n-1} \times \mathbb{R}:\left|x^{\prime}-y^{\prime}\right|>c \tau,|t-s|^{1 / 2}>c \tau\right\}$. We make the change of variables $x^{\prime}-y^{\prime}=y^{\prime \prime} \in \mathbb{R}^{n-1}$ and $t-s=s^{\prime} \in \mathbb{R}$, and we obtain that

$$
I_{1} \leq c \underset{\substack{y^{\prime \prime} \in \mathbb{R}^{n-1}, s^{\prime} \in \mathbb{R} \\\left|y^{\prime \prime}\right|>c \tau,\left|s^{\prime}\right|^{1 / 2}>c \tau}}{\int} \frac{\left|s^{\prime}\right|^{\theta}\left(\left|y^{\prime \prime}\right|+\left|s^{\prime}\right|^{1 / 2}\right)^{\alpha}}{\left|y^{\prime \prime}\right|^{n+k-1}}\left[\min \left\{\frac{1}{\left|y^{\prime \prime}\right|^{2}}, \frac{1}{\left|s^{\prime}\right|}\right\}\right]^{\gamma+1} d y^{\prime \prime} d s^{\prime} .
$$

If we set $s^{\prime}=\left(s^{\prime \prime}\right)^{2}, s^{\prime \prime} \in \mathbb{R}$, then

$$
I_{1} \leq c \underset{\substack{y^{\prime \prime} \in \mathbb{R}^{n-1}, s^{\prime \prime \prime} \\ y^{\prime \prime}|>c \tau,| s^{\prime \prime} \mid>c \tau}}{ } \frac{\left|s^{\prime \prime}\right|^{2 \theta+1}\left(\left|y^{\prime \prime}\right|+\left|s^{\prime \prime}\right|\right)^{\alpha}}{\left|y^{\prime \prime}\right|^{n+k-1}}\left[\min \left\{\frac{1}{\left|y^{\prime \prime}\right|}, \frac{1}{\left|s^{\prime \prime}\right|}\right\}\right]^{2 \gamma+2} d y^{\prime \prime} d s^{\prime \prime} .
$$


Recall the notation $\tau=\delta(x)$. Next, we rescale in $y^{\prime \prime}$ and $s^{\prime \prime}$ by seting $y^{\prime \prime}=z \tau$, $z \in \mathbb{R}^{n-1}$ and $s^{\prime \prime}=r \tau, r \in \mathbb{R}$. This yields

$$
I_{1} \leq c \tau^{-N_{0}} \iint_{\substack{z \in \mathbb{R}^{n-1}, r \in \mathbb{R} \\|z|>c,|r|>c}} \frac{|r|^{2 \theta+1}(|z|+|r|)^{\alpha}}{|z|^{n+k-1}}\left[\min \left\{\frac{1}{|z|}, \frac{1}{|r|}\right\}\right]^{2 \gamma+2} d z d r
$$

In the case when $|r| \leq|z|$,

$$
I_{1} \leq c \tau^{-N_{0}} \iint_{\substack{z \in \mathbb{R}^{n-1}, r \in \mathbb{R} \\ c<|r| \leq|z|}} \frac{|r|^{2 \theta+1}}{|z|^{n+k+2 \gamma-\alpha+1}} d z d r
$$

Let $r=r^{\prime}|z|, r^{\prime} \in \mathbb{R}$. Then $\left|r^{\prime}\right| \leq 1$ and

$$
I_{1} \leq c \tau^{-N_{0}}\left(\int_{\substack{r^{\prime} \in \mathbb{R} \\\left|r^{\prime}\right| \leq 1}}\left|r^{\prime}\right|^{2 \theta+1} d r^{\prime}\right) \int_{\substack{z \in \mathbb{R}^{n-1} \\|z|>c}} \frac{d z}{|z|^{n+N_{0}-1}} \leq c \tau^{-N_{0}},
$$

since $z \in \mathbb{R}^{n-1}$ and $n+N_{0}-1>n-1$.

When $|z|<|r|$,

$$
\begin{aligned}
I_{1} & \leq c \tau^{-N_{0}} \int_{\substack{r \in \mathbb{R} \\
|r|>c}} \frac{1}{|r|^{1+2 \gamma-2 \theta-\alpha}}\left(\int_{\substack{z \in \mathbb{R}^{n-1} \\
c<|z|<|r|}} \frac{d z}{|z|^{n+k-1}}\right) d r \\
& \leq c \tau^{-N_{0}} \int_{\substack{r \in \mathbb{R} \\
|r|>c}} \frac{d r}{|r|^{N_{0}+1}} \\
& \leq c \tau^{-N_{0}} .
\end{aligned}
$$

The last inequality holds, since $N_{0}>0$. As of now we proved that $I_{1} \leq c \delta(x)^{-N_{0}}$.

Let the second domain of integration be

$$
D_{2}:=\left\{(y, s) \in \partial \Omega \times \mathbb{R}:|\hat{x}-y|>c \tau \text { and }|t-s|^{1 / 2}<c \tau\right\}
$$


and set

$$
I_{2}:=\iint_{D_{2}}\left|\nabla^{k+1} \partial_{\text {time }}^{\gamma} I_{\text {time }}^{\theta}[E(x-y, t-s)]\right|\left(|\hat{x}-y|+|t-s|^{1 / 2}\right)^{\alpha} d \sigma_{y} d s
$$

Following the first couple of steps of the previous case, we obtain that

$$
\begin{aligned}
I_{2} & \leq c \tau^{-N_{0}} \int_{\substack{z \in \mathbb{R}^{n-1}, r \in \mathbb{R} \\
|z|>c,|r|<c}} \frac{|r|^{2 \theta+1}(|z|+|r|)^{\alpha}}{|z|^{n+k-1}}\left[\min \left\{\frac{1}{|z|}, \frac{1}{|r|}\right\}\right]^{2 \gamma+2} d z d r \\
& =c \tau^{-N_{0}} \iint_{\substack{z \in \mathbb{R}^{n-1}, r \in \mathbb{R} \\
|r|<c<|z|}} \frac{|r|^{2 \theta+1}(|z|+|r|)^{\alpha}}{|z|^{n+k+2 \gamma+1}} d z d r \\
& \leq c \tau^{-N_{0}} \iint_{\substack{z \in \mathbb{R}^{n-1}, r \in \mathbb{R} \\
|r|<c<|z|}} \frac{|r|^{2 \theta+1}}{|z|^{n+k+2 \gamma-\alpha+1}} d z d r .
\end{aligned}
$$

After the change of variables $r=r^{\prime}|z|, r^{\prime} \in \mathbb{R}$, we get that

$$
\begin{aligned}
I_{2} & \leq c \tau^{-N_{0}}\left(\int_{\substack{r^{\prime} \in \mathbb{R} \\
\left|r^{\prime}\right|<c}}\left|r^{\prime}\right|^{2 \theta+1} d r^{\prime}\right) \int_{\substack{z \in \mathbb{R}^{n-1} \\
|z|>c}} \frac{d z}{|z|^{n+N_{0}-1}} \\
& \leq c \tau^{-N_{0}},
\end{aligned}
$$

since $z \in \mathbb{R}^{n-1}$ and $n+N_{0}-1>n-1$. This proves that $I_{2} \leq c \delta(x)^{-N_{0}}$.

For the third part, let

$$
I_{3}:=\iint_{D_{3}}\left|\nabla^{k+1} \partial_{\text {time }}^{\gamma} I_{\text {time }}^{\theta}[E(x-y, t-s)]\right|\left(|\hat{x}-y|+|t-s|^{1 / 2}\right)^{\alpha} d \sigma_{y} d s
$$

where $D_{3}:=\left\{(y, s) \in \partial \Omega \times \mathbb{R}:|\hat{x}-y|<c \tau\right.$ and $\left.|t-s|^{1 / 2}>c \tau\right\}$. By (1.2.19),

$$
I_{3} \leq c \iint_{D_{3}} \frac{|t-s|^{\theta}\left(|\hat{x}-y|+|t-s|^{1 / 2}\right)^{\alpha}}{|x-y|^{n+k+1+2 \gamma}}\left[\min \left\{1, \frac{|x-y|^{2}}{|t-s|}\right\}\right]^{\gamma+1} d \sigma_{y} d s
$$

Now $|x-\hat{x}| \leq|x-y|$ for any $y \in \partial \Omega$, hence $|\hat{x}-y| \leq 2|x-y|$, and $|x-y|<c \tau$ for 
$(y, s) \in D_{3}$. Therefore

$$
I_{3} \leq c \iint_{D_{3}^{\prime}} \frac{|t-s|^{\theta}\left(|x-y|+|t-s|^{1 / 2}\right)^{\alpha}}{|x-y|^{n+k+1+2 \gamma}}\left[\min \left\{1, \frac{|x-y|^{2}}{|t-s|}\right\}\right]^{\gamma+1} d \sigma_{y} d s,
$$

where

$$
D_{3}^{\prime}:=\left\{(y, s) \in \partial \Omega \times \mathbb{R}:|x-y|<c \tau \text { and }|t-s|^{1 / 2}>c \tau\right\} .
$$

Since $x \in \Omega$ and $y \in \partial \Omega, x=\left(x^{\prime}, \varphi\left(x^{\prime}\right)+\tau\right)$ and $y=\left(y^{\prime}, \varphi\left(y^{\prime}\right)\right)$, where $x^{\prime}, y^{\prime} \in \mathbb{R}^{n-1}$, $\tau \in \mathbb{R}_{+}$and the graph of $\varphi$ is the boundary of $\Omega$. Then $|x-y| \approx\left|x^{\prime}-y^{\prime}\right|+\tau$, $d \sigma_{y} \approx d y^{\prime}$ and $I_{3}$ is majorized by a constant multiple of

$$
\iint_{D_{3}^{\prime \prime}} \frac{|t-s|^{\theta}\left(\left|x^{\prime}-y^{\prime}\right|+\tau+|t-s|^{1 / 2}\right)^{\alpha}}{\left(\left|x^{\prime}-y^{\prime}\right|+\tau\right)^{n+k+1+2 \gamma}}\left[\min \left\{1, \frac{\left(\left|x^{\prime}-y^{\prime}\right|+\tau\right)^{2}}{|t-s|}\right\}\right]^{\gamma+1} d y^{\prime} d s
$$

where

$$
D_{3}^{\prime \prime}:=\left\{\left(y^{\prime}, s\right) \in \mathbb{R}^{n-1} \times \mathbb{R}:\left|x^{\prime}-y^{\prime}\right|+\tau<c \tau \text { and }|t-s|^{1 / 2}>c \tau\right\} .
$$

Let $x^{\prime}-y^{\prime}=y^{\prime \prime}$ and $t-s=s^{\prime}$. Then

$$
I_{3} \leq c \iint_{D_{3}^{\prime \prime \prime}} \frac{\left|s^{\prime}\right|^{\theta}\left(\left|y^{\prime \prime}\right|+\tau+\left|s^{\prime}\right|^{1 / 2}\right)^{\alpha}}{\left(\left|y^{\prime \prime}\right|+\tau\right)^{n+k+1+2 \gamma}}\left[\min \left\{1, \frac{\left(\left|y^{\prime \prime}\right|+\tau\right)^{2}}{\left|s^{\prime}\right|}\right\}\right]^{\gamma+1} d y^{\prime \prime} d s^{\prime}
$$

Here $D_{3}^{\prime \prime \prime}:=\left\{\left(y^{\prime \prime}, s^{\prime}\right) \in \mathbb{R}^{n-1} \times \mathbb{R}:\left|y^{\prime \prime}\right|+\tau<c \tau,\left|s^{\prime}\right|^{1 / 2}>c \tau\right\}$. After the change of variables $s^{\prime}=\left(s^{\prime \prime}\right)^{2}, s^{\prime \prime} \in \mathbb{R}$, we obtain that

$$
I_{3} \leq c \underset{\substack{y^{\prime \prime} \in \mathbb{R}^{n-1}, s^{\prime \prime} \in \mathbb{R} \\\left|y^{\prime \prime}\right|+\tau<c \tau,\left|s^{\prime \prime}\right|>c \tau}}{\int} \frac{\left|s^{\prime \prime}\right|^{2 \theta+1}\left(\left|y^{\prime \prime}\right|+\tau+\left|s^{\prime \prime}\right|\right)^{\alpha}}{\left(\left|y^{\prime \prime}\right|+\tau\right)^{n+k+1+2 \gamma}}\left[\min \left\{1, \frac{\left|y^{\prime \prime}\right|+\tau}{\left|s^{\prime \prime}\right|}\right\}\right]^{2 \gamma+2} d y^{\prime \prime} d s^{\prime \prime}
$$


Now rescale in $y^{\prime \prime}$ and $s^{\prime \prime}$ by setting $y^{\prime \prime}=z \tau, z \in \mathbb{R}^{n-1}$ and $s^{\prime \prime}=r \tau, r \in \mathbb{R}$. Then

$$
\begin{aligned}
I_{3} & \leq c \tau^{-N_{0}} \iint_{\substack{z \in \mathbb{R}^{n-1}, r \in \mathbb{R} \\
|z|+1<c,|r|>c}} \frac{|r|^{2 \theta+1}(|z|+1+|r|)^{\alpha}}{(|z|+1)^{n+k+1+2 \gamma}}\left[\min \left\{1, \frac{|z|+1}{|r|}\right\}\right]^{2 \gamma+2} d z d r \\
& \leq c \tau^{-N_{0}} \iiint_{\substack{z \in \mathbb{R}^{n-1}, r \in \mathbb{R} \\
|z|+1<c<|r|}} \frac{d z d r}{(|z|+1)^{n+k-1}|r|^{2 \gamma-2 \theta-\alpha+1}} \\
& \leq c \tau^{-N_{0}} \int_{\substack{r \in \mathbb{R} \\
|r|>c}} \frac{d r}{|r|^{N_{0}+1}} \\
& \leq c \tau^{-N_{0}} .
\end{aligned}
$$

The integral in $r$ is bounded, since $N_{0}+1>1$ and $r \in \mathbb{R}$. This shows that $I_{3} \leq$ $c \delta(x)^{-N_{0}}$

Finally, we let the last domain of integration be

$$
D_{4}:=\left\{(y, s) \in \partial \Omega \times \mathbb{R}:|\hat{x}-y|<c \tau \text { and }|t-s|^{1 / 2}<c \tau\right\} .
$$

We also set

$$
I_{4}:=\iint_{D_{4}}\left|\nabla^{k+1} \partial_{\text {time }}^{\gamma} I_{\text {time }}^{\theta}[E(x-y, t-s)]\right|\left(|\hat{x}-y|+|t-s|^{1 / 2}\right)^{\alpha} d \sigma_{y} d s
$$

As in the case of $I_{3}$,

$$
I_{4} \leq c \tau^{-N_{0}} \iint_{\substack{z \in \mathbb{R}^{n-1}, r \in \mathbb{R} \\|z|+1<c,|r|<c}} \frac{|r|^{2 \theta+1}(|z|+1+|r|)^{\alpha}}{(|z|+1)^{n+k+1+2 \gamma}}\left[\min \left\{1, \frac{|z|+1}{|r|}\right\}\right]^{2 \gamma+2} d z d r .
$$


In the case when $|r| \leq|z|+1$,

$$
\begin{aligned}
I_{4} & \leq c \tau^{-N_{0}} \iint_{\substack{z \in \mathbb{R}^{n-1}, r \in \mathbb{R} \\
|r| \leq|z|+1<c}} \frac{|r|^{2 \theta+1}(|z|+1+|r|)^{\alpha}}{(|z|+1)^{n+k+2 \gamma+1}} d z d r \\
& \leq c \tau^{-N_{0}} \iint_{\substack{z \in \mathbb{R}^{n-1}, r \in \mathbb{R} \\
|r| \leq|z|+1<c}} \frac{|r|^{2 \theta+1}}{(|z|+1)^{n+k+2 \gamma-\alpha+1}} d z d r \\
& \leq c \tau^{-N_{0}}\left(\int_{\substack{r \in \mathbb{R} \\
|r|<c}}|r|^{2 \theta+1} d r \int_{\substack{z \in \mathbb{R}^{n-1} \\
|z|+1<c}} \frac{d z}{(|z|+1)^{n+k+2 \gamma-\alpha+1}}\right. \\
& \leq c \tau^{-N_{0}} .
\end{aligned}
$$

On the other hand, when $|z|+1<|r|$, we have that

$$
\begin{aligned}
I_{4} & \leq c \tau^{-N_{0}} \iint_{\substack{z \in \mathbb{R}^{n-1}, r \in \mathbb{R} \\
|z|+1<|r|<c}} \frac{d z d r}{(|z|+1)^{n+k-1}|r|^{2 \gamma-2 \theta-\alpha+1}} \\
& \leq c \tau^{-N_{0}}\left(\int_{\substack{z \in \mathbb{R}^{n-1} \\
|z|+1<c}} \frac{d z}{(|z|+1)^{n+k-1}}\right) \int_{\substack{r \in \mathbb{R} \\
1<|r|<c}} \frac{d r}{|r|^{2 \gamma-2 \theta-\alpha+1}} \\
& \leq c \tau^{-N_{0}},
\end{aligned}
$$

which completes the proof of (4.1.20) and of the case $0<\theta<1$ of the theorem.

Analogously to the above discussion, if $\theta=0$, it is enough to show that there exists a positive constant $c<\infty$ such that for any $(x, t) \in \Omega \times \mathbb{R}$,

$$
\int_{\mathbb{R}} \int_{\partial \Omega}\left|\nabla^{k+1} \partial_{\text {time }}^{\gamma}[E(x-y, t-s)]\right|\left(|\hat{x}-y|+|t-s|^{1 / 2}\right)^{\alpha} d \sigma_{y} d s \leq c \delta(x)^{-N_{0}}
$$

We devide the domain of integration in two parts. First, we consider the domain

$$
D^{\prime}:=\left\{(y, s) \in \partial \Omega \times \mathbb{R}:|\hat{x}-y|+|t-s|^{1 / 2}>c \tau\right\}
$$


and we set

$$
I^{\prime}:=\iint_{D^{\prime}}\left|\nabla^{k+1} \partial_{\text {time }}^{\gamma}[E(x-y, t-s)]\right|\left(|\hat{x}-y|+|t-s|^{1 / 2}\right)^{\alpha} d \sigma_{y} d s
$$

Using 1.2.13) to bound the derivatives of the fundamental solution $E$, and the fact that $|\hat{x}-y| \leq 2|x-y|$,

$$
\begin{aligned}
I^{\prime} & \leq c \iint_{D^{\prime}} \frac{\left(|\hat{x}-y|+|t-s|^{1 / 2}\right)^{\alpha}}{\left(|x-y|+|t-s|^{1 / 2}\right)^{n+k+1+2 \gamma}} d \sigma_{y} d s \\
& \leq c \iint_{D^{\prime}} \frac{d \sigma_{y} d s}{\left(|\hat{x}-y|+|t-s|^{1 / 2}\right)^{n+k+2 \gamma-\alpha+1}} .
\end{aligned}
$$

Since $\hat{x}, y \in \partial \Omega$, we can make the change of variables $y=\left(y^{\prime}, \varphi\left(y^{\prime}\right)\right)$, where $y^{\prime} \in \mathbb{R}^{n-1}$, and set $\hat{x}=\left(x^{\prime}, \varphi\left(x^{\prime}\right)\right), x^{\prime} \in \mathbb{R}^{n-1}$ (here the graph of $\varphi$ is the boundary of $\Omega$ ). Then $|\hat{x}-y| \approx\left|x^{\prime}-y^{\prime}\right|$ and

$$
I^{\prime} \leq c \underset{\substack{y^{\prime} \in \mathbb{R}^{n-1}, s \in \mathbb{R} \\\left|x^{\prime}-y^{\prime}\right|+|t-s|^{1 / 2}>c \tau}}{\int} \frac{d y^{\prime} d s}{\left(\left|x^{\prime}-y^{\prime}\right|+|t-s|^{1 / 2}\right)^{n+k+2 \gamma-\alpha+1}} .
$$

If we let $x^{\prime}-y^{\prime}=y^{\prime \prime} \in \mathbb{R}^{n-1}$ and $t-s=s^{\prime} \in \mathbb{R}$, then (4.1.22) can be rewritten as

$$
I^{\prime} \leq c \int_{\substack{y^{\prime \prime} \in \mathbb{R}^{n-1}, s^{\prime} \in \mathbb{R} \\\left|y^{\prime \prime}\right|+\left|s^{\prime}\right|^{1 / 2}>c \tau}} \frac{d y^{\prime \prime} d s^{\prime}}{\left(\left|y^{\prime \prime}\right|+\left|s^{\prime}\right|^{1 / 2}\right)^{n+k+2 \gamma-\alpha+1}}
$$

Moreover, if $s^{\prime}=\left(s^{\prime \prime}\right)^{2}, s^{\prime \prime} \in \mathbb{R}$, then

$$
\begin{aligned}
I^{\prime} & \leq c \iiint_{\substack{y^{\prime} \in \mathbb{R}^{n-1}, s^{\prime \prime} \in \mathbb{R} \\
\left|y^{\prime \prime}\right|+\left|s^{\prime \prime}\right|>c \tau}} \frac{\left|s^{\prime \prime}\right|}{\left(\left|y^{\prime \prime}\right|+\left|s^{\prime \prime}\right|\right)^{n+k+2 \gamma-\alpha+1}} d y^{\prime \prime} d s^{\prime \prime} \\
& \leq c \iiint_{\substack{y^{\prime} \in \mathbb{R}^{n-1}, s^{\prime \prime} \in \mathbb{R} \\
\left|y^{\prime \prime}\right|+\left|s^{\prime \prime}\right|>c \tau}} \frac{d y^{\prime \prime} d s^{\prime \prime}}{\left(\left|y^{\prime \prime}\right|+\left|s^{\prime \prime}\right|\right)^{n+k+2 \gamma-\alpha}} .
\end{aligned}
$$


Finally, we rescale in $y^{\prime \prime}$ and $s^{\prime \prime}$ using the change of variables $y^{\prime \prime}=z \tau, z \in \mathbb{R}^{n-1}$ and $s^{\prime \prime}=r \tau, r \in \mathbb{R}$. Hence

$$
I^{\prime} \leq c \tau^{-N_{0}} \iint_{\substack{z \in \mathbb{R}^{n-1} \\|z|+|r|>c \tau}} \frac{d z d r}{(|z|+|r|)^{n+k+2 \gamma-\alpha}} \leq c \tau^{-N_{0}}
$$

where we have used that $n+k+2 \gamma-\alpha=n+N_{0}>n,(z, r) \in \mathbb{R}^{n}$.

Denote the other domain of integration by

$$
D^{\prime \prime}:=\left\{(y, s) \in \partial \Omega \times \mathbb{R}:|\hat{x}-y|+|t-s|^{1 / 2} \leq c \tau\right\}
$$

and set

$$
I^{\prime \prime}:=\iint_{D^{\prime \prime}}\left|\nabla^{k+1} \partial_{\text {time }}^{\gamma}[E(x-y, t-s)]\right|\left(|\hat{x}-y|+|t-s|^{1 / 2}\right)^{\alpha} d \sigma_{y} d s .
$$

Using again (1.2.13) to bound the derivatives of $E$, we get that

$$
I^{\prime \prime} \leq c \tau^{\alpha} \iint_{D^{\prime \prime}} \frac{d \sigma_{y} d s}{\left(|x-y|+|t-s|^{1 / 2}\right)^{n+k+1+2 \gamma}} .
$$

By the inequality $\tau=|x-\hat{x}| \leq|x-y| \leq|x-y|+|t-s|^{1 / 2}$, which holds for any $y \in \partial \Omega$

$$
\begin{aligned}
I^{\prime \prime} & \leq c \tau^{\alpha} \iint_{D^{\prime \prime}} \frac{d \sigma_{y} d s}{\tau^{n+k+2 \gamma+1}} \\
& \leq c \tau^{-N_{0}} \cdot \tau^{-n-1}\left(\int_{\substack{y \in \partial \Omega \\
|\hat{x}-y|<c \tau}} d \sigma_{y}\right) \cdot \int_{\substack{s \in \mathbb{R} \\
|t-s|^{1 / 2}<c \tau}} d s \\
& \leq c \tau^{-N_{0}} .
\end{aligned}
$$

This shows that (4.1.19) holds for $\theta=0$, as well, hence the proof of Theorem 4.1.3 is complete.

Replacing $I_{\text {time }}^{\theta}$ with $D_{\text {time }}^{\theta}$ in Theorem 4.1.3 yields the following. 
Theorem 4.1.4. Consider a Lipschitz domain $\Omega$ in $\mathbb{R}^{n}$, and set $N_{0}^{\prime}:=k+2 \gamma+2 \theta-$ $\alpha>0$, where $k, \gamma \in \mathbb{N}_{0}, 0 \leq \theta<1,0<\alpha<1$. Then

$$
\delta^{N_{0}^{\prime}} \nabla^{k} \partial_{\text {time }}^{\gamma} D_{\text {time }}^{\theta} \mathcal{D}: B_{\alpha, p a r}^{\infty}(\partial \Omega \times \mathbb{R}) \longrightarrow L^{\infty}(\Omega \times \mathbb{R})
$$

is bounded.

Proof. According to the definition of the fractional time-derivative and integral operators $D_{\text {time }}^{\theta}$ and $I_{\text {time }}^{\theta}($ see $(1.1 .3)$ and (1.1.4)), we have that

$$
\delta^{N_{0}^{\prime}} \nabla^{k} \partial_{\text {time }}^{\gamma} D_{\text {time }}^{\theta}=\delta^{N_{0}^{\prime}} \nabla^{k} \partial_{\text {time }}^{\gamma+1} I_{\text {time }}^{1-\theta}
$$

For $0<\theta<1$, we use Theorem 4.1.3 and (4.1.19) to obtain (4.1.23). If $\theta=0$, notice that $D_{\text {time }}^{0}=\partial_{\text {time }} I_{\text {time }}^{1}=I=I_{\text {time }}^{0}$, and we can use the case $\theta=0$ of Theorem 4.1.3 to conclude the desired result.

\subsection{General case}

As of now, we have presented two endpoint cases, i.e. Theorem 4.1.2 for $p=1$ and Theorem 4.1 .4 for $p=\infty$. In this section we first give an intermediate result, then we treat the derivatives $\nabla^{k} \partial_{\text {time }}^{\gamma} D_{\text {time }}^{\theta}$ for a target space with smoothness, obtaining the expected mapping property of the double layer potential $\mathcal{D}$.

We begin with interpolating between the results of Theorems 4.1.2 and 4.1.4.

Theorem 4.2.1. Let $\Omega$ be a Lipschitz domain in $\mathbb{R}^{n}, 0<\alpha<1,1 \leq p \leq \infty$, $k, \gamma \in \mathbb{N}_{0}, 0 \leq \theta<1$ and set $N:=k+2 \gamma+2 \theta-\alpha>0$ such that $2 \gamma+2 \theta>\alpha$ for $0<\theta<1$. Then the operator

$$
\delta^{N-\frac{1}{p}} \nabla^{k} \partial_{\text {time }}^{\gamma} D_{\text {time }}^{\theta} \mathcal{D}: B_{\alpha, p a r}^{p}(\partial \Omega \times \mathbb{R}) \longrightarrow L^{p}(\Omega \times \mathbb{R})
$$


is bounded.

Proof. For $z=u+i v, 0 \leq u \leq 1$ and $v \in \mathbb{R}$, we define the family of operators

$$
T_{z}:=\delta^{N-z} \nabla^{k} \partial_{\text {time }}^{\gamma} D_{\text {time }}^{\theta} \mathcal{D}
$$

According to Theorem 4.1.4, the operator $T_{i v}=\delta^{-i v} \delta^{N} \nabla^{k} \partial_{\text {time }}^{\gamma} D_{\text {time }}^{\theta} \mathcal{D}$ is bounded from $B_{\alpha, \text { par }}^{\infty}(\partial \Omega \times \mathbb{R})$ into $L^{\infty}(\Omega \times \mathbb{R})$. Similarly, by Theorem 4.1.2, the operator $T_{1+i v}=\delta^{-i v} \delta^{N-1} \nabla^{k} \partial_{\text {time }}^{\gamma} D_{\text {time }}^{\theta} \mathcal{D}$ is bounded from $B_{\alpha, p a r}^{1}(\partial \Omega \times \mathbb{R})$ into $L^{1}(\Omega \times \mathbb{R})$. Now Theorem 3.4 from [CaTo2] implies that, for any $0<u=: \eta<1$,

$$
T_{z}:\left[B_{\alpha, p a r}^{\infty}(\partial \Omega \times \mathbb{R}), B_{\alpha, p a r}^{1}(\partial \Omega \times \mathbb{R})\right]_{\eta} \longrightarrow\left[L^{\infty}(\Omega \times \mathbb{R}), L^{1}(\Omega \times \mathbb{R})\right]_{\eta}
$$

is bounded. By part (g) of Theorem 2.3.20 (complex interpolation of Besov spaces) and Theorem 5.1.1 from [BeLö] (complex interpolation of Lebesgue spaces), the mapping property (4.2.26) further implies that

$$
T_{z}: B_{\alpha, p a r}^{p}(\partial \Omega \times \mathbb{R}) \longrightarrow L^{p}(\Omega \times \mathbb{R})
$$

is bounded for $p=\frac{1}{\eta} \in(1, \infty)$, uniformly in $v$. Since $T_{z}=\delta^{-i v} \delta^{N-\frac{1}{p}} \nabla^{k} \partial_{\text {time }}^{\gamma} D_{\text {time }}^{\theta} \mathcal{D}$, and we may take $v=0,(4.2 .27)$ implies that

$$
\delta^{N-\frac{1}{p}} \nabla^{k} \partial_{\text {time }}^{\gamma} D_{\text {time }}^{\theta} \mathcal{D}: B_{\alpha, p a r}^{p}(\partial \Omega \times \mathbb{R}) \longrightarrow L^{p}(\Omega \times \mathbb{R})
$$

is bounded for $1<p<\infty$. The endpoint cases, $p=1$ and $p=\infty$, of (4.2.28), folow from Theorems 4.1.2 and 4.1.4, respectively. Thus

$$
\delta^{N-\frac{1}{p}} \nabla^{k} \partial_{\text {time }}^{\gamma} D_{\text {time }}^{\theta} \mathcal{D}: B_{\alpha, p a r}^{p}(\partial \Omega \times \mathbb{R}) \longrightarrow L^{p}(\Omega \times \mathbb{R})
$$

for all $1 \leq p \leq \infty$, which completes the proof of the theorem. 
Remark 4.2.2. The following argument also leads to the conclusion of Theorem 4.2.1. The basic estimates (4.1.17) and (4.1.23) are equivalent with

$$
\nabla^{k} \partial_{\text {time }}^{\gamma} D_{\text {time }}^{\theta} \mathcal{D}: B_{\alpha, p a r}^{1}(\partial \Omega \times \mathbb{R}) \longrightarrow L^{1}\left(\mathbb{R} ; L^{1}\left(\Omega, \delta^{N^{\prime}} d x\right)\right)
$$

and

$$
\nabla^{k} \partial_{\text {time }}^{\gamma} D_{\text {time }}^{\theta} \mathcal{D}: B_{\alpha, p a r}^{\infty}(\partial \Omega \times \mathbb{R}) \longrightarrow L^{\infty}\left(\mathbb{R} ; L^{\infty}\left(\Omega, \delta^{N} d x\right)\right)
$$

respectively, where $N:=k+2 \gamma+2 \theta-\alpha>0, N^{\prime}:=N-1$, and $k, \gamma \in \mathbb{N}_{0}, 0 \leq \theta<1$, $0<\alpha<1$. Next we use the real interpolation method (part (h') of Remark 2.3.21) to obtain that

$$
\left(B_{\alpha, p a r}^{1}(\partial \Omega \times \mathbb{R}), B_{\alpha, p a r}^{\infty}(\partial \Omega \times \mathbb{R})\right)_{\eta, p}=B_{\alpha, p a r}^{p}(\partial \Omega \times \mathbb{R})
$$

for $0<\eta<1, \frac{1}{p}=1-\eta$. On the other hand, for the current assumptions on $\eta$ and $p$, using the real interpolation of Banach space valued $L^{p}$-spaces (Theorem 1.2.25), then the real interpolation of weighted $L^{p}$-spaces ( [Fre], Theorem 1), we have that

$$
\begin{aligned}
\left(L^{1}\left(\mathbb{R} ; L^{1}\left(\Omega, \delta^{N^{\prime}} d x\right)\right), L^{\infty}\right. & \left.\left(\mathbb{R} ; L^{\infty}\left(\Omega, \delta^{N} d x\right)\right)\right)_{\eta, p} \\
& =L^{p}\left(\mathbb{R} ;\left(L^{1}\left(\Omega, \delta^{N^{\prime}} d x\right), L^{\infty}\left(\Omega, \delta^{N} d x\right)\right)_{\eta, p}\right) \\
& =L^{p}\left(\mathbb{R} ; L^{p}\left(\Omega,\left(\delta^{N^{\prime}}\right)^{1-\eta}\left(\delta^{N}\right)^{\eta} d x\right)\right) \\
& =L^{p}\left(\mathbb{R} ; L^{p}\left(\Omega, \delta^{N-\frac{1}{p}} d x\right)\right)
\end{aligned}
$$

Consequently, (4.2.29), (4.2.30) and (4.2.31) imply that,

$$
\nabla^{k} \partial_{\text {time }}^{\gamma} D_{\text {time }}^{\theta} \mathcal{D}: B_{\alpha, p a r}^{p}(\partial \Omega \times \mathbb{R}) \longrightarrow L^{p}\left(\mathbb{R} ; L^{p}\left(\Omega, \delta^{N-\frac{1}{p}} d x\right)\right)
$$


for $p=\frac{1}{1-\eta} \in(1, \infty)$, or, equivalently,

$$
\delta^{N-\frac{1}{p}} \nabla^{k} \partial_{\text {time }}^{\gamma} D_{\text {time }}^{\theta} \mathcal{D}: B_{\alpha, \text { par }}^{p}(\partial \Omega \times \mathbb{R}) \longrightarrow L^{p}(\Omega \times \mathbb{R})
$$

for $1<p<\infty$. The endpoint cases, $p=1$ and $p=\infty$, of (4.2.33) are the two basic estimates (4.1.17) and (4.1.23), respectively, already established in Section 4.1.

Next, for $\sigma \in \mathbb{R}$, we define the lifting operator $\dot{I}_{\sigma}: \mathrm{S}^{\prime}\left(\mathbb{R}^{n}\right) \rightarrow \mathrm{S}^{\prime}\left(\mathbb{R}^{n}\right)$ by $\dot{I}_{\sigma} f:=$ $\mathcal{F}^{-1}\left(|\cdot|^{-\sigma} \mathcal{F} f\right)$, where $0 \notin \operatorname{supp} \mathcal{F} f$. The operator $\dot{I}_{\sigma}$ is often called the Riesz potential of order $\sigma$. It is well-known ([Tri4], p. 242), that $\dot{I}_{\sigma}$ maps the homogeneous isotropic Triebel-Lizorkin space $\dot{F}_{\alpha}^{p, q}\left(\mathbb{R}^{n}\right)$ isomorphically onto $\dot{F}_{\alpha+\sigma}^{p, q}\left(\mathbb{R}^{n}\right)$, for any $0<p<\infty, 0<q \leq \infty$ and $\alpha \in \mathbb{R}$. In the particular case $q=2$ and $\alpha=0$, we have that

$$
\dot{I}_{\sigma}: L^{p}(\mathbb{R}) \longrightarrow \dot{L}_{\sigma}^{p}(\mathbb{R}) \quad \text { for } \sigma>0,1<p<\infty
$$

Going further, we present a preliminary result.

Lemma 4.2.3. Let $0<\theta<1,1<p<\infty, f \in \mathrm{S}^{\prime}(\mathbb{R})$ such that $D^{\theta} f \in L^{p}(\mathbb{R})$, where $D^{\theta}$ is the fractional derivative of order $\theta$. Then $f \in \dot{L}_{\theta}^{p}(\mathbb{R})$.

Proof. According to Lemma 5.1 (v) from [Mit2], we have that

$$
\begin{aligned}
\dot{I}_{-\theta} f & =\mathcal{F}^{-1}\left(|\xi|^{\theta} \mathcal{F} f\right) \\
& =c \mathcal{F}^{-1}\left\{[1-i \operatorname{sign}(\xi)]\left[|\xi|^{\theta}(1+i \operatorname{sign}(\xi))\right] \mathcal{F} f\right\} \\
& =c(I-H)\left(D^{\theta} f\right),
\end{aligned}
$$

where $H f$ denotes the Hilbert transform of $f$, and we have used the fact that $H f=$ $c \mathcal{F}^{-1}[i \operatorname{sign}(\xi) \mathcal{F} f]$. Now $D^{\theta} f \in L^{p}(\mathbb{R})$ implies $\dot{I}_{-\theta} f \in L^{p}(\mathbb{R})$ for $1<p<\infty$. Since 
$\dot{I}_{\theta}\left(\dot{I}_{-\theta} f\right)=f$, in view of $(4.2 .34)$, we can conclude that $f \in \dot{L}_{\theta}^{p}(\mathbb{R})$ for $0<\theta<1$ and $1<p<\infty$

In what follows, we explore several useful mapping properties of the double layer potential $\mathcal{D}$.

Corollary 4.2.4. Let $\Omega$ be a Lipschitz domain in $\mathbb{R}, 0<\alpha<1$ and $1<p<\infty$. Then

$$
\mathcal{D}: B_{\alpha, p a r}^{p}(\partial \Omega \times \mathbb{R}) \longrightarrow L^{p}\left(\Omega ; \dot{L}_{\left(\alpha+\frac{1}{p}\right) / 2}^{p}(\mathbb{R})\right)
$$

is bounded.

Proof. Let us consider $f \in B_{\alpha, p a r}^{p}(\partial \Omega \times \mathbb{R})$. Then, by Theorem 4.2.1,

$$
D_{\text {time }}^{\theta}\left(\delta^{N-\frac{1}{p}} \nabla^{k} \partial_{\text {time }}^{\gamma} \mathcal{D} f\right) \in L^{p}(\Omega \times \mathbb{R})=L^{p}\left(\Omega ; L^{p}(\mathbb{R})\right)
$$

where $0 \leq \theta<1, k, \gamma \in \mathbb{N}_{0}$, and $N=k+2 \gamma+2 \theta-\alpha>0$ (also, $2 \gamma+2 \theta>\alpha$ for $0<\theta<1)$. Now applying Lemma 4.2 .3 in the time variable, we obtain that

$$
\delta^{N-\frac{1}{p}} \nabla^{k} \partial_{\text {time }}^{\gamma} \mathcal{D} f \in L^{p}\left(\Omega ; \dot{L}_{\theta}^{p}(\mathbb{R})\right)
$$

for the current assumptions on $N, k, \gamma$ and $\theta$. In the special case when $k=\gamma=0$ and $\theta=\left(\alpha+\frac{1}{p}\right) / 2$, all these conditions are verified and the conclusion of Lemma 4.2.3 becomes

$$
\mathcal{D} f \in L^{p}\left(\Omega ; \dot{L}_{\left(\alpha+\frac{1}{p}\right) / 2}^{p}(\mathbb{R})\right)
$$

which proves the corollary.

Lemma 4.2.5. Let us consider $\zeta \in C^{\infty}\left(\mathbb{R}^{n} \times \mathbb{R}\right)$ such that there exists a constant $k>0$ with $\operatorname{supp} \zeta \subseteq\left\{x \in \mathbb{R}^{n}: \operatorname{dist}(x, \partial \Omega)<k\right\} \times \mathbb{R}$, and $\zeta \equiv 1$ in a neighborhood of 
$\partial \Omega \times \mathbb{R}$. For $0<\alpha<1,1<p<\infty$ and $\Omega$ Lipschitz domain in $\mathbb{R}^{n}$,

$$
\zeta \mathcal{D}: B_{\alpha, p a r}^{p}(\partial \Omega \times \mathbb{R}) \longrightarrow L^{p}\left(\Omega ; L^{p}(\mathbb{R})\right)
$$

is bounded.

Proof. Since $B_{\alpha, p a r}^{p}(\partial \Omega \times \mathbb{R}) \subset L^{p}(\partial \Omega \times \mathbb{R})$ by definition, it is enough to show that

$$
\zeta \mathcal{D}: L^{p}(\partial \Omega \times \mathbb{R}) \longrightarrow L^{p}(\Omega \times \mathbb{R})
$$

is bounded. For $f \in L^{p}(\partial \Omega \times \mathbb{R})$,

$$
\begin{aligned}
\|(\zeta \mathcal{D}) f\|_{L^{p}(\Omega \times \mathbb{R})}^{p} & =c \int_{\mathbb{R}} \int_{\partial \Omega} \int_{0}^{1}\left|[(\zeta \mathcal{D}) f]\left(x^{\prime}, x_{n}, t\right)\right|^{p} d x_{n} d \sigma_{x^{\prime}} d t \\
& \leq c\|\mathcal{N}[(\zeta \mathcal{D}) f]\|_{L^{p}(\partial \Omega \times \mathbb{R})}^{p} \leq c\|\mathcal{N}(\mathcal{D} f)\|_{L^{p}(\partial \Omega \times \mathbb{R})}^{p} \\
& \leq c\|f\|_{L^{p}(\partial \Omega \times \mathbb{R})}^{p},
\end{aligned}
$$

where the last inequality holds cf. Theorem 4.17 in [Bro2].

Theorem 4.2.6. For $0<\alpha<1,1<p<\infty, \Omega$ Lipschitz domain in $\mathbb{R}^{n}$, and $\zeta$ as in Lemma 4.2.5,

$$
\zeta \mathcal{D}: B_{\alpha, p a r}^{p}(\partial \Omega \times \mathbb{R}) \longrightarrow L^{p}\left(\Omega ; B_{\left(\alpha+\frac{1}{p}\right) / 2}^{p}(\mathbb{R})\right)
$$

is bounded.

Proof. From Corollary 4.2.4 and Lemma 4.2.5 we have

$$
\zeta \mathcal{D}: B_{\alpha, p a r}^{p}(\partial \Omega \times \mathbb{R}) \longrightarrow L^{p}\left(\Omega ; \dot{L}_{\left(\alpha+\frac{1}{p}\right) / 2}^{p}(\mathbb{R}) \cap L^{p}(\mathbb{R})\right)
$$

is bounded, or, equivalently (by Theorem 6.3.2 from [BeLö]),

$$
\zeta \mathcal{D}: B_{\alpha, p a r}^{p}(\partial \Omega \times \mathbb{R}) \longrightarrow L^{p}\left(\Omega ; L_{\left(\alpha+\frac{1}{p}\right) / 2}^{p}(\mathbb{R})\right)
$$


is bounded. Now let $0<\alpha_{0}, \alpha_{1}<1$ such that $\alpha_{0} \neq \alpha_{1}$. Then the operator $\zeta \mathcal{D}$ : $B_{\alpha_{i}, p a r}^{p}(\partial \Omega \times \mathbb{R}) \rightarrow L^{p}\left(\Omega ; L_{\left(\alpha_{i}+\frac{1}{p}\right) / 2}^{p}(\mathbb{R})\right), i=0,1$ is bounded. Using real interpolation of parabolic Besov spaces (part (h) of Theorem 2.3.20), real interpolation of Banach space valued $L^{p}$-spaces (Theorem 1.2.25) and finally, real interpolation of isotropic Sobolev spaces (Theorem 6.4.5.(4) from [BeLö]), we obtain that

$$
\zeta \mathcal{D}: B_{\alpha^{*}, p a r}^{p}(\partial \Omega \times \mathbb{R}) \longrightarrow L^{p}\left(\Omega ; B_{\left(\alpha^{*}+\frac{1}{p}\right) / 2}^{p}(\mathbb{R})\right),
$$

where $\alpha^{*}=(1-\theta) \alpha_{0}+\theta \alpha_{1}$ and $0<\theta<1$. From this, (4.2.35) follows.

Theorem 4.2.7. For $0<\alpha<1,1<p<\infty$ and $\Omega$ Lipschitz domain in $\mathbb{R}^{n}$, the operator

$$
\mathcal{D}: B_{\alpha, p a r}^{p}(\partial \Omega \times \mathbb{R}) \longrightarrow L^{p}\left(\mathbb{R} ; B_{\alpha+\frac{1}{p}}^{p}(\Omega)\right)
$$

is bounded.

Proof. Considering two special cases $(\gamma=\theta=0, k=1$ and $\gamma=\theta=0, k=2)$ of Theorem 4.2.1, we obtain that the oparators

$$
\delta^{1-\alpha-\frac{1}{p}} \nabla \mathcal{D}: B_{\alpha, p a r}^{p}(\partial \Omega \times \mathbb{R}) \longrightarrow L^{p}(\Omega \times \mathbb{R})
$$

and

$$
\delta^{2-\alpha-\frac{1}{p}} \nabla^{2} \mathcal{D}: B_{\alpha, p a r}^{p}(\partial \Omega \times \mathbb{R}) \longrightarrow L^{p}(\Omega \times \mathbb{R})
$$

are bounded for $1 \leq p \leq \infty, 0<\alpha<1$. We denote $\left[\alpha+\frac{1}{p}\right]=: l$ and $\left\{\alpha+\frac{1}{p}\right\}=: s$. If $0<\alpha+\frac{1}{p}<1$, then (4.1.1) yields

$$
\delta^{1-s} \nabla \mathcal{D}: B_{\alpha, p a r}^{p}(\partial \Omega \times \mathbb{R}) \longrightarrow L^{p}(\Omega \times \mathbb{R}) .
$$


When $1<\alpha+\frac{1}{p}<2$, from (4.1.2) we have

$$
\delta^{1-s} \nabla^{2} \mathcal{D}: B_{\alpha, p a r}^{p}(\partial \Omega \times \mathbb{R}) \longrightarrow L^{p}(\Omega \times \mathbb{R})
$$

These two cases imply that for $l=0,1$

$$
f \in B_{\alpha, p a r}^{p}(\partial \Omega \times \mathbb{R}) \Longrightarrow \delta^{1-s} \nabla^{l+1} \mathcal{D} f \in L^{p}\left(\mathbb{R} ; L^{p}(\Omega)\right)
$$

plus natural estimates. Now applying Theorem 4.1 of [JeKe] in the spacial variable, we obtain that $\mathcal{D} f \in L^{p}\left(\mathbb{R} ; B_{l+s}^{p}(\Omega)\right)$ for any $f \in B_{\alpha, p a r}^{p}(\partial \Omega \times \mathbb{R})$, which amounts to

$$
\mathcal{D}: B_{\alpha, p a r}^{p}(\partial \Omega \times \mathbb{R}) \longrightarrow L^{p}\left(\mathbb{R} ; B_{\alpha+\frac{1}{p}}^{p}(\Omega)\right)
$$

for $0<\alpha<1,1<p<\infty$ such that $\alpha+\frac{1}{p} \in(0,1) \cup(1,2)$.

Since $\alpha+\frac{1}{p} \in(0,2)$, we are left with the case when $\alpha+\frac{1}{p}=1$. Let $\alpha_{0}, \alpha_{1} \in(0,1)$ such that $\alpha_{0}+\frac{1}{p}=\frac{1}{2}$ and $\alpha_{1}+\frac{1}{p}=\frac{3}{2}$. Then $\alpha_{0} \neq \alpha_{1}$, and (4.2.38) implies that

$$
\mathcal{D}: B_{\alpha_{0}, p a r}^{p}(\partial \Omega \times \mathbb{R}) \longrightarrow L^{p}\left(\mathbb{R} ; B_{\frac{1}{2}}^{p}(\Omega)\right)
$$

and

$$
\mathcal{D}: B_{\alpha_{1}, p a r}^{p}(\partial \Omega \times \mathbb{R}) \longrightarrow L^{p}\left(\mathbb{R} ; B_{\frac{3}{2}}^{p}(\Omega)\right) .
$$

By the real interpolation method we obtain that

$$
\mathcal{D}: B_{\alpha, p a r}^{p}(\partial \Omega \times \mathbb{R}) \longrightarrow L^{p}\left(\mathbb{R} ; B_{\alpha^{*}}^{p}(\Omega)\right),
$$

where $\alpha=(1-\theta) \alpha_{0}+\theta \alpha_{1}, \alpha^{*}=\frac{1}{2}(1-\theta)+\frac{3}{2} \theta$ and $0<\theta<1$. If $\theta=1 / 2$, then $\alpha=1-\frac{1}{p}$ and $\alpha^{*}=1$, hence

$$
\mathcal{D}: B_{\alpha, p a r}^{p}(\partial \Omega \times \mathbb{R}) \longrightarrow L^{p}\left(\mathbb{R} ; B_{1}^{p}(\Omega)\right)
$$


for $0<\alpha<1,1<p<\infty$ such that $\alpha=1-\frac{1}{p}$.

Finally, (4.2.38) and (4.2.39) yield to the desired conclusion.

Some of the general results advertised at the beginning of Section 4.2 (for any Lipschitz domain) are formulated in the next two theorems.

Theorem 4.2.8. Let $\Omega$ be a Lipschitz domain in $\mathbb{R}^{n}, 0<\alpha<1,1<p<\infty$, and $\zeta$ as in Lemma 4.2.5. Then

$$
\zeta \mathcal{D}: B_{\alpha, p a r}^{p}(\partial \Omega \times \mathbb{R}) \longrightarrow B_{\alpha+\frac{1}{p}, p a r}^{p}(\Omega \times \mathbb{R})
$$

is bounded.

Proof. According to Theorems 4.2.6 and 4.2.7, for $0<\alpha<1$ and $1<p<\infty$,

$$
\zeta \mathcal{D}: B_{\alpha, p a r}^{p}(\partial \Omega \times \mathbb{R}) \longrightarrow L^{p}\left(\Omega ; B_{\left(\alpha+\frac{1}{p}\right) / 2}^{p}(\mathbb{R})\right) \cap L^{p}\left(\mathbb{R} ; B_{\alpha+\frac{1}{p}}^{p}(\Omega)\right)
$$

is bounded. In view of the Fubini property of the diagonal Besov scale $B_{\alpha, p a r}^{p}$ (cf. Proposition 2.2.3) the proof is complete.

Theorem 4.2.9. Let $\Omega$ be a Lipschitz domain in $\mathbb{R}^{n}$ and $0<\alpha<1$. Then the caloric double layer potential

$$
\left.\mathcal{D}: B_{\alpha, p a r}^{\infty}(\partial \Omega \times \mathbb{R}) \longrightarrow B_{\alpha, p a r}^{\infty}(\Omega \times \mathbb{R})\right)
$$

is bounded.

In preparation, we state and prove a lemma of independent interest.

Lemma 4.2.10. Let $\Omega$ be a Lipschitz domain in $\mathbb{R}^{n}, 0<\alpha<1$ and consider $F$ from $C^{1}(\Omega \times \mathbb{R})$. Recall that for any $x \in \Omega$ the distance from $x$ to $\partial \Omega$ is denoted by $\delta(x)$. 
Assume that there is a positive constant $c_{0}$ such that the following holds:

$$
\sup _{(x, t) \in \Omega \times \mathbb{R}}\left[\delta(x)^{1-\alpha}|\nabla F(x, t)|+\delta(x)^{2-\alpha}\left|\partial_{t} F(x, t)\right|\right] \leq c_{0}<\infty
$$

Then there exists $c_{1}(\Omega, \alpha)>0$ such that

$$
\sup _{\substack{(x, t),(y, s) \in \Omega \times \mathbb{R} \\(x, t) \neq(y, s)}} \frac{|F(x, t)-F(y, s)|}{\left(|x-y|+|t-s|^{1 / 2}\right)^{\alpha}} \leq c_{1}(\Omega, \alpha) c_{0}<\infty .
$$

Proof. Let us denote by $e_{n}:=(0, \ldots, 0,1)$ the standard unit vector in the $n$-th direction in $\mathbb{R}^{n}$. For any $(x, t),(y, s) \in \Omega \times \mathbb{R}$ with $(x, t) \neq(y, s), r$ denotes the parabolic distance between $(x, t)$ and $(y, s)$. Then $r \approx|x-y|+|t-s|^{1 / 2}$ for any $(x, t),(y, s) \in \Omega \times \mathbb{R}$ with $(x, t) \neq(y, s)$. We shall choose a positive constant $M$ later such that the segment connecting the poits $\left(x+M r e_{n}, t\right)$ and $\left(y+M r e_{n}, s\right)$ lies inside $\Omega \times \mathbb{R}$. We observe that

$$
\begin{aligned}
|F(x, t)-F(y, s)| \leq & \left|F(x, t)-F\left(x+M r e_{n}, t\right)\right| \\
& +\mid F\left(x+\text { Mre }_{n}, t\right)-F\left(y+\text { Mre }_{n}, s\right) \mid \\
& +\mid F\left(y+\text { Mre }_{n}, s\right)-F(y, s) \mid \\
= & : I+I I+I I I .
\end{aligned}
$$

Then, in order to prove (4.2.41), it is enough to show that

$$
I \leq c_{0} c_{1}(\Omega, \alpha) r^{\alpha}, \quad I I \leq c_{0} c_{1}(\Omega, \alpha) r^{\alpha}, \text { and } I I I \leq c_{0} c_{1}(\Omega, \alpha) r^{\alpha}
$$

By the Fundamental Theorem of Calculus,

$$
I \leq \int_{0}^{1}\left|\nabla F\left(x+M_{r v e_{n}}, t\right) \cdot M r e_{n}\right| d v \leq \int_{0}^{1}\left|\nabla F\left(x+M r v e_{n}, t\right)\right| M r d v .
$$


Now using the hypothesis (4.2.40) with $\left(x+\right.$ Mrve $\left._{n}, t\right) \in \Omega \times \mathbb{R}$,

$$
I \leq M r \int_{0}^{1} \frac{c_{0}}{\delta\left(x+M r v e_{n}\right)^{1-\alpha}} d v
$$

Let $\hat{x} \in \partial \Omega$ be such that $\delta(x)=|x-\hat{x}|$. Then $\delta\left(x+M r v e_{n}\right)=\left|x+M r v e_{n}-\hat{x}\right| \geq$ $\mid x+$ Mrve $_{n}-x \mid=M r v$, hence

$$
I \leq c_{0} M r \int_{0}^{1} \frac{d v}{(M r v)^{1-\alpha}}=c_{0} c_{1}(\Omega, \alpha) r^{\alpha}
$$

which is what we wanted to prove.

In a similar way we can show that $I I I \leq c_{0} c_{1}(\Omega, \alpha) r^{\alpha}$.

In order to prove the inequality in (4.2.42) which involves $I I$, first we use the Fundamental Theorem of Calculs. Denoting $x+v(y-x)+M r e_{n}=: z$ and $t+v(s-t)=$ : $u$, we have

$$
\begin{aligned}
I I & =\left|\int_{0}^{1} \frac{d}{d v}[F(z, u)] d v\right| \\
& \leq \int_{0}^{1}\left|\left(\nabla F(z, u), \partial_{t} F(x, u)\right) \cdot(y-x, s-t)\right| d v \\
& =\int_{0}^{1}\left|\left(\nabla F(z, u),|s-t|^{1 / 2} \partial_{t} F(x, u)\right) \cdot\left(y-x, \pm|s-t|^{1 / 2}\right)\right| d v \\
& \leq \int_{0}^{1}\left|\left(\nabla F(z, u),|s-t|^{1 / 2} \partial_{t} F(x, u)\right)\right|\left|\left(y-x, \pm|s-t|^{1 / 2}\right)\right| d v \\
& \leq c r \int_{0}^{1}\left\{|\nabla F(z, u)|+r\left|\partial_{t} F(z, u)\right|\right\} d v .
\end{aligned}
$$

We next choose the positive constant $M$ such that the segment connecting the poits $\left(x+M r e_{n}, t\right)$ and $\left(y+M r e_{n}, s\right)$ lies inside $\Omega \times \mathbb{R}$. In other words we need to find $M>0$ such that $z=x+v(y-x)+M r e_{n} \in \Omega$ for all $v \in[0,1]$. Since $x=\left(x^{\prime}, x_{n}\right), y=\left(y^{\prime}, y_{n}\right) \in \Omega\left(\right.$ with $x^{\prime}, y^{\prime} \in \mathbb{R}^{n-1}$ and $\left.x_{n}, y_{n} \in \mathbb{R}\right)$, we have $x_{n}>\varphi\left(x^{\prime}\right)$ 
and $y_{n}>\varphi\left(y^{\prime}\right)$, where the graph of the Lipschitz function $\varphi: \mathbb{R}^{n-1} \rightarrow \mathbb{R}$ represents $\partial \Omega$. Some elementary calculations and the fact that $\varphi$ is Lipschitz yield

$$
\begin{aligned}
\varphi\left[x^{\prime}+\right. & \left.v\left(y^{\prime}-x^{\prime}\right)\right]-x_{n}-v\left(y_{n}-x_{n}\right) \\
& =(1-v) \varphi\left[x^{\prime}+v\left(y^{\prime}-x^{\prime}\right)\right]-(1-v) x_{n}+v \varphi\left[x^{\prime}+v\left(y^{\prime}-x^{\prime}\right)\right]-v y_{n} \\
& <(1-v)\left\{\varphi\left[x^{\prime}+v\left(y^{\prime}-x^{\prime}\right)\right]-\varphi\left(x^{\prime}\right)\right\}+v\left\{\varphi\left[x^{\prime}+v\left(y^{\prime}-x^{\prime}\right)\right]-\varphi\left(y^{\prime}\right)\right\} \\
& \leq 2 c v(1-v)\left|y^{\prime}-x^{\prime}\right| \\
& \leq \frac{c(\Omega)}{2} r<c(\Omega) r .
\end{aligned}
$$

Now if we choose $M$ to be $c(\partial \Omega)$, a constant which depends only on the Lipschitz character of $\varphi$, then $x_{n}+v\left(y_{n}-x_{n}\right)+M r>\varphi\left[x^{\prime}+v\left(y^{\prime}-x^{\prime}\right)\right]$, which amounts to $z=x+v(y-x)+$ Mre $_{n} \in \Omega$ for all $v \in[0,1]$. Moreover, if $z=\left(z^{\prime}, z_{n}\right)$ with $z^{\prime} \in \mathbb{R}^{n-1}$ and $z_{n} \in \mathbb{R}$, then

$$
\begin{aligned}
\delta(z) & \approx z_{n}-\varphi\left(z^{\prime}\right) \\
& =x_{n}+v\left(y_{n}-x_{n}\right)+M r-\varphi\left[x^{\prime}+v\left(y^{\prime}-x^{\prime}\right)\right] \\
& \geq c(\Omega) r .
\end{aligned}
$$

Returning to estimate $I I$, we obtain that

$$
\begin{aligned}
I I & \leq c r \int_{0}^{1}\left\{|\nabla F(z, u)|+\delta(z)\left|\partial_{t} F(z, u)\right|\right\} d v \\
& \leq c_{0} c r \int_{0}^{1} \frac{d v}{\delta(z)^{1-\alpha}} \\
& \leq c_{0} c r \int_{0}^{1} \frac{d v}{r^{1-\alpha}} \\
& =c_{0} c r^{\alpha}
\end{aligned}
$$


where the constant $c$ depends only on the Lipschitz character of $\Omega$ and on $\alpha$. We have shown each part of (4.2.42), hence the lemma is proved.

We are now ready to present the

Proof of Theorem 4.2.9.

According to Lemma 4.2.10, for any $F \in C^{1}(\Omega \times \mathbb{R})$,

$$
\|F\|_{B_{\alpha, p a r}^{\infty}(\Omega \times \mathbb{R})} \leq c \sup _{(x, t) \in \Omega \times \mathbb{R}}\left\{\delta(x)^{1-\alpha}|\nabla F(x, t)|+\delta^{2-\alpha}\left|\partial_{t i m e} F(x, t)\right|\right\}
$$

where $\delta(x)=\operatorname{dist}(x, \partial \Omega)$ and $0<\alpha<1$.

Let $f \in B_{\alpha, p a r}^{\infty}(\partial \Omega \times \mathbb{R})$. Then, applying Lemma 4.2 .10 for $\mathcal{D} f$, we obtain that

$$
\begin{aligned}
\|\mathcal{D} f\|_{B_{\alpha, p a r}^{\infty}(\Omega \times \mathbb{R})} & \leq c\left\|\delta^{1-\alpha} \nabla \mathcal{D} f+\delta^{2-\alpha} \partial_{\text {time }} \mathcal{D} f\right\|_{L^{\infty}(\Omega \times \mathbb{R})} \\
& \leq c\left\|\delta^{1-\alpha} \nabla \mathcal{D} f\right\|_{L^{\infty}(\Omega \times \mathbb{R})}+c\left\|\delta^{2-\alpha} \partial_{t i m e} \mathcal{D} f\right\|_{L^{\infty}(\Omega \times \mathbb{R})} \\
& \leq c\|f\|_{B_{\alpha, p a r}^{\infty}(\partial \Omega \times \mathbb{R})} .
\end{aligned}
$$

Here we have also used two special cases of Theorem 4.1.4, namely $k=1, \gamma=\theta=0$ and $k=0, \gamma=1, \theta=0$, respectively.

The main result of this chapter, for a bounded Lipschitz domain, is as follows.

Theorem 4.2.11. Let $\Omega$ be a bounded Lipschitz domain in $\mathbb{R}^{n}, 0<T<\infty$ and $0<\alpha<1,1<p \leq \infty$ such that $\left(\alpha, \frac{1}{p}\right) \in$ interior of $O A B C D E$ in the diagram below. Then

$$
\mathcal{D}:{ }_{0} B_{\alpha, p a r}^{p}(\partial \Omega \times(0, T)) \longrightarrow{ }_{0} B_{\alpha+\frac{1}{p}, p a r}^{p}(\Omega \times(0, T))
$$

is bounded. 


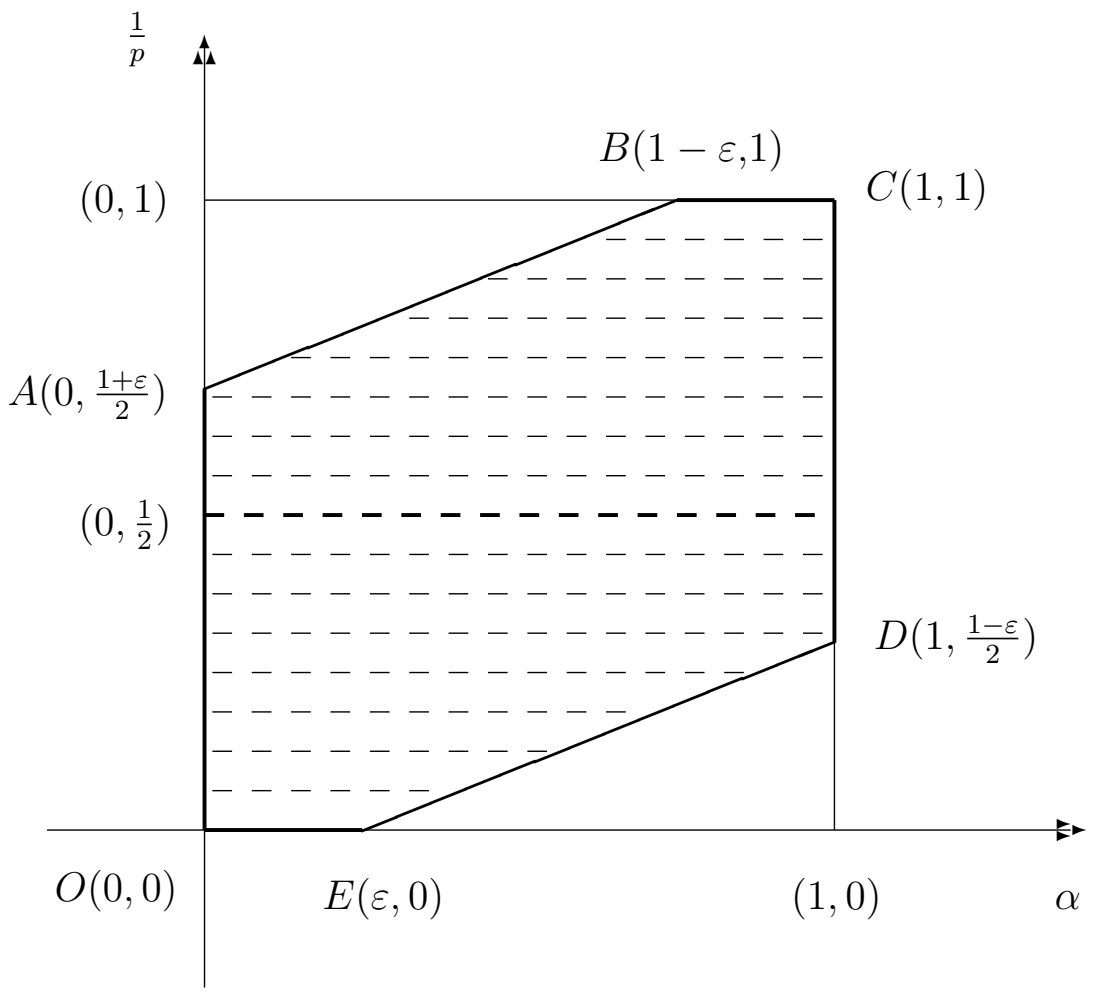

Proof. Let $f \in{ }_{0} B_{\alpha, p a r}^{p}(\partial \Omega \times(0, T))$. Then, by definition, there exists a function $F \in B_{\alpha, p a r}^{p}(\partial \Omega \times \mathbb{R})$ such that $f=\left.F\right|_{\partial \Omega \times(0, T)}$ and $F$ is supported in $\partial \Omega \times[0, \infty)$. Also, in view of the definition of the double layer potential and of the fundamental solution $E$ of the heat operator, for $(x, t) \in \Omega \times \mathbb{R}$, we have

$$
\begin{aligned}
\mathcal{D} F(x, t) & =\int_{\mathbb{R}} \int_{\partial \Omega} \partial_{\nu(y)}[E(x-y, t-s)] F(y, s) d \sigma_{y} d s \\
& =\int_{0}^{t} \int_{\partial \Omega} \partial_{\nu(y)}[E(x-y, t-s)] F(y, s) d \sigma_{y} d s .
\end{aligned}
$$

Going further,

$$
\begin{aligned}
\left.(\mathcal{D} F)\right|_{\Omega \times(0, T)}(x, t) & =\int_{0}^{t} \int_{\partial \Omega} \partial_{\nu(y)}[E(x-y, t-s)] F(y, s) d \sigma_{y} d s \\
& =\int_{0}^{t} \int_{\partial \Omega} \partial_{\nu(y)}[E(x-y, t-s)] f(y, s) d \sigma_{y} d s \\
& =(\mathcal{D} f)(x, t),
\end{aligned}
$$


since $0<s<t<T$ implies $s \in(0, T)$, and $\left.F\right|_{\partial \Omega \times(0, T)}=f$. Also, from Theorems 4.2.8 and 4.2.9, we have that for any $0<\alpha<1,1<p \leq \infty$ and $F$ in $B_{\alpha, p a r}^{p, q}(\partial \Omega \times \mathbb{R})$,

$$
\mathcal{D} F \in B_{\alpha+\frac{1}{p}, p a r}^{p, q}(\Omega \times \mathbb{R}) .
$$

We next observe that

$$
\mathcal{D} F(x, t) \equiv 0 \quad \text { for } \quad t<0 .
$$

Indeed, $t<0$ and $s<t$ (which holds, since otherwise $E(x-y, t-s)=0$ ) imply that $s<0$. Now (4.2.45) follows from the fact that $F(y, s) \equiv 0$ for $s<0$.

Consequently, recalling the spaces $\left.\square B_{\beta, p a r}^{q}(\Omega \times(0, T))\right)$ from Definition 3.1.15, we note that (4.2.43)-(4.2.45) amount to

$$
\left.\mathcal{D} f \in \square B_{\alpha+\frac{1}{p}, p a r}^{p}(\Omega \times(0, T))\right) .
$$

Finally, in view of the equaivalence between the spaces $\left.\square B_{\alpha+\frac{1}{p}, p a r}^{p}(\Omega \times(0, T))\right)$ and $\left.{ }_{0} B_{\alpha+\frac{1}{p}, p a r}^{p}(\Omega \times(0, T))\right)$ for $\left(\alpha, \frac{1}{p}\right) \in$ interior of $O A B C D E$ (by Theorem 3.1.20), we obtain that

$$
\mathcal{D}:{ }_{0} B_{\alpha, p a r}^{p}(\partial \Omega \times(0, T)) \longrightarrow{ }_{0} B_{\alpha+\frac{1}{p}, p a r}^{p}(\Omega \times(0, T))
$$

is bounded, completing the proof of the theorem. 


\section{Chapter 5}

\section{Mapping properties of the single layer potential}

In this chapter we shall prove that for a bounded Lipschitz domain $\Omega$ in $\mathbb{R}^{n}$ and $0<T<\infty$, indices $1<p<\infty$ and $0<\alpha<1$, the caloric single layer potential opetator $\mathcal{S}$ is a bounded linear map from the space ${ }_{0} B_{-\alpha, p a r}^{p}(\partial \Omega \times(0, T))$ into ${ }_{0} B_{1-\alpha+\frac{1}{p}, p a r}^{p}(\Omega \times(0, T))$ (see Theorem 5.2.7). The main idea of our approach consists of interpolating between the endpoint cases $p=1$ (Theorem 5.1.9) and $p=\infty$ (Theorem 5.1.10).

\subsection{Basic estimates}

The first part of this section contains several preliminary results needed in order to show the above mentioned endpoint cases. Then we shall discuss the mapping properties of the single layer potential in the instances when $p=1$ and $p=\infty$, respectively.

Recall that the single layer potential operator is defined by

$$
\mathcal{S} f(x, t):=\int_{\partial \Omega \times \mathbb{R}} E(x-y, t-s) f(y, s) d \sigma_{y} d s
$$


for $(x, t) \in \Omega \times \mathbb{R}$, where $E(x, t)$ is the fundamental solution of the heat operator and $\Omega$ is a domain in $\mathbb{R}^{n}$ with surface measure $d \sigma$.

We next introduce a truncated version of the single layer potential.

Definition 5.1.1. Let $\Omega$ be a domain in $\mathbb{R}^{n}$ and consider $\tilde{\psi} \in C_{c}^{\infty}\left(\mathbb{R}^{n}\right)$ and $\psi, \tilde{\tilde{\psi}} \in$ $C_{c}^{\infty}\left(\mathbb{R}^{n} \times \mathbb{R}\right)$ with the following properties: supp $\tilde{\psi} \cap \Omega \subseteq \tilde{K} \subset \Omega$,

$$
\begin{aligned}
& \text { supp } \psi \cap(\partial \Omega \times \mathbb{R}) \subseteq K \times I \text { with } K \subset \partial \Omega, I \subset \mathbb{R}, \\
& \text { supp } \tilde{\tilde{\psi}} \cap(\partial \Omega \times \mathbb{R}) \subseteq \tilde{\tilde{K}} \times \tilde{\tilde{I}} \text { with } \tilde{\tilde{K}} \subset \partial \Omega, \tilde{\tilde{I}} \subset \mathbb{R},
\end{aligned}
$$

and such that $\tilde{\psi} \equiv 1$ in $K_{\epsilon} \cap \tilde{K}$, where $K_{\epsilon}$ is a neighborhood of $K$, and $\tilde{\tilde{\psi}} \equiv 1$ in some neighborhood of $K \times I$ (assume $K \times I \subset \tilde{\tilde{K}} \times \tilde{\tilde{I}}$ ). Using the above notation, we define

$$
\tilde{\mathcal{S}} f:=\tilde{\psi} \mathcal{S}(\tilde{\tilde{\psi}} \psi f)
$$

Recall that for a domain $\Omega$ (in $\mathbb{R}^{n}$ ) and for $x \in \Omega, \delta(x)=\operatorname{dist}(x, \partial \Omega)$ represents the distance from $x$ to the boundary of $\Omega$. Also, $\nabla^{k}$ denotes any combination of mixed spacial partial derivatives of order $k$, and $I_{\text {time }}^{\theta}$ stands for the fractional integral operator defined in (1.1.4).

One of our basic estimates regarding the truncated single layer potential operator (when $p=1$ ) is as follows.

Theorem 5.1.2. Consider a Lipschitz domain $\Omega$ in $\mathbb{R}^{n}$, and set $N:=k+2 \gamma-2 \theta+$ $\alpha-2>-1$, where $k, \gamma \in \mathbb{N}_{0}, 0 \leq \theta<1,0<\alpha<1$, and $2 \gamma-2 \theta+\alpha>1$ for $0<\theta<1$. Then for any $f \in\left(B_{\alpha, p a r}^{\infty}(\partial \Omega \times \mathbb{R})\right)^{*}$

$$
\delta^{N} \tilde{\psi} \nabla^{k} \partial_{\text {time }}^{\gamma} I_{\text {time }}^{\theta} \mathcal{S}(\tilde{\tilde{\psi}} \psi f) \in L^{1}(\Omega \times \mathbb{R})
$$


with natural accompanying estimates.

Proof. First we consider the case when $0<\theta<1$.

Let $f \in\left(B_{\alpha, p a r}^{\infty}(\partial \Omega \times \mathbb{R})\right)^{*}$ and note that for each fixed $(x, t) \in \Omega \times \mathbb{R}$ we have

$$
\delta^{N} \tilde{\psi} \nabla^{k} \partial_{\text {time }}^{\gamma} I_{\text {time }}^{\theta} \mathcal{S}(\tilde{\tilde{\psi}} \psi f)(x, t)=\left\langle\delta(x)^{N} \tilde{\psi}(x) \nabla^{k} \partial_{\text {time }}^{\gamma} I_{\text {time }}^{\theta} E(x-\cdot, t-\cdot) \tilde{\tilde{\psi}}, \psi f\right\rangle
$$

Moreover,

$$
\begin{aligned}
& \left\|\delta^{N} \tilde{\psi} \nabla^{k} \partial_{\text {time }}^{\gamma} I_{\text {time }}^{\theta} \mathcal{S}(\tilde{\tilde{\psi}} \psi f)\right\|_{L^{1}(\Omega \times \mathbb{R})} \\
& =\sup _{\substack{F \in L^{\infty}(\Omega \times \mathbb{R}) \\
\|F\|_{L^{\infty} \leq 1}}} \int_{\Omega \times \mathbb{R}} F(x, t)\left\langle\delta(x)^{N} \tilde{\psi}(x) \nabla^{k} \partial_{\text {time }}^{\gamma} I_{\text {time }}^{\theta} E(x-\cdot, t-\cdot) \tilde{\tilde{\psi}}, \psi f\right\rangle d x d t \\
& =\sup _{\substack{F \in L^{\infty}(\Omega \times \mathbb{R}) \\
\|F\|_{L^{\infty} \leq 1}}}\left\langle\int_{\Omega \times \mathbb{R}} F(x, t) \delta(x)^{N} \tilde{\psi}(x) \nabla^{k} \partial_{\text {time }}^{\gamma} I_{\text {time }}^{\theta} E(x-\cdot, t-\cdot) \tilde{\psi} d x d t, \psi f\right\rangle \\
& \left.\leq\|\psi\| f \|_{\left(B_{\alpha, p a r}^{\infty}\right.}(\partial \Omega \times \mathbb{R})\right)^{*} \times \\
& \quad \times \sup _{\substack{F \in L^{\infty}(\Omega \times \mathbb{R}) \\
\|F\|_{L} \leq 1}}\left\|\int_{\Omega \times \mathbb{R}} F(x, t) \delta(x)^{N} \tilde{\psi}(x) \nabla^{k} \partial_{\text {time }}^{\gamma} I_{\text {time }}^{\theta} E(x-\cdot, t-\cdot) \tilde{\tilde{\psi}} d x d t\right\|_{B_{\alpha, p a r}^{\infty}(\partial \Omega \times \mathbb{R})} .
\end{aligned}
$$

We fix a function $F \in L^{\infty}(\Omega \times \mathbb{R})$ with $\|F\|_{L^{\infty}(\Omega \times \mathbb{R})} \leq 1$, and two pair of points $(y, s),(z, r) \in K \times I \subset \partial \Omega \times \mathbb{R},(y, s) \neq(z, r)$. Then estimating the $B_{\alpha, p a r}^{\infty}(\partial \Omega \times \mathbb{R})$ norm of the last integral comes down to proving that there exists a positive finite constant $c$ such that

$$
\begin{aligned}
& \text { A. } \begin{array}{l}
\int_{\Omega \times \mathbb{R}} F(x, t) \delta(x)^{N} \tilde{\psi}(x) \nabla^{k} \partial_{\text {time }}^{\gamma} I_{\text {time }}^{\theta} E(x-y, t-s) \tilde{\tilde{\psi}}(y, s) d x d t \\
\quad-\int_{\Omega \times \mathbb{R}} F(x, t) \delta(x)^{N} \tilde{\psi}(x) \nabla^{k} \partial_{\text {time }}^{\gamma} I_{\text {time }}^{\theta} E(x-z, t-r) \tilde{\psi}(z, r) d x d t \mid \\
\quad \leq c\left(|y-z|+|t-s|^{1 / 2}\right)^{\alpha},
\end{array}
\end{aligned}
$$


and that

B. $\sup _{(y, s) \in K \times I}\left|\int_{\tilde{K} \times \mathbb{R}} F(x, t) \delta(x)^{N} \tilde{\psi}(x)\left[\nabla^{k} \partial_{\text {time }}^{\gamma} I_{\text {time }}^{\theta} E(x-y, t-s)\right] \tilde{\psi}(y, s) d x d t\right|<\infty$, where $K \times I \subset \partial \Omega \times \mathbb{R}$ and $\tilde{K} \subset \Omega$ are compact subsets.

Since $\|F\|_{L^{\infty}(\Omega \times \mathbb{R})} \leq 1,\|\tilde{\psi}\|_{L^{\infty}(\Omega)} \leq c$, and $\tilde{\tilde{\psi}} \equiv 1$ on $K \times I$, in order to prove $\mathbf{A}$ it is enough to show that

$$
\begin{aligned}
& \int_{\Omega \times \mathbb{R}} \delta(x)^{N}\left|\nabla^{k} \partial_{\text {time }}^{\gamma} I_{\text {time }}^{\theta} E(x-y, t-s)-\nabla^{k} \partial_{\text {time }}^{\gamma} I_{\text {time }}^{\theta} E(x-z, t-r)\right| d x d t \\
& \leq c\left(|y-z|+|t-s|^{1 / 2}\right)^{\alpha}
\end{aligned}
$$

where $(y, s),(z, r) \in K \times I \subset \partial \Omega \times \mathbb{R}$ with $(y, s) \neq(z, r)$.

At this point we can drop the restriction that $(y, s)$ and $(z, r)$ are in a compact subset of $\partial \Omega \times \mathbb{R}$, and we will prove that (5.1.3) holds for any $(y, s),(z, r) \in \partial \Omega \times \mathbb{R}$, by dividing the domain of integration $\Omega \times \mathbb{R}$ in four parts, and handling each case separately. Denote $\left(|y-z|+|t-s|^{1 / 2}\right)^{\alpha}$ by $A$, and set

$$
I_{1}:=\int_{D_{1}} \delta(x)^{N}\left|\nabla^{k} \partial_{\text {time }}^{\gamma} I_{\text {time }}^{\theta} E(x-y, t-s)\right| d x d t
$$

where $D_{1}:=\left\{(x, t) \in \Omega \times \mathbb{R}:|x-y|<c A,|t-s|^{1 / 2}<c A\right\}$. We estimate $\left|\nabla^{k} \partial_{\text {time }}^{\gamma} I_{\text {time }}^{\theta} E(x-y, t-s)\right|$ using (1.2.19), obtaining

$$
I_{1} \leq c \int_{D_{1}} \delta(x)^{N} \frac{|t-s|^{\theta}}{|x-y|^{n+k+2 \gamma}}\left[\min \left\{1, \frac{|x-y|^{2}}{|t-s|}\right\}\right]^{\gamma+1} d x d t
$$

Given $x \in \Omega, y \in \partial \Omega$, we can write $x=\left(x^{\prime}, \varphi\left(x^{\prime}\right)+\tau\right)$ and $y=\left(y^{\prime}, \varphi\left(y^{\prime}\right)\right)$, where $x^{\prime}, y^{\prime} \in \mathbb{R}^{n-1}, \tau \in \mathbb{R}_{+}$, and $\varphi: \mathbb{R}^{n-1} \rightarrow \mathbb{R}$ is a Lipschitz function, whose graph is $\partial \Omega$ (if $\Omega$ is bounded, use a finite partition of unity on $\partial \Omega$ ). Then $|x-y| \approx\left|x^{\prime}-y^{\prime}\right|+\tau$ 
and $d x \approx d x^{\prime} d \tau$. Making also the change of variables $t-s=t^{\prime} \in \mathbb{R}$, (5.1.4) is equivalent with

$$
I_{1} \leq c \int_{D_{1}^{1}} \tau^{N} \frac{\left|t^{\prime}\right|^{\theta}}{\left(\left|x^{\prime}-y^{\prime}\right|+\tau\right)^{n+k+2 \gamma}}\left[\min \left\{1, \frac{\left(\left|x^{\prime}-y^{\prime}\right|+\tau\right)^{2}}{\left|t^{\prime}\right|}\right\}\right]^{\gamma+1} d x^{\prime} d \tau d t
$$

where $D_{1}^{1}:=\left\{\left(x^{\prime}, \tau, t^{\prime}\right) \in \mathbb{R}^{n-1} \times \mathbb{R}_{+} \times \mathbb{R}:\left|x^{\prime}-y^{\prime}\right|+\tau<c A,\left|t^{\prime}\right|^{1 / 2}<c A\right\}$. Вy setting $x^{\prime}-y^{\prime}=x^{\prime \prime} \in \mathbb{R}^{n}$ and $t^{\prime}=\left(t^{\prime \prime}\right)^{2}, t^{\prime \prime} \in \mathbb{R}$, we can rewrite (5.1.5) as follows.

$$
I_{1} \leq c \int_{D_{1}^{2}} \tau^{N} \frac{\left|t^{\prime \prime}\right|^{2 \theta+1}}{\left(\left|x^{\prime}\right|+\tau\right)^{n+k+2 \gamma}}\left[\min \left\{1, \frac{\left(\left|x^{\prime}\right|+\tau\right)^{2}}{\left|t^{\prime \prime}\right|^{2}}\right\}\right]^{\gamma+1} d x^{\prime \prime} d \tau d t^{\prime \prime}
$$

where $D_{1}^{2}:=\left\{\left(x^{\prime \prime}, \tau, t^{\prime \prime}\right) \in \mathbb{R}^{n-1} \times \mathbb{R}_{+} \times \mathbb{R}:\left|x^{\prime}\right|+\tau<c A,\left|t^{\prime \prime}\right|<c A\right\}$. Going further, let $x^{\prime \prime}=A u, \tau=A \kappa$ and $t^{\prime \prime}=A v$ (rescale in $x^{\prime \prime}, \tau$ and $t^{\prime \prime}$ ). Then (5.1.6) yields

$$
I_{1} \leq c A^{\alpha} \int_{D_{1}^{3}} \kappa^{N} \frac{|v|^{2 \theta+1}}{(|u|+\kappa)^{n+k+2 \gamma}}\left[\min \left\{1,\left(\frac{|u|+\kappa}{|v|}\right)^{2}\right\}\right]^{\gamma+1} d u d \kappa d v
$$

where $D_{1}^{3}:=\left\{(u, \kappa, v) \in \mathbb{R}^{n-1} \times \mathbb{R}_{+} \times \mathbb{R}:|u|+\kappa<c,|v|<c\right\}$.

Going further, first, if $|v| \leq|u|+\kappa$, we let

$$
D_{1}^{4}:=\left\{(u, \kappa, v) \in \mathbb{R}^{n-1} \times \mathbb{R}_{+} \times \mathbb{R}:|v| \leq|u|+\kappa<c\right\} .
$$

From (5.1.7), after making the change of variables $v=\tilde{v}(|u|+\kappa), \tilde{v} \in \mathbb{R}$, we obtain that

$$
\begin{aligned}
I_{1} & \leq c A^{\alpha} \int_{D_{1}^{4}} \kappa^{N} \frac{|v|^{2 \theta+1}}{(|u|+\kappa)^{n+k+2 \gamma}} d u d \kappa d v \\
& \leq c A^{\alpha}\left(\int_{\substack{\tilde{v} \in \mathbb{R} \\
\mid \tilde{v} \leq 1}}|\tilde{v}|^{2 \theta+1} d \tilde{v}\right) \int_{\substack{(u, \kappa) \in \mathbb{R}^{n-1} \times \mathbb{R}_{+} \\
|u|+\kappa<c}} \frac{\kappa^{N}}{(|u|+\kappa)^{n+k+2 \gamma-2 \theta-2}} d u d \kappa \\
& \leq c A^{\alpha} \int_{\substack{(u, \kappa) \in \mathbb{R}^{n-1} \times \mathbb{R}_{+} \\
|u|+\kappa<c}} \frac{\kappa^{N}}{(|u|+\kappa)^{n+N-\alpha}} d u d \kappa,
\end{aligned}
$$


We next let $\kappa=\kappa^{\prime}|u|, \kappa^{\prime} \in \mathbb{R}_{+}$in (5.1.8). Then

$$
I_{1} \leq a A^{\alpha}\left(\int_{\substack{u \in \mathbb{R}^{n-1} \\|u|<c}} \frac{d u}{|u|^{n-1-\alpha}}\right) \int_{\kappa^{\prime} \in \mathbb{R}_{+}} \frac{\left(\kappa^{\prime}\right)^{N}}{\left(\kappa^{\prime}+1\right)^{n+N-\alpha}} d \kappa^{\prime} \leq c A^{\alpha},
$$

wher we have used that if $\kappa^{\prime} \leq 1$, then $\kappa^{\prime}+1 \approx 1$ and $\int_{0<\kappa^{\prime} \leq 1} \kappa^{\prime N} d \kappa^{\prime}<\infty$ for $N>-1$, as well as the fact that if $\kappa^{\prime}>1$, then $\kappa^{\prime}+1 \approx \kappa^{\prime}$ and $\int_{\kappa^{\prime}<1} \frac{d \kappa^{\prime}}{\left(\kappa^{\prime}\right)^{n-\alpha}}$ is finite, since $n-\alpha>1$.

Second, if $|u|+\kappa<|v|$, we denote

$$
D_{1}^{5}:=\left\{(u, \kappa, v) \in \mathbb{R}^{n-1} \times \mathbb{R}_{+} \times \mathbb{R}:|u|+\kappa<|v|<c\right\} .
$$

From (5.1.7) we obtain that

$$
I_{1} \leq c A^{\alpha} \int_{D_{1}^{5}} \frac{\kappa^{N}}{(|u|+\kappa)^{n+k-2}|v|^{2 \gamma-2 \theta+1}} d u d \kappa d v
$$

which, after the change of variables $v=v^{\prime}(|u|+\kappa)$, yields $v^{\prime} \in \mathbb{R}$,

$$
I_{1} \leq c A^{\alpha}\left(\int_{\substack{v^{\prime} \in \mathbb{R} \\\left|v^{\prime}\right| \geq 1}} \frac{d v^{\prime}}{\left(v^{\prime}\right)^{2 \gamma-2 \theta+1}}\right) \int_{\substack{(u, \kappa) \in \mathbb{R}^{n-1} \times \mathbb{R}_{+} \\|u|+\kappa<c}} \frac{\kappa^{N}}{(|u|+\kappa)^{n+N-\alpha}} d u d \kappa .
$$

The first integral in (5.1.9) is bounded by a finite constant, since the hypothesis $2 \gamma-2 \theta>1-\alpha$ implies $2 \gamma-2 \theta>0$, while the second integral in (5.1.9) is the same as the integral in (5.1.8) which, we have seen, is bounded. Therefore $I_{1} \leq c A^{\alpha}$.

Next, we set

$$
I_{1}^{\prime}:=\int_{D_{1}} \delta(x)^{N}\left|\nabla^{k} \partial_{\text {time }}^{\gamma} I_{\text {time }}^{\theta} E(x-z, t-r)\right| d x d t
$$


where $D_{1}=\left\{(x, t) \in \Omega \times \mathbb{R}:|x-y|<c A,|t-s|^{1 / 2}<c A\right\}$, as before. Note that $|x-z| \leq|x-y|+|y-z|<c A$ and $|t-r|^{1 / 2} \leq|t-s|^{1 / 2}+|s-r|^{1 / 2}<c A$. Hence

$$
I_{1}^{\prime} \leq \int_{D_{1}^{\prime}} \delta(x)^{N}\left|\nabla^{k} \partial_{\text {time }}^{\gamma} I_{\text {time }}^{\theta} E(x-z, t-r)\right| d x d t=I_{1} \leq c A^{\alpha}
$$

where $D_{1}^{\prime}:=\left\{(x, t) \in \Omega \times \mathbb{R}:|x-z|<c A,|t-r|^{1 / 2}<c A\right\}$.

Going further, let the second domain of integration be

$$
D_{2}:=\left\{(x, t) \in \Omega \times \mathbb{R}:|x-y|<c A,|t-s|^{1 / 2}>c A\right\},
$$

and set

$$
I_{2}:=\int_{D_{2}} \delta(x)^{N}\left|\nabla^{k} \partial_{\text {time }}^{\gamma} I_{\text {time }}^{\theta} E(x-y, t-s)\right| d x d t
$$

Following the same steps as in the case of $I_{1}$, we obtain a similar inequality to (5.1.7) (only the domain of integration is different), namely

$$
I_{2} \leq c A^{\alpha} \int_{D_{2}^{1}} \kappa^{N} \frac{|v|^{2 \theta+1}}{(|u|+\kappa)^{n+k+2 \gamma}}\left[\min \left\{1,\left(\frac{|u|+\kappa}{|v|}\right)^{2}\right\}\right]^{\gamma+1} d u d \kappa d v
$$

where $D_{2}^{1}:=\left\{(u, \kappa, v) \in \mathbb{R}^{n-1} \times \mathbb{R}_{+} \times \mathbb{R}:|u|+\kappa<c,|v|>c\right\}$. After some simplifications and the change of variables $v=v^{\prime}(|u|+\kappa), v^{\prime} \in \mathbb{R}$, we get that

$$
\begin{aligned}
I_{2} & \leq c A^{\alpha} \int_{D_{2}^{1}} \frac{\kappa^{N}}{(|u|+\kappa)^{n+k-2}|v|^{2 \gamma-2 \theta+1}} d u d \kappa d v \\
& \leq c A^{\alpha}\left(\int_{\substack{v^{\prime} \in \mathbb{R} \\
\left|v^{\prime}\right| \geq 1}} \frac{d v^{\prime}}{\left(v^{\prime}\right)^{2 \gamma-2 \theta+1}}\right) \int_{\substack{(u, \kappa) \in \mathbb{R}^{n-1} \times \mathbb{R}_{+} \\
|u|+\kappa<c}} \frac{\kappa^{N}}{(|u|+\kappa)^{n+N-\alpha}} d u d \kappa .
\end{aligned}
$$

The integrals in (5.1.10) are the same as in (5.1.9) (which are bounded by a finitite constant), therefore $I_{2} \leq c A^{\alpha}$. 
For

$$
I_{2}^{\prime}:=\int_{D_{2}} \delta(x)^{N}\left|\nabla^{k} \partial_{\text {time }}^{\gamma} I_{\text {time }}^{\theta} E(x-z, t-r)\right| d x d t
$$

with $D_{2}:=\left\{(x, t) \in \Omega \times \mathbb{R}:|x-y|<c A,|t-s|^{1 / 2}>c A\right\}$, note that $|x-z|<c A$ and $c A<|t-s|^{1 / 2} \leq|t-r|^{1 / 2}+|s-r|^{1 / 2} \leq|t-r|^{1 / 2}+A$. Hence for $c>1$ (which we can assume without loss of generality), $|t-r|^{1 / 2}>c A$ and

$$
I_{2}^{\prime} \leq \int_{D_{2}^{\prime}} \delta(x)^{N}\left|\nabla^{k} \partial_{\text {time }}^{\gamma} I_{\text {time }}^{\theta} E(x-z, t-r)\right| d x d t=I_{2} \leq c A^{\alpha},
$$

where $D_{2}^{\prime}:=\left\{(x, t) \in \Omega \times \mathbb{R}:|x-z|<c A,|t-r|^{1 / 2}>c A\right\}$.

Taking the third domain of integration to be

$$
D_{3}:=\left\{(x, t) \in \Omega \times \mathbb{R}:|x-y|>c A,|t-s|^{1 / 2}<c A\right\},
$$

we consider

$$
I_{3}:=\int_{D_{3}} \delta(x)^{N}\left|\nabla^{k} \partial_{\text {time }}^{\gamma} I_{\text {time }}^{\theta} E(x-y, t-s)\right| d x d t .
$$

Following the same steps as in the case of $I_{1}$, we obtain a similar inequality to (5.1.7) (only the domain of integration is different), namely

$$
I_{3} \leq c A^{\alpha} \int_{D_{3}^{1}} \kappa^{N} \frac{|v|^{2 \theta+1}}{(|u|+\kappa)^{n+k+2 \gamma}}\left[\min \left\{1,\left(\frac{|u|+\kappa}{|v|}\right)^{2}\right\}\right]^{\gamma+1} d u d \kappa d v
$$

where $D_{3}^{1}:=\left\{(u, \kappa, v) \in \mathbb{R}^{n-1} \times \mathbb{R}_{+} \times \mathbb{R}:|u|+\kappa>c,|v|<c\right\}$. After some simplifications we obtain that

$$
\begin{aligned}
I_{3} & \leq c A^{\alpha}\left(\int_{\substack{v \in \mathbb{R}^{\prime} \\
|v|<c}}|v|^{2 \theta+1} d v\right) \int_{\substack{(u, \kappa) \in \mathbb{R}^{n-1} \times \mathbb{R}_{+} \\
|u|+\kappa>c}} \frac{\kappa^{N}}{(|u|+\kappa)^{n+k+2 \gamma}} d u d \kappa \\
& \leq c A^{\alpha} \int_{\substack{(u, \kappa) \in \mathbb{R}^{n-1} \times \mathbb{R}_{+} \\
|u|+\kappa>c}} \frac{\kappa^{N}}{(|u|+\kappa)^{n+k+2 \gamma}} d u d \kappa .
\end{aligned}
$$


To proceed, we consider two cases. First, let $\kappa \leq|u|$, and make the change of variables $\kappa=\kappa^{\prime}|u|, \kappa^{\prime} \in \mathbb{R}_{+}$. Then $\kappa^{\prime} \leq 1,|u|>c$, and we obtain that

$$
\begin{aligned}
I_{3} & \leq c A^{\alpha}\left(\int_{0<\kappa^{\prime} \leq 1} \frac{\left(\kappa^{\prime}\right)^{N}}{\left(\kappa^{\prime}+1\right)^{n+k+2 \gamma}} d \kappa^{\prime}\right) \int_{\substack{|u| \in \mathbb{R}^{n-1} \\
|u|>c}} \frac{d u}{|u|^{n+1+2 \theta-\alpha}} \\
& \leq c A^{\alpha} .
\end{aligned}
$$

Second, let $\kappa>|u|$, and set $u=u^{\prime} \kappa, u^{\prime} \in \mathbb{R}^{n-1}$. Then $\left|u^{\prime}\right|<1, \kappa>c$, and

$$
\begin{aligned}
I_{3} & \leq c A^{\alpha}\left(\int_{\kappa>c} \frac{d \kappa}{\kappa^{3+2 \theta-\alpha}}\right) \int_{\substack{u^{\prime} \in \mathbb{R}^{n-1} \\
\left|u^{\prime}\right|<1}} \frac{d u^{\prime}}{\left(\left|u^{\prime}\right|+1\right)^{n+k+2 \gamma}} \\
& \leq c A^{\alpha} .
\end{aligned}
$$

Similar to the above cases, we consider

$$
I_{3}^{\prime}:=\int_{D_{3}} \delta(x)^{N}\left|\nabla^{k} \partial_{\text {time }}^{\gamma} I_{\text {time }}^{\theta} E(x-z, t-r)\right| d x d t
$$

with

$$
D_{3}=\left\{(x, t) \in \Omega \times \mathbb{R}:|x-y|>c A,|t-s|^{1 / 2}<c A\right\} .
$$

Observe that $c A<|x-z|+|y-z| \leq|x-z|+A$, hence, for $c>1$ we have $|x-z|>c A$.

Also, $|t-r|^{1 / 2} \leq|t-s|^{1 / 2}+|s-r|^{1 / 2}<c A$. Therefore

$$
I_{3}^{\prime} \leq \int_{D_{3}^{\prime}} \delta(x)^{N}\left|\nabla^{k} \partial_{\text {time }}^{\gamma} I_{\text {time }}^{\theta} E(x-z, t-r)\right| d x d t=I_{3} \leq c A^{\alpha}
$$

where

$$
D_{3}^{\prime}:=\left\{(x, t) \in \Omega \times \mathbb{R}:|x-z|>c A,|t-r|^{1 / 2}<c A\right\} .
$$

Finally, let the fourth domain of integration be

$$
D_{4}:=\left\{(x, t) \in \Omega \times \mathbb{R}:|x-y|>c A,|t-s|^{1 / 2}>c A\right\},
$$


and, different from the previous cases, consider

$$
I_{4}:=\int_{D_{4}} \delta(x)^{N}\left|\nabla^{k} \partial_{\text {time }}^{\gamma} I_{\text {time }}^{\theta} E(x-y, t-s)-\nabla^{k} \partial_{\text {time }}^{\gamma} I_{\text {time }}^{\theta} E(x-z, t-s)\right| d x d t,
$$

and

$$
I_{4}^{\prime}:=\int_{D_{4}} \delta(x)^{N}\left|\nabla^{k} \partial_{\text {time }}^{\gamma} I_{\text {time }}^{\theta} E(x-z, t-s)-\nabla^{k} \partial_{\text {time }}^{\gamma} I_{\text {time }}^{\theta} E(x-z, t-r)\right| d x d t .
$$

We shall prove that $I_{4} \leq c A^{\alpha}$ and $I_{4}^{\prime} \leq c A^{\alpha}$, which will complete the proof of the theorem in the case when $0<\theta<1$.

To handle the difference in the integral in $I_{4}$, use the Fundamental Theorem of Calculus. Then

$$
\begin{aligned}
& \left|\nabla^{k} \partial_{\text {time }}^{\gamma} I_{\text {time }}^{\theta} E(x-y, t-s)-\nabla^{k} \partial_{\text {time }}^{\gamma} I_{\text {time }}^{\theta} E(x-z, t-s)\right| \\
& \quad \leq \int_{0}^{1}\left|\nabla^{k+1} \partial_{\text {time }}^{\gamma} I_{\text {time }}^{\theta} E\left(x-\xi_{j}, t-s\right)\right||y-z| d j,
\end{aligned}
$$

where $\xi_{j}=(1-j) z+j y$. By (1.2.19), the expression in (5.1.12) does not exceed

$$
\begin{aligned}
& c|y-z| \int_{0}^{1} \frac{|t-s|^{\theta}}{\left|x-\xi_{j}\right|^{n+k+1+2 \gamma}}\left[\min \left\{1, \frac{\left|x-\xi_{j}\right|^{2}}{|t-s|}\right\}\right]^{\gamma+1} d j, \\
& =c|y-z| \int_{0}^{1} \frac{|t-s|^{\theta}}{\left|x-\xi_{j}\right|^{n+k-1}}\left[\min \left\{\frac{1}{\left|x-\xi_{j}\right|^{2}}, \frac{1}{|t-s|}\right\}\right]^{\gamma+1} d j .
\end{aligned}
$$

We can easily show that, on the domain of integration (more precisely due to the fact that $|x-y|>c A$ ), we have $\left|x-\xi_{j}\right| \geq c \max \{|x-y|,|x-z|\}$, which implies $\left|x-\xi_{j}\right| \geq c|x-y|$. Therefore

$$
I_{4} \leq c A \int_{D_{4}} \delta(x)^{N} \frac{|t-s|^{\theta}}{|x-y|^{n+k-1}}\left[\min \left\{\frac{1}{|x-y|^{2}}, \frac{1}{|t-s|}\right\}\right]^{\gamma+1} d x d t .
$$

Since $x \in \Omega, y \in \partial \Omega$, we can write $x=\left(x^{\prime}, \varphi\left(x^{\prime}\right)+\tau\right), y=\left(y^{\prime}, \varphi\left(y^{\prime}\right)\right)$, where $x^{\prime}, y^{\prime} \in \mathbb{R}^{n-1}, \varphi: \mathbb{R}^{n-1} \rightarrow \mathbb{R}$ is a Lipschitz function such that $\Omega$ lies above the graph 
of $\varphi$ (if $\Omega$ is a bounded domain, then use a finite partition of unity on $\partial \Omega$ ), and $\tau$ is some positive real number. Then $|x-y| \approx\left|x^{\prime}-y^{\prime}\right|+\tau, \delta(x) \approx \tau$ and $d x \approx d x^{\prime} d \tau$. Also, $I_{4}$ is bounded by

$$
c A \int_{D_{4}^{1}} \tau^{N} \frac{|t-s|^{\theta}}{\left(\left|x^{\prime}-y^{\prime}\right|+\tau\right)^{n+k-1}}\left[\min \left\{\frac{1}{\left(\left|x^{\prime}-y^{\prime}\right|+\tau\right)^{2}}, \frac{1}{|t-s|}\right\}\right]^{\gamma+1} d x^{\prime} d \tau d t
$$

where $D_{4}^{1}:=\left\{\left(x^{\prime}, \tau, t\right) \in \mathbb{R}^{n-1} \times \mathbb{R}_{+} \times \mathbb{R}:\left|x^{\prime}-y^{\prime}\right|+\tau>c A,|t-s|^{1 / 2}>c A\right\}$. To simplify the integral we make the change of variables $x^{\prime}-y^{\prime}=x^{\prime \prime}, x^{\prime \prime} \in \mathbb{R}^{n-1}$ and $t-s=\left(t^{\prime}\right)^{2}, t^{\prime} \in \mathbb{R}$, and obtain that

$$
I_{4} \leq c A \int_{D_{4}^{2}} \tau^{N} \frac{\left|t^{\prime}\right|^{2 \theta+1}}{\left(\left|x^{\prime \prime}\right|+\tau\right)^{n+k-1}}\left[\min \left\{\frac{1}{\left(\left|x^{\prime \prime}\right|+\tau\right)^{2}}, \frac{1}{\left|t^{\prime}\right|^{2}}\right\}\right]^{\gamma+1} d x^{\prime \prime} d \tau d t^{\prime},
$$

where $D_{4}^{2}:=\left\{\left(x^{\prime \prime}, \tau, t^{\prime}\right) \in \mathbb{R}^{n-1} \times \mathbb{R}_{+} \times \mathbb{R}:\left|x^{\prime \prime}\right|+\tau>c A,\left|t^{\prime}\right|>c A\right\}$. Going further, let $x^{\prime \prime}=A u, u \in \mathbb{R}^{n-1} ; \tau=A \kappa, \kappa \in \mathbb{R}_{+} ;$and $t^{\prime}=A v, v \in \mathbb{R}$. Then

$$
I_{4} \leq c A^{\alpha} \int_{D_{4}^{3}} \kappa^{N} \frac{|v|^{2 \theta+1}}{(|u|+\kappa)^{n+k-1}}\left[\min \left\{\frac{1}{(|u|+\kappa)^{2}}, \frac{1}{|v|^{2}}\right\}\right]^{\gamma+1} d u d \kappa d v
$$

where $D_{4}^{3}:=\left\{(u, \kappa, v) \in \mathbb{R}^{n-1} \times \mathbb{R}_{+} \times \mathbb{R}:|u|+\kappa>c,|v|>c\right\}$.

To proceed, first consider the case when $|v| \leq|u|+\kappa$, and set $v=v^{\prime}(|u|+\kappa)$, $v^{\prime} \in \mathbb{R}$. Then

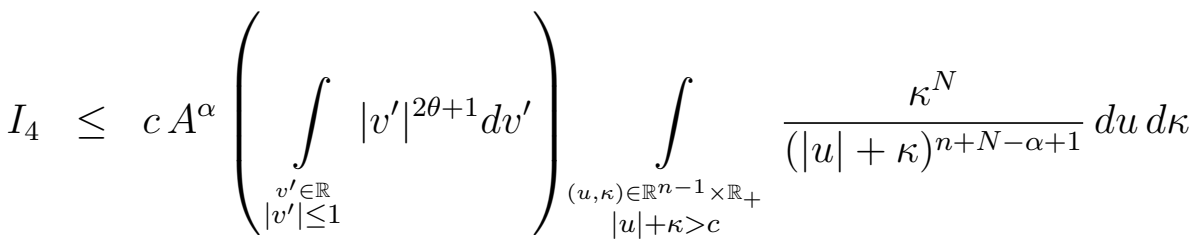

$$
\begin{aligned}
& \leq c A^{\alpha} \int_{\substack{(u, \kappa) \in \mathbb{R}^{n-1} \times \mathbb{R}_{+} \\
|u|+\kappa>c}} \frac{d u d \kappa}{(|u|+\kappa)^{n-\alpha+1}} \\
& \leq c A^{\alpha} \text {. }
\end{aligned}
$$


If $|u|+\kappa<|v|$, we set $u=u^{\prime}|v|, u^{\prime} \in \mathbb{R}^{n-1}$ and $\kappa=\kappa^{\prime}|v|, \kappa^{\prime} \in \mathbb{R}_{+}$. This yields

$$
\begin{aligned}
I_{4} & \leq c A^{\alpha}\left(\int_{\substack{v \in \mathbb{R} \\
|v|>c}} \frac{d v}{|v|^{2-\alpha}}\right) \int_{\substack{\left(u^{\prime}, \kappa^{\prime}\right) \in \mathbb{R}^{n-1} \times \mathbb{R}_{+} \\
\left|u^{\prime}\right|+\kappa^{\prime}<1}} \frac{\left(\kappa^{\prime}\right)^{N}}{\left(\left|u^{\prime}\right|+\kappa^{\prime}\right)^{n+k-1}} d u^{\prime} d \kappa^{\prime} \\
& \leq c A^{\alpha} \int_{\substack{\left(u^{\prime}, \kappa^{\prime}\right) \in \mathbb{R}^{n-1} \times \mathbb{R}_{+} \\
\left|u^{\prime}\right|+\kappa^{\prime}<1}} \frac{d u^{\prime} d \kappa^{\prime}}{\left(\left|u^{\prime}\right|+\kappa^{\prime}\right)^{n+1-2 \gamma+2 \theta-\alpha}} \\
& \leq c A^{\alpha},
\end{aligned}
$$

provided $2 \gamma-2 \theta+\alpha>1$

For the diffenence in the integral $I_{4}^{\prime}$ we use the Fundamental Theorem of Calculus to obtain that

$$
\begin{aligned}
& \left|\nabla^{k} \partial_{\text {time }}^{\gamma} I_{\text {time }}^{\theta} E(x-z, t-s)-\nabla^{k} \partial_{\text {time }}^{\gamma} I_{\text {time }}^{\theta} E(x-z, t-r)\right| \\
& \quad \leq \int_{0}^{1}\left|\nabla^{k} \partial_{\text {time }}^{\gamma+1} I_{\text {time }}^{\theta} E\left(x-z, t-\zeta_{j}\right)\right||s-r| d j
\end{aligned}
$$

where $\zeta_{j}=(1-j) r+j s$. According to (1.2.19), the expression in (5.1.13) is controlled by

$$
c|s-r| \int_{0}^{1} \frac{\left|t-\zeta_{j}\right|^{\theta}}{|x-z|^{n+k+2 \gamma+2}}\left[\min \left\{1, \frac{|x-z|^{2}}{\left|t-\zeta_{j}\right|}\right\}\right]^{\gamma+2} d j,
$$

which is equivalent with

$$
c|s-r| \int_{0}^{1} \frac{\left[\min \left\{\left|t-\zeta_{j}\right|,|x-z|^{2}\right\}\right]^{\gamma+2}}{|x-z|^{n+k+2 \gamma+2}\left|t-\zeta_{j}\right|^{\gamma+2-\theta}} d j .
$$

Simple calculations yield to the following. On the domain of integration (precisely due to the fact that $\left.|t-s|^{1 / 2}>c A\right)$ we have $|t-\zeta| \approx|t-s|(\approx|t-r|)$. Also, $|x-y|>c A$ implies $|x-z|>c A$. Therefore

$$
I_{4}^{\prime} \leq c A^{2} \int_{D_{4}^{4}} \delta^{N} \frac{\left[\min \left\{|t-s|,|x-z|^{2}\right\}\right]^{\gamma+2}}{|x-z|^{n+k+2 \gamma+2}|t-s|^{\gamma+2-\theta}} d x d t
$$


where $D_{4}^{4}:=\left\{(x, t) \in \Omega \times \mathbb{R}:|x-z|>c A,|t-s|^{1 / 2}>c A\right\}$. Since $x \in \Omega, z \in \partial \Omega$, we can write $x=\left(x^{\prime}, \varphi\left(x^{\prime}\right)+\tau\right), z=\left(z^{\prime}, \varphi\left(z^{\prime}\right)\right)$, where $x^{\prime}, z^{\prime} \in \mathbb{R}^{n-1}, \varphi: \mathbb{R}^{n-1} \rightarrow \mathbb{R}$ is a Lipschitz function such that $\Omega$ lies above the graph of $\varphi$ (if $\Omega$ is a bounded domain, then we use a finite partition of unity on $\partial \Omega$ ), and $\tau$ is some positive real number. Then $|x-z| \approx\left|x^{\prime}-z^{\prime}\right|+\tau, \delta(x) \approx \tau, d x \approx d x^{\prime} d \tau$, and

$$
I_{4}^{\prime} \leq c A^{2} \int_{D_{4}^{5}} \tau^{N} \frac{\left[\min \left\{|t-s|,\left(\left|x^{\prime}-z^{\prime}\right|+\tau\right)^{2}\right\}\right]^{\gamma+2}}{\left(\left|x^{\prime}-z^{\prime}\right|+\tau\right)^{n+k+2 \gamma+2}|t-s|^{\gamma+2-\theta}} d x^{\prime} d \tau d t,
$$

where $D_{4}^{5}:=\left\{\left(x^{\prime}, \tau, t\right) \in \mathbb{R}^{n-1} \times \mathbb{R}_{+} \times \mathbb{R}:\left|x^{\prime}-z^{\prime}\right|+\tau>c A,|t-s|^{1 / 2}>c A\right\}$. Going further, let $x^{\prime}-z^{\prime}=x^{\prime \prime}, x^{\prime \prime} \in \mathbb{R}^{n-1}$, and $t-s=\left(t^{\prime}\right)^{2}, t^{\prime} \in \mathbb{R}$, which yields

$$
I_{4}^{\prime} \leq c A^{2} \int_{D_{4}^{2}} \tau^{N} \frac{\left[\min \left\{\left|t^{\prime}\right|,\left|x^{\prime \prime}\right|+\tau\right\}\right]^{2 \gamma+4}}{\left(\left|x^{\prime \prime}\right|+\tau\right)^{n+k+2 \gamma+2}\left|t^{\prime}\right|^{2 \gamma+3-2 \theta}} d x^{\prime \prime} d \tau d t^{\prime},
$$

where $D_{4}^{2}=\left\{\left(x^{\prime \prime}, \tau, t^{\prime}\right) \in \mathbb{R}^{n-1} \times \mathbb{R}_{+} \times \mathbb{R}:\left|x^{\prime \prime}\right|+\tau>c A,\left|t^{\prime}\right|>c A\right\}$, as before. Next, let $x^{\prime \prime}=A u, u \in \mathbb{R}^{n-1}, \tau=A \kappa, \kappa \in \mathbb{R}_{+}$, and $t^{\prime}=A v, v \in \mathbb{R}$. Recall that $D_{4}^{3}=\left\{(u, \kappa, v) \in \mathbb{R}^{n-1} \times \mathbb{R}_{+} \times \mathbb{R}:|u|+\kappa>c,|v|>c\right\}$. The above change of variables yields

$$
I_{4}^{\prime} \leq c A^{\alpha} \int_{D_{4}^{3}} \kappa^{N} \frac{[\min \{|v|,|u|+\kappa\}]^{2 \gamma+4}}{(|u|+\kappa)^{n+k+2 \gamma+2}|v|^{2 \gamma+3-2 \theta}} d u d \kappa d v .
$$

In order to show that $I_{4}^{\prime} \leq c A^{\alpha}$, we first assume $|v| \leq|u|+\kappa$, and make the change of variables $v=v^{\prime}(|u|+\kappa), v^{\prime} \in \mathbb{R}$ in (5.1.14). Then the following holds.

$$
\begin{aligned}
& I_{4}^{\prime} \leq c A^{\alpha}\left(\int_{\substack{v^{\prime} \in \mathbb{R} \\
\left|v^{\prime}\right| \leq 1}}\left|v^{\prime}\right|^{2 \theta+1} d v^{\prime}\right) \int_{\substack{(u, \kappa) \in \mathbb{R}^{n-1} \times \mathbb{R}_{+} \\
|u|+\kappa>c}} \frac{\kappa^{N}}{(|u|+\kappa)^{n+N-\alpha+2}} d u d \kappa \\
& \leq c A^{\alpha} \int_{\substack{u, \kappa) \in \mathbb{R}^{n-1} \times \mathbb{R}_{+} \\
|u|+\kappa>c}} \frac{d u d \kappa}{(|u|+\kappa)^{n-\alpha+2}} \\
& \leq c A^{\alpha} \text {. }
\end{aligned}
$$


Second, assume $|v|>|u|+\kappa$, and make the same change of variables, i.e. $v=$ $v^{\prime}(|u|+\kappa), v^{\prime} \in \mathbb{R}$. Then

$$
\begin{aligned}
I_{4}^{\prime} & \leq c A^{\alpha}\left(\int_{\substack{v^{\prime} \in \mathbb{R} \\
\left|v^{\prime}\right|>1}} \frac{d v^{\prime}}{\left|v^{\prime}\right|^{2 \gamma+3-2 \theta}}\right) \int_{\substack{(u, \kappa) \in \mathbb{R}^{n-1} \times \mathbb{R}_{+} \\
|u|+\kappa>c}} \frac{\kappa^{N}}{(|u|+\kappa)^{n+N-\alpha+2}} d u d \kappa \\
& \leq c A^{\alpha} \int_{\substack{(u, \kappa) \in \mathbb{R}^{n-1} \times \mathbb{R}_{+} \\
|u|+\kappa>c}} \frac{d u d \kappa}{(|u|+\kappa)^{n-\alpha+2}} \\
& \leq c A^{\alpha},
\end{aligned}
$$

completing the proof of $\mathbf{A}$.

In order to show $\mathbf{B}$, note that, since $\|F\|_{L^{\infty}(\Omega \times \mathbb{R})} \leq 1,\|\tilde{\psi}\|_{L^{\infty}(\Omega)} \leq c$, and $\tilde{\tilde{\psi}} \equiv 1$ on $K \times I$, it suffices to prove that

$$
J_{1}:=\sup _{(y, s) \in K \times I} \int_{\tilde{K} \times \mathbb{R}} \delta(x)^{N}\left|\nabla^{k} \partial_{\text {time }}^{\gamma} I_{\text {time }}^{\theta} E(x-y, t-s) d x d t\right|<\infty,
$$

where $K \times I \subset \partial \Omega \times \mathbb{R}$ and $\tilde{K} \subset \Omega$ are compact sets.

According to (1.2.19),

$$
J_{1} \leq c \int_{\tilde{K} \times \mathbb{R}} \delta(x)^{N} \frac{|t-s|^{\theta}}{|x-y|^{n+k+2 \gamma}}\left[\min \left\{1, \frac{|x-y|^{2}}{|t-s|}\right\}\right]^{\gamma+1} d x d t .
$$

If $\Omega$ is a bounded Lipschitz domain, we can reduce matters to the graph case by choosing a finite partition of unity on $\partial \Omega$. In this scenario, $x=\left(x^{\prime}, \varphi\left(x^{\prime}\right)+\tau\right)$, $y=\left(y^{\prime}, \varphi\left(y^{\prime}\right)\right)$, where $x^{\prime}, y^{\prime} \in \mathbb{R}^{n-1}, \tau \approx \delta(x)$, and the graph of the Lipschitz function $\varphi: \mathbb{R}^{n-1} \rightarrow \mathbb{R}$ represents the boundary of $\Omega$. Moreover, since $x \in \tilde{K}$ and $y \in K$, we also have $\left|x^{\prime}\right| \leq c, \tau \leq c$, and $\left|y^{\prime}\right| \leq c$. Consequently,

$$
J_{1} \leq c \int_{\substack{\mathbb{R}^{n-1} \times \mathbb{R}_{+} \times \mathbb{R} \\\left|x^{\prime}\right| \leq c, \tau \leq c}} \frac{\tau^{N}|t-s|^{\theta}}{\left(\left|x^{\prime}-y^{\prime}\right|+\tau\right)^{n+k+2 \gamma}}\left[\min \left\{1, \frac{\left(\left|x^{\prime}-y^{\prime}\right|+\tau\right)^{2}}{|t-s|}\right\}\right]^{\gamma+1} d x^{\prime} d \tau d t .
$$


The change of variables $x^{\prime}-y^{\prime}=z \in \mathbb{R}^{n-1}$ and $t-s=r^{2}, r \in \mathbb{R}$ yield

$$
J_{1} \leq c \int_{\substack{\mathbb{R}^{n-1} \times \mathbb{R}_{+} \times \mathbb{R} \\|z| \leq c, \tau \leq c}} \frac{\tau^{N}|r|^{2 \theta+1}}{(|z|+\tau)^{n+k+2 \gamma}}\left[\min \left\{1, \frac{|z|+\tau}{|r|}\right\}\right]^{2 \gamma+2} d z d \tau d r .
$$

In the case when $|r| \leq|z|+\tau$, we let $r=r^{\prime}(|z|+\tau), r^{\prime} \in \mathbb{R}$, and obtain that

$$
\begin{aligned}
J_{1} & \leq c\left(\int_{\substack{\mathbb{R} \\
\left|r^{\prime}\right| \leq 1}}\left|r^{\prime}\right|^{2 \theta+1} d r^{\prime}\right) \int_{\substack{\mathbb{R}^{n-1} \times \mathbb{R}_{+} \\
|z| \leq c, \tau \leq c}} \frac{\tau^{N}}{(|z|+\tau)^{n+k+2 \gamma-2 \theta-2}} d z d \tau \\
& \leq c \int_{\substack{\mathbb{R}^{n-1} \times \mathbb{R}_{+} \\
|z| \leq c, \tau \leq c}} \frac{\tau^{N}}{(|z|+\tau)^{n+N-\alpha}} d z d \tau .
\end{aligned}
$$

Going further, if $|z| \leq \tau$, we make the change of variables $z=z^{\prime} \tau$, where $z^{\prime} \in \mathbb{R}^{n-1}$.

Then $\left|z^{\prime}\right| \leq 1$, and

$$
\begin{aligned}
J_{1} & \leq c \int_{\substack{\mathbb{R}^{n-1} \\
\left|z^{\prime}\right| \leq 1}} \frac{d z^{\prime}}{\left(\left|z^{\prime}\right|+1\right)^{n+N-\alpha}}\left(\int_{0<\tau \leq c} \frac{d \tau}{\tau^{1-\alpha}}\right) \\
& \leq c,
\end{aligned}
$$

where we have used the given fact that $\alpha>0$. When $|z|>\tau$, we let $\tau=\kappa|z|$, where $\kappa \in \mathbb{R}_{+}$. Then $\kappa<1$, and

$$
\begin{aligned}
J_{1} & \leq c \int_{\substack{\mathbb{R}^{n-1} \\
|z| \leq c}} \frac{d z}{|z|^{n-1-\alpha}}\left(\int_{0<\kappa<1} \frac{\kappa^{N}}{(1+\kappa)^{n+N-\alpha}} d \kappa\right) \\
& <\infty
\end{aligned}
$$

since $\alpha>0$ and $N>-1$.

On the other hand, when $|u|+\tau<|r|$, the change of variables $r=r^{\prime}(|u|+\tau)$, 
$r^{\prime} \in \mathbb{R}$ in (5.1.16) yields

$$
\begin{aligned}
J_{1} & \leq c\left(\int_{\substack{\mathbb{R}^{\mathbb{R}}\left|r^{\prime}\right|>1 \\
\left|r^{\prime}\right|^{2 \gamma-2 \theta+1}}} \int_{\substack{\mathbb{R}^{n-1} \times \mathbb{R}_{+} \\
|u| \leq c, \tau \leq c}} \frac{\tau^{N}}{(|u|+\tau)^{n+k+2 \gamma-2 \theta-2}} d u d \tau\right. \\
& \leq c \int_{\substack{\mathbb{R}^{n-1} \times \mathbb{R}_{+} \\
|u| \leq c, \tau \leq c}} \frac{\tau^{N}}{(|u|+\tau)^{n+N-\alpha}} d u d \tau .
\end{aligned}
$$

To control the integral in $r^{\prime}$, we were using the hypothesis $2 \gamma-2 \theta>1-\alpha>0$. Now, the integral with respect to $u$ and $\tau$ is the same as the last integral in (5.1.17), hence we can conclude that $J_{1}$ is bounded by a finite constant, completing the proof of $\mathbf{B}$.

This finishes the proof of Theorem 5.1.2 in the case when $0<\theta<1$.

When $\theta=0$, following the same steps as in the case $0<\theta<1$, it is enough to show that

C.

$$
\begin{aligned}
& \int_{\Omega \times \mathbb{R}} \delta(x)^{N} \mid \nabla^{k} \partial_{\text {time }}^{\gamma} E(x-y, t-s)-\nabla^{k} \partial_{\text {time }}^{\gamma} E(x-z, t-r) \mid d x d t \\
& \leq c\left(|y-z|+|t-s|^{1 / 2}\right)^{\alpha}
\end{aligned}
$$

holds for any fixed $(y, s),(z, r) \in \partial \Omega \times \mathbb{R}$, and that

$$
\text { D. } \quad \sup _{(y, s) \in K \times I} \int_{\tilde{K} \times \mathbb{R}} \delta(x)^{N}\left|\nabla^{k} \partial_{\text {time }}^{\gamma} E(x-y, t-s) d x d t\right|<\infty \text {, }
$$

where $K \times I \subset \partial \Omega \times \mathbb{R}$ and $\tilde{K} \subset \Omega$ are compact sets.

In order to show $\mathbf{C}$, as before, we divide the domain of integration $\Omega \times \mathbb{R}$ in four parts, and handle each case separately. First, let

$$
I I_{1}:=\int_{D_{1}} \delta(x)^{N}\left|\nabla^{k} \partial_{\text {time }}^{\gamma} E(x-y, t-s)\right| d x d t
$$


where $D_{1}=\left\{(x, t) \in \Omega \times \mathbb{R}:|x-y|<c A,|t-s|^{1 / 2}<c A\right\}$, as before, and we set $A=\left(|y-z|+|t-s|^{1 / 2}\right)^{\alpha}$. Using (1.2.13),

$$
I I_{1} \leq c \int_{D_{1}} \frac{\delta(x)^{N}}{\left(|x-y|+|t-s|^{1 / 2}\right)^{n+k+2 \gamma}} d x d t .
$$

Moreover, we know that $x=\left(x^{\prime}, \varphi\left(x^{\prime}\right)+\tau\right) \in \Omega, y=\left(y^{\prime} \varphi\left(y^{\prime}\right)\right) \in \partial \Omega$, where $x^{\prime}, y^{\prime} \in$ $\mathbb{R}^{n-1}, \tau \in \mathbb{R}_{+}$, and $\varphi: \mathbb{R}^{n-1} \rightarrow \mathbb{R}$ is a Lipschitz function such that $\partial \Omega$ is the graph of $\varphi$ (for a bounded domain use a finite partition of unity on $\partial \Omega$ ). Then $|x-y| \approx\left|x^{\prime}-y^{\prime}\right|+\tau, d x \approx d x^{\prime} d \tau$, and

$$
I I_{1} \leq c \int_{D_{1}^{1}} \frac{\tau^{N}}{\left(\left|x^{\prime}-y^{\prime}\right|+\tau+|t-s|^{1 / 2}\right)^{n+k+2 \gamma}} d x^{\prime} d \tau d t
$$

where $D_{1}^{1}=\left\{\left(x^{\prime}, \tau, t\right) \in \mathbb{R}^{n-1} \times \mathbb{R}_{+} \times \mathbb{R}:\left|x^{\prime}-y^{\prime}\right|+\tau<c A,|t-s|^{1 / 2}<c A\right\}$. Making the change of variables $x^{\prime}-y^{\prime}=x^{\prime \prime}, x^{\prime \prime} \in \mathbb{R}^{n-1}$ and $t-s=\left(t^{\prime}\right)^{2}, t^{\prime} \in \mathbb{R}$ yields

$$
I I_{1} \leq c \int_{D_{1}^{2}} \frac{\tau^{N}\left|t^{\prime}\right|}{\left(\left|x^{\prime \prime}\right|+\tau+\left|t^{\prime}\right|\right)^{n+k+2 \gamma}} d x^{\prime \prime} d \tau d t^{\prime}
$$

where $D_{1}^{2}=\left\{\left(x^{\prime \prime}, \tau, t^{\prime}\right) \in \mathbb{R}^{n-1} \times \mathbb{R}_{+} \times \mathbb{R}:\left|x^{\prime \prime}\right|+\tau<c A,\left|t^{\prime}\right|<c A\right\}$. Next, we set $x^{\prime \prime}=A u, u \in \mathbb{R}^{n-1} ; \tau=A \kappa, \kappa \in \mathbb{R}_{+} ;$and $t^{\prime}=A v, v \in \mathbb{R}$, to obtain that

$$
I I_{1} \leq c A^{\alpha} \int_{D_{1}^{3}} \frac{\kappa^{N}|v|}{(|u|+\kappa+|v|)^{n+k+2 \gamma}} d u d \kappa d v
$$

with $D_{1}^{3}=\left\{(u, \kappa, v) \in \mathbb{R}^{n-1} \times \mathbb{R}_{+} \times \mathbb{R}:|u|+\kappa<c,|v|<c\right\}$. Since both $\kappa$ and $|v|$ are bounded above by $|u|+\kappa+|v|$,

$$
\begin{aligned}
I I_{1} & \leq c \int_{D_{1}^{6}} \frac{d u d \kappa d v}{(|u|+\kappa+|v|)^{n+1-\alpha}} \\
& \leq c A^{\alpha},
\end{aligned}
$$

where $D_{1}^{6}=\left\{(u, \kappa, v) \in \mathbb{R}^{n-1} \times \mathbb{R}_{+} \times \mathbb{R}:|u|+\kappa+|v|<c\right\}$. 
Remaining at the first domain of integration $D_{1}$, let

$$
I I_{1}^{\prime}:=\int_{D_{1}} \delta(x)^{N}\left|\nabla^{k} \partial_{\text {time }}^{\gamma} E(x-z, t-r)\right| d x d t
$$

The conditions on $x, y, z$ and $t, s, r$ imply $|x-z|<c A$ and $|t-r|^{1 / 2}<c A$, hence

$$
I I_{1}^{\prime} \leq \int_{D_{1}^{\prime}} \delta(x)^{N}\left|\nabla^{k} \partial_{t i m e}^{\gamma} E(x-z, t-r)\right| d x d t=I I_{1} \leq c A^{\alpha}
$$

where $D_{1}^{\prime}=\left\{(x, t) \in \Omega \times \mathbb{R}:|x-x|<c A,|t-r|^{1 / 2}<c A\right\}$.

Let the second domain of integration be, as before,

$$
D_{2}=\left\{(x, t) \in \Omega \times \mathbb{R}:|x-y|<c A,|t-s|^{1 / 2}>c A\right\},
$$

and set

$$
I I_{2}:=\int_{D_{2}} \delta(x)^{N}\left|\nabla^{k} \partial_{\text {time }}^{\gamma} E(x-y, t-s)\right| d x d t
$$

Following the same steps as in the case of $I I_{1}$, we obtain that

$$
I I_{2} \leq c A^{\alpha} \int_{D_{2}^{1}} \frac{\kappa^{N}|v|}{(|u|+\kappa+|v|)^{n+k+2 \gamma}} d u d \kappa d v
$$

where $D_{2}^{1}=\left\{(u, \kappa, v) \in \mathbb{R}^{n-1} \times \mathbb{R}_{+} \times \mathbb{R}:|u|+\kappa<c,|v|>c\right\}$. Making the change of variables $v=v^{\prime}(|u|+\kappa), v^{\prime} \in \mathbb{R}$ yields

$$
I I_{2} \leq c A^{\alpha}\left(\int_{v^{\prime} \in \mathbb{R}} \frac{\left|v^{\prime}\right|}{\left(\left|v^{\prime}\right|+1\right)^{n+k+2 \gamma}} d v^{\prime}\right) \int_{\substack{(u, \kappa) \in \mathbb{R}^{n-1} \times \mathbb{R}_{+} \\|u|+\kappa<c}} \frac{\kappa^{N}}{(|u|+\kappa)^{n+k+2 \gamma-2}} d u d \kappa .
$$

If $\left|v^{\prime}\right| \leq 1$, then the first integral in (5.1.20) is bounded by a constant. If $\left|v^{\prime}\right|>1$, the same integral is majorized by a constant multiple of $\int_{v^{\prime} \in \mathbb{R},\left|v^{\prime}\right|>1} \frac{d v^{\prime}}{\left|v^{\prime}\right|^{n+k+2 \gamma-1}}$, which 
is finite, since $N=k+2 \gamma+\alpha-2>-1$ implies $k+2 \gamma>1-\alpha>0$, and $n \geq 2$. The second integral in (5.1.20) is controlled by

$$
\int_{\substack{(u, \kappa) \in \mathbb{R}^{n-1} \times \mathbb{R}_{+} \\|u|+\kappa<c}} \frac{d u d \kappa}{(|u|+\kappa)^{n-\alpha}} \leq c
$$

With $D_{2}$ as before, we set

$$
I I_{2}^{\prime}:=\int_{D_{2}} \delta(x)^{N}\left|\nabla^{k} \partial_{t i m e}^{\gamma} E(x-z, t-r)\right| d x d t
$$

Due to the fact that $(x, t) \in D_{2}$ implies $|x-z|<c A$ and $|t-r|^{1 / 2}>c A$, we have

$$
I I_{2}^{\prime} \leq \int_{D_{2}^{\prime}} \delta(x)^{N}\left|\nabla^{k} \partial_{\text {time }}^{\gamma} E(x-z, t-r)\right| d x d t=I I_{2} \leq c A^{\alpha}
$$

where $D_{2}^{\prime}=\left\{(x, t) \in \Omega \times \mathbb{R}:|x-z|<c A,|t-r|^{1 / 2}>c A\right\}$.

Taking the third domain of integration to be

$$
D_{3}=\left\{(x, t) \in \Omega \times \mathbb{R}:|x-y|>c A,|t-s|^{1 / 2}<c A\right\},
$$

we consider

$$
I I_{3}:=\int_{D_{3}} \delta(x)^{N}\left|\nabla^{k} \partial_{\text {time }}^{\gamma} E(x-y, t-s)\right| d x d t
$$

The same steps as in the case of $I I_{1}$ yield to the inequality

$$
I I_{3} \leq c A^{\alpha} \int_{D_{3}^{1}} \frac{\kappa^{N}|v|}{(|u|+\kappa+|v|)^{n+k+2 \gamma}} d u d \kappa d v
$$

where $D_{3}^{1}=\left\{(u, \kappa, v) \in \mathbb{R}^{n-1} \times \mathbb{R}_{+} \times \mathbb{R}:|u|+\kappa>c,|v|<c\right\}$. Now, evidently, $|u|+\kappa+|v| \geq|u|+\kappa$, the power in the denominator $n+k+2 \gamma>0$, and the integral in $v, \int_{v \in \mathbb{R},|v|<c}|v| d v$, is finite. Therefore 


$$
\begin{aligned}
I I_{3} & \leq c A^{\alpha} \int_{\substack{(u, \kappa) \in \mathbb{R}^{n-1}, \mathbb{R}_{+} \\
|u|+\kappa>c}} \frac{\kappa^{k+2 \gamma+\alpha-2}}{(|u|+\kappa)^{n+k+2 \gamma}} d u d \kappa \leq c A^{\alpha} \int_{\substack{(u, \kappa) \in \mathbb{R}^{n-1}, \mathbb{R}_{+} \\
|u|+\kappa>c}} \frac{d u d \kappa}{(|u|+\kappa)^{n+2-\alpha}} \\
& \leq c A^{\alpha} .
\end{aligned}
$$

With the same $D_{3}$ as before, we let

$$
I I_{3}^{\prime}:=\int_{D_{3}} \delta(x)^{N}\left|\nabla^{k} \partial_{t i m e}^{\gamma} E(x-z, t-r)\right| d x d t
$$

which is bounded by

$$
\int_{D_{3}^{\prime}} \delta(x)^{N}\left|\nabla^{k} \partial_{\text {time }}^{\gamma} E(x-z, t-r)\right| d x d t=I I_{3} \leq c A^{\alpha},
$$

where $D_{3}^{\prime}=\left\{(x, t) \in \Omega \times \mathbb{R}:|x-z|>c A,|t-r|^{1 / 2}<c A\right\}$.

Finally, let the fourth domain of integration be

$$
D_{4}=\left\{(x, t) \in \Omega \times \mathbb{R}:|x-y|>c A,|t-s|^{1 / 2}>c A\right\},
$$

and, different from the previous cases, consider

$$
I I_{4}:=\int_{D_{4}} \delta(x)^{N}\left|\nabla^{k} \partial_{\text {time }}^{\gamma} E(x-y, t-s)-\nabla^{k} \partial_{\text {time }}^{\gamma} E(x-z, t-s)\right| d x d t
$$

and

$$
I I_{4}^{\prime}:=\int_{D_{4}} \delta(x)^{N}\left|\nabla^{k} \partial_{\text {time }}^{\gamma} E(x-z, t-s)-\nabla^{k} \partial_{\text {time }}^{\gamma} E(x-z, t-r)\right| d x d t
$$

We are going to prove that $I I_{4} \leq c A^{\alpha}$ and $I I_{4}^{\prime} \leq c A^{\alpha}$, which will complete the proof of the theorem in the case when $\theta=0$. 
To handle the difference in the integral in $I I_{4}$, use the Fundamental Theorem of Calculus. Then

$$
\begin{gathered}
\left|\nabla^{k} \partial_{\text {time }}^{\gamma} E(x-y, t-s)-\nabla^{k} \partial_{\text {time }}^{\gamma} E(x-z, t-s)\right| \\
\leq \int_{0}^{1}\left|\nabla^{k+1} \partial_{\text {time }}^{\gamma} E\left(x-\xi_{j}, t-s\right)\right||y-z| d j
\end{gathered}
$$

where $\xi_{j}=(1-j) z+j y$. By (1.2.13), the integral in (5.1.21) is controlled by

$$
c|y-z| \int_{0}^{1} \frac{d j}{\left(\left|x-\xi_{j}\right|+|t-s|^{1 / 2}\right)^{n+k+1+2 \gamma}} .
$$

Since $\left|x-\xi_{j}\right| \geq c|x-y|$ (as in the case of $\left.I_{4}\right)$, we obtain that

$$
I I_{4} \leq c A \int_{D_{4}} \frac{\delta(x)^{N}}{\left(|x-y|+|t-s|^{1 / 2}\right)^{n+k+1+2 \gamma}} d x d t .
$$

Similar to the case of $I_{4}$, we have $x=\left(x^{\prime}, \varphi\left(x^{\prime}\right)+\tau\right) \in \Omega, y=\left(y^{\prime}, \varphi\left(y^{\prime}\right)\right) \in \partial \Omega$, where $x^{\prime}, y^{\prime} \in \mathbb{R}^{n-1}, \varphi: \mathbb{R}^{n-1} \rightarrow \mathbb{R}$ is a Lipschitz function such that $\Omega$ lies above the graph of $\varphi$ (if $\Omega$ is a bounded domain, then use a finite partition of unity on $\partial \Omega$ ), and $\tau$ is some positive real number. Then $|x-y| \approx\left|x^{\prime}-y^{\prime}\right|+\tau, \delta(x) \approx \tau, d x \approx d x^{\prime} d \tau$, and

$$
I I_{4} \leq c A \int_{D_{4}^{1}} \frac{\tau^{N}}{\left(\left|x^{\prime}-y^{\prime}\right|+\tau+|t-s|^{1 / 2}\right)^{n+k+1+2 \gamma}} d x^{\prime} d \tau d t
$$

where $D_{4}^{1}=\left\{\left(x^{\prime}, \tau, t\right) \in \mathbb{R}^{n-1} \times \mathbb{R}_{+} \times \mathbb{R}:\left|x^{\prime}-y^{\prime}\right|+\tau>c A,|t-s|^{1 / 2}>c A\right\}$. То simplify the integral we make the change of variables $x^{\prime}-y^{\prime}=x^{\prime \prime}, x^{\prime \prime} \in \mathbb{R}^{n-1}$ and $t-s=\left(t^{\prime}\right)^{2}, t^{\prime} \in \mathbb{R}$, and obtain that

$$
I I_{4} \leq c A \int_{D_{4}^{2}} \frac{\tau^{N}\left|t^{\prime}\right|}{\left(\left|x^{\prime \prime}\right|+\tau+\left|t^{\prime}\right|\right)^{n+k+1+2 \gamma}} d x^{\prime \prime} d \tau d t^{\prime},
$$

where $D_{4}^{2}=\left\{\left(x^{\prime \prime}, \tau, t^{\prime}\right) \in \mathbb{R}^{n-1} \times \mathbb{R}_{+} \times \mathbb{R}:\left|x^{\prime \prime}\right|+\tau>c A,\left|t^{\prime}\right|>c A\right\}$. Going further, let $x^{\prime \prime}=A u, u \in \mathbb{R}^{n-1} ; \tau=A \kappa, \kappa \in \mathbb{R}_{+} ;$and $t^{\prime}=A v, v \in \mathbb{R}$. Then

$$
I I_{4} \leq c A^{\alpha} \int_{D_{4}^{3}} \frac{\kappa^{N}|v|}{(|u|+\kappa+|v|)^{n+k+1+2 \gamma}} d u d \kappa d v
$$


where $D_{4}^{3}=\left\{(u, \kappa, v) \in \mathbb{R}^{n-1} \times \mathbb{R}_{+} \times \mathbb{R}:|u|+\kappa>c,|v|>c\right\}$. Both $\kappa$ and $v$ are bounded by $|u|+\kappa+|v|$, thus

$$
I I_{4} \leq c A^{\alpha} \int_{D_{4}^{6}} \frac{d u d \kappa d v}{(|u|+\kappa+|v|)^{n+2-\alpha}} \leq c A^{\alpha},
$$

where $D_{4}^{6}:=\left\{(u, \kappa, v) \in \mathbb{R}^{n-1} \times \mathbb{R}_{+} \times \mathbb{R}:|u|+\kappa+|v|>c\right\}$.

In a similar fashion, the difference in $I I_{4}^{\prime}$

$$
\begin{aligned}
& \left|\nabla^{k} \partial_{\text {time }}^{\gamma} E(x-z, t-s)-\nabla^{k} \partial_{\text {time }}^{\gamma} E(x-z, t-r)\right| \\
& \quad \leq \int_{0}^{1}\left|\nabla^{k} \partial_{\text {time }}^{\gamma+1} E\left(x-z, t-\zeta_{j}\right)\right||s-r| d j \\
& \quad \leq c|s-r| \int_{0}^{1} \frac{d j}{\left(|x-z|+\left|t-\zeta_{j}\right|^{1 / 2}\right)^{n+k+2 \gamma+2}},
\end{aligned}
$$

where $\zeta_{j}=(1-j) r+j s$. Note that the inequality $\left|t-\zeta_{j}\right| \geq c|t-s|$ holds for the range of parameters we consider. Also, $|x-y|>c A$ implies $|x-z|>c A$, therefore

$$
I I_{4}^{\prime} \leq c A^{2} \int_{D_{4}^{4}} \frac{\delta(x)^{N}}{\left(|x-z|+|t-s|^{1 / 2}\right)^{n+k+2 \gamma+2}} d x d t,
$$

where $D_{4}^{4}=\left\{(x, t) \in \Omega \times \mathbb{R}:|x-z|>c A,|t-s|^{1 / 2}>c A\right\}$. Similar to the case of $I_{4}^{\prime}$, we have $x=\left(x^{\prime}, \varphi\left(x^{\prime}\right)+\tau\right) \in \Omega, z=\left(z^{\prime}, \varphi\left(z^{\prime}\right)\right) \in \partial \Omega$, where $x^{\prime}, z^{\prime} \in \mathbb{R}^{n-1}$, $\varphi: \mathbb{R}^{n-1} \rightarrow \mathbb{R}$ is a Lipschitz function such that $\Omega$ lies above the graph of $\varphi$ (if $\Omega$ is a bounded domain, then use a finite partition of unity on $\partial \Omega$ ), and $\tau$ is some positive real number. Then $|x-z| \approx\left|x^{\prime}-z^{\prime}\right|+\tau, \delta(x) \approx \tau, d x \approx d x^{\prime} d \tau$, and

$$
I I_{4}^{\prime} \leq c A^{2} \int_{D_{4}^{1}} \frac{\tau^{N}}{\left(\left|x^{\prime}-z^{\prime}\right|+\tau+|t-s|^{1 / 2}\right)^{n+k+2 \gamma+2}} d x^{\prime} d \tau d t
$$

where $D_{4}^{5}=\left\{\left(x^{\prime}, \tau, t\right) \in \mathbb{R}^{n-1} \times \mathbb{R}_{+} \times \mathbb{R}:\left|x^{\prime}-z^{\prime}\right|+\tau>c A,|t-s|^{1 / 2}>c A\right\}$. To simplify the integral we make the change of variables $x^{\prime}-z^{\prime}=x^{\prime \prime}, x^{\prime \prime} \in \mathbb{R}^{n-1}$ and 
$t-s=\left(t^{\prime}\right)^{2}, t^{\prime} \in \mathbb{R}$, and obtain that

$$
I I_{4}^{\prime} \leq c A^{2} \int_{D_{4}^{2}} \frac{\tau^{N}\left|t^{\prime}\right|}{\left(\left|x^{\prime \prime}\right|+\tau+\left|t^{\prime}\right|\right)^{n+k+2 \gamma+2}} d x^{\prime \prime} d \tau d t^{\prime}
$$

where $D_{4}^{2}=\left\{\left(x^{\prime \prime}, \tau, t^{\prime}\right) \in \mathbb{R}^{n-1} \times \mathbb{R}_{+} \times \mathbb{R}:\left|x^{\prime \prime}\right|+\tau>c A,\left|t^{\prime}\right|>c A\right\}$. Going further, let $x^{\prime \prime}=A u, u \in \mathbb{R}^{n-1}, \tau=A \kappa, \kappa \in \mathbb{R}_{+}$, and $t^{\prime}=A v, v \in \mathbb{R}$. Then

$$
I I_{4}^{\prime} \leq c A^{\alpha} \int_{D_{4}^{3}} \frac{\kappa^{N}|v|}{(|u|+\kappa+|v|)^{n+k+2 \gamma+2}} d u d \kappa d v
$$

where $D_{4}^{3}=\left\{(u, \kappa, v) \in \mathbb{R}^{n-1} \times \mathbb{R}_{+} \times \mathbb{R}:|u|+\kappa>c,|v|>c\right\}$. Both $\kappa$ and $v$ are bounded by $|u|+\kappa+|v|$, hence

$$
I I_{4}^{\prime} \leq c A^{\alpha} \int_{D_{4}^{6}} \frac{d u d \kappa d v}{(|u|+\kappa+|v|)^{n+3-\alpha}} \leq c A^{\alpha}
$$

where $D_{4}^{6}=\left\{(u, \kappa, v) \in \mathbb{R}^{n-1} \times \mathbb{R}_{+} \times \mathbb{R}:|u|+\kappa+|v|>c\right\}$, which finishes the proof of $\mathbf{C}$.

To handle $\mathbf{D}$, let us denote

$$
J_{2}:=\int_{\tilde{K} \times \mathbb{R}} \delta(x)^{N}\left|\nabla^{k} \partial_{t i m e}^{\gamma} E(x-y, t-s)\right| d x d t
$$

where $(y, s) \in K \times I$. Our goal is to prove that $J_{2}<\infty$. First we note that according to $(1.2 .13)$,

$$
J_{2} \leq c \int_{\tilde{K} \times \mathbb{R}} \frac{\delta(x)^{N}}{\left(|x-y|+|t-s|^{1 / 2}\right)^{n+k+2 \gamma}} d x d t .
$$

If $\Omega$ is a bounded Lipschitz domain, use a finite partition of unity on $\partial \Omega$ to reduce matters to a graph domain case. In this situation, $x=\left(x^{\prime}, \varphi\left(x^{\prime}\right)+\tau\right), y=\left(y^{\prime}, \varphi\left(y^{\prime}\right)\right)$, where $x^{\prime}, y^{\prime} \in \mathbb{R}^{n-1}, \tau \approx \delta(x)$, and the graph of the Lipschitz function $\varphi: \mathbb{R}^{n-1} \rightarrow \mathbb{R}$ 
represents the boundary of the unbounded domain $\Omega$. Due to the extra assumptions we have, i.e. $x \in \tilde{K}$ and $y \in K$, we also have $\left|x^{\prime}\right| \leq c, \tau \leq c$, and $\left|y^{\prime}\right| \leq c$. Then

$$
J_{2} \leq c \int_{\substack{\mathbb{R}^{n-1} \times \mathbb{R}_{+} \times \mathbb{R} \\\left|x^{\prime}\right| \leq c, \tau \leq c}} \frac{\tau^{N}}{\left(\left|x^{\prime}-y^{\prime}\right|+\tau+|t-s|^{1 / 2}\right)^{n+k+2 \gamma}} d x^{\prime} d \tau d t
$$

which, after the change of variables $t-s=r\left(\left|x^{\prime}-y^{\prime}\right|+\tau\right)^{2}, r \in \mathbb{R}$, becomes

$$
\begin{aligned}
J_{2} & \leq c \int_{\substack{\mathbb{R}^{n-1} \times \mathbb{R}_{+} \\
\left|x^{\prime}\right| \leq c, \tau \leq c}} \frac{\tau^{N}}{\left(\left|x^{\prime}-y^{\prime}\right|+\tau\right)^{n+k+2 \gamma-2}} d x^{\prime} d \tau\left(\int_{\mathbb{R}^{\prime}} \frac{d r}{\left(1+|r|^{1 / 2}\right)^{n+k+2 \gamma}}\right) \\
& \leq c \int_{\substack{\mathbb{R}^{n-1} \times \mathbb{R}_{+} \\
|z| \leq c, \tau \leq c}} \frac{\tau^{N}}{(|z|+\tau)^{n+N-\alpha}} d z d \tau .
\end{aligned}
$$

Now we distinguish two cases. If $|z| \leq \tau$, we make the cange of variables $z=z^{\prime} \tau$, where $z^{\prime} \in \mathbb{R}^{n-1}$. Then $\left|z^{\prime}\right| \leq 1$, and

$$
J_{2} \leq c \int_{\substack{\mathbb{R}^{n-1} \\\left|z^{\prime}\right| \leq 1}} \frac{d z^{\prime}}{\left(\left|z^{\prime}\right|+1\right)^{n+N-\alpha}}\left(\int_{0<\tau \leq c} \frac{d \tau}{\tau^{1-\alpha}}\right)<\infty,
$$

where we have used the given fact that $\alpha>0$. In the other case, when $|z|>\tau$, let $\tau=\kappa|z|$, where $\kappa \in \mathbb{R}_{+}$. Then $\kappa<1$, and

$$
J_{2} \leq c \int_{\substack{\mathbb{R}^{n-1} \\|z| \leq c}} \frac{d z}{|z|^{n-1-\alpha}}\left(\int_{0<\kappa<1} \frac{\kappa^{N}}{(1+\kappa)^{n+N-\alpha}} d \kappa\right)<\infty,
$$

since $\alpha>0$, and the proof of $\mathbf{D}$ is complete, finishing the proof of the theorem when $\theta=0$.

As part of the proof, we also need to make sure (see (5.1.2)) that, for each fixed $(x, t) \in \Omega \times \mathbb{R}$, and for the whole range of indices

$$
\delta(x)^{N} \tilde{\psi}(x)\left[\nabla^{k} \partial_{\text {time }}^{\gamma} I_{\text {time }}^{\theta} E(x-\cdot, t-\cdot)\right] \tilde{\tilde{\psi}}(\cdot, \cdot) \in B_{\alpha, p a r}^{\infty}(K \times I),
$$


where $K \times I \subset \partial \Omega \times \mathbb{R}$.

Since supp $\tilde{\psi} \cap \Omega \subseteq \tilde{K}$ and $\tilde{\tilde{\psi}} \equiv 1$ in some neighborhood of $K \times I$, it suffices to show that

$$
\nabla^{k} \partial_{\text {time }}^{\gamma} I_{\text {time }}^{\theta} E(x-\cdot, t-\cdot) \in B_{\alpha, p a r}^{\infty}(K \times I)
$$

An idependent argument presented later (see (5.1.60) and (5.1.66)) shows that

$$
\nabla^{k} \partial_{\text {time }}^{\gamma} I_{\text {time }}^{\theta} E(x-\cdot, t-\cdot) \in \operatorname{Lip}(K \times I)
$$

which further implies that

$$
\nabla^{k} \partial_{\text {time }}^{\gamma} I_{\text {time }}^{\theta} E(x-\cdot, t-\cdot) \in L^{\infty}(K \times I)
$$

Moreover,

$$
\begin{gathered}
\sup _{\substack{(y, s),(z, r) \in K \times I \\
(y, s) \neq(z, r)}} \frac{\left|\nabla^{k} \partial_{\text {time }}^{\gamma} I_{\text {time }}^{\theta} E(x-y, t-s)-\nabla^{k} \partial_{\text {time }}^{\gamma} I_{\text {time }}^{\theta} E(x-z, t-r)\right|}{\left(|y-z|+|s-r|^{1 / 2}\right)^{\alpha}} \\
\leq c \sup _{\substack{(y, s),(z, r) \in K \times I \\
(y, s) \neq(z, r)}}\left(|y-z|+|s-r|^{1 / 2}\right)^{1-\alpha}<\infty .
\end{gathered}
$$

Now (5.1.23) and (5.1.24) imply (5.1.22), completing the proof of Theorem 5.1.2.

Our next goal is to establish a similar result to Theorem 5.1.2, with $I_{\text {time }}^{\theta}$ replaced by $D_{\text {time }}^{\theta}$, where $D_{\text {time }}^{\theta}$ represents the fractional time-derivative operator defined in $(1.1 .3)$.

Theorem 5.1.3. Consider a Lipschitz domain $\Omega$ in $\mathbb{R}^{n}$, and let $\tilde{\psi}, \tilde{\psi}, \psi$ be as in Definition 5.1.1. Set $N:=k+2 \gamma+2 \theta+\alpha-2>-1$, where $k, \gamma \in \mathbb{N}_{0}, 0 \leq \theta<1$ and $0<\alpha<1$. Then

$$
\delta^{N} \tilde{\psi} \nabla^{k} \partial_{\text {time }}^{\gamma} D_{\text {time }}^{\theta} \mathcal{S}(\tilde{\tilde{\psi}} \psi f) \in L^{1}(\Omega \times \mathbb{R})
$$

for any $f \in\left(B_{\alpha, p a r}^{\infty}(\partial \Omega \times \mathbb{R})\right)^{*}$. 
Proof. First let us consider the case when $0<\theta<1$.

Let $f \in\left(B_{\alpha, p a r}^{\infty}(\partial \Omega \times \mathbb{R})\right)^{*}$. For each fixed $(x, t) \in \Omega \times \mathbb{R}$ we have

$$
\delta^{N} \tilde{\psi} \nabla^{k} \partial_{\text {time }}^{\gamma} D_{\text {time }}^{\theta} \mathcal{S}(\tilde{\tilde{\psi}} \psi f)(x, t)=\left\langle\delta(x)^{N} \tilde{\psi}(x) \nabla^{k} \partial_{\text {time }}^{\gamma} D_{\text {time }}^{\theta} E(x-\cdot, t-\cdot) \tilde{\tilde{\psi}}, \psi f\right\rangle
$$

Moreover,

$$
\begin{aligned}
& \left\|\delta^{N} \tilde{\psi} \nabla^{k} \partial_{\text {time }}^{\gamma} D_{\text {time }}^{\theta} \mathcal{S}(\tilde{\tilde{\psi}} \psi f)\right\|_{L^{1}(\Omega \times \mathbb{R})} \\
& =\sup _{\substack{F \in L^{\infty}(\Omega \times \mathbb{R}) \\
\|F\|_{L^{\infty} \leq 1}}} \int_{\Omega \times \mathbb{R}} F(x, t)\left\langle\delta(x)^{N} \tilde{\psi}(x) \nabla^{k} \partial_{\text {time }}^{\gamma} D_{\text {time }}^{\theta} E(x-\cdot, t-\cdot) \tilde{\psi}, \psi f\right\rangle d x d t \\
& =\sup _{\substack{F \in L^{\infty}(\Omega \times \mathbb{R}) \\
\|F\|_{L^{\infty} \leq 1}}}\left\langle\int_{\Omega \times \mathbb{R}} F(x, t) \delta(x)^{N} \tilde{\psi}(x) \nabla^{k} \partial_{\text {time }}^{\gamma} D_{\text {time }}^{\theta} E(x-\cdot, t-\cdot) \tilde{\psi} d x d t, \psi f\right\rangle \\
& \leq \underset{\left.\psi\|f\|_{\left(B_{\alpha, p a r}^{\infty}\right.}(\partial \Omega \times \mathbb{R})\right)^{*} \times}{\|} \\
& \quad \times \sup _{\substack{F \in L^{\infty}(\Omega \times \mathbb{R}) \\
\|F\|_{L} \infty \leq 1}}\left\|\int_{\Omega \times \mathbb{R}} F(x, t) \delta(x)^{N} \tilde{\psi}(x) \nabla^{k} \partial_{\text {time }}^{\gamma} D_{\text {time }}^{\theta} E(x-\cdot, t-\cdot) \tilde{\psi} d x d t\right\|_{B_{\alpha, p a r}^{\infty}(\partial \Omega \times \mathbb{R})},
\end{aligned}
$$

where $(y, s) \in K \times I$.

Next we fix a function $F \in L^{\infty}(\Omega \times \mathbb{R})$ with $\|F\|_{L^{\infty}(\Omega \times \mathbb{R})} \leq 1$, and two points $(y, s),(z, r) \in \partial \Omega \times \mathbb{R},(y, s) \neq(z, r)$, which belong to $K \times I$ (from the support condition on $\psi)$. In order to contol the $B_{\alpha, p a r}^{\infty}(\partial \Omega \times \mathbb{R})$-norm of the integral above, we shall show that there exists a positive finite constant $c$ such that

$$
\begin{aligned}
& \text { A. } \int_{\Omega \times \mathbb{R}} F(x, t) \delta(x)^{N} \tilde{\psi}(x) \nabla^{k} \partial_{t i m e}^{\gamma} D_{t i m e}^{\theta} E(x-y, t-s) \tilde{\tilde{\psi}}(y, s) d x d t \\
& -\int_{\Omega \times \mathbb{R}} F(x, t) \delta(x)^{N} \tilde{\psi}(x) \nabla^{k} \partial_{\text {time }}^{\gamma} D_{\text {time }}^{\theta} E(x-z, t-r) \tilde{\psi}(z, r) d x d t \\
& \leq c\left(|y-z|+|t-s|^{1 / 2}\right)^{\alpha},
\end{aligned}
$$


and that

B. $\sup _{(y, s) \in K \times I}\left|\int_{\tilde{K} \times \mathbb{R}} F(x, t) \delta(x)^{N} \tilde{\psi}(x)\left[\nabla^{k} \partial_{\text {time }}^{\gamma} D_{\text {time }}^{\theta} E(x-y, t-s)\right] \tilde{\tilde{\psi}}(y, s) d x d t\right|<\infty$, where $K \times I \subset \partial \Omega \times \mathbb{R}$ and $\tilde{K} \subset \Omega$ are compact sets.

Since $\|F\|_{L^{\infty}(\Omega \times \mathbb{R})} \leq 1,\|\tilde{\psi}\|_{L^{\infty}(\Omega)} \leq c$, and $\tilde{\tilde{\psi}} \equiv 1$ on $K \times I$, in order to prove $\mathbf{A}$ it is enough to show that

$$
\begin{aligned}
& \int_{\Omega \times \mathbb{R}} \delta(x)^{N}\left|\nabla^{k} \partial_{\text {time }}^{\gamma} D_{\text {time }}^{\theta} E(x-y, t-s)-\nabla^{k} \partial_{\text {time }}^{\gamma} D_{\text {time }}^{\theta} E(x-z, t-r)\right| d x d t \\
& \quad \leq c\left(|y-z|+|t-s|^{1 / 2}\right)^{\alpha} .
\end{aligned}
$$

At this point we can release the restriction that $(y, s)$ and $(z, r)$ are in a compact subset of $\partial \Omega \times \mathbb{R}$, and we will prove that (5.1.3) holds for any $(y, s),(z, r) \in \partial \Omega \times \mathbb{R}$, by dividing the domain of integration $\Omega \times \mathbb{R}$ in four parts, and handeling each case separately. Denote $\left(|y-z|+|t-s|^{1 / 2}\right)^{\alpha}$ by $A$, and set

$$
I_{1}:=\int_{D_{1}} \delta(x)^{N}\left|\nabla^{k} \partial_{\text {time }}^{\gamma} D_{\text {time }}^{\theta} E(x-y, t-s)\right| d x d t
$$

where $D_{1}:=\left\{(x, t) \in \Omega \times \mathbb{R}:|x-y|<c A,|t-s|^{1 / 2}<c A\right\}$. Using (1.2.18),

$$
I_{1} \leq c \int_{D_{1}} \delta(x)^{N} \frac{|t-s|^{1-\theta}}{|x-y|^{n+2+k+2 \gamma}}\left[\min \left\{1, \frac{|x-y|^{2}}{|t-s|}\right\}\right]^{\gamma+2} d x d t
$$

Given $x \in \Omega, y \in \partial \Omega$, we can write $x=\left(x^{\prime}, \varphi\left(x^{\prime}\right)+\tau\right)$ and $y=\left(y^{\prime}, \varphi\left(y^{\prime}\right)\right)$, where $x^{\prime}, y^{\prime} \in \mathbb{R}^{n-1}, \tau \in \mathbb{R}_{+}$, and $\varphi: \mathbb{R}^{n-1} \rightarrow \mathbb{R}$ is a Lipschitz function, whose graph is $\partial \Omega$ (if $\Omega$ is bounded, use a finite partition of unity on $\partial \Omega$ ). Then $|x-y| \approx\left|x^{\prime}-y^{\prime}\right|+\tau$ and $d x \approx d x^{\prime} d \tau$. Making also the change of variables $t-s=t^{\prime}$, (5.1.28) yields

$$
I_{1} \leq c \int_{D_{1}^{1}} \tau^{N} \frac{\left|t^{\prime}\right|^{1-\theta}}{\left(\left|x^{\prime}-y^{\prime}\right|+\tau\right)^{n+2+k+2 \gamma}}\left[\min \left\{1, \frac{\left(\left|x^{\prime}-y^{\prime}\right|+\tau\right)^{2}}{\left|t^{\prime}\right|}\right\}\right]^{\gamma+2} d x^{\prime} d \tau d t
$$


where $D_{1}^{1}:=\left\{\left(x^{\prime}, \tau, t^{\prime}\right) \in \mathbb{R}^{n-1} \times \mathbb{R}_{+} \times \mathbb{R}:\left|x^{\prime}-y^{\prime}\right|+\tau<c A,\left|t^{\prime}\right|^{1 / 2}<c A\right\}$. By setting $x^{\prime}-y^{\prime}=x^{\prime \prime}$ and $t^{\prime}=\left(t^{\prime \prime}\right)^{2}$, we can rewrite (5.1.29) as follows.

$$
I_{1} \leq c \int_{D_{1}^{2}} \tau^{N} \frac{\left|t^{\prime \prime}\right|^{3-2 \theta}}{\left(\left|x^{\prime}\right|+\tau\right)^{n+2+k+2 \gamma}}\left[\min \left\{1, \frac{\left(\left|x^{\prime}\right|+\tau\right)^{2}}{\left|t^{\prime \prime}\right|^{2}}\right\}\right]^{\gamma+2} d x^{\prime \prime} d \tau d t^{\prime \prime}
$$

where $D_{1}^{2}:=\left\{\left(x^{\prime \prime}, \tau, t^{\prime \prime}\right) \in \mathbb{R}^{n-1} \times \mathbb{R}_{+} \times \mathbb{R}:\left|x^{\prime}\right|+\tau<c A,\left|t^{\prime \prime}\right|<c A\right\}$. Going further, let $x^{\prime \prime}=A u, \tau=A \kappa$ and $t^{\prime \prime}=A v$ (rescale in $x^{\prime \prime}, \tau$ and $t^{\prime \prime}$ ). Then (5.1.30) yields

$$
I_{1} \leq c A^{\alpha} \int_{D_{1}^{3}} \kappa^{N} \frac{|v|^{3-2 \theta}}{(|u|+\kappa)^{n+2+k+2 \gamma}}\left[\min \left\{1,\left(\frac{|u|+\kappa}{|v|}\right)^{2}\right\}\right]^{\gamma+2} d u d \kappa d v
$$

where $D_{1}^{3}:=\left\{(u, \kappa, v) \in \mathbb{R}^{n-1} \times \mathbb{R}_{+} \times \mathbb{R}:|u|+\kappa<c,|v|<c\right\}$.

We distinguish two cases. First, we let $|v| \leq|u|+\kappa$. In this case, if

$$
D_{1}^{4}:=\left\{(u, \kappa, v) \in \mathbb{R}^{n-1} \times \mathbb{R}_{+} \times \mathbb{R}:|v| \leq|u|+\kappa<c\right\}
$$

then (5.1.31) implies the following.

$$
\begin{aligned}
I_{1} & \leq c A^{\alpha} \int_{D_{1}^{4}} \kappa^{N} \frac{|v|^{3-2 \theta}}{(|u|+\kappa)^{n+2+k+2 \gamma}} d u d \kappa d v \\
& \leq c A^{\alpha}\left(\int_{\substack{\tilde{v} \in \mathbb{R} \\
|\tilde{v}| \leq 1}}|\tilde{v}|^{3-2 \theta} d \tilde{v} \int_{\substack{(u, \kappa) \in \mathbb{R}^{n-1} \times \mathbb{R}_{+} \\
|u|+\kappa<c}} \frac{\kappa^{N}}{(|u|+\kappa)^{n+k+2 \gamma-2 \theta}} d u d \kappa\right. \\
& \leq c A^{\alpha} \int_{\substack{(u, \kappa) \in \mathbb{R}^{n-1} \times \mathbb{R}_{+} \\
|u|+\kappa<c}} \frac{\kappa^{N}}{(|u|+\kappa)^{n+N-\alpha}} d u d \kappa,
\end{aligned}
$$

where we have made the change of variables $v=\tilde{v}(|u|+\kappa), \tilde{v} \in \mathbb{R}$. Finally, let $\kappa=\kappa^{\prime}|u|, \kappa^{\prime} \in \mathbb{R}_{+}$in (5.1.32). Then

$$
I_{1} \leq a A^{\alpha}\left(\int_{\substack{u \in \mathbb{R}^{n-1} \\|u|<c}} \frac{d u}{|u|^{n-1-\alpha}}\right) \int_{\kappa^{\prime} \in \mathbb{R}_{+}} \frac{\left(\kappa^{\prime}\right)^{N}}{\left(\kappa^{\prime}+1\right)^{n+N-\alpha}} d \kappa^{\prime} \leq c A^{\alpha},
$$


wher we have used that if $\kappa^{\prime} \leq 1$, then $\kappa^{\prime}+1 \approx 1$ and $\int_{0<\kappa^{\prime} \leq 1} \kappa^{\prime N} d \kappa^{\prime}<\infty$ for $N>-1$, as well as the fact that if $\kappa^{\prime}>1$, then $\kappa^{\prime}+1 \approx \kappa^{\prime}$ and $\int_{\kappa^{\prime}<1} \frac{d \kappa^{\prime}}{\left(\kappa^{\prime}\right)^{n-\alpha}}$ is finite, since $n-\alpha>1$

Second, let $|u|+\kappa<|v|$ and denote

$$
D_{1}^{5}:=\left\{(u, \kappa, v) \in \mathbb{R}^{n-1} \times \mathbb{R}_{+} \times \mathbb{R}:|u|+\kappa<|v|<c\right\} .
$$

From (5.1.31) we obtain that

$$
I_{1} \leq c A^{\alpha} \int_{D_{1}^{5}} \frac{\kappa^{N}}{(|u|+\kappa)^{n+k-2}|v|^{2 \gamma+2 \theta+1}} d u d \kappa d v
$$

which, after the change of variables $v=v^{\prime}(|u|+\kappa)$, yields $v^{\prime} \in \mathbb{R}$,

$$
I_{1} \leq c A^{\alpha}\left(\int_{\substack{v^{\prime} \in \mathbb{R} \\\left|v^{\prime}\right| \geq 1}} \frac{d v^{\prime}}{\left(v^{\prime}\right)^{2 \gamma+2 \theta+1}} \int_{\substack{(u, \kappa) \in \mathbb{R}^{n-1} \times \mathbb{R}_{+} \\|u|+\kappa<c}} \frac{\kappa^{N}}{(|u|+\kappa)^{n+N-\alpha}} d u d \kappa .\right.
$$

Clearly, the integral with respect to $v^{\prime}$ in $(5.1 .33)$ is bounded by a finite constant. The second integral in (5.1.33) is equivalent with the integral in (5.1.32) which, we have seen, is bounded. Therefore $I_{1} \leq c A^{\alpha}$.

Next, we set

$$
I_{1}^{\prime}:=\int_{D_{1}} \delta(x)^{N}\left|\nabla^{k} \partial_{\text {time }}^{\gamma} D_{\text {time }}^{\theta} E(x-z, t-r)\right| d x d t
$$

where $D_{1}=\left\{(x, t) \in \Omega \times \mathbb{R}:|x-y|<c A,|t-s|^{1 / 2}<c A\right\}$, as before. Note that $|x-z| \leq|x-y|+|y-z|<c A$ and $|t-r|^{1 / 2} \leq|t-s|^{1 / 2}+|s-r|^{1 / 2}<c A$. Hence

$$
I_{1}^{\prime} \leq \int_{D_{1}^{\prime}} \delta(x)^{N}\left|\nabla^{k} \partial_{\text {time }}^{\gamma} D_{\text {time }}^{\theta} E(x-z, t-r)\right| d x d t=I_{1} \leq c A^{\alpha}
$$

where $D_{1}^{\prime}:=\left\{(x, t) \in \Omega \times \mathbb{R}:|x-z|<c A,|t-r|^{1 / 2}<c A\right\}$. 
Going further, let the second domain of integration be

$$
D_{2}:=\left\{(x, t) \in \Omega \times \mathbb{R}:|x-y|<c A,|t-s|^{1 / 2}>c A\right\},
$$

and set

$$
I_{2}:=\int_{D_{2}} \delta(x)^{N}\left|\nabla^{k} \partial_{\text {time }}^{\gamma} D_{\text {time }}^{\theta} E(x-y, t-s)\right| d x d t
$$

Following the same steps as in the case of $I_{1}$, we obtain a similar inequality to (5.1.31) (only the domain of integration is different), namely

$$
I_{2} \leq c A^{\alpha} \int_{D_{2}^{1}} \kappa^{N} \frac{|v|^{3-2 \theta}}{(|u|+\kappa)^{n+2+k+2 \gamma}}\left[\min \left\{1,\left(\frac{|u|+\kappa}{|v|}\right)^{2}\right\}\right]^{\gamma+2} d u d \kappa d v
$$

where $D_{2}^{1}:=\left\{(u, \kappa, v) \in \mathbb{R}^{n-1} \times \mathbb{R}_{+} \times \mathbb{R}:|u|+\kappa<c,|v|>c\right\}$. After some elementary simplifications and the change of variables $v=v^{\prime}(|u|+\kappa), v^{\prime} \in \mathbb{R}$, we get that

$$
\begin{aligned}
I_{2} & \leq c A^{\alpha} \int_{D_{2}^{1}} \frac{\kappa^{N}}{(|u|+\kappa)^{n+k-2}|v|^{2 \gamma+2 \theta+1}} d u d \kappa d v \\
& \leq c A^{\alpha}\left(\int_{\substack{v^{\prime} \in \mathbb{R} \\
\left|v^{\prime}\right| \geq 1}} \frac{d v^{\prime}}{\left(v^{\prime}\right)^{2 \gamma+2 \theta+1}} \int_{\substack{(u, \kappa) \in \mathbb{R}^{n-1} \times \mathbb{R}_{+} \\
|u|+\kappa<c}} \frac{\kappa^{N}}{(|u|+\kappa)^{n+N-\alpha}} d u d \kappa .\right.
\end{aligned}
$$

The integrals in (5.1.34) are equivalent with those in (5.1.33), hence they are bounded by a finitite constant. This shows that $I_{2} \leq c A^{\alpha}$.

For

$$
I_{2}^{\prime}:=\int_{D_{2}} \delta(x)^{N}\left|\nabla^{k} \partial_{\text {time }}^{\gamma} D_{\text {time }}^{\theta} E(x-z, t-r)\right| d x d t
$$

with $D_{2}:=\left\{(x, t) \in \Omega \times \mathbb{R}:|x-y|<c A,|t-s|^{1 / 2}>c A\right\}$, note that $|x-z|<c A$ and $c A<|t-s|^{1 / 2} \leq|t-r|^{1 / 2}+|s-r|^{1 / 2} \leq|t-r|^{1 / 2}+A$. Hence for $c>1$ (which 
we can assume without loss of generality), $|t-r|^{1 / 2}>c A$ and

$$
I_{2}^{\prime} \leq \int_{D_{2}^{\prime}} \delta(x)^{N}\left|\nabla^{k} \partial_{\text {time }}^{\gamma} D_{\text {time }}^{\theta} E(x-z, t-r)\right| d x d t=I_{2} \leq c A^{\alpha}
$$

where $D_{2}^{\prime}:=\left\{(x, t) \in \Omega \times \mathbb{R}:|x-z|<c A,|t-r|^{1 / 2}>c A\right\}$.

Taking the third domain of integration to be

$$
D_{3}:=\left\{(x, t) \in \Omega \times \mathbb{R}:|x-y|>c A,|t-s|^{1 / 2}<c A\right\},
$$

we consider

$$
I_{3}:=\int_{D_{3}} \delta(x)^{N}\left|\nabla^{k} \partial_{\text {time }}^{\gamma} D_{\text {time }}^{\theta} E(x-y, t-s)\right| d x d t .
$$

Following the same steps as in the case of $I_{1}$, we obtain a similar inequality to (5.1.31) (only the domain of integration is different), i.e.

$$
I_{3} \leq c A^{\alpha} \int_{D_{3}^{1}} \kappa^{N} \frac{|v|^{3-2 \theta}}{(|u|+\kappa)^{n+2+k+2 \gamma}}\left[\min \left\{1,\left(\frac{|u|+\kappa}{|v|}\right)^{2}\right\}\right]^{\gamma+2} d u d \kappa d v
$$

where $D_{3}^{1}:=\left\{(u, \kappa, v) \in \mathbb{R}^{n-1} \times \mathbb{R}_{+} \times \mathbb{R}:|u|+\kappa>c,|v|<c\right\}$. One can easily deduce that

$$
\begin{aligned}
& I_{3} \leq c A^{\alpha}\left(\int_{\substack{v \in \mathbb{R} \\
|v|<c}}|v|^{3-2 \theta} d v\right) \int_{\substack{(u, \kappa) \in \mathbb{R}^{n-1} \times \mathbb{R}_{+} \\
|u|+\kappa>c}} \frac{\kappa^{N}}{(|u|+\kappa)^{n+2+k+2 \gamma}} d u d \kappa \\
& \leq c A^{\alpha} \int_{\substack{(u, \kappa) \in \mathbb{R}^{n-1} \times \mathbb{R}_{+} \\
|u|+\kappa>c}} \frac{\kappa^{N}}{(|u|+\kappa)^{n+2+k+2 \gamma}} d u d \kappa .
\end{aligned}
$$

To proceed, we consider two cases. First, we let $\kappa \leq|u|$, and we make the change of variables $\kappa=\kappa^{\prime}|u|, \kappa^{\prime} \in \mathbb{R}_{+}$. Then $\kappa^{\prime} \leq 1,|u|>c$, and (5.1.35) yields the following.

$$
\begin{aligned}
I_{3} & \leq c A^{\alpha}\left(\int_{0<\kappa^{\prime} \leq 1} \frac{\left(\kappa^{\prime}\right)^{N}}{\left(\kappa^{\prime}+1\right)^{n+2+k+2 \gamma}} d \kappa^{\prime}\right) \int_{\substack{|u| \in \mathbb{R}^{n-1} \\
|u|>c}} \frac{d u}{|u|^{n+3-2 \theta-\alpha}} \\
& \leq c A^{\alpha} .
\end{aligned}
$$


Second, let $\kappa>|u|$, and set $u=u^{\prime} \kappa, u^{\prime} \in \mathbb{R}^{n-1}$. Then $\left|u^{\prime}\right|<1, \kappa>c$, and (5.1.35) implies that

$$
\begin{aligned}
I_{3} & \leq c A^{\alpha}\left(\int_{\kappa>c} \frac{d \kappa}{\kappa^{5-2 \theta-\alpha}}\right) \int_{\substack{u^{\prime} \in \mathbb{R}^{n-1} \\
\left|u^{\prime}\right|<1}} \frac{d u^{\prime}}{\left(\left|u^{\prime}\right|+1\right)^{n+2+k+2 \gamma}} \\
& \leq c A^{\alpha} .
\end{aligned}
$$

We next consider

$$
I_{3}^{\prime}:=\int_{D_{3}} \delta(x)^{N}\left|\nabla^{k} \partial_{\text {time }}^{\gamma} D_{\text {time }}^{\theta} E(x-z, t-r)\right| d x d t
$$

with

$$
D_{3}:=\left\{(x, t) \in \Omega \times \mathbb{R}:|x-y|>c A,|t-s|^{1 / 2}<c A\right\} .
$$

Observe that $c A<|x-z|+|y-z| \leq|x-z|+A$, hence, for $c>1$ we have $|x-z|>c A$.

Also, $|t-r|^{1 / 2} \leq|t-s|^{1 / 2}+|s-r|^{1 / 2}<c A$. Therefore

$$
I_{3}^{\prime} \leq \int_{D_{3}^{\prime}} \delta(x)^{N}\left|\nabla^{k} \partial_{\text {time }}^{\gamma} D_{\text {time }}^{\theta} E(x-z, t-r)\right| d x d t=I_{3} \leq c A^{\alpha}
$$

where

$$
D_{3}^{\prime}:=\left\{(x, t) \in \Omega \times \mathbb{R}:|x-z|>c A,|t-r|^{1 / 2}<c A\right\} .
$$

Finally, let the fourth domain of integration be

$$
D_{4}:=\left\{(x, t) \in \Omega \times \mathbb{R}:|x-y|>c A,|t-s|^{1 / 2}>c A\right\},
$$

and, different from the previous cases, consider

$$
I_{4}:=\int_{D_{4}} \delta(x)^{N}\left|\nabla^{k} \partial_{\text {time }}^{\gamma} D_{\text {time }}^{\theta} E(x-y, t-s)-\nabla^{k} \partial_{\text {time }}^{\gamma} D_{\text {time }}^{\theta} E(x-z, t-s)\right| d x d t
$$


and

$$
I_{4}^{\prime}:=\int_{D_{4}} \delta(x)^{N}\left|\nabla^{k} \partial_{\text {time }}^{\gamma} D_{\text {time }}^{\theta} E(x-z, t-s)-\nabla^{k} \partial_{\text {time }}^{\gamma} D_{\text {time }}^{\theta} E(x-z, t-r)\right| d x d t .
$$

We shall prove that $I_{4} \leq c A^{\alpha}$ and $I_{4}^{\prime} \leq c A^{\alpha}$, which will complete the proof of the theorem in the case when $0<\theta<1$.

To handle the difference in the integral in $I_{4}$, we use the Fundamental Theorem of Calculus. Then

$$
\begin{aligned}
\left|\nabla^{k} \partial_{\text {time }}^{\gamma} D_{\text {time }}^{\theta} E(x-y, t-s)-\nabla^{k} \partial_{\text {time }}^{\gamma} D_{\text {time }}^{\theta} E(x-z, t-s)\right| \\
\leq \int_{0}^{1}\left|\nabla^{k+1} \partial_{\text {time }}^{\gamma} D_{\text {time }}^{\theta} E\left(x-\xi_{j}, t-s\right)\right||y-z| d j
\end{aligned}
$$

where $\xi_{j}=(1-j) z+j y$. By (1.2.18), the expression in the right-hand side of (5.1.36) is bounded by

$$
c|y-z| \int_{0}^{1} \frac{|t-s|^{1-\theta}}{\left|x-\xi_{j}\right|^{n+k+3+2 \gamma}}\left[\min \left\{1, \frac{\left|x-\xi_{j}\right|^{2}}{|t-s|}\right\}\right]^{\gamma+2} d j
$$

which is equal to

$$
c|y-z| \int_{0}^{1} \frac{|t-s|^{1-\theta}}{\left|x-\xi_{j}\right|^{n+k-1}}\left[\min \left\{\frac{1}{\left|x-\xi_{j}\right|^{2}}, \frac{1}{|t-s|}\right\}\right]^{\gamma+2} d j .
$$

We can easily show that, on the domain of integration (more precisely due to the fact that $|x-y|>c A$, we have $\left|x-\xi_{j}\right| \geq c \max \{|x-y|,|x-z|\}$, which implies $\left|x-\xi_{j}\right| \geq c|x-y|$. Therefore

$$
I_{4} \leq c A \int_{D_{4}} \delta(x)^{N} \frac{|t-s|^{1-\theta}}{|x-y|^{n+k-1}}\left[\min \left\{\frac{1}{|x-y|^{2}}, \frac{1}{|t-s|}\right\}\right]^{\gamma+2} d x d t
$$

Since $x \in \Omega, y \in \partial \Omega$, we can write $x=\left(x^{\prime}, \varphi\left(x^{\prime}\right)+\tau\right), y=\left(y^{\prime}, \varphi\left(y^{\prime}\right)\right)$, where $x^{\prime}, y^{\prime} \in \mathbb{R}^{n-1}, \varphi: \mathbb{R}^{n-1} \rightarrow \mathbb{R}$ is a Lipschitz function such that $\Omega$ lies above the graph 
of $\varphi$ (if $\Omega$ is a bounded domain, then use a finite partition of unity on $\partial \Omega$ ), and $\tau$ is some positive real number. Then $|x-y| \approx\left|x^{\prime}-y^{\prime}\right|+\tau, \delta(x) \approx \tau$ and $d x \approx d x^{\prime} d \tau$. Also, $I_{4}$ is bounded by

$$
c A \int_{D_{4}^{1}} \tau^{N} \frac{|t-s|^{1-\theta}}{\left(\left|x^{\prime}-y^{\prime}\right|+\tau\right)^{n+k-1}}\left[\min \left\{\frac{1}{\left(\left|x^{\prime}-y^{\prime}\right|+\tau\right)^{2}}, \frac{1}{|t-s|}\right\}\right]^{\gamma+2} d x^{\prime} d \tau d t
$$

where $D_{4}^{1}:=\left\{\left(x^{\prime}, \tau, t\right) \in \mathbb{R}^{n-1} \times \mathbb{R}_{+} \times \mathbb{R}:\left|x^{\prime}-y^{\prime}\right|+\tau>c A,|t-s|^{1 / 2}>c A\right\}$. Tо simplify the integral we make the change of variables $x^{\prime}-y^{\prime}=x^{\prime \prime}, x^{\prime \prime} \in \mathbb{R}^{n-1}$ and $t-s=\left(t^{\prime}\right)^{2}, t^{\prime} \in \mathbb{R}$, and obtain that

$$
I_{4} \leq c A \int_{D_{4}^{2}} \tau^{N} \frac{\left|t^{\prime}\right|^{3-2 \theta}}{\left(\left|x^{\prime \prime}\right|+\tau\right)^{n+k-1}}\left[\min \left\{\frac{1}{\left(\left|x^{\prime \prime}\right|+\tau\right)^{2}}, \frac{1}{\left|t^{\prime}\right|^{2}}\right\}\right]^{\gamma+2} d x^{\prime \prime} d \tau d t^{\prime},
$$

where $D_{4}^{2}:=\left\{\left(x^{\prime \prime}, \tau, t^{\prime}\right) \in \mathbb{R}^{n-1} \times \mathbb{R}_{+} \times \mathbb{R}:\left|x^{\prime \prime}\right|+\tau>c A,\left|t^{\prime}\right|>c A\right\}$. Going further, let $x^{\prime \prime}=A u, u \in \mathbb{R}^{n-1}, \tau=A \kappa, \kappa \in \mathbb{R}_{+}$, and $t^{\prime}=A v, v \in \mathbb{R}$. Then

$$
I_{4} \leq c A^{\alpha} \int_{D_{4}^{3}} \kappa^{N} \frac{|v|^{3-2 \theta}}{(|u|+\kappa)^{n+k-1}}\left[\min \left\{\frac{1}{(|u|+\kappa)^{2}}, \frac{1}{|v|^{2}}\right\}\right]^{\gamma+2} d u d \kappa d v
$$

for $D_{4}^{3}:=\left\{(u, \kappa, v) \in \mathbb{R}^{n-1} \times \mathbb{R}_{+} \times \mathbb{R}:|u|+\kappa>c,|v|>c\right\}$. To proceed, first consider the case when $|v| \leq|u|+\kappa$, and set $v=v^{\prime}(|u|+\kappa), v^{\prime} \in \mathbb{R}$. Then

$$
\begin{aligned}
& I_{4} \leq c A^{\alpha}\left(\int_{\substack{v^{\prime} \in \mathbb{R} \\
\left|v^{\prime}\right| \leq 1}}\left|v^{\prime}\right|^{3-2 \theta} d v^{\prime} \int_{\substack{(u, \kappa) \in \mathbb{R}^{n-1} \times \mathbb{R}_{+} \\
|u|+\kappa>c}} \frac{\kappa^{N}}{(|u|+\kappa)^{n+N-\alpha+1}} d u d \kappa\right. \\
& \leq c A^{\alpha} \int_{\substack{(u, \kappa) \in \mathbb{R}^{n-1} \times \mathbb{R}_{+} \\
|u|+\kappa>c}} \frac{d u d \kappa}{(|u|+\kappa)^{n-\alpha+1}} \\
& \leq c A^{\alpha} \text {. }
\end{aligned}
$$

If $|u|+\kappa<|v|$, we set $u=u^{\prime}|v|, u^{\prime} \in \mathbb{R}^{n-1}$ and $\kappa=\kappa^{\prime}|v|, \kappa^{\prime} \in \mathbb{R}_{+}$. Then (5.1.37) 
yields the following.

$$
\begin{aligned}
I_{4} & \leq c A^{\alpha}\left(\int_{\substack{v \in \mathbb{R}^{\prime} \\
|v|>c}} \frac{d v}{|v|^{2-\alpha}} \int_{\substack{\left(u^{\prime}, \kappa^{\prime}\right) \in \mathbb{R}^{n-1}, \mathbb{R}_{+} \\
\left|u^{\prime}\right|+\kappa^{\prime}<1}} \frac{\left(\kappa^{\prime}\right)^{N}}{\left(\left|u^{\prime}\right|+\kappa^{\prime}\right)^{n+k-1}} d u^{\prime} d \kappa^{\prime}\right. \\
& \leq c A^{\alpha} \int_{\substack{\left(u^{\prime}, \kappa^{\prime}\right) \in \mathbb{R}^{n-1}\left|\mathbb{R}_{+}\\
\right| u^{\prime} \mid+\kappa^{\prime}<1}} \frac{d u^{\prime} d \kappa^{\prime}}{\left(\left|u^{\prime}\right|+\kappa^{\prime}\right)^{n+1-2 \gamma-2 \theta-\alpha}} \\
& \leq c A^{\alpha} .
\end{aligned}
$$

This finishes the proof of the inequality $I_{4} \leq c A^{\alpha}$.

For the diffenence in the integral $I_{4}^{\prime}$, analogously to the way we treated $I_{4}$, we use the Fundamental Theorem of Calculus to obtain that

$$
\begin{aligned}
& \mid \nabla^{k} \partial_{\text {time }}^{\gamma} D_{\text {time }}^{\theta} E(x-z, t-s)-\nabla^{k} \partial_{\text {time }}^{\gamma} D_{\text {time }}^{\theta} E(x-z, t-r) \mid \\
& \leq \int_{0}^{1}\left|\nabla^{k} \partial_{\text {time }}^{\gamma+1} D_{\text {time }}^{\theta} E\left(x-z, t-\zeta_{j}\right)\right||s-r| d j,
\end{aligned}
$$

where $\zeta_{j}=(1-j) r+j s$. According to (1.2.18), the expression in the right-hand side of (5.1.38) is controlled by

$$
c|s-r| \int_{0}^{1} \frac{\left|t-\zeta_{j}\right|^{1-\theta}}{|x-z|^{n+k+2 \gamma+4}}\left[\min \left\{1, \frac{|x-z|^{2}}{\left|t-\zeta_{j}\right|}\right\}\right]^{\gamma+3} d j,
$$

which is equivalent with

$$
c|s-r| \int_{0}^{1} \frac{\left[\min \left\{\left|t-\zeta_{j}\right|,|x-z|^{2}\right\}\right]^{\gamma+3}}{|x-z|^{n+k+2 \gamma+4}\left|t-\zeta_{j}\right|^{\gamma+2+\theta}} d j .
$$

Simple calculations yield to the following. Due to the fact that $|t-s|^{1 / 2}>c A$ ), we have $|t-\zeta| \approx|t-s|(\approx|t-r|)$. Also, $|x-y|>c A$ implies $|x-z|>c A$. Therefore

$$
I_{4}^{\prime} \leq c A^{2} \int_{D_{4}^{4}} \delta^{N} \frac{\left[\min \left\{|t-s|,|x-z|^{2}\right\}\right]^{\gamma+3}}{|x-z|^{n+k+2 \gamma+4}|t-s|^{\gamma+2+\theta}} d x d t
$$


where $D_{4}^{4}:=\left\{(x, t) \in \Omega \times \mathbb{R}:|x-z|>c A,|t-s|^{1 / 2}>c A\right\}$. Since $x \in \Omega, z \in \partial \Omega$, we can write $x=\left(x^{\prime}, \varphi\left(x^{\prime}\right)+\tau\right), z=\left(z^{\prime}, \varphi\left(z^{\prime}\right)\right)$, where $x^{\prime}, z^{\prime} \in \mathbb{R}^{n-1}, \varphi: \mathbb{R}^{n-1} \rightarrow \mathbb{R}$ is a Lipschitz function such that $\Omega$ lies above the graph of $\varphi$ (if $\Omega$ is a bounded domain, then use a finite partition of unity on $\partial \Omega$ ), and $\tau$ is some positive real number. Then $|x-z| \approx\left|x^{\prime}-z^{\prime}\right|+\tau, \delta(x) \approx \tau, d x \approx d x^{\prime} d \tau$, and

$$
I_{4}^{\prime} \leq c A^{2} \int_{D_{4}^{5}} \tau^{N} \frac{\left[\min \left\{|t-s|,\left(\left|x^{\prime}-z^{\prime}\right|+\tau\right)^{2}\right\}\right]^{\gamma+3}}{\left(\left|x^{\prime}-z^{\prime}\right|+\tau\right)^{n+k+2 \gamma+4}|t-s|^{\gamma+2+\theta}} d x^{\prime} d \tau d t
$$

for $D_{4}^{5}:=\left\{\left(x^{\prime}, \tau, t\right) \in \mathbb{R}^{n-1} \times \mathbb{R}_{+} \times \mathbb{R}:\left|x^{\prime}-z^{\prime}\right|+\tau>c A,|t-s|^{1 / 2}>c A\right\}$. Going further, let $x^{\prime}-z^{\prime}=x^{\prime \prime}, x^{\prime \prime} \in \mathbb{R}^{n-1}$, and $t-s=\left(t^{\prime}\right)^{2}, t^{\prime} \in \mathbb{R}$, which yields

$$
I_{4}^{\prime} \leq c A^{2} \int_{D_{4}^{2}} \tau^{N} \frac{\left[\min \left\{\left|t^{\prime}\right|,\left|x^{\prime \prime}\right|+\tau\right\}\right]^{2 \gamma+6}}{\left(\left|x^{\prime \prime}\right|+\tau\right)^{n+k+2 \gamma+4}\left|t^{\prime}\right|^{2 \gamma+3+2 \theta}} d x^{\prime \prime} d \tau d t^{\prime},
$$

where $D_{4}^{2}=\left\{\left(x^{\prime \prime}, \tau, t^{\prime}\right) \in \mathbb{R}^{n-1} \times \mathbb{R}_{+} \times \mathbb{R}:\left|x^{\prime \prime}\right|+\tau>c A,\left|t^{\prime}\right|>c A\right\}$, as before. We next let $x^{\prime \prime}=A u, u \in \mathbb{R}^{n-1}, \tau=A \kappa, \kappa \in \mathbb{R}_{+}$, and $t^{\prime}=A v, v \in \mathbb{R}$. Then, with $D_{4}^{3}=\left\{(u, \kappa, v) \in \mathbb{R}^{n-1} \times \mathbb{R}_{+} \times \mathbb{R}:|u|+\kappa>c,|v|>c\right\}$, we obtain that

$$
I_{4}^{\prime} \leq c A^{\alpha} \int_{D_{4}^{3}} \kappa^{N} \frac{[\min \{|v|,|u|+\kappa\}]^{2 \gamma+6}}{(|u|+\kappa)^{n+k+2 \gamma+4}|v|^{2 \gamma+3+2 \theta}} d u d \kappa d v .
$$

To handle (5.1.39), we first assume $|v| \leq|u|+\kappa$, and make the change of variables $v=v^{\prime}(|u|+\kappa), v^{\prime} \in \mathbb{R}$. Then, in this case,

$$
\begin{aligned}
I_{4}^{\prime} & \leq c A^{\alpha}\left(\int_{\substack{v^{\prime} \in \mathbb{R}^{\mid} \\
\left|v^{\prime}\right| \leq 1}}\left|v^{\prime}\right|^{3-2 \theta} d v^{\prime} \int_{\substack{(u, \kappa) \in \mathbb{R}^{n-1} \times \mathbb{R}_{+} \\
|u|+\kappa>c}} \frac{\kappa^{N}}{(|u|+\kappa)^{n+N-\alpha+2}} d u d \kappa\right. \\
& \leq c A^{\alpha} \int_{\substack{(u, \kappa) \in \mathbb{R}^{n-1} \times \mathbb{R}_{+} \\
|u|+\kappa>c}} \frac{d u d \kappa}{(|u|+\kappa)^{n-\alpha+2}} \\
& \leq c A^{\alpha} .
\end{aligned}
$$


Second, we assume $|v|>|u|+\kappa$, and make the same change of variables, i.e. $v=$ $v^{\prime}(|u|+\kappa), v^{\prime} \in \mathbb{R}$. Then

$$
\begin{aligned}
& I_{4}^{\prime} \leq c A^{\alpha}\left(\int_{\substack{v^{\prime} \in \mathbb{R} \\
\left|v^{\prime}\right|>1}} \frac{d v^{\prime}}{\left|v^{\prime}\right|^{2 \gamma+3+2 \theta}} \int_{\substack{(u, \kappa) \in \mathbb{R}^{n-1} \times \mathbb{R}_{+} \\
|u|+\kappa>c}} \frac{\kappa^{N}}{(|u|+\kappa)^{n+N-\alpha+2}} d u d \kappa\right. \\
& \leq c A^{\alpha} \int_{\substack{(u, \kappa) \in \mathbb{R}^{n-1} \times \mathbb{R}_{+} \\
|u|+\kappa>c}} \frac{d u d \kappa}{(|u|+\kappa)^{n-\alpha+2}} \\
& \leq c A^{\alpha} \text {. }
\end{aligned}
$$

This finishes the proof of the inequality $I_{4}^{\prime} \leq c A^{\alpha}$, completing the proof of $\mathbf{A}$.

In order to show $\mathbf{B}$, note that, since $\|F\|_{L^{\infty}(\Omega \times \mathbb{R})} \leq 1,\|\tilde{\psi}\|_{L^{\infty}(\Omega)} \leq c$, and $\tilde{\tilde{\psi}} \equiv 1$ on $K \times I$, it suffices to show that

$$
J_{1}:=\sup _{(y, s) \in K \times I} \int_{\tilde{K} \times \mathbb{R}} \delta(x)^{N}\left|\nabla^{k} \partial_{\text {time }}^{\gamma} D_{\text {time }}^{\theta} E(x-y, t-s) d x d t\right|<\infty
$$

where $K \times I \subset \partial \Omega \times \mathbb{R}$ and $\tilde{K} \subset \Omega$ are compact sets. According to (1.2.18),

$$
J_{1} \leq c \int_{\tilde{K} \times \mathbb{R}} \delta(x)^{N} \frac{|t-s|^{1-\theta}}{|x-y|^{n+k+2 \gamma+2}}\left[\min \left\{1, \frac{|x-y|^{2}}{|t-s|}\right\}\right]^{\gamma+2} d x d t .
$$

If $\Omega$ is a bounded Lipschitz domain, we can reduce matters to the graph case by choosing a finite partition of unity on $\partial \Omega$. In this scenario, $x=\left(x^{\prime}, \varphi\left(x^{\prime}\right)+\tau\right)$, $y=\left(y^{\prime}, \varphi\left(y^{\prime}\right)\right)$, where $x^{\prime}, y^{\prime} \in \mathbb{R}^{n-1}, \tau \approx \delta(x)$, and the graph of the Lipschitz function $\varphi: \mathbb{R}^{n-1} \rightarrow \mathbb{R}$ represents the boundary of $\Omega$. Moreover, since $x \in \tilde{K}$ and $y \in K$, we also have $\left|x^{\prime}\right| \leq c, \tau \leq c$, and $\left|y^{\prime}\right| \leq c$. Consequently,

$$
J_{1} \leq c \int_{\substack{\mathbb{R}^{n-1} \times \mathbb{R}_{+} \times \mathbb{R} \\\left|x^{\prime}\right| \leq c, \tau \leq c}} \frac{\tau^{N}|t-s|^{1-\theta}}{\left(\left|x^{\prime}-y^{\prime}\right|+\tau\right)^{n+k+2 \gamma+2}}\left[\min \left\{1, \frac{\left(\left|x^{\prime}-y^{\prime}\right|+\tau\right)^{2}}{|t-s|}\right\}\right]^{\gamma+2} d x^{\prime} d \tau d t .
$$


The change of variables $x^{\prime}-y^{\prime}=z \in \mathbb{R}^{n-1}$ and $t-s=r^{2}, r \in \mathbb{R}$ yield

$$
J_{1} \leq c \int_{\substack{\mathbb{R}^{n-1} \times \mathbb{R}_{+} \times \mathbb{R} \\|z| \leq c, \tau \leq c}} \frac{\tau^{N}|r|^{3-2 \theta}}{(|z|+\tau)^{n+k+2 \gamma+2}}\left[\min \left\{1, \frac{|z|+\tau}{|r|}\right\}\right]^{2 \gamma+4} d z d \tau d r .
$$

In the case when $|r| \leq|z|+\tau$, we let $r=r^{\prime}(|z|+\tau), r^{\prime} \in \mathbb{R}$. Then

$$
\begin{aligned}
J_{1} & \leq c\left(\int_{\substack{\mathbb{R} \\
\left|r^{\prime}\right| \leq 1}}\left|r^{\prime}\right|^{3-2 \theta} d r^{\prime}\right) \int_{\substack{\mathbb{R}^{n-1} \times \mathbb{R}_{+} \\
|z| \leq c, \tau \leq c}} \frac{\tau^{N}}{(|z|+\tau)^{n+k+2 \gamma+2 \theta-2}} d z d \tau \\
& \leq c \int_{\substack{\mathbb{R}^{n-1} \times \mathbb{R}_{+} \\
|z| \leq c, \tau \leq c}} \frac{\tau^{N}}{(|z|+\tau)^{n+N-\alpha}} d z d \tau .
\end{aligned}
$$

Now we distinguish two cases. If $|z| \leq \tau$, we make the change of variables $z=z^{\prime} \tau$, where $z^{\prime} \in \mathbb{R}^{n-1}$. Then $\left|z^{\prime}\right| \leq 1$, and

$$
J_{1} \leq c \int_{\substack{\mathbb{R}^{n-1} \\\left|z^{\prime}\right| \leq 1}} \frac{d z^{\prime}}{\left(\left|z^{\prime}\right|+1\right)^{n+N-\alpha}}\left(\int_{0<\tau \leq c} \frac{d \tau}{\tau^{1-\alpha}}\right) \leq c
$$

where we have used the given fact that $\alpha>0$. In the other case, when $|z|>\tau$, let $\tau=\kappa|z|$, where $\kappa \in \mathbb{R}_{+}$. Then $\kappa<1$, and (5.1.42) implies

$$
J_{1} \leq c \int_{\substack{\mathbb{R}^{n-1} \\|z| \leq c}} \frac{d z}{|z|^{n-1-\alpha}}\left(\int_{0<\kappa<1} \frac{\kappa^{N}}{(1+\kappa)^{n+N-\alpha}} d \kappa\right) \leq c,
$$

since $\alpha>0$ and $N>-1$.

On the other hand, when $|u|+\tau<|r|$, the change of variables $r=r^{\prime}(|u|+\tau)$, 
$r^{\prime} \in \mathbb{R}$ in (5.1.41) yields

$$
\begin{aligned}
J_{1} & \leq c\left(\int_{\substack{\mathbb{R} \\
\left|r^{\prime}\right|>1}} \frac{d r^{\prime}}{\left|r^{\prime}\right|^{2 \gamma+2 \theta+1}} \int_{\substack{\mathbb{R}^{n-1} \times \mathbb{R}_{+} \\
|u| \leq c, \tau \leq c}} \frac{\tau^{N}}{(|u|+\tau)^{n+k+2 \gamma+2 \theta-2}} d u d \tau\right. \\
& \leq c \int_{\substack{\mathbb{R}^{n-1} \times \mathbb{R}_{+} \\
|u| \leq c, \tau \leq c}} \frac{\tau^{N}}{(|u|+\tau)^{n+N-\alpha}} d u d \tau .
\end{aligned}
$$

The integral with respect to $u$ and $\tau$ is equivalent with the last integral in (5.1.42), hence we can conclude that $J_{1}$ is bounded by a finite constant, completing the proof of $\mathbf{B}$. This finishes the proof of Theorem 5.1 .3 for $0<\theta<1$.

In the case when $\theta=0$, the operator we consider is the same as the one in Theorem 5.1.2 for $\theta=0$. Since Theorem 5.1.2 was already established, the proof of our theorem, when $\theta=0$, is complete.

As part of the proof, we also need to make sure (see (5.1.26)) that, for each fixed $(x, t) \in \Omega \times \mathbb{R}$, and for the whole range of indices

$$
\delta(x)^{N} \tilde{\psi}(x)\left[\nabla^{k} \partial_{\text {time }}^{\gamma} D_{\text {time }}^{\theta} E(x-\cdot, t-\cdot)\right] \tilde{\tilde{\psi}}(\cdot, \cdot) \in B_{\alpha, p a r}^{\infty}(K \times I),
$$

where $K \times I \subset \partial \Omega \times \mathbb{R}$.

Since $\operatorname{supp} \tilde{\psi} \cap \Omega \subseteq \tilde{K}$, and $\tilde{\tilde{\psi}} \equiv 1$ in some neighborhood of $K \times I$, it suffices to show that

$$
\nabla^{k} \partial_{\text {time }}^{\gamma} D_{\text {time }}^{\theta} E(x-\cdot, t-\cdot) \in B_{\alpha, p a r}^{\infty}(K \times I)
$$

First, we shall prove that $\nabla^{k} \partial_{\text {time }}^{\gamma} D_{\text {time }}^{\theta} E(x-\cdot, t-\cdot) \in \operatorname{Lip}(K \times I)$, i.e. for any

$$
\begin{aligned}
& (y, s) ;(z, r) \in K \times I \\
& \quad\left|\nabla^{k} \partial_{\text {time }}^{\gamma} D_{\text {time }}^{\theta} E(x-y, t-s)-\nabla^{k} \partial_{\text {time }}^{\gamma} D_{\text {time }}^{\theta} E(x-z, t-r)\right|
\end{aligned}
$$




$$
\leq c\left(|y-z|+|s-r|^{1 / 2}\right)
$$

If we denote the difference

$$
\left|\nabla^{k} \partial_{\text {time }}^{\gamma} D_{\text {time }}^{\theta} E(x-y, t-s)-\nabla^{k} \partial_{\text {time }}^{\gamma} D_{\text {time }}^{\theta} E(x-z, t-s)\right|
$$

by $M_{1}$, by the Fundamental Theorem of Calculus, and (1.2.18), we have that

$$
\begin{aligned}
M_{1} & \leq \int_{0}^{1}\left|\nabla^{k+1} \partial_{\text {time }}^{\gamma} D_{\text {time }}^{\theta} E(x-\Gamma(j), t-s)\right||\dot{\Gamma}(j)| d j \\
& \leq c|y-z| \int_{0}^{1} \frac{|t-s|^{1-\theta}}{|x-\Gamma(j)|^{n+k+3+2 \gamma}}\left[\min \left\{1, \frac{|x-\Gamma(j)|^{2}}{|t-s|}\right\}\right]^{\gamma+2} d j \\
& \leq c|y-z| \int_{0}^{1} \frac{|t-s|^{1-\theta}}{|x-\Gamma(j)|^{n+k-1}}\left[\min \left\{\frac{1}{|x-\Gamma(j)|^{2}}, \frac{1}{|t-s|}\right\}\right]^{\gamma+2} d j
\end{aligned}
$$

where $\Gamma:[0,1] \rightarrow \partial \Omega, \Gamma(j):=\left((1-j) y^{\prime}+j z^{\prime}, \varphi\left((1-j) y^{\prime}+j z^{\prime}\right)\right)$, and $y=\left(y^{\prime}, \varphi\left(y^{\prime}\right)\right) \in$ $K^{\prime} \subset \partial \Omega, z=\left(z^{\prime}, \varphi\left(z^{\prime}\right)\right) \in K^{\prime} \subset \partial \Omega$. We were also using that $|\dot{\Gamma}(j)| \leq c\left|y^{\prime}-z^{\prime}\right| \approx$ $|y-z|$. Since $\Gamma(j) \in \partial \Omega$, we have $|x-\Gamma(j)| \geq \operatorname{dist}(x, \partial \Omega)=\delta(x)$, therefore

$$
M_{1} \leq c|y-z| \frac{|t-s|^{1-\theta}}{\delta(x)^{n+k-1}}\left[\min \left\{\frac{1}{\delta(x)^{2}}, \frac{1}{|t-s|}\right\}\right]^{\gamma+2} .
$$

When $|t-s| \leq \delta(x)^{2}$, using the fact that $\delta(x)$ is a constant depending on $x \in \tilde{K} \subset \Omega$, we obtain that $M_{1} \leq c|y-z|$. If $\delta(x)^{2}<|t-s|$, then

$$
M_{1} \leq c|y-z| \frac{1}{\delta(x)^{n+k-1}|t-s|^{\gamma+1+\theta}} \leq c|y-z|
$$

Therefore,

$$
M_{1} \leq c|y-z|
$$

Going further, we let

$$
M_{2}:=\left|\nabla^{k} \partial_{\text {time }}^{\gamma} D_{\text {time }}^{\theta} E(x-z, t-s)-\nabla^{k} \partial_{\text {time }}^{\gamma} D_{\text {time }}^{\theta} E(x-z, t-r)\right| .
$$


By the Fundamental Theorem of Calculus, and (1.2.18), we obtain that

$$
\begin{aligned}
M_{2} & \leq|s-r| \int_{0}^{1}\left|\nabla^{k} \partial_{\text {time }}^{\gamma+1} D_{\text {time }}^{\theta} E\left(x-z, t-\zeta_{j}\right)\right| d j \\
& \leq c|s-r| \int_{0}^{1} \frac{\left|t-\zeta_{j}\right|^{1-\theta}}{|x-z|^{n+k+2 \gamma+4}}\left[\min \left\{1, \frac{|x-z|^{2}}{\left|t-\zeta_{j}\right|}\right\}\right]^{\gamma+3} d j,
\end{aligned}
$$

where $\zeta_{j}=(1-j) s+j r$. If $\left|t-\zeta_{j}\right| \leq|x-z|^{2}$, we get that

$$
M_{2} \leq c \frac{|s-r|}{|x-z|^{n+k+2 \gamma+2 \theta+2}} \leq c|s-r|^{1 / 2}
$$

where we were also using that $|x-z| \geq \delta(x)$, and $|s-r|^{1 / 2} \leq c$. On the other hand, when $|x-z|^{2}<\left|t-\zeta_{j}\right|$,

$$
\begin{aligned}
M_{2} & \leq c|s-r| \int_{0}^{1} \frac{d j}{|x-z|^{n+k-2}\left|t-\zeta_{j}\right|^{2+\gamma+\theta}} \\
& \leq c \frac{|s-r|}{|x-z|^{n+k+2 \gamma+2 \theta+2}} \\
& \leq c|s-r|^{1 / 2}
\end{aligned}
$$

where we were also using that $|x-z| \geq \delta(x)$, and $|s-r|^{1 / 2} \leq c$. Therefore,

$$
M_{2} \leq c|s-r|^{1 / 2}
$$

From (5.1.44) and (5.1.46) we immediately have that, for $0<\theta<1$,

$$
\nabla^{k} \partial_{\text {time }}^{\gamma} D_{\text {time }}^{\theta} E(x-\cdot, t-\cdot) \in \operatorname{Lip}(K \times I),
$$

which, in concert with (5.1.66), further implies that, for $0 \leq \theta<1$,

$$
\nabla^{k} \partial_{\text {time }}^{\gamma} D_{\text {time }}^{\theta} E(x-\cdot, t-\cdot) \in L^{\infty}(K \times I) .
$$


Moreover,

$$
\begin{aligned}
& \sup _{\substack{(y, s),(z, r) \in K \times I \\
(y, s) \neq(z, r)}} \frac{\left|\nabla^{k} \partial_{\text {time }}^{\gamma} D_{\text {time }}^{\theta} E(x-y, t-s)-\nabla^{k} \partial_{\text {time }}^{\gamma} D_{\text {time }}^{\theta} E(x-z, t-r)\right|}{\left(|y-z|+|s-r|^{1 / 2}\right)^{\alpha}} \\
& \quad \leq c \sup _{\substack{(y, s),(z, r) \in K \times I \\
(y, s) \neq(z, r)}}\left(|y-z|+|s-r|^{1 / 2}\right)^{1-\alpha}<\infty .
\end{aligned}
$$

Consequently, (5.1.47) and (5.1.48) imply (5.1.43), completing the proof of Theorem 5.1.3.

Remark 5.1.4. Using Theorem 5.1.2 it is possible to prove Theorem 5.1.3 with the extra assumption $2 \gamma+2 \theta+\alpha>1$, when $0<\theta<1$. In this case, for $0<\theta<1$ we can rely on the fact that $D_{\text {time }}^{\theta}=\partial_{\text {time }} I_{\text {time }}^{1-\theta}$, i.e. $\delta^{N} \nabla^{k} \partial_{\text {time }}^{\gamma} D_{\text {time }}^{\theta}=\delta^{N} \nabla^{k} \partial_{\text {time }}^{\gamma+1} I_{\text {time }}^{1-\theta}$, which yields $N=k+2 \gamma+2 \theta+\alpha-2>-1$. If $\theta=0, D_{\text {time }}^{0}=I=I_{\text {time }}^{0}$, and the theorem follows from the case $\theta=0$ of Theorem 5.1.2.

The following result is the counterpart of Theorem 5.1.2 for the other endpoint, i.e. corresponding to $p=\infty$.

Theorem 5.1.5. Let $\Omega$ be a Lipschitz domain in $\mathbb{R}^{n}, \tilde{\psi}, \tilde{\psi}, \psi$ be as in Definition 5.1.1, and set $N:=k+2 \gamma-2 \theta+\alpha-1>0$, where $k, \gamma \in \mathbb{N}_{0}, 0 \leq \theta<1,0<\alpha<1$. Then

$$
\delta^{N} \tilde{\psi} \nabla^{k} \partial_{\text {time }}^{\gamma} I_{\text {time }}^{\theta} \mathcal{S}(\tilde{\tilde{\psi}} \psi f) \in L^{\infty}(\Omega \times \mathbb{R})
$$

for any $f \in\left(B_{\alpha, p a r}^{1}(\partial \Omega \times \mathbb{R})\right)^{*}$.

Proof. First we consider the case when $0<\theta<1$. Then, for $f \in\left(B_{\alpha, p a r}^{1}(\partial \Omega \times \mathbb{R})\right)^{*}$, 
we have

$$
\begin{aligned}
& \left\|\delta^{N} \tilde{\psi} \nabla^{k} \partial_{\text {time }}^{\gamma} I_{\text {time }}^{\theta} \mathcal{S}(\tilde{\tilde{\psi}} \psi f)\right\|_{L^{\infty}(\Omega \times \mathbb{R})} \\
& =\sup _{(x, t) \in \Omega \times \mathbb{R}}\left\{\delta(x)^{N}|\tilde{\psi}(x)|\left|\left\langle\nabla^{k} \partial_{\text {time }}^{\gamma} I_{\text {time }}^{\theta} E(x-\cdot, t-\cdot) \tilde{\tilde{\psi}}, \psi f\right\rangle\right|\right\} \\
& \leq c\|\psi f\|_{\left(B_{\alpha, p a r}^{1}(\partial \Omega \times \mathbb{R})\right)^{*}} \sup _{(x, t) \in \Omega \times \mathbb{R}}\left\{\delta(x)^{N}\left\|\nabla^{k} \partial_{\text {time }}^{\gamma} I_{\text {time }}^{\theta} E(x-\cdot, t-\cdot) \tilde{\psi}\right\|_{B_{\alpha, p a r}^{1}(\partial \Omega \times \mathbb{R})}\right\} \\
& \leq c\|f\|_{\left(B_{\alpha, p a r}^{1}(\partial \Omega \times \mathbb{R})\right)^{*}}
\end{aligned}
$$

provided that there exists a positive constant $c<\infty$ such that for all points $(x, t)$ in $\Omega \times \mathbb{R}$,

$$
\left\|\nabla^{k} \partial_{\text {time }}^{\gamma} I_{\text {time }}^{\theta} E(x-\cdot, t-\cdot) \tilde{\tilde{\psi}}\right\|_{B_{\alpha, p a r}^{1}(\partial \Omega \times \mathbb{R})} \leq c \delta(x)^{-N}
$$

Observe that in order to prove (5.1.51), it suffices to show that, for $x \in \tilde{K} \subset \Omega$ and $t \in \mathbb{R}$,

A.

$$
\begin{array}{r}
\int_{\partial \Omega \times \mathbb{R}} \int_{\partial \Omega \times \mathbb{R}} \frac{\left|\nabla^{k} \partial_{\text {time }}^{\gamma} I_{\text {time }}^{\theta} E(x-y, t-s)-\nabla^{k} \partial_{\text {time }}^{\gamma} I_{\text {time }}^{\theta} E(x-z, t-r)\right|}{\left(|y-z|+|s-r|^{1 / 2}\right)^{n+1+\alpha}} \\
\left(\sigma_{y} d s d \sigma_{z} d r\right. \\
\leq c \delta(x)^{-N},
\end{array}
$$

and

B.

$$
\left\|\nabla^{k} \partial_{\text {time }}^{\gamma} I_{\text {time }}^{\theta} E(x-\cdot, t-\cdot) \tilde{\tilde{\psi}}\right\|_{L^{1}(\partial \Omega \times \mathbb{R})} \leq c \delta(x)^{-N}
$$

In order to show $\mathbf{A}$, first let us consider the domain

$$
D_{1}:=\{(y, s, z, r) \in \partial \Omega \times \mathbb{R} \times \partial \Omega \times \mathbb{R}:|x-y|<c|y-z|\}
$$


and denote the integral

$$
\iint_{D_{1}} \frac{\left|\nabla^{k} \partial_{\text {time }}^{\gamma} I_{\text {time }}^{\theta} E(x-y, t-s)-\nabla^{k} \partial_{\text {time }}^{\gamma} I_{\text {time }}^{\theta} E(x-z, t-s)\right|}{\left(|y-z|+|s-r|^{1 / 2}\right)^{n+1+\alpha}} d \sigma_{y} d s d \sigma_{z} d r
$$

by $I_{1}$. Since $x \in \Omega$ and $y, z \in \partial \Omega$, we may write $x=\left(x^{\prime}, \varphi\left(x^{\prime}\right)+\tau\right), y=\left(y^{\prime}, \varphi\left(y^{\prime}\right)\right)$ and $z=\left(z^{\prime}, \varphi\left(z^{\prime}\right)\right)$, where $x^{\prime}, y^{\prime}, z^{\prime} \in \mathbb{R}^{n-1}, \tau \in \mathbb{R}_{+}$, and $\varphi: \mathbb{R}^{n-1} \rightarrow \mathbb{R}$ is a Lipschitz function such that the graph of $\varphi$ is $\partial \Omega$ (if $\Omega$ is a bounded domain, take a finite partition of unity on $\partial \Omega)$. Therefore $|x-y| \approx\left|x^{\prime}-y^{\prime}\right|+\tau,|y-z| \approx\left|y^{\prime}-z^{\prime}\right|$, $\delta(x) \approx \tau$, and $I_{1}$ is bounded by

$$
c \iint_{D_{1}^{1}} \frac{\left|\nabla^{k} \partial_{t i m e}^{\gamma} I_{t i m e}^{\theta} E(x-y, t-s)-\nabla^{k} \partial_{\text {time }}^{\gamma} I_{t i m e}^{\theta} E(x-z, t-s)\right|}{\left(\left|y^{\prime}-z^{\prime}\right|+|s-r|^{1 / 2}\right)^{n+1+\alpha}} d y^{\prime} d s d z^{\prime} d r
$$

where $D_{1}^{1}:=\left\{\left(\left(y^{\prime}, s\right),\left(z^{\prime}, r\right)\right) \in \mathbb{R}^{n-1} \times \mathbb{R} \times \mathbb{R}^{n-1} \times \mathbb{R}:\left|x^{\prime}-y^{\prime}\right|+\tau<c\left|y^{\prime}-z^{\prime}\right|\right\}$. By the change of variables (in $r) s-r=r^{\prime}\left|y^{\prime}-z^{\prime}\right|^{2}, r^{\prime} \in \mathbb{R}^{n-1}$, we obtain that $I_{1}$ is controlled by

$$
\begin{aligned}
& \left(\int_{\mathbb{R}} \frac{d r^{\prime}}{\left(1+\left|r^{\prime}\right|^{1 / 2}\right)^{n+1+\alpha}}\right) \times \\
& \quad \times \int_{D_{1}^{2}} \frac{\left|\nabla^{k} \partial_{\text {time }}^{\gamma} I_{\text {time }}^{\theta} E(x-y, t-s)-\nabla^{k} \partial_{\text {time }}^{\gamma} I_{\text {time }}^{\theta} E(x-z, t-s)\right|}{\left|y^{\prime}-z^{\prime}\right|^{n-1+\alpha}} d y^{\prime} d s d z^{\prime},
\end{aligned}
$$

where $D_{1}^{2}:=\left\{\left(y^{\prime}, s, z^{\prime}\right) \in \mathbb{R}^{n-1} \times \mathbb{R} \times \mathbb{R}^{n-1}:\left|x^{\prime}-y^{\prime}\right|+\tau<c\left|y^{\prime}-z^{\prime}\right|\right\}$. Since the integral in $r^{\prime}$ is finite, and we can set $t-s=s^{\prime} \in \mathbb{R}$,

$$
\begin{aligned}
I_{1} & \leq c \int_{D_{1}^{2}} \frac{\left|\nabla^{k} \partial_{\text {time }}^{\gamma} I_{\text {time }}^{\theta} E\left(x-y, s^{\prime}\right)\right|}{\left|y^{\prime}-z^{\prime}\right|^{n-1+\alpha}} d y^{\prime} d s^{\prime} d z^{\prime}+c \int_{D_{1}^{2}} \frac{\left|\nabla^{k} \partial_{\text {time }}^{\gamma} I_{\text {time }}^{\theta} E\left(x-z, s^{\prime}\right)\right|}{\left|y^{\prime}-z^{\prime}\right|^{n-1+\alpha}} d y^{\prime} d s^{\prime} d z^{\prime} \\
& =: I_{1}^{\prime}+I_{1}^{\prime \prime} .
\end{aligned}
$$

According to (1.2.19), we obtain that

$$
I_{1}^{\prime} \leq c \int_{D_{1}^{2}} \frac{\left|s^{\prime}\right|^{\theta}\left[\min \left\{1, \frac{\left(\left|x^{\prime}-y^{\prime}\right|+\tau\right)^{2}}{\left|s^{\prime}\right|}\right\}\right]^{\gamma+1}}{\left(\left|x^{\prime}-y^{\prime}\right|+\tau\right)^{n+k+2 \gamma}\left|y^{\prime}-z^{\prime}\right|^{n-1+\alpha}} d y^{\prime} d s^{\prime} d z^{\prime} .
$$


Making the change of variables (in $\left.z^{\prime}\right) y^{\prime}-z^{\prime}=z^{\prime \prime}\left(\left|x^{\prime}-y^{\prime}\right|+\tau\right), z^{\prime \prime} \in \mathbb{R}$, yields

$$
I_{1}^{\prime} \leq c\left(\int_{\substack{z^{\prime \prime} \in \mathbb{R} \\ 0<c \leq\left|z^{\prime \prime}\right|}} \frac{d z^{\prime \prime}}{\left|z^{\prime \prime}\right|^{n-1+\alpha}}\right) \iint_{\left(y^{\prime}, s^{\prime}\right) \in \mathbb{R}^{n-1} \times \mathbb{R}} \frac{\left|s^{\prime}\right|^{\theta}\left[\min \left\{1, \frac{\left(\left|x^{\prime}-y^{\prime}\right|+\tau\right)^{2}}{\left|s^{\prime}\right|}\right\}\right]^{\gamma+1}}{\left(\left|x^{\prime}-y^{\prime}\right|+\tau\right)^{n+k+2 \gamma+\alpha}} d y^{\prime} d s^{\prime},
$$

where the integral in $z^{\prime \prime}$ is finite. By setting $x^{\prime}-y^{\prime}=u \in \mathbb{R}, s^{\prime}=v^{2}, v \in \mathbb{R}$, we can further conclude that

$$
I_{1}^{\prime} \leq c \int_{(u, v) \in \mathbb{R}^{n-1} \times \mathbb{R}} \frac{|v|^{2 \theta+1}\left[\min \left\{1, \frac{(|u|+\tau)^{2}}{|v|^{2}}\right\}\right]^{\gamma+1}}{(|u|+\tau)^{n+k+2 \gamma+\alpha}} d u d v .
$$

To proceed from here, first assume $|u|+\tau \geq|v|$, and set $v=v^{\prime}(|u|+\tau), v^{\prime} \in \mathbb{R}$. Then

$$
I_{1}^{\prime} \leq c\left(\int_{\substack{v^{\prime} \in \mathbb{R} \\\left|v^{\prime}\right| \leq 1}}\left|v^{\prime}\right|^{2 \theta+1} d v^{\prime}\right) \int_{u \in \mathbb{R}^{n-1}} \frac{d u}{(|u|+\tau)^{n+N-1}},
$$

where the integral in $v^{\prime}$ is bounded by a constant. Finally, let $u=u^{\prime} \tau, u^{\prime} \in \mathbb{R}$. Then we get that

$$
I_{1}^{\prime} \leq c \tau^{-N} \int_{u^{\prime} \in \mathbb{R}^{n-1}} \frac{d u^{\prime}}{\left(\left|u^{\prime}\right|+1\right)^{n+N-1}} \leq c \tau^{-N},
$$

which is what we wanted to prove.

On the other hand, if $|u|+\tau<|v|$, we let $u=u^{\prime} \tau, u^{\prime} \in \mathbb{R}$ and $v=v^{\prime \prime} \tau, v^{\prime \prime} \in \mathbb{R}$. This yields the inequality

$$
I_{1}^{\prime} \leq c \tau^{-N} \iint_{\substack{\left(u^{\prime}, v^{\prime \prime}\right) \in \mathbb{R}^{n-1} \times \mathbb{R} \\\left|u^{\prime}\right|+1<\left|v^{\prime \prime}\right|}} \frac{d u^{\prime} d v^{\prime \prime}}{\left(\left|u^{\prime}\right|+1\right)^{n+k+\alpha-2}\left|v^{\prime \prime}\right|^{2 \gamma-2 \theta+1}}
$$

Integrating first with respect to $u^{\prime}$ yields

$$
I_{1}^{\prime} \leq c \tau^{-N} \int_{\substack{v^{\prime \prime} \in \mathbb{R} \\\left|v^{\prime \prime}\right|>1}} \frac{d v^{\prime \prime}}{\left|v^{\prime \prime}\right|^{N+1}} \leq c \tau^{-N}
$$


consequently, $I_{1}^{\prime} \leq c \tau^{-N}$ in all possible cases.

To obtain the same estimate for $I_{1}^{\prime \prime}$, note that

$$
\left|x^{\prime}-z^{\prime}\right|+\tau \leq\left|x^{\prime}-y^{\prime}\right|+\left|y^{\prime}-z^{\prime}\right|+\tau<c\left|y^{\prime}-z^{\prime}\right|
$$

therefore

$$
I_{1}^{\prime \prime} \leq c \int_{D_{1}^{3}} \frac{\left|\nabla^{k} \partial_{\text {time }}^{\gamma} I_{\text {time }}^{\theta} E\left(x-z, s^{\prime}\right)\right|}{\left|y^{\prime}-z^{\prime}\right|^{n-1+\alpha}} d y^{\prime} d s^{\prime} d z^{\prime}
$$

where $D_{1}^{3}:=\left\{\left(y^{\prime}, s^{\prime}, z^{\prime}\right) \in \mathbb{R}^{n-1} \times \mathbb{R} \times \mathbb{R}^{n-1}:\left|x^{\prime}-z^{\prime}\right|+\tau<c\left|y^{\prime}-z^{\prime}\right|\right\}$. Hence $I_{1}^{\prime \prime} \leq I_{1}^{\prime}$, which, we have seen, is controlled by $c \tau^{-N}$.

Next, let the second domain of integration be

$$
D_{2}:=\{(y, s, z, r) \in \partial \Omega \times \mathbb{R} \times \partial \Omega \times \mathbb{R}:|x-z|<c|y-z|\}
$$

and denote the integral

$$
\iint_{D_{2}} \frac{\left|\nabla^{k} \partial_{\text {time }}^{\gamma} I_{\text {time }}^{\theta} E(x-y, t-s)-\nabla^{k} \partial_{\text {time }}^{\gamma} I_{t i m e}^{\theta} E(x-z, t-s)\right|}{\left(|y-z|+|s-r|^{1 / 2}\right)^{n+1+\alpha}} d \sigma_{y} d s d \sigma_{z} d r
$$

by $I_{2}$. Since $|x-z|<c|y-z|$ implies $|x-y|<c|y-z|$,

$$
I_{2} \leq I_{1} \leq c \tau^{-N}
$$

Finally, denote the complement of $D_{1} \cup D_{2}$ by $D_{3}$, i.e.

$$
D_{3}:=\{(y, s, z, r) \in \partial \Omega \times \mathbb{R} \times \partial \Omega \times \mathbb{R}:|x-y|>c|y-z|,|x-z|>c|y-z|\}
$$

and set

$$
I_{3}:=\iint_{D_{3}} \frac{\left|\nabla^{k} \partial_{\text {time }}^{\gamma} I_{\text {time }}^{\theta} E(x-y, t-s)-\nabla^{k} \partial_{\text {time }}^{\gamma} I_{\text {time }}^{\theta} E(x-z, t-s)\right|}{\left(|y-z|+|s-r|^{1 / 2}\right)^{n+1+\alpha}} d \sigma_{y} d s d \sigma_{z} d r
$$


By the Fundamental Theorem of Calculus and (1.2.19), the difference

$$
\begin{aligned}
\mid \nabla^{k} \partial_{\text {time }}^{\gamma} I_{\text {time }}^{\theta} & E(x-y, t-s)-\nabla^{k} \partial_{\text {time }}^{\gamma} I_{\text {time }}^{\theta} E(x-z, t-s) \mid \\
& \leq|y-z| \int_{0}^{1}\left|\nabla^{k+1} \partial_{\text {time }}^{\gamma} I_{\text {time }}^{\theta} E\left(x-\xi_{j}, t-s\right)\right| d j \\
& \leq c|y-z| \int_{0}^{1} \frac{|t-s|^{\theta}}{\left|x-\xi_{j}\right|^{n+k+2 \gamma+1}}\left[\min \left\{1, \frac{\left|x-\xi_{j}\right|^{2}}{|t-s|}\right\}\right]^{\gamma+1} d j \\
& \leq c|y-z| \int_{0}^{1} \frac{|t-s|^{\theta}}{\left|x-\xi_{j}\right|^{n+k-1}}\left[\min \left\{\frac{1}{\left|x-\xi_{j}\right|^{2}}, \frac{1}{|t-s|}\right\}\right]^{\gamma+1} d j,
\end{aligned}
$$

where $\xi_{j}=(1-j) y+j z$. A simple argument shows that on the domain of integration $\left|x-\xi_{j}\right| \geq c|x-y|$, hence

$$
I_{3} \leq c \iint_{D_{3}} \frac{|y-z||t-s|^{\theta}\left[\min \left\{\frac{1}{|x-y|^{2}}, \frac{1}{|t-s|}\right\}\right]^{\gamma+1}}{|x-y|^{n+k-1}\left(|y-z|+|s-r|^{1 / 2}\right)^{n+1+\alpha}} d \sigma_{y} d s d \sigma_{z} d r .
$$

Since $x \in \Omega$ and $y, z \in \partial \Omega$, we may write $x=\left(x^{\prime}, \varphi\left(x^{\prime}\right)+\tau\right), y=\left(y^{\prime}, \varphi\left(y^{\prime}\right)\right)$ and $z=\left(z^{\prime}, \varphi\left(z^{\prime}\right)\right)$, where $x^{\prime}, y^{\prime}, z^{\prime} \in \mathbb{R}^{n-1}, \tau \in \mathbb{R}_{+}$, and $\varphi: \mathbb{R}^{n-1} \rightarrow \mathbb{R}$ is a Lipschitz function such that the graph of $\varphi$ is $\partial \Omega$ (if $\Omega$ is a bounded domain, take a finite partition of unity on $\partial \Omega)$. Therefore $|x-y| \approx\left|x^{\prime}-y^{\prime}\right|+\tau,|y-z| \approx\left|y^{\prime}-z^{\prime}\right|$, $\delta(x) \approx \tau$, and

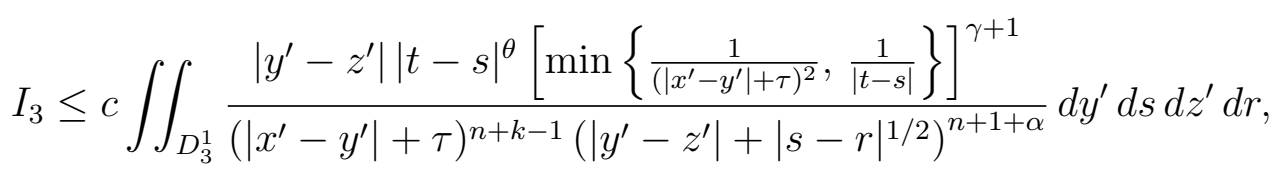

where the domain $D_{3}^{1}$ is given by

$\left\{\left(y^{\prime}, s, z^{\prime}, r\right) \in \mathbb{R}^{n-1} \times \mathbb{R} \times \mathbb{R}^{n-1} \times \mathbb{R}:\left|x^{\prime}-y^{\prime}\right|+\tau>c\left|y^{\prime}-z^{\prime}\right|,\left|x^{\prime}-z^{\prime}\right|+\tau>c\left|y^{\prime}-z^{\prime}\right|\right\}$

The change of variables $s-r=r^{\prime}\left|y^{\prime}-z^{\prime}\right|^{2}, r^{\prime} \in \mathbb{R}$ yields

$$
I_{3} \leq c\left(\int_{\mathbb{R}} \frac{d r^{\prime}}{\left(1+\left|r^{\prime}\right|^{1 / 2}\right)^{n+1+\alpha}}\right) \int_{D_{3}^{2}} \frac{|t-s|^{\theta}\left[\min \left\{\frac{1}{\left(\left|x^{\prime}-y^{\prime}\right|+\tau\right)^{2}}, \frac{1}{|t-s|}\right\}\right]^{\gamma+1}}{\left(\left|x^{\prime}-y^{\prime}\right|+\tau\right)^{n+k-1}\left|y^{\prime}-z^{\prime}\right|^{n-2+\alpha}} d y^{\prime} d s d z^{\prime}
$$


where $D_{3}^{2}$ is the domain

$$
\left\{\left(y^{\prime}, s, z^{\prime}\right) \in \mathbb{R}^{n-1} \times \mathbb{R} \times \mathbb{R}^{n-1}:\left|x^{\prime}-y^{\prime}\right|+\tau>c\left|y^{\prime}-z^{\prime}\right|,\left|x^{\prime}-z^{\prime}\right|+\tau>c\left|y^{\prime}-z^{\prime}\right|\right\}
$$

Notice that the integral in $r^{\prime}$ is finite. Going further, let $t-s=\left(s^{\prime}\right)^{2}, s^{\prime} \in \mathbb{R}$. Then

$$
I_{3} \leq c \int_{y^{\prime} \in \mathbb{R}^{n-1}} \int_{s^{\prime} \in \mathbb{R}} \frac{\left|s^{\prime}\right|^{2 \theta+1}\left[\min \left\{\frac{1}{\left|x^{\prime}-y^{\prime}\right|+\tau}, \frac{1}{\left|s^{\prime}\right|}\right\}\right]^{2 \gamma+2}}{\left(\left|x^{\prime}-y^{\prime}\right|+\tau\right)^{n+k-1}}\left(\int_{D_{3}^{3}} \frac{d z^{\prime}}{\left|y^{\prime}-z^{\prime}\right|^{n-2+\alpha}}\right) d y^{\prime} d s^{\prime},
$$

where $D_{3}^{3}:=\left\{z^{\prime} \in \mathbb{R}^{n-1}:\left|x^{\prime}-y^{\prime}\right|+\tau>c\left|y^{\prime}-z^{\prime}\right|\right\}$. Integrating with respect to $z^{\prime}$, and setting $x^{\prime}-y^{\prime}=u \in \mathbb{R}^{n-1}$, gives us

$$
I_{3} \leq c \int_{u \in \mathbb{R}^{n-1}} \int_{s^{\prime} \in \mathbb{R}} \frac{\left|s^{\prime}\right|^{2 \theta+1}}{(|u|+\tau)^{n+k+\alpha-2}}\left[\min \left\{\frac{1}{|u|+\tau}, \frac{1}{\left|s^{\prime}\right|}\right\}\right]^{2 \gamma+2} d u d s^{\prime},
$$

The right-hand side of (5.1.53) is equivalent with the right-hand side of (5.1.52), which, as we have seen earlier, is bounded by $c \tau^{-N}$. Hence $I_{3} \leq c \tau^{-N}$. What we have proved so far is as follows.

$$
\begin{aligned}
& \int_{\partial \Omega \times \mathbb{R}} \int_{\partial \Omega \times \mathbb{R}} \frac{\left|\nabla^{k} \partial_{\text {time }}^{\gamma} I_{\text {time }}^{\theta} E(x-y, t-s)-\nabla^{k} \partial_{\text {time }}^{\gamma} I_{\text {time }}^{\theta} E(x-z, t-s)\right|}{\left(|y-z|+|s-r|^{1 / 2}\right)^{n+1+\alpha}} d \sigma_{y} d s d \sigma_{z} d r \\
& \quad \leq c \delta(x)^{-N} .
\end{aligned}
$$

Next, let $I_{4}$ be the integral

$$
\iint_{D_{4}} \frac{\left|\nabla^{k} \partial_{t i m e}^{\gamma} I_{t i m e}^{\theta} E(x-z, t-s)-\nabla^{k} \partial_{t i m e}^{\gamma} I_{t i m e}^{\theta} E(x-z, t-r)\right|}{\left(|y-z|+|s-r|^{1 / 2}\right)^{n+1+\alpha}} d \sigma_{y} d s d \sigma_{z} d r
$$

where $D_{4}:=\{(y, s, z, r) \in \partial \Omega \times \mathbb{R} \times \partial \Omega \times \mathbb{R}:|t-s|<c|s-r|\}$. As in the case of $I_{1}\left(\right.$ and $\left.I_{3}\right)$, we may write $y=\left(y^{\prime}, \varphi\left(y^{\prime}\right)\right) \in \partial \Omega$ and $z=\left(z^{\prime}, \varphi\left(z^{\prime}\right)\right) \in \partial \Omega$, hence $I_{4}$ is controlled by

$$
c \iint_{D_{4}^{1}} \frac{\left|\nabla^{k} \partial_{\text {time }}^{\gamma} I_{\text {time }}^{\theta} E(x-z, t-s)-\nabla^{k} \partial_{\text {time }}^{\gamma} I_{\text {time }}^{\theta} E(x-z, t-r)\right|}{\left(\left|y^{\prime}-z^{\prime}\right|+|s-r|^{1 / 2}\right)^{n+1+\alpha}} d y^{\prime} d s d z^{\prime} d r
$$


where $D_{4}^{1}:=\left\{\left(y^{\prime}, s, z^{\prime}, r\right) \in \mathbb{R}^{n-1} \times \mathbb{R} \times \mathbb{R}^{n-1} \times \mathbb{R}:|t-s|<c|s-r|\right\}$. By the change of variables $y^{\prime}-z^{\prime}=y^{\prime \prime}|s-r|^{1 / 2}, y^{\prime \prime} \in \mathbb{R}^{n-1}$, we obtain that

$$
\begin{aligned}
I_{4} \leq c & \left(\int_{\mathbb{R}^{n-1}} \frac{d y^{\prime \prime}}{\left(\left|y^{\prime \prime}\right|+1\right)^{n+1+\alpha}}\right) \\
& \times \int_{D_{4}^{2}} \frac{\left|\nabla^{k} \partial_{\text {time }}^{\gamma} I_{\text {time }}^{\theta} E(x-z, t-s)-\nabla^{k} \partial_{\text {time }}^{\gamma} I_{\text {time }}^{\theta} E(x-z, t-r)\right|}{|s-r|^{(2+\alpha) / 2}} d s d z^{\prime} d r,
\end{aligned}
$$

where $D_{4}^{2}:=\left\{\left(s, z^{\prime}, r\right) \in \mathbb{R} \times \mathbb{R}^{n-1} \times \mathbb{R}:|t-s|<c|s-r|\right\}$. The integral with respect to $y^{\prime \prime}$ is finite, hence we can write

$$
\begin{aligned}
I_{4} \leq & c \int_{D_{4}^{2}} \frac{\left|\nabla^{k} \partial_{\text {time }}^{\gamma} I_{\text {time }}^{\theta} E(x-z, t-s)\right|}{|s-r|^{(2+\alpha) / 2}} d s d z^{\prime} d r \\
& +c \int_{D_{4}^{2}} \frac{\left|\nabla^{k} \partial_{\text {time }}^{\gamma} I_{\text {time }}^{\theta} E(x-z, t-r)\right|}{|s-r|^{(2+\alpha) / 2}} d s d z^{\prime} d r \\
=: & I_{4}^{\prime}+I_{4}^{\prime \prime} .
\end{aligned}
$$

Using (1.2.19), we obtain that

$$
I_{4}^{\prime} \leq c \int_{D_{4}^{2}} \frac{|t-s|^{\theta}\left[\min \left\{1, \frac{|x-z|^{2}}{|t-s|}\right\}\right]^{\gamma+1}}{|x-z|^{n+k+2 \gamma}|s-r|^{(2+\alpha) / 2}} d s d z^{\prime} d r
$$

where $|x-z| \approx\left|x^{\prime}-z^{\prime}\right|+\tau,\left(x=\left(x^{\prime}, \varphi\left(x^{\prime}\right)+\tau\right)\right)$, hence, setting also $s-r=r^{\prime} \in \mathbb{R}$,

$$
I_{4}^{\prime} \leq c \int_{D_{4}^{3}} \frac{|t-s|^{\theta}\left[\min \left\{\frac{1}{\left(\left|x^{\prime}-z^{\prime}\right|+\tau\right)^{2}}, \frac{1}{|t-s|}\right\}\right]^{\gamma+1}}{\left(\left|x^{\prime}-z^{\prime}\right|+\tau\right)^{n+k-2}\left|r^{\prime}\right|^{(2+\alpha) / 2}} d s d z^{\prime} d r^{\prime}
$$

where $D_{4}^{3}:=\left\{\left(s, z^{\prime}, r^{\prime}\right) \in \mathbb{R} \times \mathbb{R}^{n-1} \times \mathbb{R}:|t-s|<c\left|r^{\prime}\right|\right\}$. To simplify the above integral, let $x^{\prime}-z^{\prime}=u \in \mathbb{R}$ and $t-s=s^{\prime} \in \mathbb{R}$. Then

$$
I_{4}^{\prime} \leq c \int_{D_{4}^{4}} \frac{\left|s^{\prime}\right|^{\theta}\left[\min \left\{\frac{1}{(|u|+\tau)^{2}}, \frac{1}{\left|s^{\prime}\right|}\right\}\right]^{\gamma+1}}{(|u|+\tau)^{n+k-2}\left|r^{\prime}\right|^{(2+\alpha) / 2}} d s^{\prime} d u d r^{\prime},
$$


with $D_{4}^{4}:=\left\{\left(s^{\prime}, u, r^{\prime}\right) \in \mathbb{R} \times \mathbb{R}^{n-1} \times \mathbb{R}:\left|s^{\prime}\right|<c\left|r^{\prime}\right|\right\}$. Next, we make the change of variables $r^{\prime}=r^{\prime \prime}\left|s^{\prime}\right|, r^{\prime \prime} \in \mathbb{R}$, which yields the following.

$$
I_{4}^{\prime} \leq c\left(\int_{\substack{r^{\prime \prime} \in \mathbb{R} \\ 0<c \leq\left|r^{\prime \prime}\right|}} \frac{d r^{\prime \prime}}{\left|r^{\prime \prime}\right|^{(2+\alpha) / 2}}\right) \int_{u \in \mathbb{R}^{n-1}} \int_{s^{\prime} \in \mathbb{R}} \frac{\left|s^{\prime}\right|^{\theta-\alpha / 2}\left[\min \left\{\frac{1}{(|u|+\tau)^{2}}, \frac{1}{\left|s^{\prime}\right|}\right\}\right]^{\gamma+1}}{(|u|+\tau)^{n+k-2}} d u d s^{\prime} .
$$

The integral in $r^{\prime \prime}$ is bounded, hence, with $s^{\prime}=v^{2}, v \in \mathbb{R}$, we obtain that

$$
I_{4}^{\prime} \leq c \int_{u \in \mathbb{R}^{n-1}} \int_{v \in \mathbb{R}} \frac{|v|^{2 \theta-\alpha+1}}{(|u|+\tau)^{n+k-2}}\left[\min \left\{\frac{1}{|u|+\tau}, \frac{1}{|v|}\right\}\right]^{2 \gamma+2} d u d v .
$$

Going further, rescale in $u$ ans $v$ by letting $u=\tau u^{\prime}, u^{\prime} \in \mathbb{R}^{n-1}$ and $v=\tau v^{\prime}, v^{\prime} \in \mathbb{R}$.

Then

$$
I_{4}^{\prime} \leq c \tau^{-N} \int_{u^{\prime} \in \mathbb{R}^{n-1}} \int_{v^{\prime} \in \mathbb{R}} \frac{\left|v^{\prime}\right|^{2 \theta-\alpha+1}}{\left(\left|u^{\prime}\right|+1\right)^{n+k-2}}\left[\min \left\{\frac{1}{\left|u^{\prime}\right|+1}, \frac{1}{\left|v^{\prime}\right|}\right\}\right]^{2 \gamma+2} d u^{\prime} d v^{\prime} .
$$

To proceed from here, first assume that $\left|v^{\prime}\right| \leq\left|u^{\prime}\right|+1$ and let $v^{\prime}=v^{\prime \prime}\left(\left|u^{\prime}\right|+1\right), v^{\prime \prime} \in \mathbb{R}$.

In this setting,

$$
\begin{aligned}
I_{4}^{\prime} & \leq c \tau^{-N}\left(\int_{u^{\prime} \in \mathbb{R}^{n-1}} \frac{d u^{\prime}}{\left(\left|u^{\prime}\right|+1\right)^{n+N-1}}\right) \int_{\substack{v^{\prime \prime} \in \mathbb{R} \\
\left|v^{\prime \prime}\right| \leq 1}}\left|v^{\prime \prime}\right|^{2 \theta-\alpha+1} d v^{\prime \prime} \\
& \leq c \tau^{-N} .
\end{aligned}
$$

On the other hand, if $\left|u^{\prime}\right|+1<\left|v^{\prime}\right|$, then integrating first with respect to $u^{\prime}$, we obtain that

$$
\begin{aligned}
I_{4}^{\prime} & \leq c \tau^{-N} \int_{\substack{v^{\prime} \in \mathbb{R} \\
\left|v^{\prime}\right|>1}} \frac{1}{\left|v^{\prime}\right|^{1+2 \gamma-2 \theta+\alpha}}\left(\int_{\substack{u^{\prime} \in \mathbb{R}^{n-1} \\
\left|u^{\prime}\right|+1<\left|v^{\prime}\right|}} \frac{d u^{\prime}}{\left(\left|u^{\prime}\right|+1\right)^{n+k-2}}\right) d v^{\prime} \\
& \leq c \tau^{-N} \int_{\substack{v^{\prime} \in \mathbb{R} \\
\left|v^{\prime}\right|>1}} \frac{d v^{\prime}}{\left|v^{\prime}\right|^{N+1}} \\
& \leq c \tau^{-N} .
\end{aligned}
$$


To obtain a similar estimate for $I_{4}^{\prime \prime}$, observe that $|t-s|<c|s-r|$ implies $|t-r|<$ $c|s-r|$, hence

$$
I_{4}^{\prime \prime} \leq c \int_{D_{4}^{5}} \frac{\left|\nabla^{k} \partial_{\text {time }}^{\gamma} I_{\text {time }}^{\theta} E(x-z, t-r)\right|}{|s-r|^{(2+\alpha) / 2}} d s d z^{\prime} d r=I_{4}^{\prime} \leq c \tau^{-N}
$$

where $D_{4}^{5}:=\left\{\left(s, z^{\prime}, r\right) \in \mathbb{R} \times \mathbb{R}^{n-1} \times \mathbb{R}:|t-r|<c|s-r|\right\}$. This finishes the proof of the inequality $I_{4} \leq c \tau^{-N}$.

Next, let the second domain of integration be

$$
D_{5}:=\{(y, s, z, r) \in \partial \Omega \times \mathbb{R} \times \partial \Omega \times \mathbb{R}:|t-r|<c|s-r|\}
$$

and denote the integral

$$
\iint_{D_{5}} \frac{\left|\nabla^{k} \partial_{\text {time }}^{\gamma} I_{\text {time }}^{\theta} E(x-z, t-s)-\nabla^{k} \partial_{\text {time }}^{\gamma} I_{\text {time }}^{\theta} E(x-z, t-r)\right|}{\left(|y-z|+|s-r|^{1 / 2}\right)^{n+1+\alpha}} d \sigma_{y} d s d \sigma_{z} d r
$$

by $I_{5}$. Since $|t-r|<c|s-r|$ implies $|t-s|<c|s-r|$,

$$
I_{5} \leq I_{4} \leq c \tau^{-N}
$$

Finally, denote the complement of $D_{4} \cup D_{5}$ by $D_{6}$, i.e.

$$
D_{6}:=\{(y, s, z, r) \in \partial \Omega \times \mathbb{R} \times \partial \Omega \times \mathbb{R}:|t-s|>c|s-r|,|t-r|>c|s-r|\}
$$

and the integral

$$
\iint_{D_{6}} \frac{\left|\nabla^{k} \partial_{t i m e}^{\gamma} I_{\text {time }}^{\theta} E(x-z, t-s)-\nabla^{k} \partial_{\text {time }}^{\gamma} I_{t i m e}^{\theta} E(x-z, t-r)\right|}{\left(|y-z|+|s-r|^{1 / 2}\right)^{n+1+\alpha}} d \sigma_{y} d s d \sigma_{z} d r
$$

by $I_{6}$. By the Fundamental Theorem of Calculus and (1.2.19), the difference

$$
\left|\nabla^{k} \partial_{\text {time }}^{\gamma} I_{\text {time }}^{\theta} E(x-z, t-s)-\nabla^{k} \partial_{\text {time }}^{\gamma} I_{\text {time }}^{\theta} E(x-z, t-r)\right|
$$


is controlled by

$$
\begin{aligned}
\mid s- & r\left|\int_{0}^{1}\right| \nabla^{k} \partial_{\text {time }}^{\gamma+1} I_{\text {time }}^{\theta} E\left(x-z, t-\zeta_{j}\right) \mid d j \\
& \leq c|s-r| \int_{0}^{1} \frac{\left|t-\zeta_{j}\right|^{\theta}}{|x-z|^{n+k+2 \gamma+2}}\left[\min \left\{1, \frac{|x-z|^{2}}{\left|t-\zeta_{j}\right|}\right\}\right]^{\gamma+2} d j \\
& \leq c|s-r| \int_{0}^{1} \frac{\left[\min \left\{\left|t-\zeta_{j}\right|,|x-z|^{2}\right\}\right]^{\gamma+2}}{|x-z|^{n+k+2 \gamma+2}\left|t-\zeta_{j}\right|^{\gamma+2-\theta}} d j
\end{aligned}
$$

where $\zeta_{j}:=(1-j) r+j s$. On the domain of integration $\left|t-\zeta_{j}\right| \approx|t-s|$, therefore

$$
I_{6} \leq c \iint_{D_{6}} \frac{|s-r|\left[\min \left\{|t-s|,|x-z|^{2}\right\}\right]^{\gamma+2}}{\left(|y-z|+|s-r|^{1 / 2}\right)^{n+1+\alpha}|x-z|^{n+k+2 \gamma+2}|t-s|^{\gamma+2-\theta}} d \sigma_{y} d s d \sigma_{z} d r .
$$

Since $x=\left(x^{\prime}, \varphi\left(x^{\prime}\right)+\tau\right) \in \Omega, y=\left(y^{\prime}, \varphi\left(y^{\prime}\right)\right) \in \partial \Omega$ and $z=\left(z^{\prime}, \varphi\left(z^{\prime}\right)\right) \in \partial \Omega, I_{6}$ does not exceed a constant multiple of

$$
\iint_{D_{6}^{1}} \frac{|s-r|\left[\min \left\{|t-s|,\left(\left|x^{\prime}-z^{\prime}\right|+\tau\right)^{2}\right\}\right]^{\gamma+2}}{\left(\left|y^{\prime}-z^{\prime}\right|+|s-r|^{1 / 2}\right)^{n+1+\alpha}\left(\left|x^{\prime}-z^{\prime}\right|+\tau\right)^{n+k+2 \gamma+2}|t-s|^{\gamma+2-\theta}} d y^{\prime} d s d z^{\prime} d r
$$

where

$$
D_{6}^{1}:=\left\{\left(y^{\prime}, s, z^{\prime}, r\right) \in \mathbb{R}^{n-1} \times \mathbb{R} \times \mathbb{R}^{n-1} \times \mathbb{R}:|t-s|>c|s-r|,|t-r|>c|s-r|\right\}
$$

Making the change of variables $y^{\prime}-z^{\prime}=y^{\prime \prime}|s-r|^{1 / 2}, y^{\prime \prime} \in \mathbb{R}^{n-1}$ yields the following.

$$
\begin{aligned}
I_{6} \leq & c\left(\int_{\mathbb{R}^{n-1}} \frac{d y^{\prime \prime}}{\left(\left|y^{\prime \prime}\right|+1\right)^{n+1+\alpha}}\right) \\
& \times \int_{D_{6}^{2}} \frac{\left[\min \left\{|t-s|,\left(\left|x^{\prime}-z^{\prime}\right|+\tau\right)^{2}\right\}\right]^{\gamma+2}}{|s-r|^{\alpha / 2}\left(\left|x^{\prime}-z^{\prime}\right|+\tau\right)^{n+k+2 \gamma+2}|t-s|^{\gamma+2-\theta}} d s d z^{\prime} d r
\end{aligned}
$$

where $D_{6}^{2}:=\left\{\left(s, z^{\prime}, r\right) \in \mathbb{R} \times \mathbb{R}^{n-1} \times \mathbb{R}:|t-s|>c|s-r|,|t-r|>c|s-r|\right\}$, and the integral in $y^{\prime \prime}$ is finite. Next, integrating with respect to $r$, and setting 
$x^{\prime}-z^{\prime}=z^{\prime \prime} \in \mathbb{R}^{n-1}$, we obtain that

$$
I_{6} \leq c \int_{z^{\prime \prime} \in \mathbb{R}^{n-1}} \int_{s \in \mathbb{R}} \frac{\left[\min \left\{|t-s|,\left(\left|z^{\prime \prime}\right|+\tau\right)^{2}\right\}\right]^{\gamma+2}}{\left(\left|z^{\prime \prime}\right|+\tau\right)^{n+k+2 \gamma+2}|t-s|^{\gamma+1-\theta+\alpha / 2}} d s d z^{\prime \prime} .
$$

Going further, let $t-s=\left(s^{\prime}\right)^{2}, s^{\prime} \in \mathbb{R}$. Then

$$
I_{6} \leq c \int_{z^{\prime \prime} \in \mathbb{R}^{n-1}} \int_{s^{\prime} \in \mathbb{R}} \frac{\left[\min \left\{\left|s^{\prime}\right|,\left|z^{\prime \prime}\right|+\tau\right\}\right]^{2 \gamma+4}}{\left(\left|z^{\prime \prime}\right|+\tau\right)^{n+k+2 \gamma+2}\left|s^{\prime}\right|^{2 \gamma-2 \theta+\alpha+1}} d s^{\prime} d z^{\prime \prime} .
$$

Finally, let $z^{\prime \prime}=\tau u, u \in \mathbb{R}^{n-1}$ and $s^{\prime}=\tau v, v \in \mathbb{R}$, which yields

$$
I_{6} \leq c \tau^{-N} \int_{u \in \mathbb{R}^{n-1}} \int_{v \in \mathbb{R}} \frac{[\min \{|v|,|u|+1\}]^{2 \gamma+4}}{(|u|+1)^{n+k+2 \gamma+2}|v|^{2 \gamma-2 \theta+\alpha+1}} d v d u .
$$

In order to proceed from here, first assume $|v| \leq|u|+1$, and let $v=v^{\prime}(|u|+1)$, $v^{\prime} \in \mathbb{R}$. In this case

$$
\begin{aligned}
I_{6} & \leq c \tau^{-N}\left(\int_{\mathbb{R}} \frac{d u}{(|u|+1)^{n+N-1}}\right) \int_{\substack{v^{\prime} \in \mathbb{R} \\
\left|v^{\prime}\right| \leq 1}}\left|v^{\prime}\right|^{3+2 \theta-\alpha} d v^{\prime} \\
& \leq c \tau^{-N} .
\end{aligned}
$$

When $|u|+1<|v|$, we obtain that

$$
\begin{aligned}
I_{6} & \leq c \tau^{-N} \int_{\substack{v \in \mathbb{R} \\
|v|>1}} \frac{1}{|v|^{2 \gamma-2 \theta+\alpha+1}}\left(\int_{\substack{u \in \mathbb{R}^{n-1} \\
|u|+1<|v|}} \frac{d u}{(|u|+1)^{n+k-2}}\right) d v \\
& \leq c \tau^{-N} \int_{\substack{v \in \mathbb{R} \\
|v|>1}} \frac{d v}{|v|^{N+1}} \\
& \leq c \tau^{-N} .
\end{aligned}
$$


Summarizing the estimates obtained for $I_{4}, I_{5}$ and $I_{6}$, we have proved that

$$
\begin{aligned}
& \int_{\partial \Omega \times \mathbb{R}} \int_{\partial \Omega \times \mathbb{R}} \frac{\left|\nabla^{k} \partial_{\text {time }}^{\gamma} I_{\text {time }}^{\theta} E(x-z, t-s)-\nabla^{k} \partial_{\text {time }}^{\gamma} I_{\text {time }}^{\theta} E(x-z, t-r)\right|}{\left(|y-z|+|s-r|^{1 / 2}\right)^{n+1+\alpha}} d \sigma_{y} d s d \sigma_{z} d r \\
& \leq c \delta(x)^{-N} .
\end{aligned}
$$

Now A follows from (5.1.54) and (5.1.55).

To show $\mathbf{B}$, note that the variables $(y, s)$ are in $\operatorname{supp} \psi \cap(\partial \Omega \times \mathbb{R}) \subseteq K \times I \subset$ $\partial \Omega \times \mathbb{R}$, and $\tilde{\tilde{\psi}} \equiv 1$ in some neighborhood of $\operatorname{supp} \psi \cap(\partial \Omega \times \mathbb{R})$ (let this neighborhood be $K^{\prime} \times I^{\prime}$ ), therefore it is enough to show that

$$
\int_{K^{\prime} \times I^{\prime}}\left|\nabla^{k} \partial_{\text {time }}^{\gamma} I_{\text {time }}^{\theta} E(x-y, t-s)\right| d \sigma_{y} d s \leq c \delta(x)^{-N}
$$

where $(x, t) \in \tilde{K} \times \mathbb{R}$ is fixed, $\tilde{K} \subset \Omega$. By (1.2.19), denoting the integral in the left-hand side of (5.1.56) by $J$, we have that

$$
J \leq c \int_{K^{\prime}} \int_{I^{\prime}} \frac{|t-s|^{\theta}}{|x-y|^{n+k+2 \gamma}}\left[\min \left\{1, \frac{|x-y|^{2}}{|t-s|}\right\}\right]^{\gamma+1} d \sigma_{y} d s
$$

Since $x=\left(x^{\prime}, \varphi\left(x^{\prime}\right)+\tau\right) \in \tilde{K} \subset \Omega, y=\left(y^{\prime}, \varphi\left(y^{\prime}\right)\right) \in K \subset \partial \Omega$, the above inequality is equivalent with

$$
J \leq c \int_{\substack{y^{\prime} \in \mathbb{R}^{n-1} \\\left|y^{\prime}\right| \leq c}} \int_{\substack{s \in I^{\prime}\\}} \frac{|t-s|^{\theta}}{\left(\left|x^{\prime}-y^{\prime}\right|+\tau\right)^{n+k+2 \gamma}}\left[\min \left\{1, \frac{\left(\left|x^{\prime}-y^{\prime}\right|+\tau\right)^{2}}{|t-s|}\right\}\right]^{\gamma+1} d y^{\prime} d s
$$

The change of variables $x^{\prime}-y^{\prime}=u \in \mathbb{R}^{n-1}$, and $t-s=r^{2}, r \in \mathbb{R}$ yield

$$
J \leq c \int_{\substack{u \in \mathbb{R}^{n-1} \\|u| \leq c}} \int_{r \in \mathbb{R}} \frac{|r|^{2 \theta+1}}{(|u|+\tau)^{n+k+2 \gamma}}\left[\min \left\{1, \frac{|u|+\tau}{|r|}\right\}\right]^{2 \gamma+2} d u d r
$$


In the case when $|r| \leq|u|+\tau$, we let $r=r^{\prime}(|u|+\tau), r^{\prime} \in \mathbb{R}$. Then

$$
\begin{aligned}
J & \leq c\left(\int_{\substack{r^{\prime} \in \mathbb{R} \\
\left|r^{\prime}\right| \leq 1}}\left|r^{\prime}\right|^{2 \theta+1} d r^{\prime}\right) \int_{\substack{u \in \mathbb{R}^{n-1} \\
|u| \leq c}} \frac{d u}{(|u|+\tau)^{n+N-\alpha-1}} \\
& \leq c \int_{\substack{u \in \mathbb{R}^{n-1} \\
|u| \leq c}} \frac{d u}{(|u|+\tau)^{n+N-1}},
\end{aligned}
$$

since $(|u|+\tau)^{\alpha} \leq c(x \in \tilde{K} \subset \Omega)$. Now let $u=u^{\prime} \tau, u^{\prime} \in \mathbb{R}^{n-1}$. Then

$$
J \leq c \tau^{-N} \int_{\mathbb{R}^{n-1}} \frac{d u^{\prime}}{\left(\left|u^{\prime}\right|+1\right)^{n+N-1}} \leq c \tau^{-N} .
$$

On the other hand, when $|u|+\tau<|r|$, using again the fact that $|u|+\tau \leq c$,

$$
J \leq c \iiint_{\substack{(u, r) \in \mathbb{R}^{n-1} \times \mathbb{R} \\|u|+\tau<|r|}} \frac{d u d r}{(|u|+\tau)^{n+k+\alpha-2}|r|^{2 \gamma-2 \theta+1}}
$$

After the change of variables $u=u^{\prime} \tau, u^{\prime} \in \mathbb{R}^{n-1}$ and $r=r^{\prime} \tau, r^{\prime} \in \mathbb{R}$, then integating with respect to $u^{\prime}$, we obtain that

$$
\begin{aligned}
& J \leq c \tau^{-N} \iint_{\substack{\left(u^{\prime}, r^{\prime}\right) \in \mathbb{R}^{n-1} \times \mathbb{R} \\
\left|u^{\prime}\right|+1<\left|r^{\prime}\right|}} \frac{d u^{\prime} d r^{\prime}}{\left(\left|u^{\prime}\right|+1\right)^{n+k+\alpha-2}\left|r^{\prime}\right|^{2 \gamma-2 \theta+1}} \\
& \leq c \tau^{-N} \int_{\substack{r^{\prime} \in \mathbb{R} \\
\left|r^{\prime}\right|>1}} \frac{d r^{\prime}}{\left|r^{\prime}\right|^{N+1}} \\
& \leq c \tau^{-N} \text {, }
\end{aligned}
$$

which completes the proof of $\mathbf{B}$.

As part of the proof, we also need to ensure (see (5.1.50)) that

$$
\nabla^{k} \partial_{\text {time }}^{\gamma} I_{\text {time }}^{\theta} E(x-\cdot, t-\cdot) \tilde{\tilde{\psi}} \in B_{\alpha, p a r}^{1}(\partial \Omega \times \mathbb{R})
$$


for $x \in \tilde{K} \subset \Omega$ and $t \in \mathbb{R}$. Also, note that $(y, s) \in \operatorname{supp} \psi \cap(\partial \Omega \times \mathbb{R}) \subseteq K \times I \subset$ $\partial \Omega \times \mathbb{R}$, and $\tilde{\tilde{\psi}} \equiv 1$ in some neighborhood of $\operatorname{supp} \psi \cap(\partial \Omega \times \mathbb{R})$ (let this neighborhood be $\left.K^{\prime} \times I^{\prime}\right)$, therefore, in order to prove (5.1.57), it is enough to show that

$$
\nabla^{k} \partial_{\text {time }}^{\gamma} I_{\text {time }}^{\theta} E(x-\cdot, t-\cdot) \in B_{\alpha, p a r}^{1}\left(K^{\prime} \times I^{\prime}\right)
$$

where $x \in \tilde{K} \subset \Omega$ and $t \in \mathbb{R}$.

First, we shall prove that $\nabla^{k} \partial_{\text {time }}^{\gamma} I_{\text {time }}^{\theta} E(x-\cdot, t-\cdot) \in \operatorname{Lip}\left(K^{\prime} \times I^{\prime}\right)$, i.e. for any $(y, s),(z, r) \in K^{\prime} \times I^{\prime}$,

$\left|\nabla^{k} \partial_{\text {time }}^{\gamma} I_{\text {time }}^{\theta} E(x-y, t-s)-\nabla^{k} \partial_{\text {time }}^{\gamma} I_{\text {time }}^{\theta} E(x-z, t-r)\right| \leq c\left(|y-z|+|s-r|^{1 / 2}\right)$.

If we denote the difference

$$
\left|\nabla^{k} \partial_{\text {time }}^{\gamma} I_{\text {time }}^{\theta} E(x-y, t-s)-\nabla^{k} \partial_{\text {time }}^{\gamma} I_{\text {time }}^{\theta} E(x-z, t-s)\right|
$$

by $G_{1}$, by the Fundamental Theorem of Calculus, and (1.2.19), we have that

$$
\begin{aligned}
G_{1} & \leq \int_{0}^{1}\left|\nabla^{k+1} \partial_{\text {time }}^{\gamma} I_{\text {time }}^{\theta} E(x-\Gamma(j), t-s)\right||\dot{\Gamma}(j)| d j \\
& \leq c|y-z| \int_{0}^{1} \frac{|t-s|^{\theta}}{|x-\Gamma(j)|^{n+k+1+2 \gamma}}\left[\min \left\{1, \frac{|x-\Gamma(j)|^{2}}{|t-s|}\right\}\right]^{\gamma+1} d j \\
& \leq c|y-z| \int_{0}^{1} \frac{|t-s|^{\theta}}{|x-\Gamma(j)|^{n+k-1}}\left[\min \left\{\frac{1}{|x-\Gamma(j)|^{2}}, \frac{1}{|t-s|}\right\}\right]^{\gamma+1} d j,
\end{aligned}
$$

where $\Gamma:[0,1] \rightarrow \partial \Omega$ is defined by $\Gamma(j):=\left((1-j) y^{\prime}+j z^{\prime}, \varphi\left((1-j) y^{\prime}+j z^{\prime}\right)\right)$, and $y=\left(y^{\prime}, \varphi\left(y^{\prime}\right)\right) \in K^{\prime} \subset \partial \Omega, z=\left(z^{\prime}, \varphi\left(z^{\prime}\right)\right) \in K^{\prime} \subset \partial \Omega$. We were also using that $|\dot{\Gamma}(j)| \leq c\left|y^{\prime}-z^{\prime}\right| \approx|y-z|$. Since $\Gamma(j) \in \partial \Omega$, we have that $|x-\Gamma(j)| \geq \operatorname{dist}(x, \partial \Omega)=$ $\delta(x)$, hence

$$
G_{1} \leq c|y-z| \frac{|t-s|^{\theta}}{\delta(x)^{n+k-1}}\left[\min \left\{\frac{1}{\delta(x)^{2}}, \frac{1}{|t-s|}\right\}\right]^{\gamma+1}
$$


When $|t-s| \leq \delta(x)^{2}$, using the fact that $\delta(x)$ is a constant depending on $x \in \tilde{K} \subset \Omega$, we obtain that $G_{1} \leq c|y-z|$. If $\delta(x)^{2}<|t-s|$, then

$$
G_{1} \leq c|y-z| \frac{1}{\delta(x)^{n+k-1}|t-s|^{\gamma+1-\theta}} \leq c|y-z|
$$

Therefore,

$$
G_{1} \leq c|y-z|
$$

Next, let

$$
G_{2}:=\left|\nabla^{k} \partial_{\text {time }}^{\gamma} I_{\text {time }}^{\theta} E(x-z, t-s)-\nabla^{k} \partial_{\text {time }}^{\gamma} I_{\text {time }}^{\theta} E(x-z, t-r)\right|
$$

By the Fundamental Theorem of Calculus and (1.2.19) we obtain that

$$
\begin{aligned}
G_{2} & \leq|s-r| \int_{0}^{1}\left|\nabla^{k} \partial_{\text {time }}^{\gamma+1} I_{\text {time }}^{\theta} E\left(x-z, t-\zeta_{j}\right)\right| d j \\
& \leq c|s-r| \int_{0}^{1} \frac{\left|t-\zeta_{j}\right|^{\theta}}{|x-z|^{n+k+2 \gamma+2}}\left[\min \left\{1, \frac{|x-z|^{2}}{\left|t-\zeta_{j}\right|}\right\}\right]^{\gamma+2} d j
\end{aligned}
$$

where $\zeta_{j}:=(1-j) s+j r$. If $\left|t-\zeta_{j}\right| \leq|x-z|^{2}$, we get that

$$
\begin{aligned}
G_{2} & \leq c \frac{|s-r|}{|x-z|^{n+k+2 \gamma-2 \theta+2}} \\
& \leq c|s-r|^{1 / 2}
\end{aligned}
$$

where we were also using that $|x-z| \geq \delta(x)$, and $|s-r|^{1 / 2} \leq c$. On the other hand, when $|x-z|^{2}<\left|t-\zeta_{j}\right|$

$$
\begin{aligned}
G_{2} & \leq c|s-r| \int_{0}^{1} \frac{d j}{|x-z|^{n+k-2}\left|t-\zeta_{j}\right|^{2+\gamma-\theta}} \\
& \leq c \frac{|s-r|}{|x-z|^{n+k+2 \gamma-2 \theta+2}} \\
& \leq c|s-r|^{1 / 2}
\end{aligned}
$$


where we were also using that $|x-z| \geq \delta(x)$, and $|s-r|^{1 / 2} \leq c$. Consequently,

$$
G_{2} \leq c|s-r|^{1 / 2}
$$

From (5.1.58) and (5.1.59) we immediately have that

$$
\nabla^{k} \partial_{\text {time }}^{\gamma} I_{\text {time }}^{\theta} E(x-\cdot, t-\cdot) \in \operatorname{Lip}\left(K^{\prime} \times I^{\prime}\right)
$$

Going further, (5.1.60) implies that $\nabla^{k} \partial_{\text {time }}^{\gamma} I_{\text {time }}^{\theta} E(x-\cdot, t-\cdot) \in L^{\infty}\left(K^{\prime} \times I^{\prime}\right)$, which further implies that

$$
\nabla^{k} \partial_{\text {time }}^{\gamma} I_{\text {time }}^{\theta} E(x-\cdot, t-\cdot) \in L^{1}\left(K^{\prime} \times I^{\prime}\right)
$$

On the other hand, if we denote the main part of the $B_{\alpha, p a r}^{1}\left(K^{\prime} \times I^{\prime}\right)$ - norm, i.e.

$$
\int_{K^{\prime} \times I^{\prime}} \int_{K^{\prime} \times I^{\prime}} \frac{\left|\nabla^{k} \partial_{\text {time }}^{\gamma} I_{\text {time }}^{\theta} E(x-y, t-s)-\nabla^{k} \partial_{\text {time }}^{\gamma} I_{t i m e}^{\theta} E(x-z, t-r)\right|}{\left(|y-z|+|s-r|^{1 / 2}\right)^{n+1+\alpha}} d \sigma_{y} d s d \sigma_{z} d r
$$

by $H$, then, using (5.1.60), we obtain that

$$
H \leq c \int_{K^{\prime} \times I^{\prime}} \int_{K^{\prime} \times I^{\prime}} \frac{d \sigma_{y} d s d \sigma_{z} d r}{\left(|y-z|+|s-r|^{1 / 2}\right)^{n+\alpha}}
$$

Since $y=\left(y^{\prime}, \varphi\left(y^{\prime}\right)\right) \in K^{\prime} \subset \partial \Omega$, and $z=\left(z^{\prime}, \varphi\left(z^{\prime}\right)\right) \in K^{\prime} \subset \partial \Omega$, we have

$$
H \leq c \int_{\substack{y^{\prime} \in \mathbb{R}^{n-1} \\
\left|y^{\prime}\right| \leq c}} \int_{\substack { I^{\prime} \\
\begin{subarray}{c}{z^{\prime} \in \mathbb{R}^{n-1} \\
\left|z^{\prime}\right| \leq c{ I ^ { \prime } \\
\begin{subarray} { c } { z ^ { \prime } \in \mathbb { R } ^ { n - 1 } \\
| z ^ { \prime } | \leq c } }\end{subarray}} \int_{I^{\prime}} \frac{d y^{\prime} d s d z^{\prime} d r}{\left(\left|y^{\prime}-z^{\prime}\right|+|s-r|^{1 / 2}\right)^{n+\alpha}}
$$

The change of variables $y^{\prime}-z^{\prime}=u \in \mathbb{R}^{n-1}$ and $s-r=v \in \mathbb{R}$ yield to

$$
H \leq c \int_{\substack{u \in \mathbb{R}^{n-1} \\|u| \leq c}} \int_{\substack{v \in \mathbb{R} \\|v| \leq c}} \frac{d u d v}{\left(|u|+|v|^{1 / 2}\right)^{n+\alpha}}
$$


Finally, let $u=u^{\prime}|v|^{1 / 2}, u^{\prime} \in \mathbb{R}^{n-1}$. Then

$$
H \leq c\left(\int_{\mathbb{R}^{n-1}} \frac{d u^{\prime}}{\left(\left|u^{\prime}\right|+1\right)^{n+\alpha}}\right) \int_{\substack{v \in \mathbb{R} \\|v| \leq c}} \frac{d v}{|v|^{(\alpha+1) / 2}}<\infty .
$$

From this and (5.1.61) it follows that $\nabla^{k} \partial_{\text {time }}^{\gamma} I_{\text {time }}^{\theta} E(x-\cdot, t-\cdot) \in B_{\alpha, p a r}^{1}\left(K^{\prime} \times I^{\prime}\right)$, which is what we wanted to show. Hence the proof of Theorem 5.1.5 is complete for the case $0<\theta<1$.

When $\theta=0$, similar to the case $0<\theta<1$, it is enough to show that, for $x \in \tilde{K} \subset \Omega, t \in \mathbb{R}$,

C.

$\int_{\partial \Omega \times \mathbb{R}} \int_{\partial \Omega \times \mathbb{R}} \frac{\left|\nabla^{k} \partial_{t i m e}^{\gamma} E(x-y, t-s)-\nabla^{k} \partial_{t i m e}^{\gamma} E(x-z, t-r)\right|}{\left(|y-z|+|s-r|^{1 / 2}\right)^{n+1+\alpha}} d \sigma_{y} d s d \sigma_{z} d r \leq c \delta(x)^{-N}$

and

D.

$$
\left\|\nabla^{k} \partial_{\text {time }}^{\gamma} E(x-\cdot, t-\cdot) \tilde{\tilde{\psi}}\right\|_{L^{1}(\partial \Omega \times \mathbb{R})} \leq c \delta(x)^{-N}
$$

In order to show $\mathbf{C}$, first let us invoke that, earlier in the proof, the domain $D_{1}$ was introduced as

$$
D_{1}=\{(y, s, z, r) \in \partial \Omega \times \mathbb{R} \times \partial \Omega \times \mathbb{R}:|x-y|<c|y-z|\} .
$$

We also set

$$
I I_{1}:=\iint_{D_{1}} \frac{\left|\nabla^{k} \partial_{t i m e}^{\gamma} E(x-y, t-s)-\nabla^{k} \partial_{t i m e}^{\gamma} E(x-z, t-s)\right|}{\left(|y-z|+|s-r|^{1 / 2}\right)^{n+1+\alpha}} d \sigma_{y} d s d \sigma_{z} d r
$$


Following the same steps as in the case of $I_{1}$, we obtain that

$$
\begin{aligned}
I I_{1} & \leq c \int_{D_{1}^{2}} \frac{\left|\nabla^{k} \partial_{\text {time }}^{\gamma} E(x-y, s)\right|}{\left|y^{\prime}-z^{\prime}\right|^{n-1+\alpha}} d y^{\prime} d s d z^{\prime}+c \int_{D_{1}^{2}} \frac{\left|\nabla^{k} \partial_{\text {time }}^{\gamma} E(x-z, s)\right|}{\left|y^{\prime}-z^{\prime}\right|^{n-1+\alpha}} d y^{\prime} d s d z^{\prime} \\
& =: I I_{1}^{\prime}+I I_{1}^{\prime \prime} .
\end{aligned}
$$

where, as before, $D_{1}^{2}=\left\{\left(y^{\prime}, s, z^{\prime}\right) \in \mathbb{R}^{n-1} \times \mathbb{R} \times \mathbb{R}^{n-1}:\left|x^{\prime}-y^{\prime}\right|+\tau<c\left|y^{\prime}-z^{\prime}\right|\right\}$ and $x=\left(x^{\prime}, \varphi\left(x^{\prime}\right)+\tau\right) \in \Omega, y=\left(y^{\prime}, \varphi\left(y^{\prime}\right)\right) \in \partial \Omega$. According to (1.2.13), and using the fact that $|x-y| \approx\left|x^{\prime}-y^{\prime}\right|+\tau$, we obtain that

$$
I I_{1}^{\prime} \leq c \int_{D_{1}^{2}} \frac{d y^{\prime} d s d z^{\prime}}{\left(\left|x^{\prime}-y^{\prime}\right|+\tau+|t-s|^{1 / 2}\right)^{n+k+2 \gamma}\left|y^{\prime}-z^{\prime}\right|^{n-1+\alpha}} .
$$

Making the change of variables $y^{\prime}-z^{\prime}=z^{\prime \prime}\left(\left|x^{\prime}-y^{\prime}\right|+\tau\right), z^{\prime \prime} \in \mathbb{R}^{n-1}$ yields

$$
\begin{array}{r}
I I_{1}^{\prime} \leq c\left(\int_{\left(y^{\prime}, s\right) \in \mathbb{R}^{n-1} \times \mathbb{R}} \frac{d y^{\prime} d s}{\left(\left|x^{\prime}-y^{\prime}\right|+\tau+|t-s|^{1 / 2}\right)^{n+k+2 \gamma}\left(\left|x^{\prime}-y^{\prime}\right|+\tau\right)^{\alpha}}\right) \times \\
\times \int_{\substack{z^{\prime \prime} \in \mathbb{R}^{n-1} \\
0<c \leq\left|z^{\prime \prime}\right|}} \frac{d z^{\prime \prime}}{\left|z^{\prime \prime}\right|^{n-1+\alpha}},
\end{array}
$$

where the integral with respect to $z^{\prime \prime}$ is bounded by a finite constant. Going further, let $x^{\prime}-y^{\prime}=y^{\prime \prime} \in \mathbb{R}^{n-1}$, and $t-s=s^{\prime}\left(\left|y^{\prime \prime}\right|+\tau\right), s^{\prime} \in \mathbb{R}$. Then

$$
I I_{1}^{\prime} \leq c\left(\int_{\mathbb{R}^{n-1}} \frac{d y^{\prime \prime}}{\left|y^{\prime \prime}\right|^{n+k+2 \gamma+\alpha-2}}\right) \int_{\mathbb{R}} \frac{d s^{\prime}}{\left(1+\left|s^{\prime}\right|^{1 / 2}\right)^{n+k+2 \gamma}},
$$

where the integral is $s^{\prime}$ is finite. The change of variables $y^{\prime \prime}=\tau u, u \in \mathbb{R}^{n-1}$ yields the following.

$$
I I_{1}^{\prime} \leq c \tau^{-N} \int_{\mathbb{R}^{n-1}} \frac{d u}{(|u|+1)^{n+N-1}} \leq c \tau^{-N} .
$$

To obtain the same estimate for $I I_{1}^{\prime \prime}$, note that

$$
\left|x^{\prime}-z^{\prime}\right|+\tau \leq\left|x^{\prime}-y^{\prime}\right|+\left|y^{\prime}-z^{\prime}\right|+\tau<c\left|y^{\prime}-z^{\prime}\right|,
$$


therefore

$$
I I_{1}^{\prime \prime} \leq c \int_{D_{1}^{3}} \frac{\left|\nabla^{k} \partial_{t i m e}^{\gamma} E(x-z, s)\right|}{\left|y^{\prime}-z^{\prime}\right|^{n-1+\alpha}} d y^{\prime} d s d z^{\prime}
$$

where $D_{1}^{3}=\left\{\left(y^{\prime}, s, z^{\prime}\right) \in \mathbb{R}^{n-1} \times \mathbb{R} \times \mathbb{R}^{n-1}:\left|x^{\prime}-z^{\prime}\right|+\tau<c\left|y^{\prime}-z^{\prime}\right|\right\}$, as before.

Hence $I I_{1}^{\prime \prime} \leq I I_{1}^{\prime}$, which, we have seen, is majorized by $c \tau^{-N}$.

Next, as earlier in the proof, let the second domain of integration be

$$
D_{2}=\{(y, s, z, r) \in \partial \Omega \times \mathbb{R} \times \partial \Omega \times \mathbb{R}:|x-z|<c|y-z|\}
$$

and denote

$$
I I_{2}:=\iint_{D_{2}} \frac{\left|\nabla^{k} \partial_{t i m e}^{\gamma} E(x-y, t-s)-\nabla^{k} \partial_{t i m e}^{\gamma} E(x-z, t-s)\right|}{\left(|y-z|+|s-r|^{1 / 2}\right)^{n+1+\alpha}} d \sigma_{y} d s d \sigma_{z} d r
$$

Since $|x-z|<c|y-z|$ implies $|x-y|<c|y-z|$,

$$
I I_{2} \leq I I_{1} \leq c \tau^{-N}
$$

Finally, denote the complement of $D_{1} \cup D_{2}$ by $D_{3}$, i.e.

$$
D_{3}=\{(y, s, z, r) \in \partial \Omega \times \mathbb{R} \times \partial \Omega \times \mathbb{R}:|x-y|>c|y-z|,|x-z|>c|y-z|\}
$$

and the integral

$$
I I_{3}:=\iint_{D_{3}} \frac{\left|\nabla^{k} \partial_{t i m e}^{\gamma} E(x-y, t-s)-\nabla^{k} \partial_{t i m e}^{\gamma} E(x-z, t-s)\right|}{\left(|y-z|+|s-r|^{1 / 2}\right)^{n+1+\alpha}} d \sigma_{y} d s d \sigma_{z} d r
$$

By the Fundamental Theorem of Calculus and (1.2.13) we obtain that

$$
\begin{aligned}
\left|\nabla^{k} \partial_{\text {time }}^{\gamma} E(x-y, t-s)-\nabla^{k} \partial_{\text {time }}^{\gamma} E(x-z, t-s)\right| \\
\quad \leq|y-z| \int_{0}^{1}\left|\nabla^{k+1} \partial_{\text {time }}^{\gamma} E\left(x-\xi_{j}, t-s\right)\right| d j \\
\quad \leq c|y-z| \int_{0}^{1} \frac{d j}{\left(\left|x-\xi_{j}\right|+|t-s|^{1 / 2}\right)^{n+k+1+2 \gamma}}
\end{aligned}
$$


where $\xi_{j}=(1-j) y+j z$. A simple argument shows that on the domain of integration we have $\left|x-\xi_{j}\right| \geq c|x-y|$, hence $I I_{3}$ is controlled by

$$
c \iint_{D_{3}} \frac{|y-z|}{\left(|x-y|+|t-s|^{1 / 2}\right)^{n+k+1+2 \gamma}\left(|y-z|+|s-r|^{1 / 2}\right)^{n+1+\alpha}} d \sigma_{y} d s d \sigma_{z} d r .
$$

Since $x \in \Omega$ and $y, z \in \partial \Omega$, we may write $x=\left(x^{\prime}, \varphi\left(x^{\prime}\right)+\tau\right), y=\left(y^{\prime}, \varphi\left(y^{\prime}\right)\right)$ and $z=\left(z^{\prime}, \varphi\left(z^{\prime}\right)\right)$, where $x^{\prime}, y^{\prime}, z^{\prime} \in \mathbb{R}^{n-1}, \tau \in \mathbb{R}_{+}$, and $\varphi: \mathbb{R}^{n-1} \rightarrow \mathbb{R}$ is a Lipschitz function such that the graph of $\varphi$ corresponds to $\partial \Omega$ (if $\Omega$ is a bounded domain, take a finite partition of unity on $\partial \Omega)$. Therefore $|x-y| \approx\left|x^{\prime}-y^{\prime}\right|+\tau,|y-z| \approx\left|y^{\prime}-z^{\prime}\right|$, $\delta(x) \approx \tau$, and $I_{3}$ does not exceed

$$
c \iint_{D_{3}^{1}} \frac{\left|y^{\prime}-z^{\prime}\right|}{\left(\left|x^{\prime}-y^{\prime}\right|+\tau+|t-s|^{1 / 2}\right)^{n+k+1+2 \gamma}\left(\left|y^{\prime}-z^{\prime}\right|+|s-r|^{1 / 2}\right)^{n+1+\alpha}} d y^{\prime} d s d z^{\prime} d r
$$

where the domain $D_{3}^{1}$, as earlier in the proof, is given by

$\left\{\left(y^{\prime}, s, z^{\prime}, r\right) \in \mathbb{R}^{n-1} \times \mathbb{R} \times \mathbb{R}^{n-1} \times \mathbb{R}:\left|x^{\prime}-y^{\prime}\right|+\tau>c\left|y^{\prime}-z^{\prime}\right|,\left|x^{\prime}-z^{\prime}\right|+\tau>c\left|y^{\prime}-z^{\prime}\right|\right\}$

The change of variables $s-r=r^{\prime}\left|y^{\prime}-z^{\prime}\right|^{2}, r^{\prime} \in \mathbb{R}$ yields

$$
I I_{3} \leq c\left(\int_{D_{3}^{2}} \frac{d y^{\prime} d s d z^{\prime}}{\left(\left|x^{\prime}-y^{\prime}\right|+\tau+|t-s|^{1 / 2}\right)^{n+k+1+2 \gamma}\left|y^{\prime}-z^{\prime}\right|^{n-2+\alpha}}\right) \int_{\mathbb{R}} \frac{d r^{\prime}}{\left(1+\left|r^{\prime}\right|^{1 / 2}\right)^{n+1+\alpha}},
$$

where $D_{3}^{2}$, as before, is the domain

$$
\left\{\left(y^{\prime}, s, z^{\prime}\right) \in \mathbb{R}^{n-1} \times \mathbb{R} \times \mathbb{R}^{n-1}:\left|x^{\prime}-y^{\prime}\right|+\tau>c\left|y^{\prime}-z^{\prime}\right|,\left|x^{\prime}-z^{\prime}\right|+\tau>c\left|y^{\prime}-z^{\prime}\right|\right\}
$$

and the integral with respect to $r^{\prime}$ is finite. Going further, let $t-s=s^{\prime}\left(\left|x^{\prime}-y^{\prime}\right|+\tau\right)$, $s^{\prime} \in \mathbb{R}$. Then $I I_{3}$ is controlled by

$$
c\left[\int_{\mathbb{R}^{n-1}} \frac{1}{\left(\left|x^{\prime}-y^{\prime}\right|+\tau\right)^{n+k+2 \gamma-1}}\left(\int_{D_{3}^{3}} \frac{d z^{\prime}}{\left|y^{\prime}-z^{\prime}\right|^{n-2+\alpha}}\right) d y^{\prime}\right] \int_{\mathbb{R}} \frac{d s^{\prime}}{\left(\left|s^{\prime}\right|^{1 / 2}+1\right)^{n+k+2 \gamma+1}},
$$


where $D_{3}^{3}=\left\{z^{\prime} \in \mathbb{R}^{n-1}:\left|x^{\prime}-y^{\prime}\right|+\tau>c\left|y^{\prime}-z^{\prime}\right|\right\}$. Since the integral in $s^{\prime}$ is bounded by a constant, integrating with respect to $z^{\prime}$, and setting $x^{\prime}-y^{\prime}=y^{\prime \prime} \in \mathbb{R}^{n-1}$ implies that

$$
I I_{3} \leq c \int_{\mathbb{R}^{n-1}} \frac{d y^{\prime \prime}}{\left(\left|y^{\prime \prime}\right|+\tau\right)^{n+N-1}}
$$

which, after the change of variables $y^{\prime \prime}=\tau u, u \in \mathbb{R}^{n-1}$, becomes

$$
I I_{3} \leq c \tau^{-N} \int_{\mathbb{R}^{n-1}} \frac{d u}{(|u|+1)^{n+N-1}} \leq c \tau^{-N}
$$

What we have proved so far is as follows.

$\int_{\partial \Omega \times \mathbb{R}} \int_{\partial \Omega \times \mathbb{R}} \frac{\left|\nabla^{k} \partial_{\text {time }}^{\gamma} E(x-y, t-s)-\nabla^{k} \partial_{t i m e}^{\gamma} E(x-z, t-s)\right|}{\left(|y-z|+|s-r|^{1 / 2}\right)^{n+1+\alpha}} d \sigma_{y} d s d \sigma_{z} d r \leq c \delta(x)^{-N}$.

Next, we let

$$
I I_{4}:=\iint_{D_{4}} \frac{\left|\nabla^{k} \partial_{t i m e}^{\gamma} E(x-z, t-s)-\nabla^{k} \partial_{t i m e}^{\gamma} E(x-z, t-r)\right|}{\left(|y-z|+|s-r|^{1 / 2}\right)^{n+1+\alpha}} d \sigma_{y} d s d \sigma_{z} d r
$$

where, as earlier in the proof, $D_{4}=\{(y, s, z, r) \in \partial \Omega \times \mathbb{R} \times \partial \Omega \times \mathbb{R}:|t-s|<c|s-r|\}$. Following the same steps as in the case of $I_{4}$, we obtain that

$$
\begin{aligned}
& I I_{4} \leq c \int_{D_{4}^{2}} \frac{\left|\nabla^{k} \partial_{\text {time }}^{\gamma} E(x-z, t-s)\right|}{|s-r|^{(2+\alpha) / 2}} d s d z^{\prime} d r \\
&+c \int_{D_{4}^{2}} \frac{\left|\nabla^{k} \partial_{\text {time }}^{\gamma} E(x-z, t-r)\right|}{|s-r|^{(2+\alpha) / 2}} d s d z^{\prime} d r \\
&=: I I_{4}^{\prime}+I I_{4}^{\prime \prime},
\end{aligned}
$$

where $D_{4}^{2}=\left\{\left(s, z^{\prime}, r\right) \in \mathbb{R} \times \mathbb{R}^{n-1} \times \mathbb{R}:|t-s|<c|s-r|\right\}$, as before. Using (1.2.13) 
and the fact that $|x-z| \approx\left|x^{\prime}-z^{\prime}\right|+\tau$, we obtain the estimate

$$
I I_{4}^{\prime} \leq c \int_{D_{4}^{2}} \frac{d s d z^{\prime} d r}{\left(\left|x^{\prime}-z^{\prime}\right|+\tau+|t-s|^{1 / 2}\right)^{n+k+2 \gamma}|s-r|^{(2+\alpha) / 2}} .
$$

Let $x^{\prime}-z^{\prime}=z^{\prime \prime} \in \mathbb{R}^{n-1}$ and $s-r=r^{\prime}|t-s|, r^{\prime} \in \mathbb{R}$. Then

$$
I I_{4}^{\prime} \leq c\left(\int_{z^{\prime \prime} \in \mathbb{R}^{n-1}} \int_{s \in \mathbb{R}} \frac{d s d z^{\prime \prime}}{\left(\left|z^{\prime \prime}\right|+\tau+|t-s|^{1 / 2}\right)^{n+k+2 \gamma}|t-s|^{\alpha / 2}}\right) \int_{\substack{r^{\prime} \in \mathbb{R} \\ 0<c \leq\left|r^{\prime}\right|}} \frac{d r^{\prime}}{\left|r^{\prime}\right|^{(2+\alpha) / 2}},
$$

where the integral with respect to $r^{\prime}$ is bounded by a constant. Going further, set $t-s=s^{\prime}\left(\left|z^{\prime \prime}\right|+\tau\right)^{2}, s^{\prime} \in \mathbb{R}$. Then we obtain that

$$
\begin{aligned}
I I_{4}^{\prime} & \leq c\left(\int_{\mathbb{R}^{n-1}} \frac{d z^{\prime \prime}}{\left(\left|z^{\prime \prime}\right|+\tau\right)^{n+N-1}}\right) \int_{\mathbb{R}} \frac{d s^{\prime}}{\left(1+\left|s^{\prime}\right|^{1 / 2}\right)^{n+k+2 \gamma}\left|s^{\prime}\right|^{\alpha / 2}} \\
& \leq c \int_{\mathbb{R}^{n-1}} \frac{d z^{\prime \prime}}{\left(\left|z^{\prime \prime}\right|+\tau\right)^{n+N-1}} .
\end{aligned}
$$

Finally, the change of variables $z^{\prime \prime}=\tau u, u \in \mathbb{R}^{n-1}$ yields the estimates

$$
I I_{4}^{\prime} \leq c \tau^{-N} \int_{\mathbb{R}^{n-1}} \frac{d u}{(|u|+1)^{n+N-1}} \leq c \tau^{-N}
$$

which is what we wanted to show.

To obtain a similar estimate for $I I_{4}^{\prime \prime}$, observe that $|t-s|<c|s-r|$ implies $|t-r|<c|s-r|$, hence

$$
I I_{4}^{\prime \prime} \leq c \int_{D_{4}^{5}} \frac{\left|\nabla^{k} \partial_{\text {time }}^{\gamma} E(x-z, t-r)\right|}{|s-r|^{(2+\alpha) / 2}} d s d z^{\prime} d r=I I_{4}^{\prime} \leq c \tau^{-N}
$$

where $D_{4}^{5}=\left\{\left(s, z^{\prime}, r\right) \in \mathbb{R} \times \mathbb{R}^{n-1} \times \mathbb{R}:|t-r|<c|s-r|\right\}$, as before.

Next, as earlier in the proof, let the second domain of integration be

$$
D_{5}=\{(y, s, z, r) \in \partial \Omega \times \mathbb{R} \times \partial \Omega \times \mathbb{R}:|t-r|<c|s-r|\}
$$


and denote

$$
I I_{5}:=\iint_{D_{5}} \frac{\left|\nabla^{k} \partial_{t i m e}^{\gamma} E(x-z, t-s)-\nabla^{k} \partial_{t i m e}^{\gamma} E(x-z, t-r)\right|}{\left(|y-z|+|s-r|^{1 / 2}\right)^{n+1+\alpha}} d \sigma_{y} d s d \sigma_{z} d r
$$

Since $|t-r|<c|s-r|$ implies $|t-s|<c|s-r|$, we obtain that

$$
I I_{5} \leq I I_{4} \leq c \tau^{-N}
$$

Finally, denote the complement of $D_{4} \cup D_{5}$ by $D_{6}$, i.e.

$$
D_{6}=\{(y, s, z, r) \in \partial \Omega \times \mathbb{R} \times \partial \Omega \times \mathbb{R}:|t-s|>c|s-r|,|t-r|>c|s-r|\}
$$

and set

$$
I I_{6}:=\iint_{D_{6}} \frac{\left|\nabla^{k} \partial_{t i m e}^{\gamma} E(x-z, t-s)-\nabla^{k} \partial_{t i m e}^{\gamma} E(x-z, t-r)\right|}{\left(|y-z|+|s-r|^{1 / 2}\right)^{n+1+\alpha}} d \sigma_{y} d s d \sigma_{z} d r
$$

By the Fundamental Theorem of Calculus and (1.2.13), we get that

$$
\begin{aligned}
\left|\nabla^{k} \partial_{\text {time }}^{\gamma} E(x-z, t-s)-\nabla^{k} \partial_{\text {time }}^{\gamma} E(x-z, t-r)\right| \\
\quad \leq|s-r| \int_{0}^{1}\left|\nabla^{k} \partial_{t i m e}^{\gamma+1} E\left(x-z, t-\zeta_{j}\right)\right| d j \\
\quad \leq c|s-r| \int_{0}^{1} \frac{d j}{\left(|x-z|+\left|t-\zeta_{j}\right|^{1 / 2}\right)^{n+k+2 \gamma+2}}
\end{aligned}
$$

where $\zeta_{j}=(1-j) r+j s$. On the domain of integration $\left|t-\zeta_{j}\right| \approx|t-s|$, therefore $I I_{6}$ is majorized by

$$
c \iint_{D_{6}} \frac{|s-r|}{\left(|x-z|+|t-s|^{1 / 2}\right)^{n+k+2 \gamma+2}\left(|y-z|+|s-r|^{1 / 2}\right)^{n+1+\alpha}} d \sigma_{y} d s d \sigma_{z} d r
$$

or, equivalently, since we know that $x=\left(x^{\prime}, \varphi\left(x^{\prime}\right)+\tau\right) \in \Omega, y=\left(y^{\prime}, \varphi\left(y^{\prime}\right)\right) \in \partial \Omega$ and $z=\left(z^{\prime}, \varphi\left(z^{\prime}\right)\right) \in \partial \Omega$

$$
c \iint_{D_{6}^{1}} \frac{|s-r|}{\left(\left|x^{\prime}-z^{\prime}\right|+\tau+|t-s|^{1 / 2}\right)^{n+k+2 \gamma+2}\left(\left|y^{\prime}-z^{\prime}\right|+|s-r|^{1 / 2}\right)^{n+1+\alpha}} d y^{\prime} d s d z^{\prime} d r
$$


where, as before,

$$
D_{6}^{1}=\left\{\left(y^{\prime}, s, z^{\prime}, r\right) \in \mathbb{R}^{n-1} \times \mathbb{R} \times \mathbb{R}^{n-1} \times \mathbb{R}:|t-s|>c|s-r|,|t-r|>c|s-r|\right\} .
$$

We make the change of variables $y^{\prime}-z^{\prime}=y^{\prime \prime}|s-r|^{1 / 2}, y^{\prime \prime} \in \mathbb{R}^{n-1}$, and integrate with respect to $y^{\prime \prime}$, then with respect to $r$, to obtain the following.

$$
\begin{aligned}
I I_{6} \leq & c\left(\int_{\mathbb{R}^{n-1}} \frac{d y^{\prime \prime}}{\left(\left|y^{\prime \prime}\right|+1\right)^{n+1+\alpha}}\right) \\
& \times \int_{z^{\prime} \in \mathbb{R}^{n-1}} \int_{s \in \mathbb{R}} \frac{1}{\left(\left|x^{\prime}-z^{\prime}\right|+\tau+|t-s|^{1 / 2}\right)^{n+k+2 \gamma+2}}\left(\int_{D_{6}^{3}} \frac{d r}{|s-r|^{\alpha / 2}}\right) d z^{\prime} d s \\
\leq & c \int_{z^{\prime} \in \mathbb{R}^{n-1}} \int_{s \in \mathbb{R}} \frac{d z^{\prime} d s}{\left(\left|x^{\prime}-z^{\prime}\right|+\tau+|t-s|^{1 / 2}\right)^{n+k+2 \gamma+2}|t-s|^{\alpha / 2-1}},
\end{aligned}
$$

where $D_{6}^{3}:=\{r \in \mathbb{R}:|t-s|>c|s-r|\}$. Going further, let $t-s=s^{\prime}\left(\left|x^{\prime}-z^{\prime}\right|+\tau\right)^{2}$, $s^{\prime} \in \mathbb{R}$. Then

$$
I I_{6} \leq c\left(\int_{\mathbb{R}^{n-1}} \frac{d z^{\prime}}{\left(\left|x^{\prime}-z^{\prime}\right|+\tau\right)^{n+N-1}}\right) \int_{\mathbb{R}} \frac{d s^{\prime}}{\left(1+\left|s^{\prime}\right|^{1 / 2}\right)^{n+k+2 \gamma+2}\left|s^{\prime}\right|^{(\alpha-2) / 2}},
$$

where the integral in $s^{\prime}$ is bounded by a constant. Finally, let $x^{\prime}-z^{\prime}=z^{\prime \prime} \tau, z^{\prime \prime} \in \mathbb{R}^{n-1}$, which yields

$$
I I_{6} \leq c \tau^{-N} \int_{\mathbb{R}^{n-1}} \frac{d z^{\prime \prime}}{\left(\left|z^{\prime \prime}\right|+1\right)^{n+N-1}} \leq c \tau^{-N}
$$

The estimates we have proved for $I I_{4}, I I_{5}$ and $I I_{6}$ imply

$$
\int_{\partial \Omega \times \mathbb{R}} \int_{\partial \Omega \times \mathbb{R}} \frac{\left|\nabla^{k} \partial_{t i m e}^{\gamma} E(x-z, t-s)-\nabla^{k} \partial_{t i m e}^{\gamma} E(x-z, t-r)\right|}{\left(|y-z|+|s-r|^{1 / 2}\right)^{n+1+\alpha}} d \sigma_{y} d s d \sigma_{z} d r \leq c \delta(x)^{-N}
$$

We observe that $\mathbf{C}$ readily follows from (5.1.62) and (5.1.63). 
In order to show $\mathbf{D}$, note that the variables $(y, s)$ are in $\operatorname{supp} \psi \cap(\partial \Omega \times \mathbb{R}) \subseteq$ $K \times I \subset \partial \Omega \times \mathbb{R}$, and $\tilde{\tilde{\psi}} \equiv 1$ in some neighborhood of supp $\psi \cap(\partial \Omega \times \mathbb{R})$ (let this neighborhood be $K^{\prime} \times I^{\prime}$ ), therefore it is enough to show that

$$
\int_{K^{\prime} \times I^{\prime}}\left|\nabla^{k} \partial_{t i m e}^{\gamma} E(x-y, t-s)\right| d \sigma_{y} d s \leq c \delta(x)^{-N}
$$

where $(x, t) \in \tilde{K} \times \mathbb{R}$ is fixed, $\tilde{K} \subset \Omega$. If, temporarily, we denote the left-hand side of (5.1.64) by $L H S$, then, by (1.2.13),

$$
L H S \leq c \int_{K^{\prime}} \int_{I^{\prime}} \frac{d \sigma_{y} d s}{\left(|x-y|+|t-s|^{1 / 2}\right)^{n+k+2 \gamma}} .
$$

Taking also into account that $x=\left(x^{\prime}, \varphi\left(x^{\prime}\right)+\tau\right) \in \tilde{K} \subset \Omega, y=\left(y^{\prime}, \varphi\left(y^{\prime}\right)\right) \in K \subset \partial \Omega$, we get that

$$
L H S \leq c \int_{\substack{y^{\prime} \in \mathbb{R}^{n-1} \\\left|y^{\prime}\right| \leq c}} \int_{s \in I^{\prime}} \frac{d y^{\prime} d s}{\left(\left|x^{\prime}-y^{\prime}\right|+\tau+|t-s|^{1 / 2}\right)^{n+k+2 \gamma}}
$$

Next we let $x^{\prime}-y^{\prime}=y^{\prime \prime} \in \mathbb{R}^{n-1}$, then $t-s=s^{\prime}\left(\left|y^{\prime \prime}\right|+\tau\right)^{2}, s^{\prime} \in \mathbb{R}$, to obtain the following.

$$
\begin{aligned}
L H S & \leq c \int_{\substack{y^{\prime \prime} \in \mathbb{R}^{n-1} \\
\left|y^{\prime \prime}\right| \leq c}} \int_{s^{\prime} \in \mathbb{R}} \frac{d y^{\prime \prime} d s^{\prime}}{\left(\left|y^{\prime \prime}\right|+\tau\right)^{n+k+2 \gamma-2}\left(1+\left|s^{\prime}\right|^{1 / 2}\right)^{n+k+2 \gamma}} \\
& \leq c \int_{\substack{y^{\prime \prime} \in \mathbb{R}^{n-1} \\
\left|y^{\prime \prime}\right| \leq c}} \frac{d y^{\prime \prime}}{\left(\left|y^{\prime \prime}\right|+\tau\right)^{n+N-1}},
\end{aligned}
$$

where we have used the fact that $\left(\left|y^{\prime \prime}\right|+\tau\right)^{\alpha} \leq c$. Finally, the change of variables $y^{\prime \prime}=u \tau, u \in \mathbb{R}^{n-1}$ yields

$$
L \leq c \tau^{-N} \int_{\mathbb{R}^{n-1}} \frac{d u}{(|u|+1)^{n+N-1}} \leq c \tau^{-N}
$$


which is what we needed to show, and the proof of $\mathbf{D}$ is complete.

As part of the proof, similar to the case when $0<\theta<1$, we also need to ensure that

$$
\nabla^{k} \partial_{\text {time }}^{\gamma} E(x-\cdot, t-\cdot) \tilde{\tilde{\psi}} \in B_{\alpha, p a r}^{1}(\partial \Omega \times \mathbb{R})
$$

for $x \in \tilde{K} \subset \Omega$ and $t \in \mathbb{R}$. Note that the variables $(y, s)$ are in $\operatorname{supp} \psi \cap(\partial \Omega \times \mathbb{R}) \subseteq$ $K \times I \subset \partial \Omega \times \mathbb{R}$, and $\tilde{\tilde{\psi}} \equiv 1$ in some neighborhood of supp $\psi \cap(\partial \Omega \times \mathbb{R})$ (let this neighborhood be $K^{\prime} \times I^{\prime}$ ), therefore, to prove (5.1.65), it is enough to show that

$$
\nabla^{k} \partial_{\text {time }}^{\gamma} E(x-\cdot, t-\cdot) \in B_{\alpha, p a r}^{1}\left(K^{\prime} \times I^{\prime}\right)
$$

where $x \in \tilde{K} \subset \Omega$ and $t \in \mathbb{R}$.

First, we shall prove that $\nabla^{k} \partial_{\text {time }}^{\gamma} E(x-\cdot, t-\cdot) \in \operatorname{Lip}\left(K^{\prime} \times I^{\prime}\right)$, i.e. for any $(y, s),(z, r) \in K^{\prime} \times I^{\prime}$

$$
\left|\nabla^{k} \partial_{\text {time }}^{\gamma} E(x-y, t-s)-\nabla^{k} \partial_{\text {time }}^{\gamma} E(x-z, t-r)\right| \leq c\left(|y-z|+|s-r|^{1 / 2}\right) .
$$

Let us denote the difference

$$
\left|\nabla^{k} \partial_{\text {time }}^{\gamma} E(x-y, t-s)-\nabla^{k} \partial_{\text {time }}^{\gamma} E(x-z, t-s)\right|
$$

by $V_{1}$. Then, by the Fundamental Theorem of Calculus, and (1.2.13), we get that

$$
\begin{aligned}
V_{1} & \leq \int_{0}^{1}\left|\nabla^{k+1} \partial_{\text {time }}^{\gamma} E(x-\Gamma(j), t-s)\right||\dot{\Gamma}(j)| d j \\
& \leq c|y-z| \int_{0}^{1} \frac{d j}{\left(|x-\Gamma(j)|+|t-r|^{1 / 2}\right)^{n+k+1+2 \gamma}} \\
& \leq c|y-z| \int_{0}^{1} \frac{d j}{|x-\Gamma(j)|^{n+k+1+2 \gamma}} \\
& \leq c|y-z| \frac{1}{\delta(x)^{n+k+1+2 \gamma}} \leq c|y-z|,
\end{aligned}
$$


where $\Gamma:[0,1] \rightarrow \partial \Omega$ is giben by $\Gamma(j):=\left((1-j) y^{\prime}+j z^{\prime}, \varphi\left((1-j) y^{\prime}+j z^{\prime}\right)\right)$, and $y=\left(y^{\prime}, \varphi\left(y^{\prime}\right)\right) \in K^{\prime} \subset \partial \Omega, z=\left(z^{\prime}, \varphi\left(z^{\prime}\right)\right) \in K^{\prime} \subset \partial \Omega$. We were also using the fact that $|\dot{\Gamma}(j)| \leq c\left|y^{\prime}-z^{\prime}\right| \approx|y-z|$ and $|x-\Gamma(j)| \geq \operatorname{dist}(x, \partial \Omega)=\delta(x)$. Next, let

$$
V_{2}:=\left|\nabla^{k} \partial_{\text {time }}^{\gamma} E(x-z, t-s)-\nabla^{k} \partial_{\text {time }}^{\gamma} E(x-z, t-r)\right|
$$

Then a similar argument yields the following.

$$
\begin{aligned}
V_{2} & \leq|s-r| \int_{0}^{1}\left|\nabla^{k} \partial_{\text {time }}^{\gamma+1} E\left(x-z, t-\zeta_{j}\right)\right| d j \\
& \leq c|s-r| \int_{0}^{1} \frac{d j}{\left(|x-z|+\left|t-\zeta_{j}\right|^{1 / 2}\right)^{n+k+2 \gamma+2}} \\
& \leq c|s-r| \frac{1}{|x-z|^{n+k+2 \gamma+2}} \\
& \leq c|s-r| \frac{1}{\delta(x)^{n+k+2 \gamma+2}} \\
& \leq c|s-r|^{1 / 2},
\end{aligned}
$$

where $\zeta_{j}=(1-j) s+j r$.

From the estimates for $V_{1}$ and $V_{2}$ we immediately have that

$$
\nabla^{k} \partial_{\text {time }}^{\gamma} E(x-\cdot, t-\cdot) \in \operatorname{Lip}\left(K^{\prime} \times I^{\prime}\right)
$$

Moreover, (5.1.66) implies that $\nabla^{k} \partial_{\text {time }}^{\gamma} E(x-\cdot, t-\cdot) \in L^{\infty}\left(K^{\prime} \times I^{\prime}\right)$, which further implies that

$$
\nabla^{k} \partial_{\text {time }}^{\gamma} E(x-\cdot, t-\cdot) \in L^{1}\left(K^{\prime} \times I^{\prime}\right)
$$

On the other hand, if we denote the main part of the $B_{\alpha, p a r}^{1}\left(K^{\prime} \times I^{\prime}\right)$-norm, i.e.

$$
\int_{K^{\prime} \times I^{\prime}} \int_{K^{\prime} \times I^{\prime}} \frac{\left|\nabla^{k} \partial_{t i m e}^{\gamma} E(x-y, t-s)-\nabla^{k} \partial_{t i m e}^{\gamma} E(x-z, t-r)\right|}{\left(|y-z|+|s-r|^{1 / 2}\right)^{n+1+\alpha}} d \sigma_{y} d s d \sigma_{z} d r
$$


by $H^{\prime}$, then, following the same steps as in the case of $H$ (earlier in the proof), we obtain that

$$
H^{\prime}<\infty
$$

Now (5.1.67) and (5.1.68) imply $\nabla^{k} \partial_{\text {time }}^{\gamma} E(x-\cdot, t-\cdot) \in B_{\alpha, p a r}^{1}\left(K^{\prime} \times I^{\prime}\right)$, and the proof of Theorem 5.1.5 is complete.

In order to state the next result let us recall from (1.1.3) the definition of the fractional time-derivative operator. If we replace $I_{\text {time }}^{\theta}$ with $D_{\text {time }}^{\theta}$ in Theorem 5.1.5, we obtain the following.

Theorem 5.1.6. Let $\Omega$ be a Lipschitz domain in $\mathbb{R}^{n}, \tilde{\psi}, \tilde{\psi}, \psi$ be as in Definition 5.1.1, and $N=k+2 \gamma+2 \theta+\alpha-1>0$, where $k, \gamma \in \mathbb{N}_{0}, 0 \leq \theta<1,0<\alpha<1$. Then

$$
\delta^{N} \tilde{\psi} \nabla^{k} \partial_{\text {time }}^{\gamma} D_{\text {time }}^{\theta} \mathcal{S}(\tilde{\tilde{\psi}} \psi f) \in L^{\infty}(\Omega \times \mathbb{R})
$$

for any $f \in\left(B_{\alpha, p a r}^{1}(\partial \Omega \times \mathbb{R})\right)^{*}$.

Proof. When $0<\theta<1$, we use Theorem 5.1.5 and the fact that $D_{\text {time }}^{\theta}=\partial_{\text {time }} I_{\text {time }}^{1-\theta}$, therefore

$$
\delta^{N} \nabla^{k} \partial_{\text {time }}^{\gamma} D_{\text {time }}^{\theta}=\delta^{N} \nabla^{k} \partial_{\text {time }}^{\gamma+1} I_{\text {time }}^{1-\theta}
$$

with $N=k+2 \gamma+2 \theta+\alpha-1>0$. When $\theta=0$, it is known that $D_{\text {time }}^{0}=I=I_{\text {time }}^{0}$, and the theorem follows from the case $\theta=0$ of Theorem 5.1.5.

Before we present our next result, we give and prove two intermediate results.

Lemma 5.1.7. Let $\Omega$ be a Lipschitz domain in $\mathbb{R}^{n}, f \in\left(B_{\alpha, p a r}^{\infty}(\partial \Omega \times \mathbb{R})\right)^{*}$, and $\Phi \in C_{c}^{\infty}\left(\mathbb{R}^{n} \times \mathbb{R}\right)$ with supp $\Phi \cap(\partial \Omega \times \mathbb{R}) \subseteq \mathbb{K}$ compact, $\mathbb{K} \subset \partial \Omega \times \mathbb{R}$. Then there 
exists a constant $c=c(\mathbb{K})$ such that

$$
\begin{aligned}
& \left|\int_{\partial \Omega \times \mathbb{R}} \Phi(y, s) f(y, s) d \sigma_{y} d s\right| \\
& \quad \leq c\|f\|_{\left(B_{\alpha, p a r}^{\infty}(\partial \Omega \times \mathbb{R})\right)^{*}}\left\{\left\|\nabla_{t a n} \Phi\right\|_{L^{\infty}(\partial \Omega \times \mathbb{R})}+\left\|\partial_{t} \Phi\right\|_{L^{\infty}(\partial \Omega \times \mathbb{R})}+\|\Phi\|_{L^{\infty}(\partial \Omega \times \mathbb{R})}\right\} .
\end{aligned}
$$

Proof. Considering $\Phi \in C_{c}^{\infty}\left(\mathbb{R}^{n} \times \mathbb{R}\right)$ with supp $\Phi \cap(\partial \Omega \times \mathbb{R}) \subseteq \mathbb{K}$ compact in $\partial \Omega \times \mathbb{R}$, we obtain the following.

$$
\begin{aligned}
& \sup _{\substack{(y, s),(z, r) \in \mathbb{K} \\
(y, s) \neq(z, r)}} \frac{|\Phi(y, s)-\Phi(z, r)|}{\left(|y-z|+|s-r|^{1 / 2}\right)^{\alpha}} \\
& \leq \sup _{\substack{(y, s),(z, s) \in \mathbb{K} \\
y \neq z}} \frac{|\Phi(y, s)-\Phi(z, s)|}{|y-z|^{\alpha}}+\sup _{\substack{(z, s),(z, r) \in \mathbb{K} \\
s \neq r}} \frac{|\Phi(z, s)-\Phi(z, r)|}{|s-r|^{\alpha / 2}}
\end{aligned}
$$

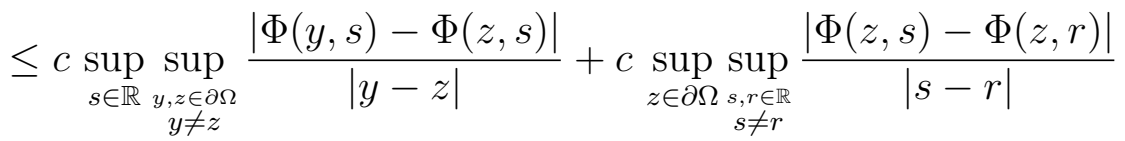

$$
\begin{aligned}
& \leq c\left\{\left\|\nabla_{\tan } \Phi\right\|_{L^{\infty}(\partial \Omega \times \mathbb{R})}+\left\|\partial_{t} \Phi\right\|_{L^{\infty}(\partial \Omega \times \mathbb{R})}\right\} .
\end{aligned}
$$

As is well-knonw,

$$
\begin{aligned}
& \int_{\partial \Omega \times \mathbb{R}} \Phi(y, s) f(y, s) d \sigma_{y} d s \mid \leq c\|f\|_{\left(B_{\alpha, p a r}^{\infty}(\partial \Omega \times \mathbb{R})\right)^{*}\|\Phi\|_{B_{\alpha, p a r}^{\infty}(\partial \Omega \times \mathbb{R})}} \\
& =\|f\|_{\left(B_{\alpha, p a r}^{\infty}(\partial \Omega \times \mathbb{R})\right)^{*}}\left\{\sup _{\substack{(y, s),(z, r) \in \mathbb{K} \\
(y, s) \neq(z, r)}} \frac{|\Phi(y, s)-\Phi(z, r)|}{\left(|y-z|+|s-r|^{1 / 2}\right)^{\alpha}}+\|\Phi\|_{L^{\infty}(\partial \Omega \times \mathbb{R})}\right\} .
\end{aligned}
$$

In view of (5.1.70) and (5.1.71), the proof is complete.

An analogue of the above result is as follows.

Lemma 5.1.8. Let $\Omega$ be a Lipschitz domain in $\mathbb{R}^{n}, f \in\left(B_{\alpha, p a r}^{1}(\partial \Omega \times \mathbb{R})\right)^{*}$, and $\Phi \in C_{c}^{\infty}\left(\mathbb{R}^{n} \times \mathbb{R}\right)$ with supp $\Phi \cap(\partial \Omega \times \mathbb{R}) \subseteq K_{0} \times I_{0}$ compact $\left(K_{0} \subset \partial \Omega, I_{0} \subset \mathbb{R}\right)$. 
Then there exists a constant $c=c(\mathbb{K})$ such that

$$
\begin{aligned}
& \left|\int_{\partial \Omega \times \mathbb{R}} \Phi(y, s) f(y, s) d \sigma_{y} d s\right| \\
& \quad \leq c\|f\|_{\left(B_{\alpha, p a r}^{1}(\partial \Omega \times \mathbb{R})\right)^{*}}\left\{\left\|\nabla_{t a n} \Phi\right\|_{L^{1}(\partial \Omega \times \mathbb{R})}+\left\|\partial_{t} \Phi\right\|_{L^{1}(\partial \Omega \times \mathbb{R})}+\|\Phi\|_{L^{1}(\partial \Omega \times \mathbb{R})}\right\} .
\end{aligned}
$$

Proof. Let $f \in\left(B_{\alpha, p a r}^{1}(\partial \Omega \times \mathbb{R})\right)^{*}$, and $\Phi \in C_{c}^{\infty}\left(\mathbb{R}^{n} \times \mathbb{R}\right)$ with $\operatorname{supp} \Phi \cap(\partial \Omega \times \mathbb{R}) \subseteq$ $K_{0} \times I_{0}$ compact, $K_{0} \subset \partial \Omega, I_{0} \subset \mathbb{R}$. Then, by Proposition 2.3.16 (Fubini property of the diagonal Besov scale), we obtain that

$$
\begin{aligned}
& \quad \int_{\partial \Omega \times \mathbb{R}} \Phi(y, s) f(y, s) d \sigma_{y} d s \mid \leq c\|f\|_{\left(B_{\alpha, p a r}^{1}(\partial \Omega \times \mathbb{R})\right)^{*}}\|\Phi\|_{B_{\alpha, p a r}^{1}(\partial \Omega \times \mathbb{R})} \\
& \leq c\|f\|_{\left(B_{\alpha, p a r}^{1}(\partial \Omega \times \mathbb{R})\right)^{*}\left\{\|\Phi\|_{L^{1}\left(K_{0} ; B_{\alpha / 2}^{1}\left(I_{0}\right)\right)}+\|\Phi\|_{L^{1}\left(I_{0} ; B_{\alpha}^{1}\left(K_{0}\right)\right)}\right\}} \\
& \leq c\|f\|_{\left(B_{\alpha, p a r}^{1}(\partial \Omega \times \mathbb{R})\right)^{*}\left\{\|\Phi\|_{L^{1}\left(K_{0} \times I_{0}\right)}\right.} \\
& \left.\quad+\iint_{K_{0}} \int_{I_{0}} \frac{|\Phi(y, s)-\Phi(y, r)|}{|s-r|^{1+\alpha / 2}} d s d r d \sigma_{y}+\iint_{I_{0}} \int_{K_{0}} \frac{|\Phi(y, s)-\Phi(z, s)|}{|y-z|^{n-1+\alpha}} d \sigma_{y} d \sigma_{z} d s\right\} \\
& =: c\|f\|_{\left(B_{\alpha, p a r}^{1}(\partial \Omega \times \mathbb{R})\right)^{*}\left\{\|\Phi\|_{L^{1}\left(K_{0} \times I_{0}\right)}+B_{1}+B_{2}\right\} .} .
\end{aligned}
$$

Observe that in order to prove the lemma it is enough to show that

$$
B_{1}+B_{2} \leq c\left\{\left\|\nabla_{t a n} \Phi\right\|_{L^{1}(\partial \Omega \times \mathbb{R})}+\left\|\partial_{t} \Phi\right\|_{L^{1}(\partial \Omega \times \mathbb{R})}\right\}
$$

If we let $\zeta_{j}:=(1-j) r+j s$, where $j \in[0,1]$, then by the Fundamental Theorem of 
Calculus,

$$
\begin{aligned}
B_{1} & \leq \int_{K_{0}} \int_{I_{0}} \int_{I_{0}} \frac{\int_{0}^{1}\left|\partial_{t} \Phi\left(y, \zeta_{j}\right)\right||s-r| d j}{|s-r|^{1+\alpha / 2}} d s d r d \sigma_{y} \\
& \leq \int_{K_{0} \times I_{0}} \int_{I_{0}} \int_{s}^{r} \frac{\left|\partial_{t} \Phi\left(y, \zeta_{j}\right)\right|}{|s-r|^{1+\alpha / 2}} d s d r d \sigma_{y} d \zeta_{j} \\
& \leq \int_{K_{0} \times I_{0}}\left|\partial_{t} \Phi\left(y, \zeta_{j}\right)\right|\left(\int_{I_{0}} \int_{I_{0}} \frac{d s d r}{|s-r|^{1+\alpha / 2}}\right) d \sigma_{y} d \zeta_{j} \\
& \leq c\left\|\partial_{t} \Phi\right\|_{L^{1}(\partial \Omega \times \mathbb{R})} .
\end{aligned}
$$

In a similar way, if $\xi_{j}=(1-j) z+j y$ for $j \in[0,1]$, then

$$
\begin{aligned}
B_{2} & \leq \iint_{I_{0}} \int_{K_{0}} \frac{\int_{0}^{1}\left|\nabla_{\tan } \Phi\left(\xi_{j}, s\right)\right||y-z| d j}{|y-z|^{n-1+\alpha}} d \sigma_{y} d \sigma_{z} d s \\
& \leq \int_{K_{0} \times I_{0}} \int_{K_{0}} \int_{z}^{y} \frac{\left|\nabla_{\tan } \Phi\left(\xi_{j}, s\right)\right|}{|y-z|^{n-1+\alpha}} d \sigma_{y} d \sigma_{z} d s d \sigma_{\xi_{j}} \\
& \leq \int_{K_{0} \times I_{0}}\left|\nabla_{\tan } \Phi\left(\xi_{j}, s\right)\right|\left(\int_{K_{0}} \int_{K_{0}} \frac{d \sigma_{y} d \sigma_{z}}{|y-z|^{n-1+\alpha}}\right) d \sigma_{\xi_{j}} d s \\
& \leq c\left\|\nabla_{\tan } \Phi\right\|_{L^{1}(\partial \Omega \times \mathbb{R})} .
\end{aligned}
$$

Consequently, (5.1.73) and (5.1.74) imply (5.1.72), hence the proof of the lemma is complete.

In the last part of this section we present two estimates, corresponding to $p=1$ and $p=\infty$, on which the following section is based. We begin with the case when $p=1$.

Theorem 5.1.9. Let $\Omega$ be a Lipschitz domain in $\mathbb{R}^{n}, N=k+2 \gamma+2 \theta+\alpha-2>-1$, where $k, \gamma \in \mathbb{N}_{0}, 0 \leq \theta<1$ and $0<\alpha<1$. Also, recall from Definition 5.1.1 the 
truncated single layer potential operator $\tilde{\mathcal{S}}$. Then

$$
\delta^{N} \nabla^{k} \partial_{\text {time }}^{\gamma} D_{\text {time }}^{\theta} \tilde{\mathcal{S}}:\left(B_{\alpha, p a r}^{\infty}(\partial \Omega \times \mathbb{R})\right)^{*} \longrightarrow L^{1}(\Omega \times \mathbb{R})
$$

is bounded.

Proof. Let $f \in\left(B_{\alpha, p a r}^{\infty}(\partial \Omega \times \mathbb{R})\right)^{*}$. Then, for $(x, t) \in \Omega \times \mathbb{R}$ fixed, we have the following.

$$
\begin{aligned}
\delta^{N} \nabla^{k} \partial_{\text {time }}^{\gamma} D_{\text {time }}^{\theta} \tilde{\mathcal{S}} f(x, t) & \\
= & \delta^{N} \nabla^{k} \partial_{\text {time }}^{\gamma} D_{\text {time }}^{\theta}[\tilde{\psi} \mathcal{S}(\tilde{\tilde{\psi}} \psi f)](x, t) \\
= & \delta(x)^{N} \nabla^{k} \partial_{\text {time }}^{\gamma} D_{\text {time }}^{\theta}\left[\tilde{\psi}(x) \int_{\partial \Omega \times \mathbb{R}} E(x-y, t-s) \tilde{\tilde{\psi}}(y, s) \psi(y, s) f(y, s) d \sigma_{y} d s\right] \\
= & \delta(x)^{N} \nabla^{k}\left[\tilde{\psi}(x) \int_{\partial \Omega \times \mathbb{R}} \partial_{\text {time }}^{\gamma} D_{\text {time }}^{\theta} E(x-y, t-s) \tilde{\tilde{\psi}}(y, s) \psi(y, s) f(y, s) d \sigma_{y} d s\right] \\
= & \delta(x)^{N} \tilde{\psi}(x) \nabla^{k} \partial_{\text {time }}^{\gamma} D_{\text {time }}^{\theta}\left[\int_{\partial \Omega \times \mathbb{R}} E(x-y, t-s) \tilde{\tilde{\psi}}(y, s) \psi(y, s) f(y, s) d \sigma_{y} d s\right] \\
+ & \delta(x)^{N} \sum_{\beta_{1}, \beta_{2} \in \mathbb{N}} \nabla^{\beta_{1}} \tilde{\psi}(x) \int_{\partial \Omega \times \mathbb{R}} \nabla^{\beta_{2}} \partial_{\text {time }}^{\gamma} D_{\text {time }}^{\theta} E(x-y, t-s) \tilde{\tilde{\psi}}(y, s) \psi(y, s) f(y, s) d \sigma_{y} d s \\
= & : A_{1}(x, t)+A_{2}(x, t)
\end{aligned}
$$

Since $A_{1}=\delta^{N} \tilde{\psi} \nabla^{k} \partial_{\text {time }}^{\gamma} D_{\text {time }}^{\theta} \mathcal{S}(\tilde{\tilde{\psi}} \psi f)$, according to Theorem 5.1.3, we have that $A_{1} \in L^{1}(\Omega \times \mathbb{R})$. On the other hand, to control $\left|A_{2}\right|$, we can use Lemma 5.1 .7 with $\psi f \in\left(B_{\alpha, p a r}^{\infty}(\partial \Omega \times \mathbb{R})\right)^{*}$ and $\Phi=\nabla^{\beta_{2}} \partial_{\text {time }}^{\gamma} D_{\text {time }}^{\theta} E(x-\cdot, t-\cdot) \tilde{\tilde{\psi}} \in C_{c}^{\infty}\left(\mathbb{R}^{n} \times \mathbb{R}\right)$. 
Then we obtain the following.

$$
\begin{aligned}
&\left|A_{2}\right| \leq c \delta(x)^{N} \sum_{\substack{\beta_{1}, \beta_{2} \in \mathbb{N} \\
\beta_{1}+\beta_{2}=k, \beta_{1} \geq 1}}\left|\nabla^{\beta_{1}} \tilde{\psi}(x)\right|\|\psi f\|_{\left(B_{\alpha, p a r}^{\infty}(\partial \Omega \times \mathbb{R})\right)^{*} \times} \\
& \times\left\{\left\|\nabla^{\beta_{2}+1} \partial_{\text {time }}^{\gamma} D_{\text {time }}^{\theta} E(x-\cdot, t-\cdot) \tilde{\tilde{\psi}}\right\|_{L^{\infty}(\partial \Omega \times \mathbb{R})}\right. \\
&+\left\|\nabla^{\beta_{2}} \partial_{\text {time }}^{\gamma+1} D_{\text {time }}^{\theta} E(x-\cdot, t-\cdot) \tilde{\psi}\right\|_{L^{\infty}(\partial \Omega \times \mathbb{R})}\left.{ }^{\beta_{2}} \partial_{\text {time }}^{\gamma} D_{\text {time }}^{\theta} E(x-\cdot, t-\cdot) \tilde{\tilde{\psi}} \|_{L^{\infty}(\partial \Omega \times \mathbb{R})}\right\} \\
&=: c \delta(x)^{N} \sum_{\beta_{1}+\beta_{2}=k, \beta_{1} \geq 1}\left|\nabla^{\beta_{1}} \tilde{\psi}(x)\right|\left\{A_{2}^{1}+A_{2}^{2}+A_{2}^{3}\right\},
\end{aligned}
$$

where we were using the fact that $\tilde{\tilde{\psi}} \equiv 1$ in some neighborhood of $K \times I$. Since $(y, s) \in K \times I$ (otherwise $A_{2}=0$ ), and $\nabla^{\beta_{1}} \tilde{\psi}(x)=0$ for $x \in K_{\epsilon} \cap \tilde{K}$, we are left considering the case when $x \notin K_{\epsilon}$ neighborhood of $K, x \in \tilde{K} \subset \Omega, \tilde{K}$ compact, and $y \in K \subset \partial \Omega, K$ compact. In a situation like this, $|x-y| \approx 1$.

When $0<\theta<1$, using (1.2.18), we obtain that

$$
\begin{aligned}
A_{2}^{1} & \leq c \sup _{(y, s) \in K \times I}\left\{\frac{|t-s|^{1-\theta}}{|x-y|^{n+3+\beta_{2}+2 \gamma}}\left[\min \left(1, \frac{|x-y|^{2}}{|t-s|}\right)\right]^{\gamma+2}\right\} \\
& \leq c \sup _{s \in I}\left\{|t-s|^{1-\theta}\left[\min \left(1, \frac{1}{|t-s|}\right)\right]^{\gamma+2}\right\} .
\end{aligned}
$$

If $t \in I$, then $|t-s| \leq c$ and

$$
A_{2}^{1} \leq c \sup _{s \in I}\left\{|t-s|^{1-\theta}\right\} \leq c
$$

If $t \in \mathbb{R} \backslash I$, then $|t-s| \geq c$ and $|t-s| \approx 1+|t|$. Also

$$
A_{2}^{1} \leq c \frac{1}{(|1+| t \mid)^{1+\gamma+\theta}}
$$


Using the same argument for $A_{2}^{2}$ and $A_{2}^{3}$ we get that, if $t \in I$,

$$
A_{2}^{2}<\infty, \quad A_{2}^{3}<\infty,
$$

and if $t \in \mathbb{R} \backslash I$,

$$
A_{2}^{2} \leq \frac{c}{(|1+| t \mid)^{2+\gamma+\theta}}, \quad A_{2}^{3} \leq \frac{c}{(|1+| t \mid)^{1+\gamma+\theta}}
$$

Now, using (5.1.76) - (5.1.79), and that $\left|\nabla^{\beta_{1}} \tilde{\psi}(x)\right|<\infty$, we obtain that

$$
\begin{aligned}
& \int_{\Omega \times \mathbb{R}}\left|A_{2}(x, t)\right| d x d t \\
& \quad \leq c\left\{\int_{\Omega \times I} \delta(x)^{N} d x d t+\int_{\Omega \times(\mathbb{R} \backslash I)} \frac{\delta(x)^{N}}{(1+|t|)^{1+\gamma+\theta}} d x d t+\int_{\Omega \times(\mathbb{R} \backslash I)} \frac{\delta(x)^{N}}{(1+|t|)^{2+\gamma+\theta}} d x d t\right\} \\
& \quad<\infty,
\end{aligned}
$$

since $N>-1$ and $1+\gamma+\theta>1$.

When $\theta=0$, based on (1.2.13) we obtain that

$$
\begin{aligned}
A_{2}^{1} & \leq c \sup _{(y, s) \in K \times I} \frac{1}{\left(|x-y|+|t-s|^{1 / 2}\right)^{n+\beta_{2}+1+2 \gamma}} \\
& \leq c \sup _{s \in I} \frac{1}{\left(1+|t-s|^{1 / 2}\right)^{n+\beta_{2}+1+2 \gamma}} \\
& <\infty .
\end{aligned}
$$

Similarly, $A_{2}^{2} \leq c$ and $A_{2}^{3} \leq c$. Consequently, in view of (5.1.75),

$$
\int_{\Omega \times \mathbb{R}}\left|A_{2}(x, t)\right| d x d t \leq c \int_{\Omega \times I} \delta(x)^{N} d x d t<\infty,
$$

since $N>-1$. We have showed that $A_{1}, A_{2} \in L^{1}(\Omega \times \mathbb{R})$, which completes the proof of Theorem 5.1.9. 
The proper analogue of the previous result, corresponding to the case when $p=$ $\infty$, is as follows.

Theorem 5.1.10. Let $\Omega$ be a Lipschitz domain in $\mathbb{R}^{n}, N=k+2 \gamma+2 \theta+\alpha-1>0$, where $k, \gamma \in \mathbb{N}_{0}, 0 \leq \theta<1$ and $0<\alpha<1$. Also, recall from Definition 5.1.1 the truncated single layer potential operator $\tilde{\mathcal{S}}$. Then

$$
\delta^{N} \nabla^{k} \partial_{\text {time }}^{\gamma} D_{\text {time }}^{\theta} \tilde{\mathcal{S}}:\left(B_{\alpha, p a r}^{1}(\partial \Omega \times \mathbb{R})\right)^{*} \longrightarrow L^{\infty}(\Omega \times \mathbb{R})
$$

is bounded.

Proof. Let $f \in\left(B_{\alpha, p a r}^{1}(\partial \Omega \times \mathbb{R})\right)^{*}$. Then, as in the proof of the previous theorem, for $(x, t) \in \Omega \times \mathbb{R}$ fixed, we have the following.

$$
\begin{aligned}
& \delta^{N} \nabla^{k} \partial_{\text {time }}^{\gamma} D_{\text {time }}^{\theta} \tilde{\mathcal{S}}(x, t) \\
& =\delta^{N} \nabla^{k} \partial_{\text {time }}^{\gamma} D_{\text {time }}^{\theta}[\tilde{\psi} \mathcal{S}(\tilde{\tilde{\psi}} \psi f)](x, t) \\
& =\delta(x)^{N} \tilde{\psi}(x) \nabla^{k} \partial_{\text {time }}^{\gamma} D_{\text {time }}^{\theta}\left[\int_{\partial \Omega \times \mathbb{R}} E(x-y, t-s) \tilde{\tilde{\psi}}(y, s) \psi(y, s) f(y, s) d \sigma_{y} d s\right] \\
& +\delta(x)^{N} \sum_{\beta_{1}, \beta_{2} \in \mathbb{N}} \nabla^{\beta_{1}} \tilde{\psi}(x) \int_{\partial \Omega \times \mathbb{R}} \nabla^{\beta_{2}} \partial_{\text {time }}^{\gamma} D_{\text {time }}^{\theta} E(x-y, t-s) \tilde{\tilde{\psi}}(y, s) \psi(y, s) f(y, s) d \sigma_{y} d s \\
& =: A_{3}(x, t)+A_{4}(x, t)
\end{aligned}
$$

Since $A_{3}=\delta^{N} \tilde{\psi} \nabla^{k} \partial_{\text {time }}^{\gamma} D_{\text {time }}^{\theta} \mathcal{S}(\tilde{\tilde{\psi}} \psi f)$, according to Theorem 5.1.6, $A_{3}$ belongs to $L^{\infty}(\Omega \times \mathbb{R})$. On the other hand, to control $\left|A_{4}\right|$, we can use Lemma 5.1 .8 with $\psi f \in\left(B_{\alpha, p a r}^{1}(\partial \Omega \times \mathbb{R})\right)^{*}$ and $\Phi=\nabla^{\beta_{2}} \partial_{\text {time }}^{\gamma} D_{\text {time }}^{\theta} E(x-\cdot,-\cdot) \tilde{\tilde{\psi}} \in C_{c}^{\infty}\left(\mathbb{R}^{n} \times \mathbb{R}\right)$.

Then we obtain the following. 


$$
\begin{gathered}
\left|A_{4}\right| \leq c \delta(x)^{N} \sum_{\substack{\beta_{1}, \beta_{2} \in \mathbb{N} \\
\beta_{1}+\beta_{2}=k, \beta_{1} \geq 1}}\left|\nabla^{\beta_{1}} \tilde{\psi}(x)\right|\|\psi f\|_{\left[B_{\alpha, p a r}^{1}(\partial \Omega \times \mathbb{R})\right]^{*} \times} \\
\times\left\{\left\|\nabla^{\beta_{2}+1} \partial_{\text {time }}^{\gamma} D_{\text {time }}^{\theta} E(x-\cdot, t-\cdot) \tilde{\tilde{\psi}}\right\|_{L^{1}(\partial \Omega \times \mathbb{R})}\right. \\
+\left\|\nabla^{\beta_{2}} \partial_{\text {time }}^{\gamma+1} D_{\text {time }}^{\theta} E(x-\cdot, t-\cdot) \tilde{\tilde{\psi}}\right\|_{L^{1}(\partial \Omega \times \mathbb{R})} \\
\left.+\left\|\nabla^{\beta_{2}} \partial_{\text {time }}^{\gamma} D_{\text {time }}^{\theta} E(x-\cdot, t-\cdot) \tilde{\tilde{\psi}}\right\|_{L^{1}(\partial \Omega \times \mathbb{R})}\right\} \\
=: c \delta(x)^{N} \sum_{\substack{\beta_{1}, \beta_{2} \in \mathbb{N} \\
\beta_{1}+\beta_{2}=k, \beta_{1} \geq 1}}\left|\nabla^{\beta_{1}} \tilde{\psi}(x)\right|\left\{A_{4}^{1}+A_{4}^{2}+A_{4}^{3}\right\} .
\end{gathered}
$$

Here we were also using the fact that $\tilde{\tilde{\psi}} \equiv 1$ in some neighborhood of $K \times I$. Note that $\delta(x)^{N}<\infty$, and $\left|\nabla^{\beta_{1}} \tilde{\psi}(x)\right|<\infty$. Since $(y, s) \in K \times I$ (otherwise $A_{2}=0$ ), and $\nabla^{\beta_{1}} \tilde{\psi}(x)=0$ for $x \in K_{\epsilon} \cap \tilde{K}$, we are left considering the case when $x \notin K_{\epsilon}$ neighborhood of $K, x \in \tilde{K} \subset \Omega, \tilde{K}$ compact, and $y \in K \subset \partial \Omega, K$ compact. In this scenario, $|x-y| \approx 1$. Using (1.2.18), we obtain that

$$
\begin{aligned}
A_{4}^{1} & \leq c \int_{K \times I} \frac{|t-s|^{1-\theta}}{|x-y|^{n+3+\beta_{2}+2 \gamma}}\left[\min \left(1, \frac{|x-y|^{2}}{|t-s|}\right)\right]^{\gamma+2} d \sigma_{y} d s \\
& \leq c \int_{I}|t-s|^{1-\theta}\left[\min \left(1, \frac{1}{|t-s|}\right)\right]^{\gamma+2} d s .
\end{aligned}
$$

If $t \in I$, then $|t-s| \leq c$ and

$$
A_{4}^{1} \leq c \int_{I}|t-s|^{1-\theta} d s<\infty .
$$

If $t \in \mathbb{R} \backslash I$, then $|t-s| \geq c$ and $|t-s| \approx 1+|t|$. Also,

$$
A_{4}^{1} \leq c \frac{1}{(|1+| t \mid)^{1+\gamma+\theta}} .
$$


Using the same argument for $A_{4}^{2}$ and $A_{4}^{3}$ we get that, if $t \in I$,

$$
A_{4}^{2}<\infty, \quad A_{4}^{3}<\infty
$$

if $t \in \mathbb{R} \backslash I$,

$$
A_{4}^{2} \leq \frac{c}{(|1+| t \mid)^{2+\gamma+\theta}}, \quad A_{4}^{3} \leq \frac{c}{(|1+| t \mid)^{1+\gamma+\theta}}
$$

Consequently, by (5.1.81) - (5.1.84) and in view of (5.1.80), we have

$$
\sup _{(x, t) \in \Omega \times \mathbb{R}}\left|A_{4}(x, t)\right| \leq c \sup _{(x, t) \in \Omega \times \mathbb{R}}\left\{1+\frac{1}{(1+|t|)^{1+\gamma+\theta}}+\frac{1}{(1+|t|)^{2+\gamma+\theta}}\right\}<\infty
$$

which is what we wanted to show. Proving that both $A_{3}$ and $A_{4}$ belong to $L^{\infty}(\Omega \times \mathbb{R})$ completes the proof of the theorem.

\subsection{General case}

Building on the previous section, first we interpolate between the endpoint cases $p=1$ (Theorem 5.1.9) and $p=\infty$ (Theorem 5.1.10) in order to obtain Theorem 5.2.1. Based on this, after several intermediate results, we obtain the mapping property of the caloric single layer potential operator on bounded Lipschitz cylinders (Theorem 5.2.7).

Recall that for a domain $\Omega$ (in $\mathbb{R}^{n}$ ) and for $x \in \Omega, \delta(x)=\operatorname{dist}(x, \partial \Omega)$ is the distance from $x$ to the boundary of $\Omega$. Also, $\nabla^{k}$ denotes any combination of mixed spacial partial derivatives of order $k$, and $D_{\text {time }}^{\theta}$ is the fractional time-derivative operator defined in (1.1.3). As before, $\tilde{\mathcal{S}}$ represents the truncated caloric single layer potential operator from Definition 5.1.1. 
Theorem 5.2.1. Let $\Omega$ be a Lipschitz domain in $\mathbb{R}^{n}$ and $1 \leq p \leq \infty$. Also, consider $N=k+2 \gamma+2 \theta+\alpha-1>0$, where $k, \gamma \in \mathbb{N}_{0}, 0 \leq \theta<1$ and $0<\alpha<1$. Then the operator

$$
\delta^{N-\frac{1}{p}} \nabla^{k} \partial_{\text {time }}^{\gamma} D_{\text {time }}^{\theta} \tilde{\mathcal{S}}:\left(B_{\alpha, p a r}^{p^{\prime}}(\partial \Omega \times \mathbb{R})\right)^{*} \longrightarrow L^{p}(\Omega \times \mathbb{R})
$$

is bounded, where $1 / p+1 / p^{\prime}=1$.

Proof. We first note that Theorems 5.1.9 and 5.1.10 imply, respectively, that the operators

$$
\nabla^{k} \partial_{\text {time }}^{\gamma} D_{\text {time }}^{\theta} \tilde{\mathcal{S}}:\left(B_{\alpha, \text { par }}^{\infty}(\partial \Omega \times \mathbb{R})\right)^{*} \longrightarrow L^{1}\left(\mathbb{R} ; L^{1}\left(\Omega ; \delta^{N-1} d x\right)\right)
$$

and

$$
\nabla^{k} \partial_{\text {time }}^{\gamma} D_{\text {time }}^{\theta} \tilde{\mathcal{S}}:\left(B_{\alpha, p a r}^{1}(\partial \Omega \times \mathbb{R})\right)^{*} \longrightarrow L^{\infty}\left(\mathbb{R} ; L^{\infty}\left(\Omega ; \delta^{N} d x\right)\right)
$$

are bounded. Now using the real interpolation method, Theorem 3.7.1 from [BeLö], then part (h') of Remark 2.3.21, we obtain that, for $0<\eta<1$,

$$
\begin{aligned}
\left(\left(B_{\alpha, p a r}^{\infty}(\partial \Omega \times \mathbb{R})\right)^{*},(\right. & \left.\left.B_{\alpha, p a r}^{1}(\partial \Omega \times \mathbb{R})\right)^{*}\right)_{\eta, p} \\
& =\left(B_{\alpha, p a r}^{\infty}(\partial \Omega \times \mathbb{R}), B_{\alpha, p a r}^{1}(\partial \Omega \times \mathbb{R})\right)_{\eta, p^{\prime}}^{*} \\
& =\left(B_{\alpha, p a r}^{p^{\prime}}(\partial \Omega \times \mathbb{R})\right)^{*}
\end{aligned}
$$

where $1 / p+1 / p^{\prime}=1$. On the other hand, by Theorem 1.2.25 and Theorem 1 from [Fre], we have that, for $0<\eta<1$ and $\frac{1}{p}=1-\eta$,

$$
\begin{aligned}
\left(L^{1}\left(\mathbb{R} ; L^{1}\left(\Omega ; \delta^{N-1} d x\right)\right),\right. & \left.L^{\infty}\left(\mathbb{R} ; L^{\infty}\left(\Omega ; \delta^{N} d x\right)\right)\right)_{\eta, p} \\
& =L^{p}\left(\mathbb{R} ;\left(L^{1}\left(\Omega ; \delta^{N-1} d x\right), L^{\infty}\left(\Omega ; \delta^{N} d x\right)\right)_{\eta, p}\right) \\
& =L^{p}\left(\mathbb{R} ; L^{p}\left(\Omega, \delta^{N-\frac{1}{p}} d x\right)\right) .
\end{aligned}
$$


Therefore, by $(5.2 .85)-(5.2 .88)$, it follows that

$$
\nabla^{k} \partial_{\text {time }}^{\gamma} D_{\text {time }}^{\theta} \tilde{\mathcal{S}}:\left(B_{\alpha, p a r}^{p^{\prime}}(\partial \Omega \times \mathbb{R})\right)^{*} \longrightarrow L^{p}\left(\mathbb{R} ; L^{p}\left(\Omega ; \delta^{N-\frac{1}{p}} d x\right)\right)
$$

for $1<p<\infty$, which is equivalent with saying that the operator

$$
\delta^{N-\frac{1}{p}} \nabla^{k} \partial_{\text {time }}^{\gamma} D_{\text {time }}^{\theta} \tilde{\mathcal{S}}:\left(B_{\alpha, p a r}^{p^{\prime}}(\partial \Omega \times \mathbb{R})\right)^{*} \longrightarrow L^{p}(\Omega \times \mathbb{R})
$$

is bounded for $1<p<\infty$. Since the endpoint cases $(p=1$ and $p=\infty)$ of (5.2.89) are already established in Theorems 5.1.9 and 5.1.10, the proof of the theorem is complete.

Relying on Theorem 5.2.1, we next describe how the operator $\tilde{\mathcal{S}}$ alone - without any derivatives on it - behaves.

Theorem 5.2.2. Let $0<\alpha<1$ and $1<p<\infty$. Then the operator

$$
\tilde{\mathcal{S}}: B_{-\alpha, p a r}^{p}(\partial \Omega \times \mathbb{R}) \longrightarrow L^{p}\left(\Omega ; \dot{L}_{(1-\alpha+1 / p) / 2}^{p}(\mathbb{R})\right)
$$

is bounded, where $\Omega$ is a Lipschitz domain in $\mathbb{R}^{n}$.

Proof. Assume $f \in B_{-\alpha, p a r}^{p}(\partial \Omega \times \mathbb{R})$. Then, by the duality result described in Proposition 2.4.5, $f$ belongs to $\left(B_{\alpha, p a r}^{p^{\prime}}(\partial \Omega \times \mathbb{R})\right)^{*}$, where $1 / p+1 / p^{\prime}=1$. Moreover, according to Theorem 5.2.1,

$$
D_{\text {time }}^{\theta}\left(\delta^{N-\frac{1}{p}} \nabla^{k} \partial_{\text {time }}^{\gamma} \tilde{\mathcal{S}} f\right) \in L^{p}\left(\Omega ; L^{p}(\mathbb{R})\right)
$$

where $N=k+2 \gamma+2 \theta+\alpha-1>0, k, \gamma \in \mathbb{N}_{0}, 0 \leq \theta<1$ and $0<\alpha<1$. Hence, by Lemma 4.2.3,

$$
\delta^{N-\frac{1}{p}} \nabla^{k} \partial_{\text {time }}^{\gamma} \tilde{\mathcal{S}} f \in L^{p}\left(\Omega ; \dot{L}_{\theta}^{p}(\mathbb{R})\right)
$$


In the special case when $k=\gamma=0$ and $\theta=(1-\alpha+1 / p) / 2$, we obtain that

$$
\tilde{\mathcal{S}} f \in L^{p}\left(\Omega ; \dot{L}_{(1-\alpha+1 / p) / 2}^{p}(\mathbb{R})\right)
$$

which proves the theorem.

The following intermediate result will play an important role shortly.

Lemma 5.2.3. For $\Omega$ Lipschitz domain in $\mathbb{R}^{n}$, and indices $0<\alpha<1,1<p<\infty$, the operator

$$
\tilde{\mathcal{S}}: B_{-\alpha, p a r}^{p}(\partial \Omega \times \mathbb{R}) \longrightarrow L^{p}\left(\Omega ; L^{p}(\mathbb{R})\right)
$$

is bounded.

Proof. Step 1. Under the assumptions of Lemma 5.2.3,

$$
B_{-\alpha, p a r}^{p}(\partial \Omega \times \mathbb{R}) \hookrightarrow L_{-1, p a r}^{p}(\partial \Omega \times \mathbb{R})
$$

According to (2.3.142), for any $1 \leq r<\infty$,

$$
B_{-\alpha, p a r}^{p}(\partial \Omega \times \mathbb{R}) \hookrightarrow B_{-1, p a r}^{p, r}(\partial \Omega \times \mathbb{R})
$$

In particular, for $r=2, B_{-\alpha, p a r}^{p}(\partial \Omega \times \mathbb{R}) \hookrightarrow B_{-1, p a r}^{p, 2}(\partial \Omega \times \mathbb{R})$, and for $2 \leq p<\infty$, due to $(2.3 .143)$

$$
B_{-1, p a r}^{p, 2}(\partial \Omega \times \mathbb{R}) \hookrightarrow F_{-1, p a r}^{p, 2}(\partial \Omega \times \mathbb{R})=L_{-1, p a r}^{p}(\partial \Omega \times \mathbb{R})
$$

This proves (5.2.90) for $2 \leq p<\infty$. Next, assume $1<p<2$. Then $2<p^{\prime}<\infty$, where $p^{\prime}=(1-1 / p)^{-1}$. Moreover, using again (2.3.143) with $2<p^{\prime}$ and (2.3.142) with $\alpha<1$, for any $0<r \leq \infty$

$$
L_{1, p a r}^{p^{\prime}}(\partial \Omega \times \mathbb{R}) \hookrightarrow B_{1, p a r}^{p^{\prime}}(\partial \Omega \times \mathbb{R}) \hookrightarrow B_{\alpha, p a r}^{p^{\prime}, r}(\partial \Omega \times \mathbb{R})
$$


In particular, for $r=p^{\prime}$ we obtain that

$$
L_{1, p a r}^{p^{\prime}}(\partial \Omega \times \mathbb{R}) \hookrightarrow B_{\alpha, p a r}^{p^{\prime}}(\partial \Omega \times \mathbb{R}),
$$

which further implies that

$$
\left(B_{\alpha, p a r}^{p^{\prime}}(\partial \Omega \times \mathbb{R})\right)^{*} \hookrightarrow\left(L_{1, p a r}^{p^{\prime}}(\partial \Omega \times \mathbb{R})\right)^{*}
$$

Identifying these dual spaces with the help of Propositions 2.4.5 and 2.4.6 yields (5.2.90) for $1<p<2$. This concludes Step 1.

Step 2. Let $\Omega$ be a Lipschitz domain in $\mathbb{R}^{n}, 1<p<\infty$ and $f \in L_{-1, p a r}^{p}(\partial \Omega \times \mathbb{R})$. Then there exist $g_{j k}, g, g_{0} \in L^{p}(\partial \Omega \times \mathbb{R})$ such that

$$
f=\sum_{j, k} \partial_{\tau_{j k}} g_{j k}+\partial_{t i m e}^{1 / 2} g+g_{0}
$$

where $\partial_{\tau_{j k}}:=\nu_{j} \partial_{k}-\nu_{k} \partial_{j}$.

Based on Proposition 2.4.6, $f$ belongs to the dual space $\left(L_{1, p a r}^{p^{\prime}}(\partial \Omega \times \mathbb{R})\right)^{*}$, where $p^{\prime}$ is the conjugate index of $p$. Next, for $b$ in $L_{1, p a r}^{p^{\prime}}(\partial \Omega \times \mathbb{R})$ we define

$$
Q b:=\left(\left\{\partial_{\tau_{j k}} b\right\}_{j k}, \partial_{\text {time }}^{1 / 2} b, b\right)
$$

and note that $\operatorname{Im} Q \hookrightarrow\left[L^{p^{\prime}}(\partial \Omega \times \mathbb{R})\right]^{N}$. Therefore, by the Hahn-Banach theorem, there exists a functional $\widetilde{f \circ Q^{-1}}$ defined on $\left[L^{p^{\prime}}(\partial \Omega \times \mathbb{R})\right]^{N}$.

In order to show that $\operatorname{Im} Q$ is closed, we pick a sequence $\{Q f\}_{r} \in \operatorname{Im} Q$ and $\left(\left\{F_{j k}\right\}_{j k}, G, H\right) \in\left[L^{p^{\prime}}(\partial \Omega \times \mathbb{R})\right]^{N}$ such that

$$
\left\|\{Q f\}^{r}-\left(\left\{F_{j k}\right\}_{j k}, G, H\right)\right\|_{\left[L^{p^{\prime}}(\partial \Omega \times \mathbb{R})\right]^{N}} \longrightarrow 0 \quad \text { as } \quad r \rightarrow \infty
$$

and show that $\left(\left\{F_{j k}\right\}_{j k}, G, H\right) \in \operatorname{Im} Q$. 
First of all $\left\|f_{r}-H\right\|_{L^{p^{\prime}}(\partial \Omega \times \mathbb{R})} \rightarrow 0$ as $r \rightarrow \infty$. Also, (2.1.56) with $\theta=1, \alpha=0$ and $\mathbb{R}^{n}$ replaced by the boundary of $\Omega$, gives us the boundedness of

$$
\partial_{\text {time }}^{1 / 2}: L^{p^{\prime}}(\partial \Omega \times \mathbb{R}) \longrightarrow L_{-1, p a r}^{p^{\prime}}(\partial \Omega \times \mathbb{R})
$$

for $1<p^{\prime}<\infty$. Therefore, $\partial_{\text {time }}^{1 / 2} f_{r} \rightarrow \partial_{\text {time }}^{1 / 2} H$ in $L_{-1, p a r}^{p^{\prime}}(\partial \Omega \times \mathbb{R})$ as $r \rightarrow \infty$. On the other hand, $\partial_{\text {time }}^{1 / 2} f_{r} \rightarrow G$ in $L^{p^{\prime}}(\partial \Omega \times \mathbb{R})$ as $r \rightarrow \infty$, hence

$$
G=\partial_{\text {time }}^{1 / 2} H
$$

In a similar fashion, this time using Corollary 2.3.22, we obtain that $\partial_{\tau_{j k}} f_{r} \rightarrow \partial_{\tau_{j k}} H$ in $L_{-1, p a r}^{p^{\prime}}(\partial \Omega \times \mathbb{R})$ as $r \rightarrow \infty$. Since $\partial_{\tau_{j k}} f_{r} \rightarrow F_{j k}$ in $L^{p^{\prime}}(\partial \Omega \times \mathbb{R})$ as $r \rightarrow \infty$, we have

$$
F_{j k}=\partial_{\tau_{j k}} H
$$

Now (5.2.91) and (5.2.92) imply that $\operatorname{Im} Q$ is closed.

Going further, by the Riesz representation theorem, there exists $\left(\left\{F_{j k}\right\}_{j k}, G, H\right)$ in $\left(\left[L^{p^{\prime}}(\partial \Omega \times \mathbb{R})\right]^{N}\right)^{*}=\left[L^{p}(\partial \Omega \times \mathbb{R})\right]^{N}$ such that

$$
\left\langle\widetilde{f \circ Q^{-1}},\left(\left\{g_{j k}\right\}_{j k}, h, k\right)\right\rangle=\sum_{j, k} \int_{\partial \Omega \times \mathbb{R}} F_{j k} g_{j k}+\int_{\partial \Omega \times \mathbb{R}} G h+\int_{\partial \Omega \times \mathbb{R}} H k
$$

for all $\left(\left\{g_{j k}\right\}_{j k}, h, k\right) \in\left[L^{p^{\prime}}(\partial \Omega \times \mathbb{R})\right]^{N}$. In particular, for $\left(\left\{g_{j k}\right\}_{j k}, h, k\right):=Q g \in$ $\left[L^{p^{\prime}}(\partial \Omega \times \mathbb{R})\right]^{N}$, where $g \in L_{1, p a r}^{p^{\prime}}(\partial \Omega \times \mathbb{R})$, we obtain that

$$
\left\langle\widetilde{f \circ Q^{-1}}, Q g\right\rangle=\sum_{j, k} \int_{\partial \Omega \times \mathbb{R}} F_{j k} \partial_{\tau_{j k}} g+\int_{\partial \Omega \times \mathbb{R}} G \partial_{t i m e}^{1 / 2} g+\int_{\partial \Omega \times \mathbb{R}} H g .
$$

Since $\widetilde{f \circ Q^{-1}}=f \circ Q^{-1}$ on $\operatorname{Im} Q,(5.2 .93)$ is equivalent with

$$
\langle f, g\rangle=\left\langle-\left(\sum_{j, k} \partial_{\tau_{j k}} F_{j k}+\partial_{\text {time }}^{1 / 2} G+h\right), g\right\rangle
$$


for all $g \in L_{1, p a r}^{p^{\prime}}(\partial \Omega \times \mathbb{R})$. Consequently, for $f \in L_{-1, p a r}^{p}(\partial \Omega \times \mathbb{R})$ there exist $\widetilde{g_{j k}}, \tilde{g}, \tilde{g_{0}} \in L^{p}(\partial \Omega \times \mathbb{R})$ such that

$$
f=\sum_{j, k} \partial_{\tau_{j k}} \widetilde{g_{j k}}+\partial_{\text {time }}^{1 / 2} \tilde{g}+\tilde{g_{0}}
$$

To justify the integration by parts from (5.2.93) to (5.2.94) (right-hand sides), we use a partition of unity and pull-back to $\mathbb{R}^{n-1} \times \mathbb{R}$, then approximation by $C_{c}^{\infty}$-functions, and the fact that $C_{c}^{\infty}\left(\mathbb{R}^{n} \times \mathbb{R}\right)$ is dense in $F_{\alpha, p a r}^{p, q}\left(\mathbb{R}^{n-1} \times \mathbb{R}\right)$ for any $0<p<\infty$, $0<q \leq \infty$ and $\alpha \in \mathbb{R}$, which covers the Sobolev spaces we are working with. This finishes the proof of Step 2.

Step 3. Consider a Lipschitz domain $\Omega$ in $\mathbb{R}^{n}, 1<p<\infty, 0<\alpha<1$ and let $f \in B_{-\alpha, p a r}^{p}(\partial \Omega \times \mathbb{R})$. Define $F:=\tilde{\tilde{\psi}} \psi f$, where $\tilde{\tilde{\psi}}, \psi \in C_{c}^{\infty}\left(\mathbb{R}^{n} \times \mathbb{R}\right)$,

$$
\begin{aligned}
& \operatorname{supp} \tilde{\tilde{\psi}} \cap(\partial \Omega \times \mathbb{R}) \subseteq \tilde{\tilde{K}} \times \tilde{\tilde{I}} \text { with } \quad \tilde{\tilde{K}} \subset \partial \Omega, \tilde{\tilde{I}} \subset \mathbb{R}, \\
& \text { supp } \psi \cap(\partial \Omega \times \mathbb{R}) \subseteq K \times I \text { with } \quad K \subset \partial \Omega, I \subset \mathbb{R},
\end{aligned}
$$

and $\tilde{\tilde{\psi}} \equiv 1$ in some neighborhood of $K \times I$. Then

$$
\mathcal{S} F=\sum_{j, k} \partial_{\tau_{j k}}\left(\mathcal{S} g_{j k}\right)+\partial_{\text {time }}^{1 / 2}(\mathcal{S} g)+\mathcal{S} g_{0}
$$

for some $g_{j k}, g, g_{0} \in L^{p}(\partial \Omega \times \mathbb{R})$ with compact support in the spacial variable.

Based on Steps 1 and 2 we know that $F \in L_{-1, p a r}^{p}(\partial \Omega \times \mathbb{R})$, hence $F$ can be represented as

$$
F=\sum_{j, k} \partial_{\tau_{j k}} g_{j k}+\partial_{\text {time }}^{1 / 2} g+g_{0}
$$

where $g_{j k}, g, g_{0} \in L^{p}(\partial \Omega \times \mathbb{R})$. Since $\operatorname{supp} F \subseteq K \times I \subset \partial \Omega \times \mathbb{R}$, if we choose $\phi \in C_{c}^{\infty}\left(\mathbb{R}^{n}\right)$ with supp $\phi \cap \partial \Omega \subseteq K^{\prime} \subset \partial \Omega$ such that $\phi \equiv 1$ in a neighborhood of $K$, 
then $\phi F \equiv F$ and

$$
\begin{aligned}
(\phi F)(x, t)= & \phi(x) \sum_{j, k} \partial_{\tau_{j k}} g_{j k}(x, t)+\phi(x) \partial_{\text {time }}^{1 / 2} g(x, t)+\phi(x) g_{0}(x, t) \\
= & \sum_{j, k} \partial_{\tau_{j k}}\left(\phi(x) g_{j k}(x, t)\right)-\sum_{j, k}\left(\partial_{\tau_{j k}} \phi(x)\right) g_{j k}(x, t) \\
& +\partial_{t i m e}^{1 / 2}(\phi(x) g(x, t))+\phi(x) g_{0}(x, t) \\
= & \sum_{j, k} \partial_{\tau_{j k}} \widetilde{g_{j k}}+\partial_{t i m e}^{1 / 2} \tilde{g}+\tilde{g_{0}}
\end{aligned}
$$

for some $\widetilde{g_{j k}}, \tilde{g}, \tilde{g_{0}} \in L^{p}(\partial \Omega \times \mathbb{R})$ with compact support in the spacial variable. Therefore,

$$
\begin{aligned}
\mathcal{S} F & =\mathcal{S}(\phi F)=\mathcal{S}\left(\sum_{j, k} \partial_{\tau_{j k}} \widetilde{g_{j k}}+\partial_{\text {time }}^{1 / 2} \tilde{g}+\tilde{g_{0}}\right) \\
& =\sum_{j, k} \partial_{\tau_{j k}}\left(\mathcal{S} \widetilde{g_{j k}}\right)+\partial_{\text {time }}^{1 / 2}(\mathcal{S} \tilde{g})+\mathcal{S} \tilde{g_{0}} .
\end{aligned}
$$

This concludes Step 3.

Step 4. Let $\Omega$ be a Lipschitz domain in $\mathbb{R}^{n}, 1<p<\infty$ and $0<\alpha<1$. Then the operator

$$
\tilde{\mathcal{S}}: B_{-\alpha, p a r}^{p}(\partial \Omega \times \mathbb{R}) \longrightarrow L^{p}\left(\Omega ; L^{p}(\mathbb{R})\right)
$$

is bounded.

Recall that $\tilde{\mathcal{S}}=\tilde{\psi} \mathcal{S}(\tilde{\tilde{\psi}} \psi f)$, where $\tilde{\psi} \in C_{c}^{\infty}\left(\mathbb{R}^{n}\right)$ with supp $\tilde{\psi} \cap \Omega \subseteq \tilde{K} \subset \Omega$, and $\tilde{\tilde{\psi}}, \psi$ are as in Step 3. In view of Step 3, in order to prove Step 4, it suffices to show that

$$
\begin{gathered}
\tilde{\psi} \partial_{\tau_{j k}}\left(\mathcal{S} g_{j k}\right) \in L^{p}(\Omega \times \mathbb{R}), \\
\tilde{\psi} \partial_{\text {time }}^{1 / 2}(\mathcal{S} g) \in L^{p}(\Omega \times \mathbb{R}),
\end{gathered}
$$


and

$$
\tilde{\psi} \mathcal{S} g_{0} \in L^{p}(\Omega \times \mathbb{R})
$$

for some $g_{j k}, g, g_{0} \in L^{p}(\partial \Omega \times \mathbb{R})$ with compact support in the spacial variable.

Observation. Note that for a function $u$ with $\operatorname{supp} u \subseteq \tilde{K} \times \mathbb{R}, \tilde{K} \subset \mathbb{R}^{n}$, such that $\mathcal{N} u \in L^{p}(\partial \Omega \times \mathbb{R})$, we have $u \in L^{p}(\Omega \times \mathbb{R})$. Indeed,

$$
\begin{aligned}
\int_{(\Omega \cap \tilde{K}) \times \mathbb{R}}|u(x, t)|^{p} d x d t & \leq \int_{\mathbb{R}} \int_{\partial \Omega \cap \tilde{K}} \int_{0}^{c}\left|u\left(x^{\prime}, x, t\right)\right|^{p} d x_{n} d \sigma_{x^{\prime}} d t \\
& \leq c \int_{\mathbb{R}} \int_{\partial \Omega}\left|\mathcal{N}(u)\left(x^{\prime}, t\right)\right|^{p} d \sigma_{x^{\prime}} d t<\infty .
\end{aligned}
$$

Now (5.2.95) and (5.2.96) follows from the above observation by setting $u=\tilde{\psi} \partial_{\tau_{j k}}\left(\mathcal{S} g_{j k}\right)$ and $u=\tilde{\psi} \partial_{\text {time }}^{1 / 2}(\mathcal{S} g)$, respectively. Note that in both cases the support of $u$ is included in $\tilde{K} \times \mathbb{R}$ and the fact that $\mathcal{N} u \in L^{p}(\partial \Omega \times \mathbb{R})$ follows from Theorem 4.16 of [Bro2], since $g_{j k}, g \in L^{p}(\partial \Omega \times \mathbb{R})$.

In order to use the above observation with $u=\tilde{\psi} \mathcal{S} g_{0}$, we need to show that $\mathcal{N} u \in L^{p}(\partial \Omega \times \mathbb{R})$. Since

$$
\mathcal{N}\left(\tilde{\psi} \mathcal{S} g_{0}\right)(x, t) \leq \tilde{\psi}_{0}\left(S g_{0}\right)(x, t) \quad \text { for all } \quad(x, t) \in \partial \Omega \times \mathbb{R}
$$

where $\tilde{\psi}_{0} \in C_{c}^{\infty}\left(\mathbb{R}^{n}\right)$, it is enough to prove that $S g_{0} \in L^{p}(\partial \Omega \times \mathbb{R})$, where the spatial variable in $S g_{0}(x, t)$ is in some compact set in $\partial \Omega$, and $g_{0} \in L^{p}(\partial \Omega \times \mathbb{R})$ with compact 
support in the spacial variable. First, we have the following estimates.

$$
\begin{aligned}
S_{1}: & =\sup _{\substack{(x, t) \in \partial \Omega \times \mathbb{R} \\
|x| \leq c}} \int_{\substack{\partial \Omega \times \mathbb{R} \\
|y| \leq c}}|E(x-y, t-s)| d \sigma_{y} d s \\
& \leq c \sup _{\substack{(x, t) \in \partial \Omega \times \mathbb{R} \\
|x| \leq c}} \int_{\substack{\partial \Omega \times \mathbb{R} \\
|y| \leq c}} \frac{1}{\left(|x-y|+|t-s|^{1 / 2}\right)^{n}} d \sigma_{y} d s \\
& \leq c \sup _{\substack{x \in \mathbb{R}^{n-1} \\
|x| \leq c}} \int_{\substack{\mathbb{R}^{n-1} \times \mathbb{R} \\
|y| \leq c}} \frac{1}{\left(|x-y|+|r|^{1 / 2}\right)^{n}} d y d r,
\end{aligned}
$$

where we have made the change in variables $t-s=r \in \mathbb{R}$. We next let $r=\tau|x-y|^{2}$, $\tau \in \mathbb{R}$ and integrate in $\tau$. Then

$$
S_{1} \leq c \sup _{\substack{x \in \mathbb{R}^{n-1} \\|x| \leq c}} \int_{\substack{\mathbb{R}^{n-1} \\|y| \leq c}} \frac{d y}{|x-y|^{n-2}} \int_{\mathbb{R}} \frac{d \tau}{\left(1+|\tau|^{1 / 2}\right)^{n}} \leq c \sup _{\substack{x \in \mathbb{R}^{n-1} \\|x| \leq c}} \int_{\substack{\mathbb{R}^{n-1} \\|y| \leq c}} \frac{d y}{|x-y|^{n-2}}
$$

Letting $x-y=z \in \mathbb{R}^{n-1}$, we obtain that

$$
S_{1} \leq c \int_{\substack{\mathbb{R}^{n-1} \\|z| \leq c}} \frac{d z}{|z|^{n-2}}<\infty
$$

Similarly,

$$
S_{2}:=\sup _{\substack{(y, s) \in \partial \Omega \times \mathbb{R} \\|y| \leq c}} \int_{\substack{\partial \Omega \times \mathbb{R} \\|x| \leq c}}|E(x-y, t-s)| d \sigma_{x} d s<\infty .
$$

Consequently, (5.2.98) and (5.2.99) imply, according to Schur's Lemma (cf., e.g., Proposition 5.1 on p. 488 in Vol. I of [Tay]) that $S g_{0}$ belongs to $L^{p}(\partial \Omega \times \mathbb{R})$, which completes the proof of Step 4 and of Lemma 5.2.3.

The relevance of Lemma 5.2.3 will be apparent from the proof of the following result. 
Theorem 5.2.4. For $\Omega$ Lipschitz domain in $\mathbb{R}^{n}$, and indices $0<\alpha<1,1<p<\infty$, the operator

$$
\tilde{\mathcal{S}}: B_{-\alpha, p a r}^{p}(\partial \Omega \times \mathbb{R}) \longrightarrow L^{p}\left(\Omega ; B_{(1-\alpha+1 / p) / 2}^{p}(\mathbb{R})\right)
$$

is bounded.

Proof. Theorem 5.2.2 and Lemma 5.2.3 imply

$$
\tilde{\mathcal{S}}: B_{-\alpha, p a r}^{p}(\partial \Omega \times \mathbb{R}) \longrightarrow L^{p}\left(\Omega ; \dot{L}_{(1-\alpha+1 / p) / 2}^{p}(\mathbb{R}) \cap L^{p}(\mathbb{R})\right),
$$

or, by Theorem 6.3.2 from [BeLö],

$$
\tilde{\mathcal{S}}: B_{-\alpha, p a r}^{p}(\partial \Omega \times \mathbb{R}) \longrightarrow L^{p}\left(\Omega ; L_{(1-\alpha+1 / p) / 2}^{p}(\mathbb{R})\right)
$$

Now let $0<\alpha_{0}, \alpha_{1}<1$ such that $\alpha_{0} \neq \alpha_{1}$. Real interpolation of two instances (i.e. for $\left.\alpha_{i}, i=0,1\right)$ of $(5.2 .100)$ yields

$$
\tilde{\mathcal{S}}: B_{-\alpha^{*}, p a r}^{p}(\partial \Omega \times \mathbb{R}) \longrightarrow L^{p}\left(\Omega ; B_{\left(1-\alpha^{*}+1 / p\right) / 2}^{p}(\mathbb{R})\right)
$$

where $\alpha^{*}=(1-\eta) \alpha_{0}+\eta \alpha_{1}, 0<\eta<1$. Here we have used Theorem 3.7.1 from [BeLö] and part (h) of Theorem 2.3.20 to interpolate between the spaces $B_{-\alpha_{i}, p a r}^{p}(\partial \Omega \times \mathbb{R})=$ $\left(B_{\alpha_{i}, p a r}^{p^{\prime}}(\partial \Omega \times \mathbb{R})\right)^{*}, 1 / p+1 / p^{\prime}=1$. For this last identity we refer the reader to Proposition 2.4.5. In order to interpolate between the target spaces we were using Theorem 1.2.25, and Theorem 6.4.5 (4) from [BeLö]. This finishes the proof of the theorem.

Our next result is similar in spirit to Theorem 5.2.4, the only difference is that this time - the smoothness of the target space is in the spacial variable (not the time variable, as it was in Theorem 5.2.4). 
Theorem 5.2.5. For $\Omega$ Lipschitz domain in $\mathbb{R}^{n}$, and indices $0<\alpha<1,1<p<\infty$, the operator

$$
\tilde{\mathcal{S}}: B_{-\alpha, p a r}^{p}(\partial \Omega \times \mathbb{R}) \longrightarrow L^{p}\left(\mathbb{R} ; B_{1-\alpha+1 / p}^{p}(\Omega)\right)
$$

is bounded.

Proof. Based on Proposition 2.4.5, for $1<p<\infty$ and $1 / p+1 / p^{\prime}=1$, we have

$$
B_{-\alpha, p a r}^{p}(\partial \Omega \times \mathbb{R})=\left(B_{\alpha, p a r}^{p^{\prime}}(\partial \Omega \times \mathbb{R})\right)^{*}
$$

That is why we may consider two special cases $(k=1, \gamma=\theta=0$ and $k=2$, $\gamma=\theta=0)$ of Theorem 5.2.1, to obtain that

$$
\delta^{\alpha-1 / p} \nabla \tilde{\mathcal{S}}: B_{-\alpha, p a r}^{p}(\partial \Omega \times \mathbb{R}) \longrightarrow L^{p}(\Omega \times \mathbb{R})
$$

and

$$
\delta^{1+\alpha-1 / p} \nabla^{2} \tilde{\mathcal{S}}: B_{-\alpha, p a r}^{p}(\partial \Omega \times \mathbb{R}) \longrightarrow L^{p}(\Omega \times \mathbb{R})
$$

are bounded for any $0<\alpha<1$ and $1<p<\infty$. As earlier, for $x \in \Omega, \delta(x)=$ $\operatorname{dist}(x, \partial \Omega)$ denotes the distance from $x$ to the boundary of $\Omega$. We denote the integer and the fractional part of $1-\alpha+1 / p$ by $l$ and $s$, respectively, i.e.

$$
\left[1-\alpha+\frac{1}{p}\right]=: l \quad \text { and } \quad\left\{1-\alpha+\frac{1}{p}\right\}=: s .
$$

Case 1. When $0<1-\alpha+\frac{1}{p}<1$, the estimate (5.2.101) yields

$$
\delta^{1-s} \nabla \tilde{\mathcal{S}}: B_{-\alpha, p a r}^{p}(\partial \Omega \times \mathbb{R}) \longrightarrow L^{p}(\Omega \times \mathbb{R})
$$

Case 2. When $1<1-\alpha+\frac{1}{p}<2$, from (5.2.102) we have

$$
\delta^{1-s} \nabla^{2} \tilde{\mathcal{S}}: B_{-\alpha, p a r}^{p}(\partial \Omega \times \mathbb{R}) \longrightarrow L^{p}(\Omega \times \mathbb{R})
$$


Based on these two cases, we can say that for any $f$ from $B_{-\alpha, p a r}^{p}(\partial \Omega \times \mathbb{R})$, $\delta^{1-s} \nabla^{l+1} \tilde{\mathcal{S}} f$ belongs to $L^{p}\left(\mathbb{R} ; L^{p}(\Omega)\right)$, where $l=0,1$. Then, according to Theorem 4.1 of $[\mathrm{JeKe}]$, we can conclude that for any $f \in B_{-\alpha, p a r}^{p}(\partial \Omega \times \mathbb{R}), \tilde{\mathcal{S}} f \in L^{p}\left(\mathbb{R} ; B_{l+s}^{p}(\Omega)\right)$, i.e.

$$
\tilde{\mathcal{S}}: B_{-\alpha, p a r}^{p}(\partial \Omega \times \mathbb{R}) \longrightarrow L^{p}\left(\mathbb{R} ; B_{1-\alpha+1 / p}^{p}(\Omega)\right)
$$

is bounded for $0<\alpha<1$ and $1<p<\infty$ such that $1-\alpha+\frac{1}{p} \in(0,1) \cup(1,2)$. Since $1-\alpha+\frac{1}{p} \in(0,2)$, we are left considering

Case 3. When $1-\alpha+\frac{1}{p}=1$. Let $\alpha_{0}, \alpha_{1} \in(0,1)$ such that $1-\alpha_{0}+\frac{1}{p}=\frac{1}{2}$ and $1-\alpha_{1}+\frac{1}{p}=\frac{3}{2}$. Then $\alpha_{0} \neq \alpha_{1}$, and (5.2.103) implies that

$$
\tilde{\mathcal{S}}: B_{-\alpha_{0}, p a r}^{p}(\partial \Omega \times \mathbb{R}) \longrightarrow L^{p}\left(\mathbb{R} ; B_{1 / 2}^{p}(\Omega)\right)
$$

and

$$
\tilde{\mathcal{S}}: B_{-\alpha_{1}, p a r}^{p}(\partial \Omega \times \mathbb{R}) \longrightarrow L^{p}\left(\mathbb{R} ; B_{3 / 2}^{p}(\Omega)\right)
$$

are bounded. By the real interpolation method, Theorem 3.7.1 of [BeLö], Theorem 2.3.20 (h), and Theorem 1.2.25, Theorem 6.4.5 (3) from [BeLö], we obtain that

$$
\tilde{\mathcal{S}}: B_{-\alpha, p a r}^{p}(\partial \Omega \times \mathbb{R}) \longrightarrow L^{p}\left(\mathbb{R} ; B_{\alpha^{\prime}}^{p}(\Omega)\right),
$$

where $\alpha=(1-\eta) \alpha_{0}+\eta \alpha_{1}, \alpha^{\prime}=\frac{1}{2}(1-\eta)+\frac{3}{2} \eta$, and $0<\eta<1$. For $\eta=\frac{1}{2}$ we get that $\alpha=\frac{1}{p}$ and $\alpha^{\prime}=1$, hence

$$
\tilde{\mathcal{S}}: B_{-\alpha, p a r}^{p}(\partial \Omega \times \mathbb{R}) \longrightarrow L^{p}\left(\mathbb{R} ; B_{1-\alpha+1 / p}^{p}(\Omega)\right)
$$

is bounded for $0<\alpha<1$ and $1<p<\infty$ such that $1-\alpha+\frac{1}{p}=1$. Now the theorem follows from (5.2.103) and (5.2.104). 
The most general mapping property of the truncated caloric single layer potential operator $\tilde{\mathcal{S}}$ (see Definition 5.1.1) is as follows.

Theorem 5.2.6. Let $\Omega$ be a Lipschitz domain in $\mathbb{R}^{n}$ and $0<\alpha<1,1<p<\infty$. Then the operator

$$
\tilde{\mathcal{S}}: B_{-\alpha, p a r}^{p}(\partial \Omega \times \mathbb{R}) \longrightarrow B_{1-\alpha+1 / p, p a r}^{p}(\Omega \times \mathbb{R})
$$

is bounded. Moreover,

$$
\tilde{\mathcal{S}}:\left(B_{\alpha, p a r}^{1}(\partial \Omega \times \mathbb{R})\right)^{*} \longrightarrow B_{1-\alpha, p a r}^{\infty}(\Omega \times \mathbb{R})
$$

is also bounded.

Proof. When $1<p<\infty$, the boundedness of (5.2.105) follows from Theorems 5.2.4, 5.2.5 and Proposition 2.2.3 (Fubini property of the diagonal Besov space).

In order to show $(5.2 .106)$, let us consider $f \in\left(B_{\alpha, p a r}^{1}(\partial \Omega \times \mathbb{R})\right)^{*}$. Then, for each fixed $(x, t) \in \Omega \times \mathbb{R}$, we have

$$
\tilde{\mathcal{S}} f(x, t)=\left\langle\left.\tilde{\psi}(x) E(x-\cdot, t-\cdot)\right|_{\partial \Omega \times \mathbb{R}} \tilde{\tilde{\psi}}, \psi f\right\rangle
$$

Before going further we show that the pairing in the right-hand side of (5.2.107) is well-defined, i.e. that for $(x, t) \in \Omega \times \mathbb{R}$

$$
\left.\tilde{\psi}(x) E(x-\cdot, t-\cdot)\right|_{\partial \Omega \times \mathbb{R}} \tilde{\tilde{\psi}} \in B_{\alpha, p a r}^{1}(\partial \Omega \times \mathbb{R})
$$

As a first step we will show that $\tilde{\psi}(x) E(x-\cdot, t-\cdot) \tilde{\tilde{\psi}} \in \operatorname{Lip}(\partial \Omega \times \mathbb{R})$, where $(x, t) \in \Omega \times \mathbb{R}$. For this it suffices to prove that

$$
|E(x-y, t-s)-E(x-z, t-r)| \leq c\left(|y-z|+|s-r|^{1 / 2}\right),
$$


where $x \in \tilde{K} \subset \Omega, t \in \mathbb{R}, y, z \in \tilde{\tilde{K}} \subset \partial \Omega$, and $s, r \in \tilde{\tilde{I}} \subset \mathbb{R}$. We denote

$$
V_{1}:=|E(x-y, t-s)-E(x-y, t-r)|
$$

and use the Fundamental Theorem of Calculus in the time variable to obtain that

$$
V_{1} \leq|s-r| \int_{0}^{1}\left|\partial_{\text {time }} E\left(x-y, t-\eta_{\theta}\right)\right| d \theta
$$

where $\eta_{\theta}:=(1-\theta) s+\theta r$. Using (1.2.13) to estimate the right-hand side of (5.2.110), then by the current assumptions on the variables involved, we get that

$$
\begin{aligned}
V_{1} & \leq c|s-r| \int_{0}^{1} \frac{d \theta}{\left(|x-y|+\left|t-\eta_{\theta}\right|^{1 / 2}\right)^{n+2}} \\
& \leq c \frac{|s-r|}{|x-y|^{n+2}} \\
& \leq c|s-r|^{1 / 2} .
\end{aligned}
$$

In a similar manner, if

$$
V_{2}:=|E(x-y, t-r)-E(x-z, t-r)|
$$

then, using the Fundamental Theorem of Calculus in the spacial variable, we obtain that

$$
V_{2} \leq \int_{0}^{1}\left|\frac{\partial}{\partial \theta} E(x-\gamma(\theta), t-r)\right| d \theta
$$

where $\gamma(\theta):=\left((1-\theta) y^{\prime}+\theta z^{\prime}, \varphi\left((1-\theta) y^{\prime}+\theta z^{\prime}\right)\right) \in \partial \Omega, y=\left(y^{\prime}, \varphi\left(y^{\prime}\right)\right), z=\left(z^{\prime}, \varphi\left(z^{\prime}\right)\right)$, and the graph of the Lipschitz function $\varphi: \mathbb{R}^{n-1} \rightarrow \mathbb{R}$ corresponds to $\partial \Omega$ (for a bounded domain we use a finite partition of unity). We can easily show that 
$\left|\frac{d}{d \theta} \gamma(\theta)\right| \leq c|y-z|$, hence, using also (1.2.13) to estimate $\nabla E$, we obtain that

$$
\begin{aligned}
V_{2} & \leq|y-z| \int_{0}^{1}|\nabla E(x-\gamma(\theta), t-r)| d \theta \\
& \leq c|y-z| \int_{0}^{1} \frac{d \theta}{\left(|x-\gamma(\theta)|+|t-r|^{1 / 2}\right)^{n+1}} \\
& \leq c|y-z| \int_{0}^{1} \frac{d \theta}{|x-\gamma(\theta)|^{n+1}} \\
& \leq c \frac{|y-z|}{\delta(x)^{n+1}} \\
& \leq c|y-z| .
\end{aligned}
$$

Consequently, (5.2.109) follows from (5.2.111) and (5.2.112). Therefore

$$
\tilde{\psi}(x) E(x-\cdot, t-\cdot) \tilde{\tilde{\psi}} \in \operatorname{Lip}(\partial \Omega \times \mathbb{R})
$$

which - due to the compact support condition on $\tilde{\tilde{\psi}}$ - further implies that

$$
\tilde{\psi}(x) E(x-\cdot, t-\cdot) \tilde{\tilde{\psi}} \in L^{1}(\partial \Omega \times \mathbb{R}) .
$$

In order to prove (5.2.108), we are left with showing that for $x \in \tilde{K} \subset \Omega$ and $t \in \mathbb{R}$

$$
V:=\int_{\tilde{\tilde{K}} \times \tilde{I}} \int_{\tilde{\tilde{K}} \times \tilde{I}} \frac{|E(x-y, t-s)-E(x-z, t-r)|}{\left(|y-z|+|s-r|^{1 / 2}\right)^{n+1+\alpha}} d \sigma_{y} d s d \sigma_{z} d r<\infty .
$$

Using (5.2.109) and a pull-back, (5.2.114) is equivalent with

$$
\begin{aligned}
& V \leq c \int_{\tilde{\tilde{K}} \times \tilde{\tilde{I}}} \int_{\tilde{\tilde{K}} \times \tilde{\tilde{I}}} \frac{1}{\left(|y-z|+|s-r|^{1 / 2}\right)^{n+\alpha}} d \sigma_{y} d s d \sigma_{z} d r \\
& \leq c \underset{\substack{\left(y^{\prime}, s\right),\left(z^{\prime}, r\right) \in \mathbb{R}^{n-1}|\tilde{\tilde{I}}\\
| y^{\prime} \mid \leq c \text { and }\left|z^{\prime}\right| \leq c}}{\left(\left|y^{\prime}-z^{\prime}\right|+|s-r|^{1 / 2}\right)^{n+\alpha}}
\end{aligned}
$$

We make the change of variables $y^{\prime}-z^{\prime}=y^{\prime \prime}|s-r|^{1 / 2}$ with $y^{\prime \prime} \in \mathbb{R}^{n-1}$ to obtain that

$$
V \leq c \int_{\mathbb{R}^{n-1}} \frac{d y^{\prime \prime}}{\left(\left|y^{\prime \prime}\right|+1\right)^{n+\alpha}}\left(\int_{\tilde{I}} \int_{\tilde{I}} \frac{d s d r}{|s-r|^{(\alpha+1) / 2}}\right)<\infty
$$


This completes the proof of (5.2.114), which, along with (5.2.113), implies (5.2.108).

Now we return to (5.2.107) and observe that by Lemma 4.2.10, in order to prove (5.2.106), it suffices to show that

$$
\tilde{\mathcal{S}}:\left(B_{\alpha, p a r}^{1}(\partial \Omega \times \mathbb{R})\right)^{*} \longrightarrow L^{\infty}(\Omega \times \mathbb{R})
$$

is bounded, and that

$$
\begin{aligned}
\sup _{(x, t) \in \Omega \times \mathbb{R}}[ & \delta(x)^{\alpha}|\langle\tilde{\psi}(x) \nabla E(x-\cdot, t-\cdot) \tilde{\tilde{\psi}}, \psi f\rangle| \\
& +\delta(x)^{\alpha}|\langle\nabla \tilde{\psi}(x) E(x-\cdot, t-\cdot) \tilde{\tilde{\psi}}, \psi f\rangle| \\
& \left.+\delta(x)^{1+\alpha}\left|\left\langle\tilde{\psi}(x) \partial_{\text {time }} E(x-\cdot, t-\cdot) \tilde{\tilde{\psi}}, \psi f\right\rangle\right|\right] \\
& \leq c\|f\|_{\left(B_{\alpha, p a r}^{1}(\partial \Omega \times \mathbb{R})\right)^{*}}
\end{aligned}
$$

First let us consider $f \in\left(B_{\alpha, p a r}^{1}(\partial \Omega \times \mathbb{R})\right)^{*}$. Then by (5.2.108) we get that

$$
\begin{aligned}
\|\tilde{\mathcal{S}} f\|_{L^{\infty}(\Omega \times \mathbb{R})} & =\sup _{(x, t) \in \Omega \times \mathbb{R}}\left|\left\langle\left.\tilde{\psi}(x) E(x-\cdot, t-\cdot)\right|_{\partial \Omega \times \mathbb{R}} \tilde{\tilde{\psi}}, \psi f\right\rangle\right| \\
& \leq \sup _{(x, t) \in \Omega \times \mathbb{R}}\left[\|f\|_{\left(B_{\alpha, p a r}^{1}(\partial \Omega \times \mathbb{R})\right)^{*}}\|\tilde{\psi}(x) E(x-\cdot, t-\cdot) \tilde{\psi}\|_{B_{\alpha, p a r}^{1}(\partial \Omega \times \mathbb{R})}\right] \\
& \leq c\|f\|_{\left(B_{\alpha, p a r}^{1}(\partial \Omega \times \mathbb{R})\right)^{*} .}
\end{aligned}
$$

This shows that the operator in (5.2.116) is bounded.

Going further, note that (5.2.117) is equivalent with saying that there are finite positive constants $c$ such that, for any $(x, t) \in \Omega \times \mathbb{R}$,
A.
$\|\tilde{\psi}(x) \nabla E(x-\cdot, t-\cdot)\|_{B_{\alpha, p a r}^{1}(K \times I)} \leq c \delta(x)^{-\alpha}$,
B.
$\left\|\tilde{\psi}(x) \partial_{\text {time }} E(x-\cdot, t-\cdot)\right\|_{B_{\alpha, p a r}^{1}(K \times I)} \leq c \delta(x)^{-1-\alpha}$,
C.
$\|\nabla \tilde{\psi}(x) E(x-\cdot, t-\cdot)\|_{B_{\alpha, p a r}^{1}(K \times I)} \leq c \delta(x)^{-\alpha}$. 
For part of $\mathbf{A}$ first we will show the following.

$$
\int_{\partial \Omega \times \mathbb{R}} \int_{\partial \Omega \times \mathbb{R}} \frac{|\nabla E(x-y, t-s)-\nabla E(x-z, t-r)|}{\left(|y-z|+|s-r|^{1 / 2}\right)^{n+1+\alpha}} d \sigma_{y} d s d \sigma_{z} d r \leq c \delta(x)^{-\alpha}
$$

where $(x, t) \in \Omega \times \mathbb{R}$. We split the domain of integration in three parts. First, let

$$
D_{1}:=\{(y, s, z, r) \in \partial \Omega \times \mathbb{R} \times \partial \Omega \times \mathbb{R}:|x-y|<c|y-z|\}
$$

and

$$
I_{1}:=\iint_{D_{1}} \frac{|\nabla E(x-y, t-s)-\nabla E(x-z, t-s)|}{\left(|y-z|+|s-r|^{1 / 2}\right)^{n+1+\alpha}} d \sigma_{y} d s d \sigma_{z} d r .
$$

We make the change of variables $x=\left(x^{\prime}, \varphi\left(x^{\prime}\right)+\tau\right) \in \Omega, y=\left(y^{\prime}, \varphi\left(y^{\prime}\right)\right) \in \partial \Omega$ and $z=\left(z^{\prime}, \varphi\left(z^{\prime}\right)\right) \in \partial \Omega$, where $x^{\prime}, y^{\prime}, z^{\prime} \in \mathbb{R}^{n-1}, \tau>0$, and the graph of the Lipschitz function $\varphi: \mathbb{R}^{n-1} \rightarrow \mathbb{R}$ is the boundary of $\Omega$ (if $\Omega$ is a bounded domain, we use a finite partition of unity on $\partial \Omega$ ). Then

$$
I_{1} \leq \iint_{D_{1}^{\prime}} \frac{|\nabla E(x-y, t-s)-\nabla E(x-z, t-s)|}{\left(\left|y^{\prime}-z^{\prime}\right|+|s-r|^{1 / 2}\right)^{n+1+\alpha}} d y^{\prime} d s d z^{\prime} d r
$$

where

$$
D_{1}^{\prime}:=\left\{\left(y^{\prime}, s, z^{\prime}, r\right) \in \mathbb{R}^{n-1} \times \mathbb{R} \times \mathbb{R}^{n-1} \times \mathbb{R}:\left|x^{\prime}-y^{\prime}\right|+\tau<c\left|y^{\prime}-z^{\prime}\right|\right\} .
$$

Setting $s-r=\left|y^{\prime}-z^{\prime}\right|^{2} r^{\prime}$ for $r^{\prime} \in \mathbb{R}$, along with the fact that $\int_{\mathbb{R}} \frac{d r^{\prime}}{\left(1+\left|r^{\prime}\right|^{1 / 2}\right)^{n+1+\alpha}}$ is finite, (5.2.119) implies that

$$
\begin{aligned}
I_{1} & \leq \iint_{D_{1}^{\prime \prime}} \frac{|\nabla E(x-y, t-s)-\nabla E(x-z, t-s)|}{\left|y^{\prime}-z^{\prime}\right|^{n-1+\alpha}} d y^{\prime} d s d z^{\prime} \\
& \leq \iint_{D_{1}^{\prime \prime}} \frac{|\nabla E(x-y, s)|}{\left|y^{\prime}-z^{\prime}\right|^{n-1+\alpha}} d y^{\prime} d s d z^{\prime}+\iint_{D_{1}^{\prime \prime}} \frac{|\nabla E(x-z, s)|}{\left|y^{\prime}-z^{\prime}\right|^{n-1+\alpha}} d y^{\prime} d s d z^{\prime} \\
& =: J_{1}+J_{2},
\end{aligned}
$$


where

$$
D_{1}^{\prime \prime}:=\left\{\left(y^{\prime}, s, z^{\prime}\right) \in \mathbb{R}^{n-1} \times \mathbb{R} \times \mathbb{R}^{n-1}:\left|x^{\prime}-y^{\prime}\right|+\tau<c\left|y^{\prime}-z^{\prime}\right|\right\}
$$

Estimating $\nabla E($ see $(1.2 .13))$ yields

$$
J_{1} \leq c \iint_{D_{1}^{\prime \prime}} \frac{1}{\left(\left|x^{\prime}-y^{\prime}\right|+\tau+|s|^{1 / 2}\right)^{n+1}\left|y^{\prime}-z^{\prime}\right|^{n-1+\alpha}} d y^{\prime} d s d z^{\prime}
$$

Furthermore, let $y^{\prime}-z^{\prime}=z^{\prime \prime}\left(\left|x^{\prime}-y^{\prime}\right|+\tau\right), z^{\prime \prime} \in \mathbb{R}^{n-1}$. Since $\int_{\substack{\mathbb{R}^{n-1} \\ 0<c \leq\left|z^{\prime \prime}\right|}} \frac{d z^{\prime \prime}}{\left|z^{\prime \prime}\right|^{n-1+\alpha}}<\infty$, from (5.2.121) we obtain that

$$
J_{1} \leq c \quad \iint_{\left(y^{\prime}, s\right) \in \mathbb{R}^{n-1} \times \mathbb{R}} \frac{1}{\left(\left|x^{\prime}-y^{\prime}\right|+\tau+|s|^{1 / 2}\right)^{n+1}\left(\left|x^{\prime}-y^{\prime}\right|+\tau\right)^{\alpha}} d y^{\prime} d s .
$$

Finally, if we set $s=s^{\prime}\left(\left|x^{\prime}-y^{\prime}\right|+\tau\right)^{2}, s^{\prime} \in \mathbb{R}$, and let $x^{\prime}-y^{\prime}=\tau y^{\prime \prime}, y^{\prime \prime} \in \mathbb{R}^{n-1}$, we can conclude that

$$
\begin{aligned}
J_{1} & \leq c \int_{y^{\prime} \in \mathbb{R}^{n-1}} \frac{d y^{\prime}}{\left(\left|x^{\prime}-y^{\prime}\right|+\tau\right)^{n-1+\alpha}}\left(\int_{s^{\prime} \in \mathbb{R}} \frac{d s^{\prime}}{\left(1+\left|s^{\prime}\right|^{1 / 2}\right)^{n+1}}\right) \\
& \leq c \tau^{-\alpha} \int_{\mathbb{R}^{n-1}} \frac{d y^{\prime \prime}}{\left(\left|y^{\prime \prime}\right|+1\right)^{n-1+\alpha}} \\
& \leq c \tau^{-\alpha} .
\end{aligned}
$$

Similarly,

$$
J_{2} \leq c \iint_{D_{1}^{\prime \prime}} \frac{1}{\left(\left|x^{\prime}-z^{\prime}\right|+\tau+|s|^{1 / 2}\right)^{n+1}\left|y^{\prime}-z^{\prime}\right|^{n-1+\alpha}} d y^{\prime} d s d z^{\prime}
$$

One can easily verify that $\left|x^{\prime}-y^{\prime}\right|+\tau<c\left|y^{\prime}-z^{\prime}\right|$ implies $\left|x^{\prime}-z^{\prime}\right|+\tau<c\left|y^{\prime}-z^{\prime}\right|$, hence

$$
J_{2} \leq c \iint_{D_{1}^{\prime \prime \prime}} \frac{1}{\left(\left|x^{\prime}-z^{\prime}\right|+\tau+|s|^{1 / 2}\right)^{n+1}\left|y^{\prime}-z^{\prime}\right|^{n-1+\alpha}} d y^{\prime} d s d z^{\prime}
$$


where

$$
D_{1}^{\prime \prime \prime}:=\left\{\left(y^{\prime}, s, z^{\prime}\right) \in \mathbb{R}^{n-1} \times \mathbb{R} \times \mathbb{R}^{n-1}:\left|x^{\prime}-z^{\prime}\right|+\tau<c\left|y^{\prime}-z^{\prime}\right|\right\}
$$

Now comparing (5.2.121) and 5.2.123), and using the estimate for $J_{1}$ (see (5.2.122)), we can conclude that $J_{2} \leq c \tau^{-\alpha}$, which, in concert with (5.2.122) and (5.2.120) implies that

$$
I_{1} \leq c \delta(x)^{-\alpha}
$$

We let the second domain of integration be

$$
D_{2}:=\{(y, s, z, r) \in \partial \Omega \times \mathbb{R} \times \partial \Omega \times \mathbb{R}:|x-z|<c|y-z|\}
$$

and we set

$$
I_{2}:=\iint_{D_{2}} \frac{|\nabla E(x-y, t-s)-\nabla E(x-z, t-s)|}{\left(|y-z|+|s-r|^{1 / 2}\right)^{n+1+\alpha}} d \sigma_{y} d s d \sigma_{z} d r
$$

Since $|x-z|<c|y-z|$ implies $|x-y|<c|y-z|$, we obtain that

$$
I_{2} \leq I_{1} \leq c \delta(x)^{-\alpha}
$$

Third, we let $D_{3}$ be the complement of $D_{1} \cup D_{2}$, i.e.

$$
D_{3}:=\{(y, s, z, r) \in \partial \Omega \times \mathbb{R} \times \partial \Omega \times \mathbb{R}:|x-y|>c|y-z|,|x-z|>c|y-z|\},
$$

and set

$$
I_{3}:=\iint_{D_{3}} \frac{|\nabla E(x-y, t-s)-\nabla E(x-z, t-s)|}{\left(|y-z|+|s-r|^{1 / 2}\right)^{n+1+\alpha}} d \sigma_{y} d s d \sigma_{z} d r .
$$


Using the Fundamental Theorem of Calculus for the difference in the numerator in (5.2.126), we obtain that

$$
\begin{aligned}
|\nabla E(x-y, t-s)-\nabla E(x-z, t-s)| & \leq|y-z| \int_{0}^{1}\left|\nabla^{2} E\left(x-\xi_{\theta}, t-s\right)\right| d \theta \\
& \leq c|y-z| \int_{0}^{1} \frac{1}{\left(\left|x-\xi_{\theta}\right|+|t-s|^{1 / 2}\right)^{n+2}} d \theta,
\end{aligned}
$$

where $\xi_{\theta}:=(1-\theta) y+\theta z$, and we were also using Proposition 1.2.2 (one could use Proposition 2.1.9 from [She2], as well) to estimate $\left|\nabla^{2} E\right|$. Due to the geometrical position of the points involved, we can easily show that $\left|x-\xi_{\theta}\right| \geq \min \{|x-y|,|x-z|\}$. Therefore,

$$
I_{3} \leq c \iint_{D_{3}} \frac{|y-z|}{\left(|x-y|+|t-s|^{1 / 2}\right)^{n+2}\left(|y-z|+|s-r|^{1 / 2}\right)^{n+1+\alpha}} d \sigma_{y} d s d \sigma_{z} d r .
$$

We make the change of variables $x=\left(x^{\prime}, \varphi\left(x^{\prime}\right)+\tau\right) \in \Omega, y=\left(y^{\prime}, \varphi\left(y^{\prime}\right)\right) \in \partial \Omega$ and $z=\left(z^{\prime}, \varphi\left(z^{\prime}\right)\right) \in \partial \Omega$, where $x^{\prime}, y^{\prime}, z^{\prime} \in \mathbb{R}^{n-1}, \tau>0$, and the graph of the Lipschitz function $\varphi: \mathbb{R}^{n-1} \rightarrow \mathbb{R}$ is the boundary of $\Omega$ (if $\Omega$ is a bounded domain, we use a finite partition of unity on $\partial \Omega$ ). Then

$$
I_{3} \leq c \iint_{D_{3}^{\prime}} \frac{\left|y^{\prime}-z^{\prime}\right|}{\left(\left|x^{\prime}-y^{\prime}\right|+\tau+|t-s|^{1 / 2}\right)^{n+2}\left(\left|y^{\prime}-z^{\prime}\right|+|s-r|^{1 / 2}\right)^{n+1+\alpha}} d y^{\prime} d s d z^{\prime} d r
$$

where the set $D_{3}^{\prime}$ is given by

$\left\{\left(y^{\prime}, s, z^{\prime}, r\right) \in \mathbb{R}^{n-1} \times \mathbb{R} \times \mathbb{R}^{n-1} \times \mathbb{R}:\left|x^{\prime}-y^{\prime}\right|+\tau>c\left|y^{\prime}-z^{\prime}\right|,\left|x^{\prime}-z^{\prime}\right|+\tau>c\left|y^{\prime}-z^{\prime}\right|\right\}$

Let $s-r=r^{\prime}\left|y^{\prime}-z^{\prime}\right|^{2}$ with $r^{\prime} \in \mathbb{R}$. Then, using the fact that $\int_{\mathbb{R}} \frac{d r^{\prime}}{\left(1+\left|r^{\prime}\right|^{1 / 2}\right)^{n+1+\alpha}}$ is finite,

$$
I_{3} \leq c \iint_{D_{3}^{\prime \prime}} \frac{1}{\left(\left|x^{\prime}-y^{\prime}\right|+\tau+|t-s|^{1 / 2}\right)^{n+2}\left|y^{\prime}-z^{\prime}\right|^{n-2+\alpha}} d y^{\prime} d s d z^{\prime}
$$


where

$D_{3}^{\prime \prime}:=\left\{\left(y^{\prime}, s, z^{\prime}\right) \in \mathbb{R}^{n-1} \times \mathbb{R} \times \mathbb{R}^{n-1}:\left|x^{\prime}-y^{\prime}\right|+\tau>c\left|y^{\prime}-z^{\prime}\right|,\left|x^{\prime}-z^{\prime}\right|+\tau>c\left|y^{\prime}-z^{\prime}\right|\right\}$

Moreover, if we set $t-s=s^{\prime}\left(\left|x^{\prime}-y^{\prime}\right|+\tau\right)$ with $s^{\prime} \in \mathbb{R}$, and take into account that $\int_{\mathbb{R}} \frac{d s^{\prime}}{\left(1+\left|s^{\prime}\right|^{1 / 2}\right)^{n+2}}<\infty$, we obtain that

$$
I_{3} \leq c \iint_{D_{3}^{\prime \prime \prime}} \frac{1}{\left(\left|x^{\prime}-y^{\prime}\right|+\tau\right)^{n}\left|y^{\prime}-z^{\prime}\right|^{n-2+\alpha}} d y^{\prime} d z^{\prime}
$$

where

$D_{3}^{\prime \prime \prime}:=\left\{\left(y^{\prime}, z^{\prime}\right) \in \mathbb{R}^{n-1} \times \mathbb{R}^{n-1}:\left|x^{\prime}-y^{\prime}\right|+\tau>c\left|y^{\prime}-z^{\prime}\right|,\left|x^{\prime}-z^{\prime}\right|+\tau>c\left|y^{\prime}-z^{\prime}\right|\right\}$

Integrating with respect to $z^{\prime}$, then making the change of variables $x^{\prime}-y^{\prime}=\tau y^{\prime \prime}$, where $y^{\prime \prime} \in \mathbb{R}^{n-1}$, yield

$$
\begin{aligned}
I_{3} & \leq c \int_{\mathbb{R}^{n-1}} \frac{d y^{\prime}}{\left(\left|x^{\prime}-y^{\prime}\right|+\tau\right)^{n-1+\alpha}} \\
& \leq c \tau^{-\alpha} \int_{\mathbb{R}^{n-1}} \frac{d y^{\prime \prime}}{\left(1+\left|y^{\prime \prime}\right|\right)^{n-1+\alpha}} \\
& \leq c \delta(x)^{-\alpha} .
\end{aligned}
$$

Consequently, $I_{1}, I_{2}$ and $I_{3}$ are bounded by some constant multiple of $\delta(x)^{-\alpha}$ (cf.

(5.2.124), (5.2.125) and (5.2.127), respectively). Therefore

$$
\int_{\partial \Omega \times \mathbb{R}} \int_{\partial \Omega \times \mathbb{R}} \frac{|\nabla E(x-y, t-s)-\nabla E(x-z, t-s)|}{\left(|y-z|+|s-r|^{1 / 2}\right)^{n+1+\alpha}} d \sigma_{y} d s d \sigma_{z} d r \leq c \delta(x)^{-\alpha}
$$

where $(x, t) \in \Omega \times \mathbb{R}$.

Our next goal is to prove that

$$
\int_{\partial \Omega \times \mathbb{R}} \int_{\partial \Omega \times \mathbb{R}} \frac{|\nabla E(x-z, t-s)-\nabla E(x-z, t-r)|}{\left(|y-z|+|s-r|^{1 / 2}\right)^{n+1+\alpha}} d \sigma_{y} d s d \sigma_{z} d r \leq c \delta(x)^{-\alpha}
$$


where $(x, t) \in \Omega \times \mathbb{R}$. In order to do so, we split the domain of integration in a different way than we did earlier in the proof. First we set

$$
D_{4}:=\{(y, s, z, r) \in \partial \Omega \times \mathbb{R} \times \partial \Omega \times \mathbb{R}:|t-s|<c|s-r|\},
$$

and

$$
I_{4}:=\iint_{D_{4}} \frac{|\nabla E(x-z, t-s)-\nabla E(x-z, t-r)|}{\left(|y-z|+|s-r|^{1 / 2}\right)^{n+1+\alpha}} d \sigma_{y} d s d \sigma_{z} d r .
$$

We make the change of variables $x=\left(x^{\prime}, \varphi\left(x^{\prime}\right)+\tau\right) \in \Omega, y=\left(y^{\prime}, \varphi\left(y^{\prime}\right)\right) \in \partial \Omega$ and $z=\left(z^{\prime}, \varphi\left(z^{\prime}\right)\right) \in \partial \Omega$, where $x^{\prime}, y^{\prime}, z^{\prime} \in \mathbb{R}^{n-1}, \tau>0$, and the graph of the Lipschitz function $\varphi: \mathbb{R}^{n-1} \rightarrow \mathbb{R}$ is the boundary of $\Omega$ (if $\Omega$ is a bounded domain, we use a finite partition of unity on $\partial \Omega$ ). Then

$$
I_{4} \leq \iint_{D_{4}^{\prime}} \frac{|\nabla E(x-z, t-s)-\nabla E(x-z, t-r)|}{\left(\left|y^{\prime}-z^{\prime}\right|+|s-r|^{1 / 2}\right)^{n+1+\alpha}} d y^{\prime} d s d z^{\prime} d r
$$

where

$$
D_{4}^{\prime}:=\left\{\left(y^{\prime}, s, z^{\prime}, r\right) \in \mathbb{R}^{n-1} \times \mathbb{R} \times \mathbb{R}^{n-1} \times \mathbb{R}:|t-s|<c|s-r|\right\} .
$$

Going further, we set $y^{\prime}-z^{\prime}=y^{\prime \prime}|s-r|^{1 / 2}$ with $y^{\prime \prime} \in \mathbb{R}^{n-1}$, and use the finiteness of $\int_{\mathbb{R}^{n-1}} \frac{d y^{\prime \prime}}{\left(\left|y^{\prime \prime}\right|+1\right)^{n+1+\alpha}}$ to obtain that

$$
\begin{aligned}
I_{4} & \leq \iint_{D_{4}^{\prime \prime}} \frac{|\nabla E(x-z, t-s)-\nabla E(x-z, t-r)|}{|s-r|^{(2+\alpha) / 2}} d s d z^{\prime} d r \\
& \leq \iint_{D_{4}^{\prime \prime}} \frac{|\nabla E(x-z, t-s)|}{|s-r|^{(2+\alpha) / 2}} d s d z^{\prime} d r+\iint_{D_{4}^{\prime \prime}} \frac{|\nabla E(x-z, t-r)|}{|s-r|^{(2+\alpha) / 2}} d s d z^{\prime} d r \\
& =: J_{3}+J_{4},
\end{aligned}
$$

where

$$
D_{4}^{\prime \prime}:=\left\{\left(s, z^{\prime}, r\right) \in \mathbb{R} \times \mathbb{R}^{n-1} \times \mathbb{R}:|t-s|<c|s-r|\right\} .
$$


According to (1.2.13),

$$
J_{3} \leq c \int_{D_{4}^{\prime \prime}} \frac{1}{\left(\left|x^{\prime}-z^{\prime}\right|+\tau+|t-s|^{1 / 2}\right)^{n+1}|s-r|^{(2+\alpha) / 2}} d s d z^{\prime} d r
$$

Next, we let $s-r=r^{\prime}|t-s|$, where $r^{\prime} \in \mathbb{R}$. Then, due to the fact that $\int_{\substack{r^{\prime} \in \mathbb{R} \\ 0<c \leq\left|r^{\prime}\right|}} \frac{d r^{\prime}}{\left|r^{\prime}\right|^{(2+\alpha) / 2}}$ is controlled by a positive finite constant,

$$
J_{3} \leq c \int_{\left(z^{\prime}, s\right) \in \mathbb{R}^{n-1} \times \mathbb{R}} \frac{1}{\left(\left|x^{\prime}-z^{\prime}\right|+\tau+|t-s|^{1 / 2}\right)^{n+1}|t-s|^{\alpha / 2}} d z^{\prime} d s .
$$

In (5.2.131) letting $t-s=s^{\prime}\left(\left|x^{\prime}-z^{\prime}\right|+\tau\right)^{2}$ for $s^{\prime} \in \mathbb{R}$ implies that

$$
\begin{aligned}
J_{3} & \leq c \int_{\mathbb{R}^{n-1}} \frac{d z^{\prime}}{\left(\left|x^{\prime}-z^{\prime}\right|+\tau\right)^{n-1+\alpha}}\left(\int_{\mathbb{R}} \frac{d s^{\prime}}{\left(1+\left|s^{\prime}\right|^{1 / 2}\right)^{n+1}\left|s^{\prime}\right|^{\alpha / 2}}\right) \\
& \leq c \int_{\mathbb{R}^{n-1}} \frac{d z^{\prime}}{\left(\left|x^{\prime}-z^{\prime}\right|+\tau\right)^{n-1+\alpha}} .
\end{aligned}
$$

Finally, the change of variables $x^{\prime}-z^{\prime}=\tau z^{\prime \prime}$ with $z^{\prime \prime} \in \mathbb{R}^{n-1}$, yields

$$
\begin{aligned}
J_{3} & \leq c \tau^{-\alpha} \int_{\mathbb{R}^{n-1}} \frac{d z^{\prime \prime}}{\left(\left|z^{\prime \prime}\right|+1\right)^{n-1+\alpha}} \\
& \leq c \delta(x)^{-\alpha} .
\end{aligned}
$$

With regard to $J_{4}$, note that $|t-s|<c|s-r|$ implies $|t-r|<c|s-r|$, hence

$$
J_{4} \leq J_{3} \leq c \delta(x)^{-\alpha}
$$

As a consequence of (5.2.130) and (5.2.133) we can conclude that

$$
I_{4} \leq c \delta(x)^{-\alpha}
$$

Let the second domain of integration be

$$
D_{5}:=\{(y, s, z, r) \in \partial \Omega \times \mathbb{R} \times \partial \Omega \times \mathbb{R}:|t-r|<c|s-r|\},
$$


and set

$$
I_{5}:=\iint_{D_{5}} \frac{|\nabla E(x-z, t-s)-\nabla E(x-z, t-r)|}{\left(|y-z|+|s-r|^{1 / 2}\right)^{n+1+\alpha}} d \sigma_{y} d s d \sigma_{z} d r .
$$

Since $|t-r|<c|s-r|$ implies $|t-s|<c|s-r|$, we obtain that

$$
I_{5} \leq I_{4} \leq c \delta(x)^{-\alpha}
$$

For the third domain of integration, we consider $D_{6}$ to be the complement of $D_{4} \cup D_{5}$, i.e.

$$
D_{6}:=\{(y, s, z, r) \in \partial \Omega \times \mathbb{R} \times \partial \Omega \times \mathbb{R}:|t-s|>c|s-r|,|t-r|>c|s-r|\},
$$

and set

$$
I_{6}:=\iint_{D_{6}} \frac{|\nabla E(x-z, t-s)-\nabla E(x-z, t-r)|}{\left(|y-z|+|s-r|^{1 / 2}\right)^{n+1+\alpha}} d \sigma_{y} d s d \sigma_{z} d r .
$$

By the Fundamental Theorem of Calculus in the time variable, and by (1.2.13),

$$
\begin{aligned}
|\nabla E(x-z, t-s)-\nabla E(x-z, t-r)| & \leq|s-r| \int_{0}^{1}\left|\partial_{\text {time }} \nabla E\left(x-z, t-\eta_{\theta}\right)\right| d \theta \\
& \leq c|s-r| \int_{0}^{1} \frac{d \theta}{\left(|x-z|+\left|t-\eta_{\theta}\right|^{1 / 2}\right)^{n+3}} .
\end{aligned}
$$

From geometrical considerations, $\left|t-\eta_{\theta}\right| \geq|t-s|$, hence

$$
I_{6} \leq c \iint_{D_{6}} \frac{|s-r|}{\left(|x-z|+|t-s|^{1 / 2}\right)^{n+3}\left(|y-z|+|s-r|^{1 / 2}\right)^{n+1+\alpha}} d \sigma_{y} d s d \sigma_{z} d r
$$

or, equivalently (using a pull-back as before),

$$
I_{6} \leq c \iint_{D_{6}^{\prime}} \frac{|s-r|}{\left(\left|x^{\prime}-z^{\prime}\right|+\tau+|t-s|^{1 / 2}\right)^{n+3}\left(\left|y^{\prime}-z^{\prime}\right|+|s-r|^{1 / 2}\right)^{n+1+\alpha}} d y^{\prime} d s d z^{\prime} d r
$$

where

$$
D_{6}^{\prime}:=\left\{\left(y^{\prime}, s, z^{\prime}, r\right) \in \mathbb{R}^{n-1} \times \mathbb{R} \times \mathbb{R}^{n-1} \times \mathbb{R}:|t-s|>c|s-r|,|t-r|>c|s-r|\right\} .
$$


Next, we let $y^{\prime}-z^{\prime}=y^{\prime \prime}|s-r|^{1 / 2}$ with $y^{\prime \prime} \in \mathbb{R}^{n-1}$. Since $\int_{\mathbb{R}^{n-1}} \frac{d y^{\prime \prime}}{\left(1+\left|y^{\prime \prime}\right|\right)^{n+1+\alpha}}$ is bounded by a positive finite constant, we obtain the following.

$$
\begin{aligned}
I_{6} & \leq c \iint_{D_{6}^{\prime \prime}} \frac{1}{\left(\left|x^{\prime}-z^{\prime}\right|+\tau+|t-s|^{1 / 2}\right)^{n+3}|s-r|^{\alpha / 2}} d s d z^{\prime} d r \\
& \leq c \iiint_{\left(z^{\prime}, s\right) \in \mathbb{R}^{n-1} \times \mathbb{R}} \frac{1}{\left(\left|x^{\prime}-z^{\prime}\right|+\tau+|t-s|^{1 / 2}\right)^{n+3}}\left(\int_{\substack{r \in \mathbb{R} \\
|t-s|>c|s-r|}} \frac{d r}{|s-r|^{\alpha / 2}}\right) d z^{\prime} d s .
\end{aligned}
$$

where

$$
D_{6}^{\prime \prime}:=\left\{\left(s, z^{\prime}, r\right) \in \mathbb{R} \times \mathbb{R}^{n-1} \times \mathbb{R}:|t-s|>c|s-r|,|t-r|>c|s-r|\right\} .
$$

Integrating with respect to $r$, then making the change of variables $t-s=$ $s^{\prime}\left(\left|x^{\prime}-z^{\prime}\right|+\tau\right)^{2}$, where $s^{\prime} \in \mathbb{R}$, yields

$$
\begin{aligned}
I_{6} & \leq c \iiint_{\left(z^{\prime}, s\right) \in \mathbb{R}^{n-1} \times \mathbb{R}} \frac{1}{\left(\left|x^{\prime}-z^{\prime}\right|+\tau+|t-s|^{1 / 2}\right)^{n+3}|t-s|^{(\alpha-2) / 2}} d z^{\prime} d s \\
& \leq c \int_{\mathbb{R}^{n-1}} \frac{d z^{\prime}}{\left(\left|x^{\prime}-z^{\prime}\right|+\tau\right)^{n-1+\alpha}}\left(\int_{\mathbb{R}} \frac{d s^{\prime}}{\left(1+\left|s^{\prime}\right|^{1 / 2}\right)^{n+3}\left|s^{\prime}\right|^{(\alpha-2) / 2}}\right) \\
& \leq c \int_{\mathbb{R}^{n-1}} \frac{d z^{\prime}}{\left(\left|x^{\prime}-z^{\prime}\right|+\tau\right)^{n-1+\alpha}} .
\end{aligned}
$$

Finally, we set $x^{\prime}-z^{\prime}=\tau z^{\prime \prime}$ with $z^{\prime \prime} \in \mathbb{R}^{n-1}$ to obtain that

$$
\begin{aligned}
I_{6} & \leq c \tau^{-\alpha} \int_{\mathbb{R}^{n-1}} \frac{d z^{\prime \prime}}{\left(\left|z^{\prime \prime}\right|+1\right)^{n-1+\alpha}} \\
& \leq c \tau^{-\alpha} .
\end{aligned}
$$

Consequently, $I_{4}, I_{5}$ and $I_{6}$ are bounded by $c \delta(x)^{-\alpha}$ (cf. (5.2.134), (5.2.135) and (5.2.136), respectively). This shows (5.2.129), which, in concert with (5.2.128) proves $(5.2 .118)$.

In order to complete the proof of $\mathbf{A}$, we need to show that, for $(x, t) \in \Omega \times \mathbb{R}$,

$$
\|\tilde{\psi}(x) \nabla E(x-\cdot, t-\cdot)\|_{L^{1}(K \times I)} \leq c \delta(x)^{-\alpha} .
$$


First we estimate $\nabla E$ using (1.2.13), then we make the change of variables $x=$ $\left(x^{\prime}, \varphi\left(x^{\prime}\right)+\tau\right) \in \tilde{K} \subset \Omega, y=\left(y^{\prime}, \varphi\left(y^{\prime}\right)\right) \in K \subset \partial \Omega$, where $x^{\prime}, y^{\prime} \in \mathbb{R}^{n-1}$, and $\Omega$ lies above the graph of the Lipschitz function $\varphi: \mathbb{R}^{n-1} \rightarrow \mathbb{R}$ (for a bounded domain we use a partition of unity). Then we obtain the following.

$$
\begin{aligned}
\|\tilde{\psi}(x) \nabla E(x-\cdot, t-\cdot)\|_{L^{1}(K \times I)} & \leq c \iint_{K \times I} \frac{1}{\left(|x-y|+|t-s|^{1 / 2}\right)^{n+1}} d \sigma_{y} d s \\
& \leq c \iint_{\substack{\mathbb{R}^{n-1 \times I} \\
\left|y^{\prime}\right| \leq c}} \frac{1}{\left(\left|x^{\prime}-y^{\prime}\right|+\tau+|t-s|^{1 / 2}\right)^{n+1}} d y^{\prime} d s \\
& \leq c \iint_{\substack{\mathbb{R}^{n-1 \times \mathbb{R}} \\
\left|y^{\prime}\right| \leq c}} \frac{1}{\left(\tau+|r|^{1 / 2}\right)^{n+1}} d y^{\prime} d r \\
& \leq c \\
& \leq c \delta(x)^{-\alpha} .
\end{aligned}
$$

Here we were also using the fact that $0<\tau \approx \delta(x) \leq c$, since $x$ belongs to the compact support $\tilde{K} \subset \Omega$ of $\tilde{\psi}$. This completes the proof of (5.2.137) and that of $\mathbf{A}$.

Folowing exactly the same steps as in the case of $\mathbf{A}$, we can show that $\mathbf{B}$ holds, as well. Similarly, the main part of the norm in the left-hand side of $\mathbf{C}$ is controlled by $c \delta(x)^{-\alpha}$. Keeping in mind that $x$ belongs to the compact support $\tilde{K} \subset \Omega$ of $\tilde{\psi}$, and by checking that $\|\nabla \tilde{\psi}(x) E(x-\cdot, t-\cdot)\|_{L^{1}(K \times I)} \leq c \delta(x)^{-\alpha}$, we immediately obtain C, completing the proof of Theorem 5.2.6.

As the last result of this chapter we present the mapping property of the single layer potential operator $\mathcal{S}$ on bounded Lipschitz cylinders, a property which will be crutial for the applications we have in mind, especially in Chapter 8. 
Theorem 5.2.7. For a bounded Lipschitz domain $\Omega \subset \mathbb{R}^{n}, 0<T<\infty$, and $1<p<\infty, 0<\alpha<1$, the caloric single layer potential operator

$$
\mathcal{S}:{ }_{0} B_{-\alpha, p a r}^{p}(\partial \Omega \times(0, T)) \longrightarrow{ }_{0} B_{1-\alpha+1 / p, p a r}^{p}(\Omega \times(0, T))
$$

is bounded.

Proof. Given $f \in{ }_{0} B_{-\alpha, p a r}^{p}(\partial \Omega \times(0, T))$, by Definition $3.2 .2(\mathrm{c})$, there exists $F \in$ $B_{-\alpha, p a r}^{p}(\partial \Omega \times \mathbb{R})$ with $\left.F\right|_{\partial \Omega \times(-\infty, T)}=f$ and support included in $\partial \Omega \times[0, \infty)$. From Theorem 5.2.6 we have

$$
\begin{gathered}
\tilde{\mathcal{S}} F \in B_{1-\alpha+1 / p, p a r}^{p}\left(\Omega_{+} \times \mathbb{R}\right), \\
(\tilde{\mathcal{S}} F)^{-} \in B_{1-\alpha+1 / p, p a r}^{p}\left(\Omega_{-} \times \mathbb{R}\right),
\end{gathered}
$$

where $\Omega_{+}=\Omega$ and $\Omega_{-}=\mathbb{R}^{n} \backslash \bar{\Omega}$. With this in hand, if we define

$$
G= \begin{cases}\tilde{\mathcal{S}} F & \text { in } \Omega_{+} \times \mathbb{R} \\ (\tilde{\mathcal{S}} F)^{-} & \text {in } \Omega_{-} \times \mathbb{R}\end{cases}
$$

then, Lemma 3.1.18 implies that

$$
G \in B_{1-\alpha+1 / p, p a r}^{p}\left(\mathbb{R}^{n} \times \mathbb{R}\right)
$$

Since the domain $\Omega$ is bounded, and $0<t<T, s<t$, one can easily see that

$$
\begin{aligned}
\left.G\right|_{\Omega \times(0, T)}(x, t) & =\left.(\tilde{\mathcal{S}} F)\right|_{\Omega \times(0, T)}(x, t)=\left.(\mathcal{S} F)\right|_{\Omega \times(0, T)}(x, t) \\
& =\left.\left(\int_{\partial \Omega} \int_{\mathbb{R}} E(x-y, t-s) F(y, s) d \sigma_{y} d s\right)\right|_{(x, t) \in \Omega \times(0, T)} \\
& =\int_{\partial \Omega} \int_{-\infty}^{T} E(x-y, t-s) f(y, s) d \sigma_{y} d s \\
& =(\mathcal{S} f)(x, t) .
\end{aligned}
$$


Our next goal is to show that

$$
\operatorname{supp} G \subseteq \mathbb{R}^{n} \times[0, \infty)
$$

First we note that for $(x, t) \in \Omega \times \mathbb{R}$

$$
(\mathcal{S} F)(x, t)=\int_{\partial \Omega} \int_{\mathbb{R}} E(x-y, t-s) F(y, s) d \sigma_{y} d s .
$$

Since $F$ is supported in $\partial \Omega \times[0, \infty)$ and $t-s>0$, it follows that $t>0$, and

$$
\operatorname{supp} \tilde{\mathcal{S}} F \subseteq \operatorname{supp} \mathcal{S} F \subseteq \Omega \times[0, \infty) \subseteq \mathbb{R}^{n} \times[0, \infty)
$$

Similarly, $\operatorname{supp}(\mathcal{S} F)^{-} \subseteq \mathbb{R}^{n} \times[0, \infty)$, therefore (5.2.140) holds.

All in all, (5.2.138), (5.2.139) and (5.2.140) are what we need in order to conclude, by definition, that $\mathcal{S} f \in{ }_{0} B_{1-\alpha+1 / p, p a r}^{p}(\Omega \times(0, T))$. 


\section{Chapter 6}

\section{Invertibility of boundary potential operators}

In the present chapter we establish the invertibility of caloric boundary potential operators on parabolic Besov spaces defined on (unbounded and bounded) Lipschitz cylinders. These properties are going to play a crutial role in solving the boundary value problems considered in Chapter 8 .

For the convenience of the reader we recall from (1.1.6) the definition of the caloric boundary potential operator $K$ : for a boundary point $(x, t) \in \partial \Omega \times \mathbb{R}$, where $\Omega$ is a Lipschitz domain in $\mathbb{R}^{n}$, we introduce

$$
K f(x, t):=\text { p.v. } \int_{\partial \Omega \times \mathbb{R}} \partial_{\nu_{y}}[E(x-y, t-s)] f(y, s) d \sigma_{y} d s,
$$

where p.v. indicates that the above integral is taken in the principal value sense. Above $\nu$ and $d \sigma$ denote, respectively, the unit normal and the surface measure on $\partial \Omega$. We next set

$$
K^{\prime}:=R \circ K^{*} \circ R
$$

where $K^{*}$ is the formal adjoint of $K$ and $R$ stands for the reflection operator in time, i.e. $R f(x, t):=f(x,-t)$ for any $(x, t) \in \mathbb{R}^{n} \times \mathbb{R}$. Recall that the double and single 
layer caloric potentials, respectively, are defined as

$$
\begin{aligned}
& \mathcal{D} f(x, t):=\int_{\partial \Omega \times \mathbb{R}} \partial_{\nu_{y}}[E(x-y, t-s)] f(y, s) d \sigma_{y} d s, \\
& \mathcal{S} f(x, t):=\int_{\partial \Omega \times \mathbb{R}} E(x-y, t-s) f(y, s) d \sigma_{y} d s,
\end{aligned}
$$

where $(x, t) \in\left(\mathbb{R}^{n} \backslash \partial \Omega\right) \times \mathbb{R}$. For the boundary trace of the caloric single layer potential we introduce the notation

$$
S f:=\left.\mathcal{S} f\right|_{\partial \Omega \times \mathbb{R}} .
$$

Throughout this chapter $I$ denotes the identity operator.

We will not make a distinction between $K$ as defined in (6.0.1) and $K_{T}$, where $K_{T} f:=\left.(K f)\right|_{\partial \Omega \times(0, T)}$. Each time, whether $K$ or $K_{T}$ is used, should be clear from the context. The same applies to the operators $K^{\prime}, \mathcal{D}, \mathcal{S}$ and $S$ defined in (6.0.2), (6.0.3) and (6.0.4).

We next invoke the fact that for any caloric function in $\Omega \times \mathbb{R}$,

$$
u=\mathcal{D}\left(\left.u\right|_{\partial \Omega \times \mathbb{R}}\right)-\mathcal{S}\left(\partial_{\nu} u\right),
$$

(cf., e.g., (2.29) in [HLM]). In particular, if $u:=\mathcal{S} f$ for some density $f$, then, taking the boundary traces of both sides in (6.0.5) and using parts (2) of Theorems 4.16 and 4.17 from [Bro2] for the trace of $\partial_{\nu} \mathcal{S}$ and $\mathcal{D}$, respectively, we obtain that

$$
K S=S K^{\prime}
$$

Note that (6.0.6) readily yields

$$
\pm \frac{1}{2} I+K=S\left( \pm \frac{1}{2} I+K^{\prime}\right) S^{-1} .
$$




\subsection{Case of an unbounded cylinder}

In this section we study different mapping properties of the boundary potential operators $\pm \frac{1}{2} I+K$, the ultimate goal being the invertibility of these operators on Besov spaces defined on an unbounded Lipschitz cylinder. First we record a few known results due to $\mathrm{R}$. Brown, and their direct consequences.

Let $\Omega$ be an unbounded Lipschitz domain in $\mathbb{R}^{n}$. According to Corollary 4.20 from [Bro2], there exists $\varepsilon>0$ such that for $2-\varepsilon<p<\infty$ the operators

$$
\pm \frac{1}{2} I+K: L^{p}(\partial \Omega \times \mathbb{R}) \stackrel{\sim}{\longrightarrow} L^{p}(\partial \Omega \times \mathbb{R})
$$

are invertible. Also, from Theorem 4.18 of [Bro2] we can conclude that there exists an $\varepsilon>0$ such that, for $1<p<2+\varepsilon$, the operators

$$
\pm \frac{1}{2} I+K: \dot{L}_{1, p a r}^{p}(\partial \Omega \times \mathbb{R}) \stackrel{\sim}{\longrightarrow} \dot{L}_{1, p a r}^{p}(\partial \Omega \times \mathbb{R})
$$

are invertible. From the same theorem we have that the operators $\pm \frac{1}{2} I+K^{*}$ are isomorphisms on $\dot{H}_{\text {par }}^{1}(\partial \Omega \times \mathbb{R})$, therefore, applying Theorem 2.7 of [KaMi], there exists an $\varepsilon^{\prime}=\varepsilon^{\prime}(\Omega, n)$ such that

$$
\pm \frac{1}{2} I+K^{*} \text { are invertible on } \dot{H}_{p a r}^{p}(\partial \Omega \times \mathbb{R}) \text { for each } 1-\varepsilon^{\prime}<p \leq 1
$$

When we applied Theorem 2.7 of [KaMi] we were using the fact that the homogeneous parabolic Hardy scale $\left\{\dot{H}_{p a r}^{p}\left(\mathbb{R}^{n} \times \mathbb{R}\right)\right\}_{0<p<\infty}$ is a complex interpolation scale (cf. Theorem 3.1 from [CaTo2]), and due to a bi-Lipschitz change of variables the same statement holds for $\left\{\dot{H}_{p a r}^{p}(\partial \Omega \times \mathbb{R})\right\}_{0<p<\infty}$, where $\Omega$ is a graph domain in $\mathbb{R}^{n}$. 
In order to continue our discussion we need an intermediate result. Inspired by the proof of Theorem B in [CoWe2] we obtain the following.

Theorem 6.1.1. Let $\Omega$ be a Lipschitz domain in $\mathbb{R}^{n}$, and indices $\frac{n+1}{n+2}<p<1$, $\alpha=(n+1)\left(\frac{1}{p}-1\right)$. Then

$$
\left(\dot{H}_{\text {par }}^{p}(\partial \Omega \times \mathbb{R})\right)^{*}=\dot{C}_{\text {par }}^{\alpha}(\partial \Omega \times \mathbb{R})
$$

Proof. Let $g \in \dot{C}_{\text {par }}^{\alpha}(\partial \Omega \times \mathbb{R})$ and $f \in \dot{H}_{\text {par }}^{p}(\partial \Omega \times \mathbb{R})$. We define the functional

$$
L f:=\int_{\partial \Omega \times \mathbb{R}} f(x, t) g(x, t) d \sigma_{x} d t .
$$

According to Definition 2.3.31, $f$ admits an atomic decomposition, i.e. $f=\sum_{j=0}^{\infty} \lambda_{j} a_{j}$ with $\operatorname{supp} a_{j} \subset \mathrm{Q}_{j},\left\|a_{j}\right\|_{L^{\infty}(\partial \Omega \times \mathbb{R})} \leq c\left|\mathrm{Q}_{j}\right|^{-1 / p}=c r_{j}^{-(n+1) / p}$ and vanishing moment condition, where $\mathrm{Q}_{j}$ are parabolic surface cubes on $\partial \Omega \times \mathbb{R}$ with sidelength $r_{j}$. Then one can easily see that

$$
\begin{aligned}
|L f| & =\left|\int_{\partial \Omega \times \mathbb{R}} \sum_{j=0}^{\infty} \lambda_{i} a_{j}(x, t) g(x, t) d \sigma_{x} d t\right|=\left|\sum_{j=0}^{\infty} \lambda_{j} \int_{\mathrm{Q}_{j}} a_{j}(x, t) g(x, t) d \sigma_{x} d t\right| \\
& =\left|\sum_{j=0}^{\infty} \lambda_{j} \int_{\mathrm{Q}_{j}} a_{j}(x, t)\left[g(x, t)-g\left(x_{0}, t_{0}\right)\right] d \sigma_{x} d t\right|
\end{aligned}
$$

where $\left(x_{0}, t_{0}\right)$ is the center of the cube $\mathrm{Q}_{j}$. Using the assumptions on the atoms $a_{j}$, as well as the fact that $g \in \dot{C}_{p a r}^{\alpha}(\partial \Omega \times \mathbb{R})$, (6.1.12) further implies that

$$
\begin{aligned}
|L f| & \leq \sum_{j=0}^{\infty}\left|\lambda_{j}\right| \int_{\mathrm{Q}_{j}}\left|a_{j}(x, t)\right|\|g\|_{\dot{C}_{p a r}^{\alpha}(\partial \Omega \times \mathbb{R})}\left(\left|x-x_{0}\right|+\left|t-t_{0}\right|^{1 / 2}\right)^{\alpha} d \sigma_{x} d t \\
& \leq c \sum_{j=0}^{\infty}\left|\lambda_{j}\right|\left|\mathrm{Q}_{j}\right|^{-1 / p} r_{j}^{\alpha} \int_{\mathrm{Q}_{j}} d \sigma_{x} d t \\
& \leq c\left(\sum_{j=0}^{\infty}\left|\lambda_{j}\right|^{p}\right)^{1 / p} .
\end{aligned}
$$


Taking the infimum over all representations of $f$ in (6.1.13), we obtain that

$$
|L f| \leq c \inf \left(\sum_{j=0}^{\infty}\left|\lambda_{j}\right|^{p}\right)^{1 / p}=c\|f\|_{\dot{H}_{p a r}^{p}(\partial \Omega \times \mathbb{R})}
$$

hence $L$ is bounded, linear and continuous. This finishes the proof of the right-to-left inclusion in (6.1.11).

Conversely, suppose $L$ is a bounded linear functional on $\dot{H}_{\text {par }}^{p}(\partial \Omega \times \mathbb{R})$.

Step 1. First we will show the existence of a function $G$, bounded on each sphere $S$ on $\partial \Omega \times \mathbb{R}$, such that $L a_{j}=\int_{\partial \Omega \times \mathbb{R}} a_{j}(x, t) G(x, t) d \sigma_{x} d t$ for each $\dot{H}_{p a r}^{p}$-atom $a_{j}$. By a sphere centered at $(x, t)$ and of radius $r>0$ we mean

$$
S_{r}(x, t):=\left\{(y, s) \in \partial \Omega \times \mathbb{R}:|x-y|+|t-s|^{1 / 2}<r\right\} .
$$

For a sphere $S$ on $\partial \Omega \times \mathbb{R}$ we let $L_{0}^{1}(S):=\left\{f \in L^{1}(S): \int_{S} f(x, t) d \sigma_{x} d t=0\right\}$. Then, for $f \in L_{0}^{1}(S)$, we easily verify that

$$
a(x, t):=f(x, t)\|f\|_{L^{1}(S)}^{-1}|S|^{1-\frac{1}{p}}
$$

is a $(p, 1)$-atom for $\dot{H}_{\text {par }}^{p}(\partial \Omega \times \mathbb{R})$ supported in $S$. Also, $\|f\|_{\dot{H}_{\text {par }}^{p}(\partial \Omega \times \mathbb{R})}<\infty$, hence $L f$ is well-defined and

$$
|L f| \leq\|L\||S|^{\frac{1}{p}-1}\|f\|_{L^{1}(S)}
$$

Therefore $L$ is a bounded linear functional on $L_{0}^{1}(S)$. Since $L_{0}^{1}(S)$ is a closed subspace of $L^{1}(S)$, by the Hahn-Banach theorem, there exists an extension of $L$, which we denote also by $L$, a bounded linear functional on $L^{1}(S)$. According to the Riesz representation theorem, there exists $g \in L^{\infty}(S)$ such that

$$
L f=\int_{S} f(x, t) g(x, t) d \sigma_{x} d t \quad \text { for all } f \in L_{0}^{1}(S)
$$


Next we show that $g$ is uniquely determined up to a constant. For this it is enough to show that

$$
\int_{S} f(x, t) g(x, t) d \sigma_{x} d t=0 \quad \forall f \in L_{0}^{1}(S) \text { implies } g \text { is a constant. }
$$

To see this we choose $h \in L^{1}(S)$. Then $h-\frac{1}{|S|} \int_{S} h(y, s) d \sigma_{y} d s \in L_{0}^{1}(S)$ and

$$
\begin{aligned}
0 & =\int_{S}\left(h(x, t)-\frac{1}{|S|} \int_{S} h(y, s) d \sigma_{y} d s\right) g(x, t) d \sigma_{x} d t \\
& =\int_{S} h(x, t)\left(g(x, t)-\frac{1}{|S|} \int_{S} g(y, s) d \sigma_{s} d s\right) d \sigma_{x} d t
\end{aligned}
$$

which implies that $g(x, t)-\frac{1}{|S|} \int_{S} g(y, s) d \sigma_{y} d s=0$ for almost every $(x, t) \in S$, i.e. $g(x, t)$ is constant for almost every $(x, t) \in S$.

Considering (6.1.14) and the uniqueness of such $g$, as well as the fact that if $a$ is a $(p, 1)$-atom for $\dot{H}_{p a r}^{p}(\partial \Omega \times \mathbb{R})$ supported in a sphere $S$, then $a \in L_{0}^{1}(S)$, we can conclude that there exists a unique $G \in L^{\infty}(S)$ such that

$$
L a=\int_{S} a(x, t) G(x, t) d \sigma_{x} d t \text { for all }(p, 1) \text {-atoms } a
$$

This conludes the proof of Step 1.

Step 2. Our next goal is to show that for $G$ as above, $G \in \dot{C}_{p a r}^{\alpha}(\partial \Omega \times \mathbb{R})$. To do so, let us consider $f \in L^{1}(S)$ with $\|f\|_{L^{1}(S)}=1$, and set

$$
b(x, t):=|S|^{1-\frac{1}{p}}\left(f(x, t)-\frac{1}{|S|} \int_{S} f(y, s) d \sigma_{y} d s\right) .
$$

One can easily verify that $b$ is a $(p, 1)$-atom for $\dot{H}_{p a r}^{p}(\partial \Omega \times \mathbb{R})$, supported in $S$, therefore, by (6.1.16), there exists a unique $G \in L^{\infty}(S)$ so that

$$
L b=\int_{S} b(x, t) G(x, t) d \sigma_{x} d t
$$


Moreover, for $b$ as in (6.1.17) and $G \in L^{\infty}(S)$ as above,

$$
\begin{aligned}
\|L\| & \geq|L b|=\left|\int_{S} b(x, t)\left(G(x, t)-\frac{1}{|S|} \int_{S} G(y, s) d \sigma_{y} d s\right) d \sigma_{x} d t\right| \\
& =|S|^{1-\frac{1}{p}}\left|\int_{S} f(x, t)\left(G(x, t)-\frac{1}{|S|} \int_{S} G(y, s) d \sigma_{y} d s\right) d \sigma_{x} d t\right| .
\end{aligned}
$$

In other words, since $\alpha=(n+1)\left(\frac{1}{p}-1\right)$,

$$
\left|\int_{S} f(x, t)\left(G(x, t)-\frac{1}{|S|} \int_{S} G(y, s) d \sigma_{y} d s\right) d \sigma_{x} d t\right| \leq\|L\||S|^{\frac{\alpha}{n+1}}
$$

The inequality (6.1.19) furher implies that, for $G \in L^{\infty}(S)$ satisfying (6.1.18),

$$
\begin{aligned}
\| G- & \frac{1}{|S|} \int_{S} G(y, s) d \sigma_{y} d s \|_{L^{\infty}(S)} \\
& =\sup _{\substack{f \in L^{1}(S) \\
\left\|f^{1}\right\|^{1}(S)}}\left|\int_{S} f(x, t)\left(G(x, t)-\frac{1}{|S|} \int_{S} G(y, s) d \sigma_{y} d s\right) d \sigma_{x} d t\right| \\
& \leq\|L\||S|^{\frac{\alpha}{n+1}}
\end{aligned}
$$

Finally, let us consider $(x, t),(y, s) \in \partial \Omega \times \mathbb{R}$. If $\|(x, t)-(y, s)\|_{\text {par }} \leq c$ for some constant c, then we choose the smallest parabolic surface ball $S, S \subset \partial \Omega \times \mathbb{R}$, with radius $r$, such that $(x, t),(y, s) \in S$. Then $\|(x, t)-(y, s)\|_{p a r} \approx r \approx|S|^{\frac{1}{n+1}}$. Using the triangle inequality in the numerator of the left-hand side in (6.1.21), then (6.1.20), we obtain that, for $G \in L^{\infty}(S)$ which fulfills condition (6.1.18),

$$
\frac{|G(x, t)-G(y, s)|}{\|(x, t)-(y, s)\|_{p a r}^{\alpha}}<\infty
$$

If $\|(x, t)-(y, s)\|_{p a r} \geq c$ for some constant $c$, then for the same $G$ as above,

$$
\frac{|G(x, t)-G(y, s)|}{\|(x, t)-(y, s)\|_{p a r}^{\alpha}} \leq c\|G\|_{L^{\infty}(S)}<\infty
$$

where $S$ is a parabolic surface ball on $\partial \Omega \times \mathbb{R}$ so that $(x, t),(y, s) \in S$. 
Based on (6.1.21) and (6.1.22) we can conclude that there exists a unique (up to a constant) $G \in \dot{C}_{\text {par }}^{\alpha}(\partial \Omega \times \mathbb{R})$ such that $L a=\int_{\partial \Omega \times \mathbb{R}} a(x, t) G(x, t) d \sigma_{x} d t$ for all $(p, 1)$-atoms $a$. Given the atomic decomposition of the space $\dot{H}_{p a r}^{p}(\partial \Omega \times \mathbb{R})$, the proof of Theorem 6.1 .1 is complete.

By dualizing (6.1.10) and using Theorem 6.1.1 we obtain the following.

Corollary 6.1.2. Consider an unbounded Lipschitz domain $\Omega$ in $\mathbb{R}^{n}$. Then there exists an $\varepsilon^{\prime}=\varepsilon^{\prime}(\Omega, n)$ such that the operators

$$
\pm \frac{1}{2} I+K \text { are isomorphisms on } \dot{B}_{\alpha, p a r}^{\infty}(\partial \Omega \times \mathbb{R}) \text { for each } 1-\varepsilon^{\prime}<p \leq 1
$$

where $\alpha=(n+1)\left(\frac{1}{p}-1\right)$.

Another important aspect of the behavior of the operators $\pm \frac{1}{2} I+K$ is analyzed in the next theorem.

Theorem 6.1.3. Let $\Omega$ be an unbounded Lipschitz domain in $\mathbb{R}^{n}$. There exists an $\varepsilon^{\prime}=\varepsilon^{\prime}(\Omega, n)>0$ such that the maps

$$
\pm \frac{1}{2} I+K \text { are isomorphisms on } \dot{H}_{\text {par }}^{1, p}(\partial \Omega \times \mathbb{R})
$$

for each $1-\varepsilon^{\prime}<p \leq 1$.

Proof. In view of the definition of the space $\dot{H}_{\text {par }}^{1, p}\left(\mathbb{R}^{n-1} \times \mathbb{R}\right)$ (see Definition 2.1.51) and of pp. $5-7$ in [Bro2], for $\frac{n+1}{n+2}<p \leq 1$ there exists an isomorphism

$$
\dot{I}: \dot{H}_{p a r}^{p}\left(\mathbb{R}^{n-1} \times \mathbb{R}\right) \stackrel{\sim}{\longrightarrow} \dot{H}_{\text {par }}^{1, p}\left(\mathbb{R}^{n-1} \times \mathbb{R}\right)
$$

where $\dot{I}$ stands for the convolution with $\tilde{E}$, i.e. $\dot{I} f:=f * \tilde{E}$, and $\tilde{E}(x, t):=$ $2(2 \pi t)^{-n / 2} \exp \left(-|x|^{2} / 4 t\right) \chi_{(0, \infty)}(t)$ for $x \in \mathbb{R}^{n-1}, t \in \mathbb{R}$. Due to a bi-Lipschitz change 
of variables, there exists an isomorphism

$$
\dot{\mathcal{I}}: \dot{H}_{\text {par }}^{p}(\partial \Omega \times \mathbb{R}) \stackrel{\sim}{\longrightarrow} \dot{H}_{\text {par }}^{1, p}(\partial \Omega \times \mathbb{R})
$$

where $\Omega$ is an unbounded Lipschitz (graph) domain in $\mathbb{R}^{n}$ and $\frac{n+1}{n+2}<p \leq 1$. Since $\dot{H}_{p a r}^{p}(\partial \Omega \times \mathbb{R})$ is a complex interpolation scale for $0<p<\infty$, as seen earlier in this chapter, (6.1.26) further implies that

$$
\dot{H}_{\text {par }}^{1, p}(\partial \Omega \times \mathbb{R}) \text { is a complex interpolation scale for } 0<p<\infty \text {. }
$$

On the other hand, from Theorem 4.18 of [Bro2] we can easily derive that the operators

$$
\pm \frac{1}{2} I+K \text { are invertible on } \dot{H}_{\text {par }}^{1,1}(\partial \Omega \times \mathbb{R})
$$

Consequently, (6.1.27) and (6.1.28), in concert with Theorem 2.7 of [KaMi], yields the following: there exists an $\varepsilon^{\prime}=\varepsilon^{\prime}(\Omega, n)$ such that the maps

$$
\pm \frac{1}{2} I+K \text { are isomorphisms on } \dot{H}_{\text {par }}^{1, p}(\partial \Omega \times \mathbb{R})
$$

for each $1-\varepsilon^{\prime}<p \leq 1$. This finishes the proof of the theorem.

We now are ready to present our main result in this section.

Theorem 6.1.4. Consider an unbounded (graph) Lipschitz domain $\Omega$ in $\mathbb{R}^{n}$. Then there exists an $\varepsilon=\varepsilon(\Omega, n)>0$ with the property that the operators

$$
\pm \frac{1}{2} I+K \text { are invertible on } \dot{B}_{\alpha, p a r}^{p}(\partial \Omega \times \mathbb{R})
$$

for each $p$ and $\alpha$ such that the pair $\left(\alpha, \frac{1}{p}\right)$ belongs to the interior of the hexagon $O A B C D E$ in the figure below. 


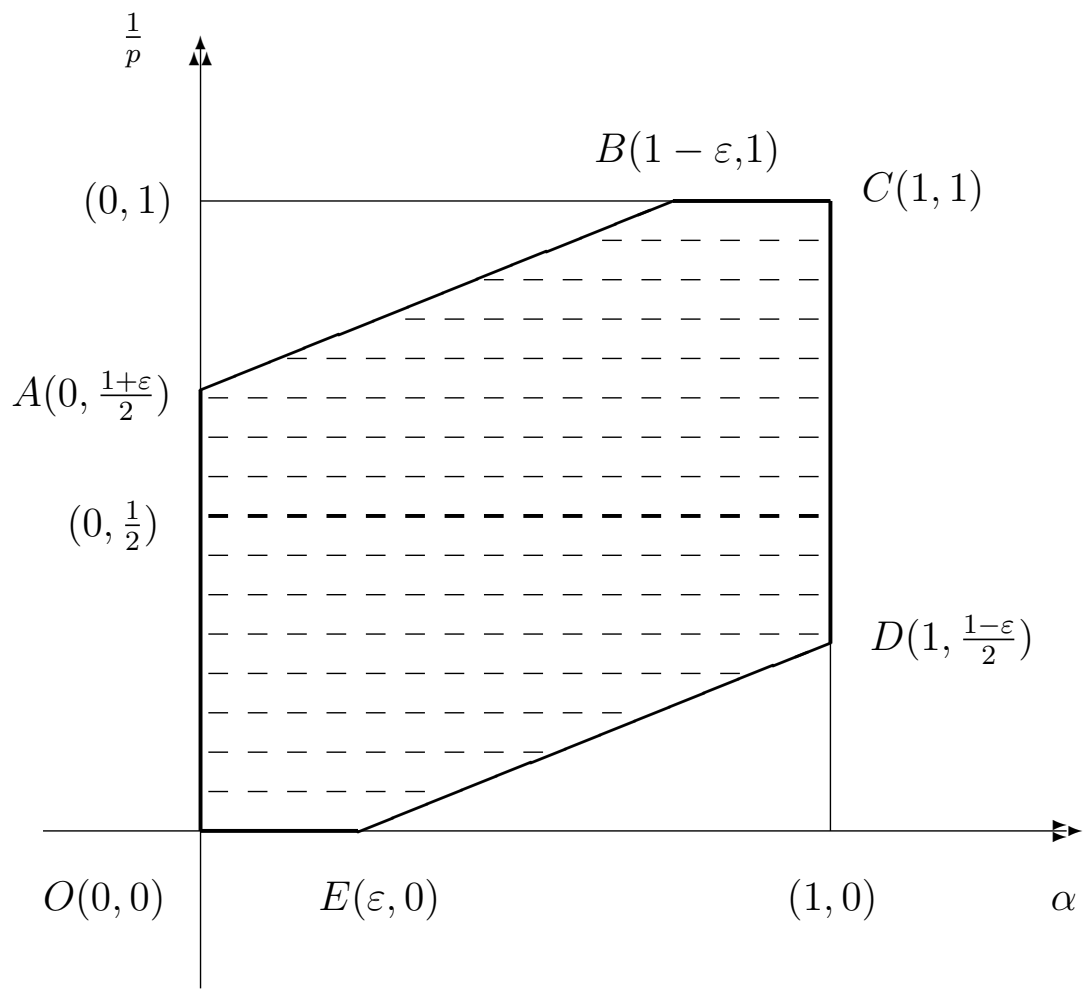

Proof. Thanks to Proposition 1.2.45 we can extend the results of Theorem 6.1.3, then, by identifying the Banach envelope of the space $\dot{H}_{\text {par }}^{1, p}(\partial \Omega \times \mathbb{R})$ (using $(2.5 .248)$ ) we conclude that there exists an $\varepsilon^{\prime}=\varepsilon^{\prime}(\Omega, n)>0$ such that

$$
\pm \frac{1}{2} I+K \text { are isomorphisms on } \dot{B}_{1-\beta, p a r}^{1}(\partial \Omega \times \mathbb{R})
$$

for $\beta=(n+1)\left(\frac{1}{p_{0}}-1\right)$ and $1-\varepsilon^{\prime}<p_{0} \leq 1$. This gives the invertibility of the operators $\pm \frac{1}{2} I+K$ on the upper horizontal segment $B C$ in the figure above.

Another observation is that complex interpolation (cf. part (b) of Remark 2.1.61) between (6.1.8) and (6.1.9) yields the following: there exists a positive $\varepsilon=\varepsilon(\Omega, n)$ so that

$$
\pm \frac{1}{2} I+K \text { are invertible on } \dot{L}_{\alpha, p a r}^{p}(\partial \Omega \times \mathbb{R}) \text { for }\left(\alpha, \frac{1}{p}\right) \in O A C D \text {. }
$$


We next consider two instances of (6.1.31) with the same $p$ and $\alpha_{1} \neq \alpha_{2}$ such that $\left(\alpha_{1}, \frac{1}{p}\right),\left(\alpha_{2}, \frac{1}{p}\right) \in O A C D$. Using real interpolation (see part (d) of Remark 2.1.61) between these two instances we obtain the existence of $\varepsilon=\varepsilon(\Omega, n)>0$ such that

$$
\pm \frac{1}{2} I+K \text { are invertible on } \dot{B}_{\alpha, p a r}^{p}(\partial \Omega \times \mathbb{R}) \text { for }\left(\alpha, \frac{1}{p}\right) \in O A C D
$$

Finally, by the complex interpolation of Besov spaces (Remark 2.1.61 c), as well as by the results $(6.1 .32),(6.1 .23)$ - corresponding to the lower horizontal segment $O E$, and (6.1.30) - corresponding to the upper horizontal segment $B C$, we obtain the desired result.

\subsection{Case of a bounded cylinder}

In the present section we focus our attention on the mapping properties of the operators $\pm \frac{1}{2} I+K$ on parabolic Besov spaces defined on bounded Lipschitz cylinders.

As a preliminary step we discuss a few invertibility results of the operators $\pm \frac{1}{2} I+$ $K$ and $\pm \frac{1}{2} I+K^{\prime}$. These are going to be very useful later on. Recall the definitions of $K, K^{\prime}, S$ from (6.0.1), (6.0.2), (6.0.4), and the identity (6.0.7).

Lemma 6.2.1. Consider a bounded Lipschitz domain $\Omega$ in $\mathbb{R}^{n}, 0<T<\infty$. Then the operators $\pm \frac{1}{2} I+K^{\prime}$ are isomorphisms on ${ }_{0} H_{\text {par }}^{1}(\partial \Omega \times(0, T))$.

Proof. We shall prove the lemma only for $\frac{1}{2} I+K^{\prime}$, since the same argument applies to $-\frac{1}{2} I+K^{\prime}$. Recall a particular case of Remark 3.2 .4 (ii) to the effect that for a bounded Lipschitz domain $\Omega$ and $0<T<\infty$,

$$
{ }_{0} H_{\text {par }}^{1}(\partial \Omega \times(0, T))={ }_{0} \dot{H}_{\text {par }}^{1}(\partial \Omega \times(0, T))+L^{2}(\partial \Omega \times(0, T))
$$


First we show that $\frac{1}{2} I+K^{\prime}:{ }_{0} H_{p a r}^{1}(\partial \Omega \times(0, T)) \rightarrow{ }_{0} H_{\text {par }}^{1}(\partial \Omega \times(0, T))$ is onto. In order to do so, let $f \in{ }_{0} H_{p a r}^{1}(\partial \Omega \times(0, T))$. On account of $(6.2 .33)$ this further implies that $f=f_{1}+f_{2}$ with $f_{1} \in{ }_{0} \dot{H}_{p a r}^{1}(\partial \Omega \times(0, T))$ and $f_{2} \in L^{2}(\partial \Omega \times(0, T))$. Since the operator $\frac{1}{2} I+K^{\prime}$ is invertible (hence onto) on both ${ }_{0} \dot{H}_{p a r}^{1}(\partial \Omega \times(0, T))$ and $L^{2}(\partial \Omega \times(0, T))$ (cf. Theorem 5.25 of $[\mathrm{Bro} 2]$ ), we obtain that there exist $g_{1} \in{ }_{0} \dot{H}_{p a r}^{1}(\partial \Omega \times(0, T))$ and $g_{2} \in L^{2}(\partial \Omega \times(0, T))$ such that

$$
\left(\frac{1}{2} I+K^{\prime}\right) g_{1}=f_{1} \quad \text { and } \quad\left(\frac{1}{2} I+K^{\prime}\right) g_{2}=f_{2}
$$

Therefore, $g:=g_{1}+g_{2} \in{ }_{0} H_{\text {par }}^{1}(\partial \Omega \times(0, T))$ with the property that $\left(\frac{1}{2} I+K^{\prime}\right) g=f$, as desired.

Second, our goal is to prove that $f \in{ }_{0} H_{\text {par }}^{1}(\partial \Omega \times(0, T))$ with $\left(\frac{1}{2} I+K^{\prime}\right) f=0$ implies $f=0$. Using again (6.2.33), $f$ can be witten as $f=f_{1}+f_{2}$ with $f_{1} \in$ ${ }_{0} \dot{H}_{\text {par }}^{1}(\partial \Omega \times(0, T))$ and $f_{2} \in L^{2}(\partial \Omega \times(0, T))$. This and the assumption $\left(\frac{1}{2} I+K^{\prime}\right) f=0$ implies that

$$
\left(\frac{1}{2} I+K^{\prime}\right) f_{1}=-\left(\frac{1}{2} I+K^{\prime}\right) f_{2}
$$

Applying (a slighty modified version of) Corollary 5 form [AlMü] to the complex interpolation scale

$$
\begin{cases}{ }_{0} \dot{H}_{p a r}^{p}(\partial \Omega \times(0, T)), & \text { for } \frac{n+1}{n+2}<p \leq 1 \\ L^{p}(\partial \Omega \times(0, T)), & \text { for } 1<p<\infty\end{cases}
$$

yields that the action of $\left(\frac{1}{2} I+K^{\prime}\right)^{-1}$ is compatible, i.e. (6.2.34) futher gives

$$
f_{1}=-f_{2}
$$

Consequently, $f=0$. This completes the proof of the lemma. 
Lemma 6.2.2. Consider a bounded Lipschitz domain $\Omega$ in $\mathbb{R}^{n}, 0<T<\infty$. Then the operators $\pm \frac{1}{2} I+K$ are isomorphisms on ${ }_{0} H_{\text {par }}^{1,1}(\partial \Omega \times(0, T))$.

Proof. Recall from (6.0.7) that

$$
\pm \frac{1}{2} I+K=S\left( \pm \frac{1}{2} I+K^{\prime}\right) S^{-1}
$$

According to Theorem 5.25 of [Bro2] the operators

$$
S:{ }_{0} \dot{H}_{\text {par }}^{1}(\partial \Omega \times(0, T)) \stackrel{\sim}{\longrightarrow}{ }_{0} H_{\text {par }}^{1,1}(\partial \Omega \times(0, T))
$$

and

$$
\pm \frac{1}{2} I+K^{\prime}:{ }_{0} \dot{H}_{\text {par }}^{1}(\partial \Omega \times(0, T)) \stackrel{\sim}{\longrightarrow}{ }_{0} \dot{H}_{\text {par }}^{1}(\partial \Omega \times(0, T))
$$

are isomorphisms. Consequently, (6.2.36) - (6.2.38) further imply that $\pm \frac{1}{2} I+K$ are invertible on ${ }_{0} H_{\text {par }}^{1,1}(\partial \Omega \times(0, T))$.

Going further, we establish the desired properties of the operators $\pm \frac{1}{2} I+K$ on parabolic Sobolev spaces.

Proposition 6.2.3. Let $\Omega$ be a bounded Lipschitz domain in $\mathbb{R}^{n}, 0<T<\infty$. Then there exists an $\varepsilon=\varepsilon(\partial \Omega)>0$ such that

$$
\pm \frac{1}{2} I+K:{ }_{0} L_{1, p a r}^{p}(\partial \Omega \times(0, T)) \stackrel{\sim}{\longrightarrow}{ }_{0} L_{1, p a r}^{p}(\partial \Omega \times(0, T))
$$

isomorphically, for $1<p<2+\varepsilon$.

Proof. Recall form Lemma 3.2.6 that for a bounded Lipschitz domain $\Omega \subset \mathbb{R}^{n}$, $0<T<\infty$ and $1<p<n+1$,

$$
{ }_{0} L_{1, p a r}^{p}(\partial \Omega \times(0, T)) \equiv \mathcal{L}_{1}^{p}(\partial \Omega \times(0, T)) .
$$


On account of Theorem 5.25 in [Bro2], given a bounded Lipschitz domain $\Omega$ in $\mathbb{R}^{n}$ and $0<T<\infty$, there exists an $\varepsilon=\varepsilon(\partial \Omega)>0$ such that the operators

$$
\begin{array}{r} 
\pm \frac{1}{2} I+K^{\prime}: L^{p}(\partial \Omega \times(0, T)) \stackrel{\sim}{\longrightarrow} L^{p}(\partial \Omega \times(0, T)), \\
S: L^{p}(\partial \Omega \times(0, T)) \stackrel{\sim}{\longrightarrow} \mathcal{L}_{1}^{p}(\partial \Omega \times(0, T))
\end{array}
$$

are invertible for $1<p<2+\varepsilon$. We also recall from (6.0.7) that $\pm \frac{1}{2} I+K=$ $S\left( \pm \frac{1}{2} I+K^{\prime}\right) S^{-1}$. With this in hand, (6.2.41) and (6.2.40) imply that given a bounded Lipschitz domain $\Omega$ in $\mathbb{R}^{n}$ and $0<T<\infty$, there exists an $\varepsilon=\varepsilon(\partial \Omega)>0$ such that

$$
\pm \frac{1}{2} I+K:{ }_{0} L_{1, p a r}^{p}(\partial \Omega \times(0, T)) \stackrel{\sim}{\longrightarrow}{ }_{0} L_{1, p a r}^{p}(\partial \Omega \times(0, T))
$$

isomorphically, for $1<p<2+\varepsilon$. This finishes the proof of Proposition 6.2.3.

The isomorphic property of the operators $\pm \frac{1}{2} I+K$ on $L^{p}$-spaces with built-in initial conditions is formulated in the following proposition.

Proposition 6.2.4. Let $\Omega$ be a bounded Lipschitz domain in $\mathbb{R}^{n}, 0<T<\infty$. Then there exists an $\varepsilon=\varepsilon(\partial \Omega)>0$ such that

$$
\pm \frac{1}{2} I+K:{ }_{0} L^{p}(\partial \Omega \times(0, T)) \stackrel{\sim}{\longrightarrow}{ }_{0} L^{p}(\partial \Omega \times(0, T))
$$

isomorphically, for $2-\varepsilon<p<\infty$.

Proof. The claim that we first make is that

$$
{ }_{0} L^{p}(\partial \Omega \times(0, T)) \equiv L^{p}(\partial \Omega \times(0, T)) .
$$

In order to see the right-to-left inclusion in (6.2.43), let $f \in L^{p}(\partial \Omega \times(0, T))$ and consider $\tilde{f}$ to be the extension (to $\partial \Omega \times \mathbb{R}$ ) by 0 of $f$, i.e. $\tilde{f}=f$ on $\partial \Omega \times(0, T)$ and 
$\tilde{f}=0$ on $\partial \Omega \times(-\infty, 0] \cup[T, \infty)$. Then by Definition 3.2.2, $f \in{ }_{0} L^{p}(\partial \Omega \times(0, T))$. The left-to-right inclusion in (3.4.72) is trivial.

We next invoke a part of Corollary 5.26 of [Bro2] to the effect that the operators $\pm \frac{1}{2} I+K$ are invertible on $L^{p}(\partial \Omega \times(0, T))$ for $2-\varepsilon<p<\infty$. With (6.2.43) in hand, this readily yields the desired conclusion.

Prior to another result that involves the operators $\pm \frac{1}{2} I+K$, we identify the dual spaces of $H_{p a r}^{p}(\partial \Omega \times I)$ and ${ }_{0} H_{p a r}^{p}(\partial \Omega \times(0, T))$ for a bounded Lipschitz domain $\Omega$.

Theorem 6.2.5. Consider a bounded Lipschitz domain $\Omega \subset \mathbb{R}^{n}$, a bounded interval $I \subset \mathbb{R}$, and indices $p$ and $\alpha$ such that $\frac{n+1}{n+2}<p<1$ and $\alpha=(n+1)\left(\frac{1}{p}-1\right)$. Then

$$
\left(H_{p a r}^{p}(\partial \Omega \times I)\right)^{*}=C_{p a r}^{\alpha}(\partial \Omega \times I)
$$

Proof. Let $g \in C_{p a r}^{\alpha}(\partial \Omega \times I)$ and $f \in H_{p a r}^{p}(\partial \Omega \times I)$. We define the functional

$$
L f:=\int_{\partial \Omega \times I} f(x, t) g(x, t) d \sigma_{x} d t .
$$

According to Definition 2.3.35, $f$ admits an atomic decomposition, i.e. $f=\sum_{j=0}^{\infty} \lambda_{j} a_{j}$ with $\operatorname{supp} a_{j} \subset \mathrm{Q}_{j},\left\|a_{j}\right\|_{L^{\infty}(\partial \Omega \times I)} \leq\left|\mathrm{Q}_{j}\right|^{-1 / p}=r_{j}^{-(n+1) / p}$ and vanishing moment condition if $\left|Q_{j}\right|<\eta$, where $Q_{j}$ are parabolic surface cubes on $\partial \Omega \times I$ with sidelength $r_{j}$. Then one can easily see that 


$$
\begin{aligned}
|L f|=\left|\int_{\partial \Omega \times I} \sum_{j=0}^{\infty} \lambda_{i} a_{j}(x, t) g(x, t) d \sigma_{x} d t\right|=\left|\sum_{j=0}^{\infty} \lambda_{j} \int_{\mathbf{Q}_{j}} a_{j}(x, t) g(x, t) d \sigma_{x} d t\right| \\
=\left|\sum_{\left|Q_{j}\right|<\eta} \lambda_{j} \int_{\mathbf{Q}_{j}} a_{j}(x, t)\left[g(x, t)-g\left(x_{0}, t_{0}\right)\right] d \sigma_{x} d t\right| \\
\\
+\left|\sum_{\left|Q_{j}\right| \geq \eta} \lambda_{j} \int_{\mathbf{Q}_{j}} a_{j}(x, t) g(x, t) d \sigma_{x} d t\right|,
\end{aligned}
$$

where $\left(x_{0}, t_{0}\right)$ is the center of the cube $\mathrm{Q}_{j}$. Using the assumptions on the atoms $a_{j}$, as well as the fact that $g \in C_{p a r}^{\alpha}(\partial \Omega \times I)$, (6.1.12) further implies that

$$
\begin{aligned}
|L f| \leq & \sum_{\left|Q_{j}\right|<\eta}\left|\lambda_{j}\right| \int_{Q_{j}}\left|a_{j}(x, t)\right|\|g\|_{\dot{C}_{p a r}^{\alpha}(\partial \Omega \times I)}\left(\left|x-x_{0}\right|+\left|t-t_{0}\right|^{1 / 2}\right)^{\alpha} d \sigma_{x} d t \\
& +\sum_{\left|Q_{j}\right| \geq \eta}\left|\lambda_{j}\right|\|g\|_{L^{\infty}(\partial \Omega \times I)}\left\|a_{j}\right\|_{L^{\infty}(\partial \Omega \times I)}\left|Q_{j}\right| \\
\leq & c \sum_{\left|Q_{j}\right|<\eta}\left|\lambda_{j}\right|\left|Q_{j}\right|^{-1 / p} r_{j}^{\alpha} \int_{Q_{j}} d \sigma_{x} d t+c \sum_{\left|Q_{j}\right| \geq \eta}\left|\lambda_{j}\right| \frac{1}{\eta^{\alpha /(n+1)}} \\
\leq & c\left(\sum_{j=0}^{\infty}\left|\lambda_{j}\right|^{p}\right)^{1 / p} .
\end{aligned}
$$

Taking the infimum over all representations of $f$ in (6.1.13), we obtain that

$$
|L f| \leq c \inf \left(\sum_{j=0}^{\infty}\left|\lambda_{j}\right|^{p}\right)^{1 / p}=c\|f\|_{H_{p a r}^{p}(\partial \Omega \times I)},
$$

hence $L$ is bounded, linear and continuous on $H_{p a r}^{p}(\partial \Omega \times I)$. This finishes the proof of the right-to-left inclusion in (6.2.44).

Conversely, suppose $L$ is a bounded linear functional on $H_{p a r}^{p}(\partial \Omega \times I)$.

Step 1. First we will show the existence of a function $G$, bounded on each sphere $S$ on $\partial \Omega \times I$, such that $L a_{j}=\int_{\partial \Omega \times I} a_{j}(x, t) G(x, t) d \sigma_{x} d t$ for each $a_{j}$, local $(p, 1)$-atom 
for $H_{p a r}^{p}(\partial \Omega \times I)$. By a sphere centered at $(x, t)$ and of radius $r>0$ we mean

$$
S_{r}(x, t):=\left\{(y, s) \in \partial \Omega \times I:|x-y|+|t-s|^{1 / 2}<r\right\} .
$$

For a sphere $S$ on $\partial \Omega \times I$ we let

$$
L_{0}^{1}(S):=\left\{f \in L^{1}(S): \text { either }|S|<\eta \text { and } \int_{S} f(x, t) d \sigma_{x} d t=0, \text { or }|S| \geq \eta\right\}
$$

where $\eta=\eta(\partial \Omega, I)$ is a fixed, sufficiently small constant. Then, for $f \in L_{0}^{1}(S)$, we easily verify that

$$
a(x, t):=f(x, t)\|f\|_{L^{1}(S)}^{-1}|S|^{1-\frac{1}{p}}
$$

is a local $(p, 1)$-atom for $H_{p a r}^{p}(\partial \Omega \times I)$ supported in $S$. Also, $\|f\|_{H_{p a r}^{p}(\partial \Omega \times I)}<\infty$, hence $L f$ is well-defined and

$$
|L f| \leq\|L\||S|^{\frac{1}{p}-1}\|f\|_{L^{1}(S)}
$$

Therefore $L$ is a bounded linear functional on $L_{0}^{1}(S)$. Since $L_{0}^{1}(S)$ is a closed subspace of $L^{1}(S)$, by the Hahn-Banach theorem, there exists an extension of $L$, which we denote also by $L$, a bounded linear functional on $L^{1}(S)$. According to the Riesz representation theorem, there exists $g \in L^{\infty}(S)$ such that

$$
L f=\int_{S} f(x, t) g(x, t) d \sigma_{x} d t \quad \text { for all } f \in L_{0}^{1}(S)
$$

Next we show that $g$ is uniquely determined up to a constant. For this it is enough to show that

$$
\int_{S} f(x, t) g(x, t) d \sigma_{x} d t=0 \quad \forall f \in L_{0}^{1}(S) \text { implies } g \text { is a constant. }
$$


To see this we choose $h \in L^{1}(S)$. Then $h-\frac{1}{|S|} \int_{S} h(y, s) d \sigma_{y} d s \in L_{0}^{1}(S)$ and

$$
\begin{aligned}
0 & =\int_{S}\left(h(x, t)-\frac{1}{|S|} \int_{S} h(y, s) d \sigma_{y} d s\right) g(x, t) d \sigma_{x} d t \\
& =\int_{S} h(x, t)\left(g(x, t)-\frac{1}{|S|} \int_{S} g(y, s) d \sigma_{y} d s\right) d \sigma_{x} d t
\end{aligned}
$$

which implies that $g(x, t)-\frac{1}{|S|} \int_{S} g(y, s) d \sigma_{y} d s=0$ for almost every $(x, t) \in S$, i.e. $g(x, t)$ is constant for almost every $(x, t) \in S$.

Considering (6.1.14) and the uniqueness of such $g$, as well as the fact that if $a$ is a local $(p, 1)$-atom for $H_{p a r}^{p}(\partial \Omega \times I)$ supported in a sphere $S$, then $a \in L_{0}^{1}(S)$, we can conclude that there exists a unique $G \in L^{\infty}(S)$ such that

$$
L a=\int_{S} a(x, t) G(x, t) d \sigma_{x} d t \text { for all }(p, 1) \text {-atoms } a
$$

This conludes the proof of Step 1.

Step 2. Our next goal is to show that for $G$ as above, $G \in C_{p a r}^{\alpha}(\partial \Omega \times I)$. To do so, let us consider $f \in L^{1}(S)$ with $\|f\|_{L^{1}(S)}=1$, and set

$$
b(x, t):=|S|^{1-\frac{1}{p}}\left(f(x, t)-\frac{1}{|S|} \int_{S} f(y, s) d \sigma_{y} d s\right)
$$

One can easily verify that $b$ is a local $(p, 1)$-atom for $H_{p a r}^{p}(\partial \Omega \times I)$, supported in $S$, therefore, by (6.2.50), there exists a unique $G \in L^{\infty}(S)$ so that

$$
L b=\int_{S} b(x, t) G(x, t) d \sigma_{x} d t
$$

Moreover, for $b$ as in (6.2.51) and $G \in L^{\infty}(S)$ as above,

$$
\begin{aligned}
\|L\| & \geq|L b|=\left|\int_{S} b(x, t)\left(G(x, t)-\frac{1}{|S|} \int_{S} G(y, s) d \sigma_{y} d s\right) d \sigma_{x} d t\right| \\
& =|S|^{1-\frac{1}{p}}\left|\int_{S} f(x, t)\left(G(x, t)-\frac{1}{|S|} \int_{S} G(y, s) d \sigma_{y} d s\right) d \sigma_{x} d t\right| .
\end{aligned}
$$


In other words, since $\alpha=(n+1)\left(\frac{1}{p}-1\right)$,

$$
\left|\int_{S} f(x, t)\left(G(x, t)-\frac{1}{|S|} \int_{S} G(y, s) d \sigma_{y} d s\right) d \sigma_{x} d t\right| \leq\|L\||S|^{\frac{\alpha}{n+1}}
$$

The inequality (6.2.53) furher implies that, for $G \in L^{\infty}(S)$ satisfying (6.2.52),

$$
\begin{aligned}
\| G- & \frac{1}{|S|} \int_{S} G(y, s) d \sigma_{y} d s \|_{L^{\infty}(S)} \\
& =\sup _{\substack{f \in L^{1}(S) \\
\|f\|_{L^{1}(S)}=1}}\left|\int_{S} f(x, t)\left(G(x, t)-\frac{1}{|S|} \int_{S} G(y, s) d \sigma_{y} d s\right) d \sigma_{x} d t\right| \\
& \leq\|L\||S|^{\frac{\alpha}{n+1}}
\end{aligned}
$$

Finally, let us consider $(x, t),(y, s) \in \partial \Omega \times I$. If $\|(x, t)-(y, s)\|_{p a r} \leq c$ for some constant c, then we choose the smallest parabolic surface ball $S, S \subset \partial \Omega \times I$, with radius $r$, such that $(x, t),(y, s) \in S$. Then $\|(x, t)-(y, s)\|_{p a r} \approx r \approx|S|^{\frac{1}{n+1}}$. Using the triangle inequality in the numerator of the left-hand side in (6.2.55), then (6.2.54), we obtain that, for $G \in L^{\infty}(S)$ which fulfills condition (6.2.52),

$$
\frac{|G(x, t)-G(y, s)|}{\|(x, t)-(y, s)\|_{\text {par }}^{\alpha}}<\infty .
$$

If $\|(x, t)-(y, s)\|_{p a r} \geq c$ for some constant $c$, then for the same $G$ as above,

$$
\frac{|G(x, t)-G(y, s)|}{\|(x, t)-(y, s)\|_{p a r}^{\alpha}} \leq c\|G\|_{L^{\infty}(S)}<\infty
$$

where $S$ is a parabolic surface ball on $\partial \Omega \times I$ so that $(x, t),(y, s) \in S$.

Based on (6.2.55) and (6.2.56) we can conclude that there exists a unique (up to a constant) $G \in \dot{C}_{p a r}^{\alpha}(\partial \Omega \times I)$ such that $L a=\int_{\partial \Omega \times I} a(x, t) G(x, t) d \sigma_{x} d t$ for all $a$ local $(p, 1)$-atoms for $H_{p a r}^{p}(\partial \Omega \times I)$. Since $G \in L^{\infty}\left(S_{j}\right)$ for every parabolic surface ball $S_{j}$ on $\partial \Omega \times I$, and $\partial \Omega \times I$ is bounded, we have $G \in L^{\infty}(\partial \Omega \times I)$. Consequently, 
$G \in C_{p a r}^{\alpha}(\partial \Omega \times I)$, and given the atomic decomposition of the space $H_{p a r}^{p}(\partial \Omega \times I)$, the proof of Theorem 6.2.5 is complete.

Inspired by a proof on pp. $11-12$ in [Bro2], we next determine the dual of ${ }_{0} H_{p a r}^{p}(\partial \Omega \times(0, T))$ for a bounded Lipschitz domain $\Omega$.

Theorem 6.2.6. Consider a bounded Lipschitz domain $\Omega \subset \mathbb{R}^{n}, 0<T<\infty$, and let $\frac{n+1}{n+2}<p<1, \alpha=(n+1)\left(\frac{1}{p}-1\right)$. Then one has

$$
\left({ }_{0} H_{p a r}^{p}(\partial \Omega \times(0, T))\right)^{*}={ }^{0} C_{p a r}^{\alpha}(\partial \Omega \times(0, T)) .
$$

Proof. First, let $g \in{ }^{0} C_{p a r}^{\alpha}(\partial \Omega \times(0, T)), f \in{ }_{0} H_{p a r}^{p}(\partial \Omega \times(0, T))$ and define

$$
L f:=\int_{\partial \Omega \times(0, T)} f(x, t) g(x, t) d \sigma_{x} d t .
$$

By the definitions of the spaces involved, there exist

$$
\tilde{g} \in C_{p a r}^{\alpha}(\partial \Omega \times \mathbb{R}) \text { such that }\left.\tilde{g}\right|_{\partial \Omega \times(0, T)}=g, \operatorname{supp} \tilde{g} \subseteq \partial \Omega \times(-\infty, T],
$$

and

$$
\tilde{f} \in H_{p a r}^{p}(\partial \Omega \times \mathbb{R}) \text { so that }\left.\tilde{f}\right|_{\partial \Omega \times(0, T)}=f, \operatorname{supp} \tilde{f} \subseteq \partial \Omega \times[0, \infty) \text {. }
$$

Since $g \in C_{p a r}^{\alpha}(\partial \Omega \times(0, T))$ and $f \in H_{p a r}^{p}(\partial \Omega \times(0, T))$, on account of Theorem 6.2.5 with $I=(0, T)$ and $(3.2 .30)$, we obtain that

$$
|L f| \leq c\|f\|_{H_{p a r}^{p}(\partial \Omega \times \mathbb{R})} \leq c\|f\|_{0 H_{p a r}^{p}(\partial \Omega \times(0, T))},
$$

hence $L$ is bounded and continuous on ${ }_{0} H_{p a r}^{p}(\partial \Omega \times(0, T))$. This proves the right-toleft inclusion in (6.2.57). 
Conversely, let $\Lambda$ be a linear functional on ${ }_{0} H_{p a r}^{p}(\partial \Omega \times(0, T))$. Since the space $H_{p a r}^{p}(\partial \Omega \times(0, T))$ is continuously embedded in ${ }_{0} H_{p a r}^{p}(\partial \Omega \times(0, T))$, we also have

$$
\Lambda \in\left(H_{p a r}^{p}(\partial \Omega \times(0, T))\right)^{*} .
$$

According to Theorem 6.2.5, if we consider a bounded Lipschitz domain $\Omega$ in $\mathbb{R}^{n}$ and $0<T<\infty$, then

$$
\left(H_{p a r}^{p}(\partial \Omega \times(0, T))\right)^{*}=C_{p a r}^{\alpha}(\partial \Omega \times(0, T)),
$$

where $\frac{n+1}{n+2}<p<1$ and $\alpha=(n+1)\left(\frac{1}{p}-1\right)$. Now (6.2.58) and (6.2.59) further imply the existence of $h \in C_{p a r}^{\alpha}(\partial \Omega \times(0, T))$ such that

$$
\Lambda(f)=\int_{\partial \Omega \times(0, T)} h(x, t) f(x, t) d \sigma_{x} d t \quad \text { for any } f \in H_{p a r}^{p}(\partial \Omega \times(0, T)) .
$$

In order to prove the left-to-right inclusion in (6.2.57), we need to show that

A. There exists a function $\tilde{g} \in C_{p a r}^{\alpha}(\partial \Omega \times \mathbb{R})$ with the properties $\left.\tilde{g}\right|_{\partial \Omega \times(0, T)}=g$ and $\operatorname{supp} \tilde{g} \subseteq \partial \Omega \times(-\infty, T]$ such that

$$
\Lambda(\tilde{f})=\int_{\partial \Omega \times \mathbb{R}} \tilde{g}(x, t) \tilde{f}(x, t) d \sigma_{x} d t
$$

for any $\tilde{f} \in H_{p a r}^{p}(\partial \Omega \times \mathbb{R})$ with $\operatorname{supp} \tilde{f} \subseteq \partial \Omega \times[0, \infty)$;

B. The map $\left({ }_{0} H_{p a r}^{p}(\partial \Omega \times(0, T))\right)^{*} \ni \Lambda \mapsto g \in{ }^{0} C_{p a r}^{\alpha}(\partial \Omega \times(0, T))$ is well-defined, linear and one-to-one.

Step 1. For a parabolic surface cube $Q \subseteq \partial \Omega \times(0, T)$ of sidelength $r$ and such that $\operatorname{dist}(\mathrm{Q}, \partial \Omega \times\{T\}) \leq \kappa r$ for some constant $\kappa>0$, we will show that $\Lambda\left(\chi_{\mathrm{Q}}\right)$ is meaningful and that

$$
\left.\mid \Lambda\left(\chi_{\mathrm{Q}}\right)\right)\left|\leq\|\Lambda\| c(\kappa) r^{(n+1)\left(\frac{1}{p}-1\right)}\right| \mathrm{Q} \mid
$$


Proof of Step 1. Consider $\tilde{\mathbf{Q}} \subseteq \partial \Omega \times(T, \infty)$ the reflection of $\mathbf{Q}$ across $\partial \Omega \times\{T\}$, and define

$$
\mathbb{A}_{Q}:=r^{(n+1)\left(1-\frac{1}{p}\right)}\left[\frac{1}{|\mathbf{Q}|} \chi_{Q}-\frac{1}{|\tilde{Q}|} \chi_{\tilde{Q}}\right] .
$$

Then the size of the support of $\mathbb{A}_{Q}$ is comparable to the size of $Q$,

$$
\left\|\mathbb{A}_{\mathbf{Q}}\right\|_{L^{\infty}(\partial \Omega \times \mathbb{R})} \leq \frac{r^{(n+1)\left(1-\frac{1}{p}\right)}}{|\mathrm{Q}|}=r^{-\frac{n+1}{p}}
$$

and

$$
\int_{\partial \Omega \times \mathbb{R}} \mathbb{A}_{Q}=0
$$

Hence, by Definition 2.3.35, $\mathbb{A}_{\mathbf{Q}}$ is a fixed constant multiple of a $(p, \infty)$-atom for $H_{p a r}^{p}(\partial \Omega \times \mathbb{R})$. Also $\mathbb{A}_{\mathbf{Q}}$ vanishes for $t<0$, therefore $\left.\mathbb{A}_{\mathbf{Q}}\right|_{\partial \Omega \times(0, T)} \in{ }_{0} H_{p a r}^{p}(\partial \Omega \times(0, T))$, which amounts to

$$
r^{(n+1)\left(1-\frac{1}{p}\right)} \frac{1}{|\mathbf{Q}|} \chi_{\mathbf{Q}} \in{ }_{0} H_{p a r}^{p}(\partial \Omega \times(0, T)) .
$$

This further implies that $\Lambda\left(\chi_{\mathrm{Q}}\right)$ is meaningful and

$$
\left\|r^{(n+1)\left(1-\frac{1}{p}\right)} \frac{1}{|\mathrm{Q}|} \chi \mathrm{Q}\right\|_{0 H_{p a r}^{p}(\partial \Omega \times(0, T))} \leq c(\kappa) .
$$

Therefore,

$$
\left|\Lambda\left(\chi_{\mathrm{Q}}\right)\right| \leq\|\Lambda\| c(\kappa) r^{(n+1)\left(\frac{1}{p}-1\right)}|\mathrm{Q}|
$$

which finishes the proof of Step 1.

Step 2. Suppose there is a cube $\mathrm{Q}_{*} \subseteq \partial \Omega \times(0, T)$ such that $\Lambda\left(\chi_{\mathbf{Q}_{*}}\right)=\int_{\mathbf{Q}_{*}} g$ for some $g \in C_{p a r}^{\alpha}(\partial \Omega \times(0, T))$. Then

$$
\Lambda\left(\chi_{\mathbf{Q}}\right)=\int_{\mathbf{Q}} g \text { for any } \mathrm{Q} \subseteq \partial \Omega \times(0, T) .
$$


Proof of Step 2. First, let $\mathrm{Q} \subseteq \partial \Omega \times(0, T)$ an adjacent cube to $\mathrm{Q}_{*}$ with $|\mathrm{Q}|=\left|\mathrm{Q}_{*}\right|$.

Then $\left|\mathrm{Q} \cup \mathrm{Q}_{*}\right| \approx\left|\mathrm{Q}_{*}\right|=r^{n+1}$ and consider

$$
a:=r^{(n+1)\left(1-\frac{1}{p}\right)}\left[\frac{1}{|\mathrm{Q}|} \chi_{\mathrm{Q}}-\frac{1}{\left|\mathrm{Q}_{*}\right|} \chi_{\mathrm{Q}_{*}}\right]
$$

Based on Definition 2.3.35, $a$ is a fixed constant multiple of a $(p, \infty)$-atom for the space $H_{p a r}^{p}(\partial \Omega \times(0, T))$, since

- $\operatorname{supp} a \subset \mathrm{Q} \cup \mathrm{Q}_{*}$,

- $\|a\|_{L^{\infty}(\partial \Omega \times(0, T))} \leq c r^{-\frac{n+1}{p}}$

- $\int_{\partial \Omega \times(0, T)} a=0$.

Then, in view of the discussion prior to Step 1 (more specifically, (6.2.60)),

$$
\begin{aligned}
\Lambda\left(r^{(n+1)\left(1-\frac{1}{p}\right)}\left[\frac{1}{|\mathrm{Q}|} \chi_{\mathrm{Q}}-\frac{1}{\left|\mathrm{Q}_{*}\right|} \chi_{\mathrm{Q}_{*}}\right]\right) \\
=r^{(n+1)\left(1-\frac{1}{p}\right)} \frac{1}{|\mathrm{Q}|} \int_{\mathrm{Q}} g-r^{(n+1)\left(1-\frac{1}{p}\right)} \frac{1}{\left|\mathrm{Q}_{*}\right|} \int_{\mathrm{Q}_{*}} g
\end{aligned}
$$

for some $g \in C_{\text {par }}^{\alpha}(\partial \Omega \times(0, T))$. We also note that $(6.2 .61)$ is equivalent with

$$
r^{(n+1)\left(1-\frac{1}{p}\right)} \frac{1}{|\mathrm{Q}|} \Lambda\left(\chi_{\mathrm{Q}}\right)-r^{(n+1)\left(1-\frac{1}{p}\right)} \frac{1}{\left|\mathrm{Q}_{*}\right|} \Lambda\left(\chi_{\mathrm{Q}_{*}}\right)
$$

Therefore, if we compare (6.2.63) with (6.2.62), and use the hypothesis to the effect that $\Lambda\left(\chi_{\mathrm{Q}_{*}}\right)=\int_{\mathrm{Q}_{*}} g$ for some $g \in C_{\text {par }}^{\alpha}(\partial \Omega \times(0, T))$, we arrive at

$$
\Lambda\left(\chi_{\mathrm{Q}}\right)=\int_{\mathrm{Q}} g \text { for } \mathrm{Q} \subseteq \partial \Omega \times(0, T) \text { adjacent to } \mathrm{Q}_{*} .
$$

One can also choose

$$
\mathrm{Q}=\frac{1}{2} \mathrm{Q}_{*}=\left\{(y, s) \in \partial \Omega \times(0, T):|x-y| \leq \frac{r}{2},|t-s| \leq\left(\frac{r}{2}\right)^{2}\right\},
$$


where $(x, t)$ is the center of $\mathbf{Q}_{*}$, and obtain the same result as above.

We next observe that for any given cube $Q \subseteq \partial \Omega \times(0, T)$ there is a sequence of cubes $\left\{Q_{j}\right\}_{j \in \mathbb{N}}$ such that $\lim _{j \rightarrow \infty} Q_{j}=Q$ and

$$
\Lambda\left(\chi_{\mathrm{Q}_{j}}\right)=\int_{\mathrm{Q}_{j}} g \quad \text { for some } g \in C_{p a r}^{\alpha}(\partial \Omega \times(0, T)) \text {. }
$$

Therefore, as $j \rightarrow \infty$,

$$
\Lambda\left(\chi_{\mathrm{Q}}\right)=\int_{\mathrm{Q}} g \text { for any } \mathrm{Q} \subseteq \partial \Omega \times(0, T) \text {, where } g \in C_{\text {par }}^{\alpha}(\partial \Omega \times(0, T))
$$

Step 3. Consider $\Lambda$ with the same significance as before. Let $g \in C_{p a r}^{\alpha}(\partial \Omega \times(0, T))$ such that

$$
\Lambda\left(\chi_{\mathrm{Q}}\right)=\int_{\mathrm{Q}} g \text { for any cube } \mathrm{Q} \subseteq \partial \Omega \times(0, T) .
$$

Then $\left.g\right|_{\partial \Omega \times\{T\}}=0$.

Proof of Step 3. We choose $Q$ to be the cube in Step $1,|Q|=r^{n+1}$. In addition to that, without loss of generality we may assume $0<r \leq 1$, since we want to send $r \rightarrow 0$ later. Then $\kappa=1$ in Step 1 , and

$$
\left|\Lambda\left(\chi_{\mathrm{Q}}\right)\right| \leq c\|\Lambda\| r^{(n+1)\left(\frac{1}{p}-1\right)}|\mathrm{Q}|
$$

Therefore, $\left|g\left(x_{r}, t_{r}\right)\right| \leq c\|\Lambda\| r^{(n+1)\left(\frac{1}{p}-1\right)}$ for $\left(x_{r}, t_{r}\right) \in \mathrm{Q}$. Consequently, as $r \rightarrow 0$, we ultimately obtain that $\left|g\left(x_{0}, T\right)\right|=0$ for $x_{0} \in \partial \Omega$, which suits our goal.

Step 4. If $g \in C_{\text {par }}^{\alpha}(\partial \Omega \times(0, T))$ with $\Lambda\left(\chi_{\mathrm{Q}}\right)=\int_{\mathrm{Q}} g$ for any $\mathrm{Q} \subset \partial \Omega \times(0, T)$, then there exists a function $\tilde{g} \in C_{\text {par }}^{\alpha}(\partial \Omega \times \mathbb{R})$ such that $\left.\tilde{g}\right|_{\partial \Omega \times(0, T)}=g$ and $\operatorname{supp} \tilde{g} \subseteq$ $\partial \Omega \times(-\infty, T]$. 
Proof of Step 4. For $(x, t) \in \partial \Omega \times \mathbb{R}$ define

$$
\tilde{g}(x, t):= \begin{cases}g(x, 0) & \text { if } t \leq 0 \\ g(x, t) & \text { if } 0<t<T, \\ 0 & \text { if } t \geq T\end{cases}
$$

On account of Step 3, $\tilde{g}$ is continuous on $\partial \Omega \times \mathbb{R}$, and, in order to prove Step 4, all we have to show is that $\tilde{g} \in C_{p a r}^{\alpha}(\partial \Omega \times \mathbb{R})$. Using the definition of this space and the hypotheses that $g \in C_{p a r}^{\alpha}(\partial \Omega \times(0, T))$, it suffices to show that for any $(x, t),(y, s) \in \partial \Omega \times \mathbb{R}$,

$$
|\tilde{g}(x, t)-\tilde{g}(y, s)| \leq c\left(|x-y|+|t-s|^{1 / 2}\right)^{\alpha} .
$$

We have several cases to analyze. In each of these cases we take $x, y \in \partial \Omega$, and we use the hypothesis that $g \in C_{p a r}^{\alpha}(\partial \Omega \times(0, T))$.

- When $t, s \leq 0$, we have

$$
\begin{aligned}
|\tilde{g}(x, t)-\tilde{g}(y, s)| & =|g(x, 0)-g(y, 0)| \\
& \leq c|x-y|^{\alpha} \\
& \leq c\left(|x-y|+|t-s|^{1 / 2}\right)^{\alpha} .
\end{aligned}
$$

- If $t, s \in(0, T)$, then

$$
\begin{aligned}
|\tilde{g}(x, t)-\tilde{g}(y, s)| & =|g(x, t)-g(y, s)| \\
& \leq c\left(|x-y|+|t-s|^{1 / 2}\right)^{\alpha} .
\end{aligned}
$$

- When $t, s \geq T$, there is nothing to prove. 
- For $t \leq 0$ and $s \in(0, T)$ we obtain that

$$
\begin{aligned}
|\tilde{g}(x, t)-\tilde{g}(y, s)| & =|g(x, 0)-g(y, s)| \\
& \leq c\left(|x-y|+|s|^{1 / 2}\right)^{\alpha} \\
& \leq c\left(|x-y|+|t-s|^{1 / 2}\right)^{\alpha} .
\end{aligned}
$$

- When $t \leq 0$ and $s \geq T$, we arrive at

$$
\begin{aligned}
|\tilde{g}(x, t)-\tilde{g}(y, s)| & =|g(x, 0)-g(y, T)| \\
& \leq c\left(|x-y|+|T|^{1 / 2}\right)^{\alpha} \\
& \leq c\left(|x-y|+|t-s|^{1 / 2}\right)^{\alpha} .
\end{aligned}
$$

- Finally, if $t \in(0, T)$ and $s \geq T$, then

$$
\begin{aligned}
|\tilde{g}(x, t)-\tilde{g}(y, s)| & =|g(x, t)-g(y, T)| \\
& \leq c\left(|x-y|+|t-T|^{1 / 2}\right)^{\alpha} \\
& \leq c\left(|x-y|+|t-s|^{1 / 2}\right)^{\alpha} .
\end{aligned}
$$

This shows (6.2.64), hence the proof of Step 4 is finished.

Step 5. Let $a$ be a $(p, \infty)$-atom for $H_{p a r}^{p}(\partial \Omega \times \mathbb{R})$, which vanishes for $t<0$. Then there exists $g \in C_{p a r}^{\alpha}(\partial \Omega \times(0, T))$ such that

$$
\Lambda\left(\left.a\right|_{\partial \Omega \times(0, T)}\right)=\int_{\partial \Omega \times(0, T)} a g .
$$


Proof of Step 5. Let $\mathrm{Q}=S \times I$ a cube in $\partial \Omega \times \mathbb{R}$ such that $\operatorname{supp} a \subset$ Q. If $I \cap(0, T))=\emptyset$, then the claim in Step 5 is obviously true. If $I \cap(0, T) \neq \emptyset$ and $I \cap(0, T)^{c} \neq \emptyset$, then consider $J \subseteq(0, T)$ an interval of lenght $r^{2}$ such that $I \cap(0, T) \subseteq J$. Also, let $\hat{\mathbf{Q}}:=S \times J$. Then the size of $J$ and $I$ coincide (i.e. $|J|=|I|$ ) and $|\hat{\mathrm{Q}}|=|\mathrm{Q}|=r^{n+1}$. If $I \subseteq(0, T)$, then we choose $J:=I$ and $\hat{\mathrm{Q}}:=\mathrm{Q}$. Going further, we introduce

$$
A:=\left.a\right|_{\partial \Omega \times(0, T)}-\left(\int_{\partial \Omega \times(0, T)} a\right) \frac{1}{|\hat{\mathbf{Q}}|} \chi_{\hat{\mathbf{Q}}} .
$$

One can easily check that $\operatorname{supp} A \subseteq \hat{\mathrm{Q}},\|A\|_{L^{\infty}(\partial \Omega \times(0, T))} \leq c r^{-\frac{n+1}{p}}$, and $\underset{\partial \Omega \times(0, T)}{\int} A=0$. Therefore, in view of Definition 2.3.35, $A$ is a fixed constant multiple of a $(p, \infty)$-atom for $H_{p a r}^{p}(\partial \Omega \times(0, T))$. On account of (6.2.60), this further implies the existsence of $g \in C_{p a r}^{\alpha}(\partial \Omega \times(0, T))$ such that

$$
\Lambda(A)=\int_{\partial \Omega \times(0, T)} A g=\int_{\partial \Omega \times(0, T)} a g-\left(\int_{\partial \Omega \times(0, T)} a\right) \frac{1}{|\hat{Q}|} \int_{\hat{Q}} g .
$$

Also, from the definition of $A$ it is immediate that $\left.a\right|_{\partial \Omega \times(0, T)}=A+\left(\int_{\partial \Omega \times(0, T)} a\right) \frac{1}{|\hat{\mathbf{Q}}|} \chi_{\hat{Q}}$, which further yields

$$
\begin{aligned}
\Lambda\left(\left.a\right|_{\partial \Omega \times(0, T)}\right) & =\Lambda(A)+\left(\int_{\partial \Omega \times(0, T)} a\right) \frac{1}{|\hat{\mathbf{Q}}|} \Lambda\left(\chi_{\hat{\mathbf{Q}}}\right) \\
& =\Lambda(A)+\left(\int_{\partial \Omega \times(0, T)} a\right) \frac{1}{|\hat{\mathbf{Q}}|} \int_{\hat{\mathbf{Q}}} g .
\end{aligned}
$$

From (6.2.65) and (6.2.66) we altogether obtain that there exists $g \in C_{p a r}^{\alpha}(\partial \Omega \times(0, T))$ 
such that

$$
\Lambda\left(\left.a\right|_{\partial \Omega \times(0, T)}\right)=\int_{\partial \Omega \times(0, T)} a g .
$$

This completes the proof of Step 5 .

Step 6. Given $g \in{ }^{0} C_{\text {par }}^{\alpha}(\partial \Omega \times(0, T))$ one has

$$
\Lambda(f)=\int_{\partial \Omega \times(0, T)} g f \quad \text { for any } f \in{ }_{0} H_{p a r}^{p}(\partial \Omega \times(0, T)) .
$$

Proof of Step 6. Let us consider $f \in{ }_{0} H_{p a r}^{p}(\partial \Omega \times(0, T))$. Then, by definition, there exists $\tilde{f} \in H_{\text {par }}^{p}(\partial \Omega \times \mathbb{R})$ such that $\left.\tilde{f}\right|_{\partial \Omega \times(0, T)}=f$ and $\tilde{f} \equiv 0$ for $t<0$. By Definition 2.3.35, $\tilde{f}$ admits an atomic decomposition, i.e. $\tilde{f}=\sum_{j=0}^{\infty} \lambda_{j} a_{j}$, where $\left\{\lambda_{j}\right\}_{j \in \mathbb{N}} \in \ell^{p}$ and $a_{j}$ 's are $(p, \infty)$-atoms for $H_{p a r}^{p}(\partial \Omega \times \mathbb{R})$. We next define

$$
\tilde{a}_{j}(x, t):= \begin{cases}a_{j}(x, t)+a_{j}(x,-t) & \text { if } t>0, \\ 0 & \text { if } t<0,\end{cases}
$$

where $(x, t) \in \partial \Omega \times \mathbb{R}$. Using the notation $\operatorname{supp} a_{j} \subset \mathrm{Q}:=S \times I$, where $S \subset \partial \Omega$ and $I \subset \mathbb{R}$, we obtain the following properties for $\tilde{a}_{j}$ :

- $\operatorname{supp} \tilde{a}_{j} \subset\{(x, t) \in \partial \Omega \times(0, \infty): x \in S, t \in I$ or $-t \in I\}$

$$
=\left\{(x, t) \in \partial \Omega \times \mathbb{R}: x \in S, t \in[I \cup(-I)]_{+}\right\},
$$

and $[I \cup(-I)]_{+} \subseteq J$ such that $|J|=r^{2}$, hence

$$
\operatorname{supp} \tilde{a}_{j} \subset \tilde{\mathrm{Q}}=S \times J, \quad \text { where }|\tilde{\mathrm{Q}}|=|\mathrm{Q}|=r^{n+1}
$$

- $\left\|\tilde{a}_{j}\right\|_{L^{\infty}(\partial \Omega \times \mathbb{R})} \leq 2 r^{-\frac{n+1}{p}}$

- eiher $|\tilde{\mathrm{Q}}|<\eta$ and $\int_{\partial \Omega \times \mathbb{R}} \tilde{a}_{j}(x, t) d \sigma_{x} d t=\int_{\partial \Omega \times \mathbb{R}} a_{j}(x, t) d \sigma_{x} d t=0$, or $|\tilde{\mathrm{Q}}| \geq \eta$. 
Above $\eta=\eta(\partial \Omega)>0$ is a fixed, sufficiently small constant. Consequently, on account of Definition 2.3.35, $\tilde{a}_{j}$ 's are fixed constant multiples of $(p, \infty)$-atoms for $H_{\text {par }}^{p}(\partial \Omega \times \mathbb{R})$ with support included in $S \times J \subseteq \partial \Omega \times[0, \infty)$. Hence, without loss of generality, we may assume that $\tilde{f}=\sum_{j=0}^{\infty} \lambda_{j} \tilde{a}_{j}$, where $\left\{\lambda_{j}\right\}_{j \in \mathbb{N}} \in \ell^{p}$ and $\tilde{a}_{j}$ 's are $(p, \infty)$-atoms for $H_{p a r}^{p}(\partial \Omega \times \mathbb{R})$ with the property that $\tilde{a}_{j} \equiv 0$ for $t<0$.

At this stage we can apply Step 5 with $a=\tilde{a}_{j}$. This yields the existence of $g \in C_{\text {par }}^{\alpha}(\partial \Omega \times(0, T))$ such that

$$
\Lambda\left(\left.\tilde{a}_{j}\right|_{\partial \Omega \times(0, T)}\right)=\int_{\partial \Omega \times(0, T)} \tilde{a}_{j} g .
$$

Using (6.2.67) and the above atomic decomposition of $\tilde{f}$, we ultimately obtain that

$$
\begin{aligned}
\Lambda(f) & =\Lambda\left(\sum_{j=0}^{\infty} \lambda_{j}\left(\left.\tilde{a}_{j}\right|_{\partial \Omega \times(0, T)}\right)\right)=\sum_{j=0}^{\infty} \lambda_{j} \Lambda\left(\left.\tilde{a}_{j}\right|_{\partial \Omega \times(0, T)}\right) \\
& =\sum_{j=0}^{\infty} \lambda_{j} \int_{\partial \Omega \times(0, T)} \tilde{a}_{j} g=\int_{\partial \Omega \times(0, T)} f g .
\end{aligned}
$$

This completes the proof of Step 6 .

The observation we make is that Steps 4 and 6 imply $\mathbf{A}$. In order to show $\mathbf{B}$, first assume that there exist $g_{1}, g_{2} \in{ }^{0} C_{\text {par }}^{\alpha}(\partial \Omega \times(0, T))$ such that

$$
\Lambda(f)=\int_{\partial \Omega \times(0, T)} g_{1} f=\int_{\partial \Omega \times(0, T)} g_{2} f
$$

for any $f \in{ }_{0} H_{p a r}^{p}(\partial \Omega \times(0, T))$. Then

$$
\int_{\partial \Omega \times(0, T)}\left(g_{1}-g_{2}\right) f=0 \quad \text { for any } f \in H_{p a r}^{p}(\partial \Omega \times(0, T)),
$$

hence $g_{1}-g_{2}=0$ on $\partial \Omega \times(0, T)$. Consequently, $\Lambda \mapsto g$ is well-defined. 
We next suppose that $\Lambda\left(f_{1}\right)=\Lambda\left(f_{2}\right)$ for $f_{1}, f_{2} \in{ }_{0} H_{p a r}^{p}(\partial \Omega \times(0, T))$. By Step 6 ,

$$
\int_{\partial \Omega \times(0, T)} g\left(f_{1}-f_{2}\right)=0 \quad \text { for } g \in{ }^{0} C_{p a r}^{\alpha}(\partial \Omega \times(0, T)),
$$

therefore $f_{1}=f_{2}$. This shows that $\Lambda$ is one-to-one. Consequently, the proof of $\mathbf{B}$ is finished, which completes the proof of Theorem 6.2.6.

After this digression we now return to analyze the behavior of the operators $\pm \frac{1}{2} I+K$ on parabolic Besov spaces defined on bounded Lipschitz cylinders.

Theorem 6.2.7. Let $\Omega$ be a bounded Lipschitz domain in $\mathbb{R}^{n}$ and $0<T<\infty$. Then there exists an $\varepsilon=\varepsilon(\partial \Omega)>0$ such that

$$
\pm \frac{1}{2} I+K:{ }_{0} B_{\alpha}^{\infty}(\partial \Omega \times(0, T)) \stackrel{\sim}{\longrightarrow}{ }_{0} B_{\alpha}^{\infty}(\partial \Omega \times(0, T))
$$

isomorphically, for $\alpha=(n+1)\left(\frac{1}{p}-1\right)$ and $1-\varepsilon<p<1$.

Proof. According to Lemma 6.2.1 the operators $\pm \frac{1}{2} I+K^{\prime}$ are invertible on the space ${ }_{0} H_{p a r}^{1}(\partial \Omega \times(0, T))$. Since ${ }_{0} H_{p a r}^{p}(\partial \Omega \times(0, T))$ is a complex interpolation scale (cf. Lemma 3.4.1), perturbing the above result, using Theorem 2.7 from [KaMi], yields the existence of $\varepsilon=\varepsilon(\partial \Omega)>0$ such that

$$
\pm \frac{1}{2} I+K^{\prime}:{ }_{0} H_{p a r}^{p}(\partial \Omega \times(0, T)) \stackrel{\sim}{\longrightarrow}{ }_{0} H_{p a r}^{p}(\partial \Omega \times(0, T))
$$

for $1-\varepsilon<p \leq 1$. Dualizing (6.2.69), with Theorem 6.2.6 in hand, we further obtain that

$$
R \circ\left( \pm \frac{1}{2} I+K\right) \circ R:{ }^{0} C_{p a r}^{\alpha}(\partial \Omega \times(0, T)) \stackrel{\sim}{\longrightarrow}{ }^{0} C_{p a r}^{\alpha}(\partial \Omega \times(0, T))
$$


for $\alpha=(n+1)\left(\frac{1}{p}-1\right)$ and $1-\varepsilon<p<1$. Here $R$ stands for the reflection operator in time, i.e. $R f(x, t):=f(x, T-t)$ for $(x, t) \in \partial \Omega \times(0, T)$. Note that

$$
R:{ }^{0} C_{\text {par }}^{\alpha}\left(\partial \Omega \times(0, T) \stackrel{\sim}{\longrightarrow}{ }_{0} C_{\text {par }}^{\alpha}(\partial \Omega \times(0, T)),\right.
$$

and $R \circ R$ gives the identity operator. We also recall from Lemma 2.3.18 the identification of the parabolic Hölder class $C_{p a r}^{\alpha}(\partial \Omega \times(0, T))$ with $B_{\alpha, p a r}^{\infty}(\partial \Omega \times(0, T))$. Consequently, (6.2.70) and (6.2.71) yield

$$
\pm \frac{1}{2} I+K:{ }_{0} B_{\alpha, p a r}^{\infty}(\partial \Omega \times(0, T)) \stackrel{\sim}{\longrightarrow}{ }_{0} B_{\alpha, p a r}^{\infty}(\partial \Omega \times(0, T))
$$

for indices $\alpha=(n+1)\left(\frac{1}{p}-1\right)$ and $1-\varepsilon<p<1$. This completes the proof of the theorem.

Another crutial property of the operators $\pm \frac{1}{2} I+K$ is discussed in the following theorem.

Theorem 6.2.8. Let $\Omega$ be a bounded Lipschitz domain in $\mathbb{R}^{n}, 0<T<\infty$, and $\alpha=(n+1)\left(\frac{1}{p}-1\right)$. Then there exists an $\varepsilon=\varepsilon(\partial \Omega)>0$ such that, for $1-\varepsilon<p<1$, $\pm \frac{1}{2} I+K:{ }_{0} B_{1-\alpha, p a r}^{1}(\partial \Omega \times(0, T)) \stackrel{\sim}{\longrightarrow}{ }_{0} B_{1-\alpha, p a r}^{1}(\partial \Omega \times(0, T))$.

Proof. We first recall from Lemma 6.2.2 that the operators

$$
\pm \frac{1}{2} I+K \quad \text { are invertible on } \quad{ }_{0} H_{\text {par }}^{1,1}(\partial \Omega \times(0, T))
$$

An independent result (Theorem 3.4.9) shows that ${ }_{0} H_{\text {par }}^{1, p}(\partial \Omega \times(0, T))$ is a complex interpolation scale for $\frac{n+1}{n+2}<p \leq 1$. With this in hand, Theorem 2.7 of [KaMi] implies that there exists $\eta=\eta(\partial \Omega)>0$ such that for $1-\eta<p \leq 1$, 


$$
\pm \frac{1}{2} I+K \quad \text { are invertible on } \quad{ }_{0} H_{p a r}^{1, p}(\partial \Omega \times(0, T)) .
$$

Taking $\mathcal{E}_{1}$-envelopes in (6.2.74), using also Proposition 1.2.45, one can conclude the existence of $\eta=\eta(\partial \Omega)>0$ such that for $1-\eta<p \leq 1$,

$$
\pm \frac{1}{2} I+K \quad \text { are invertible on } \quad \mathcal{E}_{1}\left({ }_{0} H_{p a r}^{1, p}(\partial \Omega \times(0, T))\right)
$$

Finally, let us recall from Corollary 3.3.4 that for any Lipschitz domain $\Omega$ in $\mathbb{R}^{n}$, $0<T<\infty$ and $\frac{n+1}{n+2}<p<1, \alpha=(n+1)\left(\frac{1}{p}-1\right)$, the following holds:

$$
\mathcal{E}_{1}\left({ }_{0} H_{p a r}^{1, p}(\partial \Omega \times(0, T))\right)={ }_{0} B_{1-\alpha, p a r}^{1}(\partial \Omega \times(0, T)) .
$$

Now (6.2.75) and (6.2.76) ultimately imply what we wanted to show. This finishes the proof of the theorem.

We now are ready to present the main result of this section.

Theorem 6.2.9. Consider a bounded Lipschitz domain $\Omega$ in $\mathbb{R}^{n}$ and $0<T<\infty$. Then there exists $\varepsilon=\varepsilon(\partial \Omega)>0$ such that the operators

$$
\pm \frac{1}{2} I+K:{ }_{0} B_{\alpha, p a r}^{p}(\partial \Omega \times(0, T)) \stackrel{\sim}{\longrightarrow}{ }_{0} B_{\alpha, p a r}^{p}(\partial \Omega \times(0, T))
$$

are invertible provided the point with coordinates $\left(\alpha, \frac{1}{p}\right)$ belongs to the interior of the hexagon $O A B C D E$ in the diagram below. 


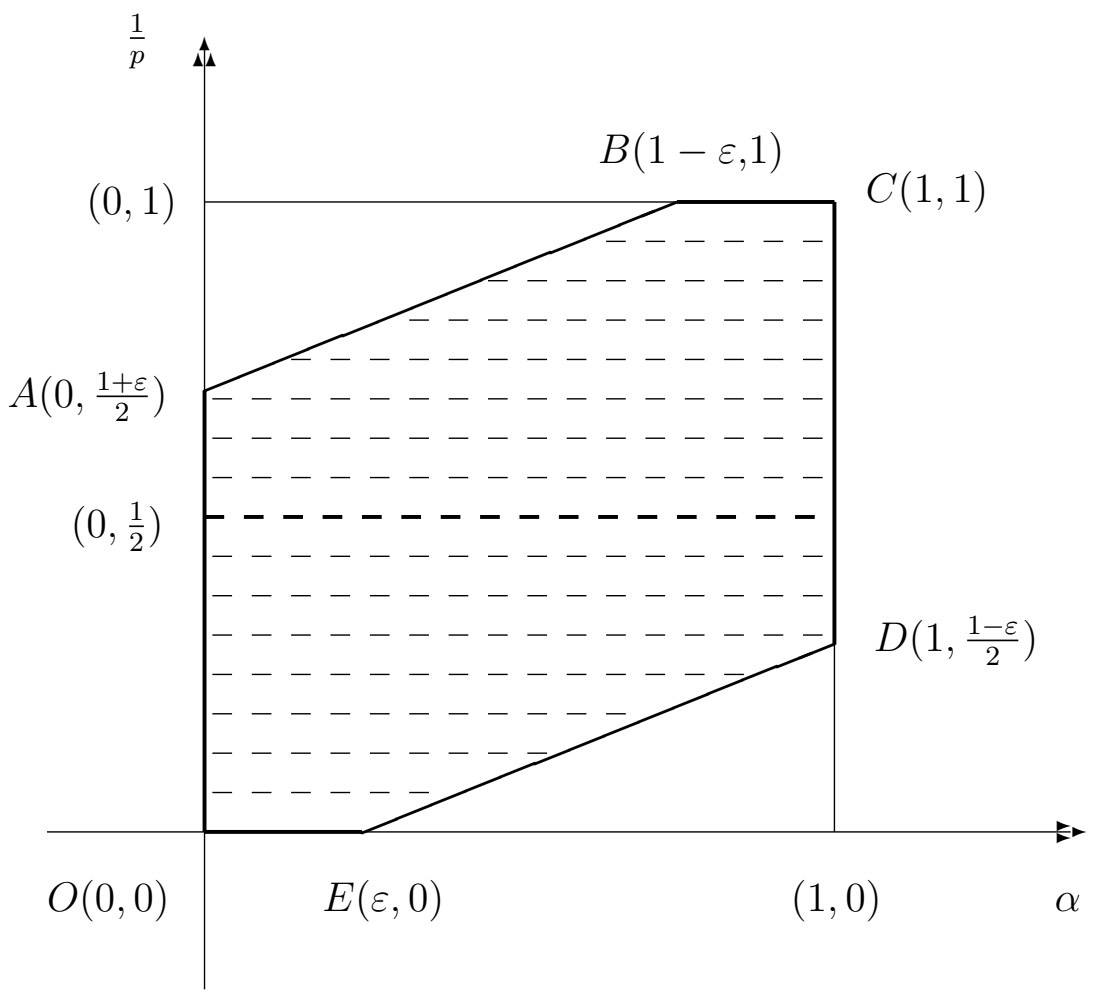

Proof. Our first observation is that comlex interpolation (Theorem 3.4.3) between the results of Propositions 6.2.3 and 6.2.4 gives the invertibility of the operators $\pm \frac{1}{2} I+K$ on parabolic Sobolev spaces ${ }_{0} L_{\alpha, p a r}^{p}(\partial \Omega \times(0, T))$, where $\Omega$ is a bounded Lipschitz domain in $\mathbb{R}^{n}$ and the pair $\left(\alpha, \frac{1}{p}\right)$ belongs to the interior of the parallelogram $O A C D$.

Second, for each fixed $p$ and $\alpha_{0} \neq \alpha_{1}$, by real interpolation between two instances of ${ }_{0} L_{\alpha_{i}, p a r}^{p}(\partial \Omega \times(0, T)), i=0,1$ (Theorem 3.4.4) we obtain that $\pm \frac{1}{2} I+K$ are isomorphisms on Besov spaces ${ }_{0} B_{\alpha, p a r}^{p}(\partial \Omega \times(0, T))$, where $\Omega$ is a bounded Lipschitz domain in $\mathbb{R}^{n}$ and the pair $\left(\alpha, \frac{1}{p}\right)$ belongs to the interior of the parallelogram $O A C D$.

Finally, according to Theorem 3.4.6 (complex interpolation), with the results of 
Theorems 6.2.7 and 6.2.8 in hand, we can conclude that

$$
\pm \frac{1}{2} I+K:{ }_{0} B_{\alpha, p a r}^{p}(\partial \Omega \times(0, T)) \stackrel{\sim}{\longrightarrow}{ }_{0} B_{\alpha, p a r}^{p}(\partial \Omega \times(0, T))
$$

for $\Omega$ bounded Lipschitz domain in $\mathbb{R}^{n}$ and $\left(\alpha, \frac{1}{p}\right) \in$ interior of $O A B C D E$. 


\section{Chapter 7}

\section{The boundary trace operator, the normal derivative and the Newtonian potential}

\subsection{Mapping properties of the trace operator}

Recall that originally, the boundary traces are taken in the nontangential limit sense, i.e. for almost every $(x, t) \in \partial \Omega \times \mathbb{R}$,

$$
\operatorname{Tr} u(x, t)=\left.u\right|_{\partial \Omega \times \mathbf{R}}(x, t):=\lim _{\substack{(y, s) \rightarrow(x, t) \\(y, s) \in \Gamma(x, t)}} u(y, s),
$$

where $\Gamma(x, t)$ is a nontangential cone at the boundary point $(x, t)$ as in (1.2.8). The main result of this section is as follows.

Theorem 7.1.1. Let $\Omega$ be a Lipschitz domain in $\mathbb{R}^{n}$ and $1<p \leq \infty, \frac{1}{p}<\alpha<1+\frac{1}{p}$.

Then the trace operator defined in (7.1.1) extends to

$$
\operatorname{Tr}: B_{\alpha, p a r}^{p}(\Omega \times \mathbb{R}) \longrightarrow B_{\alpha-\frac{1}{p}, p a r}^{p}(\partial \Omega \times \mathbb{R})
$$

as a bounded operator.

Proof. It is natural to treat the case $p=\infty$ separately. In this case, given $f \in B_{\alpha, p a r}^{p}(\Omega \times \mathbb{R})$, the intrinsic characterization of the parabolic Besov spaces 
(Lemma 2.2.4) yields

$$
f \in L^{\infty}(\Omega \times \mathbb{R}), \quad \text { and } \sup _{\substack{(X, t),(Y, s) \in \Omega \times \mathbb{R} \\(X, t) \neq(Y, s)}} \frac{|f(X, t)-f(Y, s)|}{\left(|X-Y|+|t-s|^{1 / 2}\right)^{\alpha}}<\infty
$$

where $X=\left(x^{\prime}, \varphi\left(x^{\prime}\right)+x_{n}\right), Y=\left(y^{\prime}, \varphi\left(y^{\prime}\right)+y_{n}\right), x^{\prime}, y^{\prime} \in \mathbb{R}^{n-1}, x_{n}, y_{n} \in \mathbb{R}$, and the graph of the Lipschitz function $\varphi: \mathbb{R}^{n-1} \rightarrow \mathbb{R}$ represents the boundary of $\Omega$, in case that $\Omega$ is an unbounded domain. If $\Omega$ is a bounded Lipschitz domain, we use a finite partition of unity on $\partial \Omega$.

Denote $\tilde{x}=\left(x^{\prime}, \varphi\left(x^{\prime}\right)\right), \tilde{y}=\left(y^{\prime}, \varphi\left(y^{\prime}\right)\right)$. Then (7.1.2) implies

$$
\operatorname{Tr} f \in L^{\infty}(\partial \Omega \times \mathbb{R}), \quad \text { and } \quad \sup _{\substack{(\tilde{x}, t),(\tilde{y}, s) \in \partial \Omega \times \mathbb{R} \\(\tilde{x}, t) \neq(\tilde{y}, s)}} \frac{|\operatorname{Tr} f(\tilde{x}, t)-\operatorname{Tr} f(\tilde{y}, s)|}{\left(|X-Y|+|t-s|^{1 / 2}\right)^{\alpha}}<\infty,
$$

which mean, according to the intrinsic characterization of the parabolic Besov spaces defined on the lateral side of a cylinder (Lemma 2.3.18), that $\operatorname{Tr} f \in B_{\alpha, p a r}^{\infty}(\partial \Omega \times \mathbb{R})$.

For the rest of the proof assume $1<p<\infty$.

Case 1. $\frac{1}{p}<\alpha<1$. Let $f \in B_{\alpha, p a r}^{p}(\Omega \times \mathbb{R})$ and define $\psi: \mathbb{R}_{+}^{n} \times \mathbb{R} \rightarrow \Omega \times \mathbb{R}$ by

$$
\psi\left(x^{\prime}, x_{n}, t\right):=\left(x^{\prime}, \varphi\left(x^{\prime}\right)+x_{n}, t\right)
$$

where the graph of the Lipschitz function $\varphi: \mathbb{R}^{n-1} \rightarrow \mathbb{R}$ is the boundary of $\Omega$, if $\Omega$ is an unbounded domain (in the case when $\Omega$ is a bounded Lipschitz domain, use a finite partition of unity on $\partial \Omega$ ). Using the notation

$$
\begin{array}{ll}
X=\left(x^{\prime}, \varphi\left(x^{\prime}\right)+x_{n}\right), & x=\left(x^{\prime}, x_{n}\right), \\
Y=\left(y^{\prime}, \varphi\left(y^{\prime}\right)+y_{n}\right), & y=\left(y^{\prime}, y_{n}\right),
\end{array}
$$

and Lemma 2.2.5 (the intrinsic characterization of $B_{\alpha, p a r}^{p}(\Omega \times \mathbb{R})$ ), we get that

$$
f \in L^{p}(\Omega \times \mathbb{R}) \text { and } \int_{\Omega \times \mathbb{R}} \int_{\Omega \times \mathbb{R}} \frac{|f(X, t)-f(Y, s)|^{p}}{\left(|X-Y|+|t-s|^{1 / 2}\right)^{n+2+\alpha p}} d X d t d Y d s<\infty
$$


which is equaivalent with

$$
f \circ \psi \in L^{p}\left(\mathbb{R}_{+}^{n} \times \mathbb{R}\right) \text { and } \int_{\mathbb{R}_{+}^{n} \times \mathbb{R}} \int_{\mathbb{R}_{+}^{n} \times \mathbb{R}} \frac{|f(\psi(x, t))-f(\psi(y, s))|^{p}}{\left(|x-y|+|t-s|^{1 / 2}\right)^{n+2+\alpha p}} d x d t d y d s<\infty
$$

since $|X-Y| \approx|x-y|, d X \approx d x$, and $d Y \approx d y$. In other words, $f \circ \psi$ belongs to the space $B_{\alpha, p a r}^{p}\left(\mathbb{R}_{+}^{n} \times \mathbb{R}\right)$. Then, applying the trace operator to $f \circ \psi$,

$$
\operatorname{Tr}(f \circ \psi) \in B_{\alpha-\frac{1}{p}, p a r}^{p}\left(\mathbb{R}^{n-1} \times \mathbb{R}\right)
$$

and, using again the intrinsic characterization of parabolic Besov spaces, this time for $B_{\alpha-\frac{1}{p}, p a r}^{p}\left(\mathbb{R}^{n-1} \times \mathbb{R}\right)($ Lemma 2.1.22), (7.1.4) further yields

$$
\begin{aligned}
& \operatorname{Tr}(f \circ \psi) \in L^{p}\left(\mathbb{R}^{n-1} \times \mathbb{R}\right) \text { and } \\
& \int_{\mathbb{R}^{n-1} \times \mathbb{R} \mathbb{R}^{n-1} \times \mathbb{R}} \int_{\left|\operatorname{Tr}(f \circ \psi)\left(x^{\prime}, t\right)-\operatorname{Tr}(f \circ \psi)\left(y^{\prime}, s\right)\right|^{p}} d x^{\prime} d t d y^{\prime} d s<\infty .
\end{aligned}
$$

Going further, consider $\Phi: \mathbb{R}^{n-1} \times \mathbb{R} \rightarrow \partial \Omega \times \mathbb{R}$ defined by

$$
\Phi\left(x^{\prime}, t\right):=\left(x^{\prime}, \varphi\left(x^{\prime}\right), t\right)
$$

where, if $\Omega$ is an unbounded domain, the boundary of $\Omega$ is the graph of the Lipschitz function $\varphi: \mathbb{R}^{n-1} \rightarrow \mathbb{R}$ (in the case when $\Omega$ is a bounded Lipschitz domain, use a finite partition of unity on $\partial \Omega$ ). Then (7.1.5) can be rewritten as

$$
\begin{aligned}
& \operatorname{Tr}(f \circ \psi) \circ \Phi^{-1} \in L^{p}(\partial \Omega \times \mathbb{R}) \text { and } \\
& \int_{\partial \Omega \times \mathbb{R}} \int_{\partial \Omega \times \mathbb{R}} \frac{\left|\operatorname{Tr}(f \circ \psi)\left(\Phi^{-1}(\tilde{x}, t)\right)-\operatorname{Tr}(f \circ \psi)\left(\Phi^{-1}(\tilde{y}, s)\right)\right|^{p}}{\left(|\tilde{x}-\tilde{y}|+|t-s|^{1 / 2}\right)^{n+\alpha p}} d \tilde{x} d t d \tilde{y} d s<\infty,
\end{aligned}
$$

where $\tilde{x}=\left(x^{\prime}, \varphi\left(x^{\prime}\right)\right), \tilde{y}=\left(y^{\prime}, \varphi\left(y^{\prime}\right)\right)$, and $\left|x^{\prime}-y^{\prime}\right| \approx|\tilde{x}-\tilde{y}|$. This amounts to

$$
\operatorname{Tr}(f \circ \psi) \circ \Phi^{-1} \in B_{\alpha-\frac{1}{p}}^{p}(\partial \Omega \times \mathbb{R})
$$


Finally, a closer look at $\operatorname{Tr}(f \circ \psi) \circ \Phi^{-1}$ reveals that

$$
\begin{aligned}
\operatorname{Tr}(f \circ \psi) \circ \Phi^{-1}(\tilde{x}, t) & =\operatorname{Tr}(f \circ \psi)\left(x^{\prime}, t\right)=\lim _{\substack{(y, s) \rightarrow\left(x^{\prime}, t\right) \\
(y, s) \in \tilde{\Gamma}\left(x^{\prime}, t\right) \subset \mathbb{R}_{+}^{n} \times \mathbb{R}}}(f \circ \psi)(y, s) \\
& \approx \lim _{\substack{(Y, s) \rightarrow(\tilde{x}, t) \\
(Y, s) \in \Gamma(\tilde{x}, t) \subset \partial \Omega \times \mathbb{R}}} f(Y, s)=\operatorname{Tr} f(\tilde{x}, t) .
\end{aligned}
$$

Here $\tilde{\Gamma}\left(x^{\prime}, t\right)$ is a nontangential cone in $\mathbb{R}_{+}^{n} \times \mathbb{R}$ at the boundary point $\left(x^{\prime}, t\right)$. Now the conclusion can be seen from (7.1.6) and (7.1.7).

Case 2. $1<\alpha<1+\frac{1}{p}$. We can reduce matters to the previous case, mostly due to the fact that the operator $\partial_{\text {time }}^{\theta}$, defined in (1.2.31), can be applied to and lifted from an appropriate function. Let us consider $f \in B_{\alpha, p a r}^{p}(\Omega \times \mathbb{R})$. According to Fubini's property described in Proposition 2.2.3, $f$ belongs to $L^{p}\left(\mathbb{R} ; B_{\alpha}^{p}(\Omega)\right)$, hence, by the mapping property of the trace operator in the isotropic case (see Theorem 3.1 of $[\mathrm{JeKe}])$,

$$
\operatorname{Tr} f \in L^{p}\left(\mathbb{R} ; B_{\alpha-\frac{1}{p}}^{p}(\partial \Omega)\right)
$$

On the other hand, we pick $\theta>0$ so that $\frac{1}{p}<\alpha-2 \theta<1$. Using the fact that the operator $\partial_{\text {time }}^{\theta}: B_{\alpha, \text { par }}^{p}(\Omega \times \mathbb{R}) \rightarrow B_{\alpha-2 \theta, p a r}^{p}(\Omega \times \mathbb{R})$ is bounded for $0<2 \theta<\alpha$ (Theorem 2.2.6), we obtain that

$$
\partial_{\text {time }}^{\theta} f \in B_{\alpha-2 \theta, p a r}^{p}(\Omega \times \mathbb{R})
$$

Now from the previous case we have

$$
\operatorname{Tr}\left(\partial_{\text {time }}^{\theta} f\right) \in B_{\alpha-2 \theta-\frac{1}{p}, p a r}^{p}(\partial \Omega \times \mathbb{R}),
$$

and by the Fubini property (Proposition 2.3.16),

$$
\partial_{\text {time }}^{\theta}(\operatorname{Tr} f) \in L^{p}\left(\partial \Omega ; B_{\left(\alpha-2 \theta-\frac{1}{p}\right) / 2}^{p}(\mathbb{R})\right)
$$


or, equivalently,

$$
\int_{\partial \Omega}\left\|\partial_{\text {time }}^{\theta}(\operatorname{Tr} f)(x, \cdot)\right\|_{B_{\left(\alpha-2 \theta-\frac{1}{p}\right) / 2}^{p}(\mathbb{R})}^{p} d x<\infty .
$$

Furthermore, the operator $\partial_{\text {time }}^{\theta}$ can be lifted (see second part of (1.3) from [GrKo]), to obtain that

$$
\int_{\partial \Omega}\|(\operatorname{Tr} f)(x, \cdot)\|_{B_{\left(\alpha-\frac{1}{p}\right) / 2}^{p}(\mathbb{R})}^{p} d x<\infty
$$

i.e.

$$
\operatorname{Tr} f \in L^{p}\left(\partial \Omega ; B_{\left(\alpha-\frac{1}{p}\right) / 2}^{p}(\mathbb{R})\right) .
$$

Finally, based on (7.1.8), (7.1.9) and Fubini's property, one can conclude that $\operatorname{Tr} f$ belongs to $B_{\alpha-\frac{1}{p}, p a r}^{p}(\partial \Omega \times \mathbb{R})$, as desired.

Case 3. $\alpha=1$. Here we are using both Cases 1 and 2, and interpolate between them. We could use either the real or the complex interpolation method, both yield the same result, here we present the proof with the real method.

Let $\beta \in \mathbb{R}$ so that $0<\beta<\min \left\{1-\frac{1}{p}, \frac{1}{p}\right\}$. Then $\frac{1}{p}<1-\beta<1$, and we can apply the result of Case 1, i.e.

$$
\operatorname{Tr} f: B_{1-\beta, p a r}^{p}(\Omega \times \mathbb{R}) \longrightarrow B_{1-\beta-\frac{1}{p}, p a r}^{p}(\partial \Omega \times \mathbb{R})
$$

Moreover, $1<1+\beta<1+\frac{1}{p}$, and Case 2 inplies that

$$
\operatorname{Tr} f: B_{1+\beta, p a r}^{p}(\Omega \times \mathbb{R}) \longrightarrow B_{1+\beta-\frac{1}{p}, p a r}^{p}(\partial \Omega \times \mathbb{R})
$$

Now, by the real interpolation method (see, for example, part (c) or (d) of Theorems 2.2.8 and 2.3.20), we obtain that

$$
\operatorname{Tr} f: B_{\beta^{*}, p a r}^{p}(\Omega \times \mathbb{R}) \longrightarrow B_{\beta^{*}-\frac{1}{p}, p a r}^{p}(\partial \Omega \times \mathbb{R})
$$


where $\beta^{*}=(1-\eta)(1-\beta)+\eta(1+\beta)$ and $0<\eta<1$. If $\eta=\frac{1}{2}$, then $\beta^{*}=1$ and $\operatorname{Tr} f: B_{1, p a r}^{p}(\Omega \times \mathbb{R}) \longrightarrow B_{1-\frac{1}{p}, p a r}^{p}(\partial \Omega \times \mathbb{R})$ is bounded, which completes the proof of Case 3, and the boundedness of the trace operator on parabolic Besov spaces.

In the last part of this section we turn our attention to the mapping properties of the trace operator on parabolic Besov spaces with built-in initial conditions.

Theorem 7.1.2. Let $\Omega$ be a bounded Lipschitz domain in $\mathbb{R}^{n}, 0<T<\infty$, and $1<p \leq \infty,\left(\alpha, \frac{1}{p}\right) \in$ interior of $O L P Q R$ in the following diagram.

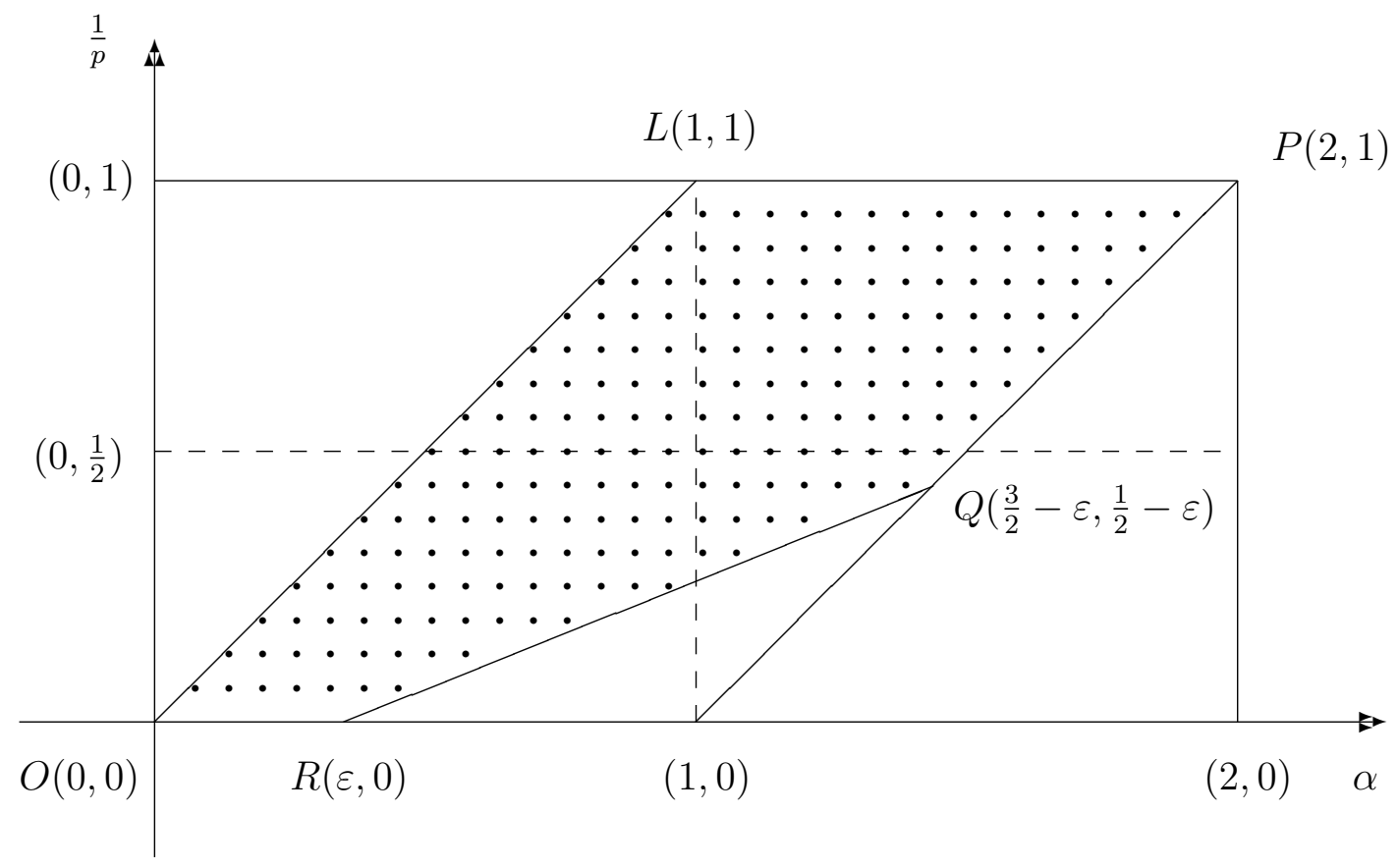

Then the trace operator defined in (7.1.1) extends to

$$
\operatorname{Tr}:{ }_{0} B_{\alpha, p a r}^{p}(\Omega \times(0, T)) \longrightarrow{ }_{0} B_{\alpha-\frac{1}{p}, p a r}^{p}(\partial \Omega \times(0, T))
$$

and

$$
\operatorname{Tr}:{ }^{0} B_{\alpha, p a r}^{p}(\Omega \times(0, T)) \longrightarrow{ }^{0} B_{\alpha-\frac{1}{p}, p a r}^{p}(\partial \Omega \times(0, T))
$$

as a bounded operator. 
Proof. (a) First observe that for the range of indices considered, based on Theorems 3.1.19 (ii) and 3.1.20, we have the equivalence

$$
{ }_{0} B_{\alpha, p a r}^{p}(\Omega \times(0, T))={ }_{\square} B_{\alpha, p a r}^{p}(\Omega \times(0, T)) .
$$

This is why letting $f \in{ }_{0} B_{\alpha, p a r}^{p}(\Omega \times(0, T))$, by Definition 3.1.15, amounts to the existence of $F \in B_{\alpha, p a r}^{p}(\Omega \times \mathbb{R})$ such that $\left.F\right|_{\Omega \times(0, T)}=f$ and $\operatorname{supp} F \subseteq \Omega \times[0, \infty)$. Now we can apply Theorem 7.1.1, which yields

$$
\operatorname{Tr} F \in B_{\alpha-\frac{1}{p}, p a r}^{p}(\Omega \times \mathbb{R})
$$

Also, it is not hard to see that

$$
\begin{aligned}
& \left.(\operatorname{Tr} F)\right|_{\partial \Omega \times(0, T)}=\operatorname{Tr}\left(\left.F\right|_{\Omega \times(0, T)}\right)=\operatorname{Tr} f, \\
& \operatorname{supp}(\operatorname{Tr} F) \subseteq \partial \Omega \times[0, \infty) .
\end{aligned}
$$

In view of Definition $3.2 .2,(7.1 .12)$ and 7.1 .13$)$ ultimately imply that

$$
\operatorname{Tr} F \in{ }_{0} B_{\alpha-\frac{1}{p}, p a r}^{p}(\partial \Omega \times(0, T)) .
$$

Part (b) of the theorem is proved in a similar fashion, using the same ingredients.

Theorem 7.1.3. Let $\Omega$ be a bounded Lipschitz domain in $\mathbb{R}^{n}, 0<T<\infty$, and $1<p \leq \infty, \alpha>0$ such that the pair $\left(\alpha-\frac{1}{p}, \frac{1}{p}\right) \in$ interior of $O A B C D E$ in the following diagram. 


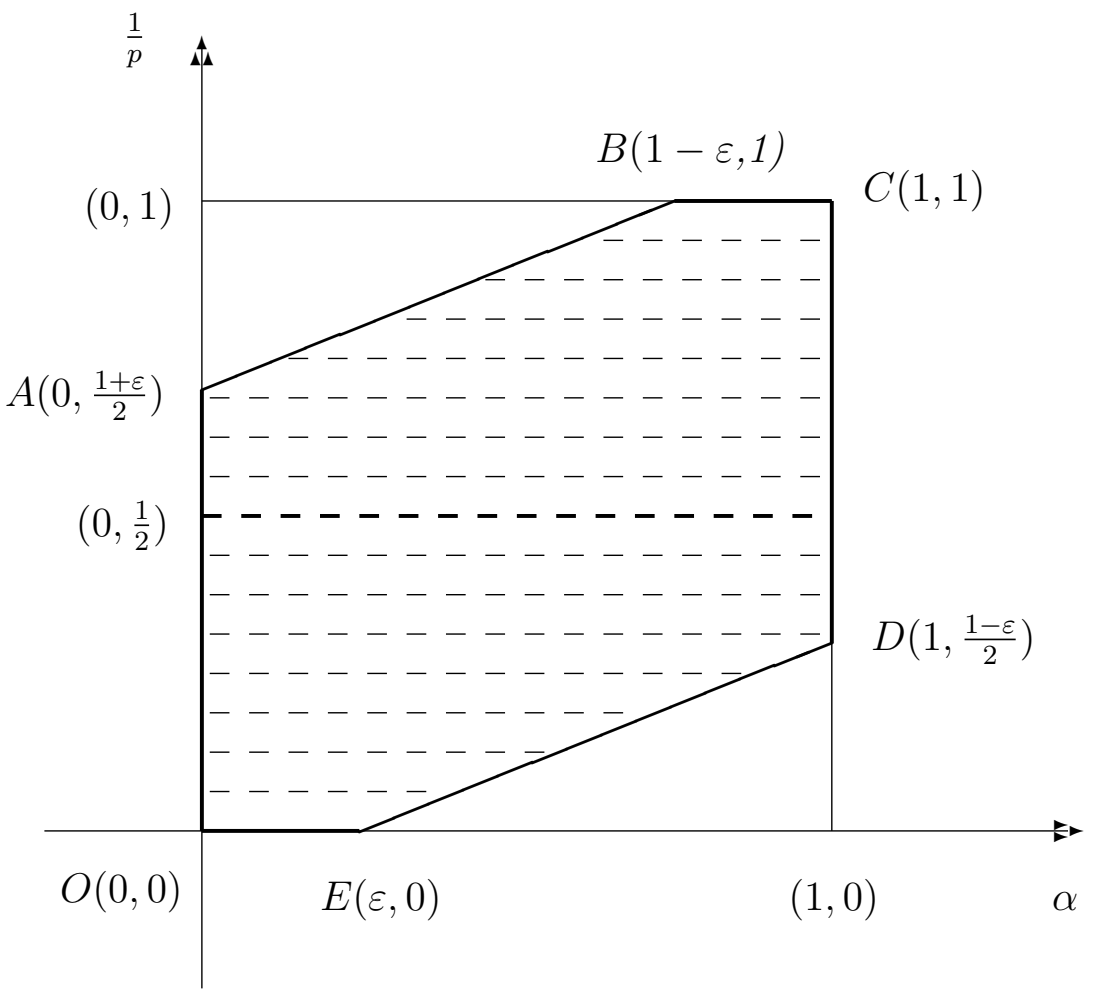

Note that, automatically, $\frac{1}{p}<\alpha<1+\frac{1}{p}$. Then the trace operator

$$
\operatorname{Tr}:{ }_{0} B_{\alpha, p a r}^{p}(\Omega \times(0, T)) \longrightarrow{ }_{0} B_{\alpha-\frac{1}{p}, p a r}^{p}(\partial \Omega \times(0, T))
$$

has a linear, continuous right-inverse.

Proof. Let us consider $f \in{ }_{0} B_{\alpha-\frac{1}{p}, p a r}^{p}(\partial \Omega \times(0, T))$ and $F:=\mathcal{D}\left[\left(\frac{1}{2} I+K\right)^{-1} f\right]$. It is meaningful to talk about the inverse of the boundary operator $\frac{1}{2} I+K$, since, according to $(6.2 .77)$, this operator is invertible on ${ }_{0} B_{\alpha-\frac{1}{p}, p a r}^{p}(\partial \Omega \times(0, T))$ as long as $\left(\alpha-\frac{1}{p}, \frac{1}{p}\right) \in$ interior of $O A B C D E$. Using the mapping properties of the caloric double layer potential (Theorem 4.2.11), we get that $F$ belongs to the Besov space ${ }_{0} B_{\alpha, p a r}^{p}(\Omega \times(0, T))$, as desired. 
On the other hand, by Theorem 4.17 (2) of [Bro2],

$$
\operatorname{Tr} F=\left(\frac{1}{2} I+K\right)\left[\left(\frac{1}{2} I+K\right)^{-1} f\right]=f
$$

which completes the proof of the theorem.

Theorem 7.1.4. Let $\Omega$ be a bounded Lipschitz domain in $\mathbb{R}^{n}, 0<T<\infty$, and $1<p \leq \infty, \alpha>0$ such that the pair $\left(\alpha-\frac{1}{p}, \frac{1}{p}\right) \in$ interior of $O A B C D E$. Note that, automatically, $\frac{1}{p}<\alpha<1+\frac{1}{p}$. Then the trace operator

$$
\operatorname{Tr}:{ }^{0} B_{\alpha, p a r}^{p}(\Omega \times(0, T)) \longrightarrow{ }^{0} B_{\alpha-\frac{1}{p}, p a r}^{p}(\partial \Omega \times(0, T))
$$

has a linear, continuous right-inverse.

Proof. Consider the reflection operator in time defined by $R f(x, t):=f(x, T-t)$ for $(x, t) \in \mathbb{R}^{n} \times(0, T)$, and let

$$
F:=R\left[\mathcal{D}\left(\left(\frac{1}{2} I+K\right)^{-1} R f\right)\right],
$$

where $f \in{ }^{0} B_{\alpha-\frac{1}{p}, p a r}^{p}(\partial \Omega \times(0, T))$. Then $R f \in{ }_{0} B_{\alpha-\frac{1}{p}, p a r}^{p}(\partial \Omega \times(0, T))$, and by the invertibility of the operator $\frac{1}{2} I+K($ cf. $(6.2 .77))$,

$$
\left(\frac{1}{2} I+K\right)^{-1} R f \in{ }_{0} B_{\alpha-\frac{1}{p}, p a r}^{p}(\partial \Omega \times(0, T)) .
$$

Moreover, according to the mapping properties of the caloric double layer potential (see Theorem 4.2.11), we have that

$$
\mathcal{D}\left(\left(\frac{1}{2} I+K\right)^{-1} R f\right) \in{ }_{0} B_{\alpha, p a r}^{p}(\Omega \times(0, T)) .
$$

Finally,

$$
F=R\left[\mathcal{D}\left(\left(\frac{1}{2} I+K\right)^{-1} R f\right)\right] \in{ }^{0} B_{\alpha, p a r}^{p}(\Omega \times(0, T))
$$


which is what we wanted to show. On the other hand, using the fact that the boundary trace of the double layer potential operator is $\frac{1}{2} I+K$ (by Theorem 4.17 of [Bro2]), we arrive at

$$
\begin{aligned}
\operatorname{Tr} F & =R\left\{\operatorname{Tr}\left[\mathcal{D}\left(\left(\frac{1}{2} I+K\right)^{-1} R f\right)\right]\right\} \\
& =R\left[\left(\frac{1}{2} I+K\right)\left(\frac{1}{2} I+K\right)^{-1} R f\right] \\
& =f .
\end{aligned}
$$

This finishes the proof of the theorem.

\subsection{The normal derivative}

In this section we give the definition of the normal derivative of a function that has low regularity. Proir to do so, we need some preliminary results.

Theorem 7.2.1. Consider a Lipschitz domain $\Omega \subseteq \mathbb{R}^{n}, 0<T<\infty$, and indices $1<p<\infty, \alpha \in \mathbb{R} \backslash\{0,2\}$. Then the heat and the adjoint heat operators

$$
\begin{aligned}
& \partial_{t} \pm \Delta:{ }_{0} B_{\alpha, p a r}^{p}(\Omega \times(0, T)) \longrightarrow{ }_{0} B_{\alpha-2, p a r}^{p}(\Omega \times(0, T)), \\
& \partial_{t} \pm \Delta:{ }^{0} B_{\alpha, p a r}^{p}(\Omega \times(0, T)) \longrightarrow{ }^{0} B_{\alpha-2, p a r}^{p}(\Omega \times(0, T))
\end{aligned}
$$

are bounded.

Proof. First, let us suppose that $f \in{ }_{0} B_{\alpha, p a r}^{p}(\Omega \times(0, T))$. Then, according to Definition 3.1.5 (a), (c), and Remark 3.1.6 (since the smoothness $\alpha$ may be positive or negative), there exists $F \in B_{\alpha, p a r}^{p}\left(\mathbb{R}^{n} \times \mathbb{R}\right)$ with support in $\mathbb{R}^{n} \times[0, \infty)$, such that $\left.F\right|_{\Omega \times(-\infty, T)}=f$. Now, from the mapping properties of the differential operators with 
respect to time and space (Theorem 2.1.16),

$$
\partial_{t} F, \Delta F \in B_{\alpha-2, p a r}^{p}\left(\mathbb{R}^{n} \times \mathbb{R}\right)
$$

Also, differentiation does not change the support condition of $F$, and

$$
\left.\left(\left(\partial_{t}-\Delta\right) F\right)\right|_{\Omega \times(-\infty, T)}=\left(\partial_{t}-\Delta\right)\left(\left.F\right|_{\Omega \times(-\infty, T)}\right)=\left(\partial_{t}-\Delta\right) f .
$$

Therefore, we may conclude that

$$
\left(\partial_{t}-\Delta\right) f \in{ }_{0} B_{\alpha-2, p a r}^{p}(\Omega \times(0, T),
$$

with appropriate norm estimates. A similar argument yields the second mapping property in the theorem.

Before we start the presentation about the normal derivative, we state and prove a lemma of independent interest, which will be needed in our further discussion.

Lemma 7.2.2. Let $(\Omega, \mu)$ be a measure space, E a separable metric space, and $1<p<\infty$. Then for any $f \in L^{p}\left(\Omega, E^{*}\right)$ there exists a linear functional $\Lambda_{f}$ from $\left(L^{p^{\prime}}(\Omega, E)\right)^{*}$, associated with $f$, such that the correspondence $f \mapsto \Lambda_{f}$ is one-to-one.

Proof. Let us consider $f$ from $L^{p}\left(\Omega, E^{*}\right)$ and define

$$
\left\langle\Lambda_{f}, g\right\rangle=\int_{\Omega}\langle f(x), g(x)\rangle_{E^{*}, E} d \mu(x) \quad \text { for } \quad g \in L^{p^{\prime}}(\Omega, E) .
$$

Then

$$
\begin{aligned}
\left|\left\langle\Lambda_{f}, g\right\rangle\right| & \leq \int_{\Omega}\|f(x)\|_{E^{*}}\|g(x)\|_{E} d \mu(x) \\
& \leq\|f\|_{L^{p}\left(\Omega, E^{*}\right)}\|g\|_{L^{p^{\prime}}(\Omega, E)}<\infty
\end{aligned}
$$


hence the operator

$$
L^{p}\left(\Omega, E^{*}\right) \ni f \longmapsto \Lambda_{f} \in\left(L^{p^{\prime}}(\Omega, E)\right)^{*}
$$

is well-defined, linear and bounded. To show that this operator is one-to-one, we consider $f \in L^{p}\left(\Omega, E^{*}\right)$ with $\Lambda_{f}=0$, and show that $f(x)=0$ for $x \in E^{*}$. For this, let us fix $e \in E$ and define $f_{e}: \Omega \rightarrow \mathbb{R}$ by $f_{e}(x)=\langle f(x), e\rangle$. For a subset $A$ of $\Omega$,

$$
0=\left\langle\Lambda_{f}, \chi_{A} e\right\rangle=\int_{\Omega}\left\langle f(x), \chi_{A}\right\rangle d \mu(x)=\int_{A}\langle f(x), e\rangle d \mu(x)=\int_{A} f_{e}(x) d \mu(x)
$$

hence there exists $S_{e} \subseteq \Omega$ with $\mu\left(S_{e}\right)=0$ such that $f_{e}(x)=0$ for every $x \in \Omega \backslash S_{e}$. Since $E$ is separable, there is a sequence $\left(e_{j}\right)_{j \in \mathbb{N}} \subseteq E$ dense in $E$. Denote $\cup_{j=1}^{\infty} S_{e_{j}}$ by $S$. Then $S \subseteq \Omega, \mu(S)=0$, and for every $x \in \Omega \backslash S, f_{e_{j}}(x)=0$, therefore $\left\langle f(x), e_{j}\right\rangle=0$ for every $j \in \mathbb{N}$ and $x \in \Omega \backslash S$. Using the fact that $\left(e_{j}\right)_{j \in \mathbb{N}}$ is dense in $E$, we can conclude that $f=0$ in $E^{*}$.

Note. In Lemma 7.2.2 with the extra assumptions that $\mu$ is a finite measure and that $E^{*}$ is separable, we can conclude that $L^{p}\left(\Omega, E^{*}\right)=\left(L^{p^{\prime}}(\Omega, E)\right)^{*}$, according to [DiUh], IV.1.

Definition 7.2.3. Given a bounded Lipschitz domain $\Omega \subseteq \mathbb{R}^{n}, 0<T<\infty$, and $1<p<\infty, 0<\alpha<1$, let us introduce the space

$$
\begin{array}{r}
{ }_{0} \mathcal{B}_{\alpha}^{p}:=\left\{(u, w) \in{ }_{0} B_{1-\alpha+1 / p, p a r}^{p}(\Omega \times(0, T)) \oplus{ }_{z} B_{-1-\alpha+1 / p, p a r}^{p}(\Omega \times(0, T)):\right. \\
\left.\quad\left(\partial_{t}-\Delta\right) u=\left.w\right|_{\Omega \times(0, T)}\right\} .
\end{array}
$$

Define

$$
\partial_{\nu}:{ }_{0} \mathcal{B}_{\alpha}^{p} \longrightarrow{ }_{0} B_{-\alpha, p a r}^{p}(\partial \Omega \times(0, T))=\left({ }^{0} B_{\alpha, p a r}^{p^{\prime}}(\partial \Omega \times(0, T))\right)^{*}
$$


by

$$
\left\langle\partial_{\nu}(u, w), f\right\rangle:= \begin{cases}\langle\nabla u, \nabla F\rangle-\langle w, F\rangle+\left\langle\partial_{t} u, F\right\rangle, & \text { if } \quad 0<\alpha+\frac{1}{p}<1, \\ \langle\nabla u, \nabla F\rangle-\langle w, F\rangle-\left\langle u, \partial_{t} F\right\rangle, & \text { if } \quad \alpha+\frac{1}{p}>1 .\end{cases}
$$

Note that since $f \in{ }^{0} B_{\alpha, p a r}^{p^{\prime}}(\partial \Omega \times(0, T))$, by Theorem 7.1.4, hence for $\left(\alpha, \frac{1}{p^{\prime}}\right) \in$ interior of $O A B C D E$, there exists $F$ from ${ }^{0} B_{\alpha+1 / p^{\prime}, \text { par }}^{p^{\prime}}(\Omega \times(0, T))$ such that $\operatorname{Tr} F=f$. Also,

$$
\left\|\partial_{\nu}(u, w)\right\|_{0} B_{-\alpha, p a r}^{p}(\partial \Omega \times(0, T)) \leq c\left(\|u\|_{0} B_{1-\alpha+1 / p, p a r}^{p}(\Omega \times(0, T))+\|w\|_{z B_{-1-\alpha+1 / p, p a r}^{p}(\Omega \times(0, T))}\right) .
$$

Remark 7.2.4. In order to show that $\partial_{\nu}(u, w)$ exists for $\alpha+\frac{1}{p}=1$ as well, it is enough to show that ${ }_{0} \mathcal{B}_{\alpha}^{p}$ is a complex interpolation scale. Applying Lemma 1.2.33 with $X_{i}={ }_{0} B_{1-\alpha+i+1 / p_{i}, p a r}^{p_{i}}(\Omega \times(0, T)) \oplus{ }_{z} B_{-1-\alpha_{i}+1 / p_{i}, p a r}^{p_{i}}(\Omega \times(0, T)), Y_{i}=\{0\}$, $D=\left(\partial_{t}-\Delta\right) u-\left.w\right|_{\Omega \times(0, T)}$ and $G=\left(\left.\Pi_{\text {par }}(\cdot)\right|_{\Omega \times(0, T)}, 0\right)$, where $\Pi_{\text {par }}$ is the caloric Newtonian potential (see Section 7.3), we obtain that

$$
\begin{aligned}
& {\left[{ }_{0} \mathcal{B}_{\alpha_{0},{ }_{0}}^{p_{0}} \mathcal{B}_{\alpha_{1}}^{p_{1}}\right]_{\theta}} \\
& =\left\{(u, w) \in{ }_{0} B_{1-\alpha+1 / p, p a r}^{p}(\Omega \times(0, T)) \oplus{ }_{z} B_{-1-\alpha+1 / p, p a r}^{p}(\Omega \times(0, T)): D(u, w)=0\right\} \\
& ={ }_{0} \mathcal{B}_{\alpha}^{p},
\end{aligned}
$$

where $0<\theta<1, \frac{1}{p}=\frac{1-\theta}{p_{0}}+\frac{\theta}{p_{1}}$ and $\alpha=(1-\theta) \alpha_{0}+\theta \alpha_{1}$. Here we were using the facts that, for the given range of indices, the spaces ${ }_{0} B_{1-\alpha+1 / p, p a r}^{p}(\Omega \times(0, T))$ and ${ }_{z} B_{-1-\alpha+1 / p, p a r}^{p}(\Omega \times(0, T))$ are complex interpolation scales (see Theorems 3.4.10 and 3.4.11).

Remark 7.2.5. For every $u \in{ }_{0} B_{1-\alpha+1 / p, p a r}^{p}(\Omega \times(0, T))$ there exists $w$ that belongs to ${ }_{z} B_{-1-\alpha+1 / p, p a r}^{p}(\Omega \times(0, T))$ such that the pair $(u, w) \in{ }_{0} \mathcal{B}_{\alpha}^{p}$. 
Indeed, take $w=\operatorname{Ext}\left(\left(\partial_{t}-\Delta\right) u\right)$. Observe that, by Theorem 7.2.1 and a duality result of spaces with built-in initial conditions (Corollary 3.1.14), for $1 / p+1 / p^{\prime}=1$,

$$
\left(\partial_{t}-\Delta\right) u \in{ }_{0} B_{-1-\alpha+1 / p, p a r}^{p}(\Omega \times(0, T))=\left({ }^{z} B_{\alpha+1 / p^{\prime}, p a r}^{p^{\prime}}(\Omega \times(0, T))\right)^{*} .
$$

Since ${ }^{z} B_{\alpha+1 / p^{\prime}, \text { par }}^{p^{\prime}}(\Omega \times(0, T))$ is a closed subspace of ${ }^{0} B_{\alpha+1 / p^{\prime}, \text { par }}^{p^{\prime}}(\Omega \times(0, T))$, by the Hahn-Banach theorem, there exists an extension of the functional $\left(\partial_{t}-\Delta\right) u$, i.e.

$$
w=\operatorname{Ext}\left(\left(\partial_{t}-\Delta\right) u\right) \in\left({ }^{0} B_{\alpha+1 / p^{\prime}, p a r}^{p^{\prime}}(\Omega \times(0, T))\right)^{*}={ }_{z} B_{-1-\alpha+1 / p, p a r}^{p}(\Omega \times(0, T)),
$$

where we have also used Theorem 3.1.11.

Definition 7.2.6. Given a bounded Lipschitz domain $\Omega \subseteq \mathbb{R}^{n}, 0<T<\infty$, and $1<p<\infty, 0<\alpha<1$, let us introduce the space

$$
\begin{array}{r}
{ }^{0} \mathcal{B}_{\alpha}^{p}:=\left\{(u, w) \in{ }^{0} B_{1-\alpha+1 / p, p a r}^{p}(\Omega \times(0, T)) \oplus{ }^{z} B_{-1-\alpha+1 / p, p a r}^{p}(\Omega \times(0, T)):\right. \\
\left.\left(\partial_{t}+\Delta\right) u=\left.w\right|_{\Omega \times(0, T)}\right\} .
\end{array}
$$

Define

$$
\partial_{\nu}:{ }^{0} \mathcal{B}_{\alpha}^{p} \longrightarrow{ }^{0} B_{-\alpha, p a r}^{p}(\partial \Omega \times(0, T))=\left({ }_{0} B_{\alpha, p a r}^{p^{\prime}}(\partial \Omega \times(0, T))\right)^{*}
$$

by

$$
\left\langle\partial_{\nu}(u, w), f\right\rangle:= \begin{cases}\langle\nabla u, \nabla F\rangle+\langle w, F\rangle-\left\langle\partial_{t} u, F\right\rangle, & \text { if } \quad 0<\alpha+\frac{1}{p}<1, \\ \langle\nabla u, \nabla F\rangle+\langle w, F\rangle+\left\langle u, \partial_{t} F\right\rangle, & \text { if } \quad \alpha+\frac{1}{p}>1 .\end{cases}
$$

Note that since $f \in{ }_{0} B_{\alpha, p a r}^{p^{\prime}}(\partial \Omega \times(0, T))$, by Theorem 7.1.3, hence for $\left(\alpha, \frac{1}{p^{\prime}}\right) \in$ interior of $O A B C D E$, there exists $F$ from $_{0} B_{\alpha+1 / p^{\prime}, \text { par }}^{p^{\prime}}(\Omega \times(0, T))$ such that $\operatorname{Tr} F=f$. Also,

$$
\left\|\partial_{\nu}(u, w)\right\|_{{ }_{0} B_{-\alpha, p a r}^{p}(\partial \Omega \times(0, T))} \leq c\left(\|u\|_{{ } B_{1-\alpha+1 / p, p a r}^{p}(\Omega \times(0, T))}+\|w\|_{z_{B_{-1-\alpha+1 / p, p a r}^{p}}^{p}(\Omega \times(0, T))}\right) .
$$


Remark 7.2.7. In order to show that $\partial_{\nu}(u, w)$ exists for $\alpha+\frac{1}{p}=1$ as well, it is enough to show that ${ }^{0} \mathcal{B}_{\alpha}^{p}$ is a complex interpolation scale. Applying Lemma 1.2.33 with $X_{i}={ }^{0} B_{1-\alpha+i+1 / p_{i}, p a r}^{p_{i}}(\Omega \times(0, T)) \oplus{ }^{z} B_{-1-\alpha_{i}+1 / p_{i}, p a r}^{p_{i}}(\Omega \times(0, T)), Y_{i}=\{0\}$, $D=\left(\partial_{t}+\Delta\right) u-\left.w\right|_{\Omega \times(0, T)}$ and $G=\left(\left.\Pi_{\text {par }}^{b}(\cdot)\right|_{\Omega \times(0, T)}, 0\right)$, where $\Pi_{\text {par }}^{b}$ is the adjoint caloric Newtonian potential (see Section 7.3), we obtain that

$$
\begin{aligned}
& {\left[{ }^{0} \mathcal{B}_{\alpha_{0}}^{p_{0}},{ }^{0} \mathcal{B}_{\alpha_{1}}^{p_{1}}\right]_{\theta}} \\
& =\left\{(u, w) \in{ }^{0} B_{1-\alpha+1 / p, p a r}^{p}(\Omega \times(0, T)) \oplus{ }^{z} B_{-1-\alpha+1 / p, p a r}^{p}(\Omega \times(0, T)): D(u, w)=0\right\} \\
& ={ }^{0} \mathcal{B}_{\alpha}^{p},
\end{aligned}
$$

where $0<\theta<1, \frac{1}{p}=\frac{1-\theta}{p_{0}}+\frac{\theta}{p_{1}}$ and $\alpha=(1-\theta) \alpha_{0}+\theta \alpha_{1}$. Here we were using the facts that, for the given range of indices, the spaces ${ }^{0} B_{1-\alpha+1 / p, p a r}^{p}(\Omega \times(0, T))$ and ${ }^{z} B_{-1-\alpha+1 / p, p a r}^{p}(\Omega \times(0, T))$ are complex interpolation scales (see Theorems 3.4.10 and 3.4.11).

Remark 7.2.8. For every $u \in{ }^{0} B_{1-\alpha+1 / p, p a r}^{p}(\Omega \times(0, T))$ there exists $w$ that belongs to ${ }^{z} B_{-1-\alpha+1 / p, p a r}^{p}(\Omega \times(0, T))$ such that the pair $(u, w) \in{ }^{o} \mathcal{B}_{\alpha}^{p}$.

Indeed, take $w=\operatorname{Ext}\left(\left(\partial_{t}+\Delta\right) u\right)$. Observe that, by Theorem 7.2.1 and a duality result of spaces with built-in initial conditions (Corollary 3.1.12), for $1 / p+1 / p^{\prime}=1$,

$$
\left(\partial_{t}+\Delta\right) u \in{ }^{0} B_{-1-\alpha+1 / p, p a r}^{p}(\Omega \times(0, T))=\left({ }_{z} B_{\alpha+1 / p^{\prime}, p a r}^{p^{\prime}}(\Omega \times(0, T))\right)^{*} .
$$

Since ${ }_{z} B_{\alpha+1 / p^{\prime}, p a r}^{p^{\prime}}(\Omega \times(0, T))$ is a closed subspace of ${ }_{0} B_{\alpha+1 / p^{\prime}, p a r}^{p^{\prime}}(\Omega \times(0, T))$, by the Hahn-Banach theorem, there exists an extension of the functional $\left(\partial_{t}+\Delta\right) u$, i.e.

$$
w=\operatorname{Ext}\left(\left(\partial_{t}+\Delta\right) u\right) \in\left({ }_{0} B_{\alpha+1 / p^{\prime}, p a r}^{p^{\prime}}(\Omega \times(0, T))\right)^{*}={ }^{z} B_{-1-\alpha+1 / p, p a r}^{p}(\Omega \times(0, T)),
$$

where we have also used Theorem 3.1.13. 
In what follows we will show that all duality pairings in (7.2.14) are well-defined. The same argument can be applied to show that the duality pairings in (7.2.16) are meaningful, as well, or one could use the properties of the reflection operator in time.

In preparation, we need an intermediate result.

Theorem 7.2.9. Consider a Lipschitz domain $\Omega$ in $\mathbb{R}^{n}, 0<T<\infty$ and indices $p$, $\alpha$ such that $1<p<\infty,-1+\frac{1}{p}<\alpha<\frac{1}{p}$. Then

$$
{ }_{0} B_{\alpha, p a r}^{p}(\Omega \times(0, T))={ }_{z} B_{\alpha, p a r}^{p}(\Omega \times(0, T))
$$

and

$$
{ }^{0} B_{\alpha, p a r}^{p}(\Omega \times(0, T))={ }^{z} B_{\alpha, p a r}^{p}(\Omega \times(0, T)) .
$$

Proof. The ingredients in the proof of (7.2.18) and (7.2.19) are the same, here we show only (7.2.18). Note that in order to prove (7.2.18), it is enough to show that (7.2.18) holds for a smaller range of indices, namely $0<\alpha<\frac{1}{p}$.

Otherwise, if $-1+\frac{1}{p}<\alpha<0$, by Theorem 3.1.13 and Corollary 3.1.14,

$$
\left(\left({ }_{0} B_{\alpha, p a r}^{p}(\Omega \times(0, T))\right)^{*}\right)^{*}=\left({ }^{z} B_{-\alpha, p a r}^{p^{\prime}}(\Omega \times(0, T))\right)^{*}={ }_{0} B_{\alpha, p a r}^{p}(\Omega \times(0, T)) .
$$

Also, from Corollary 3.1.12 and Theorem 3.1.11,

$$
\left(\left({ }_{z} B_{\alpha, p a r}^{p}(\Omega \times(0, T))\right)^{*}\right)^{*}=\left({ }^{0} B_{-\alpha, p a r}^{p^{\prime}}(\Omega \times(0, T))\right)^{*}={ }_{z} B_{\alpha, p a r}^{p}(\Omega \times(0, T)) .
$$

Comparing (7.2.20) and (7.2.21), their second term are equal to each other, due to (7.2.19) for the case when $0<-\alpha<\frac{1}{p^{\prime}}=1-\frac{1}{p}$. Hence, (7.2.18) holds for the range $-1+\frac{1}{p}<\alpha<0$. 
We now turn our attention to the case $0<\alpha<\frac{1}{p}$. It is trivial, from the definition of the spaces involved, that the right-to-left inclusion holds. Suppose $f$ belongs to the left-hand side of (7.2.18). By definition, there exists $F \in B_{\alpha, p a r}^{p}\left(\mathbb{R}^{n} \times \mathbb{R}\right)$ such that $\left.F\right|_{\Omega \times(0, T)}=f$ and $\operatorname{supp} F \subseteq \mathbb{R}^{n} \times[0, \infty)$. Let us introduce

$$
G:=F \chi_{\mathcal{O}}, \quad \text { where } \quad \mathcal{O}:=[\Omega \times(0, T)] \cup\left[\mathbb{R}^{n} \times(T, \infty)\right]
$$

Then it is not hard to see that

$$
\begin{aligned}
& \operatorname{supp} G \subseteq[\bar{\Omega} \times[0, \infty)] \cup\left[\mathbb{R}^{n} \times[T, \infty)\right], \\
& \left.G\right|_{\mathbb{R}^{n} \times(-\infty, T)}=\left.F\right|_{\Omega \times(0, T)}=f .
\end{aligned}
$$

Moreover, we have the following sequences of inequalities.

$$
\begin{aligned}
\int_{\mathbb{R}^{n}}\left\|\left(F \chi_{\mathcal{O}}\right)(x, \cdot)\right\|_{B_{\alpha / 2}^{p}(\mathbb{R})}^{p} d x & \leq \int_{\Omega}\|F(x, \cdot)\|_{B_{\alpha / 2}^{p}(\mathbb{R})}^{p} d x+\int_{\mathbb{R}^{n} \backslash \bar{\Omega}}\|F(x, \cdot)\|_{B_{\alpha / 2}^{p}(\mathbb{R})}^{p} d x \\
& \leq \int_{\mathbb{R}^{n}}\|F(x, \cdot)\|_{B_{\alpha / 2}^{p}(\mathbb{R})}^{p} d x<\infty,
\end{aligned}
$$

since $F \in B_{\alpha, p a r}^{p}\left(\mathbb{R}^{n} \times \mathbb{R}\right)$. On the other hand,

$$
\begin{aligned}
\int_{\mathbb{R}}\left\|\left(F \chi_{\mathcal{O}}\right)(\cdot, t)\right\|_{B_{\alpha}^{p}\left(\mathbb{R}^{n}\right)}^{p} d t & =\int_{0}^{T}\left\|\left(F \chi_{\mathcal{O}}\right)(\cdot, t)\right\|_{B_{\alpha}^{p}\left(\mathbb{R}^{n}\right)}^{p} d t+\int_{T}^{\infty}\left\|\left(F \chi_{\mathcal{O}}\right)(\cdot, t)\right\|_{B_{\alpha}^{p}\left(\mathbb{R}^{n}\right)}^{p} d t \\
& \leq \int_{0}^{T}\|F(\cdot, t)\|_{B_{\alpha}^{p}\left(\mathbb{R}^{n}\right)}^{p} d t+\int_{T}^{\infty}\|F(\cdot, t)\|_{B_{\alpha}^{p}\left(\mathbb{R}^{n}\right)}^{p} d t \\
& \leq \int_{\mathbb{R}}\|F(\cdot, t)\|_{B_{\alpha}^{p}\left(\mathbb{R}^{n}\right)}^{p} d t<\infty,
\end{aligned}
$$

since $F \in B_{\alpha, p a r}^{p}\left(\mathbb{R}^{n} \times \mathbb{R}\right)$. All in all, (7.2.23) and 7.2.24), along with Fubini's property, imply that

$$
G \in B_{\alpha, p a r}^{p}\left(\mathbb{R}^{n} \times \mathbb{R}\right)
$$


Finally, (7.2.22) and (7.2.25) yield the desired conclusion, i.e. that $f$ belongs to the space $_{z} B_{\alpha, p a r}^{p}(\Omega \times(0, T))$.

Returning to the duality parings, note that $\left\langle\partial_{\nu}(u, w), f\right\rangle$ is well-defined.

The pairing $\langle\nabla u, \nabla F\rangle$.

Since $F \in{ }^{0} B_{\alpha+\frac{1}{p^{\prime}}, p a r}^{p^{\prime}}(\Omega \times(0, T))$, as in Theorem 7.2.1, we can show that

$$
\nabla F \in{ }^{0} B_{\alpha+\frac{1}{p^{\prime}}-1, p a r}^{p^{\prime}}(\Omega \times(0, T)) .
$$

Similarly, for $u \in{ }_{0} B_{1-\alpha+1 / p, p a r}^{p}(\Omega \times(0, T))$ we have

$\nabla u \in{ }_{0} B_{-\alpha+1 / p, p a r}^{p}(\Omega \times(0, T))={ }_{z} B_{-\alpha+1 / p, p a r}^{p}(\Omega \times(0, T))=\left({ }^{0} B_{\alpha-1 / p, p a r}^{p^{\prime}}(\Omega \times(0, T))\right)^{*}$,

which can be seen from Theorems 7.2.9 and 3.1.11. Now we may conlude from (7.2.26) and (7.2.27) that the pairing $\langle\nabla u, \nabla F\rangle$ is meaningful.

The pairing $\left\langle\operatorname{Ext}\left(\left(\partial_{t}-\Delta\right) u\right), F\right\rangle$.

We have seen earlier (in Definition 7.2.3), that

$$
\operatorname{Ext}\left(\left(\partial_{t}-\Delta\right) u\right) \in\left({ }^{0} B_{\alpha+\frac{1}{p^{\prime}}, p a r}^{p^{\prime}}(\Omega \times(0, T))\right)^{*}
$$

Since $F \in{ }^{0} B_{\alpha+\frac{1}{p^{\prime}}, p a r}^{p^{\prime}}(\Omega \times(0, T))$, the pairing $\left\langle\operatorname{Ext}\left(\left(\partial_{t}-\Delta\right) u\right), F\right\rangle$ is well-defined.

The pairing $\left\langle\partial_{t} u, F\right\rangle$ in the case when $\alpha+\frac{1}{p}<1$.

Due to the fact that

$$
u \in{ }_{0} B_{1-\alpha+\frac{1}{p}, p a r}^{p}(\Omega \times(0, T)) \hookrightarrow B_{1-\alpha+\frac{1}{p}, \text { par }}^{p}(\Omega \times(0, T))
$$

and Fubini's property,

$$
u \in L^{p}\left(\Omega ; B_{\left(1-\alpha+\frac{1}{p}\right) / 2}^{p}(0, T)\right)
$$


Since it is clear that for any intrval $I$ in $\mathbb{R}$ and any $\alpha \in \mathbb{R}, 0<p, q \leq \infty$, the operator

$$
\partial_{t}: B_{\alpha}^{p, q}(I) \longrightarrow B_{\alpha-1}^{p, q}(I)
$$

is bounded, (7.2.28) further implies that

$$
\partial_{t} u \in L^{p}\left(\Omega ; B_{\left(-1-\alpha+\frac{1}{p}\right) / 2}^{p}(0, T)\right)
$$

One may also use Proposition 2.18 from [JeKe] to conclude (7.2.30) from (7.2.28).

Going further, based on (43) and (41) of [Tri5], the space $B_{\left(-1-\alpha+\frac{1}{p}\right) / 2}^{p}(0, T)$ can be identified with $\left(B_{\left(1+\alpha-\frac{1}{p}\right) / 2}^{p^{\prime}}(0, T)\right)^{*}$ provided $-1<\alpha+\frac{1}{p}<1$. Finally, using the abstract result described in Lemma 7.2.2,

$$
\partial_{t} u \in\left[L^{p^{\prime}}\left(\Omega ; B_{\left(\alpha+\frac{1}{p^{\prime}}\right) / 2}^{p^{\prime}}(0, T)\right)\right]^{*} .
$$

On the other hand,

$$
F \in{ }^{0} B_{\alpha+\frac{1}{p^{\prime}}, p a r}^{p^{\prime}}(\Omega \times(0, T)) \hookrightarrow B_{\alpha+\frac{1}{p^{\prime}}, p a r}^{p^{\prime}}(\Omega \times(0, T)),
$$

and by Fubini's property,

$$
F \in L^{p^{\prime}}\left(\Omega ; B_{\left(\alpha+\frac{1}{p^{\prime}}\right) / 2}^{p^{\prime}}(0, T)\right)
$$

Finally, from (7.2.31) and (7.2.32) we can see that $\left\langle\partial_{t} u, F\right\rangle$ is meaningful for $\alpha+\frac{1}{p}<1$.

The pairing $\left\langle u, \partial_{t} F\right\rangle$ in the case when $\alpha+\frac{1}{p}>1$.

Similar to the previous case,

$$
u \in L^{p}\left(\Omega ; B_{\left(1-\alpha+\frac{1}{p}\right) / 2}^{p}(0, T)\right)
$$


or, equivalently, by (43) and (41) of [Tri5], if $1<\alpha+\frac{1}{p}<3$,

$$
u \in L^{p}\left(\Omega ;\left(B_{\left(-1+\alpha-\frac{1}{p}\right) / 2}^{p^{\prime}}(0, T)\right)^{*}\right) .
$$

According to the abstract result stated in Lemma 7.2.2, this further implies that

$$
u \in\left[L^{p^{\prime}}\left(\Omega ; B_{\left(-1+\alpha-\frac{1}{p}\right) / 2}^{p^{\prime}}(0, T)\right)\right]^{*} \quad \text { if } \quad 1<\alpha+\frac{1}{p}<3 .
$$

On the other hand, in view of (7.2.29), the membership property (7.2.32) of $F$ from the previous case yields

$$
\partial_{t} F \in L^{p^{\prime}}\left(\Omega ; B_{\left(\alpha+\frac{1}{p^{\prime}}-2\right) / 2}^{p^{\prime}}(0, T)\right)
$$

From (7.2.33) and (7.2.34) we may conclude that the pairing $\left\langle u, \partial_{t} F\right\rangle$ is well-defined for $\alpha+\frac{1}{p}>1$.

In order to show that the definition of $\left\langle\partial_{\nu}(u, w), f\right\rangle$ (in Definition 7.2.3) does not depend on the particular extension $F$ of $f$, we need the following proposition.

Proposition 7.2.10. For a bounded Lipschitz domain $\Omega \subseteq \mathbb{R}^{n}, 0<T<\infty$, and $1<p<\infty, \frac{1}{p}<\alpha<1+\frac{1}{p}$, we have

$$
\left\{u \in{ }_{0} B_{\alpha, p a r}^{p}(\Omega \times(0, T)): \operatorname{Tr} u=0\right\}={ }_{z} B_{\alpha, p a r}^{p}(\Omega \times(0, T)) .
$$

Proof. The right-to-left inclusion in (7.2.35) naturally holds. Assume $u$ belongs to ${ }_{0} B_{\alpha, p a r}^{p}(\Omega \times(0, T))$ with $\operatorname{Tr} u=0$. Then, by definition, $u=\left.U\right|_{\Omega \times(0, T)}$, where $U \in B_{\alpha, \text { par }}^{p}\left(\mathbb{R}^{n} \times \mathbb{R}\right)$ with support included in $\mathbb{R}^{n} \times[0, \infty)$. Let us introduce

$$
W:=\left.U\right|_{\mathbb{R}^{n} \times(-\infty, T)}
$$


and

$$
F:=W \chi_{\Omega \times(-\infty, T)}
$$

Then $W \in B_{\alpha, p a r}^{p}\left(\mathbb{R}^{n} \times(-\infty, T)\right)$ with supp $W \subseteq \mathbb{R}^{n} \times[0, \infty)$, and we will show that $F$ also belongs to the space $B_{\alpha, p a r}^{p}\left(\mathbb{R}^{n} \times(-\infty, T)\right)$. First, observe that

$$
\begin{aligned}
\int_{\mathbb{R}^{n}}\|F(x, \cdot)\|_{B_{\alpha / 2}^{p}(-\infty, T)}^{p} d x & =\int_{\Omega}\|W(x, \cdot)\|_{B_{\alpha / 2}^{p}(-\infty, T)}^{p} d x \\
& \leq \int_{\mathbb{R}^{n}}\|W(x, \cdot)\|_{B_{\alpha / 2}^{p}(-\infty, T)}^{p} d x<\infty
\end{aligned}
$$

since $W \in L^{p}\left(\mathbb{R}^{n} ; B_{\alpha / 2}^{p}(-\infty, T)\right)$. On the other hand,

$$
\int_{-\infty}^{T}\|F(\cdot, t)\|_{B_{\alpha}^{p}\left(\mathbb{R}^{n}\right)}^{p} d t=\int_{-\infty}^{T}\left\|\left(W \chi_{\Omega}\right)(\cdot, t)\right\|_{B_{\alpha}^{p}\left(\mathbb{R}^{n}\right)}^{p} d t<\infty .
$$

An explanation is in order here. If we denote by $\widetilde{W \chi_{\Omega}}$ the extension of $W \chi_{\Omega}$ by 0 outside $\Omega$, i.e.

$$
\widetilde{W \chi_{\Omega}}(\cdot, t)= \begin{cases}W \chi_{\Omega}(\cdot, t) & \text { in } \Omega_{+}, \\ 0 & \text { in } \Omega_{-},\end{cases}
$$

then, since $W \chi_{\Omega}(\cdot, t) \in B_{\alpha}^{p}\left(\Omega_{+}\right)$for almost every $t \in(-\infty, T)$, and $\operatorname{Tr} W(\cdot, t)=$ $\operatorname{Tr} U(\cdot, t)=\operatorname{Tr} u=0$ on $\partial \Omega$, based on Lemma 3.1.17 we obtain that

$$
W \chi_{\Omega}(\cdot, t) \in B_{\alpha}^{p}\left(\mathbb{R}^{n}\right) \quad \text { for almost every } t \in(-\infty, T)
$$

On account of the Fubini property, (7.2.36) and (7.2.37), we may conclude that

$$
F \in B_{\alpha, p a r}^{p}\left(\mathbb{R}^{n} \times(-\infty, T)\right)
$$

Hence, there exists $G \in B_{\alpha, p a r}^{p}\left(\mathbb{R}^{n} \times \mathbb{R}\right)$ such that $\left.G\right|_{\mathbb{R}^{n} \times(-\infty, T)}=F$. Moreover, since the support of $F$ is included in $\Omega \times[0, T)$,

$$
\begin{aligned}
& \operatorname{supp} \subseteq[\bar{\Omega} \times[0, \infty)] \cup\left[\mathbb{R}^{n} \times[T, \infty)\right] \\
& \left.G\right|_{\Omega \times(0, T)}=\left.F\right|_{\Omega \times(0, T)}=\left.W\right|_{\Omega \times(0, T)}=\left.U\right|_{\Omega \times(0, T)}=u
\end{aligned}
$$


which amounts to

$$
F \in{ }_{z} B_{\alpha, p a r}^{p}(\Omega \times(0, T)) .
$$

Finally, with the contribution of the facts that $F, W$ and $U$ coincide on $\Omega \times[0, T)$, and $\left.U\right|_{\Omega \times(0, T)}=u$, we may deduce that $u \in{ }_{z} B_{\alpha, p a r}^{p}(\Omega \times(0, T))$.

In a similar manner, or using the reflection operator in time, given by $R f(x, t)=$ $f(x, T-t)$ for $(x, t) \in \mathbb{R}^{n} \times \mathbb{R}$, we can show the proper analogue of the above result for opposite initial conditions, formulated in the following proposition.

Proposition 7.2.11. For a bounded Lipschitz domain $\Omega \subseteq \mathbb{R}^{n}, 0<T<\infty$, and $1<p<\infty, \frac{1}{p}<\alpha<1+\frac{1}{p}$, we have

$$
\left\{u \in{ }^{0} B_{\alpha, p a r}^{p}(\Omega \times(0, T)): \operatorname{Tr} u=0\right\}={ }^{z} B_{\alpha, p a r}^{p}(\Omega \times(0, T)) .
$$

Now we are ready to show that the definition of $\left\langle\partial_{\nu}(u, w), f\right\rangle$ is independent of the particular choice of $F$.

Let $F_{1}, F_{2} \in{ }^{0} B_{\alpha+\frac{1}{p^{\prime}}, p a r}^{p^{\prime}}(\Omega \times(0, T))$ such that $\operatorname{Tr} F_{1}=\operatorname{Tr} F_{2}=f$ and set $F:=$ $F_{1}-F_{2}$. Then $\operatorname{Tr} F=0$, and Proposition 7.2.11 implies that

$$
F \in{ }^{z} B_{\alpha+\frac{1}{p^{\prime}}, p a r}^{p^{\prime}}(\Omega \times(0, T)),
$$

provided $\frac{1}{p^{\prime}}<\alpha+\frac{1}{p^{\prime}}<1+\frac{1}{p^{\prime}}$, which, naturally, holds for any $0<\alpha<1$.

Recall from Definition 7.2.3 and Remark 7.2.5 that $w=\operatorname{Ext}\left(\left(\partial_{t}-\Delta\right) u\right)$, where

$$
\left(\partial_{t}-\Delta\right) u \in\left({ }^{z} B_{\alpha+1 / p^{\prime}, p a r}^{p^{\prime}}(\Omega \times(0, T))\right)^{*} .
$$

In view of $(7.2 .39)$, the extension of the linear functional $\left(\partial_{t}-\Delta\right) u$ agrees with the functional itself, and the pairing $\langle w, F\rangle$ in (7.2.14) can be expressed as $\left\langle\left(\partial_{t}-\Delta\right) u, F\right\rangle$. 
Hence

$$
\langle\nabla u, \nabla F\rangle-\left\langle\left(\partial_{t}-\Delta\right) u, F\right\rangle+\left\langle\partial_{t} u, F\right\rangle=\langle\nabla u, \nabla F\rangle+\langle\Delta u, F\rangle=0 .
$$

The integration by parts in the last step of (7.2.40) is justified by taking dense subclasses of the function spaces involved. In a similar way we show that

$$
\langle\nabla u, \nabla F\rangle-\left\langle\left(\partial_{t}-\Delta\right) u, F\right\rangle-\left\langle u, \partial_{t} F\right\rangle=0
$$

consequently, the definition of $\left\langle\partial_{\nu}(u, w), f\right\rangle$ in (7.2.14) does not dependent of the extension $F$ of $f$.

Analogously, the definition of $\left\langle\partial_{\nu}(u, w), f\right\rangle$ in (7.2.16) is independent of the particular extension $F$ of $f$.

\subsection{The caloric Newtonian potential}

The purpose of this section is to introduce and study the parabolic Newtonian potential operator and its adjoint operator. We begin with a definition.

Definition 7.3.1. The caloric Newtonian potential $\Pi_{\text {par }}: \mathcal{E}^{\prime}\left(\mathbb{R}^{n} \times \mathbb{R}\right) \rightarrow \mathcal{D}^{\prime}\left(\mathbb{R}^{n} \times \mathbb{R}\right)$ is defined as

$$
\left(\Pi_{\text {par }} f\right)(x, t):=\int_{\mathbb{R}^{n} \times \mathbb{R}} E(x-y, t-s) f(y, s) d y d s
$$

where $E$ stands for the fundamental solution of the heat operator.

Proposition 7.3.2. For $\varphi, \psi \in C_{c}^{\infty}\left(\mathbb{R}^{n} \times \mathbb{R}\right)$ the operators

$$
\varphi \Pi_{\text {par }} \psi: L^{p}\left(\mathbb{R}^{n} \times \mathbb{R}\right) \longrightarrow L^{p}\left(\mathbb{R}^{n} \times \mathbb{R}\right)
$$


and

$$
\varphi \nabla \Pi_{\text {par }} \psi: L^{p}\left(\mathbb{R}^{n} \times \mathbb{R}\right) \longrightarrow L^{p}\left(\mathbb{R}^{n} \times \mathbb{R}\right)
$$

are bounded for $1<p<\infty$.

Proof. Assume $\operatorname{supp} \varphi \subseteq K_{1} \times I_{1}$ and $\operatorname{supp} \psi \subseteq K_{2} \times I_{2}$, where $K_{1}, K_{2}$ are compact subsets of $\mathbb{R}^{n}$, and $I_{1}, I_{2}$ are compact intervals in $\mathbb{R}$. The proof relies on Schur's Lemma (cf., e.g., Proposition 5.1 on p. 488 in Vol. I of [Tay]), hence, for (7.3.41) and (7.3.42) to hold, it is enough to show the following:

$$
\begin{gathered}
\sup _{(x, t) \in K_{1} \times I_{1}} \int_{K_{2} \times I_{1}}|\varphi(x, t)||E(x-y, t-s)||\psi(y, s)| d y d s<\infty \\
\sup _{(y, s) \in K_{2} \times I_{2}} \int_{K_{1} \times I_{1}}|\varphi(x, t)||E(x-y, t-s)||\psi(y, s)| d x d t<\infty \\
\sup _{(x, t) \in K_{1} \times I_{1}} \int_{K_{2} \times I_{1}}|\varphi(x, t)||\nabla E(x-y, t-s)||\psi(y, s)| d y d s<\infty
\end{gathered}
$$

and

$$
\sup _{(y, s) \in K_{2} \times I_{2}} \int_{K_{1} \times I_{1}}|\varphi(x, t)||\nabla E(x-y, t-s)||\psi(y, s)| d x d t<\infty .
$$

After obtaining an upper bound of the absolute value of the fundamental solution (see (1.2.13)), we make the change of variables $x-y=z \in$ some compact $K \subset \mathbb{R}^{n}$ and $t-s=r^{2}$, where $r$ is in a compact interval $I \subset \mathbb{R}$. Then we obtain that

$$
\begin{aligned}
\int_{K_{2} \times I_{2}}|E(x-y, t-s)| d y d s & \leq c \int_{K_{2} \times I_{2}} \frac{1}{\left(|x-y|+|t-s|^{1 / 2}\right)^{n}} d y d s \\
& \leq c \int_{K \times I} \frac{|r|}{(|z|+|r|)^{n}} d z d r \leq c \int_{\substack{|z|<c,|r|<c \\
(z, r) \in \mathbb{R}^{n} \times \mathbb{R}}} \frac{1}{(|z|+|r|)^{n-1}} d z d r \\
& \leq c<\infty .
\end{aligned}
$$


The same argument yields (7.3.44). As far as (7.3.45) is concerned, reasonong in a similar fashion, we obtain that

$$
\begin{aligned}
\int_{K_{2} \times I_{2}}|\nabla E(x-y, t-s)| d y d s & \leq c \int_{K_{2} \times I_{2}} \frac{1}{\left(|x-y|+|t-s|^{1 / 2}\right)^{n+1}} d y d s \\
& \leq c \int_{K \times I} \frac{|r|}{(|z|+|r|)^{n+1}} d z d r \leq c \int_{\substack{|z|<c,|r|<c \\
(z, r) \in \mathbb{R}^{n} \times \mathbb{R}}} \frac{1}{(|z|+|r|)^{n}} d z d r \\
& \leq c<\infty .
\end{aligned}
$$

Since (7.3.46) is handled in the same way as (7.3.45), the proof of the proposition is finished.

Another important property of the caloric Newtonian potential is given in the following proposition.

Proposition 7.3.3. For $1<p<\infty$ the operator

$$
\nabla^{2} \Pi_{\text {par }}: L^{p}\left(\mathbb{R}^{n} \times \mathbb{R}\right) \longrightarrow L^{p}\left(\mathbb{R}^{n} \times \mathbb{R}\right)
$$

is bounded.

Proof. According to Theorem 4.7 from [BrCe] (or [CoGu], [CoWe1]), for (7.3.47) to hold, it is enough to show that

(a) $\nabla^{2} \Pi_{\text {par }}: L^{2}\left(\mathbb{R}^{n} \times \mathbb{R}\right) \longrightarrow L^{2}\left(\mathbb{R}^{n} \times \mathbb{R}\right)$ is bounded;

(b) The kernel $k((x, t),(y, s))$ of $\nabla^{2} \Pi_{p a r}$ satisfies the following condition:

For a fixed $\left(x_{0}, t_{0}\right) \in \mathbb{R}^{n} \times \mathbb{R}$ and $r>0$, there exist $c, \beta>0, M>1$

such that, for any $(x, t) \in B_{r}\left(x_{0}, t_{0}\right)$ and $(y, s) \notin B_{M r}\left(x_{0}, t_{0}\right)$,

$$
\left|k\left(\left(x_{0}, t_{0}\right),(y, s)\right)-k((x, t),(y, s))\right| \leq c \frac{\left\|\left(x_{0}, t_{0}\right)-(x, t)\right\|_{p a r}^{\beta}}{\left\|\left(x_{0}, t_{0}\right)-(y, s)\right\|_{p a r}^{n+2+\beta}}
$$


(c) The kernel of $\left(\nabla^{2} \Pi_{p a r}\right)^{*}$ satisfies (7.3.48).

Proof of (a). If we set

$$
T_{j k} f(x, t):=\text { p.v. } \int_{\mathbb{R}^{n} \times \mathbb{R}}\left(\partial_{j} \partial_{k} E\right)(x-y, t-s) f(y, s) d y d s
$$

for $j, k=1, \ldots, n$, then evaluating the Fourier transform of the fundamental solution $E$ of the heat operator, we obtain that, for $(\xi, \tau) \in \mathbb{R}^{n} \times \mathbb{R}$,

$$
\begin{aligned}
\mathcal{F}\left(T_{j k} f\right)(\xi, \tau) & =\xi_{j} \xi_{k} \mathcal{F}(E * f)(\xi, \tau) \\
& =\xi_{j} \xi_{k}(\mathcal{F} E)(\xi, \tau)(\mathcal{F} f)(\xi, \tau) \\
& =m(\xi, \tau)(\mathcal{F} f)(\xi, \tau),
\end{aligned}
$$

where $m(\xi, \tau):=\frac{\xi_{j} \xi_{k}}{|\xi|^{2}+i \tau}$. Since $|m(\xi, \tau)| \leq 1$, from Plancherel's theorem it follows that

$$
T_{j k}: L^{2}\left(\mathbb{R}^{n} \times \mathbb{R}\right) \longrightarrow L^{2}\left(\mathbb{R}^{n} \times \mathbb{R}\right)
$$

is bounded.

Proof of (b). Note that the kernel of $\nabla^{2} \Pi_{\text {par }}$ is $k((x, t),(y, s))=\nabla^{2} E(x-y, t-s)$. For $\left(x_{0}, t_{0}\right) \in \mathbb{R}^{n} \times \mathbb{R}$ and $r>0$ fixed, and $(x, t) \in B_{r}\left(x_{0}, t_{0}\right),(y, s) \in B_{M r}\left(x_{0}, t_{0}\right)$ for some $M>1$, applying the Mean Value Theorem, we obtain the following:

$$
\begin{aligned}
\mid k\left(\left(x_{0}, t_{0}\right),(y, s)\right)- & k((x, t),(y, s)) \mid \\
\leq & \int_{0}^{1}\left|\frac{d}{d \theta}\left[k\left((1-\theta)\left(x_{0}, t_{0}\right)+\theta(x, t)-(y, s)\right)\right]\right| d \theta \\
\leq & c \int_{0}^{1}\left|x-x_{0}\right|\left|\left(\nabla^{3} E\right)\left((1-\theta)\left(x_{0}, t_{0}\right)+\theta(x, t)-(y, s)\right)\right| d \theta \\
& +c \int_{0}^{1}\left|t-t_{0}\right|\left|\left(\partial_{\text {time }} \nabla^{2} E\right)\left((1-\theta)\left(x_{0}, t_{0}\right)+\theta(x, t),(y, s)\right)\right| d \theta \\
= & : I_{1}+I_{2} .
\end{aligned}
$$


To estimate $\nabla^{3} E$ and $\partial_{\text {time }} \nabla^{2} E$, note that (1.2.13) and geometrical considerations imply that

$$
\begin{aligned}
I_{1} & \leq c\left|x-x_{0}\right| \int_{0}^{1} \frac{1}{\left\|(1-\theta)\left(x_{0}, t_{0}\right)+\theta(x, t)-(y, s)\right\|_{p a r}^{n+3}} d \theta \\
& \leq c \frac{\left|x-x_{0}\right|}{\left\|\left(x_{0}, t_{0}\right)-(y, s)\right\|_{p a r}^{n+3}} \\
& \leq c \frac{\left\|\left(x_{0}, t_{0}\right)-(x, t)\right\|_{\text {par }}}{\left\|\left(x_{0}, t_{0}\right)-(y, s)\right\|_{p a r}^{n+3}},
\end{aligned}
$$

and

$$
\begin{aligned}
I_{2} & \leq c\left|t-t_{0}\right| \int_{0}^{1} \frac{1}{\left\|(1-\theta)\left(x_{0}, t_{0}\right)+\theta(x, t)-(y, s)\right\|_{\text {par }}^{n+4}} d \theta \\
& \leq c \frac{\left\|\left(x_{0}, t_{0}\right)-(x, t)\right\|_{\text {par }}^{2}}{\left\|\left(x_{0}, t_{0}\right)-(y, s)\right\|_{\text {par }}^{n+4}} \\
& \leq c \frac{\left\|\left(x_{0}, t_{0}\right)-(x, t)\right\|_{\text {par }}}{\left\|\left(x_{0}, t_{0}\right)-(y, s)\right\|_{\text {par }}^{n+3}} .
\end{aligned}
$$

In the last inequality in (7.3.50) we have also used the fact that

$$
\left\|\left(x_{0}, t_{0}\right)-(x, t)\right\|_{p a r} \leq r=\frac{1}{M} M r \leq \frac{1}{M}\left\|\left(x_{0}, t_{0}\right)-(y, s)\right\|_{p a r} .
$$

Consequently, (7.3.48) holds for $\beta=1$ and any $M>1$.

Proof of $(c)$. Note that the kernel of the operator $\left(\nabla^{2} \Pi_{p a r}\right)^{*}$ is

$$
k^{*}((x, t),(y, s))=\nabla_{y}^{2} E(y-x, s-t) .
$$


An analogous resoning to the previous case (b) yields

$$
\begin{aligned}
\mid k^{*}\left(\left(x_{0}, t_{0}\right),(y, s)\right)- & k^{*}((x, t),(y, s)) \mid \\
\leq & \int_{0}^{1}\left|\frac{d}{d \theta}\left[k^{*}\left((1-\theta)\left(x_{0}, t_{0}\right)+\theta(x, t),(y, s)\right)\right]\right| d \theta \\
\leq & c \int_{0}^{1}\left|x-x_{0}\right|\left|\left(\nabla^{3} E\right)\left((y, s)-(1-\theta)\left(x_{0}, t_{0}\right)-\theta(x, t)\right)\right| d \theta \\
& +c \int_{0}^{1}\left|t-t_{0}\right|\left|\left(\partial_{\text {time }} \nabla^{2} E\right)\left((y, s)-(1-\theta)\left(x_{0}, t_{0}\right)-\theta(x, t)\right)\right| d \theta \\
\leq & c\left|x-x_{0}\right| \int_{0}^{1} \frac{1}{\left\|(y, s)-(1-\theta)\left(x_{0}, t_{0}\right)-\theta(x, t)\right\|_{p a r}^{n+3}} d \theta \\
& +c\left|t-t_{0}\right| \int_{0}^{1} \frac{1}{\left\|(y, s)-(1-\theta)\left(x_{0}, t_{0}\right)-\theta(x, t)\right\|_{\text {par }}^{n+4}} d \theta .
\end{aligned}
$$

From this point on one proceeds much as in the previous case, more precisely as in (7.3.49) and (7.3.50), and obtains the desired conclusion, finishing the proof of the proposition.

The result of Proposition 7.3.3, where we replace the two spacial derivatives with one time derivative, is given below.

Proposition 7.3.4. For $1<p<\infty$ the operator

$$
\partial_{\text {time }} \Pi_{\text {par }}: L^{p}\left(\mathbb{R}^{n} \times \mathbb{R}\right) \longrightarrow L^{p}\left(\mathbb{R}^{n} \times \mathbb{R}\right)
$$

is bounded.

Proof. According to Theorem 4.7 from [BrCe] (or [CoGu], [CoWe1]), it is enough to show that

(a) $\partial_{\text {time }} \Pi_{\text {par }}: L^{2}\left(\mathbb{R}^{n} \times \mathbb{R}\right) \longrightarrow L^{2}\left(\mathbb{R}^{n} \times \mathbb{R}\right)$ is bounded.

(b) The kernel $k((x, t),(y, s))$ of $\partial_{\text {time }} \Pi_{p a r}$ satisfies (7.3.48). 
(c) The kernel of $\left(\partial_{\text {time }} \Pi_{\text {par }}\right)^{*}$ satisfies $(7.3 .48)$.

Proof of $(a)$. For $(x, t) \in \mathbb{R}^{n} \times \mathbb{R}$ let us introduce

$$
(T f)(x, t):=\text { p.v. } \int_{\mathbb{R}^{n} \times \mathbb{R}}\left(\partial_{\text {time }} E\right)(x-y, t-s) f(y, s) d y d s
$$

Evaluating the Fourier transform of the fundamental solution $E$, we obtain that, for $(\xi, \tau) \in \mathbb{R}^{n} \times \mathbb{R}$

$$
\begin{aligned}
\mathcal{F}(T f)(\xi, \tau) & =\tau \mathcal{F}(E * f)(\xi, \tau) \\
& =q(\xi, \tau) \mathcal{F} f(\xi, \tau),
\end{aligned}
$$

where $q(\xi, \tau):=\frac{\tau}{|\xi|^{2}+i \tau}$. Since $|q(\xi, \tau)| \leq 1$, by Plancherel's theorem, we conclude that

$$
\partial_{\text {time }} \Pi_{\text {par }}: L^{2}\left(\mathbb{R}^{n} \times \mathbb{R}\right) \longrightarrow L^{2}\left(\mathbb{R}^{n} \times \mathbb{R}\right)
$$

is bounded.

Proof of $(b)$. Note that the kernel $k((x, t),(y, s))$ of the oprator $\partial_{t i m e} \Pi_{p a r}$ is

$$
k((x, t),(y, s))=\partial_{\text {time }} E(x-y, t-s) .
$$

For $\left(x_{0}, t_{0}\right) \in \mathbb{R}^{n} \times \mathbb{R}$ and $r>0$ fixed, and $(x, t) \in B_{r}\left(x_{0}, t_{0}\right),(y, s) \in B_{M r}\left(x_{0}, t_{0}\right)$ for some $M>1$, applying the Mean Value Theorem, we obtain the following:

$$
\begin{aligned}
\mid k\left(\left(x_{0}, t_{0}\right),(y, s)\right)- & k((x, t),(y, s)) \mid \\
\leq & \int_{0}^{1}\left|\frac{d}{d \theta}\left[k\left((1-\theta)\left(x_{0}, t_{0}\right)+\theta(x, t)-(y, s)\right)\right]\right| d \theta \\
\leq & c \int_{0}^{1}\left|x-x_{0}\right|\left|\left(\nabla \partial_{\text {time }} E\right)\left((1-\theta)\left(x_{0}, t_{0}\right)+\theta(x, t)-(y, s)\right)\right| d \theta \\
& +c \int_{0}^{1}\left|t-t_{0}\right|\left|\left(\partial_{\text {time }}^{2} E\right)\left((1-\theta)\left(x_{0}, t_{0}\right)+\theta(x, t),(y, s)\right)\right| d \theta \\
= & : I I_{1}+I I_{2} .
\end{aligned}
$$


To estimate $\nabla \partial_{\text {time }} E$ and $\partial_{\text {time }}^{2} E$, it is not hard to see that (1.2.13) and geometrical considerations give

$$
\begin{aligned}
I I_{1} & \leq c\left|x-x_{0}\right| \int_{0}^{1} \frac{1}{\left\|(1-\theta)\left(x_{0}, t_{0}\right)+\theta(x, t)-(y, s)\right\|_{\text {par }}^{n+3}} d \theta \\
& \leq c \frac{\left|x-x_{0}\right|}{\left\|\left(x_{0}, t_{0}\right)-(y, s)\right\|_{p a r}^{n+3}} \\
& \leq c \frac{\left\|\left(x_{0}, t_{0}\right)-(x, t)\right\|_{\text {par }}}{\left\|\left(x_{0}, t_{0}\right)-(y, s)\right\|_{p a r}^{n+3}},
\end{aligned}
$$

and

$$
\begin{aligned}
I I_{2} & \leq c\left|t-t_{0}\right| \int_{0}^{1} \frac{1}{\left\|(1-\theta)\left(x_{0}, t_{0}\right)+\theta(x, t)-(y, s)\right\|_{p a r}^{n+4}} d \theta \\
& \leq c \frac{\left\|\left(x_{0}, t_{0}\right)-(x, t)\right\|_{p a r}^{2}}{\left\|\left(x_{0}, t_{0}\right)-(y, s)\right\|_{p a r}^{n+4}} \\
& \leq c \frac{\left\|\left(x_{0}, t_{0}\right)-(x, t)\right\|_{\text {par }}}{\left\|\left(x_{0}, t_{0}\right)-(y, s)\right\|_{\text {par }}^{n+3}} .
\end{aligned}
$$

In the last inequality in (7.3.53) we have also used the fact that

$$
\left\|\left(x_{0}, t_{0}\right)-(x, t)\right\|_{p a r} \leq r=\frac{1}{M} M r \leq \frac{1}{M}\left\|\left(x_{0}, t_{0}\right)-(y, s)\right\|_{p a r} .
$$

Consequently, (7.3.48) holds for the kernel $k((x, t),(y, s))$ of $\partial_{t i m e} \Pi_{p a r}$, and for $\beta=1$ and any $M>1$.

Proof of $(c)$. Note that the kernel of the operator $\left(\partial_{t i m e} \Pi_{\text {par }}\right)^{*}$ is

$$
k^{*}((x, t),(y, s))=\partial_{\text {time }} E(y-x, s-t) .
$$


An analogous reasoning to the previous case (b) yields to

$$
\begin{aligned}
\mid k^{*}\left(\left(x_{0}, t_{0}\right),(y, s)\right)- & k^{*}((x, t),(y, s)) \mid \\
\leq & \int_{0}^{1}\left|\frac{d}{d \theta}\left[k^{*}\left((1-\theta)\left(x_{0}, t_{0}\right)+\theta(x, t),(y, s)\right)\right]\right| d \theta \\
\leq & c \int_{0}^{1}\left|x-x_{0}\right|\left|\left(\nabla \partial_{\text {time }} E\right)\left((y, s)-(1-\theta)\left(x_{0}, t_{0}\right)-\theta(x, t)\right)\right| d \theta \\
& +c \int_{0}^{1}\left|t-t_{0}\right|\left|\left(\partial_{\text {time }}^{2} E\right)\left((y, s)-(1-\theta)\left(x_{0}, t_{0}\right)-\theta(x, t)\right)\right| d \theta \\
\leq & c\left|x-x_{0}\right| \int_{0}^{1} \frac{1}{\left\|(y, s)-(1-\theta)\left(x_{0}, t_{0}\right)-\theta(x, t)\right\|_{p a r}^{n+3}} d \theta \\
& +c\left|t-t_{0}\right| \int_{0}^{1} \frac{1}{\left\|(y, s)-(1-\theta)\left(x_{0}, t_{0}\right)-\theta(x, t)\right\|_{\text {par }}^{n+4}} d \theta .
\end{aligned}
$$

From this point on one proceeds much as in the previous case, more precisely as in (7.3.52) and (7.3.53), and obtains the desired conclusion.

As a consequece of Propositions 7.3.2, 7.3.3 and 7.3.4 we obtain the following.

Theorem 7.3.5. For $\varphi, \psi \in C_{c}^{\infty}\left(\mathbb{R}^{n} \times \mathbb{R}\right)$ and $1<p<\infty$, the operator

$$
\varphi \Pi_{\text {par }} \psi: L^{p}\left(\mathbb{R}^{n} \times \mathbb{R}\right) \longrightarrow L_{2, \text { par }}^{p}\left(\mathbb{R}^{n} \times \mathbb{R}\right)
$$

is bounded.

Proof. First observe that

$$
\nabla^{2}\left(\varphi \Pi_{p a r} \psi\right)=\varphi\left(\nabla^{2} \Pi_{p a r}\right) \psi+\left(\nabla^{2} \varphi\right) \Pi_{p a r} \psi+(\nabla \varphi) \cdot\left(\nabla \Pi_{p a r} \psi\right)
$$

and

$$
\partial_{\text {time }}\left(\varphi \Pi_{\text {par }} \psi\right)=\varphi\left(\partial_{\text {time }} \Pi_{\text {par }}\right) \psi+\left(\partial_{\text {time }} \varphi\right) \Pi_{\text {par }} \psi
$$

Applying Propositions 7.3.3 and 7.3.4 for the first term in the right-hand side of (7.3.55) and (7.3.56), respectively, along with Proposition 7.3.2, we can conclude 
that

$$
\varphi \Pi_{\text {par }} \psi: L^{p}\left(\mathbb{R}^{n} \times \mathbb{R}\right) \longrightarrow\left\{f \in L^{p}\left(\mathbb{R}^{n} \times \mathbb{R}\right): \partial_{\text {time }} f, \nabla f, \nabla^{2} f \in L^{p}\left(\mathbb{R}^{n} \times \mathbb{R}\right)\right\}
$$

which, in view of [Gru1] (A.1), is what we wanted to show.

We next present the counterpart of the above discussion for the adjoint parabolic Newtonian potential, starting with its definition.

Definition 7.3.6. For $(x, t) \in \mathbb{R}^{n} \times \mathbb{R}$ we introduce the adjoint caloric Newtonian potential as

$$
\left(\Pi_{\text {par }}^{b} f\right)(x, t):=\int_{\mathbb{R}^{n} \times \mathbb{R}} E^{b}(x-y, t-s) f(y, s) d y d s
$$

where $E^{b}$ stands for the fundamental solution of the adjoint heat operator $\partial_{t}+\Delta$.

In order to see how $E^{b}$ is related to $E$, first, let us recall the definition of the reflection operator in time $R$, i.e.

$$
(R f)(x, t):=f(x,-t) \quad \text { for }(x, t) \in \mathbb{R}^{n} \times \mathbb{R} .
$$

One can easily check the following properties of $R$ :

$$
\begin{array}{lrl}
R \circ R=I, & R^{*}=R, \\
\partial_{t} R=-R \partial_{t}, & \Delta R=R \Delta .
\end{array}
$$

On account of Theorem 3.3.3 and Definition 3.3.1 from [Hör], we have that

$$
\left(\partial_{t}-\Delta\right) E=\delta
$$

where $E$ is the fundamental solution of the heat operator, and $\delta$ represents the Kronecker symbol. Rewriting (7.3.59), with the help of the properties of the reflection 
operator, we obtain that

$$
R\left(R \partial_{t}-R \Delta\right) E=\delta
$$

or, equivalently,

$$
\left(\partial_{t}+\Delta\right)(-R E)=R \delta
$$

Note that for any test function $\varphi \in \mathrm{S}\left(\mathbb{R}^{n} \times \mathbb{R}\right)$,

$$
\langle R \delta, \varphi\rangle=\langle\delta, R \varphi\rangle=(R \varphi)(0,0)=\varphi(0,0)=\langle\delta, \varphi\rangle .
$$

Consequently,

$$
\left(\partial_{t}+\Delta\right)(-R E)=\delta
$$

hence

$$
E^{b}(x, t)=(-R E)(x, t)=-E(x,-t)
$$

is the fundamental solution of the adjoint heat operator $\partial_{t}+\Delta$.

Some correlations between the parabolic Newtonian potential and its adjoint is formulated in the following three results.

Lemma 7.3.7. For the caloric Newtonian potential $\Pi_{p a r}$ and the adjoint caloric Newtonian potential $\Pi_{\text {par }}^{b}$, we have

$$
\Pi_{p a r}^{b}=-R \Pi_{p a r} R
$$

and

$$
\Pi_{p a r}=-R \Pi_{p a r}^{b} R,
$$

where $R$ is the reflection operator defined in (7.3.57). 
Proof. First, note that, for $(x, t) \in \mathbb{R}^{n} \times \mathbb{R}$,

$$
\left(\Pi_{\text {par }}^{b} f\right)(x, t)=-\int_{\mathbb{R}^{n} \times \mathbb{R}} E(x-y, s-t) f(y, s) d y d s .
$$

Moreover,

$$
\left(R \Pi_{\text {par }}^{b} f\right)(x, t)=-\int_{\mathbb{R}^{n} \times \mathbb{R}} E(x-y, s+t) f(y, s) d y d s .
$$

Making the change in variables $s=-r \in \mathbb{R}$ yields

$$
\begin{aligned}
\left(R \Pi_{\text {par }}^{b} f\right)(x, t) & =-\int_{\mathbb{R}^{n} \times \mathbb{R}} E(x-y, t-r) f(y,-r) d y d r \\
& =\left(-\prod_{\text {par }} R f\right)(x, t),
\end{aligned}
$$

which further implies (7.3.60). Once this is established, (7.3.61) follows from (7.3.60) via composition with $R$ from the left and from the right, using the fact that $R \circ R$ is the identity operator.

Lemma 7.3.8. One has the following:

$$
\begin{aligned}
& \left(\Pi_{p a r}^{b}\right)^{*}=-\Pi_{p a r}, \\
& \left(\Pi_{p a r}\right)^{*}=-\Pi_{p a r}^{b} .
\end{aligned}
$$

Proof. For two test functions $f$ and $g$, by Lemma 7.3.7, we obtain that

$$
\begin{aligned}
\left\langle\left(\Pi_{\text {par }}^{b}\right)^{*} g, f\right\rangle & =\int_{\mathbb{R}^{n} \times \mathbb{R}} g(x, t)\left(\Pi_{\text {par }}^{b} f\right)(x, t) d x d t \\
& =-\int_{\mathbb{R}^{n} \times \mathbb{R}} g(x, t)\left(R \Pi_{\text {par }} R f\right)(x, t) d x d t \\
& =-\int_{\mathbb{R}^{n} \times \mathbb{R}} \int_{\mathbb{R}^{n} \times \mathbb{R}} g(x, t) E(x-y,-t-s) f(y,-s) d y d s d x d t .
\end{aligned}
$$


The change of variables $-s=r \in \mathbb{R}$ yields

$$
\begin{aligned}
\left\langle\left(\Pi_{p a r}^{b}\right)^{*} g, f\right\rangle & =-\int_{\mathbb{R}^{n} \times \mathbb{R}}\left(\int_{\mathbb{R}^{n} \times \mathbb{R}} g(x, t) E(y-x, r-t) d x d t\right) f(y, r) d y d r \\
& =-\int_{\mathbb{R}^{n} \times \mathbb{R}}\left(\Pi_{\text {par }} g\right)(y, r) f(y, r) d y d r \\
& =\left\langle-\Pi_{\text {par }} g, f\right\rangle,
\end{aligned}
$$

which proves (7.3.62). As for (7.3.63), using Lemma 7.3.7, the fact that $R^{*}=R$, and (7.3.62), we obtain that

$$
\left(\Pi_{p a r}\right)^{*}=\left(-R \Pi_{p a r}^{b} R\right)^{*}=-R^{*}\left(\Pi_{p a r}^{b}\right)^{*} R^{*}=R \Pi_{p a r} R=-\Pi_{p a r}^{b},
$$

which completes the proof of the lemma.

Corollary 7.3.9. For $\varphi, \psi \in C_{c}^{\infty}\left(\mathbb{R}^{n} \times \mathbb{R}\right)$, there holds

$$
\left(\varphi \Pi_{p a r}^{b} \psi\right)^{*}=-\psi \Pi_{p a r} \varphi
$$

and

$$
\left(\varphi \Pi_{p a r} \psi\right)^{*}=-\psi \Pi_{p a r}^{b} \varphi
$$

Proof. Observe that the left-hand side of (7.3.64) can be rewritten as $\psi\left(\Pi_{p a r}^{b}\right)^{*} \varphi$, which, in concert with (7.3.62), implies (7.3.64). In a very similar manner, (7.3.63) yields (7.3.65).

Turning to the mapping properties of the adjoint parabolic Newtonian potential, we start to build on the folowing result.

Theorem 7.3.10. For $\varphi, \psi \in C_{c}^{\infty}\left(\mathbb{R}^{n} \times \mathbb{R}\right)$ and $1<p<\infty$, the operator

$$
\varphi \Pi_{\text {par }}^{b} \psi: L^{p}\left(\mathbb{R}^{n} \times \mathbb{R}\right) \longrightarrow L_{2, p a r}^{p}\left(\mathbb{R}^{n} \times \mathbb{R}\right)
$$

is bounded. 
Proof. The proof readily follows from Theorem 7.3.5 and Lemma 7.3.7.

Proposition 7.3.11. Consider $\varphi, \psi \in C_{c}^{\infty}\left(\mathbb{R}^{n} \times \mathbb{R}\right), 1<p<\infty$, and the caloric Newtonian potential $\Pi_{\text {par }}$. Then the operator

$$
\varphi \Pi_{\text {par }} \psi: L_{-2, p a r}^{p}\left(\mathbb{R}^{n} \times \mathbb{R}\right) \longrightarrow L^{p}\left(\mathbb{R}^{n} \times \mathbb{R}\right)
$$

is bounded.

Proof. Dualizing the result of Theorem 7.3.10, using (7.3.64) and the fact that

$$
\left(L_{2, p a r}^{p}\left(\mathbb{R}^{n} \times \mathbb{R}\right)\right)^{*}=L_{-2, p a r}^{p^{\prime}}\left(\mathbb{R}^{n} \times \mathbb{R}\right) \quad \text { for } \frac{1}{p}+\frac{1}{p^{\prime}}=1
$$

completes the proof of the proposition.

Theorem 7.3.12. Let $\varphi, \psi \in C_{c}^{\infty}\left(\mathbb{R}^{n} \times \mathbb{R}\right)$ and $1<p, q<\infty$. Then,

$$
\varphi \Pi_{\text {par }} \psi: L_{\alpha-2, p a r}^{p}\left(\mathbb{R}^{n} \times \mathbb{R}\right) \longrightarrow L_{\alpha, \text { par }}^{p}\left(\mathbb{R}^{n} \times \mathbb{R}\right)
$$

for $0 \leq \alpha \leq 2$, and

$$
\varphi \prod_{p a r} \psi: B_{\alpha-2, p a r}^{p, q}\left(\mathbb{R}^{n} \times \mathbb{R}\right) \longrightarrow B_{\alpha, p a r}^{p, q}\left(\mathbb{R}^{n} \times \mathbb{R}\right)
$$

for $0<\alpha<2$.

Proof. Complex interpolation (see part (e) or (h) of Theorem 2.1.60) between the results of Theorem 7.3.5 and Proposition 7.3.11 yields to (7.3.67). Furthermore, if we pick $\alpha_{0}, \alpha_{1} \in[0,2]$ such that $\alpha_{0} \neq \alpha_{1}$, then real interpolation (see part (a) of Theorem 2.1.60) of two instances of (7.3.67) implies (7.3.68). 
Theorem 7.3.13. Let $\varphi, \psi \in C_{c}^{\infty}\left(\mathbb{R}^{n} \times \mathbb{R}\right)$ and $1<p, q<\infty$. Then,

$$
\varphi \Pi_{\text {par }}^{b} \psi: L_{\alpha-2, p a r}^{p}\left(\mathbb{R}^{n} \times \mathbb{R}\right) \longrightarrow L_{\alpha, \text { par }}^{p}\left(\mathbb{R}^{n} \times \mathbb{R}\right)
$$

for $0 \leq \alpha \leq 2$, and

$$
\varphi \Pi_{\text {par }}^{b} \psi: B_{\alpha-2, p a r}^{p, q}\left(\mathbb{R}^{n} \times \mathbb{R}\right) \longrightarrow B_{\alpha, p a r}^{p, q}\left(\mathbb{R}^{n} \times \mathbb{R}\right)
$$

for $0<\alpha<2$.

Proof. The result in the theorem follows from Theorem 7.3.12 and the duality result $(7.3 .65)$

Theorem 7.3.14. Consider $\varphi, \psi \in C_{c}^{\infty}\left(\mathbb{R}^{n} \times \mathbb{R}\right)$ and $1<p, q<\infty$. Then,

$$
\varphi \Pi_{\text {par }} \psi: L_{\alpha-2, p a r, 0}^{p}\left(\mathbb{R}^{n} \times(0, \infty)\right) \longrightarrow L_{\alpha, p a r, 0}^{p}\left(\mathbb{R}^{n} \times(0, \infty)\right)
$$

for $0 \leq \alpha \leq 2$, and

$$
\varphi \Pi_{\text {par }} \psi: B_{\alpha-2, p a r, 0}^{p, q}\left(\mathbb{R}^{n} \times(0, \infty)\right) \longrightarrow B_{\alpha, p a r, 0}^{p, q}\left(\mathbb{R}^{n} \times(0, \infty)\right)
$$

for $0<\alpha<2$.

Proof. Recall from Definition 3.1.1 that

$$
L_{\alpha, p a r, 0}^{p}\left(\mathbb{R}^{n} \times(0, \infty)\right)=\left\{f \in L_{\alpha, p a r}^{p}\left(\mathbb{R}^{n} \times \mathbb{R}\right): \operatorname{supp} f \subseteq \mathbb{R}^{n} \times[0, \infty)\right\}
$$

Given a function $f \in L_{\alpha-2, p a r}^{p}\left(\mathbb{R}^{n} \times \mathbb{R}\right)$ with support included in $\mathbb{R}^{n} \times(0, \infty)$, by Theorem 7.3.12 we have

$$
\varphi \Pi_{p a r} \psi f \in L_{\alpha, p a r}^{p}\left(\mathbb{R}^{n} \times \mathbb{R}\right)
$$


Moreover, since

$$
\left(\varphi \Pi_{p a r} \psi f\right)(x, t)=\varphi(x, t) \int_{\mathbb{R}^{n}} \int_{-\infty}^{t} E(x-y, t-s) \psi(y, s) f(y, s) d y d s
$$

and $t-s>0, s \geq 0$, one can conclude that $t>0$. This, along with (7.3.73), implies that $\varphi \Pi_{p a r} \psi f \in L_{\alpha, p a r, 0}^{p}\left(\mathbb{R}^{n} \times(0, \infty)\right)$.

In a very similar manner, by the mapping property of $\varphi \Pi_{p a r} \psi$ on spaces defined on $\mathbb{R}^{n} \times \mathbb{R}$ (second part of Theorem 7.3 .12 ), we obtain (7.3.72).

Theorem 7.3.15. Consider $\varphi, \psi \in C_{c}^{\infty}\left(\mathbb{R}^{n} \times \mathbb{R}\right)$ and $1<p, q<\infty$. Then,

$$
\varphi \Pi_{\text {par }}^{b} \psi: L_{\alpha-2, p a r, T}^{p}\left(\mathbb{R}^{n} \times(-\infty, T)\right) \longrightarrow L_{\alpha, p a r, T}^{p}\left(\mathbb{R}^{n} \times(-\infty, T)\right)
$$

for $0 \leq \alpha \leq 2$, and

$$
\varphi \Pi_{\text {par }}^{b} \psi: B_{\alpha-2, p a r, T}^{p, q}\left(\mathbb{R}^{n} \times(-\infty, T)\right) \longrightarrow B_{\alpha, p a r, T}^{p, q}\left(\mathbb{R}^{n} \times(-\infty, T)\right)
$$

for $0<\alpha<2$.

Proof. Recall from Definition 3.1.1 that

$$
L_{\alpha, p a r, T}^{p}\left(\mathbb{R}^{n} \times(-\infty, T)\right)=\left\{f \in L_{\alpha, p a r}^{p}\left(\mathbb{R}^{n} \times \mathbb{R}\right): \operatorname{supp} f \subseteq \mathbb{R}^{n} \times(-\infty, T]\right\}
$$

Given a function $f \in L_{\alpha-2, p a r}^{p}\left(\mathbb{R}^{n} \times \mathbb{R}\right)$ with support included in $\mathbb{R}^{n} \times(-\infty, T]$, the first part of Theorem 7.3.13 implies that

$$
\varphi \Pi_{p a r}^{b} \psi f \in L_{\alpha, p a r}^{p}\left(\mathbb{R}^{n} \times \mathbb{R}\right)
$$

Moreover, since

$$
\left(\varphi \Pi_{p a r}^{b} \psi f\right)(x, t)=-\varphi(x, t) \int_{\mathbb{R}^{n}} \int_{-\infty}^{t} E(x-y, s-t) \psi(y, s) f(y, s) d y d s
$$


and $t-s>0, s \leq T$, one can conclude that $t<T$. This, along with (7.3.76), implies that $\varphi \Pi_{\text {par }}^{b} \psi f \in L_{\alpha, p a r, T}^{p}\left(\mathbb{R}^{n} \times(-\infty, T)\right)$. In a very similar fashion, by the mapping property of $\varphi \Pi_{\text {par }}^{b} \psi$ on spaces defined on $\mathbb{R}^{n} \times \mathbb{R}$ (second part of Theorem 7.3.13), we obtain (7.3.75).

In the last part of this section we study the ontoness of the heat (and adjoint heat) operator(s) on parabolic Besov spaces with built-in initial conditions.

Theorem 7.3.16. Let $\Omega$ be a Lipschitz domain in $\mathbb{R}^{n}, 0<T<\infty$, and $1<p<\infty$, $0<\alpha<2$. Given $f \in{ }_{0} B_{\alpha-2, p a r}^{p}(\Omega \times(0, T))$, there exists $w \in{ }_{0} B_{\alpha, p a r}^{p}(\Omega \times(0, T))$ such that

$$
\left(\partial_{t}-\Delta\right) w=f \quad \text { in } \Omega \times(0, T)
$$

and

$$
\|w\|_{0} B_{\alpha, p a r}^{p}(\Omega \times(0, T)) \leq c\|f\|_{0} B_{\alpha-2, p a r}^{p}(\Omega \times(0, T)) .
$$

Proof. Given $f \in{ }_{0} B_{\alpha-2, p a r}^{p}(\Omega \times(0, T))$, by definition, $f=\left.F\right|_{\Omega \times(0, T)}$, where $F$ is from $B_{\alpha-2, p a r}^{p}\left(\mathbb{R}^{n} \times \mathbb{R}\right)$ with support included in $\mathbb{R}^{n} \times[0, \infty)$. Let us consider $\varphi, \psi \in C_{c}^{\infty}\left(\mathbb{R}^{n} \times \mathbb{R}\right)$ so that $\varphi \equiv \psi \equiv 1$ in a neighborhood of $\bar{\Omega} \times[0, T]$, and define

$$
w=\left.\left(\varphi \Pi_{p a r} \psi F\right)\right|_{\Omega \times(0, T)} .
$$

According to $(7.3 .72), \varphi \Pi_{\text {par }} \psi F \in B_{\alpha, p a r, 0}^{p}\left(\mathbb{R}^{n} \times(0, \infty)\right)$, hence

$$
w \in{ }_{0} B_{\alpha, p a r}^{p}(\Omega \times(0, T)) .
$$


Moreover,

$$
\begin{aligned}
\left(\partial_{t}-\Delta\right) w & =\left.\left(\varphi\left(\partial_{t}-\Delta\right) \Pi_{p a r} \psi F\right)\right|_{\Omega \times(0, T)} \\
& =\left.(\varphi \psi F)\right|_{\Omega \times(0, T)} \\
& =\left.(F)\right|_{\Omega \times(0, T)} \\
& =f
\end{aligned}
$$

Here we were using the fact that $\left(\partial_{t}-\Delta\right) \Pi_{\text {par }}$ is the identity operator. This can be seen from the following. For some $g \in \mathrm{S}^{\prime}\left(\mathbb{R}^{n} \times \mathbb{R}\right)$,

$$
\begin{aligned}
\left(\mathcal{F} \Pi_{\text {par }} g\right)(\xi, \tau) & =(\mathcal{F} E)(\xi, \tau)(\mathcal{F} g)(\xi, \tau) \\
& =\frac{1}{|\xi|^{2}+i \tau}(\mathcal{F} g)(\xi, \tau), \quad(\xi, \tau) \in \mathbb{R}^{n} \times \mathbb{R} .
\end{aligned}
$$

Therefore, differentiation yields

$$
\begin{aligned}
\left(\partial_{t}-\Delta\right)\left(\Pi_{\text {par }} g\right) & =\left(\partial_{t}-\Delta\right)\left(\mathcal{F}^{-1} \mathcal{F} \Pi_{\text {par }} g\right) \\
& =\mathcal{F}^{-1}(\mathcal{F} g)=g
\end{aligned}
$$

As far as the norm estimates are concerned, note that by definition,

$$
\|w\|_{0 B_{\alpha, p a r}^{p}(\Omega \times(0, T))}=\inf \left\{\|G\|_{B_{\alpha, p a r}^{p}\left(\mathbb{R}^{n} \times \mathbb{R}\right)}:\left.G\right|_{\Omega \times(0, T)}=w, \operatorname{supp} G \subseteq \mathbb{R}^{n} \times[0, \infty)\right\}
$$

Since $\varphi \Pi_{\text {par }} \psi F$ is one of the extensions of $w$, on account of Theorem 7.3.12, we obtain that

$$
\begin{aligned}
\|w\|_{0} B_{\alpha, p a r}^{p}(\Omega \times(0, T)) & \leq\left\|\varphi \Pi_{\text {par }} \psi F\right\|_{B_{\alpha, p a r}^{p}\left(\mathbb{R}^{n} \times \mathbb{R}\right)} \\
& \leq c\|F\|_{B_{\alpha-2, p a r}^{p}\left(\mathbb{R}^{n} \times \mathbb{R}\right)} .
\end{aligned}
$$

Taking the infimum over all $F$ 's such that $\left.F\right|_{\Omega \times(0, T)}=f$ with $\operatorname{supp} F \subseteq \mathbb{R}^{n} \times[0, \infty)$, we arrive at

$$
\|w\|_{0} B_{\alpha, p a r}^{p}(\Omega \times(0, T)) \leq c\|f\|_{0} B_{\alpha-2, p a r}^{p}(\Omega \times(0, T)) .
$$


This concludes the proof of the theorem.

The adjoint counterpart of Theorem 7.3.16 is given below.

Theorem 7.3.17. Let $\Omega$ be a Lipschitz domain in $\mathbb{R}^{n}, 0<T<\infty$, and $1<p<\infty$, $0<\alpha<2$. Given $f \in{ }^{0} B_{\alpha-2, p a r}^{p}(\Omega \times(0, T))$, there exists $w \in{ }^{0} B_{\alpha, p a r}^{p}(\Omega \times(0, T))$ such that

$$
\left(\partial_{t}+\Delta\right) w=f \quad \text { in } \Omega \times(0, T)
$$

and

$$
\|w\|_{{ }_{0} B_{\alpha, p a r}^{p}(\Omega \times(0, T))} \leq c\|f\|_{{ }_{0} B_{\alpha-2, p a r}^{p}(\Omega \times(0, T))} .
$$

Proof. The same steps can be carried out as in the proof of Theorem 7.3.16, or, a different approach can be used involving the reflection operator in time $R$. In the current scenario, since we are considering a finite cylinder $\Omega \times(0, T)$, the oparator $R$ is defined as

$$
R f(x, t):=f(x, T-t)
$$

One can easily check the following properties.

$$
R \circ R=I, \quad \partial_{t} R=-R \partial_{t}, \quad \Delta R=R \Delta,
$$

and, for $1<p, q<\infty, \alpha \in \mathbb{R}^{n} \backslash\{0\}$,

$$
R:{ }_{0} B_{\alpha, p a r}^{p, q}\left(\Omega \times(0, T) \stackrel{\sim}{\longrightarrow}{ }^{0} B_{\alpha, p a r}^{p, q}(\Omega \times(0, T) .\right.
$$

The assumption $f \in{ }^{0} B_{\alpha-2, p a r}^{p}(\Omega \times(0, T))$ implies $R f \in{ }_{0} B_{\alpha-2, p a r}^{p}(\Omega \times(0, T))$, which, by Theorem 7.3.16, futher implies the existence of

$$
v \in{ }_{0} B_{\alpha, p a r}^{p}(\Omega \times(0, T)),
$$


such that

$$
\left(\partial_{t}-\Delta\right) v=R f \quad \text { in } \Omega \times(0, T),
$$

and we have the corresponding norm estimate

$$
\|v\|_{0} B_{\alpha, p a r}^{p}(\Omega \times(0, T)) \leq\|R f\|_{0 B_{\alpha-2, p a r}^{p}(\Omega \times(0, T))} .
$$

Going further, there exists $w \in{ }^{0} B_{\alpha, p a r}^{p}(\Omega \times(0, T))$ so that $R w=-v$. Then (7.3.78) can be rewritten as

$$
\left(\partial_{t}-\Delta\right)(-R w)=R f \quad \text { in } \Omega \times(0, T),
$$

or, equivalently,

$$
\left(\partial_{t}+\Delta\right) w=f \quad \text { in } \Omega \times(0, T),
$$

which is what we wanted to show. To obtain the appropriate norm estimate, note that

$$
\begin{aligned}
\|w\|_{B_{\alpha, p a r}^{p}(\Omega \times(0, T))} & \approx\|R w\|_{0 B_{\alpha, p a r}^{p}(\Omega \times(0, T))} \\
& \approx\|v\|_{0 B_{\alpha, p a r}^{p}(\Omega \times(0, T))} \\
& \leq c\|R f\|_{0 B_{\alpha-2, p a r}^{p}(\Omega \times(0, T))} \\
& \approx\|f\|_{B_{\alpha-2, p a r}^{p}(\Omega \times(0, T))} .
\end{aligned}
$$

This concludes the proof of the theorem. 


\section{Chapter 8}

\section{Boundary value problems}

In this chapter we establish the well-posedness of the Poisson problem for the heat operator with Dirichlet and Neumann boundary conditions in a bounded Lipschitz cylinder. The hexagon OABCDE retains the same significance as before, i.e.

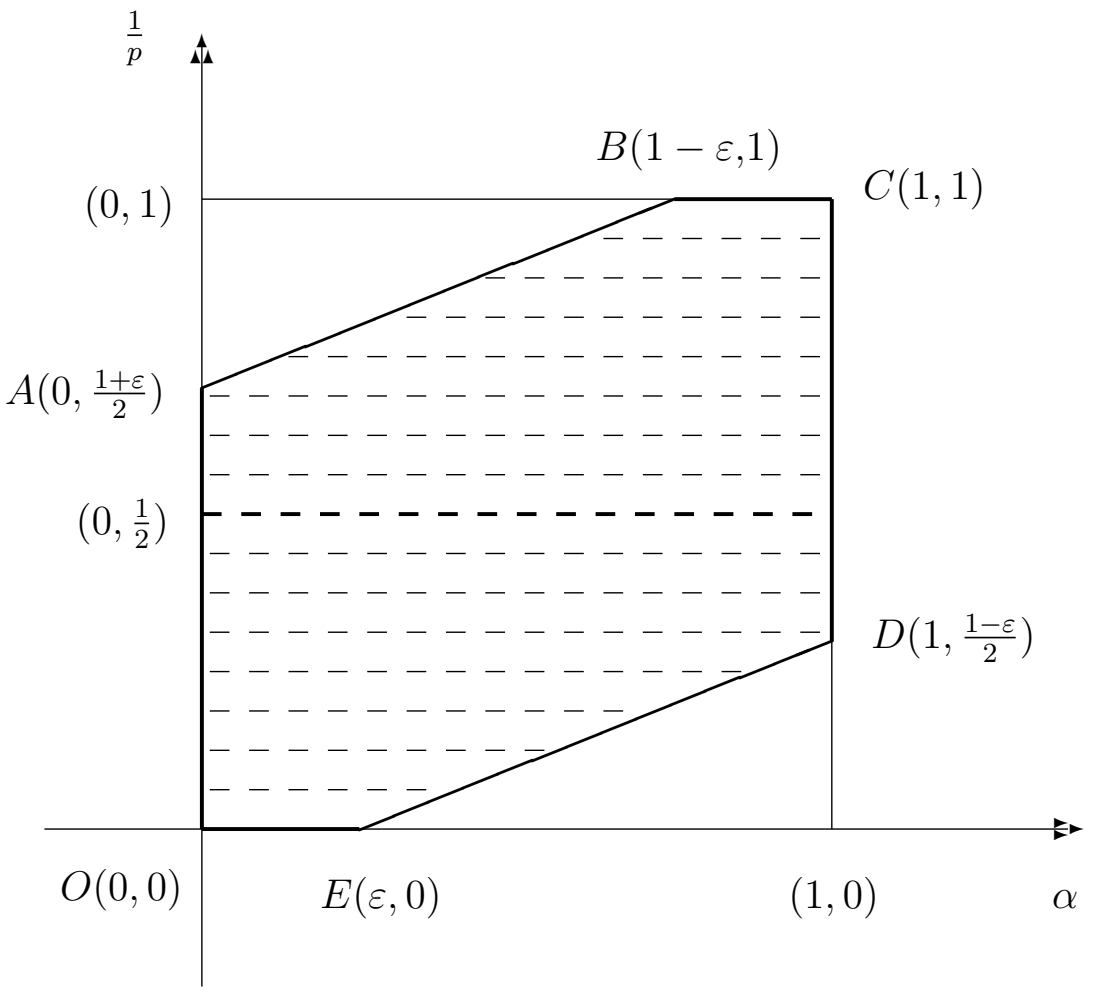




\subsection{The Poisson problem with Dirichlet boundary conditions}

Theorem 8.1.1. For each bounded Lipschitz domain $\Omega$ in $\mathbb{R}^{n}$ there exists some small $\varepsilon=\varepsilon(\partial \Omega)>0$ with the following significance. Let $0<T<\infty$ and assume that the

indices $1<p<\infty$ and $0<\alpha<1$ are such that the point with coordinates $\left(\alpha, \frac{1}{p}\right)$ belongs to the interior of the hexagon $O A B C D E$. Then there exists a unique solution $u \in{ }_{0} B_{\alpha+\frac{1}{p}, p a r}^{p}(\Omega \times(0, T))$ to the problem

$$
(\mathrm{PD})\left\{\begin{array}{l}
\left(\partial_{t}-\Delta\right) u=f \in{ }_{0} B_{\alpha+\frac{1}{p}-2, p a r}^{p}(\Omega \times(0, T)), \\
\left.u\right|_{\partial \Omega \times(0, T)}=g \in{ }_{0} B_{\alpha, p a r}^{p}(\partial \Omega \times(0, T)) .
\end{array}\right.
$$

In addition, $u$ can be represented in terms of caloric layer potentials and

$$
\|u\|_{0 B_{\alpha+\frac{1}{p}, p a r}^{p}(\Omega \times(0, T))} \leq c\left(\|f\|_{0 B_{\alpha+\frac{1}{p}-2, p a r}^{p}}(\Omega \times(0, T))+\|g\|_{0} B_{\alpha, p a r}^{p}(\partial \Omega \times(0, T))\right) .
$$

Proof. Existence and estimates. We first define

$$
u:=\Pi_{p a r} f+\mathcal{D}\left[\left(\frac{1}{2} I+K\right)^{-1}\left(g-\operatorname{Tr}\left(\Pi_{p a r} f\right)\right)\right]
$$

Then, based on the main results of Chapters $4-7, u$ solves (PD), and it belongs to the space ${ }_{0} B_{\alpha+\frac{1}{p}, p a r}^{p}(\Omega \times(0, T))$, satisfying the norm estimate (8.1.1).

Uniqueness. It suffices to show that $f \equiv 0$ and $g \equiv 0$ imply $u \equiv 0$.

Our first goal is to prove that $u \in{ }_{0} B_{\alpha+\frac{1}{p}, p a r}^{p}(\Omega \times(0, T))$ with $\left(\partial_{t}-\Delta\right) u=0$ in $\Omega \times(0, T)$, and that $\operatorname{Tr} u=0$ on $\partial \Omega \times(0, T)$ imply that $\partial_{\nu} u=0$. Considering $h \in{ }^{0} B_{1-\alpha, p a r}^{p^{\prime}}(\partial \Omega \times(0, T))$, from the existence part of Theorem 8.1.2 (which is proved independently of Theorem 8.1.1), it follows that there exists

$$
v \in{ }^{0} B_{1-\alpha+\frac{1}{p^{\prime}}, p a r}^{p^{\prime}}(\partial \Omega \times(0, T))
$$


such that

$$
\left\{\begin{array}{l}
\left(\partial_{t}+\Delta\right) v=0, \\
\operatorname{Tr} v=h .
\end{array}\right.
$$

This, in concert with the facts that $u$ vanishes at $t=0$, while $v$ vanishes at $t=T$, further implies

$$
\begin{aligned}
\left\langle\partial_{\nu} u, h\right\rangle & =\left\langle\partial_{\nu} u, \operatorname{Tr} v\right\rangle=\int_{0}^{T} \int_{\Omega}\langle\nabla u, \nabla v\rangle d x d t+\int_{0}^{T} \int_{\Omega}\left\langle\partial_{t} u, v\right\rangle d x d t \\
& =-\int_{0}^{T} \int_{\Omega}\langle u, \Delta v\rangle d x d t+\int_{0}^{T} \int_{\Omega}\left\langle\partial_{t} u, v\right\rangle d x d t \\
& =\int_{0}^{T} \int_{\Omega}\left[\left\langle u, \partial_{t} v\right\rangle+\left\langle\partial_{t} u, v\right\rangle\right] d x d t \\
& =\int_{0}^{T} \int_{\Omega} \frac{d}{d t}\langle u, v\rangle d x d t=\int_{\Omega}\left(\left.\langle u, v\rangle\right|_{t=0} ^{t=T}\right) d x=0 .
\end{aligned}
$$

Second, we will show that if $u \in{ }_{0} B_{\alpha+\frac{1}{p}, p a r}^{p}(\Omega \times(0, T))$ with $\left(\partial_{t}-\Delta\right) u=0$ in $\Omega \times(0, T), \operatorname{Tr} u=0$, and $\partial_{\nu} u=0$ on $\partial \Omega \times(0, T)$, then for any $(x, t) \in \Omega \times(0, T)$, $u(x, t)=0$. According to [Eva] (pp. $49-50)$,

$$
u(x, t)=\int_{0}^{t} \int_{\mathbb{R}^{n}} E(x-y, t-s) f(y, s) d y d s \quad \text { solves }\left(\partial_{t}-\Delta\right) u=f \text { in } \mathbb{R}^{n} \times(0, \infty) .
$$

In our case $u=u \chi_{\Omega}$, and, for $x \in \Omega, t \in(0, T)$, we have the following:

$$
\begin{aligned}
u(x, t) & =\left(\partial_{t}-\Delta\right) \int_{0}^{t} \int_{\mathbb{R}^{n}} E(x-y, t-s)\left(u \chi_{\Omega}\right)(y, s) d y d s \\
& =\int_{0}^{\infty} \int_{\Omega}\left(\partial_{t}-\Delta_{x}\right)[E(x-y, t-s)] u(y, s) d y d s \\
& =\int_{0}^{\infty} \int_{\Omega}\left(-\partial_{s}-\Delta_{y}\right)[E(x-y, t-s)] u(y, s) d y d s .
\end{aligned}
$$


This further implies that

$$
\begin{aligned}
u(x, t)= & \int_{0}^{\infty} \int_{\Omega} E(x-y, t-s)\left(\partial_{s}-\Delta_{y}\right) u(y, s) d y d s \\
& +\left.\int_{\Omega}[E(x-y, t-s) u(y, s)]\right|_{s=0} ^{s=\infty} d y \\
& -\int_{0}^{\infty} \int_{\partial \Omega} \partial_{\nu_{y}}[E(x-y, t-s)] u(y, s) d \sigma_{y} d s \\
& +\int_{0}^{\infty} \int_{\partial \Omega} E(x-y, t-s) \partial_{\nu_{y}} u(y, s) d \sigma_{y} d s .
\end{aligned}
$$

According to the assumptions we have on $u$, each term on the right-hand side of the last equality in (8.1.3) equals zero, therefore $u \equiv 0$.

The adjoint counterpart of Theorem 8.1.1 is as follows.

Theorem 8.1.2. For each bounded Lipschitz domain $\Omega$ in $\mathbb{R}^{n}$ there exists $\varepsilon=$ $\varepsilon(\partial \Omega)>0$ with the following significance. Let $0<T<\infty$ and assume that $1<p<\infty, 0<\alpha<1$ are such that the point with coordinates $\left(\alpha, \frac{1}{p}\right)$ belongs to the interior of $O A B C D E$. Then there exists a unique solution $u \in{ }^{0} B_{\alpha+\frac{1}{p}, p a r}^{p}(\Omega \times(0, T))$ to the problem

$$
(\mathrm{PD})^{*}\left\{\begin{array}{l}
\left(\partial_{t}+\Delta\right) u=f \in{ }^{0} B_{\alpha+\frac{1}{p}-2, p a r}^{p}(\Omega \times(0, T)), \\
\left.u\right|_{\partial \Omega \times(0, T)}=g \in{ }^{0} B_{\alpha, p a r}^{p}(\partial \Omega \times(0, T)) .
\end{array}\right.
$$

Furthermore, there is an integral representation formula for the solution and

$$
\|u\|_{0 B_{\alpha+\frac{1}{p}, p a r}^{p}(\Omega \times(0, T))} \leq c\left(\|f\|_{0} B_{\alpha+\frac{1}{p}-2, p a r}^{p}(\Omega \times(0, T))+\|g\|_{0 B_{\alpha, p a r}^{p}(\partial \Omega \times(0, T))}\right) .
$$

Proof. Existence and estimates. On account of the results in Chapters 4-7, it is not difficult to check that

$$
u:=\Pi_{\text {par }}^{b} f+R \circ \mathcal{D}\left[\left(\frac{1}{2} I+K\right)^{-1} \circ R\left(g-\operatorname{Tr}\left(\Pi_{\text {par }}^{b} f\right)\right)\right]
$$


solves the problem in $(\mathrm{PD})^{*}$, and that $u$ belongs to ${ }_{0} B_{\alpha+\frac{1}{p}, p a r}^{p}(\Omega \times(0, T))$, satisfying the norm estimate (8.1.4).

Uniqueness. We shall prove that $f \equiv 0$ and $g \equiv 0$ imply $u \equiv 0$.

Our first goal is to show that $u \in{ }^{0} B_{\alpha+\frac{1}{p}, p a r}^{p}(\Omega \times(0, T))$ with $\left(\partial_{t}+\Delta\right) u=0$ and $\operatorname{Tr} u=0$ imply $\partial_{\nu} u=0$.

Considering $h \in{ }_{0} B_{1-\alpha, p a r}^{p^{\prime}}(\partial \Omega \times(0, T))$, from the existence part of Theorem 8.1.1 it follows that there exists

$$
v \in{ }_{0} B_{1-\alpha+\frac{1}{p^{\prime}}, p a r}^{p^{\prime}}(\partial \Omega \times(0, T))
$$

such that

$$
\left\{\begin{array}{l}
\left(\partial_{t}-\Delta\right) v=0, \\
\operatorname{Tr} v=h .
\end{array}\right.
$$

As in the proof of Theorem 8.1.1, it is not hard to prove that $\left\langle\partial_{\nu} u, h\right\rangle=0$.

Second, we will show that if $u \in{ }^{0} B_{\alpha+\frac{1}{p}, p a r}^{p}(\Omega \times(0, T))$ with $\left(\partial_{t}+\Delta\right) u=0$, $\operatorname{Tr} u=0$, and $\partial_{\nu} u=0$, then for any $(x, t) \in \Omega \times(0, T), u(x, t)=0$. Using the fact that

$u(x, t)=\int_{0}^{t} \int_{\mathbb{R}^{n}} E^{b}(x-y, t-s) f(y, s) d y d s \quad$ solves $\left(\partial_{t}+\Delta\right) u=f$ in $\mathbb{R}^{n} \times(0, \infty)$ where $E^{b}$ is the fundamental solution of the adjoint heat operator $\partial_{t}+\Delta$, we have a similar sequence of equalities as in (8.1.2) and (8.1.3), consequently, $u \equiv 0$. This finishes the proof of the theorem. 


\subsection{The Poisson problem with Neumann bound- ary conditions}

Theorem 8.2.1. Given a bounded Lipschitz domain $\Omega$ in $\mathbb{R}^{n}$, there exists some small constant $\varepsilon=\varepsilon(\partial \Omega)>0$ with the following significance. Let $0<T<\infty$ and assume that the indices $1<p<\infty$ and $0<\alpha<1$ are such that the point with coordinates

$\left(\alpha, 1-\frac{1}{p}\right)$ belongs to the interior of the hexagon $O A B C D E$. Then there exists a unique solution $u \in{ }_{0} B_{1-\alpha+\frac{1}{p}, \text { par }}^{p}(\Omega \times(0, T))$ to the problem

$$
(\mathrm{PN})\left\{\begin{array}{l}
\left(\partial_{t}-\Delta\right) u=\left.f\right|_{\Omega \times(0, T)}, f \in{ }_{z} B_{-1-\alpha+\frac{1}{p}, p a r}^{p}(\Omega \times(0, T)) \\
\partial_{\nu} u=g \in{ }_{0} B_{-\alpha, p a r}^{p}(\partial \Omega \times(0, T)) .
\end{array}\right.
$$

In addition, $u$ can be represented in terms of caloric layer potentials and

$$
\|u\|_{0 B_{1-\alpha+\frac{1}{p}, p a r}^{p}(\Omega \times(0, T))} \leq c\left(\|f\|_{z B_{-1-\alpha+\frac{1}{p}, p a r}^{p}(\Omega \times(0, T))}+\|g\|_{0 B_{-\alpha, p a r}^{p}(\partial \Omega \times(0, T))}\right) .
$$

Generally, $\partial_{\nu} u$ is understood as the normal derivative of $u$, relative to the extension $\operatorname{Ext}\left(\left(\partial_{t}-\Delta\right) u\right)$ of $\left(\partial_{t}-\Delta\right) u$. More details on this are given in Definition 7.2.3. In Theorem 8.2.1, $\partial_{\nu} u$ means the normal derivative of $u$, relative to the functional $\left(\partial_{t}-\Delta\right) u=f$

Proof. Existence and estimates. On account of the main results in Chapters 4-7, it is simple to check that

$$
u:=\Pi_{p a r} f+\mathcal{S}\left[\left(-\frac{1}{2} I+K^{\prime}\right)^{-1}\left(g-\partial_{\nu}\left(\Pi_{p a r} f, f\right)\right)\right]
$$

solves $(\mathrm{PN})$, it satisfies (8.2.5), and that $u \in{ }_{0} B_{1-\alpha+\frac{1}{p}, p a r}^{p}(\Omega \times(0, T))$.

Uniqueness. What we need to show is that $f \equiv 0$ and $g \equiv 0$ imply $u \equiv 0$. 
First, we will show that $u \in{ }_{0} B_{1-\alpha+\frac{1}{p}, p a r}^{p}(\Omega \times(0, T))$ with $\left(\partial_{t}-\Delta\right) u=0$ and $\partial_{\nu} u=0$ imply that $\operatorname{Tr} u=0$. In order to do so, observe that

$$
\operatorname{Tr} u \in{ }_{0} B_{1-\alpha, p a r}^{p}(\partial \Omega \times(0, T))=\left({ }^{0} B_{\alpha-1, p a r}^{p^{\prime}}(\partial \Omega \times(0, T))\right)^{*}
$$

and consider $h \in{ }^{0} B_{\alpha-1, p a r}^{p^{\prime}}(\partial \Omega \times(0, T))$. Then, from Theorem 8.2.2 (whose existence part can be proved independently of our theorem), we can find $v$ from the space ${ }^{0} B_{\alpha+\frac{1}{p^{\prime}}, p a r}^{p^{\prime}}(\Omega \times(0, T))$ such that

$$
\left\{\begin{array}{l}
\left(\partial_{t}+\Delta\right) v=0 \quad \text { in } \Omega \times(0, T) \\
\partial_{\nu} v=h \quad \text { on } \partial \Omega \times(0, T)
\end{array}\right.
$$

Moreover,

$$
\langle\operatorname{Tr} u, h\rangle=\left\langle\partial_{\nu} v, \operatorname{Tr} u\right\rangle=\int_{0}^{T} \int_{\Omega}\langle\nabla v, \nabla u\rangle d x d t-\int_{0}^{T} \int_{\Omega}\left\langle\partial_{t} v, u\right\rangle d x d t
$$

On the other hand,

$$
0=\left\langle\partial_{\nu} u, \operatorname{Tr} v\right\rangle=\int_{0}^{T} \int_{\Omega}\langle\nabla v, \nabla u\rangle d x d t+\int_{0}^{T} \int_{\Omega}\left\langle\partial_{t} u, v\right\rangle d x d t
$$

which, in concert with (8.2.6), implies that

$$
\begin{aligned}
\langle\operatorname{Tr} u, h\rangle & =-\int_{0}^{T} \int_{\Omega}\left[\left\langle\partial_{t} u, v\right\rangle+\left\langle\partial_{t} v, u\right\rangle\right] d x d t \\
& =-\int_{0}^{T} \int_{\Omega} \frac{d}{d t}\langle u, v\rangle d x d t=-\int_{\Omega}\left(\left.\langle u, v\rangle\right|_{t=0} ^{t=T}\right) d x=0 .
\end{aligned}
$$

Here we have used the fact that $u$ and $v$ vanish for $t=0$ and $t=T$, respectively.

Second, to show that $u \in{ }_{0} B_{1-\alpha+\frac{1}{p}, p a r}^{p}(\Omega \times(0, T))$ with $\left(\partial_{t}-\Delta\right) u=0, \partial_{\nu} u=0$, and $\operatorname{Tr} u=0$ imply $u \equiv 0$, one proceeds much as in the proof of Theorem 8.1.1.

The counterpart of Theorem 8.2.1 for the adjoint heat operator is given below. 
Theorem 8.2.2. Given a bounded Lipschitz domain $\Omega$ in $\mathbb{R}^{n}$, there exists $\varepsilon=$ $\varepsilon(\partial \Omega)>0$ such that if $0<T<\infty$ and $1<p<\infty, 0<\alpha<1$ with the property that $\left(\alpha, 1-\frac{1}{p}\right) \in$ interior of $O A B C D E$, then there exists a unique solution $u \in{ }^{0} B_{1-\alpha+\frac{1}{p}, \text { par }}^{p}(\Omega \times(0, T))$ to the problem

$$
(\mathrm{PN})^{*}\left\{\begin{array}{l}
\left(\partial_{t}+\Delta\right) u=\left.f\right|_{\Omega \times(0, T)}, f \in{ }^{z} B_{-1-\alpha+\frac{1}{p}, p a r}^{p}(\Omega \times(0, T)), \\
\partial_{\nu} u=g \in{ }^{0} B_{-\alpha, p a r}^{p}(\partial \Omega \times(0, T)) .
\end{array}\right.
$$

Furthermore, there is an integral reprsentation formula for the solution and

$$
\|u\|_{0_{B_{1-\alpha+\frac{1}{p}, p a r}^{p}}^{p}(\Omega \times(0, T))} \leq c\left(\|f\|_{B_{-1-\alpha+\frac{1}{p}, p a r}^{p}}(\Omega \times(0, T))+\|g\|_{B_{-\alpha, p a r}^{p}(\partial \Omega \times(0, T))}\right) .
$$

Proof. Existence and estimates. The main results of Chapters 4-7 imply that

$$
u:=\Pi_{p a r}^{b} f+R \circ \mathcal{S}\left[R \circ\left(-\frac{1}{2} I+K^{*}\right)^{-1}\left(g-\partial_{\nu}\left(\Pi_{p a r}^{b} f, f\right)\right)\right]
$$

solves $(\mathrm{PN})^{*}$, it satisfies (8.2.7), and $u \in{ }_{0} B_{1-\alpha+\frac{1}{p}, p a r}^{p}(\Omega \times(0, T))$.

Uniqueness. What we need to show is that $f \equiv 0$ and $g \equiv 0$ imply $u \equiv 0$.

First, we will show that $u \in{ }^{0} B_{1-\alpha+\frac{1}{p}, p a r}^{p}(\Omega \times(0, T))$ with $\left(\partial_{t}+\Delta\right) u=0$ and $\partial_{\nu} u=0$ imply $\operatorname{Tr} u=0$. Note that

$$
\operatorname{Tr} u \in{ }^{0} B_{1-\alpha, p a r}^{p}(\partial \Omega \times(0, T))=\left({ }_{0} B_{\alpha-1, p a r}^{p^{\prime}}(\partial \Omega \times(0, T))\right)^{*},
$$

and consider $h \in{ }_{0} B_{\alpha-1, p a r}^{p^{\prime}}(\partial \Omega \times(0, T))$. Then, employing Theorem 8.2.1, we can find $v$ from the space ${ }_{0} B_{\alpha+\frac{1}{p^{\prime}}, p a r}^{p^{\prime}}(\Omega \times(0, T))$ such that

$$
\left\{\begin{array}{l}
\left(\partial_{t}-\Delta\right) v=0 \quad \text { in } \Omega \times(0, T), \\
\partial_{\nu} v=h \quad \text { on } \partial \Omega \times(0, T) .
\end{array}\right.
$$

As in the proof of Theorem 8.2.1, we can show that $\operatorname{Tr} u=0$, using the facts that $u$ and $v$ vanish for $t=T$ and $t=0$, respectively. 
Second, to show that $u \in{ }^{0} B_{1-\alpha+\frac{1}{p}, p a r}^{p}(\Omega \times(0, T))$ with $\left(\partial_{t}+\Delta\right) u=0, \partial_{\nu} u=0$, and $\operatorname{Tr} u=0$ implies $u \equiv 0$, one proceeds much as in the proof of Theorem 8.1.2. This completes the proof of the theorem. 


\section{Bibliography}

[AlMü] E. Albrecht and V. Müller, Spectrum of interpolated operators, Proc. Amer. Math. Soc. 129 (2001), no. 3, 807-814.

[BeLö] J. Bergh and J. Löfstrom, Interpolation Spaces: An Introduction, SpringerVerlag, Berlin, 1976.

[BIN] O.Besov, V.A.Il'in and S.M. Nikol'skiǔ, Integral Representations of Functions and Imbedding Theorems I-II, Winston, Wiley, Washington D. C. 1979.

[Bow1] M. Bownik, Anisotropic Hardy spaces and wavelets, Mem. Amer. Math. Soc. 164 (2003) no. 781.

[Bow2] M.Bownik, Atomic and molecular decompositons of anisotropic Besov spaces, Math. Z. 250 (2005), no. 3, 539-571.

[BoHo] M. Bownik and K.-P.Ho, Atomic and molecular decompositons of anisotropic Triebel-Lizorkin spaces, Trans. Amer. Math. Soc. 358 (2006), 1469-1510.

[BrCe] M. Bramanti and M.C. Cerutti, Commutators of singular integrals on homogeneous spaces, Boll. Un. Mat. Ital. B(7) 10 (1996), no. 4, 843-883.

[Bro1] R.M. Brown, The method of layer potentials for the heat equation in Lipschitz cylinders, Amer. J. Math. 111 (1989), no. 2, 339-379.

[Bro2] R.M. Brown, The initial-Neumann problem for the heat equation in Lipschitz cylinders, Trans. Amer. Math. Soc. 320 (1990), no. 1, 1-52. 
[BrSh1] R. M. Brown and Z. Shen, Boundary value problems in Lipschitz cylinders for three-dimensional parabolic systems, Rev. Mat. Iberoamericana 8 (1992), no. 3, 271-303.

[BrSh2] R. M. Brown and Z. Shen, A note on boundary value problems for the heat equation in Lipschitz cylinders, Proc. Amer. Math. Soc. 119 (1993), no. 2, $585-594$.

[Cal1] A.P. Calderón, Intermediate spaces and interpolation, Studia Math. (Ser. Specjalna) Zeszyt, 1 (1963), 31-34.

[Cal2] A. P. Calderón, Intermediate spaces and interpolation, the complex method, Studia Math. 24 (1964), 113-190.

[Cal3] A.P. Calderón, An atomic decomposition of distributions in parabolic $H^{p}$ spaces, Advances in Math. 25 (1977), no. 3, 216-225.

[Cal4] A.P. Calderón, Cauchy integrals on Lipschitz curves and related operators, Proc. Nat. Acad. Sci. U.S.A. 74 (1977), no. 4, 1324-1327.

[CaTo1] A.P. Calderón and A. Torchinsky, Parabolic maximal functions associated with a distribution, Advances in Math. 16 (1975), 1-64.

[CaTo2] A.P. Calderón and A. Torchinsky, Parabolic maximal functions associated with a distribution II, Advances in Math. 24 (1977), no.2, 101-171.

[CKS] D.-C. Chang, S. G. Krantz and E. M. Stein, $H^{p}$ theory on a smooth domain in $\mathbb{R}^{N}$ and elliptic boundary value problems, J. Funct. Anal. 114 (1993), no. 2, 286-347.

[Chr] M. Christ, The extension problem for certain function spaces involving fractional orders of differentiability, Ark. Mat. 22 (1984), no. 1, 63-81.

[Coi] R. R. Coifman, A real variable characterization of $H^{p}$, Studia Math. 51 (1974), 269-274. 
[CoGu] R. R. Coifman and M.de Guzmán, Singular integrals and multipliers on homogeneour spaces, Rev. Un. Mat. Argentina, 25 (1970/71), 137-143.

[CMM] R. R. Coifman, A. McIntosh and Y. Meyer, L'intégrale de Cauchy définit un opérateur borné sur $L^{2}$ pour les courbes lipschitziennes, (French) Ann. of Math. (2) 116 (1982), no. 2, 361-387.

[CoWe1] R. R. Coifman and G. Weiss, Analyse harmonique non-commutative sur certains espaces homogènes.(French) Lecture Notes in Mathematics, Vol. 242, Springer-Verlag, Berlin-New York, 1971.

[CoWe2] R. R. Coifman and G. Weiss, Extensions of Hardy spaces and their use in analysis, Bull. Amer. Math. Soc. 83 (1977), no. 4, 569-645.

[Cwi] M. Cwikel, On $\left(L^{p_{0}}\left(A_{0}\right), L^{p_{1}}\left(A_{1}\right)\right)_{\theta, q}$, Proc. Amer. Math. Soc. 44 (1974), 286-292.

[Dac1] S. Dachkovski, The Q-method and regularity problems of some integral equations and PDE's, Ph.D. Thesis, Friedrich Schiller University of Jena, 2001.

[Dac2] S. Dachkovski, Anisotropic function spaces and related semi-linear hypoelliptic equations, Math. Nachr. 248/249 (2003), 40-61.

[DaKe] B. E. J. Dahlberg and C. E. Kenig, Hardy spaces and the Neumann problem in $L^{p}$ for Laplace's equation in Lipschitz domains, Ann. of Math. (2) 125 (1987), no. 3, 437-465.

[DaTr] H.Dappa and W. Trebels, On anisotropic Besov and Bessel potential spaces, Approximation and function spaces (Warsaw, 1986), Banach Center Publ. 22, PWN, Warsaw, 1989, 69-87.

[DGT-J] W. J. Davis, D. J. H. Garling and N. Tomczak-Jaegermann, The complex convexity of quasinormed linear spaces, J. Funct. Anal. 55 (1984), no. 1, $110-150$. 
[Den] D. G. Deng, Wavelet and function spaces on Lipschitz curves, Conference on Scientific Computation 1994 (Shatin, 1994), Southeast Asian Bull. Math. 20 (1996), no. 3, 131-136.

[DeV,Sh] R.A.DeVore, R. C. Sharpley, Maximal functions measuring smoothness, Mem. Amer. Math. Soc. 47 (1984), no. 293.

[Die] J. Diestel, Geometry of Banach spaces - selected topics, Lecture Notes in Mathematics, Vol. 485. Springer-Verlag, Berlin-New York, 1975.

[DiUh] J.Diestel and J.J. Uhl, Jr., Vector Measures, American Mathematical Society, Providence, R.I., 1977.

[EsMi] L. Escauriaza and M. Mitrea, Transmission problems and spectral theory for singular integral operators on Lipschitz domains, J. Funct. Anal. 216 (2004), no. 1, 141-171.

[Eva] L. C. Evans, Partial Differential Equations, Graduate Studies in Mathematics, 19, American Mathematical Society, Providence, RI, 1998.

[Fab1] E. Fabes, The initial value problem for parabolic equations with data in $L^{p}\left(\mathbb{R}^{n}\right)$, Collection of articles honoring the completion by Antoni Zygmund of 50 years of scientific activity, IV; Studia Math. 44 (1972), 389-409.

[Fab2] E. Fabes, Layer potential methods for boundary value problems on Lipschitz domains, Potential theory-surveys and problems (Prague, 1987), 55-80, Lecture Notes in Math., 1344, Springer, Berlin, 1988.

[FaJo] E. Fabes and M. Jodeit, Jr., $L^{p}$ boundary value problems for parabolic equations, Bull. Amer. Math. Soc. 74 (1968), 1098-1102.

[FJR] E. Fabes, M. Jodeit, Jr. and N. M. Rivièr, Potential techniques for boundary value problems on $C^{1}$-domains, Acta Math. 141 (1978), no. 3-4, 165-186.

[FLR] E. B. Fabes, J.E. Lewis and N. M. Rivière, Boundary value problems for the Navier-Stokes equations, Amer. J. Math. 99 (1977), no. 3, 626-668. 
[FMM] E. Fabes, O. Mendez and M. Mitrea, Boundary layers on Sobolev-Besov spaces and Poisson's equation for the Laplacian in Lipschitz domains, J. Funct. Anal. 159 (1998), 323-368.

[FaRi] E. B. Fabes and N. M. Rivièr, Dirichlet and Neumann problems for the heat equation in $C^{1}$-cylinders, Harmonic analysis in Euclidean spaces (Proc. Sympos. Pure Math., Williams Coll., Williamstown, Mass., 1978), Part 2, pp. 179-196, Proc. Sympos. Pure Math., XXXV, Part, Amer. Math. Soc., Providence, R.I., (1979), 179-196.

[FaSa] E. B. Fabes and C.Sadosky, Pointwise convergence for parabolic singular integrals, Studia Math. 26 (1966), 225-232.

[FaSa] E. Fabes and S. Salsa, Estimates of caloric measure and the initial-Dirichlet problem for the heat equation in Lipschitz cylinders, Trans. Amer. Math. Soc. 279 (1983), no. 2, 635-650.

[Far] W. Farkas, Atomic and subatomic decompositions in anisotropic function spaces, Math. Nachr. 209 (2000), 83-113.

[FJSc] W. Farkas, N. Jacob and R. L. Schilling, Function spaces related to continuous negative definite functions: $\psi$-Bessel potential spaces, Dissertationes Math. (Rozprawy Mat.) 393, 2001.

[FJSi] W. Farkas, J. Johnsen and W. Sickel, Traces of anisotropic Besov-LizorkinTriebel spaces - a complete treatment of the borderline cases, Math. Bohem. 125 (2000), no. 1, 1-37.

[FaLe] W. Farkas and H.-G. Leopold, Chatacterizations of function spaces of generalized smoothness, Ann. Mat. Pura Appl. (4) 185 (2006), no. 1, 1-62.

[FRS] C. Fefferman, N. M. Rivière and Y.Sagher, Interpolation between $H^{p}$ spaces: the real method, Trans. Amer. Math. Soc. 191 (1974), 75-81.

[FrJa1] M. Frazier and B. Jawerth, Decomposition of Besov spaces, Indiana Univ. Math. J. 34 (1985), 777-799. 
[FrJa2] M. Frazier and B. Jawerth, A discrete transform and decompositions of distribution spaces, J. Funct. Anal. 93, No. 1 (1990), 34-170.

[FJW] M. Frazier, B. Jawerth and G. Weiss, Littlewood-Paley theory and the study of function spaces, CBMS Regional Conference Series in Mathematics, 79 (1991), Amer. Math. Soc., Providence, RI.

[Fre] D. Freitag, Real interpolation of weighted $L_{p}$-spaces, Math. Nachr. 86 (1978), 15-18.

[Fri] A. Friedman, Partial Differential Equations of Parabolic Type, PrenticeHall, Inc., Englewood Cliffs, N.J. 1964.

[GC,RF] J. Garcia-Cuerva and J.Ł. Rubio de Francia, Weighted norm inequalities and related topics, North-Holland Mathematics Studies, 116, NorthHolland Publishing Co., Amsterdam, 1985.

[GHT1] G. Garrigós, R. Hochmuth and A. Tabacco, Wavelet characterizations for anisotropic Besov spaces: cases $0<p<1$, preprint.

[GHT2] G. Garrigós, R. Hochmuth and A. Tabacco, Wavelet characterizations for anisotropic Besov spaces with $0<p<1$, Proc. Edinb. Math. Soc. (2) 47 (2004), no. 3, 573-595.

[GaTa] G. Garrigós and A. Tabacco, Wavelet decompositions of anisotropic Besov spaces, Math. Nachr. 239/240 (2002), 80-102.

[Gol] D. Goldberg, A local version of real Hardy spaces, Duke Math. J. 46 (1979), 27-42.

[GrRa] A. Grimaldi Piro and F. Ragnedda, Classical boundary value problems for integrable temperatures in a $C^{1}$ domain, Ann. Polon. Math. 54 (1991), no. $1,29-44$.

[GRN1] A. Grimaldi Piro, F. Ragnedda and U. Neri, BMO continuity for some heat potentials, Rend. Sem. Mat. Univ. Padova 72 (1984), 289-305. 
[GRN2] A. Grimaldi Piro, F. Ragnedda and U. Neri, Invertibility of some heat potentials in BMO norms, Rend. Sem. Mat. Univ. Padova 75 (1986), 77-90.

[Gri1] P. Grisvard, Commutativité de deux foncteurs d'interpolation et applications (French), J. Math. Pures Appl. (9) 45 (1966), 143-206, 207-290.

[Gri2] P. Grisvard, Équations différentielles abstraites. (French) Ann. Sci. École Norm. Sup. (4) 2 (1969), 311-395.

[Gri3] P. Grisvard, Elliptic Problems in Nonsmooth Domains, Monographs and Studies in Mathematics, 24, Pitman (Advanced Publishing Program), Boston, MA, 1985.

[Gru1] G.Grubb, Parameter-elliptic and parabolic pseudodifferential boundary problems in global $L_{p}$ Sobolev spaces, Math. Z. 218 (1995), 43-90.

[Gru2] G. Grubb, Nonhomogeneous time-dependent Navier-Stokes problems in $L_{p}$ Sobolev spaces, Differential Integral Equations 8 (1995), no. 5, 1013-1046.

[Gru3] G.Grubb, Nonhomogeneous Dirichlet Navier-Stokes problems in low regularity L $L^{p}$ Sobolev spaces, J. Math. Fluid Mech. 3 (2001), no. 1, 57-81.

[GrKo] G. Grubb and N.J.Kokholm, A global calculus of parameter-dependent pseudodifferential boundary problems in $L^{p}$ Sobolev spaces, Acta Math. 171 (1993), no. 2, 165-229.

[HPW] Y.-S. Han, M.Paluszyński and G. Weiss, A new atomic decomposition for the Triebel-Lizorkin spaces, Harmonic analysis and operator theory (Caracas, 1994), 235-249, Contemp. Math. 189, Amer. Math. Soc., Providence, RI, 1995.

[Han] R. Hanks, Interpolation by the real method between BMO, $L^{\alpha}(0<\alpha<\infty)$ and $H^{\alpha}(0<\alpha<\infty)$, Indiana Univ. Math. J. 26 (1977), no. 4, 679-689.

[Hoc] R. Hochmuth, Wavelet characterizations for anisotropic Besov spaces, Appl. Comput. Harmon. Anal. 12, No. 2 (2002), 179-208. 
[HoLe1] S. Hofmann and J. Lewis, $L^{2}$ solvability and representation by caloric layer potentials in time-varying domains, Ann. of Math. (2) 144 (1996), no. 2, 349-420.

[HoLe2] S. Hofmann and J.Lewis, The $L^{p}$ regularity problem for the heat equation in non-cylindrical domains, Illinois J. Math. 43 (1999), no. 4, 752-769.

[HLM] S.Hofmann, J. Lewis and M. Mitrea, Spectral properties of parabolic layer potentials and transmission boundary value problems in nonsmooth domains, Illinois J. Math. 47 (2003), no. 4, 1345-1361.

[Hör] L. Hörmander, The Analysis of Linear Partial Differential Operators I, 2nd etition, Springer-Verlag, 1990.

[Joh] J.E. Johnsen, The stationary Navier-Stokes equations in $L_{p}$-related spaces, Ph.D. Thesis, Mathematics Institute, University of Copenhagen, Denmark, 1993.

[JoWa] A. Jonsson and H. Wallin, Function Spaces on Subsets of $\mathbb{R}^{n}$, Harwood Academic, New York, 1984.

[JeKe] D. Jerison and C.E. Kenig, The inhomogeneous Dirichlet problem in Lipschitz domains, J. Funct. Anal. 130 (1995), 161-219.

[Kal] N. J. Kalton, Plurisubharmonic functions on quasi-Banach spaces, Studia Math. 84 (1986), no. 3, 297-324.

[KaMi] N.J. Kalton and M. Mitrea, Stability results on interpolation scales of quasi-Banach spaces and applications, Trans. Amer. Math. Soc. 350 (1998), no. 10, 3903-3922.

[KPR] N. J. Kalton, N. T. Peck and J. Roberts, An F-space sampler, London Mat. Soc. Lecture Notes 89, Cambridge University Press, 1984.

[KrMi] N. Krugljak and M. Milman, A distance between orbits that controls commutators estimates and invertibility of operators, Adv. Math. 182 (2004), no. 1, 78-123. 
[LSU] O. A. Ladyženskaja, V.A.Solonnikov and N. N. Ural'ceva, Linear and quasilinear equations of parabolic type, (Russian) Translated from the Russian by S. Smith. Translations of Mathematical Monographs, Vol. 23, American Mathematical Society, Providence, R.I. 1967.

[Las] R. Lascar, Propagation des singularités des solutions d'équations pseudodifférentielles quasi homogènes, (French) Ann. Inst. Fourier (Grenoble) 27 (1977), no. 2, 79-123.

[Lat] R.H. Latter, A characterization of $H^{p}\left(\mathbb{R}^{n}\right)$ in terms of atoms, Studia Math. 62 (1978), no. 1, 93-101.

[LaUc] R. H. Latter and A. Uchiyama, The atomic decomposition for parabolic $H^{p}$ spaces, Trans. Amer. Math. Soc. 253 (1979), 391-398.

[Leo1] H.-G. Leopold, Boundedness of anisotropic pseudodifferential operators in function spaces of Besov-Hardy-Sobolev type, Z. Anal. Anwendungen, 5 (1986), no. 5, 409-417.

[Leo2] H.-G. Leopold, Interpolation of Besov spaces of variable order of differentiation, Arch. Math. (Basel) 53 (1989), no. 2, 178-187.

[Leo3] H.-G.Leopold, On function spaces of variable order of differentiation, Forum Math. 3 (1991), no. 1, 1-21.

[LiMa] J.L. Lions and E. Magenes, Non-homogeneous boundary value problems and applications I-III, Springer-Verlag, 1972.

[LiPe] J. L. Lions and J. Peetre, Sur une classe d'espaces d'interpolation, (French) Inst. Hautes Études Sci. Publ. Math., No. 19 (1964), 5-68.

[Liz1] P.I.Lizorkin, Nonisotropic Bessel potentials. Imbedding theorems for the Sobolev space $L_{p}^{\left(r_{1}, \cdots, r_{n}\right)}$ with fractional derivatives, (Russian) Dokl. Akad. Nauk SSSR, 170, (1966) 508-511. 
[Liz2] P. I. Lizorkin, Multipliers of Fourier integrals in the spaces $L_{p, \theta}$, (Russian) Trudy Mat. Inst. Steklov, 89 (1967), 231-248.

[MeMi] O. Mendez and M. Mitrea, The Banach envelopes of Besov and TriebelLizorkin spaces and applications to partial differential equations, J. Fourier Anal. Appl. 6 (2000), 503-531.

[Mih] S. G. Mihlin, On the multipliers of Fourier integrals, (Russian) Dokl. Akad. Nauk SSSR (N.S.) 109 (1956), 701-703.

[MMP] D. Mitrea, M. Mitrea and J.Pipher, Vector potential theory on nonsmooth domains in $\mathbb{R}^{3}$ and applications to electromagnetic scattering, J. Fourier Anal. Appl. 3 (1997), no. 2, 131-192.

[Mit1] M. Mitrea, Initial boundary-value problems for the parabolic Maxwell system in Lipschitz cylinders, Indiana Univ. Math. J. 44 (1995), no.3, 797-813.

[Mit2] M. Mitrea, The initial Dirichlet boundary value problem for general second order parabolic systems in nonsmooth manifolds, Commun. in Partial Differential Equations 26 (11 \& 12), 2001, 1975-2036.

[Mit3] M. Mitrea, Envelopes of Hardy and Sobolev spaces, preprint.

[MiTa1] M. Mitrea and M.E. Taylor, Boundary layer methods for Lipschitz domains in Riemannian manifolds, J. Funct. Anal. 163 (1999), no. 2, 181-251.

[MiTa2] M. Mitrea and M.E. Taylor, Potential theory on Lipschitz domains in Riemannian manifolds: Sobolev-Besov space results and the Poisson problem, J. Funct. Anal. 176 (2000), no.1, 1-79.

[MiTa3] M. Mitrea and M.E. Taylor, Potential theory on Lipschitz domains in Riemannian manifolds: $L^{p}$ Hardy, and Hölder space results, Commun. Anal. Geom. 9 (2001), no. 2, 369-421.

[MiTa4] M. Mitrea, M. Taylor, Sobolev and Besov space estimates for solutions to second order PDE on Lipschitz domains in manifolds with Dini or Hlder continuous metric tensors, Comm. Partial Differential Equations 30 
(2005), no. 1-3, 1-37.

[Nik] S.M. Nikol'skiü, Approximation of Functions of Several Variables and Imbedding Theorems (Russian), "Nauka", Moscow, 1977, Translated from the Russian by John M. Danskin, Jr. Die Grundlehren der Mathematischen Wissenschaften, Band 205, Springer-Verlag, New York-Heidelberg, 1975.

[Peet] J. Peetre, A theory of interpolation of normed spaces, Notas de Matemática, No. 39, Instituto de Matemática Pura e Aplicada, Conselho Nacional de Pesquisas, Rio de Janeiro, 1968.

[Riv] N. M. Rivière, Singular integrals and multiplier operators, Ark. Mat. 9 (1971), 243-278.

[RiSa] N. M. Rivière and Y.Sagher, Interpolation between $L^{\infty}$ and $H^{1}$, the real method, J. Functional Analysis 14 (1973), 401-409.

[RuSi] T.Runst and W.Sickel, Sobolev spaces of fractional order, Nemytskij operators, and nonlinear partial differential equations, de Gruyter Series in Nonlinear Analysis and Applications, 3, Berlin, 1996.

[Sag] Y.Sagher, Interpolation of some analytic families of operators, Function spaces and applications (Lund, 1986), 378-383, Lecture Notes in Math., 1302, Springer, Berlin, 1988.

[Sch] H.-J.Schmeisser, Anisotropic spaces. II. Equivalent norms for abstract spaces, function spaces with weights of Sobolev-Besov type, Math. Nachr. 79 (1977), 55-73.

[ScTr] H.-J.Schmeisser and H. Triebel, Topics in Fourier Analysis and Function Spaces, A Wiley-Interscience Publication. Chichester, 1987.

[See] A. Seeger, A note on Triebel-Lizorkin spaces, Banach Center Publ. 22 (1989), 391-400.

[She1] Z.Shen, Layer potentials and boundary value problems for parabolic Lamé systems of elasticity and a nonstationary linearized system of 
Navier-Stokes equations in Lipschitz cilinders, Ph.D. Thesis, University of Chicago, 1989.

[She2] Z.Shen, Boundary value problems for parabolic Lamé systems and a nonstationary linearized system of Navier-Stokes equations in Lipschitz cilinders, Amer. J. Math. 113 (1991), no.2, 293-373.

[Ste1] E. M.Stein, Singular Integrals and Differentiability Properties of Functions, Princeton Univ. Press, Princeton, NJ, 1970.

[Ste2] E. M.Stein, Topics in Harmonic Analysis Related to the Littlewood-Paley Theory, Ann. of Math. Studies, No. 63, Princeton Univ. Press, Princeton, NJ; Univ. of Tokyo Press, Tokyo, 1970.

[SeWe] E. M.Stein and G. Weiss, Introduction to Fourier Analysis on Euclidean Spaces, Princeton Univ. Press, Princeton, NJ, 1971.

[Stö1] B.Stöckert, Remarks on anisotropic function spaces, Fourier analysis and approximation theory (Proc. Colloq., Budapest, 1976), Vol. II, 789-797, Colloq. Math. Soc. János Bolyai, 19, North-Holland, Amsterdam-New York, 1978.

[Stö2] B. Stöckert, Remarks on the interpolation of anisotropic spaces of BesovHardy-Sobolev type, Czechoslovak Math. J. 32 (107), 1982, no. 2, 233-244.

[StTr] B. Stöckert and H. Triebel, Decomposition methods for function spaces of $B_{p, q}^{s}$ type and $F_{p, q}^{s}$ type, Math. Nachr. 89 (1979), 247-267.

[Tay] M.E. Taylor, Partial differential equations I-III, Applied Mathematical Sciences, 115-117, Springer-Verlag, New York, 1996-1997.

[Tri1] H. Triebel, Fourier Analysis and Function Spaces, Taubner, Leipzig, 1977.

[Tri2] H. Triebel, Interpolation Theory, Function Spaces, Differential Operators, Second edition, Johann Ambrosius Barth, Heidelberg, 1995. 
[Tri3] H. Triebel, Complex interpolation and Fourier multipliers for the spaces $B_{p, q}^{s}$ and $F_{p, q}^{s}$ of Besov-Hardy-Sobolev type: the case $0<p \leq \infty$, $0<q \leq \infty$, Math. Z. 176 (1981), no. 4, 495-510.

[Tri4] H. Triebel, Theory of Function Spaces, Birkhäuser Verlag, 1983.

[Tri5] H. Triebel, Function spaces in Lipschitz domains and on Lipschitz manifolds. Characteristic functions as pointwise multipliers, Rev. Mat. Complut. 15 (2002), no. 2, 475-524.

[Yam] M. Yamazaki, A quazi-homogeneous version of paradifferential operators, I. Boundedness on spaces of Besov type, J. Fac. Sci. Univ. Tokyo Sect. IA Math. 33 (1986), no. 1, 131-174.

[Yos] K. Yosida, Functional Analysis, 6th edition, Springer-Verlag, New York, 1980.

[Ver1] G. Verchota, Layer potentials and boundary value problems for Laplace's equation in Lipschitz domains, Ph.D. Thesis, University of Minnesota, 1982.

[Ver2] G. Verchota, Layer potentials and regularity for the Dirichlet problem for Laplace's equation in Lipschitz domains, J. Funct. Anal. 59 (1984), no. 3, $572-611$.

[Zaf] M. Zafran, Spectral theory and interpolation of operators, J. Funct. Anal. 36 (1980), no. 2, 185-204. 


\section{Index}

$B_{\alpha, p a r}^{p, q}\left(\mathbb{R}^{n} \times \mathbb{R}\right), 77$

${ }_{0} B_{ \pm \alpha, p a r}^{p, q}(\Omega \times(0, T)), 217$

${ }_{0} B_{ \pm \alpha, p a r}^{p, q}(\partial \Omega \times(0, T)), 239$

${ }_{z} B_{\alpha, p a r}^{p, q}(\Omega \times(0, T)), 218$

$\square B_{\alpha, p a r}^{p, q}(\Omega \times(0, T)), 226$

atomic decomposition, 97, 113, 186, 189

Bessel potential space, 100

duality, 195, 196, 200, 213, 220, 246

extension oparator, 45, 57, 144, 148, 155,

247, 250, 257, 261, 264, 267, 282

Fubini property, 24, 91, 102, 141, 166

fundamental solution, 18

lifting operator, 85, 86, 88, 90

negative smoothness, 157, 159

parabolic Hölder space, 93

parabolic norm, 14 


\section{VITA}

Tunde Jakab was born on January 28, 1974 in Miercurea-Ciuc, Romania. After she attended the Marton Aron high school with Mathematics and Physics profile, she graduated from the Babes-Bolyai University in Cluj-Napoca, Romania, receiving a BS, then a MS degree in Mathematics. In 2001 she started her graduate studies in the Mathematics Department at the University of Missouri-Columbia under the guidance of Professor Marius Mitrea, and defended her dissertation in May 2006. 